- fe 


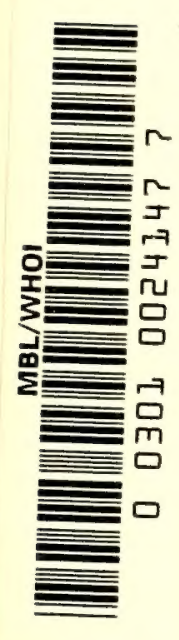





\section{“Handbook}

of

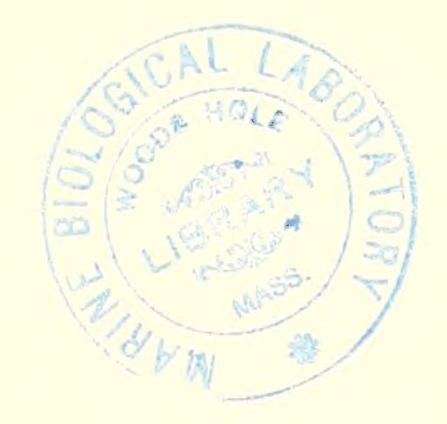

\section{Nuclear Technology"}

Summary of

the useful techniques

for the

APPLICATION OF

ATOMIC ENERGY

FOR PEACEFUL PURPOSES

Reprinted from and copyrighted by

NUCLEONICS

A McGRAW-HILL PUBLICATION

330 WEST 42ND STREET

NEW YORK 36, N. Y. 


\section{CONTENTS}

The Los Alamos Human Counter ........... 1

Glass Dosimetry .................. 5

Counting Suspensions in Liquid Scintillators .... 11

Simple Instrumentation Determines Several

Simultaneous Radioactivities .............

Fast-Neutron-Insensitive Chemical

Gamma-Ray Dosimeter ................

High-Efficiency Slow-Neutron

Scintillation Counters

Instrumentation and Controls for HRT .........

The Role of Digital Computers in Nuclear Design

$\mathrm{BF}_{3}$ Neutron Spark Counter ................

Operating Characteristics of the Spark Counter

A First Look at Fusion-Reactor Control .......

Data-Handling Stem for Nuclear-Power Tests . .

All-Transistor Cif uits for Portable Detectors ....

Zirconium - What is its Future? .............

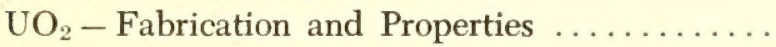

Cetting PWR on the Line ................

Research Reactors - Selection and Operation ...

How to Calculate Gamma Radiation

Induced in Reactor Materials

How Radiation Affects Materials

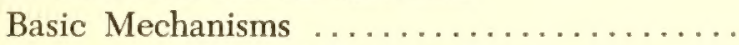

Engineering Use of Damage Data .........

Damaging Effects of Radiation -

On Solid Reactor Materials ..................

On Electronic Components ...............

On Plastics and Elastomers ............. 96

On Chemical Materials .................. 101

The Role of Critical Facilities

Critical Facilities for Reactor Design ........ 108

Critical Facilities for Basic Physics ........... 110

Critical Facilities Tomorrow .............. 111

NUCLEONICS Survey of Operating Critical

Facilities in the U. S.

Testing Nuclear-Plant Leaktightness

Pyrophoricity - A Technical Mystery

Under Vigorous Attack ..................

Fast Breeder Power Reactors -

Their Problems and Prospects

Economics of Waste Disposal .

Gamma-Irradiation Facilities

in the United States

Survey of Gamma Facilities

in U. S. and Canada - an Updating .........

Radioisotopic High-Potential,

Low-Current Sources

Beta-Ray-Excited Low-Energy X-Ray Sources ... .

Electron-Beam Sterilization of

Surgical Sutures

Radiogold Seeds for Cancer Therapy

Wear Studies of Irradiated Carbide

Cutting Tools

151
Using Neutrons for Remote Liquid-Level Gaging

Using Tracers in Refinery Control ............

Radioactive Tracers for Tagging

Special Steel Melts .

The Best Performance from Beta Gages .......

How Radiation Affects Long-Chain Polymers ....

Radioactive-Tracer Techniques

in Paper Chromatography ..............

Irradiated Males Eliminate Screwworm Flies ....

Tritium Tracing - A Rediscovery ............

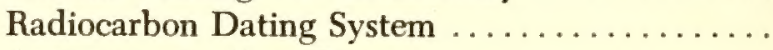

Activation Analysis Determines

Sodium Content of Aluminum Alloys ........

Nomogram for Radioisotope Buildup and Decay .

$\mathrm{X}$-Ray Critical-Absorption and

Emission Energies in kev

Gamma Rays from Thermal-Neutron Capture

Beta Emitters by Energy and Half-Life .......

Disaster Monitoring with Amateur and

Commercial Photographic Films ..........

Simple Spot Tests for Aluminum Contaminants ..

Tenth-Value Thicknesses for

Gamma-Ray Absorption

Fission-Neutron Reaction Cross Sections ........

Gamma-Ray Attenuation .................

Slow-Neutron Capture Radioisotopes

Arranged by Half-Life ...................

Stress-Strain Curves for

Reactor-Irradiated Plastics ............... 216

Weight Change in Cylindrical Shields ........ 218

Locating Compton Edges and Backscatter Peaks

in Scintillation Spectra ..................

Gamma-Ray Scattering from Thin Scatterers ....

Gamma-Ray Streaming Through an Annulus ....

Gamma Dose Rates in Cylindrical Sources ......

Gamma Attenuation with Buildup

in Lead and Iron

Radiation from Neutron-Activated

Slabs and Spheres ....................

Gamma-Ray Streaming Through a Duct ........

Gamma-Detection Efficiency of

Organic Phosphors ...................

Radionuclides Arranged by Gamma-Ray Energy

Nomogram for Radioactivity Induced

in Irradiation .........................

Thermal-Neutron Data for the Elements ........

Fission-Product Yields from $\mathrm{U}$, Th and $\mathrm{Pu}$......

Attenuation of $\gamma$-Rays from an Infinite Plane ....

Gamma-Ray Attenuation with Buildup in Water . .

Fission-Neutron Cross Sections for

Threshold Reactions ................ 246

X-Ray Production with Linear Accelerators ..... 248

Shielding-Glass Buildup Factors ............

Properties of Zircaloy-2 .................

155

158

162

165

169

176

182

185

190

196

199

200

202

204

206

208

210

211

212

214

219

220

222

223

224

226

228

229

230

232

233

236

244

245

144

149 


\section{The Los Alamos}

Human Counter

Take 140 gallons of liquid scintillator, scan it with 108 photomultipliers, shield it with 20 tons of lead . . . then you've got the essentials of a counter that will determine the gamma activity of body potassium to $5 \%$ in 100 second

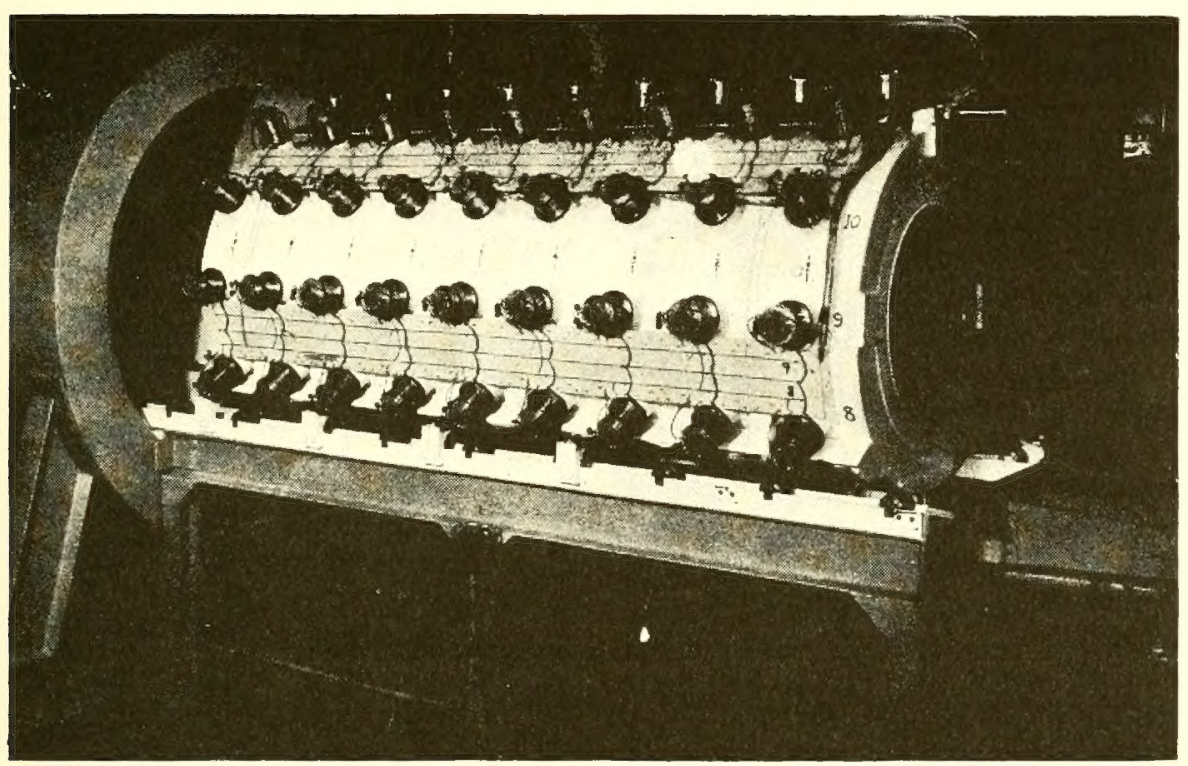

BY ERNEST C. ANDERSON, ROBERT L. SCHUCH, JAMES D. PERRINGS, and WRIGHT H. LANGHAM

Los Alamos Scientific Laboratory

University of California

Los Alamos, New Mexico

FiG. 1. Counter outside of lead shield

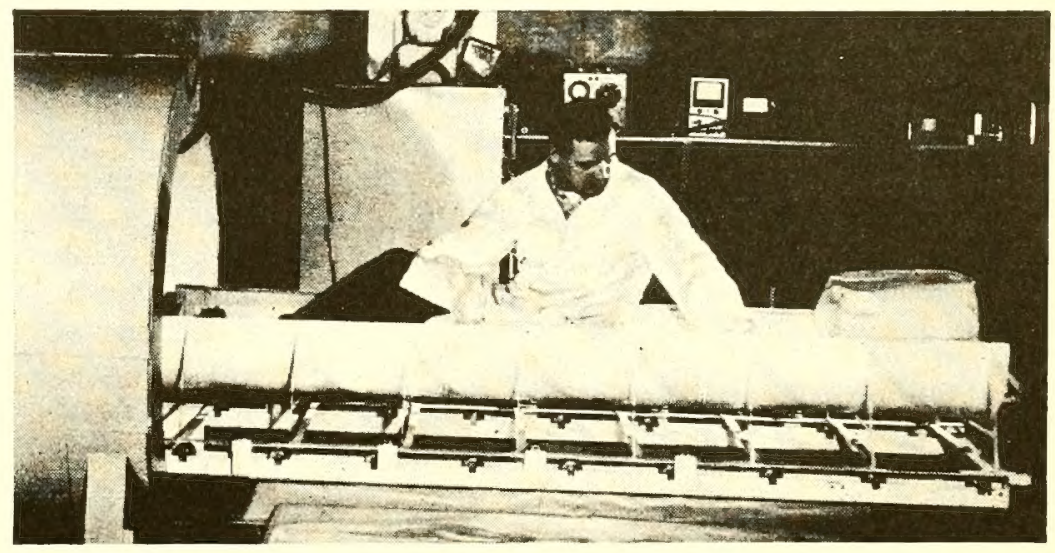

FIG. 2. General view of counter showing lead shield, loading trough, and subject about to lie down in sling that will carry him into counter 
The PRospective great InCREase in the use of nuclear reactors and radiation sources will increase the need for rapid and highly sensitive methods of monitoring for radioactivity. As more and more people are potentially exposed to contamination, genetic considerations indicate lower levels for the maximum permissible exposure. There appears to be a growing need of an instrument capable of detecting radioactivity in human beings and in foodstuffs at levels well below the recommended maximum permissible concentrations (1).

The Human Counter was designed to meet these requirements for gammaemitting nuclides. The sensitivity of the detector is $5 \times 10^{-10} \mathrm{c}$ for a nuclide emitting one gamma ray per disintegration and its sample capacity is $300 \mathrm{lbs}$. For large samples, concentrations of gamma emitters as low as $3 \times 10^{-12}$ $\mathrm{c} / \mathrm{kg}$ can be detected. The sensitivity is such that the natural $\mathrm{K}^{40}$ content of the body (about $10^{-8}$ gamma-ray curies) can be measured to a precision of 5 percent in two minutes of counting time.

\section{Description}

The Los Alamos Human Counter is essentially an enlarged and improved version of the $4 \pi$ gamma-ray detector ("dog counter") previously described (2), and of the original human counter which was a temporary modification of a neutrino detector (3). It consists of a cylindrical tank 72 in. in length by 30 in. in diameter, with an 18-in. diameter thin-walled tube (0.019-in. stainless steel) running down the axis (Fig. 1). The annular space between the tube and tank wall is filled with the scintillation solution $4 \mathrm{gm} / 1$ terphenyl and $0.1 \mathrm{gm} / 1$ 1,4-di[2-(5-phenyloxazolyl)]-benzene (POPOP) dissolved in reagent-grade toluene. The capacity of the detector is $140 \mathrm{gal}$ (about $1,000 \mathrm{lb}$ ). The inside of the tank is painted with a special reflective coating of anatase $\mathrm{TiO}_{2}$ in an epoxy resin that withstands the attack of the solution. *

Scintillations are detected by 108 photomultiplier tubes (DuMont 6292) inserted through the outer wall of the tank in 12 rows of 9 tubes each. The central cavity accommodates the subject or the sample within the sensitive shell so that the effective counting

* Glidden's Zopaque SD in Shell Chemicals Epon Formulation XA-200. angle is nearly $4 \pi$ and the counting efficiency can be made nearly independent of source position. The detector is enclosed in a 5-in.-thick 20-ton lead shield. Figure 1 shows the detector rolled out of the shield for servicing. It is mounted on heavy rails and ball-bearing rollers, so that one man can easily move it. Figure 2 shows how the subject is put into the detector. A metal trough is placed on the track on which the tank rolls so that it forms an extension of the central cavity. The subject lies down in a canvas sling which is drawn from the trough into the detector by means of a windlass.

Tubes are mounted as in the K-9 dog counter (2). Each tube views the solution through a glass window so that tubes can be changed without draining the tank. The window is cemented into the end of a steel tube with epon resin. An O-ring seal provides a tight joint between this tube and another welded into the tank wall.

Figure 3 is a schematic diagram of the method of wiring the photomultipliers. The bleeder circuits, one of which is shown, are conventional using $470-\mathrm{k}$ resistors between all stages. The last three dynodes are by-passed by $5,000-\mu \mu \mathrm{f}$ capacitors for stability at high rates. The shield is kept halfway between the cathode and first-dynode potentials. The tubes are balanced by means of an additional resistor $R$ in series with the bleeder string. This resistor adjusts the voltage drop per stage so that the output pulses of all tubes are equal for events of the same energy. An unequal voltage drop results between the anodes and tenth dynode, but the tube characteristic is independent of this voltage over a very wide range (4).

To eliminate paralleled anode load resistors and output capacitors, the anodes are tied together at the highvoltage-supply potential. (A positive supply is used to prevent spurious pulses which arise through the glass envelope when the cathode is operated at a high negative potential.) Provision is made for dividing the tubes into two banks, a convenience in testing and a necessity when counting very soft gammas. Coincidences can then be observed between the two banks to eliminate tube noise. Tube noise and spurious after-pulses are negligible for events with energies above $1 \mathrm{Mev}$, so that single-channel operation is used

\section{Background Determinations}

\begin{tabular}{ccc}
\hline $\begin{array}{c}\text { Time of } \\
\text { day }\end{array}$ & Background (cps) & $\begin{array}{c}\text { Deviation } \\
\text { from } \\
\text { average }\end{array}$ \\
\hline $12: 10$ & $447.7 \pm 2.0$ & $+2.0 \sigma$ \\
$12: 50$ & 443.3 & -0.2 \\
& 438.8 & -2.5 \\
$13: 20$ & 441.4 & -1.2 \\
& 445.2 & +0.7 \\
& 444.3 & -2.1 \\
$15: 20$ & 439.5 & +0.7 \\
$18: 30$ & 445.2 & +1.2 \\
& 446.2 & +1.0 \\
& 445.8 & $(+3.4)$ \\
& 444.9 & $(+2.7)$ \\
\cline { 2 - 3 } $20: 30$ & 450.6 & $(+4.2)$ \\
$21: 00$ & 449.1 & 452.3 \\
Average: 443.8 (excluding last three \\
\end{tabular}

in this case. The tubes in a given row are alternated between the two channels. Each channel has a single 10Meg load resistor for its entire 54 tubes and one output capacitor of $500-\mu \mu \mathrm{f}$ capacity.

The electronic circuits used to amplify, analyze and record the pulses are conventional (5). The energy resolution of the detector is sufficient to permit considerable background reduction by proper pulse-height selection, so that independently variable upper and lower discriminators are used in each channel. In addition to the scaler used for actual measurements, a precision rate meter driving a stripchart recorder is in operation at all times. While the rate meter is not accurate enough for a precise activity determination (which requires a precision of 2 eps in 400), it can be used to detect nonstatistical fluctuations in the average rate due to electrical interference, slow drifts in the electronics, etc. In practice, the apparatus has been shown to be stable to $0.5 \%$ of the background rate over periods of several hours. Because of the high counting rates (background $400 \mathrm{cps}$, average person $50 \mathrm{cps}$ of $\mathrm{K}^{40}$ ), statistical accuracy is obtained in very short times. A 100 -sec count on the average subject will determine his activity to a statistical precision of better than $5 \%$, so that long-term stability is not an essential requirement.

The table on this page shows the 
results of 14 determinations of background (100-sec counts) made during the course of a single day's operation during which time 65 subjects were measured. The last three determinations, while apparently self-consistent, are significantly higher than the previous sequence. The average of the first 11 measurements, extending over a period of six hours, is $443.8 \mathrm{cps}$. As can be seen from the last column of the table, the scatter of the individual measurements around this average appears to be statistical.

A more detailed description of the mechanical features of the counter design will be found in the report LA1717 (5).

\section{Body Potassium Content}

The Human Counter has been used to make a preliminary study of the variation of the potassium content of people as a function of their body weight and age. Potassium is naturally radioactive, the isotope responsible for the activity being $K^{40}$, abundance $0.01 \%$, half-life $1.25 \times 10^{9}$ yr (6). The decay is to $\mathrm{Ca}^{40}$ by emission of a $1.4-\mathrm{Mev}$ beta particle or to $\mathrm{A}^{40}$ by electron capture. In the latter branch of the decay scheme, a 1.45Mev gamma ray is emitted. It is this gamma ray which is used to measure the potassium. The gamma emission rate of ordinary potassium is $2.96 \mathrm{dps} /$ gm (6). Since the average person contains about $150 \mathrm{gm}$ of potassium $(7)$, the gamma emission rate from this source is expected to be about $450 \mathrm{dps}$.
At $10 \%$ efficiency, one would expect 45 cps from the potassium in the average human body.

Since potassium is largely concentrated in muscle, very little being found in bone and fat, potassium content might be expected to vary appreciably with body type. Measurements were therefore made on a group of people of both sexes whose ages varied from 4 years to 75 years, Most of the group had no occupational connection with radioactive materials. The group was "random" to the extent that it represented visitors to the Health Research Laboratory during the July, 1955 open house at Los Alamos Scientific Laboratory.

Two 100-sec counts were made on each subject and backgrounds were measured periodically throughout the day. The ratemeter trace was used as a monitor against short-term background drift. The gross counting rate observed from the subjects was usually slightly below the background with the detector empty because of soft radiation entering the open end of the shield and absorbed by the subject. (This end will be closed with a 2 -in. lead door to eliminate this effect.) Correction was made for background depression using the results of the measurement of a series of sugar phantoms of various weights. The precision of the determinations is believed to be \pm 6 cps or better in all cases.

The results of this study are shown in Fig. 4. In spite of the considerable scatter of the data, certain trends

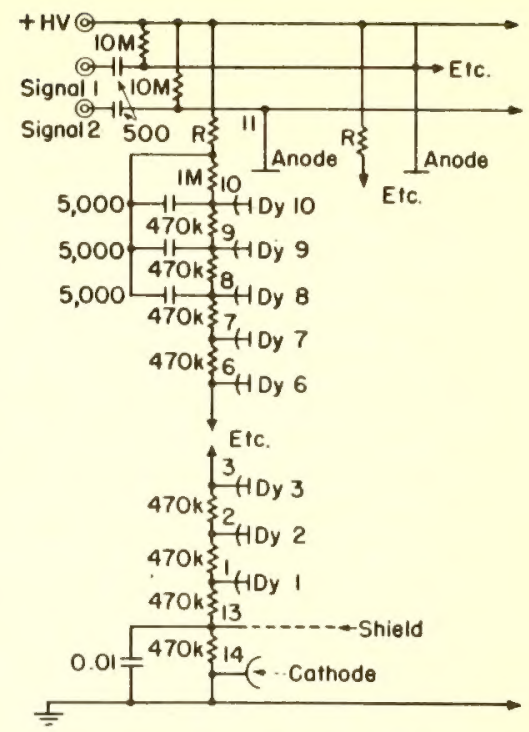

FIG. 3. Schematic of photomultiplier circuit

are evident. The group of points in the lower left portion of the graph represent children. The scatter here is probably within the statistical precision of the measurement, and may reflect a certain uniformity of body build among these subjects. There is no significant difference between girls and boys. The adult points seem to scatter downwards from the extrapolated line passing through the points for children, presumably representing the addition of fat to the basic frame. Note that the average activity of the women is significantly below that of the men and that the range of variation among adults (more than a factor of 2)

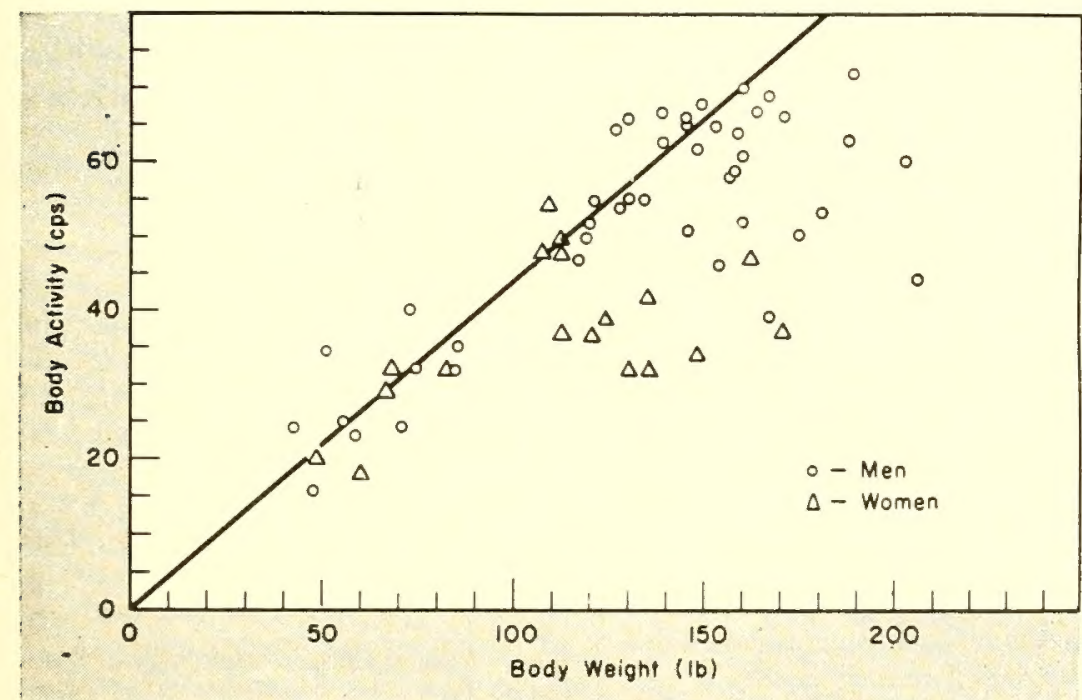

\section{Assaying Reactor Accidents}

The Human Counter could be used to determine the body dose resulting from overexposure to thermal neutrons. The neutrons activate body sodium to an extent proportional to the dose received. It is estimated that an acute exposure to thermal neutrons, resulting in a dose of $0.3 \mathrm{rep}$, would produce enough $\mathrm{Na}^{24}$ activity to be measured with this detector.

This application, which could be of interest in nuclear power plants, was pointed out to us by Dr. Austin Brues.

FIG. 4. Gamma activity of human subjects as function of body weight 
is definitely larger than experimental error. The range seems in agreement with previous results $(8-10)$. This analysis of the nature of the variations is of course a very superficial one, and a further study of the correlation of potassium activity with other parameters of the body is planned. (Deviations from the norm for natural potassium content of the body arise in certain diseases, as for example, muscular dystrophy. See the discussion at right of jobs for the human counter.)

The counting efficiency of $\mathrm{KCl}$ in a sugar phantom was found to be $8.7 \%$, compared with $10 \%$ for $\mathrm{KCl}$ alone. This indicates $13 \%$ self-absorption for the average human. The average potassium content of men, $61 \mathrm{cps}$, therefore corresponds to about $200 \mathrm{gm}$ of potassium and the women's average, $51 \mathrm{cps}$, indicates $170 \mathrm{gm}$. These numbers are larger than the value of $150 \mathrm{~g}$ given by Shohl $(\gamma)$ and the value of $140 \mathrm{~g}$ recommended by the International Commission on Radiological Protection (11); but in view of the large scatter, the difference is of little significance.

We are deeply indebted to the Los Alamos shop department for their collaboration in the design and construction. In particular, B. H. Pohlmann and R. I. Howes did an excellent job of solving the many mechanical problems involved. The whole project would have been impossible without the support and encouragement of Darol Froman. F. N. Hayes prepared the scintillation solution and I. H. Larkins assisted in the design. The work was done under the auspices of the U.S. Atomic Energy Commission.

\section{BIBLIOGRAPHY}

1. Handbook 52, National Bureau of Standards Washington, D. C., 1953

2. M. A. Van Dilla, R. L. Schuch, E. C. Anderson, Nucleonics 12, No. 9, 22 (1954)

3. C. L. Cowan, F. Reines, F. B. Harrison, E. C. Anderson, F. N. Hayes, Phys. Rev. 90, 493 (1953)

4. B. R. Linden, Nucleonics 11, No. 9, 30 (1953)

6. E. C. Anderson, R. L. Schuch, J. D. Perrings, LA-1717, (1955)

6. A. D. Suttle, Jr., W, F, Libby, Anal, Chem. 27, 921 (1955)

7. A. T. Shohl, "Mineral Metabolism," (Reinhold Publishing Corp., New York, 1939)

8. I. D. P. Wootton, M. D. Milne, E. J. King, Ann. Rev. Biochem. 23, 441 (1954)

9. R. M. Sievert, Ark. f. Fysik 3, 337 (1951)

10. F. Reines, et al., Nature 172, 521 (1953)

11. Brit. J Radiology, Supplement 6, Recom mendations of the International Commission on Radiological Protection (1955)

12. Private communication, M. A. Van Dilla, Department of Radiobiology, University of Utah

19. A. F. Stehney, W, P. Norris, H. F. Lucas, W. H. Johnston, A $m, J$. Roentgenol. 73, 774 (1955)

14. L. D. Marinelli, C. E. Miller, P. F. Gustafson, R. E. Rowland, $A m$. J. Roentgenol, 73, 661 (1955)

15. L. D. Marinelli, C. E. Miller, R. E. Rowland, J. E. Rose, Radiology 64, 116 (1955)

\section{Jobs for the Human Counter}

These five major research programs are planned for the Human Counter:

\section{Monitoring of Personnel and Foodstuffs}

The extreme sensitivity of the instrument should make it valuable for the study of possible gamma contamination from nuclear reactors, uranium mining operations, and bomb fallout. The fact that gamma activity can be detected rapidly and easily at levels below the maximum permissible concentrations means that contamination can be detected before it reaches dangerous levels, in time for remedial measures to be taken. For the monitoring of foodstuffs, forages, etc., the sensitivity, as mentioned above, is $5 \times 10^{-10} \mathrm{e}$ in samples up to $300 \mathrm{lb}$. in weight. For the monitoring of personnel, uncertainty in the natural $\mathrm{K}^{40}$ level is the limiting factor. If this uncertainty is $50 \%$ (a pessimistic estimate), the limit of detection is $5 \%$ of the permissible amount for $\mathrm{Ra}^{226}$ in equilibrium with its daughters, $3 \%$ for $\mathrm{I}^{131}$, and $0.01 \%$ for $\mathrm{Na}^{24}$ and $\mathrm{Mo}^{99}$. If the $\mathrm{K}^{40}$ can be predicted to $5 \%$ (by an isotope dilution measurement with $\mathrm{K}^{42}$ or by a correlation such as discussed below), the sensitivity is tenfold greater. This sensitivity is attainable with counting times of two minutes per sample.

\section{Studies of Body Potassium Content}

The reasons for the large variation of potassium from person to person are of considerable intrinsic interest from the viewpoint of physiological chemistry. Correlation of potassium content with body type, total body water, lean body weight, or similar parameters weuld provide a useful method of calculating the "normal" potassium value for an individual. An independent method of estimating body potassium to $5 \%$ accuracy would permit the ten-fold increase in sensitivity to other gamma emitters mentioned in the previous section.

Deviations from the norm for natural potassium content of the body are of clinical interest in certain diseases, as for example, muscular dystrophy.

\section{Human Retention of Gamma-Emitting Fission Products}

The sensitivity of the instrument is such as to permit these measurements to be made on human volunteers with amounts of material far below the permissible level. In conjunction with small animal counters similar to the Human Counter, a series of fission-product retention measurements is planned using a number of different species including mice, rats, dogs, monkeys, and men. Such a series should aid in the interpretation of animal retention studies and their extrapolation to man.

\section{Counting Beta Emitters by Bremsstrahlung}

Van Dilla, using the "dog counter" at the University of Utah, has measured large amounts of $\mathrm{Sr}^{89}$ in the dog by means of the bremsstrahlung (12). Attempts will be made to extend downward the energy range of radiations that can be detected, in the hope of obtaining better efficiency for the bremsstrahlung. The detection of bremsstrahlung will make possible measurements of such important fission products as the strontium isotopes. A very rough theoretical estimate indicates that it may be possible to reach at least the maximum permissible level of $\mathrm{Sr}^{90}$.

\section{Natural Body Gamma Activities}

Radium studies, such as those being made at the Argonne National Laboratory (13), are of considerable interest because of their bearing on the fundamental problem of the maximum permissible radiation level for man. The success of the method in this direction will depend on the establishment of an independent method for accurate potassium measurement or on the development of a coincidence technique to distinguish radium and potassium. The energy discrimination of the instrument appears to be inadequate to separate the two profitably. The limit is $5 \times 10^{-9} \mathrm{e}$ of (equilibrium) $\mathrm{Ra}$ if potassium is not independently estimated, or $5 \times 10^{-10} \mathrm{c}$ if the potassium is known to $5 \%$, assuming $2 \mathrm{~min}$ of counting time per subject. The Argonne NaI crystal counter $(14,15)$ has a reported sensitivity of $3 \times 10^{-10} \mathrm{c}$ of $\mathrm{Ra}$ (equilibrium ?) for $2 \mathrm{hr}$ of counting time and does not suffer from $\mathrm{K}^{\mathbf{4 0}}$ interference. 
FIG. 1. Arrangement for removing radiation-induced darkening of glasses by hecting

\title{
Glass Dosimetry
}

\author{
Here's a thorough study of silver-phosphate glass as a dosimeter: the effects of \\ storage temperature, how postirradiation heat treatment stabilizes fading, the \\ effect of freezing temperatures during irradiation, and other parameters
}

Production-LINE STERILIZATION of foods, drugs, and biological" materials by ionizing radiations grows increasingly probable as technical problems are solved and the number of applications increases. It is a practical and legal necessity to measure doses accurately in these radiation processes, but most of the existing dosimetric systems, which are based on ionization or calorimetric principles, do not easily lend themselves to rapid, routine, and accurate measurements.

Investigations at the Naval Research Laboratory by Schulman and associates $(1,2,3,12)$ and here at MIT (4, 13) have indicated that the silveractivated phosphate glass, originally developed at NRL $(5,6,7)$ to measure low personnel doses, has good potentialities for measuring high doses. In the measurement of high doses the optical absorption is used rather than the fluorescence properties used at the low doses.

The use of glass as a dosimeter has several advantages that make it especially attractive for use in this field. These include chemical inertness, ri-

* This paper summarizes work done by the Department of Food Technology, Mass. Inst. of Technology, on A.E.C. Contract AT(30-1)-1164 (4, 13). gidity, insolubility, small size, and permanence. A thorough investigation of those properties of silver-activated phosphate glass of importance in highlevel dosimetry is reported here.

\section{Apparatus and Methods}

The glass used was Corning \#9761. The composition is that originally specified at $\mathrm{NRL}$, namely, $50 \% \mathrm{AI}\left(\mathrm{PO}_{3}\right)_{3}$,
$25 \% \mathrm{Ba}\left(\mathrm{PO}_{3}\right)_{2}$, and $25 \% \mathrm{KPO}_{3}$, with about $8 \% \mathrm{AgPO}_{3}$ added.

Sources. The source of gamma radiation used was a multicurie $\mathrm{Co}^{60}$ source (10) with a dose rate of $\sim 1,400$ $\mathrm{rep} / \mathrm{min}$. The doses used were based upon experiments and calculations employing physical dosimetric methods and the ferrous-ferric dosimeter (10). The specific yield of the ferrous-ferric

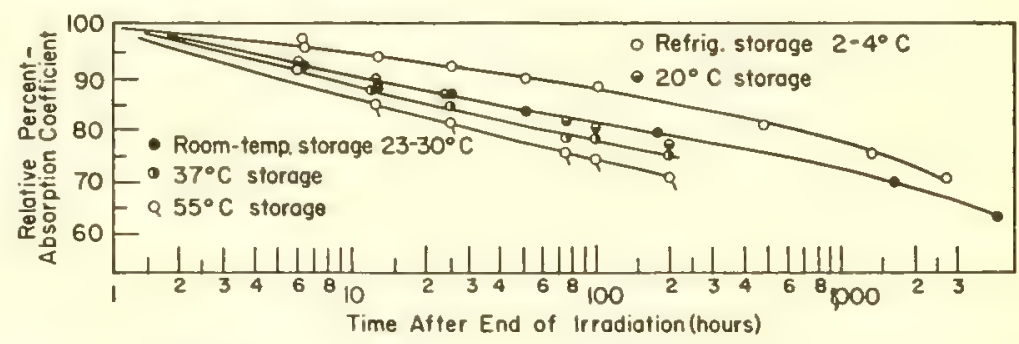

FIG. 2. Effect of storage temperature on irradiated silver-activated phosphate glasses not given heat treatment

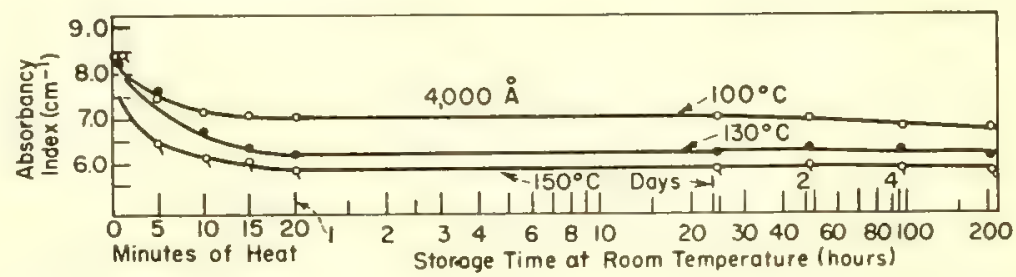

FIG. 3. Effect of heating temperature on irradiated glasses stored at room temperatures 
reaction was taken as $15.4 \mu \mathrm{M} \mathrm{Fe}^{+++} /$ liter/1,000 rep.

A Van de Graaff accelerator was used as a source of $3-\mathrm{Mev}$ electrons in some experiments. ${ }^{*}$ Doses were calculated from meter readings of current and voltage in accordance with a previous method (11).

Irradiation. When the $\mathrm{Co}^{60}$ source was used, three to six replicate glasses were used in each determination, the glasses being stacked horizontally one on top of another in a cylindrical glass ampule (diameter, $1 / 2$ in.; height, $11 / 4$ in.) with stopper. All ampules were filled with water and irradiated in fixed positions in special sample holders, to match geometry and absorber characteristics to those used in source calibration.

Measurements. Optical density was measured in a Beckman DU spectrophotometer with photomultiplier attachment. A special metal sample holder with four matched apertures to accommodate three glasses and a "blank" was used. Aperture dimensions were $0.80 \times 0.80 \mathrm{~cm}$. The "blank" aperture is used as the $100 \%$ transmission reference; the absorbancy index $\dagger$ is calculated by dividing the

* We wish to express our appreciation to J. G. Trump and K. A. Wright of the Department of Electrical Engineering, Mass. Inst. of Technology for their continuing cooperation in making available the facilities of the Van de Graaff accelerator and for calculation of the electron doses.

t The absorbancy index (AI) is related to the absorption coefficient (AC) by the factor $\log _{\text {s }} 10=2.3$. Thus, $A C=2.3 \times A I$. observed optical density by the measured thickness of the glass. Slit widths used were $0.4-0.5 \mathrm{~mm}$, and each glass was measured at four wavelengths: $3,500,4,000,4,500$, and 5,000 $\AA$. [Where data are shown only for $4,000 \AA$ see NYO-3345 (19) for more complete results.]

The absorbancy index reported here is the AI of the irradiated glass minus the AI of the nonirradiated glass.

Figure 1 shows the arrangement for erasing glasses. A Coors porcelain pipette rest (glazed except bottom) with thermocouple attached holds the glasses while in the oven and while cooling.

\section{Experimental Results}

The characteristics that follow were studied with a view toward practical utilization. The nature of these studies was also indicated by earlier results obtained by Schulman et al. $(1,3)$. More complete data are contained in NYO-3345 (13).

1. Effect of storage temperature on irradiated glasses not given heat treatment. Experiments were conducted to determine the effect of storage temperature on fading characteristics of glasses irradiated with a gamma-ray dose of $1.30 \times 10^{5}$ rep. Six glasses were stored at each of five temperatures in ${ }^{\circ} \mathrm{C}$ : room temperature $\left(23-30^{\circ}\right)$, refrigerator temperature (2$\left.4^{\circ}\right), 20^{\circ}, 37^{\circ}$, and $55^{\circ}$. No differences are evident between $20^{\circ}, 37^{\circ}$ and room temperature for about the first week of storage with the exception of the

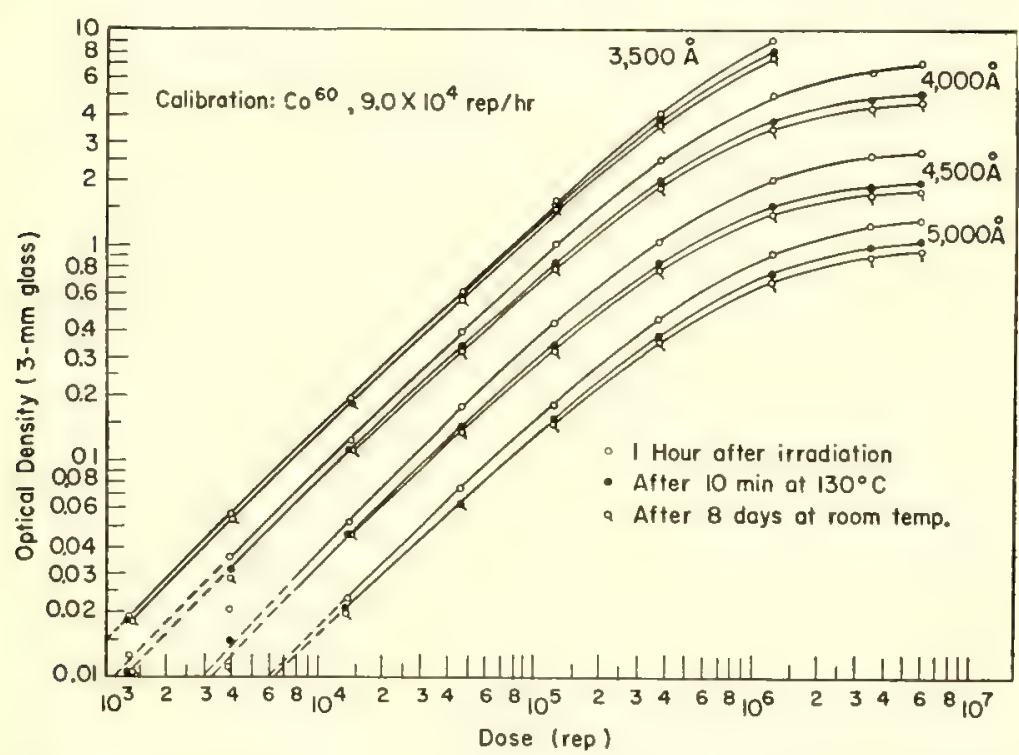

FIG. 4. Calibration of silver-activated phosphate glass irradiated with $\mathrm{Co}^{60}$ gamma rays: measured before heating, after heat freatment for $10 \mathrm{~min}$ at $130^{\circ} \mathrm{C}$, and after 8-day storage at room temperature
Probable Percentage Limits of Error*

\begin{tabular}{ccc}
$\begin{array}{c}\text { Wave- } \\
\text { length } \\
(\AA)\end{array}$ & $\begin{array}{c}\text { One glass } \\
(\%)\end{array}$ & $\begin{array}{c}\text { Mean of three glasses } \\
(\%)\end{array}$ \\
\hline 3,500 & \pm 3.3 & \pm 1.9 \\
4,000 & \pm 4.2 & \pm 2.4 \\
4,500 & \pm 4.7 & \pm 2.7 \\
5,000 & \pm 5.4 & \pm 3.1
\end{tabular}

* For Corning 9761 glass on the basis of 48 irradiations at $\sim 1.30 \times 10^{6}$ rep. Probability level is $99 \%$.

$4,000 \AA$ measurements where a small difference was noted for the $37^{\circ}$ temperature as compared to $20^{\circ}$ and room temperature (see Fig. 2).

Appreciable differences are apparent for the refrigerator and $55^{\circ}$ temperatures as compared with the other three temperatures. Thus extremes in storage temperature should be avoided. Cold and hot storage temperature retard and accelerate fading rates, respectively, of glasses not given a stabilizing heat treatment.

2. Effect of heat treatment after irradiation. Glasses irradiated with a gamma-ray dose of $3.70 \times 10^{5}$ rep were heated, $60 \mathrm{~min}$ after irradiation, to temperatures of $130^{\circ}$ and $150^{\circ} \mathrm{C}$. (Temperatures were achieved with a small precision furnace and measured by a thermocouple placed close to the glasses.) Glasses were also heated to $100^{\circ} \mathrm{C}$ in boiling water. Figure 3 presents the absorbancy indexes observed (1) immediately after heating for 5,10 , 15 , and $20 \mathrm{~min}$ at the different room temperatures and (2) after 1, 2, 4, and 8 days of storage at room temperature. The data at each temperature indicate that:

-The light absorption decreases with time and temperature of heating.

-The curves begin to flatten out after ten min of heating.

- There is little additional decrease in light absorption after about 15 or $20 \mathrm{~min}$ of heating at the three temperatures.

- The fading at room temperature is reduced considerably after the heating process, decreasing from about $8 \%$ after eight days in the $100^{\circ}-\mathrm{C}$ experiment to about $4 \%$ after eight days in the $150^{\circ}$-C experiment $(4,000 \AA)$.

These experiments indicate the usefulness of heating the glass to reduce the rate of fading after irradiation, although heating does not eliminate all 


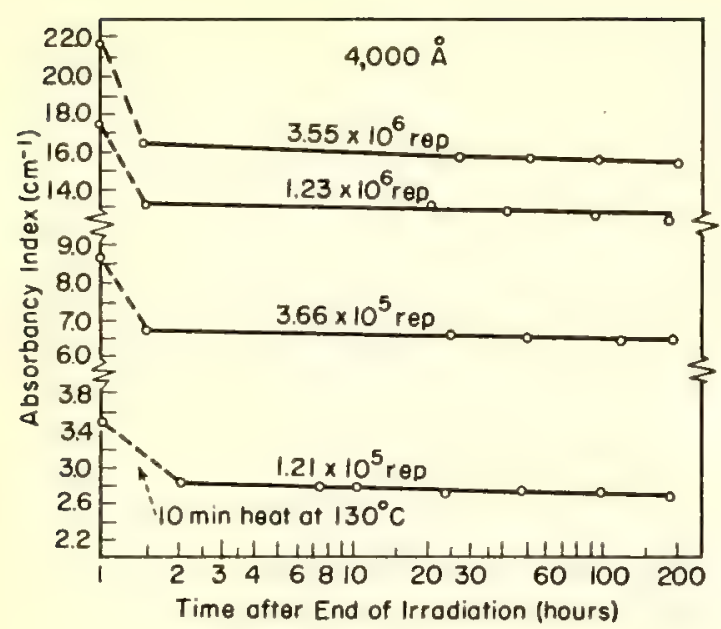

FIG. 5. Fading characteristics of silver-activated phosphate glass given different doses, heated for $10 \mathrm{~min}$ at $130^{\circ} \mathrm{C}$, and stored at roomt emperature

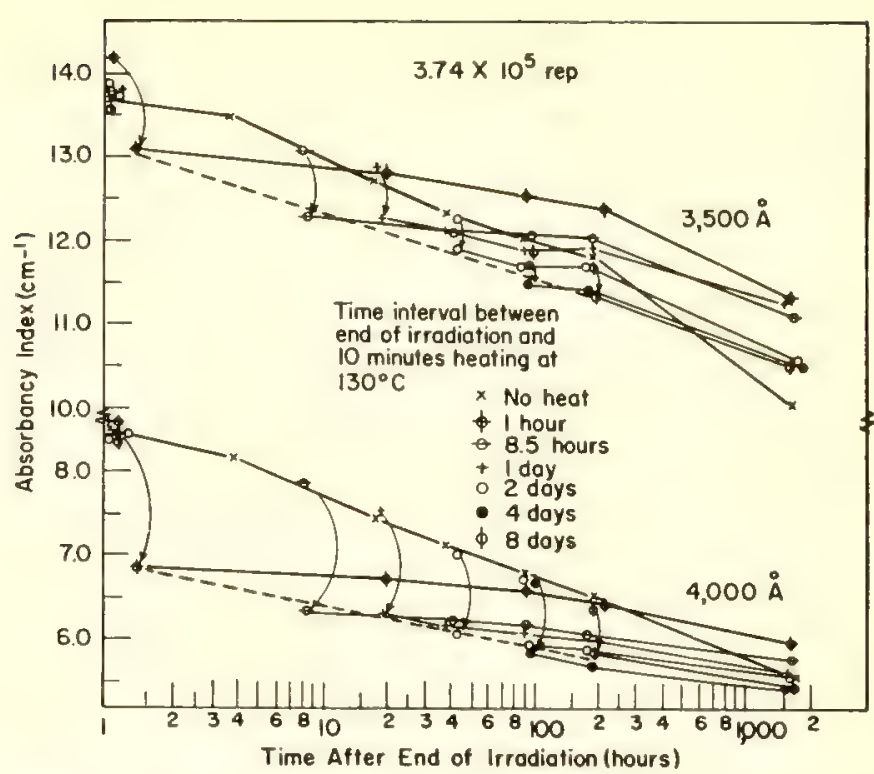

FIG. 6. Effect of heating glasses for $10 \mathrm{~min}$ at $130^{\circ} \mathrm{C}$, after different time intervals at room temperature following dose of $3.74 \times$ $10^{5}$ rep fading in glass of this particular composition.

3. Effect of storage temperature on glasses given heat treatment. A 10 min post-irradiation heating at $130^{\circ} \mathrm{C}$ has been used to minimize the effect of storage temperature such as observed in Fig. 2.

Three groups of three glasses each were irradiated with a gamma-ray dose of $3.66 \times 10^{5}$ rep. One hour after irradiation the glasses were measured and then heated for $10 \mathrm{~min}$ at $130^{\circ} \mathrm{C}$. They were then measured again. One group was stored in a refrigerator (2$4^{\circ} \mathrm{C}$ ), one at room temperature (23$26^{\circ} \mathrm{C}$ ), and the third in an oven maintained at $43^{\circ} \mathrm{C}$. Measurements were taken during an 8-day period on the three groups.

The data show no significant difference with storage temperature (except at the eighth-day measurement when a small lowering in readings for the glasses held at $43^{\circ} \mathrm{C}$ was detected). Thus an additional virtue of heat treatment after irradiation is the considerable decrease of the dependence upon storage temperature of readings made long after irradiation.

4. Calibration curves. Figure 4 shows calibration curves at four wavelengths from data obtained by irradiating glasses (six at each dose level) with gamma-ray doses ranging from about $1.3 \times 10^{3}$ rep to $7 \times 10^{6}$ rep. Sixty min after irradiation, these glasses were heated for $10 \mathrm{~min}$ at $130^{\circ} \mathrm{C}$. An extra minute was allowed for the glasses to reach the desired temperature after insertion into the oven. Following irradiation, measurements were taken before heating, within $30 \mathrm{~min}$ after heating, and after 8 days of storage at room temperature. Each point shown in Fig. 4 is the average of six measurements.

It is apparent that the slopes of the curves decrease appreciably above $\sim 3 \times 10^{5}$ rep. Consequently, sensitivity of the glass in this region is reduced. Precise experimental techniques and reproducibility of glass characteristics become increasingly important to obtain accurate results.

The percentage of fading, both during the heating period and during the 8-day storage period, decreases significantly with lower doses. In fact, when the dose is below $4 \times 10^{4} \mathrm{rep}$, fading becomes almost negligible during the 8-day period after heat treatment.

5. Fading of glasses given heat treatment after different doses. Figure 5 shows measurements of the absorbancy index at 4,000 $\AA$ observed after four different doses. The measurements were taken 1 hour after irradiation, after $10 \mathrm{~min}$ of heating at $130^{\circ} \mathrm{C}$, and at various times up to the eighth day of room-temperature storage.

A fairly good approximation to the fading for the first 8 days after irradiation is given by the equation shown above

$$
\mathrm{AI}=a \ln t+b
$$

where AI is the absorbancy index observed at a time $t$ after irradiation, and $a$ and $b$ are functions of the dose and wavelength, but are constant for a given dose and wavelength.*

6. Effect of heating glasses after different postirradiation waiting periods. In experiments described thus far heat treatment was used 1-11/2 hours after irradiation. In practice heat treatment may not be convenient if it is necessary to apply it so soon after irradiation. A series of experiments was, therefore, conducted to study the behavior of the glass when it was heated after different time intervals following irradiation.

Twenty-one glasses were irradiated with a dose of $3.74 \times 10^{5}$ rep. The glasses were separated into seven groups of three each, and all were measured one hour after the irradiation. Over a period of 8 days, each of six groups was given a 10 -min heat treatment at $130^{\circ} \mathrm{C}$, at different times after irradiation, including 8.5 hours, $1,2,4$, and 8 days, and about 2 months. The seventh group was allowed to fade at room temperature without heat treatment. All the glasses were measured at various times after heat treatment up to the eighth day following irradiation, including measurements im-

* Measurements taken at 3,500, 4,500, and $5,000 \AA$ show the same linearity (13). 
mediately following the heat treatment.

Figure 6 presents the absorbancyindex values obtained as a function of time, and includes previous data where heat treatment was made one hour after irradiation. It is evident that immediately following heat treatment the absorbancy indexes drop to values approximately linear (dashed curve) with respect to the logarithm of the time, for all the time intervals between irradiation and heating (from 1 hour to 8 days) that were tested.

However, subsequent to the initial measurement after the heat treatment the absorbancy indexes of a given group of glasses have, in general, a different linear relationship with respect to time. Fading following heat treatment proceeds at a lower rate than that shown by the curve based on measurements taken immediately following heat treatment applied at different intervals.

This would seem to suggest that the heat treatment not only eliminates some of the more unstable centers but alters the stability of some of the remaining centers so as to reduce their probability of fading. This is verified by noting that the absorbancy indexes of glasses not given heat treatment, in several cases, fall below glasses given heat treatment.

These results indicate that if heat treatment is to be applied at different time intervals following irradiation, one should not correct for the time interval by utilizing data on fading following heat treatment one hour after irradiation. Rather, it would be preferable to employ actual curves obtained by heating glasses after different time intervals. These curves appear to be fairly good linear plots with the logarithm of the time, so that measurements after two widely spaced time intervals such as one hour and one week will yield data sufficient for interpolations within this range.

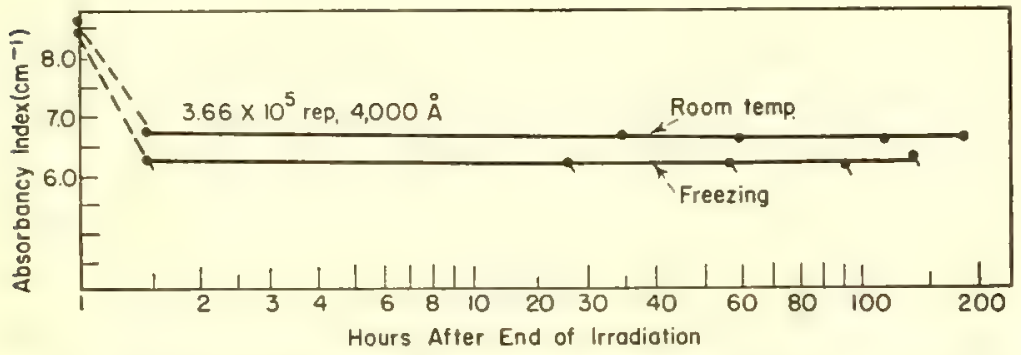

FIG. 7. Fading characteristics of glass given dose of $3.66 \times 10^{5}$ rep at two different temperatures, heat-treated for $10 \mathrm{~min}$ at $130^{\circ} \mathrm{C}$, and stored at room temperature
7. Effect of freezing temperature during irradiation. Sometimes irradiation at temperatures below $0^{\circ} \mathrm{C}$ is a means of minimizing some of the undesirable chemical and physical changes produced by radiation. Accordingly studies were initiated on the dosimetry of materials frozen while being irradiated.

Figure 7 shows the results of an experiment where two groups of glasses were given a dose of $3.66 \times 10^{5}$ rep, heated for 10 minutes at $130^{\circ} \mathrm{C}$, then stored at room temperature. One group of glasses was frozen in water during the irradiation whereas the other was irradiated at room temperature. Significant differences are apparent, with the glasses irradiated at the freezing temperature showing consistently lower readings.

The conclusion is that the silveractivated phosphate glass dosimeter can be used for frozen materials if a correction factor is applied to calibration charts constructed from results of irradiation at room temperature. Alternatively, calibration charts should be constructed from data obtained from glasses irradiated at freezing temperatures.

\section{High-temperature Erasure}

Preliminary experiments showed that exposed glasses may be "erased" (i.e., the coloration caused by irradiation removed) by application of $400-$ $500^{\circ} \mathrm{C}$ temperatures. Further experiments were conducted to determine how the sensitivity of the glass varies with repeated erasures and how many repeated erasures are possible.

Fifteen minutes at $450^{\circ} \mathrm{C}$ was found sufficient to erase these glasses after doses up to $5 \times 10^{6}$ rep.

The results obtained after five successive exposure-erasure cycles, where the dose in each exposure was $1.24 \times$ $10^{5}$ rep and the glasses were measured one hour after irradiation, are given in NYO-3345 (13). No definite increase or decrease in sensitivity of the glasses with repeated exposure-erasure cycles is observed.

A series of ten successive exposureerasure cycles was conducted in the high-dose region, doses being chosen randomly in the region of $2.5-50 \times 10^{5}$ rep. The average values for a group of three glasses are plotted on curves determined for nonerased glass from the same lot (Fig. 8). The points on the curves are marked to show the sequence of the exposure-erasure cycles. It is evident that the glass behavior remained essentially constant even after ten successive erasure-exposure cycles. Furthermore, the fading behavior observed in cycles 8,9 , and 10 remained unchanged from that of the original glass.

It seems that these glasses can be reused after erasing at high temperature; this reuse will add considerably to the value of the glass as a dosimeter.

\section{How Precise?}

Because of the large number of glasses whose optical densities were measured under identical conditions in this investigation, it has been possible to compile sufficient data for statistical analysis of the reproducibility characteristies of Corning 9761.

The table on p. 35 presents probable percentage limits of error determined from a total of 48 glasses irradiated under nearly identical conditions* over a period of two months. These glasses were all from the same lot and all received a dose of about $1.30 \times 10^{5}$ rep.

Optical density measurements on nineteen non-irradiated glasses from the same lot were also analyzed statistically. The ranges of values calculated at a $99.7 \%$ probability level were typically $0.045 \pm 0.007$ at $4,000 \AA$.

It may be concluded that no appreciable increase in precision is obtained if individual glasses are measured before irradiation with doses of $10^{5}$ rep or greater. The mean values of a few nonirradiated glasses from the same lot will suffice for the preirradiation absorption corrections. However, at the lower dose levels, where the preirradiation readings represent a larger percentage of the postirradiation read-

* Slight differences in dose due to length of irradiation or source decay were compensated by correction of the optical readings before statistical analysis. 
ings, individual preirradiation measurements are advisable.

\section{Fading Corrections}

We have demonstrated that fading occurs after irradiation, whether the glass is heated or not (although subsequent fading is reduced appreciably by heat treatment). The possibility of applying corrections to the glass measurements to compensate for fading is evident if a relationship between the rate of fading and the time after irradiation can be developed.

The nomogram in Fig. 9 provides a simple method for utilizing Eq. 1. The left-hand scale is a logarithmic time scale extending from 1 hour to 1 year.
The change of optical density (fading change) between 0 and 8 days after irradiation corresponds to the area between the curve in Fig. 4 for readings after $10 \mathrm{~min}$ of heating and the curve for readings after 8 days of storage.

The right-hand scale is graduated in linear units with unity corresponding to the 8-day period.

\section{Electron-beam Dosimetry}

Work by this laboratory (reported in the article (3) by Schulman, Klick, and Rabin) has shown the feasibility of using silver-activated phosphate glass for dosimetry 3-Mev electron beams. Since the intensity of ionization in matter falls off much more rapidly for electrons than for gamma rays* it is of importance to determine the effect of placement of the glass when it is used as a dosimeter for electron beams, and the possible effect of variation of ionization density in the glass itself because of its appreciable thickness compared with the maximum range of the electron beam.

A previous calibration of the silver-activated phosphate glass dosimeter is needed for interpretation of the changes in absorption after receiving doses of high-energy electrons. The $\mathrm{Co}^{60}$ gamma-ray calibration of the glass, Fig. 5 , can be used for this purpose. However, the relative duration of irradiation must be taken into account. For example, the Van de Graaff accelerator used in this work, delivers a $10^{6}$-rep dose in about $10 \mathrm{sec}$ whereas the $\mathrm{Co}^{60}$ source, used for calibration, delivers the same dose of gamma rays in about 12 hours. The difference in fading during the two irradiations must be taken into account. This has been done in these experiments by exposing more than one set of glasses to the same dose of electrons and measuring them at two different time intervals after irradiation.

Two stacks were assembled, each consisting of seven glasses of approximately $0.12-\mathrm{cm}$ thickness one on top of the other. The stacks were secured with Scotch tape.

* The effective range for electrons is about $0.5 \mathrm{~cm}$. for each Mev of energy in a substance of unit density.

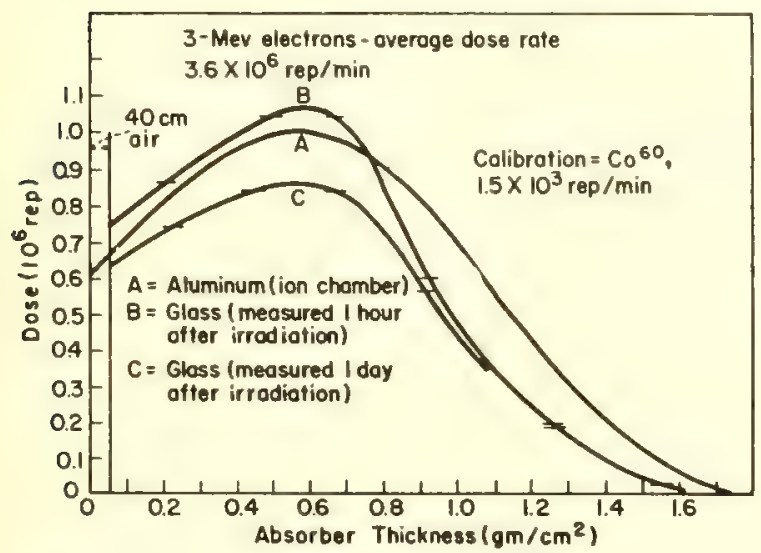

DEPTH-DOSE CURVES in glass, as defermined by selfdosimetry, compared with that in aluminum (11)
They were then irradiated with the surtaces of the glasses perpendicular to the direction of the beam. The energy of the electrons was $3 \mathrm{Mev}$ and the beam current was adjusted to deliver a dose of $10^{6}$ rep in the region of maximum ionization density (at an average dose rate of $3.6 \times 10^{6}$ $\mathrm{rep} / \mathrm{min}$ ). After irradiation, the glasses were given heat treatment of $10 \mathrm{~min}$ at $130^{\circ} \mathrm{C}$, group " $\mathrm{B}$ " receiving it one hour after irradiation and group " $\mathrm{C}$ " receiving it one day after irradiation. Measurements were taken of both groups of glasses immediately following heat treatment. Doses were determined from the calibration chart constructed from $\mathrm{Co}^{60}$ exposures (Fig. 5), by using the values obtained immediately after heat treatment applied one hour after irradiation. Depth-dose curves were drawn from apparent doses received by the top six glasses in each group. The seventh and bottom glass in each group apparently was beyond the range of the beam, as it received no detectable dose.

The abscissae in the figure are in terms of absorber thickness, obtained by multiplying the distance of penetration by the density of the material, so that materials of different density may be compared. The ionization density curves for the glass show the characteristic rise to a maximum. After the maximum is reached, the curves fall off rapidly to a value close to zero.

The "true" curve for glass should lie between curves B and $\mathrm{C}$. This is because the 1-hour and 1-day waiting periods for curves $\mathrm{B}$ and $\mathrm{C}$ are shorter and longer respectively than the irradiation times for corresponding doses involved in the $\mathrm{Co}^{60}$ calibrations.

The figure above also shows a 3-Mev-electron ionizationdensity curve for aluminum (11), with the maximum at $10^{6} \mathrm{rep}$. The three curves are similar in shape, and the maxima appear at the same absorber thickness. The maximum range in the aluminum is about $0.2 \mathrm{gm} / \mathrm{cm}^{2}$ greater than in the glass. Furthermore, the ionization density in glass as determined by both groups of glasses remains appreciably lower than that in the aluminum shortly after the maximum values are exceeded. The explanation for these differences is not known, but the differences are likely caused by a difference in the scattering properties of the aluminum and glass.

The dose rates of the Van de Graaff and $\mathrm{Co}^{60}$ sources were in a ratio of $\sim 2,000: 1$. The Van de Graaff results thus support the results of Schulman, Klick, and Rabin (3) who found no significant dependence of glass sensitivity on dose rate.

These experiments illustrate the applicability of this glass for electron-beam dosimetry and stress the importance of placement and geometry of the dosimeter system in interpreting correctly the dose received. 


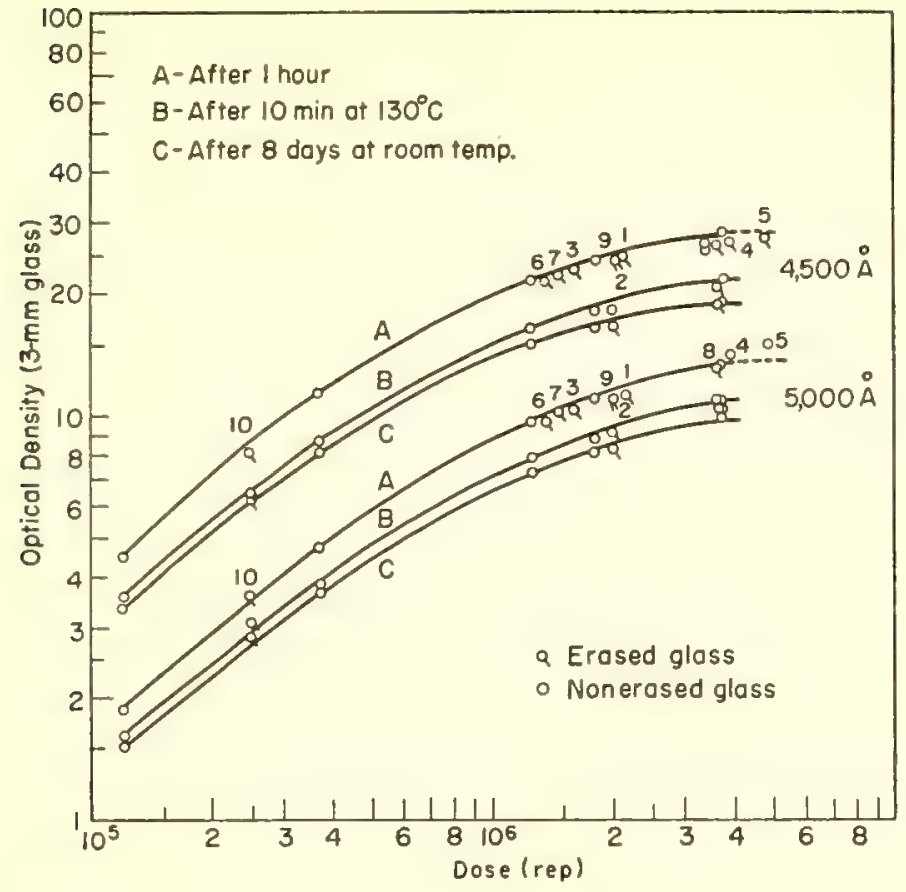

FIG. 8. Comparison of optical densities of nonerased glasses and glasses subjected to ten successive exposure-erasure cycles

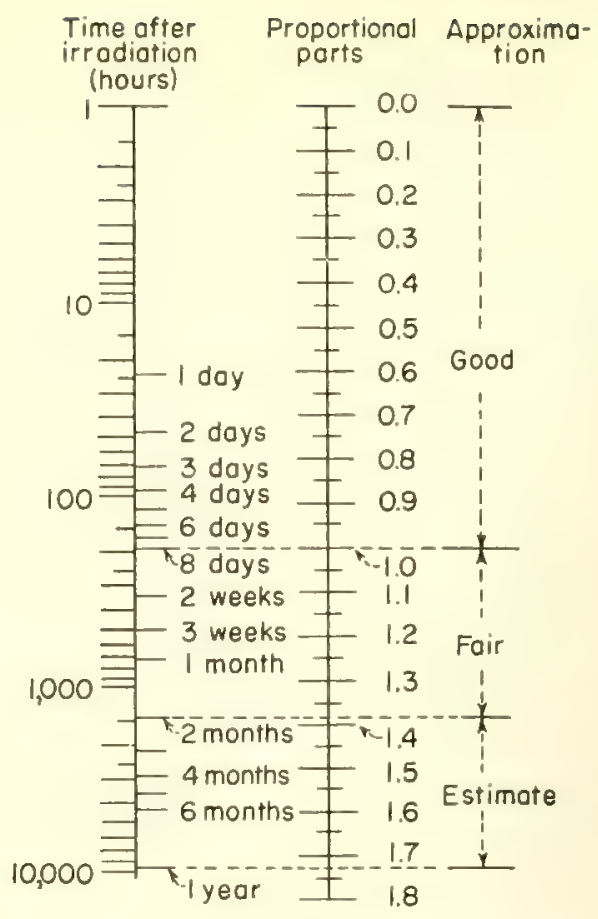

FIG. 9. Nomogram for estimating decrease in optical densities of glasses after different time intervals following irradia. tion and heating
An example can best illustrate this use of the scale. Assume that an optical density of 1.00 ( 3 -mm glass) is obtained at $4,500 \AA$, three days after the irradiation (glass was heated, $60 \mathrm{~min}$ after irradiation, for $10 \mathrm{~min}$ at $130^{\circ} \mathrm{C}$ ). The time scale shows that 3 days represents about four-fifths of the distance to 8 days. An optical density reading of 1.00 intercepts the fading area between the two curves at doses corresponding to the dose range of 4.7$5.30 \times 10^{5}$ rep. However, it intercepts the fading area four-fifths of the vertical distance between the fading curves, at about $5.15 \times 10^{5}$ rep. (Linear interpolation of the logarithmic scale is reasonably accurate for small time intervals). The total dose is, therefore, $5.15 \times 10^{5}$ rep.

Use of Fig. 4 for a calibration curve when other sources or dose rates are used must take into account fading during irradiation, if the exposure is long. The fading that takes place during irradiation is not known. However, the use of the heat treatment after irradiation will reduce appreciably the effect of the amount of time that elapses after irradiation, since it apparently eliminates the centers that are most unstable and that have a high probability of fading in the initial periods. For greatest accuracy, calibration doses should be delivered in times comparable with those to be encountered in use.

\section{Considerations for Use}

Restrictions in the use of the silveractivated phosphate glass dosimeter, such as avoidance of extreme temperatures and excessive exposure to light, are not difficult to fulfill in practice. Fading during and after exposure, and the energy dependence below $200 \mathrm{kev}$ (7) are disadvantages, but these can be minimized by various techniques and do not detract appreciably from the value of the dosimeter.

Megarep dosimetry is not precluded by the appreciable decrease in sensitivity there. It is necessary, however, to apply careful experimental techniques to attain the precision that is readily available with lower doses. The degree of precision desired is variable with different needs and the latter should dictate the experimental methods used in this high-dose region.

It would be desirable for suppliers of this glass to develop formulations that would saturate at higher dose levels than silver-phosphate glass. This would help develop glass dosimetry to its fullest potentialities.

\section{BIBLIOGRAPHY}

1. J. H. Schulman, NRL Memorandum Report 266 (1954)

Ð. H. Rabin, NRL Memorandum Report 309 (1954)

3. J. H. Schulman, C. C. Klick, H. Rabin, NuCleonice 13, No. 2, 30 (1955)

4. B. E. Proctor, S. A. Goldblith, A.E.C. Quarterly Progress Report July 1, 1954 to September 30, 1954, on Contract AT (30-1)-1164

5. J. H. Schulman, R. J. Ginther, C. C. Klick, R. S. Alger, R. A. Levy, J.A ppl. Phys, 22, 1479 (1951)

6. J. H. Schulman, W. Shurcliffe, R. J. Ginther, F. H. Attix, Nucleonics 11, No. 10, 52 (1953)

\%. J. H. Schulman, R. J. Ginther, C. C. Klick, NRL Reprint 65-52 (1952)

8. M. Eicher, Developmental study of the use of Vycor glass for gamma ray dosimetry. Research Report, Project NM 006012 . 04. 69 Vol. 12, 147-166, May 1954, Naval Medical Research Institute

9. G. E. Blair, Data on a megaroentgen dosimeter system. (Bausch and Lomb Chemical Research Lab. Report, 1955)

10. S. Davison, S. A. Goldblith, B. E. Proctor, M Karel, B. Kan, C. J. Bates, Nucleonics 11, No. 7, $22(1953)$

11. J. C. Trump, K. A. Wright, A. M. Clarke, J. Appl. Phys, 21, 345 (1950)

12. H. Rabin, W. E. Price, Nucleonics 13, No. $3,33(1955)$

13. B. E. Proctor, S. A. Goldblith, S. Davison, NYO 3345 (1955)

Contribution No, 2\%5. Department of Food Technology, Massachusetts Institute of Technology, Cambridge, Mass. 


\section{Counting Suspensions in Liquid Scintillators}

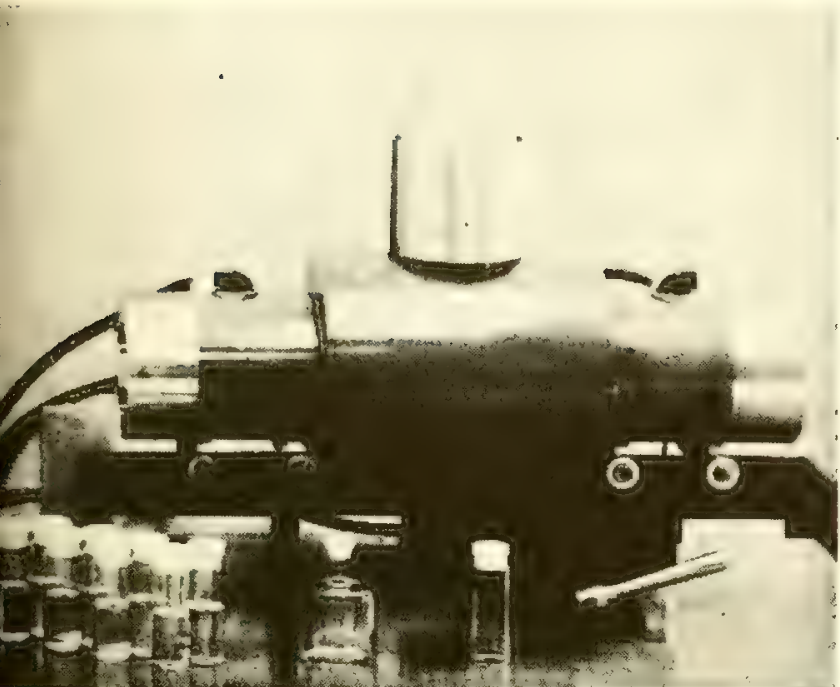

FIG. 1. Liquid scintillation counter showing sample bottles and the nonrefrigerated lead shield with light lock permitting insertion of sample without turning off high voltage
Now materials insoluble in liquid scintillators can be counted with high efficiency. Sample preparation is rapid and counting techniques are straightforward. Settling of the suspension, self-absorption, and light absorption are solvable problems

\author{
BY F. NEWTON HAYES, BETTY S. ROGERS, and \\ WRIGHT H. LANGHAM \\ Los Alamos Scientific Laboratory* \\ University of California \\ Los Alamos, New Mexico
}

Counting suspensions of solid samples in a liquid scintillator makes many compounds accessible to liquid scintillation counting for the first time. Additional advantages are ease of sample recovery through filtration and absence of conventional quenching problems. Problems present here, but not in solution counting, are selfabsorption and light scattering. However, small particle size minimizes selfabsorption and stabilizes the suspension at the same time.

We surveyed the potentialities and limitations of suspension counting for several materials and the radioisotopes $\mathrm{H}^{3}, \mathrm{C}^{14}, \mathrm{~S}^{35}$, and $\mathrm{Ca}^{15}$. The only chemical requirements were that the samples be solids insoluble in toluene. Sample preparation is discussed on p. 50.

* Work done under the auspices of the U. S. Atomic Energy Commission.

t In early phases of the study we used a 90-ml Pyrex bottle; some of the results given here were obtained that way. However, experience proved the smaller bottle better.

\section{Counting Procedures}

Here are the procedures and materials we used to count suspensions.

Counting bottles. Kimble Opticlear 10-dram vials, $29 \mathrm{~mm}$ in diameter and $85 \mathrm{~mm}$ high (including polyethylene cap), were used (Fig. 1). Maximum useful volume was about $35 \mathrm{ml} . \dagger$

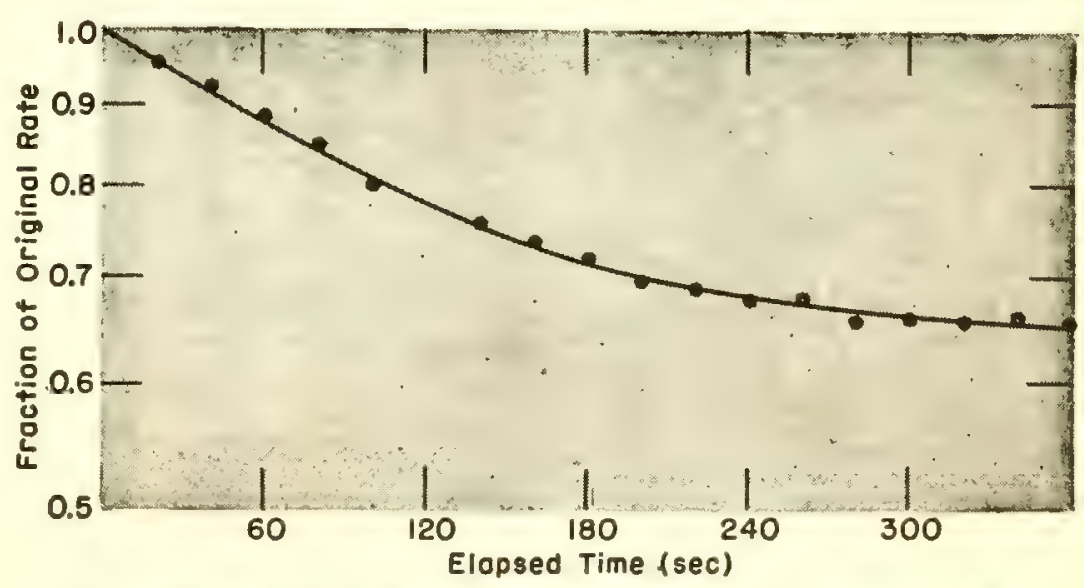

FIG. 2. Effect of settling rate on scintillation counting of a suspension of $20 \mathrm{mg}$ $\mathrm{Ca}^{45} \mathrm{C}_{2} \mathrm{O}_{4}$

liquid scintillator in common use for solution counting also was used in this study. It was $4 \mathrm{gm} / 1$ 2,5-diphenyloxazole (PPO) and 0.05 gm/l 1,4-di-[2-(5-phenyloxazolyl)]-benzene (POPOP) in toluene. It represents an excellent compromise among pulse height, economy, and ability to remain homogeneous. Since an inert suspension does not act to precipitate a dissolved solute, the latter criterion 
was not necessary here and $4 \mathrm{gm} / \mathrm{l}$ p-terphenyl might have been substituted for the PPO.

After the sample was prepared by grinding and rinsing it into the bottle, stock scintillator solution was added to bring the total volume to $25 \mathrm{ml}$.

Homogeneous internal standards. Benzenecarboxylic-C $\mathrm{C}^{14}$ acid ( $\mathrm{C}^{14}$-benzoic acid) standard (1) dissolved in toluene served as an internal-standard stock solution. Its specific activity was $96,860 \mathrm{dpm} / \mathrm{ml}$.

The internal tritium standard was a toluene solution of dihydrocholesterol5,6- $\mathrm{T}_{2}$ (2), which was carefully calibrated against standard tritium water by homogeneous liquid scintillation counting procedures. Its specific activity was $276,000 \mathrm{dpm} / \mathrm{ml}$.

Instrumentation. A Los Alamos 540 coincidence system (3) was used in this study. The upper-level discriminators were disconnected to eliminate the possibility of encountering the sometimes-useful phenomenon of loss of efficiency with increasing electronic gain. In its routine application, suspension counting will certainly make use of the desirable properties of upper-level discriminators for background reduction and even for "balance point" operation (4). Photomultipliers, shield, and sample were at room temperature.

Two lead shields, with accompanying optical systems, were used. One required that the high voltage to the photomultipliers be turned off whenever the sample was about to be removed from the counting region. A second shield (Fig. 1), which was designed for operation outside a refrigeration unit, was constructed with a shutter system that allowed sample changing to be carried out with the high voltage always remaining on. No detectable light leak to the photomultipliers occurred during sample changing.

Settings for high voltage, amplifier gain, and lower discriminator level were such that an unquenched homogeneous $\mathrm{C}^{14}$-solution would count with about $60 \%$ efficiency.

Counting techniques. Two methods were available for obtaining repeated counts on a sample. In one, the sample was shaken between each 1-min count and the results were averaged. In the other, a series of 1-min counts was obtained and the results plotted and extrapolated back to zero time. A good fit of the plot to the data substituted for the averaging used in the first method. Shaking the sample between each count was the most popular method and became quite easy with the shield that allowed the high voltage to remain on continuously. In fact, the only occasion for using the second method was with studies of settling rates.

Comparisons of the scatter in counting data with the two shields demonstrated that they were equivalent for counting rates $>1,000 \mathrm{cpm}$. Below $1,000 \mathrm{cpm}$ both shields produced a scatter somewhat beyond that expected by statistics, but the shield with the special optical shutter system was significantly better than the other. It seems reasonable to conclude from this that improved counting stability at low rates results from never allowing the photomultipliers to see light and preserving the continuity of high voltage applied across them.

Data on the efficiency with which the suspensions could be counted were obtained by counting standard suspensions prepared from assayed materials. Duplicate suspensions, to serve as backgrounds, were made from the corresponding inactive samples. Averaging, subtraction of backgrounds from active-sample results, and division by the total activity present gave the desired self-calibrated counting efficiencies. Especially in the extensive $\mathrm{C}^{14}$ studies, the procedure was to add a known quantity of the homogeneous internal standard ( $\mathrm{C}^{14}$-benzoic acid) to both the background and the active sample and recount. The resulting increases in counts were divided by the known $\mathrm{C}^{14}$ activity added, to yield internal-standard counting efficiencies.

\section{Counting Data}

It is a very useful practice in homogeneous liquid scintillation counting to determine counting efficiency by adding an internal standard after making a count. This avoids the dangerous assumption that the standard counting solution has the same counting effciency as the other samples. The internal standard corrects for the vagaries of quenching.

The ratio of suspension counting efficiency to homogeneous internalstandard counting efficiency is denoted by $f$. If $f$ is independent of scattering and absorption of light by the suspension and if it depends only on self-
TABLE 1-Suspension $f$ Values

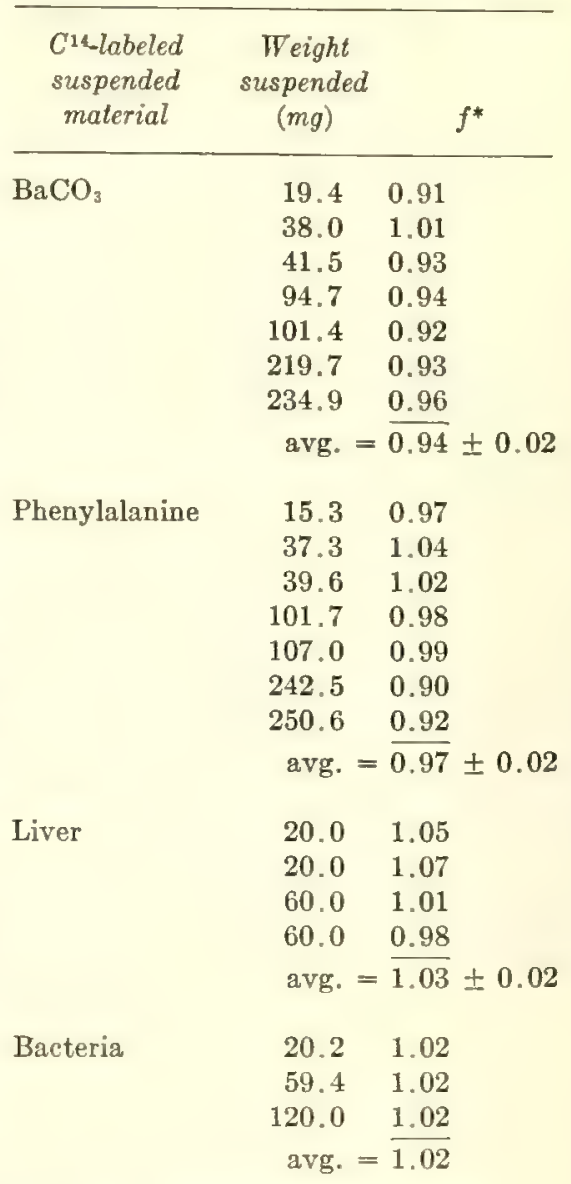

* Ratio of suspension-counting efficiency to homogeneous internal-standard counting efficiency.

absorption of the beta particles, controllable by reproducibility of grinding, then knowledge of $f$ will make the homogeneous internal standard very useful in suspension counting.

C14. Table 1 gives $f$-values for various concentrations of $\mathrm{BaCO}_{3}$, phenylalanine, liver, and bacteria when counts were made in the large bottles; $f$ is reasonably constant in each case. The average values seem to be independent of the color present in the liver samples and the high opacity in the larger concentrations of $\mathrm{BaCO}_{3}$ and phenylalanine.

These $f$-values are so close to 1 that they indicate almost no self-absorption.

Because $f$-values of about 1 were obtained for the biological materials, liver and bacteria, a study utilizing this fact was made of the counting of a variety of tissues, both lyophilized and wet. Samples of about $50 \mathrm{mg}$ were either 


\section{SAMPLE PREPARATION}

1. Barium Carbonate $\left(\mathrm{C}^{14}\right)$. The inactive material was reagent grade. C14labeled material was prepared from a suitable mixture of sucrose and standardized benzene-carboxylic-C ${ }^{4}$ acid (1) by Van Slyke-Folch wet oxidation (6) followed by routine conversion of the evolved $\mathrm{CO}_{2}$ to $\mathrm{BaCO}_{3}$.

Particles fine enough for suspension counting were made from coarse $\mathrm{BaCO}_{3}$ crystals by grinding for $3-5$ min between the ground-glass surfaces in a small tissue homogenizer. Absolute alcohol was used to wet the particles during grinding. Toluene was used to rinse them into the counting bottle. Quantities were $0.5 \mathrm{ml}$ of alcohol and $7.5 \mathrm{ml}$ of toluene. This procedure for grinding and rinsing was used for the other materials except in a few cases that will be discussed.

2. Phenylalonine $\left(\mathrm{C}^{14}\right)$. $\mathrm{C}^{14}$-labeled material of suitable specific activity was made by dilution of Tracerlab's 2-amino-3-phenylpropionic-1-C ${ }^{14}$ acid with inactive compound and precipitating it from methanol by neutralization of the hydrochloride with ammonium hydroxide. Assay of the product was obtained by Van Slyke-Folch wet oxidation, followed by direct plate counting of the derived $\mathrm{BaCO}_{3}$ in an internal-sample flow counter. This counter was carefully standardized with some of the $\mathrm{BaCO}_{3}$ mentioned previously.

3. Sodium acetate $\left(\mathrm{H}^{3}\right)$. A $1-\mathrm{ml}$ portion of a $400 \mathrm{mg} / \mathrm{ml}$ aqueous solution of tritium-labeled sodium acetate was evaporated to dryness. Grinding and rinsing were done with $8 \mathrm{ml}$ toluene. Alcohol was avoided because of its solvent power for sodium acetate.

4. Barium sulfate $\left(S^{35}\right)$. Inactive material was prepared from commercial sodium sulfate, which was dissolved in water, acidified with hydrochloric acid, and treated with barium chloride to precipitate the desired product (7). Active material was prepared by adding a suitable dilution of $\mathrm{S}^{35} \mathrm{O}_{4}{ }^{-}$in $\mathrm{HCl}$ as supplied by Oak Ridge to the aqueous sodium sulfate before treatment with barium chloride. Quantitative yields were obtained.

5. Benzidinium sulfale $\left(S^{35}\right)$. Inactive material was prepared from commercial sodium sulfate dissolved in an alcohol-water solution by adding benzidine hydrochloride to precipitate benzidinium sulfate (8). As above, for the preparation of the active material, a dilution of the Oak Ridge $\mathrm{S}^{35}$ in $\mathrm{HCl}$ was added to the solution of sodium sulfate before the precipitation.

6. Calcium oxalate $\left(\mathrm{Ca}^{45}\right)$. Inactive material was prepared by a standard procedure (9) from commercial calcium chloride. To make the labeled compound, a suitable dilution of $\mathrm{Ca}^{45}$ in $\mathrm{HCl}$ supplied by Oak Ridge was added to the above preparation before the final precipitation.

7. Calcium carbonote $\left(\mathrm{Ca}^{46}\right)$. Inert material was prepared by precipitation from an aqueous solution of calcium chloride to which was added a saturated solution of ammonium carbonate. The labeled compound was made by adding the dilution of $\mathrm{Ca}^{45}$ mentioned above before precipitation.

8. Tissues $\left(\mathrm{C}^{14}\right)$. A number of tissues, selected to give a wide range in color and composition, were taken from rats injected with $\mathrm{C}^{14}$-nitrogen mustard. These were liver, spleen, testes, muscle and bone. Homogenates were prepared by freezing the tissue in liquid nitrogen and pulverizing while still frozen.

Tissues from normal aninals were used to provide inactive material.

Initial studies were made with dried material. This was obtained by lyophilizing aliquots of the tissue homogenates for 20 to $24 \mathrm{hr}$. The water collected from this drying was assayed for any activity that may have been lost from the tissue during the process. An insignificant amount of activity was found.

The usual grinding and rinsing procedures were used at first, but later work utilized the tissue homogenates directly. The sample was ground in $2 \mathrm{ml}$ absolute alcohol and then rinsed into the counting bottle with $6 \mathrm{ml}$ toluene.

9. Bacterio $\left(\mathrm{C}^{14}\right)$. Lactobacillus arabinosus cells were harvested from a 48 -hrold synthetic culture medium (10) and washed with saline and water. They were then lyophilized for $24 \mathrm{hr}$ and yielded $260 \mathrm{mg}$ inactive material.

Labeled bacteria were obtained as above, by growing the Lactobacillus in a synthetic culture medium containing $\mathrm{C}^{14}$-nicotinic acid.

lyophilized and then suspended or suspended directly as wet tissue homogenate. Preparation of the $\mathrm{C}^{14}$-homogenates and the suspensions is described under Sample Preparation.

An efficiency for each suspension was determined by the addition of internal standard and a recount. Since this efficiency is the same as the self-efficiency of the sample, the $f$-value being consideration of the Beer-Lambert Law. With the same amount of suspended material, changing from the large bottle to the small would have the effect of increasing concentration while decreasing path length with absorption and scatter remaining the same. The small bottle numbers among its advantages: availability, cheapness, duplication of whiteness of glass, and ease of cleaning.

$\mathrm{H}^{3}$. When $42.3 \mathrm{mg}$ sodium acetate was suspended in an alcohol-free scintillator, the self-calibrated efficiency for the suspension was $3.1 \%$ and for the tritium internal standard $11.1 \%$. This $f$-value of 0.28 is evidence for an expected large degree of self-absorption of the weak beta spectrum by the particles of the suspension because of their relatively large size compared with the range of the tritium beta particles.

$\mathrm{S}^{35}$ and $\mathrm{Ca}^{45}$. The maximum beta energies of $\mathrm{S}^{35}$ (167 kev) and $\mathrm{Ca}^{45}$ (255 kev) are both greater than that of $\mathrm{C}^{14}$ (155 kev). One must then conclude from the previously mentioned $\mathrm{C}^{14}$ data that, with suitable grinding, the $f$-factors for $\mathrm{S}^{35}$ and $\mathrm{Ca}^{45}$ will be 1.0. Unfortunately, homogeneous internal standards for these isotopes were not available to check this.

Barium sulfate, benzidinium sulfate, calcium oxalate, and calcium carbonate gave very normal-appearing suspensions and seemed, from the Oak Ridge assays, to count with high efficiency, even when as much as $120 \mathrm{mg}$ of material was suspended in the counting volume.

\section{Settling Studies}

An interesting facet of the behavior of these suspensions is their settling and the accompanying drop in counting rate. This drop is presumed to be due to the drift away from $100 \%$ geometry toward $50 \%$ geometry while settling to the bottom of the counting bottle. Figure 2 is a plot of the drop in counting rate vs. elapsed time for $20 \mathrm{mg}$ of calcium oxalate in a typical experiment. At early times the drop is exponential and then levels off. Other concentrations and materials used behave this way too.

The slope of the exponential portion of the settling curve was determined for various concentrations of some of the suspensions. It was found to be reasonably independent of concentration, suggesting that the grinding process was reproducible. Average slope values were $3,640 \mathrm{sec}$ for $\mathrm{BaCO}_{3}, 1,060$ 


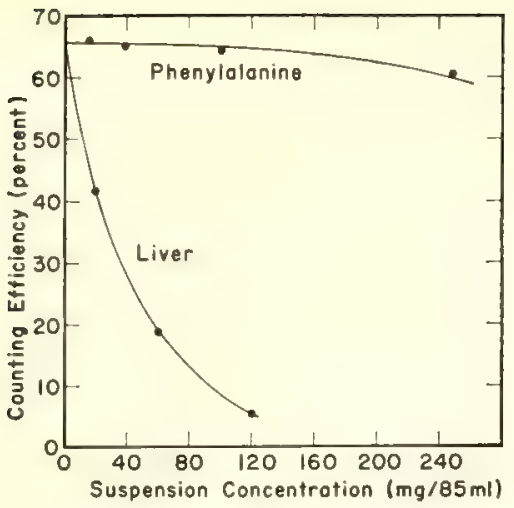

FIG. 3. Self-calibration and internal-standard counting curves for phenylalanine

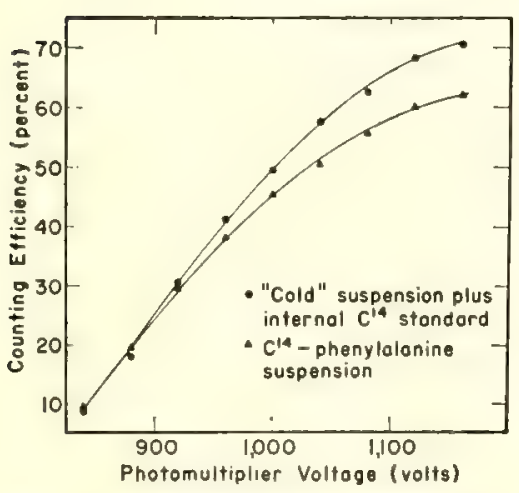

FIG. 4. Effect of light scattering and absorption on scintillation counting of suspensions

TABLE 2-Counting Lyophilized and Wet Tissues

\begin{tabular}{lcc} 
& \multicolumn{2}{c}{$\begin{array}{c}\text { Specific counting rate } \\
\text { (dpm/mg) }\end{array}$} \\
Tissue & Lyophilized & Wet \\
\hline Testes & $18.8 \pm 0.6$ & $19.0 \pm 0.4$ \\
Liver & $77.0 \pm 4.1$ & $82.0 \pm 2.8$ \\
Muscle & $23.7 \pm 0.4$ & $26.3 \pm 0.5$ \\
Spleen & $\mathbf{4 5 . 2} \pm 2.2$ & $\mathbf{4 4 . 5} \pm \mathbf{1 . 6}$ \\
Sternum & $\mathbf{1 9 . 8} \pm 1.5$ & $\mathbf{2 3 . 5} \pm \mathbf{0 . 8}$ \\
\hline
\end{tabular}

TABLE 3-Comparison of Suspension Counting with Conventional

Specific counting rate $(\mathrm{dpm} / \mathrm{mg}$ wet weight $)$

Tissue Suspension Van Slyke-Folch

$\begin{array}{lrr}\text { Whole femur } & 18 \pm 2 & 19 \pm 2 \\ \text { Liver } & 37 \pm 2 & 40 \pm 3 \\ \text { Testes } & 7 \pm 1 & 11 \pm 1 \\ \text { Spleen } & 33 \pm 2 & 44 \pm 5 \\ \text { Thigh muscle } & 25 \pm 1 & 26 \pm 1\end{array}$

sec for phenylalanine, 530 sec for barium sulfate, $570 \mathrm{sec}$ for calcium oxalate, and 310 sec for bacteria.

\section{Self-Absorption}

The suspended particle can be compared with the thin sample layer on a planchet. In the former case, there is $100 \%$ geometry between beta particles escaping the solid and the detecting medium (the liquid scintillator). But the latter case finds about $50 \%$ geometry the best realizable. A $1-\mathrm{mg} / \mathrm{cm}^{2}$ $\mathrm{BaCO}_{3}$ layer on a planchet produces a self-absorption factor of about 0.7 (5). This corresponds to an average thickness of about $2.3 \mu$ and indicates very small effective particle size for the suspension with its smaller selfabsorption. It was not established in this study whether the particles were actually as small as indicated or whether in wetting and partly permeating the particles the liquid scintillator may have reduced their absorbing dimensions.

The process of losing efficiency by self-absorption should act to degrade the beta spectrum such that the selfcalibrated and internal-standard efficiencies will be the same for high energies and different for low energies. Figure 3 is a plot of counting efficiency vs. photomultiplier voltage for $\mathrm{C}^{14}$ phenylalanine and nonradioactive phenylalanine with added $\mathrm{C}^{14}$-benzoic acid internal standard. At low voltages only the high-energy part of the $\mathrm{C}^{14}$ spectrum was being counted and the curves are almost identical. With increasing voltage the curves diverge in the direction of decreasing $f$ as low $\mathrm{C}^{14}$ energies rose above the lower discriminator and contributed to the counting efficiency.

\section{Light Absorption}

In earlier studies of homogeneous liquid scintillation counting of water samples, it was occasionally noticed on completing a count that the sample was very milky due to insufficient alcohol in the system. However, this opacity did not seem to interfere with counting.

Similarly, in this study considerable opacity due to large quantities of a white suspension did little to interfere with the passage of scintillations from solution to photomultipliers. Figure 4 demonstrates this point for phenylalanine.

The case of a colored suspension must be complicated by light absorption. Whereas, with a white suspension a photon will be reflected when striking a particle, the colored suspension must absorb it. The lower curve in Fig. 4 shows this for highly colored liver tissue.

\section{$\mathrm{C}^{14}$-Analyses of Tissue}

The biologist needs a counting method for labeled animal tissues that is rapid, reproducible, and easy. The present method was compared in these respects with Van Slyke-Folch oxidation followed by $\mathrm{BaCO}_{3}$ plate counting.

Five pooled tissue homogenates prepared as described on p. 50 were made of bone, muscle, liver, spleen, and testes taken from rats injected with $\mathrm{C}^{14}$-nitrogen mustard. Triplicate aliquots of each homogenate were counted by each of the two methods and the averaged specific counting rates are compared in Table 3. The two methods are essentially in agreement for all cases.

The difference in operator time is significant. A rough estimate of operator time on one sample of tissue homogenate would be $100 \mathrm{~min}$ for the Van Slyke-Folch oxidation and $\mathrm{BaCO}_{3}$ plate counting, 40 min for the scintillation counting of suspensions of lyophilized tissues, and $20 \mathrm{~min}$ for wet tissue homogenates. This estimate does not include time for the maintenance of equipment, etc., which probably would be about the same as operator time.

\section{BIBLIOGRAPHY}

1. D. I. Williams, F. N. Hayes, R. J. Kandel, W. H. Rogers, NucLEonics 14, No. 1, 62 (1956) 2. F. N. Hayes, R. G. Gould, Science 117, 480 (1953)

9. R. D. Hiebert, R. J. Watts, Nucleonics 11 , No. 12,38 (1953)

4. J. R. Arnold, Science 119, 115 (1954)

5. P. E. Yankwich, J. W. Weigl, Science 107, $651(1948)$

6. D. D. Van Slyke, J. Folch, J. Biol. Chem 136, 509 (1940)

7. W. C. Pierce, E. L. Haenisch, "Quantitative Analysis," 3rd ed. p. 391 (John Wiley and Sons, Inc. New York, 1948)

8. J. B. Niederl, H. Baum, J. S, McCoy, J. A Kuck, Ind. Eng. Chem. Anal. Ed. 12, 428 (1940)

g. N. H. Furman, "Scott's Standard Methods of Chemical Analysis," 5th ed. vol. 1, p. 210 (D. Van Nostrand Co., Inc., New York, 1939)

10. B. C. Johnson, "Methods of Vitamin Determination," p. 64 (Burgess Publishing Co. Minneapolis, 1948)

\section{Coming Next Month}

Scintillation Counting Today, a NUCLEONICS Special Report on the Fifth Scintillation Counter Symposium 


\section{Simple Instrumentation Determines Several Simultaneous Radioactivities}

Stepping single-channel analyzer and 5-window instrument solve many industrial problems more simply and more economically than elaborate multichannel analyzers

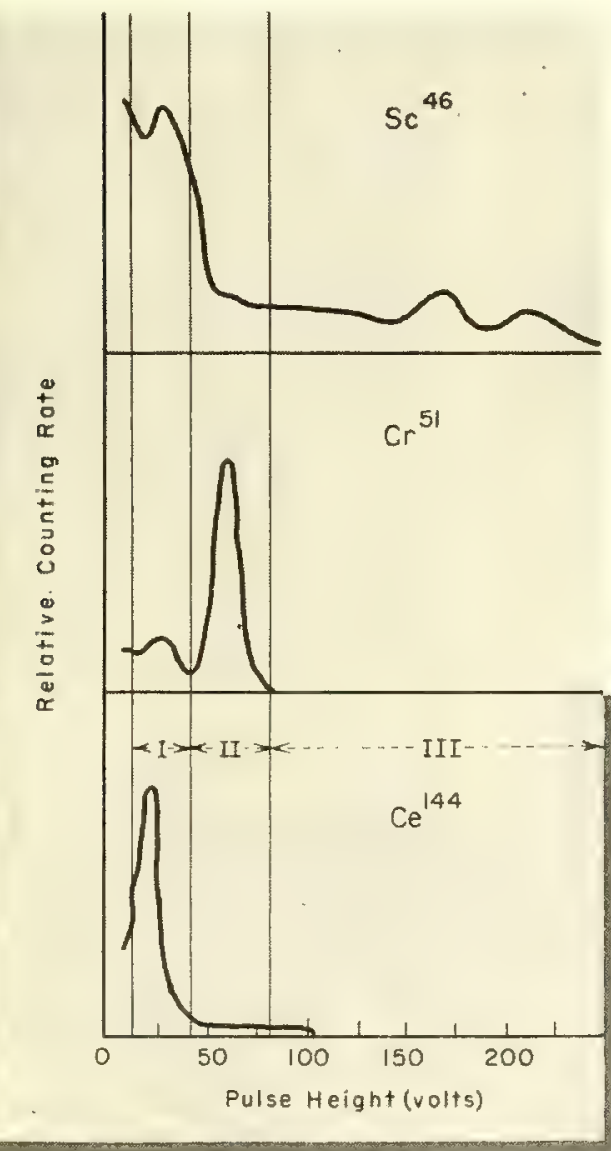

FIG. 1. Standard somples of the three tracers used simultaneously in cotalyat study gave these samma spectro with S-window enalyzer. Superimposed are windows Used in simultoneovs determinations

FIG. 2. Five-window analyzer consists of pulse-height analyzer, glow-transfer and digital register, patch panel, amplifier, and power supplies
BY J. KOHL, R. E. NATHER,

Tracerlab, Richmond, California

and V.P. GUINN

Shell Development Company, Emeryville, California

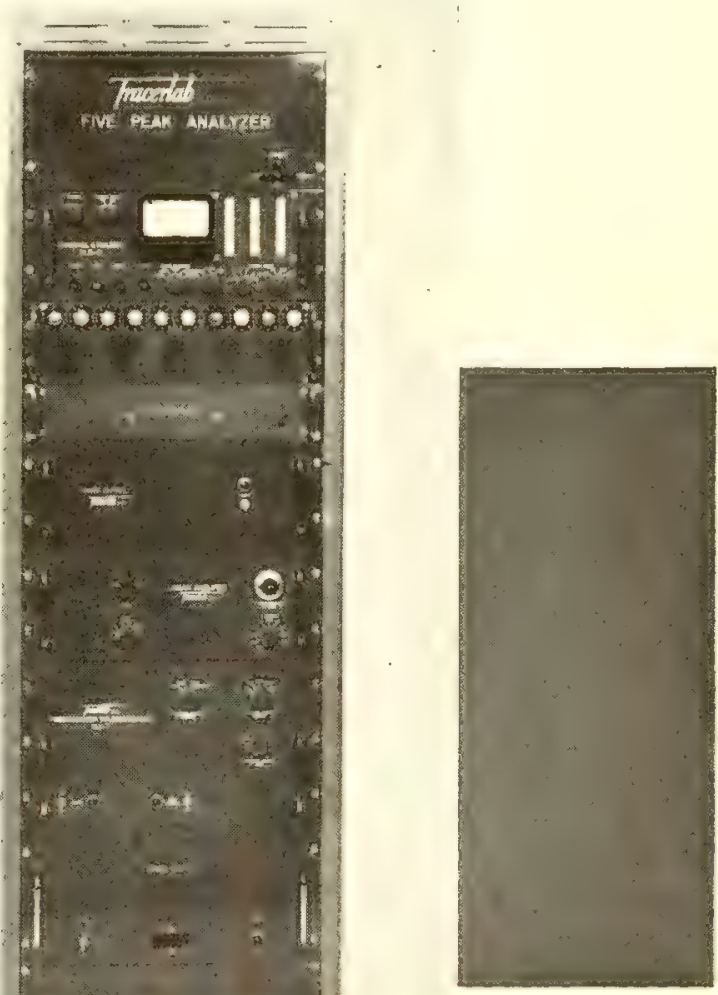


Kicksorters with small numbers of channels and stepping single-channel analyzers serve some industrial applications better than more elaborate multichannel instruments. Several instances in which the simpler instruare listed in the box at the right. We termination of mixed natural radioactivities and of mixed radiotracers.

\section{Assay for $\mathrm{K}, \mathrm{Th}, \mathrm{U}$}

It is often desirable to assay a particular radioactive ore for its relative content of the naturally occurring activities, therium, uranium, and potasfrom an oil well. If daughter products of thorium and uranium chains are in equilibrium with parents, quantitative separation using the total gamma-ray spectrum is possible. In this analysis one measures three regions of the spectrum simultaneously. The result is a set of three linear equations with three unknowns (the amount of each activity present). Calibration with pure forms of the activities determines the coefficients, and solution of the equations gives the amount of each activity. A five-window analyzer can perform such an analysis in a single run.

In experiments on limestone and sandstone, a 11/2-in.-dia, 2-in.-long $\mathrm{NaI}$ crystal surrounded by $300-\mathrm{gm}$ samples of the ground shale provided data on the concentrations of $\mathrm{U}, \mathrm{Th}$, and $\mathrm{K}$ to a maximum of $\pm 30 \%$ standard deviation with 2 -hr runs. mentation has been successfully used will discuss its use in simultaneous desium. An example is a core sample

\section{Other Successful Applications}

1. Continuous determination of $\mathbf{K}$, Th, and $U$ in oil-well formations by logging with a scintillation spectrometer coupled to a 3-channel discriminator feeding 3 ratemeters and a 3-channel recorder (9).

2. Continuous determination of the quantity of selected elements by observing their prompt gammas under neutron bombardment. Elements detectable include $\mathrm{Cl}, \mathrm{C}, \mathrm{H}, \mathrm{O}$. One can distinguish limestone from sandstone even though they both have identical total radiation intensities $(9,10)$. One can determine the difference between salt brine and oil by noting the $\mathrm{Cl}$ capture gammas. One can $\mathrm{log}$ an outflow well in a water-flooding operation to determine the location and relative volume of inflow from several different input wells injection waters of which have been tagged with different tracers (9).

3. Continuous simultaneous determination of the rate of wear of two or more parts tagged with different radioisotopes $(11,12)$.

4. Neutron irradiation and subsequent use of the spectrometer to determine: concentration of $\mathrm{Cu}$ and $\mathrm{Mn}$ in super-pure aluminum (13); concentration of $\mathrm{Cl}$ and $\mathrm{F}$ in Kel-F (14); concentration of $\mathrm{Ca}, \mathrm{Al}$, and $\mathrm{Mg}$ in cement-mill feed stocks (15); milligram quantities of $\mathrm{F}$ and microgram quantities of $\mathrm{Br}, \mathrm{Cl}$, and $\mathrm{I}$ in aqueous solutions (16).
For more complex analyses, where computations become extremely tedious, it often is necessary to call on additional machinery such as a digital computer to perform the actual data reduction. In such circumstances the automatic output of a single-channel analyzer may be of the same form as the input to the computer in the form of punched cards, punched paper tape, or magnetic tape. This simplifies and speeds up the over-all operation. A special technique has been developed, for instance, for resolving complex spectra by multiple least-squares analysis on the resulting data, which is impractical without the use of a computing machine (1). If the data are presented in a form that can be fed directly into a computer, final results can be obtained more quickly from a complex spectrum
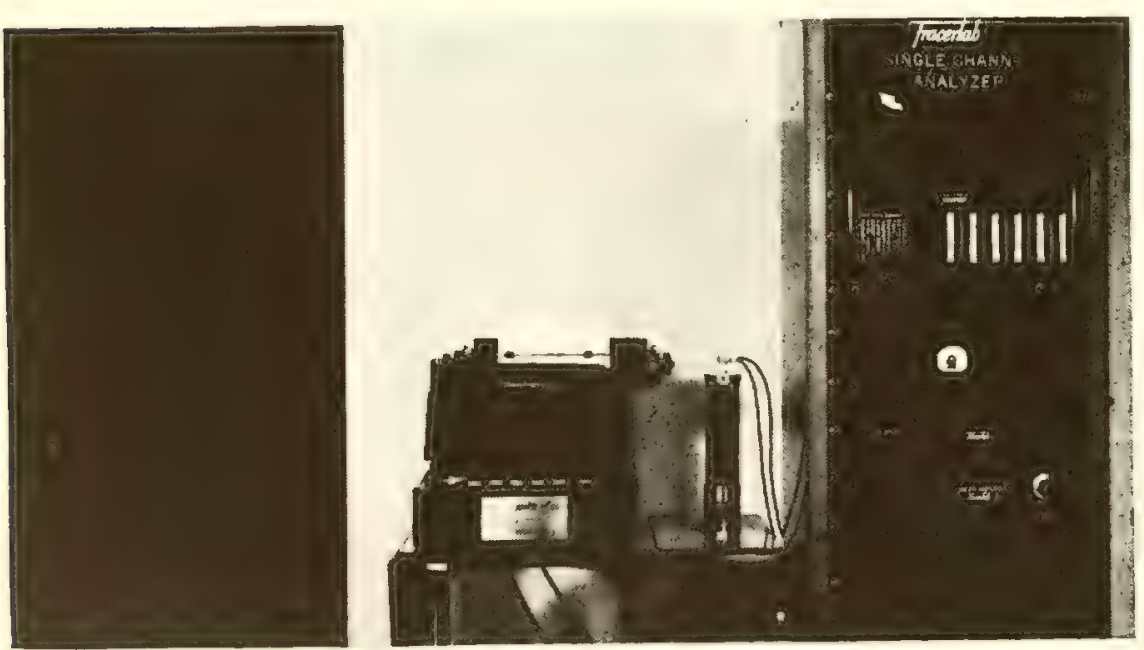

FIG. 3. Stepping single-channel analyzer offers automatic, unattended operation. Electric typewriter records window number, count, time, and time of day than from a single component by hand.

\section{Simultaneous Tracers}

Use of gamma-emitting radioisotopes as tracers in refinery-scale studies of mixing, stack loss, and aging of fluid cracking catalysts has been published recently (2-5). Tracer studies employing as little as $0.5 \mathrm{me}$ diluted with as much as 850 tons of eatalyst have been carried out successfully, by use of high-sensitivity scintillation counting. We will describe here a similar study simultaneously employing three catalysts separately tagged.

Cracking catalysts age as they are used. Their surface area may decrease from 600 to $100 \mathrm{~m}^{2} / \mathrm{gm}$, and pore volume from 0.9 to $0.3 \mathrm{~cm}^{3} / \mathrm{gm}$. When aging characteristics of several catalysts are to be compared, one should study them simultaneously because of day-to-day variation of operating conditions, feed stocks, etc.

In a recent study carried out by the Shell Development Company, the aging and stack-loss of three fresh cracking catalysts were followed simultaneously over a period of 30 days to find out which one had the best aging characteristics. The three catalysts were tagged with $\mathrm{Ce}^{144} / \mathrm{Pr}^{144}, \mathrm{Cr}^{51}$, and $\mathrm{Sc}^{46}$, respectively. Three separate tests without the spectrometer would have required many months, allowing for reasonable decay of the suitable radioisotope of shortest half-life before use of the next longer-lived isotope.

These isotopes were chosen for several reasons. They adsorb strongly and uniformly on a silica-alumina catalyst surface and are not volatilized at temperatures up to $1,000^{\circ} \mathrm{C}$. They emit gamma radiation of energies suitably 


\section{Simultaneous-Tracer Trio}

These characteristics make these radiotracers appropriate for simultaneoous use:

$$
\begin{aligned}
& \text { Isotope } \\
& \mathbf{C e}^{1+4} / \mathbf{P r}^{144} \\
& \mathbf{C r}^{51} \\
& \mathbf{S e}^{46}
\end{aligned}
$$

$$
\text { Half-life (days) }
$$

285

27

85
Gamma energy (Mev)

$$
\begin{aligned}
& 0.14 \\
& 0.32 \\
& 0.88 / 1.12
\end{aligned}
$$

$\mathrm{Ce}^{144}$ emits gammas in only about $40 \%$ of its disintegrations. Its radioactive daughter, 17-min Prit, enits essentially only 3.0-Mev beta particles. Many of these Iatter have sufficient energy to penetrate the glass wall of the sample vial and the aluminum-MgO housing of the $\mathrm{NaI}$ crystal, producing a long high-energy tail to the observed $\mathrm{Ce}^{14} / \mathrm{Pr}^{14 \star}$ gamma-ray spectrum. To minimize this a steel insert about $1 \mathrm{~mm}$ thick was used as a liner in the crystal well, improving the spectrum.

$\mathrm{Cr}^{51}$ emits 0.32-Mev. gammas in $8 \%$ of its disintegrations and emits no other radiation other than very soft $\mathbf{V}^{31} \mathbf{X}$-rays resulting from $\mathbf{K}$-capture of $\mathbf{C r}^{51}$.

$\mathrm{Sc}^{46}$ emits essentially only 0.36 -Mev betas and then two gammas in cascade.

separated for adequate resolution with a scintillation counter and pulse-height analyzer, and they possess suitable half-lives. (See box above.)

Samples removed from the commercial unit after $1.5,6$, and 39 hours, 10 and 30 days were separated by sinkfloat methods into fractions of different skeletal density, pore volume, and surface area (5). Only small fractionated samples were available (a few grams) so a 2-in. $\mathrm{NaI}$ scintillation well counter was employed-in conjunction with a Tracerlab window analyzer. Figure 1 presents the observed gammaray spectra obtained with this crystal and three standard samples, using the five-window analyzer as a point-bypoint spectrum analyzer; that is, measuring five equal channels simultaneously $(0-5,5-10,10-15,15-20,20-25$ volts, then 25-30, 30-35, etc.). From those spectra it was decided to count all of the samples in the following three channels: A, $10-40$ volts; B, $40-80$ volts; C, $80-250$ volts.
Sharper spectra could have been obtained, especially for Sc ${ }^{46}$, if a larger crystal thickness had been used. Ours was the largest well crystal available at the time. However, the 2 -in. crystal gave adequate results.

For calibration purposes pure samples of each isotope were counted. The following list shows the percentage of the net counts falling in each of the selected channels in each of the three counts:

\begin{tabular}{lcrr} 
& $\mathrm{A}$ & $\mathrm{B}$ & \multicolumn{1}{c}{$\mathrm{C}$} \\
$\mathrm{Ce}^{144}$ & 90.9 & 5.5 & 3.6 \\
$\mathrm{Cr}^{51}$ & 30.0 & 69.7 & 0.3 \\
$\mathrm{Sc}^{46}$ & 41.7 & 19.9 & 38.4
\end{tabular}

Each of the three channels contains the photoelectric peak of one of the three isotopes $\left(\mathrm{A}, \mathrm{Ce}^{144} ; \mathrm{B}, \mathrm{Cr}^{51} ; \mathrm{C}\right.$, $\left.\mathrm{Se}^{46}\right)$. Channel A also includes Compton scattering pulses from $\mathrm{Cr}^{51}$ and $\mathrm{Sc}^{46}$. Channel B includes Compton pulses from $\mathrm{Sc}^{46}$. The fraction of the pulses falling in the photopeak decreases with increasing gamma-ray energy (6).
From the above distributions, the following equations were derived:

Total $\mathrm{Ce}^{144} \mathrm{cpm}$ in sample $=1.170 \mathrm{~A}$ $-0.499 B-1.010 C$

Total $\mathrm{Cr}^{51} \mathrm{cpm}$ in sample $=-0.060$ $A+1.464 B+0.694 C$

Total $\mathrm{Sc}^{46} \mathrm{cpm}$ in sample $=-0.110$ $A+0.035 B+2.704 C$

In these equations $A$ denotes the net sample cpm in Channel $A$, etc. The standard samples had total counting rates of about $10,000 \mathrm{cpm}$, and were counted for periods of 5-20 min. The time-weighted averages of the six determinations shown in the table were the values shown in the table above. The degree of reproducibility is indicated by the fact that six determinations were made at various times over a total period of five weeks.

The data coded for the Electrodata computer were the sample and background data (gross counts and counting time) from the five-window analyzer, date of counting, and weight of sample. The computer performed all necessary calculations for some 200 samples. It provided decay-corrected cpm/gm of each isotope with its standard deviation. The computer was not necessary, but it saved considerable time.

Three-gram samples of our unknowns were counted in a standard Harshaw $\mathrm{NaI}$ well-counter crystal for 10-30 min. The over-all gamma-counting efficiencies were approximately as follows: $\mathrm{Ce}^{144}, 80 \% ; \mathrm{Cr}^{51}, 60 \%$; $\mathrm{Sc}^{46}, 40 \%$ for $0.89 \mathrm{Mev}, 30 \%$ for $1.12 \mathrm{Mev}$.

The channels were selected to minimize any effect of slight gain drifts and with 2-in. lead shielding had typical backgrounds of $110 \mathrm{cpm}, 55 \mathrm{cpm}, 65$ cpm, respectively.

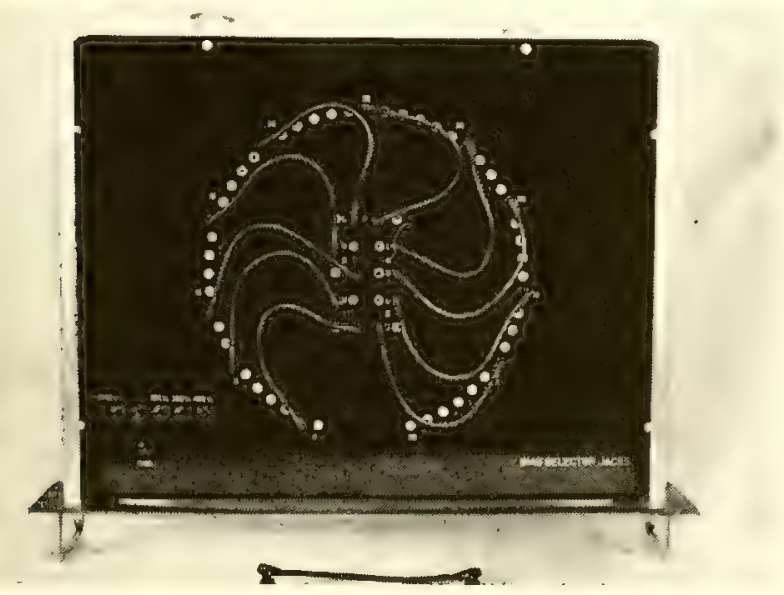

FIG. 4. Patch panel chassis contains plug-and-jack arrangement to position windows and choose their widths

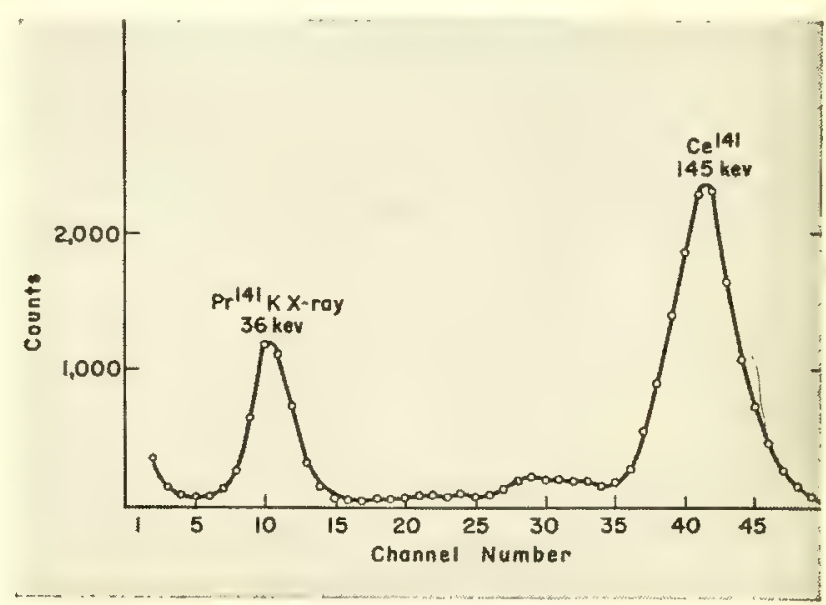

FIG. 5. Gamma spectrum of $\mathrm{Ce}^{141}$ plotted from automatically typed data of stepping analyzer ( $5 \mathrm{~min}$ per channel) 


\section{Instrumentation}

Two instruments appropriate for analyses like those just described are shown in Figs. 2 and 3. The five-window analyzer is the faster. The stepping single-channel analyzer on the other hand combines automatic printout and unattended operation.

Five-window Analyzer. The fivewindow analyzer has a nonoverloading linear amplifier, a servo-regullated highvoltage supply, and a window-discriminator chassis to perform the actual pulse-height analysis. Five separate windows are provided. Each can be located electrically to include any desired portion of the total spectrum, which normally is divided into 50 sections. Provision is made to locate each of the five windows on any group of the fifty division points (7). The narrowest possible window is a single segment, or $1 / 50$ of the spectrum, while the widest setting includes all fifty channels. The windows can be adjacent, with gaps, or overlapping.

Data are recorded completely in digital form to allow accurate mathematical treatment of the final data. Two scales-of-ten are provided for each window system to allow rapid accumulation of information. These are Ericsson glow-transfer decade counter tubes, the first of which has a resolving time of $50 \mu \mathrm{sec}$ (GC 10/D). The second is of the slower GC 10/B type. These glow tubes are followed by a fourdigit electromechanical register, so that total counting capacity is $10^{6}$ counts per window. At a rate of $30,000 \mathrm{cpm}$ there is $1 \%$ coincidence loss.

A control chassis is included. It contains a pulse generator for testing and aligning the equipment and a digital clock to provide preset time operation. A separate "patch panel" sets the windows (Fig. 4).

For certain operations such as the continuous wear-rate study mentioned in the box, the pulses at the input to the GC 10/D are fed to independent ratemeters, one for each channel.

Stepping Analyzer. The instrument of Fig. 3 was designed to permit accumulation of spectral data automatically (8). The discriminator system consists of a single window positioned by a 50 -position selector switch. The data-recording system is a specially designed scaler, accompanied by a solenoid-actuated electric typewriter. In automatic operation the window is placed at the first position, a preset

Repeated Counts of Catalyst Standards with Five-Window Analyzer

$\%$ of counls in channels

\begin{tabular}{|c|c|c|c|c|}
\hline \multirow[b]{2}{*}{ Isolope } & \multirow[b]{2}{*}{ Determination } & \\
\hline & & $A$ & $B$ & $C$ \\
\hline \multirow[t]{7}{*}{$\mathrm{Ce}^{144}$} & 1 & 91.3 & 5.1 & 3.6 \\
\hline & 2 & 91.1 & 5.3 & 3.6 \\
\hline & 3 & 91.5 & 5.1 & 3.4 \\
\hline & 4 & 91.0 & 5.5 & 3.5 \\
\hline & 5 & 91.0 & 5.4 & 3.6 \\
\hline & 6 & 90.7 & 5.6 & 3.7 \\
\hline & Wtd. avg.: & 90.9 & 5.5 & 3.6 \\
\hline \multirow[t]{7}{*}{$\mathrm{Cr}^{51}$} & 1 & 28.3 & 71.5 & 0.2 \\
\hline & 2 & 29.2 & 70.6 & 0.2 \\
\hline & 3 & 29.4 & 70.4 & 0.2 \\
\hline & 4 & 30.8 & 68.9 & 0.3 \\
\hline & 5 & 28.7 & 70.9 & 0.4 \\
\hline & 6 & 31.2 & 68.5 & 0.3 \\
\hline & Wtd. avg. & 30.0 & 69.7 & 0.3 \\
\hline \multirow[t]{7}{*}{$S c^{4 \theta}$} & 1 & 42.3 & 19.5 & 38.2 \\
\hline & 2 & 42.8 & 19.8 & 37.4 \\
\hline & 3 & 43.2 & 19.3 & 37.4 \\
\hline & 4 & 41.6 & 20.0 & 38.4 \\
\hline & 5 & 41.6 & 19.7 & 38.7 \\
\hline & 6 & 41.3 & 20.1 & 38.6 \\
\hline & Wtd. avg.: & 41.7 & 19.9 & 38.4 \\
\hline
\end{tabular}

time, preset courit, or both are chosen, and the start button is depressed. The scaler accumulates information from the first position until either the preset count or preset time is reached and the information is printed automatically by the electric typewriter on an appropriate data sheet.

Although the system is slower than a multichannel analyzer, operation without attendance makes up, to some extent, for additional time required. The analyzer can operate with a suitable automatic sample changer.

For manual operation a patch panel also is provided as in the five-window analyzer. This allows, for instance, measurement of the decay of any peak in a complex spectrum.

An illustration of the final data of the instrument is shown in Fig. 5 . The gamma ray of $\mathrm{Ce}^{141}$ and its corresponding "Compton lump" are clearly resolved from the $K \mathrm{X}$-ray line of $\operatorname{Pr}^{141}$ due to internal conversion of the soft gamma ray.

This article is based on a talk presented at the Fourth International Instruments and Measurements Conference, Stockholm, Sweden, September 18, 1956.

\section{BIBLIOGRAPHY}

1. S. D. Softky, D. H. Perkel, Private communication to A. DeHaan Jr., July 10, 1956

2. V. P. Guinn, Nucleonics 14, No. 5, 68 (1956)

3. E. Singer, D. B. Todd, V. P. Guinn, Catalyst mixing patterns in commercial catalytic cracking units. (Meeting of the American cracking units. (Meeting of the American

4. D. B. Todd, W. B. Wilson, Stack loss of catalyst from commercial catalytic cracking units. (Meeting of the American Chemical Society, Dallas, Texas, April 1956)

b. W. B. Wilson et al, Commercial performance of fluid cracking catalysts - a new technique of study. (Meeting of the American Chemical Society, Dallas, Texas, April 1956)

6. B. Crasemann, H. Easterday, NucLeonics 14, No. 6, 63 (1956)

\%. Tracerlog, No. 72, 6 (1955)

8. Tracerlog, No. 78, 2 (1956)

9. L. W. Toelke, Scintillation spectrometer well logging . (Joint Meeting of Rocky Mountain Petroleum Sections, American Institute of Mining and Metallurgical Engineers, Denver, Col. May 26-27, 1955)

10. D. B. Smith, G. R. Church, Prompt gamma rays from neutron capture as a means of oil well control. AERE $1 / \mathrm{R} 1688$ (Atomic Energy Research Establishment, Harwell, Eng., 1955)

11. A. Hundere, G, C. Lawrason, L. P O'Meara, Application of radioactive tracers to engine research. (Nuclear Engineering and Science research. (Nuclear Engineering and Science 1955) (Available from American Institute of Chemical Engineers N. Y. 36, N. Y., 30k)

12. B. D. Grozin, "International Conference on Peaceful Uses of Atomic Energy," vol. 15, p. 160 (United Nations, New York, 1956)

13. J. L. Putman, Private communication, June 13, 1956

14. P. Leveque, C. Fisher, Private communication, June 28, 1956

15. A. DeHaan, Jr., Private communicstion, Janusry 4, 1956

16. C. J. Atchison, W. H. Beamer, Anal. Chem. 28, 237 (1956) 


\title{
Fast-Neutron-Insensitive Chemical Gamma-Ray Dosimeter
}

\author{
Acid formation in stabilized chloroform or tetrachloroethylene serves as a \\ measure of gamma-ray dose that is rate-and temperature-independent and \\ linear to over $200,000 \mathrm{r}$. Adaptation to personnel monitoring is described
}

\author{
BY SANFORD C. SIGOLOFF \\ Radiobiological Laboratory, University of Texas, Austin, Texas
}

INCREASED AVAILABILITY of nuclear machines for radiobiological research has created many dosimetry problems. The ideal technique for mixed-radiation dosimetry would incorporate two dosimeters, one completely insensitive to gamma rays and the other completely insensitive to neutrons.

A useful technique for measurement of fast neutrons exists in the two-proportional-counter method (1-3). How chemical dosimetry can be used for gamma-ray measurements in the presence of neutrons is discussed here.

\section{Over-All Properties}

Both chloroform and tetrachloroethylene respond to $\mathrm{X}$ - and gamma radiation, producing stable, watersoluble acids. If a dosimeter containing these reagents* is given a known dose of radiation, the radiation-generated acids can be extracted with an acidimetric dyet, titrated with $10^{-3}$ normal sodium hydroxide, and a calibration value for the number of milliequivalents of acid per roentgen can be obtained. Evaluation of unknown-dose

\section{Chemicals + Film- Something New in Personnel Dosimetry}

A new approach to personnel monitoring for persons unlikely to receive a significant dose (e.g. secretarial help) is afforded by chemical dosimeters sensitive below $1 \mathrm{r}$. It parallels the usual film badge with a self-indicating chemical dosimeter. The film badge is developed only when the chemical dosimeter indicates the accumulation of a dose $>1 r$ or after the passage of a fairly long period, say 3 months. Weekly processing of the film badges of personnel who consistently get zero dose is thus avoided.

The necessary dosimeter is at hand in the form of alcohol-and-thiorea-stabilized tetrachloroethylene plus an acidimetric dye. Incident radiation produces acids that results in a color change in the acidimetric dye. This color change can be read with an accuracy of $20 \%$ in the range $0.5-3 r$. Complete color change from red to amber results from a $3-r$ exposure in a system having a hydrocarbon-dye ratio of $6: 1$. The dosimeter is rate- and temperature-independent; it is linear with dose up to 10,000 r. Energy dependence is like that of the high-range dosimeter described in this article.

For details see American Industrial Hygiene Association Quarterly for December, 1956. exposures is accomplished by titrating the exposed dosimeter, determining the number of milliequivalents of acid generated, and comparing with a known calibration value.

Titration techniques afford accuracy to $\pm 10 \%$. Spectrophotometric analysis can increase the accuracy to $\pm 3 \%$. A liter of solution is enough for 700 or more dose determinations.

Shelf life of these systems is excellent. Solutions have been stored at room temperature for periods greater than ten months with no decrease in their radiation sensitivity. An undesirable property of the anhydrous chloroform dosimeter is its volatility. This property precludes its use except for shortterm experimentation. For long-term exposures the less volatile tetrachloroethylene is used. Both of these systems have been flame-sealed in siliconed Neutraglas ampules and used in cooperative dosimetry efforts with other laboratories.

\section{Dosimetry Characteristics}

The chemical systems described here have many properties desirable in a

* Reagent-grade chloroform $\left(\mathrm{CHCl}_{3}\right)$ or tetrachloroethylene $\left(\mathrm{C}_{2} \mathrm{Cl}_{4}\right)$ is fractionated in a high-efficiency still to a constant refractive index. The distillate is collected in a thoroughly cleansed Pyrex container, and stabilizing agents, such as hexylresorcinol or 2,6-di-tert butyl 4 methylphenol, are added. The concentration of the stabilizing agent controls radiation sensitivity. We usually use $0.2 \%$ by weight of the stabilizing agent dissolved in the chlorinated hydrocarbon.

† Such as chlorophenol red $\left(3^{\prime}, 3^{\prime \prime}\right.$-dichlorophenol sulfonephthalein). 
gamma-ray dosimeter: linearity, rate and temperature independence, and neutron insensitivity. Details are given in Tables 1-1.

Energy dependence. The energy dependence of the anhydrous chloroform and anhydrous tetrachloroethylene dosimeters somewhat parallels that of film (4). Peak response as measured by acid production is directly related to photoelectric absorption. Energy dependence is decreased as Compton scattering begins to predominate, and the systems become energyindependent above $0.6 \mathrm{Mev}$. Energy independence down to $80 \mathrm{kev}$ is achieved when the systems are shielded with 0.5 mm oi lead.

Dose-rate dependence. Resorcinol-stabilized chloroform or tetrachloroethylene is rate-independent from $0.5 \mathrm{r} / \mathrm{hr}$ to the rates encountered during nuclear detonations. Dose-rate studies in this laboratory have been varied from $0.5 \mathrm{r} / \mathrm{hr}$ to $4,200 \mathrm{r} / \mathrm{min}$.

Temperature dependence. Both the anhydrous chloroform and tetrachloroethylene dosimeters are independent $( \pm 5 \%)$ of temperature during irradiation in the range $5-55^{\circ} \mathrm{C}$.

TABLE 1--Relative Energy Response*

\begin{tabular}{|c|c|}
\hline & $\begin{array}{c}\text { Acid yield } \ddagger \\
(m E q \times \\
\left.10^{-6} / m l / r\right)\end{array}$ \\
\hline $\begin{array}{r}\text { Effective } \\
\text { energy } \\
\text { adiation sourcet }(\text { kev })\end{array}$ & $\begin{array}{cc} & 0.5-\mathrm{mm} \\
\text { No } & \text { lead } \\
\text { lead } & \text { shield }\end{array}$ \\
\hline
\end{tabular}

\begin{tabular}{|c|c|c|c|}
\hline $\begin{array}{l}100 \mathrm{kvp} \\
(0.25 \mathrm{~mm} \mathrm{Cu} \text {, } \\
1.0 \mathrm{~mm} \mathrm{Al} \mathrm{fil-} \\
\text { tration) }\end{array}$ & 40 & 0.45 & 0.045 \\
\hline $\begin{array}{l}150 \mathrm{kvp} \\
(0.25 \mathrm{~mm} \mathrm{Cu} \text {, } \\
1.0 \mathrm{~mm} \mathrm{Al} \mathrm{fil-} \\
\text { tration) }\end{array}$ & 65 & 0.50 & 0.055 \\
\hline $\begin{array}{l}260 \mathrm{kvp} \\
(0.25 \mathrm{~mm} \mathrm{Cu}, \\
1.0 \mathrm{~mm} \mathrm{Al}, 0.5 \\
\mathrm{~mm} \mathrm{~Pb} \text { filtra- } \\
\text { tion) }\end{array}$ & 190 & 0.24 & 0.05 \\
\hline $\mathrm{Co}^{60}$ & 1,200 & 0.05 & 0.05 \\
\hline
\end{tabular}

* Hexylresorcinol-stabilized anhydrous tetrachloroethylene system.

+ Radiation rates varied from $30 \mathrm{r} / \mathrm{min}$ to $300 \mathrm{r} / \mathrm{min}$

\# Each value represents a mean obtained by titrating 20 samples; titrating was done with $10^{-3}-\mathrm{N} \mathrm{NaOH}$.
Linearity. The anhydrous tetrachloroethylene system shows a linear relationship between radiation dose and the total acids liberated by the halogenated hydrocarbon. Acid production is linear to doses greater than $200,000 \mathrm{r}$ regardless of the rate.

Neutron sensitivity. It was anticipated that the anhydrous systems would have a low response to fast neutrons. For one thing the amount of energy imparted to a unit mass of solution by one rep of fast neutrons is less than that imparted to a unit mass by 1 rep of photons. In addition, the $G$ value for similar chemical systems is lower for heavy particles than for electrons (5).

To determine the upper limit of the fast-neutron response of both systems, an alpha source was placed in a 1-ml volumetric flask containing the halogenated hydrocarbon. The disk was submerged to insure complete absorption of all alpha particles emitted. The quantity of stable acids generated per rep of alpha energy absorbed appeared to be about $1 / 10$ that produced per $r$ of gamma rays. This seems to indicate a chemical conversion efficiency of $10 \%$. It is assumed that the chemical conversion efficiencies are of the same order-of-magnitude for alpha particles, protons, and carbon recoil atoms (5).

How the energy absorbed in $1 \mathrm{gm}$ of tetrachloroethylene from 1 rep of fast neutrons compares with the energy absorbed from $1 \mathrm{r}$ of gamma rays in tissue was calculated approximately as shown in the Appendix. The calculations for the tetrachloroethylene system indicate that a maximum of $8.3 \%$ of the incident energy of 8-Mev neutrons is absorbed by the system when compared with a tissue dose. Since only $10 \%$ of the absorbed dose is utilized, the neutron response of the tetrachloroethylene system, depending on the neutron spectrum, is of the order of $0.83 \%$ or less. That is, if exposed to 1 rep of fast neutrons with no gamma rays present the dosimeter would generate $0.83 \%$ as much acid as it would from 1 rep of gammas.

\section{Field Use}

Both anhydrous systems have been tested in mixed fields of neutron and gamma radiation at the ORNL tower shielding facility, the Los Alamos prompt critical assembly, the Los Alamos Omega thermal reactor, and nuclear detonations. Comparison of
TABLE 2-Relative Dose-Rate Response*

\begin{tabular}{cc}
$\begin{array}{c}\text { Radiation rates } \dagger \\
(\mathrm{r} / \mathrm{min})\end{array}$ & $\begin{array}{c}\text { Acid yield } \ddagger \\
\left(\mathrm{mEq} \times 10^{-6} / \mathrm{ml} / \mathrm{r}\right)\end{array}$ \\
\hline 10 & 0.05 \\
100 & 0.05 \\
1,000 & 0.05 \\
2,500 & 0.05 \\
4,250 & 0.05
\end{tabular}

* Hexylresoreinol-stabilized anhydrous tetrachloroethylene system.

† Source: $\mathrm{Co}^{60}$. Total dose delivered was $10,000 \mathrm{r}$, regardless of rate.

Each value represents the average obtained by titrating 15 samples; titration was done with $10^{-3}-\mathrm{N} \mathrm{NaOH}$.

TABLE 3-Relative Temperature Dependence*

\begin{tabular}{cc}
$\begin{array}{c}\text { Temperature } \dagger \\
\left({ }^{\circ} \mathrm{C}\right)\end{array}$ & $\begin{array}{c}\text { Acid yield } \ddagger \\
\left(\mathrm{mEq} \times 10^{-6} / \mathrm{ml} / \mathrm{r}\right)\end{array}$ \\
\hline 5.0 & 0.047 \\
22.0 & 0.05 \\
37.0 & 0.05 \\
55.0 & 0.053
\end{tabular}

* Hexylresorcinol-stabilized anhydrous tetrachloroethylene system. All samples were flame-sealed in $8-\mathrm{mm}$ Neutraglassiliconed ampules.

$\dagger$ Dosimeters exposed after 4-hr equilibration in a constant-temperature water bath.

\$ Each value represents a mean of 15 titrations; titration was accomplished with $10^{-3}-\mathrm{N} \mathrm{NaOH}$.

TABLE 4-Acid production as a function of total dose*

\begin{tabular}{cc}
$\begin{array}{c}\text { Total dose } \\
(r)\end{array}$ & $\begin{array}{c}\text { Acid yield } \neq \\
\left(\mathrm{mEq} \times 10^{-6} / \mathrm{ml} / r\right)\end{array}$ \\
\hline 1,000 & 0.05 \\
10,000 & 0.05 \\
100,000 & 0.05 \\
200,000 & 0.045 \\
300,000 & 0.03
\end{tabular}

* Hexylresorcinol-stabilized anhydrous tetrachloroethylene.

$\dagger$ Dose rate $=1,000 \mathrm{r} / \mathrm{min}, \mathrm{Co}^{60}$

$\ddagger$ Each value represents a mean of 15 titrations; titration was accomplished with $10^{-3}-\mathrm{N} \mathrm{NaOH}$ 


\section{Other Gamma-Ray Dosimeters}

At present there is no gamma-ray dosimeter that has all the desired features for dosimetry work in mixed radiations. The most frequently used laboratory device is the graphite-wall $\mathrm{CO}_{2}$ flow chamber. This is primarily a dose-rate, gamma-detecting system, but it has an undesirable energy-dependent neutron response (3). The exact value of its neutron response is not known. However, this dosimeter does help obtain order-of-magnitude information.

The photographic-film dosimeter system of Ehrlich and Fitch (4) is another common method of gamma-ray detection. The fast-neutron and thermalneutron response of the film is only vaguely known, and consequently this system has limited applications for mixed dosimetry work until exact neutron-response data are obtained.

Almost every laboratory concerned with $X$-and gamma-ray dosimetry uses the Victoreen condenser-type ionization chamber. The work of Aebersold (5) reveals that the Victoreen chamber responds to neutrons but is not suitable for use as a neutron dosimeter. Variations in the neutron response between one Victoreen chamber and another may be as great as $20 \%$ (6). Since the Victoreen responds to $X$ - and gamma radiation more efficiently than it does to neutron radiation, its accuracy for gamma-ray measurements in the presence of neutrons will depend on the relative proportion of the neutrons and gamma rays.

these dosimeter systems with the $\mathrm{C}-\mathrm{CO}_{2}$ flow chamber at the tower shielding facility provided much valuable information. The $\mathrm{C}-\mathrm{CO}_{2}$ chamber measurements were made at low power and extrapolated to the actual power settings used. The chemicals indicated a gamma dose somewhat higher than the $\mathrm{C}-\mathrm{CO}_{2}$ flow chamber extrapolation; results were highly reproducible.

Calibrations at Los Alamos indicate that the thermal-neutron sensitivities of the anhydrous chloroform and tetrachloroethylene systems are $6.7 \times$ $10^{-10}$. and $1.71 \times 10^{-10} \mathrm{rep} / \mathrm{n} / \mathrm{cm}^{2}$ respectively. An experiment conducted during Operation Teapot has indicated that the maximum value for the fast-neutron response of the anhydrous chloroform system is about $2 \%$.

\section{APPENDIX}

We wish to evaluate $P$, the ratio of energy absorbed in $1 \mathrm{gm}$ of tetrachloroethylene from 1 rep of fast neutrons to the energy absorbed in $1 \mathrm{gm}$ of tissue from $1 \mathrm{r}$ of gamma rays. Now

$$
\begin{aligned}
P & =\frac{E_{a b_{s}} \text { due to } 1 \text { rep } n_{f} \text { in chemicals }}{E_{a b_{s}} \text { due to } 1 \text { rep of } \gamma \text { in tissue }} \\
& =\frac{E_{a b s} \text { due to } 1 \text { rep } n_{f} \text { in chemicals }}{E_{a b s} \text { due to } 1 \text { rep of neutrons in tissue }} \\
& =F D_{c h e m}(E) / F D_{b}(E)
\end{aligned}
$$$$
=D_{\text {chem }}(E) / D_{t}(E)
$$

where $F$ is the flux in $n / \mathrm{cm}^{2}$ needed to make a rep, and $D_{\text {chem }}(E)$ and $D_{t}(E)$ are the dose or energy absorbed per gram per $n / \mathrm{cm}^{2}$ for chemical and tissue respectively. Since

$$
D_{\text {chem }}(\mathrm{Mev} / \mathrm{gram})=\left[E \Sigma_{\mathbf{i}} \sigma_{i} f_{i} Q_{\mathbf{i}}\right]
$$

then

$$
P=\left[E \Sigma_{i} \sigma_{i} f_{i} Q_{i} \text { chemical }\right] / D_{t}(E)
$$

where $D_{\text {chem }}=\operatorname{dose}(\mathrm{Mev} / \mathrm{gm}), E=$ neutron energy ( $\mathrm{Mev}$ ), $\Sigma_{i}=$ summation over-all types of atoms present, $\sigma_{i}=$ cross section of the $i$ th type of atom $\left(\mathrm{cm}^{2}\right) f_{i}=$ average fraction of energy lost by the neutron during its collision with the ith kind of atom, (assuming isotropic center of mass scattering, $f_{i}=2 M /(m+M)^{2}$ where $m$ is the neutron mass and $M$ is the mass of the recoil atom), $Q_{i}=$ number of atoms of the element $i$ in $1 \mathrm{gm}$ of medium

From the above, since tetrachloroethylene is $\mathrm{C}_{2} \mathrm{Cl}_{4}$

$$
P=\frac{E\left[\sigma_{\mathrm{c}} f_{\mathrm{c}} Q_{\mathrm{c}}+\sigma_{\mathrm{Cl}} f_{\mathrm{cl}} Q_{\mathrm{c} 1}\right]}{D_{s}(E)}
$$

where $\sigma_{\mathrm{C}}, f_{\mathrm{C}}, Q_{\mathrm{C}}$ are appropriate values for carbon and $\sigma_{\mathrm{Cl}}, f_{\mathrm{Cl}}, Q_{\mathrm{cl}}$ are the appropriate values for chlorine. We used $Q_{\mathrm{C}}=7.2 \times 10^{21}, f_{\mathrm{c}}=0.14, Q_{\mathrm{c}} f_{\mathrm{C}}$ $=1.01 \times 10^{21}, Q_{\mathrm{cl}}=14.5 \times 10^{21}, f_{\mathrm{Cl}}=$ $0.053, Q_{\mathrm{cl}} f_{\mathrm{Cl}}=0.77 \times 10^{21} ; D_{\imath}(E)$ values are from Hurst (2).

Table 5 shows values for $P$ that are obtained by inserting the values for $\sigma_{\mathrm{C}}$ and $\sigma_{\mathrm{Cl}}(8)$. Note that the upper limit for $P$ is $8.3 \%$ at $8 \mathrm{Mev}$.

The author wishes to express appreciation for the interest shown and guidance given by G. S. Hurst, John A, Auxier, and Karl Z. Morgan of ORNL. The author is also indebted to $W . H$. Langham and $P$. Harris of Los Alamos for their calibration and evaluation of these systems.

Also, without the unlimited cooperation of the physics and engineering department of the Radiobiological Laborctory of The University of Texas and the United States Air Force. Austin, Texas, none of these studies could have been completed.

\section{BIBLIOGRAPHY}

1. G. S. Hurst, R. H Ritchie, H N Wilson. A count-rate method of measuring fast neutron tissue dose, Rev. Sei. Instr. 22, 981 (1951)

2. G. S. Hurst. An absolute tissue dosimeter for fast neutrons, Brit. J. Radiol. 27, 353 (1954)

3. G. S. Hurst, W. A, Mills, F, P. Conte, A, C Gupton. Principles and techniques of mixed radiation dosimetry-applicstion to acute lethality studies of mice with the cyclotron. lethality studies of mice with the cyclotron,
Radiation Research 4, No. 1, (1956)

4. M. Ehrlich, S. H. Fitch. Photographic x and gamms-ray dosimetry, Nocleonics 9 No. 9, (1951)

5. L. Ehrinberg, A. Saeland. Chemical dosimetry of radiations giving different ion densities. An experimental determination of $G$ values of An experimental determination of

6. P. C. Aebersold, G. A. Anslow, Fast neutron energy absorption in gases, walls, and tissue, Phys. Rev, 69, 1 (1946)

7. H. H. Rossi cited by J, W. Boag. The relative effeiency of different ionizing radiations. NBS Report $\$ 2946$ (National Buresu of Standards, U. S. Department of Commerce, Washington, D. C., 1953)

8. D. J. Hughes, J. A. Harvey. Neutron crose sections, BNL-325 (1955)

* Personal communication, G. S. Hurst, Oak Ridge National Laboratory. 


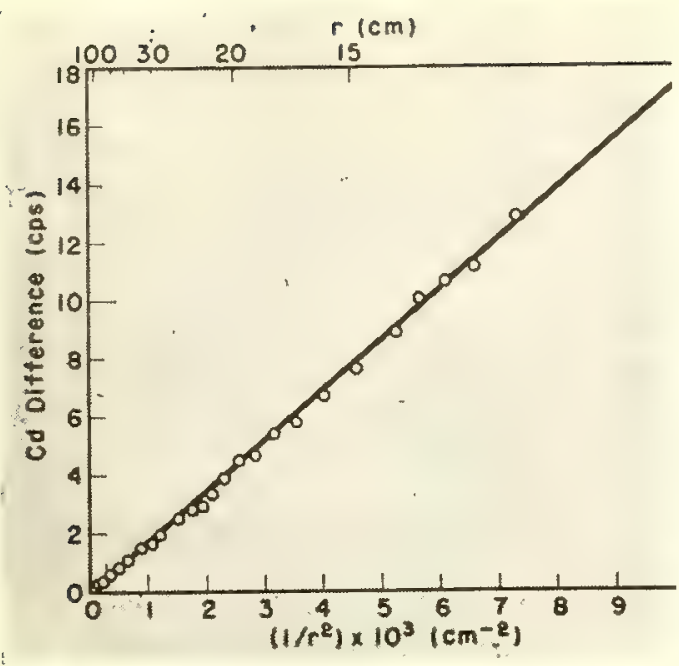

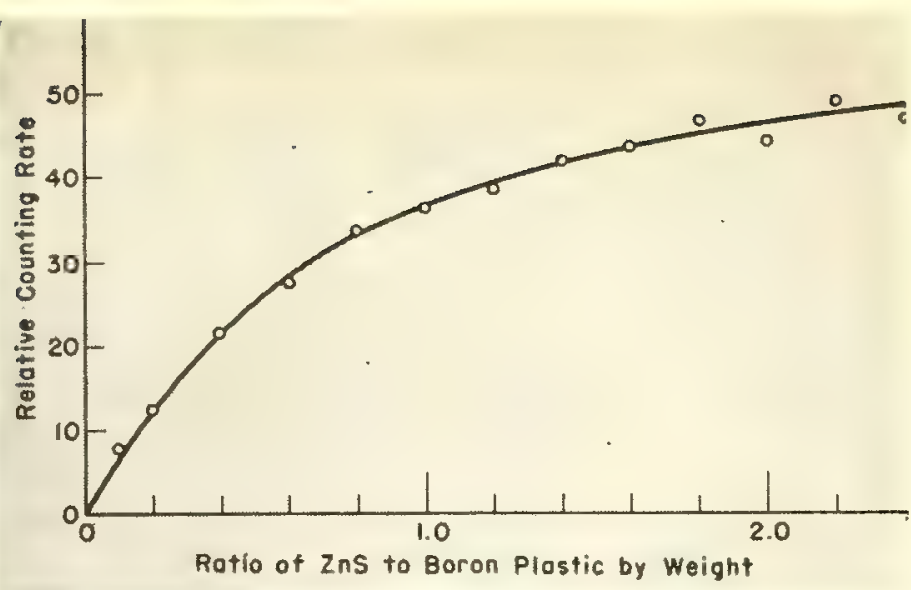

FIG. 2. Relative efficiency of neutron phosphor as a function of composition. Phosphor thickness $=1.25 \mathrm{~mm}$

FIG. 1. Counting rate for a spherical neutron source vs inverse square of the distance. Although not well shown by the figure, effect of room scattering becomes appreciable at large $r$

\section{High-Efficiency Slow-Neutron}

\section{A mixture of boron-containing plastic and $\mathrm{ZnS}(\mathrm{Ag})$ phosphor has been optimized to give slow-neutron counters with $33 \%$ efficiency yet only $1 \%$ gamma-ray contribution when counting radiations from a Po-Be source. Construction and optimization are given}

A SLOW-NEUTRON SCINTILlator such as the $\mathrm{ZnS}(\mathrm{Ag})$-boron-compound type $(1-6)$ described here operates in three principal, closely related stages:

1. A slow neutron passing through the scintillator is captured by a $\mathrm{B}^{10}$ nucleus.

2. The resultant energetic alpha particle reaches a $\mathrm{ZnS}(\mathrm{Ag})$ granule with sufficient residual energy to cause a scintillation.

3. Light from the scintillation travels to the photomultiplier photocathode and reaches it with sufficient intensity to cause a recognizable pulse at the anode.

The net efficiency of the scintillator is related to the probability that all three of these events proceed in succession, that is, to the product of the separate probabilities for each of the three events.

The probability for event 1 is $P_{c}=1-e^{-N \sigma}$, where $\sigma$ is the microscopic slow-neutron capture cross section of $\mathrm{B}^{10}$, and $N$ is the areal density of $B^{10}$ nuclei. This equation holds so long as there is little competition with slow-neutron capture in the scintillator; this has been the case with all the scintillators examined in this study. The larger $N$ is, the greater is $P_{c}$, but that as $P_{c}$ approaches unity, further increases in $N$ accomplish very little.

The probabilities for 2 and 3 are not susceptible to such direct analysis. The probability of 2 , of the resultant alpha particle reaching the $\mathrm{ZnS}(\mathrm{Ag})$, depends on the density of material, the path length involved, and the minimum residual energy required. These, in

turn, depend on secondary factors such as relative amount of $\mathrm{ZnS}(\mathrm{Ag})$ in the material, size and shape distribution of the $\mathrm{ZnS}(\mathrm{Ag})$ particles, and the minimum usable light per scintillation.

The probability of 3 , of sufficient scintillation light reaching the photomultiplier, depends on the strength of the scintillation, the transmissivity of the light path to the phototube, the

\section{Grooved Scintillators Are Best}

$A$ thin scintillator made with a grooved or corrugated surface provides more scintillating material, and hence more $B^{10}$ per unit projected area of scintillator, while maintaining a usable light transmittance. Such a scintillator was made by using a transparent Bioplastic mold, cast from a negative steel mold (see figure). The scintillator was cast at about $180^{\circ} \mathrm{C}$ and moderate pressure in the plastic mold. It was used with the grooved scintillator surface facing the photomultiplier, and with an aluminum foil covering the opposite surface. The improvement in scintillator efficiency was $\sim 60 \%$, resulting in an over-all efficiency of about $\$ 3 \%$ for

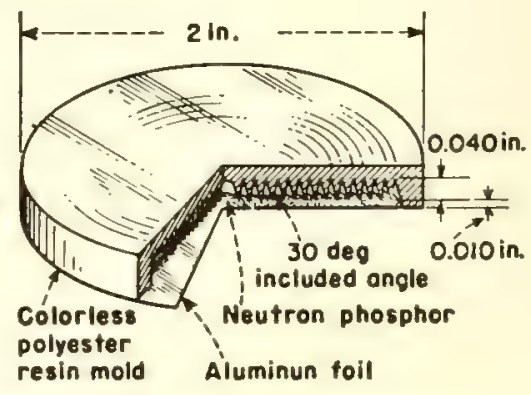

Improved slow-neutron scintillator

thermal neutrons at a discriminator setting such that $\mathrm{Po}-\mathrm{Be}$ gamma rays gave only $1 \%$ as many counts as thermal neutrons. 

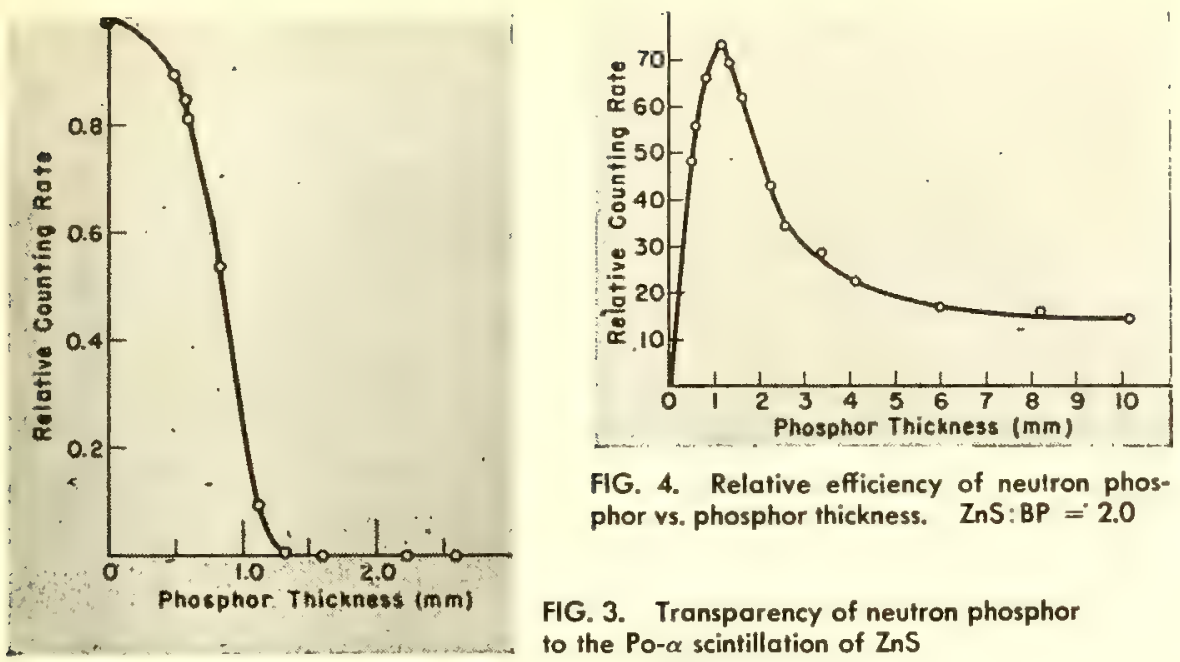

FIG. 4. Relative efficiency of neutron phosphor vs. phosphor thickness. $\mathrm{ZnS}: \mathrm{BP}=\mathbf{2 . 0}$

FIG. 3. Transparency of neutron phosphor to the Po- $\alpha$ scintillation of $\mathrm{ZnS}$

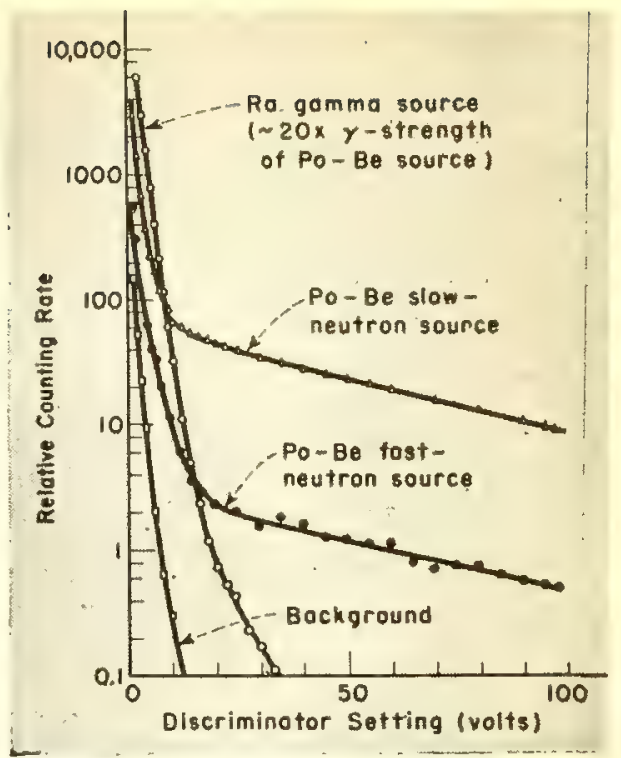

FIG. 5. Response to neutrons and gamma rays vs discrimination level

\section{Scintillation Counters}

By K. H. SUN, P. R. MALMBERG, and F. A. PECJAK

Westinghouse Electric Corp.

East Pitlsburgh, Pennsylvania

sensitivity of the photomultiplier, and the amount of background noise present. This probability is further complicated by secondary factors such as length of light path, transparencies of the media comprising the path, number of interfaces between different media in the light path, and transmissivities of such interfaces.

Consideration of these various factors shows that a change in certain of them may be favorable to one of the three main stages involved in counting a slow neutron, and unfavorable to one or both of the other two. Thus, increasing the proportion of $\mathrm{ZnS}(\mathrm{Ag})$ in the scintillator increases the probability of an energetic $\alpha$-particle reaching the $\mathrm{ZnS}(\mathrm{Ag})$, but reduces the probability for neutron capture and renders the scintillator more opaque. Because of the complexity of these many factors and their interrelationships, development and optimization of the slowneutron scintillator has been on an empirical basis.

What was optimized? We aimed primarily at high efficiency, ease of manufacture, and low cost. Although the primary consideration of merit for a scintillator is usually the counting efficiency for a specific type of radiation, other factors can be important, depending on the intended use. Examples are: sensitivity to other types of radiation, inherent noise level, usable counting life, and ruggedness.

\section{Test Equipment}

To test the scintillators we needed only slow-neutron sources and some standard electronics.

Slow-neutron sources. A slow-neutron suurce with minimal spurious radiation was required to obtain the relative efficiencies of the neutron scintillators as a function of a given design parameter, and to measure absolute efficiencies of the better scintillators. Two sources were used, each consisted of a Po-Be fast-neutron source surrounded by paraffin moderator. The Po-Be sources were 0.7-in.-diameter 0.7-in.-long cylinders and emitted $\sim 2 \times 10^{5}$ fast neutrons/sec.

In one of the slow-neutron sources, the Po-Be source was fixed at the center of a 6-in. cube of paraffin in a thin steel case. This source was used for obtaining most of the comparative data.

The second source consisted of an 8-in.-diameter sphere of paraffin in a 316 -in.-thick aluminum shell, the PoBe source being fixed at the center. J. A. DeJuren of the National Bureau of Standards calibrated the thermalneutron flux at the surface of this sphere against the Bureau's absolute-neutronflux standard, using manganese-foilactivation and cadmium-difference techniques (7). The flux was determined with an accuracy of $\pm 10 \%$, and was about 24 thermal neutrons $/ \mathrm{cm}^{2} /$ sec at the outer surface.

This spherical neutron source provides a simple $1 / r^{2}$ relationship of neutron flux to distance from source. Thus the absolute counting efficiency can be determined for a given scintillator at an arbitrary distance $r$ from the source center. Careful measurements of relative thermal neutron flux versus distance from the source center were made with the cadmium-difference method, using one of the better scintillators shielded from stray or externally reflected radiation by suitably disposed sheet cadmium. Figure 1 demonstrates that the $1 / r^{2}$ relationship does hold quite well for this source.

Circuitry. Both the 2-in. DuMont 6292 and 5-in. DuMont K1198 photo- 
multipliers were used with conventional positive-grounded dynode voltages and cathode-follower output feeding a linear amplifier, discriminator, and scaler.

\section{Optimizing the Scintillator}

An iterative procedure is generally required to optimize this type of scintillator. Parameter limits for a reasonable scintillator were established by a series of rough preliminary tests. These were followed by a more exacting series. The criteria examined most closely were sample thickness and ratio of $\mathrm{ZnS}(\mathrm{Ag})$ to boron plastic. ${ }^{*}$ Other factors investigated included particle size, type, or brand of $\mathrm{ZnS}(\mathrm{Ag})$ and its particle size, light attenuation characteristic of a fixed-composition scintillator, ratio of boric acid to glycerol in the boron plastic, and response of the scintillator to fast neutrons and gammas.

$\mathrm{ZnS}(\mathrm{Ag})$ :boron plastic ratio. Initial work showed that a high $\mathrm{ZnS}(\mathrm{Ag})$ : boron plastic (BP) ratio would be advantageous and that the optimum thickness would be $\sim 1-2 \mathrm{~mm}$. On this basis, a series of scintillators $5 \mathrm{~cm}$ in diameter $\times 1.2 \mathrm{~mm}$ thick of varying $\mathrm{ZnS}(\mathrm{Ag}): \mathrm{BP}$ ratio were compared in a slow-neutron flux. These tests, the results of which are shown in Fig. 2,

\footnotetext{
* Preparation is discussed below.
}

established that the neutron counting efficiency increases with $\mathrm{ZnS}(\mathrm{Ag}): \mathrm{BP}$ ratios up to $2.2: 1$; maximum efficiency seemed to be not far beyond that ratio. Experimentally, this ratio is limited by mixing difficulties at the higher values, where the mixtures become quite dry. For this reason, the quite-workable ratio of $2: 1$ was used subsequently.

Thickness. The variation with seintillator thickness of its transmittance for light from an alpha-particle scintillation was studied briefly. A $\mathrm{Po}^{210}$ alpha-source was covered with a $\sim 10-\mu$ layer of $\mathrm{ZnS}(\mathrm{Ag})$ and the combination used as a scintillation light source. Scintillators of $2: 1 \quad \mathrm{ZnS}(\mathrm{Ag}): \mathrm{BP} 2.4$ $\mathrm{cm}$ in diameter and of various thicknesses were interposed between this light source and the photomultiplier tube. Figure 3 shows that scintillators of this composition become rather opaque to the scintillation light when thicker than $1 \mathrm{~mm}$ or so. These measurements are at a discriminator setting such that maximum sensitivity without excessive background is obtained.

More detailed experiments on thickness versus scintillator efficiency were conducted using a series of scintillators ranging from 0.5 to $10 \mathrm{~mm}$ in thickness. The results are shown in Fig. 4 . It will be noted that a pronounced maxi- mum occurs when $\sim 1.2 \mathrm{~mm}$ thick.

Boric acid: glycerol ratio. To find the best ratio of boric acid to glycerol in the boron plastic 5 -cm-diameter $X$ 1.2-mm-thick disks having a $\mathrm{ZnS}(\mathrm{Ag})$ : $\mathrm{BP}$ ratio of $2: 1$ were used. Past a boric acid: glycerol weight ratio of $4: 1$, further increases in boric acid did not materially increase the fractional boron content nor, consequently, the counting rate. However, the higher ratios make the material less hygroscopic. Hence, the earlier decision to use a $6: 1$ ratio for most studies emerged as a good one.

Types and sizes of $\mathrm{ZnS}(\mathrm{Ag})$. Using $2: 1$ ratio of $\mathrm{ZnS}(\mathrm{Ag}): \mathrm{BP}$ and $5-\mathrm{cm}-$ diameter $\times 1.2-\mathrm{mm}$-thick disks as before, relative counting efficiencies were determined for five commercial $\mathrm{ZnS}$ (Ag) phosphors-Du Pont 2B1 (formerly 1410), Sylvania CR20 and 132, and RCA 33220A and F2030. The results indicate that the Du Pont 2B1 phosphor is somewhat superior for this particular application. The average grain size of this phosphor is about $10 \mu$.

To get some idea of the effect of particle size on efficiency, fractionated Du Pont 2B1 of grain sizes 6 and $10 \mu$, and 25- $\mu$ Du Pont 1101 were compared with the regular unfractionated $2 \mathrm{~B} 1$ phosphor. These tests showed that the unfractionated phosphor was as

\section{Preparation of Slow-Neutron Scintillators}

To make a good slow-neutron detector of the type described, the boron-containing material should be transparent and colorless, contain a large proportion of $B^{10}$, and be susceptible to intimate mixing with the $Z n S(A g)$. There are two methods that suggest themselves for the combining of $Z n S(\mathrm{Ag})$ powder with the boron-containing material. One, using the boron substance also in the powdered form, is simply to mix the two powders together and then press the mixture into a compact disk. The second is to employ a boron material which can be prepared initially in liquid form, mixing the $\mathrm{ZnS}(\boldsymbol{A} g)$ with it while liquid, and subsequently solidifying the combination. The second method, while usually more difficult, provides more intimate contact between the $\mathrm{ZnS}(\mathrm{Ag})$ and the boron compound and is the method used for most of the scintillators considered here.

A number of boron compounds fulfilling the requirements for transparency and freedom from coloration can be found, such as $\mathrm{BN}, \mathrm{B}_{2} \mathrm{O}_{3}$, or $\mathrm{H}_{3} \mathrm{BO}_{3}$. The $\mathrm{BN}$ contains more boron (43.5 wt $\%$ ) but is refractory and does not melt at temperatures below the sublimation temperature for $\mathrm{ZnS}$. Therefore, it is unusable in a liquid form. $\mathrm{B}_{2} \mathrm{O}_{3}$, containing 33.1 wt \% boron, melts at about $580^{\circ} \mathrm{C}$. It is very viscous even at $1,000^{\circ} C$ and hence difficult to manipulate. Although it is possible to lower the viscosity of molten $\mathrm{B}_{2} \mathrm{O}_{3}$ by addition of small amounts of oxides or fuorides of alkali metals, there remains the high melting temperature which tends to harm the luminescent properties of $Z n S(A g)$.

\section{Preparation of Boron Plastic (BP) and $\mathrm{HBO}_{2}$}

Two boron compounds that have proved especially suitable for this application are

1. A borate polyester plastic containing as high as $20 \%$ boron, hereafter referred to as boron plastic or $\mathrm{BP}, 2 . \mathrm{HBO}_{2}$ glass, containing about $26 \%$ boron. Both are quite readily incorporated with the $Z n S(\mathrm{Ag})$ without harming it

BP. The combination of boric acid and glycerol $\left(\mathrm{C}_{3} \mathrm{H}_{8} \mathrm{O}_{3}\right)$ to form a colorless, transparent polyester plastic has been known for some time. The molar proportions commonly used are 1:1. To increase the relative proportion of boron in this plastic, various weight ratios of boric acid to glycerol, ranging up to $10: 1$, were examined. At this high ratio, the viscosity of the polyester melt becomes high, and there is danger of charring. The boric acid: glycerol weight ratio of $6: 1$ (about $9: 1$ molar ratio) proved to be the highest that would permit ease of handling of the plastic, and is the ratio which was used for most of the work.

Boron plastic of $6: 1$ weight ratio was prepared according to the following receipt: $20 \mathrm{gm}$ of glycerol is first heated to near boiling in a round-bottom 200-ml flask. Then $120 \mathrm{gm}$ of crystalline $\mathrm{H}_{3} \mathrm{BO}_{3}$ is gradually added with continuous shaking and heating. The initial reaction is rapid, the $\mathrm{H}_{3} \mathrm{BO}_{3}$ disappearing quickly with considerable liberation of steam. As the reaction proceeds, solution of $\mathrm{H}_{3} \mathrm{BO}_{3}$ takes place more gradually. At the moment the final $\mathrm{H}_{3} \mathrm{BO}_{3}$ has been added and dissolved, the clear liquid boron plastic is poured onto a cold thick aluminum plate. The slightly cloudy and brittle plastic which results upon cooling is then stored in a dessicator in polyethylene bags until subsequent remelting and combining with $\mathrm{ZnS}(A g)$. This intermediate cooling state facilitates the many weighings necessary in combining the $B P$ and $Z n S(A g)$ in varying proportions for efficiency comparisons, but could be dispensed with in preparing a large number of scintillators of fixed composition. If the heating of the molten $B P$ is prolonged at the last stage, the cooled material will be clear and glasslike, and further heating will initiate charring. The heat delivered in the remelting stage will usually remove sufficient additional water to render the $B P$, as incorporated in the neutron scintillator, clear and colorless. Prepared 
good as other sizes. Therefore unseparated Du Pont 2B1 phosphor was used in all other tests.

\section{Scintillator Response}

The response of the scintillator to gamma rays, slow neutrons and fast neutrons is shown in Fig. 5. It is clear that gamma rays of the intensity present in the $\mathrm{Po}-\mathrm{Be}$ neutron source $(0.1$ $\mathrm{mr} / \mathrm{hr}$ when neutron flux was $24 \mathrm{n} / \mathrm{cm}^{2} /$ sec) can be easily discriminated against. Although the hydrogen content of the scintillator makes it slightly sensitive to fast neutrons, the counting efficiency for them is very small. In critical measurements of thermal-neutron fluxes, correction for fast-neutron counts can be made by $\mathrm{Cd}$ difference.

Absolute efficiency. A 2.40-cmdiameter scintillator was used in measuring the absolute efficiency of boronplastic slow-neutron scintillators of optimized thickness $(1.2 \mathrm{~mm})$ and $\mathrm{ZnS}(\mathrm{Ag})$ : BP ratio $(2: 1)$. The experiment was performed with the scintillation counter set in front of the calibrated slow-neutron source. Using the thermal neutron flux calibrated at the surface of the spherical source, together with the $1 / r^{2}$ flux dependence and the measured area of the scintillator, it was possible to evaluate the absolute efficiency straightforwardly.

With discriminator set so that the response to gamma rays from the $\mathrm{Po}$ Be source $(0.1 \mathrm{mr} / \mathrm{hr}$ when neutron flux was $24 \mathrm{n} / \mathrm{cm}^{2} / \mathrm{sec}$.) was slightly less than $1 \%$ of thermal-neutron response the efficiency of the scintillator for thermal neutrons was $21 \%$ : Note that if gamma-rays are of much greater relative intensity than from the $\mathrm{Po}-\mathrm{Be}$ source, the interference at this discrimination setting can be appreciable.

\section{Conclusions}

The scintillation counter has advantages of ruggedness, better time resolution, and superior geometrical definition when compared with $\mathrm{BF}_{3}$ counters. The boron-containing neutron scintillator developed in the present investigation is believed to be more efficient for thermal-neutron scintillation counting than other similar scintillators. A measured efficiency of $33 \%$ for thermal neutrons has been obtained with the boron-plastic-type scintillator having one corrugated surface; from this an efficiency of $37 \%$, can be estimated for a similar scintillator of the $\mathrm{HBO}_{2}$ type. This scintillator can be further and markedly improved by using pure or enriched $\mathrm{B}^{10}$ instead of natural boron, which contains only $19 \mathrm{wt}_{\%} \mathrm{~B}^{10}$.
Because of the high neutron counting efficiency of this scintillator and the large-area, thin-sheet geometry of the sensitive volume, it is possible to obtain a large solid angle for interception of neutrons from uncollimated sources.

The neutron scintillator developed here is also being used in photographic detection of neutron patterns. ${ }^{*}$ The photographic sensitivity has been found to be considerably greater than any previously known. These results will be reported later. (See p. $81-$ Ed.)

\section{* * *}

We are grateful to Michael Giuliano for his assistance in investigating the use of $\mathrm{HBO}$, in slow-neutron scintillators.

\section{BIBLIOGRAPHY}

1. K. H. Sun, Method of detecting elementary particle, U. S. Patent 2,534,932 (Applied June 19,1947-Granted December 19, 1950)

2. F. Sachs, Neutron detectors, Y-B 4-18 (1951)

3. D. E. Alburger, A slow-neutron detector, BNL-1233 (1952)

4. E. Gatti, E. Germagnoli, A. Persano, E. Zim. mer, Boron-layer scintillation neutron detectors, Nuovo Cimenio 9, No. 9, 1012 (1952)

5. P. G. Koontz, G. R, Keepin, J. E. Ashley $\mathrm{ZnS}(\mathrm{Ag})$ phosphor mixtures for neutron detection, Rev. Sci. Instr. 32, 154 (1955)

6. C. O. Muehlhause, Neutron scintillation counters, Nocleonics 14, No. 4, 38 (1956)

7. J. A. De Juren, H, Rosenwasser, Absolute calibration of the NBS standard thermalneutron density, $J$. Research Natl. Bur. Standards 62, No. 2, 93 (1954)

* In cooperation with E. O. Wollan of the Oak Ridge National Laboratory.

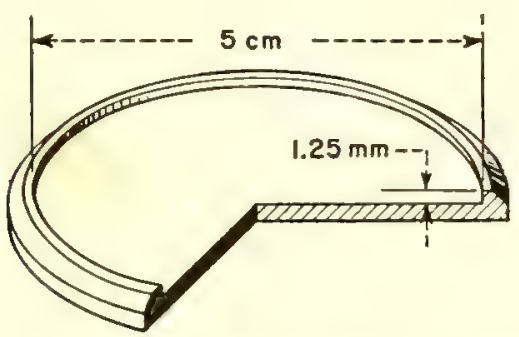

Metal mold for neutron phosphor

this way, the BP. contains about 19.5 wt \% boron and has a densily of $1.5 \mathrm{gm} / \mathrm{cm}^{3}$

$\mathrm{HBO}_{2}$. Not too well known is the fact that $\mathrm{H}_{3} \mathrm{BO}_{3}$ can be converted at moderate temperatures to a clear liquid of the approximate composition $\mathrm{HBO}_{2}$. About $5 \mathrm{hr}$ at $210^{\circ} \mathrm{C}$ is required to effect this for $200 \mathrm{gm}$ of $\mathrm{H}_{3} \mathrm{BO}_{3}$ in a covered $600-\mathrm{ml}$ beaker. The $\mathrm{HBO}_{2}$ glass prepared in this manner can be poured onto a cold aluminum plate to harden for dessicator storage. The measured boron content of this material is $25.5-26.5$ wt \% as compared with $24.7 \%$ computed for the formula $\mathrm{HBO}_{2}$.

\section{Assembly of Slow-Neutron Scintillator}

The final step in fashioning a neutron scintillator from the boron compounds just described consists of remelting, stirring in appropriate amounts of $Z n S(A g)$ powder, pouring or pressing into suitably shaped molds, and cooling. Much experimentation iranspired before the present procedures were evolved.

Using boron plastic (BP). A specific amount of BP was weighed out and remelted in an oil bath at $160^{\circ} \mathrm{C}$, the temperature at which the neutron-scintillator mixture becomes workable. A proportionate weight of $\mathrm{ZnS}(\mathrm{Ag})$, also preheated to $160^{\circ} \mathrm{C}$, was added to the melt with constant stirring and mixing. To prevent charring the boron plastic from prolonged heating, mixing was completed in one minule or less. The mixture was then transferred to a metal mold such as that shown here, covered with a $1 / 4$-in.-thick glass plate, and the excess mixture squeezed out in a small press with heavy metal platens. To prevent premature cooling and hardening of the mixture during this operation, the mold, glass cover plate, and press platens were preheated to $160^{\circ} \mathrm{C}$, and the transfer and pressing were effected as quickly as possible. Self-release of the scintillator from the mold after cooling and contracting was aided by treatment of both mold and cover glass with a mold-release agent, Dow Corning 20.

The resulting scintillator disk was backed with aluminum foil on one side to increase the light output from the opposite side, and the whole wrapped in Saran film for protection against atmospheric moisture. A transparent multipurpose type adhesive such as Cello-stick was used to improve optical contact between the Saran film and the scintillator surface. Scintillators prepared in this way were kept in a dessicator while not in use because of the hygroscopic nature of the boron plastic component. Large flat sheets of this scintillator have been made this way.

Using $\mathrm{HBO}_{2}$. The preceding method was also followed with $\mathrm{HBO}_{2}$, except that the operating temperature was kept at about $210^{\circ} \mathrm{C}$ instead of $160^{\circ} \mathrm{C}$. It was diffcult thoroughly to mix the $\mathrm{ZnS}(\mathrm{Ag})$ with the molten $\mathrm{HBO}_{2}$, because of the higher viscosity of this boron compound. One way to improve mixing of these two materials is to reduce the $\mathrm{HBO}_{2}$ to a 68-mesh (or smaller) powder and mix with the $Z n S(A g)$ powder prior to heating. $A$ second and more reproducible method was to put the previously mixed $\mathrm{ZnS}(\mathrm{Ag})$ and $\mathrm{HBO}_{2}$ powders into a mold to overflowing, cover the mold with a thick glass plate, and place in a heated press. Press temperature could be as low as $170^{\circ} \mathrm{C}$. 


\section{Instrumentation and Controls for HRT}

\author{
Here's a description of the over-all system for \\ control of the HRT as well as the components \\ that make it up. In particular several \\ temperature, level, and pressure transmitters \\ are described in detail
}

By D. S. TOOMB, Jr.

Oak Ridge National Laboralory, Oak Ridge, Tennessee

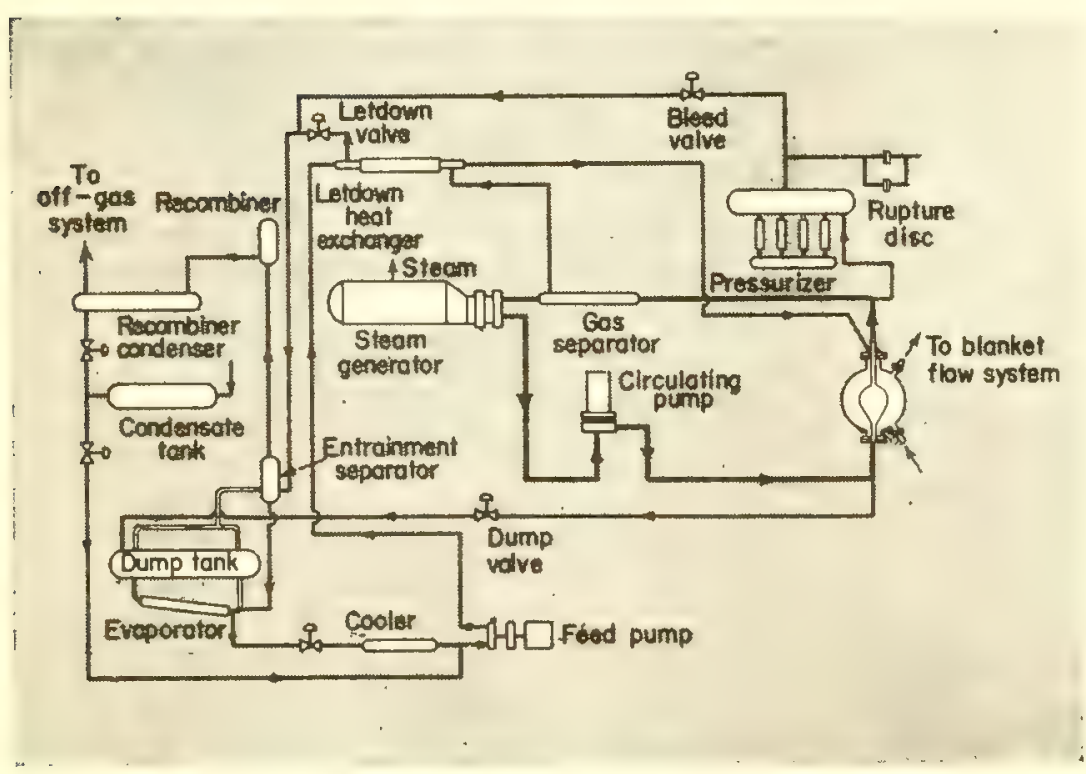

FIG. 1. Basic flow sheel for HRT. Heary lines show fuel flow
The inherent stability of the aqueous homogeneous reactor system* eliminates the need for control or safety rods. Control functions are performed by valves which regulate the fuel concentration and the rate of steam removal from the heat exchangers.

The instrumentation and controls system for the Homogeneous Reactor Test (1-3), the second experimental aqueous homogeneous reactor to be constructed under the $\mathrm{AEC}$ program for the evaluation of power reactors, was heavily influenced by the need for extreme reliability. All components exposed to the process fluid are absolutely leaktight and resistant to high levels of nuclear radiation. Special liquid-level transmitters have been developed for use under the reactor operating conditions, and all-welded construction was used broadly.

\section{Basic Flow Sheet}

Figure 1 indicates the basic flow sheet for HRT. Heat is generated in the 32-in.-diameter core vessel by fission. Steam from the heat exchanger goes to a turbine generator (or to an air-cooled condenser at power levels above the rating of the turbine). Also shown are the circulating pump that circulates the fuel at $400 \mathrm{gpm}$; and an electrically heated pressurizer that prevents boiling in the circulating stream and whose vapor space provides a surge volume to damp reactivity excursions.

Also shown is a secondary stream consisting of gas and entrained liquid removed from the nrain stream by the gas separator; this gas-liquid mixture flows to the low-pressure dump tanks and gas recombiners through the letdown valve. The liquid loss from the high-pressure system is made up by continuous feed-pump operation.

The blanket flow system is identical but circulates a different fluid (heavy water at $230 \mathrm{gpm}$ during initial operation, fertile material later).

The 60 -in-i,d. blanket vessel is designed to withstand the 2,000-psi operating pressure. But the $5 / 16$-in.-

* A homogeneous nuclear reactor is one in which the fuel material is evenly dispersed throughout the moderator as differentiated from reactors using solid fuel elements. Aqueous homogeneous reactors are desirable because the fluid state of the fuel and the properties of light and heavy water as a moderator permit the design of a system with high power density and low fuel inventory. 
thick zirconium-alloy core vessel has a much lower pressure rating, since the core-to-blanket differential pressure is controlled and is also limited by rupture dises. (Use of a thin core vessel minimizes absorption of neutrons passing into the blanket region.)

\section{Key Control Loops}

Key loops for HRT, shown in Fig. 3 , control the following:

Core pressure is controlled at 2,000 psi from sensed pressure by the proportioning of power to the pressurizer electric heaters; the blanket pressure is similarly controlled by a core-to-blanket differential-pressure signal.

Pressurizer liquid level is controlled from sensed level by pneumatic control of the letdown valve. (Electric control actions from the signals from pneumatic transmitters and transducers are derived from pressure switches. Solenoid-actuated pilot valves provide electric interlock control of the pneumatic signals to final control elements.)

Reactor power is controlled by turbine-governor (or manual) signal to a valve throttling steam from the core heat exchanger. Because of the large negative temperature coefficient and the low heat capacity, this reactor system cannot produce more heat than is being removed, except for very short times. Heat is removed by opening the steam throttling valve, which causes a decrease in the temperature of the fuel leaving the heat exchanger. Because of the negative temperature coefficient of the system, this cooler fluid entering the core causes an increase in reactivity and the fuel is reheated until it overcomes the excess reactivity.

Average core temperature is controlled by varying the concentration of the fuel solution. That is, the circulating fuel solution can be concentrated or diluted by pumping water or more concentrated fuel to the reactor core. The nominal core outlet temperature when the design power of $5 \mathrm{Mw}$ of heat is being produced is $300^{\circ} \mathrm{C}$, and the inlet temperature is about $250^{\circ} \mathrm{C}$. The inlet and outlet temperature will vary with the power extraction, while the average temperature is a function only of fuel concentration.

Blanket temperature is controlled by a signal, derived from the difference in average core and blanket temperature, that operates the steam valve of the blanket heat exchanger.

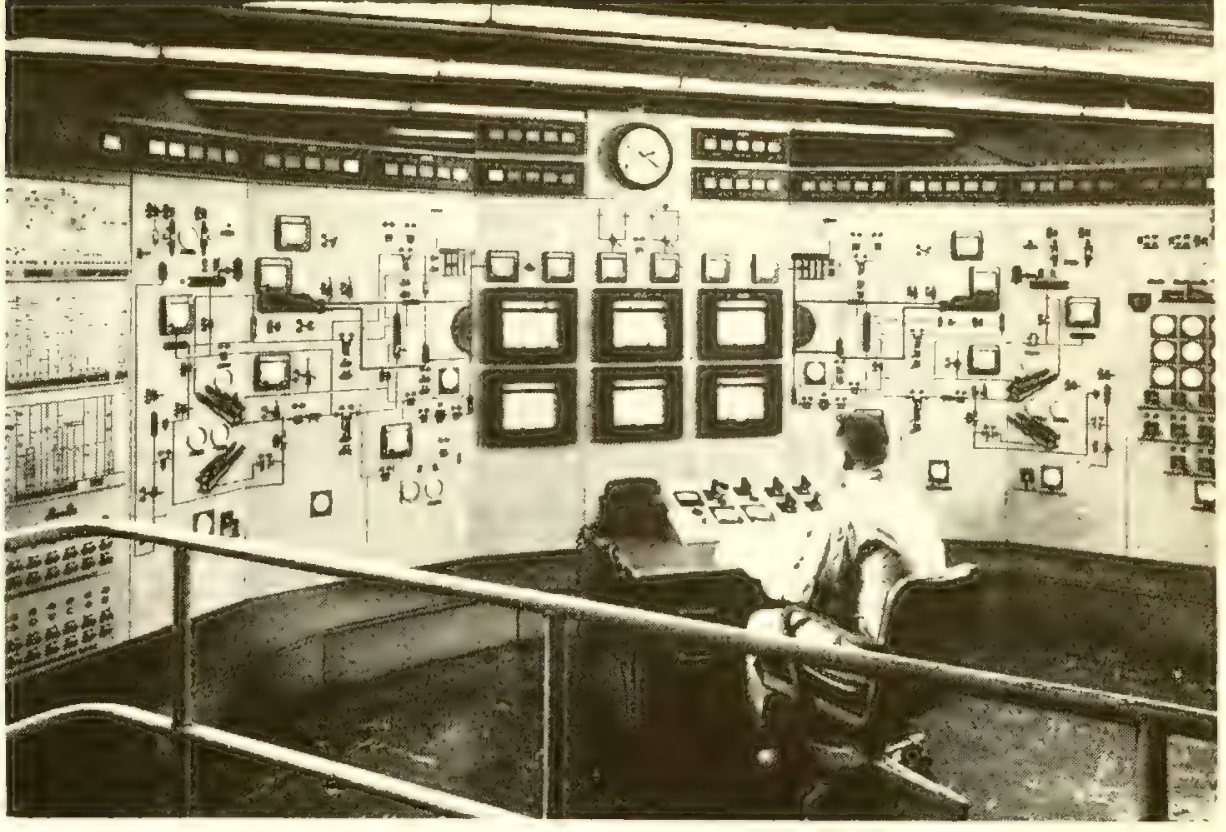

FIG. 2. Main control board and console for HRT

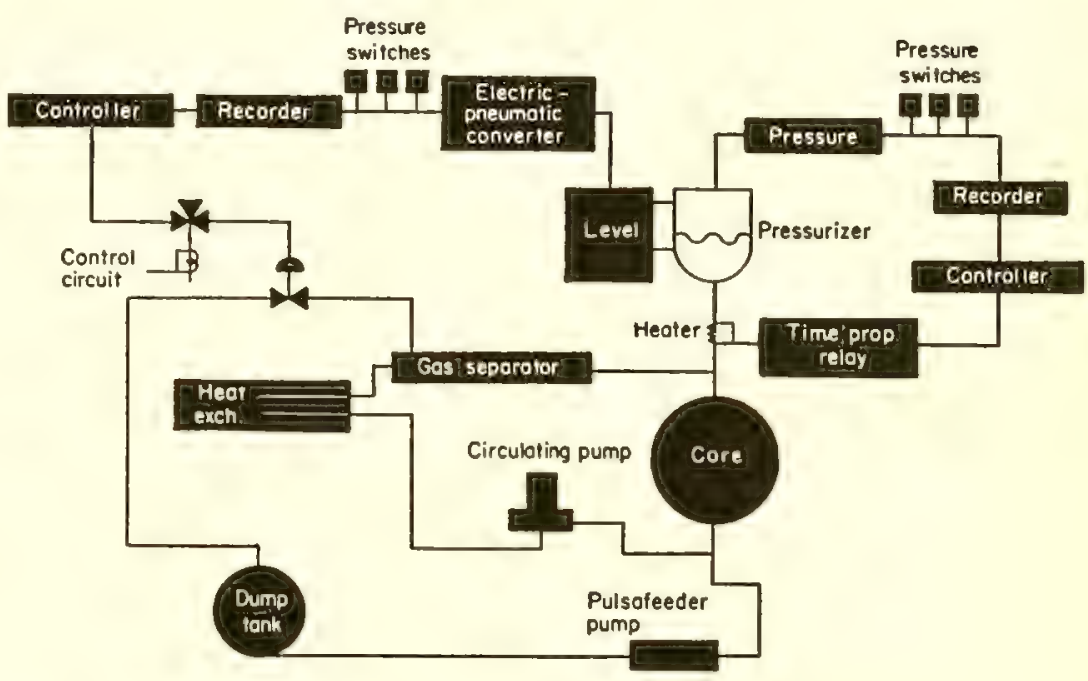

FIG. 3. Key control loops

\section{Plant Layout}

The reactor equipment exposed to the radioactive fuel and blanket solutions is located in a $54 \times 31 \times 25$-ftdeep steel tank lining an excavation. The top of this tank is flush with the building floor and ground level for economy in shielding. Conduits and piping from this reactor cell enter underground auxiliary-system galleries through a $51 / 2$-ft side shielding tank filled with aggregate and water. However, the main control room and the steam equipment are above ground.

The reactor cell tank is normally held

\section{Price Tag}

Approximately $\$ 400,000$ was spent for labor and materials for the HRT instrumentation and control systems; this was about $15 \%$ of the plant costs. Materials costs were about $\$ 100,000$ for valves and valve operators and $\$ 100,000$ for instruments and electrical control equipment. at a pressure of $0.5 \mathrm{~atm}$, so that in the very remote possibility of a brittle failure of the reactor vessel, the pressure rise would be limited to that which can be contained by the tank. The 5 -ftthick concrete roof shielding plugs are removable for remote maintenance operations.

All process and instrument lines through the shield wall are blocked by valves on signal of high shield pressure to prevent the escape of radioactivity. Electrical leads are sealed by glass-to metal seals.

Thermocouple, electrical, and air lines can be disconnected by remotely operated tools for equipment repair or removal.

All critical core and blanket system transmitters, except electrical level transmitters and thermocouples, are located in two shielded instrument cubicles located adjacent to but out- 


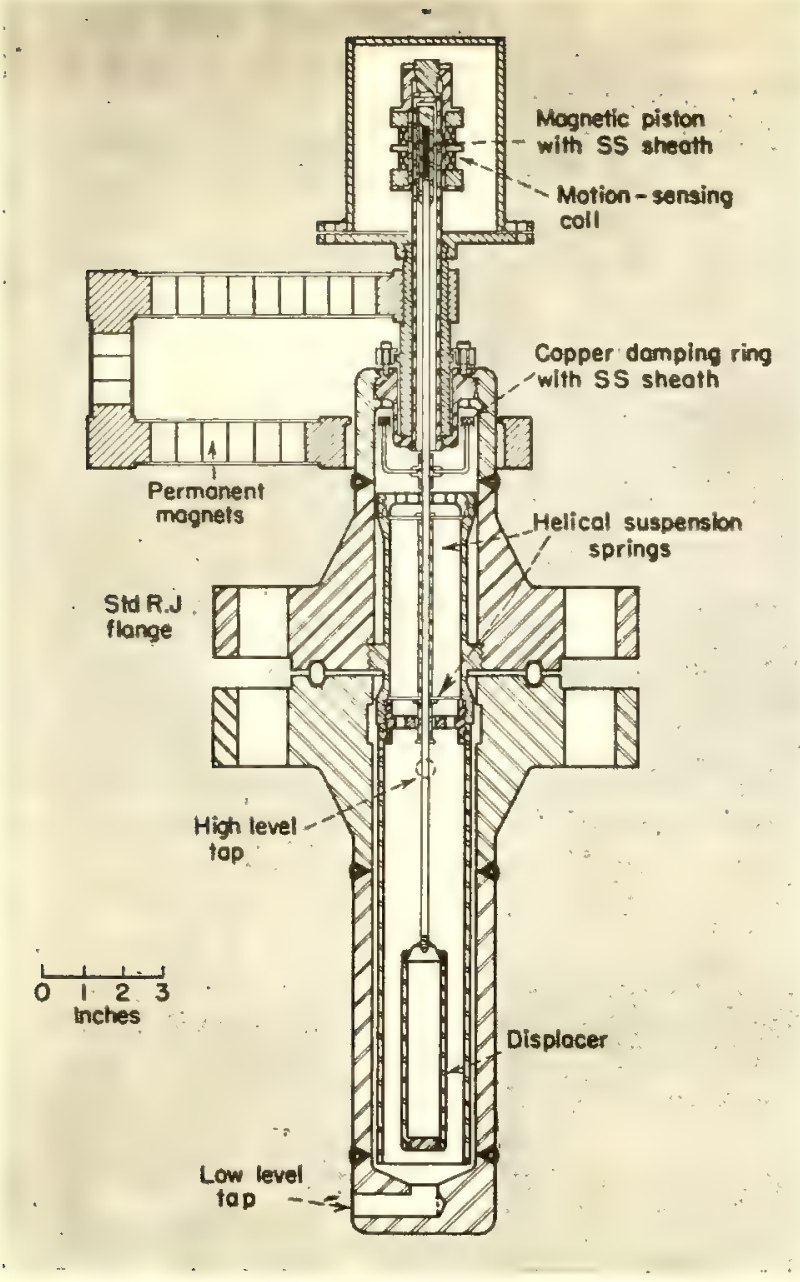

FIG. 4. Displacement-type pressurizer-level transmitter

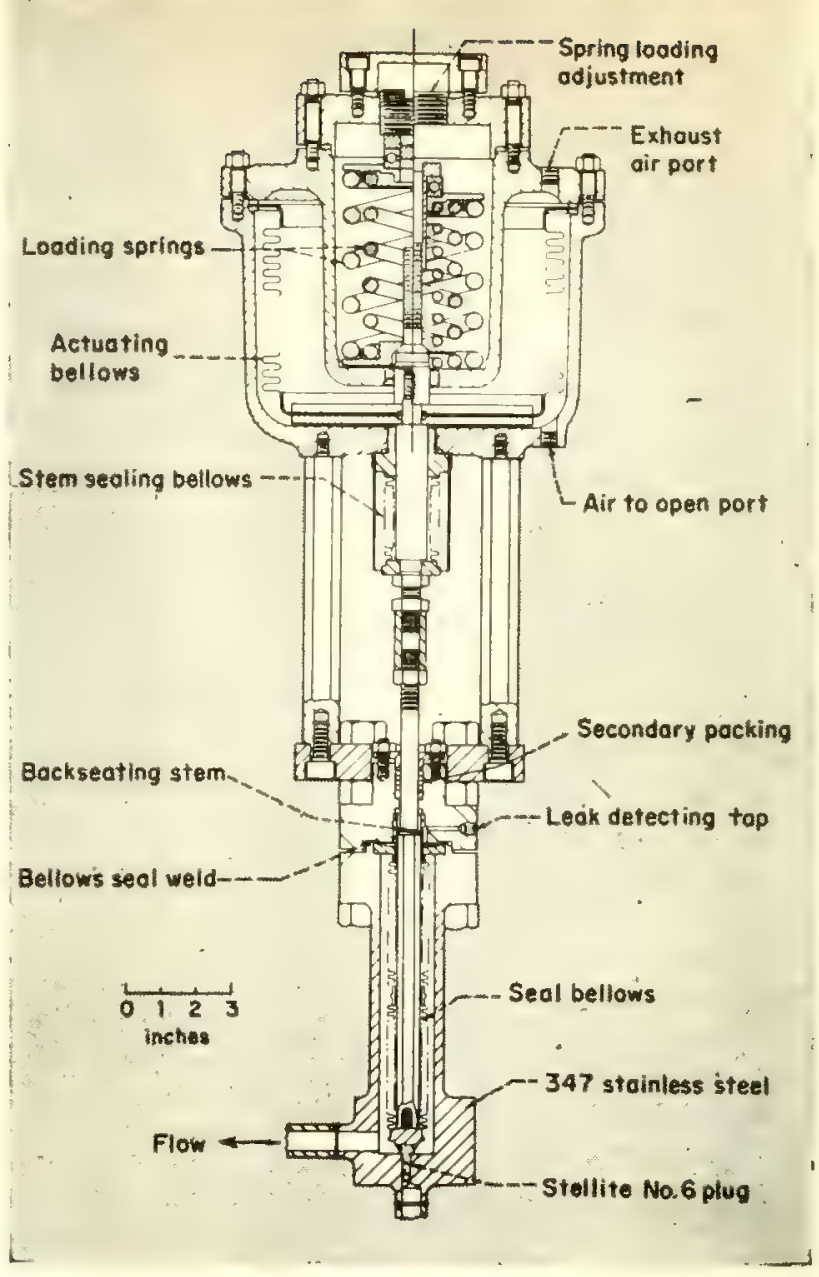

FIG. 5. Letdown .valve. Action shown is air 10 open side the main reactor tank. The instruments were located in these 5 - $\mathrm{ft}$ diameter 15 -ft-long vertical tanks to avoid opening the main tank to replace instruments, to provide a location for instrument components which could not be readily protected from the water-flooding of the main tank during remote maintenance operations, and to minimize radiation exposure. However, the cubicles are sealed and shielded to the same requirements as the reactor tank.

The cell air monitors, which will provide an alarm in case of a leak of radioactive vapor from the reactor system, are installed in one of these instrument cubicles. Cell air is circulated through a 2-in. pipe from the reactor tank, past the enclosed monitors, and then back to the cell. The blower is sized so that only 5 sec is required for cell air to reach the radiation monitors.

The neutron-sensing elements that supply signals to the radiation metering instruments are located in two 5-in.-diameter and two 3-in.-diameter aluminum tube "thimbles." These are, in turn, located in a 30-in.-diameter cylinder-26 ft long, which slopes diagonally down from just outside the main control room through the 5 -ft- thick sand-and-water radiation shield into the main reactor cell. The chamber "thimbles" are filled with water for shielding and for ready withdrawal or positioning. The 30 -in. cylinder is filled with lead shot and water to reduce the gamma irradiation of the neutron-sensing elements.

\section{Design Features}

Interesting design features of the instrumentation and controls system include the following:

(a) Key transmitters are duplicated. Signals from electrical transmitters are converted to 3 - to 15 -psi pneumatic signals by transducers. The output of the unit not controlling is displayed on an adjacent receiver gage. A valve allows either signal to be fed into the controller. Plug-in pneumatic recorders and controllers are used, and an air switch allows the loop to be manually controlled while the controller is being replaced. Pneumatic transmitters are used in control loops affecting plant safety because an inexpensive, reliable emergency control air system, in the event of power or compressor failure, is provided by nitrogen cylinders. The supply is adequate for an orderly shutdown. (b) A 48-v d-c supply from batteries is used for control-circuit power and for the operation of key pilot solenoid valves controlling the air to critical valves.

(c) During power operation a nominal 300-kva turbine-generator operated by reactor steam will be used to provide current for sustained operation.

(d) The critical dump circuit and pilot solenoid valves may be checked during operation by a test panel. Solenoid valves in this circuit are also duplicated. If a bona-fide dump signal occurs during this test, the test signals are overridden.

(e) All electrical and thermocouple leads are insulated with inorganic insulations, such as, "Fiberglas," magnesium oxide, and porcelain to resist the deteriorating radiation effects.

\section{Nuclear Instrumentation}

Nuclear instrumentation in HRT is relatively conventional, consisting of monitors for process leaks and genera] area radioactivity as well as neutron level in the core.

The gamma monitors for detecting process leaks, consist of a simple onetube, three-decade logarithmic amplifier sealed within the chamber head 
and a remote contact-making meter and multipoint recorder.* These detectors can be checked remotely by exposing a radioactive source on the actuation of a solenoid-operated shielding shutter. All channels are duplicated, and control action is initiated only upon a simultaneous signal from both channels to minimize false "scrams." However, a signal from either channel is annunciated.

For monitoring control areas for personnel protection, more stable and accurate vibrating-condenser types of electrometers are used.

Neutron-level transmitters are two Westinghouse fission chambers and two gamma-compensated ionization chambers designed by ORNL. They are conventional in design and use and will not be described here.

\section{Control Panels}

The main control board and console (Fig. 2) contain only those instruments necessary for the safe operation of the reactor. Switches and controls on the console itself are restricted to those necessary for nuclear startup, steady-state power operation, and emergency. All items are arranged in a "visual aid" form to reduce operational errors and to facilitate the training of operators. The graphic section is essentially a simplified schematic representation of the chemical process flow sheet, with instruments, control switches, and valveposition indicators located in positions corresponding to their location or function in the actual system.

Annunciators are placed in the control board directly above that instrument or that portion of the system on the graphic board with which their signal is associated.

Key measurements are displayed on "full scale" recorders in the center section of the panel and include the fuel temperature, a multipoint temperature recorder, the multiarea radiation monitoring recorder, the reactor power, the logarithmic neutron-level and countrate-meter signals, and the blankettemperature recorder.

The patch panel on the extreme left is a "jumper board," which is a schematic representation of the electrical control circuits. Provision is made for jumping certain individual contacts in the control circuit with a plug, and there are lights to indicate the position

* Manufactured by the Victoreen Instrument Co., Cleveland, Ohio.
- open or closed-of the contact in the system. The board is valuable for making control-circuits alterations necessary for experiments, as an aid in familiarizing operators with the electrical control circuitry, and, since the lights indicate contact position, as an operations aid during startup. The jumper board is placed on the main control board so that any jumper is in full view of the operator and cannot become a forgotten clip lead. Protection for sustained power operation is also offererl by the removal of startup (ircuits from control.

Data-collection instruments and the transfucers that drive the miniature pneumatic slave recorders on the graphic panel are located in an auxiliary instrument gallery benenth the main control room, as are a 548-point thermocouple patch panel, a relay panel, the nuclear amplifiers, and the nuclear instrument power supplies.

Other panels located near their respective equipment in the building include the steam control station, the turbine control panel, two sampler control panels, and a refrigeration-system control station. Standard 2-ft-wide modular eabinets and panels are used throughout to facilitate design changes.

\section{Special Components}

As HRT is similar to a fluid chemical process, the critical instrumentation and control components are liquid-level transmitters, pressure and differential-pressure transmitters, and valves. However, the difficulties involved in replacing or performing maintenance operations on process trans- mitters and valves requires that these components be extremely reliable. The design and improvement of these elements is an essential part of the development of homogeneous reactors.

The pressurizer-level transmitter (Fig. 4), developed at ORNL, consists of a 5-in.-long float-displacer suspended by two helical springs. An extension rod above the springs positions a magnetic piston in the center of a differential transformer. Vibration of the float is damped by the action of the field from permanent magnets on a one-turn copper ring.

In the transmitter the only nonwelded closure is the 2,500-psi ringjoint flange, which makes the unit amenable to remote replacement. The transmitter is self-draining - a very desirable feature in systems containing radioactive fluids. Problems not completely solved at present are zero shift due to modulus changes in the displacer supporting springs and a change in span with fluid density changes. However, both effects can be compensated at a constant operating temperature. "Constant"-modulus spring alloys used to date are not flat over the temperature range from ambient to $340^{\circ} \mathrm{C}$ and require a corrosion-resistant gold plating.

A similar type of level transmitter is used on the fuel and blanket system storage tanks and has a design pressure of 500 psi. It differs in that it has a 40-in.-long float and is damped by the interaction of two moving vanes attached to the float and a baffle attached to the housing.

The letdown valve (Fig. 5) incorpo-

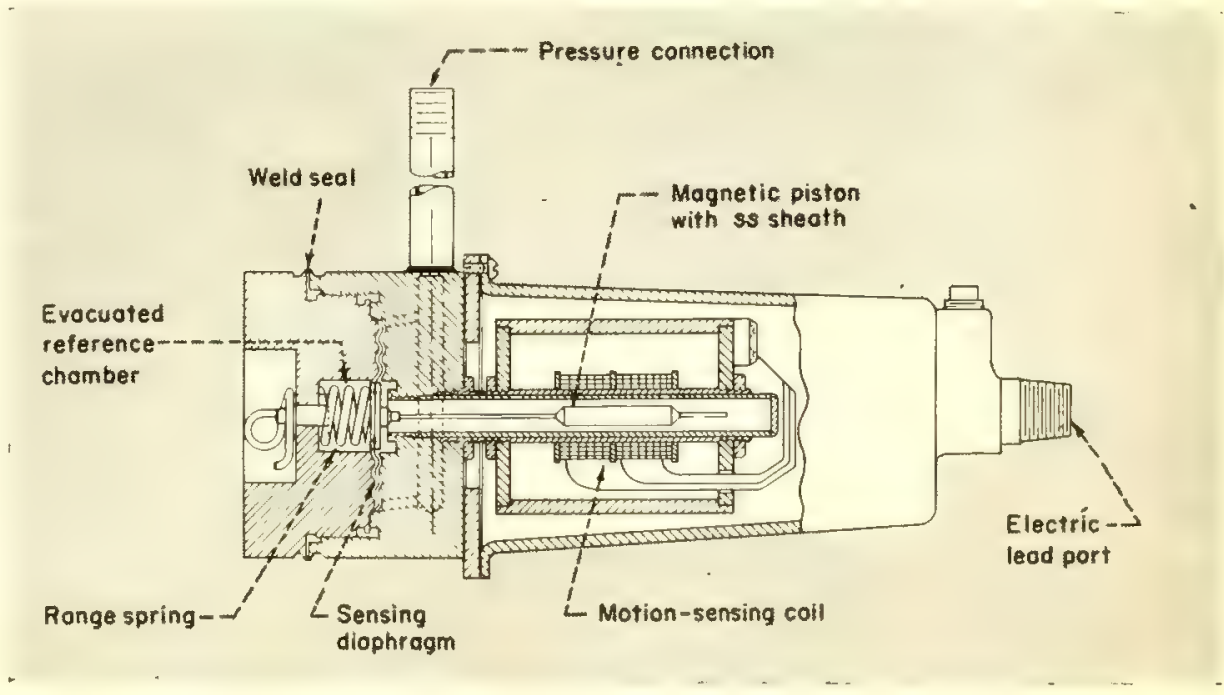

FIG. 6. Absolute pressure transmitter capable of withstanding severe overranging 


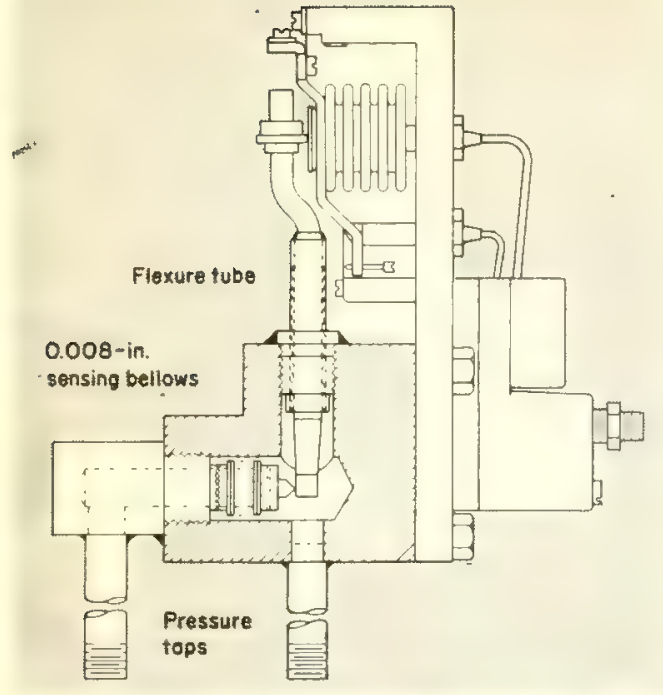

FIG. 7. Pneumatic-transmission differential-pressure cell employing a bellows sensing element

rates a two-section, three-ply stainlesssteel sealing bellows, an integral forged 347 stainless-steel seat, and a stellite No. 6 plug. The mechanically formed bellows plies are each 0.0085 -in. thick. The valve operator is all metallic so as to be radiation resistant; it uses a bellows to produce the linear shaft motion. The shaft is also sealed with a bellows to prevent air leakage into the evacuated reactor equipment cell.

The valve, ${ }^{*}$ is rated for $2,500-p s i$ service with the flow introduced under the seat; however, the downstream pressure is limited to $500 \mathrm{psi}$ by the bellows seal. The valve strokes $1 / 2$ in. and has a $C_{v}$ of 0.1. The secondary packing seal is graphited asbestos. The reversible operator $\dagger$ has a 50 -in. ${ }^{2}$ effective area and is rated for a maximum of 60 psi air.

The dump valve is a similar but larger valve designed for on-off service. This valve strokes $5 / 8$ in. and has a $C_{v}$ of 10. The trim material and sealing bellows are the same as those used in the letdown valve. The larger operator has a two-ply 321 stainless-steel bellows with an effective area of $68 \mathrm{in}^{2}{ }^{2}$ and is rated for 80 -psi actuating air pressure.

The absolute-pressure transmitter shown in Fig. 6 is a weld-sealed electrictransmission type f with an operating range of $0-300 \mathrm{in}$. of water but is capable of withstanding overranging to $2,000 \mathrm{psi}$. Overrange protection is provided by the convoluted diaphragm backup flange, which limits the dia-

* Supplied by The Fulton-Sylphon Division, Robertshaw-Fulton Controls Co.

+ Supplied by The Annin Co.

¥Valve and operator were supplied by The Fulton-bylphon Division.

T These units were supplied by The Foxboro Co. phragm motion. Such instruments may be overranged from dumps, when the contents of the high-pressure system are rapidly emptied into the storage tanks. Specifications include $140^{\circ} \mathrm{F}$ operating temperature, $1 \mathrm{mv} / \mathrm{v}$ output, $3 \%$ accuracy, and 347 stainless steel in contact with process fluids. Differential-pressure cells with similar design are also utilized.

Figure 7 illustrates a 3- to 15-psi output pneumatic-transmission differential-pressure cell which uses a bellows sensing element and an all-welded flexure tube seal. The range of the cell is \pm 50 psi with an overrange rating of \pm 100 psi. The housing design pressure of the cell, is 4,000 psi. The unit is completely weld-sealed and fabricated from 347 stainless steel.

Float switches of the type used for high or low liquid-level alarm** also have all-weld seals. The mercury switch is tripper by the action of the moving magnetic piston on a permanent magnet. All parts exposed to the process fluid or its vapors are stainless steel. The control is rated for 600 -psi service.

Another pneumatic pressure transmitter + has a range of $0-3,000 \mathrm{psi}$. Specifications provide for overrange to 3,750 psig, operating temperature of $140^{\circ} \mathrm{F}$, hysteresis of $\pm 15 \mathrm{psi}$, sensitivity of $\pm 3 \mathrm{psi}$, and weld-sealed 347 stainless steel element.

\section{Interlocks}

Extensive protective interlocking of the controls circuits is provided to prevent unsafe operating conditions. However, flexibility of operation is provided by the "jumper board" to facilitate special experimental operating conditions.

Examples of interlocked systems include the following:

Pumping of fuel instead of condensate to the reactor core is prevented by several interlocks that keep the fueladdition valve closed until the core is full of condensate and has been heated to $200^{\circ} \mathrm{C}$. This prevents power and consequent pressure surges.

For the same reuson the fuel pump is started in reverse to provide a low flow rute as protection against pumping cold solution too rapidly from the heat exchanger into the core.

\footnotetext{
S Supplied by The Foxboro Co.

** Supplied by Magnetrol Ine.

t+ This transmitter was supplied complete by The Taylor Instrument Companies.
}

To avoid dangerous thermal stresses, the control circuits do not permit the pumping of cold feed water into the heat exchangers until the level is above $50 \%$.

To give smooth startup, the fuel injection pump can only run at half speed up to a temperature of $240^{\circ} \mathrm{C}$.

The fuel feed valve will be closed and the concentration in the fuel system will be lowered by injecting condensate if the core outlet temperature becomes excessive, if the circulating pump stops, or if the power level exceeds normal.

The contents of the high-pressure systems will be automatically emptied to the low-pressure storage tanks through the "dump" valves on a signal of extremely high pressure, or a radiation leak into the steam system. Differential-pressure control between the core and blanket systems during this dump is by off-on control of valves from a differential-pressure signal.

\section{Inventory Systems}

For obtaining an accurate inventory of the fuel and moderator solutions, as required for accountability purposes with special nuclear materials, the storage and condensate tanks are weighed with pneumatic weigh cells. This was found to be the only feasible method of measuring the liquid in the 16-ft-long 15-in.-diameter horizontal storage tanks necessary because of criticality and space considerations. A pneumatic system was selected primarily because taring can be done remotely with balancing air pressures and components are less susceptible to radiation damage.

Piping to the tanks is kept flexible, to compensate for the varying loads which result from thermal expansion, by confining piping runs to horizontal planes with " $\mathrm{L}$ " and " $\mathrm{U}$ " bends in the lines.

The author wishes to acknowledge the following Oak Ridge Vational Laboratory instrumentation and control personnel whose work is reported in this paper $J, N$. Baird, M. C. Becker, A. M. Billings, J. R. Brown I. C. Gundlach, R. L. Moore, W. P. Walker (now at the University of V'irginia), $E$. Vincens, and $K$. W. West.

Dean L. R. Quarles of the University of Virginia has served as an invaluable consultant.

\section{BIBLIOGRAPHY}

1. S. E. Beall, C. E. Winters, Chem. Eng. Prop. 50, No. 5, $256(1954)$

$\therefore$ W. R. Gall, Wech. Eng., 575 (1950)

3. S. E. Bheall, J. A. Siwartout. The Homogeneou Reactor Test in "Proccedings of the Inter national Conferenre on Perceful Uses o Atomic Energy, vol. 3, p. 263 (United Nations. New York, 19.46) 


\section{The Role of}

\section{Digital Computers}

\section{in Nuclear Design}

\section{High-speed digital computers are essential}

to the modern nuclear reactor designer.

The many programs coded for nuclear problems

illuminate questions of application and cost

BY WARD C. SANGREN

John Jay Hopkins Laboratory for Pure and Applied Science

General Atomic Division, General Dynamics Corp., San Diego, California

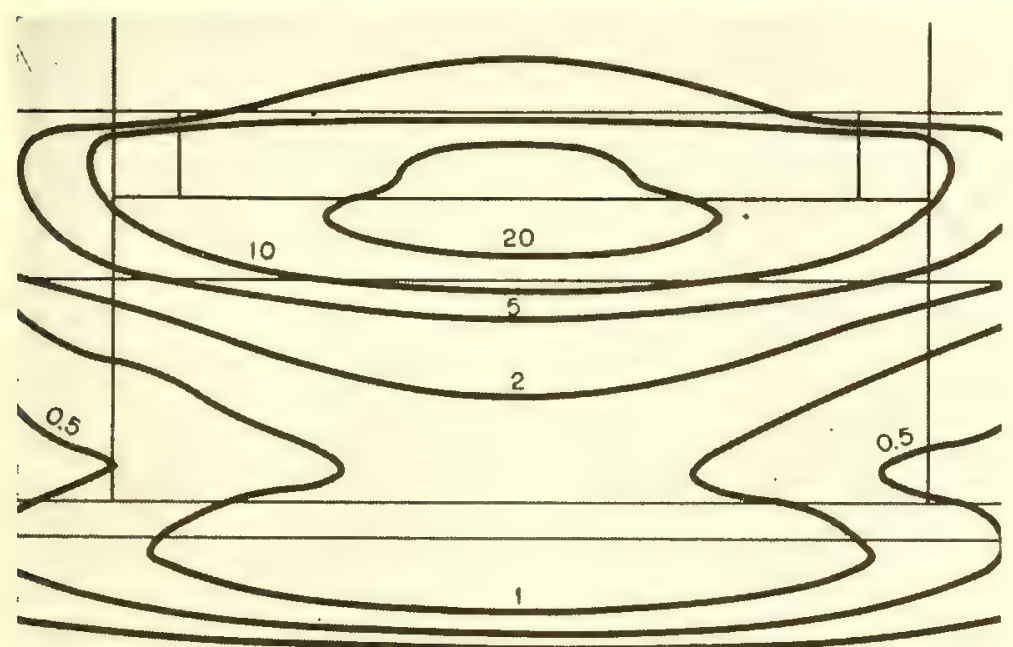

NEUTRON FLUX plot in iwo dimensions for Belgian Engineering Test Reactor typifies computer use in nuclear design. Central axis of reactor core is at top of figure and outer surface of water-shield annulus is at bottom. Top and bottom core-support plates are at right and left. Calculation by Nuclear Development Corporation of America using Datatron and new $R-Z$ reactor code
THE USEFULNESS OF digital computers for nuclear calculations was appreciated early by scientists and engineers connected with atomic laboratories. Indeed, some individuals, such as John von Neumann, played major roles in the early development of both highspeed digital computers and nuclear energy. Calculating punch-card machines, such as IBM 602, 604, and Card-Programmed Calculator, were used for reactor calculations in the late 1940's. Oak Ridge National Laboratory, Los Alamos Scientific Laboratory, and Knolls Atomic Power Laboratory ean be singled out for their early interest in such calculations.

There is little question today that nuclear reactor calculations on highspeed digital computers are vital, and will become more so, in furthering the design of nuclear reactors. Computing and mathematics groups are becoming a more essential part of the scientific or engineering installation. The recent establishment of these groups as major divisions at Brookhaven and Argonne National Laboratories emphasizes this trend.

\section{Problems and Codes}

Nuclear reactor calculations that have been programmed for digital computers are already so numerous and diverse that classification and cataloguing of the existing digital codes is not a trivial undertaking. In fact today this is equivalent to classifying nuclear reactors and all the problems involved in their design. Among the possible classification systems are those based upon the particular mathematical or numerical technique that is used; the number of independent variables, such as space dimensions; the aspect of the reactor that is being calculated; and the scientific or nuclear field from which the calculation arose.

Radkowsky and Brodsky (1) have listed and classified the digital machine codes used for reactor calculations that existed up to October, 1955. In this bibliography, reactor codes are classified under reactor physics, reactor survey, shielding, reactor kinetics, reactor burn-out, reactor engineering, or miscellaneous. The Newsletters of the Nuclear Codes Group (2, 3) have continued with the classification system of Radkowsky and Brodsky.

The classification below closely follows that of Radkowsky and Brodsky. A few comments have been added to 
indicate the scope of each category

Engineering. Calculations in this category involve engineering problems that do not involve nuclear considerations such as heat conduction, stress analysis, and flow problems. Although the most important constraints on reactor design are probably in engineering rather than in physies, relatively few codes have been written in this category. For example, even though heat limitations are of vital importance for any reactor used as a heat or power source, very little effort has been expended on codes for these problems.

Physics. Included is any calculation arising in physies, and particularly in nuclear physics that is related to reactors. In particular, most of the preliminary ealculations of parameters that are needed in the other categories are placed here (e.g., the evaluation of resonance absorption integrals to provide effective multigroup cross sections).

Shielding. These calculations are concerned with the shielding of personnel and equipment from neutrons and gamma rays. Although adequate but not excessive shielding is needed for stationary reactors, most shielding codes seem to have arisen in connection with mobile reactors, where weight is of paramount importance. These shielding calculations fall roughly into one of three mathematical categories: Monte Carlo, numerical quadrature, and numerical solution of an approximation to the Boltzmann equation.

Short-term operability. This category includes kinetic calculations, temperature-coefficient calculations, and any similar "short-term" calculations pertaining to reactor operation. These codes help determine the safety, stability, and design parameters of reactors. Most of them investigate the kinetic response of reactors to step or ramp changes in reactivity. The reactor is usually considered to respond to changes as a unit, with time as the only independent variable. The resulting equations are ordinary nonlinear, differential equations. In solving these types of problems on analog computers, it has been helpful to observe immediately the changes brought about by changes in the input parameters. This fact suggests that digital computers with immediately observable outputs, such as the cathode-ray-tube outputs of the ORACLE and IBM 704, could be profitably used in a similar manner.

Long-term operability. This cate- gory involves calculations of fissionproduct buildup, burnup of fuel and poisons, breeding or conversion ratios as a function of exposure, and core-loading life. A series of criticality calculations with appropriate intermediate calculations can be used for "long-term" codes.

Diffusion and age-diffusion. This is the largest category of reactor codes and has as its starting point the diffusion and age-diffusion equations, with various alterations. The neutron fluxes and the critical mass or radius are what is desired. The age-diffusion equation can be derived from the transport equation by expanding the angular fluxes in spherical harmonics. The age-diffusion equation results from retaining only the first two terms in this expansion. Because of the similarity to retaining the first two terms in a Legendre series expansion, this is called a $P_{1}$ approximation. $P_{3}, P_{5}$, and higher approximations can be obtained similarly.

Transport approximations. These codes start with the Boltzmann transport equation and solve for the neutron

\section{Nuclear Code Fraternities}

The Nuclear Codes Group, in existence since 1956, provides for interchange of information about nuclear codes on a nation-wide basis. At present the group has members from over forty installations, including nearly all companies and government installations interested in reactor design. Roughly half of these installations own, rent, or have ready access to large machines, and roughly half have medium machines available.

The group publishes a quarterly Newsletter containing information on the status of reactor codes at various installations. Specific information on a given program can be obtained directly from the originator or from the local representative of the computer manufacturer concerned. In addition general meetings are held semiannually concurrently with those of the American Nuclear Society. Inquiries should be directed to the chairman of the group, R. J. Graydon, Atomics International, Box 309, Canoga Park, Calif. Specific requests about the Newsletter should be sent to S. Schechter, New York Univ. AEC Computing Facility, 25 Waverly Place, New York 3, N. Y.

Two other organizations, Share and Use, made up respectively of users of the IBM's 704 and Remington Rand's 1103, have also given nuclear codes consideration. Share, the larger organization, contains about 70 installation members, of whom about 10 are concerned primarily with nuclear energy. fluxes and some critical quantity. The Boltzmann equation is a complicated integral-differential equation expressing the conservation of neutrons. The primary unknown function, the angular distribution of neutrons, can be a function of three space variables, three velocity variables, and one time variable. The physical or mathematical problem is completed when certain constants, primarily cross sections, and boundary conditions are preseribed.

Others. This category includes reactor chemistry, biology, mathematics, and codes that do not fit into the other categories.

Even with this classification system, it is very difficult to discuss comprehensively the nuclear codes that have been prepared. Among the contributing difficulties are (a) the security classification, in most cases unnecessary, of the codes and descriptions of the codes; (b) the lack of published and distributed write-ups of codes; (c) the inadequate interchange of information which has existed between workers preparing codes; (d) the newness and unsystematic development of the field; and (e) the almost continuous changes undergone by even the more successful codes.

However the bibliographies (1-3) of the nuclear codes, in addition to providing specific information about each of the available codes, do give a good feeling. for the magnitude and distribution of the effort so far. The total number of codes listed in these sources comes to about 140 . The breakdown by category is as follows:

$\begin{array}{lr}\text { Engineering } & 8 \\ \text { Physics } & 36 \\ \text { Shielding } & 10 \\ \text { Short-Term Operability } & 7 \\ \text { Long-Term Operability } & 8 \\ \text { Diffusion and Age-Diffusion } & 61 \\ \text { Transport Approximations } & 10\end{array}$

Even a casual glance at the distribution of codes shows that the greatest amounts of effort and time have been spent on solving the neutrontransport problem. More than half of all the codes are listed under the diffusion and age-diffusion category or under transport approximations. Within this family the various multigroup codes represent a particularly large investment of time and money. The two-dimensional code CURE, for example, involved over 8,000 manhours and 70 machine-hours on the 
IBM 704 preparatory to running a production problem.

\section{Neutron-Transport Codes}

Because of the special emphasis the neutron-transport codes have received, a few comments on the actual techniques involved in these calculations are in order. The usual objective of any of these calculations is to obtain the neutron fluxes and the critical parameters for a given reactor system. The over-all mathematical technique for calculating a reactor can be only indicated here. The article by Ehrlich and Hurwitz (4) illustrates the multigroup method for solving the age-diffusion equation, and the report by Carlson (5) illustrates a method for solving the transport equation. In mathematical language, the problems are reduced, in the steady-state cases, to finding the fundamental eigenvalue (a critical parameter) and eigenfunction (the neutron fluxes) of homogeneous differential-integral equations. Numerically, it is necessary, of course, to reduce the problem to finding the fundamental eigenvalue and eigenfunction of associated matrices. Although the calculations and codes actually deal with matrices, it is not particularly advantageous to think of the problems in such terms. The power technique of matrix theory is customarily used to find the eigenvalue and eigenfunction. In the language of physics, this power technique is equivalent to first guessing a source due to fission, and then computing - in continuing sequence-fluxes from a source and a source from fluxes. When succeeding sources have the same shape, the processes converge. A ratio of succeeding sources gives a value to $\nu_{c}$, the calculated average number of neutrons emitted per fission required to maintain criticality.

The number of groups in a problem refers to the number of values or intervals of energy (or velocity or lethargy) in which the neutrons are assumed to diffuse. The number of mesh points refers to the number of space points at which the flux will be evaluated. The number of regions refers to the number of materials of homogeneous composition which constitute the reactor.

The validity of multigroup calculations is best judged by comparison of the calculated results with experimental observations on actual reactors. The number and scope of such comparisons available so far are inadequate.
One may hope that future multigroup calculations will someday have a better experimental foundation.

\section{Computing Machines}

Memory. The speed, reliability and cost of digital computers are closely connected with the type of memory. At present, magnetic-core memories are used in the fastest, most reliable, and most expensive machines, such as the IBM 701 and the Remington-Rand 1103A. Cathode-ray-tube memories are cheaper and as fast but have not always proved reliable. The 701, 1103, and other Princeton-type computers all have electrostatic memories, but only MANIAC II is now being built with such a memory. The acoustic mercury-delay-line type of memory is used on the UNIVAC I and the SFAC. This acoustic memory has proved to be reliable and not too expensive, but where speed is concerned, it has the disadvantage of not having random access to the information being circulated as acoustic pulses in the delay line. Magnetic drums are also reliable and relatively cheap, but they do not have the speed advantage of random access.

The size and the speed of the memory usually determine the scope of a calculation. Ordinarily, in discussing the size of the memory, only the number of words* of the "fast" memory is mentioned. Many machines also have auxiliary, or "slow," memories consisting of magnetic drums, tapes, or disks which can store a few million words of information. The UNIVAC I has a 1,000-word memory, and the

\section{TABLE 1-Commercial Computers}

Machines Installed On order

\section{Medium machines}

$\begin{array}{lrr}\text { IBM 650 } & 560 & 1200 \\ \text { Datatron } & 55 & 55 \\ \text { UNIVAC File } & 10 & 200 \\ \text { ELECOMS } & 13 & 5 \\ \text { ALIVAC } & 16 & 5\end{array}$

\section{Large machines}

UNIVAC I and II $40 \quad 20$

UNIVAC Scientific

(1103 and 1103A)

BIZMAC

IBM 701

IBM 702

IBM 704

IBM 705
Princeton-type machines have 1,024and 2,048-word memories. Magneticdrum machines have 1,000-, 2,000- and 4,000-word memories. The latest magnetic-core machines, the 704 and $1103 \mathrm{~A}$, have $4,000-, 8,000-, 12,000$-, and will have 32,000 -word memories.

Speed. The speed of a computer can be measured in various ways. A common method is to give the access time, or fundamental machine cycle time. This time interval indicates the time it takes the central processing unit to transmit to or receive from the memory a word of information. Another method is to give the time of a typical multiplication. In a floating-point machine, this time is approximately the same whether the multiplication is carried out by means of fixed point or floating point. $\dagger$ In a fixed-point machine, a floating-point operation may take as much as twenty times as long as the corresponding fixed-point operation. Table 2 lists the number of operations per second performed by various digital machines.

Type. High-speed computers can also be separated according to whether they are one-of-a-kind machines or production models. Until 1956 one-of-akind machines, such as Whirlwind I, ORACLE, MANIAC I, ORDVAC, ILIAC, AVIDAC, SEAC, SWAC, NORC, and the MARK I, II, III and IV, played an important role in reactor calculations and, indeed, in all highspeed computing. 'The present trend is strongly toward renting or buying one of the production models built by a computer manufacturer. With the flood of machines being produced commercially now, it is unlikely that oneof-a-kind machines will again play a major part.

The figures in Table 1 give the approximate number of machines delivered and on order as of early 1957. Including one-of-a-kind machines, there are about 700 medium machines installed and 1,500 on order, and about 200 large machines installed and 250 on order.

Although any of the machines listed

* A word is a set of characters, such as 10 alpha-numeric characters or 40 binary digits, i.e., a sequence of 40 's and 1 's, which occupies one storage location and is treated by the computer as a unit.

+ In a fixed-point machine, numbers in the machine normally must be between some fixed limits, such as -1 and +1 . In a floating-point machine, a number commonly consists of both an exponent and a signed proper fraction. 
can be used for technical calculations, some, such as the 702 and 705 , were primarily intenderl for business application. Likewise, the majority of File Computers and IB.I 650's undoubtedly will not be used for technical computing.

Future machines will have fuster and larger memories and, in the immediate future at least, will cost more. (See p. 60 for more detail.)

\section{Staffing a Computer}

The size of the computing group associated with a digital computer seems to be closely proportional to the size of the machine. For example, computing groups associated with the 650 and Datatron usually contain between 3 and 20 people, while groups associated with the $704^{\circ}$ and 1103 contain between 12 and 60 people.

Much debate is centered about whether the computing should be carried out in a "closerl shop" or an "open shop." In a closed shop, all the programming or coding is carried out by a group directly associaterl with a digital computer. Problems are presented to this group by the originator, and the solution is returned to him.

In an open shop, the originator of a problem does the coding himself or supervises someone in his group who does it. In the open shop, the personnel associated directly with the machines merely operate then, prepare utility and mathematical routines and perhaps act as consultants on the use of the machine.

An appropriate compromise for groups involved in nuclear calculation is the "service shop," in which the computing group assists the engineer or scientist in whatever fashion is desired, and also carries on certain longerrange problems and programs.

As in most technical professions today, there exists a great shortage of good technical computing personnel. Even more than in most technical fields, including nuclear energy, the expansion in computing during the last five years is astounding. An estimate of 10,000 mathematicians has been marle for computing needs in 1958 . Longer-range predictions become as high as 150,000 by the late 1960 's. Inasmuch as the 1956 combined membership list of the American Mathematical Society, the Mathematical Association of America, and the Society for Industrial and Applied Mathematics contains slightly less than
10,000 names, it becomes shockingly apparent that there will not be enough trained mathematicians to go around.

Even today the majority of computing groups, including those concerned with reactor calculations, make poor use of their machines because they are unable to obtain enough welltrained personnel. It is not uncommon for an individual to waste an amount equal to his monthly salary in a few hours of machine time owing to lack of technical knowledge of either the computer or the problem.

\section{Computer Economics}

Use of a digital computer can now be obtained in four ways: purchase, rental, rental with the option to purchase, and

costs per year are between $\$ 100,000$ and $\$ 300,000$, and for large-machine installations, between $\$ 500,000$ and $\$ 1,300.000$.

\section{Cost per Problem}

The cost of performing a given nuclear calculation depends on both the character of the problem and the kind of machine used. Since the greatest effort has gone into multigroup calculations, the costs of making these kinds of calculations will be considere? in some detail.

One method of estimating the cost of using a high-speed digital computer to solve a multigroup problem is first to estimate the number of "equivalent" multiplicative operations for the prob-

TABLE 2-Computer Costs

\begin{tabular}{|c|c|c|c|c|c|}
\hline \multirow[b]{2}{*}{ Machine } & \multicolumn{2}{|c|}{ Operations/sec } & \multicolumn{3}{|c|}{ Compuler costs } \\
\hline & Fixed point & $\begin{array}{l}\text { Floating } \\
\text { point }\end{array}$ & $\begin{array}{l}\text { Rental } \\
(\$ / m o)\end{array}$ & $\begin{array}{c}\text { Purchase } \\
\text { (\$) }\end{array}$ & $\begin{array}{c}\text { Per operation } \\
(\$)\end{array}$ \\
\hline $\begin{array}{l}\text { Desk } \\
\text { calculator }\end{array}$ & $2.5 \times 10^{-2}$ & & $5 \times 10^{2}$ & & $3 \times 10^{-2}$ \\
\hline 650 , Datatron & $10^{2}$ & 15 & $4 \times 10^{3}$ & $2 \times 10^{5}$ & $6 \times 10^{-5}$ \\
\hline UNIVAC & $5 \times 10^{2}$ & $10^{2}$ & $2 \times 10^{4}$ & $10^{6}$ & $6 \times 10^{-5}$ \\
\hline $\begin{array}{l}701,1103, \\
\text { IAS type }\end{array}$ & $2 \times 10^{3}$ & $10^{2}$ & $2 \times 10^{4}$ & $10^{6}$ & $1.5 \times 10^{-5}$ \\
\hline $\begin{array}{l}704,1103 \mathrm{~A} \\
709, \mathrm{X}-301 \mathrm{G}\end{array}$ & $6 \times 10^{3}$ & $6 \times 10^{3}$ & $4 \times 10^{4}$ & $2 \times 10^{6}$ & $10^{-5}$ \\
\hline LARC & $10^{5}$ & $10^{5}$ & $10^{5}$ & $3.5 \times 10^{6}$ & $10^{-6}$ \\
\hline STRETCH & $10^{6}$ & $10^{6}$ & $2 \times 10^{5}$ & $6 \times 10^{6}$ & $2 \times 10^{-7}$ \\
\hline
\end{tabular}

the hourly rental of time on a machine at a service bureau. Medium machines rent for $\$ 2,000$ to $\$ 12,000$ per month, can be purchased for $\$ 10,000$ to $\$ 300,000$ and can be used hourly for $\$ 40$ to $\$ 200$. Large machines now rent for about $\$ 15,000$ to $\$ 55,000$ per month, can be purchased for $\$ 500,000$ to $\$ 3$,000,000 and have an hourly rate of $\$ 150$ to $\$ 750$. The monthly rental cost generally refers to a one-shift operation, with each additional shift costing $50 \%$ more.

Besides the cost of the machine itself personnel costs represent a major contribution to the total cost of operating a digital computer. A rough rule that applies fairly well to installations renting machines on a one-shift basis is that the personnel costs should be equal to the machine costs. For medium-machine installations, the total lem and then multiply by the clollar cost per multiplicative operation. A rule of thumb for the number of equivalent multiplicative operations is to count the number of multiplications and divisions and multiply by two to account for all other arithmetical and logical operations. The final column in Table 2 gives some representative costs per operation. The following word formula can be used to estimate the number of operations for a multigroup problem:

(source iterations) $\times($ groups $) \times$ (mesh points) $\times$ (operations per point) $X$ (iterations per group) $=$ operations

Before we give numerical examples, some general comments on the use of this estimate are appropriate:

1. In one dimension, the number of regions and the operations needed for 
initially calculating the constant coefficients in the differential equations can increase the estimate by a factor of two. In two and three dimensions, these factors are not significant.

2. The total number of source iterations is the product of the number of source iterations needed to converge to a particular $\nu_{c}$ (the average number of neutrons per fission required to maintain criticality) and the number of $\nu_{c}$ 's required to reach the correct value (i.e., 2.5). An adequately converged $\nu_{c}$ usually requires three to fifteen source iterations, four being very common. The number of converged $\nu_{c}$ 's required is generally between three and six.

3. The number of group iterations is dependent upon the number of mesh points for the two- and three-dimension problems. This relationship is quite complex and is largely a function of the particular iterative technique used.

4. The final product of source iter-

\section{Digital Computers of the Future}

The two largest computer manufacturers, Sperry-Rand and IBM, have each announced two new large machines. Sperry-Rand is working on the X301-G and the Livermore Automatic Research Computer (LARC), while IBM is working on the 709 and the STRETCH.

The $\mathrm{X} 301-\mathrm{G}$ and 709 will not operate significantly faster than the present $1103 \mathrm{~A}$ and 704 , but because of larger memories, more versatile orders, and advantageous input and output equipment, many problems will probably be computed in half the time now required.

The LARC is expected to be faster then the present 1103A and 704 by a factor of about 15. It will read data from its ferrite core memory at a rate of 2 $\mu \mathrm{sec}$ per word. The initial capacity of the fast memory will be 20,000 tendigit numbers. This may be substantially increased by adding supplementary fast memory units. A LARC is to be delivered to Livermore in early 1958 , while additional LARC's are on order for a year or two later. The contract price for the construction of the computer is $\$ 2,895,000$.

IBM's STRETCH, in turn, is expected to be about a factor of 10 faster than the LARC. It will also have a magneticcore-type fast memory. A data word can be read from the memory within $0.2 \mathrm{sec}$. The machine is being designed to accommodate a fast memory capacity of up to a million words. In addition, external memories (magnetic discs and magnetic tapes) may ultimately provide data in blocks up to a total capacity of 100 million words. STRETCH is being built for Los Alamos and is to be delivered about January, 1960. ations and group iterations can often be reduced (sometimes as much as a factor of 10 ) by using improved iteration techniques.

The following numerical examples are representative for criticality calculations:

(a) 1-Dimensional 3-Groups; 50-Mesh Points; 3-Regions

$(2)(20)(30)(50)(10)(4)=2.4 \times 10^{6}$ op.

(b) 2-Dimensional 30-Groups; 1,000Mesh Points; 3-Regions

$(20)(30)(1,000)(20)(50)=6 \times 10^{8} \mathrm{op}$.

(c) 3-Dimensional 10-Groups; 10,000Mesh Points; 3-Regions

$(20)(10)(10,000)(30)(100)$

$=6 \times 10^{9}$ op.

The results of these three examples can be combined with the information in Table 2 to give the final costs for the various machines.

In one-dimensional calculations, a complete criticality calculation for sample problem (a) costs, for example, about $\$ 24$ on the 704 and $\$ 144$ on the 650 or the UNIVAC I. For a single converged $\nu_{c}$, the amount is about $\$ 6$ on the 704 and $\$ 36$ on the 650 or the UNIVAC. These figures are confirmed by the running time of the Eyewash code on the UNIVAC and the 704 and the PROD II code on the 650. The computation of a converged $\nu_{c}$ averages about $20 \mathrm{~min}$ for 30 groups on the UNIVAC, about $2 \mathrm{~min}$ for 32 groups on the 704 , and about $40 \mathrm{~min}$ for 16 groups on the 650 .

In two-dimensional calculations, the cost for a full criticality calculation, such as sample problem (b), becomes almost exorbitant. Even on the 704 or $1103 \mathrm{~A}$ such a problem would cost $\$ 6,000$, while on the 650 or Datatron the cost would be over $\$ 30,000$. A single converged $\nu_{c}$, of course, would reduce the costs to about $\$ 1,200$ and $\$ 6,000$, respectively. Certainly in these problems one must attempt to minimize the number of groups, mesh points, and iterations. A three-group problem is, for example, not too expensive on the 704 but is expensive on the 650 . The running times of a two-group code on the ORACLE, the Mug II code on the UNIVAC, and the Curtiss-Wright code on the 701 bear out these costs.

The 10-group three-dimensional sample problem (c) is very expensive on present machines. If only three groups are used and a single converged $\nu_{c}$ is sought, the $70+$ price is around $\$ 5,000$.

Calculations other than multigroup calculations can also involve a large number of operations. Problems concerned, for example, with burnup. endurance, two- and three-dimensional kinetics, temperature distributions and transport approximations can all involve from $10^{6}$ to $10^{10}$ operations.

At most computing centers, problems frequently arise which are "oneshot" calculations, i.e., likely to occur only once. Experimental calculations and certain exploratory calculations often fall in this category. The question often arises as to whether hand calculations or a small or medium computer should be used rather than a large computer. The answer is very much a function of the problem, but it is even more a function of the personnel and subroutines available. Given the proper personnel and subroutines, calculations as small as $10^{3}$ operations can properly be placed on large machines.

The time needed to prepare a code is largely dependent upon the individual programmer and almost independent of the type of machine involved. The time to "debug," i.e., verify that the code is running correctly, is likewise strongly dependent upon the individual programmer and, to a much lesser extent, on the particular machine in use.

In summary, the cost of coding and debugging a given program is almost independent of machine size, while the cost per mathematical operation is much smaller for larger machines (see Table 2). The size and the repetitive nature of most reactor calculations therefore indicate the use of the fastest (and therefore generally cheapest per problem) commonly available computer. A commonly available computer is desirable because of the economic advantage of interchanging codes with other installations which are working on similar problems.

\section{BIBLIOGRAPHY}

1. A. Radkowsky, R. Brodsky. A bibliography of available digital computer codes for nuclear regctor problems, AECU-3078 (1955)

2. Nuclear Codes Group Newsletter No. 1 (AEC Computing Facility, New York University New York, 1956)

3. Nuclear Codes Group Newsletter No. 2 (AEC Computing Facility, New York University, New York, 1956)

4. H. Hurwitz, Jr., R. Ehrlich, TID-2009, Vol. 3, 13 (1953) (Classified)

5. B. Carlson. Solution of the transport equation by $S_{n}$ approximation, LA-1891 (1955) 


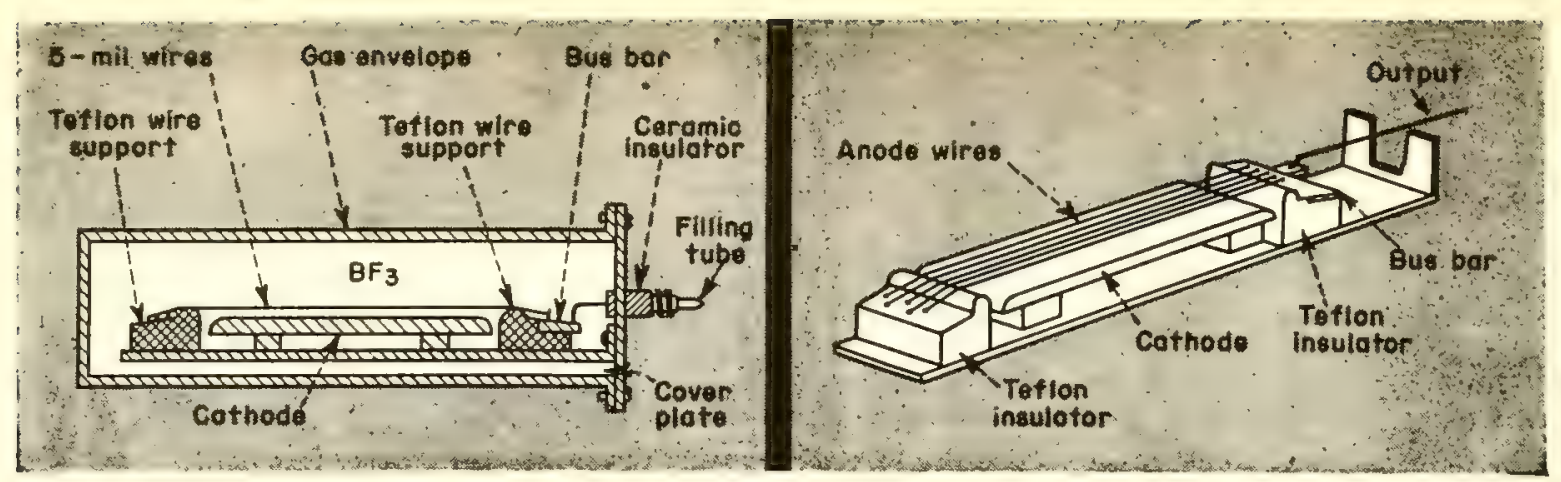

FIG. 1. Neutron-detecting spark counter

FIG. 2. Multiple-wire anode for spark counter

\title{
$\mathrm{BF}_{3}$ Neutron Spark Counter
}

\section{$A B_{3}$-filled spark counter is sensitive to neutrons yet insensitive to gammas. Cadmium shielding plus paraffin moderation make it useful for fast-neutrons too}

\author{
BY MARTIN JAY SWETNICK* and NICHOLAS G. ANTON \\ Anton Electronic Laboratories, Brooklyn, New York
}

With A ROSENBLUM spark counter (1) operated in a $\mathrm{BF}_{3}$ atmosphere we have achieved a slow-neutron detection effciency of $0.6 \%$, and the system is completely insensitive to $\mathrm{Co}^{80}$ radiation at $500 \mathrm{r} / \mathrm{hr}$. External circuitry required is limited to a high-voltage supply and a scaler.

\section{Sensitivity}

Detection efficiency is proportional to the probability of slow-neutron capture in $\mathrm{B}^{10}$ and the probability of alpha particles or lithium recoils from the $\mathrm{B}^{10}(n, \alpha) \mathrm{Li}^{7}$ reaction reaching the sensitive corona region of the electrode assembly (2). Sensitivity is not a function of the total volume of $\mathrm{BF}_{3}$ gas. The sensitive volume of the spark counter

* Present addregs: Physics Department of the University of Maryland, College Park, Maryland.

Slow-Neutron Response

\begin{tabular}{lcc}
\hline $\begin{array}{c}\text { Applied } \\
\text { voliage }\end{array}$ & $\begin{array}{c}\text { No cadmium } \\
(\text { cpm })\end{array}$ & $\begin{array}{c}\text { Cadmium } \\
(\text { cpm })\end{array}$ \\
\hline 2,000 & 10 & 0 \\
2,050 & 30 & 0 \\
2,100 & 62 & 0 \\
2,150 & 80 & 0 \\
2,200 & 115 & 4 \\
2,250 & 200 & 8 \\
2,300 & 560 & 75
\end{tabular}

extends only to the alpha-particle range. Our sensitivity is decreased by shielding of additional gas since the anode-wall distance is greater than the alpha range.

The slow-neutron-sensitive spark counter can be converted into a fastneutron-sensitive spark counter by encasing the detector in a paraffin shield to moderate fast neutrons and covering the exposed surface of the paraffin with a 2-mm cadmium sheet to absorb incident slow neutrons.

\section{Counter Construction}

Our counter is enclosed in an airtight stainless-steel cylinder filled with $\mathrm{BF}_{3}$ to $45 \mathrm{~cm} \mathrm{Hg}$ (Figs. 1 and 2). The boron in the gas is $96 \%$ enriched in $B^{10}$. The electrode assembly consists of $\mathrm{a}^{\circ}$ smooth-surfaced stainless-steel cathode plate 2 in. wide and 3 in. long and an anode array of five parallel 0.005 -in. stainless-steel wires. The wires are in a plane $1 \mathrm{~mm}$ from the cathode surface and parallel to it.

Between power supply and counter we use a two-resistor series combination to attenuate output pulses from the $\sim-200$ volts at the anode to $\sim-50$ volts. A $50-\mu \mu \mathrm{f}$ condenser couples the pulse to a scaler.

\section{Slow-Neutron Response}

The detector was exposed to a constant flux of slow neutrons from a paraffin-encased 10 -mc radium-beryllium neutron source to determine its response as a function of applied voltage. Runs were made at 50-volt intervals between 2,000 and 2,300 volts with and without cadmium shields about the detector. Although the pulse heights were observed to increase with applied voltage, all pulses had identical shapes and magnitudes at any one operating voltage. The results of the measurements are presented in the table. In the absence of neutrons, counting rate was zero over the entire voltage range we studied.

\section{Gamma Response}

With slow neutron source removed, gamma radiation response of the detector was checked by placing a $1 \mathrm{mc} \mathrm{Co}^{80}$ needle source in contact with the outer wall of the counter cylinder. The intensity of the gamma radiation at the sensitive region of the electrode assembly was computed to be $>500 \mathrm{r} / \mathrm{hr}$. The counter was found to be completely insensitive to this intense gamma radiation.

$$
\text { *** }
$$

This work was supported in part by the Bureau of Ships and the $A E C$.

\section{BIBLIOGRAPHY}

1. W. Y. Chang. S. Rosenblum, Phys. Rov. 67, 222 (1945)

8. N. K Saha, Narendra Nath, Nucueones 16, No, 6, 94 (1955) 


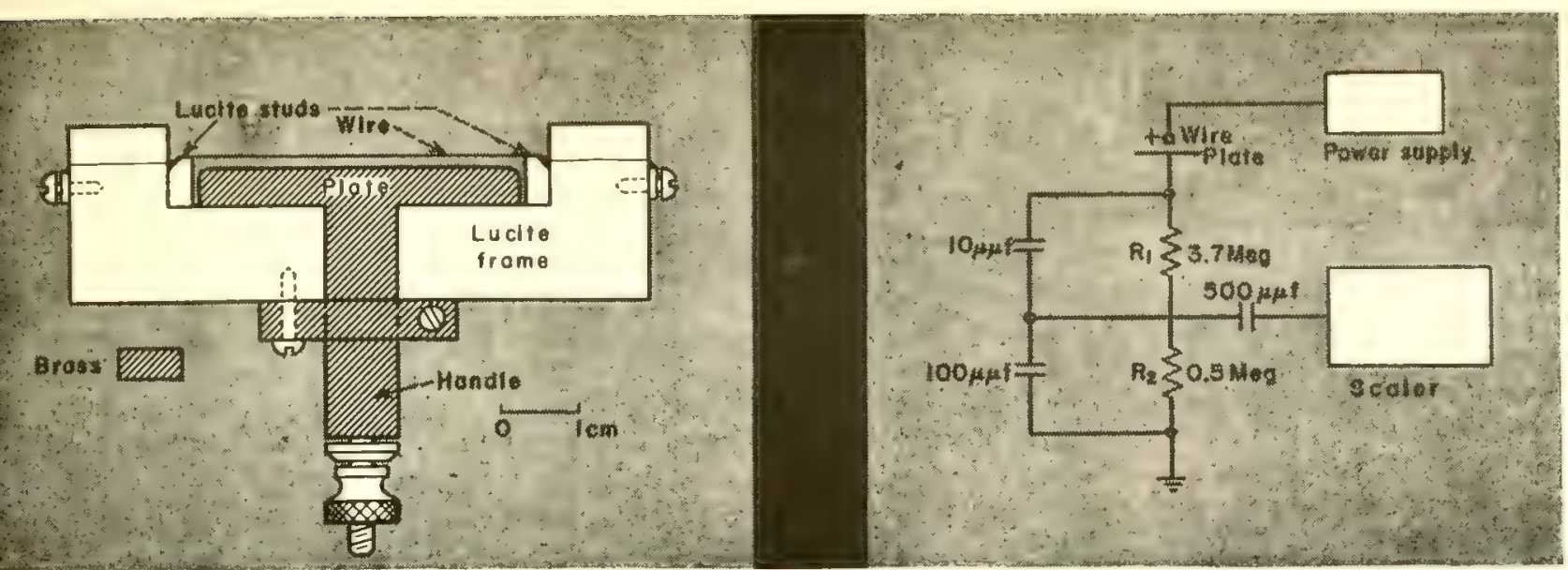

FIG. 1. Single-wire counter has wire stretched in front of metal plate. Sliding handle in frame adjusts separation
FIG. 2. Circuit used for counting spark pulses has condenser pulse divider. Sum of $R_{1}$ and $R_{2}(=R)$ determines quiescent characteristics

\title{
Operating Characteristics of the Spark Counter
}

\author{
This highly specific instrument is sensitive only to alphas. \\ With a boron plate it gives 1 count for 180 neutrons $/ \mathrm{cm}^{2}$ \\ but $<1$ count $/ \mathrm{hr}$ in a gamma field of 1 roentgen $/ \mathrm{sec}$
}

By N. K. SAHA and NARENDRA NATH

Department of Physics, University. of Delhi, Delhi, India

Alpha PARTicles have low penetrating power and ionize densely. To detect them, therefore, one should have an instrument with a thin window or no window at all, and this instrument should be sensitive to dense ionization and insensitive to the lighter ionization of beta and gamma rays. The Rosenblum spark counter meets both of these criteria. It consists of a wire or a system of wires parallel to a metal plate and maintained at such a potential that the ionization produced by an alpha particle initiates a spark discharge between the plate and the wire or wires. An efficient neutron detector is made by placing a thin layer of boron in front of the wires and detecting the alphas that result from the $\mathrm{B}^{10}(n, \alpha) \mathrm{Li}^{7}$ reaction.

Following the original idea of Greinacher (1), Chang and Rosenblum (2) designed a satisfactory alpha counter that uses the breakdown between a flat metal cathode and a fine wire anode stretched $1.5 \mathrm{~mm}$ in front of the cathode. Other workers have improved and modified the spark counter and studied its operation ( $(\mathcal{S}-7)$. A standard form has not yet emerged, however, and the mechanism is still obscure in many respects.

Conflicting reports have appeared regarding some of the counter's properties. Connor has observed a decreasing counting rate with increasing applied voltage, while Payne has observed a nearly constant rate. Similar uncertainties exist as to whether the pulse size increases or decreases with applied voltage, whether a positive temperature effect exišts, etc.

We have developed single-wire and

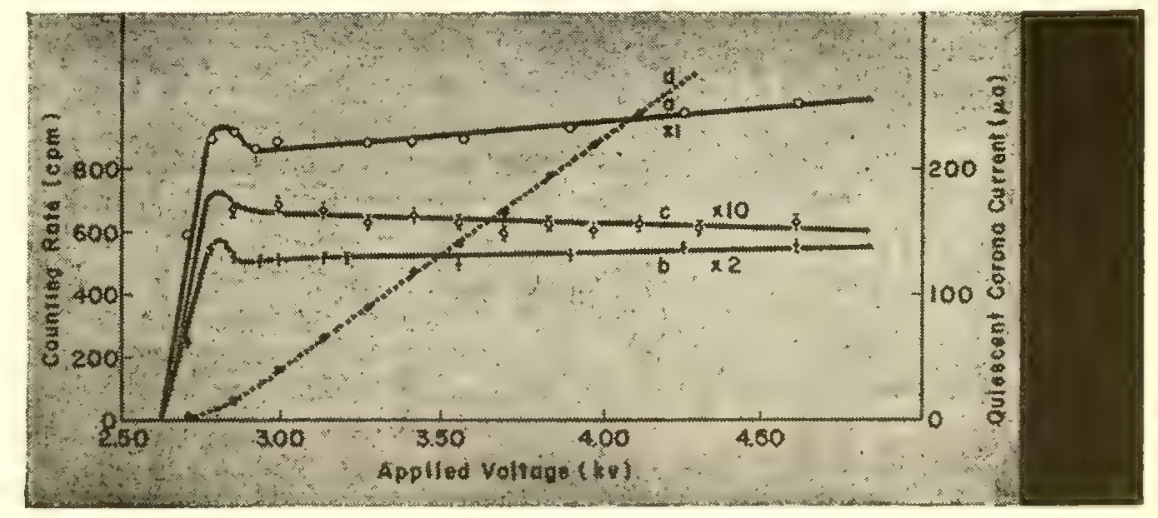

FIG. 3. Counling characteristics $(a, b, c)$ show hump where quiescent current (d) starts. With increasing source collimation slope decreases (a to $b$ to $c$ ) as oblique rays fail to reach sensitive volume 
multiple-wire counters and studied their detection of alpha particles and neutrons. A mechanism has been developed that successfully explains the counting, quiescent-current characteristics, the number-vs-distance curve for an alpha source, and our observed temperature dependence. Some apparently contradictory observations of earlier workers are satisfactorily accounted for.

\section{Single-Wire Counter}

The form of our single-wire counter is shown in Fig. 1. A slightly oxidized brass plate and two rectangular Lucite studs rest on the base of a Lucite frame. A $0.1-\mathrm{mm}$ tungsten wire is stretched over the studs and held tight by screws at both ends. The plate and the studs are accurately machined to ensure uniform separation $(\sim 1.2 \mathrm{~mm})$ between wire and plate. The plate can be moved by pushing the handle attached to it in and out. The handle can be fixed at any position by the spring-andscrew arrangement.

A positive voltage of $2-5 \mathrm{kv}$ is applied to the wire. The cathode is grounded through a pulse-quenching circuit, and a small part of the positive pulse at the cathode is taken to a Marconi TF 922 scaler for final counting. Figure 2 shows the circuit arrangement used for registering the number of sparking events.

\section{Neutron Detection}

Savel (7) was the first to use a modified form of the Rosenblum counter for measuring slow-neutron doses and alpha contaminations. Nearly simultaneously Connor (5) developed an alpha monitor using a multiple-wire system in front of a metal plate. To develop this technique for efficient neutron detection and study of $n, \alpha$ reactions, we undertook construction of a suitable spark counter. Our early experience indicates that we can detect neutrons with some confidence and reproducibility even with as weak a source as $100 \mathrm{mg}$ of radium-beryllium, that is, $\sim 10^{7} \mathrm{n} / \mathrm{sec}$.

We designed a system with 15 wires in front of a $4 \times 4-\mathrm{cm}$ flat brass plate. At first there was some difficulty getting all wires to work together, but by minor adjustments a narrow voltage region yas found in which all the wires worked satisfactorily. The counting efficiency was greatly enhanced by using many wires.

Alpha particles released by the $n, \alpha$ reaction from a very thin layer of powdered boron placed close in front of the counter wires were used to detect neutrons. The whole assembly including the neutron source was placed inside a paraffin block of $10-\mathrm{cm}$ wall thickness. With the 100-mg Ra-Be source on a line perpendicular to the plate, $7 \pm 1$ cpm was observed. When the boron layer was removed, no background counting was observed over a period of about an hour, even in the presence of the intense gamma rays $(\sim 1 \mathrm{r} / \mathrm{hr})$ from the source. In our geometry one observed pulse roughly corresponds to the passage of $20 \mathrm{n} / \mathrm{cm}^{2} / \mathrm{sec}$ in the counter area. It seems, however, that a further improvement in the efficiency of neutron counting is possible by improving the counter design and using a boron-coated cathode. Work is in progress in this direction.

\section{Counting Mechanism}

Our mechanism for the counting action can be described in terms of the spark-counter arrangement of Fig. 2 . A strong electric field exists between the wire anode and the plate cathode separated by a distance of the order of $1 \mathrm{~mm}$ in atmospheric air. $1 \mathrm{n}$ the absence of any strongly ionizing particle passing between the electrodes and for a sufficiently strong electric field, a quiescent corona current starts and with increased field is $\sim 100 \mu \mathrm{a}$. This indicates the presence of a fairly dense space-charge sheath forming a small distance from the wire in the direction of the plate. Most of the properties of the spark counter seem to depend largely on the behavior of this spacecharge sheath under various circumstances. This is a situation that does not exist in the G-M counter, where there is practically no quiescent corona current.

There is an external resistor $R=$ $R_{1}+R_{2}$ in series with the electrodes, and a corresponding drop $R I$ when a corona current $I$ flows. The effective potential drop between the electrodes is therefore

$$
V_{\text {eff }}=V_{\mathrm{a}}-R I
$$

where $V_{\mathrm{a}}$ is the applied potential. It is therefore clear that the effective electric field between the electrodes, which determines the counting behavior, is partly dependent on the external resistor. As the applied voltage is varied the corona current $I$ also changes, and therefore $V_{\text {eft }}$ may not change in the same way as $V_{\mathrm{s}}$.

Suppose an ionizing particle is now allowed to pass between wire and plate. On account of the strong electric field in the neighborhood of the wire an electron avalanche starts at once in the same region. But this will succeed in producing a spark breakdown between
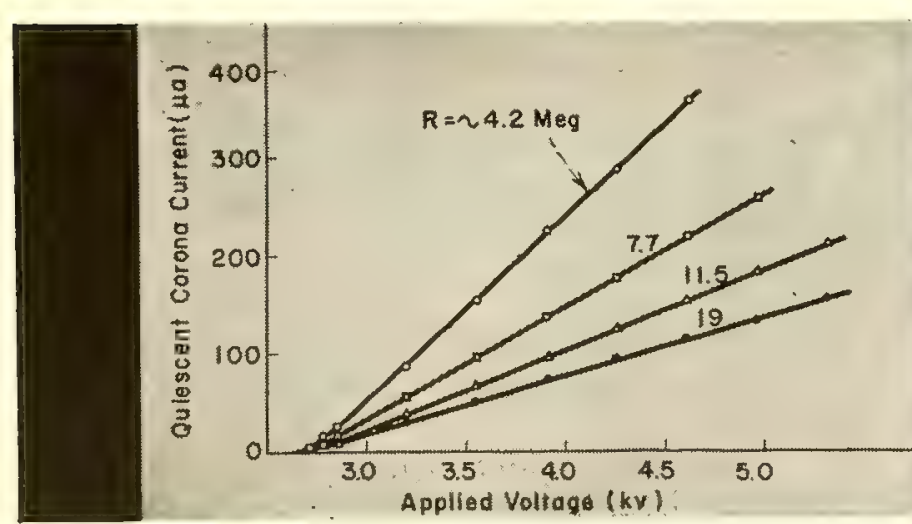

FIG. 4. Plots of I vs $V_{\mathrm{B}}$ for different values of external resistance $R$ show that corona sheath obeys equation, $V_{\text {eff }}=V_{\mathrm{a}}[1-k(R) R]$
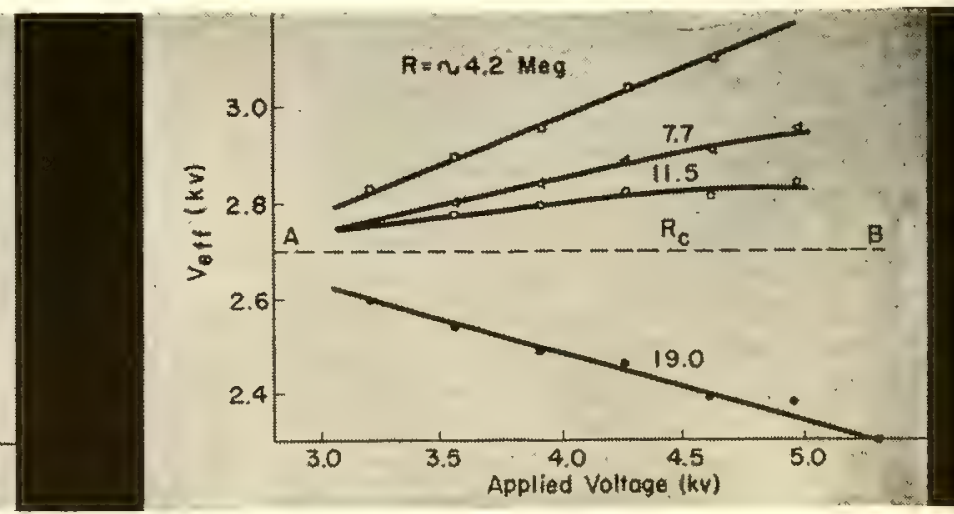

FIG. 5. When $V_{\text {eff }}$ is computed from Fig. 4, result indicates that for a critical value of $R, V_{\text {eff }}$ should be constant with changing $V_{\text {. }}$ 
the electredes only if the avalanche is sufficient to overcome the strong negative space charge present near the wire. This explains the immunity of the spark counter to beta and gamma radiations, which produce a much weaker avalanche than does a heavier ionizing particle like the alpha particle.

On further increase of $V_{\mathrm{s}}$, the $a$ priori probability for an avalanche to produce a spark breakdown, or a priori sensitivity, increases, causing an increased number of alpha particles from the source to be counted. It may happen that when $V_{\mathrm{a}}$ has increased beyond a certain limit, the corona-space-charge effect, which also increases with $V_{\mathrm{B}}$, is also very high. This might lead to a reduction in a priori sensitivity, since the limited specific ionization of some

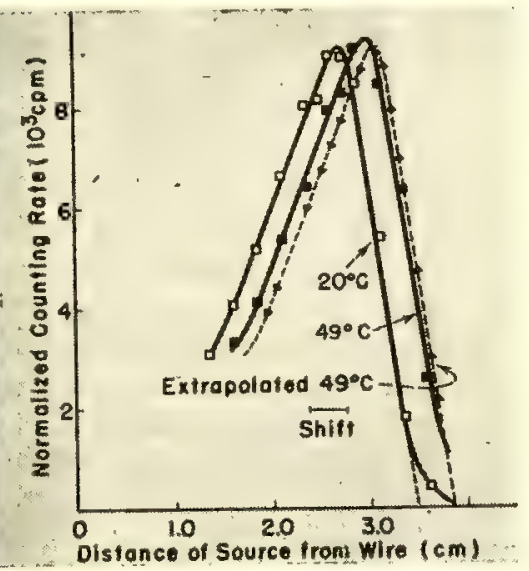

FIG. 6. Solid curves are counts observed at $20^{\circ} \mathrm{C}$ and $49^{\circ} \mathrm{C}$ as $\mathrm{Po}^{210}$ source approaches counter. Dotted curve is shifted version of $20^{\circ}$ curve

of the particles may not be sufficient to overcome the space charge and may thereby fail to produce a breakdown. We can then expect a slight fall in the counting rate although $V_{\mathrm{a}}$ is rising.

For a heavily ionizing particle like an alpha the specific ionization in the counting space depends somewhat upon the distance of the source from the counter. Sparking breakdown requires a critical concentration of ionization in the sensitive space. The variation of the source distance or the direction of incidence of the alpha particle may, therefore, cause a variation in the $a$ priori sensitivity, giving the spark counter strong directional properties.

Let us now examine the effect of $R$ and $V_{\Delta}$ on $V_{\text {ou }}$ and the counting rate of the counter. It can be seen from theoretical consideration that for a given value of $R$, an approximately linear quiescent-current characteristic exists within a certain range of $V_{\bullet}$; that is, as $V$. increases, $I$ also increases linearly. The magnitude of $I$ is, however, determined by both the value of $R$ and the dynamic resistance of the space-charge sheath. On increasing $R$ for a given $V$, the current $I$ may therefore decrease nonlinearly with $R$. We may thus represent $I$ as

$$
I=k(R) V .
$$

where $k(R)$ is the constant slope of the $I$ vs $V$, characteristic. The quantity $k(R)$ is a constant for a given $R$. Substituting in our earlier relation, one gets

$$
V_{\text {off }}=V_{\mathrm{s}}[1-k(R) R]
$$

From this equation it follows that for values of $k(R)<1 / R, V_{\text {efl }}$ will increase with $V_{\mathrm{a}}$. This will be true only for certain values of $R$. For some other (larger) values of $R, k(R)$ may exceed $1 / R$, giving a reduction in $V_{\text {off }}$ with increasing $V_{\mathrm{a}}$. If the electrode spacing is small, $k(R)$ can exceed $1 / R$ even for moderate values of $R$. The consequence of this effect will be a decreasing a priori sensitivity and a decreasing counting rate with increasing $V_{\text {. }}$. For a given electrode spacing there is therefore a critical resistor value $R_{\text {。 }}$ above which the counting rate will decrease with increasing $V_{\text {. }}$ Further, $\boldsymbol{R}_{\text {o }}$ will decrease with decreasing electrode spacing.

Experimental results on counting and quiescent-current characteristics, the number-distance curve for polonium alphas, the temperature coefficient of counting, and the detection of neutrons can be explained by the counter mechanism outlined. Some of the apparently conflicting results of earlier workers can also be accounted for.

\section{Counting Characteristics}

A polonium source on a 1-cm-diameter thin platinum disk was placed facing the counter on a line normal to the plate. The variation of the counting rate with applied voltage was observed. Figure 3 shows the result. It differs from what earlier workers have observed $(3-6)$.

The difference between our result and those of Connor and Payne can be understood as follows. Alpha rays from our extended polonium source enter the counter at many different angles. Since with increase in $V_{\mathrm{A}}$ a priori sensitivity gradually increases, some of the oblique rays that could not produce sparking breakdown at lower voltages are counted at higher voltages. This gives rise to the small positive slope of our counting characteristic. If the source is highly collimated, the directional effect on the counting rate will be practically absent, but the effect of increasing space charge and that of the external resistor as explained previously will still remain. These may result in a positive, zero, or negative slope of the counting characteristic, depending on the particular circumstances of the experiment.

It appears to us that the conditions and geometry of Connor's counting arrangement (4) were similar to ours except that he used a smaller separation between the electrodes. The latter might be responsible for a strong spacecharge effect at higher voltages. Also, the external resistor used in his case might exceed the critical value $R_{\circ}$ for his small electrode spacing. These two effects might combine to give a slow decrease in the counting rate with increasing voltage. Exact reasons can not be assigned without knowing the quiescent-current characteristics obtained in Connor's experiment. The nearly constant rate observed by Payne (6) can also be similarly understood in a general way.

To investigate the effect of source collimation we observed counting characteristics with two different collimating tubes of 1.4 - and $0.6-\mathrm{mm}$ diameters mounted over the polonium source. Figure 4 shows the results. It is clear from the three plots that the slope of the counting characteristic progressively decreases on putting the collimators in front of an extended source and even becomes negative for a highly collimated source. Clearly the absence of oblique rays for a collimated source reduces the slope. The increasing $V_{\text {off }}$ can even make the slope negative, as is probably the case for the lowest curve. The middle curve represents perhaps an intermediate case with limited collimation. The observed negative slope of the high-collimation curve is not the effect of the external resistor in our case, as will be clear from discussion further on, but should be attributed to the collimation and space-charge effects.

When examined on an oscilloscope, the counter pulses were found to be of more or less the same magnitude for a fixed applied voltage and the pulse- 
rise time $\left\langle 10^{-7} \mathrm{sec}\right.$, but the pulse height increased by about $10 \%$ when the voltage was raised from 2.6 to 4.6 . Of this increase about half took place between 2.6 and $2.9 \mathrm{kv}$ before the start of the plateau region. The rest occurred over the entire plateau region; the quiescent corona current increases with voltage, so that the effective field between the wire and the plate increases only very slightly. Indeed, depending upon the external resistor used, $V_{\text {eff }}$ can even decrease with increasing $V_{\mathrm{a}}$ in certain cases, where a fall in the pulse heights can be anticipated.

\section{Quiescent Current}

A corona discharge is set up around the anode wire as the applied voltage is raised above the counting threshold. A bluish glow in the form of a fine streak of light is observed all along the wire's length. The visible glow extends about one wire diameter toward the plate.

With the source removed we studied the variation of corona current with applied voltage by placing a microammeter in series with the cathode resistor ( $R=R_{1}+R_{2}$, Fig. 2). The quiescentcurrent characteristic for the counting arrangement described earlier is shown in Fig. 3. It is seen that the quiescent current sets in at the beginning of the hump in the counting characteristic. Then there is a very small region where it increases nonlinearly with increasing applied voltage. Beyond about $2.9 \mathrm{kv}$ it increases linearly. The start of the linear region corresponds clearly to the start of the counting plateau.

Examining the quiescent condition on an oscilloscope, we observe corona pulses, their number increasing with the applied voltage. Near the applied voltage corresponding to the hump in Fig. 3, corona pulses of comparatively large magnitude are observed and corona oscillations were loud enough to be clearly audible. Probably the slight increase in the counting rate at the hump is due to these corona oscillations. Once the corona discharge has extended over the whole length of the wire, the counting condition seems to stabilize and the observed rate settles down to the normal value.

The quiescent current characteristics were also observed for four different values of $R(4.2,7.7,11.5$, and 19 megohms). The results are shown in Fig. 4. These curves, obtained for different values of $R$, fit well with the ideas outlined earlier. For each of these curves, $V_{\text {eff }}$ can be calculated easily and the previous predictions regarding the variation of $V_{\text {erf }}$ with $V$. examined. The plots of $V_{\mathrm{eff}} \mathrm{vs} V_{\mathrm{a}} \mathrm{ob}$ tained in this way are given in Fig. 5 . Clearly the higher curves correspond to the ease when $k(R)<1 / R$ and $V_{\text {ell }}$ increases with $V_{\mathrm{s}}$. As expected, the rate of increase of $V_{\text {eff }}$ with $V_{\mathrm{a}}$ gradually falls as $R$ is increased. The bottom curve corresponds to the case where $k(R)>1 / R(R \cong 19$ megohms), and here $V_{\text {eff }}$ decreases with increasing $V_{\mathrm{s}}$. For $R$ between 11.5 megohms and 19 megohms, there should also be a critical resistance $R_{0}$ for which $V_{\text {off }}$ would remain practically constant (dotted line $A B)$ over a large range of $V_{\mathrm{s}}$. For all values of $R$ greater than $R_{c}, V_{\text {eff }}$ and hence the counting rate are expected to decrease with the applied voltage, as has been actually observed by Connor.

For all our subsequent measurements we have a total quenching resistance $R=4.2$ megohms.

\section{Rate vs Distance}

The number-distance curve for an alpha source was measured with this counter using a collimated old polonium source. Figure 6 shows the numberdistance relation at room temperature. The shape of the curve is similar to that obseryed with other alpha detectors near the end of the range. The considerable spread of the curve observed between the peak and the maximum distance at which counts were registered $(\sim 3.6 \mathrm{~cm})$ is due to our using a fairly old polonium source (8). With the source nearer to the counter there is observed, however, a remarkable fall in the counting rate with decreasing distance, making the monochromatic alpha peak singularly fine. This sudden fall seems to arise from two causes. First, the specific ionization of the alpha particles is known to fall somewhat for distances less than those corresponding to the maximum of the Bragg curve. This will amount to a reduction in a priori sensitivity and a consequent fall in the counting rate as the source approaches the counter. However, this factor does not seem to account for the rather steep fall observed. The increase in the number of oblique rays as the source is moved toward the counter seems to be the dominant cause, the a priori sensitivity being lower for the oblique rays than for the normal rays for the same working voltage. The range, however, of monochromatic alpha particles from a freshly prepared source can be determined by this counter with reasonable accuracy.

\section{Temperature Dependence}

None of the earlier workers have tried to study the temperature dependence of the spark counter. Connor has expressed the view that it is likely to be slight, but no exact data on temperature effect appear to exist.

To study the temperature dependence it is essential to allow for the apparent change in the range of particles caused by the change in the density of the surrounding air as its temperature is changed. We thought it best to study the temperature dependence by observing the number-distance curve for the polonium source at two different temperatures. The first observation was at room temperature $\left(20^{\circ} \mathrm{C}\right)$. Then the counter was placed in a thermostatically controlled volume where the temperature was maintained at $49 \pm 1^{\circ} \mathrm{C}$. The results are shown in Fig. 6, where one solid curve corresponds to room temperature and another to the higher temperature. 'The dotted curve was drawn by shifting the room-temperature curve toward higher $d$ values by an amount equal to the apparent increase in the range of the alpha particles expected at the higher temperature. The dotted curve may be called the extrapolated number-distance curve for the higher temperature.

It will be seen from the figure that a better fit between the shifted curve and the measured one might result from a simple "stretching" of the Bragg curve; that is, by increasing all abscissas by the same fraction of themselves so that extrapolated ranges (or the maxima) would coincide. We have not attempted this slightly more complicated analysis, but it appears that a small temperature effect exists.

We are greatly indebted to Professor D.S. Kothari for stimulating this work and his kind and continued interest during its progress. This work was carried out under the financial support of the Department of Atomic Energy, Government of India.

\section{BIBLIOGRAPHY}

1. H. Greinacher, Z, tech. Phys. 16, 165 (1935) W. V. Chang, S. Rosenblum, Phys. Rev, 67, $222(1945)$

8. R. D. Connor, Nature 163,540 (1949)

4. R. D. Consor, Proc. Phys. Soc. 64, 30 (1951)

5. R. D. Connor, J. Sci. Instr. 29, 12 (1952)

6. R. M. Payne, J. Sci. Instr. 26, 321 (1949)

7. M. P. Savel, Comptes Rend. 234, 2596 (1952)

8. N. K. Saha, K. L. Kaila, Indian J. Phys, 29 417 (1955) 


\title{
Fusion-Reactor Control
}

\author{
A "black-box" approach to the fusion reactor \\ predicts over-all control characteristics \\ resembling those of a conventional fission reactor
}

\author{
BY M. A. SCHULTZ \\ Westinghouse Electric Corporation, Pittsurgh, Pennsylvania
}

THE Fosion REACTOR concept has been developed to the point where there is now enough information to enable discussion of some of the associated engineering problems. For instance, R. F. Post's article "Controlled Fusion Research" (1) provides an excellent background for speculation as to what a controlled fusion reactor might eventually look like and how it might be expected to behave. Although the basic conceptual problems outlined by Post appear to be most formidable, it is quite clear that the succeeding engineering problems will easily match them in complexity.

Attempts to understand these problems can begin by comparing some of the problems with currently understood fission-reactor problems. The reactor control engineer in particular can begin to be interested in questions of stability, control, and speed of response.

Reactor stability, in the mind of the control engineer, concerns the reactor as a whole and as a component in the complete plant. Detailed effects, such as neutron-flux spatial variations in the fission reactor or plasma oscillations in the fusion reactor, are of little concern. Problems of plasma instabilities that affect the basic feasibility of a fusionreactor design [for example, the type predicted by Kruskal and Schwarzchild (2)] will presumably have been solved before the engineer is required to design a control and protective system.

These considerations lead to the "black-box" approach to over-all reactor stability in which no geometry need be specified and the performance of the "box" as a controlled power generator is given by kinetic equations in terms of a few variables.

\section{Fission-Reactor Example}

The approach used to analyze the kinetic behavior of a conventional fission reactor is a familiar example of the black-box method. The "black-box" picture of a simple fission reactor is shown in Fig. 1. The kinetic performance of such a reactor without temperature coefficient is mathematically described by the following well-known equations:

$$
\begin{gathered}
\frac{d n}{d t}=\frac{\delta k}{l^{*}} n-\frac{\beta}{l^{*}} n+\sum_{i=1}^{6} \lambda_{i} C_{i}+S \\
\frac{d C_{i}}{d t}=\frac{\beta_{i}}{l^{*}} n-\lambda_{i} C_{i}
\end{gathered}
$$

The symbols are conventional $(3,4)$.

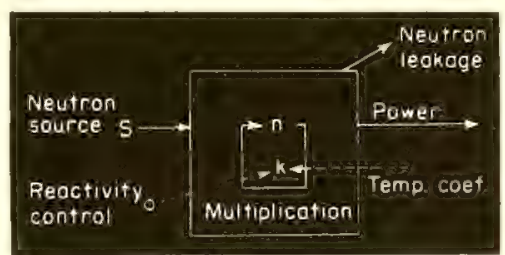

FIG. 1. Black-box representation of fission-reactor control problem. $k$ is reactivity, $n$ is neutron flux and $S$ is neutron source

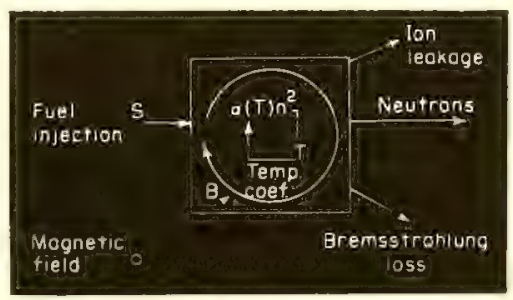

FIG. 2. Black-box representation of fusion-reactor control problem. $\alpha(T)^{2}$ is reaction rate, $T$ is reaction temperature, $B$ is magnetic fleld and $S$ is fuel injection rate
Equation 1 is a neutron-balance equation, and Eq. 2 describes the time behavior of delayed emitter concentrations. It will be noted that there is no concern about time variations in the spatial distributions of neutron flux or emitter concentrations. The equations are concerned rather with how the entire box responds as a unit with time. From these equations the frequency response or transfer function, relating output to input, can be derived. An examination of the transfer function then allows prediction about the stability of the reactor and gives insight into the general characteristics required of an external control system. This gross handling of a complex, detailed subject has been most successful for the fission reactor and consequently leads one to attempt a similar approach for the fusion reactor (see Fig. 3).

\section{Fusion-Reactor Black Box}

The corresponding "black box" for a fusion reactor in which the reaction is presumed to be confined by a magnetic field might be that of Fig. 2 . Several other configurations of black boxes can also be made, but at this stage it seems more important to illustrate the approach rather than present all of the specific concepts. The equations and constants describing this box follow Post's notation and graphs.

Two equations of roughly the same form as the fission equations can be written for a simple confined plasma system:

$$
\begin{aligned}
\frac{d E}{d l} & =15 n^{2}\langle\sigma v\rangle_{\mathrm{av}} W \\
& -0.54 \times 10^{-30} n^{2} T^{3 / 2}-\frac{K_{D_{1}} n k T}{\tau(T)}
\end{aligned}
$$




$$
\frac{d n}{d t}=-\frac{K_{D_{1}} n}{\tau(T)}-1 / 2 n^{2}\langle\sigma v\rangle_{\mathrm{avg}}+S
$$

Equation 3 is a power-balance equation that represents the rate of change of internal energy in the plasma, and Eq. 4 represents a particle-number balance. Since all that follows is concerned entirely with obtaining and interpreting the mathematical solution of these two equations, an attempt will first be made to deseribe, and hopefully to justify, the terms of the two equations and the many assumptions that have been made.

The assumption is made that some suitable configuration has been found for a fusion reactor, and this reactor will be first considered as operating on the DD reaction. None of the generalizations to be made is affected when the DT reaction is used. The deuterium ions in the "box" have a density $n$, are at an elevated temperature $T$, and are presumed to be confined in a nonspecified manner by means of a fixed constant magnetic field $B$. The process is assumed to be occurring on a per-unit-volume basis, and the over-all volume and geometry are ignored. As in the case of the fission-reactor equations, the variables can also represent either total or average values for the entire box.

$d E / d t$ is the rate of change of energy within the "box" and can be represented

\section{Definitions of Auxiliary Constants}

\section{Definition}

Auxiliary constant

\section{$D D$ reaction}

DT reaction

$\begin{array}{lll}K_{1} & 3 / 2 k & 3 / 2 k \\ K_{2} & 1 / 2 \mathrm{~W} & 1 / 4 \mathrm{~W} \\ K_{D} & \frac{K_{D_{1}} k}{\eta B}(\mathrm{Bohm}) & \text { Same as DD } \\ A & K_{2} K_{\alpha} n_{0}{ }^{2} & \text { Same as DD } \\ & -1 / 2 K_{B} n_{0}{ }^{2} T_{0}{ }^{-3 / 2} & \\ B & -2 K_{D} n_{0} T_{0} & \\ & 2 n_{0} \alpha_{0} K_{2} & \text { Same as DD } \\ & -2 K_{B} n_{0} T_{0}{ }^{3 / 2} & \\ & -K_{D} T_{0}{ }^{2} & \\ C & \frac{2}{3} \frac{K_{D}}{k} n_{0} & -\frac{2}{3} \frac{K_{D}}{k} n_{0} \\ & -1 / 2 K_{\alpha} n_{0}{ }^{2} & -1 / 4 K_{\alpha} n_{0}^{2} \\ \text { I) } & -\frac{2}{3} \frac{K_{D}}{k} T_{0} & -\frac{2}{3} \frac{K_{D}}{k} T_{0} \\ & -n_{0} \alpha_{0} & -1 / 2 n_{0} \alpha_{0} \\ E^{*} & K_{1} n_{0} & \text { Same as DD } \\ F & K_{1} T_{0} & \text { Same as DD }\end{array}$

by the rate of change of the thermal energy possessed by the system; hence, $d E / d t=d(3 / 2 n k T) / d t$, where $k=$ Boltzmann's constant. The power balance consists of two parts: first, a production term representing thermonuclear energy release, and, secondly, various losses of power by specific processes. The production term is $1 / 2 n^{2}\langle\sigma v\rangle_{\mathbf{s v g}} W$, where the constant $W$ is the energy released to the charged particles per reaction. The energy possessed by the neutrons generated in the DD reaction is presumed to be lost from the system, gainfully or otherwise, and therefore is not available to keep the reaction going. The $\langle\sigma v\rangle_{\mathrm{avs}}$ term, the average of the product of cross section and relative velocity as given in Fig. 4, is dependent on temperature and is designated hereafter as $\alpha(T)$.

The first loss term of Eq. 3 is given by $0.54 \times 10^{-30} n^{2} T^{1 / 2}$. This is the Bremsstrahlung radiation loss from the system. Here the electrons radiating are assumed to be in temperature equilibrium with the ions (the electron particle density, equals the ion particle density).

The second loss term $K_{D_{1}} n k T / \tau(T)$ is the power loss from the system by particle diffusion, where $\tau(T)$ represents the average time taken by particles beginning at the source to drift through the magnetic field to the walls of the box. This "confinement time" must be comparable to the average reaction time for a successful fusion reactor.

Other loss terms can also be imagined, such as the radiative losses caused by impurities and the possible need to provide internal power to heat up new particles inserted into the system. These other losses only add additional negative terms to Eq. 3 and are a refinement that does not alter substantially the form or the approach to the control-stability problem.

In Eq. 4 the term $K_{D_{1}} n / \tau(T)$ can be thought of in fission-reactor terms as the particle loss caused by leakage. Similarly, the second term, $1 / 2 n^{2}\langle\sigma v\rangle_{\text {ave }}$, is analogous to fuel depletion or burnup. The third term is a source or injection term which refers to the rate of addition of new particles to the system to compensate for the burnup and leakage.

It will be noted that the roles of the source term in the fission and fusion reactors are somewhat reversed. In a fission reactor as the power level is increased the effect of the source becomes smaller and smaller. In tho fusion reactor at higher power levels more and more particles are needed to keep the reaction going. Injection is probably a better term for use with fusion reactors in that the introduction of new fuel is actually implied.

\section{Mathematical Solution}

We must first consider the form of the confinement time $\tau(T)$ with respect to the temperature and the magnetic field. The confinement time is inversely proportional to an average

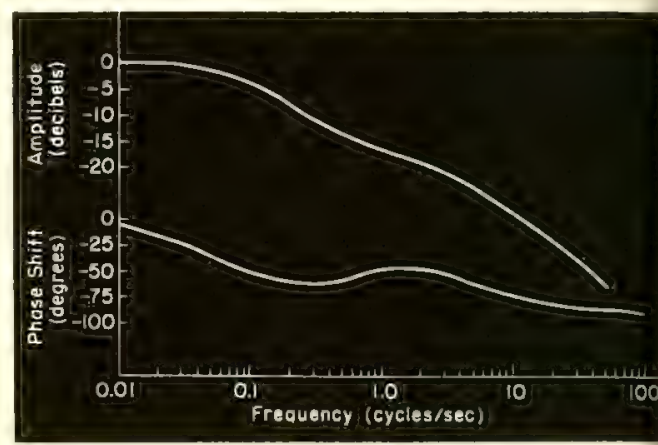

FIG. 3. Frequency dependence of transfer function $\Delta n / \Delta S$ relating time variation of ion density $n$ and injection rate $S$ for a fusion reactor operating on the DT reaction

drift velocity. Post gives two forms for this velocity-the so-called "classical" diffusion theory in which $\tau(T) \sim B^{2} T^{3 / 2} / n$ and Bohm (5) diffusion theory where $\tau(T) \sim B / T$. For our purposes either form is suitable, and we first select Bohm diffusion. Iet

$$
\tau(T)=\eta \frac{B}{T}
$$

where $\eta$ is an appropriate constant. An indication will be given later as to the effect upon stability of changing the form of the diffusion equation.

We can now rewrite Eqs. 3 and 4 in terms of the auxiliary constants defined in the table as follows:

$$
\begin{gathered}
K_{1} \frac{d}{d t} n T=K_{2} n^{2} \alpha(T)-K_{B} n^{2} T^{3 / 2} \\
\quad-K_{D} n T^{2} \\
\frac{d n}{d t}=-\frac{2}{3} \frac{K_{D}}{k} n T-1 / 2 n^{2} \alpha(T)+S
\end{gathered}
$$

These equations are nonlinear, but as in the case of the fission reastor useful solutions are obtained by linearizing them. The object here is to derive a transfer function expressing the re- 
sponse of the dependent variables $T$ and $n$ to sinusoidal variations of the injection term $S$. The characteristic equation so determined will then indicate whether the system is stable and will show the effect of the various parameters on stability. The process consists in assuming that each of the time variables, $n, T$, and $S$, is made up of a steady-state value and a small sinusoidal variation about the steadystate value:

$$
\begin{aligned}
& n=n_{0}+\Delta n(t) \\
& T=T_{0}+\Delta T(t) \\
& S=S_{0}+\Delta S(t)
\end{aligned}
$$

The variable coefficient $\alpha(T)$ also is linearized as

$$
\alpha(T)=\alpha_{0}+K_{\alpha} \Delta T
$$

We neglect second-order differentials and note that a new term $K_{\alpha}$ is introduced which is analogous to the temperature coefficient of reactivity in a fission reactor. These new variables can now be introduced into Eqs. $3 \mathrm{a}$ and 4a. With new constants, as defined in the table, we have finally

$$
\begin{gathered}
E \frac{d \Delta T}{d t}+F \frac{d \Delta n}{d t}=A \Delta T+B \Delta n \\
\frac{d \Delta n}{d t}=C \Delta T+D \Delta n+\Delta S
\end{gathered}
$$

These equations are now linear with constant coefficients; so we can take the Laplace transforms. The zero conditions are ignored as we have already subtracted the steady-state terms.

$$
\begin{gathered}
E s \Delta T(s)+F s \Delta n(s)=A \Delta T(s) \\
+B \Delta n(s) \\
s \Delta n(s)=C \Delta T(s)+D \Delta n(s)+\Delta S(s)
\end{gathered}
$$

The transfer functions for $\Delta n$ and $\Delta T$ can now be obtained directly using $\Delta S(s)$ as a forcing function.

$$
\begin{aligned}
& \frac{\Delta T(s)}{\Delta S(s)} \\
& =\frac{B-F s}{E s^{2}+(F C-E D-A) s+A D-B C}
\end{aligned}
$$

$$
\begin{aligned}
& \frac{\Delta n(s)}{\Delta S(s)} \\
& =\frac{E s-A}{E s^{2}+(F C-E D-A) s+A D-B C}
\end{aligned}
$$

These transfer functions, which are of the form

$$
\frac{s+c}{(s+a)(s+b)}
$$

start off with a finite gain at zero frequeney. They have three break points, assuming $a$ and $b$ are real, and the amplitude response ultimately falls off at a rate of $6 \mathrm{db}$ per octave. The roots can very easily be obtained once specific numerical values are used.

\section{Stability Criteria}

We now are in a position to examine the stability of the system. The system is stable if the roots of the characteristic equation (the denominator of Eqs. 9 and 10) have negative real parts. A necessary and sufficient condition for the roots of a quadratic equation to have negative real parts is that all of the coefficients have the same sign. As we know that the energy $E$

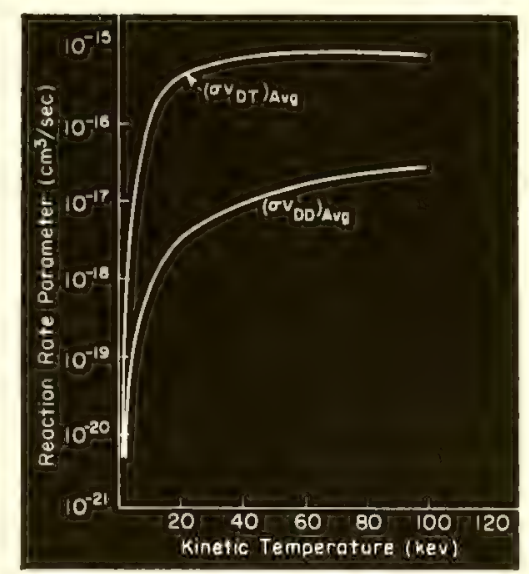

FIG. 4. Temperature dependence of reaction-rate parameter for DT and DD reactions assuming Maxwellian particle distribution. This figure from article by R. F. Post (I)

must be positive, to have a stable system it is necessary that

$$
\begin{aligned}
F C-E D-A & >0 \\
A D-B C & >0
\end{aligned}
$$

We can now see what these criteria mean in terms of the original constants. First using inequality 11 and substituting the expressions from the table, we find that, for stability,

$$
\begin{aligned}
3 / 2 k \alpha_{0}-3 / 4 k K_{\alpha} T_{0}>1 / 2 K_{\alpha} W \\
-1 / 2 K_{B} T_{0}^{-1 / 2}-\frac{2 K_{D} T_{0}}{n_{0}}
\end{aligned}
$$

This inequality reveals several interesting bits of information. It will be noted that the terms to the right of the inequality are related directly to the gain and loss terms of Eq. 3 with the last two terms representing the losses. From the negative signs it appears as though the greater the losses the higher the possibility of stability. Physically this seems reasonable, as a system with large losses is heavily damped and not capable of continuous oscillations.

Other inferences can be drawn from this inequality by examination of $K_{\alpha}$, the temperature coefficient. If $K_{\alpha}$ is negative, meaning a decreased reaction rate with an increase in temperature, the system is clearly stable, since the other pertinent constants are all positive. (From Fig. 4, $\langle\sigma v\rangle$ for the DT reaction shows a negative slope over a portion of its range. The DD reaction does not exhibit this characteristic in the indicated range. ) If $K_{\alpha}$ is zero or slightly positive, the system also appears to be stable. The maximum positive value permissible for $K_{\alpha}$ depends on $T_{0}, n_{0}$, and also $K_{D}$, which is a function of the confinement process and field strength. Without considerable refinement and good numerical data the above statements can be interpreted only as trends either toward or away from stability.

The remaining stability criterion can be obtained by substituting the original constants into inequality 12

$$
\begin{aligned}
2 K_{D} T_{0} & \left(\alpha_{0}-\frac{K_{2} K_{\alpha}}{3 k}\right) \\
+ & T_{0}^{2} K_{D}\left(\frac{2}{3} \frac{K_{D}}{k n_{0}}-1 / 2 K_{a}\right) \\
+ & 1 / 2 K_{B} \alpha_{0} n_{0} T_{0}^{-3 / 2} \\
> & K_{B} T_{0}^{3 / 3}\left(\frac{K_{D}}{k}+K_{\alpha} n_{0}\right) \\
& \quad-4 / 3 K_{2} \frac{K_{D}}{k} \alpha_{0}
\end{aligned}
$$

This inequality does not appear to have the obvious physical significance that inequality 13 does. However, if we assume that 13 imposes a lower bound on the temperature for stability, at a high temperature there seems to be a form of density limit from 14. It can be seen that at high temperatures and high densities the $K_{B} T_{0}^{3 / 2} K_{\alpha} n_{0}$ term might ultimately predominate if $K_{\alpha}$ remains positive and finite. Stableoperation then appears to have a low-temperature limit and a high-density limit at high temperature.

To indicate that the trend remains the same regardless of the form of the diffusion, we can again solve Eqs. 3 and 4 using the classical type of diffusion rather than the Bohm type. The ine- 
quality corresponding to 13 becomes

$$
\begin{aligned}
& 3 / 2 k \alpha_{0}-3 / 4 k K_{\alpha} T_{0}>1 / 2 K_{\alpha} W \\
& -\left(\frac{K_{B}+K_{D}}{2}+5 / 2 K_{D}\right) T_{0}^{-3 / 2}
\end{aligned}
$$

It is apparent that the diffusion has affected only the last term of the equation and all of the mentioned trends still are valid.

\section{Frequency Response}

Returning now to the transfer function, it is of interest to obtain order-ofmagnitude answers for the frequency response of the reactor to get a feel for the control problem. - For this step one must supply numerical values for the roots of the characteristic equation. As a convenient starting point toward obtaining numbers we will first try the DD reaction. Post's calculations (Fig. 4) suggest that fission reactors operate at a power density of about 100 watts $/ \mathrm{cm}^{3}$. At this power density it is pointed out that a DD reactor operating at $100 \mathrm{kev}$ kinetic temperature would need a particle density of $3.3 \times$ $10^{15}$ particles $/ \mathrm{cm}^{3}$. From these values the remaining constants can be obtained:

$$
\begin{aligned}
n_{0}{ }^{2} K_{B} & =6 \mathrm{watts} / \mathrm{cm}^{3} / \mathrm{kev}^{3 / 2} \\
K_{2} & =1.9 \times 10^{-13} \text { watt-sec } / \mathrm{reaction} \\
K_{D} & =1.32 \times 10^{-19} \mathrm{watt} / \mathrm{kev}^{2} \\
K_{\alpha} & =3.45 \times 10^{-19} \mathrm{~cm}^{3} / \mathrm{sec} / \mathrm{kev} \\
\alpha_{0} & =3.05 \times 10^{-17} \mathrm{~cm}^{3} / \mathrm{sec} \\
k & =1.6 \times 10^{-16} \mathrm{Watt}-\mathrm{sec} / \mathrm{kev}
\end{aligned}
$$

$K_{D}$ is obtained from the steady-state situation of Eq. 3 in which it is assumed that the diffusion losses just make up the difference between the production of the thermonuclear charged-particle energy and the Bremsstrahlung losses.

A check of inequality 13 indicates that the constants we have chosen represent an unstable system since

$$
\begin{aligned}
3 / 2 k \alpha_{0}-3 / 4 k K_{\alpha} T_{0} & >1 / 2 K_{\alpha} W \\
& -1 / 2 K_{B} T_{0}{ }^{-1 / 2}-\frac{2 K_{D} T_{0}}{n_{0}}
\end{aligned}
$$

$7.32 \times 10^{-33}-4.13 \times 10^{-33}>65.5$

$$
\times 10^{-33}-27 \times 10^{-33}-8 \times 10^{-33}
$$

It is evident that at the temperature and density chosen the positive temperature coefficient $K_{\alpha}$ is too large and quite dominates the stability picture. The depletion-loss term is small compared with the Bremsstrablung losses, and it appears as though the conditions we have chosen do not provide sufficient damping to keep the system stable. We have the option of changing $T_{0}$ or $n_{0}$ in an effort to find better conditions.
If, as is the case with the DT reaction, $K_{\alpha}$ ultimately becomes smaller or even negative as the temperature is further increased, we might hope to find stability at a higher temperature than 100 kev. Post's curves give no clue as to the magnitude of $K_{\alpha}$ for substantially higher temperatures. Attempts to improve the stability by lowering $n_{0}$ do not work within the limits of our assumptions. That is, with Bohm-type diffusion and the assumption that in the steady-state diffusion losses make up the difference between thermonuclear gain and Bremsstrahlung loss, $n_{0}$ and $K_{D}$ vary directly with each other and hence a change in density does not affect the stability of inequality 13 .

To continue our attempt to assign control speeds to the thermonuclear reactor, we will now derive the transfer function for a DT reactor operating in a range where we know the reaction has a negative temperature coefficient and where it must be stable. In this exarnple for numerical values we can again assume the fission-reactor power density of 100 watts $/ \mathrm{cm}^{3}$, a temperature of $100 \mathrm{kev}$, and an equal mixture of deuterium and tritium with the resulting total density of $4.2 \times 10^{14}$ particles $/ \mathrm{cm}^{3}$.

The basic stability equations are the same as Eqs. 3 and 4, but as shown by the table the constants are slightly different. The following values were derived from Post's curves:

$$
\begin{aligned}
\alpha_{0} & =8.2 \times 10^{-16} \mathrm{~cm}^{3} / \mathrm{sec} \\
K_{\alpha} & =-5 \times 10^{-18} \mathrm{~cm}^{3} / \mathrm{sec} / \mathrm{kev} \\
W & =5.75 \times 10^{-13} \mathrm{ev} \\
K & =14 W=1.44 \times 10^{-13} \mathrm{ev} \\
K_{D} & =4.75 \times 10^{-18} \mathrm{Watt} / \mathrm{kev}^{2} \\
A & =-0.531 \\
B & =4.71 \times 10^{-14} \\
C & =-8.09 \times 10^{12} \\
D & =-2.15 \\
E & =0.101 \\
F & =2.4 \times 10^{-14}
\end{aligned}
$$

The transfer functions can now be obtained directly, and the density function becomes

$$
\frac{\Delta n(s)}{\Delta S(s)}=0.101 \frac{s+5.27}{(s+24.3)(s+0.595)}
$$

This transfer function is plotted in Fig. 3. It is evident that it is similar to the transfer function of a fission reactor. The last break point in the transfer function occurs at approximately 4 cycles/sec. In fission-reactor terms this would correspond to a reactor hav- ing a mean neutron lifetime of about $3 \times 10^{-4}$ sec. This equivalent lifetime might cause the reactor to have a speed of response between that of a graphite-moderated fission reactor and a large water-moderated fission reactor, both of which are easily controllable.

Inasmuch as Fig. 3 shows a finite gain at zero frequency the fusion-reactor transfer function resembles that of a fission reactor having a negative temperature coefficient. (The transfer function for a fission reactor with zero temperature coefficient has infinite gain at zero frequency.) Thus all of the well-known properties of a fission reactor having a slight negative temperature coefficient would appear to carry over to this type of fusion reactor. The reactor in addition to being stable would provide a measure of self-protection against transients and would be incapable of "running away."

\section{Conclusions}

Many other interesting analogies between the two types of reactors could be derived from the equations presented here. It must be remembered, however, that the "black-box" approach must first be proved. Questions as to the effects of changing magnetic fields as well as the possibility of having moving boundary conditions have been ignored. Our attempt has been merely to show one type of engineering approach to an ultimate control problem.

However, in so far as our assumptions are valid, we have been able to establish two things: (1) because of its positive temperature coefficient the DD reaction will be unstable for temperatures up to at least $100 \mathrm{kev}$ (about $10^{9}$ $\left.{ }^{\circ} \mathrm{K}\right)$; (2) the DT reaction, on the other hand, is stable below this temperature, and indications are that the controlsystem speed requirements would not be excessive.

The author wishes gratefully to thank $J, N$. Grace for his assistance.

\section{BIBLIOGRAPHY}

1. R. F. Post, Controlled fusion research-an application of the physics of the high temperature plasmas, Rev. Mod. Phys, 28, 338 (1956)

2. M. Kruskal, M. Schwarzchild, Proc. Roy. Soc (London), A 223, 348 (1954)

3. H. Soodak, E. C. Campbell, "Elementary Pile Theory" (John Wiley \& Sons, Inc.,- New York, 1950)

4. M. A. Schultz, "Control of Nuclear Reactor and Power Plants" (McGraw-Hill Book Co. Inc., New York, 1955)

5. A. Guthrie, R. K. Wakerling, "The Characteristics of Electric Discharges in Magnetic Fields" (McGraw-Hill Book Co., Inc., New York, 1949 


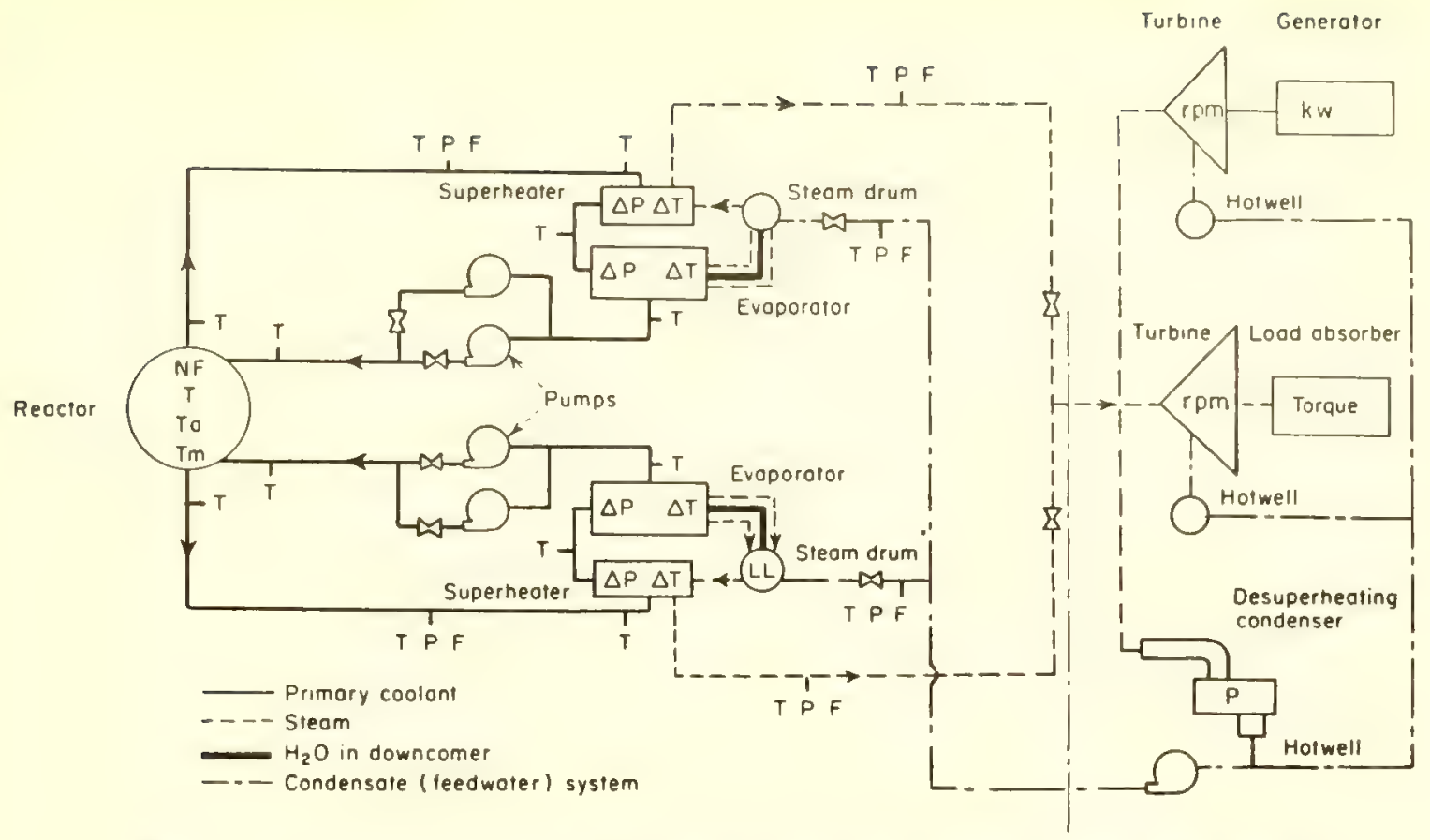

FIG. 1. Typical nuclear-power test loop showing points where sensing elements gather data. Quantities monitored in the reactor are neutron fux (NF), temperature (T), average of reactor inlet and outlet temperatures (Ta), and fuel-element-"meat" temperature (Tm)

\title{
Data-Handling System for Nuclear-Power Tests
}

\author{
Automatic data reduction is replacing hand logging and plotting. \\ This fast, accurate system gathers transient and steady-state data \\ needed for the design of efficient nuclear power plants
}

\section{BY RAYMOND A. EDWARDS}

Atomic Power Equipment Department, General Electric Company, San Jose, California

ONE OF THE CHALLENGING aspects of nuclear engineering is that a great deal of information has still to be gathered before standardization or "cookbook" plant design can take place. Interpretation of data from a chart and logging of data by an operator are time-consuming and subject to error.
Test facilities as large as the Navysponsored submarine prototypes justify the use of modern data-reduction equipment. Human operators need never handle the data until they are printed on a sheet or plotted as curves. Stripchart recorders, conventional indicators for steady-state data, and multi- channel oscillographs are losing ground to advanced methods of data reduction.

A data-reduction system has been designed for nuclear-power test facilities based on information gathered during design and operation of three prototypes. Depending on their significance, variables are read out in one or 


\section{Variable}

No. of Points

\section{Variable}

No. of Points

\section{Reactor Core}

Bulk-water temperature

Fuel-rod-meat temperature

Surface temperature

Flux measurement

In-core-boiling detectors

Channel flow

Fuel-element failure

Internal structure \& interpass

Temperature

Pressure

External mechanical region

Cooling-coil temperature

Cooling-coil flow rate

Internal-mechanisms temperature

Motor-coil temperature

Latching-solenoid temperature

External-region-of-pressure-vessel temperature

Top-hat-shielding temperature

Shield-tank-water temperature (in cooling coils)

Shield-tank-cooling-flow rate

Power Plant

Downcomer flow

Thermocouples in cooling jacket of feed-watercooled components

Pressurizer-wall-temperature distribution

Pressurizer steam quality
80

10

25

20

100

5

40

20

40

50

50

50

10

50

5

2

7

6

1
Coolant flow in main-coolant piping

Coolant pressure in loop

Steam-generator $\Delta T$

Reactor $\Delta T$

Downcomer temperature

Coolant temperature inside steam generator

Coolant temperature vertical section hot leg

Coolant temperature vertical section cold leg

$\Delta T$ vertical section hot leg

$\Delta T$ vertical section cold leg

Inlet-isolation-valve-body temperature

Outlet-isolation-valve-body temperature

Steam-generator-shell temperature

Coast-down flow measurement

Primary-coolant-pump rpm

Main-coolant-valve piston $\Delta p$

Thermal-trap temperature

Steam pressure upstream of main turbine

Steam temperature upstream of main turbine

Ahead \& astern turbine pressure

Condenser pressure

Load-absorber torque

Feedwater pressure

Turbine-generator-set-condenser pressure

Throttle-valve position

Turbine-throttle pressure

Power input to main pumps more of the following 6 sections: highspeed reducer, low-speed reducer, scram monitor, wide strip-chart recorders, 12 channel oscillograph, and temporary portable instruments. Data are all ready for feeding to a computer and can be stored efficiently so that data searches can be quick.

\section{Potential}

There are few limitations to what automatic data reduction can accomplish. Performance is limited only by the customer's imagination, his budget, and the know-how of the manufacturer of the data reducer. System simplicity will increase as confidence is gained in both reactor facilities and related instruments. 'The quantity of data required will steadily decrease to a reasonable point. This epoch may be still 5-10 years off.

\section{System Requirements}

A typical power-reactor test loop is shown in Fig. 1. On it are indicated the points where sensing elements must monitor system variables. For such a loop there is always the question,
"What are the significant data to be recorded?" A final answer is seldom available until a detailed plant-test program is completed. Still, a $90 \%$ approximation of necessary recording instrumentation can be formed rather early in the plant design. If one designs a flexible system with enough room for spare input signals, it is safe to proceed with the equipment.

Basic considerations. Several important matters should be considered at the outset: (1) Flexibility in the system must allow for instrument rearrangement and system expansion. (2) A standard input signal for the majority of the variables must be chosen early in the planning. Inputs of $0-20,0-25$, or 0-50 millivolts are convenient, for there are amplifiers that can be connected to pressure and $\Delta p$ transmitters and ionization chambers to produce linear millivolt outputs. (3) One must choose the form in which the data will appear on the final readout device; that is, in millivolts or directly in terms of the variables. (4) If a computer is available, readout on compatible magnetic tape or punched cards is desirable.
Strip-chart recorders. According to present information, the variables of Table 1 are important enough to be continuously recorded on wide stripchart recorders. If these variables are desired for transient data, they can be fed simultaneously into the high-speed data reducer.

Data reducers. All other required data, as listed in Table 2, are recorded in either the low-speed or high-speed reducers. In general the high-speed unit is totally engaged in recording reactor information and a few variables in the heat exchangers. Little emphasis is placed on determining the performance of a single component during plant operation. Such information is gathered prior to prototype startup in component-testing facilities. If singlecomponent tests are necessary during plant operation, temporary portable instrumentation is provided. The reactor is the one exception to this policy.

It can be seen that much of the listed data is relevant to the performance of the steam generators. This has been found necessary for the following reasons: (1) Due to the reactor's faster 
rate of heat output, the thermal stresses on the heat exchangers are much greater than in presently operating power plants. (2) Since the coolants used are generally corrosive to the primary system, the heat exchangers must be constantly monitored. (3) More accurate data on heat exchangers than is now available will permit us to choose the sizes of the units more accurately. Heat exchangers have been oversized by as much as $50 \%$ to insure plant performahce. Since compactness is important in nuclear power plants, accurate sizing is important.

Nuclear-power test facilities will shortly accumulate a greater amount of accurate data on heat exchangers than has been amassed in the last 25 years of conventional power-plant design.

\section{SYSTEM DETAILS}

The data-reduction system is shown in the block diagram of Fig. 2. The recording and monitoring panel records on strip charts all variables of interest to operations and design groups. The slow data reducer gives operations u typed log of 300 plant variables. The scram monitor constantly scans at high speed significant scram-circuit signals and coast-down information. The fast reducer is to record transient data and other "instantaneous" data that may be required for the IBM 704 computer.

\section{Display Panel}

A U-shaped panel for displaying the information in the system is ideal. Figure 3 is a plan view of all the recording equipment. It will be seen that the panel is divided into the following sections: health physics, reactor, primary coolant, steam system, and mechanical components.

Readout is accomplished with side-lighted name plates or Inditron lighted-filament tubes. Equipment to accomplish the readout is generally a self-balancing potentiometer and a digitizer or an integrating voltmeter.

A console desk associated with the panel serves as a communications center. On it there are a telephone, intercom system, interval timer, timemarker button, and annunciator-reset button. Another feature is an indicator on which the operator can dial any plant temperature.

A scroll on the console desk allows the operator to scan operational graphs, calibration curves, etc. This eliminates the necessity of pasting such charts on the test panel.

A useful adjunct to the panel is a centrally located graphic representation of the plant such as Fig. 1. The read- out points for the most important variables are marked on this layout, particularly for the use of operations personnel and visitors. From the points of view of both information and politics, a useful piece of information to display with the layout is reactor fullpower hours.

The panel should be designed so that modules holding from one to three recorders can be removed by merely loosening fasteners. Experience shows that test facilities suffer many changes to the panel, usually resulting in addition of instruments as well as rearrangement. Removable sliding doors at the back of the cubicles are superior to hinged doors in the cramped spaces of test sites.

\section{Strip-Chart Recorders}

Wide strip-chart recorders are preferred over all others. Operations people prefer round charts, but those are not good for getting accurate data. Operations' chief objection to strip charts is that a single day's operation is not visible as a unit. To compromise the chart speed is slowed to $1 \mathrm{in.} / \mathrm{hr}$, with some change gears available for faster chart speeds when specific tests require them. Plant operations are generally run from separate panels, but it is convenient for the operators to

\section{TABLE 2 - Variables That Should Be Fed to Fasl and Slow Reducers}

\section{Variable}

Health Physics

Area fixed gamma monitor above reactor shielding

Airborne particle gaseous monitor, maneuvering area

Area fixed neutron monitor above reactor shielding

Area particle and gaseous monitor, ventilation exhaust

No. of Points

1

1

1

1

Reactor

Period level $10^{-9}-10^{-4} \%$ power level

Period level 0-150\% power level

Count rate $10^{-2}-10^{-4} \%$ power level

Linear level $10^{-}-100 \%$ power level

Linear level $0-150 \%$ power level

Log-neutron-level recorder

Control-rod-position precision indicator

Control-rod-position recorder

Reactor temperatures

$\Delta p$, reactor (total)

Reactor inlet and outlet temperatures

$\Delta p$, reactor interpass (if applicable)

Neutron flux distribution

\section{Variable}

\section{Primary Coolant}

Flow measurements in core

Primary-coolant flow

Pressurizer liquid level and temperature (if applicable)

Pressurizer pressure

Pressurizer spray flow rate and spray-flow-inlet temperature (if applicable)

Steam Generator

Steam-generator-inlet and -outlet temperatures Superheater-inlet temperature (if applicable)

$\Delta p$ across steam-generator system

Feedwater flow rate

Steam-drum liquid level

Steam flow

Steam-drum pressure

Feedwater temperature and drum temperature

Steam flow to main turbine

Steam flow to steam condenser (heat sink)

Steam temperature at turbine throttle valve

Power Equipment

Main-turbine rpm

Turbine-generator-set output (if applicable)

Cooling-water-flow rate to rod-drive mechanism
No. of Points

$6-50$

2

1

1

1

2

2

2

2

2

2

2

1

1

1

1

4- 20 


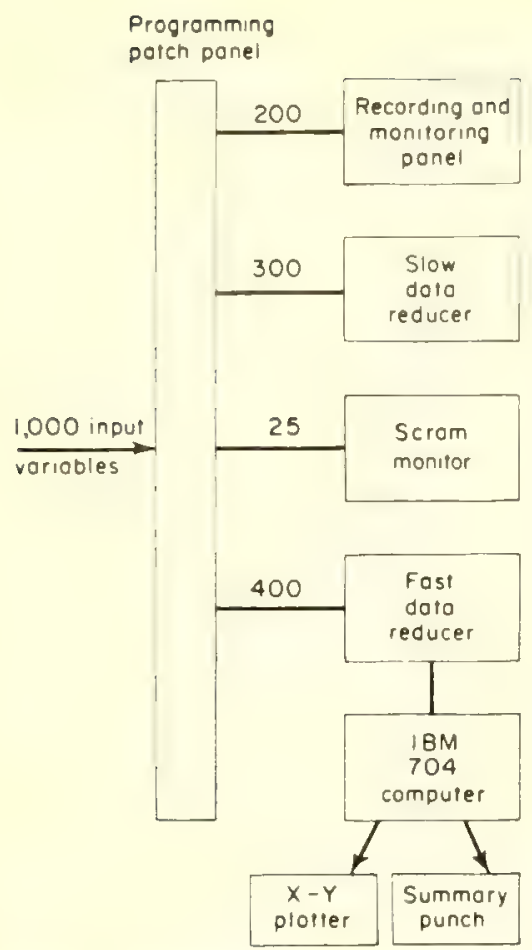

FIG. 2. Block diagram of data-reduction system in operation. Computer is not inventoried as part of system

check the results of their transient maneuvers on the strip recorders.

Recorders should have solenoidactuated time-pulse markers operated from a single button at a console. Markers that type the time on charts are inconvenient, for this equipment hides about $20 \%$ of the chart. Another feature the recorders should have is a range-suppression switch to allow fullscale pen travel for about four equal segments of the input. This is generally easy if the full-scale input was initially 4-5 millivolts or more.

Scales marked 0-100 are preferred, for exact ranges are known only late in the project's design. Moreover range suppression makes impossible the use of an exact scale marking.

\section{Fast Reducer}

A typical high-speed reducer is shown in Fig. 4.

Final design of the transient-data recorder for a test facility is a compromise. In a plant maneuver, 100150 points are of interest. Requests are common to scan all of them in $1 / 20$ sec-requiring \& speed of $2,000-3,000$ points per sec. The transient lasts about $2 \frac{1}{2} \mathrm{~min}$, the length of a flowdecay period. Another use for the fast reducer is to obtain "instantaneous" readings of its $100-150$ points at $1-\mathrm{min}$ or 5-min intervals in ordinary oper- ation. It soon becomes impractical to record and store so many data. For reasons of handling, storage, economy, and reliability the scanning speed must be reduced to a reasonable value. Dependability of systems with rates above 600 points/sec is a moot question among manufacturers of such equipment.

Computers that are comparable with the IBM 650 are usually required at nuclear testing facilities. Thus the data should be recorded in a form compatible with the computer. Magnetic tape that can be fed to an IBM 704 is advisable. A tape-to-card converter can be used with an IBM 650 .

Linearization of inputs from thermocouples should be done in the computer instead of making the data reducer capable of handling both linear and nonlinear signals.

Other features recommended for the data reducer are (1) time markers every $0.01,0.1$, or 1 sec as desired, (2) a calibration signal to check system accuracy, (3) variable scanning speed, (4) plug-in relay circuits, (5) plug-in signalcheck points, and (6) coding to prevent data mixup.

\section{Slow Reducer}

The main value of the low-speed data reducer is to make unnecessary hand logging of data and to provide at all times a typed log of general-performance data. It can also check the highspeed unit. Operation is continuous during startup and on a half-hour or one-hour printout basis otherwise.

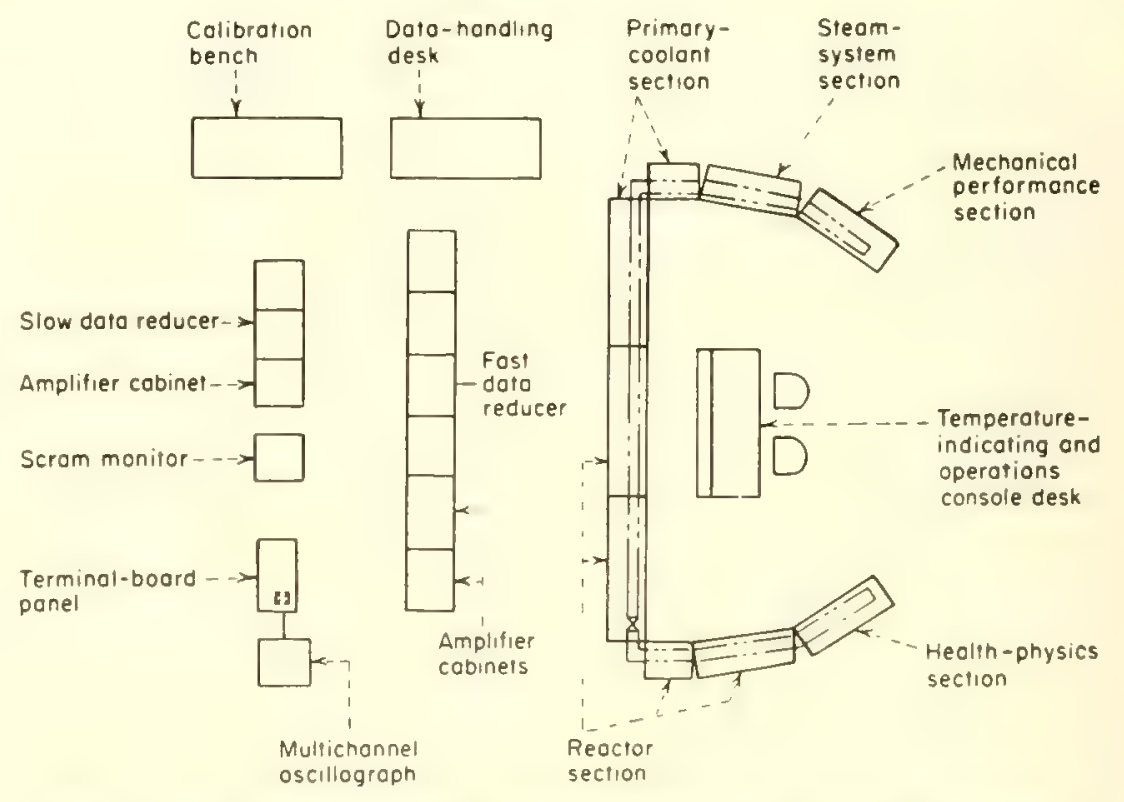

FIG. 3. Plan view of recording equipment. Legends key cabinets to block diagram of Fig. 2

The design is generally easier than that of the high-speed unit. Scanning speed is about one point/sec. Readout on the typewritten $\log$ is directly in terms of the variable. As in the highspeed unit, such features as coding, time, calibration signals, variable scanning speed, and operation on demand are included. Point-eliminate or even bank-eliminate switches are convenient when the number of inputs begins to be too great. Low-speed loggers in these plants are sometimes burdened with as many as 300-500 points.

\section{Scram Monitor}

Scram circuits to cause reactor shutdown are a constant source of concern. After a reactor scrams, the question immedia tely arises: What circuit caused the scram and what happened during shutdown? The scram monitor answers that question.

The instrument monitors the trip circuits as well as important plant variables at a speed of about 100 points/sec. Data are recorded on a continuous magnetic tape in analog form. The tape is constantly being erased. However, $21 / 2$ minutes each of printed and unprinted tape are on both sides of the recording head. If a scram occurs, the erasing mechanism stops, and the coastdown is recorded.

\section{Patch Panel}

A programming patch panel gives a high degree of flexibility to the recording facilities. It permits easy transfer 
of recording of variables from one facility to another-e.g., panel to highspeed reducer or high-speed reducer to low-speed reducer, etc. Space limitations prevent use of conventional telephone-type jack panels. It is not unusual to be handling over 1,200 variables in such a test facility.

Clamp-in patchboards are used to schedule the variables into the data reducers. Upper left and lower right quadrants of the boards contain incoming signals. The upper right quadrant connects to the low-speed reducer, and the lower left goes to the highspeed unit. A spare board permits prewiring for the next test. It is advisable to use a 2 -wire patchcord instead of a single-wire one as in other systems. This eliminates incorrect wiring of polarized inputs such as those from thermocouples. In wiring the board a close check must be made to avoid jumbled points. Pressure, temperature, and radiation channels are not interchangeable.

If a central data-reduction system serves for several prototypes, pushbutton actuation of relay banks should be considered to switch in and out the inputs from various prototypes.

\section{Oscillographs}

Even with all of this data-recording equipment it is seldom satisfactory to the responsible engineers to have all the transient data recorded "blind"; that is, on tape. Significant channels are fed also into a high-impedance pentype oscillograph so engineers can have an over-all view of trends of critical variables during a transient. Twelve channels are considered sufficient.

The attitudes of engineers dictate the need of parallel recording systems such as oscillographs and strip-chart recorders. As a rule they want to "see" the transient while it is occurring. Outside of the instrument engineers few believe that their data will be successfully recorded on the magnetic tape. When this human bottleneck has been broken, the use of chart recorders can be greatly restricted and possibly eliminated.

\section{Personnel}

The attitude of management, generally governed by unfamiliarity with this equipment, is usually a great stumbling block. Two of their most frequent statements are, "It sounds fine if you can get it to work," and

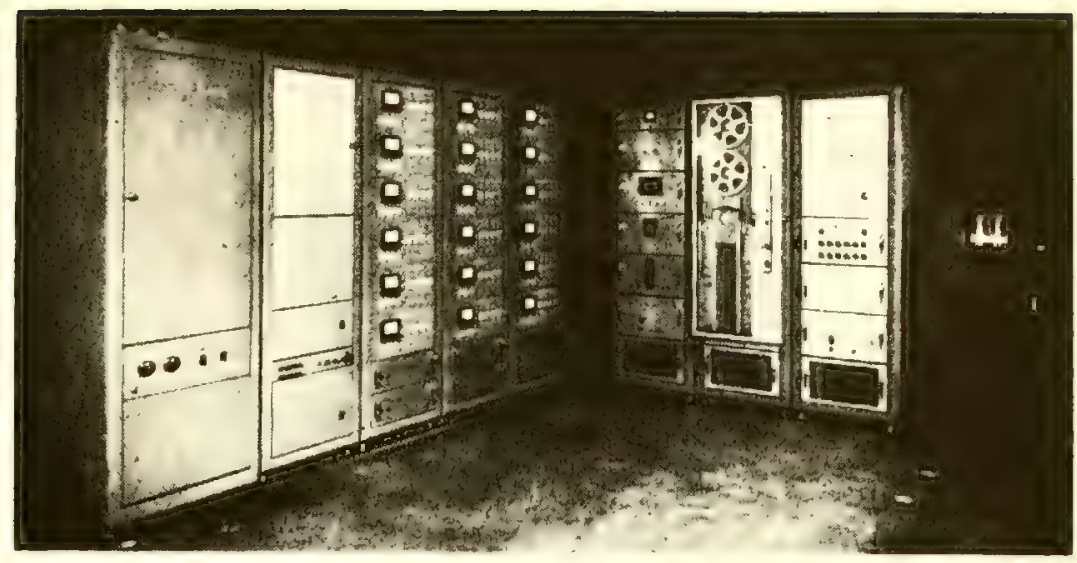

FIG. 4. High-speed data reducer used in studying prototype reactor

"What do we do with all the data?" It may be pointed out that the aircraft industry has used automatic datareduction equipment for the last 8 or 9 years.

We have two suggestions to reduce many ills: (1) Raise the level of operators who will handle this equipment to at least second-year-college people. Second-year-high-school-level operators do not operate IBM computers or simulator equipment. The complexity of data-reduction equipment is about the same as that of a computer or simulator. (2) Raise instrumentmaintenance men to a level equal to the operators'.

\section{Cost}

Data-recording equipment for a nuclear-power test facility can be expected to cost $\$ 250,000-300,000$ - about $15 \%$ of the total instrument budget. This cost is not unreasonable if you consider that the plant will cost about \$20-million and that a computer equivalent to an IBM 704 generally handles the data from such a plant. The recording instruments and data handling should not be the weak links - the sources of errors.

The cost of this instrumentation and the quantity of personnel required to produce it have always been considered high. A rule of thumb for the instrument cost in process chemical plants is that it is generally $6-10 \%$ of over-all expense. For test facilities, however, instrument costs are higher. There are large test facilities where instruments represent $25 \%$ of a total investment of \$10-million. Instrumentation in an early nuclear power plant cost about $5 \%$ of the total. This includes operational instruments as well as prototype-testing and operational instruments.

Cost of recording facilities breaks down about as follows:

Panel

Low-speed reducer

$\$ 70,000$

High-speed reducer

40,000

Scram monitor

90,000

12-channel pen-

type oscillograph

10,000

Console

3,000

TOTAL

$\$ 233,000$

Also required are thermocouples, pressure and $\Delta p$ transmitters, and ionization chambers. The types and numbers of each can vary so much from system to system that it is inaccurate to guess the cost of each group. However, experience shows that this equipment may exceed the expense of the recording equipment by as much as a factor of four.

An indication of the expense involved in supplying sensing elements and related electronics to provide suitable signals in the steam plant is as follows:

$\begin{array}{cr}65 \text { temperatures } & \$ 10,000 \\ 8 \text { pressure drops } & 6,000 \\ 1 \text { liquid level } & 800 \\ 12 \text { pressures } & 9,600 \\ 2 \text { flow rates } & 2,600 \\ 1 \text { conductivity } & 500 \\ \text { TOTAL } & \$ 29,500\end{array}$

These costs may seem high, but they are standard for transient instrumentation. It must be remembered that this $\$ 30,000$ worth of instruments is in only the steam-generation part of the plant.

Other systems such as the primarycoolant system and the reactor are even more highly instrumented and require costlier instruments because of stringent safety requirements. 


\section{Prospectors need small instruments. Canada's Department of Mines has developed 4 prototypes for search and assay that can be carried by hand or in the pocket. They are cheap and reliable}

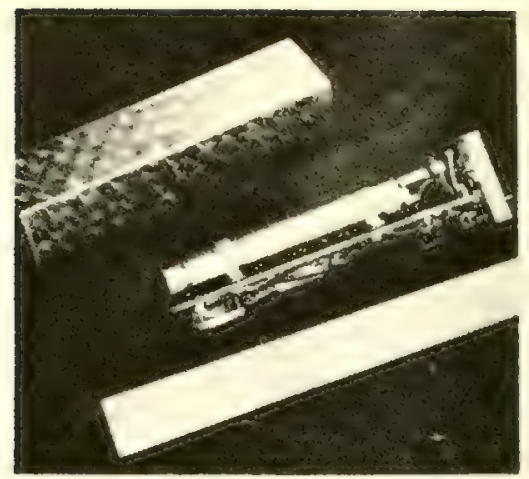

FIG. 1. G-M counter has aural indication. First model had square case

\section{All-Transistor Circuits}

By G. G. EICHHOLZ, G. E. ALEXANDER and A. H. BETTENS

Department of Mines and Technical Surveys

Ottawa, Canada

IN THE LAST FEW YEARS as uranium mining has become important among Canadian mining industries, a need has arisen for specialized radiation detectors. As a limited demand was anticipated, we undertook development of a number of portable detectors.

The characteristics of five of them are tabulated on p. 92. They have completely transistorized circuits for light weight and compactness. Circuits are kept as simple as possible, and circuit "building blocks" are standardized as much as possible. The units are intended as prototypes to stimulate commercial production.

High-voltage supplies. High-voltage circuits were largely based on a simple oscillator power supply $(1,2)$ modified to suit the particular require-

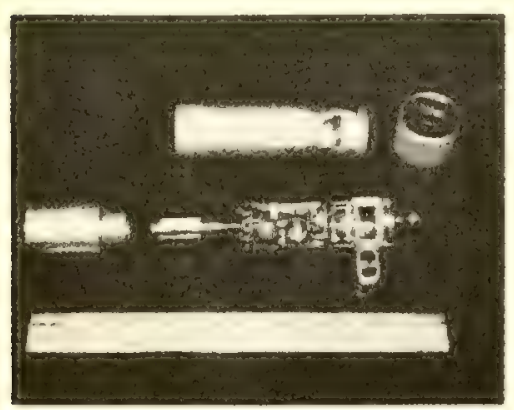

FIG. 4. Directional counter has either speaker or meter indication ments. Farlier designs with vibrator power supplies (B) have proved unsatisfactory because of limited life, large bulk, and high battery drain.

\section{Portable G-M Counter}

To avoid the bulkiness of most portable G-M counters we chose a G-M tube first and designed around it. The tube has to be large, halogenquenched for long life, and preferably low-voltage.

For a rugged, lightweight, generalpurpose survey instrument it was decided to use aural indication only. Brush BB-134 hearing-aid* and Argonne microphones were found to be small enough yet loud enough.

\footnotetext{
* Brush Electronics Co., Cleveland Ohio.
}

Two mechanical designs were made. The first was in a square box (Fig. 1), but the later model in a case of elliptical cross section fits the hand much better (Fig. 2). Both are powered by three penlight batteries, Eveready No. 912 or equivalent. The circuit consists of only an oscillator power supply with voltage tripler and direct aural indication (Fig. 3).

Tests show that it is advisable to coat the G-M tube with black paint or to wrap it with friction tape to reduce its photosensitivity.

\section{Directional G-M Counters}

Another problem was to make a directional counter for underground use in uranium mines Preliminary

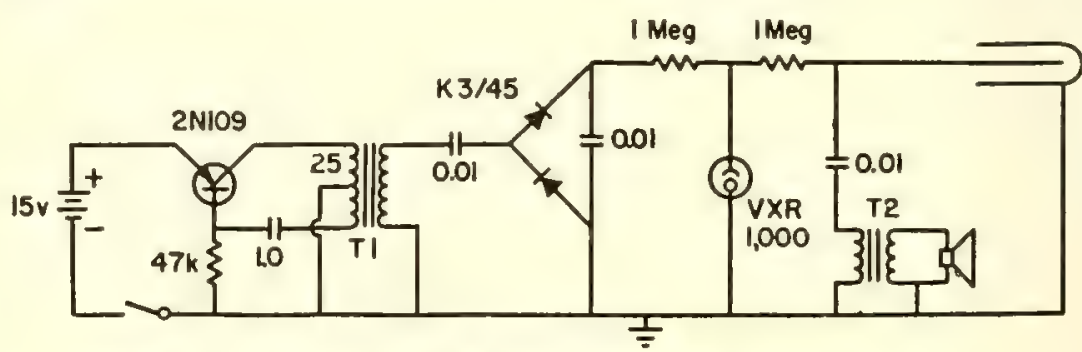

T1-Ferroxcube, Primary: 45 furns tapped at 20, Secondary: 2,000 furns. T2-Hommond 142J

FIG. 5. Speaker model of directional counter has this oscillator-doubler circuit. Life is. limited by relatively large battery drain of $8 \mathrm{mo}$ 


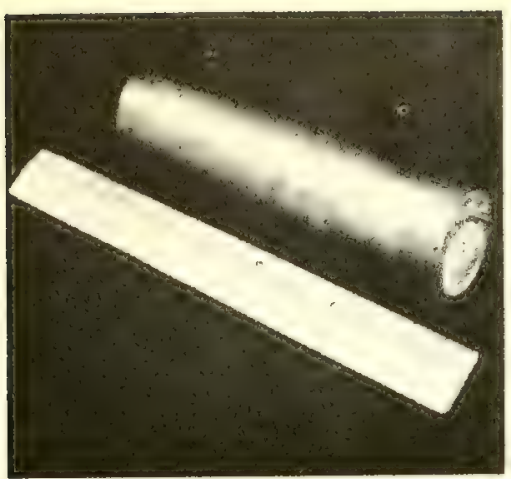

FIG. 2. Oval case makes this model of G-M counter easier to hold

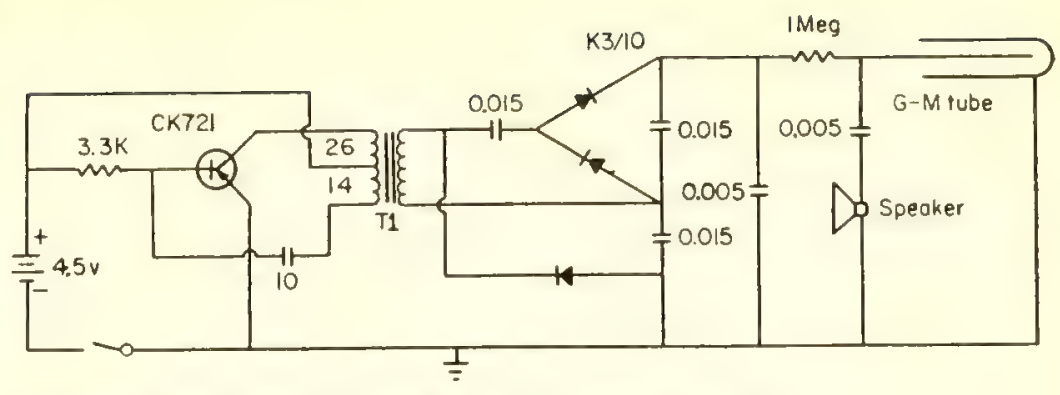

T1-Ferroxcube, Primary: 40 turns topped at 14, Secondary: 850 furns Speoker-Brush BB-134(Crysial)

FIG. 3. Circuit for portable G.M counter has transistor oscillator and voltage tripler. It is powered by three penlight batteries

\section{for Portable Detectors}

work on this subject has been described previously (3), but much further development has taken place since.

Because of the high ambient background radiation, good directionality is essential. On the other hand weight and size must be kept down to make the unit acceptable to mine staff; pocket size is desirable. One possible approach is a very small, insensitive G-M tube to reduce background. However, this means insufficient sensitivity to low-grade ores, and for this reason we chose a different approach. We use the highly localized sensitivity of an end-window G-M tube run in the "corona-spark region" $(3,4)$; with this system shielding is not excessive.

Field tests on earlier units ("spotters") have shown that cables for separate detector probes and earphones are objectionable. Therefore all re- cent units have been designed on the flashlight pattern as self-contained, tubular detectors.

Three different models have been made: (a) a simple, highly collimated detector with loud aural indication, (b) a similar unit with meter indication and interchangeable shields and (c) a less directional counter for the detection of radioactive dynamite (5). These instruments differ only in minor details; most of their mechanical features are the same. They use halogen-quenched end-window G-M tubes with a concentric lead shield at one end. The indicator is mounted on the other end. The unit is switched on and off by a knurled ring on the case.

Figure 4 is an exploded view of the directional counter with speaker indication. The tube is run at 1,200 volts; that is, above the G-M plateau. The speaker is a miniature, waterproof, permanent-magnet type made by Canadian Benaudi Ltd.* Power is supplied by a 15-volt hearing-aid battery The poor shelf life of these batteries is a weakness of the present unit, and alternatives are being investigated. The shield is a lead-filled brass container. Figure 5 shows the circuit.

Different insets can be screwed into the shield to provide different collimation angles. The tube window is protected from rock particles by a small screen.

Meter model. The directional counter with meter indication uses the same shell with the addition of a metal adapter ring to mount the meter in place of the speaker. Its circuit is shown in Fig. 6. The meter used

* 287 Metcalfe St., Ottawa, Ont.

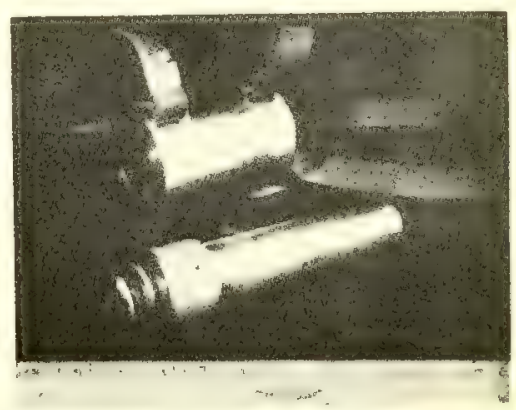

FIG. 7. Heavy shield adapts directional counter for high-grade-uranium mines

FIG. 6. Meter model uses half-wave rectifier supply and replaces speaker in circuit with transformer-coupled meter-and-rectifler system 


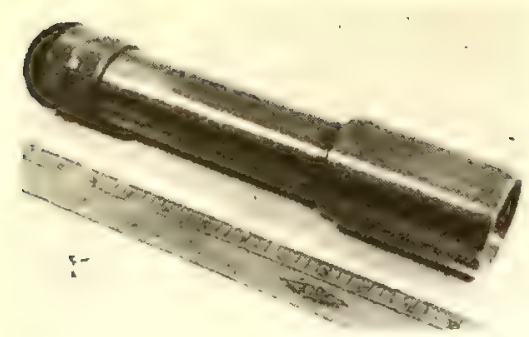

FIG. 8. Semidirectional counter locates dynamite that fails to explode

is International Instruments' Model $150 \mathrm{~W}$ microammeter, $100 \mu$ a full scale. This has proved rugged enough and easy to mount. A Benaudi rubber ring fits the adapter ring to the counter case. For greater simplicity no zerosetting control is provided, and the sensitivity must be checked periodically with a suitable standard source.

In order to adapt the counter for use in high-grade-uranium mines a special heavy shield was designed that is interchangeable with the standard shield. For easier carrying it is provided with a shoulder strap, and its use has proved feasible in field tests underground. Use of the heavy shield does not often justify its extra inconvenience, but it has proved valuable where it is needed. It is shown in Fig. 7.

\section{Semidirectional Counter}

In connection with the work on location of radioactive dynamite (see p. 170) (5) it became clear that the

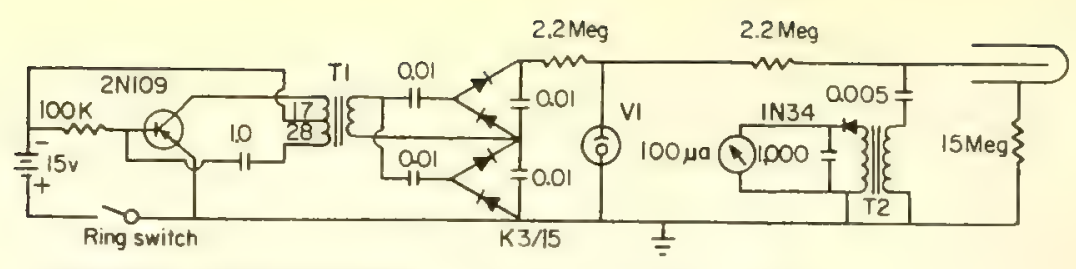

T1-Ferraxcube, Primary: 45 turns tapped of 17, Secondary, 2,000 turns T2-Output: 500 ohms, Input 50,000 (2 Hammond $142 \mathrm{~J}$ transformers) Vi-Victoreen 5950 corona regulator

FIG. 9. Circuit for semidirectional counter is similar to that of Fig. 5, but it has higher sensitivity. Hearing-aid battery supplies 15 volts directional counter could be too directional. A semidirectional counter was designed (Fig. 8). For this a slightly larger end-window tube was chosen with a larger shield opening. The circuit is shown in Fig. 9 and is seen to follow that of Fig. 5 in essentials, though its sensitivity is higher. Mechanical details had to be especially designed as the EW3H G-M tube required a longer case than the other model.

\section{Scintillation Detector}

The design of a gun-type portable scintillation counter (Fig. 10) was undertaken as it was felt that existing counters were unduly heavy and required too many expensive batteries (6). This detector uses three 935 batteries for power. The meter has five overlapping ranges and a zero-set control. The complete circuit diagram is presented in Fig. 11.

The sensitivity of the detector on its most sensitive range is such that a $100-\mathrm{gm}$ sample of ore containing $0.5 \%$ $\mathrm{U}_{3} \mathrm{O}_{8}$ will give a deflection of $6 \mu \mathrm{a}$ at a distance of 6 in. The effective battery life is governed mainly by the requirements of the oscillator, which must provide enough voltage through the quadrupler circuit to keep the corona regulator struck.

\section{Carborne Counter}

In mobile prospecting by jeep or car it is not usually possible for the driver to watch a meter or dial while navigating across country over poor roads. For that reason a scintillation unit was designed to be mounted in a car and trip an alarm whenever a predetermined radiation level is exceeded. The detector unit is self-contained and demountable and can be used as a portable counter to investigate mineral occurrences. The buzzer is driven from the car battery, and the counter has batteries that can be tricklecharged from the car battery.

The complete circuit diagram is pre-
Summary of Counter Characteristics

\begin{tabular}{|c|c|c|c|c|c|}
\hline Counter & $\begin{array}{l}\text { Simple } \\
\text { Geiger }\end{array}$ & $\begin{array}{l}\text { Direc- } \\
\text { tional }\end{array}$ & $\begin{array}{c}\text { Semidirec- } \\
\text { tional }\end{array}$ & $\begin{array}{l}\text { Scintilla- } \\
\text { tion gun }\end{array}$ & Carborne \\
\hline Sensitive element & $\begin{array}{l}\text { 20th Cen- } \\
\text { tury G10H } \\
\text { tube }\end{array}$ & $\begin{array}{l}\text { Amperex } \\
230 N \text { tube }\end{array}$ & $\begin{array}{l}20 \text { th Cen- } \\
\text { tury } \\
\text { EW3H }\end{array}$ & $\begin{array}{l}11 / 4 \times 11 / 2 \\
\text { in. } \mathrm{NaI}(\mathrm{Tl}) \\
\& 6199\end{array}$ & $\begin{array}{l}\mathrm{NaI}(\mathrm{Tl}) \& \\
6292\end{array}$ \\
\hline Operating voltage & 370 & 1,200 & 600 & & \\
\hline Weight (total)(lb) & 1.3 & 3.0 & 5.8 & 2.9 & 7.8 \\
\hline $\begin{array}{l}\text { Weight (without } \\
\text { shield) }\end{array}$ & & 1.4 & 1.8 & & $\begin{array}{l}3.4 \text { (top } \\
\text { part only) }\end{array}$ \\
\hline $\begin{array}{l}\text { Approx. sensitivity } \\
\text { range ( } \mathrm{mr} / \mathrm{hr} \text { ) }\end{array}$ & $0.03-12$ & $\begin{array}{c}0.03-0.35 \\
\text { (meter type) }\end{array}$ & $0.02-0.2$ & $0.008-10$ & $0.01-10$ \\
\hline Sensitive cone & - & $40^{\circ}$ & $60^{\circ}$ & 一 & - \\
\hline $\begin{array}{l}\text { Estimated component } \\
\text { cost }\end{array}$ & $\$ 55$ & $\$ 70$ & $\$ 75$ & $\$ 170$ & $\$ 200$ \\
\hline Battery voltage (volts) & 4.5 & 15 & 15 & 4.5 & 6.3 \\
\hline Current drain (ma) & 5 & 8 & 4 & 8 & 6 \\
\hline $\begin{array}{l}\text { Expected life (days at } \\
4 \mathrm{hr} / \text { day) }\end{array}$ & 21 & 2 & 5 & $>30$ & $\begin{array}{l}\text { (buzzer } \\
\text { extra) }\end{array}$ \\
\hline
\end{tabular}

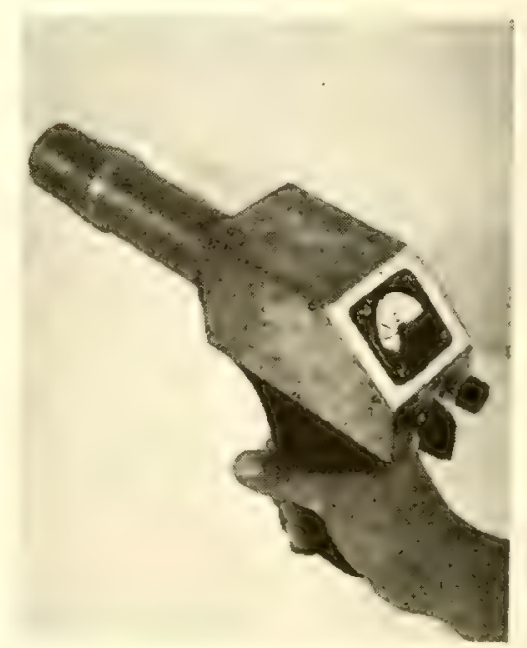

FIG. 10. Gun-style scintillation defector has zero set and 5 meter ranges 
sented in Fig. 12. The power-supply circuit is contained in a shielded section of the detector unit. A 6-pin Jones plug completes the supply circuits when the portable unit is detached from its cradle. The meter is a $1 \frac{3}{4}-\mathrm{in}$. Simpson Model 182, $100 \mu$ a full scale. The alarm system consists essentially of a Type 705 Weston Sensitrol meter relay in series with the detector meter. When the relay is tripped, it closes the buzzer circuit, which derives its power from the car battery. The relay is reset manually, and its sensitivity is controlled by the detector-range switch. Figure 13 shows the instrument.

The choice of battery posed a definite problem. Easiest solution for a carborne unit is to operate the whole system from the car battery. However, in the present case it was felt to be preferable to keep the scintillation detector self-contained for independent use. Rechargeable batteries permit long uninterrupted service. The choice of suitable lightweight batteries proved rather limited, and the type selected in the end is the Yardney Silvercel type LR-05.* These are lightweight zincsilver cells with an alkaline filling. Unfortunately they are very sensitive to overcharging, and to protect them it is necessary to charge them from the car battery while the car engine is not running. The circuit shown is intended for operation of the detector system insulated from the car body. This makes it independent of the grounding of the car battery, but the connection to plug P1 must be made as shown.

* Yardney Electric Corp., 105 Chambers Bt., N. Y., N. Y.

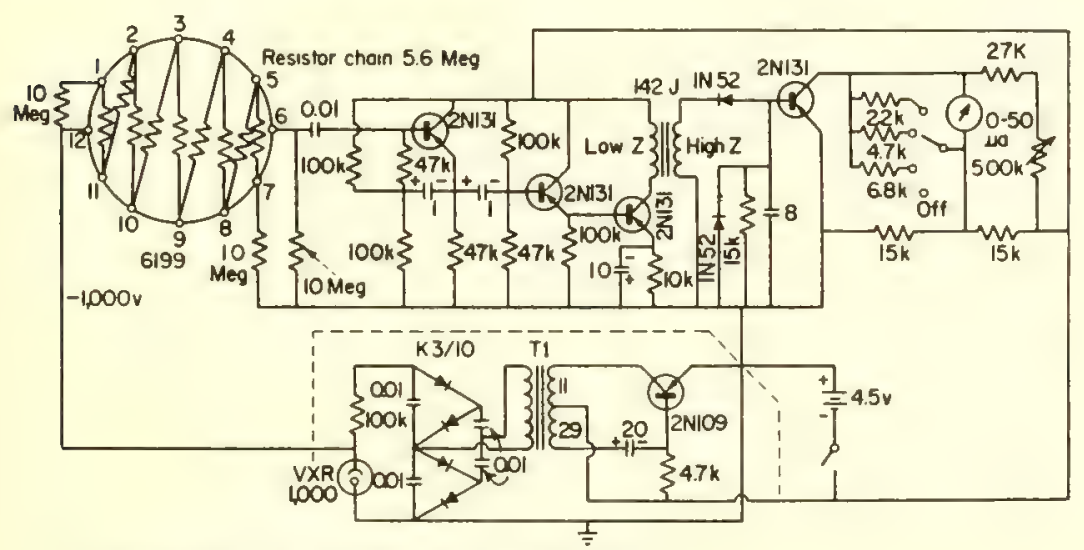

T1-Primery: 40 furns $\mathrm{No,27}$ enameled mire fopped at il furns, Secondory: 2,500 furns No.36 enameled wire

FIG. 11. Circuit for portable scintillation detector of Fig. 10

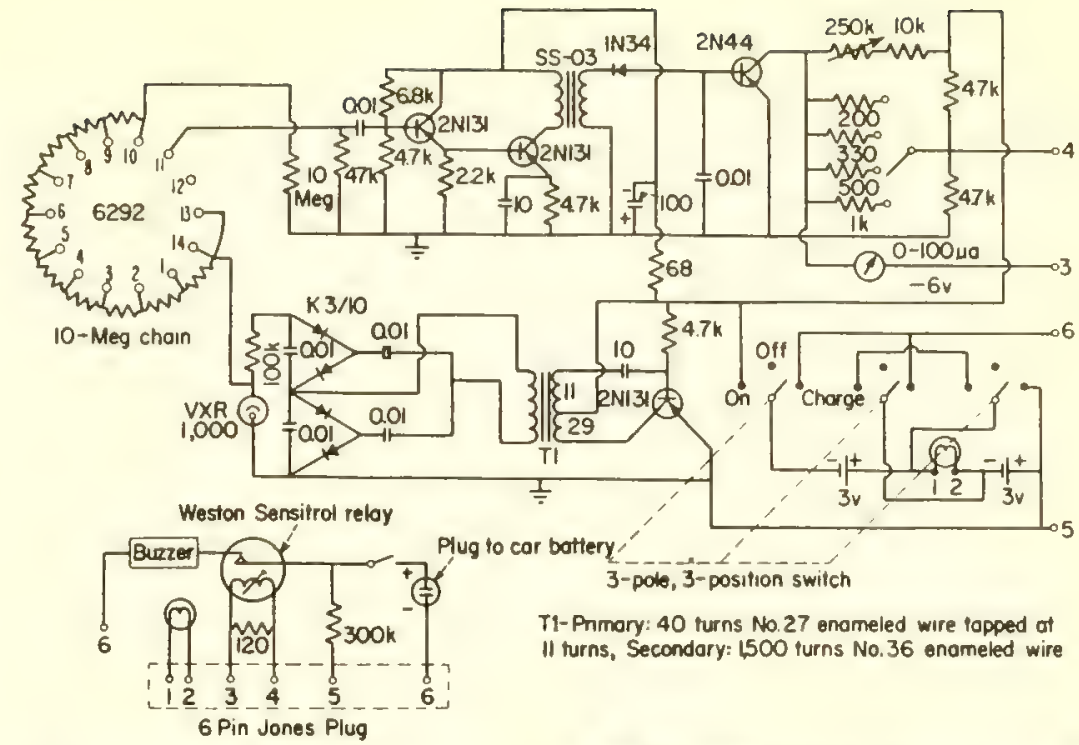

FIG. 12. Circuit for carborne unit includes buzzer operated from car battery to indicate high counting rate and rechargeable batteries in portable section

FIG. 13. Top section of carborne detector is hand-portable

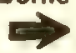

In this development mechanical design has been varied as much as possible to investigate different approaches, and further changes will probably be required for commercial production. The work has demonstrated that considerable economies are possible in size, weight, and component requirements.

There is also indication that divergence in design is to be expected as use of radioactivity spreads and higher specialization is required. Instrument cost and complexity grow steadily, but at the same time we should realize the savings that are possible by developments in simpler instrumentation.

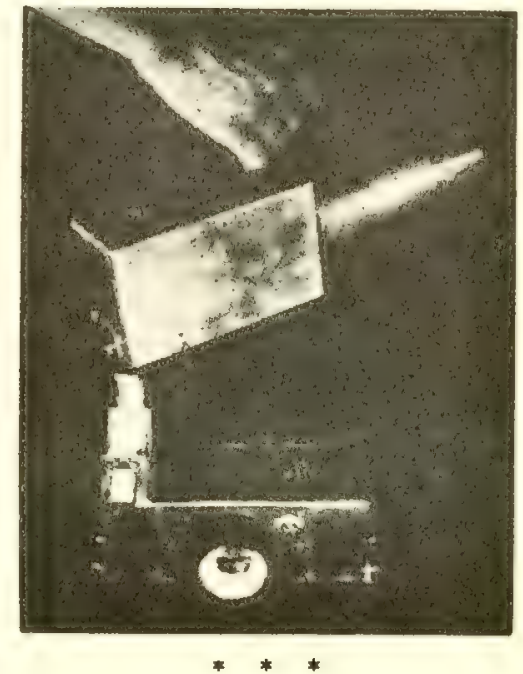

The work described here has benefited from the cooperation of many of our colleagues at the Mines Branch. Thanks are due particularly to L. S. Collett and R. B. Rowe of the Geological Survey of Canada for initial discussion of requiremenls and for assistance with field tests, to G. Meilleur for his skillful workmanship and many suggestions concerning the mechanical design of the units, to E. Hausmann of Canadian Benaudi Ltd. for the oift of a miniature speaker unit and to J. V. Krzyzewski and C. A. Josling for their assistance in the construction of the units and the drafting of circuils.

This article is based on Mines Branch Topical Report TR-1S6/56 and publiahed by permission of the Director, Mines Branch, Ottawa.

\section{BIBUOGRAPHY}

1. G. E. Alexander. A transistor high voltage poxer supply for portable Geiger counters, Mines Branch Report TR-125/54 (November 1954)

2. A. H. Bettena, G. G. Eichholz. Transistorized high voltage power supplies (in prepsration)

3. A. H. Bettens, C. Lapointe, G. G. EichholzPortable directional Geiger counters, Mines Branch Report TR-122/54 (Auguat 1954)

4. C. M. Lapointe, A. H. Bettens. U. S., Patent application Serial No. 508,724 (May 16 1955)

6. G, G. Eichholz, A. O. Smith A. Bsuer. Trans Can. In at. Mining Met. 60,59 (1857)

6. H. H. Sehwartz. Portable scintillation counters, Mines Branch Internal Report IR-183/55. (November 1955) 


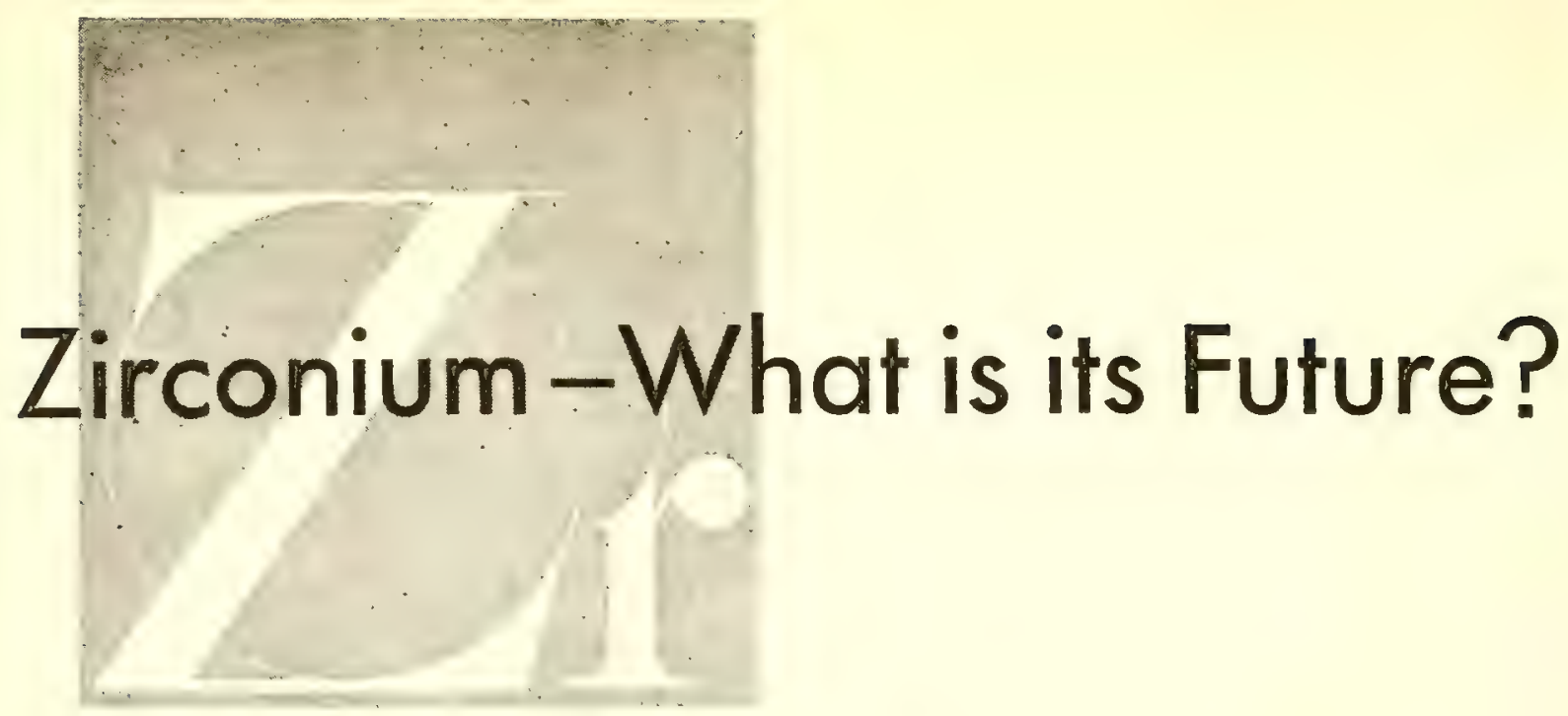

\begin{abstract}
Attracted by zirconium's low thermal-neutron cross section and good corrosion resistance, metallurgists have developed better alloys and improved fabrication techniques. Still needed-cheaper metal, more mechanical and corrosion data
\end{abstract}

The Place of zirconium in reactor engineering has been put on a much firmer basis in the last few years (NU, July '53, p. 27). In spite of its several difficult properties, metallurgists have better learned how far they can extend zirconium's mechanical and corrosion limits by processing and alloying.

Evidence of the interest in zirconium is the AEC's rapidly expanding procurement program (NU, Dec. '55, a. 13). [AEC has since requested bids, due Feb. 1, for an additional 300,000 Ib annually, bringing the annual AFC demand for reactor-grade zirconium to $1,200,000 \mathrm{lb}$.$] It has been claimed$ that the Nautilus would not have been feasible without it. As an instance of its use in power reactors, the Shippingport pressurized-water reactor uses U-Zr-alloy fuel elements clad with Zircaloy-2 (NU, Sept. '55, p. 47).

Industry's confidence in the future of zirconium is indicated by the number of companies devoting facilities and money to investigating its behavior under operating conditions and to developing fabrication techniques. A number of companies were surveyed to provide the product and price tabula- tion on pp. 6 and 7. Fabrication methods must still be perfected to allow mass production. It can be expected that prices will drop sharply in the near future - perhaps the biggest factor will be cheaper base metal through less expensive $\mathrm{Zr}$ - $\mathrm{Hf}$ separation methods.

To assess the present status of zirconium as a reactor structural material, a conference was sponsored by the Atomic Industrial Forum in New York, November 17. This article is based on the proceedings of that conference and on a NuClEonics survey of commercial products available.

\title{
Alloy Developments
}

The primary virtue of zirconium and its alloys, other than their low neutron cross sections, is their good corrosion resistance in high-temperature $\left(<680^{\circ} \mathrm{F}\right.$ ) water systems, e.g., watercooled and -moderated reactors. Zirconium-alloy systems are investigated with the aim to:

1. Develop more reproducible and predictable corrosion behavior.

2. Delay or eliminate breakaway (flaking or spalling white oxide film)after breakaway, corrosion is faster.

3. Find alloying elements that confer corrosion resistance.

4. Utilize less expensive Kroll-process sponge.

Of the binary alloying elements, Sn,
TABLE 1-Tensile Properties of Zirconium Alloys

\begin{tabular}{|c|c|c|c|c|}
\hline \multirow{2}{*}{$\begin{array}{c}\text { Alloying element } \\
(\mathrm{Zr} \text { base })\end{array}$} & \multicolumn{2}{|c|}{$\begin{array}{c}0.2 \% \text { offset } \\
\text { yield strength }\left(l b / \text { in }^{2}\right)\end{array}$} & \multicolumn{2}{|c|}{$\begin{array}{l}\text { Elongation } \\
\text { in } 1 \text { in. }(\%)\end{array}$} \\
\hline & Room temp. & $500^{\circ} \mathrm{C}$ & Room temp. & $500^{\circ} \mathrm{C}$ \\
\hline $3 \% \mathrm{Al}$ & 55,000 & 28,000 & 30 & 23 \\
\hline $3 \% \mathrm{Sn}$ & 45,000 & 24,000 & 35 & 40 \\
\hline $3 \%$ Mo & 73,000 & 30,000 & $\sim 0$ & 40 \\
\hline $3 \% \mathrm{Nb}$ & 73,000 & 30,000 & 30 & 45 \\
\hline $3 \% \mathrm{Sn}-1.5 \% \mathrm{Mo}$ & 59,000 & 30,400 & 25 & 42 \\
\hline \multirow[t]{2}{*}{$3 \% \mathrm{Sn}-3.3 \% \mathrm{Al}$} & 78,900 & 39,400 & 3 & 6 \\
\hline & \multicolumn{2}{|c|}{ Yield strength $\left(l b /\right.$ in $\left.^{2}\right)$} & \multicolumn{2}{|c|}{ Tensile strength $\left(l b /\right.$ in $\left.^{2}\right)$} \\
\hline Zircaloy-2 & 43,000 & 19,000 & 64,000 & 33,000 \\
\hline Zircaloy-3 & 37,000 & 14,000 & 60,000 & 26,000 \\
\hline
\end{tabular}


Reactor-Grade (Low-Hafnium) Zirconium Products

\begin{tabular}{cccc}
\hline INGOTS & Treatment & Size & Price \\
\hline
\end{tabular}

Allegheny Ludlum Steel Corp.*

Zr and Zircaloy Conditioned ingots

Carborundum Metals Co., Inc.

Zr and Zircaloy Double are melted; $400-600 \mathrm{lb}$; $\mathrm{min}$.

$<0.015$ wt $\%$ Hf machined or arc order $500 \mathrm{Ib}$ conditioned; Brinell hardness $=150$

Firth Sterling, Inc.

Zr or alloy

National Research Corp.

$\mathrm{Zr}$ and $\mathrm{Zr}$-base Double melted Up to $25 \mathrm{lb}$ Price on request alloys on special (Cu skull, consumorder able electrode) Are melted, poured Up to $50 \mathrm{lb}$

Price on request
Spec's

Price

Carborundum Metals Co., Inc.

$\mathrm{Zr} \quad$ Through $3 / 8$ in. on 20 mesh; 1-74 $\mathrm{lb}, \$ 22.00 / \mathrm{lb} ; \quad 75-600$, $<0.015 \mathrm{wt} \% \mathrm{Hf} \quad$ Brinell hardness $=150 \mathrm{max}$ on $\$ 18.00 ; 600-3,000, \$ 15.00$; arc-melted test ingot; max im- $>3,000, \$ 14.00$; f.o.b. Akron, purities $<0.12 \%$

N. Y.

POWDER Spec's Price

Carborundum Metals Co., Inc.

$\mathrm{Zr} \quad 20$ mesh and finer; $\mathrm{O}_{2}, \mathrm{~N}_{2}, \mathrm{H}_{2} \quad<75 \mathrm{lb}, \$ 8.35 / \mathrm{lb} ; 75-500, \$ 7.30$ $<0.02$ wt $\%$ Hf content higher than in sponge, >500, $\$ 6.40 ;$ f.o.b. Akron, N. Y. other impurities similar

CHEMICALS Price

\section{Carborundum Metals Co., Inc. \\ $\mathrm{ZrO}_{3}$ \\ $<300$ ppm Hf}

Kawecki Chemical Co.

$\mathrm{ZrO}_{2}$
$25,000 \mathrm{lb}, \$ 6.10 / \mathrm{lb} ; 25,000-50$, $000, \quad \$ 4.00 ;>50,000, \$ 3.50$; f.o.b. Akron, N. Y.

$\$ 2.20 /$ lb on contract basis (price dependent on contracted output of $75,000 \mathrm{lb}$ of oxide per month)

\begin{tabular}{lll}
\hline FABRICATED PRODUCTS Spec's & Price \\
\hline
\end{tabular}

$\mathrm{Fe}, \mathrm{Ni}$, and $\mathrm{Cr}$ are beneficial. $\mathrm{Al}$ though $\mathrm{Sn}$ decreases the corrosion resistance of pure $\mathrm{Zr}$, it counteracts the effects of nitrogen in sponge. Additions of $0.1-0.5$ wt $\% \mathrm{Fe}, \mathrm{Ni}$, or $\mathrm{Cr}$ improve corrosion resistance by delaying post-breakaway corrosion in high-temperature water and steam. In ternary alloys, $\mathrm{Fe}, \mathrm{Ni}$, or $\mathrm{Cr}$ substantially improve the corrosion resistance of $\mathrm{Zr}-\mathrm{Sn}$.

\section{Zircaloys}

Work with Sn, Fe, Ni, and $\mathrm{Cr}$ led to development of the Zircaloys. These were discussed by Stanley Kass and D. E. Thomas (Westinghouse Electric Corp.), and E. T. Hayes (Bureau of Mines).

The Zircaloys are made with sponge$\mathrm{Zr}$ base and $\mathrm{Sn}, \mathrm{Fe}, \mathrm{Ni}$, and $\mathrm{Cr}$ added in various combinations. The compositions are-Zircaloy-1: 21/2 wt $\% \mathrm{Sn}$; Zircaloy-2: 0.15 wt $\% \mathrm{Sn}, 0.12$ wt \% Fe, 0.05 wt $\%$ Ni, 0.10 wt \% Cr; Zircaloy-3: 0.25 wt $\% \mathrm{Sn}, 0.25$ wt $\% \mathrm{Fe}$.

Although the mechanical properties of the Zircaloys are poorer than some of the other possible structural materials (see Table 1), it is their corrosion resistance that is important for reactor application.

In building a reactor, selection of the particular Zircaloy composition depends on the particular application-it is not necessary to use Zircaloy-2 or -3 . Within limits (0.1-5 wt\% Sn; up to 1.1 wt \% $\mathrm{Fe}, \mathrm{Ni}$, and/or $\mathrm{Cr}$ ), corrosion resistance can be traded for strength.

The corrosion behavior of Zircaloy- 1 did not come up to expectations. However, Zircaloy-2 meets the four conditions previously stated-its corrosion resistance approaches and, under some conditions, exceeds that of the purest, unatloyed $\mathrm{Zr}$. Zircaloy-3 is the most recently developed member of the family. Sufficient data for complete evaluation are not yet available.

Zircaloy-2. The corrosion behavior of Zircaloy-2 is relatively insensitive to prior heat treatment and to slight variation in the concentrations of alloying elements.

The corrosion effect of nitrogen, deleterious at lower concentrations, is more pronounced as exposure time increases. After 70 days in $750^{\circ} \mathrm{F}$ steam, the effect is noted at $190 \mathrm{ppm}$; after 140 days, effect is observed at 120 ppm. Thus the permissible amount of nitrogen depends on the application. Similar phenomena were observed for $\mathrm{Al}$ and $\mathrm{Cu} ; 100 \mathrm{ppm} \mathrm{Pb}, 50 \mathrm{ppm} \mathrm{Mn}$,

* Fabrication and melting also done on conversion basis. 
300 ppm Be, and Co appear to have no effects.

Hydrogen is liberated during $\mathrm{Zr}$ alloy corrosion by

$$
\mathrm{Zr}+2 \mathrm{H}_{2} \mathrm{O}=\mathrm{ZrO}_{2}+2 \mathrm{H}_{2}
$$

Zircaloy-2 picks up $10-20 \%$ of the $\mathrm{H}_{2}$ produced in $750^{\circ} \mathrm{F}$ steam and $5-15 \%$ in $680^{\circ} \mathrm{F}$ water. Unalloyed $\mathrm{Zr}$ picks up $30 \%$. Incomplete data show that the corrosion rate of Zircaloy-2 containing 4-10,000 ppm hydrogen in $750^{\circ} \mathrm{F}$ steam increases slightly with increasing $\mathrm{H}_{2}$ content. Specimens containing 500-10,000 ppm hydrogen after 126 days in steam have weight gains $13 / 2$ times that for specimens containing

\section{Company Notes}

In addition to the products listed in the table, the following additional work is being done by the companies indicated:

Bridgeport Brass Co, Deep-drawn Zircaloy -2 shells are now in experimental production. Development work continues on tubing from 3 -in. extruded to $3 / 8$-in. cold. drawn. Bridgeport is cooperating with Carborundum Metals and others in a sample-preparation program; extruded billets of sponge and reactor-grade $Z_{r}$ and Zircaloy-2 are available. In '56, Bridgeport hopes to have moderate production of tubing in lengths up to $20 \mathrm{ft}$.

Kowecki Chemical Co. Independently of the AEC program, Kawecki has developed a Hf-Zr separation process. Although now done on a laboratory scale, facilities could be readily set up to produce large quantities of $\mathrm{Hf}$-free potassium zirconium fluoride and zirconium oxide and hydroxide should the demand warrant it. The process is said to be similar to the Russian method of fractional crystallization of $\mathrm{Zr}$ and $\mathrm{Hf}$ bifluorides described at Geneva in paper 634 .

Metals and Controls Corp. In fabricating fuel elements, one project is arc-melting uranium and zirconium alloys; several problems have placed $\mathrm{Zr}$ sponge in a minor role in this use. Metals and Controls is also working on a fuel element of uranium foil clad with $Z_{r}$ strip; not completely developed, it looks good for critical-assembly type of operation. A small amount of $\mathrm{Zr}_{\mathbf{r}}$ scrap is being melted down for $\mathrm{U}-\mathrm{Zr}$ ingots; it is an important factor in decreasing the price of fuel elements.

National Research Corp. A patented process for titanium shape-casting has been applied to $\mathrm{Z}_{\mathrm{r}}$; vacuum casting should offer a relatively inexpensive method of producing complex shapes with high yield. Melting (up to 80-lb capacity) and heattreating equipment are being designed and manufactured; furnaces of up to 2,000 Ib Zr melting capacity (arc, skull, or coldmold) can be furnished on order.

Nuclear Metals, Inc. Several methods have been developed for cladding fuel elements with $\mathrm{Zr}_{\mathrm{r}}$ and $\mathrm{Z}_{\mathrm{r}}$ alloys. Facilities are available for testing $\mathrm{Zr}$, e.g., its corrosion resistance and physical properties.

Wolverine Tube Div., Calumet \& Heclo, Inc. Although now primarily concerned with tube products, Wolverine has an eye on the market for products of rolling, welding, and zirconium roll-bonded to other metals.
Reactor-Grade Zirconium Products (Cont.)

FABRICATED PRODUCTS Spec's Price

\section{Bridgeport Brass Co. (Zr and Zircaloy)}

Extruded tubing

Up to $3.0 \mathrm{in}$. o.d., $1 / 4-3 / 8$ in. wall; down to 2 in. o.d., 1/8-1/4-in. wall

Wrought tubing

$11 / 2$ in. o.d., 0.100-in. wall; down to $3 / 8$ in. o.d., 0.020 -in. wall

Rod As extruded or drawn down to $3 / 4$-in. round

Chase Brass and Copper Co., Ine. ( $\mathrm{Zr}$ and Zircaloy) Extruded tubing (conversion basis) 11/2-in. min, id., 51/2-in. $\max _{\text {. o.d.; } 1 / 8-1 / 2}$ in. $\min$. wall thickness, depending on o.d.; 1-in. max. wall thickness

Firth Sterling, Inc.

Sheet bar

Forged billets

$\mathrm{Up}$ to $625 \mathrm{lb}$

Hot-rolled strip

0.100 mil min., 100 in wide, 600 -lb rolls

Cold-rolled strip 0.005 mil min. thickness $3 / 16^{-5}$ in, diameter, $1 / 4-$ 10 in. square

Hot-rolled sheet 0.1 in. thick, $36 \times 96$

$>500 \mathrm{Ib}, \$ 26.56 / \mathrm{lb}$ $<50 \mathrm{lb}, \$ 33.20 / \mathrm{lb}$; $>500 \mathrm{lb}, \$ 25.75 / \mathrm{lb}$; $<50 \mathrm{lb}, \$ 32.19 / \mathrm{lb}$ $>500 \mathrm{lb}, \$ 28.23 / \mathrm{lb}$; $<50 \mathrm{lb}, \$ 38.18 / \mathrm{lb}$

$>500 \mathrm{lb}, \$ 26.15 / \mathrm{lb}$ $<50 \mathrm{lb}, \$ 37.05 / \mathrm{lb}$

$>500 \mathrm{lb}, \$ 35 / \mathrm{lb}$;

$<50 \mathrm{lb}, \$ 60 / \mathrm{lb}$

Cold-rolled sheet

down to 0.012 in. $\mathrm{min}$ thickness

$3 / 8$ in. min. diameter; cold drawn and centerless ground to $1 / 4 \mathrm{in}$. diameter

Wire

Tubing 0.010 in. $\mathrm{min}$. diameter Extruded $-2 \frac{1}{2}-3 / 4$ in.

Price on request $>500 \mathrm{lb}, \$ 85.00 / \mathrm{lb}$; $<50 \mathrm{lb}, \$ 110.00 / \mathrm{lb}$ diameter; welded-21/2$1 / 2$ in. diameter ; both redrawn to $3 / 16$ in. diameter and 0.020 in. wall

National Research Corp.

Special $\mathrm{Zr}$ components fabricated on order; prepared to quote on shapes requiring machining and welding to close tolerance

Nuclear Metals, Inc.

Zr-clad fuel elements, mill products (wire, sheet, rods, tubing); sample quantities to large-scale production; prices on inquiry

Typical sample tubing lots $1 / 2-3 / 8$ in. o.d., $0.020-\$ 5-10 / \mathrm{ft}$

0.060 in. wall

1 in. o.d., $0.020-0.100$ in. $\$ 10-30 / \mathrm{ft}$ wall

Sylvania Electric Products, Inc.

Bid on specific items, such as fuel elements. Fabricate by powder metallurgy as well as other techniques

Calumet and Heclo, Inc., Wolverine Tube Div. (Zr and alloys)

Tubing

On individual order basis

Damascus Tube Co. (Zr, Zircaloy-2, Zircaloy-3)

Welded tubing and pipe (ASTM $1 / 2-15 / 16$ in. o,d, 0.032- Representative prices spec's or other) 0.072 wall; $1-11 \frac{1}{2}$ in., $0.032-0.083 ; 11 / 2-2 \frac{1}{2}$ in., $0.042-0.083$ per $100 \mathrm{ft}: 1-\mathrm{in}, 0 . \mathrm{d}, 18$ gage - $\$ 2,018.94 ; 1$-in. o.d., 16 gage $-\$ 2,617.96$; $3 / 4$-in. o.d., 18 gage$\$ 1,496.34 ; 3 / 4$-in. o.d., 16 gage $-\$ 1,929.14$ 
4-25 ppm. Surface hydride, cathodically applied prior to corrosion testing in $750^{\circ} \mathrm{F}$ steam, does nol appear to have any effect.

Corrosion behavior in $750^{\circ} \mathrm{F}$ steam at $1,500 \mathrm{lb} / \mathrm{in}^{2}$, with or without preoxidation in dry oxygen at $750^{\circ}$, is analogous to behavior in dry oxygen at $750^{\circ} \mathrm{F}$. Pure erystal-bar $\mathrm{Zr}$ is corrosion resistant to dry $\mathrm{O}_{2}$; however, all resistance is lost in steam.

No corrosion dependence on pressure was found in $680^{\circ} \mathrm{F}$ water $(2,705$ $\mathrm{lb} / \mathrm{in}^{2}$ ) or in $680^{\circ} \mathrm{F}$ steam at 2,500 $\mathrm{lb} / \mathrm{in}^{2}$. However, tests in $680^{\circ} \mathrm{F}$ steam at $1,500 \mathrm{lb} / \mathrm{in}^{2}$ show decreased post-breakdown corrosion rate.

The microstructure of Zircaloy-2 shows inclusions, which are presumably intermetallics of $\mathrm{Ni}$ and $\mathrm{Cr}$. Stringers also occur parallel to the rolling direction. While these do not affect mechanical properties, as measured in conventional tests, ductility is affected in operations such as shearing. Stringers maybe inert gas trapped during melting.

Zircaloy-3. Zircaloy-3 was developed to provide corrosion resistance at higher temperatures than are possible for Zircaloy -2 .

Zircaloy-2 studies show that Sn-content variation of $0-1.5 \mathrm{wt} \%$ has negligible effect on corrosion resistance. Increasing $\mathrm{Ni}, \mathrm{Fe}$, and $\mathrm{Cr}$ in the range $0.20-0.35$ wt $\%$ improves resistance. $\mathrm{Fe}$ and $\mathrm{Ni}$ can be substituted for one another and are more effective in imparting resistance than is $\mathrm{Cr}$.
The following three alloys (in wt \%) were evaluated, leading to Zircaloy-3: $\mathrm{A}-0.25 \mathrm{Sn}, 0.25 \mathrm{Fe} ; \mathrm{B}-0.50 \mathrm{Sn}, 0.40$ $\mathrm{Fe} ; \mathrm{C}-0.50 \mathrm{Sn}, 0.20 \mathrm{Fe}, 0.20 \mathrm{Ni}$. These Zircaloy-3 compositions exhibit lower post-breakdown corrosion rates than does Zircaloy-2; composition $\mathrm{A}$ is the lowest. Variations within $0.10 \%$ of nominal compositions have no effect. The maximum permissible impurity content (mainly $\mathrm{N}_{2}$ and $\mathrm{Al}$ ) appear the same as for Zircaloy-2.

That the amenability to cold-working of compositions $\mathrm{A}, \mathrm{B}$, and $\mathrm{C}$ is much better than that of Zircalloy-2 was brought out by R. L. Hoff (Superior Tube Co.). Thus the cost of fabricated products-tubing particularly-should be less than for Zircaloy-2.

\section{Reduction}

All low-hafnium zirconium supplied for $\mathrm{AEC}$ and commercial reactor use is now produced by the Kroll process. W. W. Stephens (Carborundum Metals Co.) explained how $\mathrm{ZrCl}_{4}$ is reduced by this method at Carborundum.

Quality-control costs amount to about two-thirds as much as all of the direct labor involved in manufacturing the product. Table 2 shows that the maximum allowable limits are for the most part $0.01 \%$ or lower. The exceptions are iron, carbon, oxygen, magnesium, and chlorine. The last two are removed almost completely when the metal is melted into ingots. These specifications are met through extremely rigid quality-control standards.

TABLE 2-Max. Sponge Impurities

\begin{tabular}{clll}
\hline Element & $\begin{array}{c}\text { Weight } \\
\%\end{array}$ & Element & \multicolumn{1}{c}{$\begin{array}{c}\text { Weight } \\
\%\end{array}$} \\
\hline $\mathrm{Hf}$ & $\mathbf{0 . 0 1}$ & $\mathrm{C}$ & 0.02 \\
$\mathrm{Al}$ & $\mathbf{0 . 0 0 7 5}$ & $\mathrm{N}$ & 0.01 \\
$\mathrm{~B}$ & $\mathbf{0 . 0 0 0 1}$ & $\mathrm{O}$ & 0.10 \\
$\mathrm{Cl}$ & $\mathbf{0 . 0 6}$ & $\mathrm{Li}$ & 0.0001 \\
$\mathrm{Cr}$ & $\mathbf{0 . 0 1 0}$ & Rare \\
& \multicolumn{4}{c}{ earths } & 0.0015 \\
$\mathrm{Fe}$ & 0.10 & $\mathrm{P}$ & 0.001 \\
$\mathrm{~Pb}$ & 0.005 & $\mathrm{~W}$ & 0.005 \\
$\mathrm{Mg}$ & 0.060 & $\mathrm{Zn}$ & 0.01 \\
$\mathrm{Mn}$ & 0.005 & $\mathrm{Co}$ & 0.004 \\
$\mathrm{Ni}$ & 0.007 & $\mathrm{Ca}$ & 0.003 \\
$\mathrm{Ti}$ & 0.005 & $\mathrm{Cd}$ & 0.00005 \\
$\mathrm{~V}$ & 0.005 & &
\end{tabular}

Brinell hardness $=150$ for sponge arcmelted in inert atmosphere
R. C. Dalzell (AEC Reactor Development Div.) commented that too much stress is placed on the mysteriousness of $\mathrm{Zr}$ behavior, e.g., $0.2 \%$ oxygen being damaging to mechanical properties. The standard for commercial copper is $0.03-0.05 \%$ for most products. What should be emphasized is the difficulty of keeping oxygen out of the metal, not that it is harmful.

Describing how reactor-grade zirconium specifications (Table 2) were established, Dalzell said that zirconium within these limits was found to meet requirements and that it could be produced. Thus, it was necessary to specify a great many impurities. The main goal in alloy development is to minimize zirconium's neutron cross section and then to pinpoint those impurities affecting corrosion resistance and mechanical properties. More complete data should permit less stringent specifications.

\section{Z. Fabrication Methods}

Currently, zirconium powder metallurgy is secondary in importance to arcmelting techniques. While tubular, sheet, and bar products are generally made with the arc-melted product, powder metallurgy is used for certain special products.

\section{Arc-Melted Products}

The fabrication of zirconium from ingot to finished mill products, such as forgings, billets, bars, slabs, plate, sheet, and strip, present no problems not easily mastered by mills experienced in handling stainless, high-alloy steels, and titanium for high-temperature applications.

General considerations. In his discussion of fabrication methods, W. C. Greenleaf (Allegheny Ludlum Steel
Corp.) remarked on the workability characteristics of zirconium.

The methods of fabricating $\mathrm{Zr}$ are about the same as for Ti. The crystal structure of these metals is similar to magnesium. They are less easily worked than iron or copper.

Zireonium has a wide hot-workability temperature range; above $400^{\circ} \mathrm{F}$ the tensile strength, yield strength, and hardness drop off rapidly (modulus drops only slightly); elongation rises steadily, then sharply above $750^{\circ} \mathrm{F}$ Zr hot-rolls and forges satisfactorily.

Crystal structure changes from hexagonal close-packed (alpha phase) to body-centered cubic (beta phase) at $1,580^{\circ} \mathrm{F}$. Quenching from above this temperature results in slight hardening. Zr can be stress-relief annealed by heat 
ing above $500^{\circ} \mathrm{F}$ and completely recrystallized by heating for an hour at $1,550^{\circ} \mathrm{F}$.

Almost all mill products are heated and annealed in air, but very light-gage sheet and strip (also light sections of wire and tubing) anneal better in an inert atmosphere or vacuum.

Descaling $\mathrm{Zr}$ differs from $\mathrm{Ti}$ in that any tight oxide must be removed mechanically. Machinability does not differ too much from $\mathrm{Ti}$.

Zirconium can be arc or flash-butt welded under inert atmosphere. Because of its reactivity with gases, the weld area must be shielded.

\section{Powder Metallurgy}

Four potential reactor uses of powder-metallurgy zirconium were pointed out by H. S. Kalish (Sylvania Electric Products): (a) special shapes for which large quantities of identical parts are required; (b) special alloys; for $\mathrm{Zr}$ - $\mathrm{U}$ alloys, better than $99 \%$ of theoretical density can be attained for all compositions up to $90 \% \mathrm{U}$. Zr-Be and $\mathrm{Zr}-\mathrm{MIg}$ alloys have been successfully made; (c) cermets or composite materials; a mixture of $\mathrm{Zr}$ and $\mathrm{BeO}$ powders has been compacted and sintered to vield the Zr-Be eutectic structure; (d) porous parts.

The methods of fabrication can be any of five basic techniques:

1. Cold pressing and sintering. The inherent porosity can be eliminated by further fabrication.

2. Cold pressing, presintering, coining, and annealing. The item is finished by machining. This technique minimizes waste, cuts down machining costs, and provides highdensity, high-purity material.

3. Hot pressing. A blank is hot pressed in a graphite die and machined to shape. This technique is suitable for lower volume production than the previous one. It saves considerable material and provides a high-density, high-purity material.

4. Powder rolling. This is suitable for making plates or rods. Once the powder is consolidated, the steel sheathing can be stripped and the usual fabrication techniques employed.

5. Powder extrusion. This method is successful with electrolytic zirconium powder because of its high ductility and relatively coarse particle size.

Electrolytic $\mathrm{Zr}$ powder. After the conference, J. L. Wyatt (Horizons, nc.) reported on Horizons' latest data obtained on electrolytic zirconium powder.*

Basically, the process involves the electrolysis of a fused salt bath of sodium chloride and potassium zirconium hexufluoride to produce a coarse, granular powder on a metal cathode. The product is withdrawn periodically, the deposit removed by stripping, and the salt-metal mixture purified by a simple fluosolids type of washing. Single deposits weighing up to $40 \mathrm{lb}$ have been produced on a pilotplant scale.

The majority of the material is 40 150 mesh. It is somewhat dendritic in nature, consisting of individual crystallites and tree-like agglomerates. Excellent properties are exhibited in applications involving direct extrusion from the powder and in the fabrication of component parts by powder-metallurgy techniques. $\dagger$

In Nov. '55, 18 cathodes were produced in a laboratory electrolytic cell on a continuous basis. The first cathode served as a scavenger to remove impurities normally leached out of a new crucible during initial operations. A blend of the other 17 cathodes was evaluated. Vacuum-fusion analyses showed interstitial impurities of: oxygen, $0.038 \%$; nitrogen, 0.01 ; hydrogen, 0.005 .

Nitrogen values are probably high, Kjeldahl analyses to check this are not available, but previous batches of metal were analyzed at about $0.003 \%$. A spectrographic analysis of a quartered sample of this composite is shown in Table 3. Hardness determinations on a $30-\mathrm{gm}$ as-rast button melted on a water-cooled copper hearth showed a Brinell value of 86 under a $500-\mathrm{kg}$ load. The material was too soft for the 3,000 $\mathrm{kg}$-load determination on a pellet of this size.

A melted pellet with an original thichness of 5,16 in. was cold-rolled and reduced in thickness $97 \%$ with no evidence of edge cracking. It exhibited considerable ductility thereafter.

\section{Z. Looking Ahead}

The growth of the demand for zirconium depends on many factors: government, industrial, and foreign programs; collection of basic metallurgical data; improved processing and fabricating methods; and, as a result of the former, price reduction. These factors were analyzed by N.C. Bartholomew (Carborundum Metals Co.).

AEC program. Including naval work, this program should have a minimum requirement of $600,000 \mathrm{lb}$ in 1957. Some have estimated a farfrom-conservative 2,300,000-lb demand.

Industrial power. The Atomic Industrial Forum, using 1954 as a base, predicts the nuclear power industry will be at least seven times as large in 1964 and a maximum of forty times as large. The effect will probably be something less than proportional-depending on the price of zirconium and the trend in reactor design.

* At the present time, there is no com. mercial production of electrolytic zirconium. Potential users desiring small quantities should direct inquiries to C. A Specht, Horizons Titanium Corp., Room 3418, 30 Rockefeller Plaza, New York 20 , N. $\mathbf{Y}$.

$\dagger$ H. H. Hirsch, Fabrication of zirconium by powder metallurgy techniques, Metal Progress 68, No, 6, 81 (1955).
Foreign programs. Current reactor designs abroad do not incorporate Zr. Presumably, uther countries will follow the trend to higher temperatures and $\mathrm{Zr}$. Also to be considered-urgent need for power, development of $\mathrm{Zr}$ production, and how U. S. production and transportation costs will affect foreign importing.

Chemical industry. U. S. corrosion losses are over \$5-billion per year. This should be an important market.

Sponge utilization. Improvement in yield from sponge to end product has shown marked improvement. Fabrication on a conversion basis will end - responsibility for quality and losses will be assumed by the fabricator.

Scrap recovery. Only done on a laboratory scale now, $80 \%$ recovery will bring down prices.

Improved processing. Imminent separation and reduction developments must be given careful consideration in expansion plans.

Specification changes. Current specifications are probably more stringent than necessary. This must be verified through experience.

Price. This is a function of quantity and quality. Most of the factors listed will be determinant. 


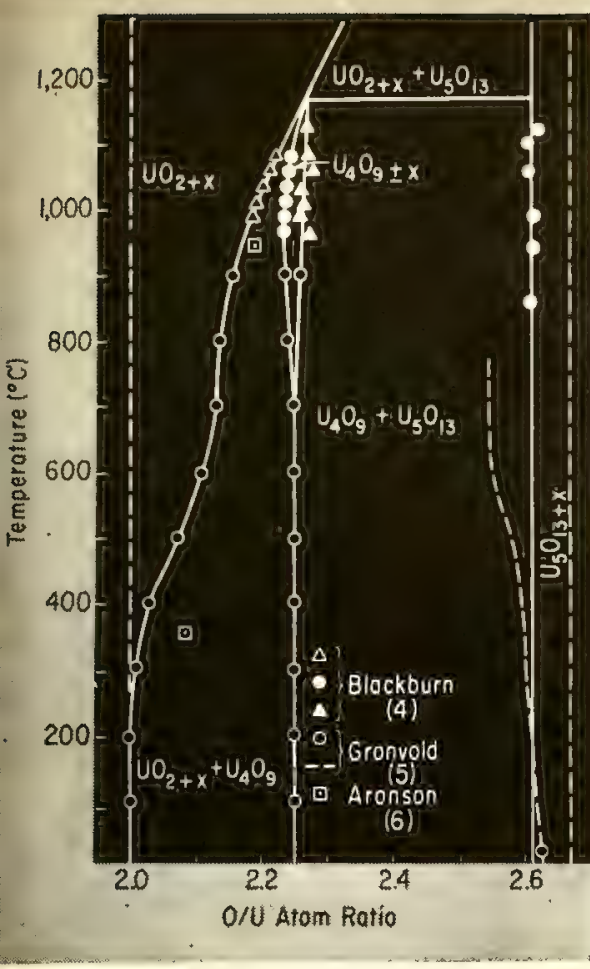

FIG. 1. Phase relationships of system $\mathrm{UO}_{2}-\mathrm{U}_{3} \mathrm{O}_{8}(2)$

\title{
$\mathrm{UO}_{2}$-Fabrication and Properties
}

\author{
Thermal and irradiation stability make uranium \\ dioxide a popular contender as a reactor fuel. \\ However, uncertainties exist in such areas as the \\ most economic fabricating methods, thermal \\ conductivity and fission-gas release
}

By O. J. C. RUNNALLS, Atomic Energy of Canada Limited

Chalk River, Ontario, Canada

\section{Properties of $\mathrm{UO}_{2}$}

to be a most satisfactory fuel for many types of power reactors. Its advantages include:

1. High melting point

2. Chemical stability with most reactor coolants

3. Compatibility with a wide variety of sheathing materials

4. Ease of fabrication to high densities

5. An isotropic structure stable under irradiation at core temperatures approaching the melting point

A disadvantage is its low thermal conductivity. Fortunately, however, the thermal conductivity in operating fuel elements appears to be little reduced even after long irradiation. The release of fission gas may impose a limitation on future fuel-element designs, but there are not sufficient data available to say this with any certainty at present.

Recent data on chemical and physical properties, fabrication and irradiation behavior of $\mathrm{UO}_{2}$ are reviewed in this article with the emphasis on those that aid in establishing the conditions required for the fabrication and operation of an economic fuel of predictable performance.
The properties of uranium oxides have been extensively reported (1-3). Only those properties that are of special interest in the design of fuel elements will be referred to here.

Composition range. The phase relationships of the system $\mathrm{UO}_{2}-\mathrm{U}_{3} \mathrm{O}_{8}(2)$ are summarized in Fig. 1. The phase boundaries appear to be reasonably well established in the $\mathrm{UO}_{2}$ to $\mathrm{U}_{4} \mathrm{O}_{9}$ region, although Roberts et al. (7) reported that the $\mathrm{UO}_{2+x}$ phase extended to at least $\mathrm{UO}_{2.28}$ at $1,077^{\circ} \mathrm{C}$, in marked disagreement with Fig. 1. The composition limits of the $\mathrm{U}_{3} \mathrm{O}_{8}$ phase are still in some doubt (2).

The tetragonal $\mathrm{U}_{3} \mathrm{O}_{7}$ phase, which is formed by the oxidation of $\mathrm{UO}_{2}$ powder or pellets below $300^{\circ} \mathrm{C}$, is not shown in Fig. 1 because it appears to be a nonequilibrium structure. For example, samples in the composition range from $\mathrm{UO}_{2.00}$ to $\mathrm{UO}_{2.25}$, which consisted of a mixture of cubic and tetragonal phases, transformed to cubic $\mathrm{UO}_{2}$ and $\mathrm{U}_{4} \mathrm{O}_{9}$ after four months at $140^{\circ} \mathrm{C}(8)$.

The calculated density of $\mathrm{UO}_{2.00}$, determined from Gronvold's measured lattice constant of $a=5.4704 \AA$, is $10.96 \mathrm{gm} / \mathrm{cm}^{3}(5)$. Cubic $\mathrm{U}_{4} \mathrm{O}_{9}$ has a higher calculated density, $11.30 \mathrm{gm} /$ $\mathrm{cm}^{3}$, indicating that the excess oxygen atoms are accommodated interstitially in the $\mathrm{UO}_{2}$-like structure (5). From Gronvold's high-temperature studies on the $\mathrm{UO}_{2+x}$ phase, where it was observed that the lattice constant decreased when the $\mathrm{O} / \mathrm{U}$ ratio was increased, a similar conclusion can be

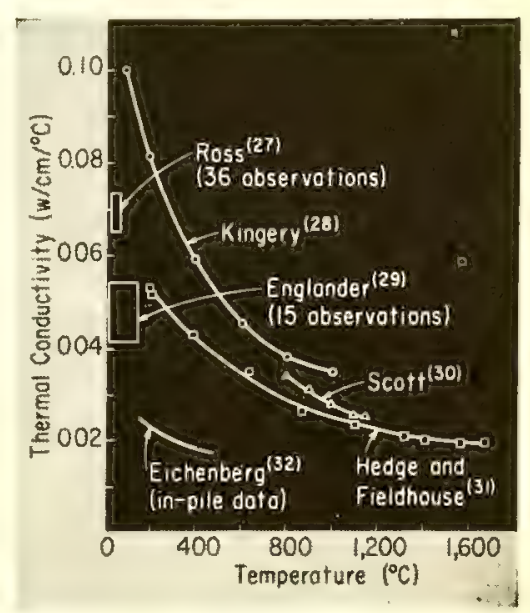

FIG. 2. Thermal conductivity of $\mathrm{UO}_{2}$ corrected to theoretical density, assuming linear dependence of conductivity on porosity 
drawn; i.e., the excess oxygen atoms must occupy interstitial positions. This is confirmed by magnetic susceptibility and electrical conductivity measurements, and by a comparison of chemical, X-ray and densimetric analyses (B).

Chemical stability. $\mathrm{UO}_{2}$ powder is readily oxidized by air, even at room temperature. The extent of oxidation depends on the particle size and on the surface area exposed.

According to Anderson et al. (9), room-temperature oxidation proceeds until the outer $50 \AA$ is oxidized, the oxygen absorbed being $0.8 \mathrm{~cm}^{3}$ at NTP per square meter of surface exposed. Finely divided powders with particle sizes of $0.1 \mu$, such as are obtained by the hydrogen reduction of ammonium diuranate at $900^{\circ} \mathrm{C}$, oxidize to a composition approaching $\mathrm{UO}_{2,25}$ after standing for one month in air at room temperature (10). Coarser powders $(1 \mu)$ are much more stable, oxidizing to only $\mathrm{UO}_{2.02}$ under similar conditions (11). Once $\mathrm{UO}_{2}$ powder has been sintered, at say $1,650^{\circ} \mathrm{C}$ in hydrogen, its oxidation rate becomes immeasurably slow. Thus, sintered $\mathrm{UO}_{2}$ pellets may be stored for long periods in air at room temperature with no fear of oxidation.

Sintered $\mathrm{UO}_{2}$ is stable in deoxygenated, high-temperature water. Pellets showed only a slight dulling of their surfaces after more than 300 days of exposure in degassed water at $343^{\circ} \mathrm{C}$ or steam at $400^{\circ} \mathrm{C}$ at neutral or high $p \mathrm{H}$ (2). When $1-3 \mathrm{~cm}^{3}$ of $\mathrm{O}_{2} / \mathrm{kg}$ was added to the water, however, a loose scale of hydrated oxide $\left(\mathrm{UO}_{3} \cdot 0.8 \mathrm{H}_{2} \mathrm{O}\right)$ formed, and the pellets lost $\sim 15 \%$ of their weight after an exposure of 8 days at $343^{\circ} \mathrm{C}$.

In water-cooled power reactors, traces of hydrogen are likely to be present in the coolant stream due to the reaction of the water with structural metals. Thus, $\mathrm{UO}_{2}$ should be stable in such systems. However, a steep temperature gradient will exist across the oxide radius, and if a hole or defect should develop in the cladding of a fuel element, the hot oxide core could be exposed to steam. Aronson's calculations (12) indicate that the core of such an element, operating near the melting point, could oxidize to $\mathrm{UO}_{2.18}$ to $\mathrm{UO}_{2.25}$. Direct evidence (19) indicates that stoichiometric $\mathrm{UO}_{2}$ irradiated in a defective sheath may, in fact, increase in $0 / U$ ratio. A sample of oxide from a defected irradiated rod, initially stoi- chiometric, was found to have a postirradiation average composition of $\mathrm{UO}_{2.06}$. Recent Chalk River tests confirmed this phenomenon. For example, a sample of oxide from the outer cooler region of a swaged, purposely defected rod changed from $\mathrm{UO}_{2.00}$ to $\mathrm{UO}_{2.21}$ during irradiation in a pressurized-water loop. As will be indicated later, an increase in $\mathrm{O} / \mathrm{U}$ ratio may have a deleterious effect on the irradiation behavior of $\mathrm{UO}_{2}$.

The chemical stability of $\mathrm{UO}_{2}$ in other coolants has been studied. The oxidation rate of $\mathrm{UO}_{2}$ in $\mathrm{CO}_{2}$ has been measured at $500^{\circ}-900^{\circ} \mathrm{C}(14)$. The $\mathrm{UO}_{2}$ was much less reactive than $\mathrm{U}$ metal. At $700^{\circ} \mathrm{C}$, the weight gain of sintered $\mathrm{UO}_{2}$ pellets of density 9.6 $\mathrm{gm} / \mathrm{cm}^{3}$ was $0.008 \mathrm{mg} / \mathrm{cm}^{2} / \mathrm{hr}$ compared with $400-560 \mathrm{mg} / \mathrm{cm}^{2} / \mathrm{hr}$ for $\mathrm{U}$ metal. Thus, there should be little

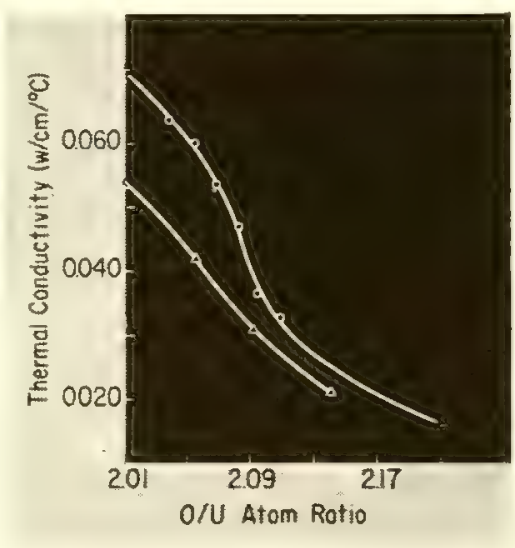

FIG. 3. Effect of $\mathrm{O} / \mathrm{U}$ ratio on thermal conductivity of $\mathrm{UO}_{2}$ at $60^{\circ} \mathrm{C}$ (27). $\mathrm{O}-\mathrm{UO}_{2}$, initial density $10.3 \mathrm{gm} / \mathrm{cm}^{3}$, sintered in $\mathrm{H}_{2}$ at $1,650^{\circ} \mathrm{C}$, oxidized at $900^{\circ} \mathrm{C}$ in $2-\mathrm{cm}-\mathrm{Hg} \mathrm{O}_{2} ; \triangle-\mathrm{UO}_{2}+$ $0.1 \% \mathrm{TiO}_{2}$ initial density $10.2 \mathrm{gm} / \mathrm{cm}^{3}$, sintered in $\mathrm{H}_{2}$ af $1,650^{\circ} \mathrm{C}$, oxidized at $900^{\circ} \mathrm{C}$ in $2-\mathrm{cm} \cdot \mathrm{Hg} \mathrm{O}_{2} ; \square-\mathrm{UO}_{2}$, density $10.6 \mathrm{gm} / \mathrm{cm}^{3}$, sintered in steam at $1,400^{\circ} \mathrm{C}$, cooled in steam

worry about the compatibility of $\mathrm{UO}_{2}$ with $\mathrm{CO}_{2}$, unless there is an appreciable increase in the reaction rate in an irradiation field.

Judging from thermodynamic data, $\mathrm{UO}_{2}$ should also be stable in liquid sodium. There is some evidence (15) that high-density stoichiometric material is compatible with $\mathrm{Na}$ or $\mathrm{NaK}$ at $600^{\circ} \mathrm{C}$.

Sintered $\mathrm{UO}_{2}$ pellets are compatible with many sheathing materials. No appreciable reaction occurs between zirconium and $\mathrm{UO}_{2}$ below $700^{\circ} \mathrm{C}(16)$. Little reaction occurs between $\mathrm{UO}_{2}$ and
TABLE 1-Thermal Expansion of $\mathrm{UO}_{2}$

\begin{tabular}{ccr}
$\begin{array}{c}\text { Memp. range } \\
\left({ }^{\circ} \mathrm{C}\right)\end{array}$ & $\begin{array}{c}\text { Mean coeff. of } \\
\text { linear expansion } \\
\left(10^{-6} /{ }^{\circ} \mathrm{C}\right)\end{array}$ & Ref. \\
\hline $20-720$ & 11.5 & 34 \\
$20-946$ & 10.8 & 5 \\
$27-400$ & 9.0 \\
$400-800$ & 11.0 & \\
$800-1,260$ & 13.0 & 35 \\
$400-900$ & 10 & 33 \\
\hline
\end{tabular}

aluminum below $500^{\circ} \mathrm{C}$ (17). No solid-state reaction has been found between solid $\mathrm{UO}_{2}$ specimens and beryllium or stainless steel at $600^{\circ}-700^{\circ} \mathrm{C}$ (11). Sintered $\mathrm{UO}_{2}$ pellets in contact with graphite plates do not react appreciably after a 10 -hr heating in argon up to $1,500^{\circ} \mathrm{C}(18)$.

Change in volume on melting. The limitation on central temperature in a $\mathrm{UO}_{2}$ fuel element has not yet been established. Many designers have set the melting-point temperature as a limit until irradiation experiments yield experience on the use of elements with molten cores. Several values for the melting point of $\mathrm{UO}_{2}$ have been published: $2,176^{\circ} \mathrm{C}(19), 2,500^{\circ}-2,600^{\circ} \mathrm{C}$ (20), $2,878 \pm 22^{\circ} \mathrm{C}(21), 2,405 \pm 22^{\circ} \mathrm{C}$ (22), $2,760 \pm 30^{\circ} \mathrm{C}(23)$ and $2,860 \pm$ $45^{\circ} \mathrm{C}$ (24). It seems reasonable to favor a value near $2,800^{\circ} \mathrm{C}$ as the true melting point.

It would be useful to know if $\mathrm{UO}_{2} \mathrm{ex}$ pands appreciably on melting to determine what void space should be provided in case the melting point is exceeded. Two measurements are under way in which $\mathrm{UO}_{2}$ will be melted in a solar furnace (25) and a carbon-electrode arc furnace with a water-cooled copper hearth (26). In both experiments, high-speed motion-picture cameras will record the solidification of a molten drop of $\mathrm{UO}_{2}$.

Thermal conductivity. Unfortunately, $\mathrm{UO}_{2}$ has such a low thermal conductivity that the advantage of its high melting point is largely offset. However, much work remains to be done before the thermal conductivity of operating $\mathrm{UO}_{2}$ fuel elements can be predicted with certainty, as indicated by the conflicting data plotted in Fig. 2.

Many thermal-conductivity measurements have been made on sintered $\mathrm{UO}_{2}$ in recent years (Fig. 2). Note that values differing by a factor of more than two have been obtained on 


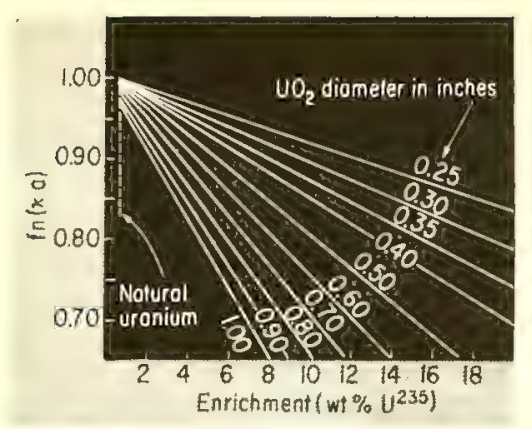

FIG. 4. Dependence of $f(x)$ on $\mathrm{UO}_{2}$ diameter and enrichment for $95 \%$ dense, solid oxide cylinders (60)

unirradiated $\mathrm{UO}_{2}$ at temperatures below $200^{\circ} \mathrm{C}$. In the range applicable to operating fuel elements, i.e., above $400^{\circ} \mathrm{C}$, the difference is only $30 \%$. However, a $30 \%$ uncertainty in the thermal conductivity results in a much larger uncertainty in calculated fuelcore temperatures, as is evident from Fig. 6.

Recently, Eichenberg (32) measured the center temperature of an assembly of $\mathrm{UO}_{2}$ pellets clad in stainless steel during irradiation in the Materials Testing Reactor. The pellets had been prepared from thermally decomposed uranyl nitrate by cold-pressing and sintering in hydrogen, the same process used for the Shippingport reactor fuel. Values of the effective thermal conductivity required to produce an observed $\mathrm{UO}_{2}$ central temperature of $150^{\circ}-600^{\circ} \mathrm{C}$ were calculated and are shown in Fig. 2. The calculated results are about a factor of three lower than the corrected Kingery data of Fig. 2 (28).

Ross (27) has measured the thermal conductivity of stoichiometric $\mathrm{UO}_{2}$ pellets at $60^{\circ} \mathrm{C}$ after irradiations up to an integrated thermal-neutron flux of $2 \times$ $10^{19} \mathrm{n} / \mathrm{cm}^{2}$. The pellets were prepared by cold-pressing and sintering $\mathrm{UO}_{2}$ powder prepared by the hydrogen reduction of ammonium diuranate. The maximum central temperature in the pellets during the irradiation did not exceed $500^{\circ} \mathrm{C}$. The measurements were made on a divided-bar type of comparative-heat-flow apparatus using Zircaloy-2 probes. Only a small decrease in thermal conductivity, $\sim 25 \%$. was observed after an irradiation of $9 \times 10^{17} \mathrm{n} / \mathrm{cm}^{2}$. Longer irradiations produced no further significant change. Additional measurements for shorter irradiations are under way to determine the irradiation level at which the decrease occurs.
There is a marked disagreement between Eichenberg's results and those of Ross. It is possible that the thermal conductivity of $\mathrm{UO}_{2}$ in a neutron flux is appreciably lower than postirradiation measurements indicate because of point defects, which might anneal out when the irradiation ended. Fuel-element tests described later do not support such a hypothesis, however.

Several laboratories have reported that a threefold decrease in thermal conductivity at $60^{\circ} \mathrm{C}$ can be produced in $\mathrm{UO}_{2}$ pellets by increasing the $\mathrm{O} / \mathrm{U}$ ratio above stoichiometric by $8 \%(S$, 15, 27). On the other hand, Thackray's values for hot-pressed $\mathrm{UO}_{2.13}$ over the temperature range $100^{\circ}-600^{\circ} \mathrm{C}(33)$ were. essentially the same as the corrected values of Kingery et al. (28) for stoichiometric $\mathrm{UO}_{2}$. Measurements by Ross (27) are given in Fig. 3. The conductivity of $0.017 \mathrm{w} / \mathrm{cm} /{ }^{\circ} \mathrm{C}$ determined for the steam-sintered sample approaches the theoretical lower limit of $0.015 \mathrm{w} / \mathrm{cm} /{ }^{\circ} \mathrm{C}$ for the $\mathrm{UO}_{2}$ lattice determined by Kingery $(3,32)$. It is not yet known whether a comparable decrease in conductivity occurs with increased $\mathrm{O} / \mathrm{U}$ ratio at higher temperatures.

Some of the apparent discrepancies in thermal-conductivity measurements may be due to variations in the fabrication technique. For example, the conductivity of stoichiometric $\mathrm{UO}_{2}$ pellets, prepared by steam-sintering at $1,400^{\circ} \mathrm{C}$ followed by hydrogen-cooling, is $25 \%$ lower than for those that were also steam-sintered but annealed in hydrogen before cooling.

Much work remains to be done before the thermal conductivity of operating $\mathrm{UO}_{2}$ fuel elements can be predicted with certainty.

Thermal expansion. The several published values for the linear thermalexpansion coefficient of $\mathrm{UO}_{2}$ are in reasonable agreement (Table 1). Murray and Livey (33) reported that the bulk density and $\mathrm{O} / \mathrm{U}$ ratio had little effect on the thermal-expansion coefficient.

\section{Fabrication of $\mathrm{UO}_{2}$}

$\mathrm{UO}_{2}$ compacts can be fabricated from a variety of starting powders by conventional ceramic processes such as cold-pressing, extrusion, slip-casting and isostatic-pressing. Dense bodies can be produced from the compacts by sintering at $1,300-2,000^{\circ} \mathrm{C}$. Other fabrication methods include rotaryswaging and loose compaction of pow- der in metal sheaths and hot-pressing in metal or graphite dies.

These processes are detailed in the following discussion.

Powder preparation. $\mathrm{UO}_{2}$ powder can be prepared by the hydrogen reduction of several compounds, e.g., $\mathrm{UO}_{3}$, $\mathrm{UO}_{3}$ hydrates, $\mathrm{U}_{3} \mathrm{O}_{8}$, ammonium diuranate, uranium oxalate and uranium peroxide, and by steam oxidation of uranium hydride or uranium metal. $\mathrm{UO}_{2}$ powders prepared by different methods show wide variations in physical characteristics such as surface area, particle density, porosity distribution and crystallite size (2, 36-39). Such variations have a marked effect on the sinterability of the powder as illustrated in Table 2.

Pellets with densities above $10 \mathrm{gm} /$ $\mathrm{cm}^{3}$ can be produced from the least $\mathrm{sin}$ terable powder shown in Table 2 , however, if high compacting pressures and long sintering times are used. The first oxide charge for the Shippingport reactor, for example, was prepared by compacting $\mathrm{UO}_{2}$ powder obtained from Mallinckrodt at 250,000 psi and sintering for $8 \mathrm{hr}$ in hydrogen at $1,675^{\circ} \mathrm{C}(41)$. The pellets varied in density from 10.2 to $10.4 \mathrm{gm} / \mathrm{cm}^{3}$.

Powders of low sinterability can be activated by wet-ball-milling before hydrogen reduction as shown in Table 2. A simpler, more cconomic method is to grind $\mathrm{UO}_{3}$ powder to particle sizes less than 1 micron in a fluid-jet mill and then to reduce in hydrogen. Pellets with densities up to $10.7 \mathrm{gm} / \mathrm{cm}^{3}$ have been produced from such powder after pressing at 40,000 psi and sintering for $1 \mathrm{hr}$ at $1,625^{\circ} \mathrm{C}(39)$.

Many laboratories have investigated the effects of chemical additives to promote more rapid sintering of $\mathrm{UO}_{2}$. Canadian workers have verified the many earlier reports on the effectiveness of a $0.1 \% \mathrm{TiO}_{2}$ addition and have found that $0.4 \% \quad \mathrm{Nb}_{2} \mathrm{O}_{5}$ has an even larger effect $(39,42)$.

The preparation of $\mathrm{UO}_{2}$ powder via the ammonium diuranate $(\mathrm{ADU})$ route has been extensively investigated (10, $39,43)$. A reproducible ADU powder was made by the batch precipitation of ADU from uranyl nitrate solution (uranium concentration of $100 \mathrm{gm} / \mathrm{l}$ at $60^{\circ} \mathrm{C}$ ) by the rapid addition of concentrated, aqueous ammonium hydroxide until a $p \mathrm{H}$ of 9 was reached. The ADU was filtered, washed with water and oven-dried in air at $200^{\circ}$ C. Higher uranium concentration, slower precipi- 
tation rate or different $p \mathrm{H}$ yielded a $\mathrm{UO}_{2}$ product that sintered to a lower density. When the precipitation was carried out by continuously mixing two liquid streams, however, the only variable that affected pellet density appreciably was the $p H$ of the mixed solutions; the optimum $p \mathrm{H}$ was $7-8$. For conversion to $\mathrm{UO}_{2}$, the ADU was loaded in trays to a depth not exceeding 2 in. and reduced in hydrogen for $1 \mathrm{hr}$ at $900^{\circ}$ C. The resulting pyrophoric powder was cooled to room temperature in hydrogen and stored in carbon dioxide for $1-2 \mathrm{hr}$, after which it could be handled and stored in air. After 30 days' storage in air, the $\mathrm{O} / \mathrm{U}$ ratio increased to 2.25 .

Several tons of $\mathrm{UO}_{2}$ powder have been produced by Eldorado Mining and Refining by both batch and continuous precipitation. Sintering tests for 1 hr at $1,625^{\circ} \mathrm{C}$ on many samples pressed at 40,000 psi have consistently produced pellets of $10.4-10.6 \mathrm{gm} / \mathrm{cm}^{3}$.

TABLE 2 - Sinterability of $\mathrm{UO}_{2}(40)$ *

\begin{tabular}{|c|c|c|}
\hline $\begin{array}{l}\text { Source of } \\
\qquad \mathrm{UO}_{2}\end{array}$ & Preparation & $\begin{array}{c}\text { Sintered } \\
\text { density } \\
\left(\mathrm{gm} / \mathrm{cm}^{3}\right)\end{array}$ \\
\hline $\begin{array}{l}\text { Mallinck- } \\
\text { rodt }\end{array}$ & $\begin{array}{l}\text { Pyrolysis of } \\
\text { uranyl nitrate } \\
\text { hexahydrate } \\
\text { (UNH) to } \mathrm{UO}_{3} \text {, } \\
\mathrm{H}_{2} \text { reduction }\end{array}$ & 7.8 \\
\hline Shattuck & $\begin{array}{l}\text { Believed similar } \\
\text { to Mallinckrodt }\end{array}$ & 8.6 \\
\hline $\begin{array}{l}\text { National } \\
\text { Lead }\end{array}$ & $\begin{array}{l}\text { Believed similar } \\
\text { to Mallinckrodt }\end{array}$ & 9.1 \\
\hline $\begin{array}{l}\text { Eldorado } \\
\text { Mining } \\
\text { \& Refin- } \\
\text { ing }\end{array}$ & $\begin{array}{l}\text { Pyrolysis of } \\
\mathrm{UNH} \text { to } \mathrm{UO}_{3}, \\
\mathrm{H}_{2} \text { reduction }\end{array}$ & 9.2 \\
\hline $\begin{array}{l}\text { Eldorado } \\
\text { Mining } \\
\text { \& Refin- } \\
\text { ing }\end{array}$ & $\begin{array}{l}\text { Pyrolysis of } \\
\mathrm{UNH} \text { to } \mathrm{UO}_{3}, \\
\mathrm{UO}_{3} \text { hydrated by } \\
\text { wet-ball-milling, } \\
\mathrm{H}_{2} \text { reduction }\end{array}$ & 10.3 \\
\hline $\begin{array}{l}\text { U.K.AEA, } \\
\text { Spring- } \\
\text { fields }\end{array}$ & $\begin{array}{l}\text { Precipitation of } \\
\text { ammonium di- } \\
\text { uranate }(\mathrm{ADU}) \\
\text { from } \mathrm{UNH}, \mathrm{H}_{2} \\
\text { reduction }\end{array}$ & 10.6 \\
\hline $\begin{array}{l}\text { Mines } \\
\text { Branch, } \\
\text { Ottawa }\end{array}$ & $\begin{array}{l}\text { Precipitation of } \\
\text { ADU from } U N H \text {, } \\
\mathrm{H}_{2} \text { reduction }\end{array}$ & $10.1-10.7$ \\
\hline $\begin{array}{l}\text { AECL, } \\
\text { Chalk } \\
\text { River }\end{array}$ & $\begin{array}{l}\text { Precipitation of } \\
\text { ADU from UNH, } \\
\mathrm{H}_{2} \text { reduction }\end{array}$ & $10.4-10.6$ \\
\hline $\begin{array}{l}* \text { All pelle } \\
\text { and sintered } \\
\text { hydrogen. }\end{array}$ & $\begin{array}{l}\text { ets cold-pressed at } \\
\text { for } 30 \mathrm{~min} \text { at } 1\end{array}$ & $\begin{array}{l}40,000 \mathrm{psi} \\
700^{\circ} \mathrm{C} \text { in }\end{array}$ \\
\hline
\end{tabular}

Although $\mathrm{UO}_{2}$ pellets of the quality required for fuel elements can be prepared from "ceramic-grade" powders at relatively low compacting pressures and sintering times, it is still not certain whether these will be less expensive than pellets made from less sinterable powders. For example, the Eldorado Company is marketing "standardgrade" $\mathrm{UO}_{2}$, prepared by the hydrogen reduction of $\mathrm{UO}_{3} \cdot 2 \mathrm{H}_{2} \mathrm{O}$ in a movingbed furnace, for $\$ 1.15 / \mathrm{lb}$ less than ADU-type $\mathrm{UO}_{2}$ (44). A careful cost analysis for each specific fuel geometry would be required to indicate whether the cost advantage of such cheaper powder would be offset by the larger investment in compacting equipment and lower furnace throughput.

ADU-type $\mathrm{UO}_{2}$ powder, made as described, offers some advantage for more economic fuel production in that it can be cold-pressed without an organic binder. It is necessary to apply a lubricant such as stearic acid to the die walls during the pressing operation to obtain crack-free compacts, which can then be charged directly to the sintering furnace without the presintering treatment normally used for binder removal.

Method of compaction. The most common method used for preparing $\mathrm{UO}_{2}$ compacts is dry-pressing in hardened-steel or tungsten-carbide dies. Usually, an organic binder such as paraffin wax, polyethylene glycol or camphor is added to increase the green strength of the compact during subsequent handling. A small amount of stearic acid, 0.2-0.4 wt \%, is often added as a die lubricant ( 39 ).

The extruision process $(37,45)$ may offer some advantage where a large length-to-diameter ratio is required or for fabricating large-diameter tubes of $\mathrm{UO}_{2}$, but no fabricator has yet chosen the technique in preference to automatic dry-pressing. Other possible processes such as slip-casting, isostaticpressing and hot-pressing have also been rejected up to now.

The compaction of $\mathrm{UO}_{2}$ powders in metal sheaths by rotary-swaging has been investigated extensively at $\mathrm{Han}-$ ford $(37,46)$ and at Chalk River (39, 47). In both laboratories it was found that, of the many powders evaluated, arc-fused $\mathrm{UO}_{2}$ could be compacted at room temperature to the highest density, about $10 \mathrm{gm} / \mathrm{cm}^{3}$, in either Zircaloy-2 or stainless-steel sheaths. Much lower densities were obtained when powders with high surface area, such as ADU-type $\mathrm{UO}_{2}$, were used. In the Hanford experiments, higher densities resulted when the oxide was swaged hot at $600^{\circ} \mathrm{C}\left(46^{\circ}\right)$.

At first sight, swaging promises a cheaper method of fabrication than the conventional sintered-pellet route. However, the swaging of fuel elements with a high ratio of diameter to sheath thickness may not be economically feasible. It seems inevitable that the trend in fuel-element development will be to decrease the sheath thickness to the minimum permitted by fabrication and irradiation experience. As an example, the diameter/sheath-thickness ratio of the $\mathrm{UO}_{2}$ fuel elements in the Shippingport reactor is $18 / 1$ (41), whereas the target in the Canadian NPD-2 reactor is $40 / 1$. Recent tests at Chalk River, with a ratio near the latter value, have shown that small cracks had formed on the inside surface of the Zircaloy-2 tube during swaging after a reduction in the element crosssectional area of only $20 \%$. One such element, which had been reduced $48 \%$ in area at $400^{\circ} \mathrm{C}$ to 0.8 -in. outside diameter and 0.025 -in. sheath thickness, was irradiated in a pressurized-water loop in the NRX reactor and split open immediately upon reactor startup. One end of the swaged specimen, which had been cut off, was metallographically examined, revealing cracks on the inside Zircaloy- 2 surface up to 0.002 in. deep. As a result of such experience, swaged $\mathrm{UO}_{2}$ has been rejected as a first charge for the NPD-2 reactor. Efforts are continuing, however, to determine if an economic process incorporating intermediate annealing steps can be evolved to produce crack-free sheaths.

The loose compacting of $\mathrm{UO}_{2}$ powder in a fuel sheath is simple in concept, but, thus far, no method has been found to exceed a packed density of 8-9 $\mathrm{gm} / \mathrm{cm}^{3}$

Sintering method. Ceramic-grade $\mathrm{UO}_{2}$ can be sintered to densities above $10 \mathrm{gm} / \mathrm{cm}^{3}$ in neutral or oxidizing atmospheres at temperatures $300^{\circ}-400^{\circ} \mathrm{C}$ lower than in reducing atmospheres. In Thackray and Murray's early work (48), for example, it was found that with an ADU-type oxide of composition $\mathrm{UO}_{2.13}$, after pressing at 20,000 psi, a density of $10 \mathrm{gm} / \mathrm{cm}^{3}$ was reached after $30 \mathrm{~min}$ in argon at $1,400^{\circ} \mathrm{C}$. Belle and Lustman $(2,3)$ have concluded from their studies on self-diffusion kinetics that densification in an 
oxidizing atmosphere occurs by the diffusion of the more mobile, excess oxygen ions, whereas in a reducing atmosphere a plastic or viscous flow model is probable. The effect of excess oxygen on the sinterability of $\mathrm{UO}_{2}$ was well demonstrated in Scott and Williams's work on warm-pressing of $\mathrm{UO}_{2}$ (49), where it was found that fine, nonstoichiometric powders could be compacted in metal dies to densities above $10 \mathrm{gm} /$ $\mathrm{cm}^{3}$ in $10 \mathrm{~min}$ at $800^{\circ} \mathrm{C}$ and 20,000 psi. When the excess oxygen was removed from the $\mathrm{UO}_{2}$ lattice by the addition of powders such as iron or uranium, the densification was inhibited.

The steam-sintering of wet-ballmilled $\mathrm{UO}_{2}$ has been studied by Arenberg and Jahn (50). To achieve high densities, the compacts were heated in $\mathrm{H}_{2}$ to $1,400^{\circ} \mathrm{C}$ before introducing a steam atmosphere. Chalder (40), on the other hand, has reported that, with ADU-type oxide, the complete cycle of heating, sintering at $1,300^{\circ}-1,400^{\circ} \mathrm{C}$ and cooling can be carried out in steam. The resulting pellets of $10.5-10.6 \mathrm{gm} /$ $\mathrm{cm}^{3}$ density usually are near $\mathrm{UO}_{2.15}$ in composition.

The major difficulty with low-temperature sintering is that the product is nonstoichiometric, unless a hydrogencooling step is introduced. Stoichiometric oxide is preferred as a reactor fuel for reasons that will be discussed in the later section on irradiation behavior. If hydrogen-cooling is a process requirement, it is not clear that there is any economic advantage over sintering in a continuously stoked furnace in a hydrogen atmosphere at $1,600^{\circ}-1,700^{\circ} \mathrm{C}$.

Although it may be fortuitous, most manufacturers prepare $\mathrm{UO}_{2}$ pellets from a "ceramic-grade" powder of high surface area in continuous rather than batch-type furnaces and in an atmosphere of dry hydrogen or cracked ammonia. Runfors et al. $(51,52)$ have reported, however, that the sintering temperature can be lowered about $100^{\circ} \mathrm{C}$ if the hydrogen is first saturated with moisture at room temperature, although the effect of the moist atmosphere on furnace life has not been established.

\section{Sheathing}

The important requirements for a fuel-element sheath are low neutroncapture cross section, good mechanical properties at working temperatures, high corrosion resistance in the hot cool-

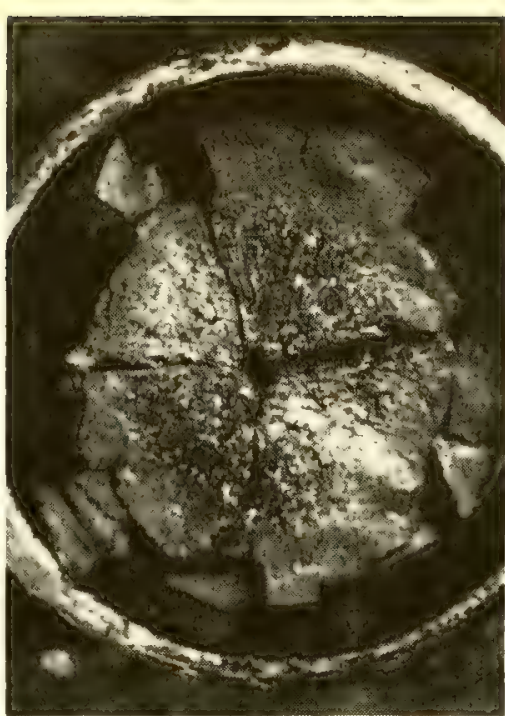

FIG. 5. Zircaloy-2 clad $\mathrm{UO}_{2}$ rod $(0.56$ in. diameter) from CR-V-e test after ir. radiation of $7,000 \mathrm{Mwd} /$ tonne $\mathrm{U}$ with surface temperature of $400^{\circ} \mathrm{C}$

ant and compatibility with the core.

For economic water-cooled reactors fueled with $\mathrm{UO}_{2}$, a zirconium alloy would appear to be the most suitable material. Stainless steel would be acceptable but for its high neutron-capture cross section. Aluminum-nickel alloys are not likely to compete with zirconium because of lower strength and lower neutron economy (53). For sodium-cooled systems, also, zirconium alloys may prove to be the best choice. In organic liquids, however, zirconium alloys are attacked by the hydrogen that is liberated when the coolant is irradiated. The most suitable material immediately available for organics at temperatures near $400^{\circ} \mathrm{C}$ is stainless steel. Stainless cladding is also likely to be used in several $\mathrm{CO}_{2}$-cooled reactors now being designed, at least until beryllium technology progresses.

The most common design of $\mathrm{UO}_{2}$ fuel element is typified by the first loading for the Shippingport reactor (41). The fuel assemblies consist of a bundle of small round rods, each rod enclosing a stack of right-cylindrical sintered pellets. The clearance between the pellets and the sheath is so specified that the pellets can be loaded easily into the tubes but will expand into near contact with the sheath when heated in the reactor. Other geometries being developed include a nested tubular type for the Plutonium Recycle Test Reactor (37) and a plate type for the second loading of the PWR (41). A rod-type element was chosen for the first loading of NPD-2 because of the satisfactory results that had been obtained from irradiation tests on such a geometry and because of the inherent stability of a cylindrical sheath when subjected to an internal pressure.
The effect of external pressure on sheath stability is of major concern, particularly in water-cooled reactors operating at coolant pressures of 1,000 $2,000 \mathrm{psi}$ where the fuel has a diameter/ sheath-thickness ratio greater than $30 / 1$. In NPD-2, for example, where the pressure is to be $1,100 \mathrm{psi}$, the proposed 1-in.-diameter, 0.025-in.-thick Zirealoy-2 sheath will deform plastically at the maximum operating surface temperature of $300^{\circ} \mathrm{C}$, forming ridges and dimples, if the fuel-sheath clearance is greater than 0.006 in. in diameter or $0.15 \mathrm{in}$. axially. To ensure accurate control of the diametral clearance, the $\mathrm{UO}_{2}$ pellets will be centerless ground to \pm 0.0005 in. before loading into carefully dimensioned sheaths. In addition, it is probable that an axial clearance of $0.2 \mathrm{in}$. will be distributed along the 18-in. fuel length by fabricating pellets with concave ends.

End caps are usually attached to $\mathrm{UO}_{2}$ fuel elements by fusion-arc welding in an inert atmosphere such as helium or argon. Resistance seamwelding combined with a Heliarc fusion weld is being developed to provide a double closure for large-diameter, thinwalled tubes (37). Electron-beam welding has also been studied as an endclosure method $(54,55)$. Since the latter process is carried out in vacuum, however, the resulting fuel element could have poorer heat-transfer properties if the oxide does not expand to contact the sheath when irradiated.

If the fuel elements are short, such as those used in PWR (41), only end spacers are required in the assembled bundle. For longer assemblies, intermediate spacers are normally incorporated. These may be wires wound in a spiral around the rod, ribs integral with the sheath, short lugs welded on, or metal grids similar to the type described by Ambartsumyan et al. (56).

\section{Irradiation Behavior}

Irradiation tests on prototype PWR elements clad in Zircaloy-2 have been reported at length (5\%). More recently, many $\mathrm{UO}_{2}$ fuel tests in the NRX reactor have been reported. Thus, within the past two years, a much clearer understanding has been obtained of the irradiation behavior of $\mathrm{UO}_{2}$. A brief summary of irradiation experience, including results from tests just completed at Chalk River, follows.

Effect of heat rating. Lewis (59) has pointed out the economic advan- 
tage of developing a $\mathrm{UO}_{2}$ fuel that has a high value of the integral

$$
\int_{\text {surface temp. }}^{\text {max. fuel temp. }} k(\theta) d \theta
$$

where $k(\theta)$ is the thermal conductivity and is a function of the temperature, $\theta$. This expression is very useful in fuel design (58) to compare the irradiation behavior of specimens of similar surface temperatures but of differing diameter, enrichment, density, heat rating and fabrication history. It can be applied without information on thermal conductivity and its variation with temperature and irradiation, the temperatures at which grain growth and melting occur or the type of sheathing used.

The integral can be obtained as a product of two terms, one determined by the measured heat-transfer rate and the other a calculated function of the specimen's properties, e.g.

$$
\begin{aligned}
\int_{T_{a}}^{T_{r}} k(\theta) d \theta & =\frac{H R}{2} \cdot \mathrm{fn}(\varkappa a, r) \\
& =(q / 4 \pi) \cdot \mathrm{fn}(\varkappa a, r)
\end{aligned}
$$

where

$$
\mathrm{fn}(\varkappa a, r)=2 \frac{I_{0}(\varkappa a)-I_{0}(\varkappa r)}{\varkappa a \cdot I_{1}(\varkappa a)}
$$

which has the value unity at $r=0$ for negligible flux depression, hence uniform heat generation; $k(\theta)=$ thermal conductivity of $\mathrm{UO}_{2}$ at temperature $\theta, T_{r}=$ temperature at radius $r, T_{s}=$ temperature at $\mathrm{UO}_{2}$ surface, $H=$ sheath-coolant surface heat flux, $R=$ radius of outside of sheath, $a=$ radius of $\mathrm{UO}_{2}, q=$ heat production per unit length of fuel, $I_{0}=$ modified Bessel function of zero order, $I_{I}=$ modified Bessel function of first order and $x=$ reciprocal of effective diffusion length in $\mathrm{UO}_{2}$.

Morison (60) has expressed the dependence of $\mathrm{fn}(\kappa a, r)$ at $r=0$ on $\mathrm{UO}_{2}$ diameter and enrichment for $95 \%$ dense, solid oxide cylinders in the convenient form shown in Fig. 4. Thus, the computation of the integral has been much simplified.

According to Robertson et al. (58) and more recent tests on sintered ADUtype $\mathrm{UO}_{2}$, cylindrical stoichiometric specimens, whose oxide surface temperature is about $400^{\circ} \mathrm{C}$ during irradiation, exhibit grain growth when $\int_{\text {surface }}^{\text {center }} k(\theta) d \theta$ exceeds $29 \pm 3 \mathrm{w} / \mathrm{cm}$. Rod-shaped elements irradiated in pressurized-water loops at Chalk River have exhibited completely satisfactory performance at ratings as high as 50 $\mathrm{w} / \mathrm{cm}$. A cross section of such a rod is shown in Fig. 5. Central melting would be expected at $55 \mathrm{w} / \mathrm{cm}$.

It was considered useful to plot several irradiation results so that values of the integral producing grain growth and melting could be predicted for solid stoichiometric cylinders with different surface temperatures. This has been done by plotting $\int_{0^{\circ} \mathrm{C}}^{T} k(\theta) d \theta$ versus $T$, a particular temperature in the fuel, as in Fig. 6. For any given irradiation, the value of $\int_{T_{*}}^{T} k(\theta) d \theta$ is the difference in ordinates for two points defined by the temperature limits, $T_{s}$ and $T$.

The point on Fig. 6 for Pellet Rod III was determined by measuring the central temperature with a thermocouple and by calculating the integral from a knowledge of the neutron flux, hence the heat output from the rod.

In plotting the points for the Canadian specimens showing grain growth (CR-V-e, X-2-O, X-2-g and Pellet Rod II), values for the integral from the oxide surface to the observed radius of grain growth were determined by a method similar to that detailed in a following paragraph. It was assumed that discernible grain growth would be produced in the $\mathrm{ADU}$-type $\mathrm{UO}_{2}$ at a temperature of $1,500^{\circ} \mathrm{C}(58)$. When no grain growth was discernible in the center of an element for comparison with the undisturbed outer area, a minor change in appearance was difficult to detect under the microscope used. Hence, the center temperature of specimens from CR-V-b and $\mathrm{X}-2-\mathrm{n}$, where no grain growth was observed, may have been as high as $1,600^{\circ} \mathrm{C}$.

The WAPD specimens 25-2-L2 and $\mathrm{X}-1-\mathrm{g} 5$, which also showed no grain growth, were made from the less reactive PWR-grade $\mathrm{UO}_{2}$. Thus, a somewhat higher temperature limit, $1,700^{\circ}$ $\mathrm{C}$, has been assumed. Specimens $\mathrm{X}-1-\mathrm{f} 5$ and $\mathrm{X}-1$-g3 showed grain growth corresponding to $2,300^{\circ} \mathrm{C}$ at the maximum-flux positions (57).

The heat outputs from specimens irradiated in the $\mathrm{CR}-\mathrm{V}$ and $\mathrm{X}-2$ loops were measured calorimetrically, and some were checked by mass-spectrometric analyses to determine burnup. Published heat fluxes for the WAPD

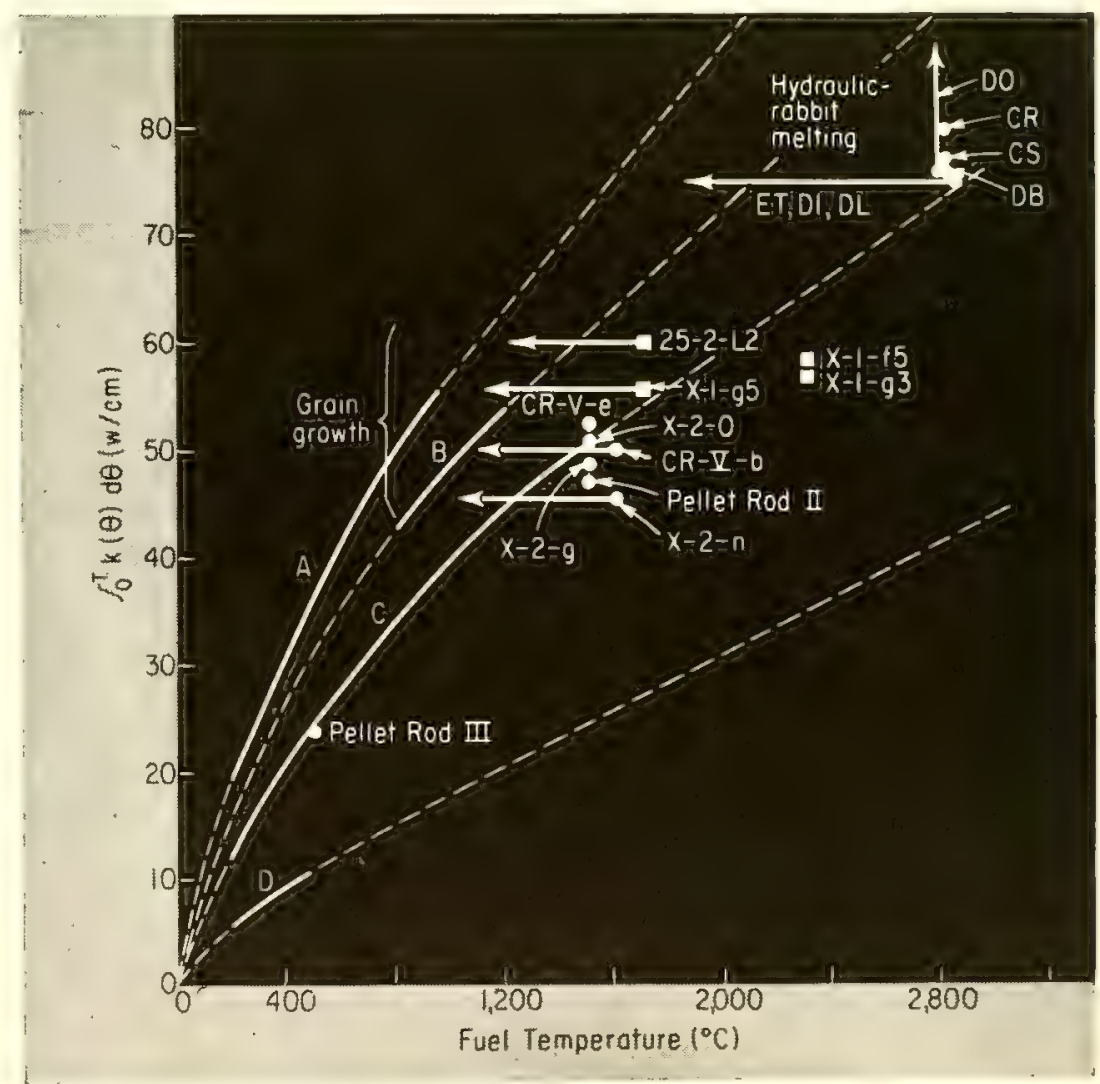

FIG. 6. Summory of irradiation tests on $\mathrm{UO}_{2}[\mathrm{O}-\mathrm{Chalk}$ River data; $\square-W A P D$ data (57)]. Solid curves A, B, C and D are values for thermal conductivity of $\mathrm{UO}_{2}$ corrected to $95 \%$ density, taken from references $28,30,31$ and 32 , respectively; dashed lines are extrapolations to $2,800^{\circ} \mathrm{C}$ 


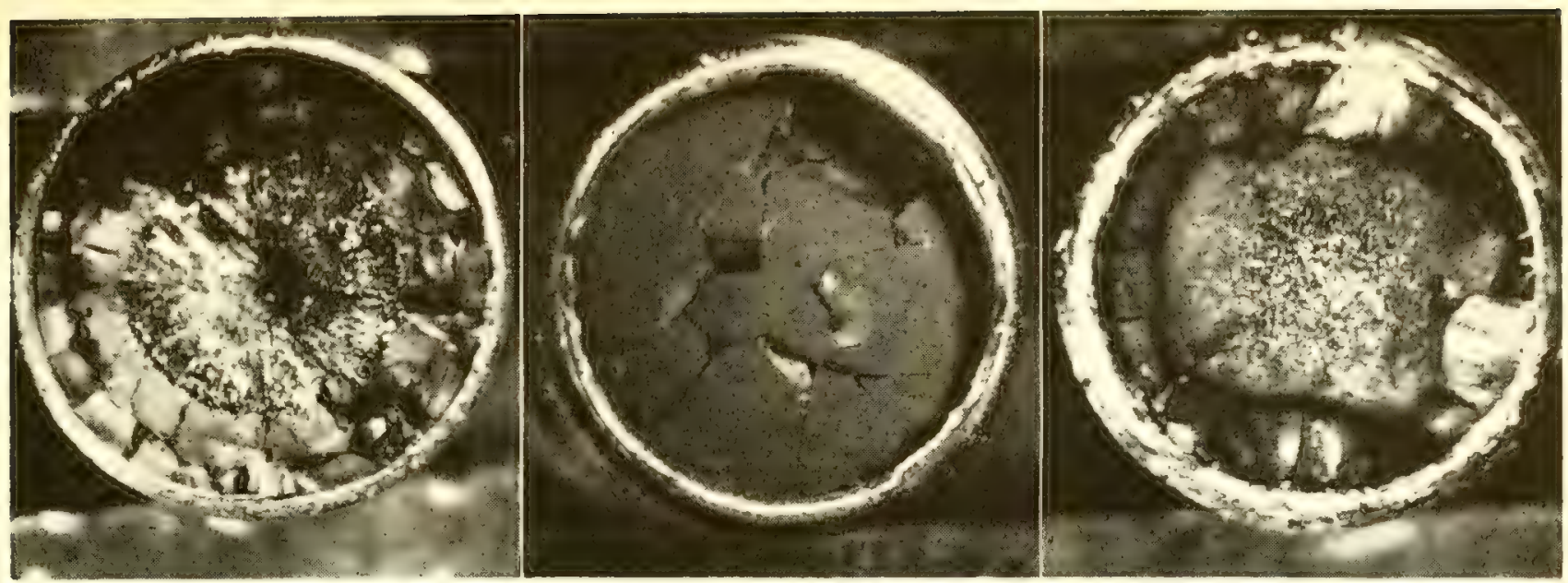

FIG. 7 Cross sections of irradiated Zircaloy-2-elad $\mathrm{UO}_{2}$ rods. At left is 0.72-in.-diameter rod irradiated for $60 \mathrm{sec}$ in NRX hydraulic rabbit; note central area that had been melted. Center and right cross sections (0.41-in.-diameter rods) show effects of $\mathrm{UO}_{2}$ composition after $\sim 4,500-M w d /$ tonne $U$ irradiation; at center is $\mathrm{UO}_{2.00}$ and at right is $\mathrm{UO}_{2.15}$ tests (57), corrected for flux gradient in the case of the $\mathrm{X}-1$ specimens, were used in calculating the integrals.

Unambiguous melting has been observed in specimens irradiated in a water-cooled facility in the NRX reactor known as the "hydraulic rabbit," where irradiations of short duration can be carried out. A cross section of a sintered $\mathrm{UO}_{2}$ specimen, $\mathrm{DB}$, clad in Zircaloy-2 and irradiated for 60 sec (Fig. 7) indicates clearly the extent of melting. The method used to apply the observed radius of melting obtained from a photograph such as Fig. 7 to the graph shown in Fig. 6 is illustrated by Fig. 8, where $\int k(\theta) d \theta$ is plotted against the fuel radius, $r$. The curve shown applies specifically to specimens $\mathrm{CR}$ and $\mathrm{DB}$, which were similar in diameter and enrichment. The oxide surface temperature of both was calculated to be $250^{\circ}$ C. Hence, $\int_{250^{\circ} \mathrm{C}}^{T_{\mathrm{m}}} k(\theta) d \theta$ is $66 \mathrm{w} / \mathrm{cm}$ for CR and $62 \mathrm{w} / \mathrm{cm}$ for $\mathrm{DB}$, at the melting temperature, $T_{\mathrm{m}}$. Specimen CR was irradiated for only 30 sec.

Later experience indicated that an irradiation time of $60 \mathrm{sec}$ was required to ensure that virtual thermal equilibrium had been reached. Thus, the integral for melting in specimen CR is probably somewhat high. Specimen DO had a larger fuel-sheath diametral clearance than the other rabbit samples. Hence, the integral might have been higher if the clearance had been comparable, as indicated by the vertical arrow on the graph. Since specimens ET, DI and DL did not melt, the ordinate of Fig. 6 could be specified but only an upper limit given to the abscissa.

Small differences in surface temperature of the oxide can be corrected for, without introducing serious errors, by using an assumed value of conductivity in the difference term alone. To plot points on Fig. 6 for specimens $\mathrm{CR}$ and $\mathrm{DB}$, a value of $14 \mathrm{w} / \mathrm{cm}$ was assumed for $\int_{0^{\circ} \mathrm{C}}^{250^{\circ} \mathrm{C}} k(\theta) d \theta$.

With the exception of sample DO, the fuel-sheath diametral clearances of all the specimens shown in Fig. 6 were small enough so that the oxide should have expanded to contact the sheath while the fuel was being irradiated. It is interesting to note that the "effective" thermal conductivity for such fuel appears approximately the same as the Hedge and Fieldhouse values (31) corrected for density.

Effect of fuel-sheath clearance. A simple model was proposed by Robertson et al. (58) to predict temperatures in fuel elements with large fuel-sheath diametral elearances. The $\mathrm{UO}_{2}$ cylinder was pictured as remaining centrally located and intact under irradiation and expanding as if it were at a uniform temperature equal to its mean temperature. The interfacial temperature drop could then be calculated from the thermal conductivity of the gas in the surrounding annulus.

It had been appreciated that the model was not physically correct, since it was unlikely that oxide pellets would remain central in the sheath and it was known that pellets would crack from the thermal stresses produced during irradiation. Evans had reported (87), for example, that cracking effectively relocated part of the original annular gap to the hotter interior of the fuel.

It was only recently, however, that the inadequacy of the model was clearly demonstrated from tests in the $\mathrm{EEC}$ loop (61) and hydraulic rabbit (62) at Chalk River. The latter tests, on 0.67-in.-diameter oxide pellets heated near the melting point in the center, indicated that the oxide surface temperature of specimens with a starting 0.017 -in. fuel-sheath diametral clearance was not more than $100^{\circ} \mathrm{C}$ higher than those with a 0.005 -in. clearance.

These results have led Robertson to suggest a more realistic model (68) in which cracked segments of oxide shift radially outward to contact the sheath, so that the interfacial temperature drop becomes mainly dependent on the interfacial pressure and the properties of the contacting surfaces. The few available irradiation data support the newer model. If the approach is valid, large differences in assembled diametral clearance could be tolerated in fuel elements where the sheath-collapse problem mentioned earlier was of no concern, since such differences would have a relatively small effect on the fuel temperature during irradiation.

Effect of $\mathrm{O} / \mathrm{U}$ ratio. Several tests $(58,64)$ have demonstrated that nonstoichiometric $\mathrm{UO}_{2}$ exhibits much more grain growth and liberates more fission gas than near-stoichiometric pellets irradiated under comparable conditions.

The most unambiguous comparison was obtained from the $\mathrm{X}-2-\mathrm{n}$ loop test where specimens, identical except for their $\mathrm{O} / \mathrm{U}$ ratio, were irradiated in adjacent positions for the same period. After irradiation, two samples of steamsintered and hydrogen-cooled $\mathrm{UO}_{2.00}$ were radially cracked with no apparent grain growth, whereas two samples of steam-sintered $\mathrm{UO}_{2.15}$ exhibited cracking and extensive grain growth, as illustrated in Fig. 7. The amount of fission gas released was $100-200$ times higher from the nonstoichiometric oxide.

The X-2-n experiment clearly indicated that the value of $\int k(\theta) d \theta$ producing grain growth is markedly lowered if extra oxygen is added to the 


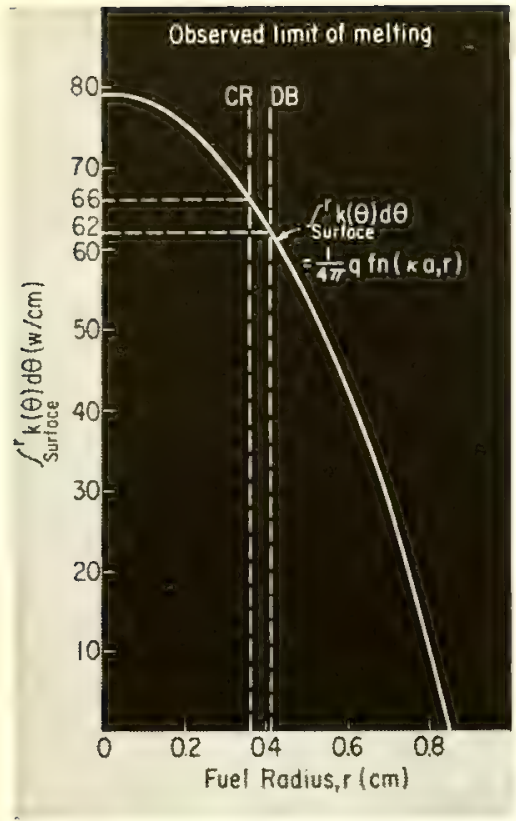

FIG. 8. Determination of integral from observed rodii of melting for specimens $C R$ and DB. Both specimens were 0.72 in. diameter and contained uranium enriched to 4.82 wt $\% U^{235}$. Tests were made in hydraulic rabbit

$\mathrm{UO}_{2}$ lattice. The oxygen self-diffusion rate is higher in $\mathrm{UO}_{2+x}$ than in $\mathrm{UO}_{2.0}(3)$; thus grain growth should proceed at lower temperatures in nonstoichiometric oxides. In addition, the thermal conductivity of $\mathrm{UO}_{2+x}$ is known to be lower at $60^{\circ} \mathrm{C}$ and hence is suspected to be lower at elevated temperatures. The liberation of gaseous $\mathrm{UO}_{3}$ by the disproportionation of $\mathrm{UO}_{2+x}$ might also produce changes.

The available irradiation results indicate that stoichiometric oxide should be chosen for optimum performance. If an element were to become defected in service, however, the core could be oxidized by coolants such as water or $\mathrm{CO}_{2}$. Thus, it is desirable to obtain irradiation experience on purposely defected test specimens, particularly at high heat ratings. Tests under way at Chalk River indicate that defected Zircaloy-2clad $\mathrm{UO}_{2}$ can be operated safely in pressurized-water coolant at values of $\int_{400^{\circ} \mathrm{C}}^{\text {center }} k(\theta) d \theta$ as high as $50 \mathrm{w} / \mathrm{cm}$.

Fission-gas release. The release of fission gases from irradiated $\mathrm{UO}_{2}$ has been studied extensively $(5 \%, 58,65-$ 67). However, the factors influencing gas release and the actual escape mechanism are still not clearly understood. Markedly different amounts of gas were evolved from samples of similar density prepared by different fabrication methods $(66)$. Even for specimens prepared by virtually the same fabrication technique in the same laboratory and receiving similar irradiations, the fissiongas release has varied appreciably.

Where grain growth has been observed, the amount of gas released in long irradiations has always been substantial; e.g., $35 \%$ of the fission-product xenon was released from rod 12 of the CR-V-e test in which $\sim 45 \%$ of the oxide exhibited grain growth during an irradiation of 7,000 Mwd/tonne $\mathrm{U}$.

Even with the most pessimistic assumptions, fission-gas release would not limit the performance of the present $\mathrm{UO}_{2}$ core in the Shippingport reactor (65). In reactors operating under lower pressures using elements with larger ratios of diameter to sheath thickness, however, the buildup of internal gas pressure might prove to be a severe limitation. On the other hand, Davies (68) has calculated that the fission-gas pressure within a typical fuel element may reach a limiting value at less than 100 atmospheres due to a "knock-on" process where free gas atoms are kinetically excited by fission fragments and reenter the oxide lattice. An experimental program is under way at Chalk River to determine the validity of the theory.

I am indebted to A. S. Bain, G. H. Chalder, W. Evans, R. G. Hart, J. A. L. Robertson, A. M. Ross and M. B. Watson for supplying information in advance of publication. I am particularly grateful to J.A. L. Robertson for many valuable discussions and for Figs, 6 and 8 .

This article is based on a paper presented before the First International Symposium on Nuclear Fuel Elements at Columbia University, Jan. 28-29, under the sponsorship of Columbia University and Sylvania-Corning Nuclear Corp.

\section{BIBLIOGRAPHY}

1. J. J. Katz, E. Rabinowitch, "The Chemistry of Uranium," National Nuclear Energy Series VIII-5 (MeGraw-Hill Book Co., New York, 1951)

2. J. Belle, B. Lustman, WAPD-184 (1957) also issued in TID-7546, p. 442 (1958)

3. J. Belle, 2nd Int. Conf. on Peaceful Uses of Atomic Energy, Paper P/2404 (1958)

4. P. E. Blackburn, Westinghouse Report 100FF942-P1 (1957)

5. F. Gronvold, J. Inorg. \& Nuclear Chem. 1 , 357 (1955)

G. S. Aronson, J. Belle, WAPD-T-573 (1957)

7. L. E. J. Roberts et al., 2nd Int. Conf, Paper P/26 (1958)

8. P. Perio, Bull. Soc. Chem. 20, 256 (1953)

9. J. S. Anderson et al., J. Chem. Soc., 3946 (1955)

10. L. C. Watson, CRL-45 (1957); also issued in TID-7546, p. 384 (1958)

11. P. Murray et al., TID-7546, p. 432 (1958)

12. J. D. Eichenberg, WAPD-167 (1957)

19. B. Lustman, Westinghouse Atomic Power Div, private communication (1957)
14. J. E. Antill et al., AERE Report M/M 168 (1957)

15. R. W. Nichols, Nuclear Eng. 3, 327 (1958)

16. M. W. Mallett et al., BMII-1028 (1955)

17. A. L. Eiss, SCNC -257 (1958)

18. L. M. Pidgeon, J. M. Toguri, University of Toronto, unpublished work (1958)

19. O. Ruff, D. Goecke, Z, angew. Chem. 24, 1459 (1911)

20. E. Friederich, L. Sittig, Z, Anorg. u. allgem. Chem. 145, $127(1925)$

21. W. A. Lambertson, M. H. Mueller, J. Am. Ceram. Soc. 36, 329 (1953)

22. R. J. Ackermann, ANL-5182 (1955)

23. L. G. Wisnyi, S. Pijanowski, KAPL-1702 (1957)

24. T, C. Ehlert, J. L. Margrave, J. Am. Ceram. Soc. 41, 330 (1958)

25. F. A. Halden, Stanford Research Institute, private communication (1958)

26. L. M. Pidgeon, University of Toronto, privaté communication (1958)

27. A. M. Ross, AECL Report CRFD-817, to be published

28. W. D. Kingery et al., J.Am.Ceram. Soc. 37, 107 (1954)

29. M. Englander, French Report CEA-79 (1951)

30. R. W. Scott, AERE Report M/R 2526 (1958)

91. J. C. Hedge, I. B. Fieldhouse, Armour Research Foundation Report GO22 D3 (1956)

32. J. D. Eichenberg, WAPD-200 (1958)

33. P. Murray, D. T Livey, "Progress in Nuclear Energy," vol V-1, p. 448 (Pergamon Press, London, 1956)

84. J. Thewlis, Acta Cryst. 6, 790 (1952)

35. M. D. Burdick, H. S. Parker, J. Am. Ceram. Soc. 39, 181 (1956)

36. J. S. Anderson, AERE Report C/R 886 (1954)

37. E. A. Evans, HW-52729 (1957); also issued in TID-7546, p. $414(1958)$

98. C. D. Harrington, TID-7546, p. 369 (1958)

39. G. H. Chalder et al., 2nd Int. Conf., Paper $\mathrm{P} / 192$ (1958)

40. G. H. Chalder, AECL Report UK/C6/115 (1957)

41. J. Glatter et al., 2nd Int. Conf., Paper $\mathrm{P} / 2380$ (1958)

49. A. H. Webster, N. F. H. Bright, Canadian Dept. of Mines and Technical Surveys Report MD-223 (1957)

43. L. C. Watson et al, AECL Report CRCE-716, Parts I-III (1958)

44. Eldorado Mining and Refining Co. Ltd., Port Hope, Ontario, Catalogue of Canadian Uranium Products (1958)

45. A. Allison, W. K. Duckworth, BMI-1009 (1955)

46. D. R. Stenquist, R. J. Anicetti, "Nuclear Metallurgy," vol. 5 (A. I.M.E., 1958)

47. D. L. Paterson, G, H. Chalder, AECL Report CRFD-759, to be published

48. R. W. Thackray, P. Murray, AERE Report M/R 614 (1950)

49. R. Scott, J. Williams, Trans. Brit. Ceram. Soc. 57, 199 (1958)

50. C. A. Arenberg, C. Jahn, J. Am, Ceram. Soc. 41, 179 (1958)

61. U. Runfors et al., 2nd Int. Conf., Paper P/142 (1958)

52. N. Schonberg et al., 2nd Int. Conf., Paper $\mathrm{P} / 182(1958)$

53. W. B. Lewis, AECL Report DL-33 (1958)

54. J. Briola, 2nd Int. Conf., Paper P/1161 (1958)

55. W. L. Wyman, W. I. Steinkamp, HW-55667 (1958)

56. R. S. A mbartsumyan et al., 2nd Int. Conf. Pарет P/2196 (1958)

57. J. D. Eichenberg et al., WAPD-183 (1957); also issued in TID-7546, p. 616 (1958)

58. J. A. L. Robertson et al., 2nd Int. Conf. Paper P/193 (1958)

59. W. B. Lewis, AECL Report DM-44 (1957)

60. W. G. Morison, Atomic Energy of Canada Ltd., un published work (1958)

61. A. S. Bain, AECL Report UKE-CR-1006 (1958)

62. J. A. I. Robertson, A, S. Bain, AECL Report CRFD-825 (1959)

6.3. J. A. L. Robertson, Atomic Energy of Canada Ltd,, private communication (1958)

64. A. S. Bain, J. A. L. Robertson, letter, submitted to J. of Nuclear Materials (1959)

65. B. Lustman, WAPD-173 (1957)

66. A. H. Booth, G. T. Rymer, AECL Report CRDC-720 (1958)

67. A. H. Booth, AECL Report CRDC-721 (1957)

68. J. A. Davies, Atomic Energy of Canada Ltd., private communication (1958) 


\title{
Getting PWR on the Line
}

\author{
With the Shippingport power station scheduled to go critical \\ in June, 1957, operating plans are now being firmed up. \\ Duquesne Light Co.'s approach to two aspects of operation- \\ cold-station startup and personnel-are described here
}

By L. R. LOVE and G. N. OLDHAM

Shippingport Power Station, Duquesne Light Company

Pittsburgh, Pennsylvania

\section{1-Startup Procedure*}

The Shippingport power station will be similar to conventional stations in that several different startup procedures will be used. These procedures will differ mainly in the starting point, with the final steps of startup being very similar.

The specific procedure to be followed in any one startup will depend on the station conditions existing at the time of the startup, which will in turn depend on the purpose and length of the previous out-age. The procedure to be presented in this paper is the cold-station startup procedure, which will be the one of longest duration, but will be used only after an extended shutdown of the reactor.

\section{Initial Station Conditions}

For this particular startup, it is assumed that station conditions are as follows:

1. All station service buses are energized from the 138-kv network.

2. The reactor coolant and associated systems are completely filled with water at ambient temperature and at a pressure less than 150 psig. The water is of the required purity except for oxygen content.

* Parts 1 and 2 of this article are based on papers presented by $L$. $R$. Love and $G$. M. Oldham, respectively, at the Tenth Annual Mechanical Engineering Conference on Nuclear Power Plants, Pittsburgh, Pa., May 1, 1956.
3. The purification system is isolated and the pressurizer spray line is open.

4. The precritical check out of reactor controls and instrumentation is completed.

5. The turbine-generator is on "motor slow roll." The generator shell is filled with hydrogen.

6. The condensate and boiler-feed systems are set up for operation with drum level slightly below normal and steam lead vents and drains open.

7. All reactor and turbine-generator plant auxiliary systems are either in service or available for service.

\section{Coolant-System Warm-Up}

With these conditions established, the first steps in the procedure are the chemical treatment of the reactor coolant for oxygen removal and the initiation of the reactor coolant system warm-up.

In the following procedure, several minor steps have been omitted for simplicity.

1. The reactor coolant pumps are started at high speed, and coolant is circulated through the loops and the pressurizer spray circuit.

2. The system is pressurized to 400 psig by the charging-system pumps. The pressurizer heaters are energized to assist the reactor-coolant pumps in raising the coolant temperature.

3. Reactor coolant is sampled and analyzed to determine its oxygen content. The required amount of hydrazine is added to the system for oxygen scavenging.

4. The coolant is then circulated for about 4 hr to permit the hydrazine-oxygen reaction to take place. During this period, the coolant pressure is maintained between 400 and 600 psig by discharging water as required. The coolant temperature is maintained between $190^{\circ} \mathrm{F}$ and $200^{\circ} \mathrm{F}$ by de-energizing the pressurizer heaters and cycling the coolant pumps as required.

5. When oxygen concentration has been reduced to the desired level, the pressurizer spray-line valve is closed and all heaters are energized. The pressurizer now assumes its true function-that of pressurizing the reactor coolant to prevent boiling in the core. From this point on, pressurizer water temperature should be at least $100^{\circ} \mathrm{F}$ but not more than $200^{\circ} \mathrm{F}$ higher than the coincident coolant temperature. The pressurizer temperature rise is limited to a maximum uniform rate of $100^{\circ} \mathrm{F} / \mathrm{hr}$.

6. The purification system is placed in service. This system was isolated previously to prevent the demineralizers from removing the hydrazine from solution.

7. Hydrogen is added to the reactor coolant so that free hydrogen is availible for scavenging the oxygen released, 
due to water decomposition, after the reactor is placed in service.

8. When the pressurizer temperature reaches $400^{\circ} \mathrm{F}$, a steam bubble is formed in the pressurizer by reducing coolant pressure to approximately 200 psig. During subsequent steps, the pressurizer level is maintained within the prescribed limits by discharging or adding water as required.

9. The reactor control-rod system is then energized and rod withdrawal begun to continue the warm-up of reactor coolant. During rod withdrawal, the neutron level and the level-change-rate instruments are closely observed to insure that the level-change rate is below the specified values. As the point of predicted criticality is approached, continuous rod withdrawal is stopped, and the rods are withdrawn in small steps until eriticality is reached. Protective devices are provided to limit the speed of approach to criticality. If the levelchange rate should exceed a predetermined value, rod motion will be stopped automatically.

10. When the reactor is critical, the control-rod position is adjusted to produce a power level equivalent to $1-2 \%$ of full power, or as required to heat reactor coolant at a rate of $100^{\circ} \mathrm{F}$ per hour. To maintain this power level, the rods must be further withdrawn at intervals to compensate for the negative temperature coefficient, which will reduce power level as temperatures increase unless reactivity is added.

11. The pressurizer heaters and spray-valve control are then placed on automatic.

\section{Turbine-Plant Start-Up}

While proceeding with the warmup of the reactor plant, the turbine-generator plant will be placed in service as follows:

1. The condenser circulating water flow is established, and the condensate system is placed in service, circulating water from the condenser hotwell through the air-ejector condenser and other heat exchangers back to the hotwell.

2. The turbine glands are sealed, and the air ejector is placed in service to evacuate the condenser.

3. The drum vents are closed when pressure reaches approximately 20 psig, and the free drains on the steam leads and the header are checked to insure that they are free of water.

4. The turbine high-pressure aux- iliary oil pump is started, and the various drains on the turbine are opened in preparation for placing the turbine on "steam slow roll."

5. When steam pressure is approximately 75 psig (reactor-coolant temperature $300^{\circ} \mathrm{F}$ ), and the condenser vacuum is at least $10 \mathrm{in}$. of $\mathrm{Hg}$, the throttle valves are opened, and steam is admitted to the turbine to place it on "steam slow roll." When steam is admitted to the turbine, the free drains on the steam leads and the header are closed.

6. If the turbine is free of rubs, the speed is increased to approximately $150 \mathrm{rpm}$, and the shaft eccentricity is checked.

7. If the shaft is true (eccentricity less than 0.001 inches), the turbine speed will be increased to $1,800 \mathrm{rpm}$ at a uniform rate in approximately $25 \mathrm{~min}$.

8. The boiler feed system is placed in service when required, and the drum level is controlled manually.

9. As the turbine speed approaches $1,800 \mathrm{rpm}$, the governor controls are observed to insure that speed is properly controlled.

10. The overspeed trip mechanism is checked by increasing turbine speed slowly to the trip point, and the throttle valves are immediately reopened.

11. Excitation voltage is applied to the generator field and is adjusted as necessary to match generator voltage with system voltage.

12. The generator is synchronized and loaded to $5-10 \mathrm{Mw}$, and voltage control is placed on automatic.

13. Half of the station service load is switched to the generator-lead station service transformer.

\section{Final Steps}

During this period, the reactor plant warmup will have continued at a rate of $100^{\circ} \mathrm{F}$ per hour, and the reactor coolant temperature will have risen to approximately $400^{\circ}$ F. During the loading of the station and the completion of the reactor coolant system warmup, the following steps are taken.

1. The turbine-generator and auxiliaries are set up for normal running operation, and the generator is loaded at specified rates in accordance with system requirements.

2. The boiler-feedwater system is placed on automatic control when flow conditions permit.

3. When average reactor coolant temperature reaches $525^{\circ} \mathrm{F}$, the rod control system is placed on automatic control.

4. The reactor coolant is sampled and analyzed for oxygen content, and hydrogen is added as required.

The Station is now capable of accepting its share of load swings as required for system regulation (determined by consumer demand). To be specific, it will be required to follow daily system load changes at an average rate of 3 Mw per minute, and to accept load swings of $20 \mathrm{Mw}$ maximum at a rate of $24 \mathrm{Mw}$ per minute. It must also handle a proportional part of the load swings imposed on the various stations by casualties on the system.

The output of the reactor will follow all but the largest load changes without immediate control-rod movement, due to the negative temperature coefficient of reactivity inherent in the core, which permits it to produce only that power which is taken from it. However, subsequent changes in control-rod position are required to maintain average temperature constant due to the change in xenon concentration in the core following load changes. Xenon, which is a fission product, absorbs neutrons. Its change in concentration with power level causes changes in reactivity.

With the type of startup described here, the time required to reach the normal operating range $(20-100 \%$ of full power) from a cold condition would be approximately $8 \mathrm{hr}$. This is considerably longer than the $5 \frac{1}{2} \mathrm{hr}$ required for a similar startup of a conventional station of this size, but as was mentioned earlier, this startup will be used infrequently. The most frequent startup is one where the reactor coolant and associated systems have been maintained at or near operating temperature during the shutdown. In a startup from this condition, the hydrazine treatment would not be necessary, and the delay introduced by the requirement for heating reactor coolant would be eliminated. This would reduce the time required for startup to approximately $1 \frac{1}{2} \mathrm{hr}$ as compared to $21 / 2 \mathrm{hr}$ required for the hot startup of a conventional station. As these comparisons of startup times indicate, the reactor plant will be as flexible and, in some instances, more flexible than a coal-fired boiler plant. Also, the startup procedure shows that the actual mechanics of reactor-plant operation are no more difficult than conventional plant operation. 


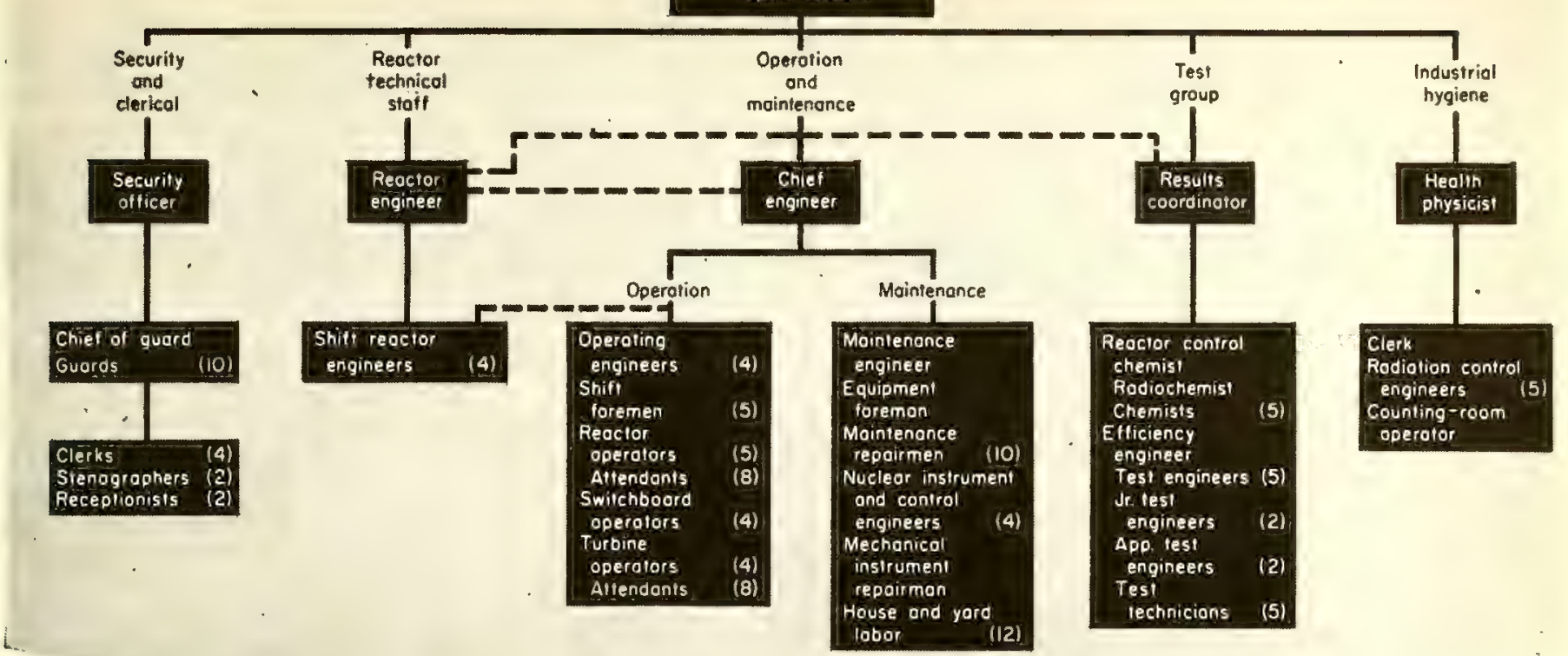

\section{2-Staffing the Plant}

The staffing Program for the Shippingport nuclear power station is more difficult than for conventional steam stations because of the lack of precedents and the lack of personnel with nuclear training within the company. Use of the station as a test facility complicates the problem.

To fill out the staff of the station, 126 people are considered necessary. Of these, 37 will be technically trained. Several of the factors leading to these high numbers are: (a) The station is the first of its kind, involving new techniques. (b) More men are necessitated by the extensive test program and by the emphasis on health and security. (c) A pool of technical manpower must be maintained to compensate for the normal turnover; this may become critical as the number of nuclear power stations grows.

\section{Training}

Except where recent college graduates are used and where individuals with specialized training, e.g., radiochemists, are required, all personnel probably can be obtained from within the company.

In addition to on-the-job training, all technical and some nontechnical men are given practical training in reactor-station operation to provide background for their own jobs and to instill an appreciation of the hazards involved. Each technical man also re- ceives theoretical nuclear training, and all personnel receive general training in health physics and security.

Most training is provided at the Westinghouse-operated Naval Reactor Facility at Arco, Idaho. At the completion of this course, about fifty men will have trained for an average of four months. Some will also be trained at the Bettis Plant in Pittsburgh.

\section{Organization}

The organization consists of five functional groups (see chart), each headed by a supervisor reporting to the superintendent. This type of organization was selected because it satisfies the program objectives of operation and test development

Operation and maintenance group is responsible for the entire plant.

Operating staff is larger than that normally found in a single-unit station by the addition of the shift reactor engineer and two attendants. The additional attendant in the turbine plant is provided because certain items requiring local operation and routine attention, such as screenhouse equipment, are remotely located. Although there are fewer items of this nature in the reactor plant, two attendants are provided since the local operations may be more time consuming due to the use of a change-room facility, which prevents the spread of radioactive contamination.
Maintenance staff is larger than in conventional station because of the high degree of station cleanliness necessary. More repaimen. are required since radioactivity levels could limit the amount of time a man can work on reactor-plant equipment. Relative inaccessibility of equipment and need for absolute leaktightness add to manpower requirements. Four instrument and control engineers are necessitated by the complicated reactor-plant instrumentation and control and the extensive test program.

Test group performs all tests and assists in evaluating new methods of operation and control. Its size is dictated by the test program. The chemical group is much larger than ordinarily due to the many unknowns in reactor-plant chemistry.

Technical staff provides technical assistance to the operating, maintenance, and test groups insofar as the reactor plant is concerned and is responsible for reactor-plant safety.

Industrial hygiene group performs routine radiation surveys of personnel, work areas, and adjacent off-site areas. This group is peculiar to a reactor plant.

Security and clerical group is responsible for security of classified documents and fissionable material.

$$
\text { * * * }
$$

This article is based on papers presented at the Tenth Annual Mechanical Engineering Conference on Nuclear Power Plants, Pittsburgh, Pa., May 1, 1956. 


\title{
RESEARCH REACTORS
}

\section{Selection and Operation}

\author{
By WILLIAM M. BREAZEALE* \\ The Babcock \& Wilcox Company, Lynchburg, Virginia
}

A RESEARCH REACTOR Is one designed primarily as a source of neutrons and gamma rays. Although a great number of designs have been proposed, all but a very few of the research reactors built so far in this country fall into one of three classes, the water boiler, the open-pool, and the tank type. The principal exceptions are the large graphite piles at Oak Ridge and Brookhaven and the graphite pile. (CP-3) at Argonne. It is unlikely that any more of this type will be built.

\section{Water Boiler}

Water boiler ( 1 ) is the designation given to the small homogeneous reactors employing a light-water solution of uranyl sulphate or uranyl nitrate contained in a stainless-steel sphere or cylinder. Cooling is accomplished by immersing stainless-steel coils carrying cooling water in the solution. The reactor operates at atmospheric pressure. The water is dissociated rather rapidly, about $2 / 3 \mathrm{ft}^{3}$ of $\mathrm{H}_{2}$ and $\mathrm{O}_{2}$ (STP) being formed per kw-hr. This gas evolution limits the operating power to $\sim 50 \mathrm{kw}$.

The chief advantages of this design are its low cost, small critical mass, and large negative temperature coefficient. The fact that the moderator and fuel are intimately mixed insures that there is no time lag in moderator expansion with increase in fuel temperature. The AEC has revealed plans for powertransient tests (2) on homogeneous reactors but no data from these tests has yet been published.

The chief disadvantages of the design are the problems associated with handling intensely radioactive and corro-

* On leave from Pennsylvania State University. sive liquids, the fact that the power level is limited to a moderate value, the danger of precipitation of the fuel in the form of insoluble uranium peroxide if the solution temperature is not carefully watched, and the necessity for recombining the $\mathrm{H}_{2}$ and $\mathrm{O}_{2}$ and storing the radioactive fission product gases until they decay sufficiently to permit discharge into the atmosphere. In the North Carolina State College design a virtue has been made of the last drawback by employing the chamber containing the radioactive gases as a neutron-free gamma-irradiation facility.

\section{Open Pool}

Open-pool reactor $(3)$ in its simplest form is an heterogeneous assembly of MTR-type fuel elements suspended in a pool of light water. The pool water serves as a coolant, moderator and shield. If power levels are restricted to $200 \mathrm{kw}$, convection cooling is satisfactory; above this level forced circulation is indicated. The upper level of open-pool operation is $2-5 \mathrm{Mw}$. Above this range the pool becomes so deep that handling of the fuel elements and samples becomes somewhat awkward. Also at these levels certain second-order effects begin to be noticeable. Chief of these is the $\mathrm{Na}^{24}$ activity. This activity arises from the production of the $\mathrm{Na}^{24}$ recoils in the aluminum by the fast-neutron reaction $\mathrm{Al}^{27}(n, \alpha) \quad \mathrm{Na}^{24}$ and by activation of the residual $\mathrm{Na}^{23}$ in the water. It becomes a serious problem as the operating power level approaches five megawatts. Considerable care is also required in returning the cooling water to the pool to insure that the $\mathrm{N}^{16}$ activity (7-sec half-life) has decayed sufficiently before it diffuses to the surface.
The advantages of the open-pool design include low cost, flexibility, and the fact that the fission products are retained in the fuel elements eliminating the necessity for radioactive gas handling and recombining equipment. The penalty paid for the extreme flexibility takes the form of a larger critical mass. A working loading will be two or three times that of the water boiler. As far as ultimate safety is concerned, experiments (4) have indicated that if the reactor is left on a rising period, corresponding to $2 \%$ or less in excess reactivity, the moderator will simply boil and limit the power rise to a level or 1 or $2 \mathrm{Mw}$. Further investigation of the transient response of heterogeneous lattices is now being carried out under AEC sponsorship, and it is possible that the results will modify the $2 \%$ figure. [See p. R5, this issue.]

\section{Tank Type}

Tank-type reactors $(5,6)$ are required when power level exceeds a few megawatts. They have a closed cooling circuit and aluminum-uranium-alloy fuel plates. The entire primary cooling circuit is shielded. Because there is no free surface it is now feasible to use heavy water as coolant and moderator. Power levels for the tank type vary from $1 \mathrm{Mw}$ for the CP-5 to $175 \mathrm{Mw}$ for the ETR now being constructed at the National Reactor Testing Station.*

The heavy-water type has a larger

* Mention should be made of the "Argonaut" a low power, tank-type research reactor which is being designed and built by the Argonne National Laboratory. This design employs graphite as reflector, light water as a moderator and coolant, and is intended for intermittent service at power levels up to $10 \mathrm{kw}$. 
lattice but a smaller critical mass, the actual ratio depending on the effectiveness of the reflectors in use. However, at a given power level the light-water type provides $5-10 \times$ greater fast-neutron flux. This is a very important consideration in the planning of radiation-damage experiments. The neutron lifetime in a heavy-water reactor is perhaps four times that in the lightwater type. Thus the period resulting from a given increase in prompt reactivity is proportionally longer and the transient correspondingly less severe. This is regarded as a safety factor. It must be added, however, that this factor is offset to a considerable degree by the fact that a given physical disturbance in a heavy-water reactor (flooding a beam hole, etc.) has a much greater effect on reactivity than in a light-water assembly:

\section{Neutron Flux}

The relation between the average flux and reactor power in a thermal reactor is given approximately by

$$
P \cong 5 \times 10^{-11} \phi_{a v} G
$$

Where $P$ is reactor power in watts, $\phi_{\Delta v}$ is average thermal flux in neutrons/ $\mathrm{cm}^{2} / \mathrm{sec}$, and $G$ is actual reactor loading in grams of U-235.

The ratio of the peak to average flux in the core depends on core geometry, reflector material, etc. but is generally 1.5-2 to 1. It must be borne in mind that in lightly loaded reactors, such as the water boiler or CP-5, insertion of an absorbing sample of appreciable size in the region of maximum thermal flux will substantially reduce this flux.

\section{Fuel Requirements}

Limitation of enrichment of export material to $20 \%$ increases the $U^{235}$ requirement relative to that needed if material enriched to $90 \%$ is available. As far as the homogeneous reactor is concerned, the increase is not great; the resonance escape probability is about 0.95 and the thermal capture cross section of $\mathrm{U}^{238}$ is only 2.5 barns. However, the increased concentration of the solution makes it even more strongly corrosive.

In the case of the heterogeneous (MTR-type) fuel elements the $U^{235}$ requirements depend strongly on the fuelelement structure. The uraniumoxide fuel elements used in the Geneva reactor contained about $50 \mathrm{wt} \%$ of $\mathrm{U}$, but this design has never been put into further production in this country. At present only Al-U-alloy fuel elements are being made and the maximum amount of $\mathrm{U}$ in fuel elements manufactured to date is about 25 wt $\%$. As a result of the weight limitation and the enrichment constraint, the present export elements can contain only $5 \mathrm{wt} \%$ of $\mathrm{U}^{235}$. The added Al to carry the $\mathrm{U}$ in the elements increases the Fermi age of the core as well as increasing parasitic capture. The extra U $U^{236}$ needed to raise the thermal utilization sufficiently to offset the greater fast leakage appreciably increases the critical mass.

\begin{tabular}{|c|c|c|c|c|c|c|}
\hline Designation & $\begin{array}{c}\text { Power } \\
(\mathrm{kw})\end{array}$ & $\begin{array}{l}\text { Average } \\
\text { thermal flux } \\
\left(\mathrm{n} / \mathrm{cm}^{2} / \mathrm{sec}\right)\end{array}$ & Moderator & Reflector & $\begin{array}{l}\text { Critical } \\
\text { mass } \\
\text { (kg) }\end{array}$ & $\begin{array}{c}\text { Total } \\
\text { requirement" } \\
\text { (kg) }\end{array}$ \\
\hline Water boiler & 50 & $\sim 1.0 \times 10^{12}$ & Light water & Graphite & 1.0 & 1.5 \\
\hline $\begin{array}{l}\text { Open-pool, } \\
10 \text {-plate } \\
\text { assemblyt }\end{array}$ & 2,000 & $\sim 1.25 \times 10^{18}$ & Light water & Light water & 3.2 & 6.0 \\
\hline $\begin{array}{l}\text { Open-pool, } \\
10 \rightarrow \text { late } \\
\text { assemblyt }\end{array}$ & $2,0,00$ & $\sim 1.4 \times 10^{13}$ & Light water & Graphite & 2.8 & 5.0 \\
\hline $\begin{array}{l}\text { Tank, } \\
\text { 10-plate } \\
\text { assembly† }\end{array}$ & 1,000 & $\sim 1.3 \times 10^{13}$ & Heavy water & Graphite & 1.5 & 3.0 \\
\hline $\begin{array}{l}\text { Tank, } \\
\text { 19-plate } \\
\text { assembly† \# }\end{array}$ & 20,000 & $\sim 1.25 \times 10^{14}$ & Light water & Beryllium & 2.8 & 5.0 \\
\hline
\end{tabular}

"Total requirement is the amount of fuel necessary to fuel the reactor and to compensate for xenon and samarium poisoning, temperature variations, the presence of experimental equipment, etc., in addition to $15 \%$ burnup.

tAlloy cores of fuel plates have 40 wt $\% \mathrm{U}$.

IThese are similar to CP-5 and MTR respectively except that the CP-5 and MTR use fully enriched fuel and thus require smaller critical masses.

However, there is good reason to believe that in the near future Al-Ualloy fuel elements containing approximately 40 wt $\% \mathrm{U}\left(10 \mathrm{wt} \% \mathrm{U}^{235}\right)$ will be available for export. A lattice made up of such elements will have a critical mass only moderately greater than one employing material enriched to $90 \%$.

The number of plates per element is determined by heat-transfer requirements; elements intended for higherpower reactors require more plates, more $\mathrm{Al}$ and consequently a larger critical mass. Between $3 / 3$ and $2 / 3$ of the $\mathrm{Al}$ in a plate is found in the cladding that protects the Al-U alloy core. The following example illustrates the difference in critical masses: a light-waterreflected-and-moderated active lattice made up of 19-plate MTR-type elements has a critical mass of about 3.5 $\mathrm{kg}$, but a lattice of 10 -plate elements under similar conditions requires only $2.8 \mathrm{~kg}$ to reach critical. Both types of elements employed in making these measurements contained the same amount of fuel, $\sim 160 \mathrm{gm}$ of $\mathrm{U}^{235} \mathrm{en}$ riched to $90 \%$.

The optimum number of plates is a compromise between critical mass and primary-coolant pumping power. The film drop (the temperature differential across the thin layer of nearly motionless water next to the surface of the plate) must not be so great that local boiling takes place. Fewer plates require a higher water velocity and more pumping power. A typical design for a 5-Mw open-pool reactor shows a film drop of $125^{\circ} \mathrm{F}$ at the hottest spot, a flow rate of $3,000 \mathrm{gpm}$ and an average rise in coolant temperature of approximately $12^{\circ} \mathrm{F}$. While the optimum number of plates is different for each power level, it has been suggested that in the interest of manufacturing economy, industry standardize on a 10plate element for research reactors which will be operated at powers less than $5 \mathrm{Mw}$ and on a 19-plate element for reactors intended for $5 \mathrm{Mw}$ or more.

The fuel requirement also is determined by the materials used for the moderator and reflector. Typical values for several reactors employing fuel enriched to $20 \%$ are shown in Table 1. Because fuel plates with alloys containing as much as $40 \mathrm{wt} \% \mathrm{U}$ will probably be available for export in the visible future, values for the heterogeneous reactors are calculated on that basis. Actually there are several thousand possible permutations and combi- 
nations (7) of moderator, reflector and fuel element; it is not possible to list here more than a small fraction of these possibilities.

In the open-pool reactor fuel can be added easily in increments of a single element to compensate for burn-up or experimental equipment and the shim rods need not control more than 7 or $8 \%$ in reactivity. In the tank type on the other hand, because the cover must be removed and replaced, changing elements is a time-consuming process and sufficient fuel is loaded at one time to allow for full burnup as well as the other effects. The shim rods must now be capable of controlling 15-20\% in reactivity.

\section{Experimental Programs}

The number of experiments that can be performed with the aid of a research reactor is much too great to list in an article of this type. Detailed descriptions of a large number of physics experiments have been given by Hughes (8) and various materials investigations have been discussed by several other authors (9).

It is possible, however, to make a general division into a group that can be carried out satisfactorily with fluxes less than $10^{13} \mathrm{n} / \mathrm{cm}^{2} / \mathrm{sec}$ and those that require higher intensities. In the first group are found the so-called physics experiments. Of particular interest to the reactor designer are investigations of Fermi age in various materials, resonance-integral measurements, crosssection determinations, and flux distributions in exponential assemblies.

On the other hand, engineering investigations of radiation damage, corrosion in the presence of radiation, fuelelement burnup, and circulating-fuel loops are performed more satisfactorily with fluxes in the neighborhood of $10^{14}$ $\mathrm{n} / \mathrm{cm}^{2} / \mathrm{sec}$. Significant results often necessitate total doses $(n v t)$ in excess of $10^{20} \mathrm{n} / \mathrm{cm}^{2}$. Such a dose requires an exposure time of nearly two weeks in a flux of $10^{14} \mathrm{n} / \mathrm{cm}^{2} / \mathrm{sec}$.

The equipment to take full advantage of neutron fluxes of the order of magnitude of $10^{14} \mathrm{n} / \mathrm{cm}^{2} / \mathrm{sec}$ is generally complicated and expensive. The samples may need special cooling during irradiation and their activity is such that removal from the reactor and subsequent handling calls for extensive shielding and rather special remote-handling equipment.

Beside work of immediate signifi- cance to the development of power reactors, the research reactor can be used for a host of experiments in physics, chemistry, and biology. Programs covering fields such as neutron diffraction, activation analysis, production of radioactive isotopes, biological irradiations, food sterilization, catalytic actions, and many others can all be carried out very satisfactorily in fluxes less than $10^{13} \mathrm{n} / \mathrm{cm}^{2} / \mathrm{sec}$ or with the gammas from spent fuel elements. An alert and imaginative staff can keep a $1-\mathrm{Mw}$ research reactor busy on such programs for many years.

\section{Operating Personnel}

The personnel associated with a research reactor can be divided into two groups, (a) those directly concerned with the operation of the reactor, and, (b) those responsible for carrying out the various experiments and tests. Normally, the second group is by far the larger and since its size and composition depend on the experimental program no detailed description of this group will be attempted here.

A minimum organization for operating a $0-5-\mathrm{Mw}$ research reactor is as follows:

Supervisor (one)-In charge of the reactor and facility operation. $\mathrm{He}$ schedules the work, oversees operation, decides whether adequate safety precautions have been taken when a new experiment is introduced into the facility, sets maintenance schedules, and in general is responsible for safe operation of the facility.

Health physicist (one per shift)-Responsible for safety of personnel. $\mathrm{He}$ maintains exposure records by means of film badges and dosimeters, examines experimental equipment to insure that personnel will not be overexposed, oversees cleanup after any spill, and is available for consultation on shielding and similar matters.

Reactor operator (one per shift) - In charge of actual operation of reactor. $\mathrm{He}$ is responsible for routine startups, maintenance of proper power level, routine instrument testing to insure that the control and safety circuits are functioning properly, and immediate supervision aimed at preventing any dangerous situations from arising.

Instrument mechanic (one)-Responsible for testing and repair of safety and control circuits and other reactor mechanisms.

Auxiliarypersonnel. One secretary, janitors, guards, or night watchmen, etc. as ordinarily found in a research installation of this size.

This organization should be expanded if members of the experimental group lack experience in working with radioactive material. For example, it may be desirable for the operating group to offer irradiation service and one technician can be employed full time for this work.

Visitors pose a problem. In most cases they should not be allowed to wander unaccompanied through the facility. If the reactor is the first of its kind near a populous area the number of visitors may justify the appointment of an assistant director to cope with them.

\section{Operating Costs}

Operating costs of the reactor and cooling system are small compared with the cost of the experimental program. Again no figures are given for the latter since its magnitude is determined by the scope of the experimental work. The principal operating costs for a $5-\mathrm{Mw}$ research reactor are as follows:

Fuel burnup. At $\$ 25 / \mathrm{gm}$ of $\mathrm{U}^{235}$ the burnup cost will be $\$ 31.25 / \mathrm{Mw}$-day. (Approximately $1.25 \mathrm{gm}$ of $\mathrm{U}^{235}$ are fissioned or are converted to $\mathrm{U}^{236}$ per Mw-day of energy released.)

Fuel rent. At $4 \%$ per annum the cost of holding $5 \mathrm{~kg}$ of $\mathrm{U}^{235}$ is $\$ 5,000$ per year.

Electric power. A $5-\mathrm{Mw}$ reactor, controls and cooling system will require about $250 \mathrm{kw}$ of electric power. (The building services are in addition to this.) Thus the electric power cost will be of the order of $\$ 85 /$ day for 24 $\mathrm{hr} /$ day operation.

Process water. Pool makeup requires $2 \mathrm{gpm}$; 2-40 gpm makeup is required for the cooling tower, depending on wet bulb temperature.

Supplies and equipment. Those peculiar to reactor operation include film badges, certain electronic items, chemicals for regenerating demineralizer, etc. Experience indicates an annual cost between $\$ 5,000$ and $\$ 10,000$ for these. Ordinary supplies and services, those normal to operation of any research establishment of this size such as telephones, pick-up truck, janitor's materials, etc., cost another \$5,000$\$ 10,000$ annually.

How costs scale. As the size of the reactor increases the operating costs go 
up in proportion. One factor sometimes overlooked is the fact that the difficulty and expense of handling irrarliated samples increases rapilly with the fux density available for irradiations. Comparatively simple remote-handling equipment which is satisfactory for use in conjunction with a $1-M w$ reactor may well be grossly inadequate to handle material from a $20-\mathrm{Mw}$ facility.

\section{Safety Considerations}

Discussions of reactor safety generally fall under three hearlings, (1) inherent self-control in the reactor, (2) reliability and effectiveness of the safety and control system, and (3) exclusion area and containment.

Self-regulation. There is little reason for building a research reactor that does not have a negative temperature coefficient. Thus all research reactors should be inherently self-limiting to some degree. The negative coefficient of reactivity is due largely to expansion of the moderator although expansion of the fuel element and increase in the mean thermal neutron temperature (determined by the temperature of the moderator) contribute to the effect. The self-limiting is of greatest importance in protecting the reactor against unforeseen actions that will cause sudden positive excursions of reactivity, and hence the time constant of the moderator is as important as its magnitude.

The homogeneous reactor has an ideal response in that the moderator temperature follows the fuel temperature practically instantaneously and there is no time lag in the reduction in reactivity. The danger in the homogeneous reactor appears to be the possibility that the containing vessel may rupture after sudden applications of positive reactivity.

On the other hand, the formation of steam between the plates of the heterogeneous reactor is delayed until the heat released in the plates is conducted into the water. After a large step increase in reactivity, the high temperature gradients necessary for a high conduction rate may allow the plates to reach the melting point before sufficient steam is formed to shut down the reactor. In the case of MTR elements immersed in light-water moderator (4) the step change required to cause melting appears to be of the order of 3 $4 \%$ in $\delta k / k$.
Scramming controls. The safety circuits operate to "scram" the reactor if the neutron flux rises above a predetermined level or if the period becomes shorter than is considered safe. The period protection usually extends four or five decarles below full power. Reliability is obtained by careful choice of components and circuits and by duplication of equipment. In addition interlocks can be provided to scram the reactor if a potentially dangerous condition should develop in the experimental equipment.

Exclusion and containment. It is conceivable that both the self-limiting features and the safety circuits might fail to protect the reactor against a positive reactivity excursion and that subsequent melting of the fuel elements would release radioactive fission products into the reactor building. To protect the general public against this eventuality, the reactor is surrounded by an exclusion area or the reactor building is made sufficiently gas tight to contain the radioactive products.

C. R. McCullough has summarized the problem of guarding against reactor accidents and the general philosophy with which the U.S. Advisory Committee on Reactor Safeguards investigates a proposed reactor installation (10). Several years ago this committee suggested the empirical relation.

$$
R=0.01 \sqrt{P}
$$

where $R$ is the radius of the exclusion area in miles and $P$ is the reactor operating power level in kilowatts. This relation postulates little containment and is really applicable only to highpower reactors. The exclusion distance determined supposedly provided sufficient time to warn and evacuate personnel downwind when the wind was light and, if it was strong, allowed time for the cloud to diffuse so that the total exposure was not too great as the radioactive cloud was blown over a person at the edge of the exclusion area. Today more detailed calculations are desired and the rule-of-thumb given above is useful only as a first approximation to be employed during the initial examination of possible reactor sites. It must be recognized that under some extreme meteorological conditions a person at the edge of an exclusion area thus determined can receive a severe overdose of gamma radiation.
More accurate parameters in terms of various atmospheric conditions have been published (11) and permissible ingestion and inhalation rates set up. The three most toxic radioactive isotopes are $\mathrm{U}^{233}, \mathrm{Pu}^{239}$, and $\mathrm{Sr}^{90}$ for which the tolerance doses* are $1,690,32$ and 1.3 micromicrograms/ cubic meter respectively. The fatal dose* is some 10,000 times this in each case. If the installation is safe from the viewpoint of these three substances, the concentrations of all other radioactive isotopes will be well within permissible limits. An exception would be the hypothetical case where these three isotopes were continuously removed and all others allowed to remain in the reactor:

Frequently a combination of exclusion area and builling containment is the least expensive solution to the problem. The building must be sufficiently strong to withstand the effects of the largest reactor power excursion that can be reasonably postulated and sufficiently gas tight so that personnel outside the exclusion area will not receive an excessive dose before they can be evacuated. The provision for some exclusion area relaxes the leakage requirements for the building and may result in appreciable cost saving.

\section{BIBLIOGRAPHY}

1. L. D. P. King in "International Conference on Peaceful L'ses of Atomic Energy." vol. 2, p. 372 (Ĺnited Nations. New lork, 1955)

2. R. H. Graham, D. G. Boyer. Reactor safety experiments, Nucleonics 14, No. 3, 45 (1956)

9. W. M. Breazeale. The swimming pool-a low cost research reactor, Nocheonica 10, No. 11, 56 (1952)

4. J. R. Dietrich in "International Conference on Peaceful Uses of Atomic Energy," vol. 13, p. 88 (United Nations, New York, 1955): W. E. Nyer, et al. Transient experiments with the SPERT-1 reactor, NoCLEONICs 14, No. 6, 44 (1956)

b. A. M. Weinberg et al. in "International Conference on Peaceful Uses of Atomic Energy," vol. 2, p. 402 (United Nations. New York 1955)

6. W. H. Zinn. ANL reactors, in "International Conference on Peaceful Uses of Atomic Energy" vol. 2, p. 456 (United Nations, New York, 1955)

7. ORNL merno C F-55-8-201

8. D. J. Hughes, "Pile Neutron Research," (Addison-Wesley Publishing Co., Boston, 1953)

9. C. D. Bopp, O. Sisman. How radiation changes polymer mechanical properties. NoCLEONics 13, No. $10(1955)$ and references at the end of this paper

10. C. R, McCullough, Mark Mills, Edward Teller in "International Conference on Peaceful Uses of Atomic Energy." vol. 13, p. 79 (United Nations, New York, 19.55)

11. J. E. Holland. Meteorology and atomic energy (Weather Bureau, U. S. Department of Commerce, Washington, D. C.. 1955)

* Tolerance dose is the maximum level that can be tolerated every day for $8 \mathrm{hr}$. equivalent to $0.043 \mathrm{rem} / \mathrm{day}$. Fatal dose is that which gives only $50 \%$ survival if the dose is acquired within an 8 -hr period. 


\section{How to Calculate}

\section{Gamma Radiation}

Induced in Reactor Materials

\section{Gamma activity induced in engineering materials,} under conditions similar to those in the ORNL graphite reactor, can be estimated with this data.

Thin specimens were irradiated in the reactor and component activities determined

\author{
By C. D. BOPP and O. SISMAN
}

Oak Ridge National Laboratory

Oak Ridge, Tennessee

Measurements were made of the gamma activity induced in a number of structural and reactor materials that were irradiated in the ORNL Graphite Reactor (1). From these data, a reasonably good estimate can be made of the activity induced in materials that are exposed under similar conditions. Table 1 has been constructed for this purpose.*

Table 1 is directly applicable to reactors of the same general class as the ORNL Graphite Reactor. For other types of reactors, one should recognize two limitations:

1. The neutron flux in the Graphite Reactor is low enough so that only for very-high-cross-section materials is depletion of the parent element signifi-

* The alternative method of estimating the activity is to calculate it from the chemical composition of the material and the neutron cross section values from the literature $(3,4)$. Except in the cases noted, activities calculated by the two methods are in good agreement. cant, except for extremely long irradiation periods.

2. Activation in the ORNL Graphite Reactor is primarily by thermal neutrons. If materials are exposed to a higher ratio of fast-to-thermal neutrons (2), $(n, p)$ and $(n, \alpha)$ reactionsoften more predominant with fast neutrons-are likely to give relatively greater contribution.

\section{Basis for Table}

The rate of change in the number of radioactive atoms per gram of material when burn-up of the parent element may be neglected, is given approximately by

$$
d n / d t=\phi \sigma N-n \lambda
$$

and the number of radioactive atoms present is

$$
n=(\phi \sigma N / \lambda)\left(1-e^{-\lambda t}\right)
$$

where $n=$ number of radioactive atoms (atoms $/ \mathrm{gm}$ ), $\phi=$ neutron flux, (neutrons $/ \mathrm{cm}^{2} / \mathrm{sec}$ ), $N=$ number of

\begin{tabular}{|c|c|c|c|}
\hline \multirow[b]{2}{*}{ a } & \multicolumn{3}{|c|}{ Component 1} \\
\hline & $t_{3,2}$ & $\begin{array}{c}\Sigma_{x}^{*} \\
\left(\mathrm{~cm}^{2} / \mathrm{gm}\right)\end{array}$ & $\begin{array}{c}E_{x} \dagger \\
(M e v)\end{array}$ \\
\hline \multicolumn{4}{|l|}{ Iron alloys } \\
\hline Armeo Iron & $2.5 h$ & $8 \times 10^{-5}$ & 1.15 \\
\hline Duriron & $2.5 \mathrm{~h}$ & $1.2 \times 10^{-3}$ & 1.15 \\
\hline 1015 & $2.5 h$ & $1.4 \times 10^{-3}$ & 1.15 \\
\hline 1030 & $2.5 \mathrm{~h}$ & $1.4 \times 10^{-3}$ & 1.15 \\
\hline 1045 & $2.5 \mathrm{~h}$ & $1.7 \times 10^{-3}$ & 1.15 \\
\hline 6150 & $2.5 \mathrm{~h}$ & $1.4 \times 10^{-3}$ & 1.15 \\
\hline \multicolumn{4}{|l|}{ Nickel alloys } \\
\hline Nickel & $2.5 \mathrm{~h}$ & $2 \times 10^{-6}$ & 1 \\
\hline Hastelloy A & $2.5 \mathrm{~h}$ & $1.0 \times 10^{-3}$ & 1.15 \\
\hline Hastelloy B & $2.5 \mathrm{~h}$ & $1.4 \times 10^{-3}$ & 1.15 \\
\hline Hastelloy C & $2.5 \mathrm{~h}$ & $3.4 \times 10^{-3}$ & 1.15 \\
\hline Hastelloy D & $2.5 \mathrm{~h}$ & $1.4 \times 10^{-3}$ & 1.15 \\
\hline Inconel & $2.5 \mathrm{~h}$ & $3.3 \times 10^{-4}$ & 1.15 \\
\hline Inconel X & $2.5 \mathrm{~h}$ & $6 \times 10^{-4}$ & 1.15 \\
\hline K Monel & $2.5 \mathrm{~h}$ & $2 \times 10^{-3}$ & 1.15 \\
\hline \multicolumn{4}{|c|}{ Stainless steels } \\
\hline Carpenter 20 & $2.5 \mathrm{~h}$ & $1.6 \times 10^{-3}$ & 1.15 \\
\hline 302 & $2.5 \mathrm{~h}$ & $1.3 \times 10^{-3}$ & 1.15 \\
\hline 303 & $2.5 \mathrm{~h}$ & $8 \times 10^{-4}$ & 1.15 \\
\hline 304 & $2.5 \mathrm{~h}$ & $8 \times 10^{-4}$ & 1.15 \\
\hline 309 & $2.5 \mathrm{~h}$ & $3 \times 10^{-3}$ & 1.15 \\
\hline 310 & $2.5 \mathrm{~h}$ & $3 \times 10^{-3}$ & 1.15 \\
\hline 316 & $2.5 \mathrm{~h}$ & $3.3 \times 10^{-3}$ & 1.15 \\
\hline 317 & $2.5 h$ & $4.2 \times 10^{-3}$ & 1.15 \\
\hline 347 & $2.5 \mathrm{~h}$ & $3 \times 10^{-3}$ & 1.15 \\
\hline 405 & $2.5 \mathrm{~h}$ & $1.0 \times 10^{-3}$ & 1.15 \\
\hline 410 & $2.5 \mathrm{~h}$ & $9.3 \times 10^{-4}$ & 1.15 \\
\hline 414 & $2.5 \mathrm{~h}$ & $9.3 \times 10^{-4}$ & 1.15 \\
\hline 430 & $2.5 \mathrm{~h}$ & $7 \times 10^{-4}$ & 1.15 \\
\hline 431 & $2.5 \mathrm{~h}$ & $1.0 \times 10^{-3}$ & 1.15 \\
\hline 446 & $2.5 \mathrm{~h}$ & $1.1 \times 10^{-3}$ & 1.15 \\
\hline 502 & $2.5 \mathrm{~h}$ & $4.3 \times 10^{-4}$ & 1.15 \\
\hline
\end{tabular}

TABLE 1-Induced Gamma

Aluminum alloys

$\begin{array}{rll}2 \mathrm{~S} & 2.5 \mathrm{~h} 2 \times 10^{-4} & 1.15 \\ 3 \mathrm{~S} & 2.5 \mathrm{~h} 1.2 \times 10^{-3} & 1.15 \\ 52 \mathrm{~S} & 2.5 \mathrm{~h} 6 \times 10^{-6} & 1.15 \\ 72 \mathrm{~S} & 2.5 \mathrm{~h}<10^{-5} & 1.15\end{array}$

Identity of the Component Activities IRON ALLOYS

The 2.5 hour component may be due both to the $\mathrm{Fe}^{66}(n, p) \mathrm{Mn}^{56}$ and the $\mathrm{Mn}^{56}$ $(n, \gamma) \mathrm{Mn}^{66}$ reactions. An upper limit on the $(n, p)$ reaction is fixed by the lowest value of this component measured for any of the iron alloys-that for Armco iron. The high values for the other iron alloys are due to their manganese content.

The 46-day component checks closely for all the iron alloys and is $50 \%$ higher than the value calculated for the $\mathrm{Fe}^{b B}(n, \gamma)$ reaction. This difference may be due to error introduced by the use of the thermal neutron cross section as an approximation for neutrons with the energy distribution that is present in the reactor. 


\begin{tabular}{|c|c|c|c|c|c|c|c|c|c|c|c|}
\hline \multicolumn{3}{|c|}{ Component $\mathcal{Z}$} & \multicolumn{3}{|c|}{ Component 8} & \multicolumn{3}{|c|}{ Component 4} & \multicolumn{3}{|c|}{ Component 5} \\
\hline$t_{38}$ & $\begin{array}{c}\Sigma_{x} \\
\left(\mathrm{~cm}^{2} / \mathrm{gm}\right)\end{array}$ & $\begin{array}{c}E_{x} \\
(\boldsymbol{M} \text { ev })\end{array}$ & $t_{1,2}$ & $\begin{array}{c}\Sigma_{x} \\
\left(\mathrm{~cm}^{2} / \mathrm{gm}\right)\end{array}$ & $\begin{array}{c}E_{x} \\
(M e v)\end{array}$ & $t_{1,2}$ & $\begin{array}{c}\Sigma_{x} \\
\left(\mathrm{~cm}^{2} / \mathrm{gm}\right)\end{array}$ & $\begin{array}{c}E_{x} \\
(M e v)\end{array}$ & $t_{1,2}$ & $\begin{array}{c}\Sigma_{x} \\
\left(\mathrm{~cm}^{2} / \mathrm{gm}\right)\end{array}$ & $\begin{array}{c}E_{x} \\
(M e v)\end{array}$ \\
\hline $45 \mathrm{~d}$ & $3.1 \times 10^{-5}$ & 1.2 & $5.3 y$ & $5.0 \times 10^{-5}$ & 1.25 & & & & & & \\
\hline $45 \mathrm{~d}$ & $2.6 \times 10^{-5}$ & 1.2 & $5.3 y$ & $6.4 \times 10^{-5}$ & 1.25 & & & & & & \\
\hline $45 \mathrm{~d}$ & $3.3 \times 10^{-5}$ & 1.2 & $5.3 y$ & $4.6 \times 10^{-5}$ & 1.25 & & & & & & \\
\hline $45 d$ & $3.3 \times 10^{-5}$ & 1.2 & $5.3 y$ & $4.6 \times 10^{-5}$ & 1.25 & & & & & & \\
\hline $45 \mathrm{~d}$ & $3.3 \times 10^{-5}$ & 1.2 & $5.3 y$ & $7.9 \times 10^{-5}$ & 1. 25 & & & & & & \\
\hline $45 \mathrm{~d}$ & $3.3 \times 10^{-5}$ & 1.2 & $5.3 y$ & $4.6 \times 10^{-5}$ & 1.25 & & & & & & \\
\hline $60 d$ & $4 \times 10^{-5}$ & 0.8 & $5.3 y$ & $8.0 \times 10^{-4}$ & 1.25 & & & & & & \\
\hline $60 \mathrm{~h}$ & $1.8 \times 10^{-4}$ & 0.3 & $60 d$ & $3 \times 10^{-5}$ & 1 & $5.3 y$ & $3.6 \times 10^{-a}$ & 1.25 & & & \\
\hline $60 \mathrm{~h}$ & $1.5 \times 10^{-4}$ & 0.3 & $60 d$ & $2.2 \times 10^{-5}$ & 1 & $5: 3 y$ & $2.7 \times 10^{-3}$ & 1.25 & & & \\
\hline $60 \mathrm{~h}$ & $6 \times 10^{-5}$ & 0.3 & $25 \mathrm{~d}$ & $8.5 \times 10^{-5}$ & 0.32 & $60 d$ & $1 \times 10^{-4}$ & 1 & $5.3 y$ & $9.5 \times 10^{-4}$ & 1.25 \\
\hline $13 \mathrm{~h}$ & $5.2 \times 10^{-4}$ & 0.51 & $60 \mathrm{~d}$ & $3.9 \times 10^{-5}$ & 1.2 & $5.3 y$ & $3.9 \times 10^{-3}$ & 1.25 & & & \\
\hline $25 \mathrm{~d}$ & $4.1 \times 10^{-6}$ & 0.32 & $60 \mathrm{~d}$ & $4.3 \times 10^{-5}$ & 1.2 & $5.3 y$ & $7.4 \times 10^{-4}$ & 1.25 & & & \\
\hline $25 \mathrm{~d}$ & $5 \times 10^{-5}$ & 0.32 & $60 d$ & $5.0 \times 10^{-5}$ & 1.2 & $115 \mathrm{~d}$ & $9.2 \times 10^{-5}$ & 1.2 & $5.3 y$ & $7 \times 10^{-4}$ & 1.25 \\
\hline $13 \mathrm{~h}$ & $2.7 \times 10^{-3}$ & 0.51 & $60 \mathrm{~d}$ & $2.7 \times 10^{-5}$ & 1.2 & $5.3 y$ & $2.1 \times 10^{-3}$ & 1.25 & & & \\
\hline $15 \mathrm{~h}$ & $2.4 \times 10^{-4}$ & 0.51 & $25 \mathrm{~d}$ & $5.5 \times 10^{-5}$ & 0.32 & $45 \mathrm{~d}$ & $3.9 \times 10^{-5}$ & 1.2 & $5.3 y$ & $1.2 \times 10^{-3}$ & 1.25 \\
\hline $25 \mathrm{~d}$ & $7.4 \times 10^{-5}$ & 0.32 & $45 \mathrm{~d}$ & $3.6 \times 10^{-5}$ & 1.2 & $5.3 y$ & $6.2 \times 10^{-4}$ & 1.25 & & & \\
\hline $25 \mathrm{~d}$ & $7.4 \times 10^{-6}$ & 0.32 & $45 d$ & $3.6 \times 10^{-5}$ & 1.2 & $5.3 y$ & $6.0 \times 10^{-4}$ & 1.25 & & & \\
\hline $25 \mathrm{~d}$ & $5.5 \times 10^{-5}$ & 0.32 & $45 d$ & $3.6 \times 10^{-5}$ & 1.2 & $5.3 y$ & $5.0 \times 10^{-4}$ & 1.25 & & & \\
\hline $25 \mathrm{~d}$ & $8.2 \times 10^{-6}$ & 0.32 & $45 d$ & $3.6 \times 10^{-5}$ & 1.2 & $120 \mathrm{~d}$ & $3.5 \times 10^{-5}$ & 1.2 & $5.3 y$ & $8.3 \times 10^{-4}$ & 1.25 \\
\hline $25 \mathrm{~d}$ & $9.8 \times 10^{-5}$ & 0.32 & $45 d$ & $3.8 \times 10^{-5}$ & 1.2 & $5.3 y$ & $1.1 \times 10^{-3}$ & 1.25 & & & \\
\hline $25 \mathrm{~d}$ & $8.5 \times 10^{-6}$ & 0.32 & $45 \mathrm{~d}$ & $3.3 \times 10^{-5}$ & 1.2 & $5.3 y$ & $7.3 \times 10^{-4}$ & 1.25 & & & \\
\hline $25 d$ & $9.6 \times 10^{-5}$ & 0.32 & $45 \mathrm{~d}$ & $3.6 \times 10^{-5}$ & 1.2 & $5.3 y$ & $9.5 \times 10^{-4}$ & 1.25 & & & \\
\hline $25 d$ & $9.6 \times 10^{-5}$ & 0.32 & $45 \mathrm{~d}$ & $2.7 \times 10^{-5}$ & 1.2 & $120 \mathrm{~d}$ & $2.9 \times 10^{-5}$ & 1.2 & $5.3 y$ & $6.1 \times 10^{-4}$ & 125 \\
\hline $25 d$ & $6.6 \times 10^{-5}$ & 0.32 & $45 \mathrm{~d}$ & $3.2 \times 10^{-5}$ & 1.2 & $5.3 y$ & $1.4 \times 10^{-4}$ & 1.25 & & & \\
\hline $25 d$ & $5.5 \times 10^{-5}$ & 0.32 & $45 \mathrm{~d}$ & $3.5 \times 10^{-5}$ & 1.2 & $5.3 y$ & $1.3 \times 10^{-4}$ & 1.25 & & & \\
\hline $25 \mathrm{~d}$ & $5.5 \times 10^{-6}$ & 0.32 & $45 \mathrm{~d}$ & $3.2 \times 10^{-5}$ & 1.2 & $5.3 y$ & $1.9 \times 10^{-4}$ & 1.25 & & & \\
\hline $25 d$ & $7.3 \times 10^{-6}$ & 0.32 & $45 \mathrm{~d}$ & $2.7 \times 10^{-5}$ & 1.2 & $5.3 y$ & $1.1 \times 10^{-4}$ & 1.25 & & & \\
\hline $25 \mathrm{~d}$ & $5.7 \times 10^{-6}$ & 0.32 & $45 \mathrm{~d}$ & $3.5 \times 10^{-5}$ & 1.2 & $5.3 y$ & $2.1 \times 10^{-4}$ & 1.25 & & & \\
\hline $25 d$ & $1.2 \times 10^{-4}$ & 0.32 & $45 d$ & $2.5 \times 10^{-5}$ & 1.2 & $5.3 y$ & $1.4 \times 10^{-4}$ & 1.25 & & & \\
\hline $25 \mathrm{~d}$ & $1.6 \times 10^{-5}$ & 0.32 & $45 \mathrm{~d}$ & $3.2 \times 10^{-5}$ & 1.2 & $5.3 y$ & $6.0 \times 10^{-5}$ & 1.25 & & & \\
\hline $15 \mathrm{~h}$ & $8.4 \times 10^{-6}$ & 2.07 & $130 \mathrm{~d}$ & $1.1 \times 10^{-7}$ & 1,2 & $5.3 y$ & $5 \times 10^{-7}$ & 1.25 & & & \\
\hline $15 \mathrm{~h}$ & $5.7 \times 10^{-6}$ & 2.07 & $45 \mathrm{~d}$ & $2.1 \times 10^{-7}$ & 1.2 & $5.3 y$ & $1.4 \times 10^{-6}$ & 1.25 & & & \\
\hline $15 \mathrm{~h}$ & $5.7 \times 10^{-6}$ & 2.07 & $120 \mathrm{~d}$ & $1.4 \times 10^{-7}$ & 1.2 & $5.3 y$ & $4 \times 10^{-7}$ & 1.25 & & & \\
\hline $15 \mathrm{~h}$ & $9.8 \times 10^{-6}$ & 2.07 & $250 \mathrm{~d}$ & $1.3 \times 10^{-5}$ & 1.12 & & & & & & \\
\hline
\end{tabular}

The activity component remaining after subtracting the shorter lived components was taken as 5.3-year $\mathrm{Co}^{80}$ and is accounted for by a cobalt content of 0.01 to $0.05 \%$ for the various alloys. In 410 and 400 stainless steels the presence of enough cobalt to account for the 5.3-year activity was confirmed by chemical analysis. This identity agrees with the average energy of the gamma radiation, about 1.0 to $1.2 \mathrm{Mev}$.

\section{NICKEL ALLOYS}

Since the 2.5-hour component was only about $10 \%$ of the total activity of the nickel sample at the time decay measurements were begun, the accuracy of resolution of this component is not better than $50 \%$. This measured activity, from $\mathrm{Ni}^{B}$ $(n, \gamma) \mathrm{Nis}$, did not check the calculated activity-the calculated value being about
3 times too large. The 2.5-hour activity for the nickel alloys is from $\mathrm{Mn}^{56}$.

The 60-70 day component is probably from the $\mathrm{Ni}^{68}(n, p) \mathrm{Co}^{68}$ reaction. After 27 hours decay time the ion chamber response for the nickel sample is about $40 \%$ from the $60-70$ day $\mathrm{Co}^{b B}$ and $60 \%$ from the 5.3-year $\mathrm{Co}^{60}$. The average value of gamma energy is $1.25 \mathrm{Mev}$ for $\mathrm{Co}^{80}$ and 0.80 $\mathrm{Mev}$ for $\mathrm{Co}^{58}$; this gives an average energy for the nickel of $0.4 \times 0.80+0.6 \times 1.25$ $=1.07 \mathrm{Mev}$, in good agreement with the value from absorption measurements, 1.1 $\pm 0.1 \mathrm{Mev}$.

Other activities are due to 26.5-day $\mathrm{Cr}^{61}$, 67-hour $\mathrm{Mo}^{98}$, and 12.8-hr $\mathrm{Cu}^{64}$. The 46-day $\mathrm{Fe}^{69}$ activity is not resolvable from the 60-70-day $\mathrm{Co}^{58}$ in the 60-day component by the present analysis, therefore the two are combined in Table 1.

\section{STAINLESS STEELS}

The 2.5-hour activity is 2.59-hour $\mathrm{Mn}^{\text {se }}$, the 46-day activity to 46 -day $\mathrm{Fe}^{59}$, and the 5.3-year activity is $\mathrm{Co}^{60}$ as discussed for the iron and nickel alloy's. The 25-day activity is due to 26.5-day $\mathrm{Cr}^{51}$. The 120-day activity is due to 113 -day $\mathrm{Ta}^{182}$ from the tantalum present in the niobium stabilizer in the 309 and 347 alloys.

\section{ALUMINUM ALLOYS}

The 2.5-hour activity is from $\mathrm{Mn}$. The 15-hour component is due to the $\mathrm{Al}^{27}$ $(n, \alpha) \mathrm{Na}^{24}$ reaction. The 250 -day $\mathrm{Zn}^{65}$ activity in $72 \mathrm{~S}$ aluminum agrees with the calculated value based on $1 \% \mathrm{Zn}$. The 45 day component in $3 \mathrm{~S}$ aluminum may be accounted for by about $0.5 \%$ iron. $5 \mathrm{ppm}$ tantalum and 1 ppm cobalt may account for the other activities. 


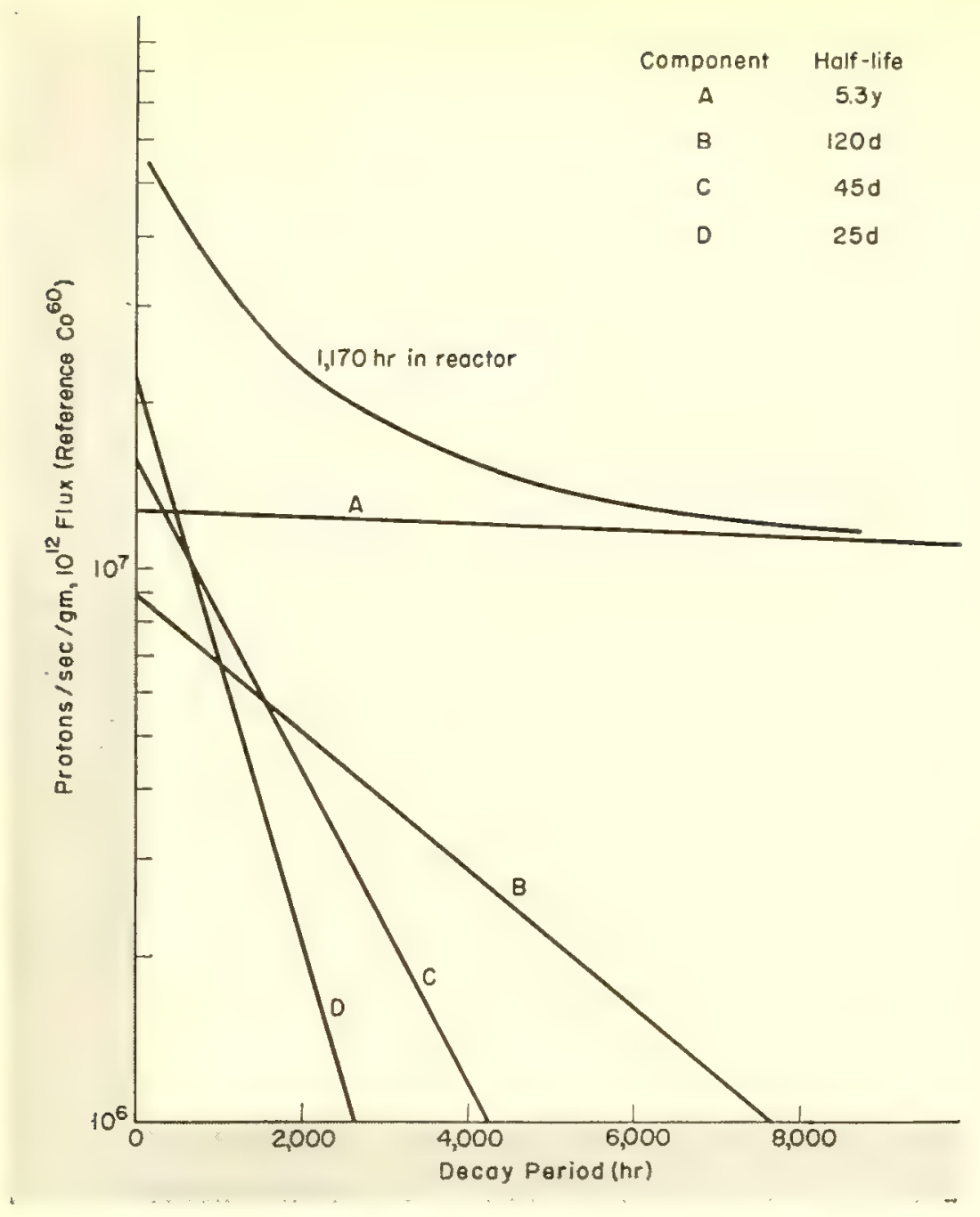

ANALYSIS of decay curve for 347 stainless steel. The 2.5 -hr component shown in Table 1 was determined by an expanded plot of the first $40 \mathrm{hr}$ decay

parent isotope atoms (atoms/gm), $\sigma=$ isotopic activation cross section $\left(\mathrm{cm}^{2}\right)$, $\lambda=0.693 / t_{32}, t_{12}=$ half-life (sec), and $t=$ irradiation time (sec).

The activity, in disintegrations per second per gram is

$$
n \lambda=\phi \sigma N\left(1-e^{-\lambda t}\right)
$$

and the specific activity $a$, in photons/ sec per gram, is

$$
a=\phi \sigma N X\left(1-e^{-\lambda t}\right)
$$

where $X=$ photons per disintegration.

It is convenient to define a macroscopic absorption cross section, $\Sigma_{x}$, such that $\Sigma_{x}=\sigma N X \mathrm{~cm}^{2} / \mathrm{gm}$ of $\mathrm{ma}$ terial. Then

$$
a=\phi \Sigma_{x}\left(1-e^{-\lambda t}\right)
$$

\section{Estimating Induced Activities}

\begin{tabular}{|c|c|c|c|}
\hline \multirow[b]{2}{*}{ Material } & \multicolumn{3}{|c|}{ Component 1} \\
\hline & $t_{32}$ & $\begin{array}{c}\Sigma_{x}^{*} \\
\left(\mathrm{~cm}^{2} / \mathrm{gm}\right)\end{array}$ & $\begin{array}{c}E_{x} \dagger \\
(M e v)\end{array}$ \\
\hline \multicolumn{4}{|l|}{ Other metals } \\
\hline Beryllium & $2.5 \mathrm{~h}$ & $7.5 \times 10^{-6}$ & 1.15 \\
\hline Cadmium & $60 \mathrm{~h}$ & $5.2 \times 10^{-4}$ & 0.40 \\
\hline Copper & $2.5 \mathrm{~h}$ & $1 \times 10^{-3}$ & 1.15 \\
\hline Lead & $2.5 \mathrm{~h}$ & $<10^{-7}$ & 1.15 \\
\hline Niobium & $115 \mathrm{~d}$ & $6.6 \times 10^{-4}$ & 1.2 \\
\hline Titanium & $2.5 \mathrm{~h}$ & $4.7 \times 10^{-5}$ & 1.15 \\
\hline Thorium & $27 \mathrm{~d}$ & $7.4 \times 10^{-3}$ & 0.3 \\
\hline Ferro-tungsten & $2.5 \mathrm{~h}$ & $1.4 \times 10^{-3}$ & 1.15 \\
\hline Zirconium & $2.5 \mathrm{~h}$ & $2.8 \times 10^{-4}$ & 1.15 \\
\hline \multicolumn{4}{|l|}{$\begin{array}{l}\text { Concrete } \\
\text { Barytes }\end{array}$} \\
\hline $\begin{array}{c}\text { Concrete } \\
\text { Brookhaven }\end{array}$ & $2.5 \mathrm{~h}$ & $9.5 \times 10^{-5}$ & 1.15 \\
\hline Cement & $2.5 \mathrm{~h}$ & $9.5 \times 10^{-4}$ & 1.15 \\
\hline $\begin{array}{l}\text { Portland } \\
\text { Concrete }\end{array}$ & $2.5 \mathrm{~h}$ & $9.5 \times 10^{-5}$ & 1.15 \\
\hline Graphite & & & \\
\hline GBF & $15 \mathrm{~h}$ & $2.7 \times 10^{-8}$ & 2.07 \\
\hline CS & $15 \mathrm{~h}$ & $3.4 \times 10^{-8}$ & 2.07 \\
\hline C-18 & $15 \mathrm{~h}$ & $4.7 \times 10^{-7}$ & 2.07 \\
\hline
\end{tabular}

Measurements were made on small
(Table 1 continued)

\section{BERYLLIUM}

From typical Be analyses it is deduced that the 80 -day component is a combination of the activities induced in $\mathrm{Zn}, \mathrm{Fe}$ and $\mathrm{Ni}$. It is difficult to resolve this portion of the curve due to the large 5.3-year component from $\mathrm{Co}^{60}$.

\section{CADMIUM}

The 60-hour component, due to 58-hour $\mathrm{Cd}^{115}$ is less than 1/2 the calculated value and is likely due to a flux depression caused by the high cross section $\mathrm{Cd}^{113}$. The 40-day component is likely 43 -day $\mathrm{Cd}^{115}$. The 250 day component is likely 250 -day $\mathrm{Zn}^{55}$.

\section{COPPER}

Since more than $95 \%$ of the photons from

cipal use for induced-activity calculations is for shielding, it is often desirable to convert the gamma flux to roentgen $/ \mathrm{hr}$. The gamma flux (photons $/ \mathrm{cm}^{2} / \mathrm{sec}$ ) is related to $\mathrm{r} / \mathrm{hr}$ by the absorption coefficient of air for the gamma radiation, the energy of the radiation, and an energy conversion factor

$$
\mathrm{r} / \mathrm{hr}=\theta E K \mu
$$
cay ensues after reactor shutdown, the activity for each component radioactive element is given by

$$
A=\phi \Sigma_{x} W\left(1-e^{-\lambda l_{1}}\right) e^{-\lambda \ell_{2}}
$$

where $A=$ photons/sec, $W=$ weight of specimen $(\mathrm{gm}), t_{1}=$ irradiation time, $t_{2}=$ decay time, and $\phi, \Sigma_{x}$, and $\lambda$ are defined as previously.

Conversion to $\mathrm{r} / \mathrm{hr}$. Since the prin- where $\theta=$ gamma flux (photons/ $\mathrm{cm}^{2}$ / sec), $E=$ energy of the photon (Mev) $K^{\prime}=$ conversion factor $=0.053\left(\mathrm{r}, \mathrm{cm}^{3}\right.$, $\mathrm{sec}) /(\mathrm{Mev}, \mathrm{hr})=1.48 \times 10^{-5} \mathrm{r}, \mathrm{cm}^{3} /$ Mev $\times 3,600 \mathrm{sec} / \mathrm{hr}$, and $\mu=$ absorption coefficient $=$ about $3.5 \times 10^{-5}$ $\mathrm{cm}^{-1}$ for photon energies from 0.5 to 2 Mev.

$E_{x}$ from Table 1 can be used for the energy in this calculation. This is an 


\begin{tabular}{|c|c|c|c|c|c|c|c|c|c|c|c|}
\hline \multicolumn{3}{|c|}{ Component 2} & \multicolumn{3}{|c|}{ Component 3} & \multicolumn{3}{|c|}{ Component 4} & \multicolumn{3}{|c|}{ Component 5} \\
\hline$t_{1 / 2}$ & $\begin{array}{c}\Sigma_{x} \\
\left(c n r^{2} / g m\right)\end{array}$ & $\begin{array}{c}E_{x} \\
(M e v)\end{array}$ & $t_{3 / 2}$ & $\begin{array}{c}\Sigma_{x} \\
\left(\mathrm{~cm}^{2} / \mathrm{gm}\right)\end{array}$ & $\begin{array}{c}E_{x} \\
(M e v)\end{array}$ & $t_{1,2}$ & $\begin{array}{c}\Sigma_{x} \\
\left(\mathrm{~cm}^{2} / \mathrm{gm}\right)\end{array}$ & $\begin{array}{c}E_{x} \\
(M e v)\end{array}$ & $t_{1,5}$ & $\begin{array}{c}\Sigma_{x} \\
\left(\mathrm{~cm}^{2} / \mathrm{gm}\right)\end{array}$ & $\begin{array}{c}E_{x_{x}} \\
(M e v)\end{array}$ \\
\hline
\end{tabular}

\begin{tabular}{|c|c|c|c|c|c|c|c|c|}
\hline $80 \mathrm{~d}$ & $1.1 \times 10^{-6}$ & 1 & $5.3 y$ & $1.7 \times 10^{-6}$ & 1.25 & & & \\
\hline $40 \mathrm{~d}$ & $2.5 \times 10^{-6}$ & 0.9 & $250 \mathrm{~d}$ & $1.6 \times 10^{-6}$ & 1.12 & & & \\
\hline $13 \mathrm{~h}$ & $7.2 \times 10^{-3}$ & 0.51 & $100 \mathrm{~d}$ & $2 \times 10^{-6}$ & 1.1 & & & \\
\hline $50 \mathrm{~h}$ & $3.5 \times 10^{-7}$ & 1.2 & $140 \mathrm{~d}$ & $5.6 \times 10^{-7}$ & 1.2 & & & \\
\hline $35 \mathrm{~h}$ & $2.3 \times 10^{-6}$ & 1.1 & $80 \mathrm{~d}$ & $1.4 \times 10^{-6}$ & 1.0 & & & \\
\hline $20 \mathrm{~h}$ & $3.2 \times 10^{-5}$ & 0.5 & $45 \mathrm{~d}$ & $3.2 \times 10^{-5}$ & 1.2 & $5.3 y$ & $2.8 \times 10^{-5}$ & 1.25 \\
\hline $20 \mathrm{~h}$ & $1.0 \times 10^{-4}$ & 0.7 & $60 \mathrm{~d}$ & $4.8 \times 10^{-4}$ & 0.74 & & & \\
\hline
\end{tabular}

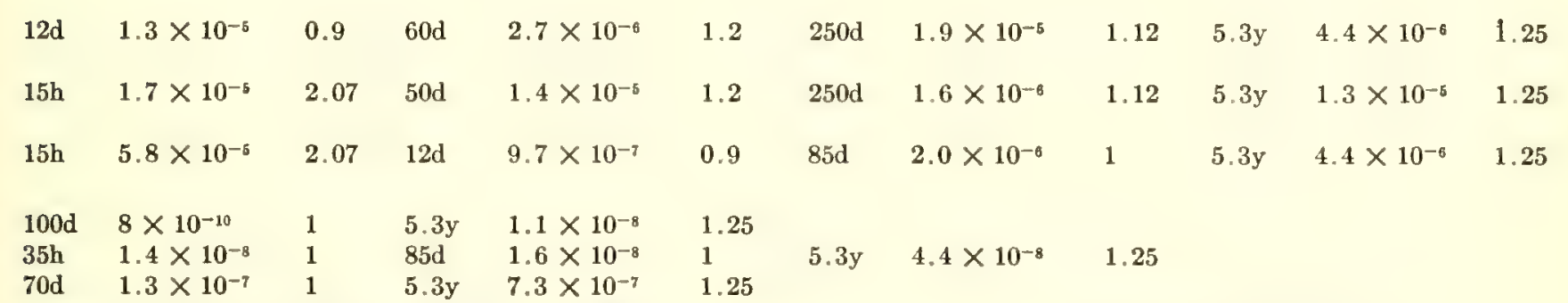

12.9-hr $\mathrm{Cu}^{84}$ result from positron annihilation, the others are neglected in the analysis and the energy taken as 0.51 Mev. The $\mathrm{Mn}^{68}$ component is quite strong in this material. The energy of the 100-day component was determined from absorption measurements.

\section{LEAD}

The induced activity is relatively low. The 2.5-hr component is likely $\mathrm{Mn}^{56}$; the 50 -hr component was not identified; the 140 day component is likely $\mathrm{Ta}^{18 \%}$.

\section{NIOBIUM}

The greater part of the activity is accounted for by a tantalum content of $1.2 \%$.

\section{IITANIUM}

Manganese likely contributes the shortlived activity. The 35-hour component may result from the reaction $\mathrm{Ti}^{48}(n, p) \mathrm{Sc}^{48}$ The 80 -day component is likely 85 -day $\mathrm{Sc}^{4}$ from the reaction $\mathrm{Ti}^{16}(n, p) \mathrm{Sc}^{16}$.

\section{THORIUM}

The greater part of the activity is 27day $\mathbf{P a}^{233}$.

\section{FERRO-TUNGSTEN}

The components are 2.5-hour $\mathrm{Mn}^{56}, 24-$ hour $\mathrm{W}^{187}, 46$-day $\mathrm{Fe}^{59}$, and 5.3-year $\mathrm{Co}^{60}$.

\section{ZIRCONIUM}

The components are 2.5-hour $\mathrm{Mn}^{68}, 17$ - hour $\mathrm{Zr}^{97}$, and 65-day $\mathrm{Zr}^{95}$.

\section{CONCRETE}

The components are 2.5-hour $\mathrm{Mn}^{56}, 14.8$ hour $\mathrm{Na}^{24}, 12.8$-day $\mathrm{Ba}^{140}, 46-\mathrm{day} \mathrm{Fe}^{69}$ (which is included in the 50-60 day component), 250-day $\mathrm{Zn}^{65}$, and 5.3-year $\mathrm{Co}^{60}$. The 85-day component was not identified. The 60-day, 140-day and 250-day components agree respectively with calculated values for $\mathrm{Fe}, \mathrm{Ba}$ and $\mathrm{Zn}$ for these materials.

\section{GRAPHITE}

The 15-hour component is likely $14.8^{-}$ hour $\mathrm{Na}^{24}$. The 35-hour and 70-100-day components are probably mixtures of several components. The 5.3-year component is likely $\mathrm{Co}^{60}$.

$* \Sigma_{x}=$ the cross section that gives the total number of photons of all energies.

$\dagger E_{x}=$ the average of the products of the energy of the photon and the number of photons of that energy that are emitted. (This figure is included for use in approximate shielding calculations.)

\section{Sample Calculation of Induced Gamma Activity}

A 5-gm thin foil of Inconel has been in the reactor for 30 days at a flux of $10^{12} \mathrm{n} / \mathrm{cm}^{2} / \mathrm{sec}$. How much gamma radiation will this specimen emit $24 \mathrm{hr}$ after reactor shutdown?

Substituting values from Table 1 in Eq. 3

Component 1 (2.5-hr half-life)

$\begin{aligned} \mathrm{A} & =\left(10^{12}\right)\left(3.3 \times 10^{-4}\right)(5)\left(1-\mathrm{e}^{-\frac{0.693 \times 30 \times 24}{2.5}}\right) \mathrm{e}^{-\frac{0.693 \times 24}{2.5}} \\ & =1.7 \times 10^{6} \text { photons } / \mathrm{sec}\end{aligned}$

Component 2 (25-day half-life)

$\mathbf{A}=\left(10^{12}\right)\left(4.1 \times 10^{-5}\right)(5)\left(1-\mathrm{e}^{-\frac{0.693 \times 30}{25}}\right) \mathrm{e}^{\frac{-0.693 \times 1}{25}}$

$=1.1 \times 10^{8}$ photons $/ \mathrm{sec}$

Conversion to $\mathrm{r} / \mathrm{hr}$

Convert component 3 of the above example to $\mathrm{r} / \mathrm{hr}$ at $10 \mathrm{~cm}$.

Here a simple configuration is assumed-a point source. The area over which the photons are distributed is $4 \pi \mathbf{r}^{2}$. The photon flux at $10 \mathrm{~cm}$ is then

Substituting in Eq. 4

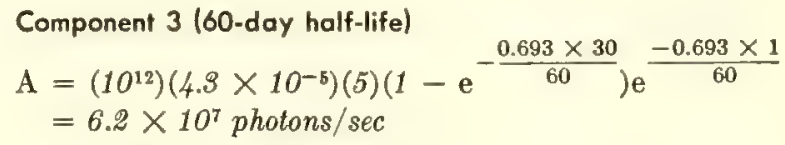

Component 4 (5.3-yr half-life)

$\begin{aligned} \mathrm{A} & =\left(10^{12}\right)\left(7.4 \times 10^{-4}\right)(5)\left(1-\mathrm{e}^{-\frac{0.693 \times 30}{5.3 \times 365}}\right) \mathrm{e}^{\frac{-0.693 \times 1}{5.3 \times 365}} \\ & =4.0 \times 10^{7} \text { photons } / \mathrm{sec}\end{aligned}$

$$
\frac{6.2 \times 10^{7}}{4 \times 3.14 \times 10^{2}}=4.9 \times 10^{4} \text { photon } / \mathrm{cm}^{2} / \mathrm{sec}
$$

$$
r / h r=\left(4.9 \times 10^{4}\right)(1.2)(0.053)\left(3.5 \times 10^{-5}\right)=0.11
$$


average energy that was determined by the decay scheme $(3,4)$ of the isotope or (if the isotope was not identified) was estimated by an absorption measurement. For greater accuracy one should, of course, make a separate calculation for each individual gamma-ray energy. In Table 2 the pertinent isotopes are listed with their photon energies and yields (4). The number of photons per disintegration is also listed.

\section{Experimental}

Specimens were prepared by cutting chips or shavings small enough to have negligible self-absorption. Irradiations were made in the ORNL Graphite Reactor for periods from 150 to $15,000 \mathrm{hr}$.

Upon removal from the reactor, the decay of gamma activity was followed with a high-pressure ionization chamber (6) (Fig. 1) until the remaining activity had a half-life greater than a year. The decay of some materials was recorded for over $20,000 \mathrm{hr}-$ most for at least $8,000 \mathrm{hr}$. Interest here is centered on the long-lived isotopes, and measurements were not begun until 3 or $4 \mathrm{hr}$ after reactor shutdown.

To assist in establishing the average energy of the radiations, absorption measurements (1) were made during the decay period by interposing sheets of lead between the specimen and a G-M tube.

Decay curves for the irradiated materials (1) were analyzed into component activities, as shown in the illustration. The identities of most of the component activities-and therefore their average energies - can be established from their half-lives and chemical analysis of the materials. Activity, $a$, was then calculated from the calibration of the ionization chamber (6). If the component was not identified, the photon energy was estimated by absorption measurements. The absorption cross section $\Sigma_{x}$ was calculated using Eq. 2. (The reactor flux was taken from reference $\%$.)

\section{BIBLIOGRAPHY}

1. C. D. Bopp, ORNL-1371 (1953)

2. C. D. Bopp, O. Sisman, ORNL-525 (1950)

s. K. Way, el al. Circular 499 (National Buresu of Standarda, U. S. Department of Commerce, Washington, D. C., 1950)

4. The Reactor Handbook, Vol, 1, Physics, AECD-3645 (1955)

5. J. S. Levin, D. J. Hughes, Nucleomics 11 No. 7,8 (1953)

6. J. W. Jones, R. T. Overman, MonC.399 (1948), AECD-2367

7. H. Jones, et al., CP-2602 (1045)
TABLE 2-Photon Yield and Energy for Isotopes of Table 1

\begin{tabular}{|c|c|c|c|c|c|}
\hline Isotope & $\begin{array}{c}\text { Half- } \\
\text { life }\end{array}$ & $\begin{array}{c}\text { Photon } \\
\text { energies } \\
\text { (Mev) }\end{array}$ & $\begin{array}{c}\text { Disintegra- } \\
\text { tions yield- } \\
\text { ing a photon } \\
(\%)\end{array}$ & $\begin{array}{c}\text { Photons } \\
\text { per } \\
\text { disintegra- } \\
\text { tion }(X)\end{array}$ & $\begin{array}{c}\text { Average } \\
\text { energy, } \\
\text { Mev } \\
\left(E_{x}\right)\end{array}$ \\
\hline $\mathrm{Na}^{24}$ & $14.8 \mathrm{~h}$ & $\begin{array}{l}1.38 \\
2.75\end{array}$ & $\begin{array}{l}100 \\
100\end{array}$ & 2.00 & 2.07 \\
\hline $\mathrm{Sc}^{46}$ & $85 \mathrm{~d}$ & $\begin{array}{l}0.88 \\
1.12\end{array}$ & $\begin{array}{l}100 \\
100\end{array}$ & 2.00 & 1.00 \\
\hline $\mathrm{Sc}^{48}$ & $44 \mathrm{~h}$ & $\begin{array}{l}1.33 \\
0.98 \\
1.00\end{array}$ & $\begin{array}{l}100 \\
100 \\
100\end{array}$ & 3.00 & 1.10 \\
\hline $\mathrm{Cr}^{\mathrm{B1}}$ & $26.5 \mathrm{~d}$ & 0.32 & 8 & 0.08 & 0.32 \\
\hline $\mathrm{Mn}^{58}$ & $2.59 h$ & $\begin{array}{l}0.84 \\
1.81 \\
2.13\end{array}$ & $\begin{array}{r}100 \\
25 \\
15\end{array}$ & 1.40 & 1.15 \\
\hline $\mathrm{Co}^{58}$ & $72 \mathrm{~d}$ & 0.81 & $\sim 100$ & $\sim 1$ & 0.8 \\
\hline $\mathrm{Fe}^{59}$ & $46 \mathrm{~d}$ & $\begin{array}{l}1.10 \\
1.30\end{array}$ & $\begin{array}{l}50 \\
50\end{array}$ & 1.00 & 1.20 \\
\hline $\mathrm{Co}^{60}$ & $5.3 y$ & $\begin{array}{l}1.17 \\
1.33\end{array}$ & $\begin{array}{l}100 \\
100\end{array}$ & 2.00 & 1.25 \\
\hline $\mathrm{Ni}^{63}$ & $2.56 \mathrm{~h}$ & $\begin{array}{l}1.01 \\
1.49\end{array}$ & $\begin{array}{r}14 \\
?\end{array}$ & 0.14 & 1 \\
\hline $\mathrm{Cu}^{84}$ & $12.9 \mathrm{~h}$ & 0.51 & 37 & 0.37 & 0.51 \\
\hline $\mathrm{Zn}^{65}$ & $250 \mathrm{~d}$ & 1.12 & 45 & 0.45 & 1.12 \\
\hline $\mathrm{Zr}^{96}-\mathrm{Nb}^{95}$ & $65 d \dagger$ & $\begin{array}{l}0.75 \\
0.72 \\
0.23\end{array}$ & $\begin{array}{r}100 \\
99 \\
1\end{array}$ & 2.00 & 0.74 \\
\hline $\mathrm{Zr}^{97}$ & $17 \mathrm{~h}$ & $\begin{array}{l}0.75 \\
0.67\end{array}$ & $\begin{array}{l}100 \\
100\end{array}$ & 2.00 & 0.71 \\
\hline $\mathrm{Mo}^{99}$ & $67 \mathrm{~h}$ & $\begin{array}{l}0.14 \\
0.18 \\
0.37 \\
0.74 \\
0.78\end{array}$ & $\begin{array}{r}10 \\
90 \\
? \\
10 \\
10\end{array}$ & 1.2 & 0.3 \\
\hline $\mathrm{Cd}^{215}$ & $58 \mathrm{~h}$ & $\begin{array}{l}0.34 \\
0.36 \\
0.52\end{array}$ & $\begin{array}{l}58 \\
42 \\
42\end{array}$ & 1.42 & 0.40 \\
\hline $\mathrm{Cd}^{115}$ & $43 d$ & $\begin{array}{l}1.30 \\
0.95 \\
0.48\end{array}$ & $\begin{array}{l}(2) ? \\
94 \\
12\end{array}$ & 1.6 & 0.9 \\
\hline $\mathrm{Ba}^{140}-\mathrm{La}^{140}$ & $12.8 \mathrm{~d} \dagger$ & $\begin{array}{l}2.9 \\
2.51 \\
1.60 \\
0.82 \\
0.52 \\
0.32 \\
0.16 \\
0.09 \\
0.03\end{array}$ & $\begin{array}{c}(6) ? \\
5 \\
95 \\
4 \\
51 \\
11 \\
70 \\
5 \\
100\end{array}$ & 2.4 & 0.9 \\
\hline $\mathrm{Ta}^{182}$ & $113 d$ & $\begin{array}{l}1.13 \\
1.22\end{array}$ & $\begin{array}{l}37 \\
57\end{array}$ & 0.94 & 1.19 \\
\hline$W^{187}$ & $24 \mathrm{~h}$ & $\begin{array}{c}0.69 \\
0.55 \\
0.48 \\
0.13 \\
0.21 \\
\dagger\end{array}$ & $\begin{array}{r}48 \\
8 \\
25 \\
8 \\
25 \\
?\end{array}$ & 1.1 & 0.5 \\
\hline $\mathrm{Pa}^{233}$ & $27 \mathrm{~d}$ & $\begin{array}{l}0.40 \\
0.08\end{array}$ & $\begin{array}{l}70 \\
30\end{array}$ & 1.00 & 0.30 \\
\hline
\end{tabular}


It has been said many times that radiation is here to stay and that man has got to learn to live with it. This usually refers to environmental radioactivity. But the same general philosophy also applies to the nuclear design specialist who needs to take radiation into account in the selection of materials and components for nuclear systems. Sometimes he's got to worry about the detrimental effects of radiation. Sometimes he's able to make use of possible beneficial effects of radiation.

Whatever the case, there's one thing that's certain-there's little data available on radiation effects and what there is is not too thoroughly understood nor available in collated form.

In this special report, the Editors of NucLoonics have assembled material that gives a picture of where we stand in our knowledge of the subject and collects data that will be useful to the nuclear designer.

\section{How Radiation Affects}

\section{Materials}

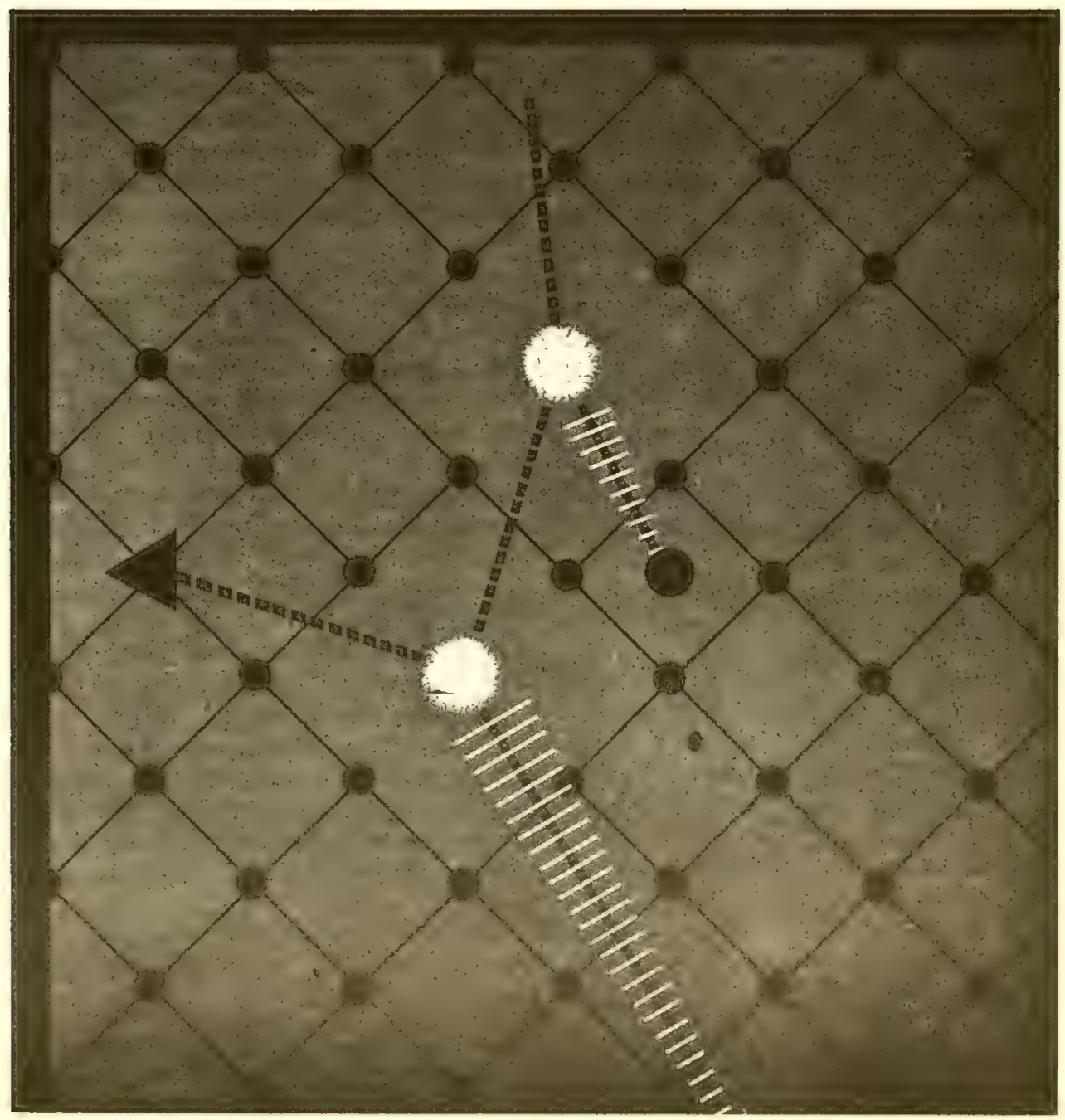




\title{
Basic Mechanisms
}

\author{
By D. S. BILLINGTON
}

Solid State Division, Oak Ridge National Laboratory, Oak Ridge, Tennessee

A NUCLEAR REACTOR can be considered, among other ways, as a copious source of high-energy radiation. This radiation can induce pronounced changes in reactor components. Under unfavorable conditions a reactor has the potential for self destruction through damage to its structural materials, even though its behavior from a nuclear standpoint is ultra safe.

\section{Why "Damage"?}

Radiation-induced changes in a solid may be deleterious from the standpoint of continued use as part of a reactor structure. This degradation has been popularly termed "radiation damage." The term "Wigner effect" is also often used, and most properly. E.P. Wigner was the first to point out the possibility of damage before it was observed experimentally in a reactor (1).

It should be noted, however, that not all radiation-induced changes in solids are harmful. Electron bombardment, for example, improves the temperature stability of polyethylene. And the increase in yield strength of a metal is not harmful, though the attendant decrease in ductility may be. In the main, damage is the principal result because materials that go into a reactor are normally in an optimal condition. Thus any change is usually deleterious.

\section{Information Needed}

The future development of nuclear reactors must depend strongly on the designers' ability to compensate for potential radiation damage. Unfortunately our state of knowledge is such that one cannot readily compile handbook-type information. There are several reasons.

First, the experimental difficulties are such that obtaining data is a slow, tedious, and potentially hazardous undertaking.

Second, the behavior of reactor materials in a radiation environment is sufficiently different from behavior in conventional environments to forbid strict interpretation in terms of the conventional environments. IVe are faced with a long period of difficult experimentation before we can accumulate sufficient data to permit an engineering "feel" for the subject. We do not have the insight or information to permit us to write specifications for materials in terms of potential radiation damage.

Third, the present availability of suitable research reactors is not sufficient to meet the demands of a comprehensive program. This situation will remain for several years.

\section{Better Flux Measurements}

A primary need is for better flux measurements. One expects damage to be influenced by the rate of introduction of defects; that is, the neutron flux. Most materials are obviously affected by the total dosage, but understanding in regard to flux is still lacking in most materials. The lack of suitable facilities has an important bearing on this point.

A qualitative theory for the radiation damage process has been developed over the past several years, that gives some insight into the mechanisms and a feel for the scope of the field. But a detailed description is not yet possible. Quantitative understanding is lacking and awaits progress in defect-solidstate knowledge. This undoubtedly will be aided by appropriate experiments in the radiation-damage field.

\section{Optimistic Outlook}

The above points, while pessimistic in tone, should not be interpreted as implying hopelessness; on the contrary the over-all situation is optimistic for most potential reactor designs. We have come a long way not only in understanding but in practical results. We have in operation a wide variety of reactors. We understand, for example, that neutron irradiation does not result in a catastrophic increase in creep rate at low fluxes as was earlier supposed.

Radiation damage is a very complex subject. In its simplest terms it is a merger of solid-state physics with nuclear physics and chemistry. Few if any of the complexities of each are left out.

\section{Breadth of Field}

The worker in the field must not only understand the object of study. He must be well informed in many aspects of reactor engineering, including shielding, production of isotopes and radioactivity, criticality studies, and flux measurements.

Problems of shielding and radiation damage are related in a complementary manner. Those who study shielding are concerned with the attenuation of nuclear radiation in its passage through solid matter. The student of radiation 


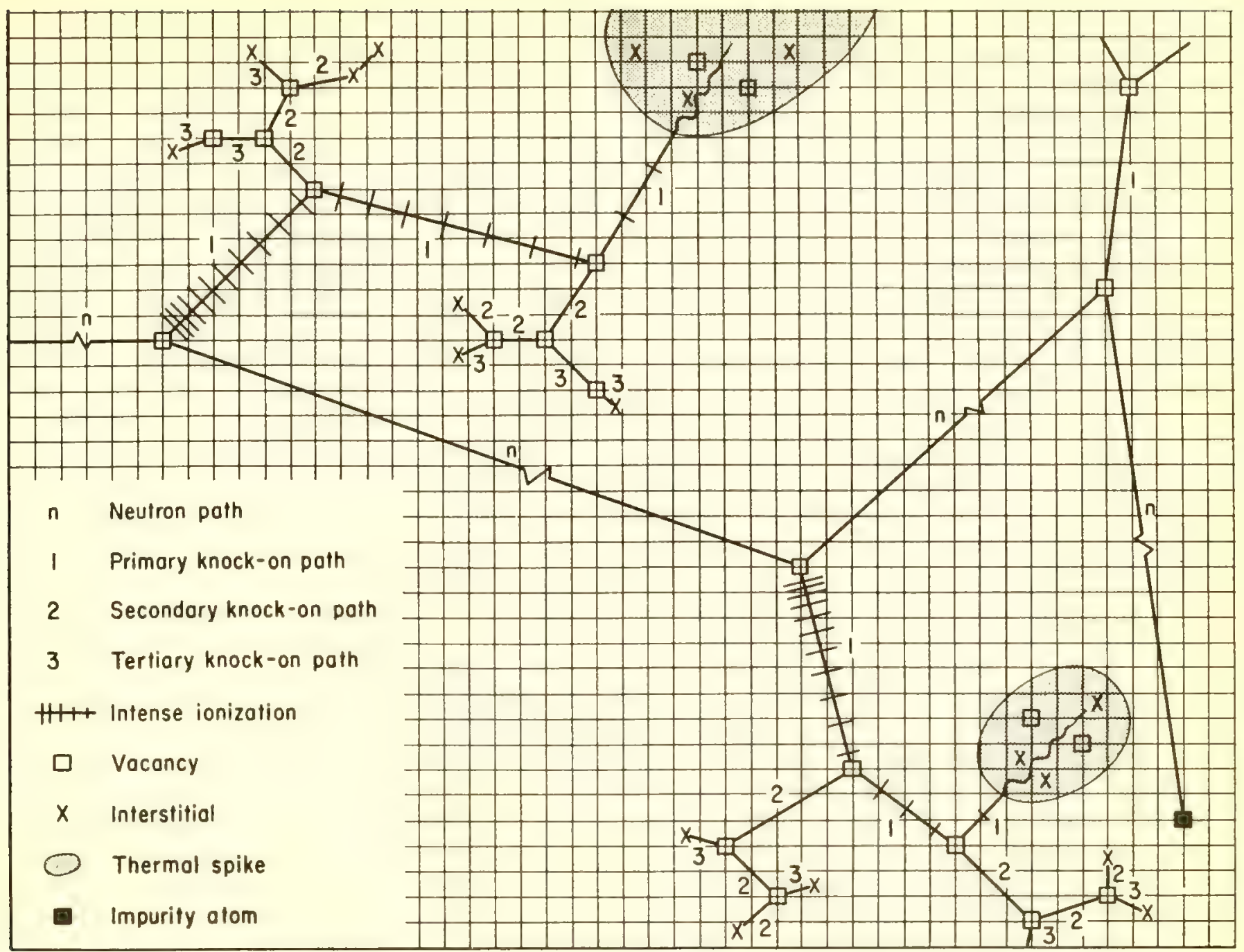

THE FIVE PRINCIPAL MECHANISMS of radiation damage are ionization, vacancies, interstitiats, impurity atoms, and thermal spikes. Here is how a neutron might give rise to each of them in coppe". Grid-line intersections are equilibrium positions for atoms

damage is concerned with what happens to the solid matter during the attenuation process. The same equations must govern many of the calculations of both workers. Practically, the attenuation process may in time render the material unsuitable for shielding purposes.

\section{DAMAGE MECHANISMS}

Radiation damage originates from the interaction of energetic radiation and matter (2). This interaction displaces atoms from their equilibrium positions and places them in nonequilibrium positions. It creates transient, local high-temperature regions. It introduces impurity atoms either through radioactive capture and decay or by inclusion of fission fragments. And it breaks chemical bonds and forms free radicals.

The results of these effects we list by the five names: vacancies, interstitials, thermal spikes, impurity atoms, and ionization effects. All will cause changes in many of the properties of matter, whether it be solid or liquid.

The first four items are of most concern to those who deal with solid reactor components. The last is of concern in liquids and gases. It is the principal topic in radiation chemistry.

\section{Estimates of Magnitudes}

Numerous theoretical estimates have been made of the number of vacancies and interstitials that are created per incident neutron and fission fragment. Sizes and durations of thermal spikes accompanying the collision process have been estimated $(3,4)$. These calculations are difficult to check experimentally. The effect per defect on properties such as electrical resistivity is not known with sufficient accuracy. Where experimental verifications have been attempted, it appears that theory leads to an overestimate of the number of defects by a factor of 2-5.

On the other hand, where the effect of radiation is to enhance solid-state reactions that are diffusion controlled, it is found that the number of defects required has been underestimated.

\section{Defect Behavior}

At the present time experiment and theory apparently agree within an order of magnitude, but closer agreement will depend in large measure on gaining additional information on the behavior of defects in general. In the case of radiation damage, it is clear that we must be dealing with a collection of defects that react with the matter and with one another in a fashion not clearly understood.

It has recently been shown that when radiation experiments are carried out at a temperature low enough to inhibit all defect motion, it is useful to consider the damaged region as resembling a frozen-in liquid $(5,6)$. Surprisingly, defect mobility has been observed at $-243^{\circ} \mathrm{C}(7,8)$. Type and extent of defect motion in a solid is a function of the type and structure of the solid containing the defects. The effect of radiation on the behavior will depend also on the latent opportunities for change 
that already exist in the solid material.

\section{Neutrons, Fission Fragments}

The nuclear particles in a reactor that cause most of the radiation damage in solids are fast neutrons and fission fragments, chiefly because the energy of each is tremendous in relation to the energy required to create a defect. The uncharged nature of the neutron means that it can interact only by direct collision. However, once a collision has taken place, the knocked-on atom in turn rapidly creates subsequent displaced atoms. The incident neutron travels many thousands of atomic spacings in the solid before it makes another collision. Thus the damage resulting from fast neutrons is widely spread through a reactor, affecting all components.

Fission fragments, possessing initially a high charge and mass must dissipate all their energy within a few microns. Therefore the damage is usually confined to the fuel volume. The total energy dissipated in radiation damage is approximately the same for both types of particles, but the spatial distribution is very different.

\section{Betas and Gammas}

Other nuclear radiations such as beta particles and gamma rays are also capable of creating damage. But in general the amount is trivial in comparison with that of fast neutrons and fission fragments. The momentum that can be transferred to a lattice atom from these radiations is small.

On the other hand, in studies of the basic nature of radiation damage, light particles are of interest because of the simpler nature of the defects that they create. It has been demonstrated in the case of electron bombardment that one can determine the energy required to create a vacancy in a solid $(9,10)$. This is a unique and useful measure- ment that is not complicated by the presence of additional defects such as thermal spikes, induced radioactivity, and impurities. These always accompany fast-neutron- or fission-fragmentinduced radiation damage.

\section{HOW DAMAGE OCCURS}

Our present knowledge of defect solid state does not allow a detailed description of how defects probably cause damage. Still we have a reasonable qualitative picture of the processes.

\section{Vacancies}

For example, it is now reasonable to assume that the intermingling of atoms in the solid state depends on vacancies and that at any given temperature there is an equilibrium number of vacancies present. The larger the number of vacancies, the more rapidly diffusion takes place. Thus creation of excessive vacancies by radiation should result in increased reaction rates at a given temperature.

Two experimental observations appear to be consistent with this viewpoint. First, a disordered $\mathrm{Cu}_{3} \mathrm{Au}$ specimen can be ordered during exposure at temperatures well below those at which ordering normally occurs (11). Second, the precipitation process in $\mathrm{Cu}-\mathrm{Be}$ alloy can be speeded up as a result of bombardment (12). This enhancement of diffusion takes place on a microscopic scale only. It has not yet been shown that appreciable changes in mass-transfer rates can be achieved under irradiation.

\section{Interstitials}

The behavior of interstitials is more difficult to understand, but the pronounced increase in yield strength that accompanies even moderate neutron irradiation of a metal leads one to believe that interstitials may be playing an important role in the hardening re-

\section{Many Things fo Many Men}

Radiation damage is somewhat unique in that it can be considered in so many different ways:

The materials engineer views the field simply as an additional environmental factor to be considered.

The metallurgist sees it as another technique for allering the properties of a metal or alloy. It belongs in a category action, though it must be acknowledged that a suitable mechanism has not yet been devised that leads to detailed understanding.

The problem of understanding the behavior of interstitials stems partly from our inability to create interstitials in close packed good metals by measures other than irradiation.

\section{Impurities}

Impurity atoms can be understood in conventional terms. Usually the number introduced is small, and the effect is small compared to other damage effects. Thus in materials suitable for reactor applications this problem usually can be neglected. In the matter of fission fragments, however, the impurity effect is of much greater magnitude. The production rate is higher, and the size and character of the fragments is such as to render them normally insoluble in the parent matrix. This leads to enhanced strain effects, and it is readily observed that a residual effect of appreciable magnitude remains after all other types of damage have been annealed. On the other hand the fuel material does not have the permanent residency of other components, so removal for reprocessing eventually corrects fission-fragment damage.

\section{Thermal Spikes}

The thermal spike presents the most controversial aspect of radiation damage. It results from that portion of the energy" that is stored in the form of displiced atoms or trapped electrons and is dissipated as heat during the collision process. The amount is comparable to that dissipated in the formation of Frenkel defects. It has been generally supposed that this energy creates a momentary region of high temperature that in a good metal may involve thousands of atoms and result in an increase in temperature of $1000^{\circ} \mathrm{K}$ for probably less than $10^{-10}$ sec. With fission fragments the number of atoms involved and the temperature increase would be correspondingly greater.

A number of low-temperature experiments have shown that the thermal spike may act as a source of vacancies and interstitials but does not directly cause radiation damage (13). In other words the thermal spike may create a frozen-in liquid-like region that upon subsequent warming may liberate vacancies and interstitials (14). Further- 
more, the effect may be overestimated in the case of metals. Its existence as a major effect in fission-fragment damage is probably better founded.

The thermal spike was first utilized to explain the disordering effect in $\mathrm{Cu}_{3} \mathrm{Au}$ (4). However, it has recently been shown that if one uses a replacement concept instead of a displacement concept, even the disordering data can be explained without a thermal spike (15).

The thermal-spike concept, while useful, will require more study before it will emerge as a quantitative tool in the interpretation of radiation damage.

\section{Variation with Material}

In any attempt to anticipate radiation damage under reactor operating conditions, it is difficult to make universally applicable generalizations. The type of damage and its extent depend strongly on the type of solid that is bombarded.

The damage process alters certain properties to a far greater extent than others. For example, in representative metals and alloys one observes sharp increases in the yield strength after modest radiation doses (16). Ductility often is reduced appreciably as well. On the other hand, one does not expect or observe significant changes in thermal or electrical conductivity when metals or alloys are bombarded at reactor ambient temperatures.

Nonmetals. Nonmetallic compounds exhibit variable behavior. Materials like $\mathrm{Al}_{2} \mathrm{O}_{3}, \mathrm{MgO}, \mathrm{NaCl}$ may show moderate electrical, thermal, and optical changes, but they do not show extensive density changes. They do show an increased susceptibility to fracture. Compounds such as quartz reveal large density and structural changes after moderate irradiations but are able to maintain macroscopic integrity $(17)$.

Semiconductors. Semiconductors are extremely sensitive to radiation. This is shown by the drastic change in electrical conductivity and in a change in the character of the conduction. The conduction may change from electron to hole conduction and vice versa, with short exposures. The nature of the change depends on the original state of the material (18).

Plastics, elastomers. Plastics and elastomers, too, are sensitive to nuclear radiation (19). The changes from moderate dosages range from complete disintegration and embrittlement to only discoloration. Quantitative data on threshold for damage and for ultimate failure are discussed in the following article of this report.

Thus, specification of a particular material and a specific property are required in order to make any estimation of probable damage. In addition to these aspects, environmental conditions will play a strong role.

\section{Effect of Temperature}

The temperature is most important in any consideration of the damage process in a crystal since the temperature will determine the subsequent behavior of the defects introduced by irradiation. Very low temperatures $\left(-253^{\circ} \mathrm{C}\right)$ will cause the defects to be completely immobile. Thus they are retained, and changes in properties like electrical conductivity that depend only on the presence of defects will be increased at low temperature. Thermal conductivity of nonmetals at low temperatures is greatly decreased because of the effect of defects on lattice conduction. This type of conduction is of chief importance at these temperatures in insulating materials.

If the subject of irradiation is either a metastable metallic or nonmetallic alloy, enhanced diffusion effects that result in the conversion of the material to the stable phase can be observed. The temperature must be high enough. to permit diffusion to take place. At low temperature these effects are repressed. The critical temperature for these effects is a function of the temperature dependence of diffusion in the material under consideration. In copper beryllium, precipitation can be observed during room-temperature irradiation, in nickel beryllium the metastability is preserved at room temperature but can be observed at $200^{\circ} \mathrm{C}$ and higher (20).

Irradiation of $\mathrm{ZrO}_{2}$ at room temperature results in the disappearance of the monoclinic phase (21). However, subsequent annealing at elevated temperatures will cause the material to revert to the stable configuration. Many additional examples of the importance of the temperature of irradiation are available in the literature (22).

\section{Other Influences}

Several other aspects of materials may influence behavior under irradi- ation. Particularly important are anisotropy, history, impurities, and surface conditions.

It has been repeatedly observed that materials possessing highly anisotropic characteristics such as metallic uranium and graphite will show an enhancement of the anisotropic properties under irradiation. The history of a given material may determine the susceptibility of a material to damage. Thus quenched or cold worked metals and alloys may show anomalous density and dimensional changes when irradiated. Small amounts of impurities of high cross section material such as boron and lithium may result in enhanced radiation effects because of the $(n, \alpha)$ reaction. The surface condition may influence the chemical reactivity induced by the irradiation.

\section{Implications}

The necessity for the control of the structure sensitivity of solids preparatory to use in a nuclear-reactor-radiation field is just as important as in any other usage where structure-sensitive properties play an important role.

Specifications of materials for reactor use require criteria that are not a part of conventional specifications since all pertinent property changes found under irradiation are not consistent with behavior in the absence of radiation.

\section{BIBLIOGRAPHY}

1. E. P. Wigner, J. Appl. Phys, 17, 857 (1946); Milton Burton, J. Phys, \& Colloid Chem. 81 611 (1947)

2. F. Seitz, Discussions Faraday Soc. 6, 271 (1949)

3. H, Brooks, KAPL-360 (1949)

4. S. Sigel, Phys. Rev. 75, 1823 (1949)

5. A. Seeger, Z. Physik 10a, 251 (1955)

6. T. H. Blewitt, private communication

7. H. G. Cooper, J. S. Koehler, J. W. Marx, Phys. Rev. 94, 496 (1954)

8. T, H. Blewitt, el al, Phys. Rev. 98, 1555 (1956)

9. E. E. Klontz, K. Lark-Horovitz, Phys. Rev. 86, 643 (1952)

10. J. M. Denny, Phys. Rev, 92, 531 (1953)

11. T. H. Blewitt, R. R. Coltman, Phys. Rev. 85, 384 (1952)

12. G. T. Murray, W. E. Taylor, Acla Med. 2,52 (1954)

13. J. W. Cleland, D. S. Billington, J. N, Crawford, Phys, Rev, 91, 238 (1953)

14. T. H. Blewitt, private communication

15. G. H. Kinchin, R. S. Pease, Jour. Nuclear Energy 1, 200 (1955)

16. T. H. Blewitt, R. R. Coltman, Phys, Rev. 82 , 769 (1951)

17. M. C. Wittels, Phys. Rev. 89, 656 (1953); W. Primak, L. H. Fuchs, P. Day, Phys, Rev, 92, 1064 (1953)

18. J. W. Cleland, J. H. Crawford, K. LarkHorovitz, F. W. Young, Phys, Rev. 83, 312 (1951); J. W. Cleland, J. H. Crawford, Phys. Rev. 95, 1177 (1954)

19. O. Sisman, C. D. Bopp, Nuczeonics 12, No. 10. $51(1955)$

go. R. H. Kernohan, D. S. Billington, A. B. Lewis, J.Appl. Phys. 27, 40 (1956)

21. M. C. Wittels, F. A. Sherrill, J. A ppl. Phys. 27, 643 (1956)

22. J. C. Wilson, D. S. Billington, J. Metals 8 , 665 (1956) 
Importance of Various Factors in Causing Radiation Damage*

\begin{tabular}{|c|c|c|c|c|c|c|c|c|c|}
\hline & $\begin{array}{c}\text { Gamma } \\
\text { Radiation }\end{array}$ & $\begin{array}{l}\text { Thermal } \\
\text { Neulrons }\end{array}$ & $\begin{array}{c}\text { Epithermal } \\
\text { Neutrons }\end{array}$ & $\begin{array}{c}\text { Fission } \\
\text { Fragments }\end{array}$ & $\begin{array}{l}\text { Dose } \\
\text { Rate }\end{array}$ & $\begin{array}{l}\text { Environ- } \\
\text { ment }\end{array}$ & $\begin{array}{l}\text { Temper- } \\
\text { alure }\end{array}$ & Stress & $\begin{array}{l}\text { Initial } \\
\text { State }\end{array}$ \\
\hline Metals, nonfissile & $\mathrm{L}$ & $\mathbf{L} \dagger$ & $\mathbf{H}$ & $\mathrm{H} t$ & $?$ & M? & M & $?$ & $\mathbf{M}$ \\
\hline Ceramics & L & $\mathrm{L} \dagger$ & $\mathbf{H}$ & $\mathrm{H} \ddagger$ & ? & $?$ & $\mathbf{M}$ & $?$ & M \\
\hline Plastics and elastomers & $\mathbf{H}$ & $\mathrm{L} \dagger$ & $\mathrm{H}$ & $\mathrm{L} \$$ & $\mathrm{H}$ ? & $\mathrm{H}$ & $\mathbf{H}$ & $\mathbf{H}$ & $\mathrm{H}$ \\
\hline Fuels & $\mathrm{L}$ & H & $\mathrm{H}$ & $\vec{H}$ & $?$ & $?$ & $\mathbf{H}$ & $?$ & $\mathbf{H}$ \\
\hline Control and shield elements & $\mathrm{L}$ & $\mathrm{H}$ & $\mathrm{H}$ & $\mathrm{L} \$$ & $?$ & ? & $\mathbf{M}$ & $?$ & $\mathrm{H}$ \\
\hline Electrical components* & $\mathbf{H}$ & $\mathrm{L} \dagger$ & $\mathbf{H}$ & $\mathrm{L} \S$ & H & H & $\mathbf{H}$ & $\mathbf{M}$ & M \\
\hline Liquids (except metals) & $\mathrm{H}$ & $\mathrm{L} \dagger$ & $\mathbf{H}$ & $\mathrm{L} \S$ & $\mathrm{L}$ & $\mathbf{L}$ & $\mathbf{H}$ & L & $\mathrm{L}$ \\
\hline
\end{tabular}

H, High; M, Medium; L, Low; ?, Unknown. * Permanent change only, for all except electrical components. † For low-cross-section materials. $\$$ When in contact with fuel. \$ Which are normally not in contact with fuel

\title{
Engineering Use of Damage Data
}

\author{
By O. SISMAN and J. C. WILSON \\ Solid State Division, Oak Ridge National Laboratory, Oak Ridge, Tennessee
}

At THIS EARLY DATE in the development of power reactors, interpretation of radiation-effects data is a most difficult task for the engineer who must incorporate the data into design. The problem is difficult because there is little data. Also, interpretation of the data in terms of unique conditions in reactors and in terms of unfamiliar properties bestowed on familiar materials by irradiation, must be made without the extensive background of service experience upon which evaluation of engineering data usually is based.

To compile a handbook of radiation effects for general use at this time would be misleading because the interpretation of the data, not the data itself, is the important consideration. This article points out the important variables and outlines a framework for interpretation of radiation-effects data for the engineer.

\section{Engineering Methods}

Normally the engineer makes an estimate of safe operating conditions. But to do this he must face such diffi- cult-to-answer questions as: When will a pressure vessel be subject to brittle fracture? When will a gasket leak? When will a bearing freeze? When will a pump stop pumping? To find answers he must look at certain readily accessible properties of materials: tensile strength, impact strength, viscosity, corrosion, etc.

The first step in establishing a tolerance radiation dose is then obvious. A tolerance change in some property or properties of the material must be established. The tolerable change may be quite large for one application and extremely small for another. The tolerable change in viscosity of the oil in which a sensitive instrument runs would have to be very small. The tolerable change in tensile strength of a structural member might be fairly large. The important point is that some tolerance must be established, no matter how small.

For unirradiated components there is usually available operating data from which to get the characteristics of operation and the expected lifetime of the component. There is almost no data of this type for irradiated components, so the effect of radiation on the component must be judged by the effect on the materials from which it is made. Often there is insufficient data to establish the radiation dose that is tolerable, but a limiting dose usually can be established below which the radiation effect is unimportant. For some materials an upper limit can be establishedabove which the material is completely unusable. The approximate radiation doses for a few important effects are presented in Fig. 1.

If it has been established that there is a problem-i.e., that the proposed irradiation dose is greater than that for which no damage has been observed and less than that for which there is no hope of using this material - then one can proceed to determine what can be done about it. In some cases the radiation-damage data may be good enough to establish a lifetime for the component in the radiation field. In other cases additional testing is indicated. Sometimes the particular material is 


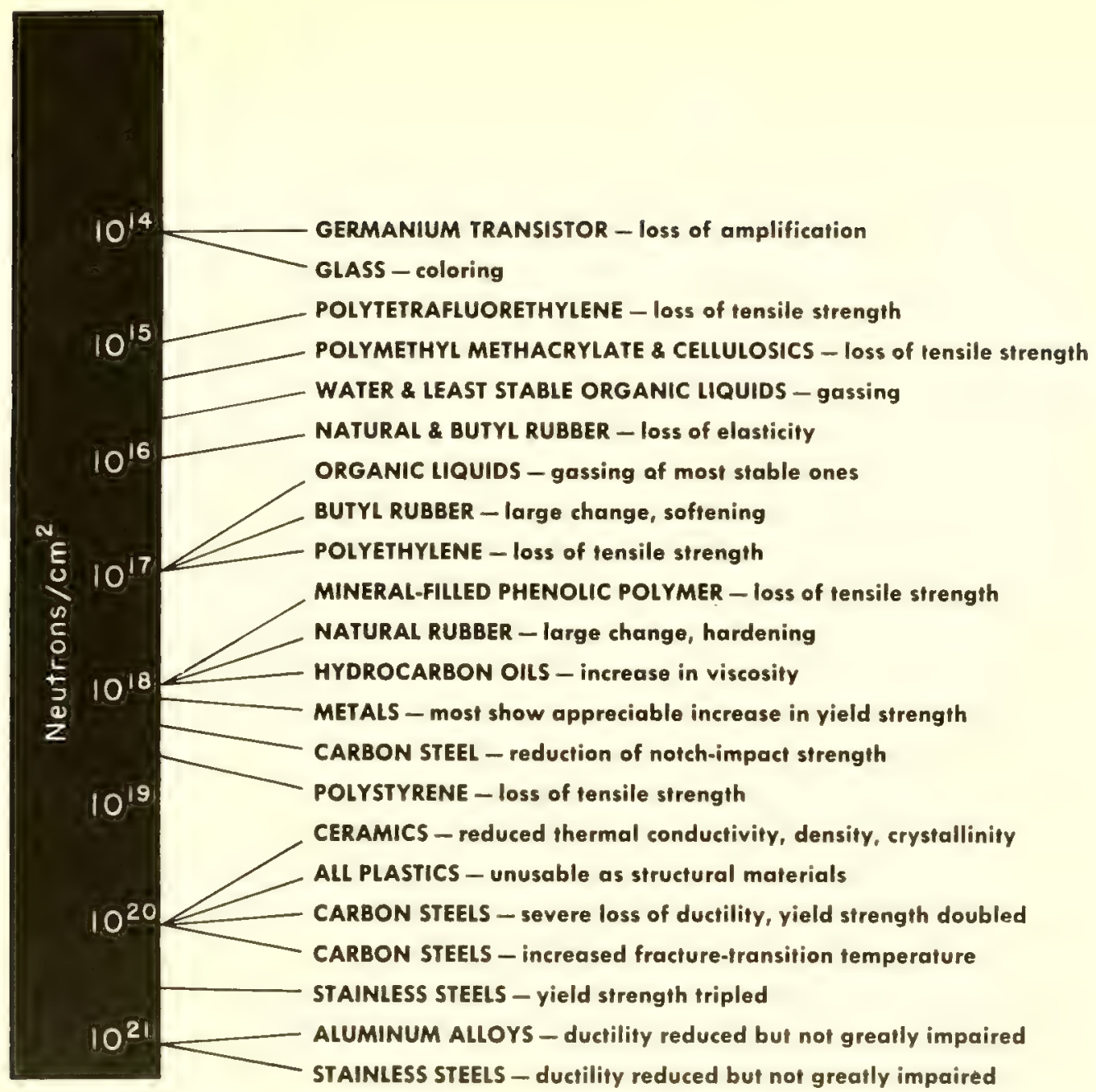

FIG. 1. Sensitivity of engineering properties to radiation. There is wide variation in effect of irradiation on various materials. Levels indicated are approximate and subject to variation. Irradiation dose is in epithermal neutrons. Indicated changes are in most cases at least $10 \%$

altogether unsuitable for a proposed use in a radiation field, and a new design is required using another material.

There are often two other alternatives: the component can be moved to a region of lower radiation intensity, or it sometimes can be shielded. In any event the problem should be carefully considered, and the more radiation-stable materials should be used where possible so as not to place an excessive burden on the designer.

\section{Structural Metals}

Structural metals usually are evaluated by strength, ductility, and impact tests. But the integrated-flux dependences of different properties are not the same. This is shown in Fig. 2. In normal structural materials the strengths (tensile and ultimate) invariably are increased with sufficient radiation at ordinary temperatures. This may not be so at higher irradiation temperatures.

The strength in creep and in fatigue have only been determined under a few conditions; these properties deserve more attention. A strength increase usually is regarded as beneficial if there is no concurrent loss in ductility. Some ASTM standards specify a maximum ultimate strength, and irradiation can cause carbon steels to exceed the maximum. In structures where some plastic flow normally is expected, increased strength.can be objectionable - it may lead to high elastic stresses that normally would be relieved by deformation or stress relaxation.

Ductility limits. Ductility (in the tension test) normally decreases after irradiation. The difficult problem is to set limits on the allowable decrease, because it is not yet possible to assess quantitatively the meaning of the ductility values. Radiation can reduce the ductility (in the tension test) below the minimum in ASTM specifications.

The loss of energy absorption in the notch-impact test and the increase in the fracture-transition temperature has been some cause for concern in irradiated steels. The minimum values specified for the notch-impact test are the result of service experience; the level of energy absorption required appears to depend on structure or heat treatment, and radiation could well act to modify the necessary, safe, minimum level requirements.

Changes in behavior. The behavior during mechanical testing of carbon and stainless steels, shown in Fig. 3 , has suggested that the response of these materials to stress is changed by radiation. For instance, stainless steels show a yield point, and the yield stress is strain-rate dependent. This has suggested that the conventional interpretation of mechanical tests may not be applicable to irradiated metals.

Another consideration in evaluating metals is that the stress, strain, and time conditions in a reactor may be different than in other structures. The fatigue properties in reactor pressure vessels may be a major factor because of the thermal-stress conditions during startup and shutdown.

\section{Plastics and Elastomers}

Since they are basically organic polymers, the changes in the physical prop- 


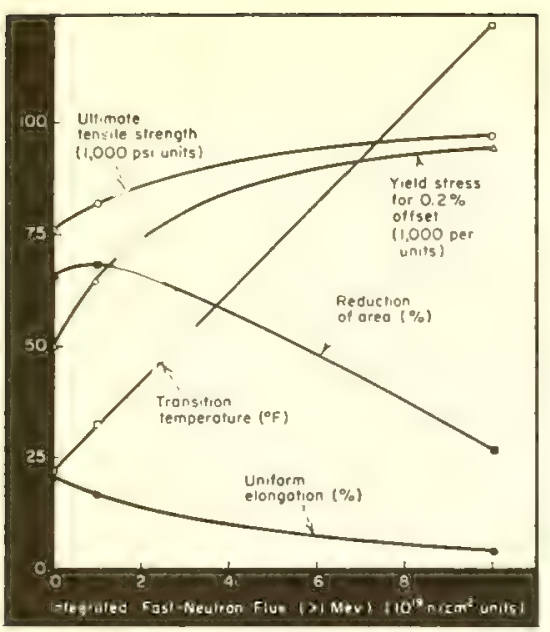

FIG. 2. Integrated-fast-neutron-flux dependence of several mechanical properties of A-212B carbon-silicon steel

erties of plastics and elastomers are reflected in the extent of two reactions of the polymer-crosslinking and cleavage. Some of the engineering properties associated with cleavage and crosslinking are summarized on page 61 .

Chemical changes. Chemically, polymers are altered quite drastically. Aside from the crosslinking and cleavage reaction, which produces most of the physical change in the material, there is usually a variety of side reactions. The production of gas is a direct result of crosslinking, but it also may come as a side reaction. Often unsaturation is produced, but double bonds may be destroyed also. Sometimes increased chemical reactivity is indicated by both increased solubility and the production of corrosive byproducts. Increased water absorption sometimes is observed. The crosslinked materials are harder to dissolve and harder to melt.

Dimensional changes. In this class of materials dimensional changes can be extremely large. In general, crosslinking causes an increase in density by reducing the specific volume of the material, and many plastics do show a large shrinkage effect. However, due to the formation of gas, there is also very often a swelling effect and a large decrease in density.

Electrical changes. Permanent electrical changes are usually not serious before mechanical failure occurs. However, while the material is in the radiation field, the electrical properties may change drastically. The change is greatest for the best insulators.

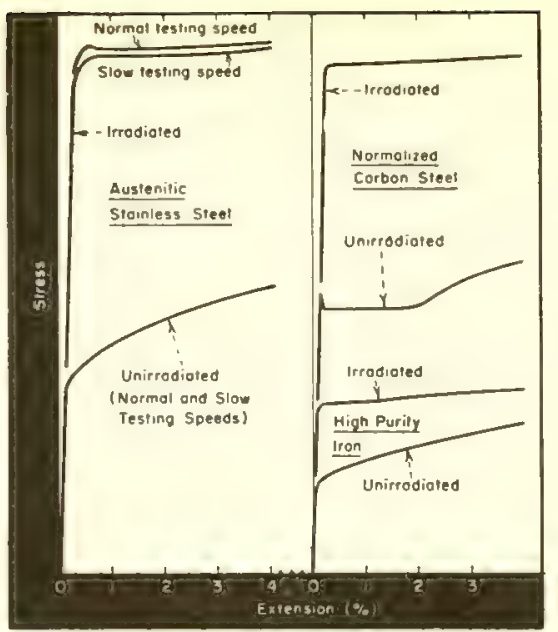

FIG. 3. Behavior of steels and iron in tensile test. Irradiation changes shape of curves and rate of work hardening

Discoloration. Many plastics discolor, and transparent materials may transmit less light. But some crystalline materials become more transparent because radiation destroys the crystallinity. The color in plastics, caused usually by decomposition products, normally cannot be removed by heat. Darkening is sometimes prevalent in the presence of oxygen when it may not occur without oxygen.

Mechanical changes. The early changes in some of the properties of plastics are often an improvement. Increased tensile strength and increased softening temperature that accompany crosslinking are desirable changes. However, some of the accompanying changes, like increased hardness and decreased elongation, may not be desirable. Even though some of the improvements in properties may occur before any serious less desirable changes, for the engineer this is usually a sign of impending radiation damage. With further irradiation the strength will decrease and embrittlement may be followed by cracking and powdering.

The elastomers that harden decrease in tensile strength to almost zero and then have a very large sudden increase in strength (Fig. 4). At this point the elastomer has been changed to a glass, and, with further irradiation, has the radiation-damage characteristics of a glass. (This rubber to glass transition can be brought about by other means too, such as lowering the temperature). For an application (a seal, for example) where a rubber need not retain its elastomeric properties, it may be considered for a much longer life in a radiation field.

\section{Ceramics}

Many materials that fall into the class of ceramics are being studied quite intensively from a fundamental standpoint, but the engineering properties are just now beginning to receive attention. For reactors there is a great deal of interest in ceramics as high-temperature materials. Here very little data is available.

As a class the ceramics are much more radiation resistant than organic materials, but will probably suffer more damage than most metals. Ceramics generally are not used where great strength is required, but not much loss in strength is seen for moderate irradiation periods. Swelling, or a decrease in density, is observed for most ceramies. But some glasses increase in density, presumably because radiation is completing the annealing processes that was not allowed to go to completion during manufacture.

Disordering, coloration. Crystalline materials are fairly readily disordered by fast-neutron bombardment, and the same materials show an early decrease in thermal conductivity. However, the thermal-conductivity change may be due at least partially to impurity atoms introduced by transmutation by thermal neutrons.

Coloration, particularly noticeable in some glasses, is caused by the formation of $F$-centers, and is readily cleared up by heat. Since much of the radiation damage in ceramics can be annealed out, it is often assumed that ceramies irradiated at high temperatures will suffer little damage. This has not yet been established experimentally.

\section{Fuels, Control Rods}

Fuels, of course, suffer heavy radiation effects because of fission. Most of the damage results from fission fragments and fast neutrons. But a large number of impurity atoms are introduced as a result of fission also. Since the fuel normally is not required to perform any structural function except holding itself together; and since, in addition, the fuel does not remain in the reactor very long, the radiationdamage requirements are not too severe. Most troubles are encountered from dimensional changes and separation of the cladding from the fuel.

Control or shield materials can be 
expected to suffer larger radiation effects than the average metal or ceramic because of the secondary particles from thermal-neutron capture. If the materials contain boron, they should be treated more as fuel elements than as structural materials. The $\mathrm{B}^{10}$ atom splits to form heavy energetic $\mathrm{Li}$ and He atoms capable of causing considerable displacement damage as do fission fragments. Two atoms result from each $\mathrm{B}^{10}$ atom split by neutron capture, and the lattice must accommodate the extra atoms.

Most of the fuels and control-rod materials are metals. Thus, most of the radiation-damage data are on metallic elements. There is an increasing interest in ceramies and liquids for this application.

\section{Semiconductors*}

Components employing semiconductors are among the most easily damaged by radiation. The amplification of a transistor, for example, is greatly reduced at a radiation level that does not affect even the least radiationstable organic material.

The effect of radiation on semiconductor components is eventually to destroy the component. The destruction of barriers in junction devices such as rectifiers and transistors usually occurs at a low integrated flux compared to the dose required to produce a comparable change in most materials. Thus the collector leakage current of a transistor and the reverse saturation current of a rectifier will change by orders of magnitude before an appreciable change can be detected in the emitter current of the transistor or forward current of the rectifier.

There are three ways in which radiation can affect the properties of a semi-

* J. C. Pigg, ORNL, personal communication.

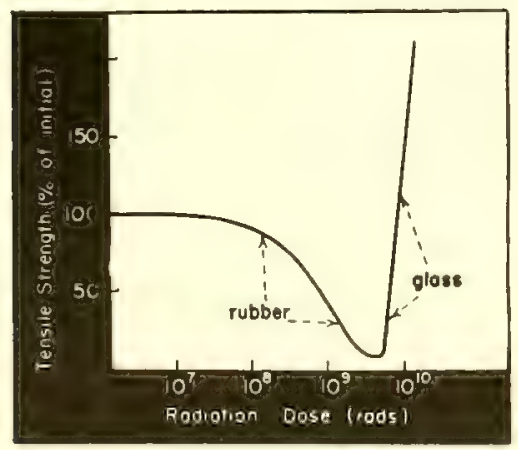

FIG. 4. Break in plot of tensile strength vs radiation dose indicates rubber-to-glass transition of natural rubber

How Organics are Changed by Radiation-Induced . . .

\section{. CROSSLINKING}

- Increases Young's modulus

- Impedes viscous flux

- Increases retardation of strain Usually cousing:

- Increased tensile strength

- Decreased elongation

- Increased hardness

- Increased softening temperature

- Decreased solubility

- Gas formation

- Fmbrittlement

- Decreased elasticity

conductor device: transmutation, photoelectric effect, and lattice disturbance. Thermal neutrons captured by the semiconductor produce excited nuclei that decay to form impurity atoms. The absorption cross sections, isotopic abundances, and decay schemes are well known for those semiconductors most widely used. Hence the effect of thermal-neutron irradiation is to introduce a predictable number of impurity atoms

Neutrons having sufficient energy to produce displaced atoms cause regions of disorder in the semiconductor crystal. This changes the carrier concentration and thus the conductivity of the material. These displacements change $n$-type germanium to $p$ type. Silicon does not change the sign of the carriers, but both $n$ and $p$ type decrease in conductivity as the number of displaced atoms increases.

\section{Liquids}

The organic liquids important to reactor technology are lubricants, conlants, hydraulic fluids, moderators, and shields. The reactions induced by radiation are much the same as for organic solids, and the stable structures are of the same general type as has been found for organic polymers. Biphenyl is the most radiation-stable organic liquid (m.p. $70^{\circ} \mathrm{C}$ ); terphenyls and substituted biphenyls are almost as good. The condensed-ring structures are also relatively radiation resistant and for some applications the alkyl benzenes have desirable properties.

Though a few liquids are decreased in viscosity, most organic liquids become more viscous by radiation-induced polymerization. Often a solid sludge is formed, and gassing is prevalent. Coking and the tendency to foam may be increased. Increased corrosion may result from some of the products.

\section{-. cleavage}

- Decreases Young's modulus

- Reduces yield stress for viscous flow Usually causing:

- Decreased tensile strength

- Increased elongation

- Decreased hardness

- Increased solubility

- Decreased elasticity Sometimes causing

- Embrittlement

- Gas formation

In many applications where liquids are used they may be circulated from an external reservoir. This dilutes the effects of irradiation and often allows the use of a liquid that otherwise might be too badly damaged; it also allows replacement of decomposed material. A difficulty with circulating systems in a neutron flux is that radioactive materials are pumped out of the reactor and require shielding. The hydrocarbon materials are very good from this standpoint because they do not become very radioactive. But corrosion products still may be bothersome.

Water or water solutions are still the primary reactor coolants and moderators and are among the most used radiation shields. The radiation-induced reactions are being studied very intensely by radiation chemists. The principal concern of the engineer is corrosion. Second is gas and sludge formation. Most water systems can stand a fair amount of radiation without serious enhancement of corrosion.

\section{Liquid Metals}

Theoretically, none of the mechanisms of radiation damage that change the properties of other materials are operable on liquid metals, and no change is expected unless some new mechanism is discovered. No radiation-induced change has ever been observed in liquid metals.

\section{Important Variables}

Engineers are only now accumulating the data needed to meet problems of nuclear design. Much of the available data are incomplete because the importance of many variables has been realized only recently. The following conditions must be carefully and completely specified for any data to be fully useful (see Table): kind and amount of radiation, rate of irradiation 
(dose rate), environment during irradiation (including temperature), and initial state of the material. Tospecify the last, one usually should know the prior history.

\section{Kind of Radiation}

The type of radiation is a very important consideration in evaluating radiation effects. In a reactor, the important classes of radiation that strike materials not in contact with the fuel are: thermal neutrons, epithermal neutrons (all energies above thermal), and gamma rays.

For metals and most ceramics, the energetic neutrons usually cause the only important changes. To adequately describe flux conditions, flux spectrum should be indicated. This is important because radiation-damage reactions occur at different neutronenergy levels - it takes neutrons of $1,000 \mathrm{ev}$ or more to displace a metal atom from its lattice to produce an interstitial and a vacancy; it takes neutrons of about $100 \mathrm{ev}$ to cause lattice expansion in a ceramic; and it takes only a few ev to break a chemical bond to cause changes in polymers.

In metals, thermal neutrons cause transmutations that may affect the properties at extreme doses-about $10^{23} \mathrm{nvt}$ for steel-depending on the elements present.

For organic materials, the gamma radiation in a reactor (especially at locations where plastics are used) may be of such an intensity as to be more important than fast neutrons. Although organics suffer from collision reactions with heavy particles, they generally suffer much larger effects from the ionization and excitation produced by the secondary particles created in the collision reaction and by the secondary electrons produced in stopping the gammas. For all but the very rigid organic solids, the change produced by radiation is proportional to the energy absorbed (regardless of source-whether it be gammas or neutrons or another kind). Thus, the radiation dose is best expressed in ergs or rads per gram of material.

Materials in contact with reactor fuel suffer greatest damage by fission fragments. Principally, they damage organics by ionization, and they damage metals by displacing atoms and by introducing impurity atoms into the lattice.

\section{Dose Rate}

If most data have been determined at radiation dose rates comparable to the dose rate for the expected service, it is reasonable to assume that the effect is proportional to the integrated dose. For large differences in dose rate an estimate of the radiation effect based on this assumption may be in error. Competing reactions may go at different rates for different levels of radiation.

Liquids. So far the dose rate appears to be unimportant for liquids. Most of the data for organic liquids is for dose rates of the order to $10^{6} \mathrm{rad} / \mathrm{hr}$ or less, and only a few special cases of inorganic liquids have received higher dose rates than this. Since the molecules in a liquid are mobile, the dose rate at which the chemical reaction rate is slower than the formation of free radicals is very high.

Solids. For organic solids, a doserate effect has been observed for a few materials, but very little data are available. One expects the dose rate effect to be more serious for the rigid materials, in which the molecules have decreased mobility, than for soft plastics and elastomers. The effect that has been observed is to increase damage per total dose for low dose rates.

Metals. For metals, no dose-rate data have been obtained. In dynamic properties such as creep or fatigue there should be a rate effect during irradiation. Dose rate may be important where several competitive processes are acting simultaneously.

\section{Environment}

There are in a reactor the normal environments considered when operating other types of machines-temperature, pressure, and surrounding fluid. Added to this is radiation.

The effect of temperature on the radiation-induced reactions is especially important in power reactors, which usually operate at high temperatures. To the engineer the most important effect of increased temperature is the annealing effect, which acts to reduce the amount of damage in some metals and ceramics. However, increased irradiation temperature does not always result in a lesser change than at lower temperatures. The ultimate strength of a carbon steel may show greater increases at higher temperatures of irradiation although the amount of increase in yield stress is reduced by the higher temperature of irradiation. Plastics and elastomers, since they are not high-temperature materials, have not been studied much at temperatures different from room temperature. Since soft and elastic polymers became hard and brittle at low temperatures, the radiation effects at low temperatures will be different from those at higher temperatures.

The effects of stress on metals during irradiation are still not known. For plastics and elastomers this effect can be very large. Elastomers will suffer "ozone damage" from radiation-produced ozone or from free radicals produced by radiation if the elastomers are stressed during irradiation. Stressed polymers generally will cleave under radiation more readily than those in the unstressed condition.

In addition to the permanent change that is caused by radiation (which is the topic of this article) there is a temporary effect on the insulation properties of materials caused by the presence of the radiation field. The resistance of a good insulator may be reduced 1,000 fold in the presence of the ionization produced by the radiation field, and an induced photo-voltage is also produced.

\section{Initial State}

The initial state of a material may have an effect on radiation-induced changes. Certain physical characteristics such as crystallinity and ordered arrangements, tend to be destroyed by radiation. In general there is a decrease in density if the substance is originally in its most compact form. On the other hand, crosslinking of polymers tends to increase the density.

In metals there seems to be a great difference in radiation effects on the mechanical properties of alloys of the same nominal composition. Whether this is a composition effect of certain elements or a function of the heattreatment and metallurgical practice is not yet known. But the fact that the susceptibility to radiation effects in similar alloys has been observed to vary by a factor of 100 (in flux) points to the importance of the initial state.

In plastics the rigidity of the material, which affects the mobility of the polymer molecule, will affect the rate of radiation damage. Unfortunately, the soft materials that are most desirable for certain applications are more easily changed than a hard material of similar chemical structure. 


\title{
On Solid Reactor Materials
}

\author{
BY J. H. KITTEL \\ Argonne National Laboratory, Lemont, Illinois
}

RAdiation HAS important effects on the engineering properties of solid materials that are used in the construction of reactors. In fuel materials only moderate irradiation produces extensive changes (1-3). Changes in nonfissionable reactor materials tend to be less drastic, particularly in the case of metals. Nevertheless reactor designers must include in their plans provisions for expected changes.

This article covers the important effects that have been found to affect the properties of moderators, structural materials, control materials, and shielding materials.

\section{Moderators}

Solid materials that are used as moderators are principally graphite, beryllium, and beryllium oxide. All of them show effects in their mechanical properties. In addition, graphite displays anisotropic dimensional changes and an increase in volume.

Graphite. The most widely used solid moderator is graphite, and changes produced in it by radiation have been studied more closely than those in any other nonfissionable material. It has been shown that the magnitude of radiation-induced changes depends to a large extent on the source of the graphite and the method of manufacture (4). The extent of radiation damage can be obtained readily by determination of the interplanar spacing, $\mathrm{C}_{0}$. Investigators are using the change in this crystal parameter of graphite powder to determine total fast flux when conducting irradiations on other materials.
Radiation produces a stronger, harder, and more brittle graphite. Compression strength and cross-breaking strength are approximately tripled after a total neutron exposure of $2.5 \times$ $10^{19} \mathrm{n} / \mathrm{cm}^{2}$ (4). These changes are not always deleterious.

More serious is the change in thermal conductivity, which may decrease as much as fiftyfold. As shown in Fig. 1, this change is markedly less when exposures are made at elevated temperatures.

Of greatest interest to reactor engineers, perhaps, are dimensional changes. Most graphites have been observed to expand along planes transverse to the axis of preferred orientation and to contract along planes parallel to this axis. In addition to this anisotropic dimensional change there is a volumetric increase that proceeds steadily with increasing exposure as shown in Fig. 2. As an example of how this knowledge affects engineering design, the MTR tank is surrounded by 1-in.-dia graphite pebbles free to move so that graphite growth can be accommodated without undue stresses on the reactor structure (5).

When graphite is irradiated in sealed containers, an additional problem may arise because of gas evolution. However, the principal gases, $\mathrm{CO}$ and $\mathrm{H}_{2}$, will be dissolved in certain container materials, such as zirconium, so that undesirably high pressures may be avoided (6). But in at least one zirconium-canned graphite reactor provision is being made to vent the gases from the cans ( 7 ).

Since all observations to date show that damage to graphite is considerably less at high temperatures than at low, one may conclude that the Sodium Reactor Experiment will produce considerably less damage in its graphite than would a similar nonpressurized water-cooled reactor operating with comparable neutron flux $(\gamma)$.

Other Moderators. Use of solid moderators other than graphite has been quite limited, but investigations have been made of radiation effects in several suitable materials. For example, beryllium, used as a reflector in the MTR, has been irradiated with fast neutron nvt's up to $1.5 \times 10^{20} \mathrm{n} / \mathrm{cm}^{2}$

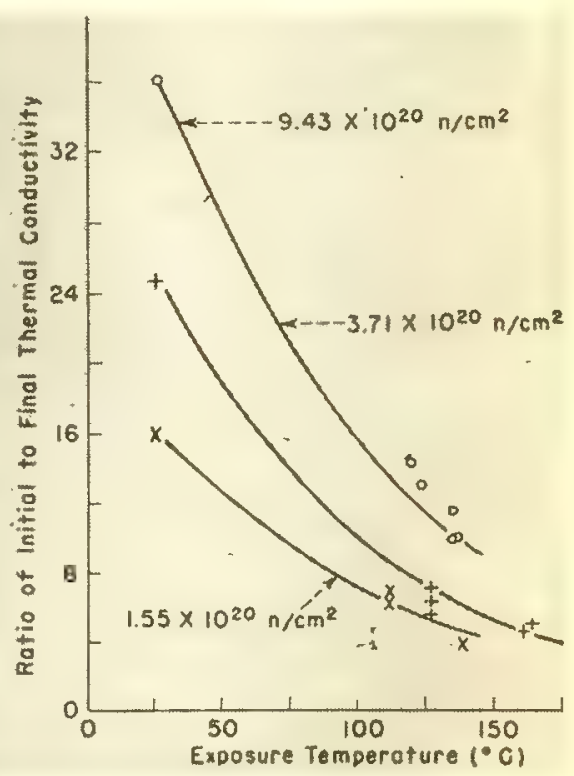

FIG. 1. Thermal conductivity of graphite decreases with irradiation, but the effect is decidedly smaller at high tem. peratures than at low (4) 


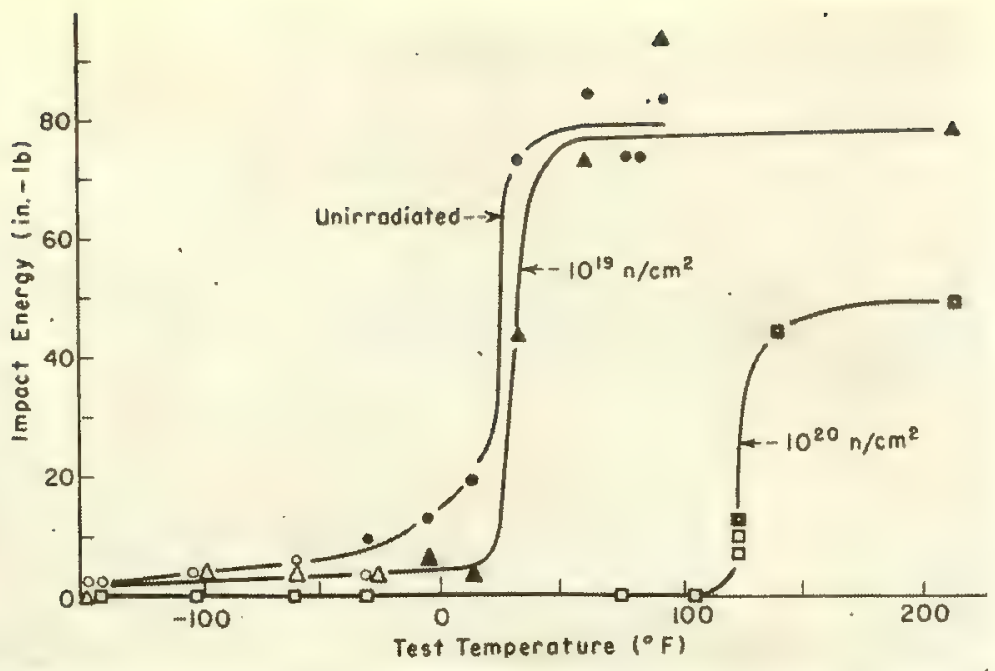

FIG. 4. Impact energy absorbed by normalized ASTM A-212 grade-B steel in breaking impact as a function of temperature. Temperature of ductile-fo-brittle frocture (break in curve) increases with increasing irradiation (15)

metal that has been studied extensively after irradiation is zirconium $(12,16)$. The metal has been studied in the annealed state and with various degrees of cold work. Experiments with arcmelted Bureau of Mines sponge zirconium and crystal-bar material show that hardness changes are greatest in annealed material and are progressively less with increasing levels of cold work. The greater changes are found in the sponge zirconium (16). Zirconium appears to recrystallize and exhibit grain growth under irradiation at temperatures not exceeding $250^{\circ} \mathrm{C}(12)$.

\section{Control Materials}

Only a limited amount of information has been published on the effects of radiation on control materials. Hafnium shows changes in its mechanical properties. Boron shows extensive damage. Cadmium shows none.

Hafnium. Hafnium, which is receiving increasing use for control rods, has been found by Westinghouse investigators to show a $9.6 \%$ increase in electrical resistivity after three months' reactor exposure, hardness increases up to $12 \mathrm{DPH}$ (diamond pyramid hardness) numbers, and density decreases up to $0.25 \%$. No significant dimensional changes are found.

Boron. Boron carbide, on the other hand, is badly damaged by radiation (17); $16.6 \%$ burnup of the $\mathrm{B}^{10}$ caused extensive cracking. $\quad \mathrm{B}_{4} \mathrm{C}$ control rods appear to have been principally used in the USSR, but no details are available on their construction (18).
Preliminary data on extruded stainless steel containing up to $5 \%$ boron irradiated to average total atom burnups up to $1.2 \%$, show no appreciable change in dimensions, although the specimens were highly embrittled (19).

Cadmium. Clad plates of silver containing $25 \%$ cadmium have been irradiated for periods up to a year. Densities and dimensions show negligible changes.

\section{Shielding Materials}

For shielding purposes investigators have studied principally concrete, iron, and boral. The effects in iron are those already discussed as occurring in carbon steels. Conflicting reports have been issued concerning concrete, and boral is relatively unaffected.

Concrete. The most widely used solid shielding material for nuclear reactors has been concrete. Effects of irradiation on this material at the exposure levels prevailing in external biological shields have not been significant (20). Some gas, mainly hydrogen, evolves under neutron irradiation. Reports differ on the effect of radiation on mechanical properties. Some experiments indicate a decrease up to $30 \%$ in crushing strength while others show an even larger increase. Several experiments have shown decreases up to $20 \%$ in the thermal conductivity.

Boral. An aluminum-boron carbide sheet material, boral, is uniquely suited for shielding where production of hard gamma rays must be avoided (21). Irradiation of this material to $0.9 \times$
$10^{20} \mathrm{n} / \mathrm{cm}^{2}$ has caused no change in its strength (22).

Attention in this article is limited to materials of greatest current interest. Untried reactor materials always should be irradiation tested. Experimenters conducting such tests should include with their specimens suitable fast-flux monitors so that the integrated fastneutron exposure can be measured and reported. One of the difficulties in comparing and evaluating present results is that many neutron exposures are reported in terms of thermal-neutron exposure, or simply by the length of exposure in a reactor.

$$
\text { * * * }
$$

This paper is based on work done under the auspices of the $A E C$.

\section{BIBLIOGRAPHY}

1. S. H. Paine, J. H. Kittel in "International Conference on the Pesceful Uses of Atomic Energy," vol. 7. P. 445 (United Nations, New York, 1956;

2. S. F. Pugh, in "International Conference on the Peaceful Uses of Atomic Energy," vol. 7 , p. 441 (United Nations, New York, 1956)

3. S. T. Konobeevsky, N. F. Pravdyuk, V. I Kutaitsev, in "International Conference on the Peaceful Uses of Atomic Energy," vol. 7. p. 433 (United Nations, New York, 1956)

4. W. K. Woods, L. P. Bupp, J. F. Fletcher, in "International Conference on the Peaceful Uses of Atomic Euergy," vol. 7. p. 455 (United Nations, New York, 1956)

5. John R. Huffman, Nocleonic8, 12, No. 4 21 (1954)

6. R. L. Carter, W. J. Greening, Evolution of gas from graphite moderator material. Preprint 319, Nuclear Engineering and Science Congress (1955)

7. W. E. Parking, in "International Conference on the Peaceful Uses of Atomic Enerky," vol. 3. p. 295 (United N\&tions, New York, 1956)

8. F. E. Faris, in "International Conference on the Peaceful Uses of Atomic Energy," vol. 7. p. 484 (United Nations, New York, 1956)

9. F. Daniels, Nucteonics 14, No. 3, 34 (1956)

10. D. S. Billington, in "International Conference on the Peaceful Uses of Atomic Energy," vol. 7, p. 421 - (United Nations, New York, 1956)

11. W. F. Murphy, S. H. Paine, Fast neutron effects on tensile and hardness properties of stainless steel. (American Society for Testing Metals-Atomic Industrial Forum Symposium on Radiation Effects on Materials. Los Angeles,-Sept. 1956)

12. S. T. Konobeevaky, N. F. Pravdyuk, V. I Kutaitsev, in "International Conference on the Peaceful Uses of Atomic Energy," vol 7, p. 479 (United Nations, New York, 1956)

13. M. B. Reynolds, J, R. Low, Jr, L. O. Sullivan, J. Meials, 7, 555 (1955)

14. C. R. Sutton, D. O. Leeser, Nucleonics 12 No. 9, 8 (1954)

15. J. C. Wilson, D. S. Billington, J. Metals, 8 665 (1956)

16. R. S. Kemper, Jr., W, S. Kelley, The effect of irradiation on the mechanical properties of arc-melted Buresu of Mines zirconium with various degrees of cold work. HW-38079 with verid

17. W. D. Valovage, Effect of irradiation on hot pressed boron carbide. KAPL-1403 (1955)

18. H. S. Isbin in "International Conference on the Peaceful Uses of Atomic Energy," vol. 3 p. 374 (1955)

19. J. D. Eichenberg, Westinghouse Atomic Power Division, personal communication (1956)

20. H, S, Dayis, Nucleonrea 13, No. 6, 60 (1955)

21. V. L. McKinney et al, Boral: A new thermal neutron shield. AECD-3625 (1954)

22. A. S. Kitzes, Oak Ridge National Laboratory, personal communication (1956) 


\title{
DAMAGING EFFECTS OF RADIATION...
}

\section{On Electronic Components}

\author{
BY R. D. SHELTON and J. G. KENNEY
}

Admiral Corporation, Chicago, Illinois

\begin{abstract}
AN EXPERIMENTAL approach to learning the effects of nuclear radiation on electrical systems can be divided into studies of materials, components, and systems. Opinions vary on the relative merits of the three approaches, but there is agreement that materials testing can be of great value in predicting the manner in which a component will fail, component testing will permit design and fabrication of a system with reasonable survival potential, and only a systems test can provide assurance that system will function.
\end{abstract}

Components testing has not been nearly as extensive as materials testing, and systems testing, because of greater complexity and lack of appropriate irradiation facilities, is practically non-

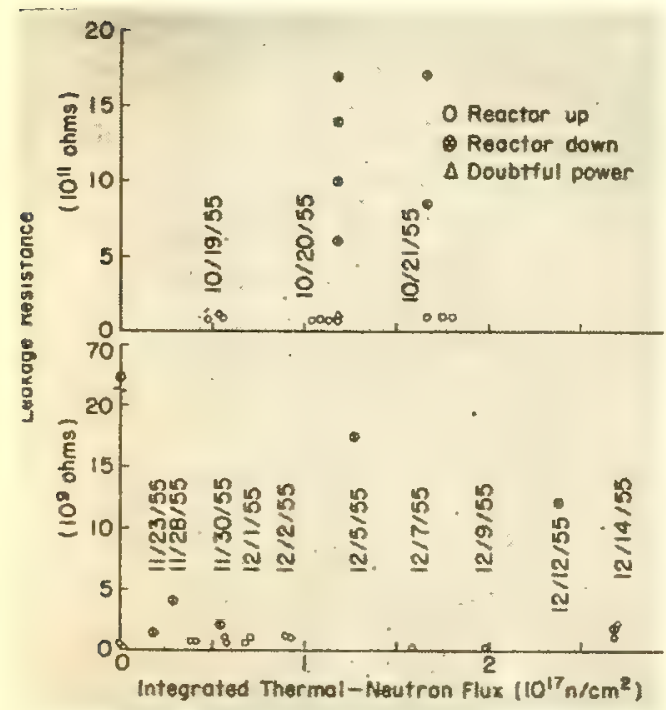

FIG. 1. Two series of observations of insulation resistance under influence of reactor radiation. Top chart shows resistance between aluminum drum and $14 \mathrm{ft}$ of wire insulated with $1 / 32$-in. polyethylene. Bottom shows similar experiment with polyvinylchloride-insulated wire. Thermal neufron flux was $6 \times 10^{11} \mathrm{n} / \mathrm{cm}^{2} / \mathrm{sec}$ existent (1-3). This article is concerned with components testing.

\section{Rate and Integral Effects}

Deleterious effects can be divided roughly into two categories. Some occur immediately when the system is placed in the radiation field and are sensitive functions of radiation flux density; these are called rate effects. Long-term degenerative effects associated with the total irradiation (the time integrals of the fluxes) are called integral effects. The damage to a given component will depend on the mode of operation during irradiation, the materials comprising the component, the spectrum of the nuclear radiation, the intensity and duration of the irradiation, and other environmental factors such as temperature and humidity.

\section{In-Pile Tests}

Under an Air Force contract, our company is investigating the effects of nuclear radiation on electronic components. From July of 1955 to July of 1956 a variety of components have been irradiated in the $\mathrm{CP}-5$ reactor of Argonne National Laboratory. Components were tested for performance before, during, and after irradiation, and fere withdrawn from the reactor after being subjected to an integrated thermal flux of $10^{18} \mathrm{n} / \mathrm{cm}^{2}$. Associated gamma and fast-neutron fluxes varied with position in the reactor, but typical values were $5 \times 10^{15}$ fast neutrons $/ \mathrm{cm}^{2}$ and $10^{18}$ gammas/ $\mathrm{cm}^{2}$.

Standard military specifications were used as the guide in determining what tests should be performed. Components to be irradiated were given preirradiation tests at the Admiral Radiation Laboratory and then transported to the reactor for in-pile testing. As a rule 18 components of a given type, but perhaps of different manufacturers, comprised a test. Six components were under active test and operating during irradiation; six were irradiated but not operated; six were not irradiated and served as a control group.

Pre-irradiation testing was conducted at room temperatures, whereas in-pile testing was conducted at $50^{\circ} \mathrm{C}$ or above, depending on the amount of cooling and on the heat dissipated by the components being tested. The reactor environment chosen for a particular test was dictated somewhat by component size and availability of reactor space. Variations of reactor power were frequent enough to demonstrate the effect of different flux levels on component behavior.

This article discusses the effects observed so far in conductors and insulators, resistors, capacitors, semiconductors, and vacuum tubes.

\section{Conductors and Insulators}

Conductors are usually metals, which are relatively resistant to radiation damage, and insulators and dielectrics are chemical compounds with far less radiation resistance. A large volume of materials research has been accomplished in the past few years and components manufacturers are becoming aware of the problems engendered by adding nuclear radiation to the environment $(4-7)$.

There are several ways in which wires and cables can be affected by nuclear radiation. The entire space in a radiation field can be thought of as an ionization chamber with charges migrating wherever there are potential differences. Beta activity produced by neutron absorption may produce "nuclear batteries" on a small scale, and 


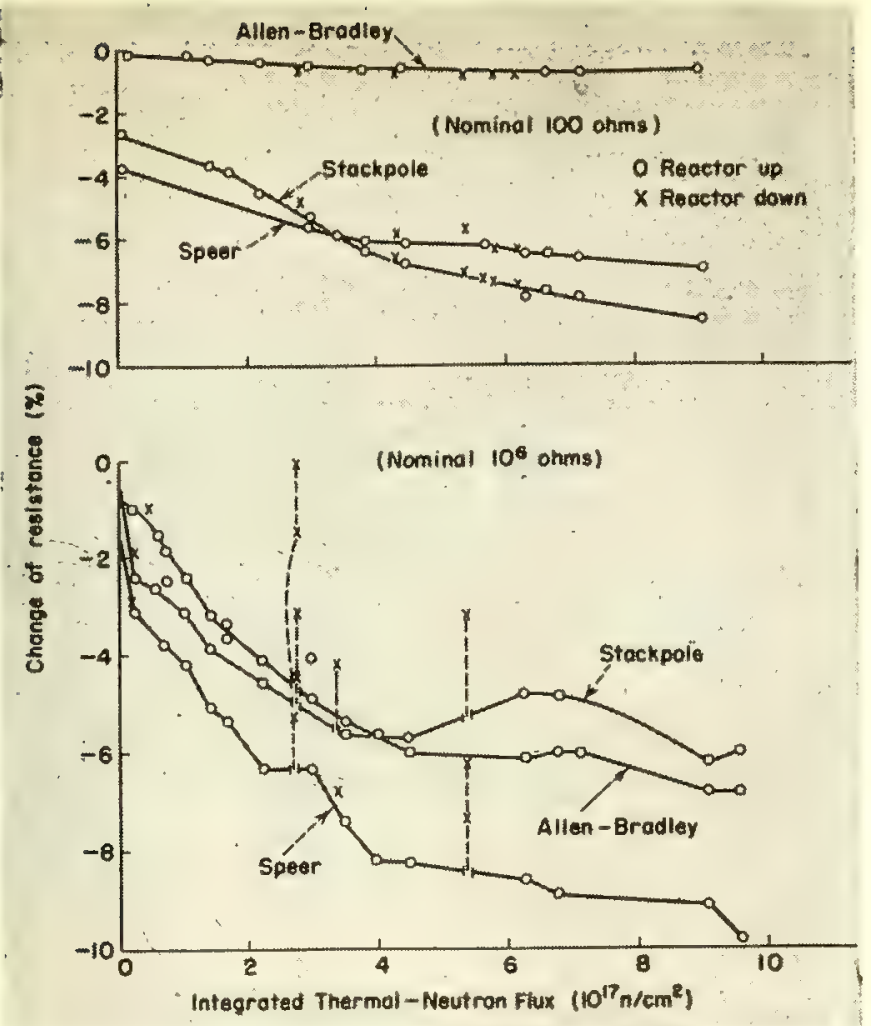

large potential gradients may be produced by charge displacement through Compton collisions $(3,8,9)$.

Figure 1 shows insulation resistance during irradiation.

\section{Resistors}

Resistors may suffer for a variety of reasons: The alternate paths afforded by ionization may reduce the effective value of large resistances subjected to high potentials. The breakdown of bonding and impregnating materials may also permit parallel conduction paths. The basic resistance material may also suffer.

Figures 2 and 3 exhibit some in-pile data on resistors. It is immediately apparent that the resistance of a component to nuclear radiation varies markedly with the manufacturer. The increase of the "rate effect" with large resistance values is expected. The initial change of resistance on insertion into the reactor results from a temperature change from 20 to $50^{\circ} \mathrm{C}$. Wire-wound power resistors showed practically no radiation damage.

\section{Capacitors}

Mica, ceramic, electrolytic, and oil capacitors were tested. Electrolytic capacitors failed in about one-tenth of the standard exposure time $\left(10^{18}\right.$ $\mathrm{n} / \mathrm{cm}^{2}$ ); oil capacitors enlarged due to gas evolution and developed leaks; and mica and ceramic capacitors showed
FIG. 2. Effect of in-pile irradiation on JAN RC-20type carbon composition resistors having nominal resistances of 100 and $10^{6}$ ohms. Recovery during reactor down time is indicated for the high-resistance elements. Irradiations were with fluxes. of 9.6 $\times 10^{12}$ thermal neutrons $/ \mathrm{cm}^{2} / \mathrm{sec}, 10^{9}$ fast neutrons/ $\mathrm{cm}^{2} / \mathrm{sec}$, and $2 \times 10^{12} \mathrm{gammas} / \mathrm{cm}^{2} / \mathrm{sec}$

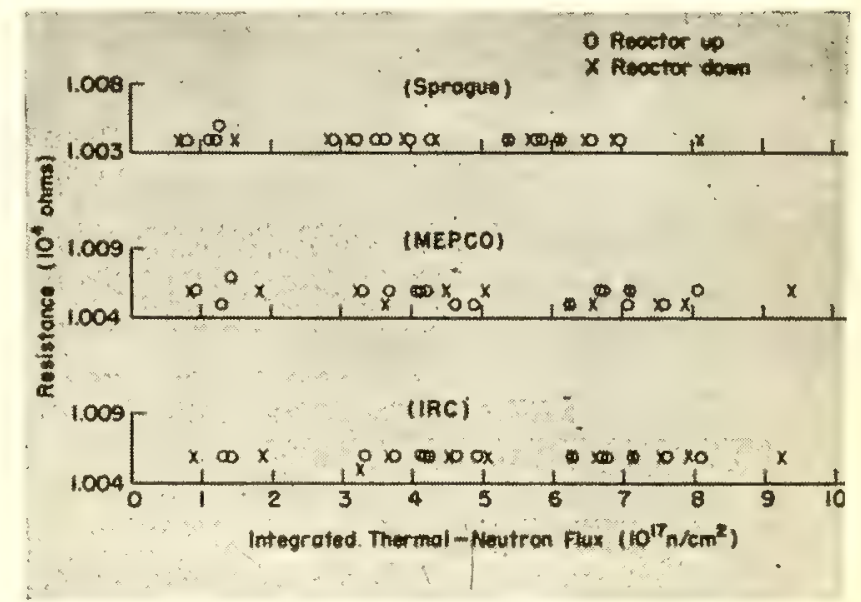

FIG. 3. Graphs demonstrate relative stability of wire-wound resistors to pile irradiation. Each point represents the average value for four resistors of nominal 10,000-ohm value. Thermal fux $6.23 \times 10^{11}$ $\mathrm{n} / \mathrm{cm}^{2} / \mathrm{sec}$

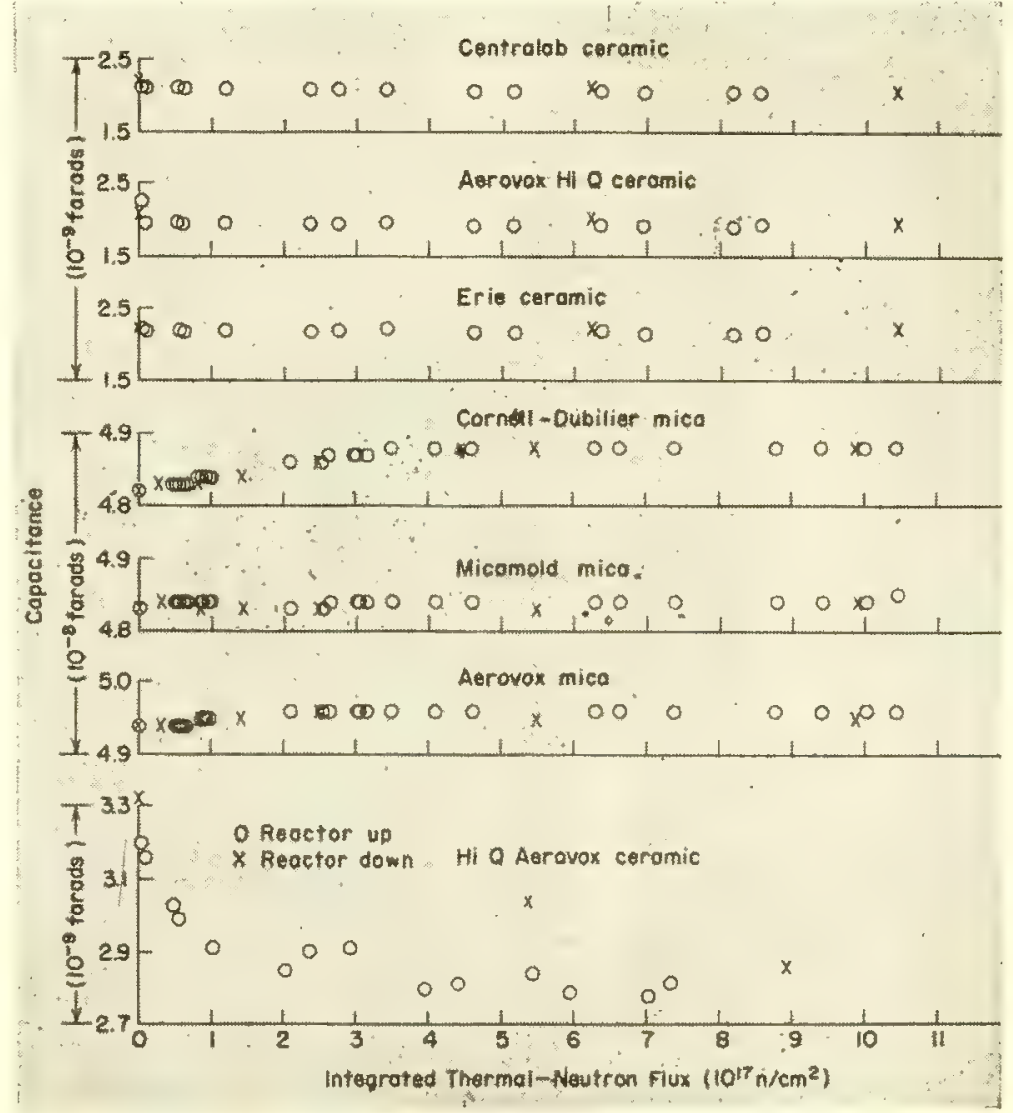

FIG. 4. Mica and ceramic capacitors show different stability under reactor irradiation. Top three charts are for CK60Y821Z ceramics. Next three are for CM61C473J micas. Bottom chart is for CK61Y152Z ceramics. Each point is an average for four copacitors. Thermal fluxes were $7 \times 10^{11} \mathrm{n} / \mathrm{cm}^{2} / \mathrm{sec}$ for first three charts, $6 \times 10^{11} \mathrm{n} / \mathrm{cm}^{2} / \mathrm{sec}$ for others 


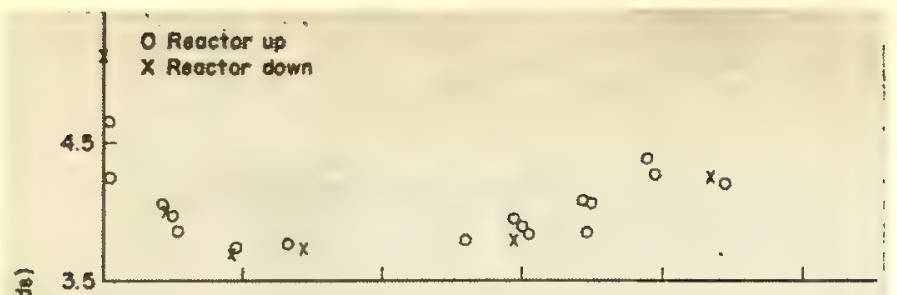

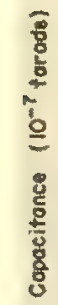

$50 \%$

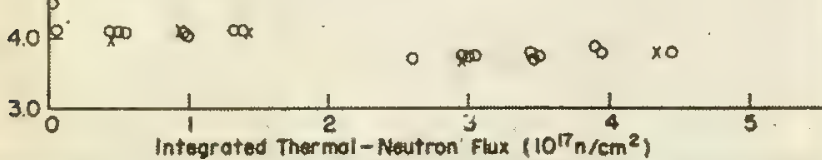

FIG. 5. Radiation effect on capacitances of type CP53B2EF504K fixed poper capacitors. Top chart shows averages for three Sprague, three Cornell-Dubilier. Center shows average for two Aerovox; bottom for one Aerovox. Thermal fux was $6.35 \times 10^{11}$ little change. The results of several experiments are shown in Figs. 4-6.

Some ceramic capacitors showed sporadic capacitance charges attributed to reactor-temperature changes in the interval from $20-50^{\circ} \mathrm{C}$. Other capacitors have much smaller temperature coefficients and are only slightly affected by the same temperature changes.

Measurement of small capacitances in a reactor is made difficult by the capacitance contributed by the connecting cables, which changes throughout the irradiation, and by increased dissipation factors, which make impedance measurements more difficult.

\section{Semiconductors}

Because of both the growing application of transistors and their great susceptibility to radiation damage, they have been studied as thoroughly as any type of component. The low damage threshold also has meant that weaker sources (thus more potential investigations) could be used in the study.

The effect of solar radiation on $p-n$ junctions has been reported $(10,11)$, and gamma radiation is expected to behave in a like manner. In addition the regular structure of the crystals will be disturbed by collisions, and donors and acceptors will be created by neutron capture and subsequent beta decay.

The effect of nuclear radiation on the forward and backward current of semiconductor rectifiers, as indicated to date by this program is indicated in Fig. 7 and the table. The 1 N58 ger- manium rectifiers supplied by Sylvania and CBS Hytron suffered drastic changes in forward and backward current characteristics before reaching an integrated thermal flux of $10^{17} \mathrm{n} / \mathrm{cm}^{2}$. The silicon rectifiers suffered similar damage except for two manufactured by Microwave and one manufactured by Bomac. These three remained in operation during the entire test. Their successful operation indicates that semiconductor rectifiers can be developed (or present-type modified) for more severe radiation environment.

\section{Vacuum Tubes}

Vacuum tubes vary greatly in materials, size, complexity, and resistance to radiation. The most common causes of failure appear to be the deterioration of the glass envelope with subsequent gas problems, and cathode deterioration, sometimes associated with the presence of gas in the tube. More evidence is needed before a positive statement can be made, but it appears that the principal problem is to keep gas from inside the tubes.

Several tube types survived an irradi-

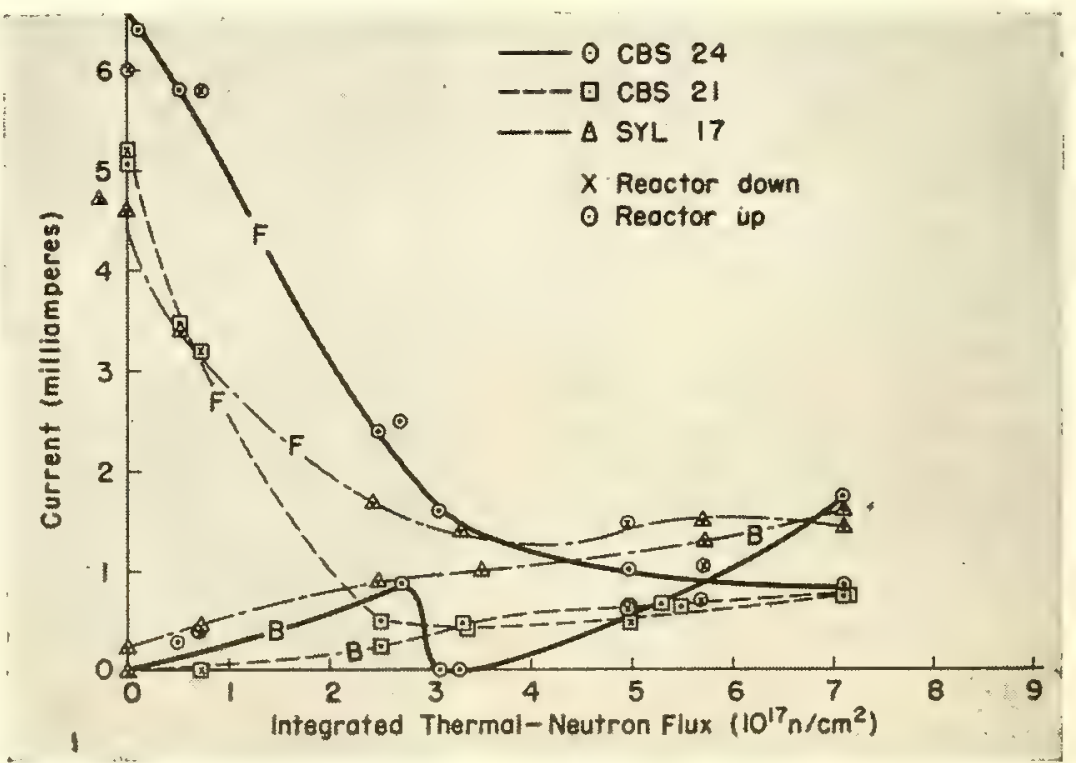

FIG. 7. Germanium rectifiers show this response to reactor radiation. Curves are for two CBS and one Sylvania crystal of type IN58. Forward currents, shown by curves labeled "F," were measured with applied potential of 1 volt; backward currents with potential of 10 volts. Thermal-neutron flux was $0.3 \times 10^{12} \mathrm{n} / \mathrm{cm}^{2} / \mathrm{sec}$. One other Sylvania crystal was considered destroyed at $3.05 \times 10^{17} \mathrm{n} / \mathrm{cm}^{2}$ when its backward current rose above $5 \mathrm{ma}$ 
Forward and Backward Currents of 1N21 Silicon Rectifiers under Reactor Irradiation*

\begin{tabular}{|c|c|c|c|c|c|c|c|c|c|c|c|c|c|c|c|c|c|c|}
\hline \multirow{3}{*}{$\begin{array}{l}\text { Dale } \\
\text { and } \\
\text { time }\end{array}$} & \multirow{3}{*}{$\begin{array}{l}\text { Reac- } \\
\text { tor } \\
\text { up or } \\
\text { down }\end{array}$} & \multirow{3}{*}{$\begin{array}{l}\text { Integrated } \\
\text { thermal flux } \\
\left(10^{17} \mathrm{n} / \mathrm{cm}^{2}\right)\end{array}$} & \multicolumn{4}{|c|}{ Bomac } & \multicolumn{4}{|c|}{ Microwave } & \multicolumn{4}{|c|}{ Sylvania } & \multicolumn{4}{|c|}{ Kentron } \\
\hline & & & \multicolumn{2}{|c|}{ Unit 1} & \multicolumn{2}{|c|}{ Unit 2} & \multicolumn{2}{|c|}{ Unit 3} & \multicolumn{2}{|c|}{ Unit 4} & \multicolumn{2}{|c|}{ Unit 5} & \multicolumn{2}{|c|}{ Unit 6} & \multicolumn{2}{|c|}{ Unit $\gamma$} & \multicolumn{2}{|c|}{ Unit 8} \\
\hline & & & $\begin{array}{c}F \\
(m a)\end{array}$ & $\begin{array}{c}B \\
(\mu a)\end{array}$ & $\begin{array}{c}F \\
(m a)\end{array}$ & $\begin{array}{c}B \\
(\mu a)\end{array}$ & $\begin{array}{c}F \\
(m a)\end{array}$ & $\begin{array}{c}B \\
(\mu a)\end{array}$ & $\begin{array}{c}F \\
(m a)\end{array}$ & $\begin{array}{c}B \\
(\mu a)\end{array}$ & $\begin{array}{c}F \\
(m a)\end{array}$ & $\begin{array}{c}B \\
(\mu a)\end{array}$ & $\begin{array}{c}F \\
(m a)\end{array}$ & $\begin{array}{c}B \\
(\mu a)\end{array}$ & $\begin{array}{c}F \\
(m a)\end{array}$ & $\begin{array}{c}B \\
(\mu a)\end{array}$ & $\begin{array}{c}F \\
(m a)\end{array}$ & $\begin{array}{c}B \\
(\mu a)\end{array}$ \\
\hline \multicolumn{19}{|l|}{$1 / 3 / 56$} \\
\hline $9: 45$ & $d$ & 0. & 42 & 120 & 32 & 115 & 38 & 400 & 38 & 200 & 16 & 2,200 & 39 & 13 & 44 & 86 & 32 & 210 \\
\hline $10: 30$ & $\mathbf{u}$ & 0.014 & 44 & 105 & 35 & 107 & 38 & 380 & 37 & 200 & 19 & 2,300 & 40 & 14 & 44 & 21.5 & 32 & 180 \\
\hline $10: 45$ & d & 0.019 & 17 & 390 & 35 & 110 & 38 & 540 & 37 & 165 & 19 & 2,600 & 40 & 18.5 & 44 & 20.5 & 32 & 380 \\
\hline $11: 30$ & $\mathrm{~d}$ & 0.036 & 15.5 & 390 & 33 & 110 & 40 & 415 & 38 & 175 & 205 & 3,000 & 40 & 18 & 45 & 23.5 & 32 & 360 \\
\hline $12: 30$ & $\mathrm{~d}$ & 0.045 & 17.7 & 380 & 37 & 105 & 39 & 600 & 38 & 175 & 23 & 3,300 & 40 & 16.3 & 45 & 21.5 & 33 & 360 \\
\hline $1: 30$ & $\mathbf{u}$ & 0.061 & 12 & 380 & 36 & 140 & 38 & 500 & 37 & 200 & 26 & 3,700 & 40 & 21 & 45 & 27 & 33 & 390 \\
\hline $2: 50$ & $\mathbf{u}$ & 0.090 & 17.5 & 300 & 36 & 135 & 39 & 600 & 38 & 170 & 26 & 4,800 & 40 & 26 & 46 & 30 & 33 & 390 \\
\hline $3: 45$ & d & 0.120 & 17.5 & 610 & 37 & 140 & 40 & 590 & 38 & 180 & 29 & 4,700 & 38 & 25 & 135 & 26 & 27 & 380 \\
\hline \multicolumn{19}{|l|}{$1 / 4 / 56$} \\
\hline $8: 40$ & $\mathbf{u}$ & 0.416 & 17.5 & 610 & 35 & 137 & 37 & 875 & 38 & 197 & 31 & 9,000 & 33 & 215 & 125 & 34 & 400 & 1.1 \\
\hline $10: 15$ & $u$ & 0.450 & 14 & 975 & 38 & 137 & 33 & 620 & 24 & 290 & 35 & 8,000 & 35 & 8,500 & 125 & 40,000 & 450,000 & 400,000 \\
\hline $11: 25$ & $\mathrm{u}$ & 0.475 & 14 & 7,800 & 38 & 140 & 37 & 620 & 38 & 300 & 28 & 5,800 & 38 & 7,400 & 125 & 45,000 & 480,000 & 480,000 \\
\hline $2: 00$ & $\mathrm{u}$ & 0.531 & & $* *$ & 38 & 340 & 39 & 620 & 39 & 300 & & $* *$ & & $* *$ & & ** & & $* *$ \\
\hline $3: 00$ & $\mathrm{u}$ & 0.552 & & & 36 & 310 & 38 & 620 & 38 & 295 & & & & & & & & \\
\hline $4: 00$ & $\mathbf{u}$ & 0.574 & & & 35 & 300 & 37 & 600 & 37 & 295 & & & & & & & & \\
\hline \multicolumn{19}{|l|}{$1 / 5 / 56$} \\
\hline $8: 40$ & d & 0.868 & & & 35 & 280 & 36 & 560 & 37 & 250 & & & & & & & & \\
\hline 10:00 & $\mathbf{u}$ & 0.872 & & & 31 & 280 & 32 & 660 & 33 & 320 & & & & & & & & \\
\hline $11: 00$ & $\mathrm{u}$ & 0.893 & & & 30 & 290 & 31 & 560 & 32 & 260 & & & & & & & & \\
\hline $12: 00$ & $\mathrm{u}$ & 0.915 & & & 32 & 280 & 32 & 640 & 32 & 240 & & & & & & & & \\
\hline $1: 00$ & $\mathrm{u}$ & 0.936 & & & 33 & 290 & 36 & 660 & 37 & 235 & & & & & & & & \\
\hline $2: 00$ & $\mathbf{u}$ & 0.958 & & & 35 & 300 & 37 & 580 & 37 & 280 & & & & & & & & \\
\hline $3: 00$ & $\mathrm{u}$ & 0.980 & & & 36 & 300 & 37 & 660 & 38 & 280 & & & & & & & & \\
\hline $4: 00$ & d & 1.001 & & & 36 & 300 & 37 & 590 & 38 & 230 & & & & & & & & \\
\hline
\end{tabular}

* 1 volt applied for both forward and backward currents. ** At backward current $>5$ ma, rectifier was considered destroyed.

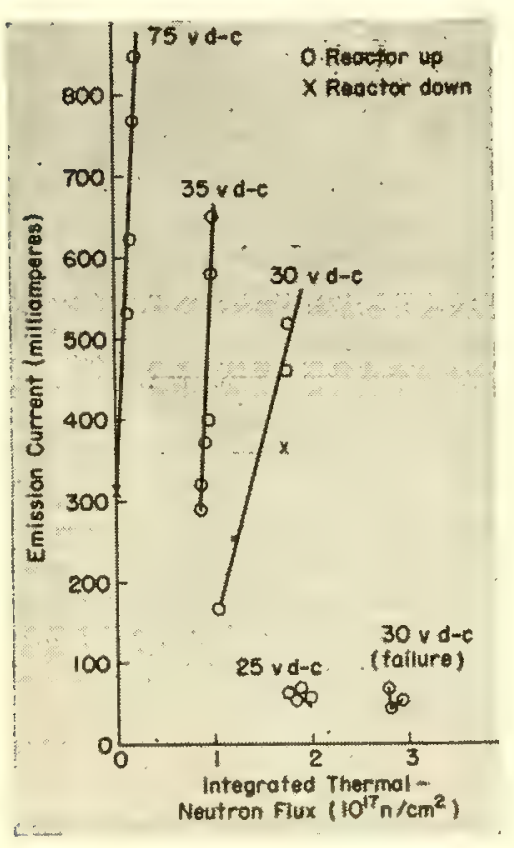

FIG. 8. Curves of emission current vs integral thermal fux demonstrate failure of 5R4WGB tubes. At failure, plate current decreased with either increase or decrease of plate voltage from 30 volis d-c. Points represent averages for 6 tubes ation to $10^{18} \mathrm{n} / \mathrm{cm}^{2}$ with little or no apparent damage. Some examples are cathode-ray tubes $3 J P 1,3 J P 7$, and 3JP12; gaseous voltage regulators $0 \mathrm{~A} 2 \mathrm{WA}, 0 \mathrm{~B} 2 \mathrm{WA}$, and 5651 ; pentagrid converter $6 \mathrm{BE} 6$; twin diodes $6 \mathrm{AL} 5$ and 6080 ; and subminiature types 5718 , 5896, and 6C4.

Other tubes failed rapidly. Examples are a beam-power $829-B$, which failed before $10^{17} \mathrm{n} / \mathrm{cm}^{2}$; orthicons and vidicons, which suffered glass damage below $10^{17} \mathrm{n} / \mathrm{cm}^{2}$; rectifiers $5 \mathrm{R} 4 \mathrm{WGB}$, four of six failing before $4 \times 10^{17} \mathrm{n} / \mathrm{cm}^{2}$. Figure 8 shows the behavior of the $5 \mathrm{R} 4 \mathrm{WGB}$ rectifiers before failure. Metal-enclosed tubes are under test.

The radiation resistance of a given component type varies widely from manufacturer to manufacturer, and there is hope that only minor changes in materials and design will greatly improve many components. Tests on resistors, semiconductor devices, and vacuum tubes support this view.

The gas problem in vacuum tubes can be solved by studying seals and glasses. Some of the tubes that failed most rapidly contained boron in the glass.

It is unfortunate that most component manufacturers regard their formulas as private data, and it is difficult to determine what materials and processes cause trouble.

The data quoted above resulted from the efforts of the radiation-effects group of Admiral Corporation; program director is $E$. R. Pfaff.

\section{BIBLIOGRAPHY}

1. E. R. Pfaff, R. D. Shelton, The effects of nuclear radiation on electronic components. Admiral Corporation (1955, "56)

2. NoCLEONICB 14, No. 7,33 (1956)

9. C. C. Robinson, American Aviation, 19, No. 26, $53(1956)$

4. A. E. Javitz, Electrical Manufacturing, 65 , No. 6, 85 (1955)

5. Effects of radiation on dielectric materials ONR Symposium Report ACR-2. (December, 1954)

6. O. Sisman, C. Bopp, Physical properties of irradiated plastics. ORNL-928

7. R. D. Shelton, The problem of electronic components in nuclear power plants. Aeronautical Electronics Conference Proceedings, Dayton, Ohio (1956)

8. A. Thomas, Nucleonics 13, No, 11, 129 (1955)

9. J. R. Milliron, Electrical Manufacturing \$6, No. 5, $125(1955)$

10. H. Thanos, R. Weber, Investigation of solar cells. Admiral Report (August 5, 1955)

11. Bell Telephone Laboratories advertisement, Electronies 29, No. 1, 78 (1956) 


\section{On Plastics and Elastomers}

\section{BY ROBERT HARRINGTON \\ Hanford Atomic Products Operation General Electric Company \\ Richland, Washington}

STUDY of How radiation affects solid materials incorporated in various components of a reactor is necessary because of the changes induced. The design engineer is confronted with new materials selection problems.

The solid constructional materials most susceptible to change under radiation bombardment are the molecular solids to which the plastics and elastomers or rubber-like materials belong.

\section{Radiation Source}

As it is believed that the changes induced in organic materials are essentially the same for any type of ionizing radiation with respect to energy absorbed, gamma radiations from spent fuel elements and cobalt-60 isotope sources were used as being representative of ionizing radiations in general. However, it may be possible that this relationship is not valid over a wide range of energy absorption levels and rates.

Effects of irradiation rates are shown in Table 1. These imply that materials in a radiation field of a few hundred $\mathrm{r} / \mathrm{hr}$ would not be altered as much at any given total dose as they would be at the dose rates used here.

\section{Procedure and Results}

The experimental work was designed primarily as a sereening process whereby promising materials could be selected for more comprehensive study as dictated by specific applications. However, in some instances sufficient data were obtained for design purposes.
Ultimate elongation and tensile strength properties were measured in accordance with ASTM D 412-51T using three Die C specimens for each exposure. A 180-deg bend test was also made after the exposures by folding the broken tensile specimen back on itself with positive finger pressure applied to insure the specimen sides were in contact. Hardness and elasticity measurements were not taken for the more rigid ethylene materials. Properties were measured at $25^{\circ} \mathrm{C}$ and $50 \%$ relative humidity after a 1 -hr conditioning period.

Table 2 summarizes the changes in properties of plastics and elastomers induced by various doses of gamma radiation from spent fuel elements.

\section{Discussion}

The data show how plastics and

TABLE 1-How Polymer Properties are Changed by $5 \times 10^{7} \mathrm{r}$ from $\mathrm{Co}^{60}$ Compared with the Same Dose from Spent Fuel Elements*

Percent change in properties

Material and source Hardness Elasticity Elongation Tensile Weight

Silastic 152

Cobalt-60

Spent fuel elements

Alathon $4 B K 20$

Cobalt- 60

Spent fuel elements

SE-361

Cobalt- 60

Spent fuel elements

Hycar 1001

Cobalt-60

Spent fuel elements

$$
\begin{aligned}
& 46.0 \\
& 36.0
\end{aligned}
$$

$$
-51.8
$$$$
-40.7
$$$$
\begin{aligned}
& -77.3 \\
& -68.2
\end{aligned}
$$$$
\begin{array}{r}
3.7 \\
-8.8
\end{array}
$$$$
-0.09
$$

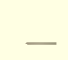

$$
-84.3
$$$$
-10.8
$$$$
-59.0
$$$$
-19.7
$$

* Incident rates: $\mathrm{Co}^{80} \approx 3.7 \times 10^{8} \mathrm{r} / \mathrm{hr}$ in $25^{\circ} \mathrm{C}$ air; spent fuel elements $\approx 10^{5} \mathrm{r} / \mathrm{hr}$ in $15^{\circ} \mathrm{C}$ air. 
and SE-550. The properties of these materials undergo very nearly the same changes, but at any given radiation dose, the SE-550 is much more flexible and appears in better over-all condition.

Weight and thickness measurements do not indicate the condition of a material. Teflon at an exposure of $5 \times$ $10^{6} \mathrm{r}$ had not changed in either weight or thickness but was extremely brittle and crumbly.

Elasticity appears to offer the same advantages as does elongation. However, because of the scale used ( 0 to 100), materials having an initial elasticity of 6 , which lowers to 3 after exposure, have essentially undergone less change than those materials that change from 30 to 15 -the over-all change being a $50 \%$ reduction in elasticity. Also, in the first case because of the low values, a larger error is introduced because of the measurement method.

Materials. The styrene rubber PR 408-70 is affected less than the other materials investigated. This is due to the presence of the styrene, which is able to absorb greater amounts of energy from radiation without being altered to the same degree as the other materials. The acrylics, neoprenes, and nitrile classes all appear to be about equally damaged.

Teflon is the poorest of all the materials evaluated. It is severely damaged at $5 \times 10^{6}$ r. Kel-F elastomer and Hycar 2202 (butyl type) both softened and became tacky, indicating poor suitability in radiation fields.

The remainder of the ethylene materials appear to be in the same class as the acrylies, neoprenes, and nitriles on the basis of the longer exposures. However, intermediate doses affected the ethylenes in a different manner; properties were either upgraded or degraded depending upon the amount of exposure received.

The vinyls appear to sustain less damage than all other materials except the styrene-type rubber. The silicone elastomers as a class are inferior to all other classes except the fluorocarbons and butyl. However, within the silicone class, the phenyl types are superior to the methyl and vinyl types owing to the presence of the benzene ring.

Data presented for all exposures at $5 \times 10^{6} \mathrm{r}$ show conclusively that most materials are significantly affected by this dose, and shorter exposures are needed before the complete representation of property changes can be shown.

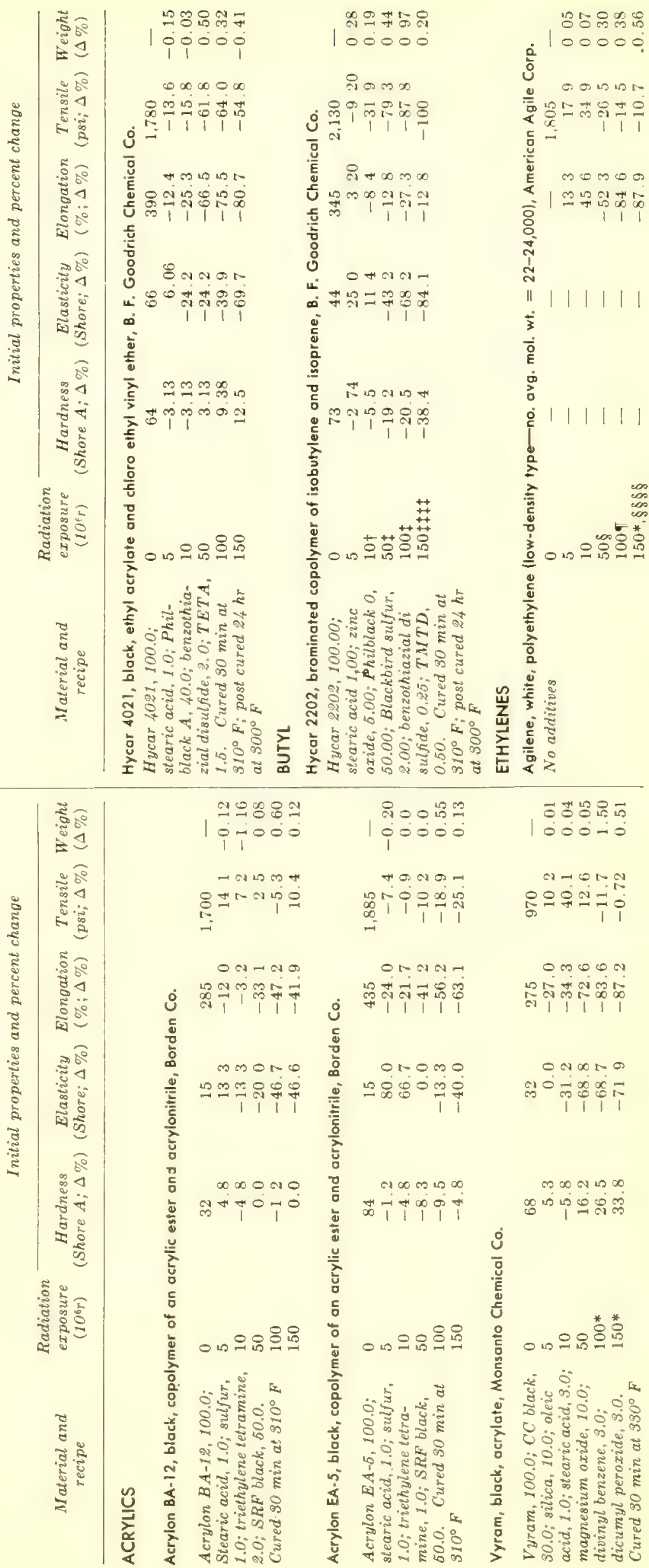



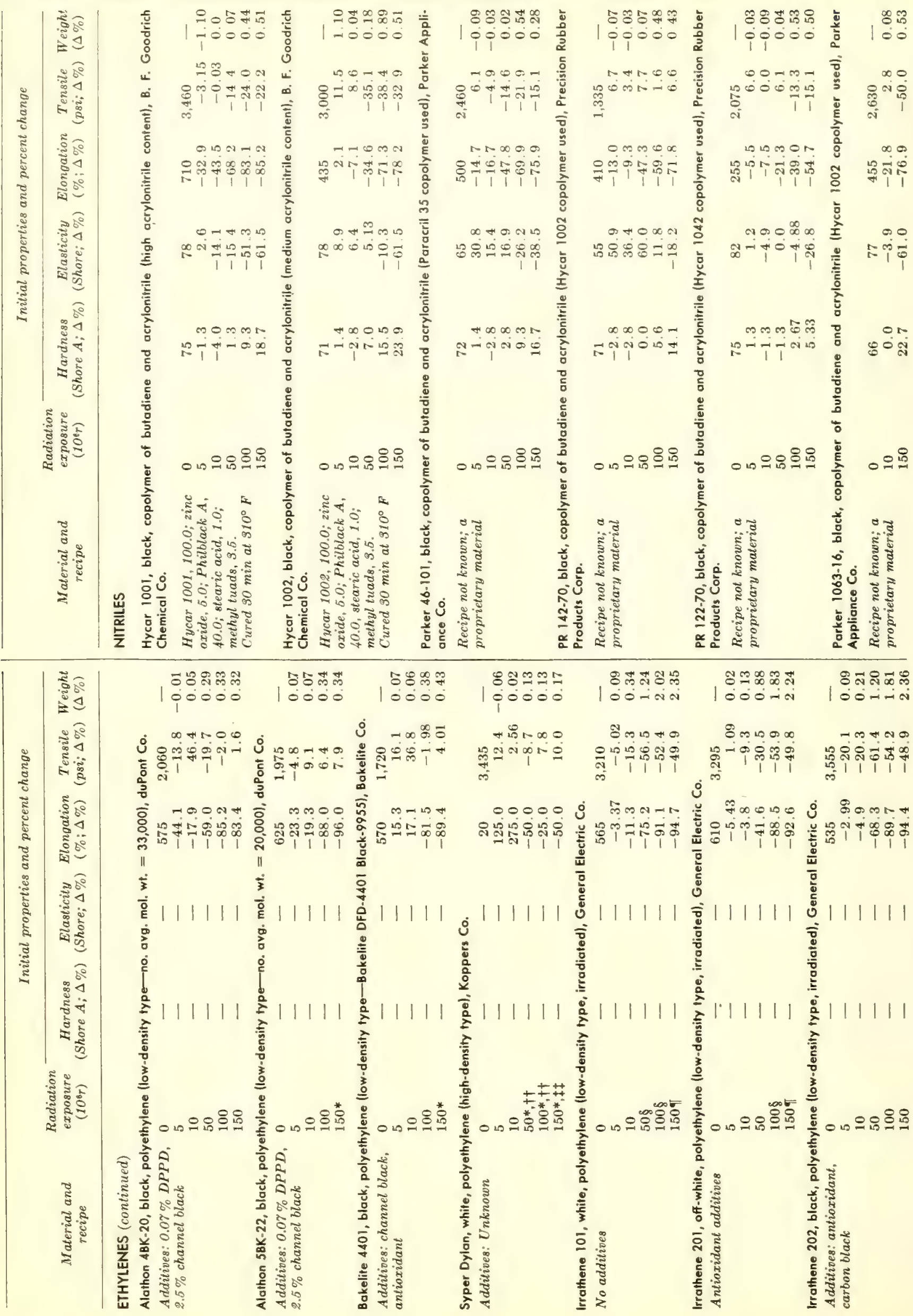


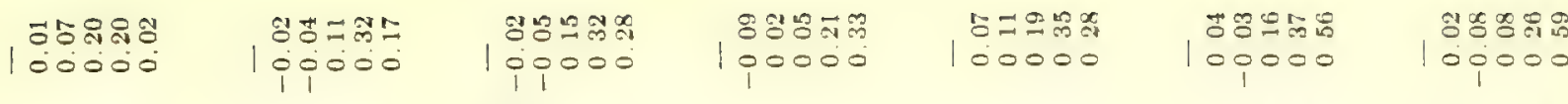

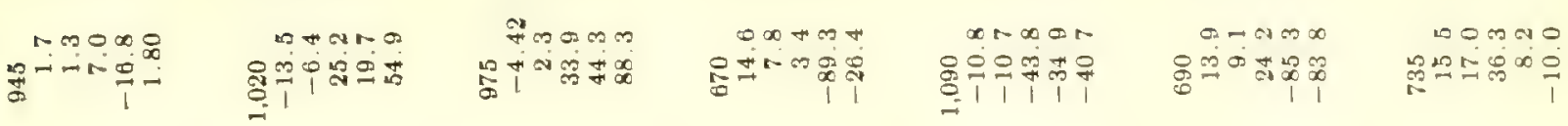

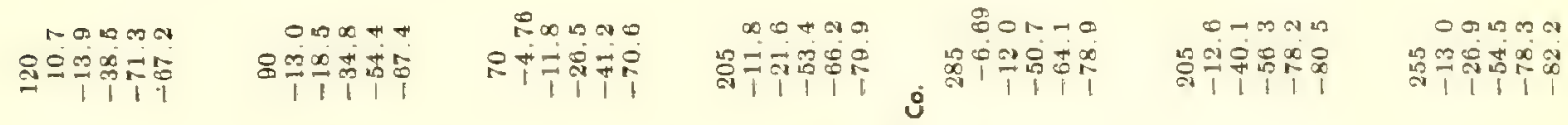

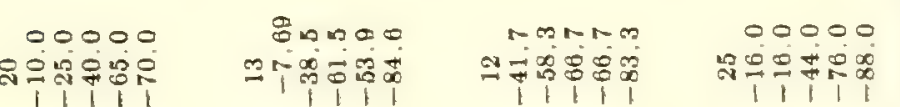

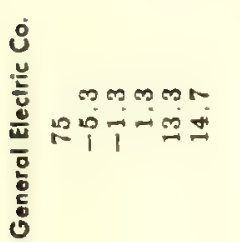
高

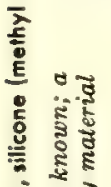

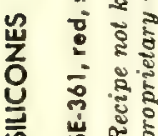

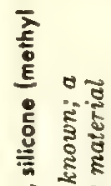

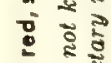

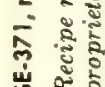

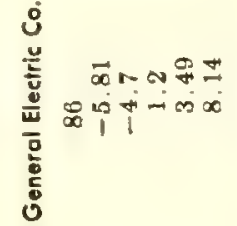

$\stackrel{-}{a}$

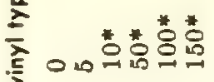

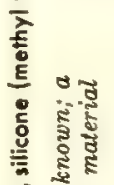

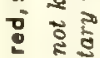

क्ष. is

पू omm

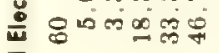

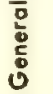

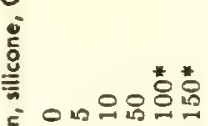

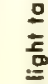
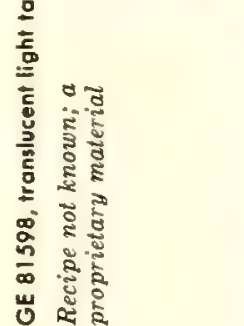

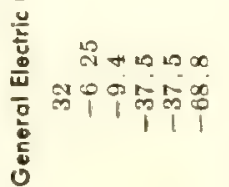

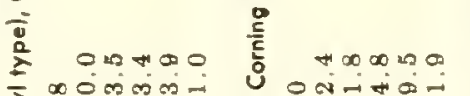
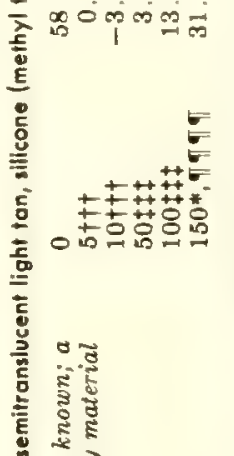

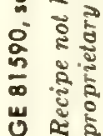

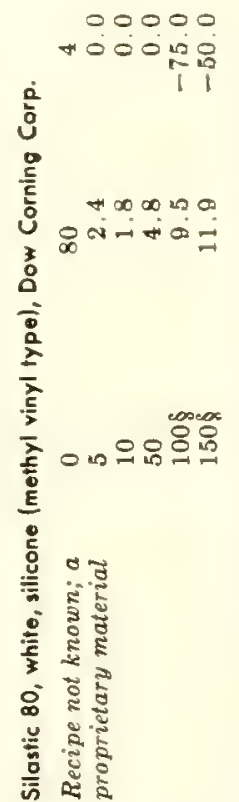

豙

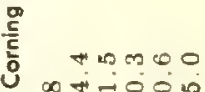

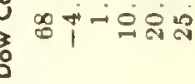

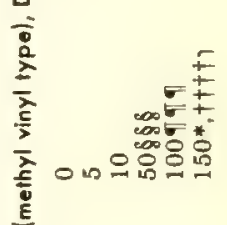

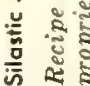

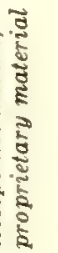

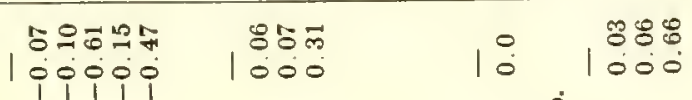

แேเッ

๑๐

कृ

$\infty \& \infty \infty \pi$

औัต

- $N \infty \infty$

j

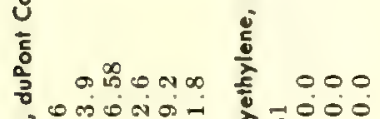

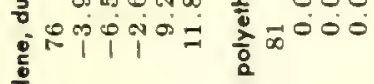

है

总

岸岀

焉的施

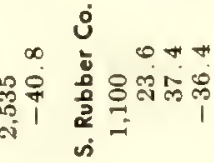

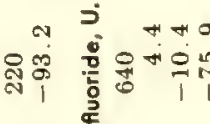

tั $\cos$

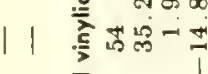

듬

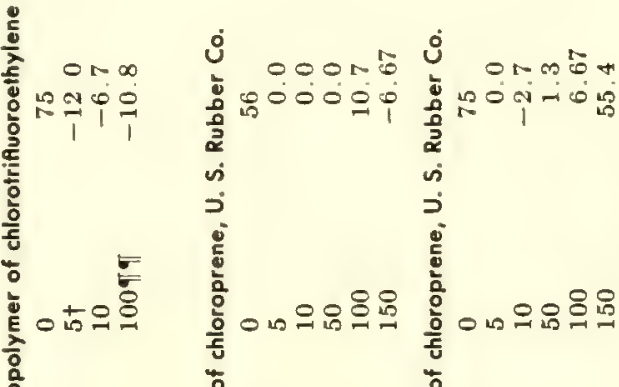

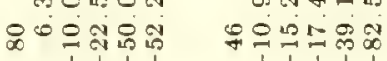

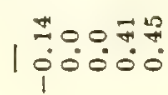

nosom 落二 卷菜

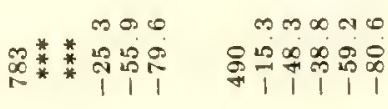
舟 $1+\infty=\infty$

కอำ

0000

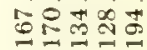

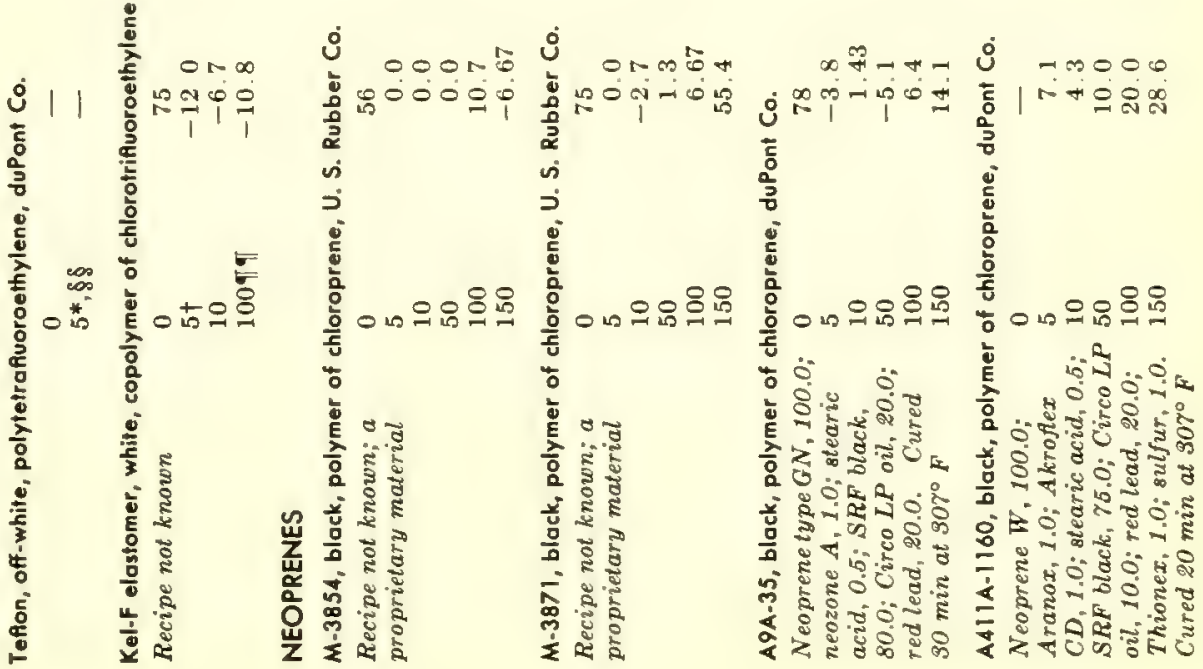




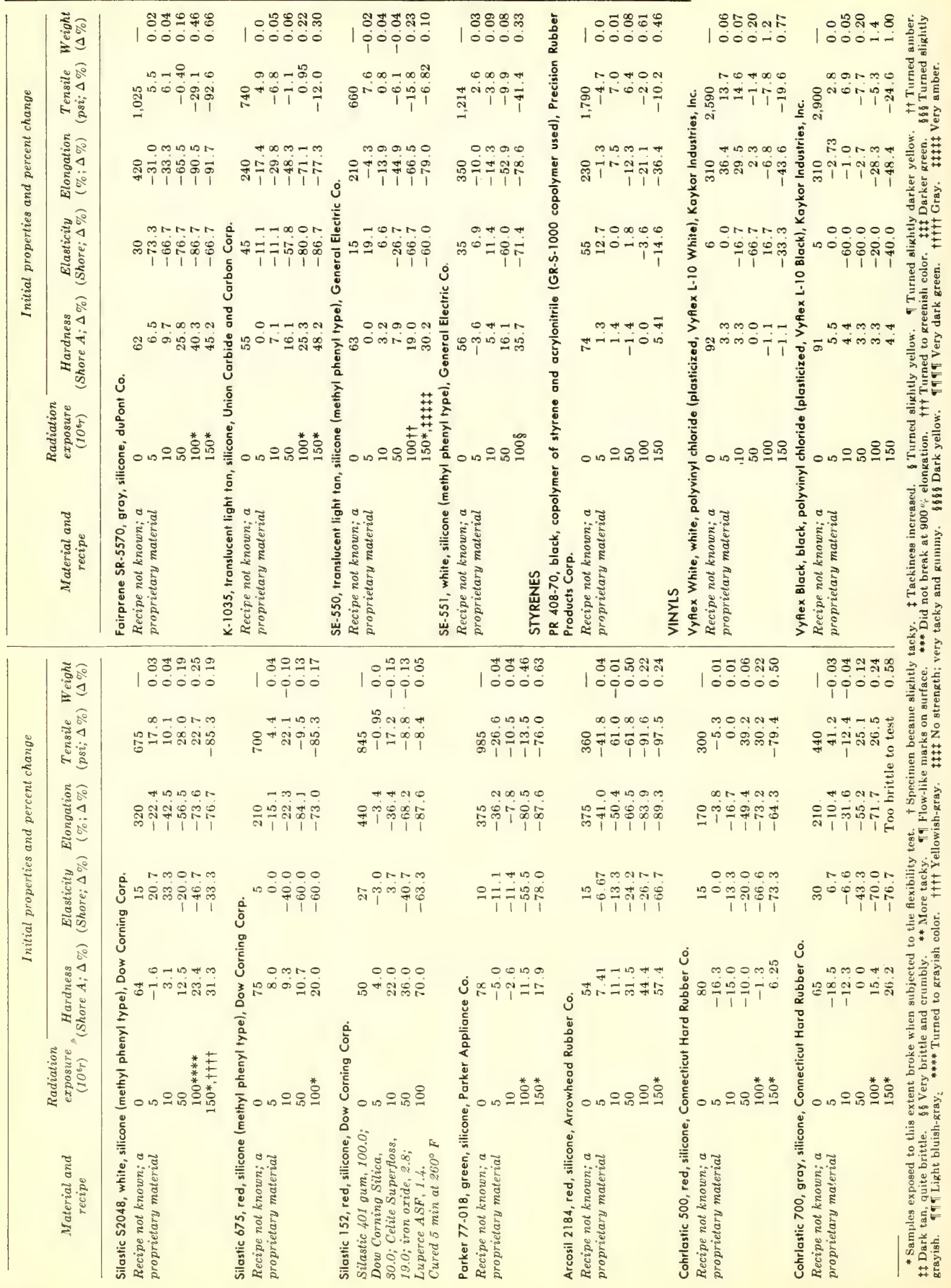




\section{On Chemical Materials}

By J. C. BRESEE, J. R. FLANARY, J. H. GOODE, C. D. WATSON, and J. S. WATSON

Chemical Technology Division, Oak Ridge National Laboratory, Oak Ridge, Tennessee

IMPORTANT IN THE REPROCESSING Of reactor fuels is the effect of radiation damage on process reagents and organic materials of construction, especially for short-cooled fuels where the fissionproduct radiation is very intense. The magnitude of the chemical and physical changes produced by radiation is sufficient to affect the usefulness, and even the feasibility, of some materials used in the recovery of uranium, thorium, and other materials from irradiated reactor fuels. This article presents the details of how gamma radiation damages organic protective coatings and gaskets.

To simulate fission-product radiation, a cobalt-60 gamma source was used for these tests. Average gamma energy is $1.2 \mathrm{Mev}$. The source provided an intensity of $1.2-3.6 \times 10^{4} \mathrm{r}$ per minute in air; the maximum of total radiation accumulated by the test specimens was about $10^{9} \mathrm{r}$.

The specimens. Radiation stability of many industrial materials has been tested on a laboratory scale to aid in selecting materials for the construction and operation of radiochemical processing plants. The materials were acquired by: (a) accepting samples from vendors who solicited ORNL, and (b) nonexhaustive queries to vendors whose products appeared to be of interest.

Materials that apparently exhibit poor radiation stability in these tests need not be considered inferior products; slight variations in composition (such as the nature or content of plasticizers, binders, fillers, pigments, etc.) in many cases may affect radiationstability properties quite drastically. Often a poorly rated product easily could be made to test higher by a slight change in raw materials or processing procedure.

The tests are empirical and have been developed only for immediate compari- son purposes. Standard methods have not been established for radiation stability, chemical resistance or decontamination tests, and because of the variety and number of samples a few

\section{Chemical Tests on Protective Coatings}

Quantitative measurements were made to determine how radiation affects the chemical resistance and chemical decontaminability of protective coatings.

Resistance. Standard chemical-resistance tests were run on irradiated and unirradiated specimens. Cold-rolled steel immersion rods ( $3 / 8$ in. dia, 6-in. long) with one rounded end were coated with the paint to be tested and were irradiated to various levels of accumulated radiation. Specimens that did not "fail" (from visual observations) were immersed in several chemical reagents after an accumulated dose of about $10^{9} \mathrm{r}$ in air. The reagents were 3 molar nitric, sulfuric and hydrochloric acids, 9 molar sodium hydroxide, and an organic solvent (methyl isobutyl ketone or "hexone"). The tests were continued until failure, or for a total of 9 days, and the results with irradiated specimens were compared with those for unirradiated specimens.

A second phase of the chemical resistance studies occurred in the process of preparing specimens for decontamination tests. Protective coatings were applied to aluminum, steel, and concrete panels and were irradiated to various levels of accumulated radiation. The test panels then were contaminated with $0.1 \mathrm{ml}$ fission products in $6 \mathrm{M} \mathrm{HNO}$. Many specimens that satisfactorily resisted 3 molar $\mathrm{HNO}_{3}$ during immersion tests were attacked by the contaminating acid, so several decontamination studies were rerun with 3 molar contaminating acid.

Decontaminability. The chemical decontamination procedure consisted of scrubbing specimens that withstood the contaminating acid with water, $3 \mathrm{M} \mathrm{HNO}$, and in most cases concentrated citric acid. After each wash the surface of the specimens were monitored and a decontamination factor (DF) was calculated. The process of contamination and decontamination was repeated three times or until the coating failed.

The tests were performed in series, and essentially only those coatings that "passed" the previous tests were subjected to later ones. Thus, only a few of the total number of coatings studied were carried through the decontamination tests.

In many cases, mounted coatings were zradiated on two or three different types of surfaces-aluminum, steel, and concrete. The series of tests was necessary since the stability of an irradiated coating was strongly infuenced by the surface to which it was applied. Similar tests were performed for each type of coated panel. 


\section{Manufacłurers of Organics Tested}

\section{UNMOUNTED COATINGS}

A-89-A-Black-Gordon-Lacey Chemical Products Co., N. Y., N. Y.

Amercoat Strip, Amerplate (T-Locked, Black and White)-Amercoat Corp., South Gate, Cal.

Brevon-Black-Atlas Powder Co., $N$. Chicago, Ill.

GE Cocoon-R. M. Hollingshead Corp., Camden, N.J.

Tygofilm-Blue-- $C$. S. Stoneware, $A k$ ron, $O$.

Flame-sprayed polyethylene-Powder Weld Process Co., Brooklyn, N. Y.

\section{MOUNTED COATINGS}

Alkaloy-550, Amphesive-801, Ampreg-E, Neobon, Zerox-110-Atlas Mineral Products Co., Houston, Texas.

Amercoat-23, 33, 44, 55 and 1574 SE-Amercoat Corp., South Gate, Cal.

Barrett silicone-Barrell Varnish Co., Cicero, Ill.

Corrosite-22-Corrosite Corp., $N . Y$, N. Y.

Duralon-36- $C$.S. Stoneware, Akron, $o$.

DuPont White-DuPont Co., Inc., Wilnington, Del.

products may have received incorrect ratings.

\section{Protective Coatings}

The most important uses of protective coatings in radiochemical processing are to: (a) prevent corrosion of auxiliary process equipment (equip-
Dyna-clad-Merchants Chemical Co.,

Inc., N. Y., N. Y.

Epon-395-Glidden Co., Chicago, Ill.

Epon-1001-Shell Chemical Corp., N. Y., N.Y.

Nukemite-40-Nukem Products Corp., Buffalo, N. Y.

Phenoline-3, Polyclad SealcoatCarboline Co., St. Louis, Mo.

Prufcoat-Prufcoat Laboratory, Inc., Cambridge, Mass.

Solar Silicone Alkyd-Solar Corp., Milwauke, Wis.

Ucilon-United Chromium, Inc., N.Y., N. Y.

\section{GASKETS AND ELASTOMERS}

Polyethylene, plain and carbonfilled-American Agile Co., Cleveland, $O$.

Polystyrene-Dow Chemical Co., Midland, Mich.

Teflon-Du Pont Co., Inc., Wilmington, Del.

Kel-F-Kellex Corp., N. Y., N.Y. Silicone rubber 12602 and $12603-$ General Electric Co., Waterford, N. Y. Hycar rubber PA-21 and OR-25B. F. Goodrich Chemical Co., Cleveland, $O$.

ment not normally in contact with main process streams), and (b) improve the decontaminability of auxiliary equipment, the outside surface of main process equipment, and other surfaces such as the walls and floors of highactivity cells.

Coatings applied in various ways

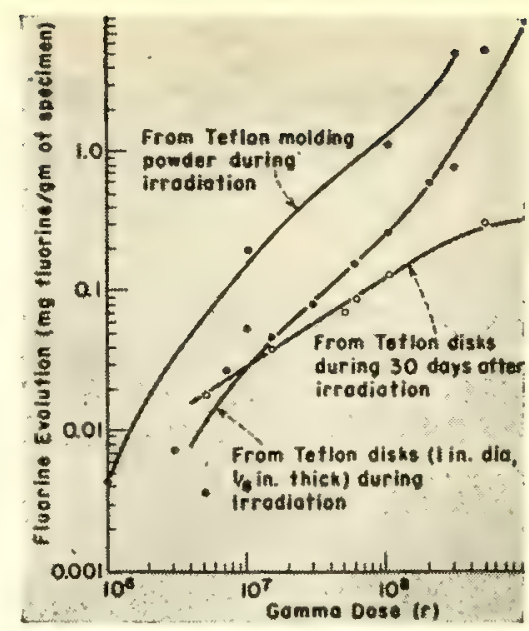

FIG. 1. Fluorine evolution from Teflon during and after irradiation

were irradiated with the $\mathrm{Co}^{60}$ gamma source. For convenience, several types of coatings that in actual use would be subjected to radiation while mounted on a surface were irradiated in an unmounted sheet form. These include stripcoats, a flame-sprayed polyethylene coating, and examples of "T-locked" plastic sheet. All other coatings were applied to either panels (aluminum, steel or concrete) or steel immersion rods.

The acceptability or failure of an irradiated coating depends on the requirements of its use. In the same way, the types of tests used to study the effect of gamma radiation vary with the projected use of the coating.

Unmounted coatings. For the three types of coatings irradiated in sheet form, the strength of a coating-to-sur-

TABLE 1-How a Gamma Dose of $1.05 \times 10^{9} \cdot *$ Damages Unmounted Coatings

\begin{tabular}{|c|c|c|c|c|c|}
\hline Coaling & Polymer base & Color change & Flexibility & 180-deg-bend test & Remarks \\
\hline \multicolumn{6}{|l|}{ Strip coats } \\
\hline A-89-A & Vinyl & Black-unchanged & Stiff & Breaks & Sample curled \\
\hline Amercoat Strip & Vinyl & White to gray & Stiff & Breaks & Sample curled \\
\hline Brevon & Vinyl & Black-unchanged & Stiff & Breaks & Sample curled \\
\hline GE Cocoon & Vinyl copolymer & Orange to black & Flexible & Bends & Tacky surface \\
\hline Tygofilm & Vinyl copolymer & Blue to gray & Stiff & Breaks & Not curled \\
\hline \multicolumn{6}{|l|}{ Plastic sheets } \\
\hline $\begin{array}{l}\text { Amer Plate } \\
\text { (T-locked, black) }\end{array}$ & Vinyl & Black-unchanged & Stiff & Breaks & Not curled \\
\hline $\begin{array}{l}\text { Amer Plate } \\
\text { (T-locked, white) }\end{array}$ & Polyethylene & White to amber & Stiff & Breaks & Not curled \\
\hline $\begin{array}{l}\text { Polyethylene } \\
\text { (flame-sprayed) }\end{array}$ & Polyethylene & Red, yellow, and blue to darker shades & Stiff & Breaks & Not curled \\
\hline
\end{tabular}


TABLE 2- How Gamma Radiation Affects Protective Coatings

\begin{tabular}{|c|c|c|c|c|}
\hline Coaling & Polymer base & Surface & $\begin{array}{l}\text { Total gamma dose } \\
\qquad\left(10^{8} r\right)\end{array}$ & Visual obsertations \\
\hline Alkaloy -550 & Furane & $\begin{array}{l}\text { Concrete panel } \\
\text { Steel immersion rod }\end{array}$ & $\begin{array}{l}11.3^{*} \\
10^{*}\end{array}$ & $\begin{array}{l}\text { No visible change } \\
\text { No blistering or checking }\end{array}$ \\
\hline Amercoat-23 & Vinyl chloride & $\begin{array}{l}\text { Concrete panel } \\
\text { Steel panel } \\
\text { Steel panel-wet }\end{array}$ & $\begin{array}{c}12.3 \\
5 \\
10\end{array}$ & $\begin{array}{l}\text { Blistered, some cracks } \\
\text { Blistered } \\
\text { One large blister on panel surface }\end{array}$ \\
\hline Amercoat-33 & Vinyl chloride & $\begin{array}{l}\text { Aluminum panel } \\
\text { Concrete panel } \\
\text { Steel panel-wet }\end{array}$ & $\begin{array}{l}2.4 \\
12.2 \\
10\end{array}$ & $\begin{array}{l}\text { Blistered } \\
\text { Blistered, some cracks } \\
\text { Many pinpoint blisters }\end{array}$ \\
\hline Amercoat-44 & Vinyl & Steel panel-wet & 10 & Many pinpoint blisters \\
\hline Amercoat-55 & Vinyl & $\begin{array}{l}\text { Aluminum panel } \\
\text { Concrete panel } \\
\text { Steel panel } \\
\text { Steel panel-wet }\end{array}$ & $\begin{array}{r}2.4 \\
12.3 \\
5 \\
10\end{array}$ & $\begin{array}{l}\text { Blistered } \\
\text { Small blisters } \\
\text { Blistered } \\
\text { Blistered }\end{array}$ \\
\hline Amercoat-1574-SE & Epoxy & $\begin{array}{l}\text { Concrete panel } \\
\text { Steel panel-wet }\end{array}$ & $\begin{array}{l}12^{*} \\
10^{*}\end{array}$ & $\begin{array}{l}\text { Tacky, some brittleness, discoloration } \\
\text { Color streaked, some hardening }\end{array}$ \\
\hline Amphesive- 801 & Modified phenolic & $\begin{array}{l}\text { Concrete panel } \\
\text { Steel immersion rod }\end{array}$ & $\begin{array}{l}11.3^{*} \\
10^{*}\end{array}$ & $\begin{array}{l}\text { No visible change } \\
\text { Drastically embrittled }\end{array}$ \\
\hline Ampreg-E & Polyester resin & $\begin{array}{l}\text { Concrete panel } \\
\text { Steel immersion rod-wet }\end{array}$ & $\begin{array}{l}10^{*} \\
2.3\end{array}$ & Layer of crystals on surface of coating \\
\hline Barrett Silicone & Silicone-alkyd & $\begin{array}{l}\text { Concrete panel } \\
\text { Steel immersion rod } \\
\text { Steel panel }\end{array}$ & $\begin{array}{l}8^{*} \\
8^{*} \\
8^{*}\end{array}$ & Blistered \\
\hline Corrosite-22 & Vinyl & $\begin{array}{l}\text { Aluminum panel } \\
\text { Concrete panel }\end{array}$ & $\begin{array}{r}2.4 \\
12.3\end{array}$ & $\begin{array}{l}\text { Blistered, cracked } \\
\text { Dark orange spots (borderline case) }\end{array}$ \\
\hline Duralon-36 & Furane & $\begin{array}{l}\text { Concrete panel } \\
\text { Steel immersion rod }\end{array}$ & $\begin{array}{l}10^{*} \\
10^{*}\end{array}$ & $\begin{array}{l}\text { Brittle } \\
\text { Entire surface badly blistered }\end{array}$ \\
\hline Dupont White & Vinyl chloride & Steel panel-wet & 10 & Entire surface badly blistered \\
\hline Dyna-clad & Vinyl chloride & Steel panel-wet & 10 & \\
\hline Epon-1001 & Epoxy & $\begin{array}{l}\text { Steel immersion rod } \\
\text { Steel panel } \\
\text { Steel panel-wet } \\
\text { Concrete panel } \\
\text { Steel immersion rod } \\
\text { Steel panel }\end{array}$ & $\begin{array}{r}8^{*} \\
8^{*} \\
10^{*} \\
12^{*} \\
8^{*} \\
8^{*}\end{array}$ & $\begin{array}{l}\text { Color, change from gray to green, hardened } \\
\text { Tacky, some brittleness, splotchy } \\
\text { diseoloration } \\
\text { Hardened but no cracks or blisters }\end{array}$ \\
\hline Neobon & Neoprene & $\begin{array}{l}\text { Concrete panel } \\
\text { Steel immersion rod } \\
\text { Steel immersion rod-wet } \\
\text { Steel panel }\end{array}$ & $\begin{array}{c}10^{*} \\
10^{*} \\
2.3 \\
10^{*}\end{array}$ & $\begin{array}{l}\text { Hardened on tip of rod } \\
\text { Some crystallization below surface, much } \\
\text { harder surfäce }\end{array}$ \\
\hline Nukemite-40 & Vinyl & $\begin{array}{l}\text { Aluminum panel } \\
\text { Concrete panel } \\
\text { Steel immersion rod } \\
\text { Steel panel-wet }\end{array}$ & $\begin{array}{l}3.5 \\
12.3^{*} \\
10^{*} \\
10\end{array}$ & $\begin{array}{l}\text { Blistered } \\
\text { Surface soft, roughened } \\
\text { Few small blisters, color much darkened }\end{array}$ \\
\hline Phenoline-3 & Phenolic & $\begin{array}{l}\text { Concrete panel } \\
\text { Steel immersion rod }\end{array}$ & $\begin{array}{l}10 \\
10\end{array}$ & $\begin{array}{l}\text { Visible cracks } \\
\text { Visible cracks }\end{array}$ \\
\hline Polyclad Sealcoat & Vinyl chloride & Steel panel-wet & 10 & Surface badly wrinkled \\
\hline Prufcoat & Styrene & $\begin{array}{l}\text { Concrete panel } \\
\text { Steel immersion rod } \\
\text { Steel panel wet }\end{array}$ & $\begin{array}{r}10 \\
10 \\
1\end{array}$ & $\begin{array}{l}\text { Hard, brittle craters, no blisters, dark- } \\
\text { ened layer under surface } \\
\text { Checked, cracked, blistered } \\
\text { Paint cracked from panel }\end{array}$ \\
\hline Solar silicone alkyd & Silicone-alkyd & $\begin{array}{l}\text { Concrete paniel } \\
\text { Steel immersion rod } \\
\text { Steel panel }\end{array}$ & $\begin{array}{l}8^{*} \\
8^{*} \\
8^{*}\end{array}$ & \\
\hline Ucilon & Vinyl chloride & Steel panel-wet & 10 & Surface blistered, \\
\hline Zerox-110 & Styrene butadiene & $\begin{array}{l}\text { Concrete panel } \\
\text { Steel immersion rod-wet }\end{array}$ & $\begin{array}{c}11.3^{*} \\
2.3\end{array}$ & Extreme checking, darkened \\
\hline
\end{tabular}

* Discontinued before failure; all others were discontinued because of failure. 


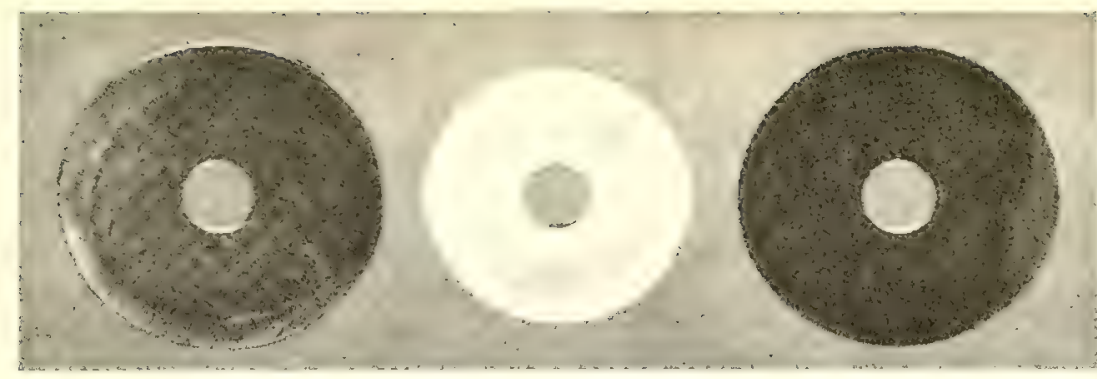

FIG. 2. Disassembled 347 stainless steel test flanges show corrosion resulting from contact with Teflon disk (center) during $10^{8} \mathrm{r}$ irradiation immersed in $50 \% \mathrm{HNO}_{3}$ for five days at $20^{\circ} \mathrm{C}$

face bond normally is quite weak (for stripcoats, deliberately so), and radiation-caused changes in the bond are less important than changes in the physical properties of the coating. Gamma damage to unmounted coatings is shown in Table 1.

The main requirements for a radiation-resistant stripcoat are that the coating surface retain its integrity and the film its flexibility so that decontamination can be carried out by removing the coating after irradiation. In four of the five stripcoat tests, the surface remained intact, but the film became brittle and broke during a bending test; these stripcoats could not, be removed readily after $10^{9} \mathrm{r}$.

The fifth strip coat, G. E. Cocoon, remained flexible but the surface became very tacky. There is a pösibility that the film porosity increased with irradiation, but no quantitative tests were carried out to establish this point.

The proposed use of both T-locked coatings and flame-sprayed polyethylene called for flexibility of the coatings. The unmounted tests indicated that both types were seriously embrittled by exposure to $10^{9} \mathrm{r}$. However, applications without the need for flexibility (e.g., a T-locked surface for a concrete cell wall) would be possible provided that the sheet porosity had not increased. Again, porosity tests were not carried out.

Mounted coatings. Table 2 presents the results of screening studies of mounted protective coatings. It shows the gamma dose at which the coating failed or the maximum dose applied to the coating without failure.

The influence of the surface to which the coating is applied on the stability of the coating is seen by comparing the

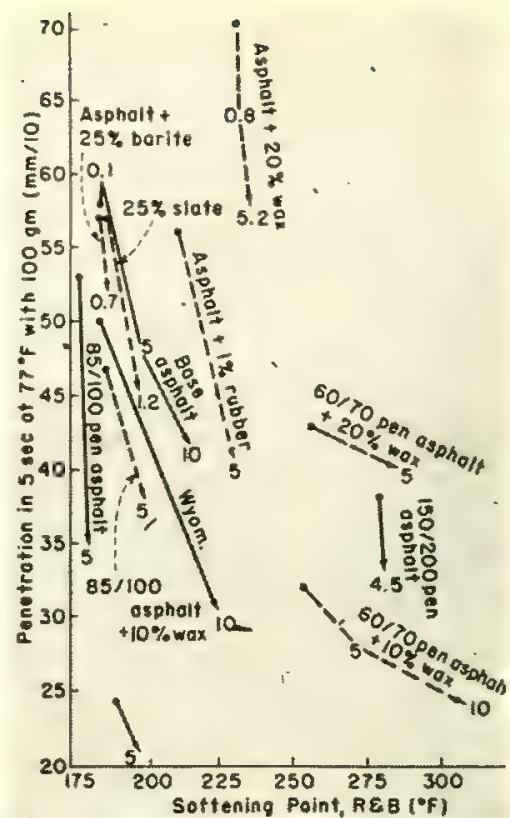

FIG. 3. Asphalts are hardened by exposure to gamma radiation-softening point and penetration change. Note that origin of each line is property of unirradiated sample; broken line denotes blend. Numbers show total dose, in $10^{8} \mathrm{r}$

performance of a single coating type on several surfaces. The differences are often striking. For example, the vinyl coating Amercoat-33, which failed on an aluminum panel after $2.4 \times 10^{8} \mathrm{r}$, did not fail on a concrete panel until $1.22 \times 10^{9} \mathrm{r}$-over five times the ga mma dose.

TABLE 3-How Gamma Radiation Affects the Chemical Resistance and Decontaminability of Mounted Coatings

\begin{tabular}{|c|c|c|c|c|c|c|c|c|}
\hline \multirow[b]{2}{*}{ Coating } & \multirow[b]{2}{*}{ Surface } & \multirow{2}{*}{$\begin{array}{c}\text { Gamma } \\
\text { dose } \\
\left(10^{8} r\right)\end{array}$} & \multirow[b]{2}{*}{ Reagents } & \multicolumn{5}{|c|}{ Results* } \\
\hline & & & & Acid & Base & Solvent & $C / D \dagger$ & $D F \ddagger$ \\
\hline \multirow[t]{2}{*}{ Alkaloy-550 } & Concrete & 11.3 & $\mathrm{HNO}_{3}-\mathrm{FP}$ & & & & $\mathbf{R}$ & $25-200$ \\
\hline & Steel & 10 & $\mathrm{HNO}_{3}, \mathrm{NaOH}$, hexone & $\mathrm{R}$ & $\mathrm{R}$ & $\mathrm{R}$ & & \\
\hline Amercoat-33 & Aluminum & 1.0 & $\mathrm{HNO}_{3}-\mathrm{FP}$ & & & & $\mathbf{R}$ & $70-117$ \\
\hline Amphesive-801 & Steel & 10 & $\mathrm{HNO}_{3}, \mathrm{NaOH}$, hexone & $\mathrm{N}$ & $\mathrm{N}$ & $\mathrm{N}$ & & \\
\hline \multirow[t]{2}{*}{ Barrett silicone } & Concrete & 8 & $\mathrm{HNO}_{3},-\mathrm{FP}$ & & & & $\mathbf{R}$ & 12 \\
\hline & Steel & 8 & $\mathrm{HNO}_{3}, \mathrm{HCl}, \mathrm{NaOH}$, hexone, $\mathrm{HNO}_{3}-\mathrm{FP}$ & $\mathrm{N}$ & $N$ & $N$ & $\mathbf{N}$ & \\
\hline Corrosite-22 & Aluminum & 1.0 & $\mathrm{HNO}_{3} \cdot \mathrm{FP}$ & & & & $\mathrm{R}$ & 560 \\
\hline \multirow[t]{2}{*}{ Duralon-36 } & Concrete & 10 & $\mathrm{HNO}_{3},-\mathrm{FP}$ & & & & $\mathrm{R}$ & $40-200$ \\
\hline & Steel & 10 & $\mathrm{HNO}_{3}, \mathrm{NaOH}$, hexone, $\mathrm{HNO}_{3}-\mathrm{FP}$ & $\mathbf{R}$ & $\mathbf{R}$ & $N$ & & \\
\hline Epon-395 & Steel & 8 & $\mathrm{HNO}_{3}, \mathrm{NaOH}, \mathrm{HCl}, \mathrm{H}_{2} \mathrm{SO}_{4}$, hexone & $\mathrm{R}$ & $\mathbf{R}$ & $\mathbf{R}$ & $\mathrm{R}$ & 200 \\
\hline \multirow[t]{2}{*}{ Epon-1001 } & Concrete & 12 & $\mathrm{HNO}_{3},-\mathrm{FP}$ & & & & $\mathrm{R}$ & 5 \\
\hline & Steel & 8 & $\mathrm{HNO}_{3}, \mathrm{NaOH}, \mathrm{HCl}$, hexone, $\mathrm{HNO}_{3}-\mathrm{FP}$ & $\mathbf{R}$ & $\mathbf{R}$ & $\mathrm{N}$ & $\mathbf{N}$ & \\
\hline Neobon & Steel & 10 & $\mathrm{HNO}_{3}, \mathrm{NaOH}$, hexone, $\mathrm{HNO}_{3}-\mathrm{FP}$ & $\mathbf{R}$ & $\mathbf{R}$ & $\mathrm{N}$ & $\mathrm{R}$ & $3-8$ \\
\hline Nukemite-40 & Steel & 10 & $\mathrm{HNO}_{3}, \mathrm{NaOH}$, hexone & $\mathbf{N}$ & $\mathbf{N}$ & $\mathrm{N}$ & & \\
\hline \multirow[t]{2}{*}{ Solar silicone alkyd } & Concrete & 8 & $\mathrm{HNO}_{3}-\mathrm{FP}$ & & & & $\mathrm{R}$ & $8-15$ \\
\hline & Steel & 8 & $\mathrm{HNO}_{3}-\mathrm{FP}$ & & & & $\mathrm{N}$ & \\
\hline $\begin{array}{l}\text { * Symbols for re } \\
\dagger C / D \text { is the pro } \\
\ddagger D F \text { is deconta }\end{array}$ & $\begin{array}{l}=\text { resistant } \\
\mathrm{NO}_{3} \text {-fission }\end{array}$ & $\begin{array}{l}\mathrm{N}=\mathrm{no} \\
\text { roduct }\end{array}$ & $\begin{array}{l}\text { esistant. } \\
\text { ntamination and decontamination. }\end{array}$ & & & & & \\
\hline
\end{tabular}




\begin{tabular}{|c|c|c|c|c|c|c|c|c|}
\hline $\begin{array}{c}\text { Gamena dose } \\
\left(10^{8} r\right)\end{array}$ & $\begin{array}{l}\text { Specific gravity } \\
\quad\left(\left(\mathrm{m} / \mathrm{cm}^{3}\right)\right.\end{array}$ & $\begin{array}{l}\text { Tensile strength } \\
\quad\left(10^{3} \mathrm{psi}\right)\end{array}$ & $\begin{array}{l}\text { Shear strength } \\
\quad\left(10^{3} \mathrm{psi}\right)\end{array}$ & $\begin{array}{c}\text { Elongation } \\
(\%)\end{array}$ & $\begin{array}{c}\text { Breaking energy } \\
\text { (ft-lb per in. } \\
\text { of notch) }\end{array}$ & $\begin{array}{c}\mathrm{H}_{2} \mathrm{O} \text { absorbed } \\
(\%)\end{array}$ & $\begin{array}{l}\text { Haze } \\
(\%)\end{array}$ & $\begin{array}{c}\text { Hardness } \\
\text { (Rockwell } R)\end{array}$ \\
\hline \multicolumn{9}{|l|}{ Polyethylene } \\
\hline 0 & 0.914 & 1.56 & 1.89 & 325 & 11.2 & 0.026 & 1.72 & -8.5 \\
\hline 1 & 0.920 & 1.64 & 1.81 & 28 & 5.6 & 0.035 & 1.83 & 14.3 \\
\hline 11 & 0.921 & 1.90 & 1.91 & 7.6 & 2.9 & 0.027 & 98 & 49.2 \\
\hline \multicolumn{9}{|c|}{ Polyethylene, $1 \%$ carbon filled } \\
\hline 0 & & 1.59 & 1.76 & 450 & 10.3 & & & -6 \\
\hline 5 & & 1.65 & 2.09 & $10-30$ & 3.1 & & & 34 \\
\hline 10 & & 1.80 & 2.15 & $10-30$ & 2.9 & & & 66 \\
\hline \multicolumn{9}{|l|}{ Polystyrene } \\
\hline 0 & 1.049 & 4.64 & 5.9 & 0.75 & 0.45 & 0.054 & 12.5 & 124 \\
\hline 0.01 & & 4.2 & 5.5 & 0.78 & 0.29 & & & 124 \\
\hline 0.1 & & 5.4 & 6.1 & 1.03 & 0.23 & & & 124 \\
\hline 1 & 1.051 & 3.3 & 5.6 & 0.45 & 0.30 & 0.089 & 8.3 & 122 \\
\hline 10 & 1.053 & 5.0 & 5.6 & 0.99 & 0.19 & 0.089 & 14.2 & 121 \\
\hline \multicolumn{9}{|l|}{ Tefion } \\
\hline 0 & 2.14 & 3.76 & 3.01 & 175 & 2.65 & 0.0053 & 5.5 & \\
\hline 1 & 2.21 & Failed & 0.483 & Failed & 0.37 & 0.014 & 14.6 & \\
\hline 10 & 2.19 & Failed & 0.094 & Failed & 0.30 & 0.345 & & \\
\hline \multicolumn{9}{|l|}{ Kel-F } \\
\hline 0 & & 2.55 & 3.41 & 264 & & & & \\
\hline 0.01 & & 2.40 & 3.65 & 230 & & & & \\
\hline 0.1 & & 1.67 & 1.85 & 73 & & & & \\
\hline 1 & & Failed & Failed & Failed & & & & \\
\hline
\end{tabular}

In most cases the greater stability of coatings on concrete may be attributed to: (a) the greater chemical resistance of concrete to attack by gaseous and liquid decomposition products from the coating, and (b) the absorption of these products in the porous concrete structure. In the case of vinyl coatings, the chemical attack that takes place is from halide acids.

Of the 23 mounted coatings tested, only four polymer-base types did not fail at the maximum doses used: furanes, epoxys, silicone-alkyds, and one modified phenolic specimen. Eight coatings that exhibit radiation stability, plus three examples of low-irradiated vinvl coatings and one neoprenebase coating, were tested for chemical stability and decontaminability after irradiation.

Table 3 shows that six of the irradiated coatings are not resistant to the organic solvent hexone. In addition, several lacking solvent resistance also do not resist either acids or a base. Two other coatings, the vinyl-based Amercoat-33 and Corrosite-22, show good decontaminability but fail at higher gamma doses $\left(2.4 \times 10^{8} \mathrm{r}\right)$. The silicone-alkyd coating is difficult to decontaminate on concrete and fails on a steel-panel surface during the process of its contamination.

Two types of coatings, one each of the furane-based and epoxy-based coating (Alkaloy-550 and Epon-395), show excellent chemical resistance and decontaminability. The other two coatings of similar polymer base, Duralon36 and Epon-1001, both lack solvent resistance after irradiation.

\section{Gasket Materials}

Gasket materials were irradiated in leakproof containers lowered into a vessel surrounded by $\mathrm{Co}^{60}$ slugs. Radiation intensity varied, but only the total dose of each specimen is recorded in Table 4.

In early tests, corrosive gases containing fluorine were detected being evolved from irradiated Teflon. Tests were made to determine the quantity of fluorine evolved from Teflon disks (1 in. dia., 1/8 in. thick) and Teflon molding powder during irradiation, the quantity of degraded fluorine within the irradiated disks that was released during the 30-day period immediately after irradiation, and the effects of these gases on stainless steel flanges carrying $50 \% \quad \mathrm{HNO}_{3}$ solutions. To measure the quantity of fluorine evolved, the specimens were immersed in a $\mathrm{NaOH}$ solution during irradiation, and then the solution was analyzed for fluoride content. No attempt was made to determine in what compounds the fluorine was evolved.

In many cases only one reading could be obtained from each specimen; so several specimens were necessary for each series of tests. Since perfectly uniform specimens could not be obtained, only changes in properties that are greater than the variations in the specimens could be detected.

Results. The most evident observation is the difference between the radiation stability of halogenated polymers, Teflon and Kel-F, and nonhalogenated polymers, polyethylene and polystyrene (see Table 4). Both Teflon and Kel-F evolve corrosive gases and are degraded completely by irradiation to $10^{8} \mathrm{r}$, while polyethylene and polystyrene appear very resistant to irradiation to and beyond this level.

Polyethylene, polystyrene. The only major difference between the behavior of polyethylene and polystyrene is the marked increase in the hardness of polyethylene after irradiation. Polystyrene is the most resistant gasket material tested and probably would be satisfactory for use at radiation levels up to $10^{9} \mathrm{r}$. But the rate of hardening of polyethylene may limit its use to ra- 
diation dosages of about $10^{9}$ roentgen.

Since inorganic fillers often make organic plastics more resistant to radiation, a $1 \%$-carbon-filled polyethylene was tested to compare this material with natural polyethylene. But no difference in stability could be detected.

Corrosive gases. Corrosive gases evolving from gaskets during irradiation not only indicate deterioration of the gasket, but the gases also may damage flanges or other equipment. No evolution from Teflon is detected at radiation levels less than $10^{6} \mathrm{r}$ (see Fig. 1). The appreciable quantity of fluorine given off by the Teflon disks during the 30-day period after irradiation and the difference between the quantity of fluorine evolved from the disks and the molding powder show that released fluorine is accumulated within the specimen before diffusing through the surface. Data on halide evolution from polymonochlorotrifluoroethylene and more complete data on changes in the physical properties of Kel-F are reported elsewhere (1).

Flange corrosion. Figure 2 shows how these gases affect stainless steel flanges immersed in $50 \% \mathrm{HNO}_{3} . \mathrm{A}$ light brown area is noted on the flange where the steel had been in contact with Teflon. Microscopic examination reveals masses of tiny pits; all other surfaces of the flange remain as bright as before the test. Control specimens of stainless steel in the gamma field (without contact with Teflon) were corroded by $50 \% \mathrm{HNO}_{3}$ at a rate less than $1 \mathrm{mil} / \mathrm{yr}$. No difference could be detected between the corrosion rates of the control specimens with and without irradiation.

Hycar, silicone rubber. As shown in Table 5, the elastomeric properties of Hycar and silicone rubber are stable at radiation levels not greater than $10^{7} \mathrm{r}$. However, between $10^{7} \mathrm{r}$ and $10^{8} \mathrm{r}$ there are considerable changes in the properties of both materials, largely embrittlement processes. After $10^{8} \mathrm{r}$ both types of silicone rubber deteriorate to a material with approximately the same mechanical properties.

\section{Asphalts and Tars}

The need for a cheap method of indefinitely storing radioactive waste solutions resulting from the reprocessing of spent reactor fuels prompted an investigation of impervious lining materials for earthen basins. Asphalts and tars, because of cheapness and ease

TABLE 5- How Gamma Radiation Affects Gasket Materials

\begin{tabular}{|c|c|c|c|c|c|c|}
\hline \multirow{3}{*}{$\begin{array}{c}\text { Gamma } \\
\text { dose } \\
(r)\end{array}$} & \multirow{2}{*}{\multicolumn{2}{|c|}{ Tensile strength }} & \multirow{2}{*}{\multicolumn{2}{|c|}{ Elongation }} & \multicolumn{2}{|c|}{ Hardness } \\
\hline & & & & & & \\
\hline & $\left(10^{3} p s i\right)$ & (\% change) & $(\%)$ & (\% change) & (Shore) & change) \\
\hline \multicolumn{7}{|c|}{ Silicone 12602} \\
\hline 0 & 0.277 & & 58 & & 62 & \\
\hline $10^{4}$ & 0.245 & -12 & 50 & -14 & 65 & 4.8 \\
\hline $10^{5}$ & 0.260 & -6.1 & 62 & 6.9 & 66 & 6.5 \\
\hline $10^{7}$ & 0.282 & 1.8 & 25 & -57 & 74 & 19 \\
\hline $10^{8}$ & 0,151 & -46 & 5 & -91 & 95 & 53 \\
\hline \multicolumn{7}{|c|}{ Silicone 12603} \\
\hline 0 & 0.555 & & 36 & & 84 & \\
\hline $10^{4}$ & $0.5+0$ & -2.7 & 36 & 0 & 84 & 0 \\
\hline $10^{6}$ & $\longrightarrow$ & - & 一 & - & 85 & 1.2 \\
\hline $10^{7}$ & 0.528 & -4.9 & 25 & -31 & 87 & 3.6 \\
\hline \multirow[t]{2}{*}{$10^{3}$} & 0.135 & -76 & 5 & -86 & 86 & 2.4 \\
\hline & $B$ & $B$ & $B$ & $A$ & $A$ & $A$ \\
\hline
\end{tabular}

Hycar OR-25*

\begin{tabular}{|c|c|c|c|c|c|c|c|c|c|c|}
\hline 0 & 2.74 & 2.26 & & & 275 & 365 & & & 72 & \\
\hline $10^{4}$ & 1.88 & 2.33 & -32 & 3.1 & 230 & 371 & -16 & 1.6 & 77 & 6.9 \\
\hline $10^{6}$ & 2.42 & 2.40 & -12 & 6.2 & 255 & 358 & -7 & -1.9 & 78 & 8.3 \\
\hline $10^{7}$ & 2.59 & 2.64 & -5 & 17 & 208 & 185 & -24 & -49 & 81 & 13 \\
\hline $10^{8}$ & 1. 63 & 2.04 & -40 & -9.7 & 35 & 32 & -87 & -91 & 92 & 28 \\
\hline \multicolumn{11}{|c|}{ Aycar PA-21 * } \\
\hline 0 & 1.36 & 1.57 & & & 190 & 145 & & & 71 & \\
\hline $10^{4}$ & 1. 29 & 1.40 & -5.1 & -11 & 155 & 130 & -18 & -10 & 70 & -1.4 \\
\hline $10^{6}$ & 1.33 & 1.40 & -2.2 & -11 & 150 & 130 & -21 & -10 & 73 & -2.9 \\
\hline $10^{7}$ & 1.33 & 1.38 & -2.2 & -12 & 135 & 106 & -29 & -27 & 72 & 14 \\
\hline $10^{8}$ & 0.81 & 0.97 & -40 & -38 & 50 & 41 & -74 & -72 & 80 & 13 \\
\hline
\end{tabular}

* Two series of tests ( $A$ and $B$ ) were made with these materials.

of application, were selected for tests of radiation damage and chemical resistivity.

As shown in Fig. 3 and Table 6, asphalts and tars retain their ductility and are hardened (but not excessively) with increasing exposure up to $10^{9} \mathrm{r}$ total dose. From the slopes of the lines, there is evidence that excessive hardness may occur at $10^{10} \mathrm{r}$.

Asphalts and tars evolve gases (principally hydrogen) during irradiation. The asphalt assumes a vesicular or honeycombed structure of individual cells that probably would not develop leaks in actual service. Irradiated tars exhibit this structure to a slight extent. Both are adequately resistant to neutralized radioactive wastes up to $200^{\circ} \mathrm{F}$, but attack by acid wastes is severe.

Thus, asphalts and tars (if a suitable cheap radiation-resistant reinforcing material for the tar can be found) can be used to contain radiochemical wastes in earthen basins if: (a) the total dose does not greatly exceed $10^{9} \mathrm{r}$, (b) the wastes are neutralized, and (c) the temperature of the stored waste is not permitted to rise above $200^{\circ} \mathrm{F}$.

It is estimated that an earthen basin of $10^{6} \mathrm{gal}$ capacity can be constructed with a $1 / 2$-in.-thick asphalt lining, covered with $2 \mathrm{ft}$ of compacted earth and complete with roof, for $3-5 \mathrm{c} / \mathrm{gal}$ of stored waste.

\section{Ion Exchange Resins}

Preliminary work has established the order of magnitude of radiation damage to ion exchange resins as shown by a loss in capacity. While the capacity loss is possibly the most important factor in the use of ion exchange resins for radiochemical processing at high activity levels, other effects that must be considered include changes in the physical and chemical character of the material. Figures 4 and 5 show the effect of $\mathrm{Co}^{60}$ gamma rays on the capacity of Dowex A-1 anion exchange resin and on Dowex 50 cation exchange resin as a function of the energy absorbed. A third resin, Dowex 30 , a sulfonated phenolic cation exchange resin, shows a capacity loss of only $1 \%$ per watt-hr of energy absorbed per gram as compared 
TABLE 6- How Gamma Radiation Affects Coal Tar Products*

\section{Characteristic}

Roofing pitch

Gamma dose, $r$

Softening point (ASTM D36), ${ }^{\circ} \mathrm{F}$

Softening point (ASTM D61), ${ }^{\circ} \mathrm{F}$

Penetration at $77^{\circ} \mathrm{F}, 100 \mathrm{gm}, 5 \mathrm{sec}$, $\mathrm{mm} / 10$

Penetration at $32^{\circ} \mathrm{F}, 200 \mathrm{gm}, 60 \mathrm{sec}$, $\mathrm{mm} / 10$

Penetration at $115^{\circ} \mathrm{F}, 50 \mathrm{gm}, 5 \mathrm{sec}$, $\mathrm{mm} / 10$

Ductility at $77^{\circ} \mathrm{F}, 5 \mathrm{~cm} / \mathrm{min}$, $\mathrm{cm}$

Ductility at $90^{\circ} \mathrm{F}, 5 \mathrm{~cm} / \mathrm{min}, \mathrm{cm}$

Ash, \%

\begin{tabular}{|c|c|c|c|c|c|c|c|}
\hline 0 & $5 \times 10^{8}$ & 0 & $5 \times 10^{8}$ & 0 & $5 \times 10^{8}$ & 0 & $5 \times 10^{3}$ \\
\hline- & - & 199 & 203 & 192 & 197 & 237 & 248 \\
\hline 158 & 161 & - & - & 169 & 168 & - & - \\
\hline 5.5 & 5 & 9 & 8.5 & 0 & 0 & 15 & 7.5 \\
\hline 0 & 0 & 1 & 1 & 0 & 0 & 5 & 0.5 \\
\hline 84 & 70 & 26 & 24 & 1.5 & 1.5 & 31 & 22.5 \\
\hline 67 & 17.5 & 2 & 2 & 0 & 0 & 3 & 1.5 \\
\hline - & - & - & - & 1.5 & 1 & - & - \\
\hline 0.08 & $1.04 \dagger$ & 30.7 & 28.2 & 16.9 & 16.8 & 29 & 295 \\
\hline
\end{tabular}

* Test specimens supplied by the Barrett Div. of Allied Dye and Chemical Corp., Edgewater, N. J.

† Similar to ferric oxide residues, probably due to metallic contamination.

to the losses of $15-40 \%$ for Dowex $\mathrm{A}-1$ and Dowex 50. The variations in capacity loss demonstrate that radiation damage can perhaps be minimized with the proper choice of resin, provided that the chemical stability of the resin is satisfactory (2).

Decomposition of Dowex $50 \mathrm{~W}$ resin by alpha bombardment is observed when $\mathrm{Am}^{241}$ is isolated and concentrated by cation exchange. During loadings of about $0.5 \mathrm{gm} \mathrm{Am}$ per liter of resin, darkening, presumably charring, of the resin bed is observed within 24 hr (3). But this phenomenon does not deleterrously affect the cation-exchange procedure.

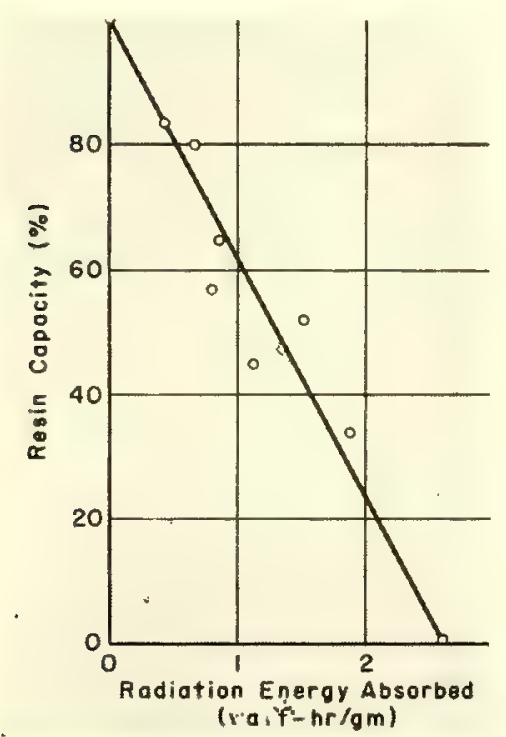

FiG. 4. Capacity loss of Dowex A-1 anion-exchange resin (quaternary amine polystyrene) caused by $\mathrm{Co}^{60}$ gammas

\section{Aqueous Solutions}

The chemical effects produced by ionizing radiations on aqueous solutions can be divided into two parts: (a) indirect action of $\mathrm{H}$ and $\mathrm{OH}$ radicals formed by water decomposition reacting with solute particles and with one another, and (b) direct action of radiation on solute particles followed by chemical change. The primary mechanism of water decomposition is

$$
\begin{aligned}
\mathrm{H}_{2} \mathrm{O} & \rightarrow \mathrm{H}+\mathrm{OH} \\
2 \mathrm{H}_{2} \mathrm{O} & \rightarrow \mathrm{H}_{2}+\mathrm{H}_{2} \mathrm{O}_{2}
\end{aligned}
$$

The radicals $H$ and $O H$ are available for reaction with solutes or with one another to form $\mathrm{H}_{2}$ and $\mathrm{H}_{2} \mathrm{O}_{2}$. In

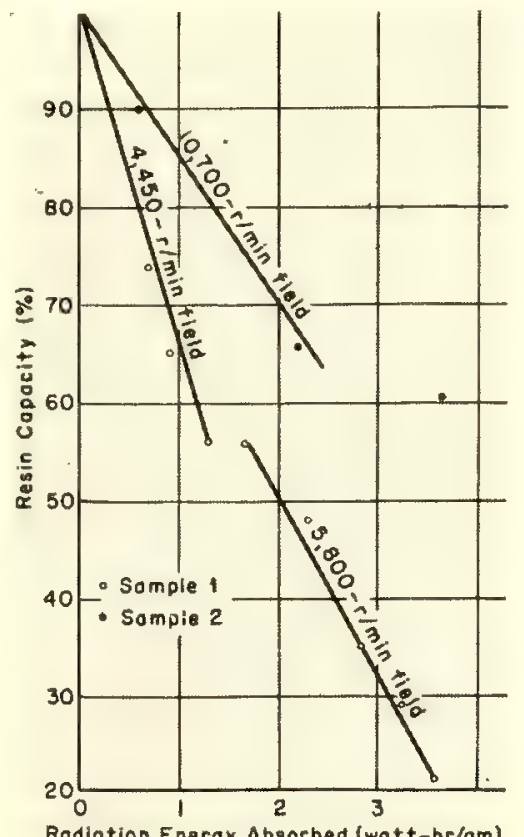

FIG. 5 (right). Effect on Dowex 50 cation-exchange resin (sulfonated polystyrene) regions of low ionization density, most radicals react with solutes, while in regions of high ionization density, radicals recombine to form the products shown in Eq. 2. Since $\mathrm{H}, \mathrm{OH}, \mathrm{H}_{2} \mathrm{O}_{2}$, and the intermediate perhydroxy radical $\mathrm{HO}_{2}$ are either strong oxidizing or reducing agents, oxidation or reduction reactions occur with many of the solutes present. In the direct absorption of radiation by the solute, oxidation or reduction results, since electrons are transferred or removed in the process.

Chromiumvi ions, for example, are reduced to trivalent chromium in acidic acetic acid-sodium acetate buffered solutions when subjected to intense gamma radiations. The reduction rate of a $0.5 \mathrm{M}$ chromate solution buffered with $4.0 \mathrm{M}$ acetate is $2.35 \pm 0.15$ millimoles/keal at $p \mathrm{H} 4$ in a gamma field of about $10^{7} \mathrm{r} / \mathrm{hr}$. The decomposition rate is reduced to $0.51 \pm 0.15$ millimoles/kcal by the addition of $0.5 \mathrm{M}$ sodium bromide as a "protector" (4).

The authors wish to acknowledge the assistance of the following in carrying out the radiation-damage test program for protective coatings and gaskels: A. J. Hoiberg, Lion Oil Co., a division of Monsanto Chemical Co.: G. A. West, F, L. Rogers, G. R. Guinn, R. J. HeNamee, Union Carbide Nuclear Co. $(O R N L)$; M. Tyner, Jr., Univ. of Florida; J.N.Hunsberger of $E, I$. du Pont de Nemours \& Co.; and the various companies that supplied specimens.

\section{BIBLIOGRAPHY}

1. Ind. Eng. Chem:45, 2549 (1953)

D. G. I. Cathers, "Radiation Damage to Radiochemical Processing Reagents," Paper 584. Geneva Conference on Peaceful L'ses of Atomic Energy (1955)

3. R. H. Rainey, ORNL, personal communication $(1456)$

4. R. L. Andelin, E. L, Anderson, IDO- $1+347$ (1955) 
Today's nuclear technology depends on critical facilities for information in four important areas: 1) reactor design, 2) weapons-systems design, 3) criticality limits on processing operations and 4) basic physics data.

$B y$ far the largest number of the critical facilities in the $U$. S. are devoted to reactor design. In these the experimenter seeks to achieve a delicate balance between neutron production and loss over the full range of reactor operating conditions.

For processing-operation critical experiments, the emphasis is on setting safety limits to ensure that batches of fissionable materials will never support a self-sustaining reaction in operations such as storage and shipping, isotope separation, chemical processing of irradiated fuel and fertile material, and fabrication of fuel elements. Through critical experiments, safety factors are established for systems of varying geometry, composition, fuel concentration and degree of coupling.

In contrast, weapons criticals are concerned with the understanding of supercritical assemblies with very short neutron lifetimes. Hence such experiments concentrate on determining the static and kinetic behavior of unmoderated nuclear systems.

Critical facilities that perform any of these first three functions can also be used to obtain basic physics information. In addition much useful data has come from "pure-geometry" critical assemblies constructed especially for physics experiments.

\section{Criticall Facilities for Reactor Design}

By W. C. REDMAN, Argonne National Laboratory, Lemont, Illinois

IN MANY WAYS the critical facility bears the same relation to reactor design as the wind tunnel does to aircraft design. Just as the aeronautical engineer at a certain point in the design process looks to the wind tunnel for erucial information so the nuclear engineer must check out his proposed reactor design in a critical facility before he can confidently begin construction of the fullscale reactor.

In principle, a complete knowledge of all of the pertinent neutron cross sections and yields as a function of energy would enable the reactor designer to calculate the neutron distribution in space and energy and thereby specify the reactor configuration in all detail. In practice, the large gaps in our knowledge of the nuclear interaction parameters and the limited accuracy of many of the existing data make the use of refined theories and improved calculational techniques of questionable value in reactor design.
For example, theoretical calculations predicted a reference design loading for EBIVR of 42 fuel assemblies with a probable error of $\pm 3 \%$ in $k$. As such calculations go this one would appear reasonably accurate; however, in terms of fuel loading $+3 \%$ correspunds to almost a factor of two in fuel loadsome 40 extra fuel assemblies. For EBWR the observed loading fell just within the probable error, requiring a total of 81 assemblies. A much more accurate estimate for the loading could have been given by a critical experiment-and in fact this is what the first EBWR runs were used to do (NU, July' 57,60 ).

A critical experiment can be compared to a computer in which a Monte Carlo type of calculation is performed. The appropriate neutron cross sections are inherent in the system being studied, and the life histories of a tremendous quantity of neutrons are "calculated" simultaneously. Thus critical experiments afford a means of obtaining required answers without a knowledge of the neutron cross sections. Analysis of the results of critical experiments enables the reactor theorist to arrive at better parameter values for use in reactor-design calculations.

\section{Experimental Objectives}

Critical experiments serve primarily to yield information on the nuclear performance of the heart of the reactor system-namely, the reactor core and reflector.

Working within the limits set by heat-transfer, metallurgical and nuclear considerations, the critical experimenter seeks an optimum design. Variables typically at his disposal include quantity and enrichment of fuel, amount of moderator, dimensions of the reactor, lattice spacing of fuel channels, arrangement of fuel in the channel, area of coolant channel around fuel, physical properties and pressure of 
coolant and parasitic absorber present as cladding and structure.

Changes in design that improve heat transfer, such as decrease in coolantchannel flow length, increase in size of channel, flux flattening, decrease in moderator-fuel ratio and subdivision of the fucl for increased heat-transfer surface, usually affect the neutron economy adversely. Consequently, a combination of heat-transfer and critical experiments is desirable to achieve a reliable core design.

\section{Typical Program}

The duration of the critical experiments depends upon several factors including the novelty of the reactor design, the importance of reliability in operation, the amount of pertinent data otherwise accumulated prior to startup and pressure to achieve early operation of the reactor.

Exponential experiments. If time permits and if materials that make possible a reasonable simulation of the proposed design are available, a series of exponential experiments may first be initiated to explore nuclear characteristics of assemblies having a range of values for lattice spacing, clustering, fuel concentration, enrichment, etc. Exponential experiments involve a thermal-neutron sourcé plus an assembly containing only a fraction of the material required for a critical assembly with its self-sustaining chain reaction. Generally, an exponential program includes only a measurement of buckling of a particular system although the availability of a high-intensity neutron source, such as a thermal column of a power reactor, makes possible the measurement of lattice parameters and neutron spectra. The main advantage of an exponential assembly is that the system is operated well below criticality and consequently requires no elaborate instrumentation, control and shielding systems. The disadvantage is that the accuracy of information observed is limited and results of measurements of nonuniform systems require extensive analysis and involve uncertainties in interpretation.

Critical experiments. Once a tentative configuration and size for the core have been chosen either by calculation or by exponential experiments, detailed knowledge of the nuclear characteristics of the proposed design is determined through a series of critical experiments.
The initial phase of a typical program will include:

- determining the cold, clean critical mass by step-wise addition of the selected materials

- determining reflector savings from measurements of radial and axial flux distributions

- evaluating the factors comprising $k_{\infty}$ from observed fission, capture and flux patterns in a representative lattice cell

- measuring the migration area $\left(M^{2}=\tau+L^{2}\right)$ by adding distributed nuclear poisons or making dimensional changes in the core.

The program might then turn to an investigation of reactivity coefficients. Experimental evaluation of the temperature effects on reactivity generally involves a combination of actual heating of the entire assembly over a limited range and heating of a sample of fuel to an appreciably higher temperature. Some indirect effects, such as density changes, can be simulated by substitution of materials; for example, Styrofoam or organic materials can be used to mock up the reduced density of water at high temperature. The magnitude of other reactivity effects occurring during operation of a power reactor, such as fuel burnup, fissionproduct production (particularly $\mathrm{Sm}^{149}$ and $\left.\mathrm{Xe}^{135}\right)$, and formation of various plutonium isotopes, is normally calculated.

Next the critical experimenter's attention may turn to evaluating controlrod effectiveness. The cold, clean critical is loaded with rods of the proper composition, number, size, shape and location to give the required reactivity margin. The rods are generally added individually or in symmetric groups and the system restored to criticality after each addition. The selection of rods is also influenced by considerations of heat removal so that the determination of power-production patterns is an important part of this stage of the program.

Zero-power experiments. The measurement of detailed flux-distribution patterns for various rod configurations expected throughout the core life is carried out in the zero-power system. Since zero-power experiments may use assemblies of the actual fuel elements and control rods or perhaps the actual core itself (see p. 53), they are well suited for locating possible hot spots. Distributed neutron absorbers are introduced to permit the various rod positions anticipated in actual power operation, and differential calibration curves for the rods are obtained during the course of the flux-mapping program. Flux flattening by nonuniform distribution of fuel can also be investigated at this time.

Other important information that can be obtained from critical-assembly measurements includes:

- The interrelation between the location and strength of the startup source and the location and sensitivity of the instruments for control.

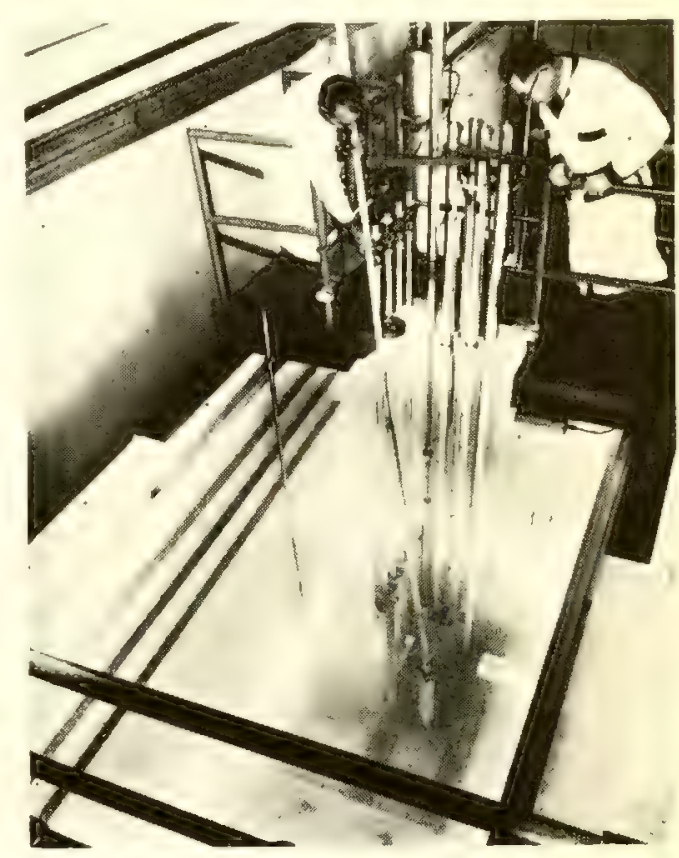

ASSEMBLING an experimental core in Babcock \& Wilcox's pool-type facility

- Measurement of neutron and gamma radiation intensities to determine the effectiveness and heating of the shield.

- Neutron economy (conversion or breeding ratio).

The extensive manipulation of the components in a critical assembly requires that the induced activity be kept low. Moreover the materials and design generally preclude operation at very high temperatures. Consequently, critical experiments are not used for investigation of effects requiring high neutron fluxes and/or high temperatures. Thus a program of critical experiments gives no information on heat transfer, radiation damage or kinetic behavior at high power density. 


\section{Critical Facilities for Basic Physics}

By H. C. PAXTON, Los Alamos Scientific Laboratory, Los Alamos, New Mexico

Critical assemblies that are intended to supply basic reactor physics data are distinguished from their reactor-development counterparts primarily in degree of complexity. The types of measurements-for example, determining critical configurations and observing related neutron and fission characteristics, time behavior and effects of perturbations-cannot differ significantly. Thus the general facility requirements are the same. There must be provision for safe operation without sacrifice of convenience. For satisfactory measurements, assemblies must be reproducible, while reasonably flexible, and instrumentation must be reliable.

The "clean-critical" assembly is the basic source of reactor-physics data, whether the fissionable material is present in solution or slurry, as a lattice, or as a single piece of metal. The object is a complete understanding of elementary assemblies, to serve as a springboard for the design of practical, more complex reactor systems, either steady power producers or nuclear explosives, or to provide nuclear safety guidance.

A complete experimental survey of a certain class of clean criticals involves an overwhelming number of parameters; these describe such things as the nature and distribution of the fissionable materials, moderators and other diluents, and the type, thickness and shape of the reflector. In practice, experimental surveys are limited in scope and depend upon parallel computing programs to complete the survey by means of interpolation and extrapolation. Indeed, computing schemes are potentially so valuable for reactor design and for filling in basic nuclear safety data that verifying them and providing input parameters for them have become major preoccupations of critical-assembly organizations.

Although the same critical facility can be used for all types of basic physics measurements, one can distinguish three major categories:

Critical surveys. There is no question that the simplified analytical schemes - such as diffusion theory for thermal reactors and the extrapolatedend-point method for fast systems- are the work horses of reactor design. However, these schemes depend for their success on the proper choice of certain parameters (e.g., Fermi age, resonance escape, etc.) that are best obtained from critical surveys of simple geometries.

Experimental critical-mass surveys useful for this purpose are illustrated by compilations intended for nuclear safety guidance. Even more pertinent to reactor development are moderated lattices of fissionable material, which are customarily interpreted in terms of simplified calculations. In this class

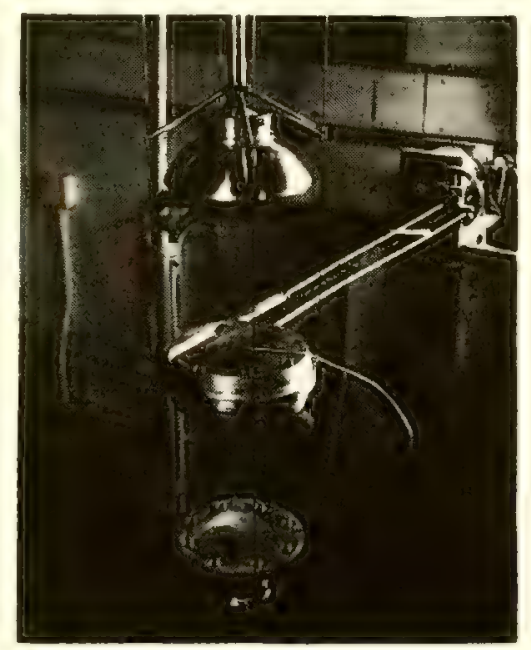

"JEZEBEL," a bare Pu sphere, is typical of "simple-geometry" critical assemblies used at Los Alamos to accumulate physics data

are the Brookhaven and Westinghouse water-moderated lattices of slightly enriched uranium and the Argonne heavy-water moderated lattices of $\mathrm{U}^{235}$ and various diluents.

Generally, assemblies for this purpose should have a simple unambiguous description at critical, instrumentation for measuring neutron multiplication during approach to critical, and instrumentation for period measurement to calibrate controls so that correction can be made for their influence.

Detailed computation checks. Simple critical-mass data are insufficient to verify detailed analytical schemes such as multigroup, transport or Monte Carlo programs; these schemes and their input data require checking against all available properties of clean critical assemblies before they are ready for use with practical reactors.

Some satisfying confirmations of detailed calculations, for instance, have been made for the $S_{n}$ transport method by observing the properties of simple fast-neutron assemblies of $\mathrm{U}^{235}, \mathrm{Pu}^{239}$, and $\mathrm{U}^{233}$ metal.

In addition to requirements stated earlier for good critical-mass data, assemblies for checking detailed computations must provide internal neutron detectors to map flux and neutron spectra. Other useful measurements such as the Rossi alpha, pulsed-source data and the reactivity effects of changes in core density make further demands on the assembly and its instrumentation.

Input-parameter measurements. Frequently reactor-calculation parameters may best be had by direct observation on clean critical assemblies. Usually the critical system is modified so that the resulting change in critical conditions is a measure of the parameter in question. For instance ORNL measured $\eta$ for $\mathrm{U}^{233}$ by comparing critical dilute aqueous solutions of $\mathrm{U}^{233}$ and $\mathrm{U}^{235}$ in spherical containers of the same size.

Since the ratio of the just-critical fuel concentrations depends primarily upon $\eta\left(\mathrm{U}^{233}\right), \eta\left(\mathrm{U}^{235}\right)$ and the thermalabsorption cross sections of $\mathrm{U}^{233}, \mathrm{U}^{235}$, and $\mathrm{H}, \eta\left(\mathrm{U}^{233}\right)$ could be obtained in terms of the other better-known parameters.

Occasionally the critical facility will be used solely as a radiation source for an input-parameter experiment in much the same way as a test reactor is used to feed a thermal column. Thus the radiation may be studied on one day as an important property of the assembly, then used the next day for effects measurements. An example is, first, measurement of the spectrum of leakage neutrons from Godiva (the bare $U^{235}$ metal assembly) and, next, application of the neutrons to biological measurements. Similarly, the study of dynamic behavior of Godiva through prompt critical led to its use as a pulsed neutron source. 


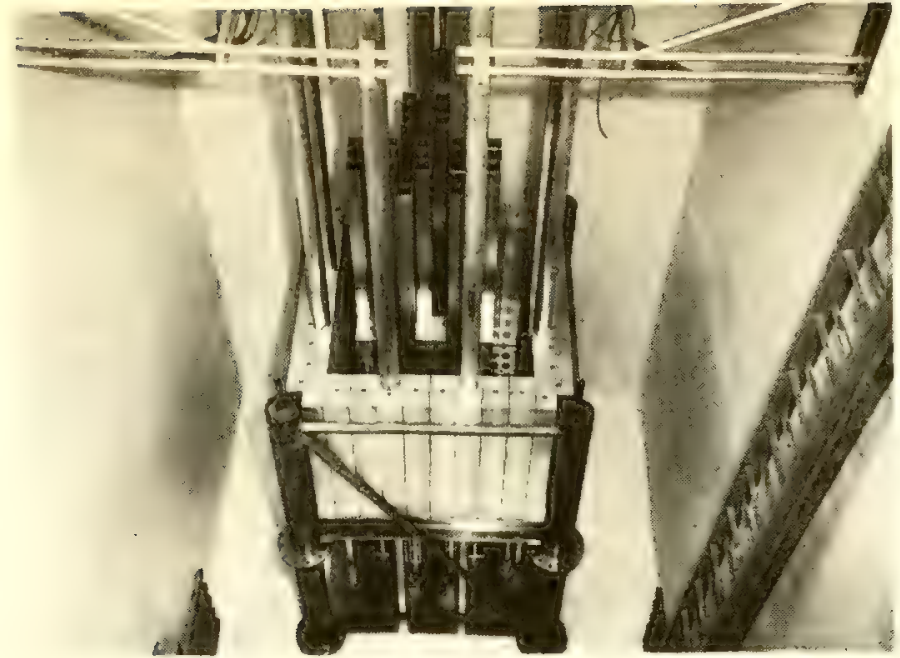

NEW TREND has been introduced by Engineering Test Reactor Critical Facility shown here. ETRC will be retained as permanent part of ETR operation to check out proposed test-reactor runs

\section{Critical Facilities Tomorrow}

Critical facilities are slated to become more rather than less important in the affairs of the reactor designer. As a means of turning out specific design information the critical experiment is proving superior to its rival, the multigroup computing-machine program. Outstanding reactor theorists such as B. Spinrad of Argonne and M. C. Edlund of Babcock \& Wilcox state quite frankly that, compared with detailed theoretical calculations, the reactor design information from critical facilities is cheaper and more accurate and takes less time to get.

To date most of the critical experiments supporting prototype reactor designs have been done in the national laboratories. The future should see private industry take over the greatest part of the task of developing specific design information of this kind; this will leave the national laboratories free to concentrate on more fundamental research.

In using critical facilities for reactor design the industry will very likely adopt the strategy used for the Army Package Power Reactor (APPR). Here critical experiments were performed in two distinct phases: First a highly flexible arrangement was used (at ORNL) to establish "ball-park" estimates for the critical mass and the reactivity available for control and shim. With this information the fuelelement design was frozen and a set of elements fabricated. These elements were assembled together with the actual APPR-1 core support structure and control-rod drives to form a "zeropower" experiment in Alco Products' critical facility (see p. 53). This second step gave refined figures for the critical loading and allowed optimization of core performance.

For the ball-park phase of critical experimentation future private reactor designers will need all-purpose facilities flexible enough to mock up a wide variety of concepts; eventually these will include advanced design concepts operating at extreme temperatures and heat flux. Larger concerns will find it profitable to operate their own flexible facilities. Others, who cannot justify full-time use of a facility, will look to organizations like Battelle Memorial Institute, Nuclear Development Corp. of America (NDA) and Alco Products, which are in the business of hiring out critical-facility services.

The second-stage zero-power experiments will be much more an individual problem for each reactor. These experiments may very well be carried out in the reactor vessel itself using as much of the actual core structure as can be safely frozen. (This has already

\footnotetext{
Meet the Prophets

The predictions on this page represent NUCLEONICS' summary of a collective crystal-gazing session on the subject of the future of critical facilities attended by Dixon Callihan (ORNL), Joel Chastain (Battelle), M. C. Edlund (B\&W), G. A. Linenberger (AGN), John Noakes (Alco), Hugh C. Paxton (Los Alamos), W. C. Redman (Argonne), D. V. Williams $(B \& W)$ and Roy Zimmerman (NDA).
}

been done with EBWR; see NUCLEonics July '57, 62.) Preferably the tests would be conducted at temperatures and pressures close to the design conditions. Since no heat flux is involved, fuel elements at this stage would not have to be real working models but only reasonably exact facsimiles; much of the fabrication expense could be saved by omitting welds and using looser tolerances for the mock-up elements.

Another problem that is just beginning to be studied is that of determining core characteristies at very high burnup and for recycled fuel. Zero-powertype experiments will probably be used to get this information; facsimile fuel elements containing radioactive fuel and fission products may be necessary.

Beyond the area of reactor design, critical facilities will find new applications as auxiliary facilities supporting routine reactor operations. Perhaps the first example of this is the Engineering Test Reactor critical facility (see figure). The ETRC has been retained as a permanent fixture to check out scheduled core loadings for the ETR before putting them in the ETR itself. Similarly every power reactor of the future may well come equipped with a modest on-site critical assembly to be used for routine measurements. These might include double-checking new fuel elements as they arrive from the manufacturer (presumably the manufacturer will have already checked the shipment in his own standard critical assembly), evaluating the reactivity worth of used elements and predicting the effects of modifications in core loading. 


\section{NUCLEONICS Survey of}

\begin{tabular}{|c|c|c|c|c|c|c|c|c|}
\hline Owner: & Alco & $\mathrm{AEC}$ & $\mathrm{AEC}$ & $\mathrm{AEC}$ & $\mathrm{AEC}$ & B\&W & $\mathrm{AEC}^{2}$ & $\mathrm{AEC}^{2}$ \\
\hline Designer: & Vitro & ANL & ANL & ANL & ANL & owner & Bettis & Bettis \\
\hline Location: & $\begin{array}{l}\text { Schenectady, } \\
\text { N. Y. }\end{array}$ & NRTS, Idaho & Lemont, III. & Lemont, Ill. & Lemont, Ill. & Lynchburg, Va. & $\begin{array}{l}\text { Pittsburgh, } \\
\text { Pa. }\end{array}$ & $\begin{array}{l}\text { Pittsburgh, } \\
\text { Pa. }\end{array}$ \\
\hline Name: & none & ZPR-3 & ZPR-4' & ZPR-5 & ZPR-7 & none & TRX & SFR-FA \\
\hline Startup: & $8 / 26 / 56$ & $10 / 20 / 55$ & $5 / 31 / 57$ & $6 / 28 / 56$ & $7 / 3 / 57$ & $1 / 15 / 57$ & 1953 & 1954 \\
\hline Copital investment: & $\$ 300,000$ & n.a. & n.a. & n.a. & n.a. & $\$ 700,000$ & & \\
\hline Use or operating charge: & $\$ 600 /$ day & n.a. & n.a. & n.a. & n.a. & n.a. & & \\
\hline Number of cells: & 1 & 1 & 1 & 1 & 1 & 3 & 1 & 1 \\
\hline \multicolumn{9}{|l|}{ Applications - } \\
\hline basic physics: & $\rightarrow$ & 3 & 2 & 2 & - & 3 & & \\
\hline bosic reactor physics: & 2 & 2 & 1 & 1 & 2 & 2 & & \\
\hline $\begin{array}{l}\text { specific reactor design: } \\
\text { specific reactor }\end{array}$ & 1 & 1 & - & 一 & 1 & 1 & & \\
\hline $\begin{array}{l}\text { operation \& safety: } \\
\text { Specific reactor designs }\end{array}$ & - & - & - & - & - & 一 & & \\
\hline investigated to date: & APPR-1 & $\begin{array}{l}\text { EBR-1 } \\
\text { EBR-2 }\end{array}$ & 一 & - & EBWR & $\begin{array}{l}\text { Indian Pt., } \\
\text { MSR, LMFRE }\end{array}$ & - & $\mathrm{S} 3 \mathrm{~W}, \mathrm{S4W}$ \\
\hline $\begin{array}{l}\text { Available for hire: } \\
\text { Materials available- }\end{array}$ & yes & yes 1 & yes ${ }^{1}$ & yes ${ }^{1}$ & yes 1 & yes & & \\
\hline fuel: & $\mathrm{UO}_{2}-\mathrm{SS}$ & $\mathrm{U}, \mathrm{Pu}$ & $\mathrm{U}$ & U & $\mathrm{UO}_{2}, \mathrm{ThO}_{2}$ & $\mathbf{U}, \mathrm{Th}$ & & \\
\hline moderator: & $\mathrm{H}_{2} \mathrm{O}$ & - & $\mathrm{H}_{2} \mathrm{O}$ & $\mathrm{H}_{2} \mathrm{O}$ & $\mathrm{D}_{2} \mathrm{O}$ & $\mathrm{H}_{2} \mathrm{O}$ & & \\
\hline coolant: & - & Al & $\mathrm{H}_{2} \mathrm{O}$ & Al & $\mathrm{D}_{2} \mathrm{O}$ & $\mathrm{H}_{2} \mathrm{O}, \mathrm{Bi}$ & & \\
\hline reflector: & $\mathrm{H}_{2} \mathrm{O}$ & U & $\mathrm{H}_{2} \mathrm{O}$ & $\mathrm{H}_{2} \mathrm{O}$ & $\mathrm{D}_{2} \mathrm{O}$ & - & & \\
\hline structure: & SS & $\mathrm{SS}, \mathrm{Zr}$ & $\mathrm{Al}$ & SS & Al & - & & \\
\hline control: & - & - & - & - & - & - & & \\
\hline References: & APAE-21 & - & ANL-5379 & ANL-5547 & ANL-5715 & - & & \\
\hline \multirow{2}{*}{$\begin{array}{l}\text { Owner: } \\
\text { Designer: }\end{array}$} & GE & AEC & $\mathrm{AEC}^{2}$ & $\mathrm{AEC}^{2}$ & $\mathrm{AEC}^{2}$ & $\mathrm{AEC}^{2}$ & $\mathrm{AEC}_{2}$ & $\mathrm{AEC}$ \\
\hline & owner & HAPO & KAPL & KAPL & KAPL & KAPL & KAPL & KAPL \\
\hline \multirow{7}{*}{$\begin{array}{l}\text { Location: } \\
\text { Name: } \\
\text { Startup: } \\
\text { Capital investment: } \\
\text { Use or operating charge: } \\
\text { Number of cells: } \\
\text { Applications- }\end{array}$} & $\begin{array}{l}\text { Pleasanton, } \\
\text { Calif. }\end{array}$ & $\begin{array}{l}\text { Richland, } \\
\text { Wash. }\end{array}$ & $\begin{array}{l}\text { Schenectady, } \\
\text { N. Y. }\end{array}$ & $\begin{array}{l}\text { Schenectady. } \\
\text { N. Y. }\end{array}$ & $\begin{array}{l}\text { Schenectady, } \\
\text { N. Y. }\end{array}$ & $\begin{array}{l}\text { Schenectady, } \\
\text { N. Y. }\end{array}$ & $\begin{array}{l}\text { Sehenectady, } \\
\text { N. Y. }\end{array}$ & $\begin{array}{l}\text { Sebenectady, } \\
\text { N. Y. }\end{array}$ \\
\hline & none & PCTR & PPA & ATR & PMA & FPR & CWA & PTR \\
\hline & $9 / 6 / 57$ & $10 / 25 / 55$ & 1948 & 1954 & 1954 & 1956 & 1957 & $4 / 10 / 58$ \\
\hline & $\$ 4,000,000$ & $\$ 250,000$ & & & & & & $\$ 2,000,000$ \\
\hline & n.a. & $\$ 300 /$ day & & & & & & n.. . \\
\hline & 2 & 1 & 1 & 1 & 1 & 1 & 1 & 1 \\
\hline & & & & & & & & \\
\hline basic physics: & 3 & 4 & & & & & & - \\
\hline basic reactor physics: & 2 & 2 & & & & & & $\begin{array}{l}1 \\
2\end{array}$ \\
\hline $\begin{array}{l}\text { specific reactor design: } \\
\text { specific reactor }\end{array}$ & 1 & 1 & & & & & & 2 \\
\hline $\begin{array}{l}\text { operation \& safely: } \\
\text { Specific reactor designs }\end{array}$ & - & 3 & & & & & & - \\
\hline $\begin{array}{l}\text { Specific reactor designs } \\
\text { investigated to date: }\end{array}$ & Dresden & PRTR & - & SAR & - & SAR & $\mathrm{DIG}$ & 一 \\
\hline \multirow{2}{*}{$\begin{array}{l}\text { Available for hire: } \\
\text { Materials available- } \\
\text { fuel: }\end{array}$} & yes & yes $^{d}$ & & & & & & no \\
\hline & $\mathrm{UO}_{2}$ & $\mathrm{U}-\mathrm{Al}, \mathrm{UO}_{2}-\mathrm{Pb}$ & & & & & & $\mathbf{U}$ \\
\hline moderator: & $\mathrm{H}_{2} \mathrm{O}$ & C & & & & & & $\mathrm{H}_{2} \mathrm{O}$ \\
\hline coolant: & $\mathrm{H}_{2} \mathrm{O}$ & - & & & & & & $\mathrm{H}_{2} \mathrm{O}$ \\
\hline reflector: & $\mathrm{Be}$ & $\mathrm{C}$ & & & & & & $\mathrm{H}_{2} \mathrm{O}$ \\
\hline structure: & $\mathrm{Al}$ & $\mathrm{C}$ & & & & & & SS, Zircaloy \\
\hline control: & boral & $\mathrm{Cd}-\mathrm{U}$ & & & & & & B-steel \\
\hline References: & GEA 6595-2 & HW-32791 & & & & & & n.a. \\
\hline
\end{tabular}

\section{Explanatory Notes}

Capital Investment-estimated total investment Use or operating charge-charge to user in case of commercial facilities; estimated operating cost in case of government facilities; fuel charges not included; day $=8 \mathrm{hr}$

Applications-what the facility is used for in order of importance

D.a. - not a vailable

Alco-Alco Products, Inc.

A1W-first generation Westinghouse reactor for aircraft carriers

ANL-Argonne National Laboratory (operated by Univ, of Chicago for $\mathrm{AEC}$ )
APPR-1-Army Package Power Reactor, Ft. Belvoir, $\mathrm{Va}$.

ARE-Aírcraft Reactor Experiment (dismantled) B\&W-The Babcock \& Wilcox Co.

Bettis-Bettis Field (operated by Westinghouse Electric Corp. for $\mathrm{AEC}$ )

BMI-Battelle Memorial Institute

BNL-Brookhaven National Laboratory (operated by Associated Universities, Inc. for AEC) BSR - Bulk Shielding Reactor, Oak Ridge, Tenn. CANEL-Connecticut Aircraft Nuclear Engine Laboratory

CE-Combustion Engineering, Inc.

D 1 G- first generation General Electric reactor for destroyers
EBR-Experimental Breeder Reactor, NRTS, EBWR-Experimental Boiling Water Reactor Lemont, Ill.

ETR-Engineering Test Resctor, NRTS, Idaho GCRE - Gas Cooled Reactor Experiment, NRTS GD-General Dynamic Corp., John Jay Hopkins Laboratory

GE-General Electric Co., Vallecitos Atomic Laboratory GEANP-General Electric Aircraft Nuclear Propulsion

HAPO-Hanford Atomic Products Operation (operated by General Electric Co, for AEC)

KAPL-Knolls Atomic Power Laboratory (operated by General Electric Co. for AEC) 


\section{Operating Critical Facilities in the U. S.}

\begin{tabular}{|c|c|c|c|c|c|c|c|c|c|c|}
\hline $\mathrm{AEC}^{2}$ & $\mathrm{AEC}^{2}$ & $\mathrm{AEC}^{2}$ & $\mathrm{AEC}^{2}$ & BMI & $A E C$ & $\mathrm{CE}$ & $\mathrm{CE}$ & GD & AEC & $\mathrm{AEC}$ \\
\hline Bettis & Bettis & Bettis & Bettis & owner & BNL & KAPL & owner & owner & GEANP & GEANP \\
\hline $\begin{array}{l}\text { Pittsburgh, } \\
\text { Pa. }\end{array}$ & $\begin{array}{l}\text { Pittsburgh. } \\
\text { Pa. }\end{array}$ & $\begin{array}{l}\text { Pittsburgh, } \\
\text { Pa. }\end{array}$ & $\begin{array}{l}\text { Pittsburgh, } \\
\text { Pa. }\end{array}$ & $\begin{array}{l}\text { W. Jefferson, } \\
\text { Ohio }\end{array}$ & Upton, N. Y. & $\begin{array}{c}\text { Windsor, } \\
\text { Conn. }\end{array}$ & $\begin{array}{l}\text { Windsor, } \\
\text { Conn. }\end{array}$ & $\begin{array}{l}\text { San Diego, } \\
\text { Calif. }\end{array}$ & $\begin{array}{l}\text { Evendale } \\
\text { Ohio }\end{array}$ & NRTS, Idaho \\
\hline PWR-FA & RPF & S5W-FA & AIW-FA & none & none & FCE & ACE & none & none & LPTF \\
\hline \multirow[t]{9}{*}{1954} & 1956 & 1956. & 1956 & $1 / 2 / 57$ & $8 / 15 / 55$ & $7 / 16 / 56$ & $11 / 27 / 56$ & $8 / 15 / 57$ & $8 / 30 / 54$ & $4 / 11 / 58$ \\
\hline & & & & $\$ 500,000$ & $\$ 300,000$ & n.a. & n.a. & n.a. & $\$ 1,700,000$ & $\$ 1,300,000$ \\
\hline & & & & $\$ 500 /$ day & $\$ 5,000 /$ day & n.a. & n.8. & n.8. & n.s. & n.8. \\
\hline & 1 & 1 & 2 & 1 & 2 & 1 & 1 & 1 & 2 & 2 \\
\hline & & & & & & & & & n.e. & n.a. \\
\hline & & & & - & - & - & - & - & & \\
\hline & & & & 2 & 1 & 1 & 2 & 2 & & \\
\hline & & & & 1 & 2 & 2 & 1 & 1 & & \\
\hline & & & & - & 3 & 一 & - & - & & \\
\hline \multirow[t]{9}{*}{ Shippingport } & - & S5W & AlW & GCRE & $\begin{array}{l}\text { MRR. NRR. } \\
\text { LMFRE }\end{array}$ & $\mathrm{glC}$ & SIO & TRIGA & n.a. & n.8. \\
\hline & & & & yes & no & ses & yes & yes & no & no \\
\hline & & & & $\mathbf{U}$ & $\mathbf{U}$ & $\mathrm{U}$ & $\mathbf{U}-\mathbf{Z} \mathbf{r}$ & $\mathrm{U}$ & & a.a. \\
\hline & & & & $\mathrm{H}_{2} \mathrm{O}, \mathrm{Be}, \mathrm{C}_{2} \mathrm{H}_{4}$ & $\mathrm{H}_{2} \mathrm{O}, \mathrm{C}$ & $\mathrm{C}_{2} \mathrm{H}_{4}$ & $\mathrm{H}_{2} \mathrm{O}$ & $\mathrm{ZrH}$ & & \\
\hline & & & & $\mathrm{Al}, \mathrm{C}_{2} \mathrm{H}_{4}$ & $\mathrm{H}_{2} \mathrm{O}$ & $\mathrm{C}_{2} \mathrm{H}_{4}$ & $\mathrm{H}_{2} \mathrm{O}$ & - & & \\
\hline & & & & $\mathrm{H}_{2} \mathrm{O}, \mathrm{Be}, \mathrm{C}_{3} \mathrm{H}_{4}$ & C, $\mathrm{H}_{2} \mathrm{O}$ & $\mathrm{C}_{2} \mathrm{H}_{4}$ & $\mathrm{H}_{2} \mathrm{O}$ & C & & \\
\hline & & & & SS, Al & $\mathrm{Al}, \mathrm{SS}$ & $\mathrm{Al}_{\mathrm{r}} \mathrm{Zr}$ & $\mathrm{Zr}_{\mathrm{r}}$ & $\mathbf{Z r}$ & & \\
\hline & & & & B, C, W, Cd & SS, B-steel, Cd & - & - & - & & \\
\hline & & & & TID-4500 & - & - & - & - & n.a. & n. $\mathbf{a}$. \\
\hline USAF & Martin & NDA & AEC & AEC & $A E C$ & $\mathrm{AEC}^{2}$ & AEC & AEC & USAF & Westinghouse \\
\hline $\begin{array}{l}\text { GE/Lock- } \\
\text { heed }\end{array}$ & owner & owner & ORNL & ORNL & ORNL & ORNL & Phillips & Phillips & CANEL & owner \\
\hline $\begin{array}{l}\text { Dawsonville, } \\
\text { Ga. }\end{array}$ & $\begin{array}{l}\text { Baltimore, } \\
\text { Md. }\end{array}$ & $\begin{array}{c}\text { Pawling, } \\
\text { N. Y. }\end{array}$ & $\begin{array}{l}\text { Oak Ridge, } \\
\text { Tenn. }\end{array}$ & $\begin{array}{c}\text { Oak Ridge, } \\
\text { Tenn. }\end{array}$ & $\begin{array}{l}\text { Oak Ridge, } \\
\text { Tenn. }\end{array}$ & $\begin{array}{c}\text { Oak Ridge, } \\
\text { Tenn. }\end{array}$ & $\begin{array}{l}\text { NRTS, } \\
\text { Idaho }\end{array}$ & $\begin{array}{l}\text { NRTS, } \\
\text { Idaho }\end{array}$ & $\begin{array}{l}\text { Middletown, } \\
\text { Conn. }\end{array}$ & Waltz Mill, Pa. \\
\hline CER & none & NDA-CX & West & East & South & PCA & $\mathrm{RMF}$ & ETRC & none & WREC \\
\hline $6 / 9 / 58$ & $12 / 10 / 57$ & $1 / 22 / 58$ & $12 / 20 / 50$ & $2 / 4 / 51$ & $3 / 20 / 58$ & 1958 & $2 / 11 / 55$ & $5 / 20 / 57$ & $3 / 12 / 58$ & $12 / 8 / 57$ \\
\hline n.a. & $\$ 400,000$ & n. $\mathbf{a}_{\text {. }}$ & $\$ 400,000$ & $\$ 500,000^{\circ}$ & $\$ 300,000$ & & n.a. & $\$ 398,000$ & D. $\mathbf{a}_{\text {w }}$ & $\$ 450,000$ \\
\hline n.a. & $\$ 700 / \mathrm{dey}$ & a.a. & $\$ 600 /$ day & $\$ 600 /$ day & $\$ 600 /$ day & & $\$ 500 /$ day & $\$ 500 /$ day & n.a. & $\$ 800-1,200 / \mathrm{day}$ \\
\hline 1 & 2 & 2 & 1 & 1 & 1 & 1 & 1 & 1 & 2 & 2 \\
\hline - & - & 4 & 3 & 3 & 2 & & 2 & 一 & - & - \\
\hline - & 2 & 3 & 2 & 2 & 1 & & 1 & 2 & 2 & 2 \\
\hline 1 & 1 & 1 & 4 & 1 & 4 & & 一 & 1 & 1 & 1 \\
\hline - & 一 & 2 & 1 & 4 & 3 & & 一 & 3 & 一 & - \\
\hline RER & APPR-1 & PRR & $\begin{array}{r}\text { APPR-1, } \\
\text { BSR-2 }\end{array}$ & ARE & - & ORR & 一 & ETR & n.a. & Yankee, WTR \\
\hline yest & $\begin{array}{l}\text { yes } \\
\text { n.a. }\end{array}$ & yes & yes $^{5}$ & yes $^{6}$ & yes $^{6}$ & & yes & yes 7 & $\begin{array}{l}\text { no } \\
\text { n.8. }\end{array}$ & yes \\
\hline U-AI & & $\mathbf{U}-\mathbf{A} \mathbf{1}$ & $\begin{array}{l}\mathrm{U}, \mathrm{UO}_{2}\left(\mathrm{NO}_{3}\right)_{2} \\
\quad \mathrm{UO}_{2} \mathrm{~F}_{2}\end{array}$ & $\mathbf{U}, \mathbf{U F}_{\mathbf{4}}$ & $\begin{array}{l}\mathrm{U}, \mathrm{UO}_{2}\left(\mathrm{NO}_{3}\right)_{2} \\
\quad \mathrm{UO}_{2} \mathrm{~F}_{2}, \mathrm{U}-\mathrm{Al}\end{array}$ & & $\mathrm{U}$ & $\mathbf{U}$ & & $U$ \\
\hline $\mathrm{H}_{2} \mathrm{O}$ & & $\begin{array}{c}\mathrm{D}_{2} \mathrm{O}, \mathrm{C} \\
\mathrm{H}_{2} \mathrm{O}\end{array}$ & $\mathrm{H}_{2} \mathrm{O}$ & $\mathrm{Be}, \mathrm{C}, \mathrm{C}_{2} \mathrm{H}_{4}$ & $\mathrm{H}_{2} \mathrm{O}, \mathrm{D}_{2} \mathrm{O}$ & & $\mathrm{H}_{2} \mathrm{O}$ & $\mathrm{H}_{2} \mathrm{O}$ & & $\mathrm{H}_{2} \mathrm{O}, \mathrm{D}_{2} \mathrm{O}$ \\
\hline $\mathrm{H}_{2} \mathrm{O}$ & & $\mathrm{D}_{2} \mathrm{O}, \mathrm{H}_{2} \mathrm{O}$ & - & - & - & & $\mathrm{H}_{8} \mathrm{O}$ & $\mathrm{H}_{2} \mathrm{O}$ & & $\mathrm{H}_{2} \mathrm{O}, \mathrm{D}_{2} \mathrm{O}$ \\
\hline $\mathrm{H}_{2} \mathrm{O}$ & & $\mathrm{C}, \mathrm{D}_{2} \mathrm{O}$ & $\mathrm{H}_{2} \mathrm{O}$ & $\mathrm{Be}, \mathrm{C}, \mathrm{C}_{2} \mathrm{H}_{4}$ & $\mathrm{H}_{2} \mathrm{O}, \mathrm{D}_{2} \mathrm{O}$ & & $\mathrm{H}_{2} \mathrm{O}$ & $\mathrm{Be}, \mathrm{BeO}$ & & - \\
\hline Al & & $\mathrm{Al}$ & - & SS, Al & - & & AI, SS & $\mathrm{Al}, \mathrm{SS}$ & & SS, Al \\
\hline $\mathrm{Cd}$ & & $\mathrm{Cd}$ & - & - & - & & $\mathrm{Cd}, \mathrm{B}_{4} \mathrm{C}$ & B, $\mathrm{Ni}, \mathrm{Cd}$ & & - \\
\hline LAC-143 & MND-1157 & NDA-3002 & - & NEPA-1769 & - & & IDO-16108 & IDO-16332 & n.s. & WCAP-311 \\
\hline
\end{tabular}

LMFRE-Liquid Metal Fueled Reactor Experiment

Lockheed-Lockheed Aircraft Corp.

Lockheed-Lockheed Aircraft Corp.
Martin-The Martin Co. (formerly The Glenn L.

Martin Co.)

MRR-Medical Research Reactor, Upton, N. Y.

MSR-Merchant Ship Reactor, NS Savannah

NDA-Nuclear Development Corp. of America

NRR-Naval Research Reactor, Washington,

D. C.

NRTS-National Reactor Testing Station

ORNL-Oak Ridge National Laboratory (oper-

ated by Union Carbide Nuclear Co.)

Phillips-Phillips Petroleum Co.

PRR-Pawling Research Reactor, Pawling, N. Y.
PRTR-Plutonium Recycle Test Reactor, Richland, Wash.

SAR-Submarine Advanced Reactor, West Milton, N. Y. and USS Triton

S1C-first generation Combustion Engineering reactor for submarines

S3W, S4W, S5W-third, fourth, fifth generation Westinghouse reactor for submarines

Vitro-Vitro Engineering Co. Div, Vitro Corp. of America

WTR-Westinghouse Test Reactor, Waltz Mill, $\mathrm{Pa}$.

\section{Footnotes}

1 There have been at least two cases in the past, and another pending, in which Argonne's critica facilities have been made available under special circumstances.

"Although these facilities are listed as "unclassified" in the "Twenty-fourth Semiannua Report" of the AEC, NUCLEonica was unable to get further details from the contractor or AEC.

s This facility may be available for use in connection with AEC or other governmental programs. 4 U. S. Air Force permission required.

Possibly.

- Plus $\$ 1,000,000$ for Be

7 It is available to experiment sponsors (in ETR) for the purpose of deriving data from testing of prototypes of their experiments. 


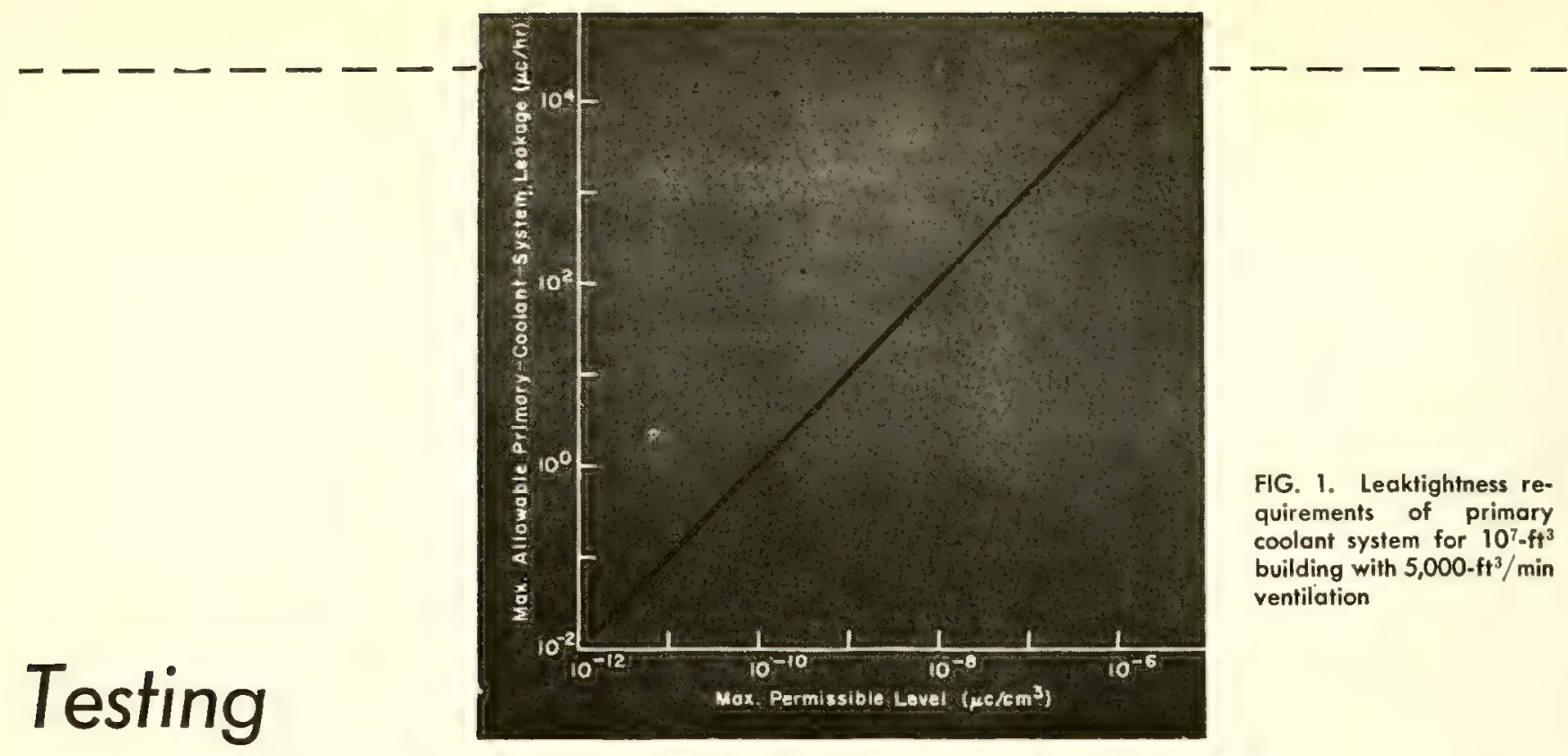

\section{Nuclear-Plant Leaktightness}

By J. P. VERKAMP and S. L. WILLIAMS

Knolls Alomic Power Laboratory, ${ }^{*}$ General Electric Company, Schenectady, New York

\section{LEAKTIGHTNESS PROGRAM SHOULD INCLUDE:}

- Determination of how extensive a program can be justified

- Design of components and connections for cleaning and inspecting facility, minimum-size welds, and convenient-size subassemblies

- Enforceable cleanliness and inspection program

- Leaktightness requirements in all component specifications and all subassembly work; sequence operations to permit several rechecks of leak-susceptible components

- Extra care to insure sound welds in component and subassembly work and final system assembly

- Integration and sequencing of over-all fabrication, inspection, testing, and assembly work

Althodgh LEAKAGE Control has been an important consideration in engineering and constructing remote nuclear power plants, greater emphasis on leaktightness will be necessary for plants located in populated areas. Since the use of sewers, streams, or the atmosphere for dissipating leakage and medical surveillance of the local popu-

* Operated for the U. S. Atomic Energy Commission by the General Electric Co. lation are impractical, a leaktightness program must be instituted covering design, fabrication, and final assembly of the plant.

\section{Design}

In designing piping for nuclear plants, particular attention should be paid to selecting components that will minimize leaks. For example, socket weld fittings have been used on small piping as a general construction practice to facilitate field assembly. But welding these fittings to obtain leaktight joints is frequently difficult, and the fittings do not lend themselves to radiographic testing after installation.

Early pressurized-water systems required a number of auxiliary systems composed of numerous small pipe connections. Here, careful selection of 
These Methods

Are Used

For Tests

\section{Here's How They Compare}

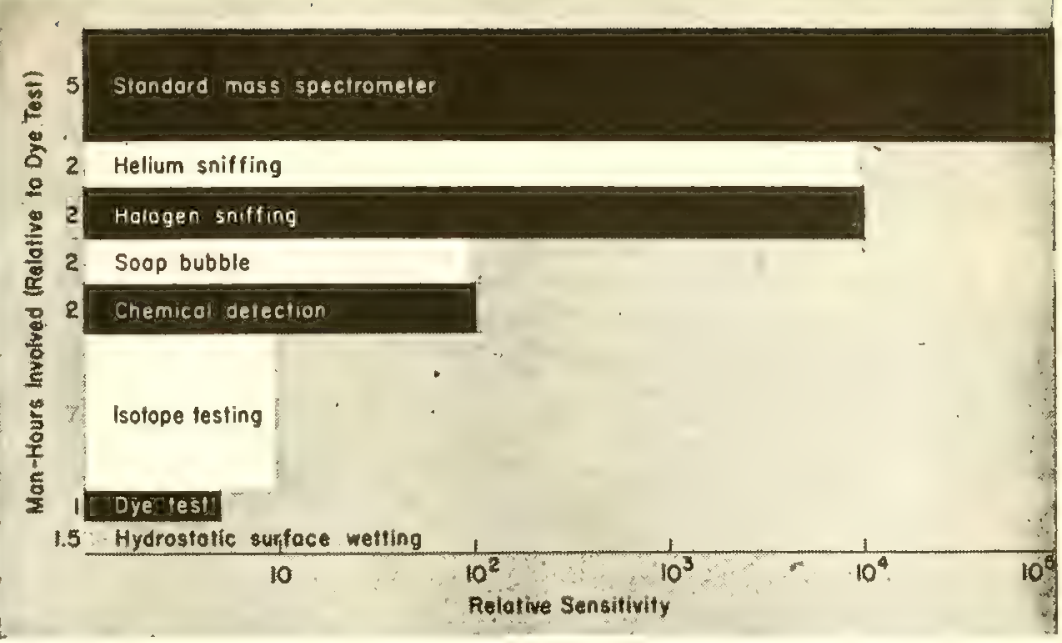

MASS SPECTROMETER - HOOD TEST. Tests entire surface; sums total leakage; can be used to localize leaks.

Procedure: Draw 50-micron vacuum in test volume; introduce helium into rubber or plastic bag over test surface. Draw sample of evacuated gas into mass spectrometer. Ultimate sensitivity reached by evacuating all gas through tester. Leak pin-pointed by using fine helium jet as blanket.

Comments: Sensitivity depends on proportion of gas passing through tester; properly applied, method develops full inherent sensitivity of tester. When attempting full sensitivity on large volume, response is slow and outgassing is time consuming; cleanliness requirements stringent to avoid outgassing problems.

MASS SPECTROMETER - SNIFFER TEST. Useful step in approaching high degree of leaktightness.

Procedure: Pressurize test volume with helium and air so that pressure differential drives helium through leaks; helium concentration is $20 \%$ or greater. Probe outer surface with nozzle in short hose leading to tester; mark leaks. When leaks are repaired, repeat test.

Comments: Efficiency depends on operator's experience and diligence; careful probing is tedious. Cleanliness is essential to avoid masking leaks.

MASS SPECTROMETER - BOOT TEST. Localizes individual leak; permits rapid repetitive testing of parts for which specialized boots can be developed.

Procedure: Introduce helium into test volume; apply cup or boot to portion of outside surface; evacuate gas between boot and surface of tester.

Comments: Sensitivity comparable to previous method. Response unaffected by size or configuration of test volume; almost as rapid as sniffer test. Outgassing time short. Cleanliness only sufficient to avoid masking leaks being sought since large-volume outgassing problems are avoided. Probably does not develop full tester sensitivity; sealing boot to test surface is major problem. Single boot not applicable to every test.

HALOGEN SNIFFING. High-sensitivity test.

Procedure: Pressurize system to 100 psig with halogen-air mixture; generally use halogenated hydrocarbons, e.g., Freon-12 at $20 \%$ conc. Cover test surface with probe at $1 / 2 \mathrm{in} / \mathrm{sec}$. Fan draws continuous air samples into detector containing halogen-vapor-sensitive element. Portable control unit contains power supply, amplifier, indicator, and loud-speaker.
Comments: Less expensive than mass-spectrometry testing, but less satisfactory; may leave traces of halogen in system; sensitivity to airborn contaminants gives false leak indications under field conditions.

SOLUBLE-DYE TEST. Visual observation of leak.

Procedure: Fill system with water containing fluorescent dye, e.g., Calco uranine B-4315 (American Cyanamid); much smaller fluorescent-dye concentration with $u-v$ light pin points leak than possible with ordinary dye. Raise system to $1.5 \times$ design pressure and hold for 6-8 hr. Depressurize and inspect under $u-v$.

SURFACE-WETTING TEST. Hydrostatic water test; generally used for component and subassembly tests where volumes are relatively low.

Procedure: Fill system with water to $1.5 \times$ design pressure. Isolate system. Drop in pressure indicates leak rate. Detect leaks by noting weeping at surface.

\section{CHEMICAL DETECTION.}

Procedure: Introduce tracer compound inside test volume, explore outside with "detector." Suitable test is white fumes given off by acid-base reaction between ammonia and carbon dioxide or hydrogen chloride; or halogen torch, whereby halogenated hydrocarbon as tracer imparts green color to torch flame.

Commenrs: Several chemical methods have about same sensitivity.

SOAP-BUBBLE TEST. Finds larger leaks in assembled system before employing more sensitive methods.

Procedure: Pressurize test volume with air. Paint leakage areas with soap solution. After repairing leaks, repeat.

Comments: Relatively high sensitivity with proportionally low expenditure of man-hours.

RADIOISOTOPES. Used after repairing major leaks.

Procedure: Fill system with radioisotope solution ( $\mathrm{Rb}^{86}$ often used); initial concentration generally about $0.01 \mu \mathrm{c} / \mathrm{ml}$ of water. Using weld numbers as guide, wrap all pipe welds, valve bonnets, vent plugs, etc. with absorbent $3 / 4$-in.-wide absorbent tape. Raise filled system to $1.5 \times$ design pressure and hold for $6-8 \mathrm{hr}$; then generally cycle several times, depressurize system, remove tapes, and count. With tapes giving $150 \mathrm{cpm}$ or more with standard-geometry G-M tube, welds are inspected and repaired.

Comments: This is variation of standard water hydrostatic testing procedure to achieve greater sensitivity. 


\section{A Case Study_Leaktesting a Sodium-Cooled Plant}

A KAPL-operated sodium plant (5) with a primarycoolant volume of several hundred cubic feet has shown unusually high freedom from leaks. To provide the plant with a primary coolant system as leaktight as possible, an all-metal, all-welded system is used.

Previous experience with sodium showed that where high system-tightness standards were set, the occurrence of leaks during operation was reduced to near the vanishing point. Since some seemingly insignificant leaks are actually relatively large perforations that are partially masked by occlusions and will enlarge under operating conditions, any detectable leak was assumed to represent a flaw that should be repaired. Therefore, with components, and where possible with entire systems, massspectrometer-quality tightness was required.

\section{Leak Specifications}

Since the assembled system might not be accessible for high-quality mass-spectrometer testing, an over-all component, subassembly, and field weld-testing program was substituted. The program assured that no individual leak would exceed the lower limit detectable by the mass spectrometer leaktester under field conditions. This assured that the system would remain leaktight during operation and placed an approximate ceiling on over-all system leakage.

The program specified that: (a) all small components tested to $0.005 \mathrm{mefh}\left(\mathrm{ft}^{3} / \mathrm{hr}\right.$ at 1 -micron $\mathrm{Hg}$ ) and large components. to values consistent with their volumes; (b) all small subassemblies tested to $0.005 \mathrm{mcfh}$ and large subassemblies to values consistent with their volumes before inclusion in the system; and (c) all field welds tested to $0.005 \mathrm{mcfh}$ during assembly. Since there were about 100 subassemblies and field welds, the final system would be tight to about $1 \mathrm{mch}$ over-all leakage.

\section{Inspection Procedure}

Rigorous handling, inspection, and testing procedures of all components and subassemblies were instituted:

1. Component manufacturers supplied an outline of their cleaning procedures, leaktesting schedules, and packaging procedures. Leaktesting was conducted at several steps during manufacture since a leak in an inaccessible part of a component is difficult to discover and repair after assembly. Cleanliness, essential in the lowleak-rate ranges, must be maintained for repeat and subassembly tests; thus, packaging for shipment is important.

2. Inspection on receipt of components included recleaning as necessary and retesting-for leaks.

3. The subassemblies were inspected and leak checked just prior to inclusion in the system since many of the subassemblies and components were fabricated in the vendor's shops and because fit-up work at the construction site involved some handling and cutting of subassemblies.

4. Finally, the field welds were checked by the massspectrometer boot technique. piping components was necessary to obtain a leakproof system. Butt welds or other closures satisfactory for welding fabrication will greatly reduce leaktesting and repair work after assembly.

\section{Leaktesting}

The degree of tightness required for a nuclear system can be appreciated from the table and Fig. 1. As an example, the maximum leak rate for fission gases that have a maximum permissible level of $2 \times 10^{-6} \mu \mathrm{c} / \mathrm{cm}^{3}$ (based on $\mathrm{Xe}^{135}$ ) would be $2 \times 10^{4}$

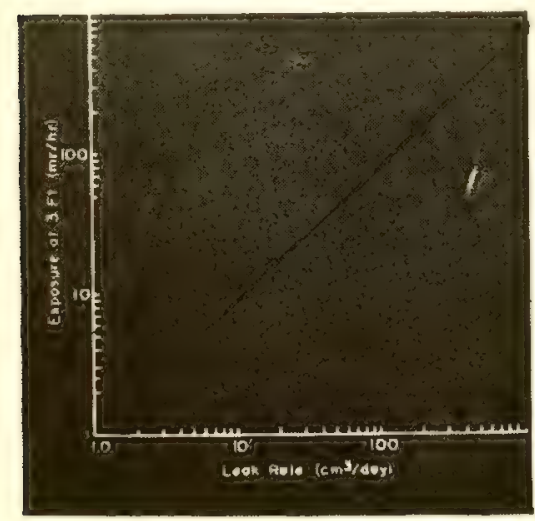

FIG. 2. Radiation exposure $3 \mathrm{ft}$ from point-source leak vs. leok rate $\mu \mathrm{c} / \mathrm{hr}$ in a $10^{7}-\mathrm{ft}^{3}$ building (1). Although the activity of fission gases varies with reactor power and previous operating history, an allowable leakage rate of $10^{-6} \mathrm{~cm}^{3} / \mathrm{sec}$ is conceivable. Such low leak rates require specialized techniques for rapid measurement.

Excessive leakage can produce a direct radiation hazard during maintenance. Figure 2 shows the dose rate that might be expected from leaks in a sodium-cooled plant.

Applications of the various leaktesting methods vary with the type of heattransfer fluid and the materials of construction used. The leak-detection methods that have been used for pressurized-water and liquid-metal systems are summarized on p. 34 .

\section{Sensitivity}

The graph (p. 55) does not indicate ultimate sensitivity that can be attained by modification and the expenditure of greater amounts of time. For example, the dye or chemical-detection tests could be improved by using absorbent strips, as in the radioisotope test, but man-hours would beincreased.

Mass spectrometry is capable of the greatest sensitivity (4). The unit espe- cially designed for helium leaktesting attains a sensitivity of 1 part helium in 200,000 parts air, thus being capable of detecting a leak of about $2 \times 10^{-4}$ mefh (micron $\mathrm{ft}^{3} / \mathrm{hr}-\mathrm{a}$ leak rate of 1 mefh is such that when applied to $1 \mathrm{ft}^{3}$ under high vacuum, the pressure rises

Max. Permissible Levels in Air*

\begin{tabular}{|c|c|}
\hline Radioisotope & $M P L\left(\mu \mathrm{c} / \mathrm{cm}^{3}\right)$ \\
\hline $\mathrm{H}^{3}$ & $2 \times 10^{-5}$ \\
\hline $\mathrm{Na}^{24}$ & $2 \times 10^{-6}$ \\
\hline$K^{42}$ & $2 \times 10^{-6}$ \\
\hline $\mathrm{Cr}^{51}$ & $8 \times 10^{-6}$ \\
\hline $\mathrm{Mn}^{86}$ & $4 \times 10^{-6}$ \\
\hline $\mathrm{Xe}^{135}$ & $2 \times 10^{-6}$ \\
\hline $\mathrm{Po}^{210}$ & $7 \times 10^{-11}$ \\
\hline U (natural) & $1.7 \times 10^{-11}$ \\
\hline $\mathrm{Pu}^{239}$ & $2 \times 10^{-12}$ \\
\hline \multicolumn{2}{|c|}{$\begin{array}{l}\text { Beta-gamma emit- } \\
\text { ter (isotope un- } \\
\text { known) }\end{array}$} \\
\hline $\begin{array}{l}\text { Alpha emitter (iso- } \\
\text { tope unknown) }\end{array}$ & $5 \times 10^{-12}$ \\
\hline $\begin{array}{l}\text { * Reproduced from } \\
\text { cook, U. S. Dept. He } \\
\text { Welfare (1954). }\end{array}$ & $\begin{array}{l}\text { Radiological Hand- } \\
\text { ealth, Education and }\end{array}$ \\
\hline
\end{tabular}


The final leaktest of subassemblies uncovered only two detectable leaks, one in a 2-in.-pipe elbow, the other in a section of 2 -in. pipe. A preliminary vacuum test of the completed system indicated it met desired tightness. No leaks occurred during the first six months operation.

Other factors contributing to system tightness were design and welding. The primary coolant system is designed to contain relatively few small pipes or fittings. The system was amenable to subdivision into a relatively small number of subassemblies and components. For strength as well as leaktightness, all welds were subjected to a dye-penetrant test of first and last pass, followed by radiographic examination. A mass-spectrometer test also indicates soundness of a weld.

In addition to fabricating techniques, choice of materials is important to minimize leaks because of the possibility of flaws. For example, in using nickel-bearing stainless steels, the unstabilized types give "cleaner" welds; the stabilizers, such as $\mathrm{Cb}$ and $\mathrm{Ti}$, tend to cause carbide inclusions in the welds. Where a stabilized type is required, tight specifications on carbon and stabilizer content may be desirable. Commercial specifications on type-347 stainless call for stabilizer not less than ten times the carbon content and not more than $1.0 \%$. Where needed, 347 stainless has been procured with $0.06 \%$ carbon, max., and columbium eight times carbon.

To minimize fissures and cracks in welds, the composition of the weld should be such that it contains about $4 \%$ ferrite. By specifying the proper filler-rod composition, the weld composition can be made nearly as desired. For example, in welding tube-mill products of 347 stainless, which commonly runs well on the austenitic side (no ferrite), a filler rod of $8-10 \%$ ferrite produced welds containing the desired $3-4 \%$ ferrite.

However, the type of weld, preparation, and weld dimensions, as well as material composition, must be considered in estimating the final weld composition. Trial welds are the best method of obtaining the necessary ferrite composition data. Another indirect means of controlling welds through material specifications is obtained by specifying weld electrode coatings. For example, where ease and rapidity of welding is important, a titania-bearing coat is used on electrodes. The titania increases the fluidity of the flux; however, the rapid welding and reduced puddling resulting from the use of such a coating sometimes is considered to contribute to slag inclusions in welds. If weld quality is paramount, a straight lime-coated rod should be considered.

Since the skill of the welder is most important in producing good welds, attention should be given to details in welder qualification tests. Tests should be used that are representative of the work to be done. The use of dye-penetrant tests to assure soundness of first and last pass of a weld is good practice and reduces the amount of repair time. Radiographic inspection of the completed weld is highly desirable. In thick material requiring many weld passes, more frequent testing should be done.
1 micron of mercury per hour due to atmospheric in-leakage). To obtain this ultimate sensitivty, the surface to be tested must be scrupulously clean and extreme care must be exercised in the test procedure. These requirements may be met under special conditions, but for most practical field work, $0.005 \mathrm{mcfh}$ is considered the limit.

\section{Testing Procedure}

A progressive type of leak-detection procedure making use of several tests gives the best results.

Hydrostatic procedure with water generally is used for testing components and subassemblies. The soap-bubble test is attractive for finding the large leaks in the assembled system before employing more sensitive methodslarge leaks mask the smaller ones. In one large pressurized-water nuclear system, $\sim 63 \%$ of the pre-operational leaks were discovered by this method in 15 days. After repairing the leaks found by the bubble test, a hydrostatic water test should be performed on highpressure systems; exposure of the system to 1.5 times the design pressure may open additional leaks.

The system is now ready for a high- sensitivity leak test, e.g, halogen or helium sniffing or standard mass-spectrometer testing. The choice largely depends on the degree of tightness required.

The most satisfactory procedure has been to localize leaks by standard mass-spectrometer testing of various portions of the system under vacuum. Then the suspect areas can be worked over using the spectrometer as a helium sniffer. If a higher degree of tightness is required, the boot technique should be employed.

Omitting the standard mass-spectrometer testing for leak localization appears to result in prolongation of the helium-sniffing test. Providing the operator with a known leak to search out markedly increases his diligence.

Although this type of rigorous leaktightness program increases the cost of individual components and subassemblies, it offisets a comparable expense in rework time after the system is completed. The specific advantages gained in a program of this type, rather than relying on corrective measures after assembly, are:

1. The responsibility for achieving a particular degree of leaktight- ness is properly divided between designer, manufacturer, fabricator, and constructor.

2. Unpredictable delays in placing the system in operation after assembly are avoided.

3. A specific leaktightness goal can be systematically approached.

Rather rigid test requirements were of necessity placed on the first nuclear power-plant prototypes. However, as methods and materials improve and experience is gained, considerable simplification of the leaktightness procedures should result.

\section{***}

The authors thank R. A. Koehler of the General Electric Co., General Engineering Laboratory, and G. E. Halm of Knolls Atomic Power Laboratory, who were associated with the authors on leaktightness problems and who graciously checked the manuscript for accuracy.

\section{BIBLIOGRAPHY}

1. Radiological Handbook, U. S. Dept. of Health, Education and Welfare (1954)

A. O. Nier, C. M. Stevens, A. Hustralid. T. A. Abbott, J. Appl. Phys. 18, 30 (1947)

3. R. B. Jacobs, H. F. Zuhr, J. Appl. Phys. 18, 34 (1947)

4. W. G. Worcester, E. G. Doughty, High vacuur leak testing with the mass spectrometer, presented at AIEE convention, Detroit, June, 1946 (AIEE Tech. Paper 26-142)

5. Liquid-Metals Handbook, NAVEXOS P-733 (1952) 


\section{Mystery Under Vigorous Attack}

between the lower and upper explosive limits. It is important to note that actual combustion involves an airflammable-vapor reaction and not an air-flammable-liquid reaction. Experiments indicate that free burning of metals in air takes place at temperatures well above the metal melting point, at which temperatures the metal vapor pressure is well within a range comparable with that of flammable liquids undergoing combustion.

When a small amount of flammable liquid is burned, the heat of combustion raises the temperature of the liquid to its boiling point where it remains constant until all of the liquid has vaporized. A very recent experiment involving free-burning tests of small magnesium specimens indicated that combustion took place while the $\mathrm{Mg}$ temperature was very close to the boiling point. Such data again suggest a close analogy between the mechanisms respectively involved in combustion of flammable liquid and flammable metals. (Recent high-speed movies of an $\mathrm{Mg}$ wire burning in oxygen showed that combustion rates along the wire were erratic and that oxidation was accompanied by periodic small localized highenergy explosions, the causes of which are uncertain.)

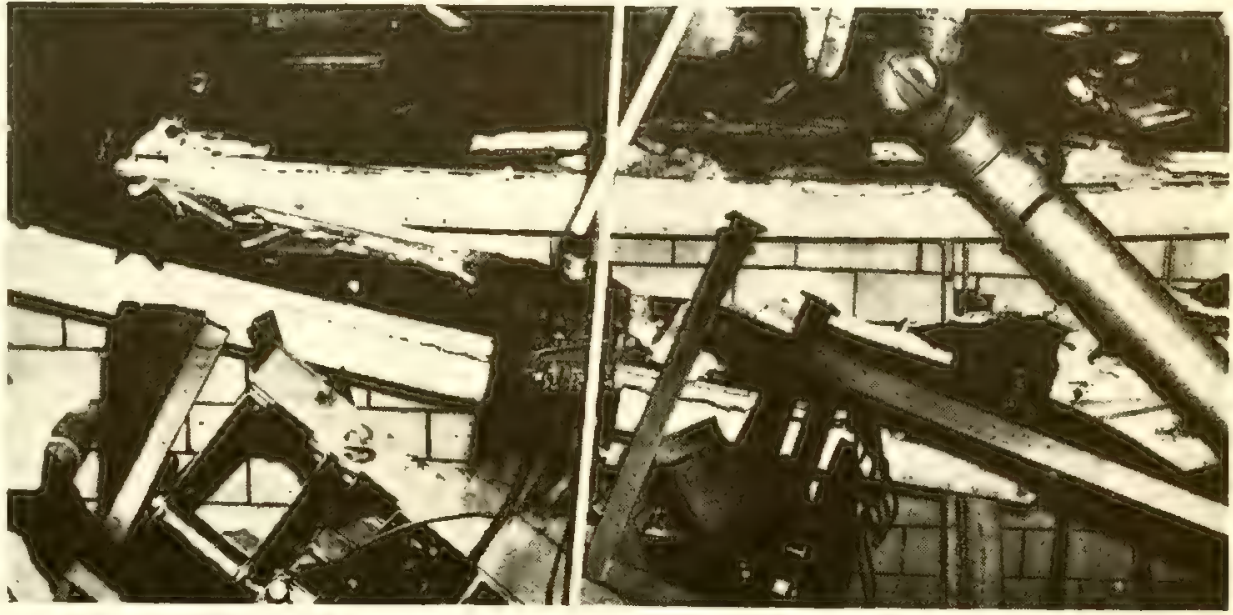

FIG. 3. Close-up of damage in metallurgical laboratory after a thorium explosion in July 1956. Tile is part of wall of ground-floor room; above it is floor of second. story lab room where explosion took place; apparatus seen diagonally in lower left is part of a calciner that had stood on part of floor that dropped: standing next to it had been a drum containing major part of thorium scrap to be burned to oxide. Triggered by initial blast, this material exploded in its wake
When a fire occurs on the surface of a large amount of oil, initially at room temperature and contained in a tank, liquid temperatures at the surface are again at the boiling point while liquid at the base of the tank may remain at the initial temperature. Such fires have been extinguished through use of

\section{AEC's Three-Pronged Attack on Pyrophoricity}

Following two recent serious and unusual explosions, involving metallic $Z \mathbf{r}$ (I) and Th (2) (see Incidents listed on p. 30), AEC efforts to study unusual metal fires and explosions were intensified and metal-pyrophoricity research activities expanded.

1. A fundamental research program covering the mechanics of metal pyrophoricity is being conducted at Argonne National Laboratory.

2. A "crash" program aimed at rapidly determining specific methods to ensure safe handling of metallic zirconium was recently initiated by the Bureau of Mines at Pittsburgh (see box on p. 95 for details).

3. Very-high-temperature metal-water reactions are the subject of classified research at a third location.

From these three programs AEC expects to obtain fundamental information permitting increased safety in the manufacture and utilization of such heavy pyrophoric metals as uranium, plutonium, thorium, hafnium, and zirconium. Incidental to the major purposes of this research, it is probable that some fundamental information will also be derived relative to theoretical approaches for attaining oxidation and waterresistant alloys as well as affording a better understanding of the conditions under resistant alloys as well as affording a better understanding of the conditions under or initiated. agitation, permitting replacement of relatively hot liquid oil at the surface with cool oil from subsurface locations.

In the case of massive pyrophoric metals, the surface is similarly rapidly cooled by conduction of heat to subsurface levels at a rate far greater than can take place between quiescent layers of oil. This ability to dissipate heat accounts in large measure for the difficulty in obtaining and sustaining combustion of such specimens without continued external application of heat. In view of the extremely high temperatures required for massive metal combustion, plus the fact that heat-radiation losses take place at a rate proportional to the fourth power of the temperature, the rarity of massivemetal fires is not surprising.

\section{Massive-Metal Combustion}

Spontaneous ignition of any massive pyrophoric metal is very rare. How- 
ever, it has occurred in air at room temperatures in the case of $\mathrm{Pu}, \mathrm{U}$, and $\mathrm{Th}$ with initial metal temperatures ranging from that of dry ice to red heat. Witnesses generally agree that these incidents are accompanied by slow but noticeable-flaking off of the metal surface with sporadic emanation of localized sparking.

From these observations it is apparent that combustion of abnormally pyrophoric massive metals includes a "built-in" mechanism for converting a solid metal surface into an extremely fine, brittle, and chemically reactive powder, and that this mechanism is accelerated during auto-oxidation of the powder. Since ignition of massive metal has occurred at quite low temperatures, combustion in air of the surface powder must involve a highly exothermic reaction that more than offsets the heat loss from the metal surface. Thus, a basic mechanism contributing to ultimate burning of massive metal must involve both physical aspects (e.g., stress, brittleness, cracks, voids, etc.) and chemical aspects (initiating and secondary combustion).

\section{Powder Combustion}

The large difference in observed pyrophoricity of metal powders and massive metal proceeds from differences in secondary factors influencing combustion efficiency, rather than from any basic difference in the reaction mechanisms involved. To illustrate the magnitude of these secondary "efficiency" factors, identical volumes of solid metal and powder are compared in the table on p. 96 .

The table shows that metal powders possess a much greater surface area on which to generate heat, have a much smaller mass of metal in which to dissipate this heat, and have a much smaller percentage of total surface area available for loss of heat by radiation to external surroundings. All of these factors combine to increase the probability of metal combustion.

Effect of mass. Unlike solid metal (in which heat can be internally transferred by the relatively efficient process of direct heat conduction), escape of any heat from within metal powders must largely take place by radiation through multiple insulating layers of air. Thus, accumulation of heat generated within metal powders by slow oxidation becomes increasingly probable with increasing size of the powder

\section{Unusual Metal Fires and Explosions}

The following unusual metal fires or explosions that occurred in the $U$. $S$. atomic enery program bave for the most part been taken from a much more comprehensive list of such incidents $(12,13)$.

It should be noted that such incidents have been relatively infrequent in the largescale handling of pyrophoric metals over a period of years in the atomic energy program.

In reviewing such incidents, it is of interest to note that increased pyrophoricity can apparently be "built into" a metal at the time of its initial manufacture or may be subsequently acquired as a surface phenomenon.

\section{Zirconium Incidents}

I Up to May 1955 no serious fires had been encountered during storage of scrap-7 turnings, chips, plates, rods, etc. Such scrap had been stored (pending contemplated future recovery) in segregated open-top bins as shown in Figs. 1 and 2 . Severa days after a heavy rain, a fire of unknown origin took place in one of the bins with flames extending $100 \mathrm{ft}$ into the air. Shortly afterwards, contents of other (but not necessarily adjoining) bins suddenly and intermittently flared up. Material in all bins soon became involved and $159,000 \mathrm{lb}$ of $\mathrm{Zr}$ were consumed. The heat was sufficiently intense to crack windows and ienite wood located over 150 feet away. Particles of burning $\mathrm{Zr}$ were carried over one-quarter of a mile through the air. [This took place at Bettis laboratory, Pittsburgh.]

About 5 years ago some water-wet scrap $\mathbf{Z r}$ powder in wooden barrels was placed in outside storage pending development of scrap-recovery processes. During the next several years, a few minor spontaneous fires broke out in this material. In January 1956, the material in several deteriorated wooden barrels was wet with water and repackaged in steel drums.

In May 1956, employees working in the area noted that one of the steel drums lying on its side contained a black material "similar to carbon dust." Just exactly what happened there is uncertain, but a spontaneous explosion occurred accompanied by streaks of red fire with black smoke extending 100 ft into the air. A pronounced concussion wave was noted and sound of the blast heard several miles away. Two employees were killed, one having been blown 80 ft through the air, and a third lost an arm.

The drum contained $\mathrm{Zr}$, probably in the form of a fine powder.

Using extensive precautions, the remaining drums of scrap $\mathrm{Zr}$ were subsequently burned. During this operation, one of the drums exploded in a manner shown in Fig. 4. [This incident took place at the Y-12 area, Oak Ridge.]

I Two men died and two others were seriously lajured in 1954 in a spontaneous explosion initiated during removal of the friction-top lid from a polythene-bag-lined, one-gallon metal can containing $Z r$ powder $16 \%$ wet with water. $A$ ball of flame enveloped the entire area, accompanied by a definite concussion wave.

If $\mathbf{A}$-Ib sample of carbon-tetrachloride-moistened powdered $\mathrm{Zr}$ was placed in a glass flask, vacuum applied, and the flask very gently heated with a Bunsen burner. The $\mathrm{Zr}$ suddenly began to heat up and detonated with a blinding fash. The explosion was attributed to a small amount of water.

\section{Uranium Incidents}

If In June 1956, three flat $U$ plates $(1 / 4 \times 3 \times 14 \mathrm{in}$ ), made by powder metallurgical means, were observed to have "blown up" to approximately 3-in.-diameter roda. The "rods" were removed from the building awaiting disposal. The following morning, one of the plates exploded and "took off like a rocket," hitting a tree 30 f away. The remaining plates were pierced with ballets but no explosions occurred. It was the opinion of company personnel that the plates had not been pressed to fall density and that there was an incomplete decomposition of the hydride in the $U$ powder, causing a bulld-up of Internal hydrogen pressure.

I In January 1955, an attempt was made to roll two 1,000-lb U slabs to 0.01-in. thick strip. After inital heating to $1,150^{\circ} \mathrm{F}$ in a lithium-carbonate-potassiumcarbonate bath, several $30 \%$ reductions were made by rolling. It was observed that heavy work passes had caused overheating. The strip, then $3 / 4$ in. thick, was cooled to $1,200^{\circ} \mathrm{F}$ before entering the four finishing stands. The strip again ercesdrel heated during the next three reductions and became so ductile on entering the fourth stand that it palled into two parts and a cobble resulted. The strip at this atage was cherry red, but by the time it had been removed to the mill floor it was observed to increase in temperature to a white heat followed by melting and burning

II February 1956, a technician was attempting to roll a plate consisting of Zr-clad U, which, in turn, was clad in a low-carbon-steel jacket. During preheating. the furnace temperature control (which had been set to $1,450^{\circ}$ F) failed, allowing the temperature to rise to $1,800^{\circ} \mathrm{F}$. During subsequent rolling, molten Fe- $\mathrm{Zr}$ eatectle alloy within the steel jacket was forced to one end of the strip where It burst into flames as it sprayed ont over an area approximately $10 \mathrm{ft}$ wide, $10 \mathrm{ft}$ high, and $25 \mathrm{ft}$ long. One employee was serionsly injured.

If In the eariy program for the large-scale manufacture of metallic $U$, fine powder was allowed to collect under roughly $25 \mathrm{ft}$ of water. At approximately one-month intervals, and without prior warning, a geyser about $30 \mathrm{ft}$ high would suddenly develop over the powder and then immediately subside.

I A series of cases is known in which massive pieces of metallic $U$, Pu, and Th have displayed unusual pyrophoricity, e.g., spontaneously igoitins at room temperature. Spontaneous fires in $U$ chips are, however, much more common and in one case ignition occurred 6 months after the chips had been placed ta storase. One investigator of spontaneous fires in briquetted $U$ chips opened a drum filled wit briquettes that had been in ontside storage for several weeks. Alter noting that the drum contents were normal and at approximately room temperature, be was wamed by an operator to stand back. A few seconds later, a fiame shot to a helght of aboat 
$25 \mathrm{ft}$ and then immediately subsided. Upon reinspecting the drum interior, he noted that all of the briquettes were at an incandescent temperature.

A series of incidents have been experienced in which $U$ and $T i$ alloys have displayed explosive surface films following acid treatment $(12,14)$. Studies at Argonne National Laboratory showed that such explosions could be averted through use of adequate fuoride ion concentrations in nitric acid efching baths. Witnesses have de scribed metal-surface explosions of this type as involving a brilliant flash of white light, accompanied by a sound similar to that of a 22-gage rifte shot.

\section{Thorium Incidents}

I For several years scrap Th powder had been disposed of by burning in successive small amounts. In July 1956, employees were engaged in burning scrap Th powder that had prevlously been washed with several aqueous solutions and vacuum-dried 3 days earlier. Some of the Th was placed in a special hood and ignited without incident. An employee took a "golf-ball-size" piece of Th from a metal pail containing $30-40 \mathrm{lb}$, replaced the pail lid, and placed the piece on a small Th fire. An immediate sharp explosion blew the employee $20 \mathrm{ft}$ across the room. Almost immediately, a second blast involving the Th in the pail was accompanied by a jet of orange fire and a big cloud of dust. A third explosion occurred in a nearby vacuum dryer containing about $7 \mathrm{lb}$ of moist Th powder. One employee suffered fatal burns,
whlle three others suffered serious injuries. The extent of property damage is evident in Fig. 3. [This took place at Sylvania Electric Company's Metallurgica laboratory, Bayside, N. Y.]

In preparing an experimental charge for making metallic Th in a reduction bomb, mirer was being used to blend metallic calcium dry zinc chloride, and dry thorium fuoride. After several revolutions of the mixer, the operator opened the mixer vent and, noting that dust and gas were escaping, decided to call his foreman. A second operator closed the vent, started the mixer, and soon heard a rumbling noise, followed by a sudden burst of flame covering a 45 -deg angle and extending parallel to the floor for $40 \mathrm{ft}$. Of the eight persons injured by the blaze, two subsequently died. Reason for initiation of the reduction reaction in the blender is uncertain and unprecedented.

It was subsequently found that the calcium used was particularly reactive. In one test a marked, but unexplained, temperature rise occurred when some -50 mesh calclum fines were left standing in an argon atmosphere.

\section{Miscellaneous Incidents}

IOn June 16, 1954, employees of a non-AEC high-energy-fuel laboratory were sampling 15 drums of "bag fines" Mg powder, which were opened in a special room that had been purged with nitrogen until the oxygen content had dropped below 1\%. During sampling of the fifth dnen, the porder ignited suddenly. The flame shot out from the drum, immediately subsided, and the operators left the room after replacing the drum cover. From an external observation window, the employees noticed a gradual darkening of the drum's exterior, moving down to within $2-4$ in. of the drum bottom. The following day the drum was opened and contained a definite yellow coloration, which was presumed due to formation of magnesium nitride.

I A massive block of metallic barium was cut into $3 / 4$-in.-square pieces while submerged in kerosene. During attempts to remove residual kerosene with carbon tetrachloride (an operation that had been performed many times before without incident) violent reaction dispersed glass fragments and burning barium over the immediate area. Similar explosions have also been suffered when $\mathrm{Na}$, $\mathrm{U}$, and $\mathrm{Zr}$ were treated with carbon tetrachloride.

9 Trouble had been experienced in getting a Kroll process reduction of ZrCl with Mg to go to completion. When the furnace was opened up, a slate grey material was noted on the surface, which was thought to consist of $\mathbf{Z r}$, $\mathbf{M g}$, and $\mathbf{M g C l}$. A sample of this material, roughly $1 / 4$ in.-thick and 8 in.-square, was removed for test and was totally inert when scratched with a file or hit with a hammer. A piece of the sample melted under an oxyacetylene flame but showed no pyrophoric properties. Samples were then placed in water and slight evolution of gas noted. The following day an were then placed in water and slight evolution of gas noted. The following day an
attempt was made to further wash the samples in several changes of water. While under 5 in. of water and without any prior evidence of reaction, an explosion oc curred that shattered the laboratory bench, threw the technician against the wall, and blew out a window $25 \mathrm{ft}$ away. Portions of the water-washed sample blown to the floor ignited and "spit" when stepped upon. Small samples were sub. sequently tested and found to contain $\mathrm{Mg}, \mathrm{Zr}$, and $1 \% \mathrm{C}$.

\section{Crash Program Aims}

Objective of. $A E C$ 's crash program on $Z$ r sensitivity is to determine those properties of the various $Z_{r}$ forms and alloys that affect inflammability and explosiveness, and the reaction mechanisms involved in the sensitization of the metal. The study will assess the hazards involved in $\mathrm{Zr}$ manufacture, handling, storage, and shipping. $H f$ and $T i$ will be examined on a limited scale to establish relative sensitivity of the three melals.

The experimental approach to the problem will be to determine flammability and explosive characteristics of various types of sponge, process materials, powders and metallic scrap to establish relative standards and regions of sensitivity for the various materials. To do this, tests will determine the sensitivity of the material to friction and impact forces, flame or radiation, lemperature and high velocity shoch waves under controlled environmental conditions. Detailed studies will weigh effect of particle size, surface area (and, in sponge, density and extent of agglomeration), impurities and process contaminants such as $F$ e and other metals, salts, carbon, $O$, $N, H$, water, etc. mass and with decreasing particle size.

Several interesting observations have been made of the effect of powder mass on pyrophoricity. Dust obtained by fling a zirconium-titanium alloy never ignited when dispersed over a bench, butalways ignited spontaneously whenever small layers were accumulated. $\mathrm{Zr}$ powder dispersed over a red hot plate slowly oxidized, while a layer of the same powder thrown on the same plate immediately ignited and burned violently. Small layers of very fine U powder under water appear inert, while larger amounts tend to "ball up" following which spontaneous ignition is virtually certain.

The foregoing and other reported work $(3,4)$ suggest that for a metal powder of particular particle size there exists a definite minimum quantity of metal that must be exceeded for spontaneous ignition. Following the same line of reasoning, any metal capable of reacting exothermically with oxygen should also be capable of spontaneously igniting in air if the metal is sufficiently finely divided and if a sufficient quantity is present. Experimental work (5) tends to confirm this suspicion for a. series of metals (including iron, copper, and tungsten).

In many respects spontaneous ignition of metal powders is similar to spontaneous ignition of oily rags, while self-sustaining burning of massive metal is more nearly comparable to burning of ordinary flammable liquids and solids.

\section{Powder Explosions}

In general, the combustion in air of flammable vapors generates much less heat but a much greater volume of gases than does combustion of powdered pyrophoric metals. Since explosion pressure is roughly proportional to the product of the heat generated and the ultimate gas volume, an increase in explosion pressure is expected if the metals tested contain dissolved gases (notably hydrogen). Comparison of air-metal and air-metal-hydride dust explosion tests (6) tends to confirm this suspicion. Maximum explosion pressures attainable upon detonation of air-powdered-metal mixtures are approximately equal to the maximum attainable by detonation of flammablevapor-air mixtures $(\gamma)$.

Vapor or powdered-metal explosion pressures obviously vary with the materials involved, the ratio of mixture 


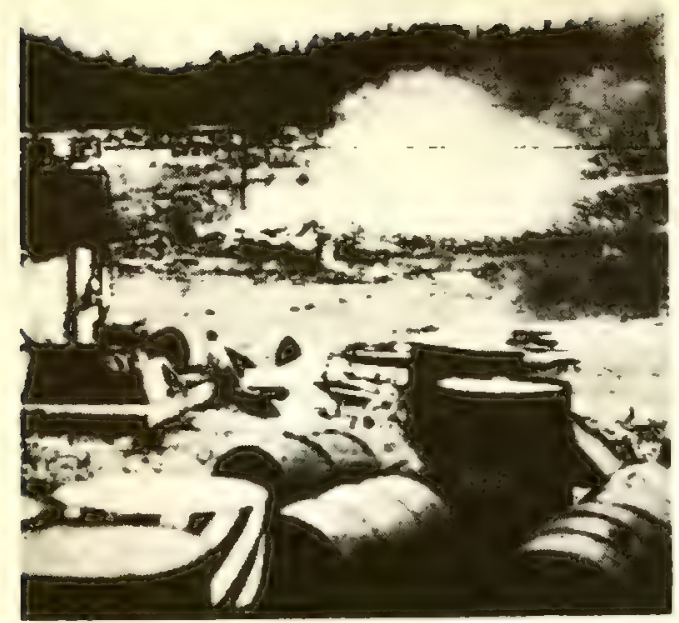

FIG. 4. Flare-up of $\mathrm{Zr}$ serap during disposal operation in May 1956. After two men had been killed and a third lost an arm while attempting to re-crate scrap $\mathrm{Zr}$ for removal, it was decided to burn remaining scrap cautiously in outdoor storage area

components, and the initial pressure. With initial mixtures at optimum proportions and atmospheric pressure, maximum explosion pressures are roughly 0-150 psig.

Effect of particle size. Qualitatively, the ease of ignition and explosion pressures attainable with metal dusts decrease rapidly with increasing particle size. The maximum particle size that will just support combustion following dispersion in air has not been adequately investigated. The amount of energy required to initiate-explosion of fine metal powders dispersed in air is often surprisingly low (6). In some cases the energy involved in dispersing the powder is sufficient to initiate spontaneous explosions.

\section{Oxides Reduce Pyrophoricity}

Although pyrophoric metals burn in oxygen, the pyrophoricity of many metals, e.g., $U(8)$, can often be dramatically reduced using oxygen. As an example, finely divided iron powder, prepared by hydrogen reduction of iron formate, will often ignite when first exposed to air. If, however, after preparing such powders, the hydrogen is replaced with an inert gas into which small amounts of dry oxygen are slowly fed while the furnace is being cooled to room temperatures, an iron powder is obtained that may be handled in air without ignition. A similar commercial process reduces pyrophoricity of highly porous $\mathrm{Ti}$ and $\mathrm{Zr}$ sponge produced by the Kroll process.

The ability of oxide coatings to reduce apparent metal pyrophoricity varies considerably among different metals and even among specimens of the same metal. Both the physical and chemical properties of oxide coatings may change markedly with increasing temperatures. Some metals such as $\mathrm{Ti}$ and $\mathrm{Zr}$ are capable of dissolving large quantities of surface oxides when heated above phase transformation temperatures, while other metals such as $\mathrm{Al}$ and $\mathrm{U}$ do not absorb significant quantities of surface oxides even when the metal is heated to its melting point.

Several investigators, while admitting that it does not make sense from a thermodynamic viewpoint, have found by test that $\mathrm{Zr}$ powders containing up to $25 \%$ oxide are more readily ignited than similar powders relatively free of oxides. The role played by surface oxides in the metal pyrophoricity picture is obviously important, complex, often erratic, and confusing.

Much information is available in the chemical literature on the oxidation of metals by oxygen, air, and water. Much is also known about the effects of oxide films on metal oxidation rates. The amount of heat liberated from combustion of specific metals can be accurately predicted, as can many factors influencing the probability of a reaction (such as temperature, pressure, etc.).

Despite this profusion of information, very little is known about the specific sequence of physical and chemical steps occurring during metal burning. Even less is known about reaction-rate variables during intermediate steps preceding and during metal combustion. This is particularly true when moisture and metal contaminants are involved.

The study of many unusual pyrophoric-metal incidents $(12,13)$, indicates apparently several independent mechanisms may contribute to metal pyrophoricity, and that surface oxides, stress, moisture, and contaminants are major facets deserving evaluation.

\section{Moisfure Increases Pyrophoricity}

Metal pyrophoricity is strongly influenced by moisture. Metals such as $\mathrm{Na}$ and $\mathrm{K}$ can react violently with water (9), as is well known. In the case of $\mathrm{U}$, the high-temperature reaction with steam is much more violent than with oxygen (10).

Studies have indicated that the reaction between some metals (e.g., powdered $\mathrm{Al}$ or $\mathrm{Mg}$ ) and water is theoreti- cally capable of producing slightly more explosive energy than nitroglycerin or TNT. Russian scientists reported (11) attainment of close-to-theoretical energy releases during tests aimed at preliminary evaluation of the use of water-metal reactions as highexplosives.

Many methods are known for inducing water-metal explosions (12). A few violent water-metal explosions have accidentally occurred in industry (notably during arc melting of $\mathrm{Ti}$ in watercooled crucibles). However, the rarity of such occurrences suggests that explosions of this type are only attainable under a very narrow set of conditions. That they can occur at all, however, justifies current $\mathrm{AEC}$ research in this area.

Two recent reports $(12,13)$ provide strong circumstantial evidence that pyrophoricity of $\mathrm{U}$ and $\mathrm{Zr}$ can sometimes be very materially increased by prior quiescent exposure to moisture at room temperature. Research is currently under way to determine the mechanism by which metal surfaces are pyrophorically influenced by water.

\section{Stress Increases Pyrophoricity}

There exists fragmentary but impressive evidence that pyrophoricity is increased when the metal is under stress. This stress can be physical or chemical. Uranium lathe chips, for example, appear to be very much more susceptible to spontaneous ignition than annealed metal of the same dimensions in sheet form. Several incidents (14) suggest a connection between stress and metal-surface explosions in nitric acid. Sudden release of elastic

Combustion of Metals: Solids vs Powders

Powder-to-
solid ratio of
Property

Combustion surface area

13,300

Metal mass involved

0.524

Total-surface: total-mass ratio

Fraction of total surface area exposed to external surroundings

0.000118

* Based on cubic packing of spherical particles $1 \mu$ in dia. 
strain can, under suitable conditions, cause adiabatic heating of metallic $\mathrm{Ti}$ fragments to their ignition temperature in air (15).

That pyrophoricity is increased by metal brittleness would be expected. For example, brittle alloys are used in lighter flints.

$\mathrm{Na}-\mathrm{K}$ and $\mathrm{Ti}-\mathrm{Zr}$ alloys are more pyrophoric and have lower melting points than the pure constituent metals. This suggests that metal contaminants that lower melting points can, in some cases, add to metal pyrophoricity.

Sheet Zr has ignited while under nitric acid when the sheet accidentally touched the graphite container. This suggests that surface contaminant occlusions can (in part through electrolytic effects) substantially alter metal pyrophoricity.

\section{Significance of Hydrogen}

The metal fire and explosion vagaries studied covered a wide range of temperatures, metal purities, atmospheres, surface-to-mass-ratios, etc. The only factor that appeared to be commonly involved in all of these incidents was hydrogen, i.e., all of the metals involved had either been made by hydride decomposition (and could therefore be suspected of retaining some hydrogen) or had at some time been exposed to water in some form.

The evidence of the significance of hydrogen was often dramatic. Thus, $\mathrm{U}$ chips often ignited in moist air but seemed nonpyrophoric in dry air. Metal spontaneously ignited (sometimes even in the absence of air) after prolonged storage under moist argon or water. Hydrogen concentrations on machined U surfaces sometimes ranged up to 50 times the published room temperature solubility of hydrogen in $U$. Brittle, pyrophoric uranium hydride has been identified following U exposure to water, etc.

While it is known that hydrogen on and below the metal surface is not the sole cause of pyrophoric anomalies, it is reasonably certain that it is a major common contributing cause. The various mechanisms by which hydrogen derived from water can enter and migrate within a metal $(16,18)$ are of less immediate interest than the ultimate effect of such hydrogen on pyrophoricity and the fate of the oxygen left after hydrogen removal from water.

It is thought that the principal effects of hydrogen on metal pyrophor- icity include increasing internal stress $(17,18)$, promoting metal surface fragmentation (in part through effects on brittleness, accentuation of grain boundary defects, and through subsurface gas evolution under heat), and reduction of surface-oxide protection of the metal.

Moesel's theory. According to a theory proposed by F. C. Moesel, three possible reactions occur: (a) metal plus moisture form metal hydride and hydrogen peroxide; (b) metal plus oxygen, in the presence of moisture, form metal oxide and additional peroxide; (c) metal plus hydrogen peroxide form metal peroxide or at least associate with each other by physical adsorption or absorption, which makes this reaction imminent. A metal fire in the absence of air would be the reaction of metal peroxide (or metal and hydrogen peroxide) with adjacent metal to form metal oxide with a liberation of heat. With a sufficient buildup in metal peroxide concentration, this reaction could be explosive. In the presence of air, the dispersion resulting from the primary explosion would result in a secondary explosion involving the oxidation of not only the earlier formed metal hydride, but also of the residual metal in the dispersed particles.

The general theory involved in spontaneous ignition of metal powders has been previously discussed. However, a number of incidents show that moist metal powders can, under some conditions, literally explode (either in the presence or absence of air), instead of simply igniting and quiescently burning. Mixed hydride and peroxide on the metal surface is one of several possible high-energy sources capable of spontaneously and very rapidly raising powder temperatures to a high degree.

In the discussion of massive-metal pyrophoricity theories, several mechanisms were covered relative to fragmentizing of heated metal surfaces. The same principles are thought to be even more applicable to metal powders.

Finally, the Russian experimenters obtained high-energy water-metal explosions under conditions comparable to hot fragmentized powdered metal (11). Thus, both theory and experience justify considerable precautions when handling or heating moist, finely divided powders with the degree of concern increasing with increasing quantities and decreasing average particle size.
Experience and experiments have shown that when water contacts molten pyrophoric metals, rapid generation of steam is, under quite narrow conditions, occasionally accompanied by violently explosive water-metal reactions. In the case of high-meltingpoint pyrophoric metals, experiments have shown that when water-metal explosions are attained, only a small percentage of the molten metal reacts with water. From this fact it can be assumed that the critical conditions are disrupted during the explosion. It is thought that during that very brief interval in which the very-high-temperature liquid metal can remain in contact with water, a combination of mechanical and exothermic chemical forces cause rapid spewing of finely divided and exceedingly hot metal particles into water (12). The basic mechanism involved in liquid metalwater explosions is viewed as being very similar to, but more severe than, the moist-metal-powder explosions.

\section{BIBLIOGRAPHY}

1. Nocheonics 14, No. 6,24 (1956)

2. Nochenics 14, No. 7, R12 (1956)

3. H. C. Anderson, L. H. Belz. Factors controlling the combustion of zirconium powders, J. Electrochem. Soc. 100, 240 (1953)

4. W. R. DeHollander, "Spontaneous Combustion of Zirconium," Memorandum to D. F: Hayes (1956) (Included as Appendix C to Ref. 13)

5. B. Kopleman, N. B. Compton. Spontaneous combution of metal powders, Metal Progr. 61,77 (1953)

6. I, Hartmann, J. Nagy, M. Jacobson, "Exploaive characteristics of titanium, zirconium, thorium, uranium, and their hydrides" (Bureau of Mines Report of Investigations 4835,1951 )

7. E. W. Cousins, P. E. Cotton. Design of closed vessels to withstand internal explosions, Chem. Ëng. 58, No. 8,133 (1951)

8. U. S. Patents Nos. $2,667,668$ and $2,667,669$ (1954)

9. "Liquids Metals Handbook; Sodium-NaK, Supplement" (Superintendent of Documents, Government Printing Office, Washington 25. D. C., 1955)

10. J. Katz, E. Rabinowitch, "The Chemistry of Uranium," National Nuclear Energy Series VIII-5 (McGraw-Hill Book Co., Inc., New York, 1951)

11. Zhurnal prikladnoy khimii, 19, No. 4, p. 371 (1946)

12. R. B. Smith. The fire properties of uranium. TID-8011, (1956)

13. R. B. Smith, Zirconium fire and explosion hazard evaluation. TID.5635 (1956)

14. "Recent Titanium-Nitric Acid Explosions" (AEC Accident and Fire Prevention Information, September 13, 1954)

15. "Handbook on Titanium Metal," 7th ed., p. 26 (Titanium Metals Corp, of America, New York, 1953)

16. D. S. Gibbs, H. J. Svec, J. Am. Chem. Soc. 75, 6,052 (1953)

17. S. S. Sidhu. Neutron diffraction studies of Hafnium-Hydrogen and Titsnium-Hydr`gen Systems," ANL-5501 (1956)

18. T. P. Smith, L. W. Eastwood, D. J. Carney, C. E. Sims, "Gabes in Metals" (American Society for Metsls 1953)

19. Explosions involving pickling of zirconium uranium alloys, WAPD-84 (1953) 


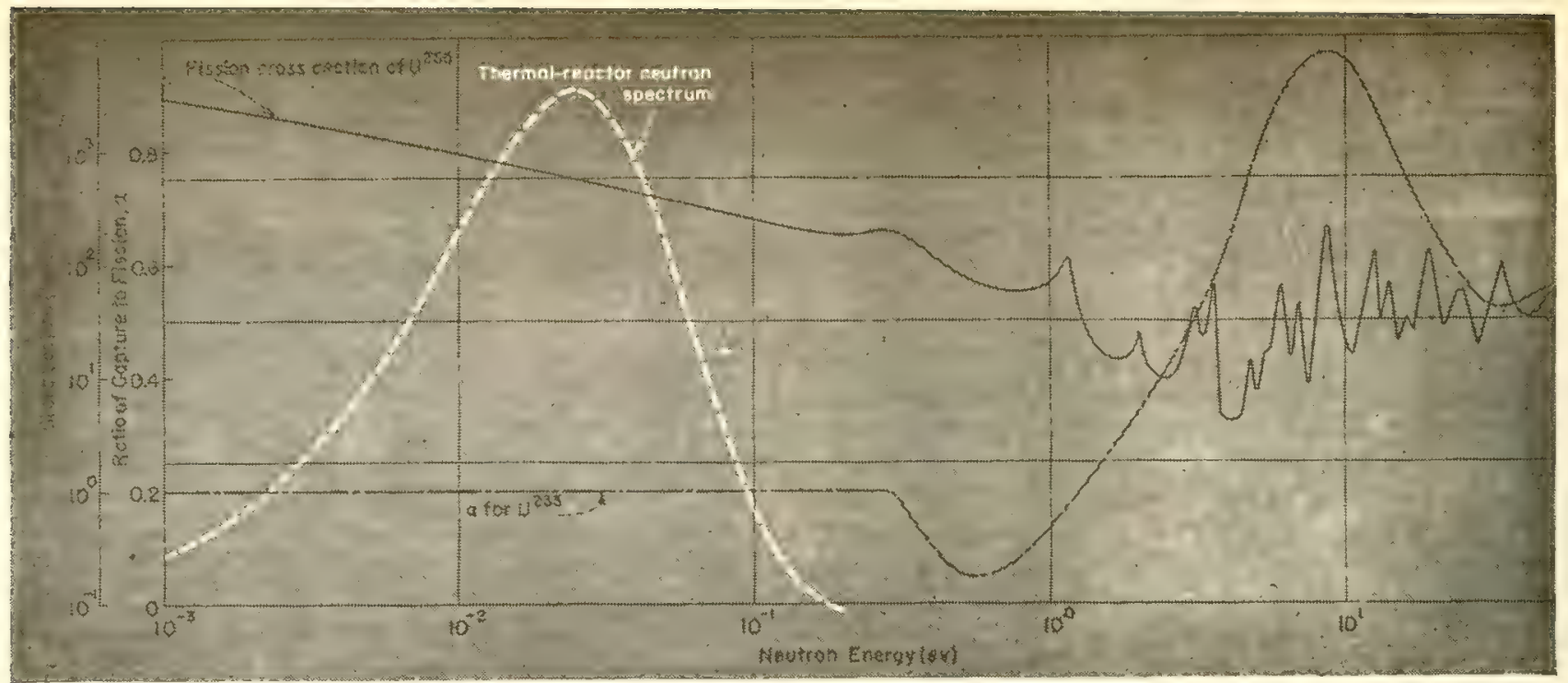

AT THERMAL ENERGIES $\alpha$ is moderate, fission cross section high. Thus breeding ratio is not quite 1.0 but critical mass is low

\section{Fast Breeder Power Reactors}

\section{Aside from their long-term role as plutonium breeders fast reactors could become competitive as power producers. At the moment four major difficulties stand in the way}

BEcause the fast-breeder power reactor is the only reactor type that can breed $\mathrm{Pu}$ from $\mathrm{U}^{238}$ it will certainly find a place in any future largescale atomic energy economy. How large this role will be, however, will depend not so much on the ability of the fast reactor as a breeder but more on its ability to compete successfully with other reactor concepts as a producer of commercial power. At present four basic difficulties tend to make the fast-reactor concept less attractive for large-scale power plants:

1. Need for operation at a high core-power density.

2. Problems of sodium technology.

3. High fuel costs because of large inventory and frequent reprocessing.

4. Criticisms have been made concerning fast-reactor safety.

\section{High Power Density}

High power density is an intrinsic feature of the fast power reactor and calls for heat-transfer performance not usually encountered in industry. Because the active core of a fast reactor is inherently smaller than the core of a thermal reactor for the same total power, the power density in the fast-reactor core must be an order of magnitude greater than the power density in a typical thermal reactor. To accommodate this high power den- sity, high heat-transfer rates must be provided for in the core design.

In addition to using liquid metal as a coolant, the designer must use finely divided fuel to provide enough surface area for heat transfer. Thus, fuel-element thicknesses and coolantchannel widths are small in a fast power reactor. In the Enrico Fermi Fast Breeder Reactor, for instance, a fuel-element assembly consists of 144 fuel pins, 0.158 in. in diameter, 30 in. long, spaced $0.021 \mathrm{in}$. apart.

In addition to being thin the fuel pins must be made and assembled accurately.

Since the power density is never uniform over the core and since dimensions and physical properties of individual elements will vary to some extent from the precise design values, the operating temperatures of some elements in the core will always be significantly above the average value. The average fuel operating temperature (and thereby the thermal efficiency) is then limited by the temperatures of these "hot elements."

Thermal efficiency can be improver by making the fuel-element temperature distribution as uniform as possible. Fast-reactor designers minimize the effect of spatial power variation by rearranging core materials and differentially orificing coolant flow channels.
The effect of variations in the properties and dimensions of individual fuel elements can be offset only by specifying strict design tolerances for the fuel-element components. Thus the basic parts of a fast-reactor core are not only smaller but must be fabricated and positioned with greater precision than would be required in thermal reactors having the same thermal efficiency. The additional expenses for constructing and maintaining the more finely divided core represent an economic penalty for the fast-reactor concept.

\section{Sodium Technology}

Liquid-metal cooling is another necessary feature of fast-power-reactor designs. A fast reactor needs a coolant that does not moderate neutrons and has good heat-transfer properties. Liquid metals in general fulfill both these requirements. Solium in particular has outstanding heat-transfer properties and does not attack containers made of widely used structural materials like low-carbon iron, stainless steel and Inconel. In addition the melting point of sodium is low enough to be used with orlinary structural materials, and its boiling point is high enough to allow a high thermal efficiency. For these reasons all fast power reactor designs use sodium (or 


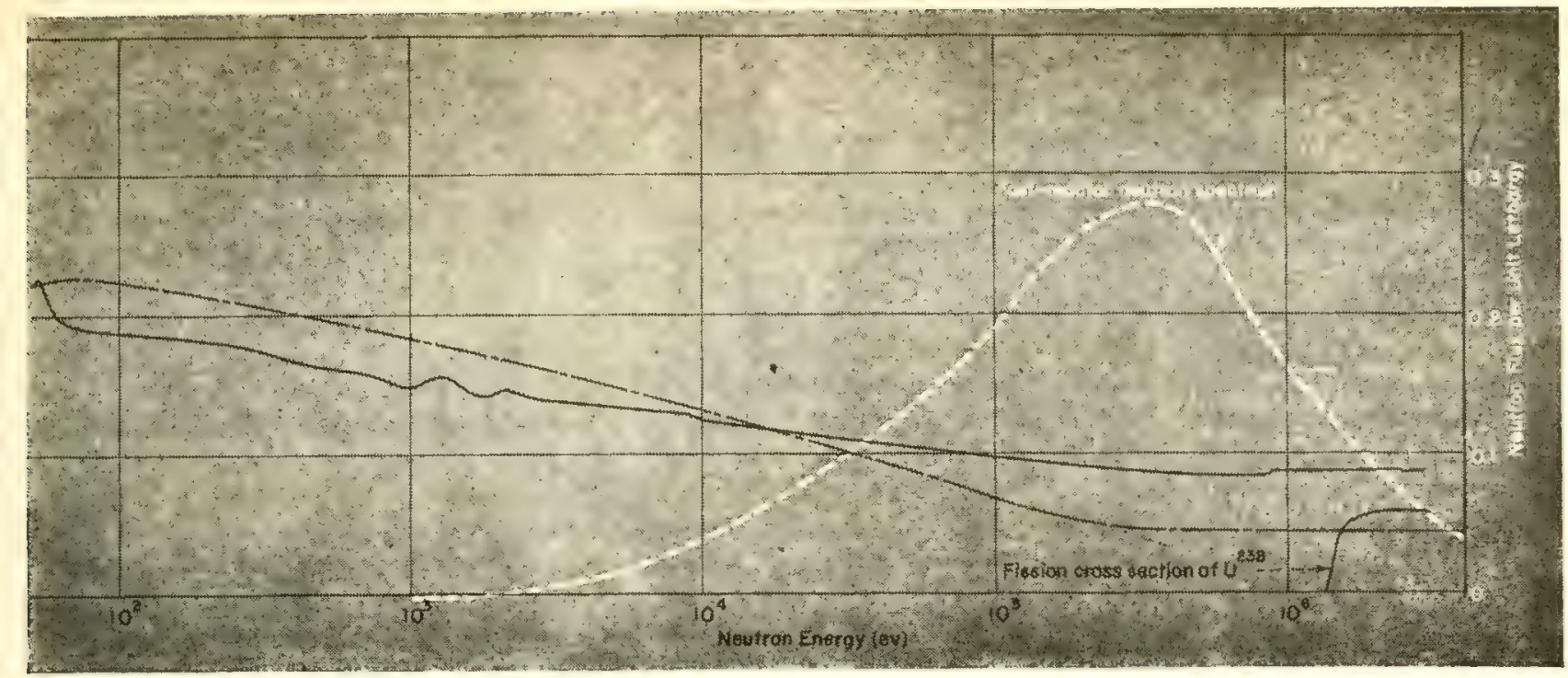

... AT HIGH ENERGIES $\alpha$ is small, fission cross section low. Thus breeding ratio is greater than 1.0 but critical mass is high

\section{Their Problems and Prospects}

sodium-potassium as the coolant.

Although enough information exists today to indicate that a full-scale sodium-cooled reactor could be constructed and made to work, no one has yet demonstrated that the operation of such a system would be feasible in a commercial power plant. Systems having primary sodium and secondary water cooling, compared with systems with only water circuits, require heat exchanger and piping welds of much greater reliability. Liquid-metal system components, pumps, valves, and instruments are beginning to appear on the commercial market, but they need considerably more engineering development before they can be used with as much confidence as the more familiar components used in water systems.

To prevent plugging and corrosion in the coolant channels and piping, the amount of sodium oxide present in the system must be kept small. The control of this contaminant over a long period of time may prove to be a major problem for large sodium systems.

The scarcity of experience with sodium systems is summed up in the realization that the EBR-I, a $1-M w$ experimental system, is the only unclassified sodium-cooled reactor that has been operated anywhere in the world to date.

However, the reactor programs of the United States and Great Britain should soon correct this situation. The Sodium Reactor Experiment, a joint venture by the $\mathrm{AEC}$ and North American Aviation, will go critical the first part of this year. The British have scheduled the start-up of the world's first fast power reactor at Dounreay, Scotland, in 1958. The EBR-II, the American counterpart of the Dounreay reactor, is to begin operation in 1959, and, finally, the EFFBR, the first large-scale fast power reactor, is planned for 1960 . The considerable experience obtained in operating these large sodium systems should answer many of the questions about the feasibility of sodium technology in industry.

\section{Fuel Inventory and Reprocessing}

The fast-power-reactor concept also implies high fuel inyentory and extra fuel-reprocessing costs. The critical mass of fissionable material of a fast reactor is larger than the mass of a comparable liquid-cooled thermal reactor by a factor of two or so. Since a large fuel inventory represents a large capital investment the material efficiency of a reactor ( $\mathrm{kw}$ of electrical output per $\mathrm{kg}$ of fuel) is an important factor in determining the economic feasibility of a reactor. Although fast systems have high thermal efficiency they by nature have low material efficiency so that the over-all or "economic" efficiency of a fast system could easily be smaller than that of a water-cooled thermal system.

In addition the concentration of fissionable material in the meat of a fast-reactor fuel element is roughly
$20 \%$ as compared with 1 or $2 \%$ for a thermal-reactor fuel element. Because of radiation damage, both kinds of elements would have to be removed from the reactor for reprocessing after not more than a number of fissionable atoms equal to about $2 \%$ of all the atoms (fissionable and nonfissionable) present have been burned up (1). This amount of burnup would consume almost all of the initial fuel charge in a thermal element (assuming enough breeding to keep the reactor critical) but not more than $10 \%$ of the initial fuel churge present in a fast-reactor element. Thus a fuel atom may have to be reprocessed more than 10 times before it is finally burned in a fast reactor.

These extra reprocessing operations will certainly add to the costs of operating a fast reactor directly, through the costs of the operations themselves and, indirectly, through the larger inventory requirement and fuel-loss charges associated with reprocessing.

On the other hand, unlike the thermal reactor the fast reactor is insensitive to fission-product poisons. Fuel burnup in a fast reactor is therefore limited more by radiation damage to the fuel than by available excess reactivity. Moreover the reprocessing need not remove all of the fission products and therefore can be much cruder. However, it seems unlikely that these advantages will outweigh the disadvantage of multiple reprocessing.

A possible way around this difficulty is being explored by the AEC in 
the Los Alamos molten-plutonium reactor experiment. But this reactor concept has extreme design requirements and is regarded as something that may prove feasible only in the distant future.

At the present time the fastreactor concept seems to be burdened with excessive fuel-reprocessing costs which may or may not be eventually eliminated. In any case the fast reactor will always be faced with the extra expenses associated with its large fuel inventory.

\section{Safety}

The recent Power Reactor Development Corp. (PRDC) hearings have

\section{Principles of Fast Breeders}

A fast reactor is one in which the fission events are caused by fast neutrons-neutrons with energies close to $1 \mathrm{Mev}$. Thus, a fast-reactor designer must choose a configuration of materials that gives a neutron born in fission a good chance of being captured in fissionable material before it can either be captured elsewhere or be slowed down through scattering. The neutron cross section of materials in general (and of fissionable materials in particular) limit the design choice to one basic type-an enriched, unmoderated, core of fuel diluted as little as possible by other materials.

The absence of a hydrogenous moderator and the careful restriction of the amount and kind of materials used in the core are necessary to keep the neutron spectrum fast. Thus, the use of light elements in a fast-reactor core is always avoided. Structural and coolant materials should be held to a minimum since the addition to the core of any material other than fuel tends to soften the neutron spectrum. For instance, in going from the EBR-I (a fast experimental reactor) to the Enrico Fermi Fast Breeder Reactor (a power reactor) the average neutron energy is depressed from $0.5 \mathrm{Mev}$ to $0.25 \mathrm{Mev}$ by the addition to the core of materials necessary for cooling and breeding.

Since the fast-neutron fission cross section of $U^{235}$ is only a few times greater than the absorption cross section of $U^{238}$, enriched fuel must be used to make a fast reactor critical. In addition, the fast reactor, to achieve criticality, needs a greater total amount of $U^{235}$ than does a liquid-cooled thermal reactor. The reason is that the fast fission cross section of $U^{235}$ is roughly $\mathbf{4 0 0}$ times smaller than its thermal fission cross section. Thus if the two kinds of reactors were to have the same core volume, the critical mass of $U^{23 s}$ for the fast reactor would be many times larger than the critical mass of the thermal reactor.

However, a fast-reactor core will always be smaller than a thermal-reactor core producing the same total power. The fast-reactor core is smaller to begin with since it has no moderator component. In addition, the designer makes the core still smaller to reduce the critical mass and to keep the neutron spectrum fast. The first effect is purely geometrical; the second holds because a large core will be more diluted with nonfuel materials. The minimum practical core size is fixed by the amount of power per unit of core that can be tolerated from heat-transfer considerations.

It turns out that compared with a liquid-cooled thermal reactor of the same power the optimum fast reactor, although it has a much smaller core volome, still has a significantly larger critical mass. A fast reactor will then always operate at a higher power per unit volume of core and at a lower power per unit weight of invested fissionable material.

A primary reason for building fast power reactors is that breeding of $U^{2 s}$ is only possible in a fast reactor. The breeding ratio (number of fissionable atoms created per fissionable atom destroyed) in a thermal reactor is less than $\mathbf{1 . 0}$ for the $\mathrm{U}^{235}$-fuel$\mathrm{U}^{238}$-blanket and the $\mathbf{P u}^{239}$-fuel-U $\mathbf{U}^{239}$-blanket combinations.* The fast reactor has a breeding ratio near 1.2 for $U^{235}-U^{239}$ and of at least 1.5 for $\mathbf{P u}^{230}-\mathbf{U}^{238}$.

The fast-reactor breeding ratio is larger in general because of the smaller absorption of neutrons in structurals. For $\mathbf{P u}^{239}-\mathbf{U}^{238}$ and $\mathbf{U}^{238} \cdot \mathbf{U}^{238}$, in particular, it is larger because of the fast fissions in $U^{239}$ and the high value of $\alpha$ (ratio of the nonfission capture to fission cross sections in the fissionable isotope) for thermal neutrons in $\mathrm{Pu}^{230}$ and $\mathbf{U}^{233}$.

To obtain the breeding ratios quoted above, the average energy of the neutrons in the fast reactor must be kept above $0.10 \mathrm{Mev}$. Below this energy the value of $\alpha$ increases rapidly, reducing the breeding gain. (See figure on p. 112)

* It is true that the breeding ratio in a thermal reactor can be greater than 1.0 where $\mathrm{U}^{2 s}$ is the fuel and thorium is the fertile material but we are concerned here with $\mathrm{U}^{228}$. that the undesirable features of the EBR-I are not basic to fast-reactor designs and that a small amount of additional knowledge in this field would point the way to fast-reactor designs that are safe.

There are at number of characteristics peculiar, or sometimes believed to be peculiar, to fast reactors that are often cited by those who argue that this class of reactors is, in fact, inherently unsafe. They are cited here along with the counterarguments.

Short prompt lifetime. Perhaps the most conspicuous of these characteristics is that the prompt-neutron lifetime of the fast reactor is very short, at least a hundred times smaller than the prompt-neutron lifetime of a typical thermal reactor. Thus, if the reactivity ever exceeds prompt-critical, the power in a fast reactor will begin to rise with a period a hundred times faster than that in a thermal reactor.

Although the advocates of fast systems admit that the power excursion resulting from a prompt-critical reactivity would be much more severe for a fast reactor, they point out that the nature of fast reactors permits designs that make a prompt-critical situation very unlikely.

Because fast reactors are insensitive to fission-product poisons and have a large critical mass, the excess reactivity required to operate for a given number of megawatt days is much smaller for a fast reactor than for a thermal reactor. A large thermal power reactor requires an excess reactivity of the order of ten dollars. The needs of a comparable fast power reactor can be satisfied by an excess reactivity of less than one dollar, which is below the prompt-critical region. Thus, unlike thermal-reactor designs, the total amount of excess reactivity built into a fast reactor is less than the amount required to make the reactor go prompt-critical. Fast-reactor designers feel that under these circumstances it is difficult to imagine how a prompt-critical situation can arise without postulating a series of very unlikely events.

Even if by some unforeseen combination of circumstances the reactor should exceed prompt-critical, fast-reactor designers predict that nothing too serious would happen. NDA (Nuclear Development Corporation of America) has carried out calculations for APDA (Atomic Power Development Associates, Inc.) which show that even 
under quite pessimistic conditions the total energy released during a promptcritical power surge would probably not be enough to rupture the reactor vessel.

Moderator accident. Another characteristic of fast reactors that might influence safety is that the reactivity of a fast reactor will increase if a molerator is somehow introduced into the system. For instance, if the sodium in a fast-reactor coolant system were partially replaced by some material containing hydrogen-say oil or water-the reactivity would increase and might conceivably go beyond prompt-critical. Designers believe, however, that fast-reactor systems can be constructed in ways that will eliminate this possibility.

The EFFBR designers, for instince, have severely restricted the use of hydrogenous materials anywhere in the building. Since it is difficult to see how even the small amount of material that will be available could get into the sodium system, the EFFBR designers believe that for their machine the moderator hazard is negligible.

Positive temperature coefficient. The observation that the EBR-I had a prompt positive temperuture coefficient is sometimes interpreted to mean that all fast reactors will have prompt positive temperature coefficients. Those familiar with the physics and the history of the EBR-I believe that the opposite is true. They contend that the observed positive cuefficient most likely originates from the bowing of the fuel elements caused by radial temperature gradients present during reactor operation. One can estimate the amount of bowing that should occur at a given reactor power, and this turns out to be about the right amount to explain the observed temperature coefficient. Since no one can suggest any alternative effects to explain the observations, ${ }^{*}$ it is generally believed that bowing was the sole cause of the EBR-I positive temperature coefficient. Such an effect clearly depends on particular details of the core design and has nothing to do with characteristics of fast reactors in general.

Resonance instability. The EBR-I experiments also revealed another, less well known type of instability, called resonance instability. This kind of reactor instability is characterized by spontaneous oscillations of the reactor power at certain discrete reactor oper-

\section{Strategies for Exploiting Our Atomic Resources}

From a technical point of view there are two possible strategies that could be used to extract energy from the world's resources of uranium and thorium. One could use fast reactors exclusively or one could use mostly thermal reactors with a few fast reactors for breeding purposes. Economic feasibility will determine which scheme will eventualiy be used. It is too soon to say which system will ultimately prove superior. At the moment, however, for reasons presented elsenhere in this article thermal reactors seem to have the edge.

If the thermal machines do indeed win out, the atomic power industry may develop along the following lines: In the beginning all commercial power will be generated by thermal converters using $U^{239}$ or thorium as raw materials. Since thermal reactors can breed successfully on a $U^{233}-T h$ cycle and there would be presumably no economic advantage to using fast reactors for this purpose, thermal reactors alone will continue to be used to exploit thorium energy resources. Thorium deposits are believed to represent about half of the total atomic-energy potential; therefore on this basis, thermal thorium converters will account for roughly half of the reactor population. Thermal reactors converting natural uranium cannot breed and so require periodic additions of fissionable material. At first $U^{23 s}$ obtained by enriching natural uranium will be used for this purpose.

Later on Pu produced by a fast reactor will become cheaper than $\mathbf{U}^{23 s}$ produced by enrichment. At this point the fast breeder will take over the function of supplying fissionable material to the uranium-converting thermal power reactors. The breeding ratio of the thermal converter is close enough to 1.0 so that a single fast breeder can supply a number of thermal reactors. Thus if the converter has a breeding ratio of 0.9 and the fast reactor has a ratio of 1.5 the fast reactor can keep five thermal converters in business, each at a power level equal to that of the fast reactor. Thus the primary function of a fast-breeder power reactor of the future will be to feed fissionable material to thermal reactors which in turn will generate practically all the power.

In this way the total energy available in the world's resources of $\mathbf{U}^{223}$ can be completely utilized. Although in such a scheme the number of fast reactors would be in the minority, their presence is absolutely escential. Without them most of the energy stored in $U^{298}$ would be inaccessible. For this reason, regardless of which reactor type proves more economical, the development of satisfactory fast breeder power reactors is a job which must be done.

ating conditions. Again, since experience with this kind of behavior is limited to the EBR-I, there has been a tendency to associate resonance instability with fast reactors. However, recent studies indicate that the resonant behavior of the EBR-I, like the positive temperature coefficient, is caused by specific design features and would not be a general characteristic of fast reactors.

In general, resonance behavior of a physical system depends on the existence of a feed-back relationship between two dynamic variables associated with the system. In a reactor the power influences the reactivity, and the reactivity in turn determines the power so that these two variables form a feedback loop. Because of this feed-back mechanism reactor systems may have resonances at certain well-defined operating conditions - when the reactor is operated at certain combinations of

* At one time the Doppler effect in the reactor fuel was considered as a possible source of a prompt positive temperature coefficient. However enough information is now available to discount this possibility. For the EBR-I at best only $5 \%$ of the observed positive temperature coefficient can be explained by Doppler effect. In a large power reactor like the EFFBR, because of the higher ratio of $U^{238}$ to $U^{235}$, the Doppler effect will give a negative temperature coefficient. power level and coolant flow, the reactor power will begin to oscillate at a definite frequency without any external cause. If the reactor power is increased beyond the resonance value the amplitude of the power oscillations will increase exponentially with time. The reactor would then be intrinsically unstable in this region. However, the reactor would be completely safe as long as its power level is kept below the resonance value.

Bethe incorporated in his PRDC testimony a thorough analysis of the theory and detection of resonances in reactor systems (3). He concludes that a reactor resonance always requires the existence of a large delayed negative temperature coefficient of reactivity. Such coefficients would probably be associated with the heating of structural components outside the core by convection. Bethe points out that this kind of difficulty would be hard to foresee in the design stage and might not be uncovered until the reactor is built and tested. (For instance, no one has yet explained the physical origin of a delayed negative temperature coefficient in the EBR-I.)

Because the existence of a delayed negative temperature coefficient depends on the details of the individual 
reactor design, Bethe argues that resonances, in principle, could occur in either thermal or fast reactors. However, he also points out that the conditions for a resonance are more likely to be satisfied in an unmorlerated smallcore reactor than in a moderated large-core reactor. Since fast reactors generally fit the former description and thermal reactors the latter, resonances might be expected to crop up more frequently in fast systems.

Bethe feels confident that in either kind of reactor system resonances can be avoided by proper reactor design. Further observations planned by the AEC on EBR-I should provide valuable information for such designs.

Supercriticality upon meltdown. The final criticism of fast reactors is that the large amount of fissionable material contained in a fast-reactor core might through some accident redistribute itself to form a highly supercritical mass. For instance, if all the enriched material in the EFFBR were assembled in a solid ball and then surrounded by a good neutron reflector, the resulting mass would be six times critical.

This hazard is distinctly a characteristic of fast reactors. Thermal reactor fuel is always more diluted because of lower $\mathrm{U}^{235}$ enrichment or the presence of a moderator. In fast reactors, only the geometric dispersion of the fuel for cooling purposes prevents the reactor from being supercritical. For this reason, it is essential to make sure that the fuel in a fast reactor can never assemble in one large mass.

Such a situation might conceivably result if the core of the reactor were to melt during a severe power excursion. The fact that a partial meltdown did occur in the core of EBR-I (4) might lead to concern for the safety of all fast reactors in this respect.

Two important aspects of the EBR-I incident argue against this:

First, the meltdown accident occurred when the reactor was deliberately used in an unsafe way to test the positive temperature coefficient.

A full-scale power reactor would not have a positive temperature coefficient and would be designed so that operation under "experimental circumstances" would be impossible.

Second, the EBR-I meltdown did not result in the formation of a critical mass. Boiling sodium entrapped in the core is believed to have forced the molten uranium apart, preventing the formation of a critical mass. $\mathrm{Ap}$ parently the melting of uranium in the presence of sodium, which can vaporize, does not lead to a supercritical assembly but tends instead to shut down the reactor.

The most serious potential hazard associated with a meltdown arises from a situation not present in the EBR-I incident. At the moment, as far as safety is concerned, the fast-reactor concept's biggest worry is the possibility that a supercritical meltdown might occur following the loss of the sodium coolant. Even if the reactor were immediately shut down with the safety rods, in the absence of coolant the fission-product afterheat would melt the core in a few minutes. Since there would be no sodium vapor to exert pressure, the molten core would collapse under gravity and might form a critical mass in the bottom of the reactor container.

Dr. Bethe and Dr. Tait of the British Atomic Research Establishment at Harwell, have made rough upper-limit calculations of the energy that might be released by such an accident. The most realistic value obtained so far is a total energy release equal to $1,000 \mathrm{lb}$ of TNT. Although Bethe points out that this figure may be a gross overestimate, the magnitude of this result has stimulated a large effort to obtain more information.

The AEC has initiated a comprehensive program at Argonne designed to arrive at a basic understanding of meltdown phenomena.

In addition APDA has asked Nuclear Metals, Inc., Cambridge, Mass., to do experiments similar to those planned by ANL and has also undertaken further theoretical studies.

Meanwhile fast-reactor designers are trying very hard to minimize the chances of sodium-coolant loss and are also building in features to prevent a nuclear event if a coolant-loss meltdown should occur.

In the EFFBR secondary containment is provided around all primarycoolant-system piping and vessels so that a leak in the primary system cannot cause excessive loss of sodium. In the event of loss of pumping power the design allows sufficient natural-convection cooling for the decay heat. A large reservoir of sodium above the core appreciably increases the time required for a leak to drain the core. Other design provisions allow for direct emergency action to restore cooling if somehow the core were to be completely drained.

To limit the consequence of a meltdown a special meltdown container has been built into the lower sodium plenum. If a meltdown should occur the molten fuel is expected to collect in the container in a noncritical geometry. However, there is some concern that the molten fuel might refreeze in the coolant channels in the blanket region beneath the core and never reach the meltdown container. Depending on results of additional analysis PRDC may abandon the lower blanket in the interests of safety.

In summary, the question of fastreactor safety is most likely one that can be eliminated by proper design. What constitutes proper design, however, is not yet completely understood.

\section{Outlook}

We may conclude that, as things stand today, the feasibility of the fastreactor concept as a basic power producer suffers from both technical and economic difficulties. Hopefully, the purely technical problems may quickly be eliminated; the economic questions, however, are likely to remain.

The combined efforts of the AEC and PRDC can be expected to demonstrate in the near future that properly designed fast reactors are as safe to operate as thermal reactors. Also experience with the large sodium systems now being constructed may prove before long that the industrial use of sodium coolant is technically and even economically within reach. On the other hand, it is more doubtful that the fast reactor can escape the economic penalty of the extra fuel reprocessing. Higher expenses for core fabrication and fuel inventory appear to be permanent economic disadvantages.

In its favor the fast reactor has the one fundamental advantage of a breeding ratio substantially greater than 1.0 . Thus, the value of the extra Pu produced by a fast breeder must exceed the additional expenses of building and operating a fast breeder if this kind of reactor is to compete with thermal reactors for the job of large-scale commercial power production.

\section{BIBLIOGRAPHY}

1. W. B. Lewis, Nocleonice 14, No. 10, 28 (1956)

\&. NUCLEONICs 15, No. 2, R2 (1957)

3. H. A. Bethe. Reactor safety and oscillator tests, APDA-117 (1956)

4. NuCLFonics 16, No, 1, 84 (1957) 


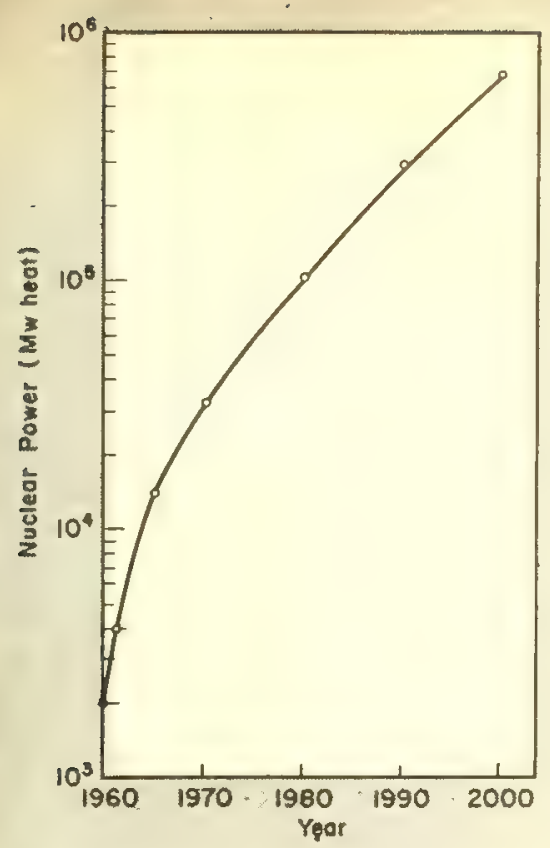

FIG. 1. Predicted U. S. nuclear power (heat) generation, assuming 8 -fold increase by 2000 , and half of plants built in $\mathbf{2 0 0 0}$ are nuclear

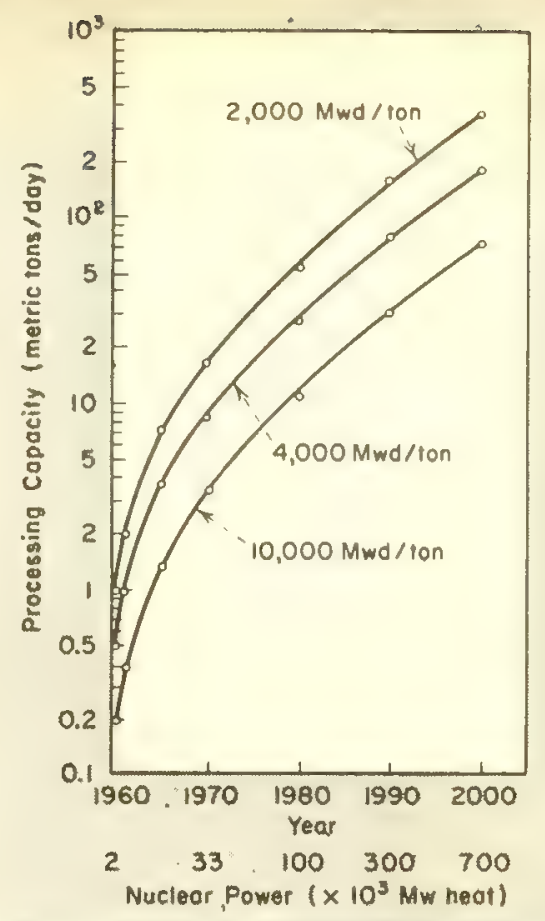

FIG. 2. Radiochemical-processing-capacity requirements. Average irradia. tion level (Mwd/ton) of spent reactor fuel is taken as parameter

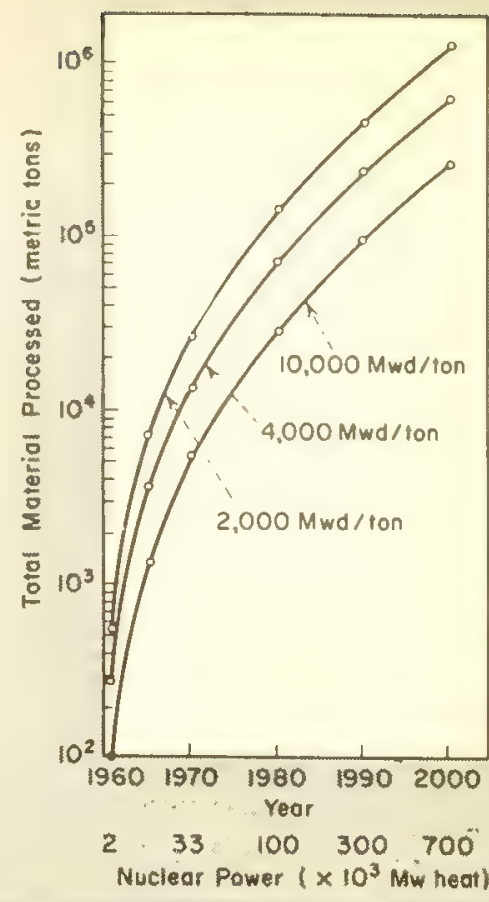

FIG. 3. Predicted total spent fuel processed. Average irradiation level of spent fuel (Mwd/ton) is taken as parameter

\section{Economics of Waste Disposal}

\section{By the year 2000, accumulated fission-product activity may be greater than}

\section{$3 \times 10^{11}$ curies. To dispose of it, the authors estimate a cost}

\section{of $\$ 1.60-\$ 64 / \mathrm{gal}$ will be permissible for the predicted nuclear-power economy}

By H. R. ZEITLIN,* E. D. ARNOLD, and 5. W. ULLMANN

Chemical Technology Division, Oak Ridge National Laboratory, Oak Ridge, 'T'ennessee

IN A NUCLEAR-POWER ECONOMY, the cost of disposing of reactor fission-product waste will be significant. To determine the magnitude of the waste-disposal problem and to define the limits within which the solution must be found, we have studied three aspects:

1. How much waste will accumulate by the year 2000 ?

2. What fraction of the cost of nuclear power can be economically allocated to waste disposal?

3. What are the optimum wastestorage (cooling) period and shipment weight (including shield) for minimum cost?

\footnotetext{
* Preaent addregs: Argonne Nationa Laboratory, Lemont, Ill.
}

\section{Predicted Accumulated Activity}

Based on a predicted nuclear power economy growth rate, calculations have been made to determine as a function of time the magnitude of several quantities important to the radiochemicalprocessing and waste-disposal industries. Included are such quantities as required processing capacity, buildup of activity of important fission products, and accumulated volume of highactivity liquid wastes.

If it is assumed that the installed electrical plant capacity of the United States will increase eight-fold during the next 50 years $(1,2)$, that there will be $500 \mathrm{Mw}$ of installed nuclear electric capacity in 1960, and that half of all new plants built in the year 2000 will be nuclear plants, the installed nuclear plant capacity, $N(\mathrm{Mw})$, at time $T$ (years after 1960) will be

$$
N=5,800\left(1.09^{T}-1\right)+500
$$

Assuming a thermal efficiency for the reactor system of $25 \%$, the heat power requirements will be four times as great $N(\mathrm{MW}$ heat $)=23,200\left(1.09^{T}-1\right)$
$+2,000$

This equation is plotted in Fig. 1.

Differential equations were set up for the simultaneous growth and decay of various fission products being produced by an expanding nuclear economy. The solutions give the accumulated 


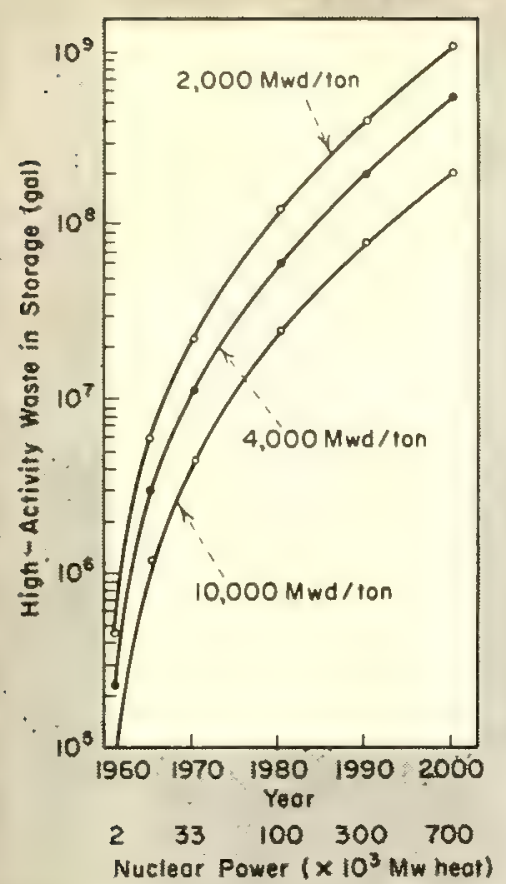

FIG. 4. Accumulated volume of highactivity waste. Curves are based on $820 \mathrm{gal}$ of high-activity waste per ton of fuel processed

curies of a fission product at any time between 1960 and 2000. It should be emphasized that the activities plotted are not only the activities to be found in waste-disposal tanks but include fission products present in the reactors and in fuel being stored prior to processing. This is especially important for the shorter-lived isotopes such as $\mathrm{Ba}^{160}$.

In Figs. 2 and 3 are plotted predicted processing-capacity requirements. Figures 4-6 show accumulated radioactive wastes, and Fig. 7 the accumulated plutonium activity. Accumulated activity means the integrated production of fission-product activity with time minus loss by decay and by neutron capture while in the reactor.

TABLE 1-Cost of Tank Storage of Liquid Waste

\begin{tabular}{cccc}
\hline \multicolumn{4}{c}{ Unit cost $(\$ /$ gat $/ y r)$} \\
$\begin{array}{c}\text { Rate for } \\
\text { fixed charge } \\
(\%)\end{array}$ & $\begin{array}{l}\text { Fixed } \\
\text { charge }\end{array}$ & $\begin{array}{c}\text { Direct } \\
\text { charge }\end{array}$ & Total \\
\hline \multicolumn{5}{c}{ chitial investment } \\
\hline $0.25 /$ gal inis \\
15 & 0.038 & 0.003 & 0.041 \\
12 & 0.030 & 0.003 & 0.033 \\
4 & 0.010 & 0.003 & 0.013 \\
$\$ 2.00 /$ gal initial investment & \\
15 & 0.30 & 0.003 & 0.30 \\
12 & 0.24 & 0.003 & 0.24 \\
4 & 0.08 & 0.003 & 0.08 \\
\hline
\end{tabular}

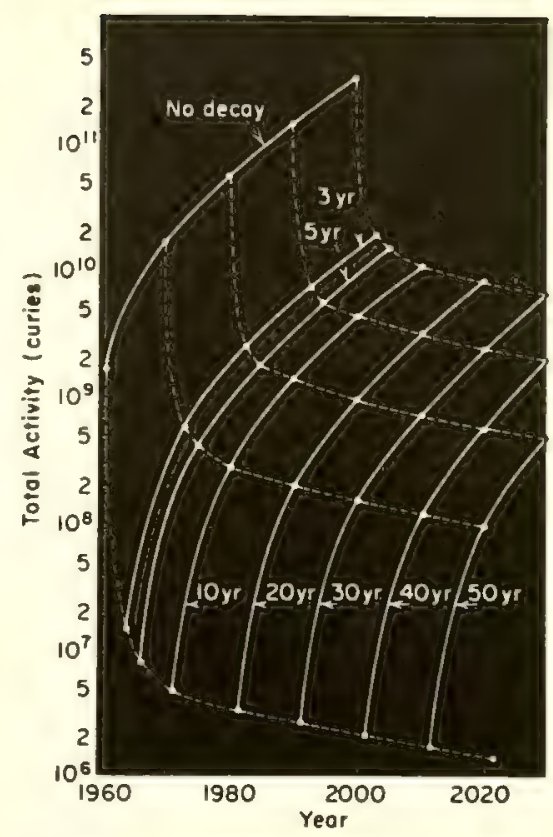

FIG. 5. Accumulated activity. Dashed decay lines are used to determine accumulated octivity following any specifled cooling period

Capture is negligible, making the curves essentially independent of reactor design and operating conditions. The activity can be considered as being dumped into and accumulated in a common "sink."

With respect to the waste-disposal problem, the amount of plutonium in storage is several orders of magnitude less than the $\mathrm{Cs}^{137}$ and $\mathrm{Sr}^{90}$ in storage. Thus plutonium becomes the limiting factor on permitted dispersal of aged wastes only after removal of $\mathrm{Cs}^{137}$ and $\mathrm{Sr}^{90}$.

\section{Allowable Waste-Disposal Cost}

The limits on the tolerable cost per initial gallon of liquid radiochemical process waste can be defined as a function of

1. Reactor operating and design characteristics.

2. The radiochemical separations process employed.

3. The fraction of 8 mill $/ \mathrm{kwh}$ power that is allocated to the disposal of wastes.

It has been said that ultimate disposal of radioactive liquid wastes, particularly those resulting from the processing of spent reactor fuels, would place a significant economic burden on the future nuclear power economy (\$). Waste-disposal costs of the order of $\$ 1$ per initial gallon of liquid wastes resulting from the standard solvent-extrac-

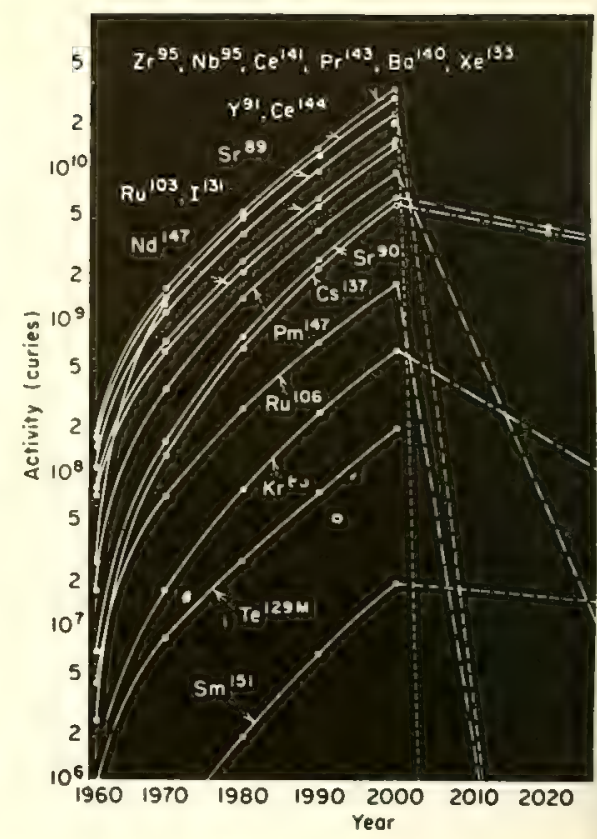

FIG. 6. Accumulated activity of important fission products in predicted nuclear economy. Dashed lines indicate decay of accumulated activity

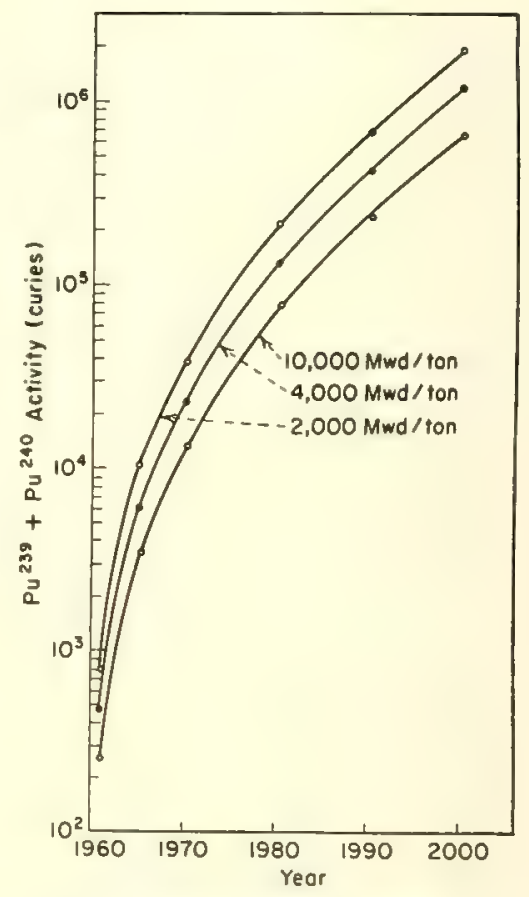

FIG. 7. Accumulated plutonium activity. Curves are based on $1 \%$ process loss of plutonium

tion separations processes seemed to be an upper limit. These cost limits do hold for certain reactor-processing complexes, but are for the most part not in accord with the characteristics of the contemplated nuclear power economy.

It is our purpose here to indicate the allowable cost limits in the more genera] case, taking pertinent reactor-process- 
TABLE 2-Fission-Product Activities at Discharge from Reactor

Curie/watt reactor power for operating times of:

\begin{tabular}{|c|c|c|c|c|c|c|}
\hline \multirow[b]{2}{*}{ Nuclide } & \multirow[b]{2}{*}{$\lambda_{i}$} & \multirow[b]{2}{*}{$Y_{i}$} & \\
\hline & & & 80 days & 160 days & 320 days & 400 days \\
\hline $\mathrm{Ba}^{140}\left(\mathrm{La}^{140}\right)$ & 0.0541 & 0.063 & 0.052 & 0.053 & 0.053 & 0.053 \\
\hline $\mathrm{Nb}^{95}$ & 0.0198 & 0.064 & 0.042 & 0.051 & 0.054 & 0.054 \\
\hline $\mathrm{Zr}{ }^{06}$ & 0.0107 & 0.064 & 0.031 & 0.044 & 0.052 & 0.053 \\
\hline $\mathrm{Ce}^{144}\left(\operatorname{Pr}^{144}\right)$ & 0.00239 & 0.061 & 0.0087 & 0.016 & 0.027 & 0.031 \\
\hline $\mathrm{Cs}^{187}$ & $5.75 \times 10^{-6}$ & 0.059 & $2.5 \times 10^{-1}$ & $4.5 \times 10^{-4}$ & $8.9 \times 10^{-4}$ & $1.1 \times 10^{-3}$ \\
\hline$R u^{108}\left(R h^{106}\right)$ & 0.0019 & 0.0038 & $4.5 \times 10^{-6}$ & $8.3 \times 10^{-4}$ & $1.5 \times 10^{-3}$ & $1.7 \times 10^{-3}$ \\
\hline
\end{tabular}

TABLE 3-Activity in Liquid Waste for Various Cooling Times*

\begin{tabular}{|c|c|c|c|c|c|c|c|c|}
\hline \multirow[b]{2}{*}{ Nuclide } & \multirow[b]{2}{*}{$\begin{array}{c}\gamma \text { energy } \\
(M e v)\end{array}$} & \multirow[b]{2}{*}{$\begin{array}{c}\text { Gamma } \\
\text { yield } \\
(\%) \dagger\end{array}$} & \multicolumn{6}{|c|}{$\gamma$ curies/gal for cooling time of: } \\
\hline & & & $\begin{array}{c}100 \\
\text { days }\end{array}$ & $\begin{array}{l}500 \\
\text { days }\end{array}$ & $\begin{array}{l}1,000 \\
\text { days }\end{array}$ & $\begin{array}{l}3,000 \\
\text { days }\end{array}$ & $\begin{array}{c}10,000 \\
\text { days }\end{array}$ & $\begin{array}{c}30,000 \\
\text { days }\end{array}$ \\
\hline \multirow[t]{3}{*}{$\mathrm{La}^{140}$} & 3.0 & 1 & 0.068 & & & & & \\
\hline & 2.5 & 5.4 & 0.37 & & & & & \\
\hline & 1.5 & 94 & 6.4 & & & & & \\
\hline $\mathrm{Nb}^{96}$ & 0.75 & 100 & 240 & 0.084 & & & & \\
\hline $\mathrm{Zr}^{96}$ & 0.73 & 100 & 560 & 7.4 & 0.035 & & & \\
\hline \multirow{3}{*}{$\operatorname{Pr}^{144}$} & 2.2 & 1 & 7.7 & 3.1 & 1.0 & $1.4 \times 10^{-4}$ & & \\
\hline & 1.5 & 2 & 15.4 & 6.2 & 2.0 & $2.8 \times 10^{-4}$ & & \\
\hline & 0.7 & 4 & 30.8 & 12 & 4.0 & $5.6 \times 10^{-4}$ & & \\
\hline $\mathrm{Cs}^{187}$ & 0.66 & 92 & 31 & 31 & 29 & 23 & 18 & 6.2 \\
\hline \multirow[t]{5}{*}{$\mathrm{Rh}^{106}$} & 2.4 & 0.25 & 0.11 & 0.053 & 0.020 & & & \\
\hline & 1.6 & 0.5 & 0.22 & 0.11 & 0.041 & & & \\
\hline & 1.0 & 2 & 0.86 & 0.41 & 0.16 & & & \\
\hline & 0.9 & 1 & 0.43 & 0.21 & 0.08 & & & \\
\hline & 0.6 & 12 & 5.2 & 2.5 & 0.97 & & & \\
\hline
\end{tabular}

* Based on 400-day irradiation, $25 \mathrm{Mw} /$ ton, and $800 \mathrm{gal} /$ ton waste.

$\dagger$ Percentage of decays of nuclide yielding gammas of stated energy.

ing-cost variables into consideration.

Figure 8 gives the allowable disposal cost per initial gallon of waste as a function of the burnup of the spent reactor fuel that is being processed. These calculations are based on an electrical power cost of 8 mills $/ \mathrm{kwh}$. The curves were derived using values representative of the reactor and processing characteristics now being proposed for an economic (low-enrichment) nuclear power economy. A similar curve using values of the variables representative of a premium power (high-enrichment) economy is plotted in Fig. 9.

Allowable costs for waste disposal can vary from $\$ 0.80$ to $\$ 80$ per gallon for a low-enrichment economic nuclear power economy and from $\$ 0.08$ to $\$ 4.80$ for a high-enrichment power economy, depending on the reactor and process characteristics and on the fraction of the total cost of power allocated to waste disposal.

Although both high-activity and low- activity wastes result from processing reactor fuels, and low-activity wastes will presumably be cheaper to dispose of, no differentiation was made in Fig. 8. Therefore, on the assumption that onefourth of the allocated portion of the total power cost went for low-activity disposal, another series of curves shown in Fig. 10 was determined to indicate the allowable cost of disposing of lowactivity wastes.

The shaded area in Fig. 8 probably well represents an average of the operating characteristics of reactors and chemical processes that will exist in an economic low-enrichment nuclear power economy. From this area, it can be shown that as the allocated portion of the total power cost varies from $1 \%$ to $10 \%$ (i.e., $0.08-0.80 \mathrm{mill} / \mathrm{kwh}_{\text {。 }}$ ), the allowable cost for waste disposal will vary between $\$ 1.60$ and $\$ 64$ per gallon.

Since there are many areas in the economic picture of nuclear power that may be in error to $3 \%$ or more, an allocated cost for waste disposal of $3 \%$ does not appear unreasonable. Therefore, a cost range of $\$ 5-\$ 20$ per gallon of initial high-activity waste may be a better basis to use in determining the direction that waste-disposal research and development should follow.

It is obvious that to reduce the stringent economic requirements dictated to the disposers of radioactive wastes (i.e., geologists, biologists, oceanographers, sanitary engineers, designers of tanks, pits, and fancy gadgets for treatment of wastes, etc.) it is necessary to design reactors with even increasing processing-cycle times and design chemical processes with ever decreasing volumes of wastes per unit weight of reactor fuel processed.

\section{Storage and Shipping Costs}

One of the potentially attractive schemes for the ultimate disposal of radioactive waste is simply to pour the waste into pits. The best geological location for these pits may not coincide with the optimum location for a processing plant and transport, will be required.

It may be necessary or desirable to remove some fission products from the waste, particularly the long-lived ac-

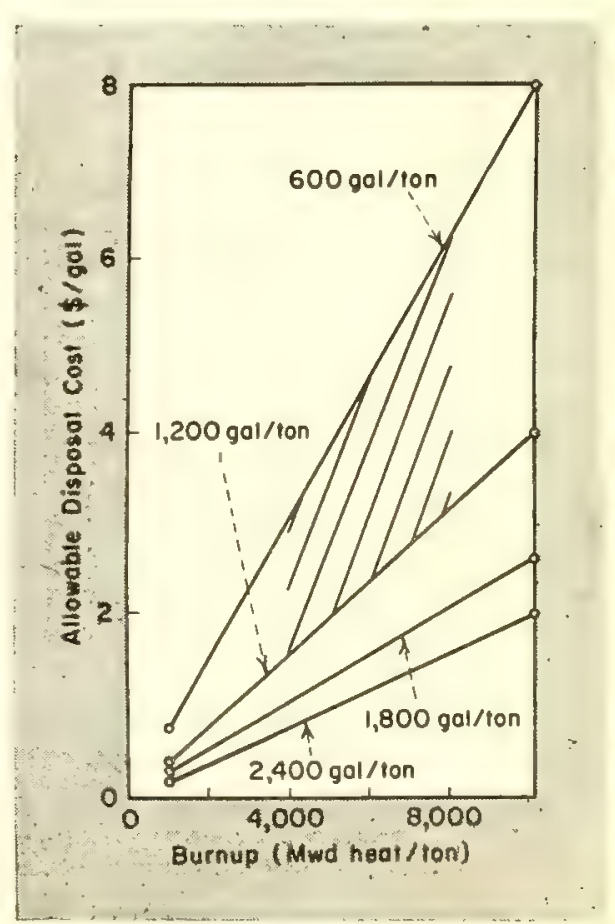

FIG. 8. Allowable waste disposal cost as function of burnup and processwaste volume $-0.08 \mathrm{mill} / \mathrm{kwh}_{\text {. }} 11 \%$ power costl allocated to disposal 
TABLE 4-Storage and Shipping Costs as Functions of Cooling Time

\begin{tabular}{|c|c|c|c|c|c|c|}
\hline \multirow{2}{*}{$\begin{array}{c}\text { Cooling time } \\
(y r)\end{array}$} & \multicolumn{3}{|c|}{ Slorage cost $(\$ / g a l)$} & \multicolumn{3}{|c|}{ Shipping cost (\$/gal) } \\
\hline & $\$ 0.019 / \mathrm{gal} / \mathrm{yr}$ & $\$ 0.041 / \mathrm{gal} / \mathrm{yr}$ & $\$ 0.30 / \mathrm{gal} / \mathrm{yr}$ & $200 \mathrm{mi}$ & $500 \mathrm{mi}$ & $1,000 \mathrm{mi}$ \\
\hline 0.5 & 0.006 & 0.02 & 0.15 & 1.70 & 3.16 & 5.46 \\
\hline 5 & 0.06 & 0.20 & 1.50 & 0.74 & 1.37 & 2.38 \\
\hline 6 & 0.08 & 0.25 & 1.80 & 0.55 & 1.02 & 1.77 \\
\hline 7 & 0.09 & 0.29 & 2.10 & 0.49 & 0.91 & 1.58 \\
\hline 10 & 0.13 & 0.41 & 3.00 & 0.44 & 0.82 & 1.42 \\
\hline 15 & 0.19 & 0.61 & 4.50 & 0.42 & 0.78 & 1.35 \\
\hline 20 & 0.26 & 0.82 & 6.00 & 0.41 & 0.76 & 1.32 \\
\hline 30 & 0.39 & 1.23 & 9.00 & 0.40 & 0.74 & 1.29 \\
\hline
\end{tabular}

tivities, prior to ground disposal. The influence of fission-product removal on shipping costs will depend on which elements are removed and to what extent. Since no firm flowsheet has been generally accepted for this step, its effect on waste-disposal economics is not considered here.

As waste is cooled, the cost of storage increases, and the cost of shipping decreases by virtue of having to ship less shield weight. The optimum cooling time will depend on the irradiation history of the fuel, the cost of storage, and the cost of shipping.

Storage costs. Tank storage of liquid wastes may be an essential step

in a scheme for ultimate waste disposal. Storage reduces the heat generation and radiation problems attendant with shipping and subsequent processing. As an example, the rate of heat generfission-product saturation) at $25 \mathrm{Mw} /$ ton is shown in Fig. 11 as a function of cooling time; 800 gal of waste per ton have been assumed.

To determine an optimum storage time to be employed in the over-all storage-shipment-treatment-disposal cycle, an estimate of the cost of storing a unit volume per unit time is necessary. The following assumptions were made: ation for fuel irradiated a long time (to
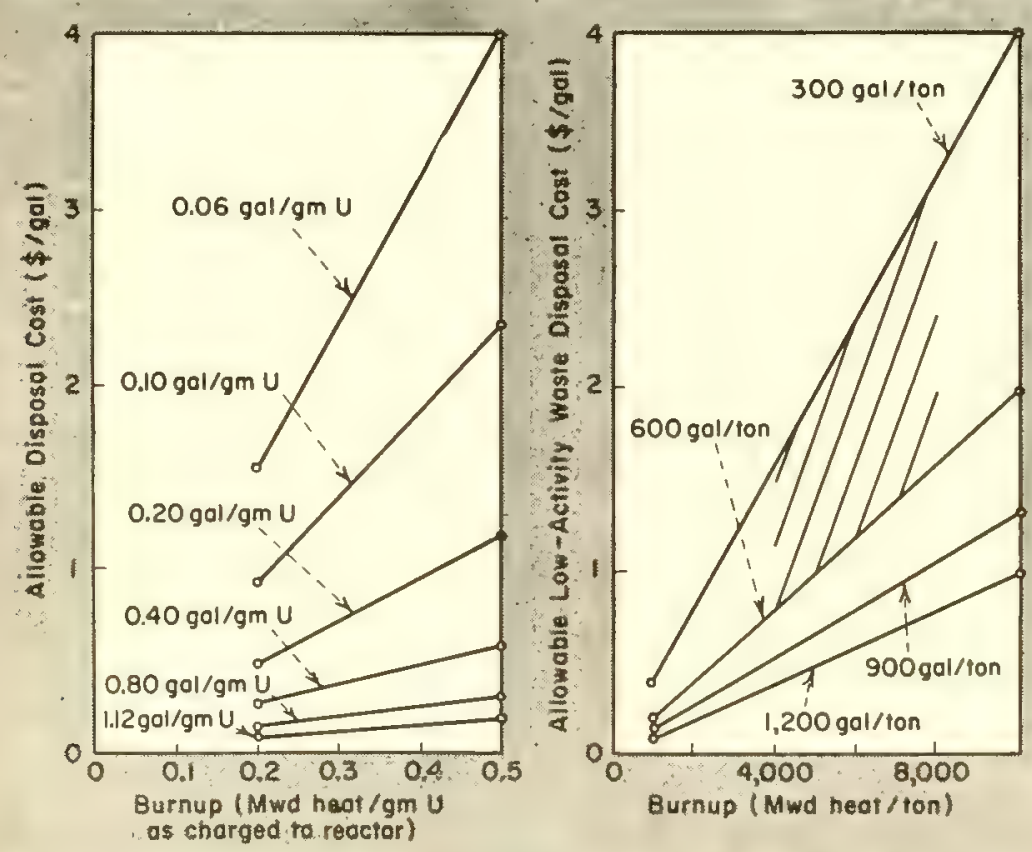

FIG. 9. Allowable waste disposal cost for highly enriched (premium power) reactors-1\% power cost allocated to disposol

FIG. 10. Allowable disposal cost for low-activity waste-based on allocation of $0.25 \%$ power cost to waste disposal

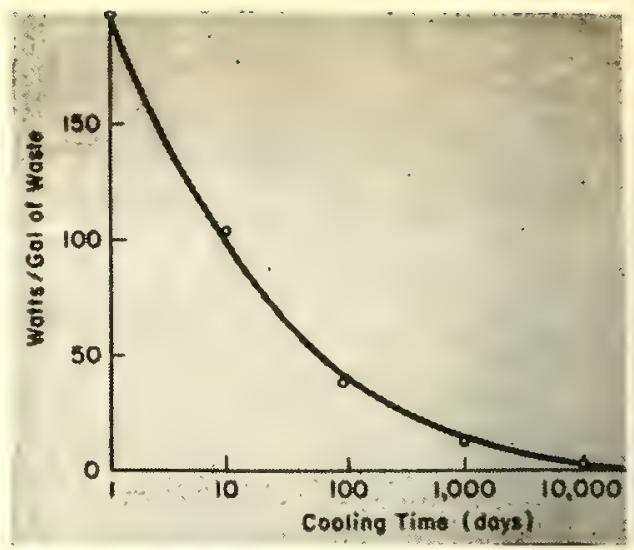

FIG. 11. Waste-heat generation of discharged reactor fuel. Based on infinite irradiation time of $25 \mathrm{Mw} /$ ton and $800 \mathrm{gal}$ waste per ton

1. Lifetime of underground storage tank of $50 \mathrm{yr}$

2. Purex-type waste

3. Tank farm operating personnel of $2 \mathrm{men} / \mathrm{shift}$

Fixed charges were calculated for initial investments of $\$ 0.25$ and $\$ 2$ per gallon at three different annual rates: $12 \%$ and $15 \%$ per year represent the range used by utility companies to write off investments, including profit, taxes, and interest on capital; $4 \%$ per year might be the rate for a government-owned burial site ( $2 \%$ amortization plus $2 \%$ average interest).

The cost of land [( $\$ 5,000 /$ acre $) /$ $(1,000,000 \mathrm{gal} /$ acre $)=\$ 0.005 / \mathrm{gal}]$ was neglected compared to the initial cost of tankage.

Direct operating costs (based on a $20,000,000$-gal form that has reached steady state) will be

2 men/shift $\times 4$ shifts $\times \$ 4500 /$ man-yr

$$
\begin{aligned}
20,000,000 \mathrm{gal} & \\
& =\$ 0.0018 / \mathrm{gal} / \mathrm{yr}
\end{aligned}
$$

Allowing for $67 \%$ overhead, the direct charge will be $\$ 0.003 / \mathrm{gal} / \mathrm{yr}$. The calculated unit charges for the six cases are given in Table 1.

Shielding for shipment. The number of curies of a fission product per watt of reactor operating power will be

$$
\begin{aligned}
\frac{\text { curies }}{\text { watt }}= & \frac{3.1 \times 10^{10} \mathrm{fiss} / \mathrm{sec} / \text { watt }}{3.7 \times 10^{10} \mathrm{dps}} \\
& \times\left(Y_{i}\right)\left(1-e^{-\lambda_{i} t_{0}}\right)\left(e^{-\lambda_{i t_{0}}}\right) \\
& =0.84 Y_{i}\left(1-e^{-\lambda_{i t_{0}}}\right) e^{-\lambda_{i t_{0}}}
\end{aligned}
$$

where $Y_{i}=$ fraction fission yield, $\lambda_{i}=$ decay constant $\left(\right.$ day $\left.^{-1}\right), t_{0}=$ reactor operating time (days), and $t_{c}=$ cooling time (days).

Based on Project Separations(4) tab- 

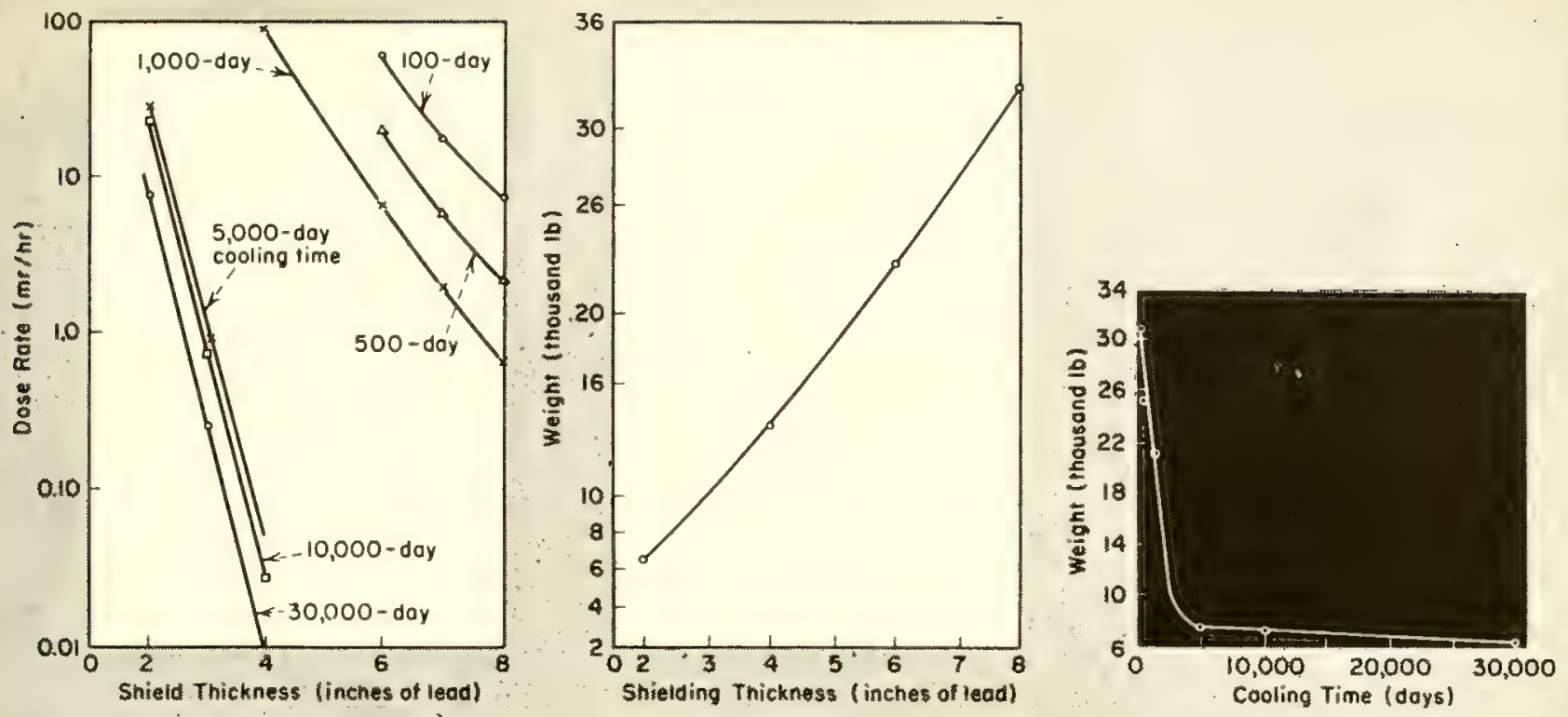

FIG. 12. Dose rate at car wall for vorious cooling periods and shield thicknesses. Bosed on 400-day irradiotion to $10,000 \mathrm{Mwd} /$ ton

ulation a typical reactor specific power of ${ }^{\prime} 1,000 \mathrm{Mw} / 40$ ton $=25 \mathrm{Mw} /$ ton is assumed. On this basis, the reactor operating time would be $80,160,320$, and 400 days for $2,000,4,000,8,000$, and $10,000 \mathrm{Mwd} /$ ton, respectively.

Fission products to be considered for the shielding calculations are $\mathrm{Ba}^{140}$ $\left(\mathrm{La}^{140}\right), \mathrm{Zr}^{95}, \mathrm{Nb}^{95}, \mathrm{Ce}^{144}\left(\operatorname{Pr}^{144}\right), \mathrm{Cs}^{137}$

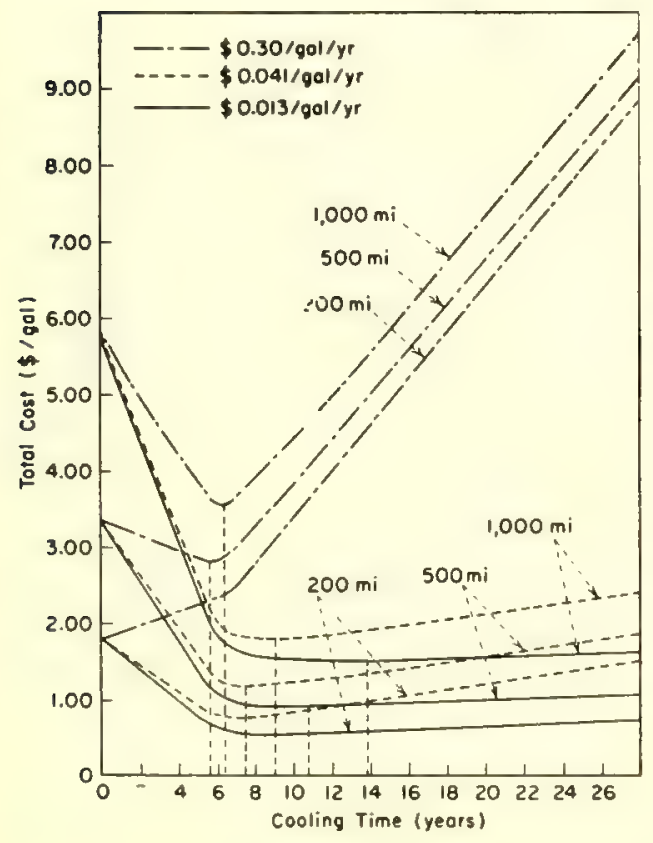

FIG. 13. Weight of spherical lead shipping carrier (4-ft i.d. and 250-gal nominal capacity) as function of shield thickness
FIG. 14. Shield weight to give $10-\mathrm{mr} /$ hr surface dose $1250 \mathrm{gal}$ in 4-fi-i.d. sphere; $800 \mathrm{gal}$ waste per ton, 400 -day irradiation to $10,000 \mathrm{Mwd} /$ ton) and $\mathrm{Ru}^{106}\left(\mathrm{Rh}^{106}\right)$. Table 2 gives the number of curies of each of these fission products present at time of discharge from the reactor for various reactor operating times.

Since $800 \mathrm{gal}$ of high-activity waste per metric ton and a specific power of $25 \times 10^{6}$ watts have been assumed, curies $/$ gal $=A($ curies $/$ watt $) \times 2.5 \times$ $10^{7} / 800=3.1 \times 10^{4} A$. The specific activities are listed in Table 3 for 400 day irradiation at $25 \mathrm{Mw}$ /ton.

Shielding calculations were made for a 4-ft i.d. carrier holding $250 \mathrm{gal}$ of waste from fuel irradiated 400 days $(10,000 \mathrm{Mwd} /$ ton $)$. A maximum permissible dose rate of $10 \mathrm{mr} / \mathrm{hr}$ at the wall of a 9-ft-wide railroad car was the basis for determining the shield thickness.

Figure 12 shows the total dose rate at the car wall as a function of the cooling time.

Combined cooling and shipping costs. For a fixed irradiation, the combined cost of storage and shipping

FIG. 15. Combined shipking and storoge costs for storage costs of $\$ 0.013$, $\$ 0.041$, and $\$ 0.30 / \mathrm{gal} / \mathrm{yr}$. Based on $250 \mathrm{gal}$ shipped in 4-ft-i.d. sphere, 800 gal waste per ton spent fuel, 400-day irradiation to $10,000 \mathrm{Mwd} /$ ton will be a function of cooling time and shipping distance.

Carrier weights are plotted as a function of shield thickness in Fig. 13 for a 250-gal waste capacity. Using the shield thicknesses from Fig. 12 that give a $10-\mathrm{mr} / \mathrm{hr}$ tolerance dose (for 400-day, 10,000-Mwd/ton irradiation), carrier weights are plotted as a function of cooling time in Fig. 14. It is assumed that the empty carrier must be returned to its point of origin and that carrier cost and handling charges would not be significantly influenced by cooling time compared to freight charges.

The freight charges per ton of carrier (round trip) are based on the following rates: $\$ 1.40, \$ 2.60$, and $\$ 4.50$ for oneway distances of 200,500 , and 1,000 miles. The optimization is carried out for these three distances and for unit storage costs of $-\$ 0.013, \$ 0.041$, and $\$ 0.30 / \mathrm{gal} / \mathrm{yr}$ from Table 1 . The basic costs appear in Table 4 and the combined results in Figs. 15-17.

\section{BIBLIOGRAPHY}

1. J. A. Lane, Nucleonics 12, No, 10, 65 (1954) 1. Palmer C. Putam. "Energy in the Future" (D. Van Nostrand Co., New York, 1953)

3. A. B. Joseph. The status of land disposal of atomic reactor waste, presented at the Nuclear Engineering and Science Congress, Cleveland. Dec. 12-16, 1955

4. "Project Separation." Processing of Spent Fuels, AEC survey at MIT (1954) 


\section{A NUCLEONICS Survey-}

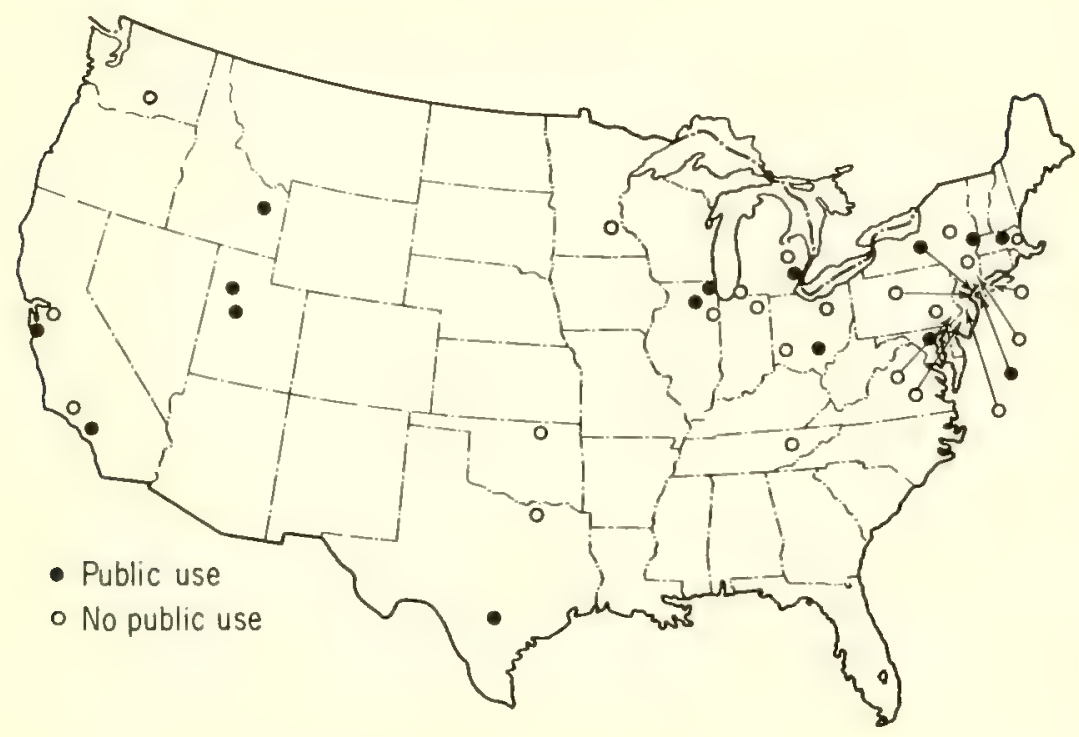

\section{Gamma-Irradiation Facilities in the United States}

By R. HOBART ELLIS, Jr., Associate Editor, NUCLEONICS

About 40 high-level isotopic irradiation facilities are currently in use by schools and industrial and research establishments of the United States. They include a total of $\sim 350,000$ curies of $\mathrm{Co}^{60}$ and nine spent-fuel installations. These are among the results of a NUCLEONICS survey of such facilities. Some of the results are tabulated on the facing page.

The survey supplements an earlier bibliography of similar information (NU, Nov. '57, p. 170). No attempt has been made to include medical installations, particle-irradiation facilities or other sources of high-energy photons such as accelerators and $\mathrm{X}$-ray generators.

Many for public use. Of the facilities listed 20 indicate that they are available for public use, and 27 say that they are not. In some cases the answers were qualified to indicate that while the facility is generally used only by the owner company, there is no real objection to inquiries for service irradiation.

Only eight facilities indicate that clearance is required for their use. Twenty-nine can accommodate classified experiments.

Largest source of the group is the Oak Ridge storage facility (NU,
March '57, p. 98), currently rated at 200,000 curies of $\mathrm{Co}^{60}$. This facility is no longer available for public use.

\section{Rapid Development}

The irradiator field is in a state of rapid change and development. So many companies have established their own facilities and so many of these offer service irradiations to outsiders that the Atomic Energy Commission has bowed out of the business. As of last March 31 it no longer offers gamma irradiations where industrial facilities are adequate for the purpose. $\mathrm{Co}^{60}$ prices have been reduced and placed on a new schedule that gives advantage to large-quantity orders. The current range is $\$ 2-5$ per curie, replacing a former range of $\$ 2-10$ per curie ( $N U$, Sept. '57, p. 32). The price of $\mathrm{Cs}^{137}$, often suggested as a substitute for $\mathrm{Co}^{60}$ and widely used in Britain, has been reduced by a much larger factorfrom $\$ 14$ to $\$ 1-2$ per curie (NU, May '58, p. 27). This isotope will soon be available in increased quantities from Oak Ridge.

At least one irradiator is planned to use more $\mathrm{Co}^{60}$ than all current sources combined. This High Intensity Food Irradiator (HIFI) will be installed at the Quartermaster Corps's Sharpe
General Depot at Lathrop, Calif. It is likely to contain $\sim 2$-million curies. The current annual $\mathrm{Co}^{60}$ production rate in the United States of $300,000 \mathrm{c} / \mathrm{yr}$ will have to be increased several fold if it is to supply irradiators of this magnitude. Additionally an AEC-sponsored study will evaluate the potential demand for large irradiators, and this may inspire further construction (NU, April '58, p. 24).

\section{Lack of Standardization}

A serious difficulty in surveying the field is a need for vocabulary. It is probably impossible to find commonly recognized units for size, prices and irradiation capacity.

Consider, for example, the matter of size. The curie of $\mathrm{Co}^{60}$ is adequate for cobalt sources, but it does not permit comparison with photon radiation from other sources. The situation becomes further complicated if one tries to include particle accelerators. The survey questionnaire asked for the radiation power in watts, but few of the people answering had this figure available.

Prices for service irradiations are another potential source of confusion. They have probably not become important to irradiation people yet, but they will be more important when radiation becomes a commercial commodity. Few people sent prices in terms of the units offered: $\$ / \mathrm{hr}, \$ / \mathrm{hr}$ $/ \mathrm{ft}^{3}$ and $\$ /$ megarad-lb.

Irradiation capacity would be an interesting figure of merit, but no generally acceptable figure appears to be available to describe it. Few facilities were prepared to estimate their outputs in megarad-lb/hr.

A set of suggested standards. It appears possible and desirable to define a set of quantities that would make irradiation facilities comparable. Preferably the set would include all types of irradiators-accelerators as well as isotopic sources. A usable set would appear to be the following: total radiation power (watts), potentially useful radiation (total radiation power less the amount inevitably lost by absorption in source and shielding), radiation efficiency (per cent of the useful radiation delivered to the sample in a particular geometry), radiation cost (dollars per megarad-lb). Other useful units could be derived; for example, the irradiation rate or production capacity in megarad-lb/hr. The irradiation field is growing at a rate that indicates the need for some standardization of this kind. 
Facility

Location

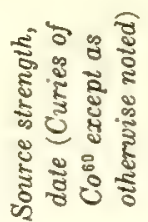

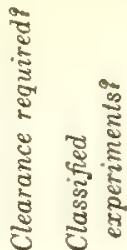

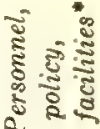

Argonne National Laboratory

Rack M-1

Rack M-2

Storage rack

Atomics International

Battelle Memorial Institute

Brookhaven National Laboratory

Budd Co., Nuclear Systems Div.

California Research Corp.

Cities Service R \& D Co.

Columbia University

Continental Oil Co.

Dugway Proving Ground

Spent-fuel facility

Large $\mathrm{Co}^{60}$ facility

Small $\mathrm{Co}^{60}$ facility

Electronic Products Co.

Esso Research \& Engineering Co.

B. F. Goodrich Co.

Hanford Atomic Prod. Operation $\mathrm{Co}^{60}$ dry facility

Reactor-fuel-storage basin

Hughes Research Laboratories

Inland Testing Laboratories

Knolls Atomic Power Laboratory

Linde Co.

Magnolia Petroleum Co.

The Martin Co.

MIT, Dep't of Food Technology

National Reactor Testing Station

University of Michigan

Fission Products Laboratory

Phoenix Laboratory

Minnesota Mining \& Mfg. Co.

$\mathrm{Co}^{60}$ facility

Naval Air Material Center

University of Notre Dame

Ghormley-Hochanadel

Underground

Oak Ridge National Laboratory

Radiation Applications, Inc.

Rensselaer Polytechnic Inst.

Sinclair Research Laboratories

Socony Mobil Oil Co. ๆ

Southwest Research Inst.

Stanford Research Inst.

Stevens Inst. of Technology

The Texas Co.

Worcester Foundation for

Experimental Biology

Wright Air Development Center Spent-fuel facility

Lemont, Illinois

(12 MTR elements)

(8 MTR elements)

(0-12 MTR elements)

Canoga Park, Calif. 1,100 Sept. '54

2,400 June '58

$\sim 25,000$

Upton, New York

Philadelphia, $\mathrm{Pa}$.

Richmond, Calif.

Cranbury, N. J.

New York, N. Y.

Ponca City, Okla.

Dugway, Utah

$10,000-15,000 \dagger$

590 Nov. '57

$\sim 5,000 \quad$ May' 57

1,400 Sept. '57

(8 MTR elements)

(8 MTR elements)

3,160 April '58

30 April '58

Mount Vernon, N. Y

$\sim 80$

Linden, N. J.

Brecksville, Ohio

Richland, Wash.

17,600 Nov.' 57

1,378 June '55

$3.5 \quad 1.47$

5.2 Yes No Yes PCOR

0.31 .47 Yes No Yes PCOR

1.51 No No Yes PCLR

$1 \sim 700$ Yes No Yes PCOMLR

$\begin{array}{llll}1.25 & 1 & \text { No No Yes PLR }\end{array}$

$\begin{array}{lllll}0.75 & 320 & \text { Yes No Yes PCWTMBL }\end{array}$

$\begin{array}{lll}0.24 & 0.014 & \text { No No Yes PCL }\end{array}$

1.6500 No No No PWMLR

$\begin{array}{llllll}0.4 & 12 & \text { No No No PC }\end{array}$

0.53 No No No PWTOML

2 1.0 Yesł Yes Yes PBR

$0.225 \quad 0.5 \quad$ Yes $\ddagger$ Yes Yes PR

$0.003 \quad 0.05$ Yes Yes Yes PO

- $\quad$ - Yes Yes Yes PCOM

10440 No No Yes PWMBLR

0.190 .54 No No No LR

2,000 - $\quad-\quad$ No Yes Yes PCL

(Fuel elements) $\quad-\quad 3 \quad$ No Yes Yes PCL

$\mathrm{Co}^{80}$ - 10 No Yes Yes PCL

Culver City, Calif.

Morton Grove, Ill.

Schenectady, N. Y.

Tonawanda, N. Y.

Dallas, Texas

Baltimore, Md.

Cambridge, Mass.

NRTS, Idaho

Ann Arbor, Mich.

St. Paul, Minn.

500 Mar. '57 $0.4 \quad 0.04$ Yes No Yes PC

62,000 Oct. '57 $10 \quad 4,000$ Yes No Yes PCWOMBLR

$3,440 \quad 1952$

4,280 April '58

20

15 June '57

5,150 Oct.'57

850 Nov. '54

(Fuel elements)

2,800 June '58

5,100 June '58

$2.5 \quad 400$

0.008

0.38

0.220 .05 No No No PCOML

101,600 Yes $\S$ No No PO

MTR elements)

600 Sept.'5

Philadelphia, $\mathrm{Pa}$.

Notre Dame, Ind.

Oak Ridge, Tenn.

L. I. City, N. Y.

Troy, N. Y.

Harvey, Illinois

Pennington, N. J.

San Antonio, Texas

Menlo Park, Calif.

Hoboken, N. J.

Beacon, N. Y.

1,400 Jan. '58

0.265700

700
20

Yes

No Yes PCOLR

12

Yes

No No PCWTMLR

Shrewsbury, Mass.

Wright-Patterson

$$
\text { A. F. B., Ohio }
$$

* $\mathrm{P}=$ personnel to conduct experiments, $\mathrm{C}=$ contract research, $\mathrm{W}=$ viewing window, $\mathrm{T}=$ closed-circuit television,

$\mathrm{O}=$ other viewing equipment, $\mathrm{M}=$ manipulators, $\mathrm{B}=$ conveyor, $\mathrm{L}=$ liquid-flow equipment, $\mathrm{R}=$ refrigeration equipment.

$\dagger$ Varying amounts of Co ${ }^{10}$. Facility can handle any amount up to 25,000 curies.

$\ddagger$ If time is available, public use can be arranged through the

\begin{tabular}{|c|c|c|c|c|c|c|c|}
\hline 1,200 & Apr. '58 & 0.6 & 0.0018 & No & No & No & $\mathrm{P}$ \\
\hline 1,193 & Aug. '57 & 0.6 & 0.0018 & No & No & No & $\mathrm{P}$ \\
\hline \multicolumn{2}{|c|}{$\sim 200,000$} & 2.5 & 1 & No & No & Yes & PR \\
\hline 1,350 & Dec. '56 & 0.36 & 6.75 & Yes & No & Yes & PCMLR \\
\hline 900 & Apr. '58 & 0.3 & 35 & Yes & No & No & PCWMLR \\
\hline \multicolumn{2}{|c|}{ MTR elements) } & 1.5 & 1 & No & No & No & WMBL \\
\hline 一 & - & 2 & - & No & No & No & WMLR \\
\hline 8,800 & Jan. '57 & 20 & 180 & Yes & No & Yes & PCWM \\
\hline 2,500 & May'58 & 0.6 & 1 & Yes & No & Yes & PCMBLR \\
\hline 300 & Oct. '56 & $\sim 0.17$ & 1.5 & No & No & Yes & PCOLR \\
\hline 7,500 & June '58 & 1 & & No & 一 & - & PWML \\
\hline $15 t$ & Mar. '58 & - & - & Yes & No & No & PW \\
\hline 000 & Oct.' 57 & 0.8 & 1.8 & No & No & Yes & PWTM \\
\hline
\end{tabular}

Commandant, Quartermaster Food and Container Institute, 1819 West Pershing Rd., Chicago 9, Ill.

\& AEC may grant permission for experiments that private facilities can not handle.

- Facility is not yet in operation.

\|F Facility is available for biological research. Arrangements can be made through Dr. Ralph Dorfman at low cost or none. 


\section{Survey of Gamma Facilities in U. S. and Canada-an Updating}

The following table brings up to date the list of industrial gamma facilities that we published a year ago (NU, July '58, p. 108). Together the two lists describe all of the industrial gamma facilities of the U. S. and most of those of Canada as they exist today.

The NuCLEoNics card file of gamma facilities is kept continuously up to date. New cards are added as we hear of new facilities; changes are entered as we hear them. In addition we have asked each of the facilities that was listed at year ago whether there should be any change in its list- ing. Only one change was called to our attention; a boldface line identifies this change in the present table. Information about General Electric's ANP facility in Cincinnati has been hard to get because of security regulations. Otherwise the combined list is complete and accurate to the best of our knowledge.

Miss Leida Blanco of the NUCLEONICs staff receives credit for most of the gathering and tabulation of the data.

Gamma-Facility Characteristics-Answers to a NUCLEONICS Questionnaire

Facility

Location

\section{New Listings}

Admiral Corp.

Aerojet-General Corp

General Electric Co. (Gen'l Eng'g Lab.)

Lockheed Aircraft Corp

Southern Research Inst.

State-Federal Screwworm

Eradication Program

Union College

U. S. Dept. of Agriculture $\S$

Univ. of Denver

Univ. of Georgia

Univ. of Florida

(College of Engineering)

(Agricultural Experimental Station)

Univ. of Louisville

Univ. of Minnesota

Univ. of Vermont

Canadian Facilities

Atomic Energy of Canada Ltd.

(Irradiation cell)

Defense Research Board, Defense

Research Chemical Laboratories

Univ. of Saskatchewan

(Source 1)

(Source 2)

\section{Changed Listings}

B. F. Goodrich

(WADC) (BFG)

Chicago, Ill.
Azusa, Calif
Schenectady, N. Y.
Dawsonville, Ga.
Birmingham, Ala.
Sebring, Fla.
Schenectady, N. Y.
New Orleans, La.
Denver, Colo.
Athens, Ga.
Gainesville, Fla.

Louisville, $\mathbf{K y}$.

Minneapolis, Minn.

Burlington, Vt.

20,000
10,000

Jan. '57

Dec '58
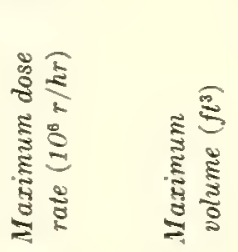

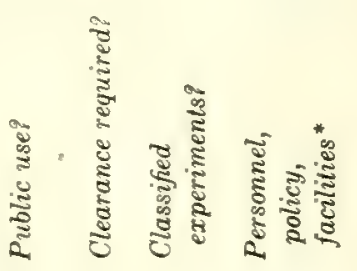

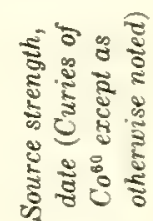

10,000 Jan.'59

(Fuel elements)

790 Aug. '57

3,600\| Nov. '58

1,120 May ' 58

1,000 June '57

2,000 June' 59

145 Dec. '57

835

6,400

1,100

2,000

1,017

April '58

May '58

Aug. '58

Oct. '57

April '58

0.2300

$\sim 1 \quad 0.25 \dagger$

Yes No Yes PCWML

$0.46 \quad 2,000$

0.3

$-$

No Yes Yes -

Yes No Yes PCWTL

Nof Yes Yes PC

Yes No Yes PCOM

$0.05 \quad 0.098$

No No No MB

$\begin{array}{ll}0.4 & 0.01\end{array}$

$0.7 \quad 0.28$

No No No PCLR

$0.5 \quad 0.21$

Yes No No PCWR

$0.0448 .6 \times 10^{-4}$ No No No $P$

\section{$0.45 \quad 0.0112$}

Yes No No PC

$\sim 0.15 \sim 0.25$

Yes No No PC

0.3130 .004

Yes No No PCR

$1.21,500$

Yes No No PCWMLR

Yes No No PCWT

\begin{tabular}{|c|c|c|c|c|c|c|c|c|}
\hline Ottawa, Ont. & 3,750 & Jan. '59 & 0.35 & 0.13 & Yes & No & No & PCBLR \\
\hline \multirow{3}{*}{$\begin{array}{l}\text { Ottawa, Ont. } \\
\text { Saskatoon, Sask. }\end{array}$} & 1,500 & 1958 & 0.07 & - & No & Yes & Yes & $\mathrm{PW}$ \\
\hline & 90 & Jan. '58 & 0.1 & 70 & No & No & No & PCOLR \\
\hline & 1,100 & July '5! & 0.1 & 0.15 & No & No & No & PCOLR \\
\hline \multicolumn{9}{|l|}{ Brerksville, Ohio } \\
\hline & 1,085 & June '58 & 0.435 & 0.017 & No & No & No & MIR \\
\hline & 1,600 & & $\sim 0.28$ & 0.35 & No & No & No & MLR \\
\hline \multicolumn{2}{|c|}{$\begin{array}{l}\text { eriments, } C=\text { contract re- } \\
=\text { closed-circuit television, } \\
=\text { manipulators, } B=\text { con }- \\
=\text { refrigeration equipment. } \\
\text { st dose rate. Several other }\end{array}$} & \multicolumn{7}{|c|}{$\begin{array}{l}\text { volumes also available. } \\
\pm \text { Available to government subcontractors. } \\
\mid 6 \mathrm{Co}^{60} \text { units varying from } 450 \text { to } 660 \text { curies. } \\
\text { \$ Southern Utilization Rextarch and Development Uivision } \\
\text { | Presently being instulled. }\end{array}$} \\
\hline
\end{tabular}




\title{
Radioisotopic High-Potential,
} Low-Current Sources

\author{
Batteries using an $\mathrm{Sr}^{90}-\mathrm{Y}^{90}$ source and a polystyrene insulator provide up to \\ 7,000 -volt charging potential at $40 \mu \mu \mathrm{a}$. Smaller, lighter, and less expensive to \\ operate than chemical batteries, they offer long life under extreme conditions
}

\section{BY JOHN H. COLEMAN}

Radiation Research Corporation, West Palm Beach, Florida

Generation of electric energy by collecting the beta particles emitted from a radioactive source with an electrode that is insulated from the source by a solid dielectric has been found feasible with polystyrene as the insulator.

The principle of operation of directconversion devices in general can be illustrated by generators utilizing a vacuum dielectric $(1-5)$. Two of these devices will be described preliminary to a discussion of models incorporating solid dielectrics.

\section{Vacuum-Dielectric Devices}

As early as 1913, Moseley (1) attained a high electric potential by collecting the beta particles emitted

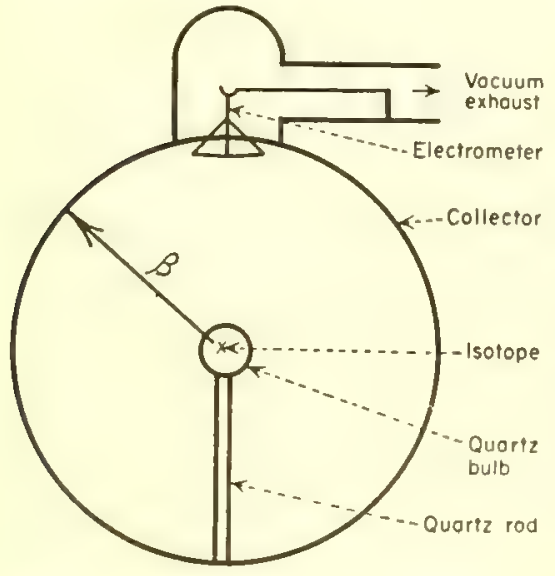

FIG. 1. Moseley's vacuum generator from a radioactive source suspended in a vacuum. Moseley's apparatus, Fig. 1 , consisted of two concentric spheres with a vacuum connection to the outer sphere for evacuation. The center sphere or source electrode was a quartz bulb containing approximately 20 millicuries of radium. The wall of the bulb was sufficiently thin to enable penetration of the emitted beta particles but thick enough to absorb the alphas.

This source of beta electrons was supported by a thin insulating quartz rod attached to the inside wall of the outer sphere that collected the electrons. The inner and outer sphere were covered with a conducting coating of silver, forming the anode and cathode, respectively. Any potential difference between the electrodes was measured by an attractive disk-type electrometer located in the mouth of the vacuum connection.

On evacuation of the interelectrode space, Moseley repeatedly measured around $150 \mathrm{kv}$ on the source electrode before internal flashover discharged the device. A simple capacity-charging relationship was noted after each discharge for a beta charging current of about $10^{-11}$ ampere.

In 1952, Linder (2) published results on two vacuum devices similar to Moseley's, using a $\mathrm{Sr}^{90}-\mathrm{Y}^{90}$ fissionproduct source. In one device, with

Specifications of Solid-Dielectric Batteries

\begin{tabular}{|c|c|c|}
\hline & \multicolumn{2}{|c|}{ Battery model } \\
\hline & $B-50$ & $D-50$ \\
\hline Source & $10-\mathrm{me} \mathrm{Sr} r^{90}-\mathrm{Y}^{90}$ & $10-\mathrm{mc} \mathrm{Sr}^{90}-\mathrm{Y}^{90}$ \\
\hline Insulator & Polystyrene & Polystyrene \\
\hline Collector & Aluminum & Aluminum \\
\hline Shield & Lead & Lead \\
\hline Potting compound & Biwax & Polystyrene \\
\hline Output current ( $\mu \mu \mathrm{a})$ at zero voltage & 40 & 40 \\
\hline Current-collector efficiency & $33 \%$ & $33 \%$ \\
\hline Maximum charging voltage (volts) & 6,000 (after 2 months) & 7,000 (after 2 weeks) \\
\hline Total volume (in. ${ }^{3}$ ) & 4 & 1 \\
\hline
\end{tabular}




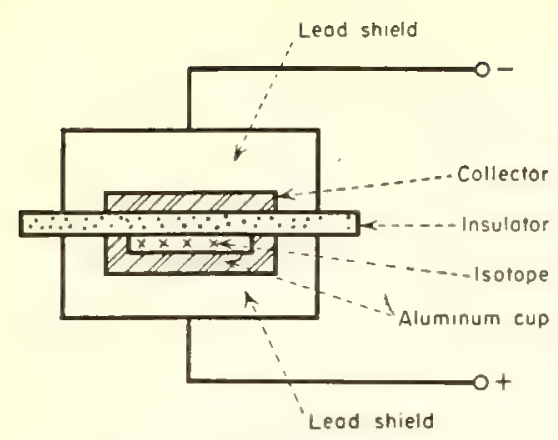

FIG. 2. First experimental model of generator with solid insulator

improved vacuum techniques and electrode design, a potential difference of $365 \mathrm{kv}$ was obtained. In the other device, a direct electrical connection to the source electrode was made through an external insulator that formed part of the vacuum enclosure. A somewhat lower voltage was obtained with this device due to surface leakage on the external part of the insulator exposed to the atmosphere. A capacitycharging curve across a resistive load was measured by Linder for an equivalent circuit of a beta current source, a capacity, and an internal resistance in parallel with the external load. The internal resistance was a combination of surface conduction across the insulator bushing and some conduction through the vacuum by cold emission or residual gas.

\section{Solid Dielectric}

In 1951 , a project was started at this laboratory to develop a solid insulator that could be substituted for the vacuum dielectric in the direct-conversion generator to increase reliability.

The literature at that time stated, however, that insulators were both lowered in electrical resistivity (6) and physically degraded ( $(\gamma)$ by ionizing radiation. These effects are not limitations in the vacuum generators since insulator bushings can be shielded from the radiation. On the other hand, when a solid is substituted for a vacuum, the radiation must penetrate the solid dielectric itself for operation of the device.

Thus, to determine the feasibility of a solid-dielectric type of radioactive battery, a test program was started on commercially available insulators.

\section{Experimental Data}

For geometrical simplicity, tests were made using flat sheets of insula-

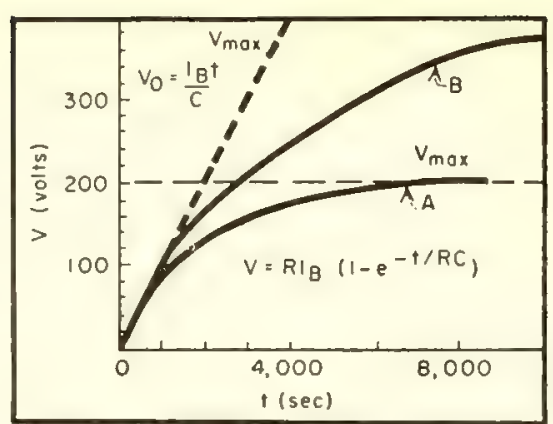

FIG. 3. Charging voltage vs time for 0.002 -in. polystyrene after irradiation for few minutes (A) and several hours (B)

tion between the faces of cylindrical source and collector electrodes, as shown in Fig. 2. As a beta source, 25 $\mathrm{mc}$ of $\mathrm{Sr}^{90}-\mathrm{Y}^{90}$ were deposited in an electrode formed by an aluminum cup covered with aluminum foil. An aluminum disk, opposite this source electrode, collected the beta particles after penetration of the insulator. Lead shielding was used around the aluminum collector and aluminum source electrode to reduce the bremsstrahlung below tolerance at the surface.

In operation, the beta current collected through a 0.002-in.-thick polystyrene insulator was measured with an electrometer to be about $50 \times 10^{-12}$ ampere at zero voltage. The total beta current emitted from 25-me $\mathrm{Sr}^{90}-\mathrm{Y}^{90}$ is $300 \times 10^{-12}$ ampere; however, one half of the current is emitted in an opposite direction from the collector and is absorbed in the aluminum cup. The remaining loss of current is due to absorption in the thin aluminum sheet covering the cup and in the insulator, itself.

Charging voltage. The open-circuit charging voltage was measured as a function of time by an electrometer

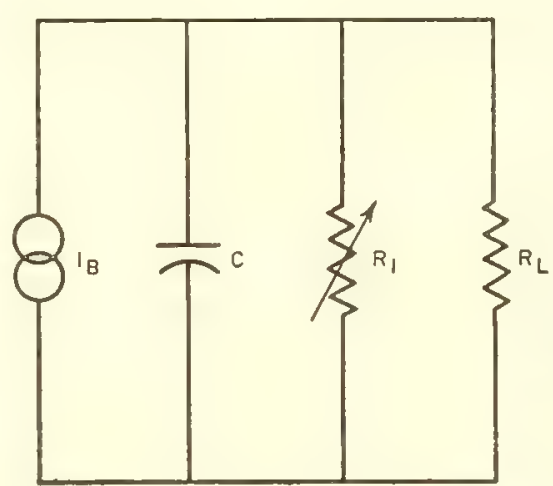

FIG. 5. Equivalent circuit of battery using solid dielectric that increases in resistance with irradiation time

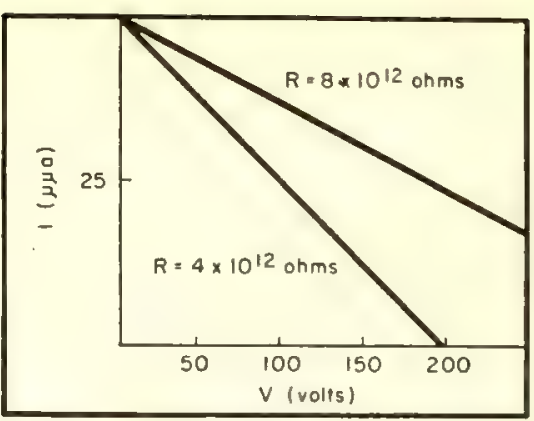

FIG. 4. Current-voltage characteristics of 0.002-in. polystyrene sheet under conditions of Fig. 3

voltmeter with negligible capacity. The resulting voltage, $V$, (the lower curve in Fig. 3) for the 0.002 -in.-thick polystyrene insulator showed an exponential rise with time, $t$, to a maximum of 200 volts.

As in the case of the vacuum generators of Moseley and Linder, capacity charging of this type is given by the equation

$$
V=R I_{B}\left(1-e^{-t / R C}\right)
$$

where $R$ is the total internal and external resistance in parallel, $C$ is the total capacity, and $I_{B}$ is the beta current. The maximum charging voltage, $V_{\mathrm{msx}}$, for $t \gg R C$ is

$$
V_{\max }=R I_{B}
$$

Next, the initial rate of charge is found by expansion of Eq. 1 for $t \ll R C$ to be independent of $R$ and given by

$$
V_{0}=\frac{I_{B}}{C} t
$$

Thus, from Eq. 2, for $V_{\max }$ of 200 volts and $I_{B}$ of $50 \times 10^{-12}$ ampere, $R$ is $4 \times 10^{12}$ ohms.

Irradiation effect on insulator. After several hours, a second measurement of the charging voltage as a function of time was made, and $V_{\max }$ was found to have increased to 400 volts, as shown by the upper curve of Fig. 3, indicating an increase in insulation resistance to $8 \times 10^{12}$ ohms while the initial charging rate remained the same. Consequently, a direct measurement of internal resistance, $R_{j}$, was made by applying an external voltage to the generator terminals and recording the resulting current with the electrometer.

The current-voltage characteristics for an unused 0.002-in.-thick polystyrene sheet is shown by the lower curve of Fig. 4. The straight line indicated an ohmic resistance of $R_{I}=$ 


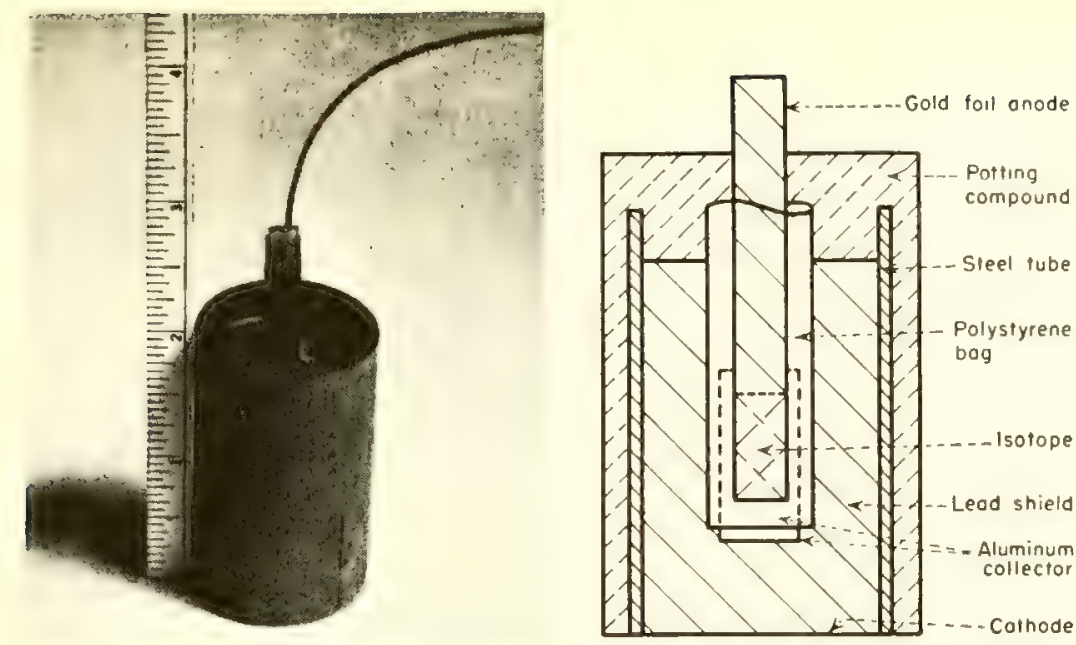

FIG. 6. Model B-50 generator. Assembled, unpotted battery at left

$4 \times 10^{12} \mathrm{ohms}$, which can be calculated by inverse of the slope or

$$
R_{I}=\frac{V}{I-I_{B}}
$$

On continued irradiation, the currentvoltage characteristic (upper curve of Fig. 4) showed a decrease in slope or increase in resistance to $R_{I}=8 \times 10^{12}$ ohms, both values of $R$ agreeing with the results in Fig. 3.

Next, to measure this apparent change in resistance after irradiation, a fresh sheet was inserted between the electrodes of Fig. 2 and a constant voltage applied while the source was covered with an absorber. The absorber was removed after the normal polarization current decreased to a low value (the corresponding volume resistivity $\rho=10^{18}$ ohm-cm) and the induced current recorded. The calculated resistivity was found first to drop to about $10^{16} \mathrm{ohm}-\mathrm{cm}$, decrease further for a few minutes to a minimum of about $10^{15} \mathrm{ohm}-\mathrm{cm}$, and then actually increase in resistance after one day with approximately the square root of the time. Details of this effect were given in a previous paper (8).

Thus, the equivalent circuit in Fig. 5 of a generator using a solid insulator can be deduced from Eq. 1 and from the observed induced conductivity effect in solid insulation. This circuit is seen to be similar to the vacuum generator except that the internal resistance $R_{I}$ is a variable, being a func. tion of the total time of irradiation and of the incident beta-current density. All good insulation (i.e., unirradiated $\rho>10^{16}$ ohms-cm) showed this effect; however, an accelerated life test using a $250-m c$ source eliminated all plastic insulators but polystyrene due to physical degradation. For example, the fluorocarbons Teflon and Kel-F increased in resistance for a few weeks, then became brittle, and shorted the electric circuit; polyethylene increased in resistance for a week, then began to decrease due to slow degradation. Polystyrene, on the other hand, has increased in resistance for over $1 \mathrm{yr}$.

On the basis of these results, the test source of Fig. 2 was potted in plastic and demonstrated in January, 1952, as a radioactive battery. Since that time, models B-50 and D-50 have been built, reducing the size and weight and increasing the safety for the same current output of $50 \mu \mu \mathrm{a}$.

\section{Battery Design, Characteristics}

Model B-50 is shown in Figs. 6 and 7. A beta source of $10 \mathrm{mc}$ of $\mathrm{Sr}^{90}-\mathrm{Y}^{90}$ is welded between two strips of 0.0005 -in.thick gold foil, 2 in. long and $1 / 4$ in. wide. The active area extends $1 / 4$ in. from the bottom end and is surrounded

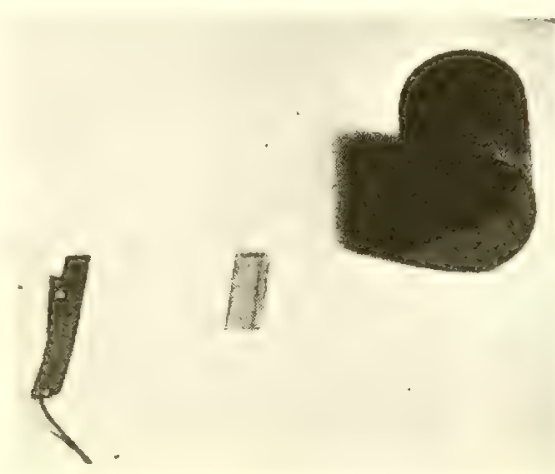

FIG. 7. Parts of B-50: shield, insulator, and gold-encased source by a "U"-shaped aluminum collector, $1 / 8$ in. thick, which is supported by a lead shield.

A slot for insertion of the source and insulator was formed by pouring the lead around a stainless-steel jig, which held the aluminum collector in position in a steel tube. The jig was removed after the lead cooled and the source was placed in two polystyrene bags and inserted in the slot. One bag, $3 / 8$ in. wide and $11 / 4$ in. long, was inside a larger bag, $1 / 2$ in. wide and $13 / 8$ in. long. Both bags were made by folding 0.005-in. Plax polystyrene along the long dimension and heat sealing along a $1 / 8$-in., "L"-shaped border, leaving the top open. "Biwax" potting compound was poured around the outside of the unit, and the output characteristics were measured.

First, the average output current for twenty-five batteries at zero voltage was found to be $I_{B}=40 \times 10^{-12}$ ampere out of a total available current of $120 \times 10^{-12}$ ampere, a $33 \%$ currentcollection efficiency. Most of the $67 \%$ loss was due to absorption in the gold foil of most of the $\mathrm{Sr}^{90}$ beta particles and some of the weaker $\mathrm{Y}^{90}$ beta particles.

The initial rate of charge of the output voltage was then measured across an electrometer voltmeter to be 1.2 volts/sec and the capacity was calculated for Eq. 3 to be $C=30 \mu \mu \mathrm{f}$. This capacity is slightly higher than the capacity of $25 \mu \mu \mathrm{f}$ measured on a bridge, since a high-resistance contact to the surface of the insulator by ionization of the air between the source and collector reduces the effective spacing between the source and collector electrode. Also, polarization current and trapped beta particles were found to be a significant part of the current when the voltage was changed. For example, it was necessary to wait several minutes on shorting the terminals before the current reduced to its original value at zero voltage.

The maximum charging voltage across an electrostatic voltmeter after two months was increased from a few hundred volts to 6,000 volts, which from Eq. 2 corresponds to an internal insulator resistance of $1.5 \times 10^{14} \mathrm{ohms}$. Model B-50 had a higher currentcollection efficiency than the original unit of Fig. 2, since the collector electrode surrounds the source. The dense gold source electrode, however, in- 
creased the bremsstrahlung and, consequently, the shielding.

Model D-50. In the third model, D-50 (Figs. 8 and 9), the radioisotope is deposited directly in the polystyrene insulator, which is machined in the form of a cup $3 / 16$ in. in diameter and $3 / 8$ in. long with a $1 / 32-$ in. wall. Contact is made to the isotope source by a 0.005 -in.-diameter monel-wire loop, which is soldered to a 1/8-in.diameter copper rod held in a $1 / 4$-in.diameter polystyrene plug. A solvent such as toluene is used to seal the open end of the capsule to a groove in the plug after the isotope is inserted. The plug and capsule fit in a cylindrical aluminum collector $3 / 8$ in. in diameter and $3 / 4$ in. long that is held in the lead shielding and copper tube. The wax potting compound used in the B-50 was replaced with polystyrene to reduce surface leakage. When the complete battery was assembled, swab tests were made to detect any radioisotope leakage. The measured radiation of the D-50 was approximately the same as model B-50; however, the volume, including shielding, was reduced from 4 in. $^{3}$ to 1 in. $^{3}$ by the elimination of the gold source electrode. In the D-50, the only dense material within range of the beta particles is the monel wire, which has a small cross-section.

Next, the electrical characteristics were taken as in the case of the B-50. The current at zero voltage was again $40 \mu \mu \mathrm{a}$; the battery capacity was approximately $5 \mu \mu \mathrm{f}$ for a charging rate of 5 volts/sec across an electrometer, which added $3 \mu \mu \mathrm{f}$; and the maximum voltage was 7,000 volts after two weeks shelf life. This beta current represents a $33 \%$ collection efficiency of the $120 \times 10^{-12}$ ampere emitted from the isotope. Most of the lost current in this case is absorbed in the $1 / 32$-in.thick insulator wall. This thickness, however, increases the breakdown voltage to approximately 30,000 volts. For special applications where the maximum voltage required is only a few hundred volts, the insulator thickness can be correspondingly reduced to obtain an increase in current (at a sacrifice of mechanical strength). Also, for lower current applications, the shielding can be decreased for the same external radiation.

The output voltage decreased when a battery was exposed to room atmosphere in a fully charged condition for
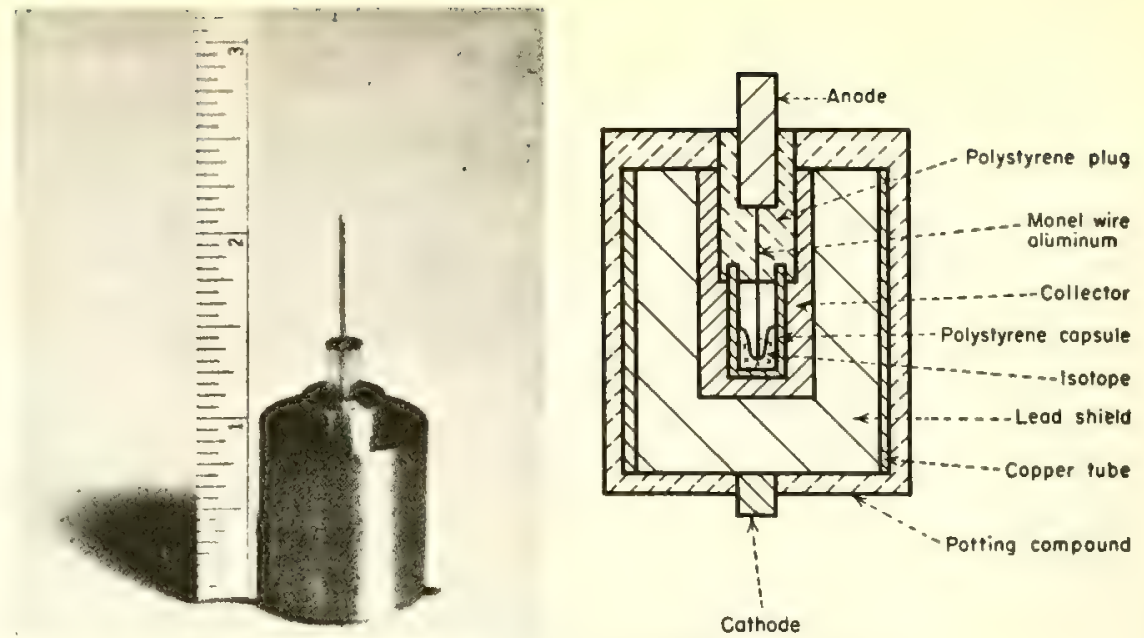

FIG. 8. Model D-50 generator. Assembled, unpotted battery at left

more than a few hours due to electrostatic precipitation of dust and, consequently, surface conduction across the external insulation. Contamination of the polystyrene surface was less serious than in the case of the wax potting compound. In either case, however, the dust could be removed with soap and water followed by a thorough drying.

Development work is continuing on improving the mechanical seals to decrease the possibility of any isotope leakage. It appears, however, that the present geometry gives about the minimum volume with a 10 -me source for an external radiation that is below tolerance at the surface. For lower currents, the shielding can be scaled down accordingly.

\section{Applications}

Batteries using radioisotopes as an energy source offer, in principle, the advantages of long life under extreme

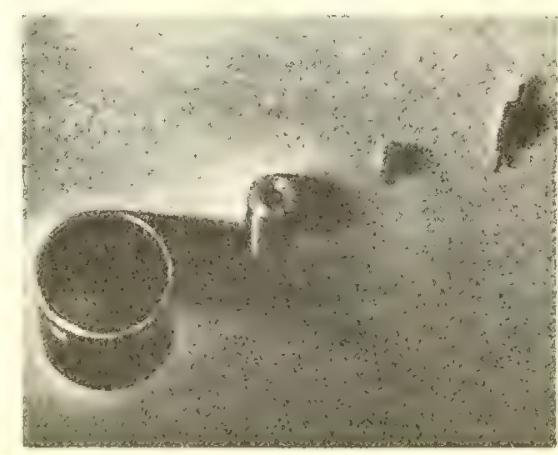

FIG. 9. Parts of D-50: shield, aluminum collector, polystyrene cup insulator, and polystyrene plug with wire anode lead operating conditions, as the nuclear process itself is essentially unaffected by temperature or pressure. The half-life of the $\mathrm{Sr}^{90}-\mathrm{Y}^{90}$ isotope is approximately $25 \mathrm{yr}$; however, a wide choice of half-lives is available. The extrapolated life of the polystyrene insulation from the 250 -mc tests has exceeded $25 \mathrm{yr}$ for the 10 -mc batteries, or $250 \mathrm{yr}$ for a 1-mc battery. As no chemical reactions or solid-state effects are used in the power generation, the insulation appears to be the only critical feature.

Thus, for applications requiring small currents, such as ionization chambers, these batteries should offer the advantages of smaller size, less weight, and lower cost, ${ }^{*}$ since most of the power available from conventional chemical batteries is not used. Also, in applications requiring operation over a period of many years at low temperatures, these batteries appear to be more reliable than batteries that depend completely or in part on a chemical reaction.

\section{BIBLIOGRAPHY}

1. H. G. J, Moseley, Proc. Roy. Soc. (London) A88 471 (1913)

2. E. G. Linder, S. M. Christian, J. Appl. Phys. 23, 1213 (1952)

3. E. G. Linder, Phys. Rev. 71, 129 (1947)

4. I. A. Lobanev, A. P. Beliahov, Compt. Rend. Acad. Sci. URSS 47, 332 (1945)

5. P. H. Miller, Phys. Rev. 69, 666 (1944)

6. R Hofstadter, Nucl Eonics 4 No. 4, 2 (1949)

7. O. Sisman, C. D. Bopp, ORNL-928 (1951)

8. J. H. Coleman, D. Bohm, J. Appl. Phys. 24. 497 (1953)

* It is estimated that an ionization chamber power supply producing up to 10,000 volts with a charging current of 10 $\mu \mu$ a could be manufactured in quantity for under five dollars at the current price of Sro0 of fifty cents per me. 

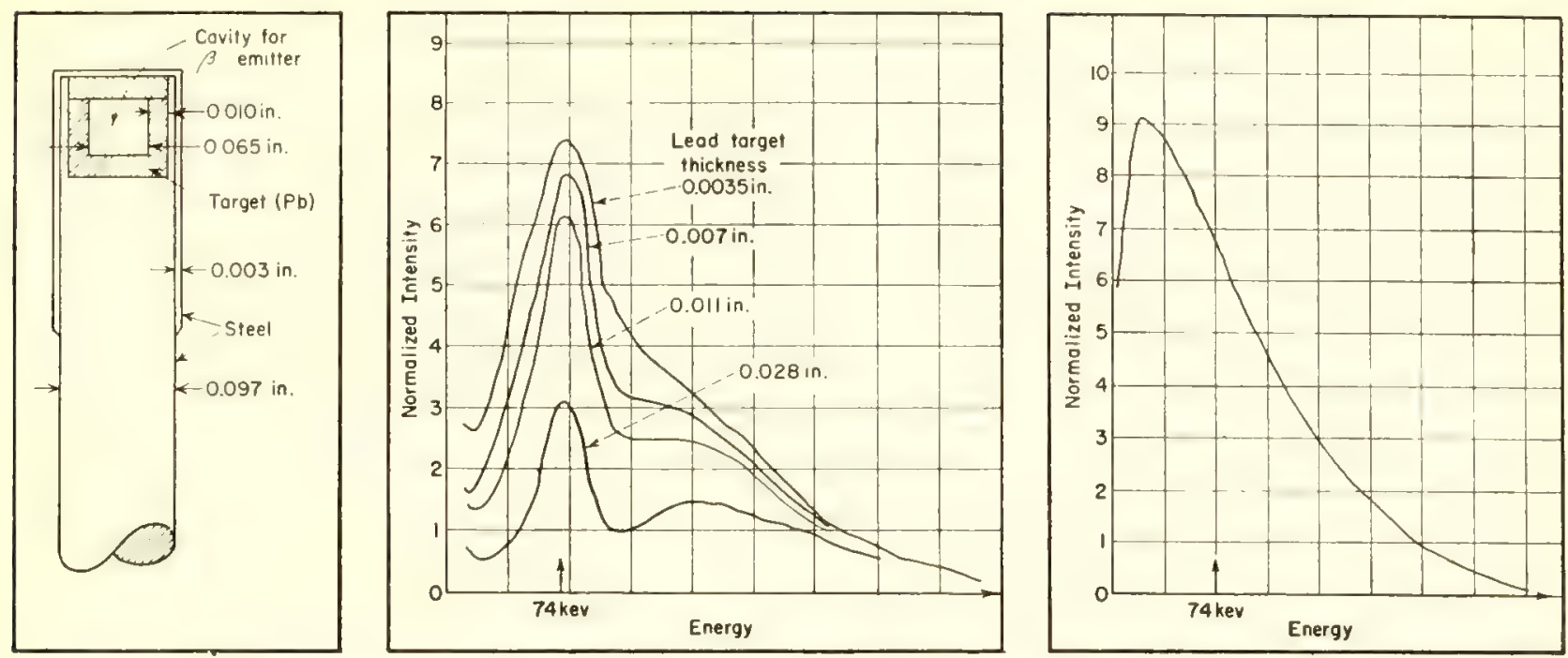

Transmission-target source, with $\mathrm{Sr}^{90} \mathrm{Y}^{90}$ betas, gives spectra in center for various target thicknesses and at right for bremsstrahlung

\title{
Beta-Ray-Excited
}

Low-Energy X-Ray Sources

\author{
Beta-ray excitation provides $X$-rays in the range below $400 \mathrm{kev}$ where gamma \\ emitters are lacking. Although present units only yield up to $1 / 10 \mathrm{mc}$ of
} $X$-rays per mc of beta particles, their portability and stability recommend them for radiography, thickness determinations, and X-ray fluorescence analysis

\author{
By L. REIFFEL \\ Armour Research Foundation of the Illinois Institute of Technology \\ Chicago, Illinois
}

Prompted BY THE LACK of low-energy ( $<400 \mathrm{kev}$ ) gamma-emitting nuclides, for several years we have been exploring the possibility of using beta-ray-excited X-ray sources. Such sources are constructed using pure beta-ray emitters to bombard target nuclei much in the same fashion that electrons in an $\mathrm{X}$-ray tube bombard the anode; the two processes are essentially identical in the physics involved. Although radioactive sources cannot compete with electronic $\mathrm{X}$-ray sources where very high intensity is required, their zero power consumption, small size and portability, long-time stability, and other advantages would be of value in many applications.

Using $\mathrm{Sr}^{90}-\mathrm{Y}^{90}$ beta emission, we have demonstrated the practicality of such sources. This article discusses the characteristics of various source designs and the possibilities for future development.

These sources are needed because of the small number of low-energy-emission radioisotopes in the classical $\mathrm{X}$-ray region. Among the few useful emitters in this region are thulium and iron-55. Thulium, which emits 84-kev and $52-\mathrm{kev}$ radiation, is available at high specific activity but, unfortunately, has an inconveniently short half-life of 129 days. It has been developed for radiography* and absorptiometry. Iron-55 emits, by $K$-capture, a 6 -kev $\mathrm{X}$-ray. It has a conveniently long life -2.9 years-but its energy is unfortunately somewhat

* S. Untermyer, Nucheonics 12, No. 5 , 35 (1954). 
low for many purposes. It has found limited application in exciting fluorescence $\mathrm{X}$-ray spectra from the lighter elements. A considerable gap exists, therefore, in available energies.

Fortunately there is a rather wide choice of pure beta-ray emitters, ranging from the strontium-90-yttrium-90 chain, emitting $0.6-\mathrm{Mev}$ and $2.2-\mathrm{Mev}$ betas with an effective half-life of 20 years, down to materials such as phosphorus-32 with a $1.7-\mathrm{Mev}$ beta and a 14-day half-life or calcium-45 with a 0.25 -Mev beta and 152-day half-life. In all, there are approximately ten beta emitters useful for the production of $\mathrm{X}$-rays by the methods to be described.

\section{Mechanisms}

Two mechanisms are active in producing $\mathrm{X}$-radiation when the betas from such isotopes are allowed to interact with target nuclei. The first involves simple acceleration of charge during the beta absorption process and results in bremsstrahlung radiation. The second radiation source is principally the result of ionization in the $K$ shell of target atoms with subsequent emission of characteristic or $K$-radiation. Characteristic radiation may be excited directly in an interaction between a beta particle and a target atom or indirectly when the target atom absorbs bremsstrahlung radiation formed elsewhere in the target material.

For thin targets, the efficiency of bremsstrahlung production is proportional to the square of the atomic number of the target material and to the first power of the beta-ray energy. For energies of the order of the $\mathrm{P}^{32}$ beta and for a lead target, quantum efficiencies of the order of $10 \%$ have been measured when only $\mathrm{X}$-rays in excess of $50 \mathrm{kev}$ are considered. The effectiveness of such a mechanism therefore compares reasonably well with the branching ratio in the decay schemes of materials such as thulium. The efficiency of direct excitation of fluorescence radiation by beta bombardment had not been studied in detail. It is complicated by indirect excitation previously mentioned. This aspect of the performance of the sources is undergoing further measurement.

\section{Source Design}

Various configurations can be used for beta-ray excited X-ray sources. Among others, liquids can be mixed
2-REFLECTION-TARGET SOURCE
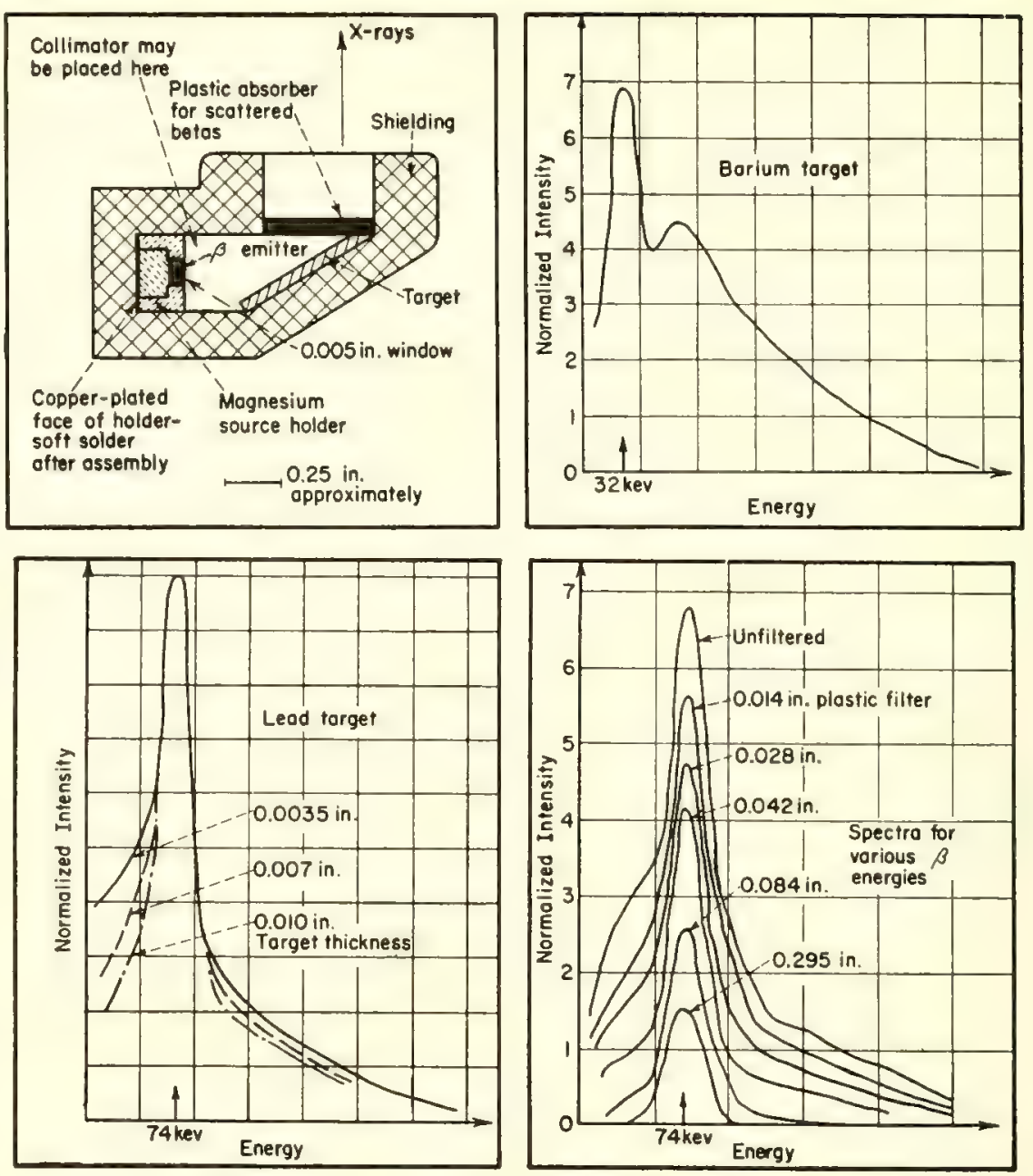

Reflection-forget source, with $\mathrm{Sr}^{90}=\mathrm{Y}^{90}$ betas, provides spectra at bottom left for various thicknesses of lead target and top right for barium target; spectra for various beta energies, bottom right, were obtained with lead target, plastic filters, and $\mathrm{Sr}^{90}-\mathrm{Y}^{90}$ source

\section{3-MONOENERGETIC X-RAY SOURCE}

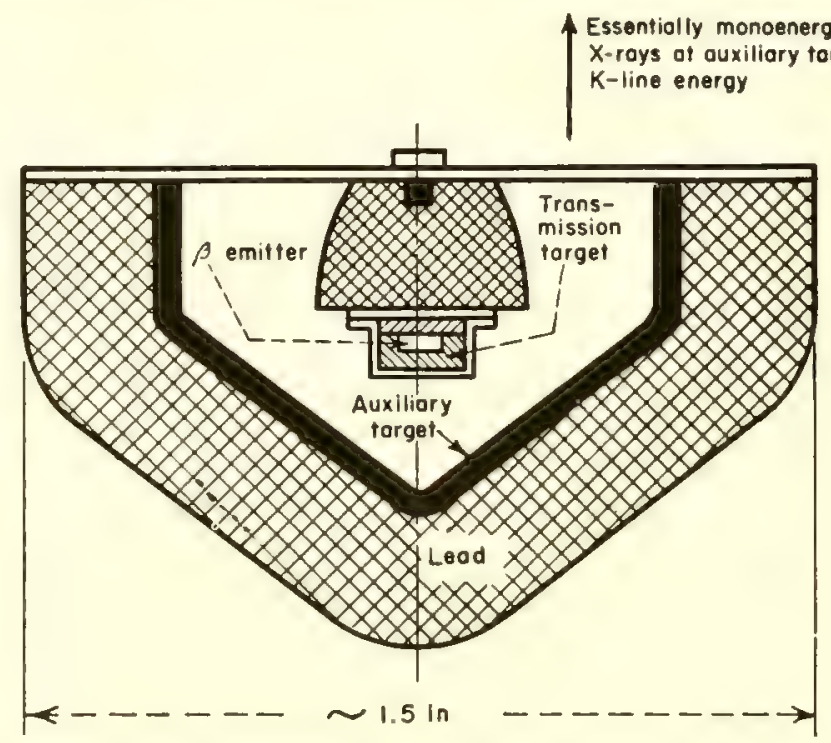

Source provides almost completely monoenergetic radiation with energy dependent only upon auxiliary target material 
with the beta emitter material, or solid powders can be intermixed much in the manner that neutron sources are constructed, or a core of beta-ray emitter can be surrounded by a casing of target material. Still another configuration that lends itself readily to changing the energy of the X-rays emitted by the source employs a reflection target wherein the beta emitter is separated from a target surface in almost direct analogy to an $\mathrm{X}$-ray tube.

A typical $\mathrm{X}$-ray transmission target (casing target) configuration and a typical reflection target configuration are shown in Figs. 1 and 2, together with spectra obtained using $\mathrm{Sr}^{90} \mathrm{Y}^{90}$ as the beta emitter. Various target thicknesses were used to illustrate the effect of conversion of bremsstrahlung radiation to characteristic radiation in each case. The curves are all normalized to the same peak intensity, and the data were obtained with the usual sweeptype differential-pulse-height analyzer. The measured energy spectrum is of course smeared by the finite resolution of the sodium-iodide scintillation-counter detector. The actual spectrum would exhibit a very sharp spike at $73.8 \mathrm{kev}$ in the case of lead.

Since the intensity of the bremsstrahlung continuum varies rapidly with beta-ray energy, it is evident that to secure monoenergetic X-ray sources low-energy betas should be employed. Figure 2 displays the result of filtering the betas from $\mathrm{Sr}^{90} \mathrm{Y}^{90}$ through various thicknesses of Lucite. It is noteworthy that the ratio of the $K$-line intensity to the continuum increases with decreasing beta-ray energy; also the integrated intensity decreases with decreasing energy. The bottom curve of this series shows the lead fluorescence excited by the bremsstrahlung present due to the beta source structure itself and demonstrates another interesting source configuration.

To obtain a source of monoenergetic radiation, the wavelength of which may be varied over wide limits, it is only necessary to provide a source such as illustrated in Fig. 1 with a second target so positioned that it can be bombarded by the $\mathrm{X}$-radiation from the source. A typical configuration is shown in Fig. 3.

\section{Applications}

The wide range of energy spectra available from the several source designs, in combination with the wide

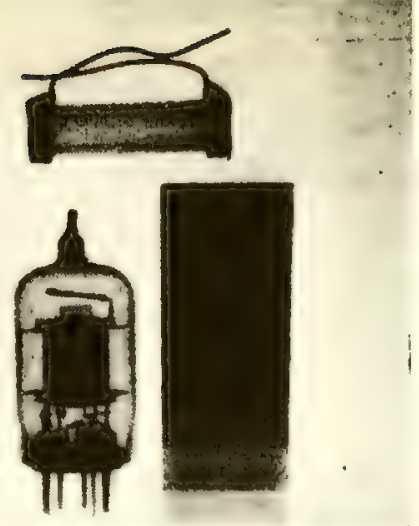

TYPICAL RADIOGRAPH using 10-me $\mathrm{Sr}^{90}$ $Y^{90}$ source, lead torget, and type $K$ flm objects are aluminum step wedge $(0.050$ in. steps), resistor, and electronic tube

range of effective half-lives, opens a number of areas of possible utility.*

The sources may be used for radiography of thin sections or light materials. For radiography, it appears feasible to obtain carrier-free activity such that 1 curie of strontium can be incorporated in a $1.5-\mathrm{mm}$ focal spot. For emitters of shorter half-life, the specific activities would be correspondingly higher. Fairly good definition and comparatively short exposure times will be possible with a full-scale strontium source now under construction. Radiographs of full density should be obtainable through $1 / 2$ in. of aluminum at 4-min exposure times and source-tofilm distances of about $1 \mathrm{ft}$ using type $\mathrm{K}$ film. Intensification screens and faster films would reduce exposures correspondingly. The sources alone are not sufficiently intense for medical radiography, but it appears entirely possible that compact image intensifiers (either electronic or solid-state types) may be used to advantage, thereby making such applications feasible. The possibility of placing the source within the object to be radiographed may be advantageous. The possible utility of the sources in medical therapy has not been examined.

Either the continuous spectrum from a source emitting primarily bremsstrahlung or a line-spectrum source of known properties may be used in thickness gaging over a fairly wide range of thicknesses. There appear to be certain advantages in using $\mathrm{X}$-ray sources in preference to beta-ray sources in

* Patents have been applied for on various phases of this work. some gaging applications, but this aspect of the problem needs further exploration. The continuous spectrum is ideally suited to density measurements in liquids, gas-liquid mixtures, and slurries. High-speed density determinations to better than $1 \%$ accuracy have been achieved with automatic instrumentation.

The line spectra obtainable from these sources are admirably suited to absorptiometry, particularly for coating control or for additive concentration control, as well as for chemical analysis. The energy of the incoming radiation may be set at the critical absorption edge of the element of interest and thus render the system preferentially sensitive to variations in this element. Such a technique appears promising, for example, in silveremulsion thickness control where antimony radiation may be used to probe silver content of the emulsion as it is deposited without producing sufficient exposure to fog the film.

With either the $\mathrm{X}$-rays from such sources or beta rays directly, X-ray fluorescence analysis in certain simple systems can be achieved. Here the low intensity of the sources, compared to X-ray machines, may be at least partially compensated for by the use of Ross balanced-filter techniques to obtain large effective apertures and still retain the required energy selection properties of the detector.

In most of the applications examined, with the exception of radiography, the sources have employed millicurie amounts of beta emitter to achieve adequate counting rates in the detectors, which are usually scintillation counters exhibiting close to $100 \%$ efficiency in the energy range of interest. The yield per millicurie of beta emitter will, of course, vary with the details of the source configuration. For a typical transmission-target design, a nominal value of $1 / 10$ me of $\mathrm{X}$-rays per millicurie of beta particles may be used to estimate the required activities. For reflection targets, this figure must be reduced by about one order of magnitude, i.e., $10 \mu \mathrm{c} / \mathrm{mc}$. These rough figures apply to bremsstrahlung spectra for emitters such as $\mathrm{P}^{32}$ and $\mathrm{Y}^{90}$. The ratio of bremsstrahlung to fluorescence radiation may be estimated from the curves previously discussed.

Further details of developments using the techniques sketched here will be reported at a future time. 


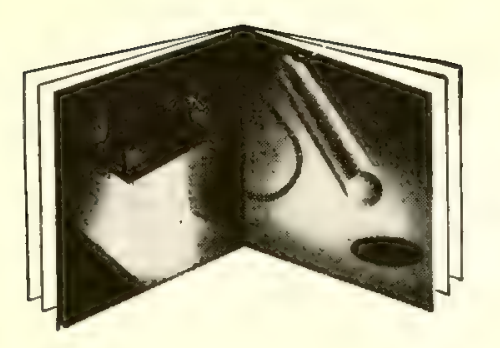

APPLIED

\title{
RADIATION
}

CASE HISTORY

NO. 1

\section{Electron-Beam Sterilization of Surgical Sutures}

\author{
Substituting radiation for heat results in a stronger product, \\ a better package, simpler manufacture and continuous processing. \\ Savings in these areas help pay for more expensive sterilization
}

By CHARLES ARTANDI and WALTON VAN WINKLE, Jr.
Ethicon, Incorporated, Somerville, New Jersey

ELECTRONS FROM A microwave linear accelerator are routinely sterilizing surgical sutures in Ethicon's Somerville, N. J. plant, the world's largest producer of surgical sutures (NU, Feb. '58, p. 27). Catgut sterilization using heat has always presented difficult problems. Our commercial electron sterilization has grown out of a five-year research program to find a better way.

For our purposes the best radiation source is the linear accelerator, or linac. Ours has been installed in a special split-level building with appropriate conveyor facilities and monitoring equipment. We monitor the accelerator, the process and the product, in some instances with methods that we have invented for our own needs. It is hard to estimate our costs, but it is plain that electron sterilization is expensive. Nevertheless it has brought us many benefits including better packaging, simpler manufacture and even customer appeal. Figure 1 shows a tray of sutures on its way to sterilization.

\section{Catgut Sterilization}

Surgical sutures are of two main types: absorbable and nonabsorbable. The absorbable variety, known as "catgut," is derived from connective-tissue layers of the intestines of beef and sheep. It is with this type of material that the suture industry has always had sterilization problems.

Classically sterilization has always used heat-high enough temperature for a long enough time kills all bacteria and other forms of microbial life. Unfortunately catgut sutures are composed of an animal protein, collagen, and like all proteins it is "cooked" by heat. This cooking reduces tensile strength, pliability and other important properties of the suture. To avoid damage as much as possible, the practice has been to dehydrate the sutures for 8-12 hr at $100-110^{\circ} \mathrm{C}$, then immerse them in an anhydrous hydrocarbon bath and sterilize at $156^{\circ} \mathrm{C}$ for an hour. This must be done before sealing the container. After sterilization, an aseptic process is used to fill the container with sterile tubing fluid, rehydrate the suture and seal the container. This process requires manufacturing with operating-room precautions.

Heat-sterilized sutures are always packaged in glass tubes-the only economical material suitable for the high sterilizing temperatures. Getting the suture in the operating room requires breaking the glass container with attendant hazards of broken glass near the operative field.

Another objection to heat sterilization is that it is a batch process. Thus it is a bar to continuous flow of materials and automation. A method that permits conveyor-belt operation makes possible automation and leads to manufacturing economies in several areas.

It is obviously desirable to find some sterilization method that will:

- Minimize damage to the suture

- Permit sterilization in the final, sealed container

- Allow use of new and more convenient packaging

- Lend itself to continuous operation.

\section{Exploration}

As early as 1949 it was well established that electron-beam irradiation is capable of killing microorganisms at doses that do not adversely affect many materials. Subsequent work established the dose parameters necessary to sterilize sutures and similar objects (1). However, the large capital investment required and the lack of definitive experience with the commercial use of electron accelerators, beta, and gamma radiation deterred most industries from extensive development work.

Early in 1953 the problems peculiar to the suture industry prompted us to undertake a detailed feasibility study of radiation sterilization of sutures. We rented a $2-\mathrm{Mev} 0.5-\mathrm{kw}$ Van de Graaff accelerator from High Voltage 


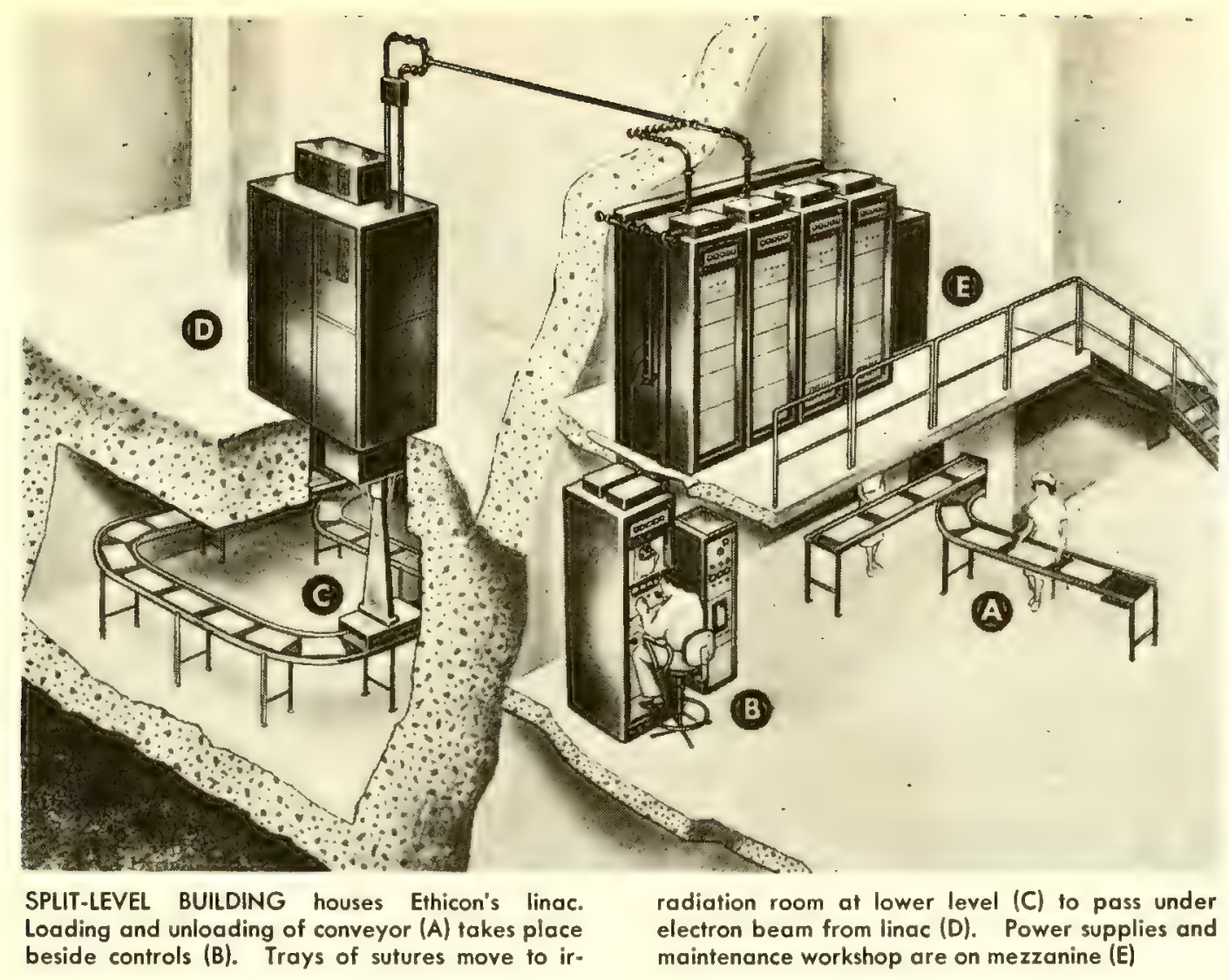

Engineering Corp. and embarked on an extensive research project.

We established the minimum killing dose for over 150 different species of microorganisms and found the maximum dose that could be safely tolerated by catgut. We chuse 2.5 megarads as our sterilizing dose. This is $40 \%$ above the minimum to kill the most resistant microorganism. Catgut can tolerate 5.0 megarads before showing properties inferior to heat-sterilized gut.

Although we knew that the $2-\mathrm{Mev}$
Van de Graaff would not have enough production capacity, we later bought the unit for our expanding research program in the field of irradiation. We have since installed the linac that we use routinely for sterilization. Currently we are installing a 3-Mev Van de Graaff.

Setting specifications. As our investigations progressed we began to have more enthusiasm for radiation sterilization. Simultaneously we began establishing our requirements.

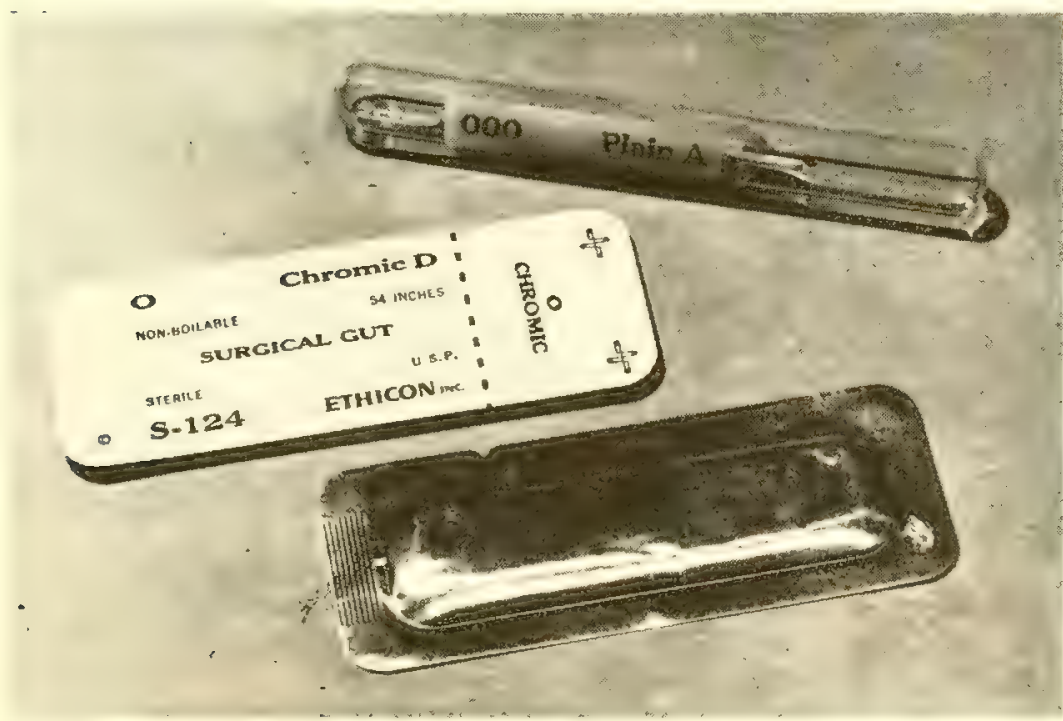

OLD AND NEW PACKAGES. Aluminum-foil package shown in front and back views at bottom is more convenient than glass package it replaced

If radiation is to be used in production-line sterilization, the source must satisfy the following requirements:

- Adequate dose delivery

- Complete and uniform coverage of the product

- Adequate penetration

Dose is determined mainly by beam current. Coverage is ensured by the proper scan width, which is determined by scanning coil current. Penetration is determined by accelerating voltage or, more generally, by electron energy. The energy must be great enough for complete penetration.

Total penetration is the depth of complete absorption of electrons. For our purpose we define "useful penetration" as the thickness such that exit dose is equal to entrance dose (Fig. 2). With monoenergetic electrons the entrance dose is $\sim 60 \%$ of the maximum in this energy range.

Having met the penetration requirement one seeks a source delivering the largest number of electrons of a specified energy per unit time. Usually beam current then determines production capacity. For each scan width, beam current tells us at what rate the product can move under the source for a specific minimum dose.

If one is dealing with a homogeneous material like grain or a fluid, dimensions can be varied for maximum beam utilization. However, the majority of 


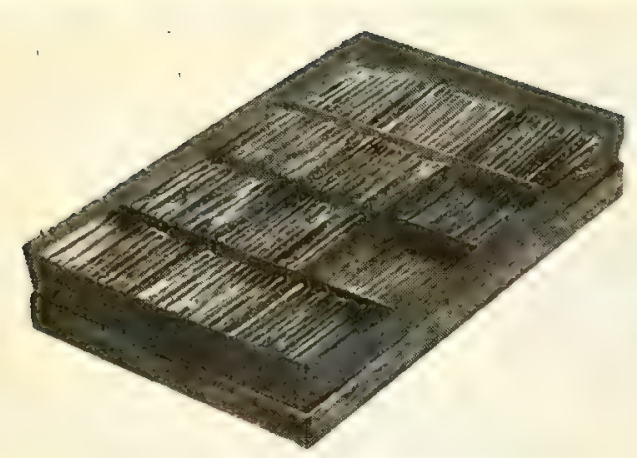

FIG. 1. SUTURES ON TRAY, ready for irradiation, are arranged to make most efficient use of electrons as tray passes under beam

objects to be sterilized are not homogeneous and have fixed dimensions. Therefore penetration has to be set according to the densest part of the object, resulting generally in reduced beam utilization.

Consideration of these points in relation to the product we proposed to sterilize indicated the need for an energy of 4-7 Mev and an output of at least $2.5-3.0 \mathrm{kw}$.

\section{Radiation Source}

Gamma emitters like $\mathrm{Co}^{60}$ and waste fission products provide penetration considerably greater than our needs. However, their low dose rates necessitate very long dwell times for adequate dose. This creates some complex engineering problems to provide accurate and controllable dose. High-power $\mathrm{X}$-ray machines also offer adequate penetration, but their power output is low and they pose many design problems. Van de Graaff accelerators above 3 Mev are of tremendous size and comparatively low output.

The source that appeared to meet our needs of adequate energy with bigh power output was the linac (2).

Operating requirements for a commercially used linear accelerator are vastly different from those of universities and research laboratories. Commercial sterilization demands continuous uninterrupted performance with unvarying beam characteristics. Low maintenance costs and ease of operation are highly desirable, of course.

Throughput. The production capacity of a machine can be given in several ways: (a) in kilowatts, (b) in mass processed per hour at a certain dose (1 megarad equals $4.50 \mathrm{kw}-\mathrm{sec}$ of radiation totally absorbed in $1 \mathrm{lb}$ of product; at 2.5 megarads a $1-\mathrm{kw}$ device is capable of irradiating $320 \mathrm{lb}$ in an hour), (c) in area coverage per minute for a certain dose (1-ma beam current delivers 1 megarad to the useful-penetration thickness of $1,676 \mathrm{in}^{2} / \mathrm{min}$ ).

The $100 \%$-utilization capacities of our three machines are as given in the table on this page.

Using irradiation from one side only, the maximum utilization of ionizing energy cannot be more than about $60 \%$. This is the area under the equal-entrance-and-exit-dose line in Fig. 2. The area above this line represents about $24 \%$ of the total ionizing energy. This part is really wasted as overdose in the sterilization process. The tail end beyond the useful penetration, representing about $16 \%$, is also completely lost because it leaves the product.

Another important consideration is the efficiency with which an electron beam can be used. It is convenient to define three efficiencies, each corresponding to one of the dimensions of the product. Thus the scan efficiency is the fraction of the scan in which the beam hits the product. The loading

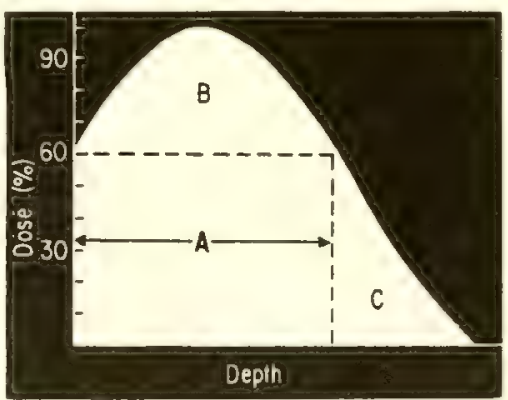

FIG. 2. PENETRATION CURVE measured with stack of slides shows depth of "useful penetration" (A) and area of useful dose, area of overdose (B) and area of wasted dose (C)

efficiency is the fraction of time in which the conveyor motion places a part of the load under the beam. The penetration efficiency is that portion of the beam (measured in the direction of electron motion) that is usefully employed as indicated by Fig. 2. Our 2-Mev Van de Graaff and our linac both have $80 \%$ scan efficiency and $95 \%$ loading efficiency. Penetration efficiency of the Van de Graaff is 15-25\%, and that of the linac is $20-40 \%$.

\section{Facility Design}

We decided to house both the 2-Mev Van de Graaff and the linac in the same area and place them close to our older

\section{Capabilities of Three Accelerators}

Van de Graaffs Linac

\begin{tabular}{llll}
\hline $\begin{array}{l}\text { Beam energy } \\
\text { (Mev) }\end{array}$ & 2 & 3 & 5 \\
Beam power (kw) & 0.5 & 3.0 & 2.1 \\
$\begin{array}{l}\text { Capacity } \\
\text { lb } / \mathrm{hr} \text { at } \\
3 \text { megarads }\end{array}$ & 160 & 960 & 672 \\
$\begin{array}{c}\text { in } 2 / \text { min at } \\
3 \text { megarads }\end{array}$ & 167 & 667 & 280 \\
\hline
\end{tabular}

sterilization facilities. This provides a logical flow of materials through our manufacturing operation and permits the greatest flexibility for any later mechanization we want to undertake.

The accelerator building is separate from the main manufacturing building but connected to it by an enclosed passageway. This permits eventual expansion of the manufacturing area in the direction of irradiation facilities and at the same time maintains our normal process flow.

Split-level building. Consultations among our engineers, the architect and the accelerator builder led to design of a split-level accelerator building shown in the figure on p. 87. On the lowest level (partly underground) are two irradiation rooms each surrounded by $7 \mathrm{ft}$ of concrete. This thickness was based on calculations of probable hard $\mathrm{X}$-rays generated by electrons striking the metal conveyor belt and other objects.

Half a story higher and in front of the target room is the production area for loading and unloading conveyor belts. We have only recently extended the conveyor system to lead into the manufacturing area. Operating controls and monitoring devices are also on this floor. About $3 \mathrm{ft}$ higher, above the target rooms, are the accelerator rooms.

The highest level is half a story above the production area. This is a mezzanine that houses the high-frequency amplifiers and the power supply for the linac. Maintenance workshop and testing instruments are also on the mezzanine.

Access to both accelerator rooms and target rooms is through a passage with two right-angle turns. These turns effectively block all stray radiation.

Installation of our linac began in late 1957, and we started the checkout early in 1958. 


\section{Monitoring}

Each installation has its own monitoring problems that are peculiar to the process at hand. We cannot afford a single instance of lack of sterility in our product. We want to measure dose, scan width and penetration as it affects our product under processing conditions. We prefer continuous monitoring; a second choice is intermittent monitoring.

Problems. We had to consider control of machine parameters. With a linac this problem is a bit more complicated than with electrostatic accelerators. In an electrostatic accelerator like a Van de Graaff, controls are fairly simple. Beam current and scan-coil current can be read on ammeters. Accelerating voltage, which is very stable, can be measured on an electrostatic voltmeter.

After determination of maximum allowable variations in any of the three important parameters these changes can be fed into a computer circuit. This circuit gives a signal whenever the irradiation dose is reduced by a certain amount as a result of changes in any combination of parameters. The signal can be recorded or used to actuate a marking device or kickoff mechanism in conjunction with the conveyor belt.

Linear accelerators present a more difficult problem because the energy of the electrons can not be measured directly. Electron pulses are accelerated by an $\mathrm{r}-\mathrm{f}$ traveling wave (2). Energy is affected by field strength and frequency of the $r-f$ wave. Peak frequency is affected by various factors such as changes in line voltage, drift

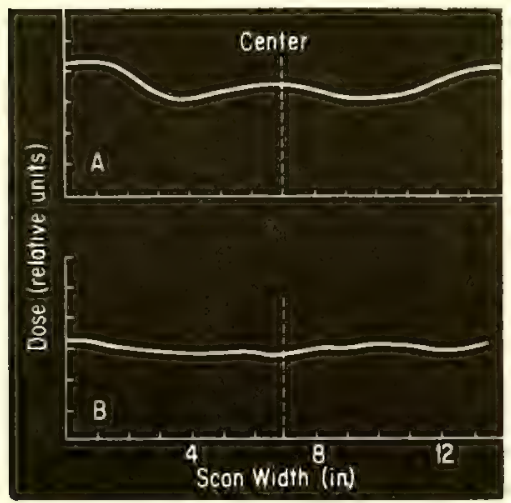

FIG. 3. SCAN CURVES show lack of lateral uniformity in dose (A), which preceded adjustments of magnet pole pieces and more uniform scan that followed (B) in phase, defocusing, etc. Any change in energy of the $r-f$ wave affects penetration, scan width and dose.

Monitoring can be achieved in a manner like that for the Van de Graaff, except that a frequency parameter has to be used instead of voltage. Scanwidth changes can be measured by metal absorbers placed at each end of the scan. Two are spaced at cach end $\sim 7 / 8$ in. apart and wired to separate ammeters. The inner pair are always touched by the scanned beam. The outer pair normally are not. If the scan widens, both sets of absorbers record current; if it narrows, neither does.

A meter on the exit window records the electron current that the window absorbs. In general the more current absorbed in the window, the lower the energy of the electron beam.

For preventive trouble shooting we find it important to monitor focus-coil currents, operating and pulse frequencies, vacuum, r-f power, line voltage, power-supply voltage and acceleratorroom radiation level.

Conveyor speed must be accurately controlled at the point of irradiation. In normal operation our conveyor speed is $15-18 \mathrm{in} . / \mathrm{min}$, and it is continuously measured. For maximum beam utilization our conveyor system is designed so that the product is irradiated continuously without spaces or gaps in the flow of material.

Linac operation. Our only way of measuring linac energy is to absorb the beam completely in a stack of dosimeter slides. From the readings of the slides in proper order, useful penetration is calculated and converted into electron energy. An energy of 1 Mev implies a penetration of $0.130 \mathrm{in}$. in unit-density material. Plotting individual readings against thickness produces an ionization-distribution curve.

Scan uniformity with the linac was at first unsatisfactory. Dose range within the 14 -in. scan was as great as 1.0 to 1.2 megarads around a nominal dose of 2.5 megarads. After proper adjustments in the pole pieces of the scanning mechanism the range was reduced to $0.5-0.7$ megarads (Fig. 3). This nonuniformity across the scan is still greater than that of the Van de Graaff, but for a sterilization process we can live with it if the average dose is right.

Another problem is beam-energy fluctuation during runs. Initially we had some large and many small fluctu- ations. We learned to take care of the large ones by keeping the machine continuously adjusted to optimum operational settings. The many little fluctuations are still with us, but they do not affect our processing greatly. On a few occasions when the energy was gradually dropping off over several days in spite of the fact that the machine was operating at the right settings, we could predict impending major klystron trouble through our dosimetry. A continuous energy indicator on the linac would be a major improvement.

With a year's experience under production conditions, we can say that we required about 6 months to obtain

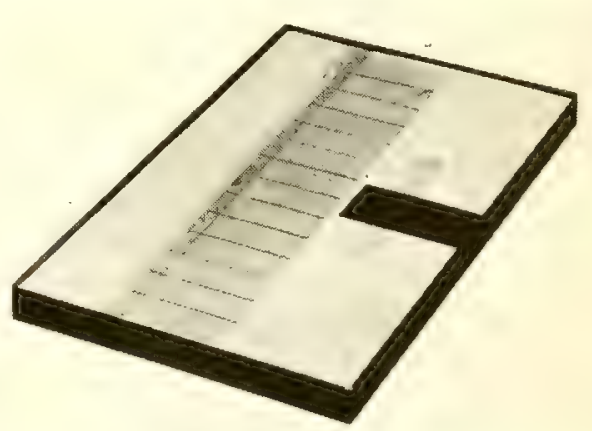

FIG. 4. PRODUCTION TRAY for lesting machine output carries row of slides to test scan uniformity and stack of slides in cutaway slot to test penetration

efficient and consistent operation of the equipment.

Process monitoring. We have been using ceric-cerous dosimetry (3) as a primary standard, and blue cellophane (4) and rigid vinyl film (5) as secondary standards. Rigid vinyl film as a dosimeter is suitable for the following measurements: (a) total and useful penetrations, (b) ionization distribution, (c) absorption in nonhomogeneous objects, (d) scan width and uniformity.

At the beginning of each shift on the linac a production tray is put through (Fig. 4). It contains a row of 14 rigid vinyl slides to test scan width and uniformity and a stack of 60 slides, which is thick enough to absorb the beam completely. Each slide measures $3 \times$ $1 \times 0.015 \mathrm{in}$. The slides are placed on a $3 / 4$-in. plywood board to reduce backscatter. The row of 14 slides are placed side by side across the board with their long dimensions in the direction of travel. They are numbered consecutively so that their positions 
will not be forgotten. A cutout provides a place for the stack of 60 .

After irradiation the slides are removed, developed and read. The fourteen slides across the tray tell us whether (a) the scan width is adequate to cover the product, (b) the dose is right, (c) the dose is acceptably uniform within the scan width. From the stack, we get the depth of useful penetration.

The procedure is similar for the 2 Mev Van de Graaff machine except that only four slides are needed for scan width and 20 for penetration.

As a spot check for dose, a single slide on plywood in the center of a tray is sent through the conveyor system every hour.

Product monitoring. A rigid vinyl slide is placed under the product in the direction of travel, on each tray. The slides are numbered consecutively, and the numbers also identify the trays. The slides are removed after irradiation, developed and read at their lightest point. Only if this reading indicates a sterilizing dose is the tray released for sterility sampling. Otherwise the material has to be salvaged.

\section{Package Design}

We are now packaging our radiationsterilized sutures in the aluminum-foil packages shown on the cover, on page 87 and in Fig. 5. We devoted a great deal of effort to investigation of aluminum-foil-plastic laminates. The resulting package is impermeable, attractive, and easy to open by tearing. It takes irradiation well. An apparent disadvantage is that it is opaque. However, our field evaluation showed that there is no preference for a transparent package. Ease of opening and good identification are found much more important.

Our first packaging ideas revolved around plastics because they are transparent like glass tubes. We determined the irradiation characteristics of a large number of plastic films. Simultaneously we studied the permeabilities of these films. We had to select a plastic that could withstand irradiation and, more importantly, that was impermeable to the fluids inside and outside the package. Inside the package is $90 \%$ isopropyl alcohol to make the catgut pliable. The packages are stored in a jar containing $97 \%$ isopropyl alcohol and $1 \%$ formaldehyde. This storage solution is intended to sterilize and to maintain the sterility of the outside of the packages. Any significant penetration of formaldehyde to the inside will destroy the catgut. Kel-F $\mathbf{5 0 0}$ was the only plastic film that satisfied our strict requirements, and it proved too expensive.

\section{What Does It Cost?}

While it is difficult to adjust cost figures from one irradiation operation to another, the elements of cost can be identified and some rough estimates can be made.

Capital costs are high. Available accelerators range from about $\$ 50,000$ to nearly $\$ 200,000$ depending primarily on power output. Monitoring and control equipment can range from as low as $\$ 5,000$ to as high as $\$ 75,000$. Finally the facility costs will depend on the

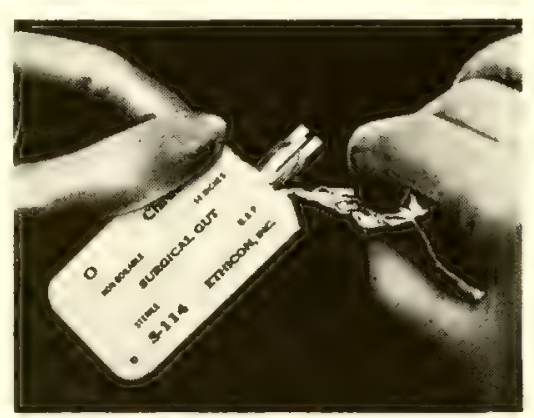

FIG. 5. OPENING NEW PACKAGE is quick, easy and safe. There is no glass to cut the nurse or contaminate the operative field

type of construction and the space requirements. In turn, the space requirements and type of construction depend on accelerator type. For instance, a 3-Mev Van de Graaff machine requires a minimum of $20 \times 25 \mathrm{ft}$ with at least $30 \mathrm{ft}$ vertical headroom. A modern linac requires much less space, particularly in the vertical direction.

The principal operating expenses are labor and maintenance. A single accelerator needs a competent technician for operation and at least one additional technician to assist in maintenance. Additional labor is required for loading and unloading the product on conveyor belts.

Maintenance and repair costs vary with the type of accelerator. Frobably the highest cost is experienced with the linear accelerator and will average about $\$ 12.50$ per operating hour. This includes only replacement parts. Most of our linac breakdowns are associated with failures of high-voltage diodes, thyratrons and klystrons. The accelerator tube can cause trouble if misaligned or if the beam is not properly focused. Generally the repairs take from a few minutes to a couple of hours. Vacuum troubles take one or two days, but they happen very seldom.

Shutdowns on the Van de Graaff are mainly caused by loosening or breakdown of the belt, breakdown of belt spacers or equipotential rings. Shutdowns are very infrequent, but they require one day for repairs because the tank has to be removed in each case.

Electron-beam sterilization is not cheap. However, it is unfair to make direct cost comparisons between electron-beam sterilization and other forms of sterilization. Some objects can be sterilized only by electron beam. Newer forms of packaging may be possible only with this new method of sterilization.

Developments now under way in high-frequency amplifiers promise a major reduction in both initial and operating costs. This plus the cost advantages of mechanization may well bring the cost of radiation sterilization to that of heat.

\section{Safety}

Our whole installation is designed for maximum safety. Shielding is adequate for nearly complete absorption of all radiation. The doors leading to the target and accelerator rooms are locked and interlocked with the machine. Opening of any of these doors will turn off the machine instantaneously. When the machine is turned on, there is a delay in starting up. During this delay a horn sounds repeatedly to warn people that irradiation is about to begin. Through a maze-and-mirror system, the target room can be easily inspected.

The highest radiation level we have detected in any area occupied by personnel is less than $0.5 \mathrm{mr} / \mathrm{hr}$. Under normal operating conditions the radiation level is about one tenth of this.

\section{BIBLIOGRAPHY}

1. R. E. Реpper, N. T. Buffa, V. L. Chandler. Relative resistances of microorganisms to cathode rays, III. Bacterial spores, A ppl. Microbiol. 4, 150 (1956)

2. Linear accelerators gain in use as radiation facilities, NUCLEONics 14, No. 11, 166 (1956)

3. J. Weiss. Chemical dosimetry using ferrous and ceric sulfates, NUCLEONICg 10, No. 7, 28 (1952)

4. E. J. Henley. Gamma-ray dosimetry with cellophane-dye systems, NoCLEONICs 12, No. 9 , 62 (1954)

5. C. Artandi, A. A. Stonehill. Polyvinyl chloride-new high-level dosimeter, NuczEoNics 16, No, 5, 118 (1958) 
RADIOGOLD SEEDS and linear sources developed during the past three years (1-5) have displaced radon completely in radiation therapy at The Ohio State University Medical Center. These sources are now used advantageously in many types of cases in which radioactive cobalt was formerly applied $(6-10)$.

In external appearance the $A u^{188}$ seeds are indistinguishable from the usual radon seeds, and they can be utilized in the same manner and with the same apparatus. The half-life of $\mathrm{Au}^{198}$ is 2.70 days, which is about 0.7 of the half-life of radon (3.83 days). The energy of the gamma radiation emitted by $\mathrm{Au}^{198}$ is $411 \mathrm{kv}$; it is monoenergetic in contrast with the range of energies between $184 \mathrm{kv}$ and $2,198 \mathrm{kv}$ for the dozen different gamma rays emitted by radon and its disintegration products (11). Further biological studies $(1,2)$ are under way to determine the relative merits of the differences in half-lives and gamma-ray energies of $A u^{198}$ and radon.

Sources for interstitial applications should not be confused with radioactive gold in the colloidal state which is now being used in some medical centers for the treatment of pleural and peritoneal carcinomatosis. In contrast to colloidal $\mathrm{Au}^{198}$, in which the beta radiation emitted by the radiogold is used primarily, gold seeds containing $\mathrm{Au}^{198}$ are employed only because of the gamma rays emitted from the radiogold within them. The seeds are usually superior because of the relatively homogeneous dosage of ionizing radiation that may be achieved when discrete sources of gamma rays are properly spaced in accordance with well-established physical principles. Dosimetry is highly uncertain whenever radioactive sources are injected that are mainly dependent on the emission of beta particles for whatever effectiveness they may have. The $\mathrm{Au}^{198}$ seeds are, therefore, essentially similar to radon seeds, where much of the beta radiation is removed by filtration.

Advantages of $\mathrm{Au}^{198}$ seeds over radon

- Julius F. Stone Research Associate Professor of Medical Biophysics. seeds for applications in therapy include:

1. Radiogold seeds of any strength can be cut instantly, even at the time the decision to use them is made (e.g. in the operating room), whereas radon seeds usually must be ordered a day or more in advance of the time they are to be used.

2. Radiogold seeds are very uniform in strength, but wide variations in strengths are often found in radon seeds.

3. Because gold is a metal there is no danger of leakage of gas from $\mathrm{Au}^{198}$ seeds.

4. The beta particles emitted by $\mathrm{A} \mathrm{u}^{198}$ with maximum energy of $970 \mathrm{kv}$ are almost completely filtered out, in contrast to the beta particles emitted by radon disintegration products with maximum energy of $3,170 \mathrm{kv}$.

5. The facilities and equipment required for manufacturing seeds loaded with $A u^{198}$ are simple and inexpensive.

6. Problems of protection are greatly simplified in the preparation and handling of $\mathrm{Au}^{198}$ seeds because the half-value layer in lead for the gamma rays is only $2.8 \mathrm{~mm}$, in contrast to $14 \mathrm{~mm}$ for radon.

\section{Preparation of Sources}

Each Monday there arrives in Columbus via air express a package shipped by the Oak Ridge National Laboratory earlier that day. This package contains several pure gold wires $\dagger 0.2-0.3 \mathrm{~mm}$ in diameter and $85 \mathrm{~mm}$ long that have been irradiated during the previous week in a flux of about $1-5 \times 10^{11}$ thermal neutrons/ $\mathrm{cm}^{2} / \mathrm{sec}$ in a nuclear reactor. In the radioisotope laboratory the radioactive wires are slid into inert gold tubing $\dagger$ about $0.4 \mathrm{~mm}$ inside and $0.8 \mathrm{~mm}$ outside diameter. This nonradioactive outer gold tubing absorbs almost all of the beta particles and passes about $90 \%$ of the $411-\mathrm{kv}$ gamma rays emitted by the radioactive gold wire contained within it.

Seeds are cut to any desired strengths with a simple cutter. The device consists of a lead block with a central bore

+ Supplied by the American Platinum Works, Newark, New Jersey. into which the encased radioactive gold wire is introduced and from which it may be extruded to any desired length from 1 to $40 \mathrm{~mm}$, preadjusted by a micrometer. The gamma radiation of the part of the encased radioactive gold wire pushed outside of the lead shield to be cut off with the cutter, is measured with a radiation survey meter equipped with an ionization chamber ("Cutie-Pie" type).

Calibration of seed strength. The survey meter is placed in a fixed position at such a distance from the cutter that a swing of the needle to 20 scale divisions on the $50 \mathrm{mr} / \mathrm{hr}$ full-scale range of the meter will result in a $\mathrm{Au}^{198}$ seed which will deliver 1,000 gamma roentgens-total-at-one-centimeter (designated "rtcm") during complete decay. This is approximately the same strength as the $1,112 \mathrm{r}$ given off during lifetime decay of $1 \mathrm{mc}$ of radon enclosed in $0.5 \mathrm{~mm}$. of platinum. The cutter is portable and is often taken to the operating room where $\mathrm{Au}^{198}$ seeds are calibrated and cut in a few seconds

Details of design of the cutter, of the method of calibration of the sources, and of dosimetry with the $\mathrm{Au}^{198}$ seeds will be published separately.

Following publication $(1,2)$ of the method developed here for making sources containing $\mathrm{Au}^{198}$, Sinclair (12) briefly described sources made from cylinders of gold sealed in a platinum beta-particle absorber before exposure to the neutron flux in a nuclear reactor. The method described here for preparing $\mathrm{Au}^{198}$ seeds would appear to be a superior one in the following respects:

1. The radiation therapist has all of the advantages of on-the-spot adjustability of strengths of sources provided by a radon plant without the many well-known disadvantages associated with the establishment and operation of a radon plant. The method described by Sinclair does not permit adjustment of strengths of cources at the point of usage, nor does it enable the therapist to compensate for decay of radioactivity by making longer seeds or by using successively thicker radioactive gold wires irradiated in the same container in the 
nuclear reactor, during the latter part of the week they are received.

Radiogold seeds received from a distant point of preparation will always put the therapist to the same disadvantages that now prevail for those physicians who receive radon seeds from commercial sources. The variation of source strengths to provide the flexibility required for individualization of patterns to meet the multitudinous clinical variations encountered in practice, is not possible with sources of initially fixed strengths.

2. It was shown previously $(3,5)$ that the leakage of the undesirable beta particles through the thinned parts of the ends of $\mathrm{Au}^{198}$ seeds prepared here is insignificant in comparison with the escape of highly energetic and penetrating beta particles from a radon seed obtained from a commercial source. Measurements made by Sinclair revealed a beta-particle emission from the platinum-sheathed $\mathrm{Au}^{108}$ sources he prepared that differed only slightly from that of radon seeds.

3. The cost of nonradioactive gold for sheathing material to absorb the same fraction of the beta particles emitted from the enclosed radiogold is only about one-fourth as great as that for the platinum sheaths used by Sinclair. Moreover, the expense incurred for the precision machining and welding that is required to encapsulate the gold within the platinum is avoided in the method devised here.

\section{Clinical Uses}

Radiogold seeds may be utilized to form permanent implantations by means of the same applicators that are now widely used for radon seeds. Alternatively, $\mathrm{Au}^{198}$ seeds may be loaded into Nylon tubing that is threaded through tumors and withdrawn at the end of the exposure time, which is usually 7 days. An advantage of implanting $\mathrm{Au}^{198}$ seeds permanently is that it is unnecessary to load the seeds into Nylon tubing. However, permanent implantations by free-hand techniques have not resulted in the regular patterns of sources that are usually obtained readily when the seeds are first enclosed in Nylon tubing.

Another advantage of the Nylontube method is that errors in alignment of the pattern during implantation can be corrected by pulling the Nylon tubes into the proper position, as indicated by roentgenograms. Also, no foreign body remains in the tissue after the
Nylon tubes are withdrawn at the end of a week. Experiences at this medical center have demonstrated that the Nylon-tube technique is generally to be preferred to the permanent implantation of the seeds.

Improved implantation method. The Nylon-tube implantation method, previously developed here for use with $\mathrm{Co}^{60}(8-10)$, has recently been improved in several respects. Since details of this new Nylon-tube technique will be reported independently by Henschke, only the principal features of it will be outlined briefly here. First, in place of the thickwalled, relatively stiff Nylon tube formerly employed at The Ohio State University Medical Center, a thinwalled, narrow Nylon tube is now used. In this new Nylon tube, the radioactive sources are held firmly in place by the resiliency of the Nylon without the need for the metallic spacers used formerly. These new tubes are much more flexible and can be bent more readily than the previous tubes. Moreover, should the loaded suture break inadvertently, the radioactive sources cannot fall out as they could with the old method.

A second major improvement in the Nylon-tube technique has been evolved and is now used in many cases. The Nylon tubes are not loaded with the radioactive sources prior to implantation; instead, Nylon tubes loaded with dummy metal seeds, or thin stainlesssteel wires are inserted in the tumor area at the time of operation. Roentgenograms are then made and an appropriate distribution for the radioactive sources is determined from the spatial relationships. Nylon tubes are loaded with the radioactive sources accordingly and are pulled into position after attachment to the dummyloaded Nylon tubes or to the stainlesssteel wires. This substitution of the Nylon tubes loaded with active sources for the ones containing the dummy sources, or the wires, causes little discomfort and is usually done satisfactorily without anesthesia.

It will be apparent that this technique enables the surgeon to prepare a patient during operation for subsequent implantation with the radioactive seeds. The radiation therapist can then design an appropriate pattern and insert it at a time and place convenient for him. This technique has proved mutually satisfactory to the surgeon and the radiotherapist members of the team here.
Clinical experience. Fifty-one applications of $A u^{198}$ seeds clinically in 46 patients thus far have included 31 permanent implantations, 12 Nylontube implantations, 2 intrauterine applications, 2 intraesophageal applications, 2 nasopharyngeal applications, 1 intraoral mold, and 1 skin mold.

No untoward or unusual reactions have been noted in any case in which the $\mathrm{Au}^{198}$ seeds have been used. The clinical regressions of tumor tissue have been comparable to those observed with other methods of interstitial irradiation. And they have been consistent with the preliminary animal experiments in which it was demonstrated that it is possible to deliver a cancerocidal dose to tumor tissue with $\mathrm{Au}^{198}$ seeds alone $(1,2)$. However, the preferred practice here usually is to use $\mathrm{Au}^{198}$ seeds in combination with high-voltage external irradiation. In some cases two insertions of radiogold seeds spaced 1-8 weeks apart have been used to deliver the total dose over a longer period.

\section{* * *}

This study was supported, in part, by a grant-in-aid from the Ohio Department of Health, the Ohio State University Development Fund, the Julius F. Stone Fund for Medical Research, and cancer research grant C-1899 from the National Cancer Institute approved by the National Advisory Cancer Council.

\section{BIBLIOGRAPHY}

1. W. G Myers, B. H. Colmery, Jr. Radioactive Au-198 in gold seeds for cancer therspy, Cancer Research 12, 285 (1952)

2. B. H. Colmery, Jr. Preparation of seeds of radiosctive gold-198 and their use in cancer therspy (Thesia, The Otio State Univeraity, 1951)

s. W. M. McLellon. The use of radiosotive gold-198 for the treatment of malignancies (Thesis, The Ohio State Univeraity, 1952)

4. W. G. Myers. Applications of artificial radioisotopes in interstitial radiation therapy. Presented March 5, 1952 at the Second National Cancer Conference, Cincinnati, Ohio. In press

5. W. G. Myers, B. H. Colmery, Jr. W. M. McLellon. Radioactive gold-198 for gamms radiation therspy, Am. J. Roentgenol. Radium Therapy Nuclear $M$ ed., in press

6. W. G. Myers. Radioactive needles containing cobalt-60, Science 107, 621 (1948)

7. W. G. Myers, Applications of artificially radiosetive isotopes in therapy: $I$. cobslt-60. $A$ m. J. Roentgenol. Radium Therapy 60, 816 (1948)

8. J. L. Morton., G. W. Callendine, Jr., W. G. Myers. Radioactive cobalt-60 in plastio tubing for interatitial radiation therapy, Radiology 66, 553 (1951)

9. A. G. James, F. D. Williams, J. L. Morton. Radiosetive cobalt in nonresectsble head and neck cancer, Cancer 4, 1333 (1951)

10. A. G. James, R. D. Williams, J. L. Morton. Radioactive cobalt as an adjunct to cancer Burgery, Surgery 30, 95 (1951)

11. R. D. Evans. Radioactivity unitg and standarda, Nucleonica 1, No. 2, 32 (1947)

12. W. K. Sinclair. Artificial radioactive sources for interstitial therapy, Brit. J. Radiolooy 25, 417 (1952) 


\title{
Wear Studies of Irradiated Carbide Cutting Tools
}

\begin{abstract}
Isotope-tracer measurements, more accurate than microscopic examination, show a constant rate of tool wear. With proper choice of isotope and specific activity, G-M and autoradiographic techniques can be used under normal machining conditions
\end{abstract}

By B. COLDING* and L.-G. ERWALL $\dagger$ Royal Institute of Technology

Stockholm, Sweden

NEUTRON IRRADIATION of carbide machining-tool tips provides a sensitive and accurate method of following cutting-tool wear.

In normal operation, tool wear proceeds more or less continuously. Microscopic examination, which necessitates interrupting the machining, cannot demonstrate this constant rate of wear. Consequently, it is necessary to machine until the cutting edge breaks down or until the wear has reached a given amount. A microscopic investigation, involving the data in Table 1 , would require at least nine determinations at three cutting speeds because of the poor accuracy of the method.

Merchant, using radioisotopes, has shown $(1,2)$ that a linear relationship exists between tool wear and length of machining time. Our investigations show that the transfer of irradiated tool material to chips and distribution of the material on the work piece can be accurately measured by Geiger-Müller and autoradiographic methods. However, counting-rate and health-precaution considerations demand the proper isotope selection and optimum specific activity.

\section{Irradiation of Tips}

Two carbide tips--qualities 1 and 2 in Table 1 -were irradiated in a neu-

- Division of Mechanical Technology.

† Division of Physical Chemistry.
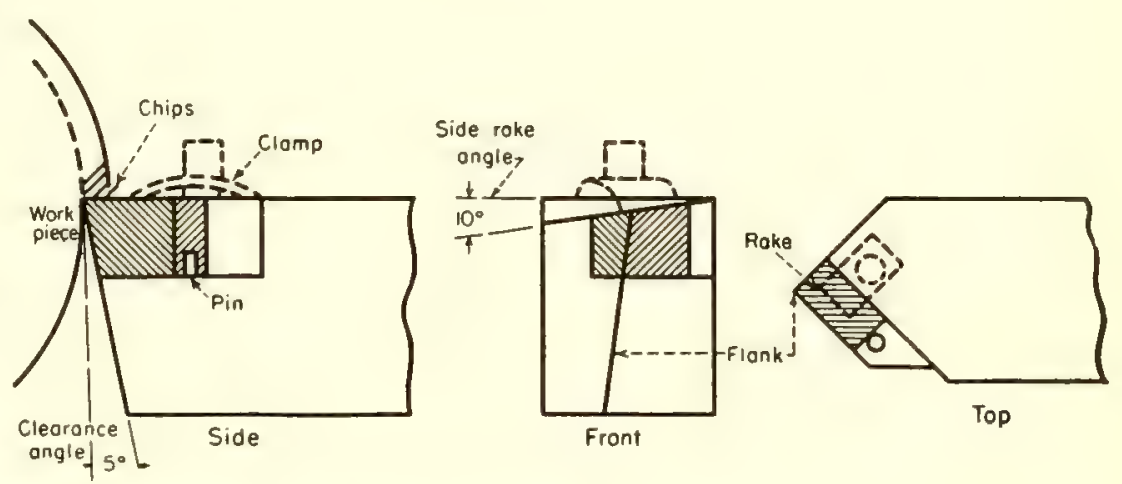

FIG. 1. Carbide-tip cutting tool characteristics

TABLE 1-Machining Specifications for Test Runs

Carbide tool composition

Quality no. 1

Quality no. 2

\begin{tabular}{cccc}
$\mathrm{Co}(\%)$ & $\mathrm{TiC}(\%)$ & $\mathrm{TaC}(\%)$ & $\mathrm{WC}(\%)$ \\
\hline 7.2 & 3.5 & 2.5 & 86.8 \\
9.3 & 4.7 & - & 86.0
\end{tabular}

Work-piece data

Billet dia. $200 \mathrm{~mm}$, Brinell hardness $220 \mathrm{~kg} / \mathrm{mm}^{2}$

\begin{tabular}{ccccc}
$\mathrm{C}(\%)$ & $\mathrm{Mn}(\%)$ & $\mathrm{Si}(\%)$ & $\mathrm{P}(\%)$ & $\mathrm{S}(\%)$ \\
\hline $0.58-0.60$ & $0.80-1.10$ & $0.20-0.24$ & $0.04(\max )$. & $0.04(\max )$.
\end{tabular}

Cutting data

Depth of cut $=2.0 \mathrm{~mm}$

Feed $\quad=0.5 \mathrm{~mm} / \mathrm{rev}$

Cutting speed $=100 \mathrm{~m} / \mathrm{min}$

Tool geometry

\begin{tabular}{ll}
\hline Nose radius & $=1.0 \mathrm{~mm}$ \\
Clearance angle & $=5^{\circ}$ \\
Side rake & $=10^{\circ}$ \\
Side cutting edge angle & $=45^{\circ}$
\end{tabular}

Side cutting edge angle $=45^{\circ}$

Nose angle $\quad=90^{\circ}$

Machine: Skoda SUR Lathe $260 \times 2,000 \mathrm{~mm}$ 


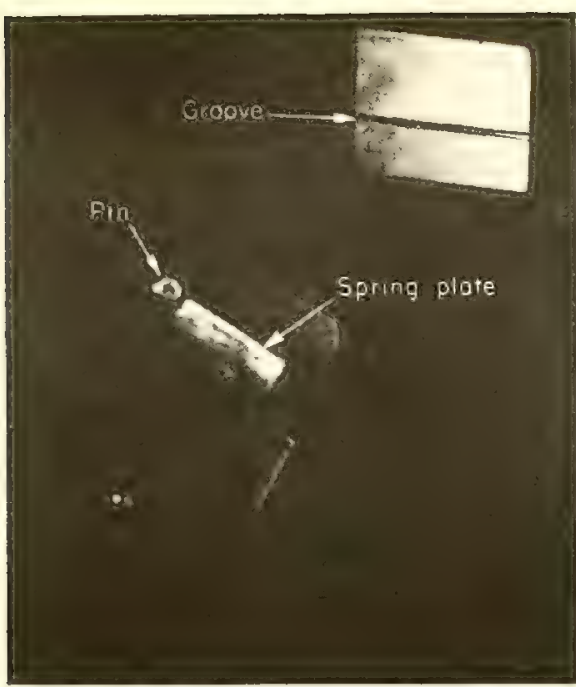

FIG. 2. Carbide tip (left) and tool shaft

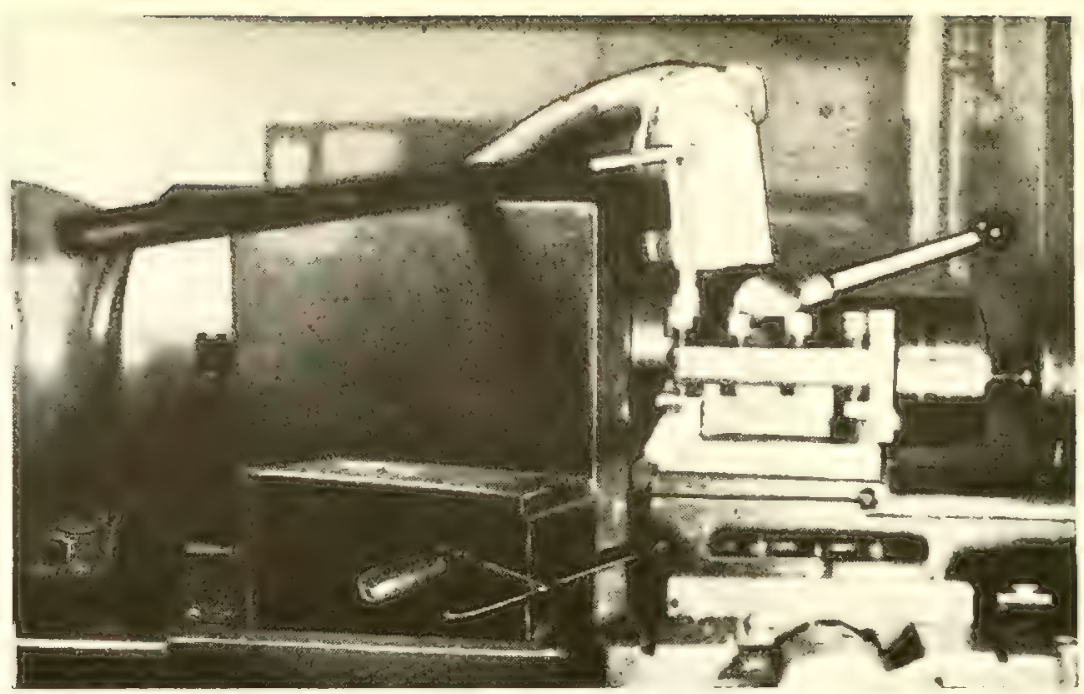

FIG. 3. Lathe with radiation shield and dust extractor in front tron flux of $10^{12} \mathrm{n} / \mathrm{cm}^{2} / \mathrm{sec}$ for $1 \mathrm{hr}$. The isotopes $\mathrm{Co}^{60}, \mathrm{Ti}^{51}(3), \mathrm{W}^{185}, \mathrm{~W}^{187}$ and $\mathrm{Ta}^{182}$ were formed.

Choice of isotopes. The following points have to be considered:

1. The isotope must be formed in sufficient amount during irradiation.

2. The isotope must emit radiation suitable for detection.

3. The isotope must have a half-life long enough to allow for transportation from the pile and machining tests.

4. The half-life should, on the other hand, be short enough to minimize the need for handling precautions.

5. If possible, the isotope should be a pure beta emitter to avoid the health hazards involved in gamma emission.

Only the tungsten isotopes are formed in sufficient amount during a short irradiation (a few days) to require consideration. The nuclear data are given in Table 2 (4).

Both isotopes fulfill requirements 1-3. Requirement 5 is better satisfied by $W^{185}$. However, 4 greatly favors $\mathrm{W}^{187}$ because its 24.1 -hr half-life necessitates precautions against health hazards for only one week or so. Furthermore, the shorter half-life and higher neutron-capture cross section of $W^{187}$ require a shorter irradiation time. Thus, $W^{187}$ is preferred for the present work.
Tungsten-187 is formed with a target-element cross section of 10.2 barns. Thus, only very short irradiations in a high flux of thermal neutrons are necessary for production of high activities.

Mechanical effects of irradiation. Prolonged bombardment in a high flux of fast neutrons may be supposed to cause certain changes in the crystal structure (6) and thus in the mechanical properties of carbide tips. However, if the irradiation takes place in the thermal shield of the pile, where the flux of fast neutrons is low, the risk of structural changes may be supposed to besmall. In this case, mechanical properties are considered unchanged ( 8 ).

\section{Desired Specific Activity}

Preliminary experiments had shown that transfer of at least $5 \mathrm{mg}$ of tip material could be expected from the rake (see Fig. 1) during a period of $20 \mathrm{~min}$. This corresponds to a total of 2,000 meters of chips.

The G-M measurements were planned to be made with a $10 \%$ geometry (as calibrated with $\mathrm{UX}_{2}$ ) and with a mosaic of small pieces of chip with a total length of $100 \mathrm{~mm}$. Thus, the specific activity of the carbide tips necessary for a counting rate of 1,000 $\mathrm{cpm}$ is $20 \mathrm{mc} / \mathrm{gm}$.

Now each tip weighed about 35

TABLE 2-Tungsten Isotope Dato

\begin{tabular}{|c|c|c|c|}
\hline Isotope & Half-life & $\beta$-energy (Mev) & $\gamma$-energy $(M e v)$ \\
\hline W-185 & $76 \mathrm{~d}$ & $0.68, \quad 0.48$ & $-(6)$ \\
\hline$W-187$ & $24.1 \mathrm{~h}$ & $1.33(30 \%), 0.63(70 \%)$ & 0.696 , etc. \\
\hline
\end{tabular}

$\mathrm{gm}$; thus, a total of 1.4 curies was necessary for two tips. This necessitates rather rigorous precautions as to health hazards, so an activity of only $10-\mathrm{mc} / \mathrm{gm}$ tip material was chosen, giving a counting rate of $500 \mathrm{cpm}$.

\section{Machining Procedure}

To reduce irradiation costs, the tips were irradiated before they were fastened to the shafts. Complications would be expected if the activated tips were attached to the shaft with the usual soldering and polishing method. Therefore a clamping method was used.

Figure 2 shows one of the cemented carbide tips ground to shape and ready for mounting on the tool shaft. The edge of the spring plate is pressed against the groove cut across the top surface of the tip. The pin holds the tip in position during machining.

Turning is carried out in the normal manner except that the tips are handled by remote control. Operators are protected by a shield and a fan to remove radioactive dust (Fig. 3 ). A filter in the fan, checked after the work is finished, exhibits no contamination.

The feed is engaged for $30 \mathrm{sec}$. The tool is then unfastened and mounted in position for photographing the top surface of the tip. The last chips to be formed during each 30 -sec operation are collected and measured with the G-M counter. The tip of quality no. 1 -was used for a total of 35 runs of $30 \mathrm{sec}$ each, and no. 2 , for 43 runs.

\section{Preparation of Chips}

The turning chips, almost flat to start with, become more and more 
curved towards the end of the cuttingtool life.

The flat chips are cut into pieces of 2-cm length. Five pieces are placed side by side to form a square on a Perspex disk. This is centered under an end-window $\mathrm{G}-\mathrm{M}$ tube

The curved chips are broken into small pieces of a few millimeters length. These pieces are built up into a mosaic of approximately the same area as the groups of flat chips.

The exact width, total length, and thickness of each group of chips is measured. The activity of one of the groups was followed for a few days, giving a half-life of $24 \mathrm{hr}$.

\section{Activity Measurement}

To calculate the absolute amount of material transferred to the chips, one of two methods can be used:

1. The specific activity of the carbide tips can be calculated from irradiation and nuclear data, if the counter efficiency is known.

2. A weighed piece of the same material as the tip, used as a reference source, is irradiated together with the tip. The piece is dissolved in a suitable chemical agent. A known part of the solution is evaporated, and the activity is measured under identical geometrical arrangements as for the measurements of the chips. Dividing all activity figures for the turnings with this figure gives directly the corresponding amount of radioactive material.

In the present work, the second method is used. The first method gives a check of the results.

Reference sources. As the activity of the small reference pieces is too high to be measured without dilution, these are dissolved in molten sodium nitrite, the tungstate dissolved in water, and $1 / 1,000$ of the solution evaporated on an iron disk with the same area and bottom thickness as the turnings, thus making backscattering corrections unnecessary.

The activity of the reference sources is measured under the same geometrical arrangements as for the chips. These operations are performed two days after the other work. Because of this, the reference-source activities are followed for a long period to permit an exact extrapolation.

The curves show that a small amount of a long-lived activity was present in the sources. After complete decay of the $W^{187}$, this remaining activity is determined and subtracted from the total source activity.

A linear semi-logarithmic relation between activity and time results. The half-life values for $W^{187}$ calculated from these curves are 23.9 and $24.2 \mathrm{hr}$, respectively. These are very close to the accepted figure of $24.1 \mathrm{hr}$ (4).

\section{Calculations and Results}

For each group of turnings, a total of about 3,000 counts was made, giving a statistical error of $2 \%$. All radioactivity figures are corrected for the deadtime of the counting equipment, for the background, and for the halflife of $W^{187}(24.1 \mathrm{hr})$.

A separate experiment was made to find the relation between area and counting rate (to correct for the differences in area of the chips). This experiment showed that, within the range of areas used $\left(230-350 \mathrm{~mm}^{2}\right)$, this relation is linear. No corrections for the backscattering of the beta particles are needed since the thickness of the chips gave saturation backscattering [backscattering factor $=1.45(3)$ ].

The beginning of each run is fixed as "zero time." All radioactivity figures are corrected back to that time.

The corrected counting-rate figures for the chips are divided by the counting rate of the corresponding reference source, giving the weight of the carbidetip material transferred during a certain period. The length of this period can be calculated from the cutting data (including the cutting ratio, i.e., the ratio chip thickness before:after removal), and thus the material transferred in $\mathrm{gm} / \mathrm{min}$ can be determined.

Figure 4 shows that the material transferred per minute from the tip of quality no. 2 is almost constant. Quality no. 1 shows a large transfer during an initial period of $3 \mathrm{~min}$, followed by an almost constant wear.

The total amount transferred, found by integrating the curves of Fig. 4, is given in Fig. 5. As may be expected, the curve for quality no. 1 is a straight line; for quality no. 2 , the relation is linear after the initial period.

All the above figures of counting rate correspond to material transferred from the rake side of the carbide tip. Only for one group of turnings was the transfer from the flank also measured. For this group of chips, the rake-toflank ratio is 3.5 .

The chips corresponding to $14.5 \mathrm{~min}$ turning with quality no. 2 gave an extraordinarily high counting rate (9,000 cpm, the ordinary being 400 $\mathrm{cpm})$. This was due to one piece. A small piece of the carbide tip had broken off and cut a groove in the chip,

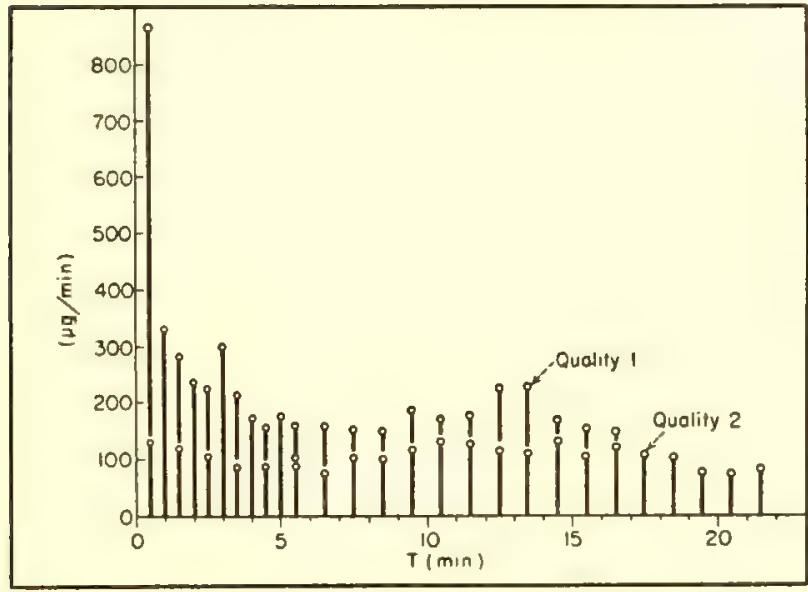

FIG. 4. Rate of material transfer from rake side to turnings

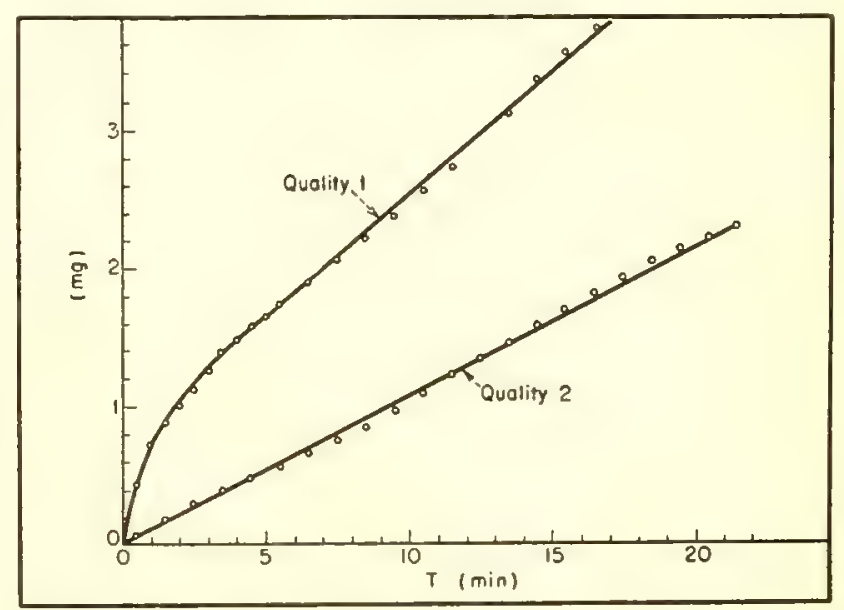

FIG. 5. Tolal wear of rake side as function of time 

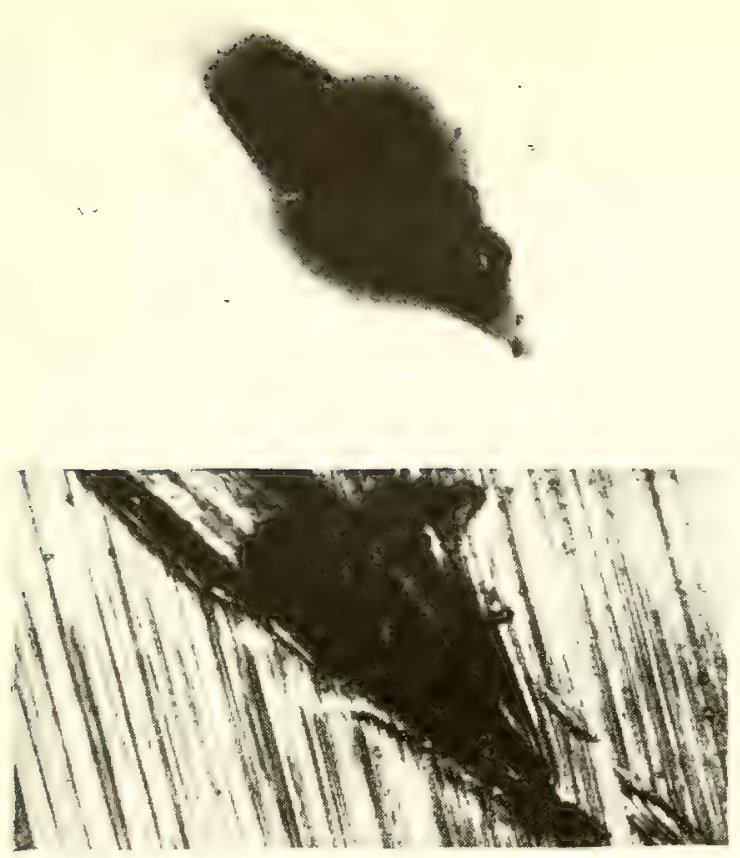

FIG. 6. Chip having abnormally high activity because of broken off piece of carbide tip. (Top) Autoradiograph (7). (Bottom) Micrograph

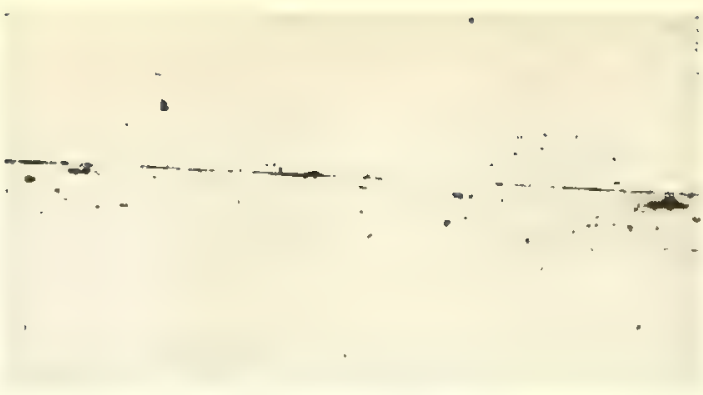

FIG. 7. Autoradiograph of work piece after turning (film: llford C)

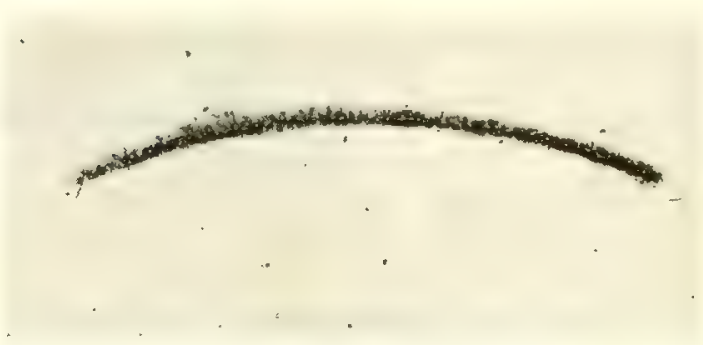

FIG. 8. Autoradiograph of chip formed after 30-sec turning (film: Ilfex) where it finally stuck. The size of this piece, calculated from the activity, was $15 \mu \mathrm{g}$. An autoradiograph [Kodak autoradiographic plate (7)] and a micrograph are shown in Fig. 6.

Counting efficiency. From irradiation and nuclear data, the specific activity of the tips immediately after the irradiation is $25 \mathrm{mc} / \mathrm{gm}$ tungsten (corresponding to $10 \mathrm{mc} / \mathrm{gm}$ during the experiments). Comparison of this figure with the activity of the reference sources, corrected for the difference in time, gives a total efficiency for the counting equipment of $12 \%$, which within the limits of error is equal to the $10.5 \%$ found when using a $U_{2}$ standard source. Of course, since the neutron flux is not known exactly, this check is only approximate. The error in the flux is probably $<20 \%$ (3).

Autoradiographs. Because of the relatively low activity, autoradiographs of the work piece and turnings were not distinct. A work-piece autoradiograph is shown in Fig. 7, and another (one of the turnings), in Fig. 8.

From Fig. 7, it is seen that the material transfer to the work piece is distributed partly evenly (the faint lines) and partly discontinuously (the black spots). This region of spots and marked lines corresponds to start or finish of a run. This is verified by the fact that the axial distance between two such regions (one of which is shown) is equal to the length of the transfer of the tool during a 30-sec run.

The autoradiograph of the turning, Fig. 8 , is very faint but shows no marked discontinuities.

\section{Discussion}

This investigation confirms that, with these materials and cutting data there exists a linear relationship between the rake transfer and the turning time. If this relationship is obtained with other materials and data, it will be possible to make a comparison between different qualities of carbide tips without machining until the cutting edge of the tip breaks down.

A material-transfer-versus-time curve could be constructed after only a few runs of 30 sec each. The slope of this graph gives an idea of the cuttingtool life. However, if a group of curves of the second order, which do not intersect, is obtained, a comparison can be made by measuring the slopes.

According to Merchant, the accuracy of the radioactive method is better than that of conventional methods, and, moreover, the cost of making a test is relatively low. Merchant has also shown that, when using cutting fluid, more than $95 \%$ of the activity is attached to the turnings.

Merchant made the radioactivity measurements on all turnings formed during a very short period by means of a gamma-sensitive G-M tube, thus giving the sum of the activities on both sides of the turnings. The present method, however, gives the amount of material transferred to one side only, thus permitting separate investigation of each side.

The authors wish to express their thanks to Professors $R$. Woxen, head of the Div. of Mechanical Technology, O. Lamm, head of the Div. of Physical Chemistry, and O. Andersson, acting Professor at the Div. of Mechanical Technology for their kind interest in this work and for placing laboratory facilities at our disposal, and Messrs. T. Westermark and $G$. Aniansson for preliminary calculations and valuable discussions. Thanks are also due to Mr. C. Lindberg for skillful technical assistance.

The nucleonic equipment used in this work was placed at our disposal by the Swedish Atomic Committee.

The irradiations were made by $A E R E$ Harwell, England, and the valuable help of $M r . J . L$. Putman is also gratefully acknowledged.

Health precautions were supervised by Dr. S. Benner of the Radiophysical Department, Karolinska Institutet, Stockholm.

Further research on this method will be carried out at the Div. of Mechanical Technology, this being made possible by the State Council of Technical Research who have kindly placed funds at our disposal for this investigation.

\section{BIBLIOGRAPHY}

1. M. E. Merchant, E. J. Krabacher, J. Applied Phys, 22, 1,507 (1951)

2. M. E. Merchant, H. Ernst, E. J. Krabacher Paper presented for ASME, June, 1952, Cincinasti, Ohio

3. J. L. Putman, personal communication

4. AERE Catalogue No. 2 (Harwell, Eng., 1950)

4. Amendment to AERE Catalorue No. 2 (1952)

5. Amendment to AERE Catalogue IJo

7. L.-G. Erwall, M. Hillert, Research, 4, 242 (1951) 
Using Neutrons for Remote

Liquid-Level

Gaging

First tests of liquid-level measurement using a fast-neutron source and a new slowneutron detector show that method works well for hydrogenous and other light liquids. Less shielding is needed than with a gamma gage; advantage of completely external measuring is retained. Accuracy of new method is $\pm 2 \mathrm{~mm}$

\author{
By S. BARNARTT and K. H. SUN \\ Westinghouse Research Laboratories \\ East Pittsburgh, Pennsylvania
}

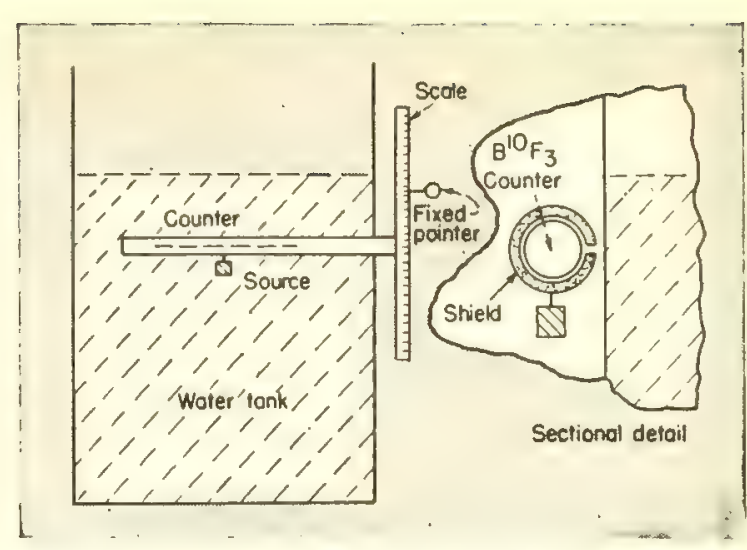

FIG. 1. Experimental set-up for determining liquid-level by neutron radiation uses rectangular brass tank, $30-\mathrm{cm}$ square by $50-\mathrm{cm}$ high, and movable counter with neutron source attached. Inset shows shield for $\mathrm{B}^{10} \mathrm{~F}_{3}$ counter, consisting of two concentric $\mathrm{Cd}$ tubes, each $0.4-\mathrm{mm}$ thick, flled with $2.5 \mathrm{-mm}$ of boron carbide

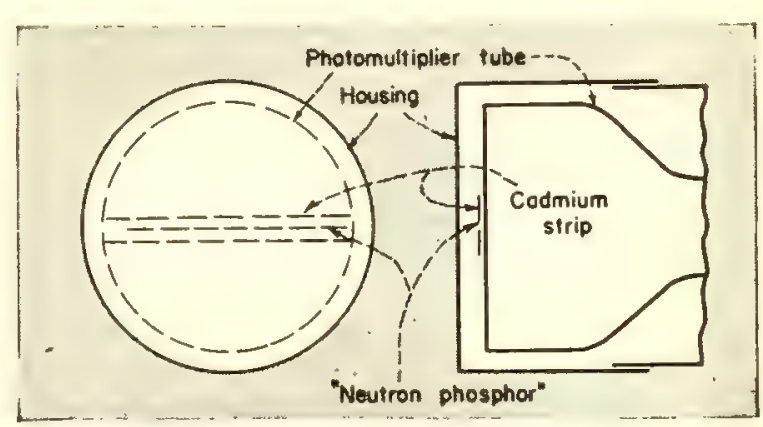

FIG. 2. Slow-neutron detector is made of "neutronphosphor" strip placed against face of DuMont type $K$ 1198 photomultiplier tube. Neutron phosphor is made by embedding silver-activated zine sulfle particles in glycerylborate polyester. Strip is $11-\mathrm{cm}$ long by $0.15-\mathrm{cm}$ wide by $0.12 \mathrm{~cm}$ thick. Codmium sheet $1.2-\mathrm{cm}$ wide and $0.12-\mathrm{cm}$ thick surrounds phosphor. Entire assembly is encased in light-tight aluminum housing
The feasibility of Using neutron radiation in a remote liquid-level gage is the subject of this article. The equipment used for the study and results for different geometries are explained. Measuring liquid level in a container by nuclear radiation depends on the fact that radiation is transmitted with less loss through gas than through liquid, or on the fact that backscattering occurs to a greater degree in liquid. Several level gages based on differential transmission of gamma rays have been described $(1-8)$. Other gages utilize back-scattering of $\gamma$-rays $(1,9,10)$. However, the paucity of actual data published prevents comparing the precision of the various $\gamma$-ray devices.

Using neutron radiation for measuring liquid level has received much less attention than using $\gamma$-radiation, although neutron gaging appears to be more attractive because less and consequently lighter shielding is required. Neutron gaging is especially attractive for liquids containing hydrogen or other low-atomic-weight elements, since collisions with these elements quickly slow down neutrons, and slow neutrons can be detected much more efficiently than fast ones. Thus a convenient remote liquid-level gage can be made by placing a fast-neutron source near a slow-neutron counter, and moving the assembly vertically just outside the liquid container. The fast neutrons that enter the liquid are slowed down, and some of them are scattered back and detected by the counter. As the detector is moved from just above the liquid level to just below it, a sudden increase in count is obtained. This method of determining level was suggested in 1945 by Hare (11), although no data have appeared to indicate what positioning of source and counter is most effective or how accurately the level may be measured. These factors were investigated in the following experiments. The results indicate that the neutron-radiation method may be suitable for practical applications.

\section{How Experiment Was Done}

Essential parts of the apparatus used in this study are shown schematically in Fig. 1. The liquid (tap water) was in a rectangular brass tank with 0.16 $\mathrm{cm}$ walls. The source was in a nickel tube $2.5-\mathrm{cm}$ long $\times 0.6-\mathrm{cm}$ diameter. It produced $8 \times 10^{5}$ fast neutrons/ sec from $400 \mathrm{mc}$ of polonium with 


\section{Counting-rate curves show effect of relative}

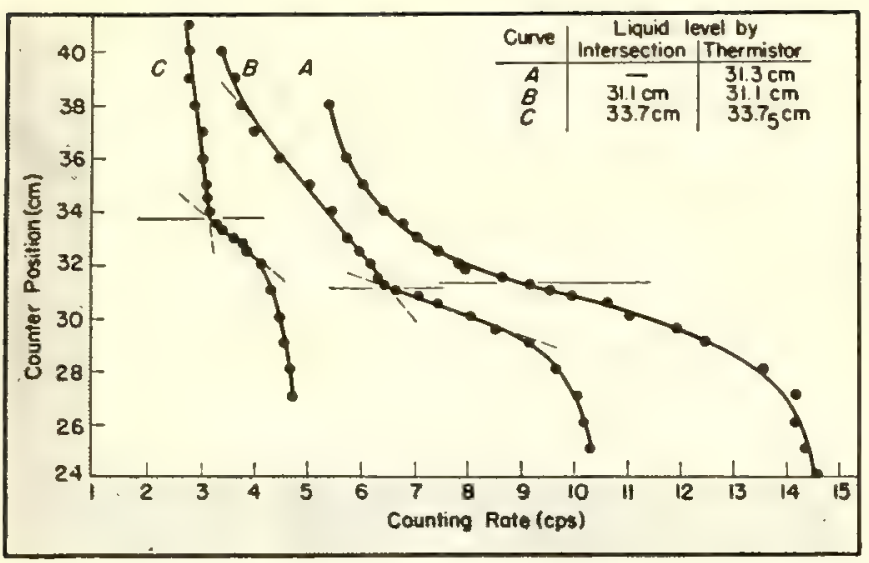

FIG. 3. Source moves with detector, at fixed distance below detector. Distances below are as follows: for curve $\mathbf{A - 2 . 0} \mathrm{cm}$; for curve B. $6.2 \mathrm{~cm}$; for curve C $-10.8 \mathrm{~cm}$

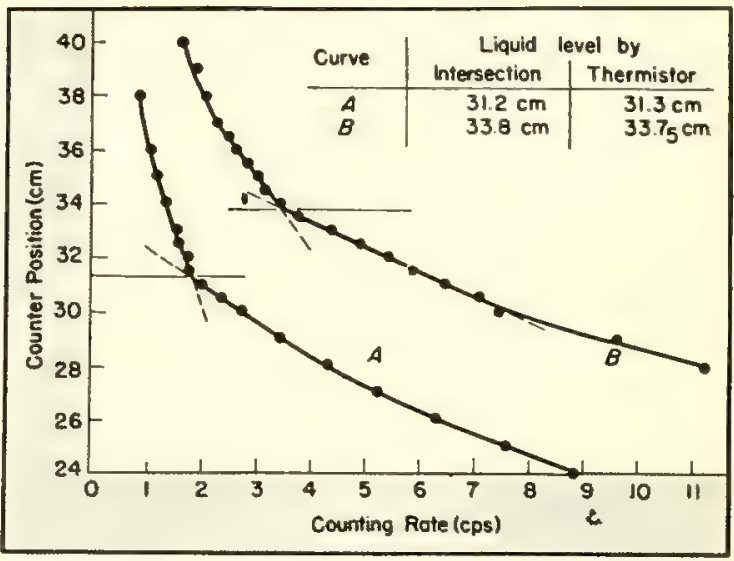

FIG. 4. Source is stationary $15-\mathrm{cm}$ below liquid level as detector moves. Higher counting rates for $B$ are caused by block of paraffin wax $6-\mathrm{cm}$ thick covering source beryllium. Safe distance from this source is $56 \mathrm{~cm}$ for continuous exposure, based on a maximum dose of 20 fast neutrons $/ \mathrm{cm}^{2} / \mathrm{sec}$. :

The counter could be moved vertically just outside the tank wall, carrying a centimeter scale. As the scale traveled past a fixed pointer, counter position was indicated to $\pm 0.2 \mathrm{~mm}$.

Two types of neutron counters used. One type of neutron counter used was a boron trifluoride counter $2.5 \mathrm{~cm}$ in diameter containing $\mathrm{B}^{10} \mathrm{~F}_{3}$ gas, at 40 $\mathrm{cm} \mathrm{Hg}$. The effective counting surface was a horizontal slot 15 -cm long $X$ 0.2-cm wide. As indicated in Fig. 1, the remaining area of the counter tube was covered with a shield that absorbed thermal neutrons and provided some collimation of the neutrons diffusing through the tank wall to the counter. Electrical pulses from the counter were fed into and recorded by a Tracerlab Ampliscaler. This counter was used in all experiments except where otherwise noted.

The other counter used was the scintillation counter depicted in Fig. 2. The detecting element was a "neutron phosphor" made by embedding silveractivated zinc sulfide particles in a glyceryl-borate polyester containing naturally occurring boron (12). This counter will be described in detail in a future issue of Nucleonics. The neutron phosphor was placed horizontally against the face of a photomultiplier tube. Photomultiplier tube output was fed into a cathode follower, linear amplifier, discriminator and scaler. Although the active area of the scintillation counter was about half that of the boron-trifluoride counter, the scintillation counter was more efficient-it yielded more than double the count of the boron-trifluoride counter under comparable conditions.

Liquid-level measurements. An independent liquid-level measurement was obtained in each experiment by a thermistor $1 \mathrm{~mm}$ in diameter. This was fixed to the counter, so that the thermistor moved vertically inside the tank wall as counter position was changed. Constant voltage was applied across the thermistor. Thermistor current was relatively high in air and much lower when the thermistor was cooled by the water. Thus, as the thermistor was lowered towards the water, the position where the current suddenly decreased corresponded to the liquid level. The probable error in this measurement was estimated to be $< \pm 1 \mathrm{~mm}$.

\section{Counting-Rate Curves}

Curves of neutron counting rate vs counter position for various arrangements of source and counter are shown in Figs, 3-6. Counter position is plotted as the ordinate. The scale refers to an arbitrary zero that is very close to the bottom of the water tank. Liquid level, as determined by the thermistor, is shown as a horizontal line (the points below the line correspond to readings opposite the liquid).
There is a definite discontinuity in the curve at the water level for most of the geometries studied. In the more favorable cases, where the curve is linear on either side of the sharp break, intersection of the two lines can be fixed to $\pm 1 \mathrm{~mm}$ or closer. In all these cases the liquid level as indicated by the point of intersection on the curve agreed with the thermistor determination to $\pm 1.5 \mathrm{~mm}$ or better. This agreement is within experimental error.

Source below counter. Figure 3 shows data obtained when the source is moved with the counter a fixed distance below it. With the source very close to the counter $(2.0-\mathrm{cm}$ away, curve $A$ ), the counting rate increases rapidly as the counter approaches the liquid level from above, resulting in a smooth sigmoidal curve. This type of curve is an undesirable one, although the point of inflection appears to be close to the liquid level. The sharp rise in count as the water level is closely approached from above results from the fact that, when the source is just opposite the water level and is moving downward, the number of neutrons being confined by the water is increasing rapidly. When the source is placed somewhat farther below the counter (6.2-cm away, curve $B$ ), a discontinuity in the counting rate appears at the liquid level. With the source relatively far away $(10.8 \mathrm{~cm}$, curve $C)$, the counting rate increases very slowly as the counter approaches the liquid from above; therefore as the water level is 


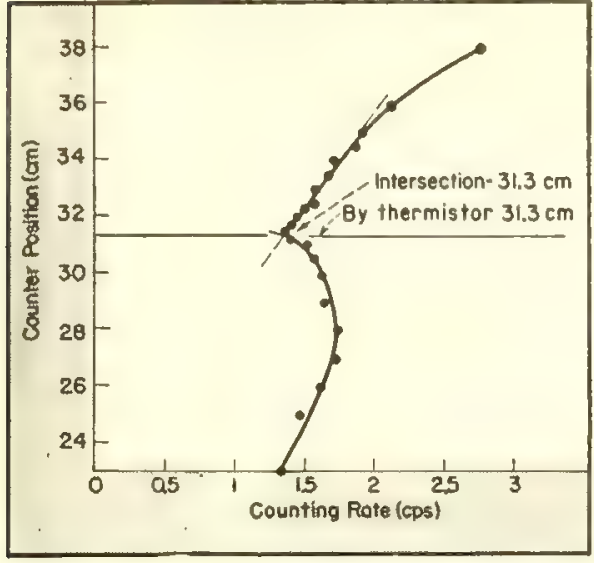

FIG. 5. Source is stationary at $15-\mathrm{cm}$ above liquid level. Low rise in counting rate as detector moves past liquid level is undesirable

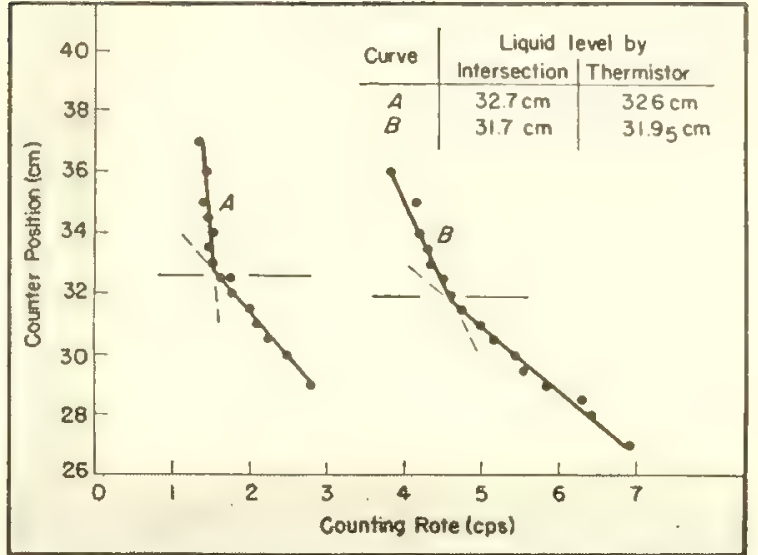

FIG. 6. For $A$, source was inside the liquid, stationary at $15-\mathrm{cm}$ below the level. For $B$, source moved with detector, $8.3-\mathrm{cm}$ above it. Thicker-walled tank was used passed, the rise in count, although not as great as before, is quite sharp.

Behavior similar to that shown on curve $C$ is expected if the source were to be fixed outside the tank relatively far below the water level, with the region around the level being scanned by the counter. This is confirmed by Fig. 4, curve $A$, for which the fastneutron source was fixed to the outside of the tank $15 \mathrm{~cm}$ below the level. Curve $B$ in this figure shows the results when a block of paraffin wax, shaped to fit symmetrically over the source, was placed against the tank, covering the source with approximately $6 \mathrm{~cm}$ of wax. The paraffin produced essentially a slow-neutron source of greater intensity, but otherwise the curve obtained was similar to that with the fast-neutron source. Principle advantage of converting a fast-neutron source to a slow one appears to be that less radioactive material is needed.

Since the slow neutrons in the liquid inside the tank largely determine the counting-rate curve shape, an equally sharp break at the liquid level is expected on placing the stationary source inside the liquid rather than outside of it. This was confirmed by experiment.

Source above counter. The previous results demonstrate that when the source is positioned below the counter, it should not be placed too close to it. Experiments with the source above the counter indicate that the source should not be too far away in this case. With the center of the source as close as 2.0 cm above the counting element, the counting curves showed sharp discontinuities at the liquid level and resembled those in Fig. 4. Figure 5 presents the case where the source is stationary and relatively far $(15 \mathrm{~cm})$ away from the liquid level-note the minimum corresponding to the liquid level. The important point demonstrated here is that the rise in counting rate as the counter passes down under the level is relatively small. This undesirable feature would be expected to occur also when the source is moved with the counter but fixed at a relatively large distance above it.

The previous results were obtained with a brass container having a wall thickness of only $0.16 \mathrm{~cm}(1 / 16 \mathrm{in}$ ). It was expected that a thick container wall would tend to make the discontinuity in neutron count at the liquid level less sharp. There are two reasons for this: the detector does not pass as closely to the liquid-gas interface; and the neutrons are scattered within the container wall. Figure 6 presents data obtained when the tank-wall thickness was increased by placing a steel sheet $1.27-\mathrm{cm}(1 / 2-$ in. $)$ thick against the brass wall. The scintillation counter was used in these experiments. Curve $A$ was obtained with the source inside the liquid, $15 \mathrm{~cm}$ below the level and $15 \mathrm{~cm}$ inside the tank wall. The break in the curve is sharp and indicates the level accurately. With the source $8.3 \mathrm{~cm}$ above the counter, curve $B$ in this figure, the break in the curve is not as well defined. These examples illustrate the need for determining the best relative location of the source for a given thick-walled container. For thin-walled containers there is a rather wide choice of source locations.

\section{Conclusions}

The level of a hydrogenous liquid in a tank can be measured remotely to $\pm 2 \mathrm{~mm}$ or better from outside the container by means of a fast-neutron source attached to a slow-neutron counter. The source may be positioned below but not too close to the counter, or above the counter but not too far away from it. Equal precision is attainable when the source is stationary and either opposite the liquid outside the tank or inside the liquid.

We are grateful to $P . R$. Malmberg for helpful advice, and to $M$. Giuliano and $\boldsymbol{P}$. Szydlik for assistance with some of the measurements.

\section{BIBLIOGRAPHY}

1. D. G. C. Hare, U. S. Patent 2,323,128 (June 29,1943 )

2. L. S. McCaslin, Oil Gas $J$. 46, No. 21, 100 (1947)

3. A. Wolf, U. S. Patent 2,456,233 (1948)

4. D. M. McCuteheon, Am. Foundryman 15, No. 6,35 (1949)

5. F, S. McCarthy, G. A. Rice, J. Electrochem. Soc, 97, $249(1950)$

6. J. E. Johnston, Brit. Cast Iron Research Assoc. J. Research and Develop. 3, 523 (1950)

7. R. Meakin, J. Sci. Instr. 28, 372 (1951)

8. J. E. Jacobs, R. F. Wilson, Electronics 24, No, $10,172(1951)$

9. D. G. C. Hare, U. S. Patent 2,348,810 (May $16,1944)$

10. J. H. Kunkel, Petroleum Engr. 16, No. 12, 155 (1945)

11. D. G. C. Hare, U. S. Patent 2,378,219 (June $12,1945)$

12. K. H. Sun, et, al., Phys. Rev, 95, 600 (1954) 


\section{Using Tracers}

in Refinery

Control

Flow rate, volume, flow pattern, efficiency of separation and mixing, and leakage-all can be determined easily with tracers where other methods fail

\author{
By D. E. HULL \\ California Research Corporation \\ Richmond, California
}

Significant IMPROVEMENTs in product yield and quality, and safer operations result from using radiotracers in refinery control. The radiotracer applications described here are only a fraction of those studied.

Radioisotopes are valuable in petroleum refining in two different manners: as fixed radiation sources, and as tracers. Level gages are the most common use of radioactive sources in the refinery. Because $\gamma$-rays can penetrate several feet of petroleum stock, plus the container walls, both source and detector can be installed outside the container. Many types of radioactive level gages $(1-3)$ are available.

A newer use for radiation sources is in devices for analyzing hydrocarbons for their $\mathrm{C} / \mathrm{H}$ ratio (4) or for their $\mathrm{S}$ content (5). These are based on the different radiation-absorption characteristics of different elements.

In contrast to sources, radiotracers have not been widely exploited as implements for refinery control.

\section{Tracers Used}

Most frequently the material to be traced in refining is a liquid stream. The tracer radioisotope should be preferably a $\gamma$-emitter; its half-life must be comparable with test duration; and it must be physically and chemically compatible with what it traces. Tracers most commonly used are triphenylstibine containing $\mathrm{Sb}^{124}$ and $\mathrm{Co}^{60}$ naphthenate for oil streams, and $\mathrm{Cs}^{134}$-salts for water streams. For solid-material tracing, $\mathrm{Zr}^{95}$ is used. Its refractory properties mean that cracking catalyst can be traced. Gas tracers would be very useful in refinery tests, but no completely satisfactory isotope is available.

\section{1-Flow Rate by Integral Count}
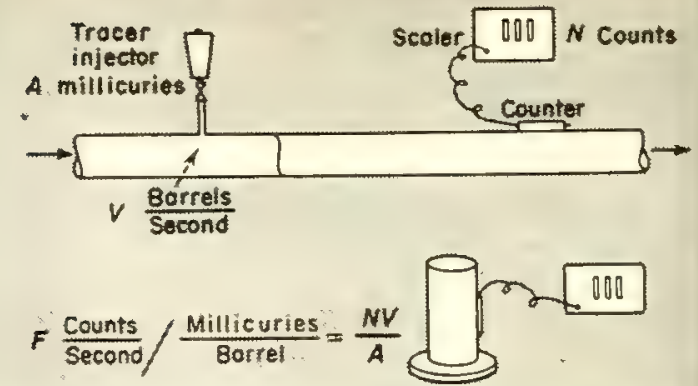

\section{Measuring Flow Rates}

In the continuous processing that is the backbone of present-day refining methods, flow-rate knowledge and control are essential.

Two-point method. The general method (Fig. 2) for measuring liquid or gas flow rates is timing a surge of tracer between two points separated by a determinable volume (6). This condition is most easily satisfied on a straight section of pipe of known dimensions, free from branch connections. The radiotracer is injected quickly, at a point close to the section where it will be timed, so that sharply defined peaks in counting rate are obtained as the tracer passes the counters. The counters at the two points are connected together into a single amplifier so that they both record on the same chart. The volume between the two points, divided by the time between peaks, gives the flow rate directly.

Integral-count method. A new tracer method (Fig. 1) for flow uses but one detector and eliminates the need to know the volume between two points ( 7$)$. It is based on the principle that the integral number of $\gamma$-rays registered by a G-M counter on a pipe passing a definite quantity of radioisotope is inversely proportional to flow velocity. This integrated count is independent of the variation in isotope concentration along the stream, as long as the flow rate is constant. This principle can be demonstrated mathematically; it was first experimentally validated in field pipeline tests. A given batch of tracer, observed at points so far apart that extensive spreading had occurred during transit from one to the other, was found to give the same total number of counts while passing each point.

To translate the integral count into an absolute determination of flow rate, it is necessary to calibrate the counting set-up on the particular type and size of pipe involved. This is done by filling a cut section of the pipe with a radiotracer solution at known concentration and measuring the counting rate on a counter tube attached to the pipe in the same way as in the field measurements. The dimensions of this calibration factor, $F$, are

$$
F=(\text { counts } / \mathrm{sec}) \div(\mathrm{mc} / \text { barrel })
$$

These can be transposed into

$$
F=(\text { counts } / \mathrm{mc}) \times(\text { barrels } / \mathrm{sec})
$$

With the calibration factor known, a measurement of the 


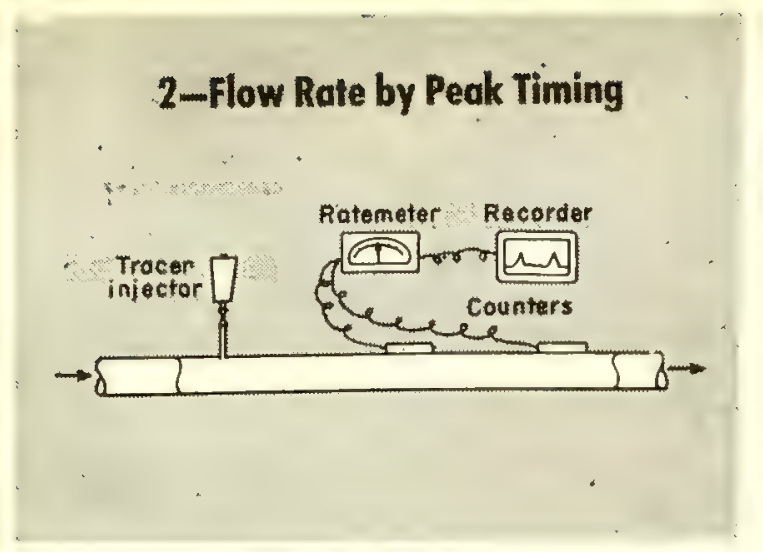

integral number of counts during passage of a known number of millicuries permits calculating the flow rate in barrels per second.

Solid flow rate. Timing a burst of counts is also used in measuring solid flow rates in circulating-catalyst systems as sketched in Fig. 3 (8). In the catalytic-cracking process, catalyst beads accumulate coke and are reactivated in a furnace. In this continuous cycle, circulation speed must be controlled. No satisfactory instrument has been available for gas-lift plants. Circulation speed now is measured

\section{3-Flow Rate in Catalytic Cracker}

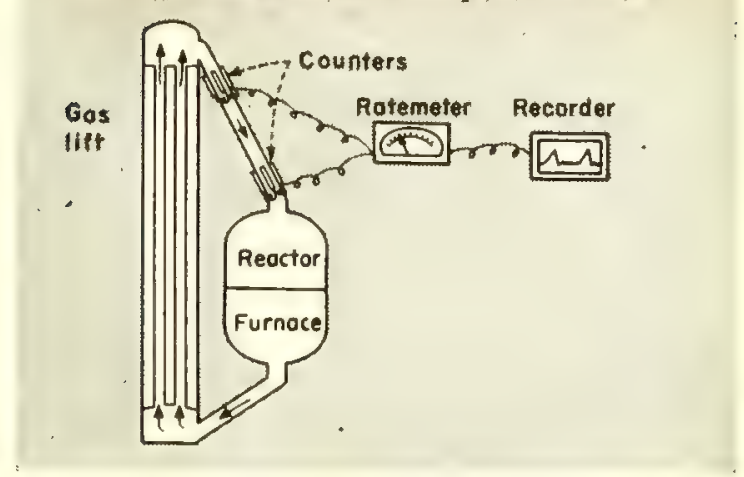

reliably in several such plants using tracer beads impregnated with $\mathrm{Zr}^{95}$. The seal leg is surrounded by two rings of $\mathrm{G}-\mathrm{M}$ counters. A radioactive bead passing these detectors produces two bursts of counts, about a minute apart. Dividing the calculated seal-leg capacity by the time interval gives catalyst circulation rate. This method does not give continuous readings because it depends on individual beads randomly spaced. However, two or three radioactive beads in a total of perhaps $20 \times 10^{9}$ give readings with satisfactory frequency.

\section{Measuring Volume}

Liquid volume in a closed system may be difficult to calculate from container dimensions, or, because of foaming or a turbulent surface, calculated volume may not correspond to liquid volume. A radiotracer can be used if there is a mixing action, either by external circulation or by internal stirring.

For example, $\mathrm{H}_{2} \mathrm{SO}_{4}$ volume is desired in an alkylation plant where extensive intermixing between acid and hydrocarbon prevents a well-defined level from forming ( $(\gamma)$. Volume is found by adding a known quantity of $\mathrm{Cs}^{134}$ in $\mathrm{H}_{2} \mathrm{SO}_{4}$. After the tracer is mixed in, a sample is drawn out and counted in comparison with a standard prepared by dilution. From tracer concentration and total quantity added, total volume of $\mathrm{H}_{2} \mathrm{SO}_{4}$ is found.

Exponential method. Another method is applicable to tanks through which there is a known, constant flow. (See Fig. 4.) A tracer batch is put into the incoming line. Mathematical treatment, assuming complete mixing of the incoming stream with the vessel contents, predicts that tracer concentration in the tank falls off exponentially with a rate determined by throughput, $R$, and volume, $V$, as follows

$$
-\frac{d \ln C}{d t}=\frac{R}{V}
$$

where $C$ is the counting rate.

The alkylation plant also has been checked by this method (7). Sample radioactivity at various times is measured and plotted on semi-log coordinates. Known throughput rate divided by slope of this plot gives acidphase volume.

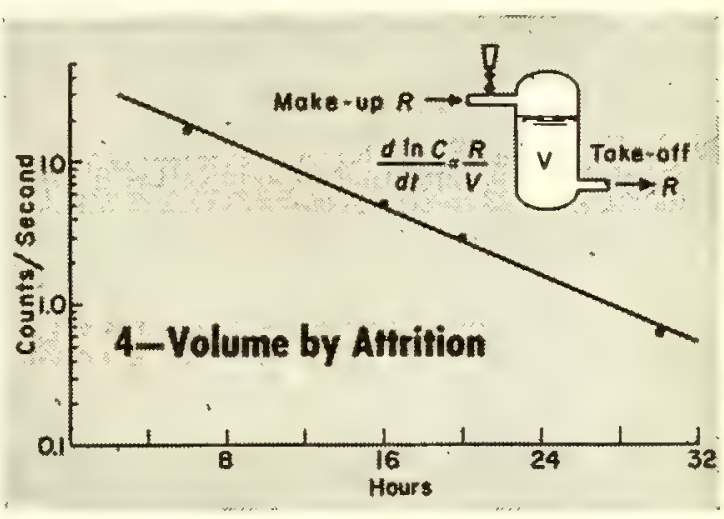

Circulating-loop volume. Volume of a circulating-loop system can be determined by still another method, in case mixing is slow compared to circulation rate. A concentrated slug of tracer, quickly injected, is observed in repeated cycles. Time between successive tracer-peak appearances, multiplied by circulation rate, gives the total volume of the circulating liquid. This is illustrated in the vacuum-distillation-column trace on Fig. 5. Part of the tracer was returned repeatedly through a recycle line. The initial peak was followed after an interval by an attenuated peak. After three or four circuits, the tracer peaks were spread out and merged into the background.

This principle also has been applied to determining total quantity of catalyst in the catalytic cracker. Average time required for a complete circuit through the system multiplied by the flow rate (determined from the same beads, as described above), gives total quantity. 


\section{5-Flow Pattern in Vacuum Distillation}

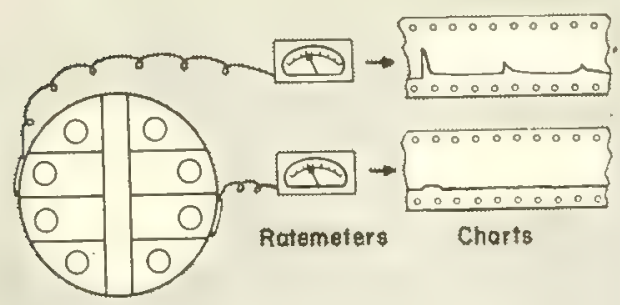

aubble tray

\section{Tracing Flow Patterns}

Petroleum-stock movement in refining operations frequently follows a complex pattern. Sometimes relative partition of a stream among two or more paths may be a matter of some technical or economic importance about which quantitative information is difficult to obtain. In this situation, radiotracers can be used advantageously to gather the information.

Flow distribution in a large vacuum-distillation column (9) was investigated as shown in Fig. 5. Each distillation tray is divided by baffles into eight channels. Overhead stream color, plus the presence of heavy metals, raised the suspicion that bubble caps in some trays were not flooded, permitting unwashed vapor to carry entrained liquid up the column. Such partial flooding could be caused by nonuniform liquid flow over the trays. Flow uniformity was tested during operation by injecting radioactive triphenylstibine into the column. After the injection the response of eight G-M counters was observed simultaneously. Each was attached outside the column opposite one of the eight channels on a single tray. Four of the counters, on one side of the column, showed a strong response as the tracer flowed down; the other four, on the opposite side, showed little or no response. The conclusion was that liquid was flowing over the trays on only one side, leaving the bubble caps on the other side open to pass unscrubbed vapor up the column.

Solid flow pattern. Tracer applications in catalyticcracking plants have yielded valuable operating data that could not be obtained in any other way. Catalyst-bead flow patterns have been determined with radioactive beads (7). Effectiveness of an intricate baffle system in promoting uniform flow through a large sloping pipe at the bottom of a furnace in one cracker (Fig. 3) was a disputed subject.

The controversy finally was settled by placing a pair of G-M counters, one at each end of the pipe, successively on the top, on the bottom, and on each side of the pipe and observing the speed and relative number of radioactive beads that followed these paths. The great difference in speed and number of transits between the different paths showed that the baffles did not serve their intended function.

In the same plant, relative load carried by the gas lift through three parallel pipes was measured by attaching the counters in turn to each of the pipes and observing the number of radioactive bead transits for several hours.

In a large regenerating furnace in a similar plant, tracer beads were introduced in different paths near the periphery. Differences in bead flow rate down the furnace sides were noted and used as a basis for adjusting bottom orifice plates to obtain more uniform flow.

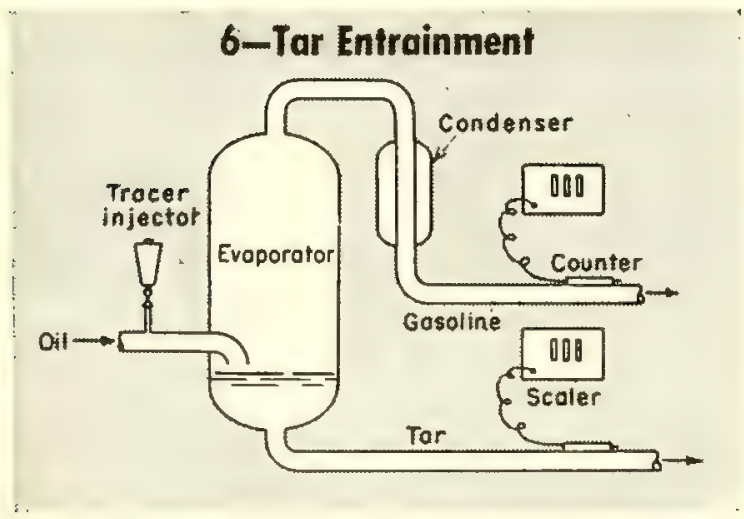

\section{Tracing Tar Entrainment}

In contrast to the wide and varied radiotracer applications to operations that involve stock movement or mixing (see the section on "Determining Mixing" on next page), only a few applications have been made to operations in which petroleum-stock components are separated. One study (Fig. 6) using this kind of tracer application was of tar entrainment in evaporator and distillation-column overhead streams. Tar cannot be tolerated in refined products; also, it must be excluded from furnace-tube feed to reduce coke formation and increase the plant operating time.

A plant-scale test was made of tar entrainment in an evaporator in a thermal-cracking plant (7). $\mathrm{Co}^{60}$-naphthenate, as a tar tracer, was injected in a short surge into the evaporator feed. A counter attached to the pipe carrying the still bottoms to the tar tanks recorded the activity of the bulk of the radiocobalt. Another counter attached to the overhead stream from the evaporator showed the cobalt activity in that stream. By integrating the counts on both streams and making corrections for the different flow rates, a quantitative measure was obtained of how much tar was going into the distillate. Values ranging from zero up to a few per cent, depending on operating conditions, were found. 


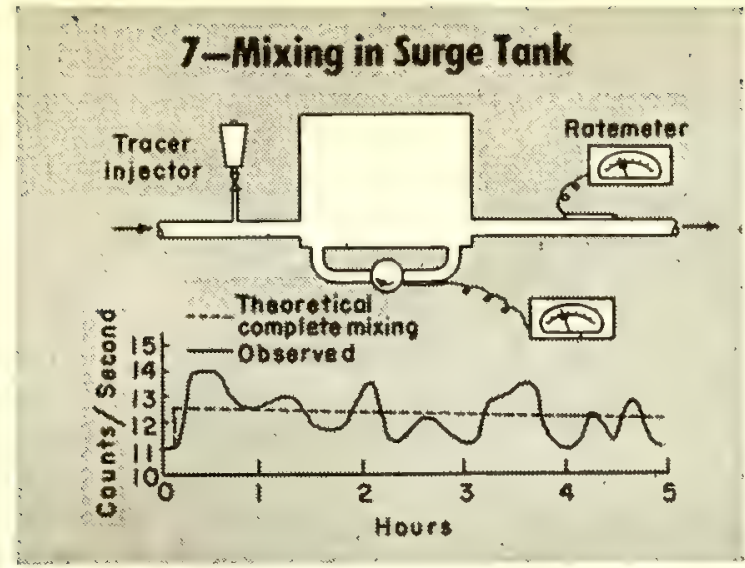

\section{Defermining Mixing}

Stock blending is a refining operation that has been followed widely with radiotracers. A radioisotope is incorporated in one component of a blend, and mix activity is observed by sampling or by placing a counter in the mixture. Large fluctuations diminish until constant activity is reached, showing that mixing is complete.

A study of mixing in a 50,000-barrel surge tank in a thermal-cracking-plant feed line illustrates the technique (7). Feed comes from two sources and varies in viscosity and other properties. Tank contents are mixed by pumping the liquid through a side loop at $10^{5}$ barrels/day. Surge-tank effectiveness in minimizing feed variations was studied (Fig. 7) by injecting Sb ${ }^{124}$-triphenyl into the intake line and watching the activity at the loop and the outgoing line. " The tracer was introduced into the tank in a volume of 50 barrels. During 5.5 hours, $13.1 \%$ of the labeled batch left the tank, compared with $17.0 \%$ expected. How-

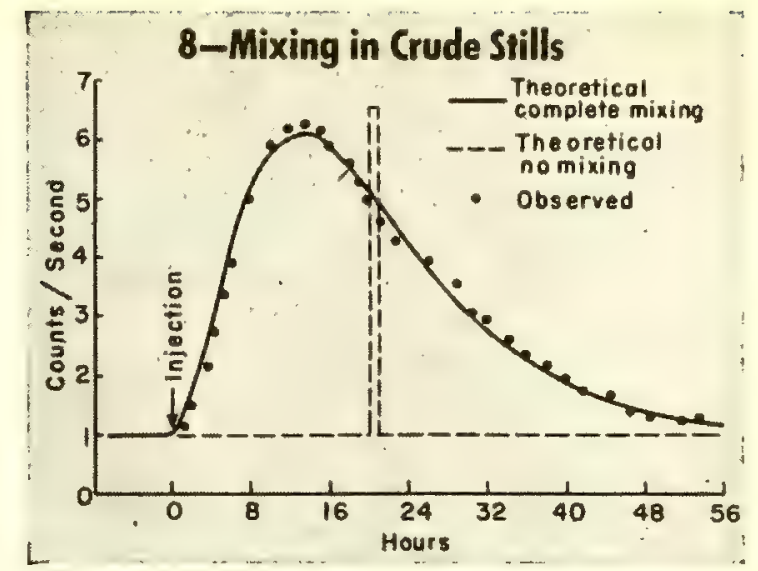

ever, fluctuations of $0-0.22 \%$ of labeled batch in the effluent showed that mixing was considerably less than ideal.

Tor in crude stills. In another refinery application, segregation of tar bottoms from different crudes fed to a distillation battery of four stills in series was studied (7). When switching to a new crude, $\mathrm{Co}^{60}$-naphthenate was added to the feed. The tar line leaving the battery was monitored for several days with a G-M counter. Activity began to appear in the outgoing stream only two hours after injection; whereas, if the stocks had followed a pistondisplacement pattern through the stills, it should have been delayed for 21 hours. The tracer spread out into a broad, diffuse wave, with a peak at 14 hours and a tail persisting into the third day. The curve expected if complete mixing occurred in each still (Fig. 8) closely approximated the observed data, showing that the mixing was not much short of ideal. Thus, it was not possible to effect the desired sharp break between tars from the two crudes.

\section{Leak Testing}

Diversion of any liquid stream from one channel to another can be detected readily with radiotracers. Under the proper conditions such leaks can be measured quantitatively. Leakage between cross-streams in a heat exchanger (Fig. 9) offers an excellent example of this technique (7).

Tracer is introduced in a short surge into the heatingstream inlet. G-MI counters are attached to the exit pipes of both the heating medium and process streams. Appearance of a tracer wave at the counter on the process stream indicates a leak. Leak size is measured by the number of counts in this tracer peak compared to the other peak. Leaks of a few tenths of $1 \%$ are detectable.

\section{9-Leak Test in Heat Exchanger}

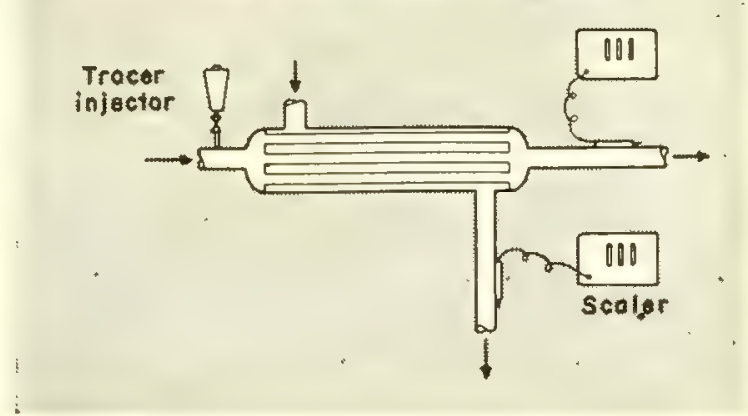

Many of the processes described in this paper are patented or patent applications have been filed on them. This article is based on a paper presented at the Berkeley, Calif. meeting of the American Association for the Advancement of Science, December, 1954.

\section{BIBLIOGRAPHY}

1. M. A. Elliott, U. S. Patent 2,506,585 (1950) 2. D. G. C. Hare, U. S. Patent 2,323,128 (1943) S. D. P. Thornton, Pet. Proc. 5, 941 (1950) 4. R. B. Jacobs, L. G. Lewis, Oil and Gas J. 52, No. 21,128 (1953)
5. H. K. Hughes, J. W. Wilczewski, Anal. Chem. 26, 1889 (1954)

6. G. H. Metcalf, U, S. Patent $2,631,242$ (1853) 7. Californis Research Corporation (unpublished)

8. D. E. Hull. R. R. Bowles, Oil and Gas J. B1, No. 46, 295 (1953)

9. G. Gester, et. Gl."Pet. Proc. 8, 550 (1953) 


\section{To trace steel melts of critical composition, such as for rexctor construction, radioisotopes offer several advantages over customary chemical analysis: time involved negligible, little plant space necessary, melt identification definite}

\section{By DAVID L. DOUGLAS}

Chemistry and Chemical Engineering Section, Knolls Atomic Power Laboratory* Schenectady, New York

The SECRET OF success in many industrial fields lies in the use of special steels or other alloys. With alloy steels, some particular property, such as corrosion resistance or high-temperature strength, can be essential to the successful operation of the device under consideration. Nowhere is this more true than in the field of reactor technology.

The usual method of operation is to order the alloy steel as a special melt and then, by way of insuring that no substitutions are made, to set up an extensive inspection and analysis program to follow the steel from the mill to finished article. Clearly this is both expensive and uncertain, the latter since one cannot commit all the steel to analysis and still have a finished product. As an answer to this problem, inclusion of a radioactive tracer in the original steel melt would appear to be ideal; not only would the laboratory space committed be negligible, but a 5-min check would suffice where a chemical analysis involves $15 \mathrm{man}$-hr.

* Operated by the General Electric Co. for the Atomic Energy Commission.
It is axiomatic that the amount of activity added must be kept as small as is consistent with the required detection sensitivity. Because several tons of the steel are likely to be accumulated in a warehouse or in the final product assembly, the maximum permissible specific activity, in consideration of the personnel hazard, is of the order of millicuries per ton.

\section{Discussion}

The important limits that constrain the selection of the nature and amount of the radioactive tracer employed are discussed next.

Detector sensitivity. To obtain satisfactory levels of sensitivity with readily available detection equipment, the emitted radiations must be gamma rays of energy greater than about 0.3 Mev or beta particles of energy greater than about $0.5 \mathrm{Mev}$. Thus low-energy beta-emitting and electron-capturing nuclides are eliminated. The discussion of detection sensitivity must refer to the specific problem in terms of the smallest fabricated part whose activity is to be measured. In this article, this will be taken as $1 \mathrm{gm}$.

The efficiency of radiation detection is related directly to geometry and range of the radiation, i.e., to the amount of material that the detector can "see." Since destruction of the sample by dissolution is prohibited, gamma emitters are much to be preferred over beta emitters on account of the limited range of beta particles in dense solids such as steel. Scintillation counters are the preferable detectors because of their high efficiencies for gamma rays.

Radioisotope availability. The radioisotope should be easy to obtain, e.g., sold by the AEC.

Metallurgy. Chemical and physical properties of the steel must not be altered by the tracer. (In reactor applications, nuclear properties must be considered too.)

One method of tagging is that the tracer be an isotope of one of the constituents of the alloy. Another is that the actual amount of material added be so small, $0.001 \%$ or less, that the properties of the alloy are not altered. The added material must mix thoroughly in the melt and have no tendency to collect in a slag or on the walls of the crucible.

Time. A lower limit of a few months on the half-life of the activity is set by the fact that it will usually be necessary to "follow" the steel for about a year. Shorter half-lives would mean inordinately high activity levels at the beginning of the period. The upper limit may be important if the purchaser is required by the steel mill to be re- 
sponsible for (i.e., to purchase) the mill scrap. In any event, prudence requires as short a half-life as possible. One year would seem to be a reasonable upper limit.

All of these requirements are met very satisfactorily by the nuclide tantalum-182. Its nuclear characteristics $(1,2)$ are 110 -day half-life, $0.53-\mathrm{Mev}$ betas, and 1.1-, 1.2-Mev and other gammas. One of the outstanding advantages of tantalum-182 is that it is readily available in very high specific activities - up to 50 curies/gm. This means that entirely insignificant amounts of the element would have to be added.

\section{Experimental}

Simple experiments have been carried out to determine the lowest specific activity required for easy "tracing" of a steel and the dose rate to be expected in the neighborhood of a large accumulation of the tagged metal.

Tracer preparation. Two pieces (60.6 and $111 \mathrm{mg}$, respectively) of 25-mil tantalum wire were irradiated in the reactor at Brookhaven National Laboratory for an equivalent nvt of $9 \times 10^{14}$ flux-seconds. The tantalum content was determined spectrographically and found to be more than $98 \%$ Qualitative analysis showed no impurities in quantity sufficient to produce appreciable radioactivity on irradiation.

A few milligrams from each specimen were dissolved in hydrofluoric acid and the absolute beta activity determined by counting an aliquot of each of the diluted solutions. Since the decay scheme of $\mathrm{Ta}^{182}$ is not known $(1,2)$, it was decided to use absolute beta counting for standardization rather than the less reliable gamma counting techniques. The aliquots were evaporated to dryness on gold-coated Pliofilm*

* Rubber hydrochloride, sold by Reed Laboratories, Akron, Ohio. mounts (less than $0.7 \mathrm{mg} / \mathrm{cm}^{2}$ ), and the activity determined with $4 \pi$ counters (3) of two different designs-both of which have been successfully used for several months at KAPL and checked against standard activities supplied by the National Bureau of Standards. The activation results are given in Table 1.

With identical aliquots of these same solutions, the counting efficiency of the scintillation counter for $\mathrm{Ta}^{182}$ was determined. This counter is a standard instrument in which the detector is a $\mathrm{NaI}(\mathrm{Tl})$ crystal $1 / 4 \mathrm{in}$. thick and 2 in. in diameter. The background with lead shielding averages about $55 \mathrm{cpm}$. Accordingly, the $\mathrm{Ta}^{182}$ counting effciency under the experimental conditions is $6.5 \pm 0.1 \%$.

Preparation and sampling of billets. As stated previously, two cylindrical billets of mild steel were vacuum cast in graphite molds by induction heating. Dimensions of the as-cast billets were 6.1-cm diameter, and 19.5-cm length. The tantalum wires were added before melting. Expected (calculated) specific activities are given in Table 2 .

To be certain that the activity was uniformly distributed throughout the metal, chips were collected by machining about 10 mils off the radius of each billet, and by drilling three 1/4-in.diameter holes equally spaced along the axis of each billet. A 1-gm sample of each lot of chips was mounted on cardboard and counted with the scintillation counter. From the average of these determinations, the "observed specific activities" were obtained and are tabulated in Table 1.

In view of the considerable differences between the chip mounts and the standard mount used to determine the counter efficiency, the agreement between the calculated and observed specific activities is considered satis- factory. Further, the agreement between chip samples from any one billet showed that the activity was distributed uniformly to within $3 \%$ or better.

The original billets were "counted" with a standard scintillation probe* and with a beta-gamma survey meter; results are shown in Table 2. Also shown are the survey-meter counting rates observed with pieces of the billets weighing approximately $1 \mathrm{lb}$. Measurements were made with the detectors all but touching the sample.

Since an estimation of the radiation dose rate requires a knowledge of the activity as a function of thickness of tagged metal, a series of disks of varying thickness were cut from each of the billets. These disks were then counted in the scintillation counter, care being taken to maintain a fixed distance between the top of the disk and the bottom of crystal can.

The data for disks from billet No. 2 are presented in Table 3 . The "efficiencies" are the usual ratio of observed counts to total activity in the sample. The "relative counting efficiencies" are ratios of the observed counting rates to those expected if the sample were counted with the efficiency of the 50-mil disk. These efficiencies are then a measure of the self-absorption of the radiation by the metal.

The efficiencies are presented in graphical form in the illustration on page 18 . The value for the 2.00-in. disk was obtained by extrapolation. Clearly this measurement is approximate in that it takes no account of scattering by the walls of the counter

\footnotetext{
* An example is the RCL scintillatior head, mark 10, model 10, Radiation Counter Laboratories, Chicago, Ill.

† For example, alpha-beta-gamma survey meter, model SU-5, Tracerlab, Inc., Boston. Mass.
}

TABLE 1-Specific Activity Data*

\begin{tabular}{|c|c|c|c|}
\hline \multirow[b]{2}{*}{ Billet } & \multirow{2}{*}{$\begin{array}{l}\text { Weight } \\
\text { of Ta } \\
\text { wire } \\
(m g)\end{array}$} & \multirow{2}{*}{$\begin{array}{c}\text { Tolal } \\
\text { activity } \\
\text { (curies) }\end{array}$} & Specific activity (curies/gm) \\
\hline & & & Calculated \\
\hline
\end{tabular}

No. $155.35 .21 \times 10^{-6} 1.15 \times 10^{-9} 1.28 \pm 0.03 \times 10^{-9}$ No. $295.19 .06 \times 10^{-6} 2.00 \times 10^{-9} 2.44 \pm 0.07 \times 10^{-9}$

* All activities corrected for decay.
TABLE 2-Results of Survey of "Tagged" Billets

\begin{tabular}{lcc}
\hline $\begin{array}{c}\text { Place counted and } \\
\text { physical arrangement }\end{array}$ & $\begin{array}{c}\text { Scintillation } \\
\text { probe }(\mathrm{cpm})\end{array}$ & $\begin{array}{c}\text { Beta-gamma } \\
\text { survey meter }(\mathrm{cpm})\end{array}$ \\
\hline Background & 3,550 & $\sim 200$ \\
Billet 1-end on & 6,150 & 1,700 \\
Billet 1-side & 7,030 & \\
Billet 2-end on & 12,100 & 3,900 \\
Billet 2-side & 13,700 & 800 \\
$529 \mathrm{gm}$ of billet 1 & - & 1,500 \\
$567 \mathrm{gm}$ of billet 2 & - & \\
\hline
\end{tabular}


TABLE 3-Counting Efficiencies vs Thickness for Billet No. 2

\begin{tabular}{|c|c|c|c|c|c|c|}
\hline $\begin{array}{l}\text { Nominal } \\
\text { thickness } \\
\quad \text { (in.) }\end{array}$ & $\begin{array}{l}\text { Weight } \\
(g m)\end{array}$ & $\begin{array}{c}\text { Surface } \\
\text { densily } \\
\left(\mathrm{gm} / \mathrm{cm}^{2}\right)\end{array}$ & $\begin{array}{c}\text { Tolal } \\
\text { activity } \\
\text { (curies) }\end{array}$ & $\begin{array}{c}\text { Gamma } \\
\text { counting } \\
\text { rate } \\
(\text { cpm })\end{array}$ & $\begin{array}{c}\text { Efficiency } \\
(\%)\end{array}$ & $\begin{array}{c}\text { Relative } \\
\text { counting } \\
\text { efficiency } \\
(\%)\end{array}$ \\
\hline 0.050 & 30.7 & 1.14 & $0.614 \times 10^{-7}$ & 12,275 & 9.0 & 100 \\
\hline 0.100 & 59.7 & 2.03 & $1.19 \times 10^{-7}$ & 20,544 & 7.75 & 94 \\
\hline 0.250 & 153.1 & 5.21 & $3.03 \times 10^{-7}$ & 44,712 & 6.65 & 73 \\
\hline 0.500 & 311 & 10.6 & $6.22 \times 10^{-7}$ & 71,732 & 5.2 & 63 \\
\hline 1.00 & 567 & 19.3 & $11.34 \times 10^{-7}$ & 92,778 & 3.7 & 45 \\
\hline 1.25 & 720 & 24.5 & $14.4 \times 10^{-7}$ & 98,665 & 3.1 & 37 \\
\hline 1.5 & 878 & 29.9 & $17.56 \times 10^{-7}$ & 102,962 & 2.6 & 32 \\
\hline 1.75 & 1,031 & 35.1 & $20.62 \times 10^{-7}$ & 107,575 & 2.35 & 28 \\
\hline 2.00 & 1,184 & 40.3 & $23.65 \times 10^{-7}$ & & & 23 (est) \\
\hline
\end{tabular}

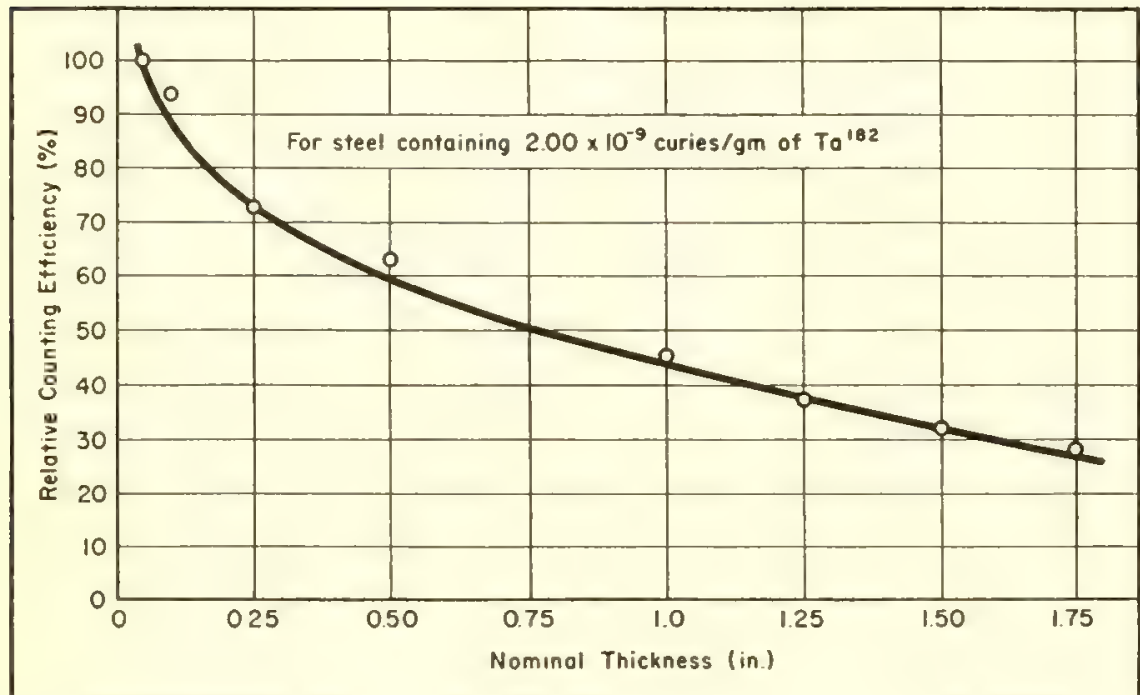

RELATIVE counting efficiency vs thickness

shield and other effects; however, it is more than sufficiently accurate for the purpose at hand.

Dose-rate factor. The preceding activity measurements have all involved counting rates determined with a scintillation counter and are not readily converted into dose rates. To obtain a factor for converting activity in curies into dose rate, the following simple experiment was carried out:

The dose rate was measured at various distances from a nominal 1-mc $\mathrm{Ta}^{182}$ source obtained from Oak Ridge National Laboratory. A $4 \pi$ count of an aliquot of the solution established the total absolute beta activity of the source as $0.76 \mathrm{mc}$. The instrument used in the dose-rate measurement was a recently calibrated "Cutie Pie" gamma survey instrument.*

The results for the range of interest were: $0.2 \mathrm{mr} / \mathrm{hr}$ at 2.0 meters; $0.4 \mathrm{mr} /$ hr at 1.5 meters; $0.9 \mathrm{mr} / \mathrm{hr}$ at 1.0

Note that the inverse square la obeyed to a first approximation.

\section{Conclusions}

The experiments show that steels containing amounts of $\mathrm{Ta}^{182}$ of the order of $1 \times 10^{-9}$ curies $/ \mathrm{gm}$ ( $1 \mathrm{mc} /$ metric ton) can be readily identified with counting equipment commonly available in radiochemical laboratories. For large pieces $-1 \mathrm{lb}$ and greater-a beta-gamma survey meter is sufficient. Smaller parts require the use of a scintillation counter.

What remains is to calculate the minimum specific activity that meets our detection condition, viz., ability to detect the activity in $1 \mathrm{gm}$ of metal after $1 \mathrm{yr}$, and, using this, to estimate the maximum probable dose rates.

* For example, radiation survey meter. model SU-1E, Tracerlab, Boston, Mass.
With a sample-to-background activity ratio of unity and a background of $50 \mathrm{cpm}$, the required final specific activity is $0.31 \times 10^{-9}$ curies/gm. Thus the initial specific activity is $2.5 \times 10^{-9}$ curies/gm or $2.5 \mathrm{mc} /$ ton.

In estimating the radiation dose rate in the neighborhood of a large accumulation of such steel, we assume the particularly severe case of a hollow sphere 1-in. thick and 1 meter in radius. The total mass of such a sphere would be about $2.5 \times 10^{6} \mathrm{gm}$, the total activity about $6.3 \mathrm{mc}$. At the center of the sphere, the dose rate would be $6.3 \times(0.9 / 0.76) \times 0.45=3.4 \mathrm{mr} / \mathrm{hr}$, where the second factor is the dose-rate and the third is the relative efficiency for metal 1-in. thick. This dose rate is only about half that deemed safe for day in and day out exposure (4). An inspection of the relative efficiencies in Table 3 shows that significantly higher dose rates would not be obtained by increasing the thickness of the sphere. Since an inverse-square law relates dose rate to distance from the source, decreasing the sphere radius, while maintaining the same mass, does not increase dose rate.

The conclusion is that it is entirely safe, insofar as personnel are concerned, to employ radioactive tracers in amounts of the order of $1 \mathrm{mc} / \mathrm{ton}$ for tagging special melts of ferrous or other alloys. However, it should be realized that this activity is 220 times the maximum activity of contaminated serap steel $(10 \mathrm{dpm} / \mathrm{gm}) \mathrm{re}$ leased by the AEC and considered as technically nonhazardous to sensitive industries.
The assistance of the following individuals and departments of the Knolls Atomic Power Laboratory is gratefully acknowledged: Mr. F. D. Nicol and ather members of the Manufacturing Unit for many helpful discussions pointing up the importance of the problem. Dr. D.W. White and Mr. R. V. Gray of the Metallurgy Section for preparing the steel billets: Special Material Shop for machining the billets; and Mrs. A. C. Mewherter of the Chemistry Section for assistance in carrying out the radiochemical work.

\section{BIBLIOGRAPHY}

1. K. Way, et al., Nuclear dats, NBS Circular 499 (National Bureau of Standards, Washington. D. C., 1950)

2. J. M. Hollander, D. Perlman, G. T. Seaborg Revs. Mod. Phys. 25, 469 (1953)

3. C. J. Borkowski, Conference on Absolute Beta-Counting, Preliminary Report No. 8, Nuclear Science Series, National Research Council, Washington, D. C. (Oct. 1950); Aral. Chem, 21, 348 (1949)

4. "Radiological Health Handbook," issued by the Federal Security Agency, Public Health Service, Environmental Health Center, Cincinnati, Ohio 
For measurement of . . .

\section{THICKNESS}

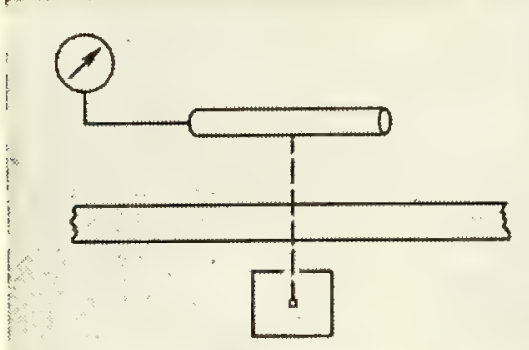

BY TRANSMISSION

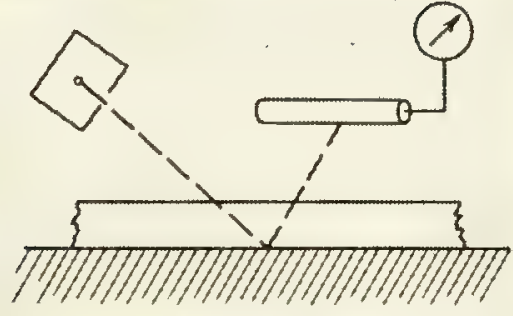

BY REFLECTION

\section{CONCENTRATION}

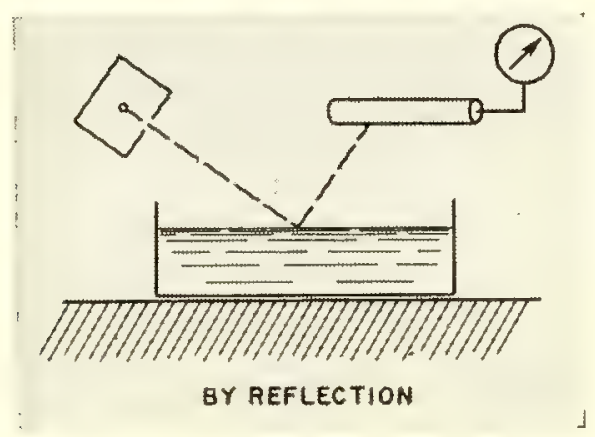

\section{... this article tells how to get ...}

\section{The Best Performance from Beta Gages}

By L. R. ZUMWALT

Tracerlab, Inc.

Berkeley, California

INDUSTRIAL PROCESS controls with beta gages as the primary indicators can be made most useful when the characteristics of the gages are known quantitatively. This article presents a means of analyzing the application of a beta gage to any particular process.

Thicknesses of continuously moving materials and the concentrations of process solutions are the quantities most commonly measured with beta gages $(1-5)$. Measurements are based on two principles. The absorption of beta radiation as it passes through a material provides an indication of the material's thickness. The degree of backscattering of beta rays from a substance can be used to determine either thickness or concentration.

\section{Mathematical Formulation}

A quantitative analysis of beta-gage sensitivity, accuracy, and response time requires the mathematical formulation of beta-ray transmission and reflection.

Approximate relationships between the observed current from the ionization chamber and the thickness or con- centration (variables that deternine the number of beta particles entering the ionization chamber) are given in this section.

Thickness-by-transmission. When the material measured is between the radiation source and the ionization chamber

$$
\Delta I=I-I_{0}=-I_{0}\left(1-e^{-\mu_{a} x}\right)
$$

With reference (meter zeroed) at $x=x^{\prime}$

$$
\begin{aligned}
\Delta I=I-I_{x^{\prime}} \\
\quad=-I_{0} \mathrm{e}^{-\mu_{a} x^{\prime}}\left(1-e^{-\mu_{a}\left(x-x^{\prime}\right)}\right)
\end{aligned}
$$




\section{SYMBOLS}

$I=$ ionization current with material of thickness $x$, amperes

$x=$ thickness in units of mass per unit area, $\mathrm{mg} / \mathrm{cm}^{2}$

$I_{0}=$ ionization current with material of thickness 0 (in thickness measurements) or ionization current backscattered by the solvent when it contains no solute (in concentration measurements), amperes

$\Delta I=$ change in ionization current caused by material, amperes

$\mu_{a}=$ absorption coefficient, $\left(\mathrm{mg} / \mathrm{cm}^{2}\right)^{-1}=0.693 \mathrm{~m} / R_{\theta}$, empirically determined; due to scattering effects, it will show some dependence on the atomic number of the material being measured

$R_{o}=$ maximum range of beta particles, $\mathrm{mg} / \mathrm{cm}^{2}$

$=412 E^{1.265-0.220 \log _{10} E}$ (when $E<3 \mathrm{Mev}$ )

$=542 E-133$ (when $E>$ $0.8 \mathrm{Mev}$ )

$E=$ maximum energy beta emitted, Mev

$m=$ a number from 6 to 13 , depends on the geometry of the setup and on the beta particle energy spectrum characteristic of the source isotope

$I_{A}=$ ionization current obtained for a saturation thickness of $\mathbf{A}$

$I_{B}=$ ionization current from $B$ with zero thickness of $A$

$\mu_{b}=$ reflection coefficient, $(\mathrm{mg} /$ $\left.\mathrm{cm}^{2}\right)^{-1}=f_{\mu_{a}}$ (empirically)

$f=$ empirical factor

$=2.5$ to 3

$Z_{A}=$ effective atomic number of $A$

$Z_{B}=$ effective atomic number of $B$

$n=$ empirical exponent

$=0.7$ to 0.8

$x_{0}=$ saturation thickness, $\mathrm{mg} /$ $\mathrm{cm}^{2}$
$K=$ a constant dependent on setup $\left(K \approx 4 \times 10^{-4}\right.$ in author's experiments)

$Z$ = effective atomic number of solute

$Z_{\text {s }}$ = effective atomic number of solvent

$c=$ concentration of solute dissolved in a low-atomicnumber solvent (water or organic), weight per cent

$j=$ empirical exponent $(j \approx$ $0.9_{6}$ in author's experiments)

$k=$ empirical exponent $(k \approx$ $0.9_{0}$ in author's experiments)

$M=$ meter reading, $\mu \mathrm{a}$

$R=$ load resistance from ionization chamber anode to ground, ohms

$g_{m}=$ amplification $=$ effective transconductance of electrometer tube amplifier system, $\mu \mathrm{a} /$ volt

$S_{x}=$ sensitivity of thickness gage, $\mu \mathrm{a} / \mathrm{mg} / \mathrm{cm}^{2}$

$S_{\varepsilon}=$ sensitivity of concentration gage, $\mu \mathrm{a} / \mathrm{wt} \%$

$\delta x=$ uncertainty in thickness $x$

$\delta c=$ uncertainty in concentration $c$

$\delta M=$ uncertainty in meter reading

$N=$ number of betas entering ionization chamber per second

$C$ = capacity of ionization circuit, farads

$q_{\mathrm{avg}}=$ average charge produced by a beta particle in ionization chamber, coulombs/ beta particle

$\sigma_{1}=$ standard deviation of ionization current, $I$, through $R$

$\left(q^{2}\right)_{a v \varepsilon^{3 / 2}}=$ root-mean-square deviation of $q$

$\sigma_{M}=$ standard deviation of meter reading due to statistical fluctuations

$\tau=$ equilibrium time, sec
Thickness-by-reflection. When a thin material, $A$, is laid over a thick material, $B$ (usually thick enough to cause saturation backscattering from $B)$, and beta particles backscatter into the ion chamber

$\Delta I=I-I_{B}=\left(I_{A}-I_{B}\right)\left(1-e^{-\mu_{b} x}\right)$

For saturation backscattering

and

$$
\frac{I_{A}}{I_{B}}=\left(\frac{Z_{A}}{Z_{B}}\right)^{n}
$$

$$
\begin{aligned}
\Delta I=I & -I_{B} \\
& =I_{B}\left[\left(Z_{A} / Z_{B}\right)^{n}-1\right]\left(1-e^{-\mu_{b} x}\right)
\end{aligned}
$$

Saturation thickness is

$$
x_{s} \approx 116 E^{0.67}
$$

Concentration-by-reflection. When the beta particles received in the ionization chamber have been backscattered from a solution (liquid or solid) of at least saturation thickness

$$
\Delta I=I-I_{0}=I_{0} K\left(Z-Z_{0}\right) c^{k}
$$

where

$$
Z,<Z
$$

\section{Accuracy}

The usefulness of the gage is determined by the over-all accuracy with which measurements can be made.

As an example, assume that measurements are being made using a $\mathrm{d}-\mathrm{c}$ electrometer bridge amplifier circuit with a $d$-c microammeter as the indicating meter. If the meter is zeroed for $\Delta I=0$ and the polarity is such that a positive reading is obtained, then

$$
M=\left|\Delta I R g_{m}\right|
$$

Sensitivity. For each of the three types of gages, the sensitivity is

Thickness-by-transmission

$$
S_{x}=\frac{d M}{d x}=I_{0} R g_{m} \mu_{a} e^{-\mu_{0} x}
$$

Thickness-by-reflection

$$
\begin{aligned}
S_{x}= & \frac{d M}{d x} \\
& =\left|I_{B} R g_{m}\left[\left(Z_{A} / Z_{B}\right)^{n}-1\right] \mu_{b} e^{-\mu_{b} x}\right|
\end{aligned}
$$

Concentration-by-reflection

$$
S_{c}=\frac{d M}{d c}=I_{0} R g_{m} k K\left(Z-Z_{\bullet}\right)^{i} c^{k-1}
$$

Precision. Two factors influence the precision of measurement: (1) The uncertainty in the meter reading associated with its inherent accuracy; and, (2) The fluctuation in the meter due to statistical fluctuations in the number of beta particles and the 


\section{Calculated Characteristics of Three Types of Beta Gages}

\begin{tabular}{|c|c|c|c|c|c|c|c|c|}
\hline Type of Gage & $\begin{array}{c}\Delta I \\
(a m p)\end{array}$ & $\begin{array}{c}S_{x} \\
\left(\mu a / m g / \mathrm{cm}^{2}\right)\end{array}$ & $\begin{array}{c}S_{e} \\
(\mu a / w t . \%)\end{array}$ & $\delta x / x$ & $\delta c / c$ & $\sigma_{x} / x$ & $\sigma_{c} / c$ & $\stackrel{T}{(s e c)}$ \\
\hline $\begin{array}{l}\text { Chickness-by- } \\
\text { transmission }\end{array}$ & $1.72 \times 10^{-10}$ & 3.3 & - & $6.1 \times 10^{-3}$ & 一 & $1.6 \times 10^{-8}$ & - & 12.9 \\
\hline $\begin{array}{l}\text { Thickness-hv- } \\
\text { reflection }\end{array}$ & $1.85 \times 10^{-10}$ & 5.8 & 一 & $6.9 \times 10^{-8}$ & - & $1.9 \times 10^{-8}$ & - & 12.7 \\
\hline $\begin{array}{l}\text { Concentration-by- } \\
\text { reflection }\end{array}$ & $4.8 \times 10^{-11}$ & 一 & 17.3 & - & $2.3 \times 10^{-2}$ & - & $0.66 \times 10^{-2}$ & 10.4 \\
\hline
\end{tabular}

associated number of ion pairs (electric charge) that appear in the ionization chamber. The larger of the two factors determines the gage's over-all accuracy.

The accuracy of the measurement of thickness or concentration is related to the uncertainty in the meter reading by

$$
\delta x=\frac{\delta M}{S_{x}} \quad \text { or } \quad \delta c=\frac{\delta M}{S_{c}}
$$

In the case of a ratemeter-type circuit, the fractional standard deviation of the ionization current due to the random nature of radioactive decay is $(6)$

$$
\frac{\sigma_{I}}{I}=\frac{1}{\sqrt{2 N R C}}=\frac{1}{\sqrt{2 I R C / q_{\text {ovg }}}}
$$

Since there is a statistical fluctuation in the charge produced by an individual beta particle, the expected fractional standard deviation of $I$ is somewhat greater than that given above, and is

$$
\frac{\sigma_{I}}{I}=\left(\frac{\sqrt{\left(q^{2}\right)_{a v o}}}{q_{a v o}}\right) \frac{1}{\sqrt{2 I R C / q_{a v o}}}
$$

The standard deviation of the meter reading due to statistical fluctuations is

$\sigma_{M}=\sigma_{I} R g_{m}=\frac{I R g_{m}}{\sqrt{2 I R C / q_{a v \theta}}}\left(\frac{\sqrt{\left(q^{2}\right)_{a v o}}}{q_{a v \theta}}\right)$

The fractional standard deviations of thickness and concentration are

$\frac{\sigma_{x}}{x}=\frac{\sigma_{M}}{x S_{x}}=\frac{g_{m}}{x S_{x}} \sqrt{\frac{I R q_{a v 0}}{2 C}}\left(\frac{\sqrt{\left(q^{2}\right)_{a v o}}}{q_{a v g}}\right)$ and

$$
\frac{\sigma_{c}}{c}=\frac{g_{m}}{c S_{c}} \sqrt{\frac{I R q_{a v o}}{2 C}}\left(\frac{\sqrt{\left(q^{2}\right)_{a v o}}}{q_{a v 0}}\right)
$$

Over-all accuracy. In the determination of thickness and concentration with the three types of gages, the fractional uncertainties associated with inherent meter accuracy ( $\delta$ 's) and statistical fluctuations ( $\left.\sigma^{\prime} s\right)$ of the radiation source are
Thickness-by-transmission

$$
\begin{aligned}
& \frac{\delta x}{x}=\frac{\delta M}{x S_{x}}=\frac{e^{\mu_{a} x} \delta M}{\mu_{a} x I_{0} R g_{m}} \\
& \left.\frac{e_{x} \xi^{3 \mu_{a} x}}{x}=\frac{\sqrt{\left(q^{2}\right)_{a v o}}}{q_{a v o}}\right)
\end{aligned}
$$

Thickness-by-reflection

$$
\begin{aligned}
& \frac{\delta x}{x}=\frac{e^{\mu_{b} x} \delta M}{\mu_{b} x I_{B} R g_{m}\left[\left(Z_{A} / Z_{B}\right)^{n}-1\right]} \\
& \frac{\sigma_{x}}{x}=\frac{e^{\mu_{b} x} \sqrt{\left(Z_{A} / Z_{B}\right)^{n}-}\left[\left(Z_{A} / Z_{B}\right)^{n}-1\right] e^{-\mu_{b} x}}{\mu_{b} x\left[\left(Z_{A} / Z_{B}\right)^{n}-1\right] \sqrt{2 I_{B} R C / q_{\text {avg }}}} \\
& \left(\frac{\sqrt{\left(q^{2}\right)_{a v g}}}{q_{a v q}}\right)
\end{aligned}
$$

Concentration-by-reflection

$$
\begin{aligned}
& \frac{\delta c}{c}=\frac{\delta M}{I_{0} R g_{m} k K\left(Z-Z_{s}\right)^{j} c^{k}} \\
& \frac{\sigma_{c}}{c}=\frac{\sqrt{K\left(Z-Z_{*}\right)^{j} c^{k}+1}}{k K\left(Z-Z_{*}\right)^{j} c^{k} \sqrt{2 I_{0} R C / q_{\text {avo }}}} \\
& \left(\frac{\sqrt{\left(q^{2}\right)_{a v g}}}{q_{\text {avo }}}\right)
\end{aligned}
$$

The gross accuracy of measurement is determined principally by the larger of the two uncertainties. More precisely, the over-all fractional uncertainty is the root-mean-square value of the two fractional uncertainties. In general, the meter accuracy uncertainty is proportional to the source strength, the amplification, and the load resistance; but the source fluctuation uncertainty is.inversely proportional to the square-root of both source strength and time constant $R C$. In an optimum design these variables would be chosen so that the two uncertainties are about equal.

\section{Response Time}

In the design of automatic controls that rely on beta-gage measurements, the gage's time of response is important. The equilibrium time for a rate meter to go from a rate of 0 to $N$ particles per second is (6)

$$
\tau=R C[1 / 2 \ln (2 N R C)+0.394]
$$

where $\tau$ is the time required for the exponentially decreasing difference between the actual and true counting rate reading to be less than the probable error due to statistical fluctuations.

It is, however, more rational to define equilibrium time in terms of the standard error rather than the probable error, and

$$
\tau=1 / 2 R C \ln (2 N R C)
$$

For thickness gage applications it is interesting to estimate the equilibrium time in going from counting rate $N_{1}$ to $N_{2}$ or ionization current $I_{1}$ to $I_{2}$. The equilibrium time (defined in terms of the standard error) in this case is

$$
\begin{gathered}
\tau=\frac{1}{2} R C \ln \left[\frac{2\left(N_{2}-N_{1}\right)^{2}}{N_{2}} R C\right] \\
\tau=\frac{1}{2} R C \ln \left[\frac{2\left(I_{2}-I_{1}\right)^{2}}{I_{2}} \frac{R C}{q_{a v}}\right]
\end{gathered}
$$

or

It may be noted that $\tau$ is zero when

$$
I_{2}-I_{1}=\sqrt{I_{2}} / \sqrt{2 R C / q_{a v g}}
$$

This occurs when the change in ionization current in going from $I_{1}$ to $I_{2}$ is equal to the standard deviation expected for an ionization current of magnitude $I_{2}$.

In terms of $\Delta I$ and $I$, where thickness or concentration changes from 0 to $x$ or $c$, the equilibrium time is

$$
\tau=1.15 R C \log _{10}\left[\frac{2(\Delta I)^{2}}{I} \frac{R C}{q_{\mathrm{av} \sigma}}\right]
$$

One of the first steps in the design of a beta gage consists of choosing a time constant $R C$ such that suitable equilibrium times are obtained for the changes in $I$ involved in making the measurement of thickness or concentration.

\section{Example}

To illustrate the application of the quantitative relationships to the evaluation of an industrial beta gage, con- 
sider a series of uses where the gage is adapted to be a thickness-by-transmission gage, a thickness-by-reflection gage, and a concentration-by-reflection gage.

Equipment. The gage detector is a cylindrical ionization chamber with a thin stainless-steel window for betaparticle entry. The ionization current from the chamber is measured by a stable, one-stage, bridge-type d-c amplifier.

The very small ionization current $\left(\sim 2 \times 10^{-9}\right)$ is put through a large resistance $\left(\sim 5 \times 10^{9}\right.$ ohms $)$ so the signal produced by the total source is 10 volts. This negative voltage is opposed by a positive voltage used for adjusting the operating point of the bridge circuit. The net sum of these voltages is applied to the grid of the electrometer tube as a signal in excess of its proper bias voltage. The electrometer tube forms one arm of a bridge (balanced when the proper bias voltage alone is applied to the electrometer tube). A variable sensitivity microammeter is set across the bridge to indicate any unbalance. The fullscale reading of the microammeter (at maximum sensitivity) is $20 \mu \mathrm{a}$, and the inherent "accuracy" of the meter, $\delta M$, is about $0.4 \mu \mathrm{a}$. The effective transconductance of the electrometer tube in the bridge circuit is about $80 \mu \mathrm{a} /$ volt. The time constant of the gage is $2.0 \mathrm{sec}$.

The beta particles from strontium-90 of maximum energy $0.6 \mathrm{Mev}$ are almost completely absorbed by the combination of absorbers comprising the stainless-steel source cover and the window of the ionization chamber. Therefore, the effective beta radiation from strontium-90 is actually that from its daughter, yttrium-90, which emits beta particles with a maximum energy of $2.2 \mathrm{Mev}$.

Coefficients. With this arrangement, it is found that for a

Thickness-by-transmission,gage

$$
\mu_{a}=0.0045\left(\mathrm{mg} / \mathrm{cm}^{2}\right)^{-1}
$$

Thickness-by-reflection gage

$$
\mu_{b}=0.012\left(\mathrm{mg} / \mathrm{cm}^{2}\right)^{-1}
$$

Concentration-by-reflection gage

$$
K=4 \times 10^{-4}
$$

Calculation. The table shows the characteristics calculated for three types of beta gages:

Thickness-by-transmission gage. Thickness measured is $20 \mathrm{mg} / \mathrm{cm}^{2}$ and
$I_{0}=2 \times 10^{-9} \mathrm{amps} . \quad$ Source $\approx 10 \mathrm{mc}$.

Thickness-by-reflection gage. A $10 \mathrm{mg} /$ $\mathrm{cm}^{2}$ piece of plastic $\left(Z_{A} \approx 5\right)$ laid on a thick iron plate $\left(Z_{B}=26\right)$ is measured and $I_{B}=2 \times 10^{-9}$ amps. Source $\approx$ $10 \mathrm{mc}$.

Concentration-by-reflection gage. A 1.0 weight per cent solution of lead $(Z=82)$ in water $(Z, \approx 7)$ is measured. $I_{0}=2 \times 10^{-9}$ amps. Source $\approx 20 \mathrm{mc}$.

A value of $q_{a v g}=1.6 \times 10^{-16}$ coulombs is estimated from a consideration of the ionization chamber geometry and data on the specific ionization of beta particles. It is assumed that $\sqrt{\left(q^{2}\right)_{\text {ave }}}$ is approximately equal to $q_{a v o}$.

Certainty. From the table it can be seen that in each case, the "accuracy" of the meter, rather than the fluctuations due to the radioactive source, is the limiting factor in the measurements' uncertainty. Assuming that we do not to change the load resistor and thus the response characteristic of the gage, and assuming that it is impractical to use a more accurate microammeter, there remain two means for improving the certainty of measurement.

The first alternative involves increasing $g_{m}$ by changing the electronic circuitry of the beta gage to form a more elaborate amplifier. An increase in amplification will not affect $\sigma_{x} / x$ or $\sigma_{c} / c$. Thus the increase of $g_{m}$ will be effective in decreasing the uncertainty only up to the point that $\sigma_{x} / x$ or $\sigma_{\varepsilon} / c$ becomes larger than $\delta x / x$ or $\delta c / c$.

The second possibility entails increasing $I_{0}$ or $I_{B}$ by increasing the strength of the source of beta radiation. The value of $\sigma_{x} / x$ or $\sigma_{c} / c$ will decrease in this case along with $\delta x / x$ or $\delta c / c$, but less rapidly because of their inverse square root and first power dependence, respectively, on ionization current.

The subject matter of this article constituted a portion of the lecture on Refiection Beta Gages presented on A pril 21, 1959, for the course "Advanced Radioisotope Techniques in Industry" sponsored by the Oak Ridge Institute of Nuclear Studies.

\section{BIBLIOGRAPHY}

1. J. R. Carlin, Electronics 22, 110 (1949)

\&. C. W. Clapp and S. Berstein, Gen. Elec. Rev. 63, 31 (1950); Trans. Am. Inst. Elec. Enots. $69,488(1950)$

9. J. R. Carlin, Rubber Age and Synthetics 66, 173 (1949)

4. W. R. Dixon, NRC-2358 (National Research Council, Chalk River, Canada, 1951)

5. J. Kohl, Pelroleum Refiner 31, $117^{*}(1952)$

6. L. I. Schiff, R. D. Evans, Reo, Sci. Instr. 7, 456 (1936) 


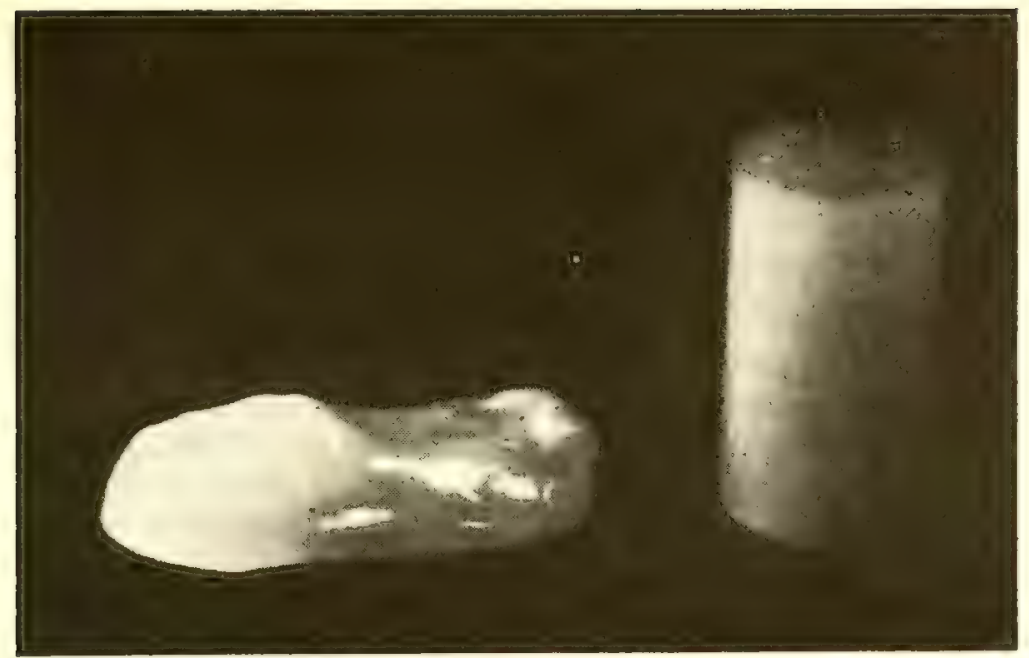

FIG. 1. Effect of irradiation on heat-resistant $\left(120^{\circ} \mathrm{C}\right)$ properties of polyethylene. Control sample at left; shortly-irradiated sample at right

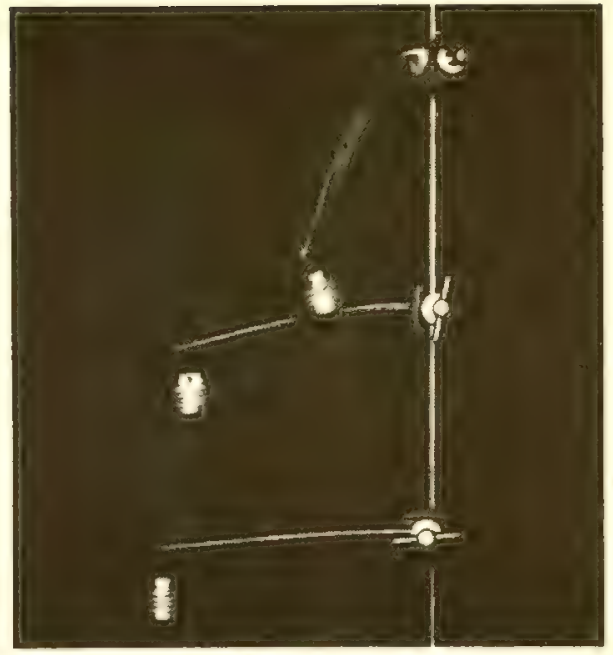

FIG. 2. Elastic properties of irradiated poly. ethylene at $150^{\circ} \mathrm{C}$. Top rod had received 0.6 units irradiation; middle rod, 3 units; bottom rod, 12 units

\section{How Radiation Affects Long-Chain Polymers}

Data showing effects of radiation-induced crosslinking or degradation of plastics indicate that the effects can be used in polymer research and for industrial processes. Rough cost estimates define commercial feasibility

\section{BY A. CHARLESBY}

Alomic Energy Research Establishment

Harwell, England

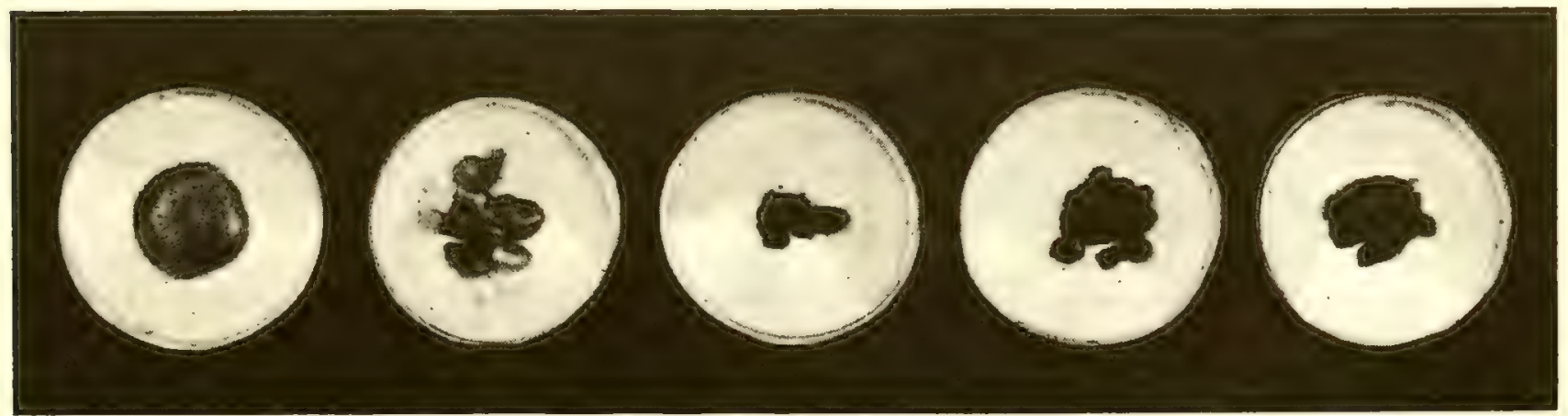

FIG. 3. Transformation of polyethylene from a melting to a non-melting polymer at $190^{\circ} \mathrm{C}$. Samples held at femperature for $13 / 2$ hours. From left to right, somples received $0,0.04,0.05,0.09$, and 0.14 units of radiation 
whose formation is induced by radiation. The crosslinking profoundly affects melting and solubility.

\section{Polyethylene}

At room temperature polyethylene has a white appearance reminiscent of paraffin wax, to which it has a certain chemical analogy (apart from molecular length). It can be cold-drawn. It begins to soften at about $70^{\circ} \mathrm{C}$, and becomes a viscous transparent liquid at about $115^{\circ} \mathrm{C}$. It is readily soluble in many organic compounds at temperatures of about $80^{\circ} \mathrm{C}$. These properties are a serious disadvantage in many applications.

During irradiation, polyethylene can be crosslinked readily, and primary bonds can form between adjacent carbon atoms $(5)$. After a radiation dose of about 0.05 units or 2-million roentgen (amount depends on molecular weight) it no longer melts, because it is transformed partly into a threedimensional network. This cffect is shown in Fig. 1.

After irradiation up to 1 unit there is no obvious change in the physical properties at room temperature; after doses up to 10 units it becomes progressively more flexible; above this it stiffens again and may transform into brittle glass-like material. In this region it is almost entirely amorphous, even at room temperature. It develops a strong yellow or brown tint similar to that observed in many polymers after irradiation.

By irradiation of specimens under certain conditions for about 4 units, polyethylene can be converted into a new type of polymer that is flexible, amorphous and largely transparent at room temperature. The amorphous character is retained down to very low temperatures. This material is so very different from the more usual polyethylene that a new name, "setylene," might well be used to emphasize its thermoset character.

Melting. It is misleading, perhaps, to refer to a radiation-produced rise in the melting point if by this is meant the removal of crystallinity. In ordinary polyethylene, the molecules are bound together largely by Van der Waals forces in the crystalline regions, which comprise about two-thirds of the specimen at room temperature. When these melt, the solid polymer is converted to a liquid. Radiation does not increase the melting point of these crystalline regions-instead there is a slight decrease (6). The crosslinks produced by radiation hold the molecules together even when crystallinity is destroyed above $115^{\circ} \mathrm{C}$. The irradiated polymer then is transformed into a flexible amorphous material that has certain rubber-like properties as shown in Fig. 2.

The melting properties of slightly irradiated polyethylene are shown in Fig. 3. Small cubes were placed in the reactor, irradiated to varying extents, and then were heated to $190^{\circ} \mathrm{C}$ for $90 \mathrm{~min}$. The unirradiated cubes fused together into a liquid that subsequently solidified to the shape shown. The same was true of polyethylene irradiated for varying dosages up to about 0.04 or 0.05 units, though to a less marked extent because the small amount of crosslinking that had occurred increased the average molecular weight sufficiently to produce a very viscous liquid that did not flow readily. Longer irradiations prevented the cubes from fusing together and they could be separated readily.

The transition at about 0.05 units of radiation corresponds to about 0.5 crosslinks per molecule. This figure is lower than the minimum that might be expected (namely one crosslink per molecule) for gel formation. The low transition occurs because the molecules are of different sizes and the longer molecules have a greater chance of having at least one crosslink. These longer molecules link to form a nonfusible network that retains the shorter molecules that have not been crosslinked. The shorter molecules can be

\section{"Memory" Effect}

An interesting property of lightly-irradiated polyethylene can be referred to as the "memory" effect. If the polymer is crosslinked very slightly (abcut 1.5 crosslinks per molecule) and then heated above the usual melting point, it is extremely flexible and can be deformed very considerably. If this modified shape is cooled, crystallization occurs and the molecules become locked in their new position. The specimen then resembles polyethylene molded into this new shape. However, on reheating the specimen above the melting point, the crystallites melt and the few crosslinks present return the specimen to its original shape. The process can be repeated any number of times, and the time of storage in the distorted form is irrelevant.

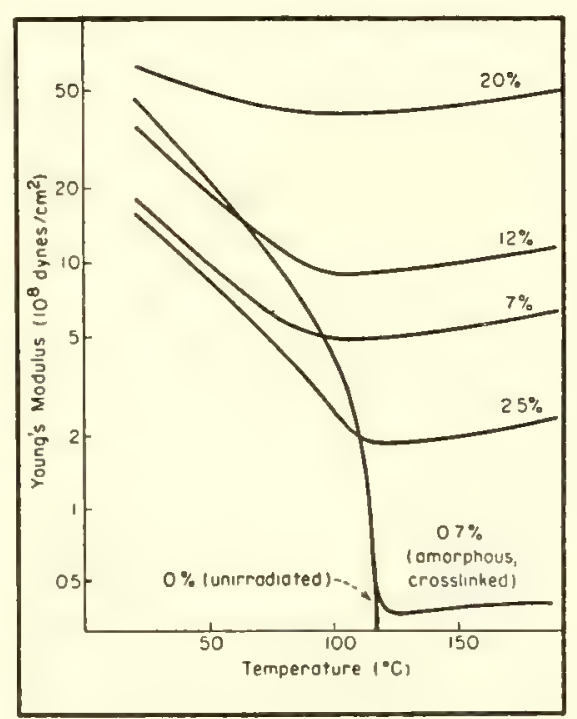

FIG. 4. Elastic properties of irradiated polyethylene. Percentages give proportions of carbons crosslinked

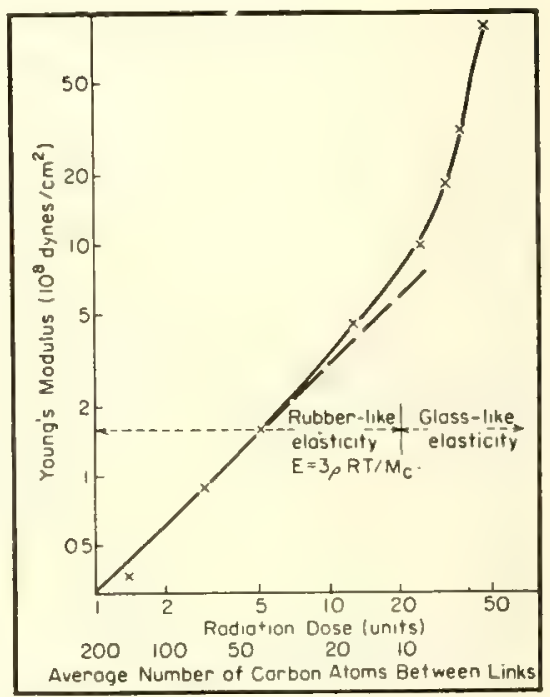

FIG. 5. Elastic properties of amorphous polyethylene of $150^{\circ} \mathrm{C}$ 
removed from the network by solubility tests.

Elastic properties. Figure 4 shows the change in elastic properties of polyethylene after various irradiation doses (7). At first there is a drop in Young's modulus until the usual melting point is reached (about $115^{\circ} \mathrm{C}$ ). Unirradiated polyethylene becomes a viscous liquid. Irradiated polyethylene is transformed into a transparent and elastic material that is amorphous in character. The elastic properties above this temperature are found to follow the theoretical formula for amorphous rubber-like elasticity that has been deduced from entropy considerations

$$
E=3 \rho R T / M_{c}
$$

where $E$ is Young's modulus, $\rho$ is the density, $R$ is the gas constant, $T$ is the absolute temperature, and $M_{c}$ is the average molecular weight between crosslinks. Since density of crosslinking is directly proportional to radiation dose, $E$ is proportional to the degree of irradiation over a wide range of doses. The curves of Fig. 5 show that the formula fails only for very high crosslinking densities where elasticity corresponds to that of a glasslike structure.

Once crystallinity has been destroyed by radiation, the density of an amorphous structure can be determined in terms of both crosslinking density and temperature. The results can be expressed in terms of an equation very reminiscent of a simplified Van der Waals equation

$$
\left(P+P_{0}\right)\left(V-V_{0}\right)=R T[1-\beta(c)]
$$

where $P$ and $P_{0}$ are the external and internal pressures, $V$ is the volume of polyethylene per gram mole of $\mathrm{C}_{2} \mathrm{H}_{4}$, and $V_{0}$ is the volume at $0^{\circ} \mathbf{K}$. The $\operatorname{term} \beta(c)$ is a function of the degree of crosslinking $c$, and approximates to $c$ (the proportion of carbons crosslinked).

This equation of state applies approximately to other crosslinked polymers in the amorphous state.

Solubility. The solubility characteristics of many long-chain polymers are affected considerably by radiation.

Ordinary polyethylene is readily soluble in many organic materials at temperatures above $70^{\circ} \mathrm{C}$. A very slight amount of crosslinking (corresponding to less than 0.03 units of radiation) does not greatly affect this property, since molecules are only linked together in small numbers to alter the molecular weight distribution. A somewhat larger radiation dose (about 0.05 units), corresponding to about 0.5 crosslink per molecule, links a large number of molecules together into one large molecule or gel that is insoluble in all usual organic solvents. The remainder of the specimen (the molecules that have not been linked into the structure) can still be removed by solvents, but the amount of this soluble fraction or sol is limited. Further irradiation gives a rapidly decreasing amount of soluble material. From the relationship between the sol fraction and the radiation dose, useful information can be derived as to the molecular weight distribution in the original polymer.

In solvents, crosslinked polyethylene swells to an extent that depends on its temperature and degree of crosslinking. Considerable swelling can be produced in lightly-linked specimens at temperatures above the usual melting point.

Other paraffinic structures. The effect of radiation on other paraffinic structures has also been studied (8). Paraffin wax and paraffin molecules of known molecular weight from heptane $\left(\mathrm{C}_{7} \mathrm{H}_{16}\right)$ to hexatriacontane $\left(\mathrm{C}_{36} \mathrm{H}_{74}\right)$ in both liquid and solid state have been successfully crosslinked into an infusible gel. The amount of radiation required to do this is inversely proportional to the molecular chain length. In all cases the energy absorbed is about 25 ev per crosslink.

\section{Polystyrene}

Insolubility of the product and changes in the softening point show that polystyrene can be crosslinked by ionizing radiation. Radiation doses to achieve these effects are far greater than for polyethylene despite the much larger number of monomer units in the average polystyrene molecule. For example, a polystyrene molecule containing an average of about 8,000 carbon atoms in the main chain of the molecule requires 1 unit of radiation per crosslink, whereas a polyethylene chain of the same chain length would only need about 0.02 units to achieve the same amount of crosslinking. The difference can be ascribed to the benzene ring in styrene. Such a ring stabilizes many organic compounds against the effects of radiation. The resonant levels of the benzene structure act as a sink and dissipate the added energy that otherwise might produce crosslinking or degradation.

It can be shown directly that the stabilizing effect is due to the resonating benzene ring. Molecules consisting of either a naphthyl or decalyl ring structure to which similar paraffinic side chains are attached, have been irradiated. The former, which has a resonant structure, requires far more radiation to induce gel formation than the latter, although there is no significant difference in the rate of energy absorption by the two types of molecule.

Surface oxidation. As in polyethylene, surface oxidation occurs when polystyrene is irradiated in the presence of air. After considerable irradiation, a brown powder is formed that contains substantial amounts of oxygen. Unlike the oxidation product on the surface of polyethylene, this material is not sticky and can be removed readily. Apart from this slight oxidation the only obvious change to irradiated polystyrene rods is a yellow coloration that increases rapidly with radiation dose.

Melting. Figure 6 shows how irradiated polystyrene rods withstand high temperature. In spite of the slightness of crosslinking, the irradiated specimens retain their shape and do not melt or flow when heated to $250^{\circ} \mathrm{C}$. At the same temperature, the unirradiated specimen forms a viscous liquid. For a radiation dose (0.6 units) corresponding to only 0.6 crosslinks per molecule (1 crosslink per 6,000 carbon atoms) there is a marked effect in the melting character of the polymer. It would be expected that at least 1 crosslink per chain is needed to obtain an infusible network. However, the wide variation in chain lengths means that most of the longer molecules will contain at least one link and will form a network that can withstand the high temperature while retaining the shorter molecules within itself.

Solubility. Results obtained on solubility and swelling of crosslinked polystyrene are of considerable interest in polymer research $(9,10)$.

For a small amount of radiation and crosslinking, the molecular weight distribution is altered with a corresponding change in the viscosity of the product when dissolved. For such radiation doses certain molecules be- 
come linked together in pairs, threes, etc. As radiation dose is increased, a point is reached where a network of theoretically infinite extent is formed that includes a large number of the original molecules linked together into one gigantic molecule. By definition, this is insoluble and is referred to as the gel. Molecules not linked into this structure are still soluble and form the sol fraction. With further radiation and increased crosslinking, the gel fraction increases while the sol fraction diminishes. It can be shown that if only crosslinking occurs, the soluble fraction tends to zero with increasing radiation dosage, whereas, if some degree of random main chain fracture also is produced, the sol fraction tends to a finite amount characteristic of the ratio of chain fracture to chain crosslinking. For polystyrene, the curves of Fig. 7 indicate that little or no main chain fracture occurs under radiation.

Several theoretical studies have been made of the variation of gel fraction with crosslinking density-the latter being accurately proportional to radiation dose. The relation is found to depend on the initial molecular weight distribution. Experimental measurements of the gel fraction in polystyrene crosslinked by pile radiation have been combined with this theory to obtain information on this molecular weight distribution.

Irradiated polystyrene also has been used in connection with studies of swelling of crosslinked polymers. A crosslinked polymer will swell in compounds that are usually solvents for the uncrosslinked molecules. The solvent molecules (benzene, toluene) enter the network and try to force the molecules apart. This tendency is resisted by the elastic properties of the crosslinked network. With higher degrees of radiation (and, hence, of crosslinking) the swelling is reduced.

An approximate theory of this effect has been worked out (11). Irradiation provides the polymers of varying and known degrees of crosslinking that are necessary for full experimental analysis. Figure 8 shows that the theory is remarkably accurate over a wide range of degrees of crosslinking, in spite of several approximations made in the theoretical relationships. For example, the theory does not allow for the existence of a soluble fraction nor for the difference in crosslinking between

gel and sol (the gel will tend to contain a higher proportion of crosslinked polymers). A detailed analysis shows that the errors due to these various factors tend to cancel each other out so that the excellent agreement observed is to some extent fortuitous.

By calibrating the swelling of a series of polymers crosslinked by irradiation it should prove possible to provide laboratory standards of crosslinking for use in industrial and research laboratories dealing with polymer behavior.

\section{Rubber}

Rubber can be considered a naturally occurring polymer that, for most practical purposes, has to be vulcanized (crosslinked). This crosslinking can be achieved by exposure to ionizing radiation alone, without introducing sulfur or accelerators and without heat treatment (12). The process can take place in the absence of oxygen, and Fig. 9 shows that the degree of crosslinking is proportional to the radiation. The efficiency of crosslinking (number

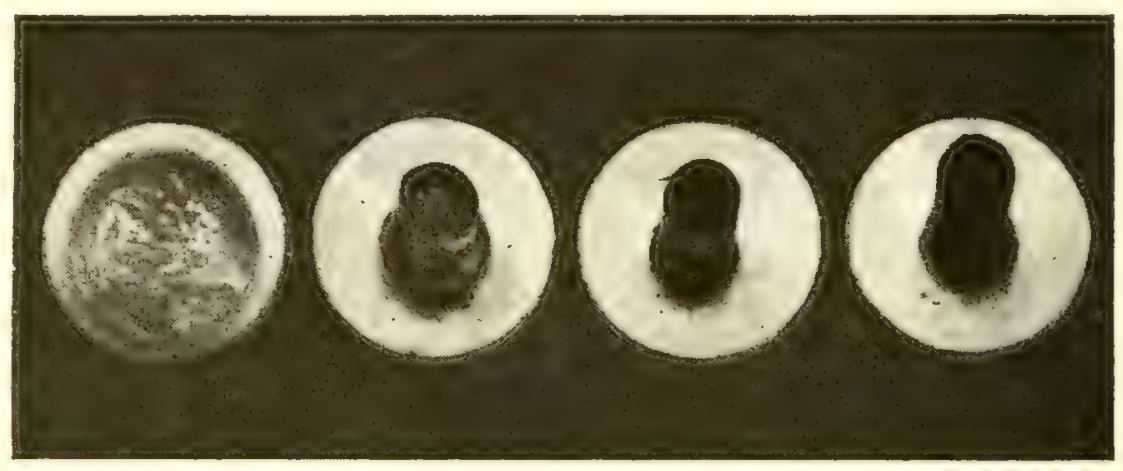

FIG. 6. Effect of heat on irradiated polystyrene. From left to right, samples received $0,0.6,2$, and 6 units of radiation. All samples were heated at $250^{\circ} \mathrm{C}$ for 30 minutes

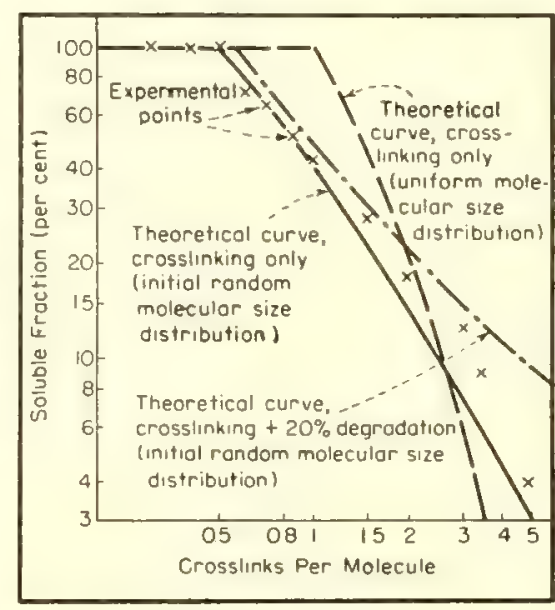

FIG. 7. Solubility decrease of irradiated polystyrene

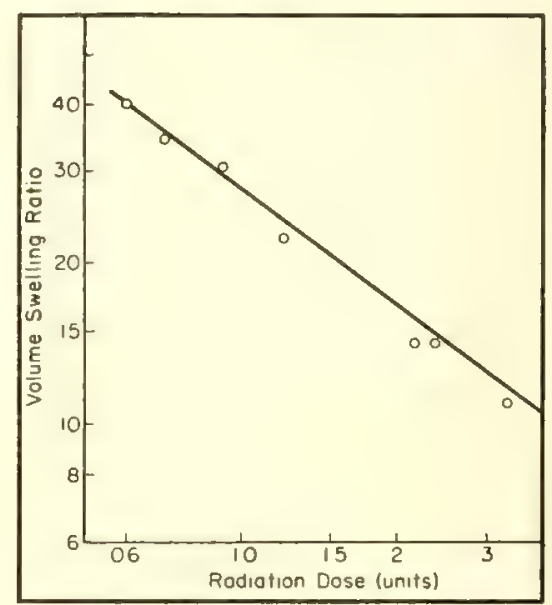

FIG. 8. Decrease in swelling in tolvene of polystyrene crosslinked by radiation 
will retain this orientation. The material produced will have different properties parallel and perpendicular to the direction of stretch.

\section{Other Polymers}

Numerous other long-chain polymers can be crosslinked by high-energy radiation; these include Nylon, polyvinyl acetate, polyvinyl alcohol, gutta percha, and Neoprene. Nylon, for example, becomes far more brittle and is less extensible after irradiation. With an adequate amount of crosslinking, creep largely can be suppressed.

The property of crosslinking is not confined to long-chain polymers with carbon atoms in the main chain. Figure 10 shows how lubricating oil solidifies. Silicones have been successfully crosslinked, the a mount of radiation required being smaller for the longer molecules, as is to be expected if crosslinking occurs at random. The crosslinked polymer formed is transparent and does not flow even at fairly elevated temperatures.

\section{CHAIN DEGRADATION}

The individual molecules in polytetrafluoroethylene, polymethyl methacrylate, polyisobutylene, and cellulose ąre degraded by high-energy radiation. The changes have varying effects.

\section{Polytetrafluoroethylene}

The essential difference between this polymer and polyethylene consists in the substitution of fluorine for hydrogen atoms on the carbon atoms of the main chain. The inert character of PTFE can be ascribed to the strong bond between the fluorine and carbon atoms.

If the crosslinking of polyethylene is due to the breaking of $\mathrm{C}-\mathrm{H}$ bonds and the removal of hydrogen having active carbon atoms that can crosslink, it is to be expected that the possibility of crosslinking in PTFE will be diminished greatly by the high stability of the C-F bonds. This behavior is, in fact, observed. On irradiation, PTFE tends to break up, and gases (particularly $\mathrm{CF}_{4}$ ) are released (18). Energy absorbed in the molecules breaks the $\mathrm{C}-\mathrm{C}$ bonds in the main chain rather than the stronger $\mathrm{C}-\mathrm{F}$ bonds.

Figure 11 shows that after 10 units of radiation, solid blocks of PTFE decompose to a coarse powder. Thin layers of the same material are more resistant. A detailed analysis (based

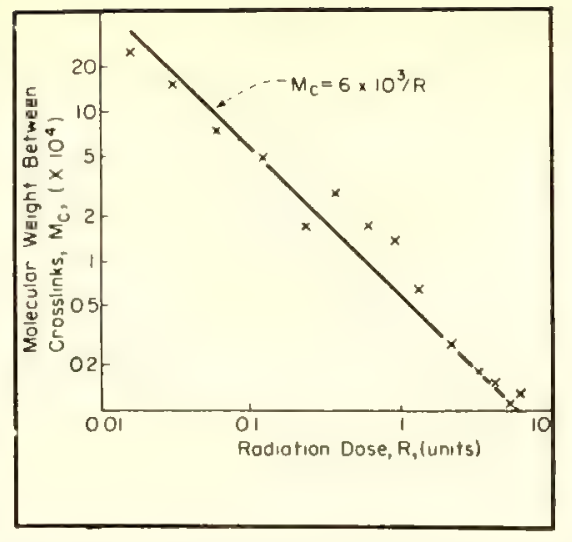

FIG. 9. Effect of radiation on crosslinking of rubber

on weight changes during irradiation) shows that weight losses are proportional to the surface area and to the square of the radiation dose.

These results are consistent with the hypothesis that chain fracture occurs at random, producing groupings the shortest of which are eventually evolved as $\mathrm{CF}_{4}$, provided they are produced close to the surface. Similar gases produced deeper within the specimen cannot escape, and the internal pressure of these gases (together with the weakening due to the reduction of

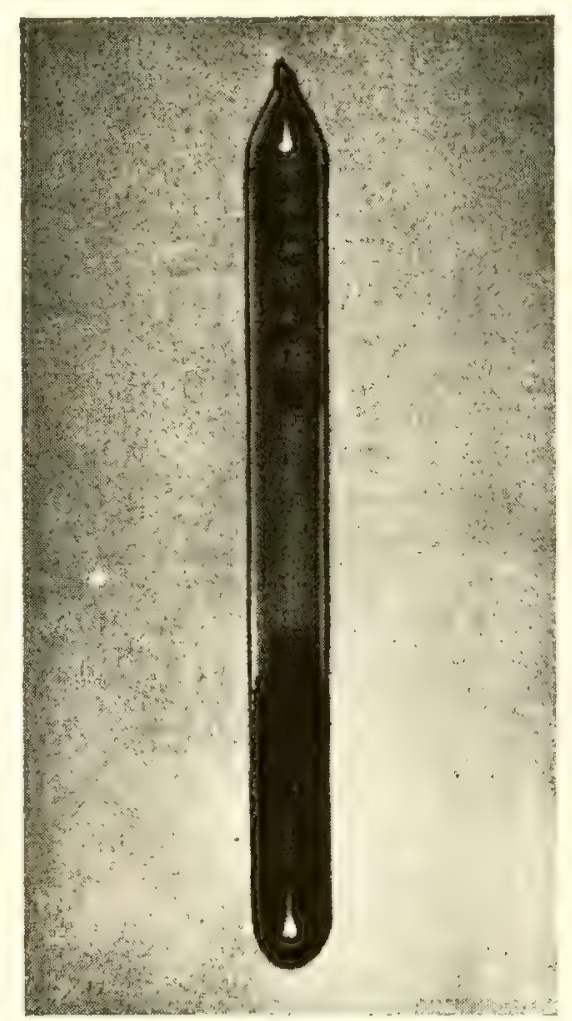

FIG. 10. Radiation-induced solidification (by crosslinking) of lubricating oil into a yellow, bubbled mass molecular weight arising from the fracture of the main chain) breaks the material into fragments.

The irradiated material softens at a lower temperature, and this may offer considerable processing advantages as compared with the more usual form of polytetrafluoroethylene.

\section{Polymethyl Methacrylate}

Polymethyl methacrylate shows most strikingly the degradative effects produced on the main and side chains by exposure to high-energy radiation (14, 15).

When exposed to small radiation doses (of the order of 0.5 units or less) specimens of the polymer in the form of rods or blocks appear to be almost unaffected; the only obvious physical changes are a slight yellow coloration and an increased brittleness. After irradiations of the order of one unit at about $80^{\circ} \mathrm{C}$., the polymer swells into a foam or mass of bubbles consisting mainly of hydrogen, carbon monoxide and carbon dioxide, which are the main breakdown products of the side chain. Increases in volume of the order of eight times can be produced.

Figure 12 shows that if irradiation is stopped before the foaming stage is reached, and the specimen is subsequently heated, the same process of foaming takes place. The higher the radiation dose, the lower is the temperature needed to produce this effect, at least for temperatures above about $70^{\circ} \mathrm{C}$.

The explanation is that radiation produces side-chain fracture at random throughout the specimen, and the resultant gases are retained in the solid until a combination of increased pressure (due to the temperature rise) and weakened polymer structure cause bubble formation. Bubbles do not occur near the surface; the gases produced there can escape.

This behavior may be of interest in connection with research into the diffusion of gases in solids and the formation of nuclei. The process is, in some respects, analogous to the formation of bubbles in boiling liquids; but it has the advantage (from the point of view of the investigator) that it takes place more slowly and can be stopped at any stage by sudden cooling.

Viscosity measurements of irradiated material give a measure of the molecular weight of the polymer. Molecular 
weight is found to decrease rapidly with radiation dose as

$$
M_{v}=K /\left(R+R_{0}\right)
$$

where $M_{v}$ is the viscosity average molecular weight (deduced from viscosity measurements), $K$ is a constant, $R$ is the radiation dose and $R_{0}$ is a small correction factor that can be considered as the "virtual" radiation dose needed to produce the initial molecular weight from an infinite chain. Therefore, measurement of viscosity appears to offer an extremely convenient means of estimating radiation doses of from 1-million roentgens upwards. All that is required is to irradiate a small piece of Perspex, dissolve it in a suitable solvent, and measure the viscosities of various concentrations at a given temperature. This method of measuring intrinsic viscosity is used often in polymer science and can give very reproducible results. The irradiated specimen may be kept for considerable periods before testing.

The two processes of side-chain and main-chain degradation are closely related, each main chain fracture being associated with the decomposition of approximately one side chain. The energy required per main-chain fracture is about $60 \mathrm{ed}$. Main-chain fracture takes place uniformly throughout the specimen; the absence of a surface effect is demonstrated by viscosity measurements. On the other hand, bubbling does not take place at a distance of about $1 \mathrm{~mm}$ from the surface. It has been shown that this is due to the evolution of the gas produced by side-chain fracture and that this process takes place during irradiation. The gases produced by irradiation can be retained in the polymer for considerable periods prior to bubbling by heating.

One can envisage a possible use for this irradiated polymer for heat insulation purposes. Blocks of irradiated polymethyl methacrylate could be placed around the vessel to be insulated. When heated, bubble formation would occur and insulating material would be produced in situ. The absence of bubbles in the outer layers would result in a material having a tough skin.

\section{Polyisobutylene}

Polyisobutylene has methyl groups replacing some of the hydrogen atoms in polyethylene. It degrades rapidly when irradiated and can be converted readily to a viscous liquid. At first thought this is surprising because we would expect hydrogen atoms to be evolved and, thus, active bonds to be made available for crosslinking. Diffraction data show that the molecular chain in polyisobutylene is arranged very differently from that in most longchain polymers, probably due to steric hindrance of the relatively large side groups. Therefore, the main chain is in a state of strain, and this weakness renders it more liable to fracture under radiation.

\section{APPLICATIONS}

The fields in which use of highenergy radiation can be applied to produce changes in long-chain molecules appear to be considerable.

Polymer research. It is possible to use radiation to produce polymers

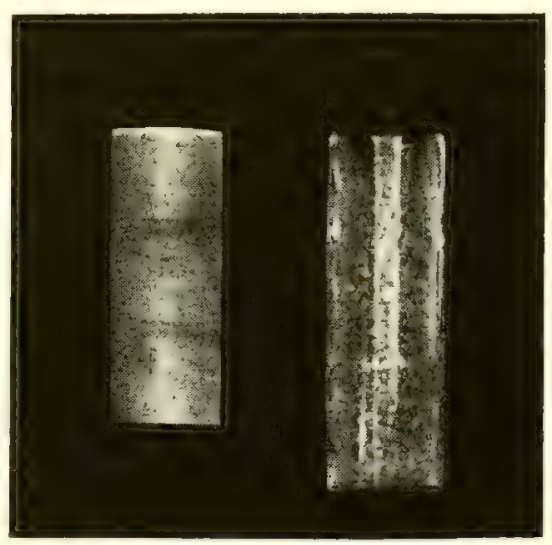

FIG. 11. Radiation-induced degradation of a solid rod of polytetrafluoroethylene into a coarse powder

(crosslinked to any required extent) and study their properties as a function of the degree of crosslinking. By using small radiation doses, a known degree of chain branching can be produced, and the effect of this on viscosity, softening or melting points, elastic properties, etc., then can be investigated quantitatively.

Where radiation-induced chain degradation occurs, it becomes possible to produce polymers of known molecular weight and of a given molecular weight distribution (namely, that corresponding to random chain fracture). This method has already been used to study the relationship between viscosity molecular weight and intrinsic viscosity for polyisobutylene and polymethyl methacrylate.

A further field is in the quantitative study of the ability of various types of bonding to resist the effect of highenergy radiation. The influence of the benzene ring in polystyrene has been mentioned, and other structures are being studied. This is of particular interest in such fields as copolymerization and in studies of the role played by sulfur and carbon black in rubber vulcanization.

The process is not limited to longchain polymers. It can be used to modify the properties of shorter molecules, such as those present in oils, although the radiation doses needed are much greater.

The method also can be applied to biological systems. A fuller understanding can be expected only when studies of simpler long-chain molecules

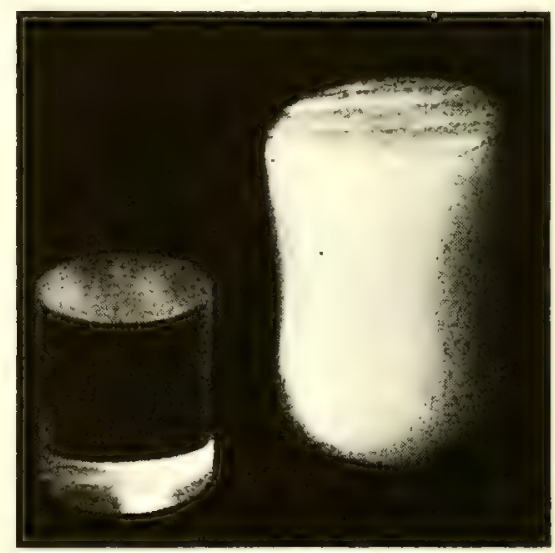

FIG. 12. After the slightly irradiated rod of polymethyl methacrylate (left) has been heated to about $120^{\circ} \mathrm{C}$, it is expanded by bubble formation

(in which the chemical and physical structures are known) permit making useful predictions of the mechanism of radiation effects.

Commercial possibilities. Apart from its use in research work, the possibility of producing crosslinked, degraded, and foamed material under accurately controlled conditions by a purely physical process can well have valuable commercial possibilities. In the long run, these will depend on the cost of irradiation, the enhanced value of the material, and the comparative cost of the competitive chemical process (where one is available).

There are two stages in which the process can be introduced. In the 
first stage, specialized materials of high intrinsic value when treated will be processed on a limited scale. Particularly, this method will be used to treat materials whose properties cannot readily be suitably modified by other techniques. In the second stage, irradiation may be in direct competition with existing chemical techniques. The time scale will be determined mainly by the availability and comparative costs of the various methods of obtaining high-energy radiation.

Sources. Since the phenomena observed occur equally well whatever the source of primary high-energy radiation (electrons, $\mathrm{X}$-rays, $\gamma$-rays, fast neutrons, etc.), the most suitable form of radiation will depend on the cost of the source and its ability to produce radiation of the most useful penetrating power. High-energy electrons obtained from linear accelerators have penetrations of only a few millimeters and, thus, seem most suitable for thin specimens. Gamma radiation is so penetrating that fairly considerable thicknesses of material are necessary if full use is to be made of the energy available. The use of reactors appears feasible. Irradiations can be carried out in the shield, thus making use of energy otherwise wasted. However, installation of conveyor belts or similar methods of feeding specimens into reactors, and post-irradiation storage in the immediate vicinity of the reactor might raise engineering objections.

Economics. Use of high-energy radiation on a commercial scale appears to be promising in a number of fields. In many cases the required radiation doses run into at least several million roentgens. It is too early to make any reliable calculations of costs, but very rough estimates concerned with the use of fission products may be of interest as an order-of-magnitude indication.

Assume that fission products will be available at $d$ dollars per curie (including cost of installation and operation) and have a half-life of $y$ years. Furthermore, consider a radiation treatment needing $r$ million roentgens, and consider that the plant is designed to have an efficiency $\eta$ (i.e., the proportion of emitted radiation captured by the irradiated specimens). A gamma source of $10^{5}$ curies, emitting $\gamma$-rays of $E \mathrm{Mev}$, will emit $\left(3.7 \times 10^{10} \times E\right.$ $\left.\times 10^{6} \times 10^{5}\right) \mathrm{ev} / \mathrm{sec}$, or $\left(2.3 \mathrm{E} \times 10^{9}\right)$ ergs/sec. This is equivalent to about $\left(3 E \times 10^{7}\right) \quad$ roentgen/sec for the materials generally dealt with, and will process $(30 \mathrm{E \eta} / \mathrm{r}) \mathrm{gm} / \mathrm{sec}$, or about $(1,000 \mathrm{E \eta} / r)$ tons/yr in full operation. The cost of treatment will be $10^{5} d / y$, or $(100 d r / y E \eta)$ per ton.

For example, if we assume an installed and operating cost of $\$ 1 /$ curie $(d=1)$, a treatment needing 1-million roentgens $(r=1)$, a source of half-life 10 years $(y=10)$, a gamma source emitting 1-Mev $\gamma$-rays $(E=1)$, and an over-all efficiency of $10 \%$, the cost of processing will be $\$ 100 /$ ton, or $5 \phi / 1 b$.

This estimate is very rough indeed; somewhat higher radiation doses are needed for many polymers. About 2- to 3-million roentgens are required to initiate gel formation in many types of polyethylene. The cost of producing, installing, and operating fissionproduct sources is not known, nor is the efficiency with which radiation can be absorbed. However, these figures indicate that costs are not necessarily exhorbitant for certain modifications in plastics.

In the cases of oils or shorter chain compounds in which several units of pile radiation are needed to produce useful changes, the costs are much higher. Two units of reactor radiation (or 100-million roentgens) would, on these bases, cost about $\$ 5 / \mathrm{lb}$. It is for this reason that the more promising commercial applications of crosslinking lie in the field of long-chain polymers. This is where small degrees of crosslinking result in marked and often desirable changes in physical properties.

A considerable need for sources of high-energy radiation appears to be arising for a variety of industrial purposes. Design of equipment to provide such sources at an economical cost is most desirable. The modification of polymer properties is only one of the possible applications of such sources.

They also could be used for sterilizing pharmaceuticals and foodstuffs.

\section{CONCLUSIONS}

The ability to crosslink or degrade long-chain polymers by high-energy radiation (under accurately controllable physical conditions) offers many interesting lines of research. The amount of information obtained to date is limited mainly by the effort devoted to this interesting new subject. Large-scale industrial use depends on the availability of suitable sources of radiation. The many applications possible can be discovered best by close collaboration between industrial scientists and radiation physicists and chemists.

\section{BIBLIOGRAPHY}

1. A. Charlesby. Effect of high energy radia tion on long chain polymers, Nature 171, 167 (1953)

2. E. J. Lawton, A. M. Bueche, J. S. Balwit. Irradiation of polymers by high energy radiation, Nature 172, 76, (1953)

3. O. Sisman, C. D. Bopp. Physical properties of irradiated plastics, ORNL-928 (1951)

4. Nucheonici 8, No. 6, 36 (1951)

6. A. Charlesby. Crosslinking of Polythene by pile radiation, Proc. Roy. Soc. (London) A, 215, 187 (1952)

6. A. Charlesby, M. Ross. Effect of crosslinking on the density and melting of Polythene, Proc. Roy. Soc. (London) A, 217, 122 (1953)

7. A. Charlesby, N. H. Hancock. Effect of crosslinking on the elagtic properties of Poly thene, Proc. Roy. Soc. (London) A, 218, 245 (1953)

8. A. Charlesby- Crosslinking and degradation of paraffin chains by high energy radiation. Proc. Roy. Soc. (London) A, 222, 60 (1954)

9. A. Charlesby. The solubility and molecular weight distribution of crosslinked polystyrene, J. Polymer, Sci. 11, 513 (1953)

10. A. Charlesby. The swelling properties of polystyrene crosslinked by high energy radiation, J. Polymer. Sci. 11, 521 (1953)

11. P. J. Flory, J. Phys. Chem. 46, 132 (1942)

12. A. Charlesby. The crosslinking of rubber by pile radiation, Atomics \$, 12 (1954)

13. A. Charlesby. The decomposition of polytetrafuoroethylene by pile radiation, AERE M/R-978 (Atomic Energy Research Establishment. Harwell, Eng*, 1952)

14. A. Charlesby, M. Ross. Breakdown of methyl methacrylate polymer by high energy radiation, Nature 171, 1153 (1953)

16. M. Ross, A. Charlesby. Effect of pile radiation on polymethyl methacrylate I, Atomics 4, (1953)

16. NUCLEONICA 11, No. 8,15 (1953) 


\title{
Radioactive-Tracer Techniques
}

in Paper Chromatography

\author{
Associating one or more radioisotopes with one or more substances separated \\ on a paper chromatogram offers the possibility of quantitative,
}

as well as qualitative, component analysis. A simple automatic device is used to

scan the paper radiometrically and locate the labeled substance

By F. P. W. WINTERINGHAM,* A. HARRISON, and R. G. BRIDGES

Department of Scientific and Industrial Research

Pest Infestation Laboratory, Slough, England

The USE of paper chromatography as a micro-analytical tool is well established. In many instances, paper chromatography is capable of separating substances in amounts below the limits of chemical detection. In other cases, the resolving powers of paper chromatography may be undiscovered or unexploited because of the lack of suitable chemical methods of detection.

The application of radiotracer techniques to paper chromatography may permit the separated components not only to be located and characterized but to be estimated quantitatively. In the combined technique, the principle is to associate one or more suitable radioisotopes with one or more of the components of the mixture, either before or after chromatography. The components separated on the chromatogram are then located and estimated by their associated radioactivity. In some cases, the radioactivity may be used for characterizing or identifying a particular component when its association may be made to depend upon a specific chemical reaction.

Locating and estimating the radio-

* Fellow of the Commonwealth Fund of New York. Present Address: Agricultural Experiment Station, University of California, Berkeley, Calif, activity is done by systematically scanning the paper chromatogram with a Geiger-Müller counter, for example, or by clamping whole or part of the paper chromatogram to a photographic plate in the dark. In the photographic method, the radioactive zones, if sufficiently intense, will have autoradiographed themselves.

The scanning method is capable of great sensitivity and is quantitative, while the photographic method is sometimes more convenient in certain qualitative work.

\section{Preparing the Chromatograms}

The association of a suitable radioisotope with one or more bands of the resolved components of the paper chromatogram may be brought about in any one of three ways.

1. Labeling of mixture before paper chromatography. The mixture for chromatography may already contain one or more labeled components as in tracer experiments. For example, Benson et al. (1) studied the path of radiocarbon in the photosynthetic incorporation of this element by respiring plant cells. Labeled intermediates were located and estimated on paper chromatograms by autoradiographic and counting techniques.
The metabolism of a radioactive bromine analog of DDT absorbed by susceptible and DDT-resistant houseflies was studied $(2,3)$ by the combined techniques in which paper chromatograms were scanned by the methods to be described.

Keston, Udenfriend, and Levy (4) analyzed an unlabeled mixture of amino acids by treating the mixture with a labeled reagent before applying paper chromatography. The paper chromatograms were cut up into small sections which were mounted in turn below a Geiger-Müller tube and the measured rate of count plotted against distance along the strip. In this way they were able to determine quantitatively glutamic acid, serine, glycine, and alanine in a mixture of a mino acids.

Another example in which the mixture already contained labeled components is illustrated by radiochromatogram $A$ in the chart opposite. A paper chromatogram was run on a protein hydrolysate prepared from wheat grown on a solution containing $\mathrm{S}^{35}$. It was scanned radiometrically and the radiochromatogram shown was obtained by plotting the $\mathrm{S}^{35}$ activity against the $R_{f}$ value. $\dagger$ The two peaks correspond to the cystine and methionine biosynthesized in the plant. 


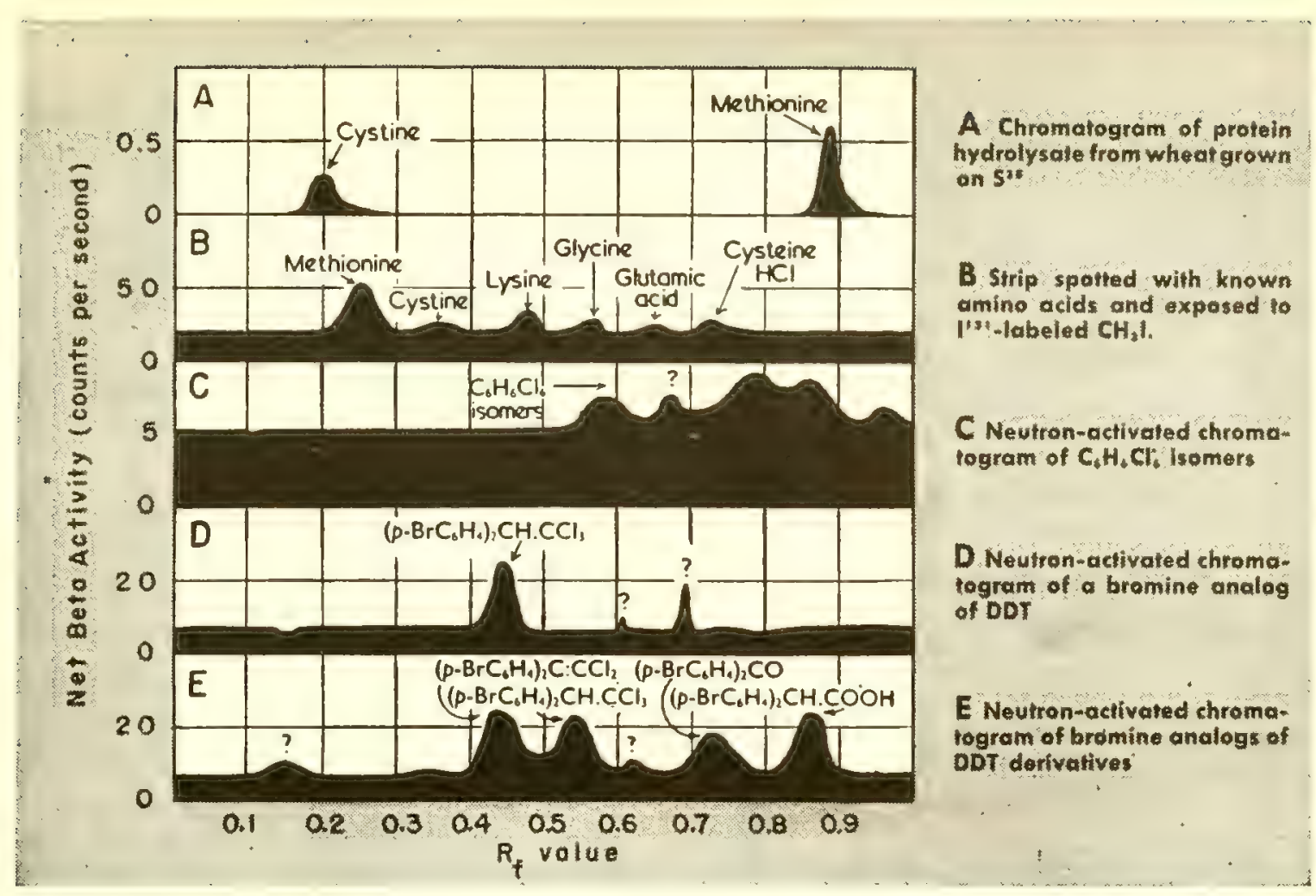

2. Treatment of the paper chromatogram with a labeled reagent. The possibilities of this method have received little attention. We have made experiments on the possible location of certain amino acid groups By methylation with I ${ }^{131}$-labeled methyl iodide. No attempt has been made to apply this particular reaction as a quantitative tool, and its description here is merely included to illustrate the principles of the method which, it is believed, has cunsiderable potentialities.

The important condition for the successful application of this method is that a labeled reagent be chosen which will selectively react with one or more of the compounds separated on the paper chromatogram but not with the paper material itself.

A strip of Whatman No. 1 paper was spotted with solutions of different amino acids, dried, and exposed to methyl iodide- $\mathrm{I}^{131}$ vapor at room temperature. Methylation of the amino acids resulted in the liberation of $I^{131}$ in the amino acid zones, particularly in the methionine zone, probably as a result of the formation of the methyl methionine sulphonium iodide. After

† The $R$, value is the distance traveled by the individual band divided by the distance traveled by the solvent front. pumping off the excess methyl iodide, the paper was scanned radiometrically. The plotted radiochromatogram is shown as $B$ above. The high background was apparently due to some methylation of the paper.

3. Neutron activation of the paper chromatogram. In this method, the finished chromatogram undergoes neutron irradiation in a reactor after the residual solvent is dried off. For the success of this method, the separated components must contain or be associated with an element of suitable activation cross section so that it can be readily assayed against any background radioactivity of the paper chromatogram itself.

Fortunately, neither carbon, hydrogen, nor oxygen of cellulose possesses significant neutron activation cross sections in this respect, and it was found that in washed Whatman No. 1 paper the wide range of trace metals almost certainly present did not give rise to any serious effects under the moderate conditions of some experimental irradiations in the Harwell reactor.

This method has been applied successfully to the radioactivation of bromine containing organic compounds by taking advantage of the
$\mathrm{Br}^{81}(n, \gamma) \mathrm{Br}^{82}$ reaction. For example, an inactive bromine analog of DDT and three derivatives were separated on a reversed phase paper chromatogram (3). The chromatogram was then irradiated for three days in the Harwell reactor at a flux of $10^{10} \mathrm{n} / \mathrm{cm}^{2} /$ sec and was scanned a few days later. The resulting radiochromatogram obtained is shown by $E$ above. Only the DDT analog was present on the chromatogram scanned in $D$. The small unidentified additional peaks may have been due to activated chromatographed impurities or to contamination.

In the method of neutron activation, one important point must be borne in mind. The energy of recoil from the gamma emission in the $(n, \gamma)$ reaction is usually more than sufficient to rupture any chemical bond between the target atom and the rest of the molecule. The induced radioactivity, therefore, can only be associated with the component and cannot be used as a tracer in further tests with the eluted substance.

Another complication may be due to the volatile nature of the recoil-freed isotope which can result in loss of activity and contamination of other parts of the chromatogram. For example, in the neutron activation of 


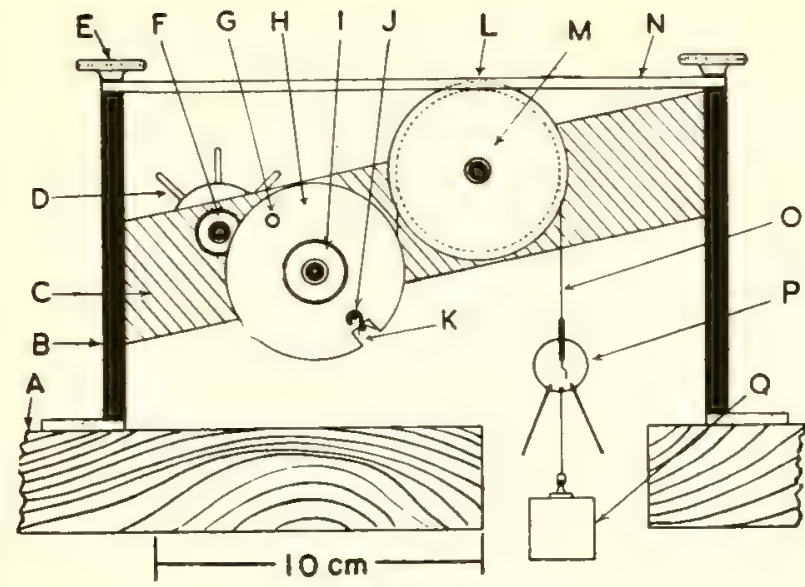

\section{Front elevation}

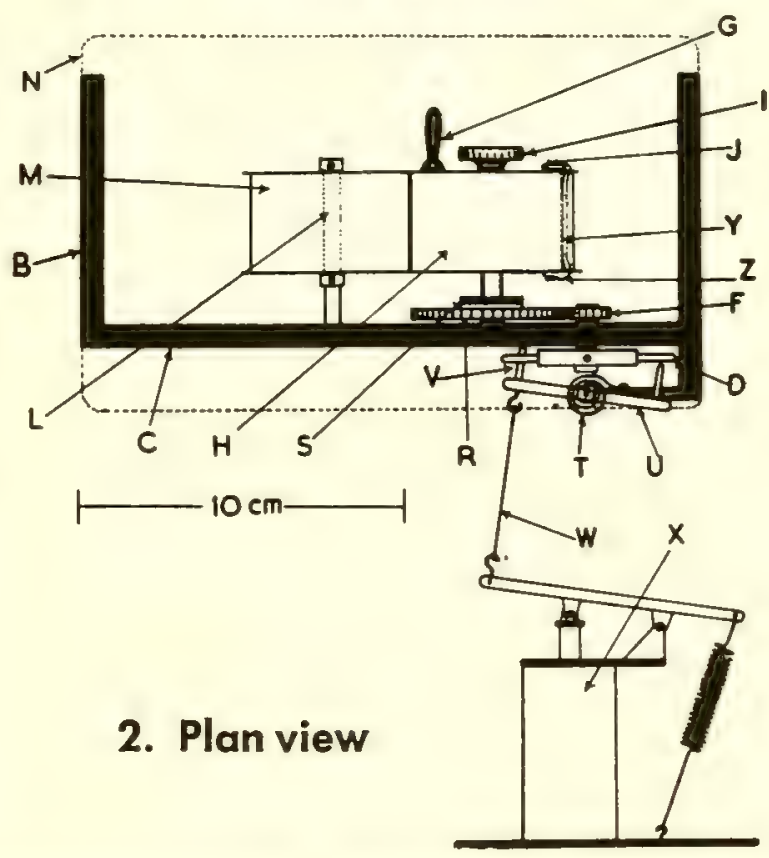

\section{How Automatic Scanner Works}

Paper strip $O$ is wound around $5.05-\mathrm{cm} \operatorname{drum} H$ and passes over 5.05 -cm idler roller $M$ under tension due to $100 \mathrm{gm}$ weight $Q$ that is fastened to the free end of strip $O$ by means of "bull-dog" clip $P$. Each drum possesses a 3-mm flange on both sides. The other end is held to drum $H$ by spring-steel wire $Y$ passing through drum slot $K$. The wire is anchored to the drum $H$ at $J$ and screwed on the opposite side at $Z$. Drum $H$ drives an eight-bladed escapement wheel $D$ by a $4: 1$ gear $F$-R so that 38 of 1 revolution of $D$ allows strip $O$ to advance, under tension, almost exactly $0.5 \mathrm{~cm}$. The diameter of drum $H$ is such that the changing thickness of paper on the unwinding drum has a negligible effect on the distance the paper moves with each escapement movement. The mean value of this distance is exactly $0.5 \mathrm{~cm}$ for the average chromatogram. Roller $M$ is.mounted exactly below a $13 / 8$-inch $\times 0.5-\mathrm{cm}$ slot $L$ cut in the platform $N$ so that a transverse $0.5-\mathrm{cm}$ section of strip $O$ is exposed through the slot.

The over-all effect is that for every escapement movement consecutive $0.5-\mathrm{cm}$ sections of the paper strip are exposed to the thin end-window WI of the Geiger-Müller tube GM which is housed inside the lead castle $L C$. Milled nuts $E$ allow the platform to be removed for cleaning purposes, etc. Heavy frame $B$ is screwed to the edge of laboratory bench $A$ through which a suitable hole is cut for the descending strip $O$. Drum and roller shafts run in phosphor-bronze bearings mounted in bearing plate $C$.

To date, scanning has been limited to beta counting, the Geiger-Müller tube being relatively insensitive to the gamma rays from bromine-82, iodine-131, etc. Platform $N$ must be sufficiently thick to eliminate all beta particles from neighboring but unexposed sections of the strip, an important factor in the resolving power of the scanner; 16-inch steel plate has been found to be satis- organic bromine compounds, the recoil-freed bromine is probably present in part as hydrogen bromide. It was found, however, that if the paper strip were rolled up with a second plain strip of paper, the resulting contamination was negligible.

One possible application is to the analysis of a mixture of the isomers of hexachlorocyclohexane, the gamma isomer of which is a well-known insecticide. A mixture containing $10 \mu \mathrm{g}$ of the $\alpha, \beta, \gamma$, and $\delta$ isomers was chromatographed under the conditions used for the DDT analogs (3). The chromatogram was then irradiated in a flux of $10^{11} \mathrm{n} / \mathrm{cm}^{2} / \mathrm{sec}$ for one week and scanned a fortnight later. The radiochromatogram obtained is shown in $C$, the peaks of which are caused by
$\mathrm{S}^{35}$ arising from the chlorine of the partly separated isomers by the $\mathrm{Cl}^{35}(n, p) \mathrm{S}^{35}$ reaction. This particular application is being investigated further.

\section{Radiometric Scanning}

Having prepared the paper chromatogram so that the resolved components are labeled or associated with a suitable radiotracer, the distribution of the tracer must be determined quantitatively.

Fink et al. (5) have located $\mathrm{I}^{131}$ labeled compounds by placing the chromatogram against an X-ray plate in the dark so that the radioactive zones could be located autoradiographically after fixing and developing the exposed plate. This method does not lend itself easily to quantitative work, and it is far less sensitive than counting techniques.

It has been estimated (6) that, to obtain a reasonable contact autoradiograph of a $\mathrm{Br}^{82}$-labeled compound, a radioactive disintegration density of the order of $10^{2}$ disintegrations per $\mathrm{cm}^{2}$ (of paper chromatogram, in this case) would be required. If this figure were obtained, say, in a 24 -hour exposure, it would correspond initially to about 0.5 microcuries of $\mathrm{Br}^{82}$. One hundredth of this activity, or $5 \times 10^{-3}$ microcuries, could be determined to within $\pm 5 \%$ by Geiger counting for one minute using typical apparatus.

A simple method of scanning a paper chromatogram is to cut it up into small equal sections and mount them, in 
factory. Platform and frame are stainless steel, the drums aluminum.

Left prong $V$ of escapement rocker $U$ normally engages a blade of escapement wheel $D$ under the tension of a small spring $T$ attached to the rocker and frame. The armature of solenoid $X$ momentarily pulls back rocker $U$ via connecting rod $W$ against the spring; when rocker $U$ returns to normal position, $D$ rotates $1 / 8$ of a complete revolution but no more. Friction clutch $S$ becomes disengaged by relaxing milled nut $I$ so that drum $H$ is free of $R$ and, hence, the escapement mechanism. New strips can then be wound up by means of handle $G$.

Lead castle $L C$ is in two parts to facilitate handling. Tube $G M$ is placed exactly over $L$. The lower half of $L C$ is then lowered into position so that prongs $P R$ engage the GM flange. The top half of $L C$ is then placed in position so that contact disk $C D$ engages anode $A N$. High-voltage and ground connections'are then made at $A L$ and $E T$. Insulating glass tube $G L$ is flared at the lower end to guide $A N$ onto $C D$.

Solenoid $X$ is simply the electromechanical register unit taken from a type 1003 timing unit which is available in many laboratories equipped for beta counting. Its operation requires $50-\mathrm{v}$ d-c pulses which can be drawn from the timing unit at intervals of $1 / 2$ or 1 second, or $1 / 2$ or 1 minute. By inserting additional scale-of-two units, solenoid $X$ can be made to operate at 2-, 4 -, or 8-minute intervals, etc. The probe unit amplifies pulses from tube $G M$ and feeds them to a type $1138 \mathrm{~A}$ ratemeter.

The $0-100 \mathrm{mv}$ potential difference across the output potentiometer is proportional to the mean rate of count; alternatively the ratemeter output may be obtained as a $0-5$-ma current in the same circuit. This output is fed to a pen-recording milliammeter or to a self-balancing potentiometer. The rate of count is thus automatically plotted against distance along the strip.

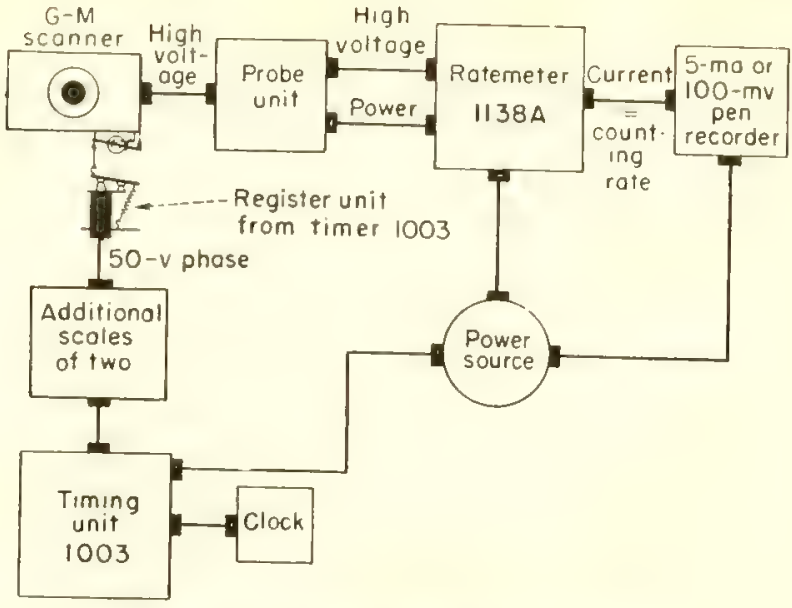

\section{Unit-to-unit connections}
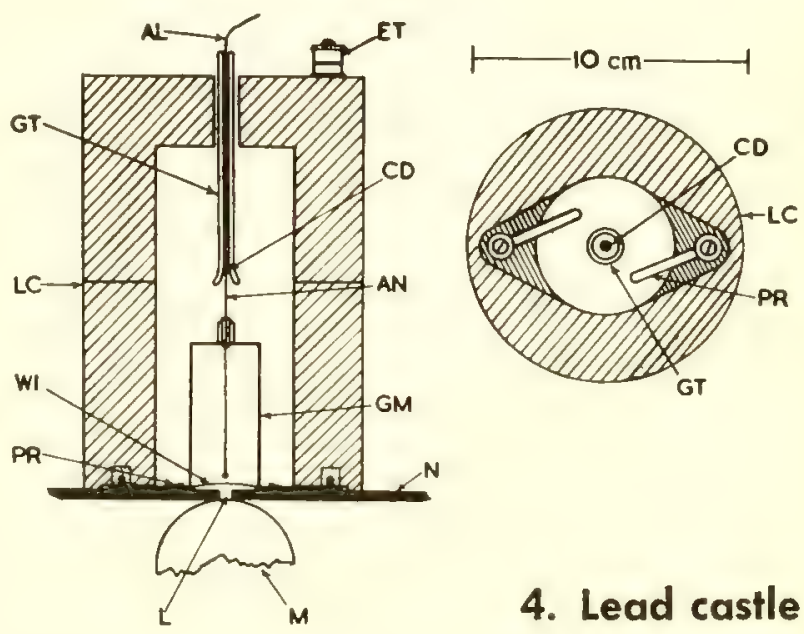

4. Lead castle turn, below an end-window Geiger tube. This method is very tedious, and unless each section is cut exactly and mounted precisely, errors will result.

Automatic scanning device. The four diagrams and text above show the components and working order of a device for automatic scanning of $1 \frac{1 / 8}{8}$ inch unidimensional paper chromatograms. Whatman No. 1 paper is conveniently available in rolls of this width. Two-dimensional chromatograms are scanned by cutting the sheet into the equivalent number of strips as described later.

The advantages of this device are: 1) The paper chromatogram is autoratically scanned in a geometrically uniform manner. 2) The movement of the paper is controlled by a simple escapement mechanism that is operated by conventional timing equipment. 3) Provision is made for plotting radiochromatograms automatically. 4) All parts of the scanning unit are easily accessible for cleaning purposes, decontamination, etc.

In our experience, the milliammeter recorder was more suitable than the self-balancing potentiometer because the tendency of the latter to overbalance gave rise to fluctuations greater than the intrinsic statistical fuctuations of particle counting. The statistical fluctuations of the ratemeter output for a given mean rate of count can be modified by the variable capacitance of the integrating circuit of the ratemeter. The corresponding inte- grating time must be less than the time intervals of the scanner or the ratemeter will not be able to keep pace. On the other hand, the integrating time must be sufficiently large for the statistical fluctuations in the counting rate to be small compared to the radioactivity peaks of the chromatogram.

\section{Quantitative Interpretation}

A separated component will normally be distributed over several sections of the paper chromatogram. Ideally, the counting geometry will be the same for each section. The weight of the component is invariably small compared to the weight of the paper, so that self-absorption of the beta particles assayed will depend only 
TABLE 1-Effect of Paper Density Variation on Self-absorption

\begin{tabular}{|c|c|c|}
\hline Isolope & $\begin{array}{c}\text { Proportion of } \\
\text { beta particles } \\
\text { unabsorbed by } \\
\text { paper of mean } \\
\text { density } 8.785 \\
m g / \mathrm{cm}^{2}\end{array}$ & $\begin{array}{l}\text { Variation of self- } \\
\text { absorption correc- } \\
\text { tion factor corre- } \\
\text { sponding to } \pm 10 \% \\
\text { tariation in paper } \\
\text { density }\end{array}$ \\
\hline $\mathrm{C}^{14}$ & 0.366 & $\pm 7.4 \%$ \\
\hline $\mathrm{S}^{35}$ & 0.431 & $\pm 6.5 \%$ \\
\hline $\mathrm{Br}{ }^{82}$ & 0.787 & $\pm 2.4 \%$ \\
\hline$I^{131}$ & 0.828 & $\pm 1.9 \%$ \\
\hline $\mathrm{Cl}^{36}$ & 0.843 & $\pm 1.7 \%$ \\
\hline $\mathbf{P}^{32}$ & 0.957 & $\pm 0.5 \%$ \\
\hline
\end{tabular}

upon the density or weight per unit area of the paper strip. For practical purposes, the density can be considered constant.

On this basis, the total weight $w$ of the labeled component is proportional to the sum total of the net rates of count (corrected for background, radioactive decay, deadtime losses, etc.) of the relevant sections, i.e.

$$
w=K \Sigma a
$$

where $a$ is the corrected net rate of count of each relevant section. The constant $h$ depends upon the specific radioactivity of the original component and upon the over-all counting efficiency.

In practice it has been found convenient to determine $K$ by scanning a chromatogram run with a known weight of compound of known specific activity. Its position also serves to identify the peaks of the original chromatogram.

When the radiochromatogram is plotted as net rate of count against distance along the strip, $w$ is proportional to the area enclosed by the relevant part of the curve (see below). With the scanner described, it has been found convenient to measure the areas plotted on the recorder by means of a planimeter. In comparing different radiochromatograms quantitatively, it is important that they be plotted on this basis since the total radioactivity is the product of the mean rate of count (over the relevant sections) and the distance in an absolute sense over which the radioactivity is spread. For the purposes of identification, however, the $R$, value is the important property, and the radiochromatogram should then be plotted as net rate of count against $R_{f}$ value. Other factors of significance in quantitative work are discussed below.

Decay corrections. Usually the time taken to scan a unidimensional paper chromatogram is small compared to the half-life of the isotope being assayed. Thus corrections for decay may be applied to the measured areas as a whole and based on the mean time of scanning. When the time of scanning is not relatively small, the time scale corresponding to each part of the strip must be recorded and different corrections applied according to position on the strip.

Geometry effects. Ideally, the geometrical distribution of radioactivity would be the same for every exposed section of the paper chromatogram. In practice, variations occur, especially in strips cut from a two-dimensional sheet when the radioactive zone would not be expected to be in the middle region of the section, except by chance.

The beta-particle sensitivity of an end-window-type Geiger tube varies according to the position of the source below the window; the more off center the source, the less the sensitivity. For this reason, Boursnell (7) has used a large-area window tube for scanning. In unidimensional chromatograms, however, the radioactive zones appear to be sufficiently similar to permit assays based on adequate controls with known amounts of radioactive material.

For two-dimensional chromatograms, a tube of larger window area than the one illustrated on page 55

TABLE 2-Radiometric Assay of $\mathrm{Br}^{82}$-labeled Derivatives Separated on Paper Chromatograms

\begin{tabular}{|c|c|c|c|}
\hline $\begin{array}{l}\text { Compound on } \\
\text { mixture }\end{array}$ & $\begin{array}{l}\text { Weight applied } \\
\text { to chromatogram } \\
\text { (micrograms) }\end{array}$ & $\begin{array}{c}\text { Recovery } \\
\text { by scanning } \\
(\%)\end{array}$ & Remarks \\
\hline$\left(p-\mathrm{BrC}_{6} \mathrm{H}_{4}\right)_{2} \mathrm{CH} . \mathrm{CCl}_{3}$ & 0.2 & 109 & \multirow{6}{*}{$\begin{array}{l}\text { Labeled compound applied } \\
\text { singly }\end{array}$} \\
\hline$"$ & 1.0 & 104 & \\
\hline " & 1.0 & 111 & \\
\hline$"$ & 2.0 & 105 & \\
\hline$"$ & 5.0 & 85 & \\
\hline$"$ & 5.0 & 92 & \\
\hline$\left(p-\mathrm{BrC}_{6} \mathrm{H}_{4}\right)_{2} \mathrm{CH} \cdot \mathrm{CCl}_{3}$ & 1.0 & 111 & \multirow{5}{*}{ Applied as a labeled mixture } \\
\hline$+\left(p-\mathrm{BrC}_{6} \mathrm{H}_{4}\right)_{2} \mathrm{C}: \mathrm{CCl}_{2}$ & 0.5 & 97 & \\
\hline$\left(p-\mathrm{BrC}_{6} \mathrm{H}_{4}\right)_{2} \mathrm{CH} . \mathrm{CCl}_{3}$ & 50 & 107 & \\
\hline$+\left(p-\mathrm{BrC}_{6} \mathrm{H}_{4}\right)_{2} \mathrm{C}: \mathrm{CCl}_{2}$ & 50 & 118 & \\
\hline$+\left(p-\mathrm{BrC}_{6} \mathrm{H}_{4}\right)_{2} \mathrm{CH} \cdot \mathrm{COOH}$ & 50 & 100 & \\
\hline$\left(p-\mathrm{BrC}_{6} \mathrm{H}_{4}\right)_{2} \mathrm{C}: \mathrm{CCl}_{2}$ & 20 & 126 & \multirow{4}{*}{$\begin{array}{l}\text { Applied as a mixture; acti- } \\
\text { vated after chromatogra- } \\
\text { phy }\end{array}$} \\
\hline$+\left(p-\mathrm{BrC}_{6} \mathrm{H}_{4}\right)_{2} \mathrm{CO}$ & 20 & 62 & \\
\hline \multirow[t]{2}{*}{$+\left(p-\mathrm{BrC}_{6} \mathrm{H}_{4}\right)_{2} \mathrm{CH} . \mathrm{COOH}$} & 20 & 90 & \\
\hline & Average recovery & $\longdiv { 1 0 1 . 2 }$ & \\
\hline
\end{tabular}


of Whatman No. 1 paper was $\pm 3.9 \%$; mean density was $8.785 \mathrm{mg} / \mathrm{cm}^{2}$

The effect of a $\pm 10 \%$ variation on the self-absorption and on the corresponding corrections in the assay of some typical isotopes was calculated by means of Libby's self-absorption equation (8). The results are shown in Table 1. Even in the case of the soft beta emitters, it is unlikely that this variation will seriously impair quantitative work.

Resolution. When two or more labeled components are incompletely resolved, one must decide arbitrarily how to divide the area of the plotted radiochromatogram. Alternatively, an ingenious technique developed by Keston et al. (4) may be useful. In this method, one of the unresolved components is labeled by means of a second isotope of sufficiently different radiation characteristics to enable its boundary on the chromatogram to be determined by selective scanning.

Corrections for recorder drum speed, rate of scanning, and background. The radiochromatogram is plotted by the recorder as counting rate (ordinate) against distance along the strip (abscissa). In Eq. 1, the activity or rate of count $a$ will be constant (neglecting statistical fluctuations and decay) for each exposed section; $\mathbf{\Sigma} a$ is therefore proportional to the area $A$ enclosed by each radioactivity peak of the radiochromatogram. Thus $w=K^{\prime} A$, where $K^{\prime}$ depends upon the over-all counting efficiency (observed rate of count per absolute disintegration rate) and upon the units in which $A$ is measured.

In practice, it is convenient to adjust the recorder drum speed (provision is made for this in the majority of recorders) so that radiochromatograms plotted from $R_{f}=0$ to $R_{f}=1$ at different scanning rates will be represented by equal or comparable distances along the recorder paper. The scanning rate will be varied (by altering the timer-controlled intervals between pulses fed to the scanner solenoid) according to the level of activity on the paper strip. For example, as discussed later, low activities require a lower scanning rate than will high activities for the same statistical accuracy.

Corrections for differing recorder drum speeds and scanning rates in quantitative work are then made in the following manner: Suppose, in the calibration of the apparatus for quan- titative work, a known weight $w$ of a labeled substance is scanned. Let $s$ be the scanning rate, $d$ the recorder drum speed, and $r$ the rate of count corresponding to unit scale (ordinate) of the recorder. Let $A$ be the area enclosed by the radioactivity peak corresponding to the labeled substance of the radiochromatogram. 'This area on the recorder paper may be measured in any convenient unit (to suit a particular planimeter, for example).

Area $A$ must be corrected for decay in the usual way and for background, which is simply the observed counting rate over that part of the strip free of labeled material. Then $K^{\prime}=w / A$.

A second strip containing an unknown weight $w^{\prime}$ of the same labeled substance is now scanned at a rate $s^{\prime}$ and recorder drum speed $d^{\prime}$. Let $A^{\prime}$ be the corresponding net area on the radiochromatogram, and $r^{\prime}$ the rate of count equivalent to unit scale of the recorder. It follows that

$$
w^{\prime}=K^{\prime} A^{\prime} \frac{d s^{\prime} r^{\prime}}{d^{\prime} s r}
$$

In the apparatus described above, $r^{\prime} / r$ will be $10^{ \pm_{n}}$, where $n$ is $0,1,2,3$, etc., corresponding to the sensitivity ranges of the ratemeter. The fraction $s^{\prime} / s$ may be more conveniently replaced by $t / t^{\prime}$, where $t$ is the timer interval or time of exposure of each section, and will therefore be a simple multiple of one second or of one minute.

Preçision in quantitative work. In radiometric assays, one rarely tries to determine a labeled substance by calculating the absolute disintegration rate from the observed counting rate. Assays are usually based on the rate of count observed with a known weight of the substance under standardized conditions.

In the methods described here, assays are similarly based on radiochromatograms obtained with known weights of labeled material. Under these circumstances, mean recoveries are complete, and precision or reproducibility becomes the important point. The principal factors affecting precision, apart from manipulative errors such as micropipetting on to the paper strip, are self-absorption, statistical errors (9) inherent in all random particle counting, and geometry.

The effects of self-absorption have been discussed. The statistical coefficient of variation of a net count obtained as the difference between the total count $N_{T}$ and the background count $N_{B}$ over the same interval of time is

$$
\pm 100 \sqrt{ } N_{T}+\bar{V}_{B}^{-} /\left(N_{T}-N_{B}\right)
$$

It can be shown that the coefficient of variation of an assay by scanning is

$$
\pm 100 \sqrt{d\left(A_{T}+A_{B}\right)} /\left(A_{T}-A_{B}\right)
$$

where $A_{T}$ and $A_{B}$ are the total and background areas in $\mathrm{cm} / \mathrm{cps}$ and $d$ is the recorder drum speed in $\mathrm{cm} / \mathrm{sec}$.

The particular contribution of geometrical factors to variation, such as an asymmetrical distribution of active material over an exposed section, has not been investigated.

Data are available, however, on the over-all variation due to all errors. including those due to manipulation, incomplete chromatographic resolution, etc., as a result of some resolution experiments with $\mathrm{Br}^{82}$-labeled derivatives. Single labeled substances or simple mixtures were resolved on reversed phase paper chromatograms (3) and scanned. In one case, the separated components were activated after chromatography. All recoveries were estimated in terms of a reference strip run with a known weight of pure substance and scanned. The results are shown in Table 2. They are not intended to demonstrate the sensitivity of the methods. In all cases, fairly active samples were used, and weights of the order of $1 / 100$ or $1 / 1,000$ of those given could have been assayed with comparable accuracy.

The authors are indebted to Mr. W. K. Cordaroy for constructing the escapement mechanism and scanning frame and to the E.R.D. Engineering Company for constructing the specially designed lead castle.

This paper was first presented in London to the Physical Methods Group of the Society of Public Analysts in May, 1951, and acknowledgments are due to the editors of The Analyst for permission for its publica. tion in NucLEoNics. The contribution is made by permission of the Department of Scientific and Industrial Research.

\section{BIBLIOGRAPHY}

1. A. A Benson, J A. Bassham, M. Calvin T. C Goodale, V. A. Haas, W. Stepka, J. Amer Chem. Soc. 72, $1710(1950)$

2. F. P. W. Winteringham, P. M. Loveday, A Harrison, Nature 167, 106 (1951)

3. F. P. W. Winteringham, A. Harrison, R. G Bridges, Nature 166, 999 (1950)

4. A. S. Keston, S. Udenfriend, M. Levy, J. A mer. Chem. Soc. 69, 3151 (1947)

5. 1R. M. Fink, C. E. Dent, K. Fink, Nature 160, 801 (1947)

6. F, P. W. Winteringham, J. Chem. Soc. S416 (1949)

\%. J, C. Boursnell, Nature 165, 399 (1950)

8. W. F. Libby, Anal. Chem. 19, 2 (1947)

9. I. J. Rainwater, C. S. Wu, Nocheonics 1, No. 2, $60(1947)$ 


\title{
Apruto \\ RADIATION CASE HISTORY Irradiated Males
}

NO. 3

\author{
In 16 months a fly factory in a converted airplane hangar \\ furned out three billion sterile flies to eliminate a costly \\ menace to cattle herds of Southeastern ranches
}

By MERRILL E. JEFFERSON, Department of Agriculture, Washington, D. C.

Thanks To Radiation, cattle herds of Southeastern United States are no longer menaced by the screwworm fly. A fly factory capable of producing 100 million sterile flies a week overwhelmed the natural fly population with sterile males, raised and sterilized in captivity and then released from airplanes.

So successful was the method that the factory discontinued production last November 14-mission completedseveral months ahead of the anticipated schedule. Appropriations of $\sim \$ 10$-million from the Federal Government and Southeastern States supported the effort to eliminate a pest costing Southeastern livestock producers an average of $\$ 20$-million a year.

\section{Life of the Fly}

The red-eyed, blue-bodied screwworm fly (Callitroga hominivorax) can kill a full-grown steer in $\mathbf{1 0}$ days. The female lays its eggs in any open wound of a warm-blooded animal. The eggs hatch and tiny larvae (screwworms) burrow into the wound and gouge out a pocket in the flesh.

After they have lived for four or five days and done their damage to the host animal, the larvae drop to the ground and develop into flies. The total life cycle is only $21-28$ days, and because the insects are subtropical, a cold winter kills off the pests. They remain a menace, however, when they are introduced into mild climates like those of the southern states, the West Indies, Mexico and South America. The flies migrate northward each season or spread by movement of infested animals. They can survive the winter in South America, Mexico, Florida and extreme southern parts of the Southwest.

\section{Eradication}

Usual methods of insect elimination are not good enough for the screwworm problem. You can treat all domestic animals with ointments and sprays. These, however, are

FIG. 1. GROUND FLOOR OF FLY FACTORY is where pupae develop into sterile flies ofter hatching on floor above

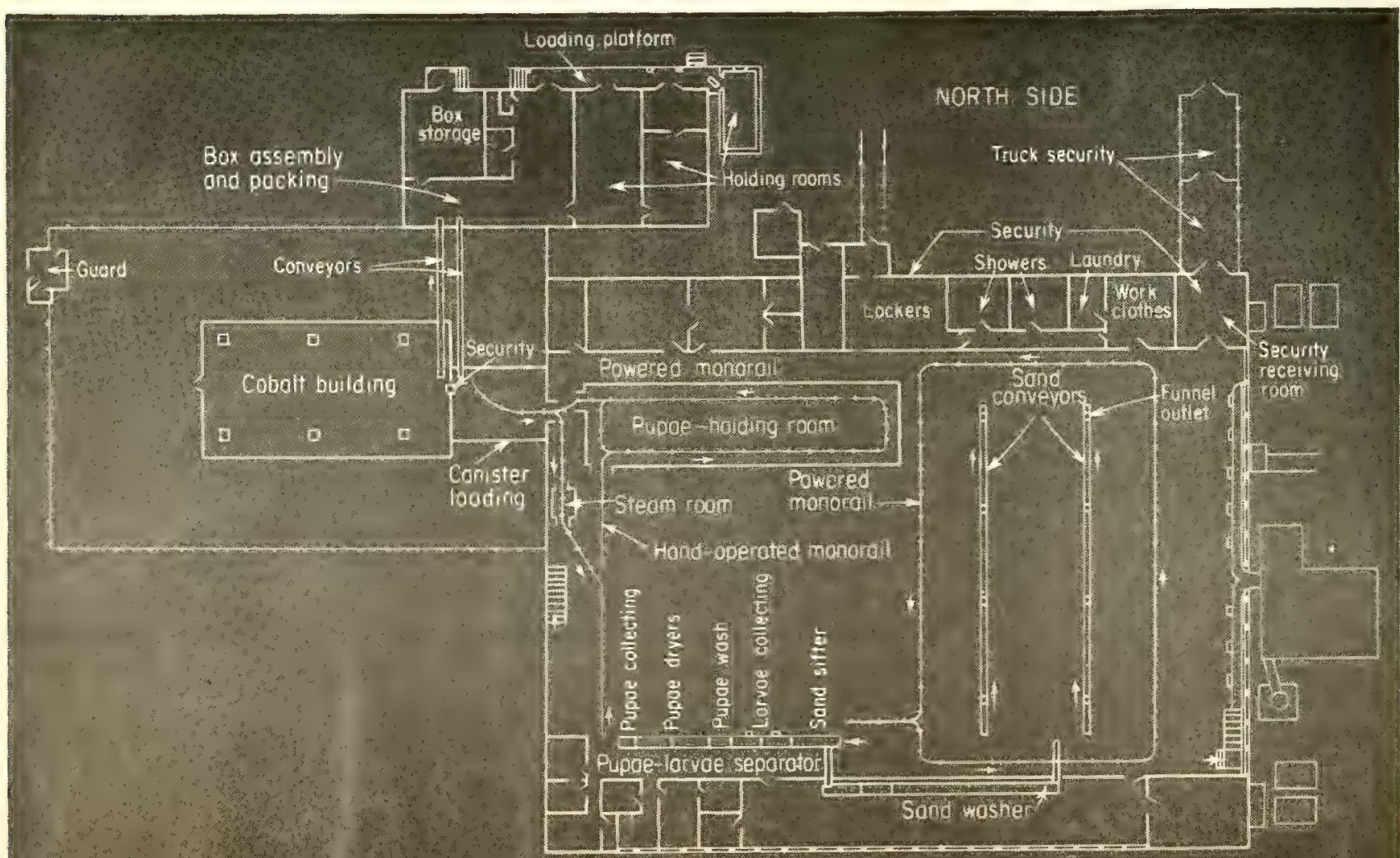




\section{Eliminate Screwworm Flies}

\section{Sterile-Male Method-Past and Future}

Aside from its accomplishments in the Southeastern U. S., the sterile-male method of pest eradication has had successes elsewhere. It has been proposed for many problems and is being used for some of them.

The first great success was elimination of the screwworm fly on the island of Curaçao in 1954. A pilot test reduced screwworm incidence by $70 \%$ in a 2,000 -squaremile area of Florida in 1957 before the full-scale attack was launched.

In cooperation with the government of Mexico, the U.S. Department of Agriculture has done some promising research work with this method against the Mexican fruit fly.

Belgium is working on a program to apply the sterilemale method to eradication of the tsetse fly in its African colonies, and a facility in Hawaii is directed at several fruit flies. The females mate more than once, but the mathematics of the method show that if sterile males make enough eggs sterile, the method may work with such insects (2). It has also been stated that such pests as small rodents might surrender to this kind of assult (2).

good for only a few days to a week at a time, and even if you clean up your herd completely, you can expect reinfestation from such animals as deer, raccoons and rabbits.

Eradication is accomplished by causing females to mate with sterile males (2). Afterward the female lays her sterile eggs, unaware that she has been frustrated in her natural purpose and apparently satisfied; she does not breed a second time.

The method depends for its success on rearing and sterilizing adequate numbers. In the Florida operation this has required an average of 50 million flies a week. A maximum of 70 million a week was reached, and full plant capacity was 100 million a week.

The fly factory is a converted World War II B-17 hangar at Sebring, Fla. It measures $160 \times 200 \mathrm{ft}$ and had operations on two floors. The ground floor is shown in Fig. 1. Three million fertile flies in a darkened adult colony produced larvae, which came to maturity on the upper floor and dropped through funnels into sand trays for pupation in the pupae-holding room. Pupation requires $\sim 8 \mathrm{hr}$. After that pupae were held for 5.5 days at $80^{\circ} \mathrm{F}$ and $95 \%$ relative humidity to mature.

During the maturing period the trays remained at body temperature and moved slowly along as food was added and waste removed. Food requirements indicate the magnitude of the operation: 40 tons of ground meat, 9,600 gal of water, 4,500 gal of beef blood, $65 \mathrm{gal}$ of plasma, and $35 \mathrm{gal}$ of honey were required each week.

Sexual sterility was produced by exposing the pupae to $8,000 \mathrm{r}$ of $\mathrm{Co}^{60}$ radiation two days before appearance of adult flies. Early research and tests showed that sterile

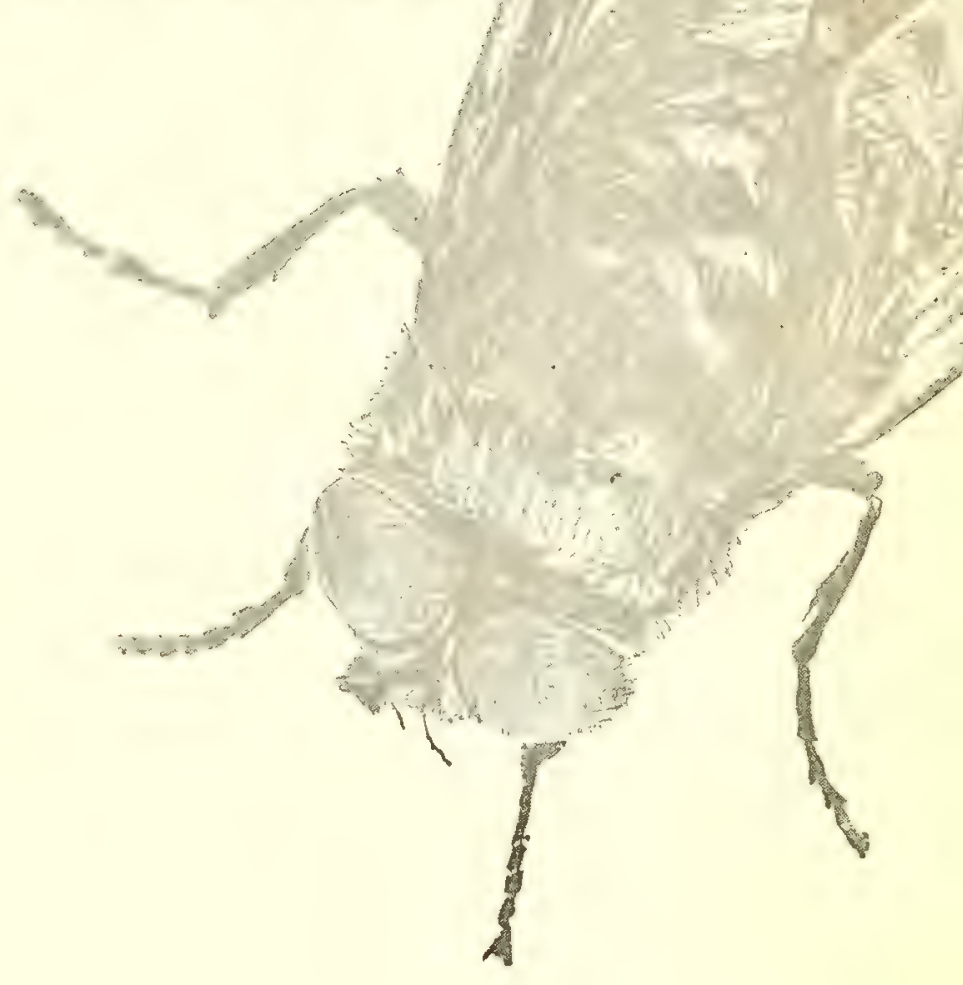

males could be obtained with a dose of $5,000 \mathrm{r}$ while $10,000 \mathrm{r}$ had no adverse effect on development of the pupae (3-5); $8,000 \mathrm{r}$ sterilizes the females among the released flies as well as the males to assure that there is no inadvertent contribution to the healthy population.

Elaborate precautions assured that none of the fertile flies escaped from the fly factory. These included changes of clothes and showers for all employees as they left work.

\section{Distributing the Flies}

Once reared and sterilized the fly battalions traveled to their theatres of operation in small Cessna 172 airplanes. Each plane carried 1,000 boxes of flies and flew for 5-6 hr each day over prearranged patterns in Florida and parts of Alabama and Georgia.

Pilots flew at 1,500 feet while insect dispersers loaded automatic ejectors. The ejectors dropped the boxes and opened them at a rate that dispersed 100-800 flies to each square mile of terrain. An air-conditioned station wagon carried flies to places that were hard to reach by plane.

To relieve anxieties of the human population, each box was marked to indicate the harmlessness of box and contents. Screwworm flies don't bite or look for picnic tables.

\section{Irradiators}

The radiation source used for early field tests was a cylindrical arrangement of $\mathrm{Co}^{60}$ slugs designed and built by Oak Ridge National Laboratory (6). This source was quite satisfactory and served as the basis for an irradiator of greater capacity and somewhat more uniform field.

Figure 2 shows one of the $\mathrm{Co}^{60}$ units at Sebring. The 


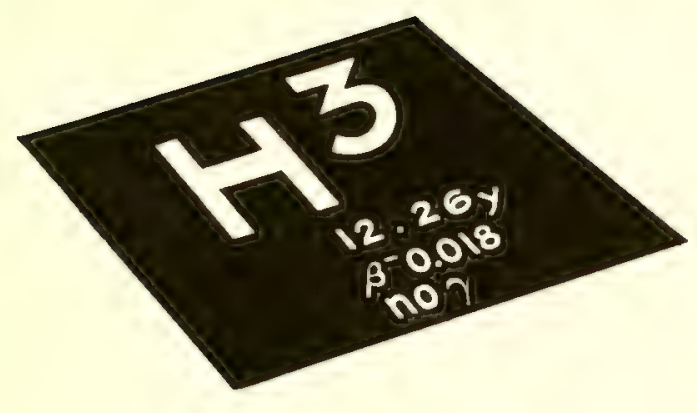

\title{
Tritium Tracing -A Rediscovery
}

\author{
Better labeling techniques, more sensitive detection and lower price \\ bring new usefulness to tritium. Good half-life and versatility make \\ this radiohydrogen valuable to biologists, chemists and geologists
}

Tritiom Has recently been "rediscovered" as a tracer. Three developments have contributed:

(a) Tritium is cheaper. In recent years the price has dropped by a factor of 50 -from $\$ 100$ to $\$ 2$ per curie.

(b) Detection is more efficient. In particular, liquid-scintillator counting techniques have made possible fullefficiency counting without self-absorption. This conquers the greatest technical difficulty-detection of the very weak (18 kev max) beta particles.

(c) Labeling is easier and more versatile. Recoil labeling that makes use of energetic tritons from the $\mathrm{Li}^{6}(n, \alpha) \mathrm{T}$ reactions in a nuclear reactor is effective for a wide variety of compounds, and discharge-tube labeling promises a means of using the same technique with simpler apparatus $(1,2)$. More recently a still simpler, still more versatile technique has been developed by K. E. Wilzbach at Argonne National Laboratory. This is gas-exposure labeling in which tritium serves as both a radiation source and the inserted label (3). Discharge-tube labeling and gas-exposure labeling have the impressive advantage that they can be carried out with simple apparatus.

There are other contributing causes for the tritium revolution. Tritium is a very useful tracer for biologists and organic chemists because it is the only radioactive form of hydrogen, the most common element in organics.

In spite of technical difficulties, tritium was used as a tracer before World War II. With the accelerated development of radioactivity techniques carbon-14 displaced it as the most used radiotracer for organics because of $\mathrm{C}^{14} \mathrm{~s}$ easier detection and labeling. The current revolution is bringing tritium back into prominence and forcing scientists to reread prewar publications. Lower costs and wider use are making it possible to exploit many of its advantages: useful half-life, high specific activities, low cost, etc. Even the weakness of its betas is sometimes an advantage, particularly in producing high-resolution radioautographs (see box p. 64).

Ten experts in the use of tritium offered a detailed picture of the state of the science at a recent symposium sponsored by the New England Nuclear Corp., Atomic Associates, and Packard Instrument Co. They spoke on labeling techniques, detection, health-physics considerations, and uses in the pharmaceutical industry, petroleumreservoir engineering, biochemistry, and radioautography.

\section{TRITIUM LABELING}

Tritium's usefulness as a tracer has been greatly increased by the gas-exposure labeling technique developed by K. E. Wilzbach and described by him at the symposium. The compound to be labeled is exposed to tritium gas, which serves a double function: beta particles activate either gaseous tritium or the molecules of the compound, and this promotes exchange between hydrogen in the molecule and gaseous tritium. (It is not clear whether the mechanism is activation of tritium or activation of the exposed molecule, but the difference is not important to people who want to use it.)

Other tritium labeling methods are the tritium-recoil method (1) and chemical synthesis and exchange techniques, which can be used in combination with gas exposure and recoil. Gas exposure has the advantages of smaller radiation damage and simpler laboratory techniques.

In both gas-exposure and recoil labeling the product usually contains small amounts of high labeled impurities. Removal of these by-products requires efficient purification procedures. These lead to most of the effort involved in using the two methods. 


\section{Gas-Exposure Procedure}

Good gas-exposure labeling requires isotopic purity, relatively high tritium pressures $(\sim 1 \mathrm{~atm})$ and large surface area in the exposed sample. Wilzbach uses tritium of about $90 \%$ isotopic purity. The presence of $\mathrm{He}^{3}$, formed by tritium decay, has no apparent effect on the process, but accumulation of hydrogen and methane makes it unwise to reuse the gas. Higher pressures increase both the activation and the tritium available for exchange; however, present techniques limit pressures to just below atmospheric (Fig. 1).

For safety and economy it is advantageous to use a limited amount of gas and a small sample. Subsequent dilution is better than using a large sample to start with. Since the tritium beta has a range of only $0.7 \mathrm{mg} / \mathrm{cm}^{2}$, it is best to use solids in finely powdered form and to shake liquids. Room temperature is generally satisfactory, but some compounds display better labeling at reduced temperatures.

Apparatus. Figure 1 shows a typical reaction vessel and the pumping system used to introduce tritium. Samples consisting of $0.1-1 \mathrm{gm}$ of liquid or powdered organics are introduced into the $6-\mathrm{ml}$ vessel through the constriction, which is subsequently sealed off. Volatile samples can be introduced into a similar vessel from the Toepler pump.

After the vessel has been filled with sample and placed on the pumping apparatus, $\sim 2 \mathrm{~cm}^{3}$ (5 curies) of tritium are introduced from a storage bulb or by distillation from uranium tritide. The vessel is then pulled off at the capillary. Shaking promotes the reaction with liquid samples, and with solids it is customary to rotate the tube and distribute the sample as well as possible over the walls.

The length of the gas exposure depends on the extent of radiation damage. This is expressed in terms of $G_{-\mathbf{M}}$, the number of molecules destroyed per 100 ev absorbed. Unfortunately this number has been measured for only a limited number of organic compounds, but the available values have been tabulated (4). Its value determines the length of the optimal exposure. $G_{-\mathrm{s}}$ ranges between 1 and 10 , and optimal exposures are usually 10-30 curie-days per millimole.

Incorporation of tritium is expressed in terms of $G_{\mathrm{T}}$, the number of tritium

\section{Gas-Exposure Labeling-Apparatus and Results}

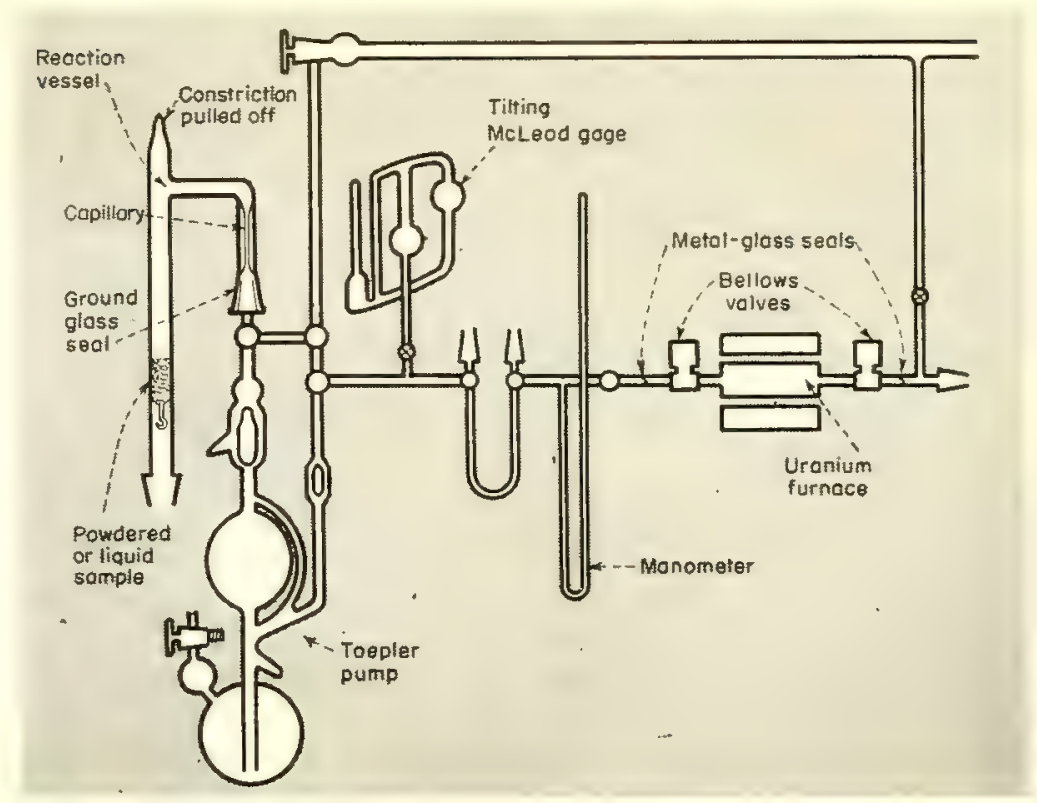

FIG. 1. System above permits introduction of gaseous tritium and powdered or liquid samples into vessel at upper left. Tritium comes from storage bulb mounted on U-tube or by distillation from uranium tritide

\section{TABLE 1-Tritium Labeling by Gas Exposure}

\begin{tabular}{|c|c|c|c|c|c|c|c|c|}
\hline \multirow[b]{2}{*}{$\begin{array}{l}\text { Compound } \\
\text { exposed }\end{array}$} & \multirow[b]{2}{*}{$\begin{array}{c}\text { Sample } \\
\text { weight } \\
(\mathrm{gm})\end{array}$} & \multirow[b]{2}{*}{$\begin{array}{c}\text { Exposure } \\
(\text { days })\end{array}$} & \multirow[b]{2}{*}{$\begin{array}{l}\text { Amount of } \\
\text { tritium } \\
\text { (curies) }\end{array}$} & \multicolumn{3}{|c|}{ Tritium incorporated } & \multirow{2}{*}{\multicolumn{2}{|c|}{ Values of $G_{T}$}} \\
\hline & & & & $\begin{array}{l}\text { Total } \\
(m c)\end{array}$ & $\begin{array}{l}\text { Labile } \\
(m c)\end{array}$ & $\begin{array}{l}\text { In pure } \\
\text { product } \\
(\mathrm{mc} / \mathrm{gm})\end{array}$ & & \\
\hline Toluene & 0.86 & 2.9 & 7.5 & 42.7 & None & 22.2 & 0.22 & 0.10 \\
\hline$n$-Heptane & 1.37 & 9.8 & 6.9 & 17.5 & None & 1.3 & 0.27 & 0.003 \\
\hline Benzoic acid & 1.31 & 5.0 & 6.4 & 156 & 40 & 14.0 & 0.57 & 0.067 \\
\hline Sucrose & 4.0 & 6.7 & 14.0 & 593 & 480 & 5.0 & 0.72 & 0.024 \\
\hline Cholesterol & 1.88 & 4.8 & 7.2 & 335 & 90 & 64.3 & 1.11 & 0.40 \\
\hline Digitoxin & 0.50 & 5.8 & 7.5 & 438 & 182 & 90 & 1.16 & 0.13 \\
\hline
\end{tabular}

\section{TABLE 2-Distribution of Tritium in Toluene}

The specific activities of these toluene derivatives ...

... indicate this tritium distribution in gas-exposure-labeled toluene

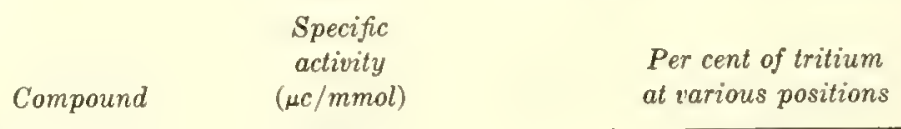

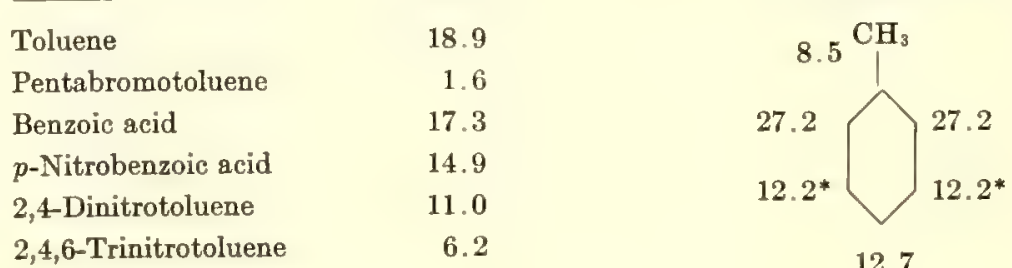

* By difference. 
atoms incorporated per $100 \mathrm{ev} a b$ sorbed. For purposes of computation it is assumed that all of the tritium radiation is absorbed by the sample. The results of several exposures and the values of $G_{T}$ for both the crude mixture and the purified parent are shown in Table 1. Comparable $G$ values for recoil labeling are smaller by a factor of $10^{5}$, which indicates that radiation damage for the same amount of labeling can be expected to be considerably smaller when the gas-exposure technique is used. As an example, it has been reported that ribonuclease and lysozyme can be labeled without great loss of enzymatic activity (5).

Position of label. Wilzbach has investigated the positions assumed by the label in toluene. The method is to separate derivative fractions and determine the amount of activity that remains with each one. From the data one can determine the fraction of the total toluene activity that is associated with each of the hydrogen positions in the toluene molecule. The results of these investigations are shown in Table 2.

\section{Conventional Labeling}

When the special nature of the labeled molecule does not require the special techniques of gas exposure or tritium recoil, simpler methods produce compounds of high specific activity. These include reduction, exchange, hydrolysis, and synthesis by means of intermediates.

Reduction. This is the method of choice if a precursor is available that has a double bond or a reducible group such as a carbonyl radical. Activity as great as $58 \mathrm{c} /$ millimole is possible by this method, although one could not expect to keep such a compound under control. However, curies per millimole are possible even after dilution with carrier hydrogen.

Finding a suitable solvent is a problem. In polar solvents exchange between the tritium and solvent hydrogen reduces the eventual activity by a large factor. Modification of the compound before the reduction and subsequent restoration to the desired form often simplifies the problem.

Simple exchange. This method is limited by the fact that labels that go

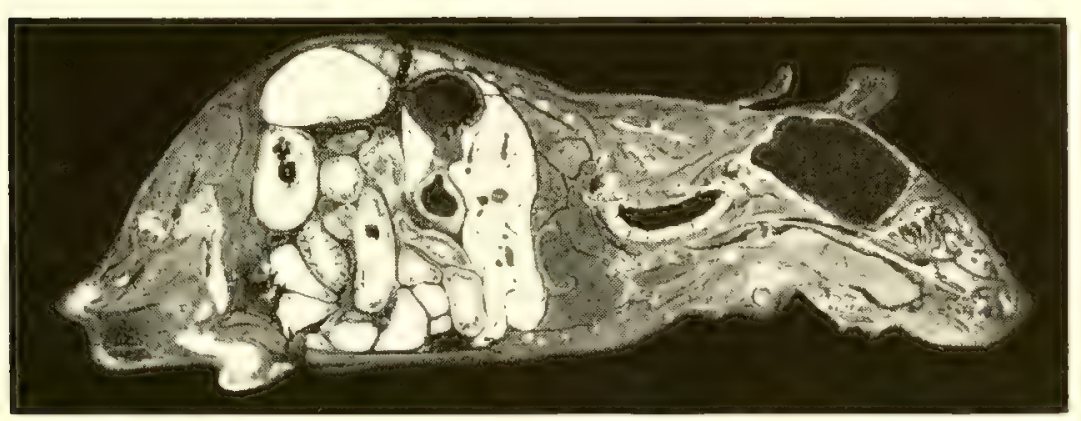

\section{Tritium Radioautography}

Spectacular print* shows distribution of tetracycline in a mouse sacrificed 20 min after intravenous injection. Concentration is high where this figure is light. Brain at right shows low, while kidney at upper left shows high concentration. This exposure required 2.5 months.

Short range of tritium betas produces resolution that is unusually high for radioautographs. In microscopic enlargements individual cells are frequently distinguishable.

At the tritium symposium Walter L. Hughes gave figures to indicate the specific activity required in thymadine to label a mammalian cell: one locus requires 5 silver grains, which, at $5 \%$ efficiency, require 100 disintegrations, which might occur during a 1-month exposure. This is $\sim 10^{-9}$ curies per locus. Taking into account the composition of the average cell, one finds that one needs 0.2 curies per mole of thymadine.

* Torsten André, Farmakologiska Avdelningen, Kungl. Veterinärhögskolan (1956) published as supplementum 142 to Acta Radiologica. in easily come out easily; if a tracer is easily prepared, it is likely to be unstable, and vice versa. The method is useful if the exchange-labeled molecule is quickly modified to a stable form after the initial labeling.

Catalyzed reactions. Acid-catalyzed reactions are useful for aromatic compounds. The usefulness is limited by the tendency toward sulfonation. Other types of molecules, notably acetone and fatty acids, can be labeled by base- and metal-catalyzed reactions, respectively.

Hydrolysis. The following equations illustrate labeling by hydrolysis. As applied to benzene derivatives this method has the advantage of providing specific labeling. Reactions with the Grignard reagent, illustrated by the first two equations, have rather general applicability.

$$
\begin{gathered}
\mathrm{RMgX}+\mathrm{H}_{2}^{3} \mathrm{O} \rightarrow \mathrm{RH}^{3}+\mathrm{H}^{3} \mathrm{OMgX} \\
\mathrm{SOCl}_{2}+\mathrm{H}_{2}^{3} \mathrm{O} \rightarrow \mathrm{SO}_{2}+2 \mathrm{H}^{3} \mathrm{Cl} \\
\mathrm{H}^{3} \mathrm{Cl}+\mathrm{RMgX} \rightarrow \mathrm{RH}^{3}+\mathrm{MgXCl} \\
\mathrm{CaC}_{2}+2 \mathrm{H}_{2}^{3} \mathrm{O} \rightarrow \\
\mathrm{H}^{3} \mathrm{C} \equiv \mathrm{CH}^{3}+\mathrm{Ca}\left(\mathrm{OH}^{3}\right)_{2} \\
\mathrm{KCN}+2 \mathrm{H}_{2}^{3} \mathrm{O} \rightarrow \mathrm{H}^{3} \mathrm{COOK}_{3} \mathrm{NH}_{3}^{3} \\
\mathrm{Cl}_{3} \mathrm{CCHO}+\mathrm{NaOH}^{3} \rightarrow \\
\mathrm{Cl}_{3} \mathrm{CH}^{3}+\mathrm{HCOONa}
\end{gathered}
$$

\section{TRITIUM DETECTION}

The current "rediscovery" of tritium as a tracer arises in part from development of new sensitive methods for detecting it. The beta particles have a maximum energy of only $18 \mathrm{kev}$, which means that few of them will penetrate even the thinnest window. Even more important, almost any tritium sample, no matter how thin, will absorb most of its own beta energy.

Two counting techniques conquer the difficulty effectively-liquid scintillation and gas counting.

The more powerful of the two is liquid scintillation counting, which has been widely developed in the past few years $(6-12)$. The sample is dissolved or suspended in the scintillating liquid or gel. Thus there is a minimum of self-absorption and essentially no barrier between sample and detector. Beta energy is converted immediately into light, and the light can transverse the scintillating substance without the absorption experienced by beta rays. Such a system will recognize as little as $25 \mu \mu \mathrm{c}$ of tritium. In a recent extension of the liquid-scintillator technique, leaf patterns have been squeezed 


\section{Tritium or Carbon-14?}

Since most organics are made up of hydrogen, carbon, and oxygen, and since there is no usable radioactive oxygen, organic tracers must be tagged with tritium or $\mathrm{C}^{14}$. Both are weak beta emitters so the same instrumentation for detection and assay is applicable to both.

F. Marott Sinex, chairman of the recent symposium, made a comparison of tritium and $\mathrm{C}^{14}$ for tracer purposes. Here are some of his figures:

\section{Carbon-14}

Tritium

Maximum beta energies

$$
0.158 \text { Mev } 0.018
$$

Dose from 1 me uniformly distributed in a $70-\mathrm{kg} \operatorname{man}$ :

$$
0.039 \mathrm{rep} / \mathrm{day} \quad 0.0044
$$

Cost of the isotope. $\mathrm{C}^{14}$ is sold at Oak Ridge in solid $\mathrm{BaCO}_{3}$ at specific activities up to $80 \mathrm{mc} / \mathrm{gm}$. Tritium is sold as the pure gas except for the $\mathrm{He}^{3}$ decay product.

$$
28 \$ 1 \mathrm{mc} \quad 0.002
$$

Cost of labeled compounds. For example, here are the price of tritium-labeled leucine and $\mathrm{C}^{14}$-labeled amino acids:

$$
800 \$ / \mathrm{mc} \quad 35
$$

Specific activities. Tritium's much shorter half-life (12.3 yr compared with $5,600 \mathrm{yr}$ ) indicates that it takes much fewer atoms to make a millicurie than is the case for $\mathrm{C}^{14}$. Thus maximal specific activities for the two are

$$
5 \text { curies/mole } 700
$$

into filter paper that contains the scintillator (13).

In the other method one introduces tritium-labeled gases including tritium gas itself into gas counters to reduce absorption. This leads to two types of counting: conventional G-M counting with the tritium in the counter initiating the discharges and gaseous scintillation counting $(14,15)$. (See Table 3, page 67.)

\section{Liquid Counting}

Today's techniques for liquid-scintillation counting require a solution that has five components aside from the sample that is to be counted. This solution is viewed by two photomultipliers operated in coincidence to reduce background. Further background reduction is accomplished with pulse-height discrimination.

Scintillating solutions. The five components of a good scintillating solution are (a) an aromatic solvent, (b) a fluorescent dye, (c) a wavelength shifter, (d) a diluent in which the sample can be dissolved, and (e) naphthalene as a light restorer.

Solvents that are frequently used to hold the dye are toluene, xylene, triethylbenzene, ethylhexene, etc. Preferably one uses a solvent that will not attack Lucite, since Lucite is the most convenient container because of its high transparency. Terphenyl and diphenyloxazole (PPO) are the most useful dyes.
The purpose of the wavelength shifter is to absorb the light emitted by the dye at 3,500-4,000 angstroms and re-emit it at wavelengths closer to the phototube peak of $\sim 4,600$ angstroms. Most commonly used is POPOP, standing for phenyl-oxyzol-phenyl-oxyzolphenyl.

Samples that do not dissolve readily in the basic three-component mixture can be introduced after introduction of an appropriate diluent. Water can be introduced at the cost of increased light absorption, and quartenary amine bases make it possible to dissolve carbon dioxide and proteins. Some of the light lost upon introduction of diluents is restored to the detection system when napthalene is added.

Electronics. Even when most of the energy of a tritium beta is efficiently converted into light in a scintillator it still produces a relatively weak pulse at the output of a photomultiplier. A counting system that is sensitive enough to detect these pulses is sensitive to many background pulses. The two devices that are used to reduce background are coincidence counting of two phototubes looking at the same scintillator and pulse-height discrimination. Coincidence counting eliminates essentially all of the thermal-noise pulses, which have energies comparable to those that originate from tritium scintillations. Discrimination against high-energy pulses eliminates most of the pulses that originate from non- tritium radiation absorbed in the scintillator. Two-channel pulse height counting makes possible simultaneous counting of the two most useful organic tracer nuclides, tritium and $\mathrm{C}^{14}$. An upper channel counts only $\mathrm{C}^{14}$, and a lower one counts tritium and some $\mathrm{C}^{14}$ pulses as well.

Improvements in liquid-counting equipment are reducing technical difficulties. Figure 2 shows one example, an automatic sample changer that enables long counting runs without any exposure of sensitive elements to light. This saves time since extensive dark adaption is required for low-noise counting.

\section{Other Assay Techniques}

Most non-scintillation methods for tritium assay depend on conversion of the labeled compound into a suitable gas. Methods that have been found useful for conversion and assay are described by Fig. 3 and Table 3 , and the bibliography (p. 67). Charles V. Robinson deseribed these methods at

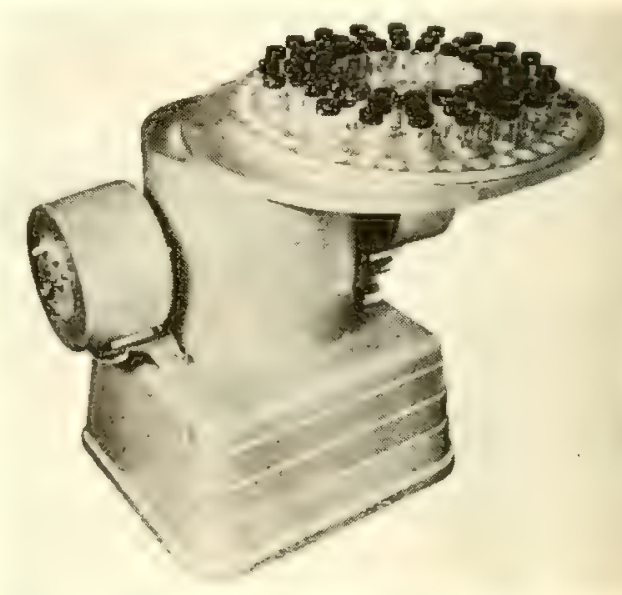

FIG. 2. Automatic sample changer for liquid scintillation counting permits long runs without interruption by exposure to external light

the symposium and prepared the figure, table, and bibliography.

Radioautography. Photographic detection is also used with tritium betas, principally in making radioautographs. An example is the figure shown in the box on page 64 . The low energy of the particles is at once an advantage and a disadvantage. The short range in emulsions leads to extremely fine resolution. On the other hand, exposures of several months are 
frequently required to produce recognizable darkening.

\section{APPLICATIONS}

The usefulness of tritium as a tracer comes from the presence of hydrogen in most organic substances and the existence of tritium and tritium-labled compounds as gases. Four of the symposium speakers discussed practical tracer uses of tritium.

\section{Biology and Drugs}

The manufacturer of pharmaceuticals uses tracers primarily to study new drugs. The things he wants to know about them are (a) chemical and biological stability, (b) site and extent of absorption, (c) translocation and distribution in body fluids and organs as functions of time, (d) biological halflife, (e) places of permanent or semipermanent storage, (f) biological transformations and methods of excretion and (e) modes of biochemical and pharmacological action.

Tritium labeling by exchange methods is possible with most drugs. Testing is performed largely in animal experiments, and assay is by liquidscintillator counting and radiosutographs.

In new-drug studies, tests to find an appropriate labeling technique and then to test the quality of the label are always fundamental. A label can arrive at a spot through movement of the whole molecule, transfer of part of the molecule in a metabolic process or merely by exchange of the tritium atom with the hydrogen of another molecule.

One must also evaluate isotopic effects. Tritium can move preferentially in chemical or biological systems as the result of two things: (a) the different strengths of hydrogen and tritium bonds and (b) the different mobilities exhibited by atoms containing hydrogen and their counterparts containing tritium.

\section{Petroleum Engineering}

Oil geologists find tritium useful in studying fluid flow in both laboratory experiments and underground exploration. Tritium has several advantages over other tracers: (a) a cost that is a small fraction of the total cost of a test, (b) weak radiation that presents no health hazard, (c) relatively long life, (d) detection techniques that can be used in the field, (e) absence of

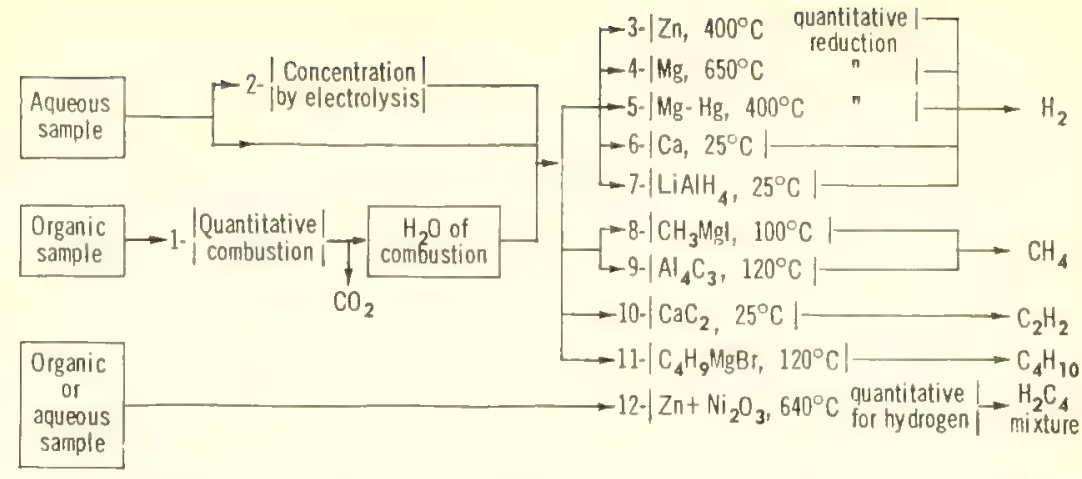

FIG. 3. Conversion methods for gas counting of tritium. Table and references on the next page list users, methods, and publications. Numbers associated with conversions above appear in fifth column of the table. References should not be confused with story bibliography on this page

appreciable tritium in naturally occurring gases and (f) the ability to incorporate it as a label in the fluid that is to be traced, which is usually water or a hydrocarbon.

In a "five-spot" technique oil is taken from a central producing well while four other wells are used as injection wells. Water or gas forced down an injection well will force oil to the site of the production well. Different tracers, used together, indicate which of the injection wells are effective. Tritium-labeled gases that are useful are tritium itself, $\mathrm{CH}_{4}^{3}$ and $\mathrm{C}_{2} \mathrm{H}_{6}^{3}$. Other radioactive gases that are frequently used are $\mathrm{C}^{14} \mathrm{H}_{4}, \mathrm{C}_{2}^{14} \mathrm{H}_{6}$ and $\mathrm{Kr}^{85}$. Dilution factors as great as $10^{13}$ are currently usable.

Many nonradioactive gas tracers have been used in the past. They include dyes, boron compounds, ammonia, salts, helium and carbon monoxide. However, such tracers present many difficulties: (a) failure to behave as the fluid to be traced, (b). lack of sensitivity, (c) high cost and (d) need for laboratory techniques that cannot be used in the field. In the use of helium there is inconvenient background due to naturally occurring helium. Carbon monoxide presents a special health hazard

If the flooding fluid is water, tritiumlabeled water is the ideal tracer. Other long-lived tracers are usable if they are chemically complex so that they do not react with the materials they encounter.

Another special problem is the labeling of gases held in underground storage reservoirs. The technique is appropriate for measuring the size of the reservoir and finding leaks that can waste the stored gas or poison nearby mines. Currently reservoirs are tagged at levels that permit tracing their gas for 25-30 yr. This requires about 1 c per million cubic feet

Cost of homogeneous labeling is $\$ 2-3$ per milion cubic feet. The cost per injection includes similar costs of a few hundred dollars for isotope procurement and calibration, respectively, and an injection cost of a few thousand dollars.

This article is based on material presented at the Symposium on Tritium in Tracer Applications, Hotel Statler, New York, November 22, 195\%.

The speakers were $F$. Marott Sinex, chairman, Boston University School of Medicine, Kenneth $E$. Wilzbach, Argonne National Laboratory, Seymour Rothchild, New England Nuclear Corp., James R. Arnold, Princeton University, Charles V. Robinson, New England Medical Center, John S. Handloser, Brookhaven National Laboratory, Jay $F$. Snell, Pfizer Therapeutic Institute, Donald $R$ Carr, Isotopes, Inc., Maxwell Eidinoff, Sloan Kettering Institute, Walter L. Hughes, Brookhaven National Laboratory.

\section{BIBLIOGRAPHY}

1. F. S. Rowland, R. Wolfgang, NECLEONICs 14, No. 8, $58(1956)$

2. R. Wolfgang, T. Pratt, F, S. Rowland, $J$. A $m$ Chem. Soc. 78, 132 (1956)

3. K. E. Wilzbach, J. Am. Chem. Soc. 79, 1013 (1957)

4. B. M. Tolbert, R. M. Lemmon, Rodiation Research 3, 52 (1955)

5. D. Steinberg et al, Science 126, 447 (1957)

6. F. N. Hayes, B. S. Rogers, W. Langham, NUCLEONICS 14, No. 3,48 (1956)

7. NUCLEONICB 14, No. 1, 74 (1956)

8. G. T. Okita, J. Spratt; G. V. LeRoy, NUCLEONICs 14, No. 3, 76 (1956)

9. B. L. Funt, NUCLEONICs 14, No, 8, 82 (1956)

10. C. G. White, S. Helf, NUCLEonics 14, No. 10 46 (1956)

1. G. T. Okita, J. J. Kabara, F. Richardson G. V. LeRoy, Nucleonics 16, No. 6, 111 (1957)

19. M. Blau, Nucleonics, 15, No. 4, 91 (1957)

13. J-C. Roucayrol, E. Oberhauser, R. Schussler, NUCLEONICS 15, No, 11, 104, 1957

14. J. Northrop, R. Nables, NocLEonics 14, No. 4. $36(1956)$

15. C. Eggler, C. Huddleston, NUCleonics 14 No. $4,34(1956)$ 


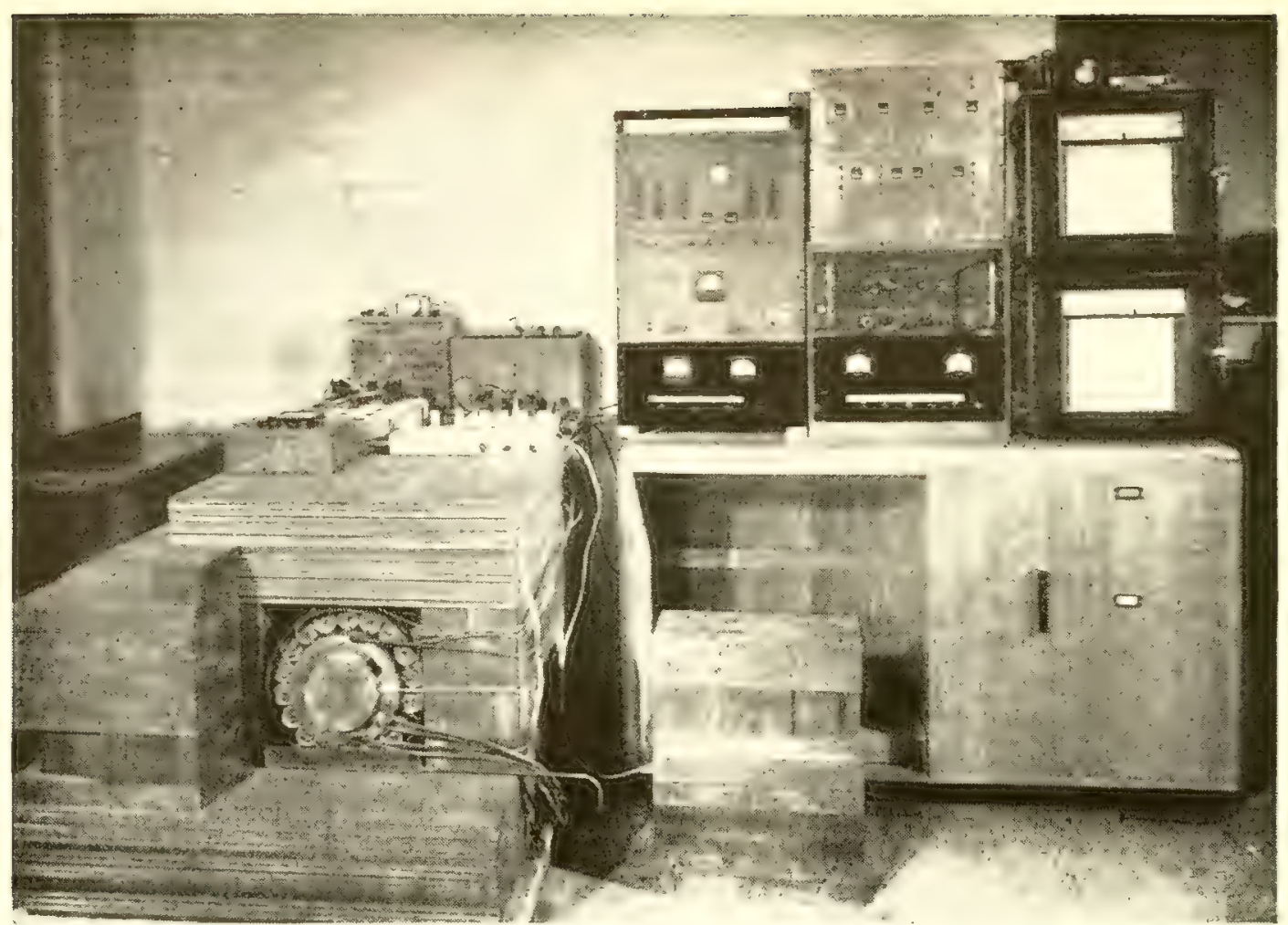

FIG. 1. Over-all view of counting system with front of iron shield removed to show counters

\section{Radiocarbon Dating System}

\section{Dating range has been extended to 45,000 years and accuracy of "living-carbon" assay increased to $0.3 \%$ by carefully instrumented system centered on a 7.7 -liter proportional counter filled with up to 3 atmospheres of pure $\mathrm{CO}_{2}$}

BY G. J. FERGUSSON

Dominion Physical Laboratory

Lower Hutt, New Zealand

AN IMPROVED METHOD for radiocarbon dating has been developed to supplant the solid-sample method (1) previously used here $(2,3)$. The system (Fig. 1) centers around a well-shielded 7.7-liter proportional counter filled with up to 3 atmospheres of especially purified $\mathrm{CO}_{2}$. The output pulse heights are analyzed electronically. In addition, pulses are sorted according to whether or not they are in coincidence with a surrounding ring of $\mathrm{G}-\mathrm{MI}$ counters. This identifies most of cosmic-ray background.

For "living" carbon the counting rate is $37.5 \mathrm{cpm}$ above a background of $9.8 \mathrm{cpm}$ for a counter-filling pressure of $1 \mathrm{~atm}$ and $103 \mathrm{cpm}$ above $14 \mathrm{cpm}$ background for a filling pressure of 3 atmospheres. For most problems a filling pressure of 1 atmosphere appears to offer the best compromise between accuracy and man-hours of work per sample. When increased accuracy is desired a 3-atmosphere filling is used.

The maximum age that can be measured is arbitrarily defined here as the age for which the net sample count is equal to four times its statistical standard deviation. This definition has also been used by several other workers (4). For a 1-day count on sample and on background the maximum age is 35,000 yr for 1 atmosphere and 42,000 yr for 3 atmospheres filling pressure. For a 2-day count on sample and on background with 3-atmosphere filling, maximum age is $45,000 \mathrm{yr}$.

\section{Apparatus in General}

Carbon in the sample to be dated is converted to $\mathrm{CO}_{2}$, which is then purified according to a procedure developed by 
Rafter.* The purification procedure is basically similar to that of Je Vries and Barendsen (5). It involves appropriate washings, absorption on lime at $700-750^{\circ} \mathrm{C}$, and then re-evolution at a higher temperature $\left(800-900^{\circ} \mathrm{C}\right)$. Electronegative impurities are also absorbed on the lime but are notre-evolved even at $1,000^{\circ} \mathrm{C}$. The purified $\mathrm{CO}_{2}$ is then passed into the proportional counter.

A low background counting rate is achieved by extensive gamma-ray shielding. Surrounding the proportional counter with a ring of anticoincidence Geiger-Müller counters (Fig. 2) identifies counts due to cosmic-ray mesons. The unshielded background of the proportional counter is approximately 2,000 cpm while its bachground when operating in the equipment is only 10-14 cpm. This background counting rate is checked periodically by filling the counter with $\mathrm{CO}_{2}$ prepared from coal (more than 50,000 yr old).

Pulses from the counter are linearly amplified and then electronically sorted into four groups depending on their amplitude, as shown in Fig. 3. The pulses in each of these groups are then sorted into two groups, depending on whether they are or are not in coincidence with a pulse from the ring of G-M counters; thus the sorter has eight outputs. The energy distribution of coincidence pulses is shown by the number of counts in the four coincidence channels, and the energy distribution of anticoincidence pulses is displayed in the four anticoincidence channels.

\section{Counter Construction}

The proportional counter, constructed from 5-in.-o.d. copper tubing, is $28 \mathrm{in}$. long. Previous experience had shown that, of the readily available materials suitable for counter construction, copper had the lowest proportion of radioactive impurities.

The anode is 4 -mil tungsten wire with a sensitive length of $213 / 4$ in. At one end of the counter the anode connection is brought out through a Pyrex insulator and is provided with a guard ring. At the other end, the anode has a long glass insulator, and no guard ring is provided. The ends of the counter are made approximately hemispherical to prevent them from flexing

*T. A. Rafter, New Zealand J. Sci. Technol. (in press).

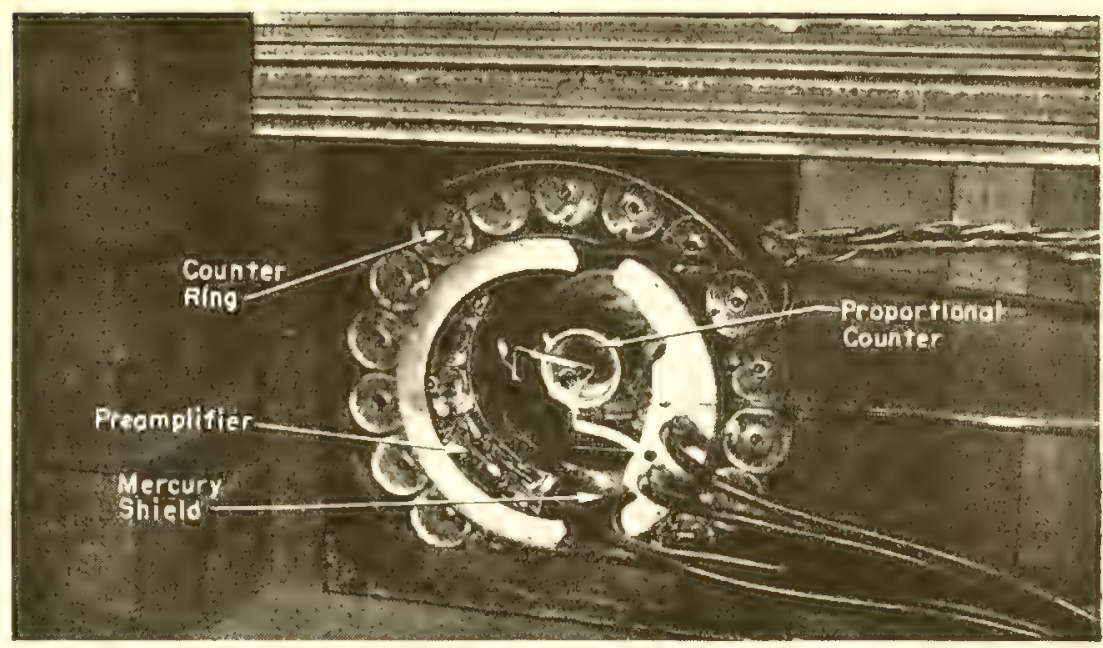

FIG. 2. Counter ring surrounds proportional counter, visible with cover removed. High voltage for proportional counter is supplied from banks of stable $671 / 2-\mathrm{r}$ batteries to anode and cathode in equal + and - parts respectively. Thus only two end insulators have to stand full counter voltage (up to $10,000 \mathrm{v}$ ); maximum voltage elsewhere is 1/2 that. Small variations in voltage or gain are corrected by changing cathode voltage. Preamplifiers shown use $46 \mathrm{AK} 5$ 's in a ring-of-3 feedback-sfabilized amplifier with cathode-follower output; gain is 40 . Heaters are $d$-c powered in series; plate supplies are heovily filtered. Locating preamplifier within $\gamma$-ray shield results in short counter leads and good electrical shielding when the pressure is changed and to reduce dead space. The sensitive volume is $80 \%$ of the $7.7-1$ total.

\section{Filling the Counter}

The proportional counter remains permanently in position in the gammaray shield. A glass pipeline connects it to the filling system next door.

The Pyrex filling system is evacuated by a mercury diffusion pump backed by a two-stage rotary pump. The pres- sure in the system can be reduced quickly to $10^{-4} \mathrm{~mm} \mathrm{Hg}$. Adequate "condensation traps" are provided to allow for experimentation with final purification and filling procedures. The system is also arranged so that the most time-consuming operations, distillation of $\mathrm{CO}_{2}$ and pumping the counter, can proceed simultaneously. Provision is also made for storing the sample being removed from the counter.

After chemical purification the $\mathrm{CO}_{2}$

\section{Possible Dating Methods-Why $\mathrm{CO}_{2}$ ?}

This table indicates some of the comparisons involved in our choice of proportional counting of $\mathrm{CO}_{2}$ as the basis of our radiocarbon dating system. It appeared to offer the best compromise of conflicting requirements.

\begin{tabular}{|c|c|c|c|c|c|}
\hline Method & $\begin{array}{c}C^{14} \\
\text { detection } \\
\text { efficiency } \\
(\%)\end{array}$ & $\begin{array}{c}\text { Residual } \\
\text { background }\end{array}$ & $\begin{array}{l}\text { Possibility of } \\
\text { radioactive } \\
\text { contamination }\end{array}$ & $\begin{array}{l}\text { Man-hours per } \\
\text { sample }\end{array}$ & I.F.† \\
\hline Solid-sample ( 1 ) & 5 & & $\begin{array}{l}\text { High, inorganic } \\
\text { preps, solid }\end{array}$ & High, $3 \times \mathrm{CO}_{2}$ & Yes \\
\hline $\begin{array}{l}\mathrm{CO}_{2} \text { gas pro- } \\
\text { portional }\end{array}$ & Up to 100 & $\begin{array}{l}\text { Relatively high; } \\
\text { one C per mole- } \\
\text { cule means large } \\
\text { counter }\end{array}$ & $\begin{array}{l}\text { Low, only gase- } \\
\text { ous contami- } \\
\text { nants, i.e., Rn }\end{array}$ & $\begin{array}{l}\text { Lowest; convert } \\
\mathrm{C} \text { to } \mathrm{CO}_{2} \text { first } \\
\text { in all methods }\end{array}$ & No* \\
\hline $\begin{array}{l}\text { Hydrocarbon } \\
\text { gas propor- } \\
\text { tional }(13,14)\end{array}$ & Up to 100 & Better than $\mathrm{CO}_{2}$ & $\begin{array}{l}\text { Low, but more } \\
\text { reagents than } \\
\mathrm{CO}_{2}\end{array}$ & High & Yes \\
\hline $\begin{array}{l}\text { Liquid } \\
\text { scintillation (15) }\end{array}$ & Up to 100 & $\begin{array}{l}\text { Best, small } \\
\text { volume }\end{array}$ & $\begin{array}{l}\text { Medium; organic } \\
\text { preps, liquid }\end{array}$ & High & Yes \\
\hline$\overline{\dagger \text { Possibility of } i}$ & isotopic fract & ctionation. & $0 \%$ transfer of $\mathrm{CO}_{2}$ & & \\
\hline
\end{tabular}




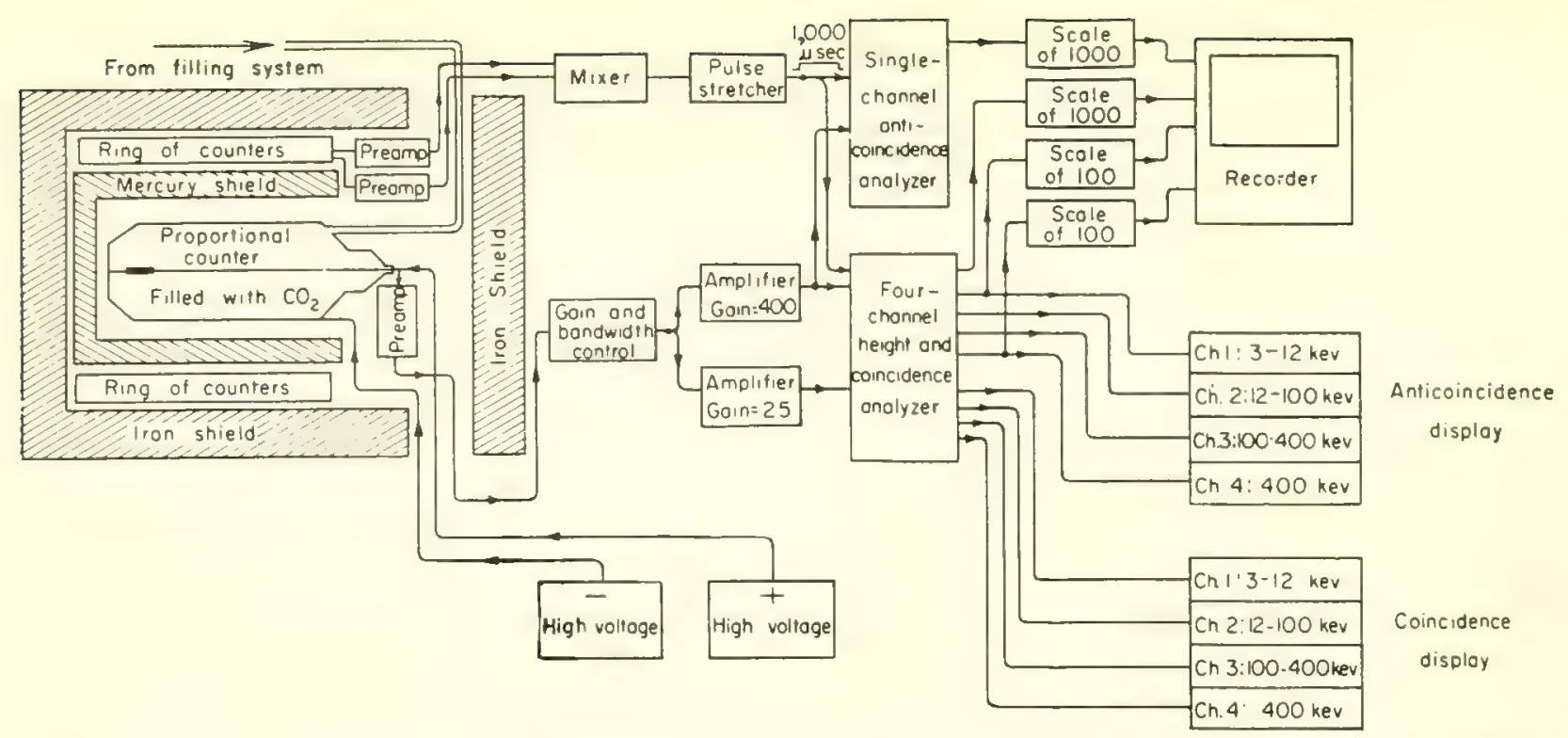

FIG. 3. Circuitry outside of shield begins with gain control, normally set at $-12 \mathrm{db}$. Bandwidth control, normally a 40 - $\mu$ sec differentiator, is to conirol frequency response for optimum signal-to-noise. Parallel main amplifiers are ring-of-3 feedback-stabilized; ratio of their gains is accurately set at 16:1 by control in lower-gain amplifier. Four-channel analyzer has four discriminators with cancelling circuits arranged so that only highest discriminator triggered records. Two lower-level discriminators are fed by high-grain amplifier, higher two by low-gain amplifier. Energy loss in counter corresponding to each channel is shown. The analyzer also sorts pulses according to whether or not they are in coincidence with a pulse from the surrounding counter ring. Coincidence events are largely due to cosmic-ray mesons. Pulse stretcher in ring output insures coincidence pulse is present even if meson passes through $G-M$ counter recovery. Parallel single-channel analyzer checks four-channel anticoincidence count

sample is removed from the chemical system as "dry ice," having been frozen with liquid oxygen. It is contained in a $100-\mathrm{cm}^{3}$ flask provided with a stopcock so that it can be removed from the chemical-purification system and joined to the counter-filling system without admission of air. At this stage the sample still contains traces of air trapped in the frozen $\mathrm{CO}_{2}$ and traces of water, both of which would upset its counting characteristics.

On the counter-filling system the $\mathrm{CO}_{2}$ is distilled from one condensation trap to another and then pumped. The process is repeated until the amount of gases, noncondensable in liquid oxygen (mainly air), is less than $0.1 \mathrm{ppm}$ of the $\mathrm{CO}_{2}$. One distillation is usually sufficient.

The frozen $\mathrm{CO}_{2}$ is now allowed to expand and pass into the counter through a $\mathrm{P}_{2} \mathrm{O}_{5}$ drying tube that also acts as an efficient dust filter. It is essential that drying be carried out last; if drying precedes distillation, sufficient water vapor will be outgassed from the glass to affect counting characteristics.

The counter is filled to the desired pressure $(78-230 \mathrm{~cm} \mathrm{Hg})$ when the counter temperature is $18^{\circ} \mathrm{C}$ as measured by a resistance thermometer with an accuracy of $34^{\circ} \mathrm{C}$. At any other counter temperature the filling pressure is changed so that the mass of gas in the counter is constant from one filling to the next to 1 part in 1,000 . The working voltage of the proportional counter as a function of filling pressure for a gas gain of 2,000 is shown in Fig. 4 .

\section{Shield and Counter Ring}

To prevent gamma rays from reaching the counter, an 8-in.-thick iron shield is provided. This shield is built of mild steel plate and machined cast-iron blocks, the space provided for the proportional counter and ring of meson counters being $14 \times 14 \times 36$ in. A layer of mercury 1 in. thick surrounds the counter to provide additional

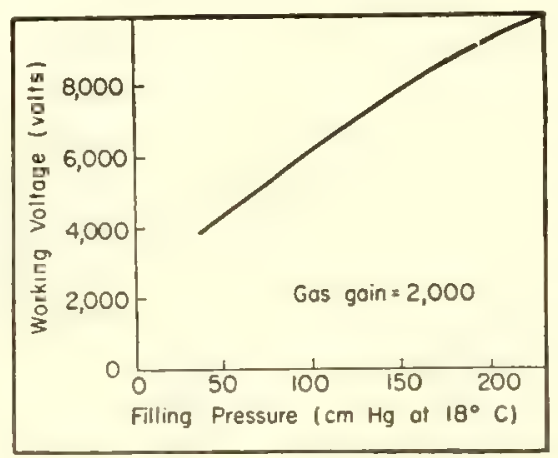

FIG. 4. Working voltage of the proportional counter as a function of $\mathrm{CO}_{2}$ filling pressure for a gas gain of 2,000 shielding and also absorb some of the gamma rays from radioactive contaminants in the iron. The arrangement of these components is shown in Figs. 1-3.

Between the mercury shield and the iron shield is the ring of $18 \mathrm{G}-\mathrm{M}$ counters, each 2 in. o.d. and 35 in. long. The ring of counters is divided horizontally into two segments. This division enables a faulty counter to be detected quickly by a comparison of the counting rates of the segments. Each segment has its own preamplifier that provides a $900-\mu$ sec quenching pulse. Deadtime of the counters is $600 \mu \mathrm{sec}$ so that $900 \mu \mathrm{sec}$ is about the minimum quenching-pulse duration that can be used.

The total counting rate of the ring of counters is $2,600 \mathrm{cpm}$, so a $4 \%$ loss of anticoincidence counting rate occurs due to the total deadtime of the ring of counters. The size of the ring of counters is thus as large as is practicable unless the counting rate were to be reduced by operating underground to reduce the meson flux.

\section{Associated Electronics}

The counter voltage supply, amplifiers, and pulse-height analyzers are described in Figs. 2 and 3.

The output of the pulse-height analy- 
zer is counted as follows: each anticoincidence channel output is counted by a scale-of -4 and a mechanical register, each coincidence channel by a scale-of10 and a register.

Recording equipment provides information about the progress of the counting during overnight operation. When the scale-of-1,000 that counts the anticoincidence output of the single-channel analyzer reaches full-count it resets. This actuates a pulse-forming circuit that feeds a signal to a single-pen millivolt recorder and thus marks the recorder paper. The paper moves at a constant $3 \mathrm{in.} / \mathrm{hr}$, so the counting rate at any time can be calculated from the spacing between marks.

Similarly, the counting rates of the lowest and the highest anticoincidence channels are recorded using scales-of100. The pulses in all four anticoincidence channels are added together and recorded also, using a scale-of $-1,000$.

Until more recording equipment arrives only these most important counting rates can be recorded.

Temporary faults that affect the counting rates will be apparent from the record. The appropriate period can be deleted so that an erroneous answer is not obtained for the counting rate.

It is worth noting the high standard of performance demanded of the electronic equipment and the proportional counter if the correct counting rate is to be obtained. The statistical accuracy possible from this equipment is approximately $0.1 \mathrm{cpm}$, i.e., $6 \mathrm{cph}$.

The voltage applied to the proportional counter is 10,000 volts for a 3-atmosphere filling. A count will be registered for all pulses greater than $2 \mathrm{mv}$ at the anode of the counter. A small rapid change in counter anode voltage, due to corona discharge or current leakage across the insulators, can cause spurious pulses. To avoid this, counter voltage must not change more than 1 part in 5,000,000 per $100 \mu$ sec. If such pulses do occur the counting rate in channel 1 will change, the other channels not being affected. The counting rates from the other channels can thus be used for calculating sample activity for this particular run.

Spurious anticoincidence pulses can also result from a change in efficiency of the ring counters and pulse analyzer. The coincidence counting rate is 600 cpm, so that $0.02 \%$ change in ringcounter or pulse-analyzer efficiency will

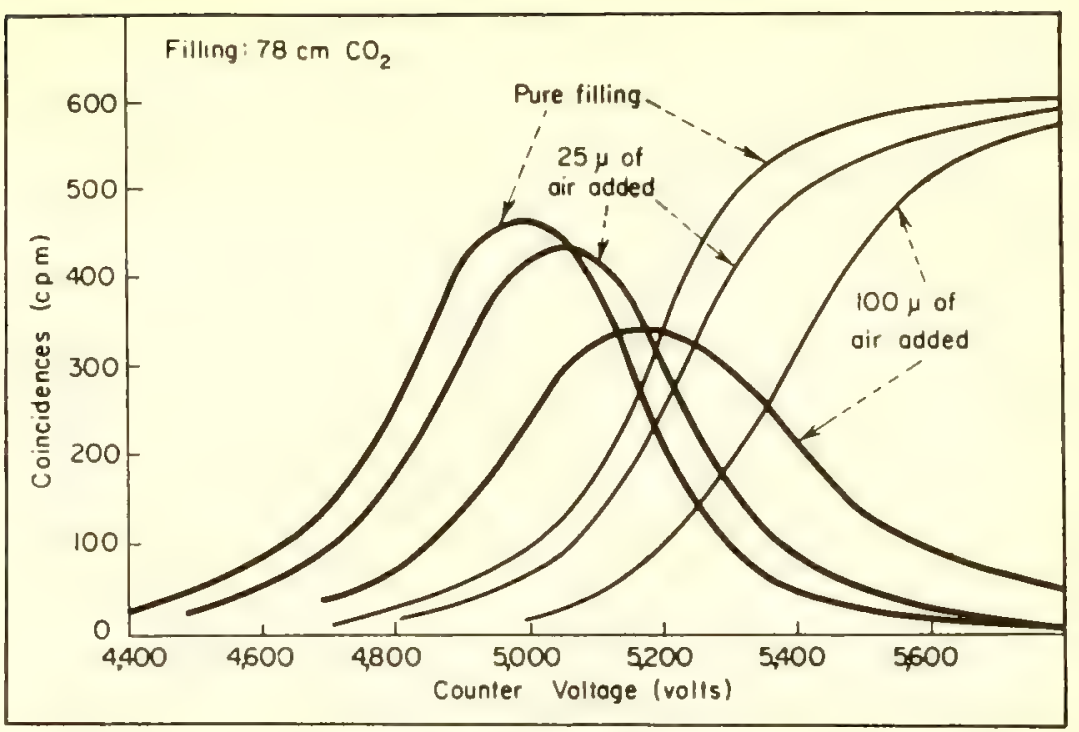

FIG. 5. Curves used to check for electronegative impurities give coincidence counts in channel 1 (heavier lines) and channels $2+3+4$ (lighter lines) as a function of counter voltage

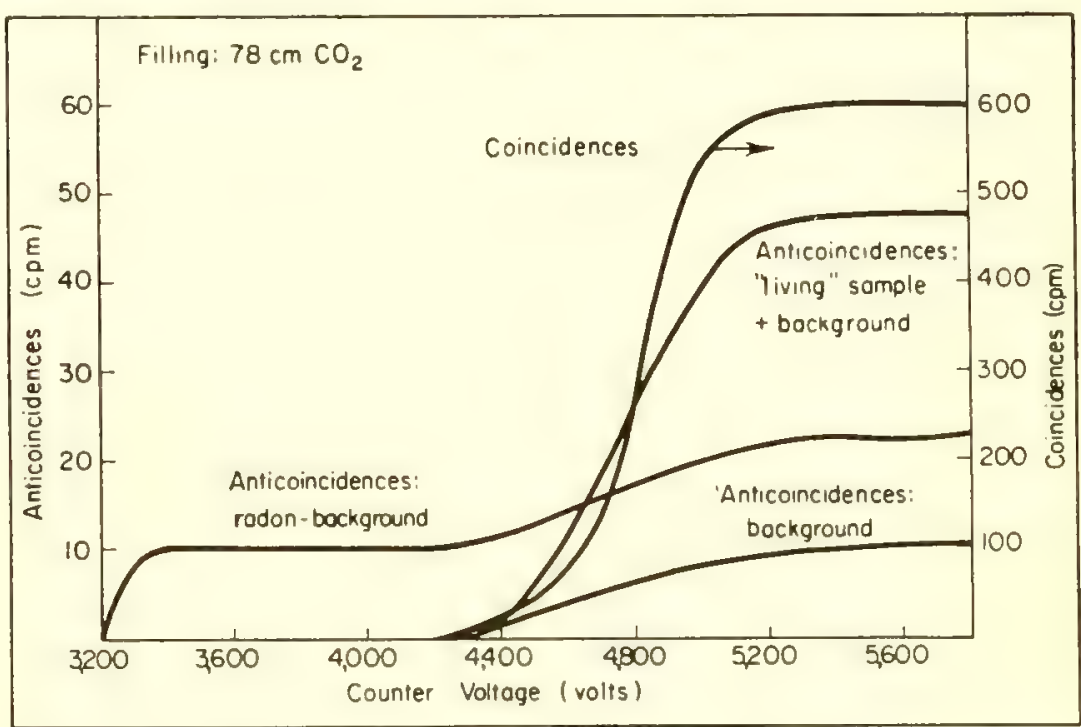

FIG. 6. Total coincidence and anticoincidence counting rates as a function of proportional-counter voltage for a $78-\mathrm{cm} \mathrm{CO} \mathrm{CO}_{2}$ filling. Flat plateaus suggest low electron attachment

\section{$\mathrm{CO}_{2}$ as a Counter Gas}

The results in our laboratory show that previous difficulties with $\mathrm{CO}_{2}$ as a counter gas were due to electronegative impurities and not to the $\mathrm{CO}_{2}$ itself. These electronegative impurities were separated from the $\mathrm{CO}_{2}$ but have not yet been identified. For reliable proportional counting, the loss of electrons due to electronegative impurities should be less than a few per cent. To keep the electron loss less than $1 \%$, the concentration of $O_{2}$ must be less than 1 in $10^{6}$, the concentration of $\mathrm{Cl}_{2}$ must be less than 1 in $10^{7}$.

When we first considered using $\mathrm{CO}_{2}$ we were not very optimistic in view of the measurements of English and Hanna (11). These workers were unable to get saturation even with the purest $\mathrm{CO}_{2}$ commercially available, indicating that electron attachment was taking place. Freedman and Anderson (12) had used a $\mathrm{CO}_{2}$-filled proportional counter for biglogical applications, but the plateau slope of $10 \%$ again indicated electron attachment. De Vries and Barendsen (5) have applied $\mathrm{CO}_{2}$ counting to radiocarbon dating, using a small counter. 


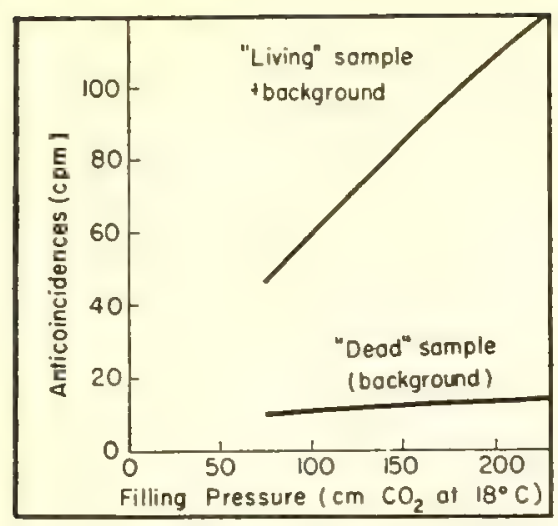

FIG. 7. Change in anticoincidence rate with filling pressure

cause $0.12-\mathrm{cpm}$ change in the anticoincidence counting rate

Thus very careful design and construction of counting equipment is essential to achieve the reliable counting required for this dating method.

\section{Checking Counter Performance}

The pulse amplitude distribution of the coincidence pulses provides a chech on the $\mathrm{CO}_{2}$ with respect to electronegative impurities. The coincidence pulses are used because they are due to the cosmic-ray meson flux across the counter, which is essentially constant, apart from variations of a few per cent caused by barometric pressure changes. The anticoincidence pulse distribution is not very suitable because of the large change with different samples and also because of its lower counting rate.

The number of coincidence pulses from the amplifier between 7.5 and 30 volts and the number greater than 30 volts (i.e., channel 1 and the sum of channels 2,3, and 4) are plotted versus counter voltage in Fig. 5. A small amount of electronegative gas displaces these curves as shown. The counter voltage at which a pair of curves cross can be quickly checked, since both curves are steep there. A quick and accurate check of $\mathrm{CO}_{2}$ purity is thus possible.

The counter voltage at the cross point will repeat from one filling to the next within 5 volts in 5,500 for a $78-\mathrm{cm}$ filling. If $1 \%$ of the electrons are being lost due to electron attachment, the cross-over point will occur at a gas gain $1 \%$ greater than for no loss. A $1 \%$ change in gas-gain corresponds to a counter-voltage change of 216 volts. Thus from one filling to the next the change in electron loss due to electron attachment is less than $2 \%$.

\section{Distribution of Anticoincidence Events}

Channel

1

2

3

4

Source

(\% of total counts in each channel)

Residual counter background

$\mathrm{C}^{14}$ disintegrations from $\mathrm{CO}_{2}$ in counter

$\mathrm{CO}^{60}$ source outside shield

Radon in counter in equilibrium with daughters

\begin{tabular}{rrrr}
14 & 61 & 22 & 3 \\
13 & 75 & 12 & 0 \\
9 & 62 & 29 & 0 \\
6 & 32 & 18 & 44 \\
\hline
\end{tabular}

Observations of the shape of the curve of channel 1 with change of filling pressure suggests that not only is the change in electron loss less than $2 \%$, but that the actual electron loss is less than 2\%. Further work with other filling gases is needed to prove this.

Total coincidence and anticoincidence counting rates plotted versus applied voltage in Fig. 6 show flat alpha and beta plateaus. The flat beta plateau suggests that the counter is $100 \%$ efficient for ionization occurring in the sensitive volume. This conclusion is also supported by a comparison of the total meson rate and the cross sectional area for the proportional counter and for smaller G-M counters known to be better than $99.8 \%$ efficient for detection of mesons. End effects must also be small because of the excellent plateau of the counter, but as a precaution, these have been eliminated by measuring the counting rate of living carbon using different anode lengths with the same style of anode terminations.

The choice of energy range covered by each channel was made so that the lower limit of the lowest channel falls on the beta plateau while the highest channel includes only those pulses due to alpha rays. The boundaries of channel 2 were chosen so that approximately $75 \%$ of the $\mathrm{C}^{14}$ disintegrations fall in this band.

The change in anticoincidence counting rate with change of filling pressure is shown in Fig. 7 for a "dead"-carbonsample filling and for a "living"-carbon-sample filling.

\section{Background}

Some radon is always present in each filling. This radon arises from a trace of radium in the lime employed in the final step of the chemical purification.
The amount present is proportional to the time the $\mathrm{CO}_{2}$ is left absorbed on the lime. The pulses due to alpha rays from radon and its daughter products are recorded in channel 4. However, beta rays from daughters of radon produce pulses in channels 1,2 and 3. The number of these pulses bear a fixed relation to the number of pulses in channel 4, and a correction can be applied.

The table on this page shows the distribution of anticoincidence events in the four channels for several sources of radioactivity, measured for normal operating conditions.

The remaining background counting rate of the proportional counter is the sum of four components due to counter contamination, mesons not detected by the counter ring, gamma rays from radioactive conta mination of the shield, and gamma rays associated with the cosmic rays. Previously, when G-M counters have been used, it has not been possible to investigate the relative contributions of these four components. The use of a proportional counter, combined with different filling pressures, now enables the following information to be obtained.

1. Alpha and beta rays from radioactive contamination of counter matericls. An alpha counting rate of 0.33 cpm is obtained when the counter is filled with radon-free $\mathrm{CO}_{2}$. The source of this alpha rate is almost certainly traces of the elements of the U and Th series in the copper wall of the counter. Thus, beta rays will also be present, and owing to their greater range, their contribution to the background counting rate will be approximately five times the alpha counting rate. Thus the counting rate due to radioactive impurities in the copper of the counter will be approximately $2 \mathrm{cpm}$. The 
area of the cathode surface of the sensitive volume of the counter is $2,100 \mathrm{~cm}^{2}$, so that the alpha rate is $1.6 \times 10^{-4}$ $\mathrm{cm}^{2} / \min$, which agrees closely with the lowest figures reported by Bearden (6). It is therefore unlikely that this background component could be decreased appreciably by trying to obtain radioactively purer material for the construction of the counter.

2. Cosmic-ray mesons not detected by the ring of counters. Calculations based on the geometrical relationship of the proportional counter and the surrounding ring of counters indicate that approximately $2 \mathrm{cpm}$ of the background counting rate will be due to mesons not detected by the ring of counters. With more counters in the ring this component could be eliminated at the expense of a slight increase in equipment complexity.

3. Gamma rays from radioactive contaminants in the gamma-ray shield. It has been noted (Fig. 7) that the background counting rate increases with filling pressure for "dead" $\mathrm{CO}_{2}-$ being 10, 12, and $14 \mathrm{cpm}$ for 1, 2, and 3 atmospheres respectively. This could be due either to a radioactive contaminant in the gas or to an increase in efficiency of detection of gamma rays. The relative efficiency of detection of a strong gamma ray source outside the gamma ray shield was therefore measured as a function of pressure of $\mathrm{CO}_{2}$ in the counter. An increase of $30 \% /$ atmosphere was observed.

The $2 \mathrm{cpm}$ due to counter contaminants and the $2 \mathrm{cpm}$ due to undetected mesons will be independent of pressure. The variation of the remainder of the background counting rate with pressure is then 6,8 , and $10 \mathrm{cpm}$ for 1,2 , and 3 atmospheres respectively. As this agrees with the change of gammaray efficiency with pressure it may be concluded that, at 1 atm filling pressure, $6 \mathrm{cpm}$ are due to gamma rays passing through the counter.

The gamma-ray shield provides 8 in. of iron and $1 \mathrm{in}$. of mercury as shielding around the counter. The background counting rate at $1 \mathrm{~atm}$ filling pressure is reduced from 14 to $10 \mathrm{cpm}$ by the addition of the mercury, so a reduction of $4 \mathrm{cpm}$ is due to absorption in the mercury of $\gamma$ rays from the iron. One in. of mercury will have a shielding factor of approximately 6 , so less than 1 cpm of the background will be due to $\gamma$ rays from the iron.

There are two reasons for believing that the gamma rays from radioactive contaminants in the mercury cause only a small $(<5 \%)$ contribution to the background counting rate. Firstly, Kulp and Tryon, in their experiments with a mercury shield ( 7 ), obtained only a small improvement between the use of commercial-grade mercury and triply distilled mercury. Secondly, the $400 \mathrm{lb}$ of mercury used in our shield was obtained in batches from several sources of supply. Batches were compared in lots of $50 \mathrm{lb}$ for radioactive contamination in a low-background Geiger-counter arrangement. No difference between the various batches was detectable.

4. Gamma rays associated with the cosmic-ray flux passing through the gamma-ray shield. The background counting rate appears to depend on barometric pressure, but unfortunately statistical variations of the counting rate and the small percentage change of barometric pressure prevent this cor- relation from being established accurately. The pressure coefficient observed for mesons (coincidence counts from counter) is $-2 \% / \mathrm{cm} \mathrm{Hg}$. For the total background counting rate (anti-coincidence counts from counter) it is $(-3 \pm 1) \% / \mathrm{cm} \mathrm{Hg}$. Therefore, it is reasonable to assume that the remaining $5 \mathrm{cpm}$ of background due to gamma rays is associated with cosmic rays.

The amount of radon and thoron present in the atmosphere is known to be dependent on the barometric pressure. The possibility of this variation affecting the counting rate has been eliminated by continuous flushing of the space between the proportional counter and the mercury shield with nitrogen gas.

If the preceding reasoning is correct, a considerable decrease in the background counting rate should be obtained by setting up the equipment underground so as to reduce the components ( 2 and 4 ) associated with cosmic rays. At $100 \mathrm{ft}$ underground, background components associated with cosmic rays would be reduced suffciently to be negligible compared to shield and counter contaminant background. The background counting rate of the equipment would then be approximately $3 \mathrm{cpm}$.

The author wishes to acknowledge the collaboration of T.A. Rafter who developed the chemical procedures for burning the samples and purifying the $\mathrm{CO}_{2}$, and of W. $\mathrm{MCC}$ Cabe for his careful preparation of the numerous samples that have been necessary during developmental work. Construction of nearly all electronic equipment has been by $K$. A. Bargh whose attention to detail has contributed to its reliability. This paper is published with permission of the Director of the Dominion Physical Laboratory.

\section{BIBLIOGRAPHY}

1. W. F. Libby, "Radiocarbon Dating" (Uni veraity of Chicago Press, Chicago, III., 1952)

2. G. J. Fergusson, Nevo Zealand J. Sci. Technol. B35, 90 (1953)

S. T. A. Rafter, New Zealand J. of Sci. Technol. B35, 64 (1953)

4. E. C. Anderson, H, Levi, Kol, Danske Videnskab. Selskab., Matfys Medd., 27, No. 6 (1952)

5. H. L. DeVries, G. W. Barendsen, Physica 19, 987 (1953)

6. J. A. Bearden, Rev. Scr. Instr. 4, 271 (1933)

7. J. L. Kulp, L. E. Tryon, Rev. Sci. Inatr 33, $296(1952)$

8. F. N. Hayes, D. L. Williams, B. Rogers, Phys. Rev. 92, 512 (1953)

9. M. Blau, E. S, Deevey, M, S. Gross, Science 118, 1 (1953)

10. J. L. Kulp, Atomics 4, 96 (1953)

11. W. N. English, G. C. Hanna, Can. J. Phys. 31, 768 (1953)

12. A J Freedman, E, C Anderson, NoCLEONIC8 10, No. 8, 57 (1952)

19. A. R. Crathorn, Nature 172, 632 (1953)

14. H. Barker, Nalure 172, 631 (1953)

1B. J. R. Arnold, Science 119, 155 (1954) 


\section{Activation Analysis}

\section{Determines Sodium Content}

\section{of Aluminum Alloys}

\section{Precision better than $1 \%$, a limiting accuracy of $0.0001 \%$, and an average standard deviation of $0.00024 \%$ are obtained in the range $0.01-0.04 \%$ sodium when neutron-activation analysis is applied to aluminum alloys}

BY ROBERT C. PLUMB and

RALPH H. SILVERMAN

Aluminum Research Laboratories Aluminum Company of America New Kensington, $\mathrm{Pa}$.

NeUtron-ACTIVATION-ANALYSIS principles and techniques, as applied to determining the sodium content of aluminum alloys, are presented by this article. After outlining the procedure, details of the irradiation, chemical separation, and measurement steps are given. The precision and accuracy obtained and the problems overcome are presented.

\section{Analysis Principles}

Analysis by neutron activation has been described by many authors (1-4). In its simplest form, it consists of exposing samples containing an unknown amount of an element together with standards containing a known amount of that element to a neutron flux and comparing the activity produced in each. In the general case a variety of radioisotopes could be produced from elements in the samples. Therefore, the radioisotopes being determined must be separated chemically. A salient feature of activation analysis is that the separation need not be quantitative but must yield a product of radiochemical purity, i.e., free from other radioisotopes. A large quantity of nonradioactive carrier may be added to the irradiated material to facilitate the separation. The comparison method of activation analysis described here has a distinct advantage over many other comparison methods in that primary standards, such as pure salts, can be used.

There are several possible shortcomings in activation analysis. It is generally assumed that neutron flux through reasonably sized samples is constant. However, in detailed consideration, there may be variations in neutron flux over the irradiation container dimensions from inhomogeneities in the neutron source and from attenuation of neutrons by the samples them-

\section{Chemical Analysis Method}

Determination of sodium in aluminum is customarily made by a spectrochemical comparison of samples with standards whose sodium content has been determined by a sinter-leach method (5).

Completeness of sodium removal from aluminum by the sinter-leach method has been open to question. There has been no direct proof of the validity of the chemical determination because of the difficulty of preparing synthetic metal standards of known sodium concentration and the prohibitively large blanks encountered in complete sample dissolution procedures. Neutron activation analysis appears to have sufficient sensitivity and inherent accuracy to be applied as an independent method of analysis. 

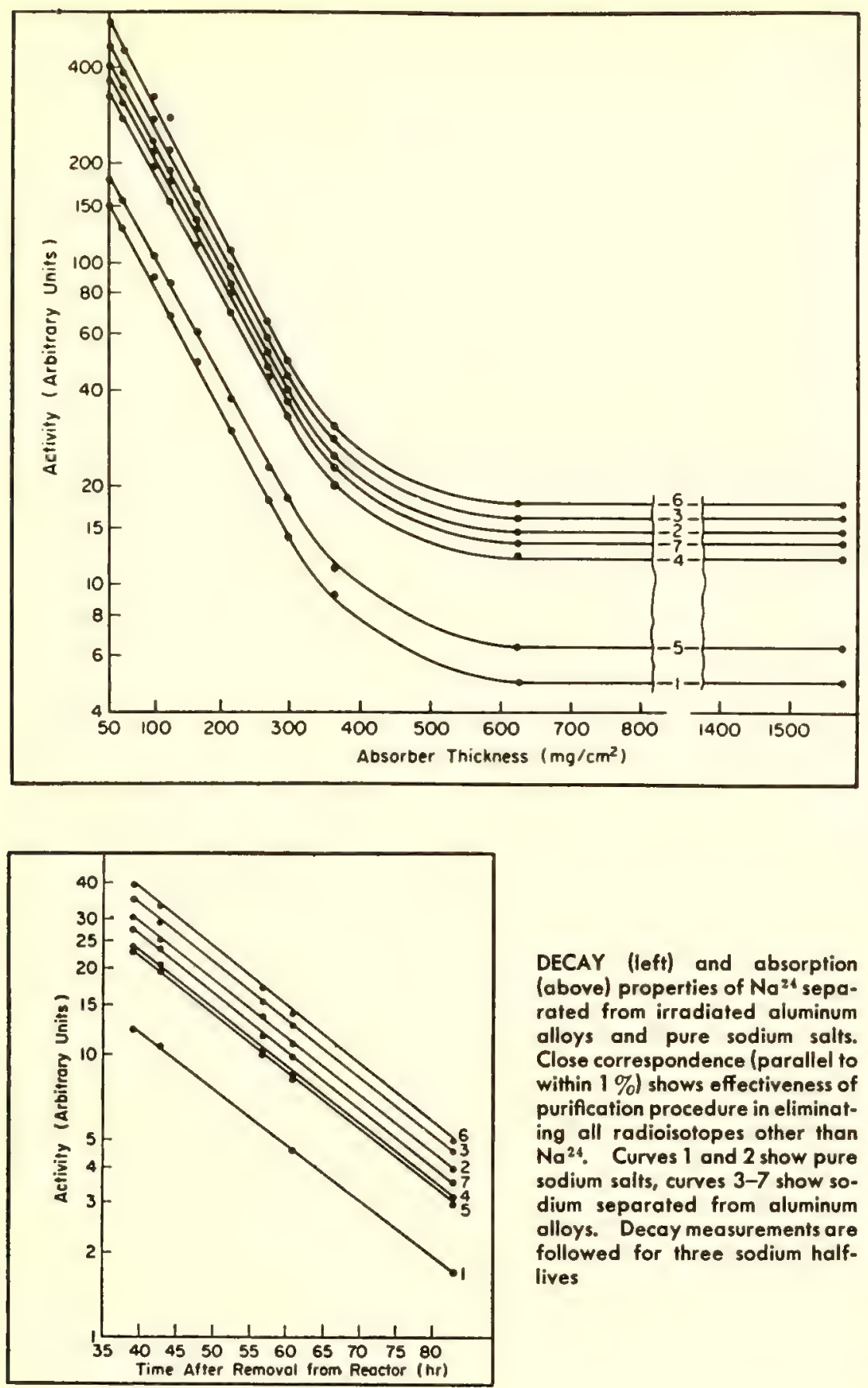

DECAY (left) and absorplion (above) properties of $\mathrm{Na}^{24}$ separated from irradiated aluminum alloys and pure sodium salts. Close correspondence (parallel to within $1 \%$ ) shows effectiveness of purification procedure in eliminating all radioisotopes other than Na24. Curves 1 and 2 show pure sodium salts, curves $3-7$ show sodium separated from aluminum alloys. Decay measurements are followed for three sodium halflives

selves. Similarly, the reactions having the highest cross section for thermal neutrons are of the $(n, \gamma)$ type, but under actual irradiation conditions nonthermal neutrons may cause transmutation reactions [such as $(n, \alpha)$ and $(n, p)]$; producing radioisotopes of the element in question from other elements originally present in the sample.

\section{Experimental Details}

All the samples analyzed were cut from existing spectrochemical stand- sodium salt than did aluminum. A $2 \mathrm{~S}$ aluminum tube 5-mm i.d. and 1-in. long, crimped at each end and cleaned with Alcoa R-5 Bright Dip (U. S. patent $2,650,157$ ) was adopted.

Samples were irradiated in a standard aluminum irradiation can (0.75-in. i.d. $\times 2.875$-in. long) for one week at the Oak Ridge $\mathrm{X}-10$ reactor.

Irradiation conditions. Irradiation of aluminum alloys leads, in general, to the production of $\mathrm{Na}^{24}$ from sodium $(n, \gamma)$, aluminum $(n, \alpha)$, and magnesium $(n, p)$. Specific activity of the final salt is given by $\left(K \times \mathrm{Na}+K^{\prime} \times \mathrm{Al}+\right.$ $\left.K^{\prime \prime} \times \mathrm{Mg}\right) /(\mathrm{Na}+$ carrier $\mathrm{Na})$, where the $K$ 's are activation constants, and the element symbol stands for the weight of the element involved.

In the first work, the contribution of $\mathrm{Na}^{24}$ activity from transmutation reactions put a serious limitation on the sensitivity and precision. By activating samples of super-purity aluminum and magnesium, positions in the reactor that reduce $K^{\prime}$ and $K^{\prime \prime}$ to very small values were found. The apparent sodium content of the super-purity aluminum as determined after exposure in several positions was:

$\begin{array}{cc} & \begin{array}{c}\text { Apparent content from } \\ \text { Reaclor position }\end{array} \\ A^{27}(n, \alpha) \mathrm{Na}^{24} \text { reaction } \\ 14-\mathrm{A}-14 & 0.014 \% \mathrm{Na} \\ 13-\mathrm{H}-7 & 0.005 \% \mathrm{Na} \\ 14-\mathrm{Z}-20 & 0.00019 \% \mathrm{Na}\end{array}$

All further activations were carried out in positions 14-Z-19 through 14-Z-21 in which super-purity aluminum gave from $0.00014-0.00020 \%$ sodium. The lower flux in these positions (as compared to the maximum flux available in the reactor) was of no serious consequence.

After irradiating the samples, the sodium formed is chemically separated and its activity determined. The procedures for these steps will be presented after first considering the irradiation conditions in more detail. Specifically, the flux received by samples in different positions in the container will be examined.

Flux variations. Two experiments were used to detect and evaluate flux variations over the can dimensions that might occur from flux inhomogeneities in the irradiation can's immediate vicinity.

Liners of tin were placed inside the cans, the cans irradiated, and the specific activity at various points on the liner determined. There was a 
maximum variation in flux of $3 \%$ from one end of the can to the other. In further experiments, quantities of sodium carbonate were irradiated in the top, middle, and bottom of a can. The specific activities obtained indicated a flux variation of less than $1 \%$.

The most likely source of error from attenuation within the can would be self-shielding by the aluminum alloy samples. In order to determine whether or not this was an important factor, the array of disks used in an analysis was simulated by cylindrical coils of aluminum foil $(0.001 \mathrm{in}$.) $2-\mathrm{cm}$ in height and $1-\mathrm{cm}$ in radius. The coils were irradiated and the flux variation within them determined by counting 14 -in. circles punched from various positions along a radius. The variation with radius at half the height of the coil was about $5 \% / \mathrm{cm}$.

From the foregoing variations, it can be shown that the average flux on a disk in the middle of the eylinder differs from the average flux on a disk at the end of the cylinder by less than $1 \%$.

Sodium separation. Aliquots from the $\mathrm{Na}_{2} \mathrm{CO}_{3}$ and $\mathrm{NaHCO}_{3}$ standards are carried through a process identical to the following one for obtaining radiochemically pure sodium from the irradiated aluminum alloys. The alloy is dissolved with $\mathrm{HCl}$ and $\mathrm{H}_{2} \mathrm{O}_{2}$ in the presence of 5-mg of carrier $\mathrm{Na}$ (as $\mathrm{NaCl}$ ). The residue after the highsilicon alloys are dissolved is collected and the Si volatilized as $\mathrm{SiF}_{4}$. The remaining residue is then dissolved and added to the $\mathrm{HCl}$ solution. Precipitation of the $\mathrm{Na}$ as sodium zinc uranyl acetate follows. The precipitate is filtered, then redissolved in hot water. As a general clean-up, heavy metals are then precipitated as sulfides. This step also eliminates the possibility of Zn contamination by exchange with the reagent, or $\mathrm{Cu}$ contamination by co-precipitation. The sodium is reprecipitated, and the resulting salt is used directly for the counting measurements. The figures on page 30 show the effectiveness of the purification procedure.

Activity determination. The counting measurements were carried out using an end-window Geiger tube. Enough counts were taken to obtain less than $1 \%$ statistical error. To eliminate self-absorption errors, all $\beta$ radiation was filtered out with a 600 $\mathrm{mg} / \mathrm{cm}^{2} \mathrm{Al}$ filter and only $\gamma$ rays were counted. To eliminate backscattering errors, the samples were counted in identical containers. Counting measurements were made at rates no higher than 10,000 counts per minute, and experimentally determined coincidence corrections were applied.
Sodium Content of Aluminum Alloys

\begin{tabular}{|c|c|c|c|c|}
\hline $\begin{array}{c}\text { Aluminum } \\
\text { Alloy }\end{array}$ & $\begin{array}{l}\text { Previously } \\
\text { assigned } \\
\text { \% sodium }\end{array}$ & $\begin{array}{l}\text { Neutron } \\
\text { activation } \\
\% \text { sodium }\end{array}$ & $\begin{array}{c}\text { Number of } \\
\text { delerminations }\end{array}$ & $\begin{array}{c}\text { Standard } \\
\text { deviation } \\
\text { (\%) }\end{array}$ \\
\hline $3 \mathrm{~A}$ & $\begin{array}{l}0.0014 \\
0.002 \\
0.008 \\
0.0006 \\
0.005 \\
0.032 \\
0.018 \\
0.023 \\
0.016\end{array}$ & $\begin{array}{l}0.0017 \\
0.0024 \\
0.0107 \\
0.0007 \\
0.0064 \\
0.0352 \\
0.0199 \\
0.0267 \\
0.0189\end{array}$ & $\begin{array}{l}2 \\
4 \\
2 \\
1 \\
2 \\
4 \\
2 \\
2 \\
2\end{array}$ & $\begin{array}{c}0.00007 \\
0.00016 \\
0.00000 \\
- \\
0.00010 \\
0.00083 \\
0.00000 \\
0.00007 \\
0.00016\end{array}$ \\
\hline $7.5 \% \mathrm{Si}$ & $\begin{array}{l}0.002 \\
0.032\end{array}$ & $\begin{array}{l}0.0015 \\
0.0307\end{array}$ & $\begin{array}{l}1 \\
2\end{array}$ & $\overline{0.00000}$ \\
\hline 220 & $\begin{array}{l}0.001 \\
0.006 \\
0.011 \\
0.026\end{array}$ & $\begin{array}{l}0.0005 \\
0.0061 \\
0.0125 \\
0.0265\end{array}$ & $\begin{array}{l}2 \\
2 \\
2 \\
4\end{array}$ & $\begin{array}{l}0.00000 \\
0.00007 \\
0.00045 \\
0.00040\end{array}$ \\
\hline 2024 & $\begin{array}{l}0.017 \\
0.013 \\
0.006 \\
0.0025\end{array}$ & $\begin{array}{l}0.0168 \\
0.0130 \\
0.0063 \\
0.0027\end{array}$ & $\begin{array}{l}4 \\
2 \\
2 \\
2\end{array}$ & $\begin{array}{l}0.00025 \\
0.00040 \\
0.00010 \\
0.00000\end{array}$ \\
\hline 7075 & 0.006 & 0.0054 & 2 & 0.00016 \\
\hline
\end{tabular}

The activity from the uranium in the sodium zinc uranyl acetate salt, equivalent to about $0.0003 \%$ sodium, was determined by means of a blank and corrected for. The relative activities of the primary standards and the unknown samples were read from the decay curves at a particular time.

\section{Results and Conclusions}

Since in positions 14-Z-19 through 14-Z-21, the super-purity aluminum gave from 0.0001 to $0.0002 \%$ sodium, the blank resulting from the transmutation reaction can be no larger than about $0.0002 \%$. Conversely, the sodium content of that sample is between the limits 0.0000 and $0.0002 \%$ bat is indeterminate. A correction of about $0.0001 \%$ (1/2 the observed blank) was made in each set of determinations. The uncertainty in the blank results in a limiting accuracy of $\pm 0.0001 \%$ sodium.

Precision and accuracy of method. In the table, the results of several anslyses are given. Many analyses were performed in multiplicate. In the range below $0.01 \%$ sodium, the average standard deviation was $0.00009 \%$; from $0.01-0.02 \%$ sodium, the average standard deviation was $0.00020 \%$; and from $0.02-0.04 \%$ sodium it was $0.00042 \%$. It is apparent from the observed precision that random errors that might be associated with variation in flux over the can dimensions, or with self-shielding by the aluminum alloy samples, and statistical errors associated with the counting measurements, did not exceed $1 \%$.

It is seen from the table that activation analysis gives results in excellent agreement with the previously assigned values for the standards. The technique of activation analysis appears to be capable of producing sufficiently precise and accurate results to be useful not only for directly calibrating primary analytical standards but for general application as an umpire-grade method in cases where requirements of sensitivity or limitations in form or size of sample preclude other methods.

\section{BIBUOGRAPHY}

1. W. A. Brooksbank, G. W. Leddicotte, H. A. Mahlmsn, J. Phys. Chem. 57, 815 (1953)

\&. A. A. 8males, Atomie 8, 2, 55 (1953)

5. W. Herr, Angew. Chem. 11, 679 (1952)

4. G. W. Leddicotte, 8. A Reyuolds, NucuroN IC8 8, 3, 62 (1951)

6. H. V. Churchill, et. al. "Chemical Analysis of Aluminum," (Aluminum Company of America, Pittsburgh, Pa, 1850) 


\section{Nomogram for}

\section{Radioisotope Buildup and Decay}

By J. R. STEHN and E. F. CLANCY

Knolls Atomic

Power Laboratory*

Schenectady

New York

Calculations of build-

up or decay of radio-

isolopes involve the

lerms $e^{-069 M / T_{32}}$ or

$\left(1-e^{-0.683 / T_{3,6}}\right)$ and

often are inconvenient or tedious. This nomogram speeds such compulations.

By plocing a straighted 30 on appropriale positions on the half-life and time of decay or irradiation scales, decay factors or builduploward-saluration facfors for a particular iso. lope can be read at the straightedge inlersection with the scale on the right. One presaution: be sure to state hall. life and time of cooling or irradiation in the same units.

Example A: For a radioisolope whose half-life is 300 days (half-life scale) and whose porent is irradiated for 30 days (time scale), from the buildup-factor scale it can be seen that the rodioisolope achieres $0.066(6.6 \%)$ of its sofuration activity in a given neutron fux.

Example B: After the radioisotope considered in example $A$ has been removed from the noutron flux for a period of 190 days lime scale), from the decay scale it is seen that its radioactivity has decayed to - foctar at $0.64(64 \%)$ at what if was at the time of remoral.

* Operated by the General Electric Co. for the U. S. Atomir Energy Commission.

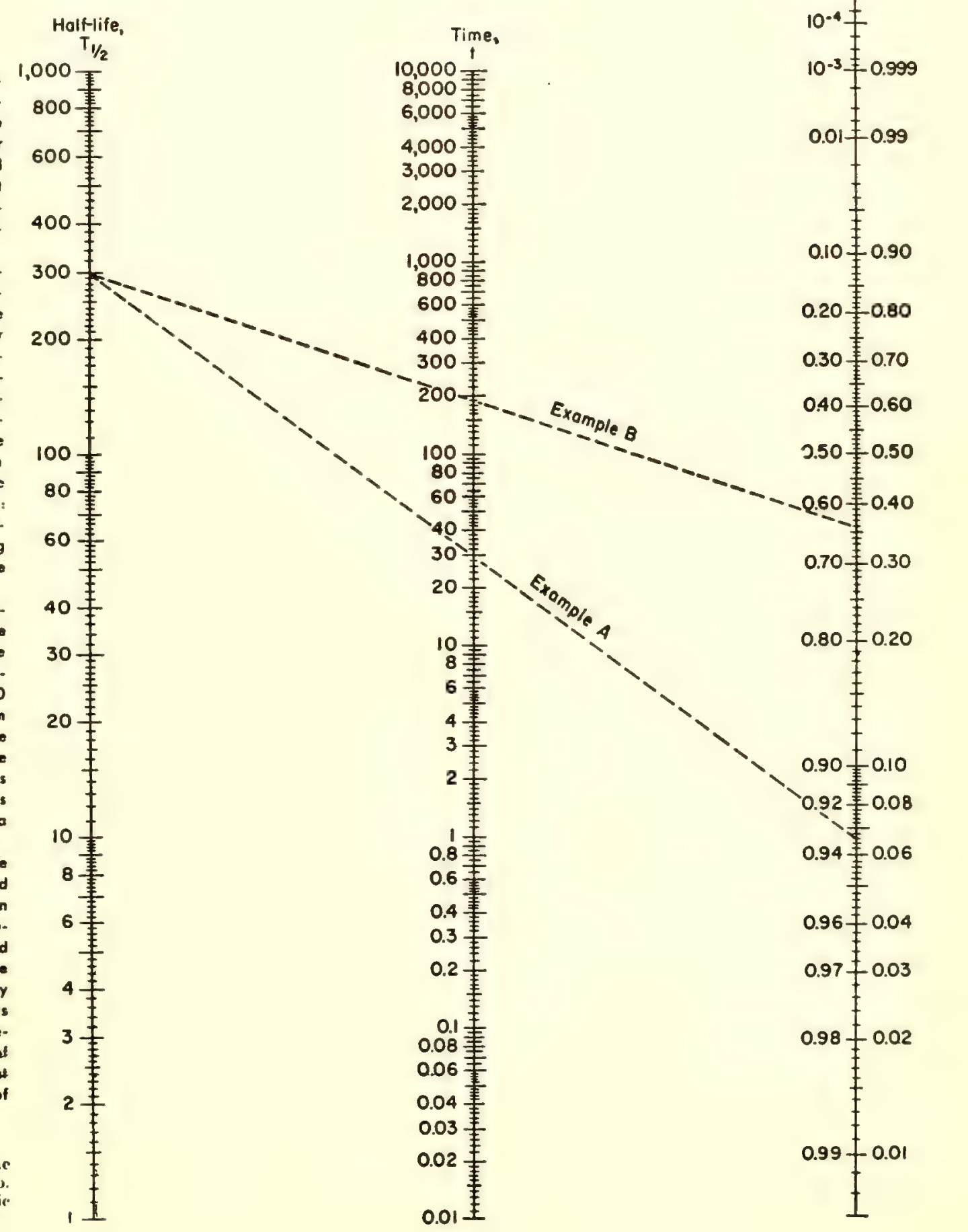




\section{X-Ray Critical-Absorption and Emission Energies in kev}

\section{By S. FINE and C. F. HENDEE Philips Laboratories \\ Irvington on Hudson, New York}

Increased use of energy-proportional detectors for $\mathrm{X}$-rays has created a need for a table of energy values of $K$ and $L$ absorption and emission series.

The table presented here includes all elements. Most values were obtained by a conversion to kev of tabulated experimental wavelength values (1-3); some are from previous energyvalue compilations $(4,5)$. Where a choice existed, the value chosen was the one derived from later work. Certain values were determined by interpolation, using Moseley's law. (All this is annotated in footnotes.)
The conversion equations relating energy and wavelength used are (6)

$E(\mathrm{kev})=(12.39644 \pm 0.00017) / \lambda(\AA)$ $=12.39644 / 1.002020 \lambda(\mathrm{kX}$ unit $)$

In computing values the number of places retained sufficed to maintain the uncertainty in the original source value. The values in the table have been listed uniformly to $1 \mathrm{ev}$. However, chemical form may shift absorption edges as much as $10-20 \mathrm{ev}(4,5)$.

To discover computational errors a fit was made to Moseley's law. In general the values were consistent, however there were a few irregularities due to the deviation of some input values (1). These were retained in the body of the table but a set of values calculated to fit better are footnoted.

The authors wish to express their appreciation to W. Parrish for helpful suggestions and to $H$. Kasper for performing the computation in connection with this work.

\section{BIBLIOGRAPHY}

4. Y Cauchois, H. Hulubei "Tables de Constantes et Donnees Numeriques, I, Longueurs D'Onde des Emissions X et des Discontinuites D'Absorption X" (Hermann et Cie, Paris France, 1947)

2. A. H. Compton and S. K. Allison, "X-rays in Theory and Experiment" (D. Van Nostrand Co., Inc., New York, 1951)

\$. C. E. Moore, "Atomic Energy Levels," NBS 467 (National Bureau of Standards, U. S Department of Commerce, Washington, D. C. 1949)

4. Y. Cauchois, J, phys, radium 13, 113 (1952)

b. R. D. Hill, E. L. Church, and J. W. Mihelich, Rev. Sci. Instr. 23, 523 (1952)

6. J. W. M. DuMond, E. R. Cohen, Phys. Rev。 82, 555 (1951)

X-Ray Critical-Absorption and Emission Energies in kev

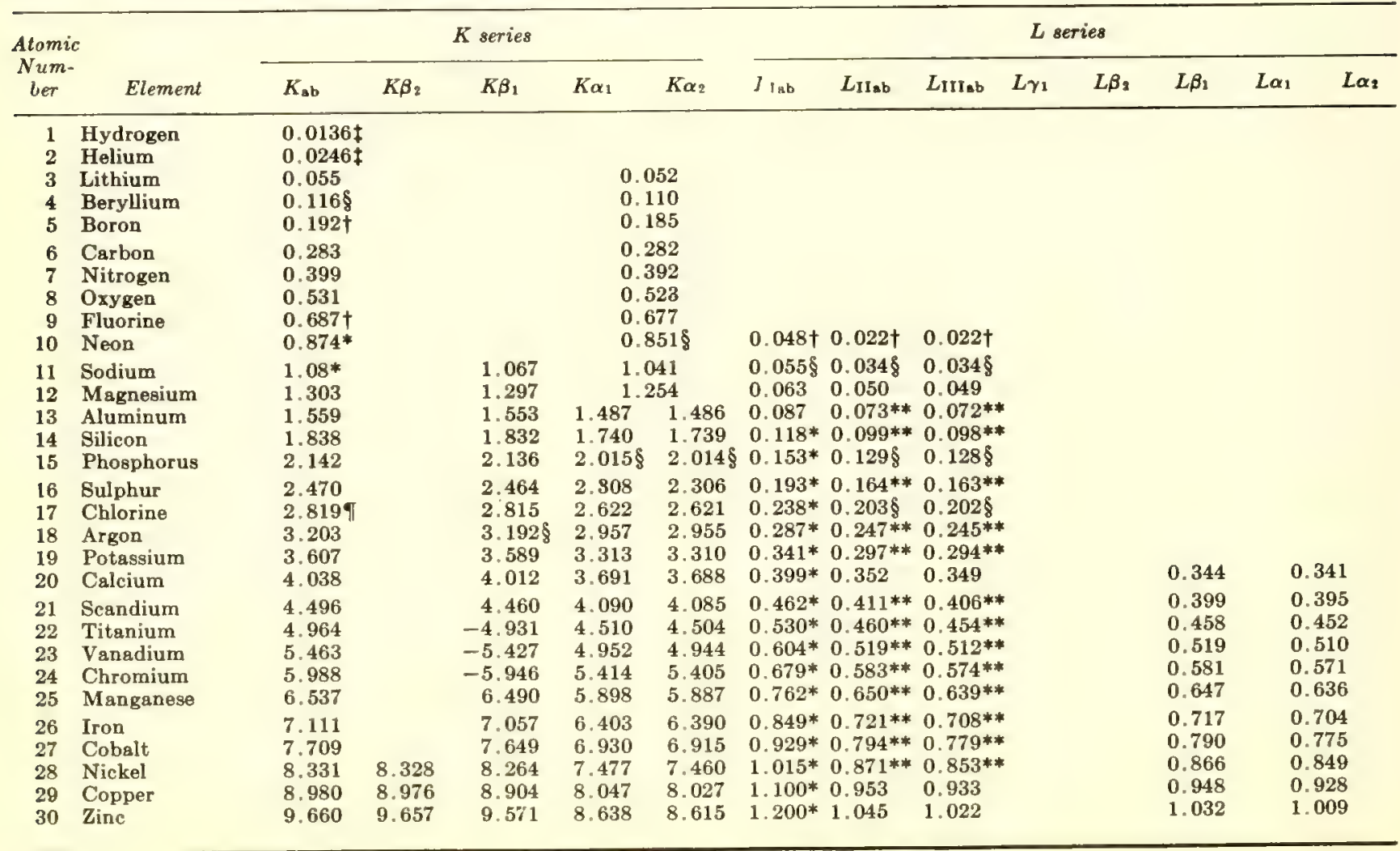




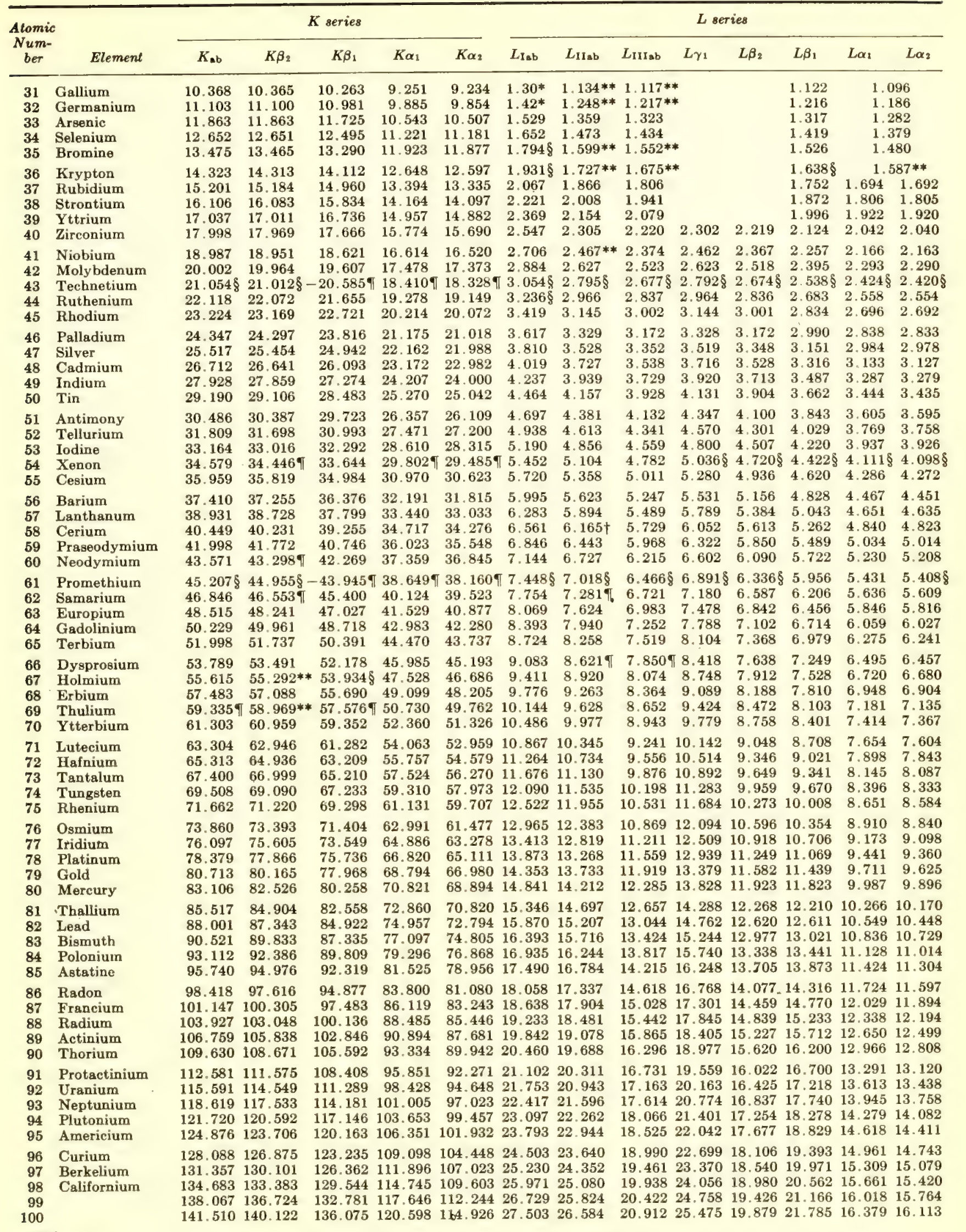

For $Z \leq 69$, values without aymbols are derived from (1). Values prefixed with a - sign are $K \beta_{t+1}$.

For $Z \geq 70$, absorption-edge values are from (4) in the case of $Z=70-83,88,90$, and 92 ; remaining absorption edges to $Z=100$ are obtained from these by least-squares quadratic fitting. All emission values for $Z \geq 70$ are derived from the preceding absorption edges, and others based on (4), using the transition relations $K \alpha_{1}=K_{\mathrm{ab}}-L_{1 I I}, K \alpha_{2}=K_{\mathrm{ab}}-L_{\mathrm{II}}, K \beta_{1}=K_{\mathrm{ab}}-M_{\mathrm{III}}$, etc.

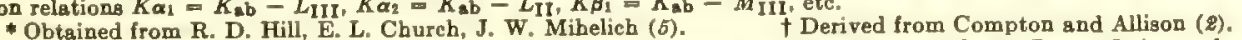

The

$K \alpha_{1}=18.370, K \alpha_{2}=18.250, K \beta_{1}=20.612 ; Z=54, K \alpha_{1}=29.779, K \alpha_{2}=29.463, K \beta_{1}=34.398 ; Z=60, K \beta_{2}=43.349 ; Z=61, K \alpha_{1}=38.726, K \alpha_{3}=$

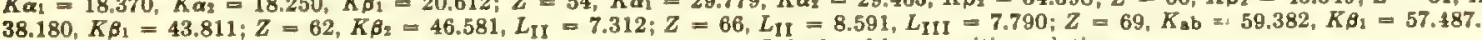

Calculated by method of least squares. ** Calculated by transition relations. 


\section{Gamma Rays from Thermal-Neutron Capture}

\author{
BY PHILLIP S. MITTELMAN \\ and ROBERT A. LIEDTKE \\ Nuclear Development Associates, Inc. \\ White Plains, New York
}

Gamma RAYs from absorption of thermal neutrons in a reactor and its shield are often of major importance to the shield designer. Table on p. 51 lists all available data on these gamma rays in useful form. Not all elements are listed since not all have been measured.

In all cases, the target material is listed. Usually this is the natural element, but in some instances single isotopes are tabulated as indicated by the mass number following the element name. In these instances, the capture cross section applies only to the isotope indicated. Tabulated $(n, \gamma)$ cross sections were usually taken from applicable papers of the Kinsey-BartholomewWalker group (1-14). If no such reference existed, the cross section was obtained from AECU-2040 (35).

Gamma rays emitted by an element after thermal-neutron absorption fall into two classes: "capture" and "decay" gamma rays. Capture gamma rays originate when a nucleus, having absorbed a thermal neutron, releases the binding energy of the neutron (5-10 Mev). Usually this energy is given off promptly $\left(\sim 10^{-14} \mathrm{sec}\right)$ in the form of one or more gamma rays. "Decay" gamma rays are produced if the deexcited nucleus is still unstable, and decays by beta and gamma emission to a stable nucleus; pertinent information is tabulated in the last column.

Capture-gamma-ray spectra are tabulated by four major types. Three are illustrated by Figs. 1-3 and described in accompanying captions. A fourth "type," that for which the gamma rays are weak or nonexistent because particle emission is the favored mode of deexcitation, is necessarily not illustrated. Boron-10 and lithium-6 are the only representatives of that class included here (because of importance to shielding design).

In addition to spectral classification, the number of photons emitted in

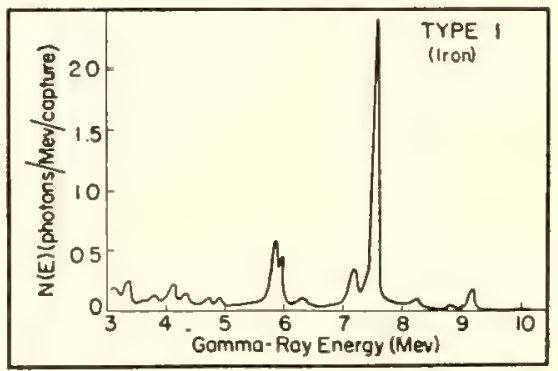

FIG. 1. Type 1 capture-gamma-ray spectrum shows few gamma roys. Groundstate-transition line dominates. Most of de-excitation energy is carried by single 6-8-Mer gamma rays

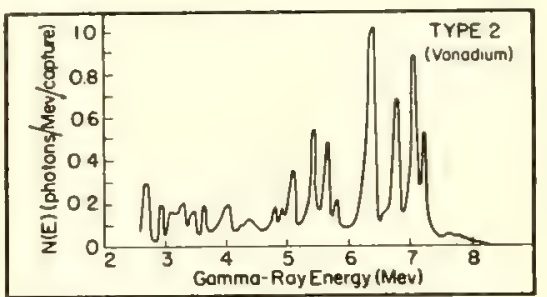

FIG. 2. Type 2 capture-gamma-ray spectrum shows many gamma ráys. Distinct line structure is evident. This is typical of light and medium-weight elements with fairly large level spacing between levels and transitions between levels as likely as transitions to ground

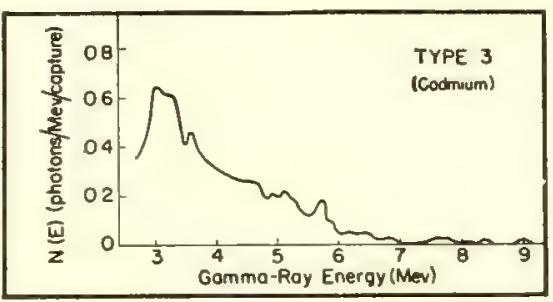

FIG. 3. Type 3 capfure-gamma-ray spectrum shows many gamma rays, no line strucfure below $5 \mathrm{Mev}$. This is typical of heavier elements; they have high level density and overlapping highest levels. Distribution peaks below half maximum energy

various energy intervals per 100 captures is tabulated. In some cases sufficient data were available to determine the total number of photons in each energy interval. In other cases, only the intensity of the resolved lines could be determined. For spectral types 1 and 2 the difference is small, but for type- 3 spectra the difference is significant. Fortunately, sufficient data were available to determine the number of unresolved photons for most type-3 spectra. For those elements for which the number of unresolved photons has been determined, the spectral type is marked with an asterisk (*).

This article is based largely on NDA $10-99$ prepared under contracl with Pratt and Whilney Aircraft Division of L'nited Aircraft Corporation. Table has becn reoised and brought up to date by $R$. A. Lirdike under AEC contract AT(30-1)862C,

\section{BIBLIOGRAPHY}

1. B. B. Kinsey, G. A. Bartholomew, W. H. Walker, Phys. Rev. 82, 380 (1950)

2. B. B. Kinsey, G A. Bartholomew, W. H. Walker, Phys, Rev, 89, 519 (1951)

S. B. B. Kinsey, G. A. Bartholomew, W. H. Walker, Phys. Rev. 85, 1012 (1952)

4. B. B. Kinsey, G, A. Bartholomew, Phys. Rev. 89,375 (1953)

5. G. A. Bartholomew, B. B. Kinsey, Phys, Rev。 89,386 (1953)

6. G. A. Bartholomew, B. B. Kinsey, Phys. Rev. 90, 355A (1953)

7. G. A. Bartholomew, B. B. Kinsey, Phys. Rev. 93, 1434(E) (1954)

8. B. B. Kinsey, G. A. Bartholomew, W. H. Walker, Can. J. Phys. 29, 1 (1951)

9. G. A. Bartholomew, B. B. Kinsey, Can. J. Phys. 31, 49 (1953)

10. B. B. Kinsey, G. A. Bartholomew, Can. J. Phys, 31, 537 (1953)

11. B. B. Kinsey, G. A. Bartholomew, Can. J. Phys. 31, 901 (1953)

12. G. A. Bartholomew, B. B. Kinsey, Can. J. Phys. 31, 927 (1953)

13. G. A. Bartholomew, B. B. Kinsey, Car, J. Phys. 31, 1025 (1953)

14. B. B. Kinsey, G. A. Bartholomew, Can. J. Phys. 31, 1051 (1953)

15. T. H. Braid, Nuclear Science Abstracts, 7, No. 6B, New Nuclear Data (1953); Phys. Rev. 90, 355A, (1953)

16. T. H. Brsid, Phys. Rev. 91, 442 (1953)

17. H. T. Motz, Phys. Rev. 90, 355A (1953), Nuclear Science Abstracts, 7, No. 6B, New Nuclear Data (1953)

18. H. T. Motz, Phys, Rev. 93, 925A (1954)

19. R. W. Pringle, G. Isford, Phys. Rev. 83, 467, (1951)

20. R. W. Pringle, Phys. Rev. 87, 1016 (1952)

21. W. A. Resrdon, R. W. Krone, R. Stump, Phys. Rev. 91, 334 (1953)

22. W. A. Reardon, R. W. Krone, R. Stump. Phys. Rev. 91, 442A (1953)

29. W. Thornton, et al, Phys, Rev, 86, 604A (1952)

24. C. O. Mueblhause, Phys. Rev. 79, 277 (1950)

25. R. E. Bell, L. G. Elliot, Phys. Rev. 79, 282 (1950)

26. F. Ajzenberg, T. Lauritien, Revs. Mod. Phys. 24, 321 (1952)

27. M. Reier, M. H. Shamos, Phys. Rev. 95, 636A (1954) also BNL-1667

28. A. Recksiedler, B. Hamermesh, Phys. Rev., 95, 650 (1954)

\$9. A. Recksiedler, B. Hamermesh, Phys. Rev. 96, 109 (1954)

\$O. B. Hamermesh, R. J. Culp, Phys, Rev. 92, 211L (1953)

31. B. Hamermesh, V, Hummel, Phys. Rev, 88, 916 (1952)

32. B. Hamermesh, V. Hummel, Phys. Rev. 83, 663 (1951)

\$\$. J. M. Hollander, I. Perlman, G. T. Seaborg Revs. Mod. Phys, 25, 469 (1953)

54. P. Chudom and C. O. Muehlhause, Plutonium Project Report CP-3801, Pg. 25

s5. Neutron Cross Section, AECU 2040 


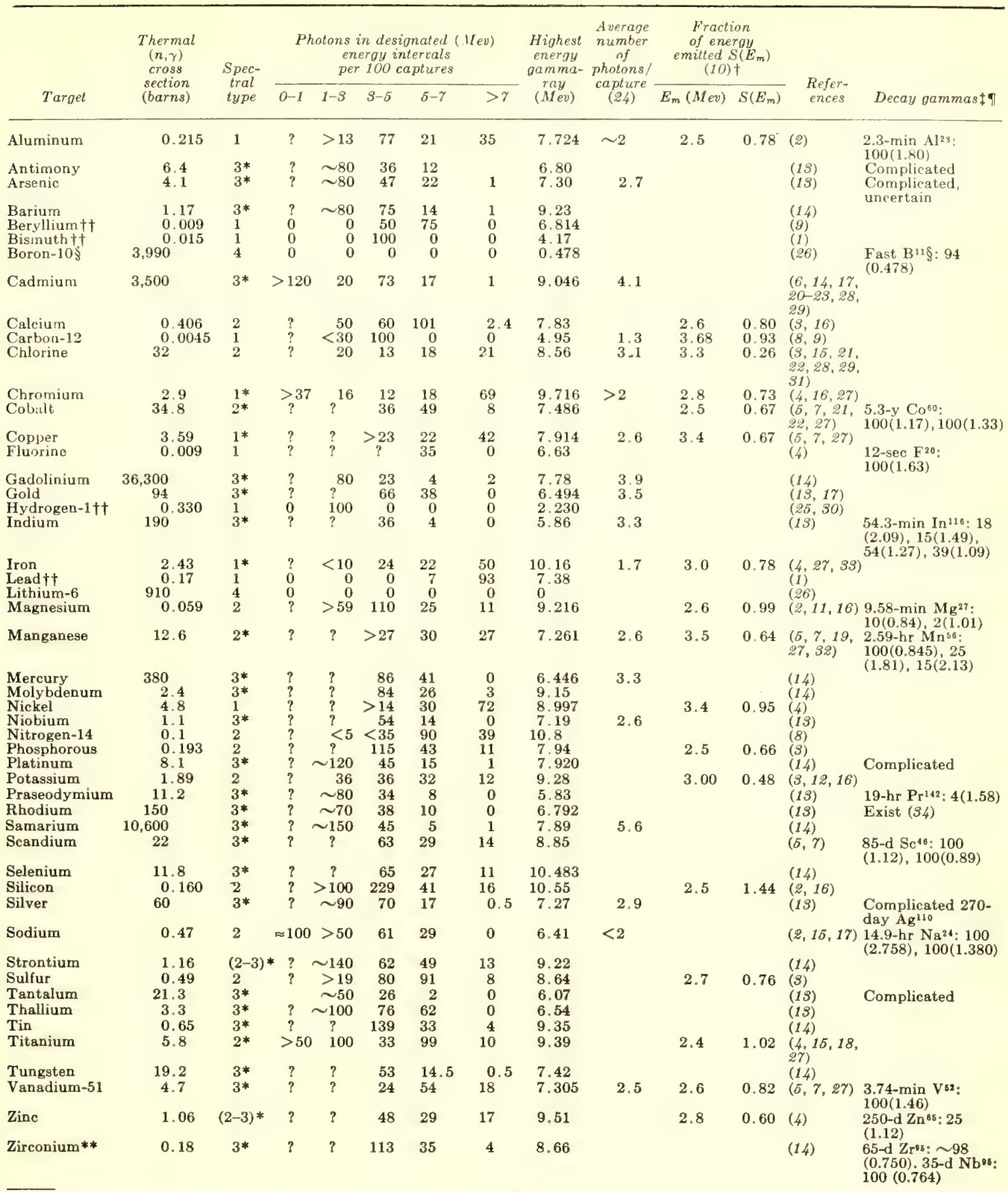

* For elements thus marked, number of unresolved photons has been determined; for all others only the intensity of resolved lines is given.

† Fraction, $S$, of available energy emitted by gamma rays with energy greater than $E_{m} E_{m}$ is the minimum gamma-ray energy observed in the measurement, it has no deep significance.

‡"Complicated" means decay gammas above $750 \mathrm{kev}$ are claimed to exist but the multiplicity of isotopes and/or the complezity of the decay schemes render estimations of intensities difficult. See reference (38) for data.

I Notation for decay gamma rays is as follows: half-life of decay-gamma emitter is given first, then number of decay gamma rays of each energy $>750 \mathrm{kev}$ per 100 neutron captures. Energy is in parentheses. Thus "9.58-min Mg"7: 10 (0.84), 2 (1.01)" means isotope emits $100.84-\mathrm{Mev}$ and $21.01-\mathrm{Mev}$ decay gamms rays per 100 captures.

Boron-10 decay gamma is from $\mathrm{Li}^{7}$ following alpha decay of $\mathrm{B}^{11}$.

** Capture-gamma-ray intensities for zirconium may be off by $50 \%$.

†† For these elements, the highest-energy gamma ray is the only gamma ray present in the highest occupied energy interval. For lead, in addition, the seven 5-7-Mev gamma rays are all $6.73 \mathrm{Mev}$; for beryllium, in addition, the fify 3-5-Mev gammas are all 3.37 Mov. 
Isotope Characteristics

\section{Beta Emitters}

\section{by Energy and}

\section{Half-Life}

\section{BY NAOMI A. HALLDEN}

Analytical Branch

Health and Safety Laboratory

$U$, S. Atomic Energy Commission

New York, N. Y.

THIS TABLE can help identify unknown beta emitters whose half-life and beta energy have been determined by standard laboratory techniques. It also is a guide to beta-emitting isotopes for applications requiring specific halflives and energies. (A similar table for gamma emitters is in preparation.) A more detailed compilation of nuclesr data, such as National Bureau of Standards Circular 499 and Supplements, should be consulted for details of these emitters and their decay.

Emitters of conversion electrons and positrons as well as emitters of beta rays are included since all these particles produce similar effects when absorption methods are used to determine energy. Where an isotope can decay by emission of beta particles of different energies, the emitter is listed in the energy group corresponding to each beta, provided its contribution to total beta activity is greater than $5 \%$. This is the lower limit of detection of typical absorption technique (Harley and Hallden, NU, Jan. '55, p. 32). All the betas from one emitter will lie in the same half-life interval.

Only isotopes with half-lives greater than $6 \mathrm{hr}$ are listed; in general, a shorter half-life limits identification by the methods described.

Daughters with shorter half-lives than their parents are listed in italic under the half-life of the parent. In the natural series, the short-lived daughters are listed under the half-life of the -nearest antecedent having a half-life over $6 \mathrm{hr}$. $\mathrm{U}^{237}(0.1-0.3 \mathrm{Mev}$, $5-10 y)$ should be in italic.

All data used in this compilation are from National Bureau of Standards Circular 499. including Supplements 1, 2, and $\$$.

\begin{tabular}{|c|c|c|c|c|}
\hline Half-life & $E_{\max }($ Hev) & $0.1-0.3$ & $0.3-0.5$ & $0.5-0.7$ \\
\hline $6-12 \mathrm{hr}$ & & $\mathrm{Tm}^{166} \mathrm{Bi}^{204}$ & $\begin{array}{l}f^{135} \mathrm{Eu}^{132} \mathrm{Ta}^{180} \\
\mathrm{~T}^{190} \mathrm{~Pb}^{212} \mathrm{~Pa}^{234}\end{array}$ & $\begin{array}{l}\mathrm{Fe}^{32} \mathrm{Zn}^{62} \mathrm{Pd}^{201} \\
\mathrm{El}^{171} \mathrm{Ta}^{180} \mathrm{PD}^{212}\end{array}$ \\
\hline $12 \mathrm{hr}-1 \mathrm{~d}$ & & $\begin{array}{l}\mathrm{K}^{43} \mathrm{Br}^{76} \mathrm{Pd}^{182} \mathrm{Pr}^{142} \\
\mathrm{~Tb}^{134} \mathrm{Au}^{193}\end{array}$ & $\begin{array}{l}\left.\right|_{N p^{236}} ^{133} \mathrm{Ce}^{135} \text { Ir }^{194} \\
\end{array}$ & $\begin{array}{l}\mathrm{Cu}^{44} \mathrm{Ga}^{72} \mathrm{NO}^{98} \\
\mathrm{H}^{130} \mathrm{Pt}^{197}\end{array}$ \\
\hline $1-3 d$ & $\mathrm{Lu}^{170} \mathrm{Ta}^{177}$ & $\begin{array}{l}\mathrm{Zn}^{72} \mathrm{No}^{99} \mathrm{Ba}^{135} \mathrm{Re}^{184} \mathrm{Os}^{193} \\
\mathrm{Th}^{231} \mathrm{~Pa}^{232} \mathrm{~Np}^{238} \mathrm{~Np}^{239}\end{array}$ & $\begin{array}{l}\mathrm{Cu}^{67} \mathrm{As}^{76} \mathrm{Br}^{77} \mathrm{Br}^{12} \\
\mathrm{Cd}^{113} \mathrm{Sn}^{121} \mathrm{Cs}^{129} \\
\mathrm{~T}^{200} \mathrm{~Np}^{239}\end{array}$ & $\begin{array}{l}\mathrm{Sc}^{48} \mathrm{As}^{71} \mathrm{Ga}^{72} \\
\mathrm{As}^{77} \mathrm{w}^{187} \mathrm{~Np}^{239}\end{array}$ \\
\hline $3-5 d$ & $T b^{137}$ & $\begin{array}{l}\mathrm{Te}^{119} \mathrm{Te}^{132} \mathrm{Eu}^{145} \mathrm{~Tb}^{157} \\
\mathrm{Yb}^{175} \mathrm{Pt}^{193}\end{array}$ & $D y^{166} \mathrm{~V}^{175} \mathrm{Au}^{190}$ & $\left.S^{47} Y^{57}\right|^{124}$ \\
\hline $5-10 d$ & & $\begin{array}{l}\operatorname{Cs}^{131} T^{153} \mathrm{~T}^{167} \mathrm{Lu}^{171} \\
\mathrm{LU}^{172} \mathrm{Lu}^{177} \mathrm{U}^{237}\end{array}$ & $\begin{array}{l}\mathrm{I}^{131} \mathrm{Xe}^{133} \mathrm{Ho}^{163} \\
\mathrm{Er}^{169} \mathrm{Lu}^{177}\end{array}$ & $\begin{array}{l}\left.\mathrm{Hb}^{32}\right|^{131} \mathrm{Cs}^{132} \\
\mathrm{~Tb}^{181} \mathrm{~Pb}^{309}\end{array}$ \\
\hline $10-13 d$ & $4^{190}$ & $\mathrm{Ba}^{131}$ & $\mathrm{Ba}^{140} \mathrm{Nd}^{147} \mathrm{~Pb}^{211}$ & \\
\hline $13-15 d$ & & $\mathrm{Os}^{191} \mathrm{Ra}^{225}$ & $\mathrm{Cs}^{136} \mathrm{Eu}^{149}$ & \\
\hline $15-20 d$ & & & $E u^{136}$ & \\
\hline $20-30 d$ & $w^{179}$ & $T^{234} \mathrm{~Pa}^{233}$ & $\mathrm{Ce}^{142}$ & $\mathrm{Ce}^{141} \mathrm{~Pa}^{233}$ \\
\hline $30-40 d$ & & mos & & \\
\hline $40-50 d$ & $R b^{303}$ & $\mathrm{Fe}^{39} \mathrm{Hg}^{203}$ & $\mathrm{Fe}^{50} \mathrm{Ru}^{203} \mathrm{HI}^{2 \mathrm{I}}$ & $R u^{10 s}$ \\
\hline $50-100 d$ & & $S^{35}$ N69s T-16e & $\begin{array}{l}\mathrm{Sc}^{46} \mathrm{Co}^{58} \mathrm{Zr}^{93} \mathrm{Sb}^{124} \\
\mathrm{~T}^{164} \mathrm{w}^{184}\end{array}$ & $S b^{124} \mathrm{~Tb}^{160} \mathrm{t}^{192}$ \\
\hline $100-150 d$ & $\mathbf{w}^{162}$ & $T^{170}$ & & $T a^{182}$ \\
\hline $150-200 d$ & & $\mathrm{Ca}^{45} \mathrm{Lu}^{194}$ & & $\mathrm{Lu}^{174}$ \\
\hline $200-250 \mathrm{~d}$ & & & $Z_{n}=$ & \\
\hline $250 d-1 y$ & $\mathrm{Ru}^{106} \mathrm{As}^{110} \mathrm{~Tb}^{158}$ & $\mathrm{Co}^{58}$ & $\operatorname{Co}^{144}$ & $M^{110}$ \\
\hline $1-2 y$ & $\operatorname{Ta}^{171}$ & Eust & $\sin ^{121}$ & \\
\hline $2-3 y$ & $\operatorname{Cs}^{134}$ & $S^{12 S}$ & & $\mathrm{Ma}^{22} \mathrm{SO}^{225} \mathrm{Cs}^{24}$ \\
\hline $3-5 y$ & & $\mathrm{P}^{147} \mathrm{Lx}^{172}$ & & \\
\hline $5-10 y$ & $\mathrm{Ra}^{228} \mathrm{Pu}^{241}$ & $E u^{184} u^{297}$ & $\mathrm{Co}^{\circ}$ & $\operatorname{Cd}^{123} \mathrm{Eu}^{154}$ \\
\hline $10-20 y$ & $H^{3}$ & & & \\
\hline $20-30 y$ & $\mathrm{~Pb}^{210} \mathrm{Ac}^{227}$ & & & $5 t^{90}$ \\
\hline $30-50 y$ & & & & $\operatorname{Cs}^{137}$ \\
\hline $50-100 y$ & $\mathrm{Ni}^{63} \mathrm{Sm}^{151}$ & & & \\
\hline$>100 y$ & $\begin{array}{l}\mathrm{Pd}^{107} \mathrm{Nd}^{150} \mathrm{Re}^{187} \\
\mathrm{Ac}^{227} \mathrm{Re}^{228}\end{array}$ & $\begin{array}{l}C^{14} R^{27} \mathrm{TC}^{99} 1^{129} \mathrm{Cs}^{235} \\
T b^{231} \mathrm{~Pa}^{230} \mathrm{~Tb}^{234} \mathrm{~Np}^{238}\end{array}$ & $A w^{242}$ & $B e^{10}$ \\
\hline
\end{tabular}




\begin{tabular}{|c|c|c|c|c|c|c|c|c|c|c|c|c|c|c|c|}
\hline $0.7-0.9$ & $0.9-1.1$ & $1.1-1.3$ & $13-1.5$ & $1.5-1.7$ & $1.7-1.9$ & 2.9 & $\begin{array}{l}2.1- \\
2.3\end{array}$ & $\begin{array}{l}2.3- \\
2.5\end{array}$ & $\begin{array}{l}2.5- \\
2.7\end{array}$ & \begin{tabular}{|l|}
$2.7-$ \\
2.9
\end{tabular} & $\begin{array}{l}2.9 \\
3.1\end{array}$ & \begin{tabular}{|l|}
$3.1-$ \\
3.3
\end{tabular} & \begin{tabular}{|l|}
$3.5-$ \\
3.7
\end{tabular} & $\begin{array}{l}3.7- \\
3.9\end{array}$ & $\begin{array}{l}3.9 \\
4.2\end{array}$ \\
\hline $\begin{array}{l}\mathrm{Ga}^{68} \mathrm{Rb}^{82} \mathrm{Te}^{827} \\
\mathrm{Sm}^{136}\end{array}$ & $\begin{array}{l}\mathrm{I}^{1335} \mathrm{Xe}^{135} \mathrm{Er}^{173} \\
\mathrm{Ta}^{176}\end{array}$ & $\begin{array}{l}\mathrm{Se}^{33} \mathrm{~S}^{992} \\
\mathrm{~Pa}^{234}\end{array}$ & $1^{13 s} E_{1}{ }^{171}$ & $A c^{228}$ & $G e^{77} T^{208}$ & $T m^{100}$ & $\begin{array}{l}P d^{101} \\
B i^{252}\end{array}$ & & $M^{52}$ & $C u^{62}$ & $y^{93}$ & $s r^{91}$ & & & $6 a^{66}$ \\
\hline $2 n^{69} \mathrm{Gd}^{139}$ & $\begin{array}{l}\mathrm{Co}^{55} \mathrm{Ga}^{72} \mathrm{Y}^{87} \mathrm{Pd}^{109} \\
\mathrm{I}^{130} \mathrm{Eu}^{157} \mathrm{Am}^{242}\end{array}$ & $\mathrm{Rh}^{100}$ & $\mathrm{Na}^{\mathrm{Na}^{24}} \mathrm{CO}^{53} \mathrm{Ga}^{72} \mathrm{Nb}$ & ${ }_{N 6}^{90} E U^{157}$ & & $\begin{array}{l}K^{42} \\
\operatorname{Re}^{188}\end{array}$ & $\begin{array}{l}Z r^{97} \\
\mid t^{199}\end{array}$ & & $\begin{array}{l}6 a^{72} \\
\mathrm{~Tb}^{134}\end{array}$ & & $\mathrm{Rh}^{100}$ & $\begin{array}{l}\mathrm{Ga}^{72} \\
\mathrm{Br}^{76}\end{array}$ & \begin{tabular}{l|}
$K^{42}$ \\
$A g^{132}$
\end{tabular} & & \\
\hline $\begin{array}{l}\mathrm{Ni}^{37} \mathrm{Rh}^{105} \mathrm{ln}^{115} \\
\mathrm{Sm}^{153}\end{array}$ & $\begin{array}{l}\mathrm{Ge}^{69} \mathrm{Ga}^{72} \mathrm{Cd}^{115} \\
\mathrm{Pm}^{149} \mathrm{Au}^{198}\end{array}$ & $0 s^{193}$ & $\mid \begin{array}{ll}\mathrm{Sc}^{44} \mathrm{As}^{76} \mathrm{Sb}^{122} \mathrm{Te}^{132} \\
\mathrm{Lat}^{140} \mathrm{Ce}^{143} \mathrm{~W}^{187} \mathrm{Nop}^{238}\end{array}$ & $2 n^{72} \operatorname{La}^{140}$ & $H 0^{166}$ & \begin{tabular}{|l|}
$S b^{122}$ \\
$T e^{131}$
\end{tabular} & $\begin{array}{l}Y^{90} \\
L^{140}\end{array}$ & & $\begin{array}{l}\mathrm{Cu}_{u^{68}} \mathrm{Ca}^{72} \\
\mathrm{As}^{76}\end{array}$ & $\mathrm{As}^{72}$ & & $\begin{array}{l}\mathrm{Ga}^{72} \\
\mathrm{As}^{76}\end{array}$ & & & \\
\hline \multirow[t]{2}{*}{$\mathrm{Te}^{\mathrm{X} 27} \mathrm{l}^{132} \mathrm{~Pb}^{214}$} & $21^{89} \mathrm{Re}^{186}$ & $\begin{array}{l}R b^{100} S b^{127} \\
B i^{210}\end{array}$ & 1124 & $B i^{214}$ & $H 0^{165}$ & & $\begin{array}{l}1^{124} \\
1^{132}\end{array}$ & $P_{r}{ }^{140}$ & & & $R s^{100}$ & $B i^{214}$ & & & \\
\hline & $\mathrm{Ca}^{47} \mathrm{Ag}^{112}$ & $B i^{213}$ & & & & & & $\begin{array}{l}S_{n^{235}} \\
P^{124}\end{array}$ & & $A s^{72}$ & $s b^{118}$ & & & & \\
\hline \multirow[t]{2}{*}{ |226 Nd ${ }^{187}$} & $\mathrm{Ba}^{\mathrm{s}} \mathrm{aO}$ & 123 & $\mathrm{Nb}^{92} \mathrm{La}^{140} \mathrm{Tl}^{207} \mathrm{~Pb}^{211}$ & $L a^{140}$ & & & $L a^{140}$ & & & & & & & & \\
\hline & $\mathrm{Pr}$ iss & & & & $p^{32}$ & & & & & & & & & & \\
\hline \multirow[t]{4}{*}{$V^{48} \mathrm{As}^{74} \mathrm{Rb}^{86}$} & $\mathrm{As}^{74} \mathrm{~Pa}^{230}$ & & $\mathrm{Ta}^{178}$ & & Rus & & & $E y^{156}$ & & & & & & & \\
\hline & & $F^{223}$ & & & & & & $\mathrm{~Pa}^{234}$ & & & & & & & \\
\hline & & & & & $T e^{129}$ & & & & & & & & & & \\
\hline & & & & $\gamma^{91} \mathrm{Cd}^{115}$ & & $n^{114}$ & & & & & & & & & \\
\hline$T e^{127}$ & $\mathrm{Sb}^{124}$ & & $\cos ^{36} \mathrm{Si}^{89}$ & $\mathrm{Sb}^{124}$ & & & & $S b^{124}$ & & & & & & & \\
\hline \multirow[t]{3}{*}{$\operatorname{Tm}^{170}$} & $T \mathrm{~m}^{170}$ & & $\mathrm{Sn}^{123}$ & & & & & & & & & & & & \\
\hline & $\mathrm{Rh}^{102}$ & $\mathrm{Rh}^{102}$ & & & $G a^{68}$ & & & & & & & & & & \\
\hline & & & & & & & $\begin{array}{l}R b^{806} \\
A g^{110}\end{array}$ & & & & $\mathrm{Pr}^{144}$ & & $R b^{106}$ & & \\
\hline \multicolumn{16}{|l|}{$T 1^{204}$} \\
\hline \multirow[t]{3}{*}{$\mathrm{Kr}^{85} \mathrm{Eu}^{232}$} & & & & $E U^{132} A c^{228}$ & Eu ${ }^{154}$ & & & & & & & & & & \\
\hline & & $B i^{210}$ & & & & & $y^{90}$ & & & & & & & & \\
\hline & & $\mathrm{Cs}^{137}$ & & & & & & & & & & & & & \\
\hline $\mathrm{Cl}^{3 \mathrm{~B}}$ & $\left.A\right|^{26}$ & & $K^{40} N p^{238}$ & $A c^{228}$ & & & & $P a^{234}$ & & & & & & & \\
\hline
\end{tabular}


By G. M. CORNEY and $H . M$. CLEARE Research Laboratories Eastman Kodak Company Rochester, New York

\section{TABLE 1-Exposure in Roentgens of 1,000-kvp X-rays to Produce Various Densities}

\begin{tabular}{|c|c|c|c|c|c|c|c|}
\hline \multirow[b]{2}{*}{ Kodak film } & \multirow{2}{*}{$\begin{array}{c}\text { Kodak } \\
\text { developer }\end{array}$} & \multicolumn{6}{|c|}{ Roentgens } \\
\hline & & $D=0.5$ & $=1.0$ & $=1.5$ & $=2.0$ & $=2.5$ & $=3.0$ \\
\hline Super Panchro- & Kodak Rapid & & & & & & \\
\hline \multirow[t]{2}{*}{ Press, Type B } & X-ray & 1.3 & 4.3 & 11. & 30. & 85. & 280 . \\
\hline & DK-60a & 1.7 & 7.6 & 27. & 87. & 280 . & 1,000 . \\
\hline \multirow[t]{3}{*}{ Super-XX } & Kodak Rapid & & & & & & \\
\hline & X-ray & 1.4 & 4.7 & 13. & 35 . & 110 & 440 . \\
\hline & DK-60a & 1.9 & 9.8 & 38. & 140 . & 470 & 1,300 . \\
\hline \multirow[t]{3}{*}{ Verichrome } & Kodak Rapid & & & & & & \\
\hline & X-ray & 1.9 & 5.4 & 14. & 43. & - & - \\
\hline & DK-60a & 2.1 & 6.9 & 22. & 83. & - & - \\
\hline $\begin{array}{l}\text { Kodalith Ortho, } \\
\text { Type } 2\end{array}$ & Kodalith & 1,100 & 1,400 & - & 1,700 . & - & 1,900 . \\
\hline
\end{tabular}

\section{Disaster Monitoring with Amateur and Commercial Photographic Films}

IN THE EVENT of an atomic disaster, widely distributed stand-by dosimeters might be extremely valuable, even if they gave only approximate indications. Photographic films ordinarily stored in commercial stocks and homes might fulfill this need.

With this emergency in mind, the sensitivities to penetrating radiation were determined for several popular films: Kodak Super Panchro-Press Film, Type B; Kodak Super XX Panchromatic Film; Kodak Verichrome Film; and Kodalith Ortho Film, Type 2. X-ray films were not included because their sensitivities to penetrating radiation have been published (1-3).

\section{Film Calibration}

Film sensitivities, in roentgens, were determined for the unfiltered primary radiation from a $1,000-\mathrm{kvp}$ resonanttransformer $\mathrm{X}$-ray machine and were checked with radium gamma rays. These sensitivities also apply reason- ably well to radiation generated at voltages up to several $\mathrm{Mev}$ and to the gamma rays of radium and cobalt- 60 , and therefore should also apply to atomic bomb prompt radiation (4).

Photographic materials are less sensitive to these hard radiations, e.g., 1,000 -kvp $\mathrm{X}$-rays, than to softer radiations. Therefore, calibrations made with 1,000-kvp X-rays would cause softer radiations to be overvalued. Specifically, such a calibration would cause overvaluation of any residual radiation from an atomic bomb (4) to which the film may have been exposed. The magnitude of the error would be difficult to estimate. The extent of the exposure to the residual radiation would, in general, be unknown. Further, different types of film vary in their relative sensitivities to hard and soft radiation. Silver-bromide photographic materials would be expected to have a spectral sensitivity curve to $\mathrm{X}$ - or gamma rays similar to that given by Wilsey (1), but the ratio of maximum to minimum sensitivity depends upon the specific material. Therefore, the dosage values obtained with recovered film should be regarded as "approximate maximum dosages."

To calibrate the films, samples in light cardboard holders were placed at a series of distances from the X-ray focal spot and exposed simultaneously to $1,000-\mathrm{kvp} \mathrm{X}$-rays. The intensity ( $r / \mathrm{min}$ ) for one film location was determined to within $10 \%$ (b) with a Victoreen condenser-type r-meter. From this, the dosage to each film of a series could be computed. The films were developed in deep tanks, for the times shown in Table 1; fixation, washing, and drying followed normal procedure.

A few points for each film were checked by exposing films to known radium gamma-ray dosages. These agreed with the $\mathrm{X}$-ray measurements to within $\pm 15 \%$. The difference between the two determinations included 


\section{Response of Film to 1,000-kvp X-Rays}

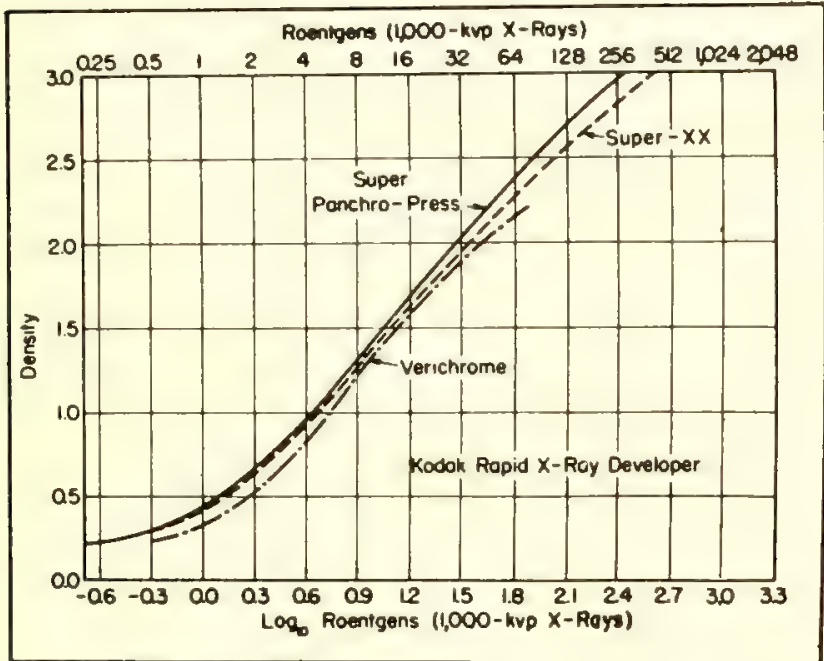

EACH DOSE-DENSITY CURVE represents average of several independent determinations. (Table 1 gives roentgens to produce various specific densities.) Super Panchro-Press Film, Type B, and Super-XX Panchromatic Film, useful up to 300$1,000 \mathrm{r}$, are preferable for dosage estimates; Verichrome film

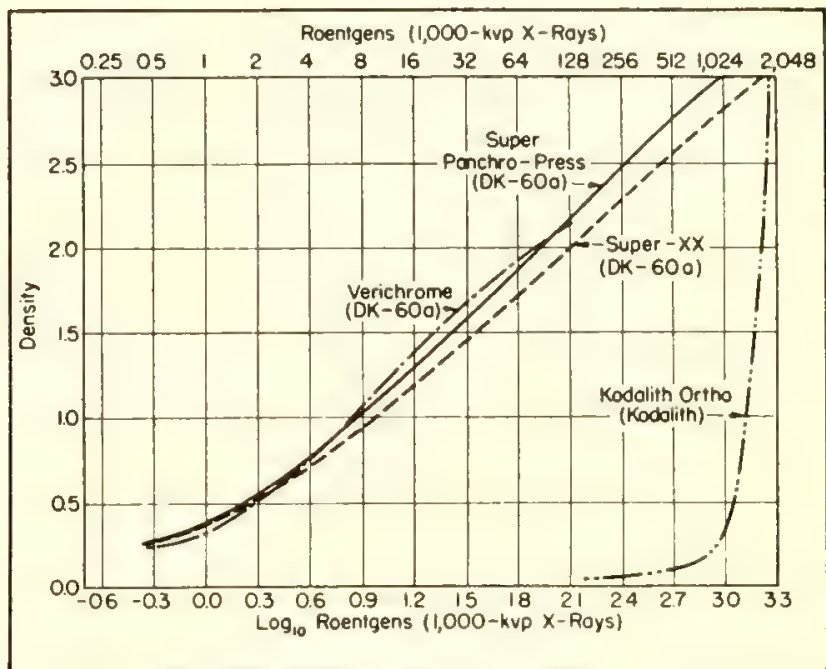

cannot be recommended for densities above about 2.0, corresponding to about $100 \mathrm{r}$ of hard $\mathrm{X}$-rays. Very high contrast of Kodalith Ortho Film, Type 2, makes it suitable to estimate only if exposure exceeds $1,000 \mathrm{r}$. If processed film is clear, exposure was probably $<1,000 \mathrm{r}$; if black, probably $>1,000 \mathrm{r}$ the difference in film sensitivity to the two radiations, errors in ionization measurements at $1,000 \mathrm{kvp}$, and experimental variations.

\section{Practical Considerations}

In the absence of a densitometer, estimates of film densities of less than 2.0 may be made with a photoelectric exposure meter, or a Kodak Densiguide may be used for visual comparisons. Such methods are mere stopgaps and are not adequate substitutes for the use of a conventional densitometer.

Monitoring by means of common photographic films recovered from a disaster area will give only approximate results. Dosage evaluations will be performed with films of different ages that may have been stored under widely differing conditions; development techniques will be difficult to standardize under disaster conditions; exposure to relatively soft radiation before and during recovery will affect

\section{TABLE 2-Development and Darkroom Illumination Recommendations}

\begin{tabular}{|c|c|c|c|c|}
\hline \multirow[b]{2}{*}{ Kodak Film } & \multicolumn{3}{|c|}{ Development times $(\min )$ at $68^{\circ} \mathrm{F}$} & \multirow[b]{2}{*}{$\begin{array}{c}\text { Maximum darkroom } \\
\text { illumination }\end{array}$} \\
\hline & $\begin{array}{c}\text { Kodak Rapid } \\
\text { X-ray Dev. }\end{array}$ & $\begin{array}{c}\text { Kodak Dev. } \\
D K-60 a\end{array}$ & $\begin{array}{l}\text { Kodalith } \\
\text { Dev. }\end{array}$ & \\
\hline \multirow{2}{*}{\multicolumn{5}{|c|}{$\begin{array}{l}\text { Verichrome } \\
\text { Super Panchro- }\end{array}$}} \\
\hline & & & & \\
\hline Press, Type B & 5 & 6 & - & Total darkness \\
\hline Super-XX & 5 & 5.5 & - & Total darkness \\
\hline $\begin{array}{l}\text { Kodalith Ortho, } \\
\text { Type } 2\end{array}$ & - & 一 & $2.25 \dagger$ & Kodak Wratten Series $1 \mathbf{A}^{*}$ \\
\hline
\end{tabular}

the results. Despite these sources of error, results of this crude monitoring procedure might prove of value in the absence of more reliable data.

$$
\text { * * * }
$$

This article is Communication No. 1696 from the Kodak Research Laboratories.

\section{BIBLIOGRAPHY}

1. R. B. Wilsey, Radiology 66, 229 (1951)

2. M. Ehrlich, S, H. Fitch, Nocreonics 9, No. 3 $5 ; 9$, No, 6, 12 (1951)

3. L. S. Taylor, Brit. J. Radiol. 24, 67 (1951)

4. "Effects of Atomic Wespons" (Superintendent of Documents, Washington 25, D. C. 1950)

6. H. O. Wyckoff, G. H. Aston, E. E. Smith, Brì J. Radiol. 27, 325 (1954) 


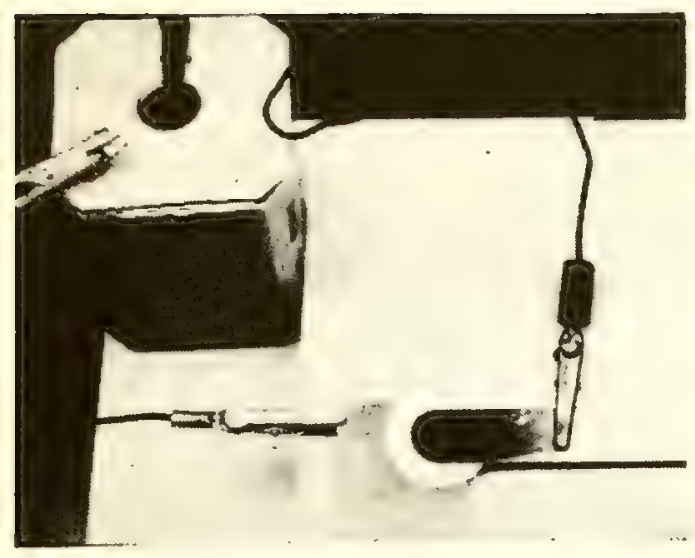

SPOT-TEST setup for electrographic and colorimetric tests

\title{
Simple Spot Tests for
}

\section{Aluminum Contaminants}

\author{
By H. S. HILBORN and R. C. PUGH \\ Pile Materials Division, Technical Division, Savannah River Laboratory \\ E. I. du Pont de Nemours \& Company, Augusta, Georgia
}

\section{Preparation and Properties of Reagents}

\section{Electrolytes}

Acetic acid: $10 \%$ - and $5 \%$-by-volume solution in water

Nitric acid: $0.1 N, 1 N$, and $8 N$ solutions

Potassium nitrate: $10 \%$-by-weight solution in water

Potassium sulfate: $5 \%$-by-weight solution in water

\section{Indicators*}

Cacotheline (saturated aqueous solution): Reacts with acidic stannous solutions to produce violet color. Other reducing ions, solutions, or compounds should be absent to assure specific test. Certain oxides and ions also interfere, e.g., colored metallic ions (arsenic, antimony, molybdenum, and uranium)

Dimethylglyoxime (1\% by weight in ethyl alcohol): In neutral or acetic acid solutions, produces red color with nickel. Large amounts of oxidizing substances and iron and copper may interfere

Diphenylcarbazide ( $1 \%$ by weight in ethyl alcohol): Gives violet to blue color with mercury in weakly acid solutions. Chlorides and iron, copper, and nickel interfere in neutral or weakly acid solutions. In $8 N$ nitric acid, test is specific but sensitivity is greatly reduced. Also gives violet color with molybdenum. Should be made up fresh each day

a-Tolidine solution $(0.1 \mathrm{gm}$ o-tolidine and $0.5 \mathrm{gm}$ ammonium thiocyanate in $5 \mathrm{ml}$ acetone): Blue color with copper and red-brown with iron. Mercury and strongly oxidizing metal salts interfere. Should be made up fresh each day

p-Dimethylaminobenzylidenerhodanine (saturated solution in ethyl alcohol): Red-violet with mercury. Large amounts of chloride interfere. Copper may interfere with test in neutral or acetate-buffered solutions

Potassium thiocyanate ( $10 \%$ by weight in water): When used with $8 \%$-by-volume hydrochloric acid solution and $25 \%$-by-weight solution of sodium thiosulfate in water, gives pink to deep red with molybdenum. If solutions are added slowly with moderate time lapse between additions, blue color may develop. Mercury and other metals or compounds that consume thiocyanate interfere. Certain acids form complexes with molybdates and interfere

Potassium ferrocyanide ( $3 \%$ by weight in water): Blue with iron and red-brown with uranium; test for one is interfered with by other. Copper and other metals that form colored compounds with ferrocyanide also interfere. Strong reducing agents turn iron compound white

Potassium iodide ( $10 \%$ by weight in water): Gives yellow color with lead. Mercury, copper, tin, and any material that produces free iodine interfere. Indicator solution should be colorless

Rubeanic acid (dithio-oxamide) ( $0.5 \mathrm{gm}$ in $100 \mathrm{ml}$ of ethyl alcohol; of this solution, $5 \mathrm{ml}$ are diluted to $100 \mathrm{ml}$ with water): Gives olive-green to black spot with copper. Nickel interferes

Quinalizarin solution (saturated ethyl alcohol solution): Gives blue color with magnesium. Aluminum may interfere if alkali concentration is not sufficient

* For information on sensitivities of indjcators, and interfering ions, compounds, etc., see bibliography references.
Since corrosion of aluminum components in a nuclear reactor may be dangerous and expensive, it is essential that harmful inclusions be identified and traced to their source. The tests reported here for impurities in aluminum combine electrographic sampling (1-4) and colorimetric spot tests (5).

Colorimetric spot tests, well established methods for qualitative analysis, are now a recognized field in microchemistry. Feigl (5) lists over 200 spot tests for over 40 metals, including rare-earth elements. Electrographic sampling is a spot-testing innovation; it has been applied recently $(1-4)$ to identifying both metallic and nonmetallic constituents in alloys.

The reagents required for the tests described here are, in most instances, those described by Feigl. The equipment, consisting of clips, wire, 6-volt dry cell, cathodes, and filter paper, is compact and portable. Tests are easy and convenient, facilitating inspection in the field. For information on indicator sensitivities, interfering ions, etc., see references.

\section{BIBLIOGRAPHY}

1. E. R. Caley, Museum Neros 16, No. 5, 9 (1937) 2. M. S. Hunter, J. R. Churchill, R. B. Mears. Electrographic methods of surface analysis. Metal Progr. 42, 1070 (1942)

S. American Society for Teating Materials, symposium on Rapid Methods for the Iden tification of Metals (S.T.P. No. 98)

4. R. Jirkovsky, Chem. Abstracts 25, 5640 (1931)

5. F. Feigl, "Spot Tests," 4 th. ed., vol. 1 (Elsevier Publishing Co., New York, 1954)

6. M. H. Brown, W. W. Binger, R. H. Brown Mercury and Its Compounds-a Corrosion Hasard, Corrosion 8, 155 (1952)

7. J. R. Churchill. Mercury and Its Compoundo-a Corrosion Hazard, Corrosion 8, 430 (1952) 


\section{Spot-Test Procedure}

1-Area of aluminum to be tested is cleaned, if necessary using soap and water, alcohol, or acetone

2 - Sample to be tested is made anode of galvanic cell by connecting it to positive terminal of 6-volt dry cell. Adequate connection is made by alligator clip or by resting specimen on plate connected to dry cell

3-Prepare cathode by connecting negative terminal to stainless-steel or aluminum electrode of proper size and shape to fit area to be tested

4-Prepare pad of 2-4 thicknesses of Whatman No. 50 filter paper

5-Moisten pad with electrolyte, which should penetrate all layers of pad and cover an area at least as large as that to be tested

6-Place moistened pad on test area and cathode on top of it. Hold cathode without movement so that it exerts steady pressure against pad and specimen. Current flow through pad causes metal ions to leave specimen and be deposited in pad

7-After 30-60 sec, remove cathode and pad; pad now contains "negative" of test area

8-Develop negative by spotting exposed surface of pad with proper indicator to form characteristic colored precipitate or complex with metal ions removed from specimen surface

\section{Precautions and Suggestions}

1-So that the tests be satisfactory, it is necessary that: (a) all electrical contacts be good; (b) moistened filter pad be in good contact with test area; (c) cathode be in contact with pad over test area; (d) sufficient time be allowed for ion transfer to take place; (e) in case of pits, indentations, and pinholes, electrolyte fill cavity before test is started

2-In some instances, good results are obtained by mixing electrolyte and indicator before passing current through cell

3-Increased current flow usually makes test more positive by causing more metal ions to collect in filter pad; optimum time depends on individual case and is affected by size and distribution of inclusions

4-Amount of pressure applied to cathode and pad has some effect; moderately heavy finger pressure is best when using filter pad of type described; pressure should be constant with no movement of cathode

5-Cross check on results, using another test or indicator if available, is desirable

\section{Specific Tests for Contaminants}

\begin{tabular}{cc}
$\begin{array}{c}\text { Contaminant } \\
\text { and electrolyle }\end{array}$ & $\begin{array}{c}\text { Indicalor and color } \\
\text { for positive test }\end{array}$ \\
\hline
\end{tabular}
$\left(\mathrm{K}_{2} \mathrm{SO}_{4}\right)$ cyanide light to dark blue) or o-tolidine solution (Cu-blue; $\mathrm{Fe}$ red brown)

Lead (10\% acetic Potassium iodide acid or $0.1 N$ (yellow)

$\mathrm{HNO}_{8}$ )

Magnesium $\left(\mathrm{K}_{2} \mathrm{SO}_{4}\right)$-test 1

Quinalizarin solution (red-violet or red indicates $\mathrm{Al}$; blue, $\mathbf{M g}$ )

$\mathrm{Mg}\left(\mathrm{K}_{2} \mathrm{SO}_{4}\right)-$ test 2

Quinalizarin solution

Mercury $\left(\mathrm{K}_{2} \mathrm{SO}\right.$ 。 and $\mathrm{HNO}_{3}$ )

Molybdenum in "Molykote"molybdenum disulfide $(0.1 \mathrm{~N}$ $\mathrm{HNO}_{3}$ or $\mathrm{K}_{2} \mathrm{SO}_{4}$ )

Nickel plating ( $\mathrm{K}_{2} \mathrm{SO}$, or $10 \%$ acetic acid)

Tin $(5 \%$ acetic acid)

Copper flash on stainless steel $\left(\mathrm{K}_{2} \mathrm{SO}_{4}\right)$

Copper inclusions $\left(\mathrm{K}_{2} \mathrm{SO}_{4}\right)$

Uranium in U-Al alloys $\left(\mathrm{KNO}_{3}\right)$

Uranium exposed in pits and pinholes in $\mathrm{Al}$ sheath (10\% acetic acid)

Diphenylcarbazide (deep blue to purple) or $p$-dimethylaminobenzylidenerhodanine (red-violet) potassium thiocyanate, and sodium thiosulfate (pink to deep red or blue); or diphenylcarbazide (lavender to deep purple) Dimethylglyoxime (bright red)

Cacotheline (lavender to purple) Rubeanic acid (dithiooxamide)

(olive-green to black)

tion (light to dark blue) or rubeanic acid (olive-green to black)

Potassium ferrocyanide (brown)

Potassium ferrocyanide (brown stain)
Successful in identifying iron inclusions in tubing, bar stock, and vessels. With ferrocyanide, depends on amount of iron present and time of current flow; with otolidine, $\mathrm{Cu}$ and $\mathrm{Fe}$ can be detected simultaneously if they occur discretely some distance from each other

Useful in testing for removal of powder used as lubricant on extruded stock and in detecting inclusions in tubes

After spotting filter pad with indicator, pad is held over mouth of bottle of concentrated ammonium hydroxide until indicator turns blue; then over glacial acetic acid until indicator turns pink-lavender Follows same general procedure except that after spotting with indicator, sufficient sodium hydroxide is added to dissolve aluminum hydroxide and form aluminate ion; solution has faint blue tint; insoluble magnesium hydroxide remains in place and retains darker-blue color indicating $\mathrm{Mg}$. Both tests useful on tubes and other extruded stock on which $\mathrm{Mg}$ was lubricant and on $\mathrm{Mg}$ - $\mathrm{Al}$ alloys

Hydrochloric acid,

Strongly recommended that this test, because of severe attack on specimen, be last applied. Try electroly tesin order: $\mathrm{K}_{2} \mathrm{SO}_{4}$, $1 N \mathrm{HNO}_{3}$, and $8 N \mathrm{HNO}_{3}$. Test is only moderately successful since it is difficult to dissolve $\mathrm{Hg}(6,7)$ without seriously attacking specimen or cathode

Successful with Molykote used as lubricant on hot-pressed $\mathrm{Al}$ pieces. First indicator consists of reagents added in order listed; color depends on time intervals between addition of each reagent

Satisfactory for determining if corrosion pit or indentation has penetrated through $\mathrm{Al}$ sheath to nickel plating on underside Detects remnants of tin solders left on $\mathrm{Al}$ sheeting after corrosion testing

Ideal for identifying very thin copper plating on stainless steel. Rubeanic acid must be used since $\mathrm{Fe}, \mathrm{Cr}$, and $\mathrm{Ni}$ interfere with other indicators

Identifies copper inclusions in $\mathrm{Al}$ tubing, bar stock, and fabricated vessels
Not satisfactory if alloy has been treated to produce protective layer of aluminum oxide on surface

For determining if clad $U$ is exposed 


\section{Tenth-Value Thicknesses for Gamma-Ray Absorption}

BY JOHN MOTEFF

Aircraft Nuclear Propulsion Department

General Electric Company

Cincinnati, Ohio

Figures 1-3 on this page provide data useful in gamma-ray shielding calculations (1). Included are graphs of: tenth-value thicknesses, energy at which narrow-beam absorption coefficients are a minimum, and flux equivalent to $1 \mathrm{r} / \mathrm{hr}$.

Tenth-value thicknesses (Fig. 1) were calculated from total absorption coefficients tabulated in G. R. White's tables (2) using $L_{1 / 0}=0.904 / \mu$. (Note: Pb tenth-values are wrong in Fig. 4, Stanford Res. Inst. report, "Industrial uses . . . fission products" and Fig. 4, p. 57, NU, June '54.)

Energy range is that of principal interest to reactor and isotope work. For lead and tungsten the curves can be straight-line extrapolated to get values from 0.1 to $0.2 \mathrm{Mev}$, but the $0.116-\mathrm{Mev} K$ absorption edge in uranium makes extrapolation difficult.

The thickness to attenuate by a factor other than ten can be gotten by multiplying the tenth-value thickness by the common logarithm of the desired factor; thus, half-value thicknesses are $\log _{10} 2=0.3010$ times tenthvalue thicknesses.

The use of tenth-value layers assumes "good," narrow-beam absorption geometry; that is, that attenuation is exponential with equal attenuation in equal thicknesses. For broad-beam poor-geometry absorption, build-up of scattered radiation has to be considered $(1,3)$. However, good-geometry values are a guide and give a lower limit for transmitted intensity.

Figure 2 is based on Table 6 of ref. (3). Figure 3 , flux equivalent to $1 \mathrm{r} / \mathrm{hr}$, is based on the absorption coefficient of air (given in ref. (3), Table 10) and assumes that $1 \mathrm{r}=6.77 \times 10^{4} \mathrm{Mev} / \mathrm{cm}^{3}$ of std. air, based on $32.5 \mathrm{ev} /$ ion pair.

\section{BIBLIOGRAPHY}

1. J. Moteff. Miscellaneous data for shielding calculations, APEX 176 (1954)

2. Gladys R. White. X-ray attenuation coeff. cients from $10 \mathrm{kev}$ to $100 . \mathrm{Mev}$, NBS 1003 (1952)

8. U. Fano, Nucleoncs 11, No. 8, 8 and No. 9 , 55 (1953)

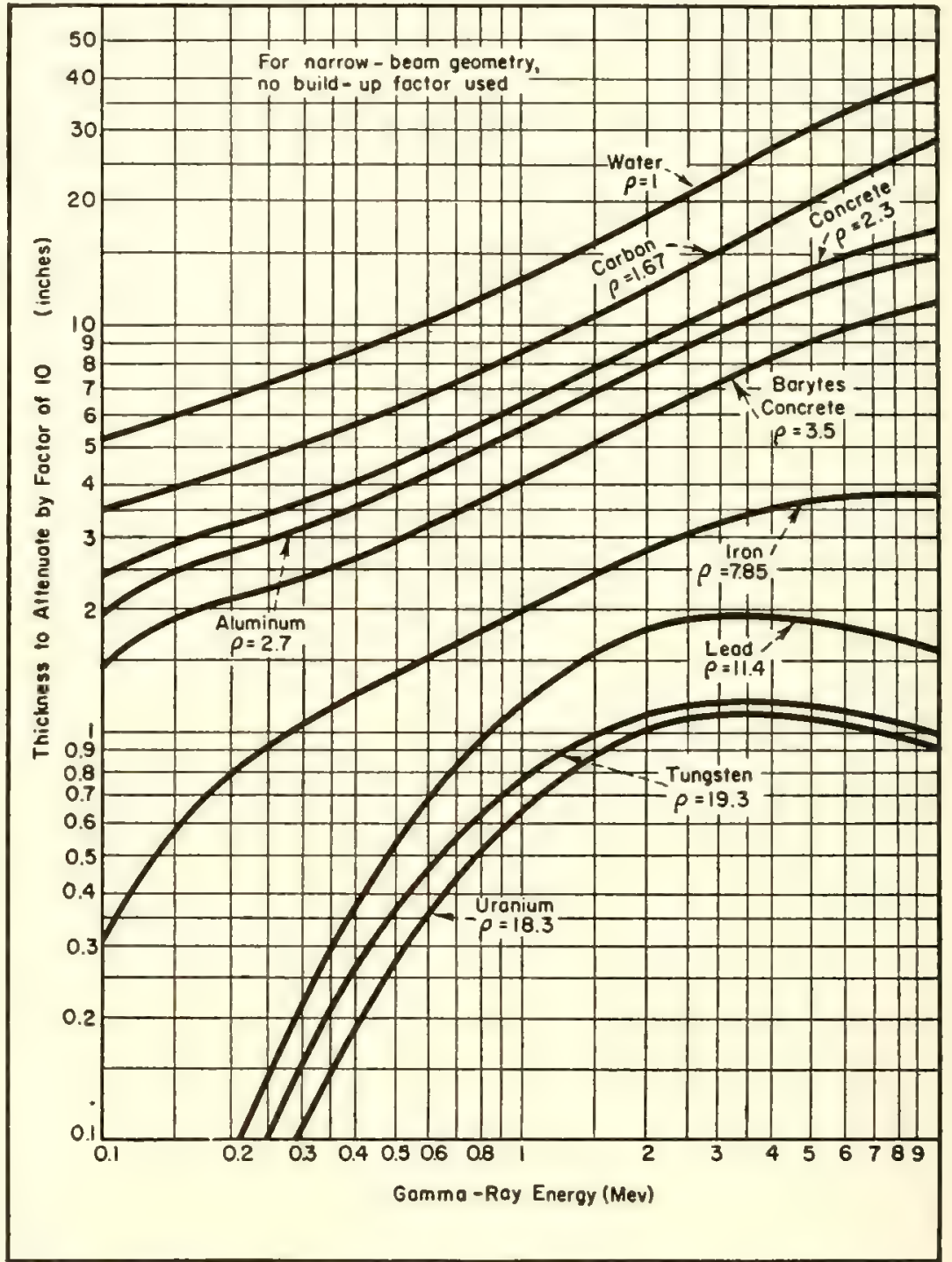

FIG. 1. Thickness to attenuate narrow beam of gamma rays by a factor of 10

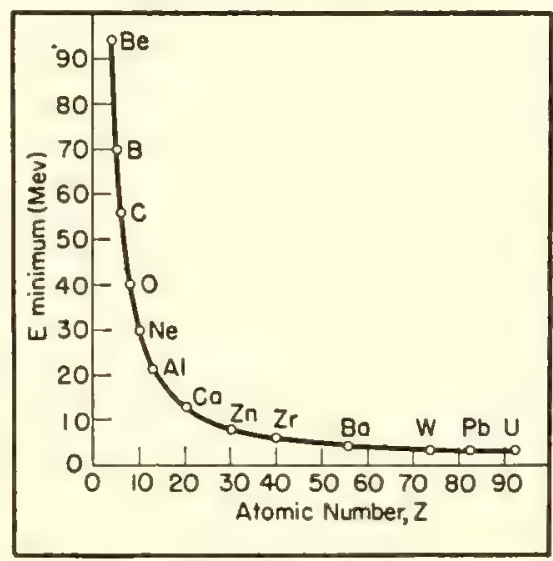

FIG. 2. Energy at which narrow-beam absorption coefficients are o minimum

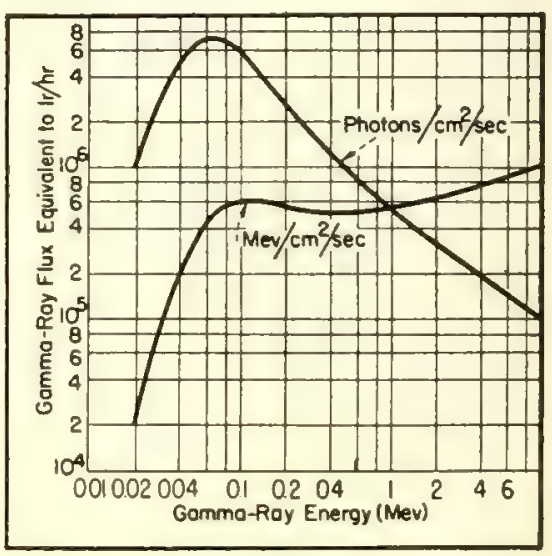

FIG. 3. Gamma-ray flux equivalent to $1 \mathrm{r} / \mathrm{hr}$ as a function of gamma-ray energy 


\section{Fission-Neutron}

\section{Reaction}

\section{Cross Sections}

\section{By W. INTHOFF}

Laboratory for Nuclear Chemistry* Chalmers University of Technology Goteborg, Sweden

HUGHeS HAS SHOWN (1) how yields of $(n, p)$ and $(n, \alpha)$ reactions induced by fission-spectrum neutrons can be calculated with fair accuracy (about a factor 2) for lighter elements. This nomogram facilitates such calculations.

The first scale indicates the atomic number $Z$ of the target element. The second scale gives the threshold energy $\boldsymbol{E}_{\boldsymbol{T}}$ as derived from

$$
E_{T}=-Q(A+1) / A
$$

where $A$ is the mass number of the target nuclide and $Q$ the reaction energy. A great number of $Q$ values are tabulated $(2,3)$, otherwise they can be calculated from the atomic masses (4). $Q$ values for $(n, p)$ reactions may also be calculated from the total beta-disintegration energies $Q\left(\beta^{-}\right)$(5) according to:

$$
Q(n, p)=0.782-Q\left(\beta^{-}\right)
$$

The third scale gives the value of $\bar{\sigma} \times 10 / A^{3 / 3}$, where $\bar{\sigma}$ is the mean value of the activation cross section for fission neutrons in millibarns.

EXAMPLES: For the reaction $\mathrm{P}^{31}$ $(n, p) \mathrm{Si}^{31}$ the $Q$ value is $-0.97 \mathrm{MeV}$, hence $E_{T}=0.97 \times 32 / 31=1.00 \mathrm{MeV}$. By drawing a straight line from $Z=15$ on the first scale through $E_{T}=1.00$ on the second scale one gets $\bar{\sigma} \times 10 / A^{3 \hat{s}}=$ 8.4 on the third scale. As $A=31$ the resulting cross-section is $\bar{\sigma}=8.5$ milli-

* Operated by the Swedish Atomic Energy Commission.

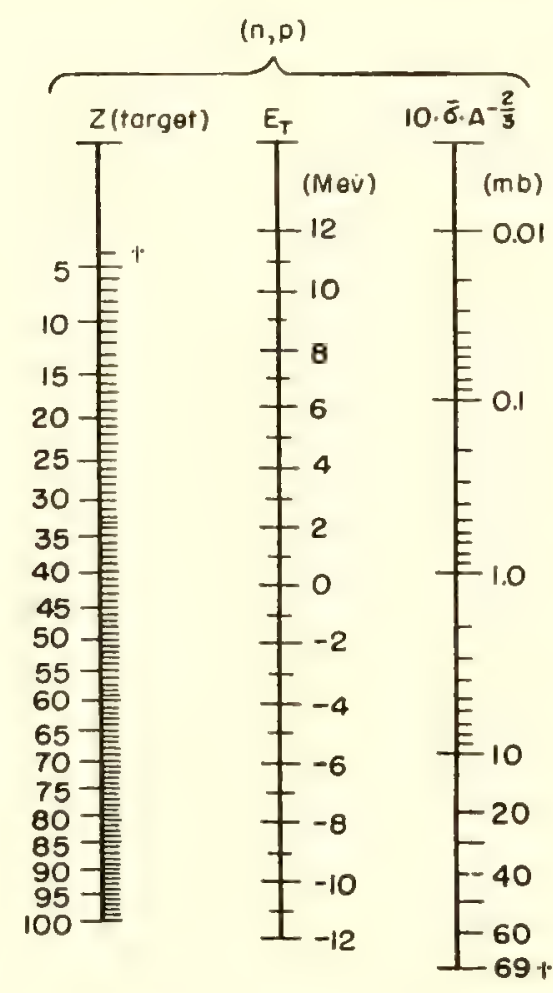

† Scale should not be extrapolated beyond this point-theory no longer holds; e.g.. $\mathrm{B}^{10}(n, a) \mathbf{L i}^{7}$.

barns. The experimental value is 19 $\mathrm{mb}(1)$.

For the reaction $\mathrm{Cl}^{35}(n, \alpha) \mathrm{P}^{32}$ one gets $\bar{\sigma}=3.5 \mathrm{mb}$, in fair agreement with the experimental value of $3.0 \mathrm{mb}$.

The calculations of Hughes are based on the assumption, that the energyspectrum of the fast neutrons has the form

$$
N(E)=\dot{e}^{-E} \sinh \sqrt{2 E}
$$

This is valid for an unmoderated spectrum of fission neutrons as available inside of uranium lumps in a reactor. In this case it is also possible to use the thermal-neutron flux as an approximation for the fast-neutron flux.

In the irradiation channels through the moderator of a reactor the flux of fast neutrons is somewhat smaller than the thermal flux, depending on the relative position of target and uranium rods. Moreover there are deviations of the actual spectrum from the fission spectrum given above. This was shown by the measurements of $(n, \alpha)$

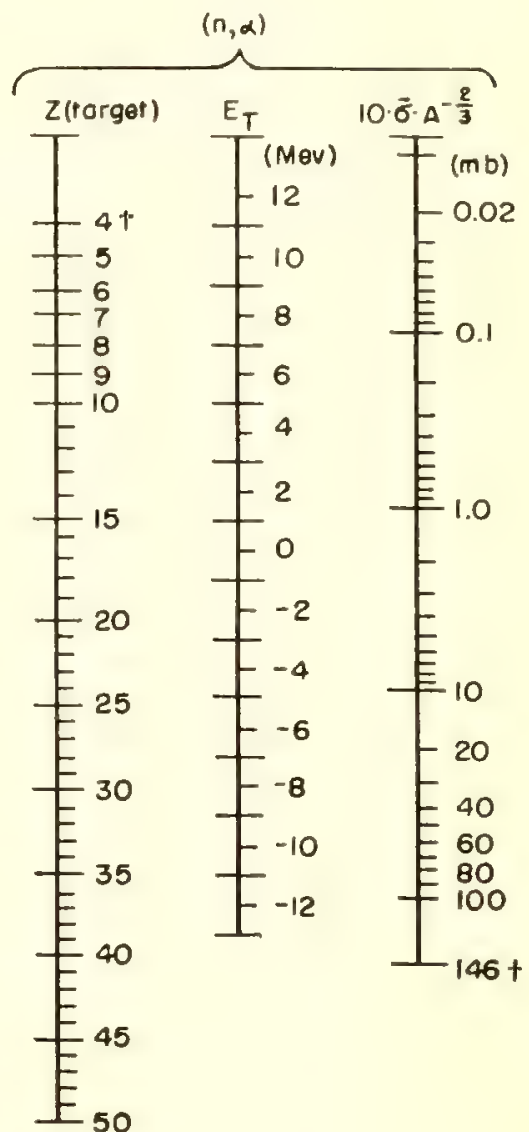

cross-sections of heavier elements by Saeland et al. (6). For $(n, p)$ reactions these deviations are negligible. For $(n, \alpha)$ reactions, however, the results of Saeland et al. indicate that the above formula for the fission spectrum, and hence the nomogram presented here, can be used without corrections only for $Z<20$. For heavier nuclei the nomogram gives values of $\bar{\sigma}$ that are too high.

The author is indebted to Dr.E. Saeland, JENER, Norway, for making JENER report No. 27 available before publication, and to the head of this laboratory, Dr, K. E. Zimen, for helpful discussions.

\section{BIBLIOGRAPHY}

1. D. J. Hughes, "Pile Neutron Research," ch. 4 (Addison-Wesley, Cambridge, 1953)

2. K. T. Bainbridge, "Experimental Nuclear Physics," vol. 1, part V, E. Segre, ed. (Wiley and Sons, New York, 1953)

and Sons, New York, 1953) "LandoltBōrnstein Zablenwerte und Funktionen" 6. Aut. Berlin (Springer) 1952, Bd I, Teil 5.

4. N. Metropolis, G. Reitwiesner. Table of atomic masses, AEC NP 1980 (1948)

5. R. W. King, Revs. Mod. Phys, 26, 327 (1954)

6. E. Saeland, et al. JENER No, 23 (1954) and No. $27(1955)$ 


\title{
Gamma-Ray Attenuation
}

\author{
BY DAVID G. CHAPPELL \\ Knolls Atomic Power Laboratory* \\ General Electric Co. \\ Schenectady, N.Y.
}

IN CALCULATING atTENUATION of gamma-rays, one must determine the mass absorption coefficient, $\mu_{m}$. The $\mu_{m}$ for a number of elements has been calculated, (1). The value for other elements is obtained by interpolation. Hullings did this for five energy groups (2). Recently Moteff extended the list of available energy plots to 25 elements ( 3 ). However, complete tables of $\mu_{m}$ from 0.5 to $6 \mathrm{Mev}$, the usual range of interest, for all elements are still unavailable. The plot of $\mu_{m}$ vs $\mathrm{Z}$ below facilitates interpolation to any atomic number and energy. The data is taken primarily from Moteff (\$).

Energies greater than $6 \mathrm{Mev}$ have been neglected since for these energies and for $Z=30-94 \mu_{m}$ is defined quite well by the 6-Mev curve, the locus of minimum absorption coefficient. This is generally used for shielding calculations for any energy above 6 Mev (4). For $Z=1-29, \mu_{m}$ determined from the chart is slightly high for energies greater than $6 \mathrm{Mev}$.

The nomogram on p. 41 can be used to convert $\mu_{m}$ to the linear absorption coefficient, $\mu$, by multiplying by the density of the shield material, $\rho$. If $\mu$ is connected to the shield thickness, $x$, the shield attenuation factor for a point source with unit build-up, $e^{-b}$, is obtained $(b=\mu x)$. The approximate build-up factor, $B$, for most elements, but not lead, up to $3 \mathrm{Mev}$ is $1+b$ and for lead over the same range $1+1 / 2 b$. The corrected attenuation factor is $B \times e^{-b}$. (This applies for atomic numbers greater than that of iron but definitely not for light elements like aluminum.)

Source strength $\left(\gamma / \mathrm{cm}^{2} / \mathrm{sec}\right)$ for an infinite plane source is approximately $\lambda / 2 \times Q_{v}$, where $Q_{v}$ is the specific activity, $\gamma / \mathrm{cm}^{3}$, of the soyrce material. If $\mu$ is determined for the source $\gamma$-ray, $\lambda$ can be read on the adjacent scale.

\section{BIBLIOGRAPHY}

1. G. R. White NBS 1003 (1952)

2. M. K. Hullings, ORNL, CF 53-2-266 (1953)

3. J. Motef, APEX 176 (1954)

4. R. Stephenson, "Introduction to Nuclear Engineering," D. 185 (McGraw-Hill Book Co., Inc, New York, 1954).

* Knolls Atomic Power Laboratory is operated by the Genersl Electric Co. for the U. S. A tomic Energy Commission under Contract W-31-109 Eng-52. 


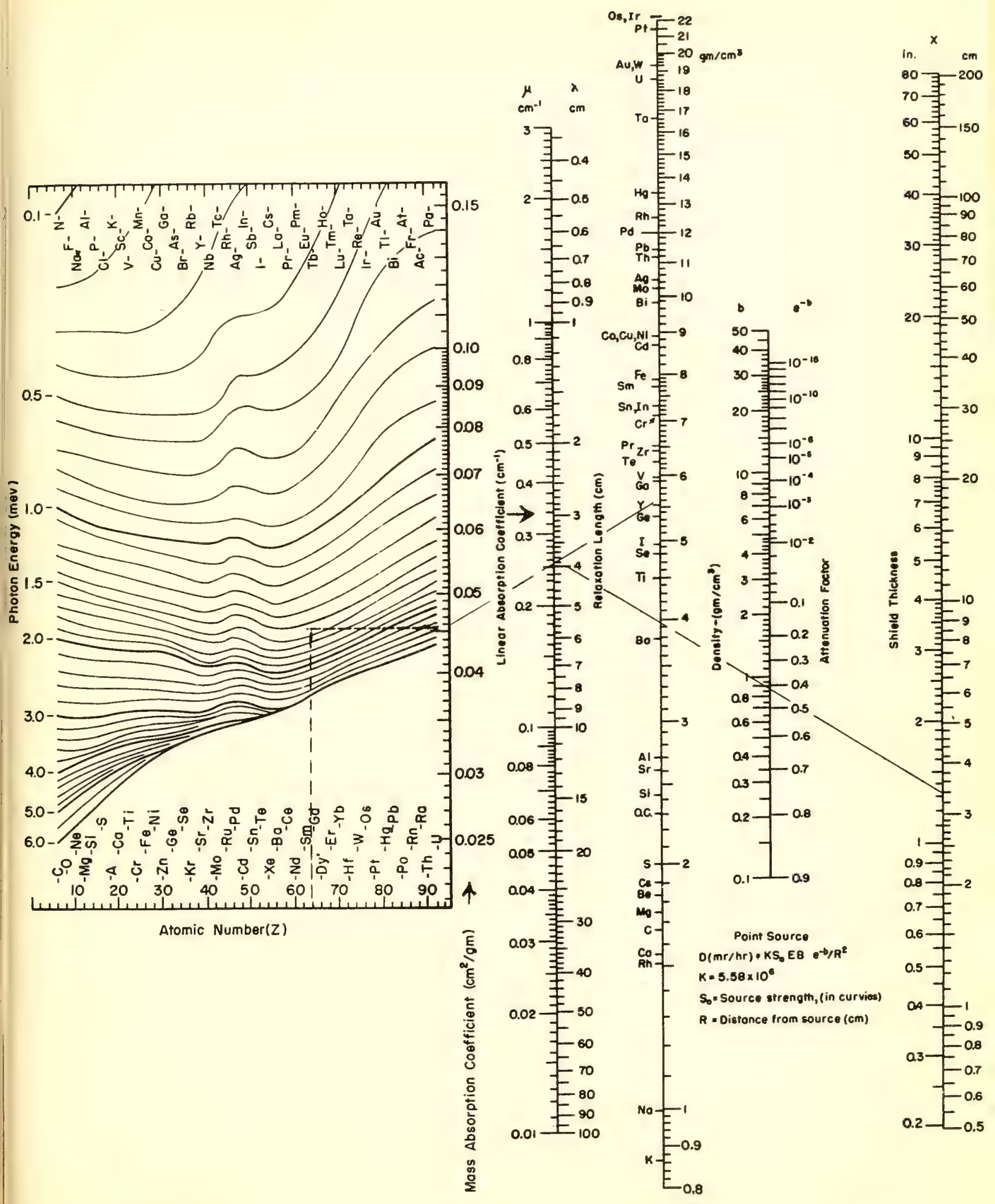




\section{Slow-Neutron Capture Radioisotopes}

\section{Arranged by Half-Life}

By R. C. GEIGER and R. C. PLUMB

Aluminum Company of America

New Kensington, $\mathrm{Pa}$.

The accompanying table lists, in order of increasing half-life, the isotopes which are known to be produced by slow-neutron capture, excluding transmutation reactions. The halflives of other isotopes of the same element that can be similarly produced by neutron irradiation are listed in parentheses after each entry. The table is useful in the identification of isotopes as, for example, for qualitative detection of trace-level impurities in a neutron-irradiated sample.

In practice, all of the isotopes of a given element will not be observed after neutron irradiation. One must consider the neutron energies involved, the cross sections for the particular reactions, the irradiation and decay times, and the relative efficiencies of the particular detection system for the various radiations and arrive at some estimate as to which isotopes will be detected under a given set of conditions.

To identify an unknown isotope completely, the usual procedures of characterizing the energy of the radiation and the chemistry of the element by carrier techniques are desirable.

This table has been compiled from data in BNL-325, Neutron cross sections (July 1, 1955).

\begin{tabular}{|c|c|c|c|c|c|}
\hline$t_{3 \xi}$ & Isotope & $t_{32}$ & Isotope & $t_{32}$ & Isolope \\
\hline $0.03 \mathrm{sec}$ & $\mathrm{B}^{12}$ & $3.6 \mathrm{~min}$ & $\mathrm{Cr}^{55}(27.8 \mathrm{~d})$ & $23.3 \min$ & $\mathrm{Th}^{233}$ \\
\hline 0.85 & $\mathrm{Li}^{8}$ & | $3.6^{\circ}$ & $\operatorname{Gd}^{161}(230 \mathrm{~d}, 18.0 \mathrm{~h})$ & 23.5 & $\mathrm{U}^{239}\left(2.4 \times 10^{9} \mathrm{y}, 7.1\right.$ \\
\hline 2.5 & $\operatorname{Er}^{171}(9.4 \mathrm{~d}, 7.5 \mathrm{~h})$ & 3.76 & $V^{52}$ & & $\left.\times 10^{8} \mathrm{y}\right)$ \\
\hline 7.4 & $\mathrm{~N}^{16}$ & 3.9 & $\mathrm{Xe}^{137}(5.3 \mathrm{~d}, 9.13 \mathrm{~h})$ & 24 & $\mathrm{Sm}^{155}(400 \mathrm{~d}, 47 \mathrm{~h})$ \\
\hline 11 & $F^{20}$ & | 4.2 & $\mathrm{Tl}^{206}(2.7 \mathrm{y})$ & 25 & $\begin{array}{l}\mathrm{Se}^{83}(123 \mathrm{~d}, 18 \mathrm{~s}, 57 \mathrm{~m} \\
\quad 17 \mathrm{~m}, 67 \mathrm{~s})\end{array}$ \\
\hline 13 & $\begin{array}{l}\operatorname{In}^{116}(49 \mathrm{~d}, 54.1 \mathrm{~m} \\
\quad 72 \mathrm{~s})\end{array}$ & $\begin{array}{l}4.5 \\
5.0\end{array}$ & $\begin{array}{l}R h^{104}(44 s) \\
S^{37}(87 d)\end{array}$ & 25 & $\mathrm{Te}^{131}(110 \mathrm{~d}, 58 \mathrm{~d}$ \\
\hline 18 & $\begin{array}{c}\mathrm{Se}^{77}(123 \mathrm{~d}, 57 \mathrm{~m}, \\
17 \mathrm{~m}, 67 \mathrm{~s}, 25 \mathrm{~m})\end{array}$ & $\begin{array}{ll}\mid & 5.14 \\
\mid & 5.5\end{array}$ & $\begin{array}{l}\mathrm{Cu}^{66}(12.8 \mathrm{~h}) \\
\mathrm{Hg}^{205}(47 \mathrm{~d})\end{array}$ & 25.0 & $\begin{array}{l}72 \mathrm{~m}, 30 \mathrm{~h}) \\
\mathrm{I}^{128}\end{array}$ \\
\hline 20 & $\operatorname{Sc}^{46}(85 \mathrm{~d})$ & 5.8 & $\mathrm{Te}^{51}$ & 31 & $\mathrm{Pt}^{199}(4.3 \mathrm{~d}, 18 \mathrm{~h})$ \\
\hline 24.2 & $\operatorname{Ag}^{110}(2.3 \mathrm{~m}, 270 \mathrm{~d})$ & 6.6 & $\mathrm{Nb}^{94}$ & 37.5 & $\mathrm{Cl}^{38}\left(3.08 \times 10^{5} \mathrm{y}\right)$ \\
\hline $\begin{array}{l}29 \\
40\end{array}$ & $\mathrm{O}^{19}$ & 8.5 & $\mathrm{Ca}^{49}(152 \mathrm{~d}, 4.8 \mathrm{~d})$ & 40 & $\mathrm{Sn}^{123}(112 \mathrm{~d}, 14.5 \mathrm{~d}$, \\
\hline $\begin{array}{l}40 \\
44\end{array}$ & $\begin{array}{l}\mathrm{Ne}^{23} \\
\mathrm{Rh}^{104}(4.5 \mathrm{~m})\end{array}$ & $\begin{array}{l}9.5 \\
10\end{array}$ & $\begin{array}{l}\mathrm{Mg}^{27} \\
\mathrm{Sn}^{125}(112 \mathrm{~d}, 14.5 \mathrm{~d}\end{array}$ & & $\begin{array}{llll}250 \mathrm{~d}, & 27.5 \mathrm{~h}, 130 \\
\mathrm{~d} .10 \mathrm{~m}, 10 \mathrm{~d}\end{array}$ \\
\hline 57 & $\begin{array}{l}\mathrm{Ge}^{77}(11.3 \mathrm{~d}, 82 \mathrm{~m} \\
12 \mathrm{~h})\end{array}$ & & $250 \mathrm{~d}, 27.5 \mathrm{~h}, 40 \mathrm{~m}$, & & \\
\hline 67 & $\begin{array}{l}\mathrm{Se}^{83}(123 \mathrm{~d}, 18 \mathrm{~s}, 57 \mathrm{~m} \\
\quad 17 \mathrm{~m}, 25 \mathrm{~m})\end{array}$ & 10.4 & $\begin{array}{c}>400 \mathrm{~d}) \\
\mathrm{Co}^{60}(5.28 \mathrm{y})\end{array}$ & $=0$ & $43 \mathrm{~d}, 53 \mathrm{~h}, 2.9 \mathrm{~h})$ \\
\hline 72 & $\begin{array}{l}\mathrm{In}^{114}(49 \mathrm{~d}, 54.1 \mathrm{~m} \\
13 \mathrm{~s})\end{array}$ & $\begin{array}{l}14.3 \\
16.4\end{array}$ & $\mathrm{Mo}^{101}(6.9 \mathrm{~h}, 2.8 \mathrm{~d})$ & 52 & $\begin{array}{l}\mathrm{Zn}^{69}(250 \mathrm{~d}, 13.8 \mathrm{~h} \text {, } \\
2.2 \mathrm{~m})\end{array}$ \\
\hline $1.3 \mathrm{~min}$ & $\begin{array}{l}\mathrm{Sb}^{124}(2.8 \mathrm{~d}, 21 \mathrm{~m} \\
60 \mathrm{~d})\end{array}$ & $\begin{array}{l}16.4 \\
17\end{array}$ & $\begin{array}{l}\mathrm{Se}^{81}(123 \mathrm{~d}, 18 \mathrm{~s}, 57 \mathrm{~m}, \\
\quad 67 \mathrm{~s}, 25 \mathrm{~m})\end{array}$ & $\begin{array}{l}54.1 \\
57\end{array}$ & $\begin{array}{l}\operatorname{In}^{116}(49 \mathrm{~d}, 72 \mathrm{~s}, 13 \mathrm{~s}) \\
\mathrm{Se}^{81}(123 \mathrm{~d}, 18 \mathrm{~s}, 17 \mathrm{~m}, \\
\quad 67 \mathrm{~s}, 25 \mathrm{~m})\end{array}$ \\
\hline 1.3 & $\operatorname{Dy}^{165}(2.32 \mathrm{~h})$ & 17.8 & $\mathrm{Rb}^{88}(19.5 \mathrm{~d})$ & 72 & $\mathrm{Te}^{129}(110 \mathrm{~d}, 58 \mathrm{~d}$ \\
\hline 1.4 & $\operatorname{Ir}^{192}(74 \mathrm{~d}, 19.0 \mathrm{~h})$ & 18 & $\operatorname{Br}^{80}(4.6 h, 35.9 h)$ & & $110 \mathrm{~d}, 9.3 \mathrm{~h}, 33 \mathrm{~d}$, \\
\hline 2.2 & $\mathrm{Zn}^{71}(250 \mathrm{~d}, 13.8 \mathrm{~h}$, & | 20.2 & $\mathrm{Ga}^{70}(14.2 \mathrm{~h})$ & & $30 \mathrm{~h}, 25 \mathrm{~m})$ \\
\hline 2.27 & $\begin{array}{l}52 \mathrm{~m}) \\
\mathrm{Al}^{28}\end{array}$ & 21 & $\begin{array}{l}\mathrm{Sb}^{124}(2.8 \mathrm{~d}, 1.3 \mathrm{~m} \\
\quad 60 \mathrm{~d})\end{array}$ & 77 & $\begin{array}{c}\mathrm{Kr}^{87}(9.4 \mathrm{y}, 4.4 \mathrm{~h} \\
\quad 34.5 \mathrm{~h})\end{array}$ \\
\hline $2.3 \mathrm{~min}$ & $\operatorname{Ag}^{108}(270 \mathrm{~d}, 24.2 \mathrm{~s})$ & $122 \min$ & $\operatorname{Pd}^{111}(13.6 \mathrm{~h}, 17.0 \mathrm{~d})$ & $82 \mathrm{~min}$ & $\mathrm{Ge}^{75}(11.3 \mathrm{~d}, 57 \mathrm{~s}, 12 \mathrm{~h})$ \\
\hline
\end{tabular}




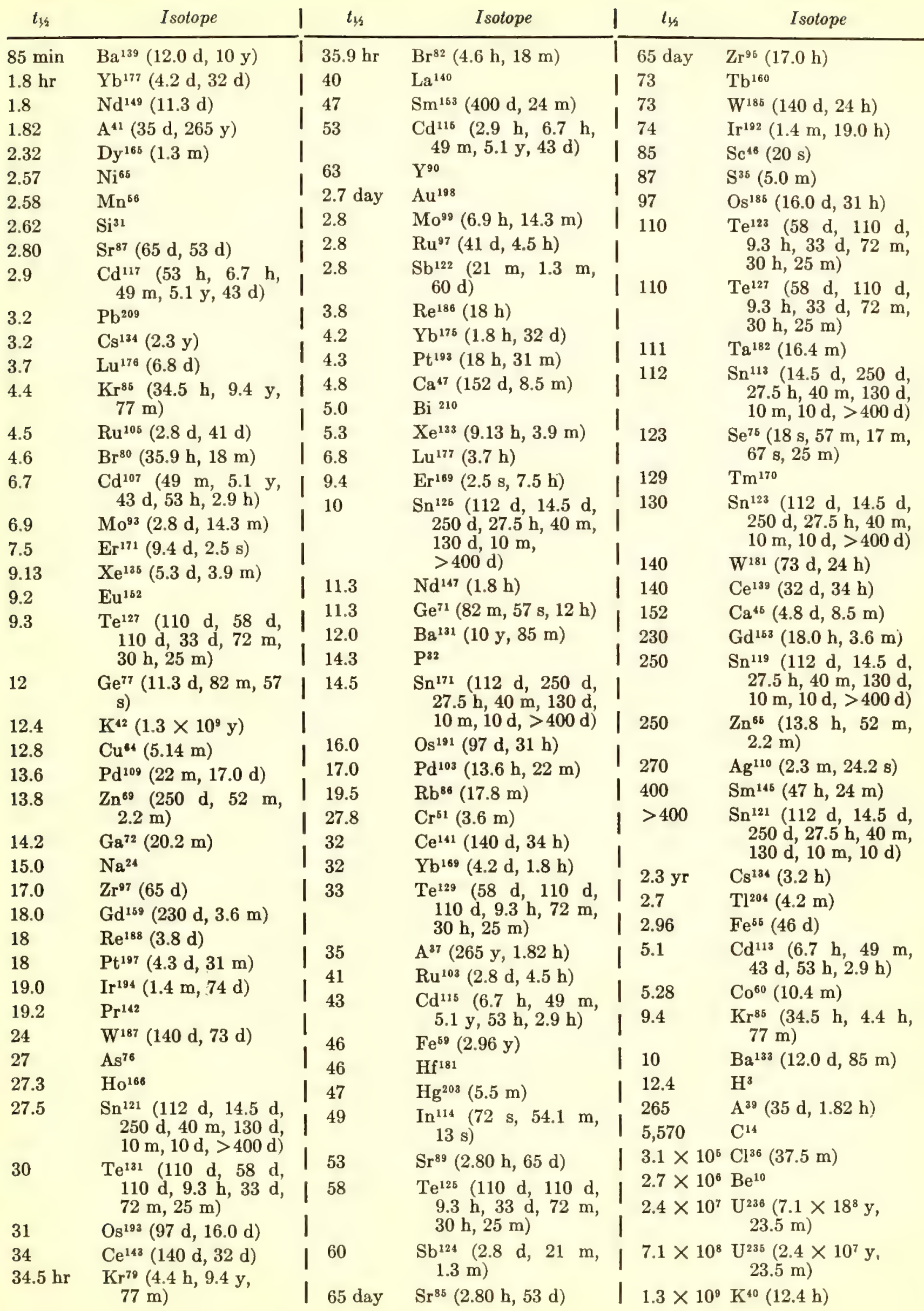




\title{
Stress-Strain Curves for Reactor-Irradiated Plastics
}

\author{
By C. D. BOPP and O. SISMAN \\ Oak Ridge National Laboratory \\ Oak Ridge, Tennessee
}

These DatA were taken on commercial plastics irradiated in the ORNL graphite reactor. A discussion has been given in ORNL-928, ORNL-1373, and Nucleonics (July '55, p. 28; Oct. '55, p. 51).

Tests were run on a Baldwin universal testing machine with recording extensiometer according to ASTM standard procedure D638-49T. Testing speed was $0.05 \mathrm{in} . / \mathrm{min}$ to 0.02 strain and then increased to $0.20 \mathrm{in}$./ min. Test specimens were $1 / 2 \mathrm{in}$. wide, 116-1/4 in. thick, and strain (in./in.) was measured over a 2-in. gage length. Specimens were pretest conditioned to $25^{\circ} \mathrm{C}$ and $50 \%$ relative humidity. The plastics included here are: Amphenol (unmodified polystyrene), Styron 475 (black-pigment-filled polystyrene), Royalite (styrene-acrylonitrile copolymer), Polythene (polyethylene), Fluorothene (polymonochlorotrifluoroethylene), Teflon (polytetrafluoroethylene), Catalin(unfilled phenol-formaldehyde plastic), paper-base and asbestosfiber-base Bakelite (phenol-formaldehyde), Haveg (phenolic-bonded asbestos), Karbate (phenolic-bonded graphite), Nylon FM 3003 (polyamide), Saran (vinyl-vinylidene chloride polymer), Butacite (polyvinyl butyral),
Beetle (urea formaldehyde polymer), Melmac (melamine formaldehyde polymer), Plaskon Alkyd (alkyd polyester), Lucite (poly methyl methacrylate), Ameroid (casein plastic), CR-39 (allyl diglycol carbonate polyester), Vinylite (vinyl chloride acetate polymer), Cibanite (aniline formaldehyde), Polectron (polyvinyl carbazole), Duralon (furan resin with asbestos and carbon black filler), Pyralin (cellulose nitrate), Plastacele (cellulose acetate)-other cellulosics behave similarly-Geon 2046 (polyvinyl chloride), Selectron 5038 (polyester), and Formvar (polyvinyl formal).
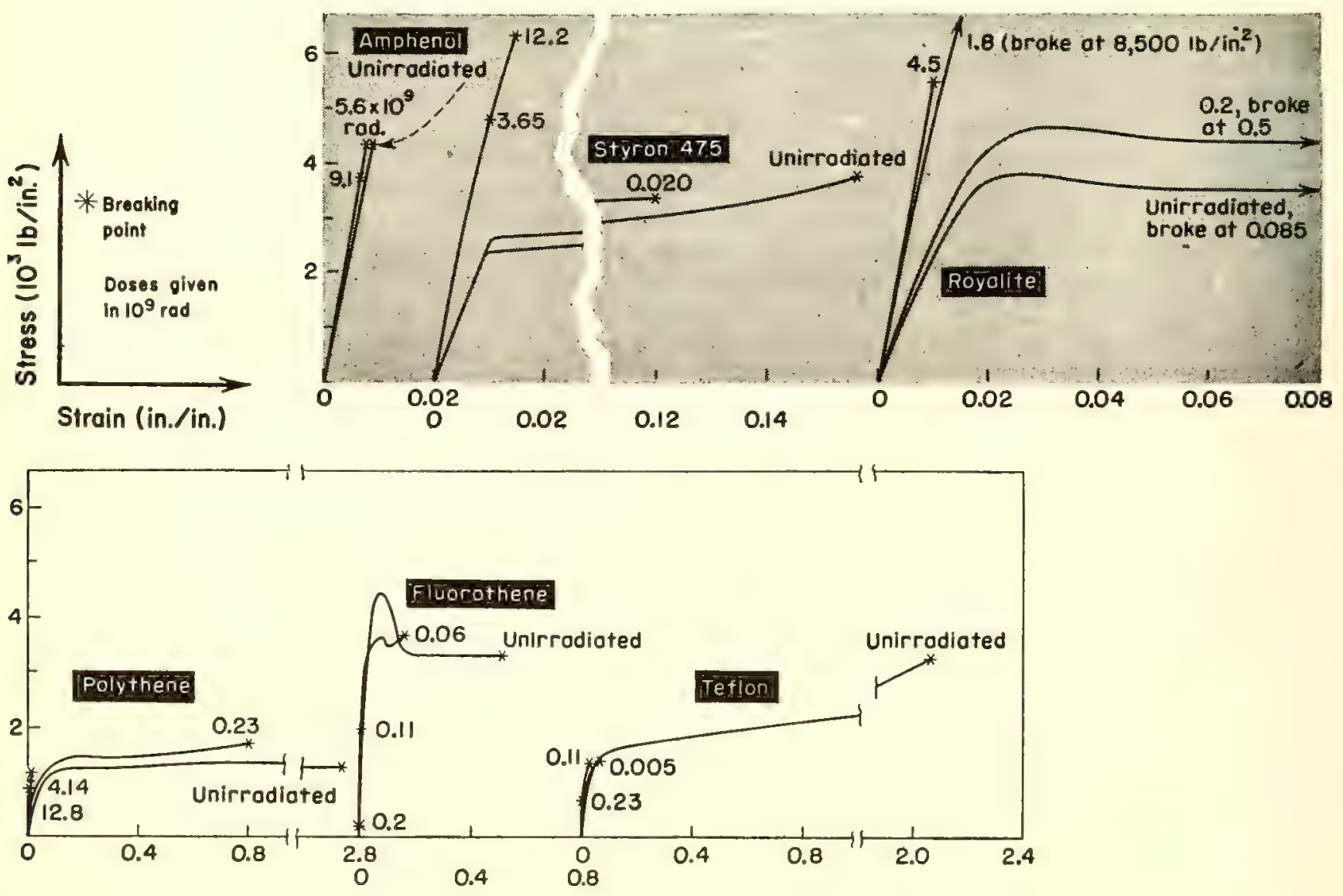

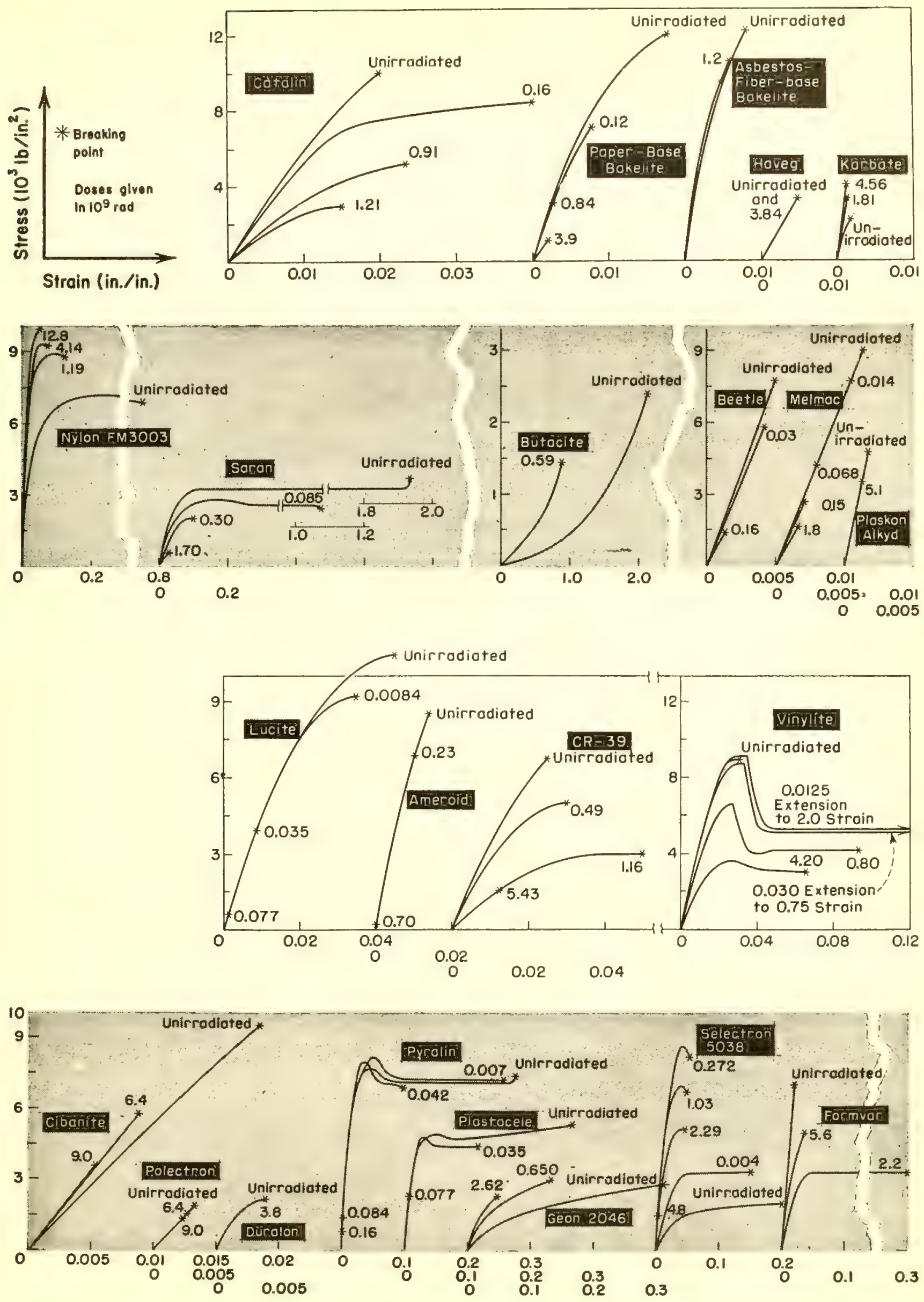


\section{Weight Change in Cylindrical Shields}

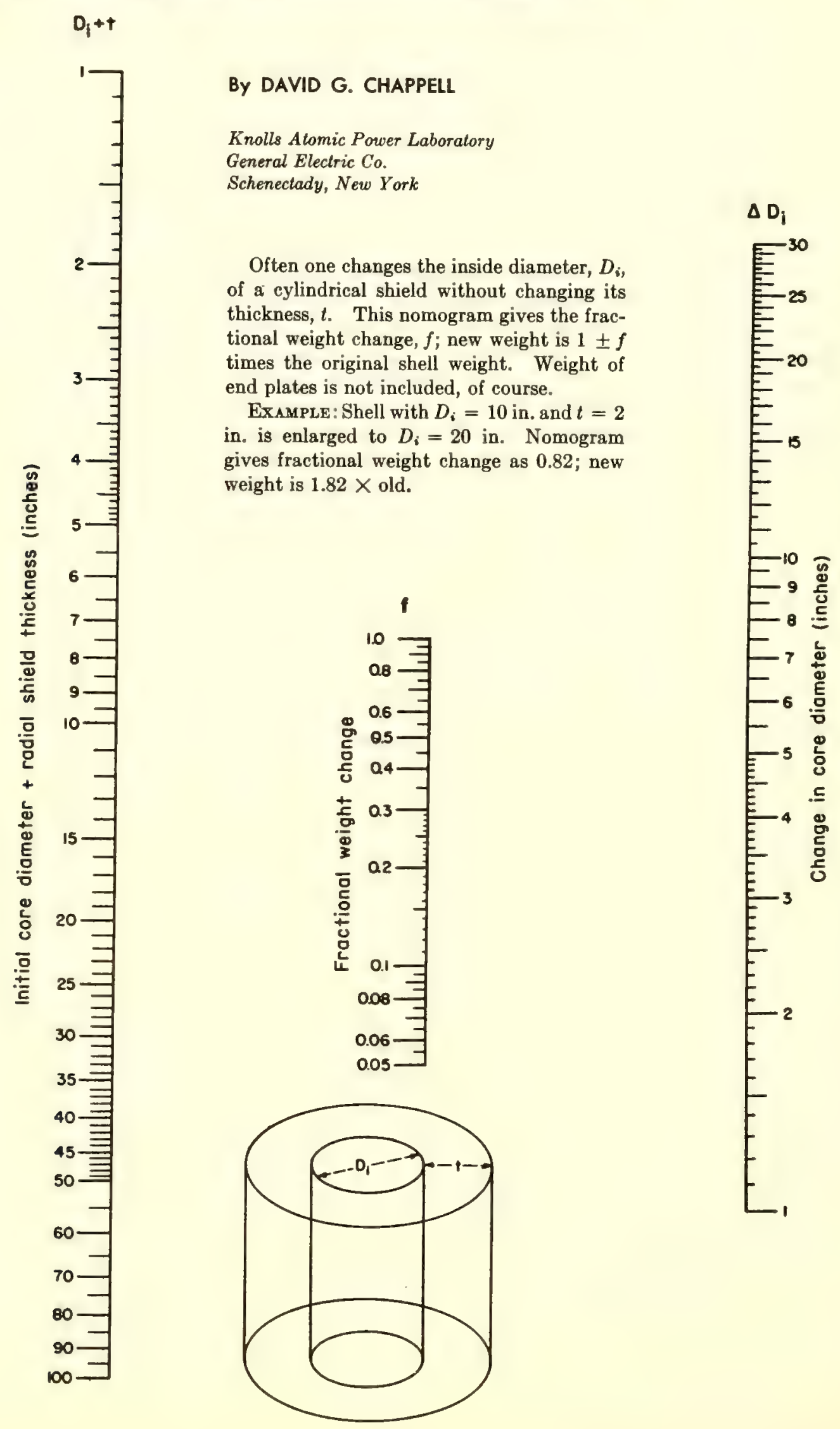




\section{Locating Compton Edges and}

\section{Backscatter Peaks in Scintillation Spectra}

BY B. CRASEMANN and H. EASTERDAY

Department of Physics, ${ }^{*}$ University of Oregon, Eugene, Oregon

IN A sCintillation spectrum, each photopeak is accompanied by a characteristic Compton distribution and a backscatter peak. Figure 1 shows these features in the pulse-height distribution of $0.51-\mathrm{Mev}$ annihilation radiation, obtained with a cylindrical $13 / 2 \times 1$-in, NaI(Tl) crystal. This article reviews the behavior of these characteristic features.

\section{Compton Edge}

The Compton edge corresponds to the maximum energy that an incident photon can impart to an electron in the scintillation crystal by Compton scattering. If the scattered photon escapes from the crystal, a pulse is obtained whose height corresponds to the energy received by the electron in this process. The energy of the Compton edge, $\widehat{E}_{\text {coms }}$, for an incident photon of energy $E_{\gamma}$ is given by

* This work was supported by the National Science Foundation.

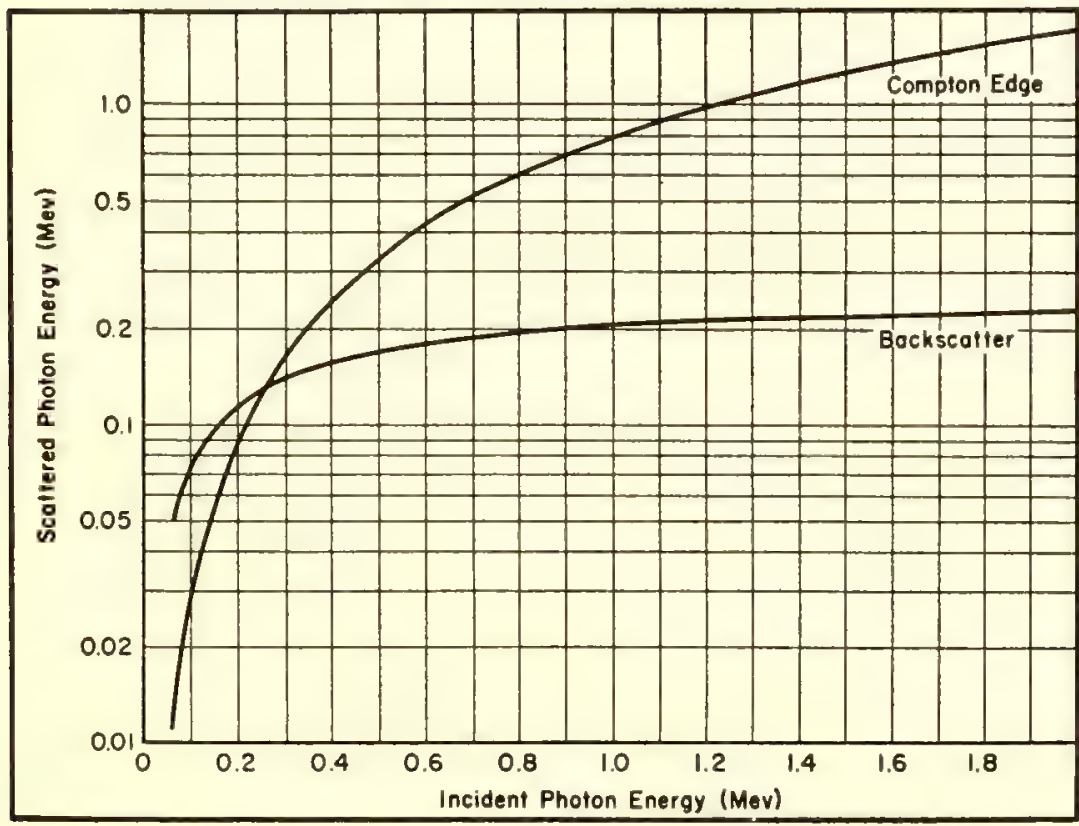

FIG. 2. Compton-edge and backscatter energies vs incident photon energy

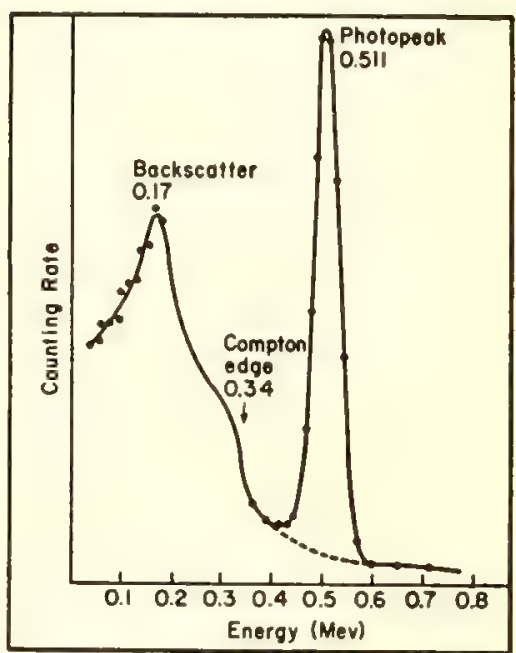

FIG. 1. Scintillation spectrum of 0.511 . Mev annihilation radiation

tion of Compton-edge and backscatter energies can be avoided by use of Fig. 2 , which gives these quantities for incident gammas up to $2 \mathrm{Mev}$.

The Compton edge is more prominent in the spectrum of high-energy gamma rays since the cross section for Compton scattering decreases more slowly with energy than the cross section for the production of photoelectrons, and the escape probability for scattered photons is larger. On the other hand, the backscatter peak becomes less noticeable as the incident gamma energy increases: with increasing energy, the total Compton crosssection decreases, and the angular distribution of the secondary photons from Compton events becomes more peaked in the forward direction, so that backscattering becomes less.

With larger scintillation crystals, the Compton edge becomes less pronounced since the escape probability for scattered photons is reduced. The backscatter peak does not decrease with larger crystals; however, the effect can be diminished by the use of a crystal container of low atomic number and a thin glass cover. The number of Compton events in the photomultiplier face can be reduced drastically by allowing the radiation to enter the crystal parallel to the tube face. 


\section{NUCLEONICS DATA SHEET No. 14}

Shielding Constants

\section{Gamma-Ray Scattering from Thin Scatterers}

BY DAVID G. CHAPPELL

Knolls Atomic Power Laboratory*

General Electric Company, Schenectady, New York

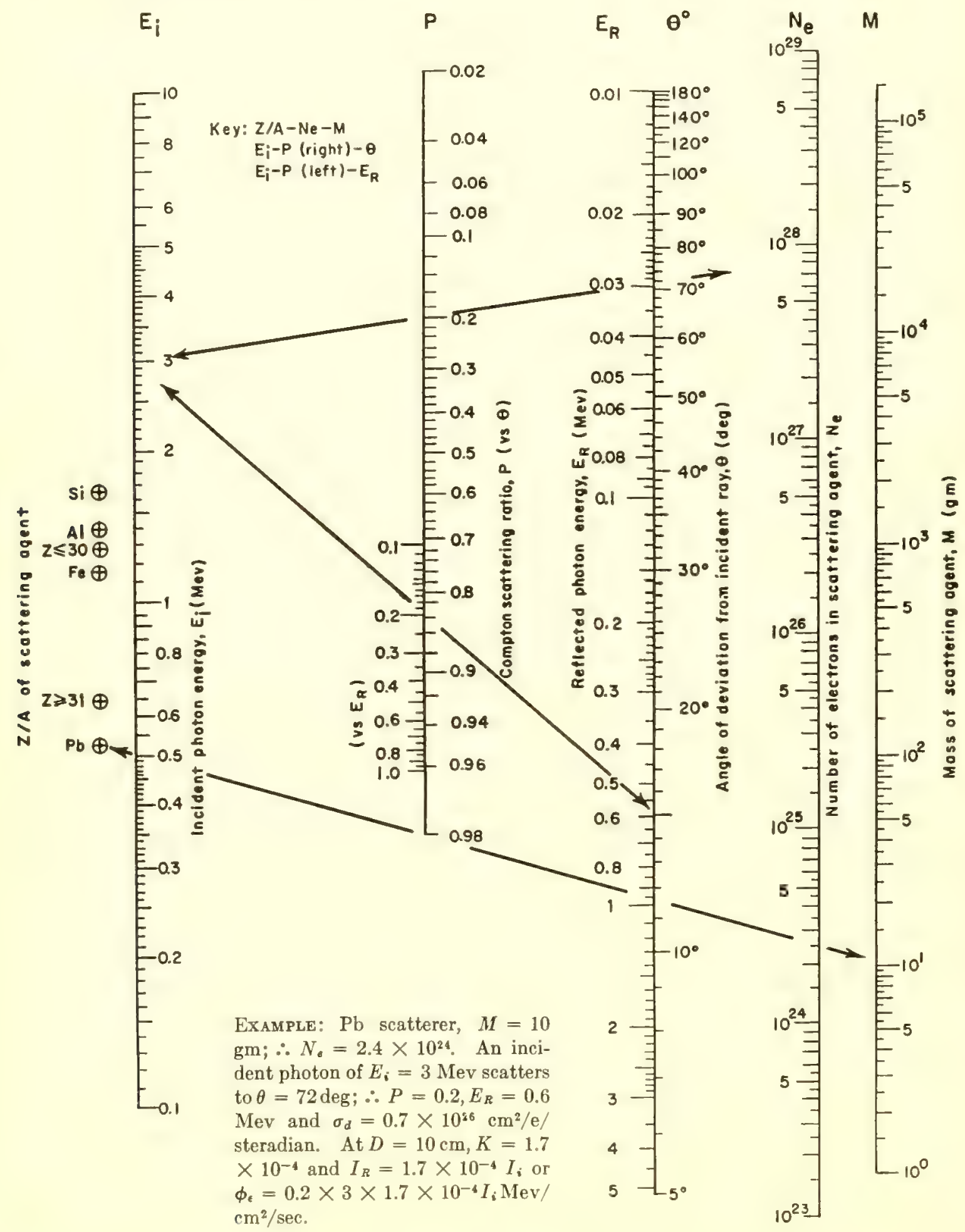


These nomograms permit rapid estimation of the photon flux reflected from a thin object; this is often required in shield design.

A photon flux $I_{i}\left(\gamma / \mathrm{cm}^{2} / \mathrm{sec}\right)$ of energy $E_{i}$ (Mev) per photon, incident upon a scatterer thin with respect to the relaxation length of the incident or scattered photon $(t<\lambda)$, will scatter through an angle $\theta$ to a receiver $R$ at distance $D(\mathrm{~cm})$ with an intensity $I_{R}$ $\left(\gamma / \mathrm{cm}^{2} / \mathrm{sec} /\right.$ steradian $)$ and energy $E_{R}$ (Mev) per photon and an intensity given by

$$
I_{R}=N_{e} \sigma_{D} D^{-2} I_{i}=K I_{i}
$$

The energy flux, $\phi_{\epsilon}\left(\mathrm{Mev} / \mathrm{cm}^{2} / \mathrm{sec} /\right.$ steradian), at $R$ is

* Knolls Atomic Power Laboratory is operated by the General Electric Co. for the U. S. Atomic Energy Commission.

$$
\phi_{\epsilon}=E_{R} I_{R}=P E_{i} K I_{i}
$$

The scattered photon energy, $E_{R}$, is given by the Compton equation

$P=E_{R} / E_{i}$

$$
=\left[1+\frac{E_{i}}{0.51}(1-\cos \theta)\right]^{-1}
$$

The probability per electron of scattering the incident photon through angle $\theta$ is given by the Klein-Nishina equation

$\sigma_{D}=d \sigma / d \Omega$

$$
=\left(P-P^{2} \sin ^{2} \theta+P^{3}\right) r_{e}{ }^{2} / 2
$$

where the classical electron radius $r_{s}=2.82 \times 10^{-13} \mathrm{~cm}$ and $\sigma_{D}$ is in $\mathrm{cm}^{2} /$ steradian per electron. One must multiply by the number of electrons in the scattering medium, $N_{0}=6.02 \times 10^{23}$ $M Z / A$, to get the total scattering probability.
Large objects must be subdivided into pieces of such size that $I_{i}$ and $\theta$ can be assumed constant over each region of subdivision. Each region is then considered to be a point scatterer defining a new set of parameters $M, D$, and $\theta$, and the scattered intensities from all subdivisions are summed.

If small values of $M$ are used, $K$ may go off-scale; in this case $N_{\text {c }}$ can be multiplied by a convenient factor of 10 and $K$ divided by the same factor to obtain the corrected value of $K$.

The value of $K$ is not particularly sensitive to $Z / A$. However, the average value $Z / A$ for $Z=2-30$ is plotted at $Z \leq 30$ and for $Z / A$ for $Z=31-91$ at $Z \geq 31$. This provides for seattering media for which only an approximate composition is known.

I wish to acknowledge the assistance of Miss Joyce Berger, who drew the nomograms.

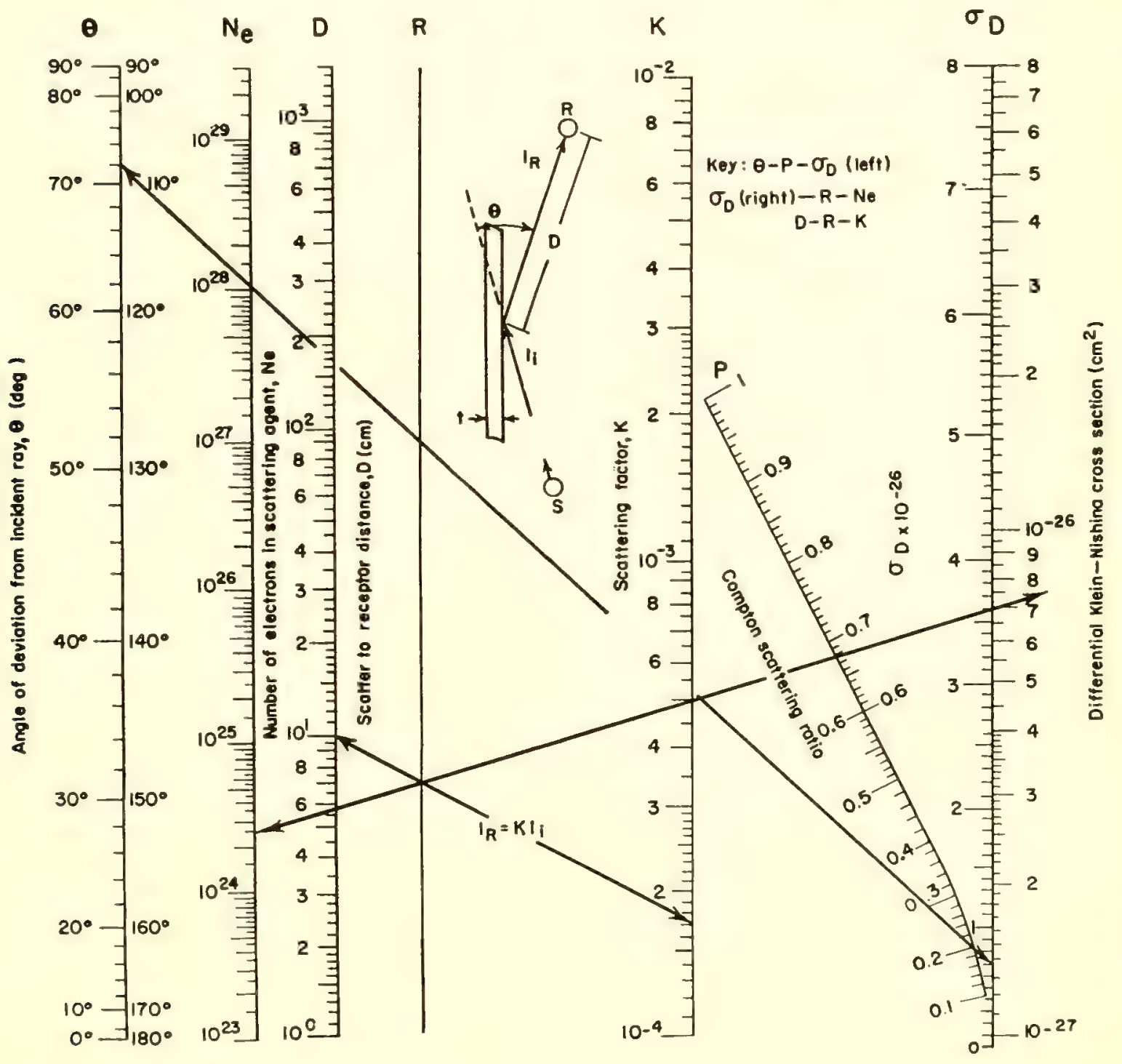




\section{Gamma-Ray Streaming Through an Annulus}

\section{By DAVID G. CHAPPEL}

Knolls Atomic Power Laboratory*

General Electric Co.

Scheneclady, New York

Practical desian of biological shields for reactors often involves accounting for the gamma flux that streams through the annular space between a pipe and the shield. This nomogram permits rapid and accurate determination of the geometrical attentuation of the gammaray flux from an emitting surface onto which the annulus abuts perpendicularly. Scattering effects are not ac- counted for. The formula for the attenuation is

$$
A=K D_{0}^{1 / 2} \Delta D^{3 / 6} L^{-2}
$$

where $K$ is $2.8 / 8 \pi, 5.6 / 8 \pi$ and $7.2 / 8 \pi$ for spherical, cosine, and Fermi emitters $\ddagger$ respectively. The other symbols are shown in the diagram accompanying the nomogram. The formula is valid only if $L \gg \Delta D$, the usual situation.

Example: Annulus with $D_{0}=4.5 \mathrm{in}$., $\Delta D=0.02$ in. $(\Delta R=0.01$ in. $)$, and $L=10$ in. abuts perpendicularly onto a gamma-emitting surface. As shown the nomogram gives $A=7.0 \times 10^{-6}$ for a spherical emitter, $1.8 \times 10^{-5}$ for a Fermi emitter. Attenuation for a cosine emitter is twice that for a spherical or $14 \times 10^{-8}$.

* Under Contract No. W-31-109-ENG-52. +C. W. Wende. The computation of radiation hazards. TNX Report 7, p. 45.

$\ddagger$ When source density increases with depth and there is self-absorption the radiation passing through the interface will be enhanced in the forward direction. A good approximation to the distribution is given by the Fermi formula,

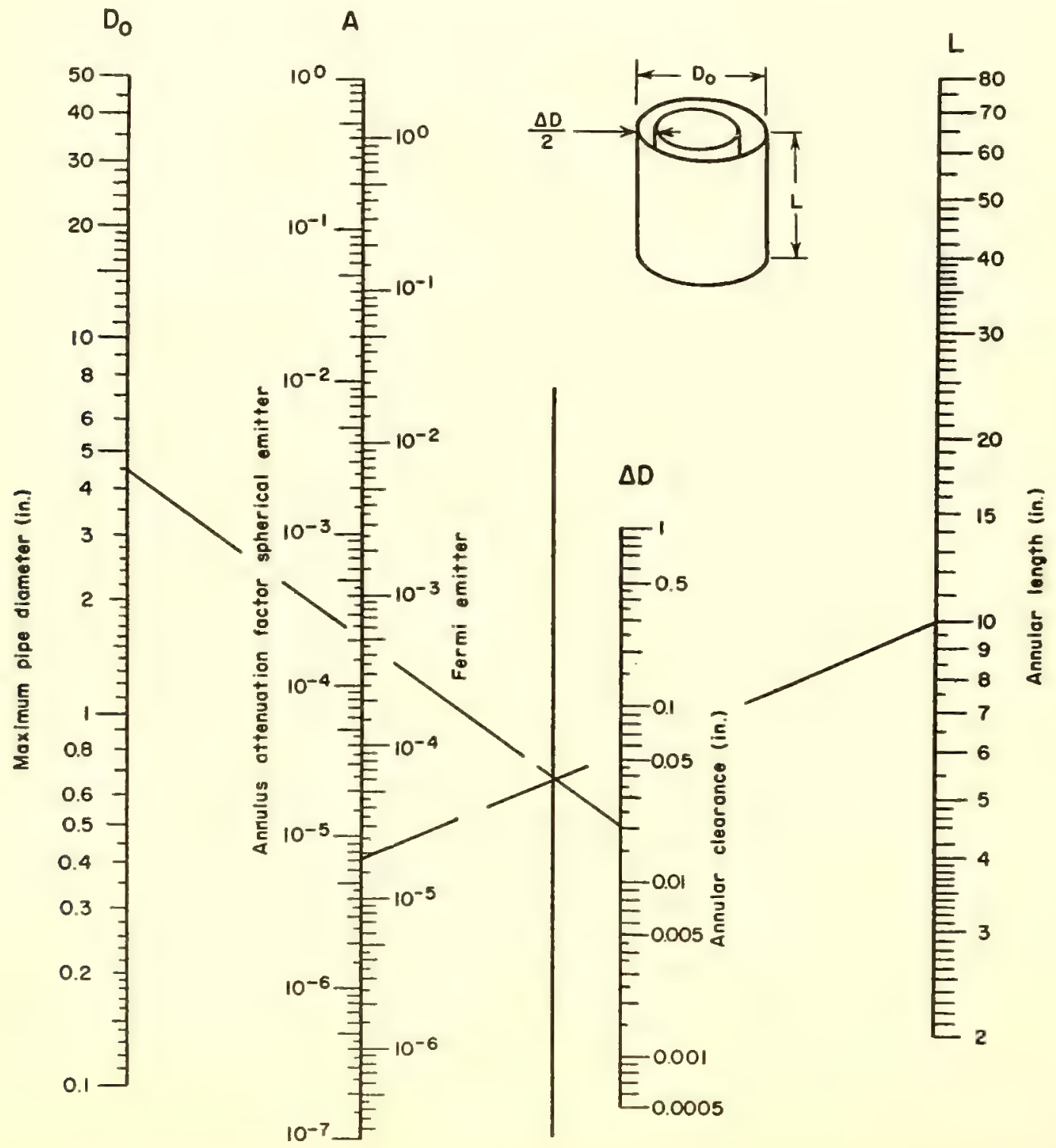




\title{
Gamma Dose Rates in Cylindrical Sources
}

\author{
BY DONALD R. SMITH \\ and \\ Air Force Institute of Technology, Dayton, Ohio
}

\author{
MARVIN C. ATKINS \\ Wright Air Development Center, Daylon, Ohio
}

What gamara-Rat exposure dose rate* will you find at the center of a given cylindrical $\mathrm{Co}^{\mathrm{BO}}$ source? How should you design such a source for a required flux in a given volume? Figures 1 and 2 are designed to answer such questions. By appropriate conversion they can be used for other gamma emitters. The computations are an extension of earlier work $(1,2)$.

Figure 1 is a plot showing the gamma dose rate to be expected at the centers of cylindrical sources of various sizes and activities. Self-absorption and backscatter are neglected. To use the graph for other gamma-emitters one must multiply the ordinates by $M / 14.7$, where $M$ is the exposure dose rate in $\mathrm{r} / \mathrm{hr} 1 \mathrm{ft}$ from a 1-c point source of the other isotope.

The following useful rules will be found to follow from this method of source design:

1. For a specified total activity, an increase in the flux by a factor of 10 will decrease the useful volume by $a$ factor of $31.6\left(1,000^{1 / 2}\right)$ if the same relationship between the average radius and height is maintained.

2. For a specified central dose rate an increase in the total activity by a factor of 10 will increase the useful in-

- The International Committee on Radiation Units has recommended introduction of the terms "exposure dose" (measured in roentgens) and "absorbed dose" (measured in rads) to reduce the confusion between the radiation field and the energy absorbed in any exposure.

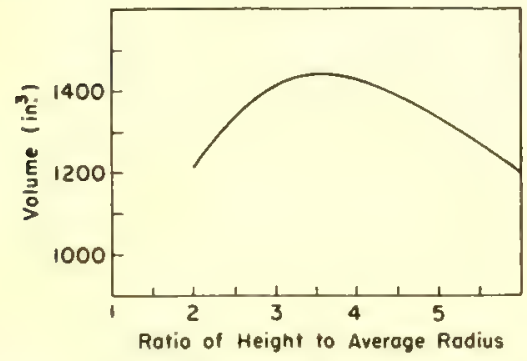

FIG. 2. Volume of $10,000-c$ cobait source with central flux of $5 \times 10^{6} \mathrm{r} / \mathrm{hr}$ as a function of ratio of height to average radius

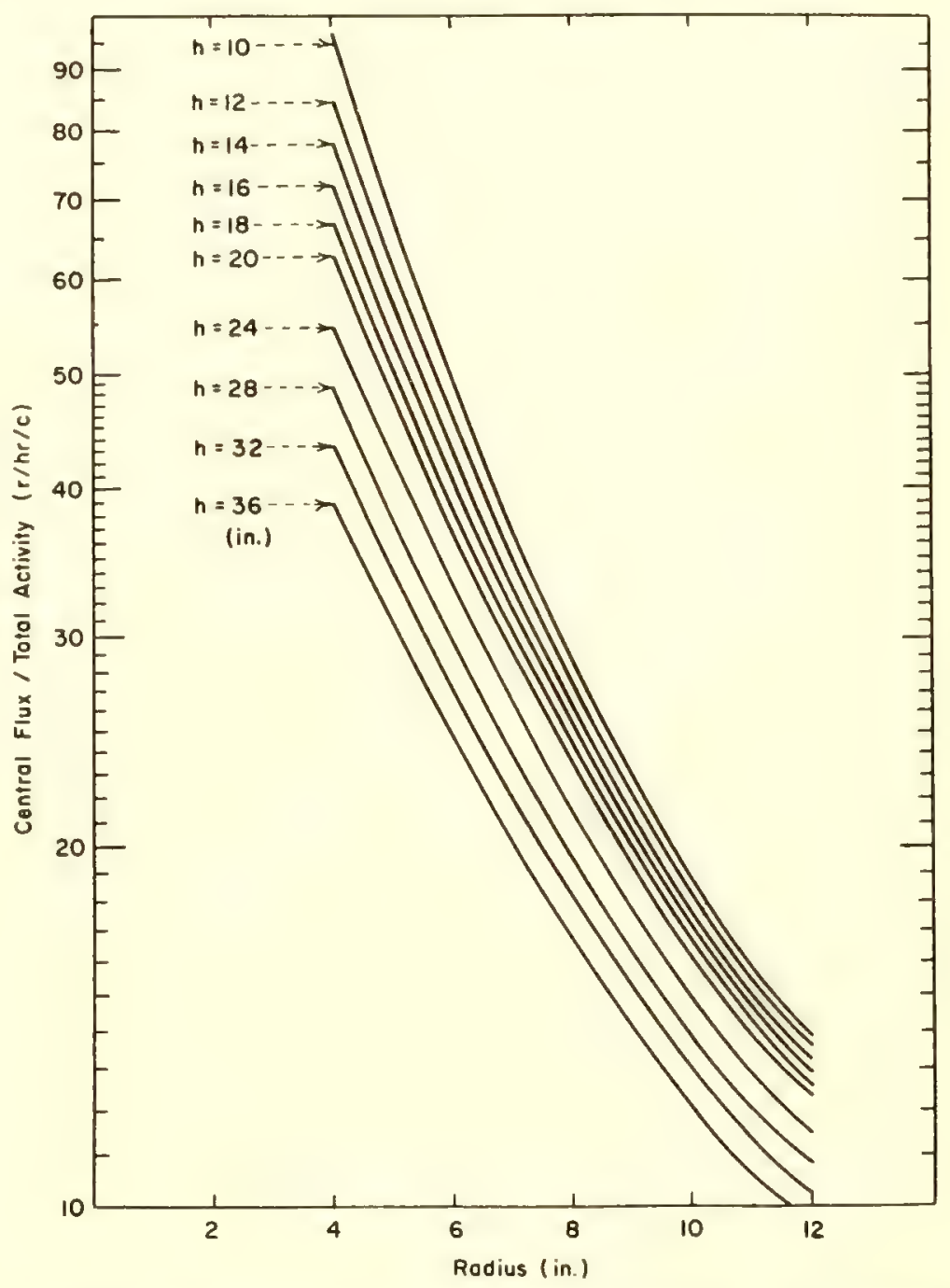

FIG. 1. Exposure dose rate of center of cylindrical cobalt source is a function of activity, height, and radius. Ordinate gives flux per total activity

ternal volume by a factor of 31.6 if the radius-height ratio is maintained.

Example. "Select dimensions to give maximum interior volume of a $10,000-\mathrm{c}$ cobalt source if the central flux is to be $5 \times 10^{5} \mathrm{r} / \mathrm{hr}$." Since $5 \times 10^{5} / 10,000=50$, we follow the $50-\mathrm{r} / \mathrm{hr} / \mathrm{c}$ line across the graph to find appropriate combinations of dimensions. Usable values are $h=24$, $b=4.4$, etc. In Fig. 2 we plot the volume as a function of $h / b$. The maximal volume is found to be 1,440 in. ${ }^{3}$ for $h / b=3.6$. The closest integral values that correspond to this volume and ratio are $h=18$ and $b=5 \mathrm{in}$. From Fig. 1 the central dose rate in a $10,000-\mathrm{c}$ source of these dimensions would be $5.02 \times 10^{5} \mathrm{r} / \mathrm{hr}$.

\section{BIBLIOGRAPHY}

1. D. L. Loughborough, A. E. Juve, J. R. Beatty, J. W. Born. A study of the effects of nuclear radiation on elastomeric compounds and compounding materials, Wright Air Development conter Technieal) Report 55-58, pp. 6, 76 Center

8. J. G. Lewis, J. V. Nehemias, D. E. Harmer J. J. Martin, Nocleonics 18. No. 1, 40 (1954) 


\section{Shielding}

Gamma Attenuation with Buildup in Lead and Iron

BY DAVID G. CHAPPELL

Knolls Atomic Power Laboratory, General Electric Company, Schenectady, New York
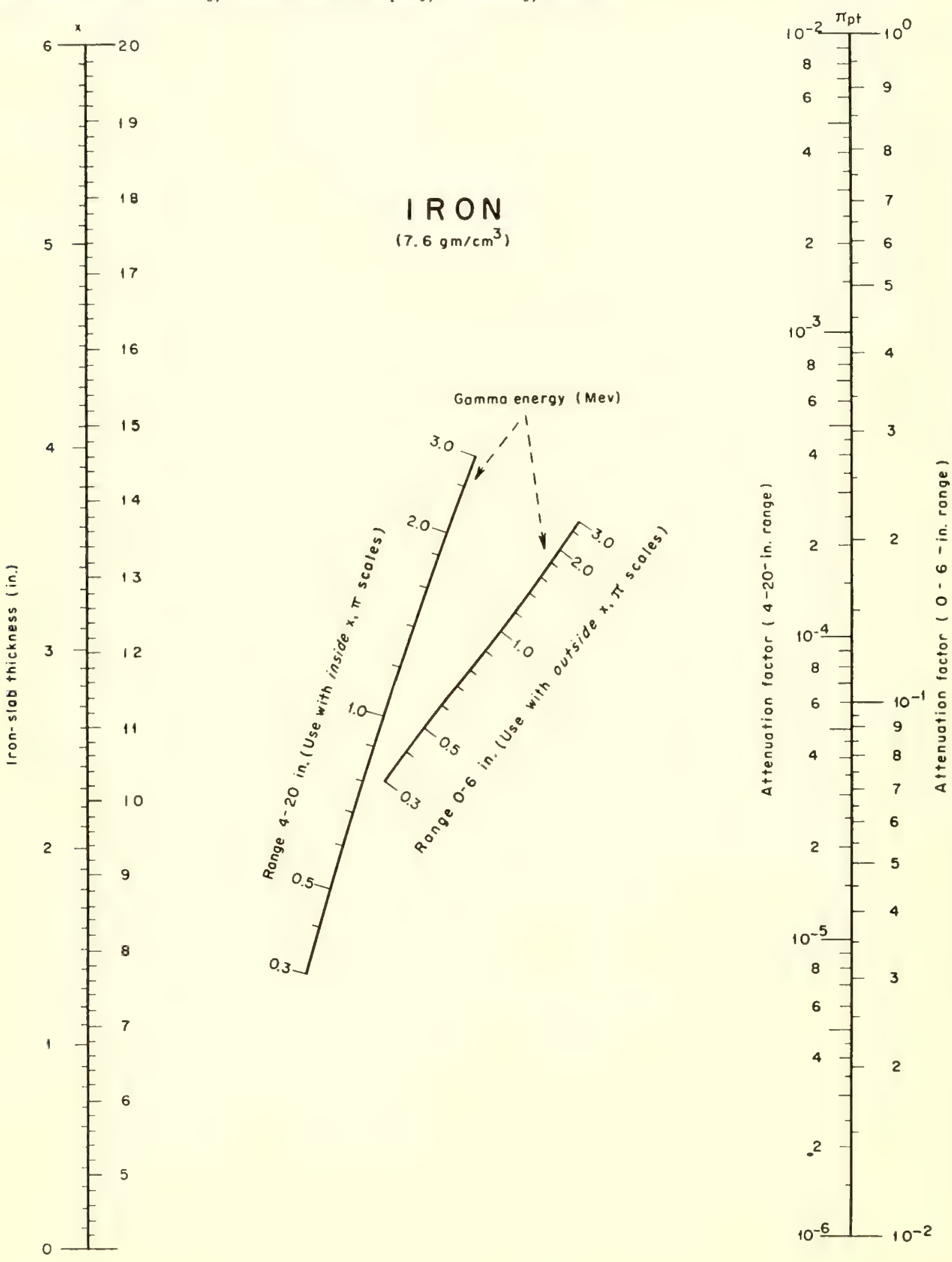
These Nomograms give attenuation factors for lead and iron shielding slabs used with point sources of gamma radiation.

The nomograms include the effect of buildup-the scattering of degraded

* Miscellaneous data for shielding calculations. Compiled by John Moteff. A PEX 176 (1954).

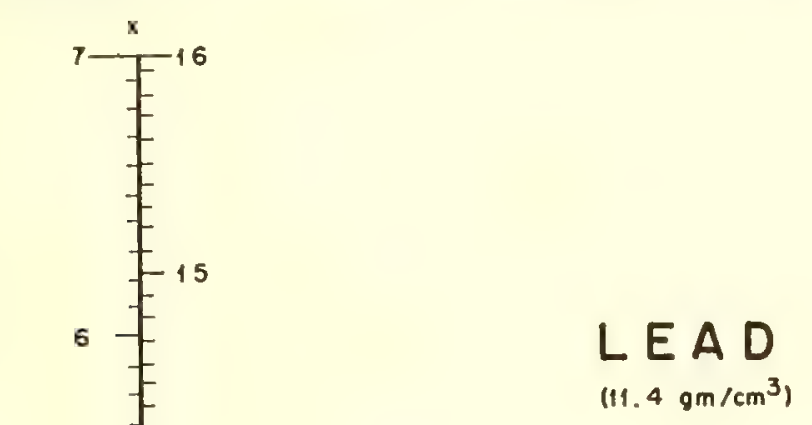

radiation into the beam from all points of the absorber. Absorption coefficients and buildup factors are from APEX 176.*

Over most of the range, accuracy is $10 \%$ or better. At extreme positions accuracy is better than $25 \%$.

Example. How thick must a lead shield be to reduce the dose from a point source of $1.0-\mathrm{Mev}$ gammas by a factor of $2 \times 10^{-8}$ ? We draw a line from the number $2 \times 10^{-8}$ at the right through 1.0-Mev on the proper gammaenergy scale. Reading on the appropriate side of the slab-thickness scale we find the answer: $9.67 \mathrm{in}$.

I wish to acknowledge the assistance of $M$ iss Joyce Berger, who drew the nomograms.

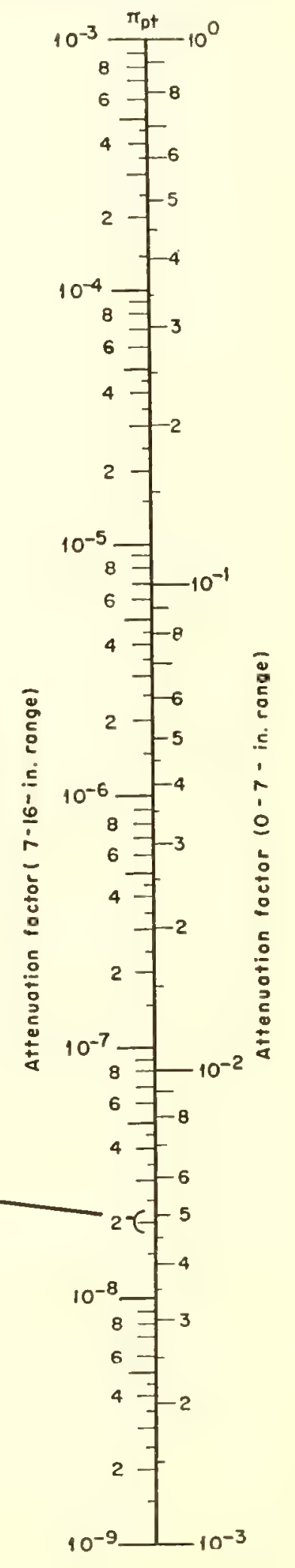




\section{Radiation from Neutron-Activated Slabs and Spheres}

\author{
By W. B. - LEWIS \\ Atomic Energy Division \\ Phillips Petroleum Co. \\ Idaho Falls, Idaho
}

THE RADIATION from an object activated by irradiation in a neutron flux that is uniform and isotropic depends on the manner in which the induced activity is distributed through the sample volume and on the absorption coefficient of the object for the radiation under consideration. The distribution of induced activity depends on the shape of the sample and on the coefficient of absorption for neutrons.

The effective activity, defined as the ratio of the amount of radiation leaving the surface to the total amount induced in the object, can be determined by solving two problems sequentially. First, the distribution of the radioactive nuclides must be determined. Second, the fraction of radiation reaching the surface from an element of volume must be calculated, and an integration made over the entire volume.
These problems have been solved for two simple cases: (1) a sphere of radius $a$ and (2) an infinite slab of halfthickness $a$. Three quantities are displayed: the density distribution $Z$, the effective activity $s$, and contours of constant s. Figure 1 shows these for spheres, and Fig. 2 for slabs.

Analytical expressions can be obtained for four quantities. These are tabulated below. The general functions $s\left(B_{n}, B_{r}\right)$ are obtained by interpolation.

The contours of $s\left(B_{n}, B_{r}\right)$ offer a quick means to evaluate the effective activity. It is necessary only to evaluate $B_{n}$ and $B_{r}$ from the physical constants of the sample and locate the corresponding point on the map. The accuracy of the value obtained is about $\pm 10 \%$. The contours cannot be used when both $B$ 's are close to unity. In such cases recourse to numerical inte- gration is necessary, or, better yet, just irradiate and see what happens.

Example: Suppose we irradiate a typical iridium sphere having the following characteristics: mass $=800 \mathrm{mg}$, $\mu_{n}=30.8 \mathrm{~cm}^{-1}, \mu_{r}=8.0 \mathrm{~cm}^{-1}, a=0.204$ $\mathrm{cm}, b_{n}=6.08, b_{r}=1.63, B_{n}=0.9977$, $B_{\mathrm{r}}=0.805$.

Since $b_{n}$ is large, most of the activity is produced in a thin outer shell, and since $b_{r}$ is fairly large, the efficiency of recovery should be about $50 \%$. The problem is complicated by the large range of gamma energies. There are 11 gammas listed for Ir with energies ranging from 0.136 to $0.613 \mathrm{Mev}$. The value chosen for $\mu_{r}$ is a good estimate for four gammas that account for $80 \%$ of the total energy: $0.296,0.308,0.316$, $0.468 \mathrm{Mev}$.

Values of 8 are 0.65 from Fig. 1 and 0.59 from numerical integration.

Quantities Important in Evaluating Neutron-Induced Radiation

\begin{tabular}{|c|c|c|c|}
\hline Quantity & Value for sphere & & Value for slab \\
\hline$Z\left(b_{n}, x\right)$ & $\frac{e^{-b_{n}}}{2}\left[\cosh b_{n} x+\left(1+b_{n}\right) \frac{\sinh b_{n} x}{b_{n} x}\right]$ & $-\frac{b_{n}\left(1-x^{2}\right)}{4 x} E$ & 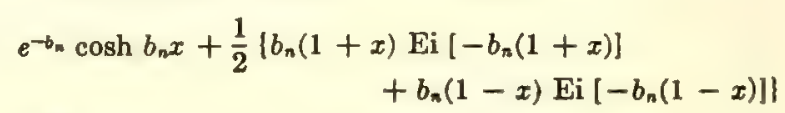 \\
\hline$Z_{a v o}\left(b_{n}\right)$ & $\frac{3}{8 b_{n}^{2}}\left[2 b_{n}^{2}-1+\left(2 b_{n}+1\right) e^{\left.-2 b_{n}\right]}\right]$ & & $\frac{e^{-2 b_{n}}}{2}+\frac{1}{4 b_{n}}\left(1-e^{-2 b_{n}}\right)+b_{n} \operatorname{Ei}\left(-2 b_{n}\right)$ \\
\hline$s\left(0, B_{r}\right)$ & $\frac{3}{8 b_{r}^{8}}\left[2 b_{r}^{2}-1+\left(2 b_{r}+1\right) e^{\left.-2 b_{r}\right]}\right.$ & & $\frac{e^{-2 b_{r}}}{2}+\frac{1}{4 b_{r}}\left(1-e^{-2 b_{r}}\right)+b_{r} \mathrm{Ei}\left(-2 b_{\varepsilon}\right)$ \\
\hline$s\left(1, B_{r}\right)$ & $\frac{1}{2}+\frac{1}{4 b_{r}}\left(1-e^{-b_{r}}\right)$ & & $\frac{1}{2}\left(1+e^{-2 b_{r}}\right)+b_{r} \operatorname{Ei}\left(-2 b_{r}\right)$ \\
\hline $8\left(B_{n}, 0\right)$ & 1 & & 1 \\
\hline$s\left(B_{n, 1}\right)$ & 0 & & 0 \\
\hline
\end{tabular}




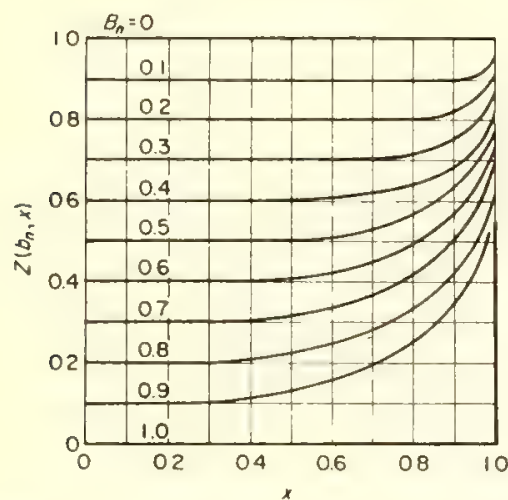

FIG. 1. Quantities needed for evaluation of radiation from neutron-activated sphere. $Z\left(b_{n}, x\right)$ is plotted as a function of $x$ for different values of the porameter $B_{n}$ (see table of symbols). $s$ for sphere

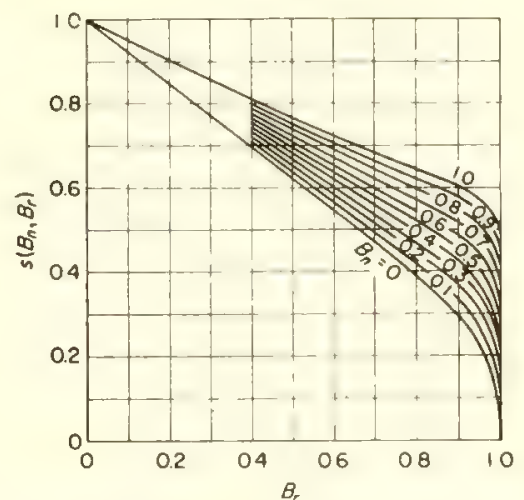

Constont-s contours, sphere

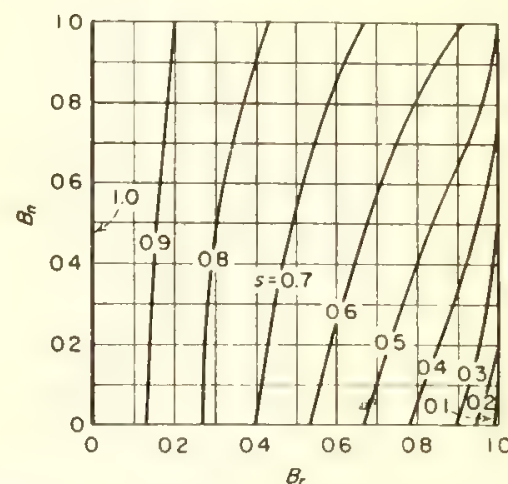

Values of $s\left(B_{n}, B_{r}\right)$ are plotted as functions of $B_{r}, B_{n}$ being the parameter. Contours of constant $s$ are shown on a map of $B_{n}$ and $B_{\text {, }}$

$Z\left(b_{n}, x\right)$ for slab

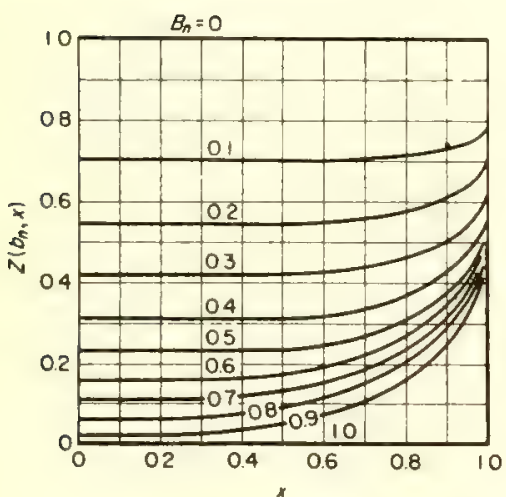

FIG. 2. Quantities $Z\left(b_{n}, x\right)$ and $s$ and contours of constant $s$ that are used to determine radiation from irradiated slabs are dis- $s$ for slob

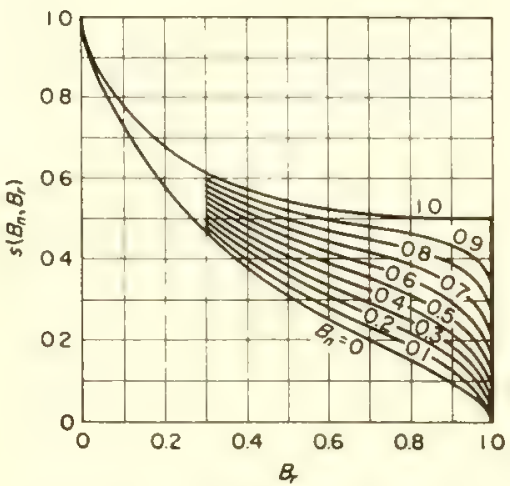

played in same manner as those for sphere that are displayed above in Fig. 1

\section{Symbols}

$a=$ significant length (radius of sphere, half-thickness of slab)

$x a=$ distance from center of sphere or midplane of slab to volume element

ha $=$ distance from volume element to surface element

$\mu=$ absorption coefficient

$n=$ subscript referring to neutrons

$r=$ subscript referring to induced radiation

$b=\mu a$

$B=1-e^{-b}$

$E=\int_{b(1-x)}^{b(1+x)} e^{-y} d y / y \equiv \operatorname{Ei}[-b(1+x)]-\operatorname{Ei}[-b(1-x)]$

$\phi=$ neutron flux $\left(\mathrm{n} / \mathrm{cm}^{2} / \mathrm{sec}\right)$

$\phi t=$ integrated neutron flux $\left(\mathrm{n} / \mathrm{cm}^{2}\right)$

$Z\left(b_{n}, x\right)=$ distribution function for induced activity
$=\int_{S} e^{-b_{n}{ }^{A}} \cos \theta d S / 4 \pi h^{2} a^{2}$

$\rho=$ actual volume density of radioactivity $\left(\mathrm{dps} / \mathrm{cm}^{3}\right)=\mu_{n} \phi t Z$ $\sigma\left(B_{n}, B_{r}\right)=$ surface density of radioactivity of equivalent thin shell $\left(d p s / \mathrm{cm}^{2}\right)=\int_{\nabla} e^{-b_{r} h} \rho\left(B_{n}, x\right) \cos \theta d V / 4 \pi h^{2} a^{2}$

$B\left(B_{n}, B_{r}\right)=$ ratio of equivalent-surface activity to total induced activity

$=\int_{S} \sigma d S / \int_{V} \rho d V=\sigma_{a v g} S / \rho_{a v g} V$

$\sigma_{\text {avo }}=\int_{S} \sigma d S / S$

$\rho_{\text {avg }}=\int_{V} \rho d V / V$

$Z_{\text {avg }}=\rho_{\text {avg }} / \mu_{n} \phi t$ 


\title{
Gamma-Ray Streaming Through a Duct
}

\author{
BY DAVID G. CHAPPELL
}

Knolls Atomic Power Laboralory, General Electric Co., Schenectady, New York

Practicar design of biological shields for radiation sources often involves accounting for the gamma flux that streams through a cylindrical duct that penetrates the shield.

This nomogram permits rapid determination of the geometrical attenuation of the gamma flux streaming through the duct from an emitting surface upon which the duct abuts perpendicularly. Scattering effects are not accounted for.

The formula for the attenuation ${ }^{*}$ is

$$
A=\frac{K}{4}\left(\frac{D}{L}\right)^{2}
$$

where $K$ is $35,1,1.268$ for a spherical, cosine and Fermi emitter, respectively; $D$ is the diameter of the duct; and $L$ is the duct length in identical units. The formula is valid if $L \gg D$, i.e., long, thin, straight cylindrical ducts for which $(D / 2)^{2}$ is negligible compared with $L^{2}$.

Example: 0.8-in-diameter duct penetrates 21-in.-thick shield and abuts perpendicularly onto a cosine emitting surface. As shown, the nomogram gives $A=3.5 \times 10^{-4}$ for a cosine emitter, $4.6 \times 10^{-4}$ for a Fermi emitter. Attenuation for a spherical emitter is half that of the cosine emitter, or $1.8 \times 10^{-4}$.

$I$ wish to acknowledge the assistance of Miss Joyce Berger, who drew the nomogram. Knolls Atomic Power Laboratory is operated by the General Electric Co. for the $U$. S. Atomic Energy Commission under coniract No. W-31-109 Eng. 52.

* "Reactor Shielding Design Manual," T. Rockwell III, editor, p. 290 (McGrawHill Book Co., New York, 1956).

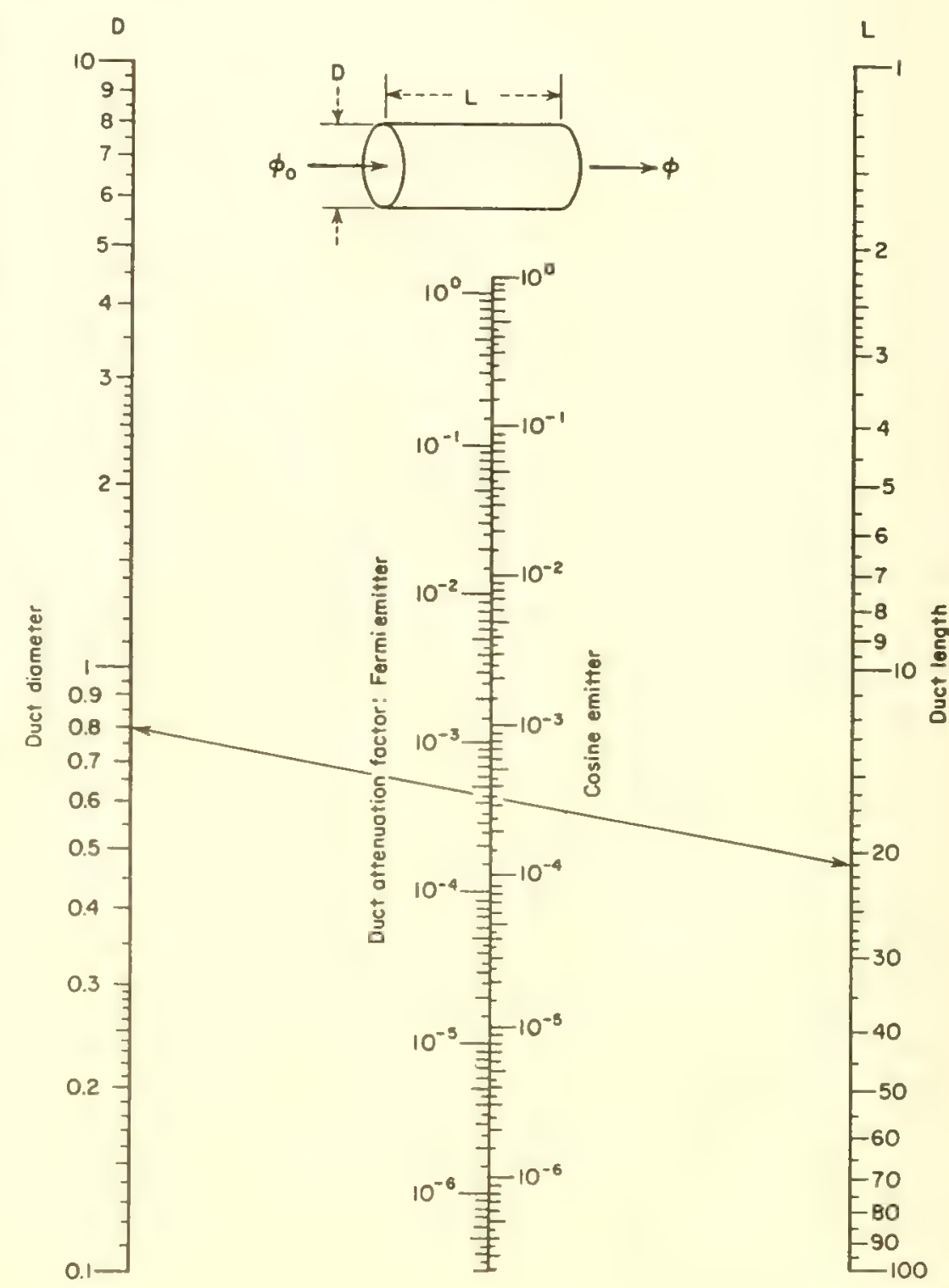




\section{Gamma-Detection Efficiency of Organic Phosphors}

\author{
BY K. I. ROULSTON \\ and S. I. H. NAQVI \\ Department of Physics \\ University of Maniloba \\ Winnipeg, Canada
}

This Nomogram and the accompanying curves make possible a rapid determination of the detection efficiency of an organic phosphor.

For detection a certain minimum energy must be transferred to a Compton electron. The cross section can be calculated by integrating the differential Compton cross section from the corresponding minimum photon scattering angle to $180 \mathrm{deg}$.

One starts with the minimum acceptable energy transfer (discriminatorbias setting). From Fig. 1 one then finds the cross section for detectable energy transfer, $\sigma$. To determine the corresponding absorption coefficient $\mu$ $(=\rho \sigma)$ we use the three nomogram scales at left. The nomogram then determines the efficiency $\left(1-e^{-\mu x}\right)$ from $\mu$ and the thickness $x$.

The method assumes equality of total linear absorption coefficient and detection absorption coefficient. This is generally justified by crystal geometries in which a photon that is scattered by an event not leading to detection cannot escape from the crystal without traversing the remainder of the path lengths.

With 25 -kev bias we observe effjciencies that correspond to our curve above $200 \mathrm{kev}$. Below $200 \mathrm{kev}$ the cross section is larger than calculated, apparently due to multiple collisions.

Example: Discriminator bias is at $25 \mathrm{kev}$, and we are detecting 200-kev photons in a 10 -cm-thick plastic phosphor. From the curves we find a detection cross section of 0.27 barns. A representative phosphor has $\rho=0.34$ $\times 10^{24}$ electrons $/ \mathrm{cm}^{3}$. With this assumption we find a detection efficiency of $60 \%$.

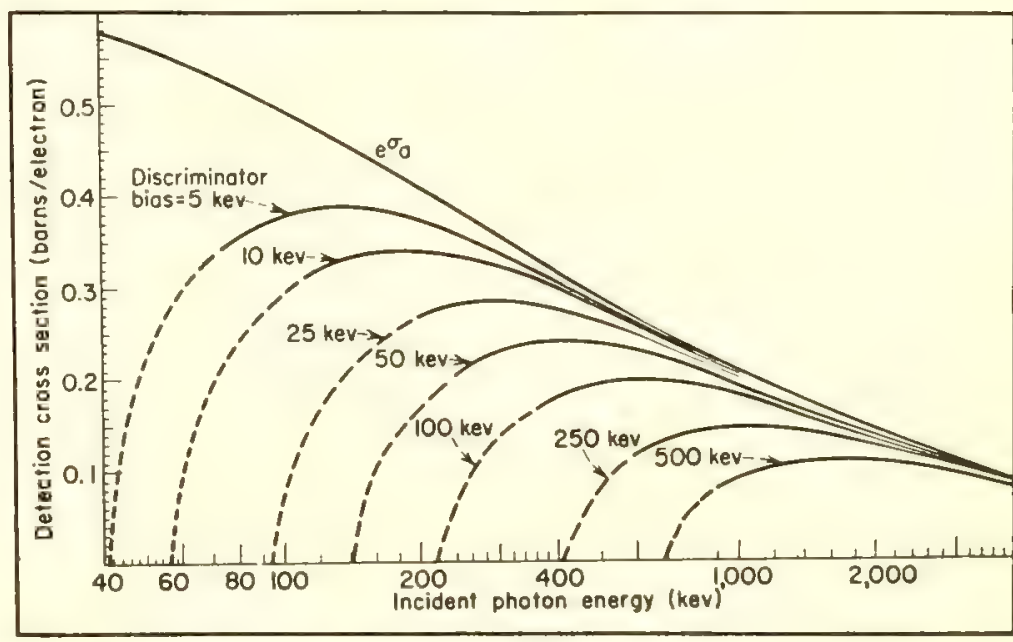

FG. I. Detection cross section vs incident photon energy

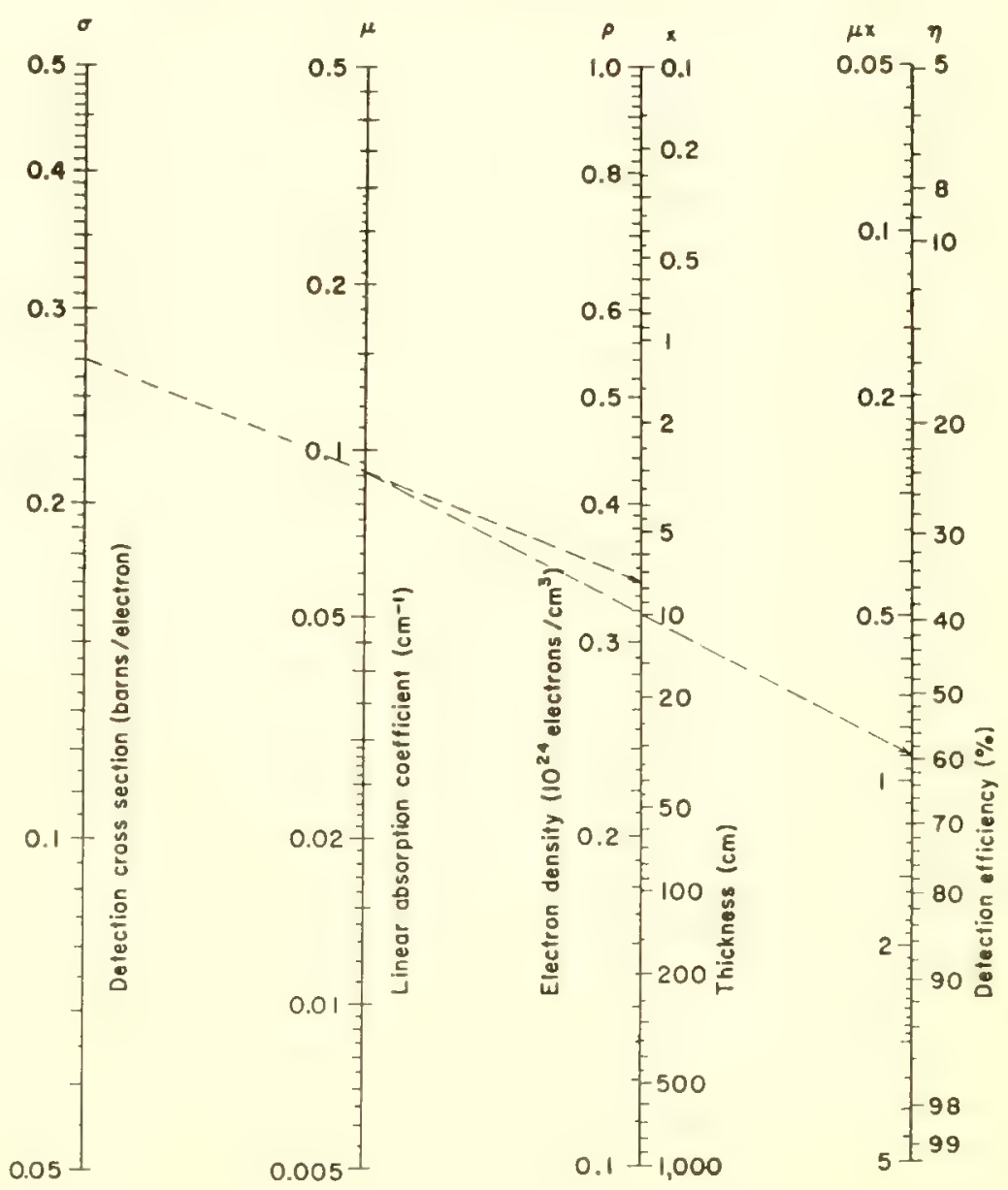




\title{
Radionuclides Arranged by Gamma-Ray Energy
}

\author{
BY GILBERT W. SMITH and DONALD R. FARMELO \\ Curtiss-Wright Corp., Quehanna, Pennsylvania
}

The table presented here lists radionuclides in order of the energies of their emitted photons. The listing covers the literature to October, 1956, as given by the Table of Isotopes (1) and the annual cumulations of nuclear data in Nuclear Science Abstracts.

The following criteria have been used in selecting the items:

- Nuclides derived from $n, \gamma$ reactions and fission products having $>\sim 0.5 \%$ yield are included.

- Half-lives or precursor half-lives are a few minutes or longer.

- Gammas $<10 \%$ of the total activity of any nuclide are omitted.

For use in identifying nuclides by gamma-scintillation spectrometry, the list includes other data along with photopeak energy. The list is also useful in finding photon energies for calibration purposes.

Photopeaks must not be confused with other peaks (2): (a) Pair-production peaks appear 0.51 and $1.02 \mathrm{Mev}$ below the photopeak and are especially bothersome if the photopeak occurs above $1.5 \mathrm{Mev}$. (b) Annihilation radiation appears at $0.51 \mathrm{Mev}$ in positron emitters. (c) Compton collisions that scatter the photon at $180 \mathrm{deg}$ create a peak at the following energy: $E=E_{0} /$ $\left(1+3.9137 E_{0}\right)$ with both energies in Mev (3). (d) The energy of the $K_{\alpha}$
$\mathrm{X}$-ray line in kev can be computed from its wave length $\lambda$ in angstroms as $12.34 / \lambda$.

Radiochemical separations may be necessary before using the table if a complex mixture has more than 5 photopeaks. Half-life, detection efficiency, daughter activities, and other properties can be used to resolve situations of this kind.

\section{BIBLIOGRAPHY}

1. J. M. Hollander, I. Perlman, G. T. Seaborg. R so8. Mod. Phys. 15, 469 (1953)

2. P. R. Bell "The Scintillstion Method"" in "Beta and Gamma-Ray Spectroscopy," K. Siegbahn, ed. (Interscience Publishers, New York, 1955)

9. B. Crasemann, H. Esaterday, nuclfontcs 14 No, 6, $63(1956)$

\section{Radionuclides by Increasing Gamma-Photon Energy}

\begin{tabular}{|c|c|c|c|c|c|c|c|c|c|c|c|}
\hline $\begin{array}{l}\text { Pholo- } \\
\text { peak } \\
\text { energy } \\
\text { (Mev) }\end{array}$ & Nuclide & $\begin{array}{l}\text { Half- } \\
\text { life }\end{array}$ & $\begin{array}{c}\text { Production } \\
\text { cross } \\
\text { section* } \\
\text { (barns) or } \\
\text { fission } \\
\text { yield }(\%)\end{array}$ & $\begin{array}{l}\text { Photon } \\
\text { yield } \\
\text { per } \\
\text { disinte- } \\
\text { gration } \\
(\%)\end{array}$ & Daughler & $\begin{array}{l}\text { Photo- } \\
\text { peak } \\
\text { energy } \\
(M e v)\end{array}$ & Nuclide & $\begin{array}{l}\text { Half- } \\
\text { life }\end{array}$ & $\begin{array}{c}\text { Production } \\
\text { cross } \\
\text { section* } \\
\text { (barns) or } \\
\text { fission } \\
\text { yield (\%) }\end{array}$ & $\begin{array}{l}\text { Photon } \\
\text { yield } \\
\text { per } \\
\text { disinte- } \\
\text { gration } \\
(\%)\end{array}$ & Daughler \\
\hline 0.037 & $\mathrm{Br} r^{80 m}$ & $4.58 \mathrm{~h}$ & $2.9 \mathrm{~b}$ & 100 & $\mathrm{Br} r^{80}$ & $0.113 \mathrm{c}$ & $\mathbf{E} r^{171}$ & $7.5 \mathrm{~h}$ & $9 \mathrm{~b}$ & & $\operatorname{Tm}^{111}$ \\
\hline 0.040 & $\mathrm{Rh}^{103 m}$ & $57 \mathrm{~m}$ & $2.9 \%$ & 100 & $\mathrm{Rh}^{103}(\mathrm{st}) \dagger$ & $0.123 \mathrm{c}$ & $\mathrm{Eu}^{164}$ & $16 \mathrm{y}$ & $420 \mathrm{~b}$ & & $\mathrm{Gd}^{154}(\mathrm{st})$ \\
\hline 0.047 & $\mathrm{~Pb}^{210}$ & $22 y$ & & $\sim 100$ & $\mathrm{Bi}^{210}$ & 0.128 & $\mathrm{Cs}^{184 m}$ & $3.2 \mathrm{~h}$ & $0.016 \mathrm{~b}$ & 100 & $\mathrm{Cs}^{384}$ \\
\hline 0.049 & $\mathrm{Br}^{80 m}$ & $4.58 \mathrm{~h}$ & $2.9 \mathrm{~b}$ & 100 & $B r^{80}$ & 0.129 & $\mathrm{Os}^{191}$ & $16.0 \mathrm{~d}$ & $8 \mathrm{~b}$ & 100 & $\operatorname{Ir}^{181}(\mathrm{st})$ \\
\hline 0.052 & $\mathrm{Rh}^{104 m}$ & $4.4 \mathrm{~m}$ & $12 \mathrm{~b}$ & 100 & $\mathbf{R h}^{104}$ & 0.132 & $\mathrm{Hf}^{181}$ & $46 \mathrm{~d}$ & $10 \mathrm{~b}$ & & $\mathrm{Ta}^{181 m, m_{1}}$ \\
\hline 0.058 & Gd $^{158}$ & $18 \mathrm{~h}$ & $4 b$ & & $\mathrm{~Tb}^{159}(\mathrm{st})$ & 0.134 & $\mathrm{Ce}^{141}$ & $290 \mathrm{~d}$ & $6.0 \%$ & 30 & $\operatorname{Pr}^{144}$ \\
\hline $0.068 c \ddagger$ & $\mathrm{T}^{182}$ & $112 \mathrm{~d}$ & $21 b$ & & $\mathrm{~W}^{182}$ (st) & $0.136 \mathrm{c}$ & $\mathrm{Se}^{75}$ & $127 d$ & $26 \mathrm{~b}$ & & $\mathrm{As}^{75}$ \\
\hline 0.077 & $\mathrm{Pt}^{197}$ & $10 \mathrm{~h}$ & $1.1 \mathrm{~b}$ & 100 & $\mathrm{Au}^{187}(\mathrm{st})$ & 0.140 & $\mathrm{Te}^{99 m}$ & $6.04 \mathrm{~h}$ & $5.8 \%$ & 99 & $\mathbf{T e}^{99}$ \\
\hline 0.080 & $\mathrm{Ho}^{18 s}$ & $27.3 \mathrm{~h}$ & $60 \mathrm{~b}$ & & $\mathrm{Er}^{146 m}$ & 0.142 & $C e^{141}$ & $32.8 \mathrm{~d}$ & $0.3 b, 5.7 \%$ & 67 & $\operatorname{Pr}^{141}(s t)$ \\
\hline 0.081 & $\mathrm{Xe}^{138}$ & $5.27 d$ & $6.5 \%$ & & $\mathrm{Cs}^{133}(\mathrm{st})$ & 0.147 & $\mathrm{Te}^{131}$ & $24.8 \mathrm{~m}$ & $0.22 \mathrm{~b}, 2.9 \%$ & 55 & $I^{181}$ \\
\hline 0.084 & $\begin{array}{c}T_{m}^{170} \\
Y^{170 m}\end{array}$ & $129 d$ & $125 b$ & & $\mathrm{Yb}^{170}(\mathrm{st})$ & $\begin{array}{l}0.150 \\
0.150\end{array}$ & $\begin{array}{l}\mathrm{Cd}^{118 m,} \\
\mathrm{Kr}^{\mathrm{Bbm}}\end{array}$ & $\begin{array}{l}49 \mathrm{~m} \\
4.36 \mathrm{~h}\end{array}$ & $\begin{array}{c}0.2 b \\
0.1 b, 1.1 \%\end{array}$ & 100 & $\begin{array}{l}\mathrm{Cd}^{111 m_{1}} \\
\mathrm{Kr}^{8 b}\end{array}$ \\
\hline 0.084 & $T^{228}$ & $1.9 \mathrm{y}$ & & 28 & $\mathrm{Ra}^{284}$ & 0.155 & $\operatorname{Re}^{188}$ & $16.9 \mathrm{~h}$ & $70 \mathrm{~b}$ & & $\mathrm{Os}^{188}(\mathrm{st})$ \\
\hline 0.087 & $\mathrm{Pd}^{109}$ & $13.6 \mathrm{~h}$ & $12 \mathrm{~b}$ & & $\mathrm{Ag}^{109 m}$ & 0.16 & $\mathrm{Ba}^{140}$ & $12.8 \mathrm{~d}$ & $4.0 \mathrm{~b}, 6.3 \%$ & 60 & $\mathrm{La}^{140}$ \\
\hline 0.089 & $\begin{array}{c}\mathrm{Lu}^{176 m-} \\
\mathrm{H}^{178 m}\end{array}$ & $\begin{array}{l}3.7 \mathrm{~h}- \\
\text { short }\end{array}$ & $40 \mathrm{~b}$ & 100 & $\mathrm{Hf}^{178}(\mathrm{st})$ & $\begin{array}{l}0.16 \\
0.162\end{array}$ & $\begin{array}{l}\mathrm{Xe}^{131 m_{\mathrm{q}}} \\
\mathrm{Ba}^{1 \mathrm{ag}}\end{array}$ & $\begin{array}{l}12.0 \mathrm{~d} \\
85.0 \mathrm{~m}\end{array}$ & $\begin{array}{c}2.9 \% \\
0.5 b, 6.2 \%\end{array}$ & $\begin{array}{l}\S \\
26\end{array}$ & $\begin{array}{l}\mathrm{Xe}^{181}(\mathrm{st}) \\
\mathrm{La}^{139}\end{array}$ \\
\hline 0.089 & $\mathrm{Te}^{127 m}$ & $110 \mathrm{~d}$ & $0.09 \mathrm{~b}$ & & $\mathrm{Te}^{127}$ & 0.176 & $\mathrm{Se}^{8 s}$ & $25 \mathrm{~m}$ & $0.004 \mathrm{~b}$ & & $\mathrm{Br}^{83}$ \\
\hline 0.092 & $\mathrm{Nd}^{147}$ & $11.3 \mathrm{~d}$ & $1.8 \mathrm{~b}, 2.6 \%$ & & $\mathrm{Pm}^{147}$ & 0.177 & $\mathrm{Te}^{131 m}$ & $30 \mathrm{~h}$ & $0.42 \%$ & & $\mathrm{Te}^{131}$ \\
\hline 0.093 & $T_{h}^{234}$ & $24.1 d$ & & 20 & $\mathrm{~Pa}^{2 a 4}$ & 0.180 & $\mathrm{Ta}^{182 m}$ & $16.5 \mathrm{~m}$ & $0.03 \mathrm{~b}$ & 100 & $\mathrm{Ta}^{182}$ \\
\hline 0.094 & $\mathrm{U}^{285}$ & $7.1 \times 10^{8} \mathrm{y}$ & & & $\mathrm{Th}^{231}$ & 0.184 & $\mathrm{U}^{286}$ & $7.1 \times 10^{8} y$ & & & $T_{h}^{231}$ \\
\hline 0.100 & $S m^{153}$ & $47 \mathrm{~h}$ & $140 \mathrm{~b}$ & & $\mathrm{Eu}^{163 m}$ & 0.188 & $\mathrm{Ra}^{226}$ & $1622 \mathrm{y}$ & & 6 & $\operatorname{Rn}^{222}$ \\
\hline 0.103 & $\mathrm{Gd}^{153}$ & $236 d$ & $<125 \mathrm{~b}$ & & $\mathrm{Eu}^{158}(\mathrm{st})$ & 0.190 & In $114 m$ & $49 \mathrm{~d}$ & $56 \mathrm{~b}$ & 100 & $\mathrm{Cd}^{114}$ \\
\hline 0.105 & $\mathrm{~Pa}^{232}$ & $27.4 \mathrm{~d}$ & $\sim 50 \mathrm{~b}$ & & $\mathrm{U}^{232}$ & 0.191 & $\mathrm{Mo}^{101}$ & $14.6 \mathrm{~m}$ & $0.20 \mathrm{~b}, 5.4 \%$ & & $\mathrm{Te}^{101}$ \\
\hline 0.105 & $\mathrm{Sm}^{165}$ & $2.4 \mathrm{~m}$ & $5.5 \mathrm{~b}$ & 100 & $\mathrm{Eu}^{165}$ & 0.206 & $\mathrm{Lu}^{177}$ & $6.8 \mathrm{~d}$ & $4 \times 10^{3} \mathrm{~b}$ & & $\mathrm{Hf}{ }^{177}$ (st) \\
\hline 0.106 & $\mathrm{Te}^{189 m}$ & $33.5 \mathrm{~d}$ & $.015 b, 0.34 \%$ & 100 & $\mathrm{Te}^{129}$ & $0.216 \mathrm{c}$ & $\mathrm{Hf}^{180 \mathrm{~m}}$ & $5.5 \mathrm{~h}$ & $75 \mathrm{~b}$ & 100 & $\mathrm{Hf}^{180}$ \\
\hline 0.110 & $\mathrm{Te}^{186 \mathrm{~m}}$ & $58 d$ & $5 \mathrm{~b}$ & 100 & $\mathrm{Te}^{125}$ (st) & 0.231 & $\mathrm{Te}^{122}$ & $77.7 \mathrm{~h}$ & $4.4 \%$ & 40 & $I^{132}$ \\
\hline 0.112 & $\mathrm{Lu}^{177}$ & $6.8 \mathrm{~d}$ & $4 \times 10^{2} b$ & & $\mathrm{Hf}^{177}(\mathrm{st})$ & 0.234 & $\mathrm{Xe}^{183 m}$ & $2.3 d$ & $6.5 \%$ & 100 & $X e^{133}$ \\
\hline
\end{tabular}




\begin{tabular}{|c|c|c|c|c|c|c|c|c|c|c|c|}
\hline $\begin{array}{c}\text { Photo- } \\
\text { peak } \\
\text { energy } \\
\text { (Mev) }\end{array}$ & Nuclide & $\begin{array}{l}\text { Half- } \\
\text { life }\end{array}$ & $\begin{array}{c}\text { Production } \\
\text { cross } \\
\text { section } \\
\text { (barns) or } \\
\text { fission } \\
\text { yield }(\%)\end{array}$ & $\begin{array}{l}\text { Photon } \\
\text { yield } \\
\text { per } \\
\text { disinte- } \\
\text { gration } \\
(\%)\end{array}$ & Daughter & $\begin{array}{l}\text { Photo- } \\
\text { peak } \\
\text { energy } \\
(\text { Mev) }\end{array}$ & Nuclide & $\begin{array}{l}\text { Half- } \\
\text { life }\end{array}$ & $\begin{array}{l}\text { Production } \\
\text { cross } \\
\text { section } \\
\text { (barns) or } \\
\text { fission } \\
\text { yield }(\%)\end{array}$ & $\begin{array}{l}\text { Pholon } \\
\text { yield } \\
\text { per } \\
\text { disinte- } \\
\text { gration } \\
(\%)\end{array}$ & Daughter \\
\hline 0.239 & $\mathrm{~Pb}^{212}$ & $10.6 \mathrm{~h}$ & & $\sim 80$ & $B i^{212}$ & 0.673 & $I^{132}$ & $2.26 \mathrm{~h}$ & $600 \mathrm{~b}$ & & $\mathrm{Xe}^{132}(\mathrm{st})$ \\
\hline $0.244 c$ & $\mathrm{Eu}^{152}$ & $13 y$ & $7 \times 10^{3} b$ & & $\mathrm{Gd}^{152}(\mathrm{st})$ & 0.69 & $W^{187}$ & $23.9 \mathrm{~h}$ & $34 b$ & & $\mathrm{Re}^{187}$ \\
\hline 0.246 & $\mathrm{Cd}^{111 m_{1}}$ & $49 m$ & $0.2 \mathrm{~b}$ & 100 & $\mathrm{Cd}^{111}(\mathrm{st})$ & 0.7 & $\mathrm{Te}^{1 \mathfrak{d} i}$ & $24.8 \mathrm{~m}$ & $0.22 b$ & 45 & $I^{131}$ \\
\hline 0.250 & $\mathrm{Xe}^{18 s}$ & $9.13 \mathrm{~h}$ & $5.9 \%$ & 100 & $\mathrm{Cs}^{136}$ & 0.72 & $\mathrm{Zr}^{96}$ & $65 d$ & $0.10 \mathrm{~b}, 6.3 \%$ & 99 & $\mathrm{Nb}^{05}$ \\
\hline 0.25 & $\mathrm{Sm}^{156}$ & $24 \mathrm{~m}$ & $5.5 \mathrm{~b}$ & 100 & $\mathrm{Eu}^{168}$ & 0.726 & $R u^{108}$ & $4.5 \mathrm{~h}$ & $0.7 b, 0.85 \%$ & 100 & $\mathrm{Rh}^{105 m}$ \\
\hline $0.26 c$ & $\mathrm{Ge}^{77}$ & $12 \mathrm{~h}$ & $0.2 \mathrm{~b}$ & & $\mathrm{As}^{77}$ & 0.740 & $\mathrm{Mo}^{99}$ & $68 \mathrm{~h}$ & $0.13 b, 6.1 \%$ & 20 & $\mathrm{Tc}^{90}$ \\
\hline 0.265 & $\mathrm{Ge}^{75}$ & $82 \mathrm{~m}$ & $0.5 b$ & & $\mathrm{As}^{75}(\mathrm{st})$ & 0.764 & $\mathrm{Nb}^{95}$ & $35 \mathrm{~d}$ & $15 b, 6.3 \%$ & 100 & $\mathrm{Mo}^{95}(\mathrm{st})$ \\
\hline $0.265 \mathrm{c}$ & $\mathrm{Se}^{75}$ & $127 \mathrm{~d}$ & $26 \mathrm{~b}$ & & $\mathrm{As}^{75}$ & $0.77 \mathrm{c}$ & $B r^{8 z}$ & $35.9 \mathrm{~h}$ & $2.6 b$ & & $K_{r^{32}}(\mathrm{st})$ \\
\hline 0.279 & $\mathrm{Hg}^{203}$ & $47 d$ & $3.8 b$ & 100 & $\mathrm{Tl}^{203}(\mathrm{st})$ & 0.775 & $\mathrm{Zr}^{97}-$ & $17.0 \mathrm{~h}-$ & $6.1 \%$ & 100 & $\mathrm{Nb}^{97}$ \\
\hline 0.284 & $I^{181}$ & $8.05 \mathrm{~d}$ & $2.9 \%$ & & $X e^{1 H m_{2} m_{1}}$ & & $\mathrm{Nb}^{97 m}$ & $60 \mathrm{~s}$ & & & \\
\hline 0.285 & $\mathrm{Pm}^{149}$ & $50 \mathrm{~h}$ & $1.3 \%$ & & $\mathrm{Sm}^{149}(\mathrm{st})$ & 0.796 & $\mathrm{Cs}^{134}$ & $2.3 y$ & $26 \mathrm{~b}$ & $\sim 80$ & $\mathrm{Ba}^{131}(\mathrm{st})$ \\
\hline 0.290 & $\mathrm{Ce}^{143}$ & $33 \mathrm{~h}$ & $1.0 \mathrm{~b}, 5.4 \%$ & & $\operatorname{Pr}^{143}$ & 0.829 & $\mathrm{~Pb}^{211}$ & $36.1 \mathrm{~m}$ & & 13 & $\mathrm{Bi}^{211}$ । \\
\hline 0.305 & $\mathrm{Kr}^{85 m}$ & $4.36 \mathrm{~h}$ & $0.1 \mathrm{~b}, 1.1 \%$ & & $\mathrm{Kr}^{85}$ & $0.83 \mathrm{c}$ & $\mathrm{Ga}^{73}$ & $14.3 \mathrm{~h}$ & $4.6 \mathrm{~b}$ & & $\mathrm{Ge}^{72}(\mathrm{st})$ \\
\hline 0.307 & $T c^{101}$ & $14 \mathrm{~m}$ & $5.4 \%$ & & $R u^{101}(\mathrm{st})$ & 0.835 & $\mathrm{Mn}^{64}$ & $291 d$ & & 100 & $\mathrm{Cr}^{64}(\mathrm{st})$ \\
\hline $0.31 \mathrm{c}$ & $\mathrm{Pa}^{233}$ & $27.4 d$ & $50 \mathrm{~b}$ & & $\mathrm{U}^{283}$ & 0.835 & $\mathrm{Ga}^{72}$ & $14.3 \mathrm{~h}$ & $3.4 b$ & 100 & $\mathrm{Ge}^{72 m}$ \\
\hline $0.316 \mathrm{c}$ & $\mathrm{Ir}^{192}$ & $74.4 d$ & $700 \mathrm{~b}$ & & $P t^{192}(8 t)$ & 0.845 & $\mathrm{Mn}^{66}$ & $2.58 \mathrm{~h}$ & $13.4 \mathrm{~b}$ & 100 & $\mathrm{Fe}^{68}(\mathrm{st})$ \\
\hline 0.32 & $\mathrm{Nd}^{147}$ & $11.3 \mathrm{~d}$ & $1.8 b, 2.6 \%$ & & $\mathrm{Pm}^{147}$ & 0.88 & $\mathrm{Sc}^{46}$ & $84 d$ & $12 \mathrm{~b}$ & 99.5 & $\mathrm{Ti}^{46}(\mathrm{st})$ \\
\hline 0.325 & $\mathrm{Cr}^{51}$ & $27 d$ & $11 \mathrm{~b}$ & 9 & $V^{51}(s t)$ & $0.91 \mathrm{c}$ & $\mathbf{R b}^{\mathbf{8 8}}$ & $17.8 \mathrm{~m}$ & $0.14 b$ & & $\mathrm{Sr}^{88}(\mathrm{st})$ \\
\hline 0.326 & $\mathrm{Sn}^{125}$ & $9.5 \mathrm{~m}$ & $0.2 b$ & & $\mathrm{Sb}^{128}$ & 0.95 & $\mathrm{Cd}^{115}$ & $53 \mathrm{~h}-$ & $1.1 \mathrm{~b}$ & & $\operatorname{In}^{115}(\mathrm{st})$ \\
\hline $0.33 \mathrm{c}$ & Ir ${ }^{194}$ & $19 \mathrm{~h}$ & $130 \mathrm{~b}$ & & $\mathrm{Pt}^{194}(\mathrm{st})$ & & In $115 m$ & $4.5 \mathrm{~h}$ & & & \\
\hline 0.335 & $\mathrm{Cd}^{116}$ & $53 \mathrm{~h}-$ & $1.1 \mathrm{~b}$ & & $\operatorname{In}^{118}(\mathrm{st})$ & 0.95 & $\mathrm{Se}^{83}$ & $25 \mathrm{~m}$ & $0.004 b$ & & $\mathrm{Br}^{83}$ \\
\hline & In $^{115 m}$ & $4.5 \mathrm{~h}$ & & & & 0.96 & $\mathrm{Mo}^{101}$ & $14.6 \mathrm{~m}$ & $0.20 \mathrm{~b}, 5.4 \%$ & & $T e^{101}$ \\
\hline 0.35 & $\mathrm{Bi}^{211}$ & $2.16 \mathrm{~m}$ & & 16 & $\left.\mathrm{~T}\right|^{207}$ & $0.96 c$ & $\mathrm{~Tb}^{180}$ & $73 \mathrm{~d}$ & $44 b$ & & $\mathrm{Dy}^{180}(\mathrm{st})$ \\
\hline 0.36 & $\mathrm{Gd}^{160}$ & $18 \mathrm{~h}$ & $4 \mathrm{~b}$ & & $T b^{169}(\mathrm{st})$ & $1.0 \mathrm{c}$ & Dy ${ }^{186}$ & $2.32 \mathrm{~h}$ & $2.7 \times 10^{3} \mathrm{~b}$ & & $\mathrm{Ho}^{185}(\mathrm{st})$ \\
\hline 0.364 & $I^{131}$ & $8.05 \mathrm{~d}$ & $2.9 \%$ & 80 & $X \mathrm{e}^{131 m_{2} m_{1}}$ & 1.08 & $\mathrm{Rb}^{\mathrm{86}}$ & $18.7 \mathrm{~d}$ & $0.7 \mathrm{~b}$ & 20 & $\mathrm{Sr}^{86}(\mathrm{st})$ \\
\hline 0.37 & $\mathrm{Ni}^{86}$ & $2.56 \mathrm{~h}$ & $2.6 \mathrm{~b}$ & 15 & $\mathrm{Cu}^{86}$ (st) & 1.097 & $\mathrm{Fe}^{68}$ & $46 d$ & $0.9 b$ & 50 & $\mathrm{Co}^{59}(\mathrm{st})$ \\
\hline 0.37 & $\mathrm{Se}^{\mathrm{Bs}}$ & $25 \mathrm{~m}$ & $0.004 \mathrm{~b}$ & & $\mathrm{Br}^{83}$ & 1.11 & $\mathrm{Zn}^{65}$ & $245 \mathrm{~d}$ & $0.5 b$ & 45 & $\mathrm{Cu}^{85}$ (st) \\
\hline 0.388 & $\mathrm{Sr}^{87 m}$ & $2.8 \mathrm{~h}$ & $1.3 \mathrm{~b}$ & 100 & $\mathrm{Sr}^{87}$ & 1.11 & $\mathrm{Sc}^{48}$ & $84 d$ & $12 \mathrm{~b}$ & 99.5 & $\mathrm{Ti}^{46}(\mathrm{st})$ \\
\hline 0.393 & $\mathrm{Sn}^{113}$ & $118 d$ & $1.3 \mathrm{~b}$ & & $\mathrm{In}^{112 m}$ & $1.12 \mathrm{c}$ & $\mathrm{Eu}^{151}$ & $16 y$ & $4.2 \times 10^{2} \mathrm{~b}$ & & $\mathrm{Gd}^{134}(\mathrm{st})$ \\
\hline $0.41 \mathrm{c}$ & $\mathbf{K} \mathbf{r}^{87}$ & $78 \mathrm{~m}$ & $2.7 \%$ & & $R b^{87}$ & 1.12 & $\mathrm{Ni}^{65}$ & $2.56 \mathrm{~h}$ & $2.6 \mathrm{~b}$ & 30 & $\mathrm{Cu}^{65}$ (st) \\
\hline 0.412 & $\mathrm{Au}^{198}$ & $2.69 \mathrm{~d}$ & $96 \mathrm{~b}$ & 99 & $\mathrm{Hg}^{198}$ & $1.12 \mathrm{c}$ & $\mathrm{Ta}^{182}$ & $112 \mathrm{~d}$ & $21 b$ & & $\mathrm{~W}^{182}(\mathrm{st})$ \\
\hline 0.44 & $\mathrm{Zn}^{88 m}$ & $13.8 \mathrm{~h}$ & $0.1 \mathrm{~b}$ & 100 & $\mathrm{Zn}^{89}$ & 1.172 & $\mathrm{Co}^{\circ 0}$ & $5.27 y$ & $20 \mathrm{~b}$ & 99 & $\mathrm{Ni}^{80}$ (st) \\
\hline $0.444 \mathrm{c}$ & $\mathrm{Hf}^{180 \mathrm{~m}}$ & $5.5 \mathrm{~h}$ & $75 b$ & 100 & $\mathrm{Hf}^{1 \mathrm{BO}}$ & $1.27 \mathrm{c}$ & $\operatorname{In}^{118 m}$ & $54 \mathrm{~m}$ & $150 \mathrm{~b}$ & 75 & $\operatorname{In} 116$ \\
\hline 0.45 & $I^{128}$ & $25 \mathrm{~m}$ & $6.3 b$ & 7 & $\mathrm{Xe}^{128}(\mathrm{st})$ & 1.27 & $I^{135}$ & $6.75 \mathrm{~h}$ & $6.3 \%$ & & $\mathrm{Xe}^{138.126 m}$ \\
\hline $0.468 \mathrm{c}$ & $\operatorname{Ir}^{192}$ & $74.4 \mathrm{~d}$ & $700 \mathrm{~b}$ & & $\mathrm{Pt}^{192}$ (st) & 1.277 & $\mathrm{Na}^{22}$ & $2.60 \mathrm{y}$ & & 100 & $\mathrm{Ne}^{22}$ (st) \\
\hline 0.479 & $\mathrm{Be}^{7}$ & $52.9 \mathrm{~d}$ & & 11 & $\mathrm{Li}^{9}(\mathrm{st})$ & 1.29 & $A^{41}$ & $109 \mathrm{~m}-$ & $0.53 b$ & 99 & $\mathrm{~K}^{41}(\mathrm{st})$ \\
\hline 0.48 & $W^{187}$ & $23.9 \mathrm{~h}$ & $34 \mathrm{~b}$ & & $\operatorname{Re}^{187}(\mathrm{st})$ & & $K^{41 m}$ & short & & & \\
\hline 0.490 & $\mathrm{La}^{140}$ & $40.0 \mathrm{~h}$ & $8.4 \mathrm{~b}$ & & $\mathrm{Ca}^{140}(\mathrm{st})$ & 1.295 & $\mathrm{Fe}^{50}$ & $45.0 \mathrm{~d}$ & $0.9 \mathrm{~b}$ & 50 & $\mathrm{Co}^{59}(8 \mathrm{t})$ \\
\hline 0.498 & $R u^{103}$ & $39.8 \mathrm{~d}$ & $1.2 b, 2.9 \%$ & 99 & $\mathrm{Rh}^{103 \mathrm{~m}}$ & 1.332 & $\mathrm{Co}^{80}$ & $5.27 y$ & $20 \mathrm{~b}$ & 99 & $\mathrm{Ni}^{80}(\mathrm{st})$ \\
\hline 0.513 & $\mathrm{Sr}^{85}$ & $65 d$ & $1.0 \mathrm{~b}$ & 100 & $R b^{85 m}$ & 1.36 & $\mathrm{Ho}^{168}$ & $27.3 \mathrm{~h}$ & $60 \mathrm{~b}$ & 11 & $\mathrm{Er}^{186 m}$ \\
\hline 0.513 & $\begin{array}{c}\mathrm{Ru}^{108}- \\
\mathrm{Rh}^{108}\end{array}$ & $\begin{array}{r}1 \mathrm{y}- \\
30 \mathrm{~s}\end{array}$ & $0.38 \%$ & 11 & $\mathrm{Pd}^{108}(\mathrm{st})$ & $\begin{array}{l}1.38 \\
1.4\end{array}$ & $\begin{array}{l}\mathrm{Na}^{24} \\
\mathrm{~V}^{32}\end{array}$ & $\begin{array}{l}14.90 \mathrm{~h} \\
3.77 \mathrm{~m}\end{array}$ & $\begin{array}{l}0.56 b \\
4.5 b\end{array}$ & 100 & $\begin{array}{l}\mathrm{Mg}^{24}(\mathrm{st}) \\
\mathrm{Cr}^{52}(\mathrm{st})\end{array}$ \\
\hline 0.52 & $\mathrm{Si}^{31}$ & $2.65 \mathrm{~h}$ & $0.11 b$ & & $P^{\prime \prime}(s t)$ & $1.44 c$ & $\mathrm{Cs}^{188}$ & $32.9 \mathrm{~m}$ & $5.7 \%$ & 100 & $\mathrm{Ba}^{138}(\mathrm{st})$ \\
\hline 0.52 & $\mathrm{Xe}^{125 m}$ & $15.6 \mathrm{~m}$ & $6.3 \%$ & 100 & $\mathrm{Xe}^{135}$ & 1.49 & $\mathrm{Ni}^{65}$ & $2.56 \mathrm{~h}$ & $2.6 \mathrm{~b}$ & 15 & $\mathrm{Cu}^{\mathrm{Bb}}$ (st) \\
\hline 0.53 & $I^{138}$ & $20.9 \mathrm{~h}$ & $6.5 \%$ & 94 & $\mathrm{Xe}^{138,132 m}$ & 1.53 & $\mathrm{~K}^{42}$ & $12.44 \mathrm{~h}$ & $1.0 \mathrm{~b}$ & 20 & $\mathrm{Ca}^{42}$ (st) \\
\hline 0.53 & $\mathrm{Nd}^{147}$ & $11.3 \mathrm{~d}$ & $1.8 b, 2.6 \%$ & & $\mathrm{Pm}^{147}$ & 1.57 & $\mathrm{Cl}^{38}$ & $37.3 \mathrm{~m}$ & $0.6 b$ & 31 & $A^{38}(s t)$ \\
\hline 0.537 & $\mathrm{Ba}^{140}$ & $12.8 \mathrm{~d}$ & $6.3 \%, 4.0 \mathrm{~b}$ & 30 & $\mathrm{~L} \mathrm{a}^{140}$ & 1.60 & $\mathrm{Cl}^{38}$ & $37.3 \mathrm{~m}$ & $0.6 b$ & 31 & $A^{38}(s t)$ \\
\hline 0.541 & $\mathrm{Br}$ & $35.7 \mathrm{~h}$ & $3.5 b$ & & $\mathrm{Kr}^{\mathrm{s2}}$ (st) & 1.60 & $\mathrm{La}^{140}$ & $40.3 \mathrm{~h}$ & $8.4 \mathrm{~b}$ & & $\mathrm{Ce}^{140}(\mathrm{st})$ \\
\hline 0.549 & $\mathrm{As}^{76}$ & $26.5 \mathrm{~h}$ & $4.2 \mathrm{~b}$ & 50 & $\mathrm{Se}^{78}(\mathrm{st})$ & 1.61 & $\operatorname{Pr}^{142}$ & $19.21 \mathrm{~h}$ & $10 \mathrm{~b}$ & & $\mathrm{Nd}^{142}(\mathrm{st})$ \\
\hline 0.55 & $\mathrm{~S} r^{91}$ & $9.7 \mathrm{~h}$ & $5.8 \%$ & & $Y^{91}$ & 1.78 & $A l^{28}$ & $2.27 \mathrm{~m}$ & $0.23 \mathrm{~b}$ & 100 & $\mathrm{Si}^{28}(\mathrm{st})$ \\
\hline $0.56 c$ & $\mathrm{Ge}^{77}$ & $12 \mathrm{~h}$ & $0.2 b$ & & $\mathrm{As}^{77}$ & 1.8 & $I^{135}$ & $6.75 \mathrm{~h}$ & $6.3 \%$ & & $\mathrm{Xe}^{125,136 m}$ \\
\hline 0.564 & $\mathrm{Sb}^{122}$ & $2.75 \mathrm{~d}$ & $7 \mathrm{~b}$ & & $\mathrm{Te}^{122}(\mathrm{st})$ & 1.81 & $\mathrm{Mn}^{58}$ & $2.58 \mathrm{~h}$ & $13.4 \mathrm{~b}$ & 30 & $\mathrm{Fe}^{\mathrm{sb}}(\mathrm{st})$ \\
\hline 0.605 & $\mathrm{Sb}^{124}$ & $60 \mathrm{~d}$ & $2.5 b$ & 50 & $\mathrm{Te}^{184}(\mathrm{st})$ & $1.85 \mathrm{c}$ & $\mathrm{Rb}^{88}$ & $17.8 \mathrm{~m}$ & $0.14 b$ & & $\mathrm{Sr}^{68}(\mathrm{st})$ \\
\hline $0.609 \mathrm{c}$ & $\mathrm{Bi}^{214}$ & $19.7 \mathrm{~m}$ & & & $\mathrm{Po}^{214}$ & 2.15 & $\mathrm{Cl}^{20}$ & $37.3 \mathrm{~m}$ & $0.6 \mathrm{~b}$ & 47 & $A^{38}(\mathrm{st})$ \\
\hline $0.62 \mathrm{c}$ & $\mathrm{Br}^{82}$ & $35.9 \mathrm{~h}$ & $2.6 \mathrm{~b}$ & & $K r^{82}(s t)$ & 2.62 & $\mathrm{~T} 1^{208}$ & $3.1 \mathrm{~m}$ & & 100 & $\mathrm{~Pb}^{208}$ (st) \\
\hline 0.624 & $\mathrm{Ru}^{108}$ & $1 \mathrm{y}-$ & $0.38 \%$ & 12 & $\mathrm{Pd}^{108}(\mathrm{st})$ & 2.76 & $\mathrm{Na}^{24}$ & $14.90 \mathrm{~h}$ & $0.56 \mathrm{~b}$ & 100 & $\mathrm{Mg}^{24}(\mathrm{st})$ \\
\hline & $\mathrm{Rh}^{106}$ & $30 \mathrm{~s}$ & & & & $2.8 \mathrm{c}$ & $\mathrm{Rb}^{88}$ & $17.8 \mathrm{~m}$ & $0.14 b$ & & $\mathrm{Sr}^{88}(\mathrm{st})$ \\
\hline 0.637 & $I^{131}$ & $8.05 d$ & $2.9 \%$ & $\begin{array}{r}9 \\
97\end{array}$ & $\begin{array}{l}X e^{131 m} \\
\mathrm{Ag}^{110}\end{array}$ & \multirow{3}{*}{\multicolumn{6}{|c|}{$\begin{array}{l}\text { * Thermal-neutron cross section of target atom. } \\
\dagger(s t)=\text { stable element. } \\
+c=\text { complex spectrum. }\end{array}$}} \\
\hline 0.657 & $\mathrm{Ag}^{110 m}$ & $270 \mathrm{~d}$ & $\begin{array}{l}2.8 b \\
5.9 \%\end{array}$ & $\begin{array}{l}97 \\
92\end{array}$ & $\begin{array}{l}\mathrm{Ag}^{110} \\
\mathrm{~J}^{13}{ }^{137}\end{array}$ & & & & & & \\
\hline 0.662 & $\begin{array}{l}\mathrm{Cs}^{137}- \\
\mathrm{Ba}^{197 m}\end{array}$ & $\begin{array}{c}33 \mathrm{y}- \\
2.6 \mathrm{~m}\end{array}$ & $5.9 \%$ & 92 & & & & & & & \\
\hline
\end{tabular}




\title{
Nomogram for Radioactivity Induced in Irradiation
}

\author{
BY EDWARD C. FREILING \\ V. S. Nanal Radiological Defense Inaboratory \\ San Francisco, Califonia
}

Frequint calculations in radioactivity laboratories answer such questions as this: If a sample is irradiated in a flux for a specified time, how many atoms are transmuted? How much radioactivity is induced?

The known quantities of one problem may be the unknowns of another: If a certain irradiation produces a measured activity, how much of a particular nuclide was originally present? In terms of an observed activity and half-life, what is the capture cross section?

This nomogram can be used for determinations of this type. It summarizes the relationships among:

1. Total exposure $(\phi \times t)$

2. Product of amount of material in sample $(n)$ and activation cross section per atom $(\sigma)$

3. Amount of material transmuted (N)

4. Amount of resulting activity

5. Decay constant $(\lambda)$ or halflife $(\tau)$.

Example: Activation analysis of a sample of $\mathrm{U}^{238}$ exposed to $10^{17}$ thermal neutrons $/ \mathrm{cm}^{2}$ produced $1,500 \mathrm{dpm}$ of 2.34-day $\mathrm{Np}^{239}$. The nomogram shows that there are $7 \times 10^{6}$ atoms of $\mathrm{Np}^{239}$ in the sample. A second line from $\phi t$ to $N$ shows that the target material had a total cross section of $10^{-10}$ molebarns. Since the thermal-neutron capture cross section of $\mathrm{U}^{238}$ is 2.8 barns, there were $3.6 \times 10^{-11}$ moles of $U^{238}$, or $8.5 \times 10^{-8} \mathrm{gm}$.

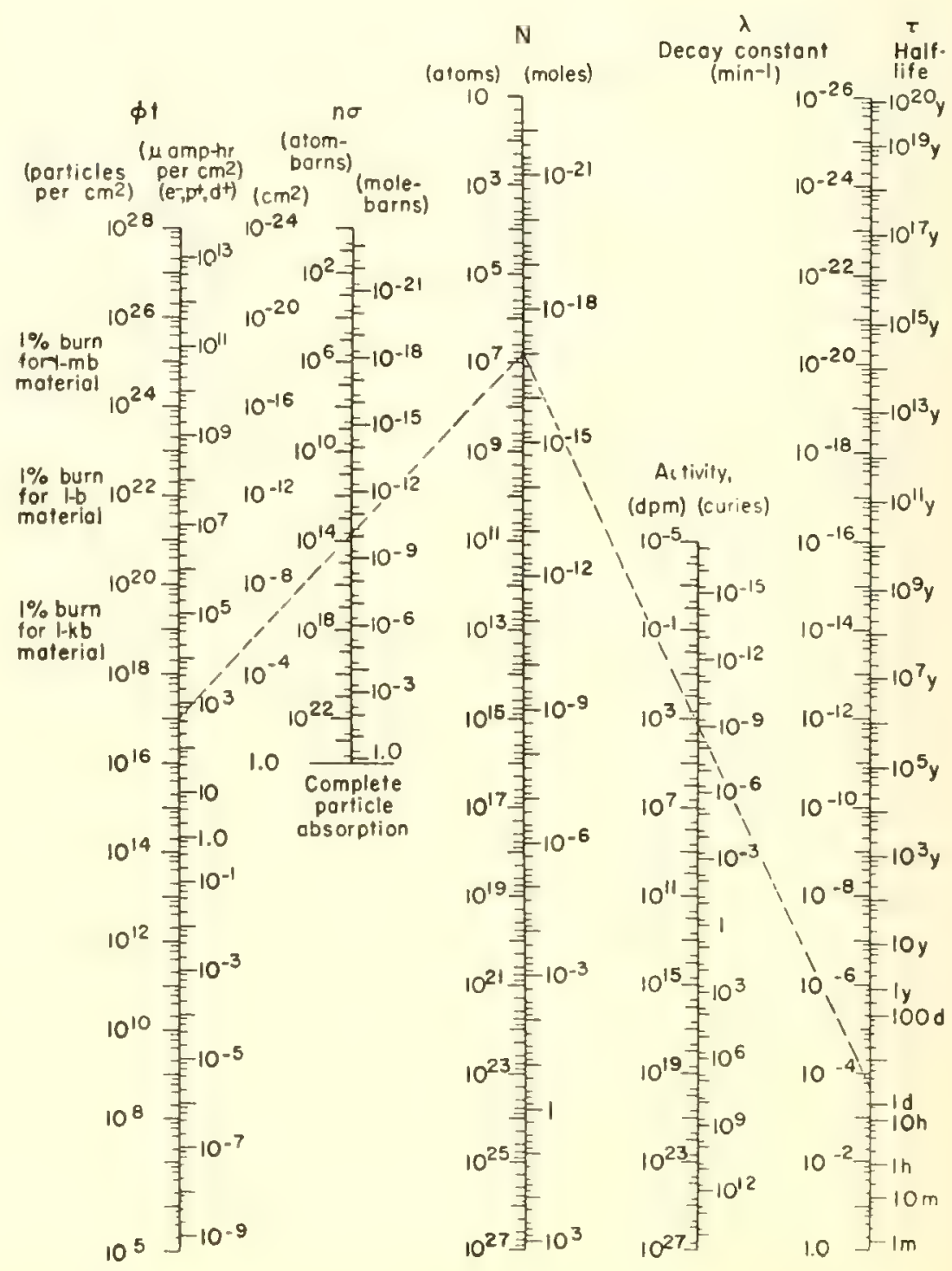




\section{Thermal-Neutron Data For the Elements}

\author{
BY M. Y. DAVIS and D. T. HAUSER \\ Hanford Laboralories Operation \\ General Electric Company \\ Richland, Washington
}

The widespread acceptance of an earlier thermal-neutron-data tabulation by Kroeger (1) has stimulated this updated compilation. Intended primarily for calculations related to reactor studies, it should also prove useful in neutron-source and shielding studies.

The material, for the most part, is found in the references or calculated using standard techniques, such as are presented by Glasstone and Edlund ( 2 ). The cross sections are the $2,200-\mathrm{m} / \mathrm{sec}$ cross sections. Non-1/v cross sections are corrected to yield a result equivalent to a $1 / v$ cross section in a Maxwellian thermal-neutron flux.

The average value for the cosine of the scattering angle, $\bar{\mu}_{0}$, is calculated from $\bar{\mu}_{0}=2 / 3 A$, where $A$ is the mass of the scattering nucleus.

The average logarithmic energy decrement, $\xi$, is found from the approximation $\xi=2 /(A+2 / 3)$ for the elements with mass larger than 10. For the lighter elements, $\xi=1+$ $\left[(A-1)^{2} / 2 A\right][\ln (A-1) /(A+1)]$.

The diffusion coefficient is $D=L^{2} \Sigma_{a}$ or $D=\lambda_{0} / 3\left(1-\bar{\mu}_{0}\right)$, where $\lambda_{1} /\left(1-\bar{\mu}_{0}\right)$ is the transport mean free path. The transport cross sections and mean free path were deleted to allow space for the resonance integral, the moderation ares and the diffusion coefficient.

The cross sections given in the table are the absorption (a), scattering (s) and total (t) cross sections.

This work was performei under the auspices of the U.S. Atomic Eneroy Commission.

\section{BIBLIOGRAPHY}

1. H. R. Kroeger, Neclionics 5, No. 4, 51 (1949)

8. 8. Glasetone, M. C. Edlund, "The Elements of Nuclear Resotor Theory" (D. Van Noetrand, Princeton, 1952)

3. D. J. Hughes, J. A. Harvey, Neutron Croes Beotions, BNL-325 (1955)

4. J. F. Stehn, E. F. Clsnoy, General Eleotrio Chart of the Nuolides, 5th ed. (1956)

8. "The Reactor Handbook, Vol. 1. Physios," AECD-3645 (1955)

6. R. L. Macklin, H. 8. Pomeranee, Resonance Capture Integrals, in "Progress in Nuclear Energy," vol. 1 (MoGraw-Hill Book Co., New York, 1956)

7. E. Critoph, CRRP-653 AECL No. 350 (1956)

8. H. Noumann. Effeotive oosttering masses for mixtures, HW-48814 (1957)

\begin{tabular}{|c|c|c|}
\hline \multirow{2}{*}{\multicolumn{2}{|c|}{$\begin{array}{l}\text { Aromic no. } \\
\text { Oiffosion } \\
\text { cootf. } \\
\text { (cm) }\end{array}$}} & 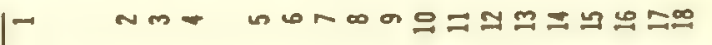 \\
\hline & & 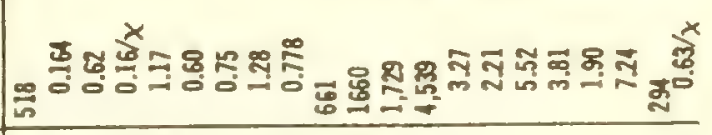 \\
\hline \multicolumn{2}{|c|}{$\begin{array}{c}\text { Diffusion } \\
\text { length } \\
\text { (em) }\end{array}$} & 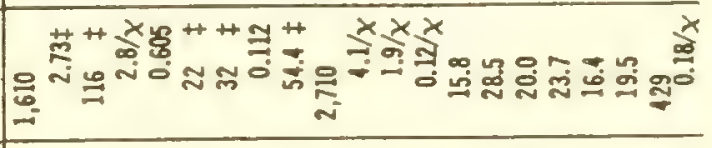 \\
\hline \multicolumn{2}{|c|}{$\begin{array}{l}\text { Resonancf: } \\
\text { Integral : } \\
\text { (barns) }\end{array}$} & స్ㅇㅇ융 \\
\hline \multirow{3}{*}{ 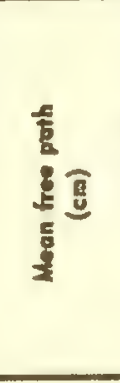 } & से & 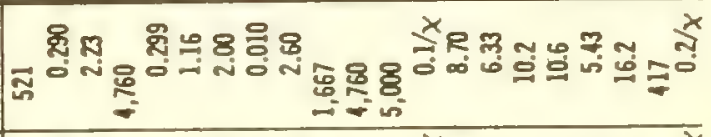 \\
\hline & $x^{\infty}$ & 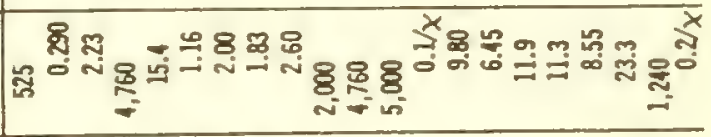 \\
\hline & $<$ & 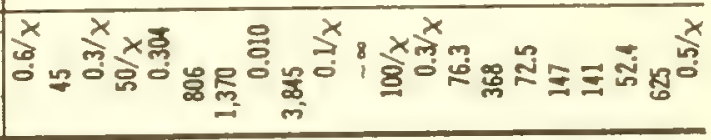 \\
\hline \multirow{3}{*}{ 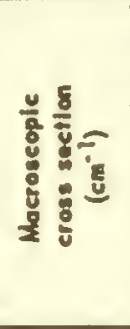 } & $\tilde{w}$ & 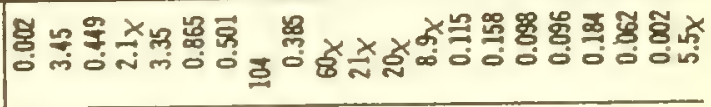 \\
\hline & $w^{\infty}$ & శ్ \\
\hline & $\omega^{\infty}$ & 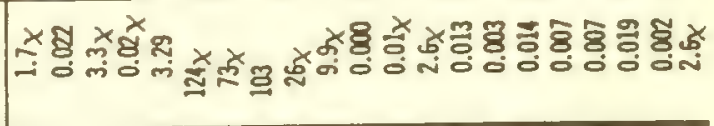 \\
\hline \multirow{3}{*}{ 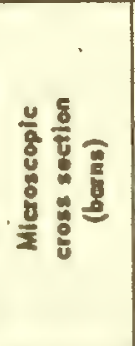 } & 5 & 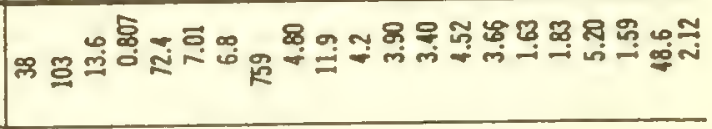 \\
\hline & $0^{\circ}$ & m \\
\hline & $0^{\circ}$ & 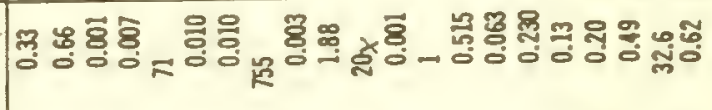 \\
\hline \multicolumn{2}{|c|}{ us } & 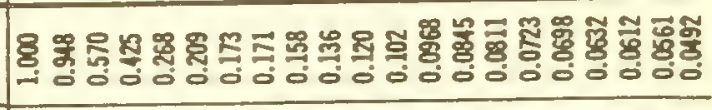 \\
\hline & İ & 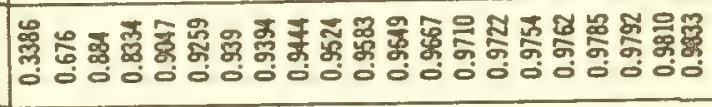 \\
\hline \multicolumn{2}{|c|}{$\begin{array}{c}\text { Nuelei } \\
\text { pot unit vol. } \\
\left(x 10^{2} 4 \mathrm{em}^{3}\right)\end{array}$} & 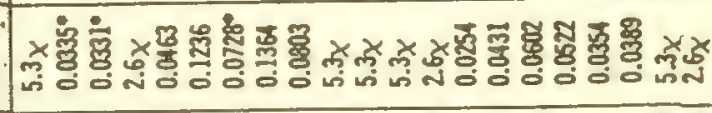 \\
\hline \multicolumn{2}{|c|}{ Density } & 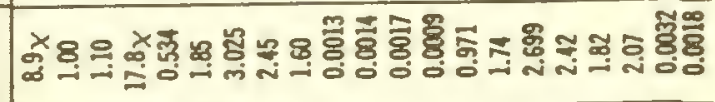 \\
\hline \multicolumn{2}{|c|}{$\begin{array}{l}\text { Atrovile of } \\
\text { mole. wgt. }\end{array}$} & 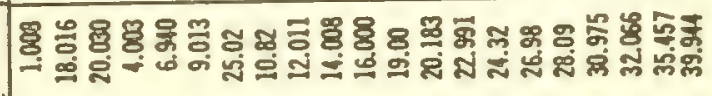 \\
\hline \multirow{2}{*}{\multicolumn{2}{|c|}{$\begin{array}{l}\text { Elemont of } \\
\text { compousd } \\
\text { Arciilc ino. }\end{array}$}} & エ \\
\hline & & 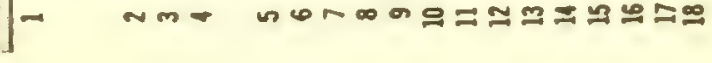 \\
\hline
\end{tabular}




\section{Thermal-Neutron Data For the Elements}

Atomic no.

\begin{tabular}{|c|c|c|c|c|}
\hline $\begin{array}{l}\text { Diffusion } \\
\text { cosff. } \\
\text { (cm) }\end{array}$ & 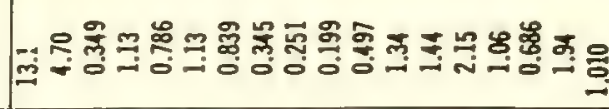 & 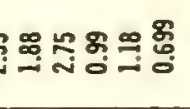 & 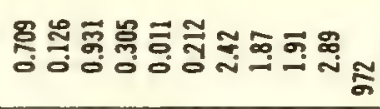 & 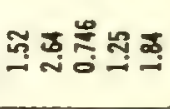 \\
\hline $\begin{array}{c}\text { Diffusion } \\
\text { length } \\
(\mathrm{cm})\end{array}$ & 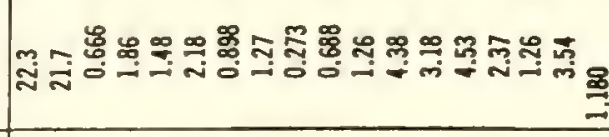 & 要点 & 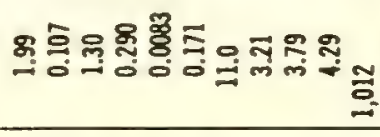 & 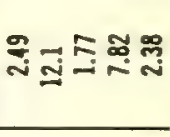 \\
\hline $\begin{array}{l}\text { Resononce } \\
\text { integral } \\
\text { (borns) }\end{array}$ & 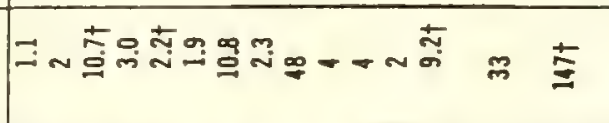 & 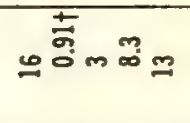 & 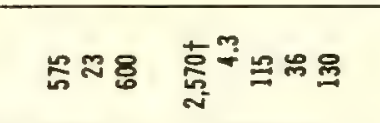 & $\stackrel{\sim}{\sim}=$ \\
\hline
\end{tabular}

\begin{tabular}{|c|c|c|}
\hline \multirow{3}{*}{ 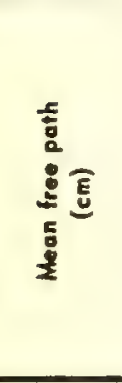 } & & 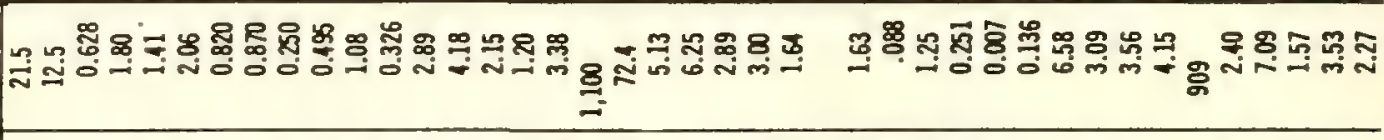 \\
\hline & 2 & 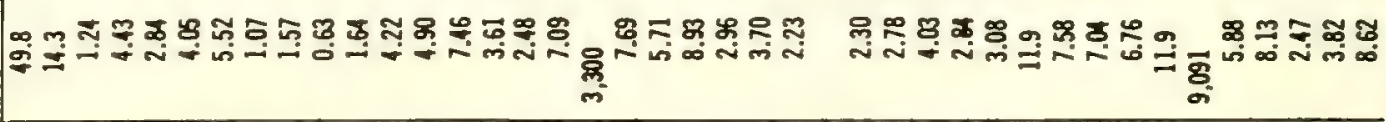 \\
\hline & $\sum^{\infty}$ & 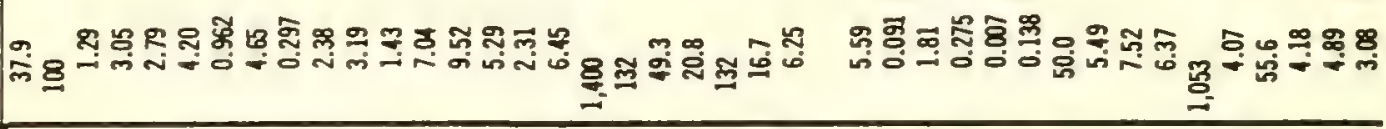 \\
\hline \multirow{3}{*}{ 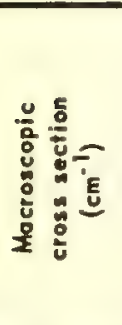 } & $\tilde{W}$ & 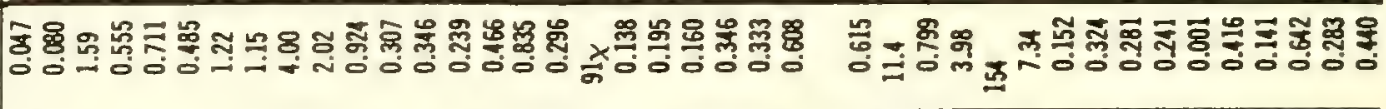 \\
\hline & $w$ & 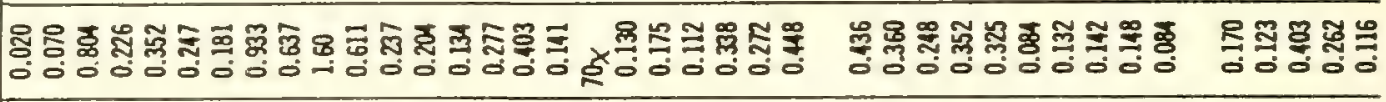 \\
\hline & & 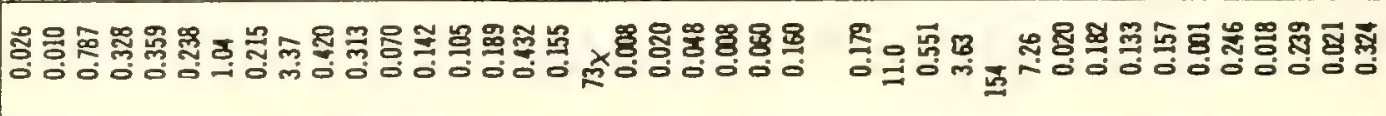 \\
\hline \multirow{3}{*}{ 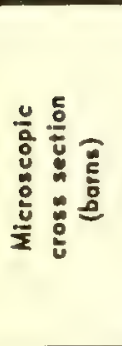 } & 5 & 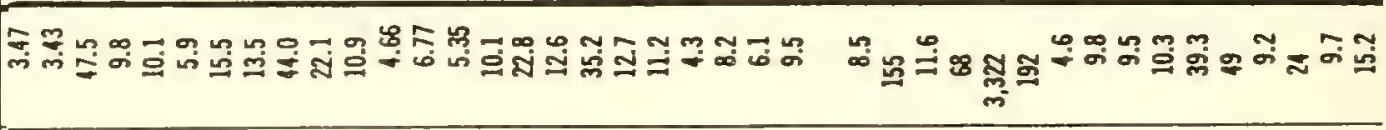 \\
\hline & $b^{6}$ & 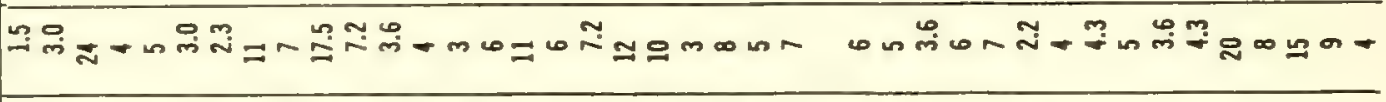 \\
\hline & $0^{\circ}$ & 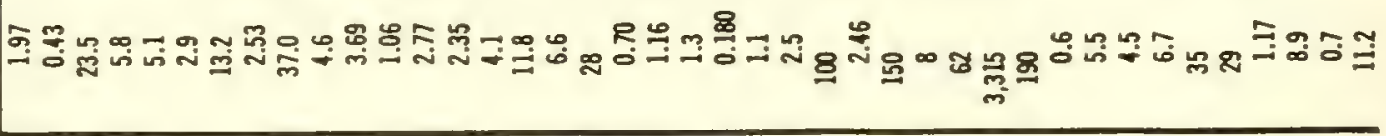 \\
\hline & un & 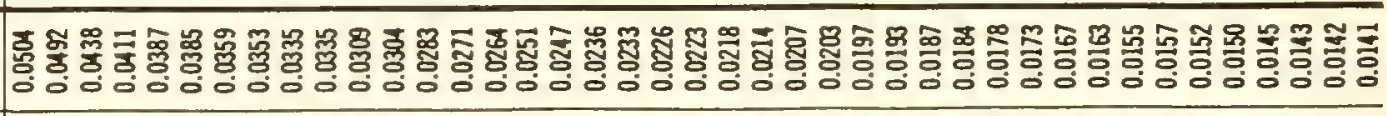 \\
\hline & $\frac{3}{1}$ & 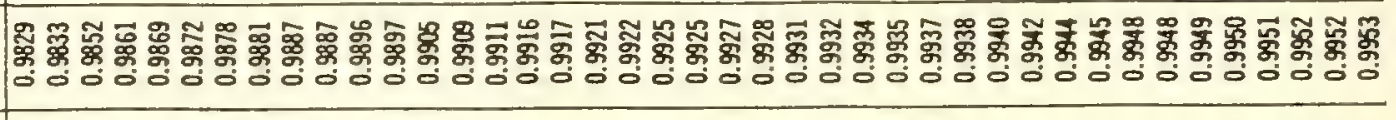 \\
\hline \multicolumn{2}{|c|}{$\begin{array}{l}\text { Nuclei } \\
\text { per unit vol. } \\
\left(\times 10^{2} 4 \mathrm{~cm}^{3}\right)\end{array}$} & 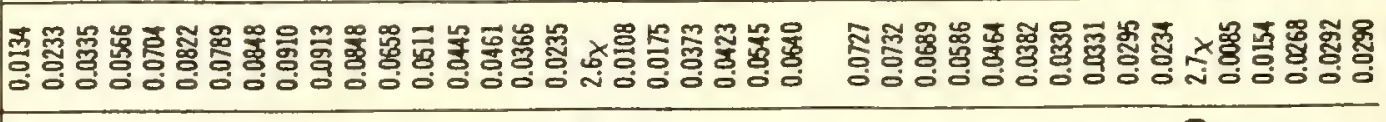 \\
\hline \multicolumn{2}{|c|}{$\begin{array}{c}\text { Density } \\
(\mathrm{gm} / \mathrm{cm})\end{array}$} & 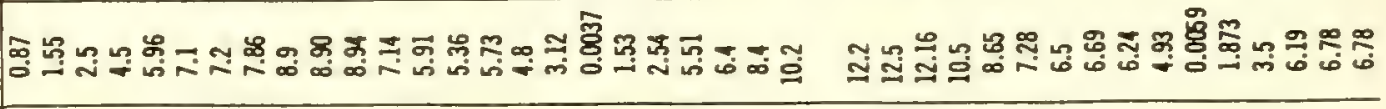 \\
\hline \multicolumn{2}{|c|}{$\begin{array}{l}\text { Atomic or } \\
\text { mole. wgt. }\end{array}$} & 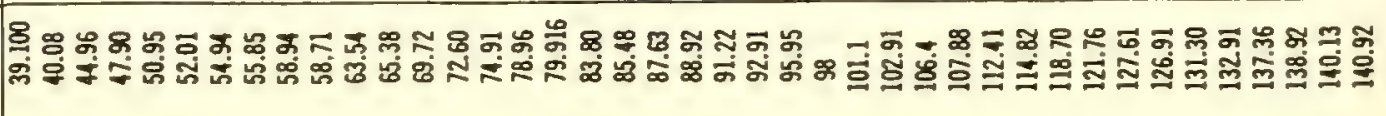 \\
\hline \multicolumn{2}{|c|}{$\begin{array}{l}\text { Element of } \\
\text { compound }\end{array}$} & × \\
\hline
\end{tabular}




\title{
Fission-Product Yields from
}

\author{
BY SEYMOUR KATCOFF \\ Chemistry Department, Brookhaven National Laboratory, Upton, New York
}

Fission of a Heavy nucleus, such as $\mathrm{U}^{236}$, by thermal neutrons results in two fission fragments that recede from each other with a total kinetic energy of about $170 \mathrm{Mev}$. In general, their masses are unequal - the most probable heavy mass is around 139 , and the most probable light mass is around 95 . However, products have been found in detectable amounts throughout the mass region 72-161.

The percent probability per fission of forming a given nuclide, or a given chain, is defined as its fission yield. Since the fission fragments are formed with an excess of neutrons, they undergo a series of $\beta^{-}$decays, in a "chain," until they attain stability (Table 2, p. 82).

Fission yields can be determined by radiochemical or mass-spectrometric means.
In the radiochemical method, the number of fissions is usually determined by direct fission counting of a small aliquot of the fissile material exposed simultaneously to the same neutron flux as the main sample. The latter is radiochemically analyzed for the radionuclides of interest. Their absolute disintegration rates can be determined by $\mathbf{4} \pi$ counting or by gas-phase counting. However, in most of the older work the beta counting was done with end-window G-M counters, and correction to disintegration rates was accurate only to $\pm 10 \%$. Frequently the number of fissions was not determined directly in each experiment; only the relative yields of nuclides were measured and $\mathrm{Ba}^{140}$ was used as the standard.

The mass-spectrometric method has been applied to measuring fission yields of stable and long-lived isotopes of $K_{r}$,
$\mathrm{Rb}, \mathrm{Sr}, \mathrm{Zr}, \mathrm{Mo}, \mathrm{Ru}, \mathrm{Xe}, \mathrm{Cs}, \mathrm{Ba}, \mathrm{Ce}, \mathrm{Nd}$ and Sm. The absolute number of atoms of each nuclide can be determined by the isotope-dilution technique (1). The number of fissions can be determined by measuring the change in the $\mathrm{B}^{10} / \mathrm{B}^{11}$ ratio in a $\mathrm{BF}_{3}$ flux monitor irradiated simultaneously in the same neutron flux as the fission sample. Conversion to fissions is effected by use of the known ratio of $\mathrm{B}^{10}-\mathrm{absorption}$ cross section to the fission cross section. Many of the yields from $U^{3 s 5}$ thermalneutron fission were measured in this way (2). In most of the other massspectrometric work to date, only the relative yields have been measured.

In compiling these tables, first priority was given to mass-spectrometric data since these are more accurate $( \pm 3 \%$ ) than most of the radiochemical data $( \pm 10 \%)$. Where

\section{Fission Yields from . . .}

The fission-yield data of Table 2 (p. 82) are plotted versus mass number in the $U^{236}$ curve (circles). The sum of the yields under each peak is very close to $100 \%$, as expected for binary fission. This requirement of summation to $100 \%$ each, for the light and heavy groups of fission products, was used as an aid in normalizing some of the data in Table 1 (p. 80). Such a procedure was necessary because few of the fission yields have been measured accurately on an absolute basis.

Small "fine-structure" peaks are clearly shown in some of these yield-vs-mass curves. For $\mathrm{U}^{235}$, these peaks are at masses 100 and 134 ; they result from a preference for the formation of fragments with a closed shell of 82 neutrons.

The $U^{235}$ curves illustrate the effect on mass distribution of increasing the neutron energy above thermal. The greatest change is the increase in the probability of symmetric fission. For 14-Mevneutron fission, the increase is about 100-fold; for a fission-neutron spectrum, the increase is four-fold. The other changes are: a small drop in the peak yields and a moderate increase in the most asymmetric modes of fission, i.e., a rise in the wings of the yield-vs-mass curve.

The other two sets of curves show fission-product distributions from thermal-neutron fission of $U^{233}$, and $P u^{239}$ and from fission-neutron fission of ' $T h^{232}$ and $\mathrm{U}^{238}$.

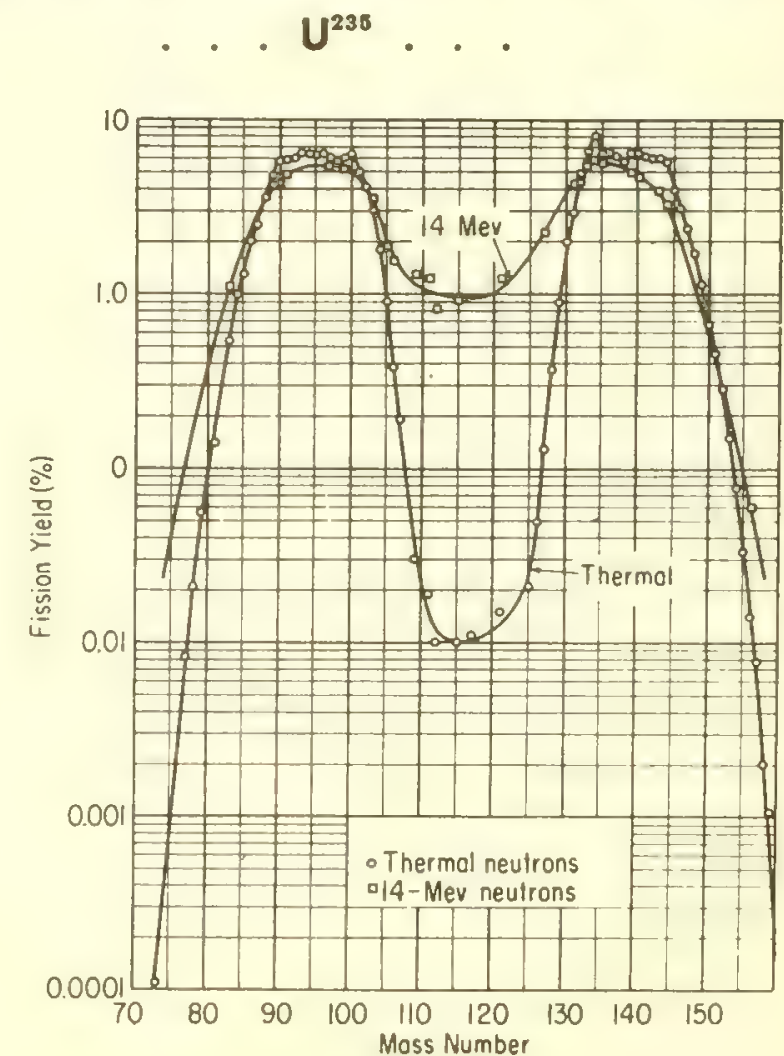




\section{$\mathrm{U}$, Th and $\mathrm{Pu}$ \\ 1-Graphs of Fission Yields \\ 2-Tables of Fission Yields \\ 3-Tables of $U^{235}$ Decay Chains and Yields}

duplicate results were about equally reliable, these were averaged, as indicated in the references to the tables. When one value was considered superior to the others, only that one was selected. No attempt was made to correct any of the values for the transfer of yield from one mass chain to the next lower one by delayed-neutron emission. However, all of the yields have been corrected for known perturbations resulting from neutron capture by the fission products.

It has been shown $(3,4)$ in studies of nuclear-charge distribution in fission that in most cases the last two or three members of a fission-product chain are formed almost exclusively from decay of precursors. In general, only small fractions of the yields of the last two members result from direct formation as primary fission fragments. There- fore a fission yield measured for any of the later members of a chain usually represents the total yield of that chain. In a few cases this rule is violated. For example, the total $\mathrm{U}^{235}$ thermal-neutron-fission yield of $86-\sec \mathrm{I}^{136}$ is $3.1 \%$ (Table 2), but the yield for the entire mass -136 chain is $6.46 \%$. The difference $(6.46 \%-3.1 \%)$ is accounted for by some direct formation in fission of the next chain member, stable $\mathrm{Xe}^{136}$.

Occasionally the fission yield of a product is lower than that of the corresponding total chrin yield because of chain branching to isomers. For example, the total yield of $\mathbf{R b}^{\mathrm{s}}$ and of total mass 85 is $1.30 \%$ (Table 2), but the yield of $10.3-\mathrm{yr} \mathrm{Kr}^{85}$ is only $0.293 \%$. The difference $(1.30-0.293 \%)$ represents the yield of the isomeric 4.4-hr $\mathrm{K}^{\mathrm{Bsm}}$. In some cases, differences between values for different fission prod- ucts within a chain are due to experimental error.

The thermal-neutron-fission yields of nearly all shielded nuclides are extremely low because these nuclides cannot result from decay of neutron-rich precursors $(3,4)$. The yields $(5)$ for 36-hr $\mathrm{Br}^{82}, 19-\mathrm{day} \mathrm{Rb}^{86}, 23-\mathrm{hr} \mathrm{Nb}^{86}$, $25-\min I^{128}$ and $12.6-h r I^{130}$ from $U^{236}$ fission are all about $3 \times 10^{-5}$ to $5 \times$ $10^{-4 \%}$. The yields (5) of $13-$ day $\mathrm{Cs}^{836}$ are somewhat larger: $0.006 \%$ from $U^{225}$, $0.05 \%$ from $\mathrm{Pu}^{239}$ and $0.12 \%$ from U233. All attempts to detect radioactive products that might result from ternary fission, such as 53-day $\mathrm{Be}^{7}$, $3 \times 10^{6}$-yr $\mathrm{Be}^{10}, 87$-day S ${ }^{35}, 4.8$-day $\mathrm{Ca}^{47}$ and 46-day $\mathrm{Fe}^{59}$, have failed (6). The upper limits range from $1 \times 10^{-1} \%$ to $4 \times 10^{-4} \%$. The only well established ternary fission product is $\mathrm{He}^{4}$, which has been observed as an $\alpha$-parti-
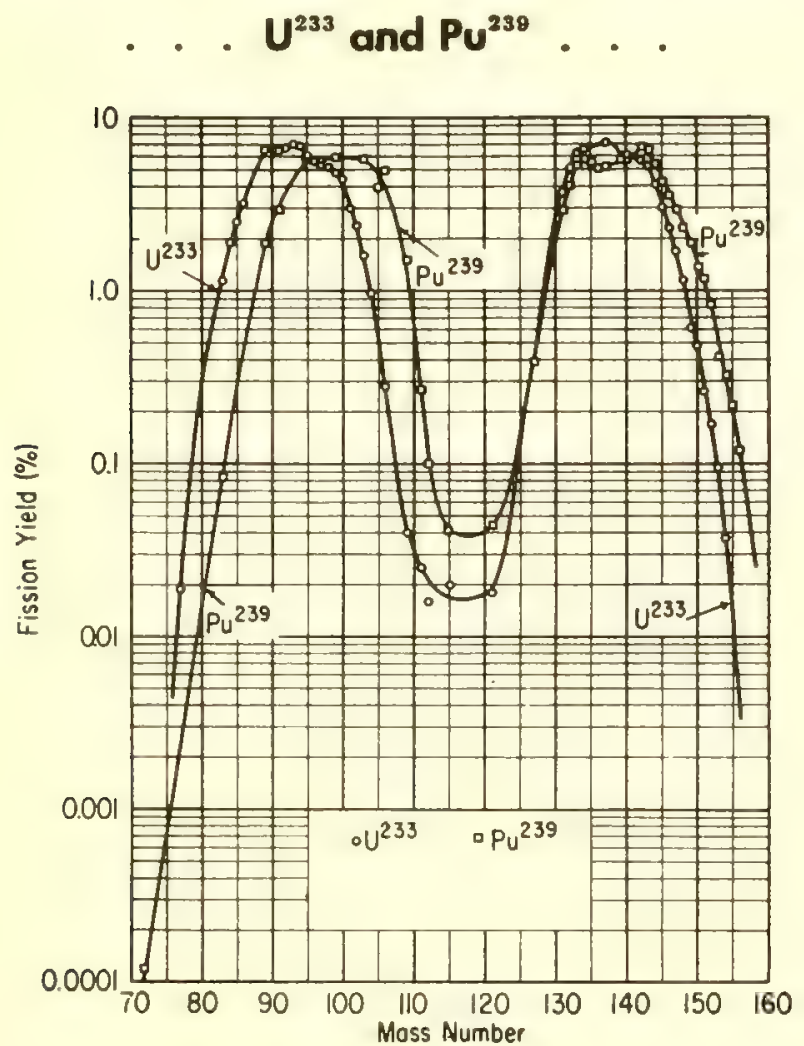
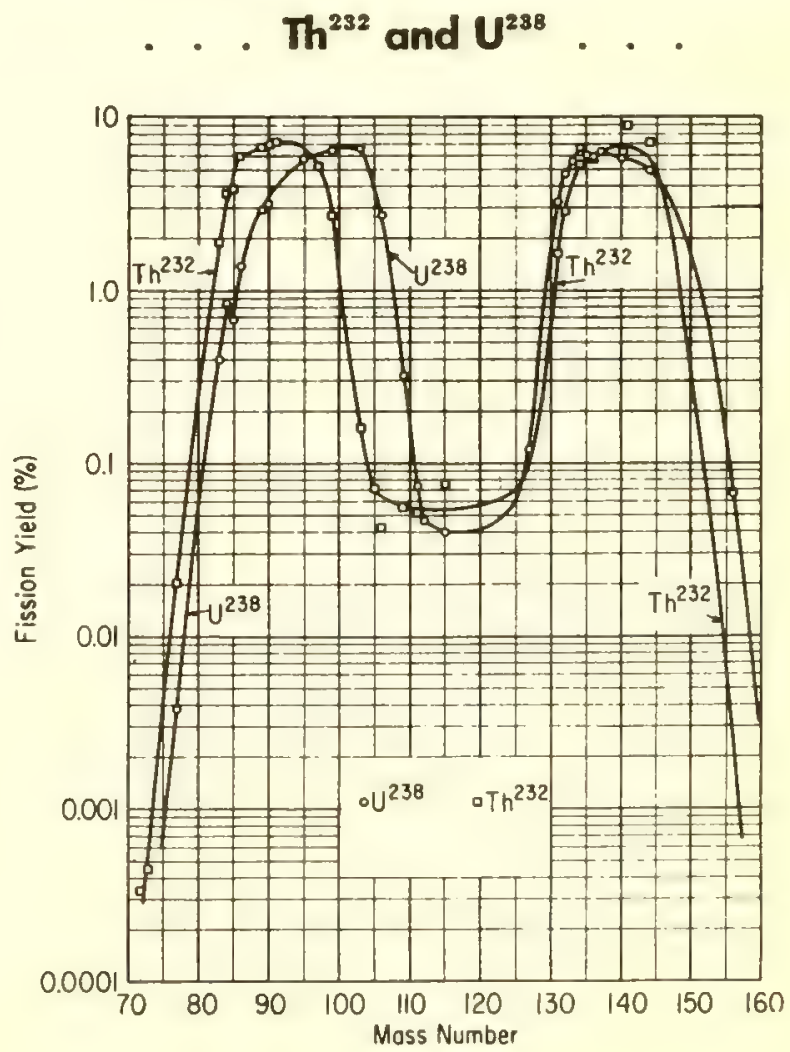
DATA SHEET continued

cle in $\sim 0.3 \%$ of thermal-neutron fissions of $\mathrm{U}^{233}, \mathrm{U}^{235}$ and $\mathrm{Pu}^{239}$.

Prior to 1953 most yields from the thermal-neutron fission of $\mathrm{U}^{235}$ were measured relative to $\mathrm{Ba}^{110}$, whose yield had been determined absolutely as $6.17 \%$ (7). In Table 2 (p. 82) these yields have been renormalized to $6.44 \%$ for $\mathrm{Ba}^{140}$. This seems to be a more reliable value obtained from massspectrometric data, which is also supported by recent radiochemical measurements. Those yields that were messured relative to $\mathrm{Mo}^{99}$ were normalized to a $6.06 \%$ value for the latter. (See references 16, 23 and 51 of Table 2.) A few of the more recent radiochemical yield determinations (e.g., $\mathrm{Sr}^{89}, \mathrm{Mo}^{99}$, $\mathrm{Xe}^{133}$ and $\mathrm{Ba}^{140}$ ) are comparable to the mass-spectrometric measurements.

No half-lives shorter than $1 \mathrm{sec}$ are listed in Table 2. Such very short periods would be absent even were it not for the experimental difficulty of identifying them. As Brolley et al. (8) have shown from studies of gross $\beta$-ray and $\gamma$-ray fission-product activities, no periods below a few tenths of a second are formed in appreciable yield.

The values for the half-lives and the branching ratios that are given were selected from "Table of Isotopes" by Hollander, Perlman and Seaborg [Ret. Mod. Phys. 25, 469 (1953)]; "Nuclear Science Abstracts" (1953-1957); and a survey of the most recent literature.

Special thanks are due the following for oupplying unpublished data and for making helpful suggestions: $J . D, K n i g h t, A, C$. Pappas, R. A. Sharp, E. P. Steinberg, H. G. Thode, R. H. Tomlinson, A.C.Wahl and W. Seelmann-Eogebert. This work was performed under the auspices of the U.S. Atomic Energy Commission. The tables are to appear in the "Handbook of Nuclear Enoineering," copyright 1958 by Addison-Wesley Publishing Co., Inc.

\section{BIBLIOGRAPHY}

1. M. G. Inghram in "Annual Review of Nuclear Seience," vol. 4, p. 81 (Annual Reviewa, Inc. Stanford, 1954)

2. J. A. Petruska, H. G. Thode, R. H. Tomlinson, Can. J. Phys, 33, 693 (1955)

3. L. E. Glendenin, C. D. Coryell R. R. Edwards in "Radiochemical Studies: The Fission Products," C. D. Coryell, N. Sugarman, eds. National Nuclear Energy Series IV-9, p. 489 (McGraw-Hill Book Co., New York, 1951)

4. A. C. Pвppas in "Proceedings of the International Conference on the Pesceful Uses of Atomic Energy," vel. 7, p. 19 (United Nations, New York, 1956)

6. See refo. 19 and $1 \theta$ of Table 8 ; aloo, T. J. Kennett, H. G. Thode, Phys, Rev. 103, 323 (1956)

6. K. F. Flynn, L. E. Glendenin, E. P. Steinberg. Phys. Rev. 101, 14 g2 (1956)

7. E. P. Steinberg. M. S. Freeman, ref. 1 of Table 2. D. 1378

8. J. E. Brolley, Jro, D. H. Cooper, W. S. Hall, M. S. Livingston, L. K. Scblacks, Phys. Rev. 83, 90 (1951)
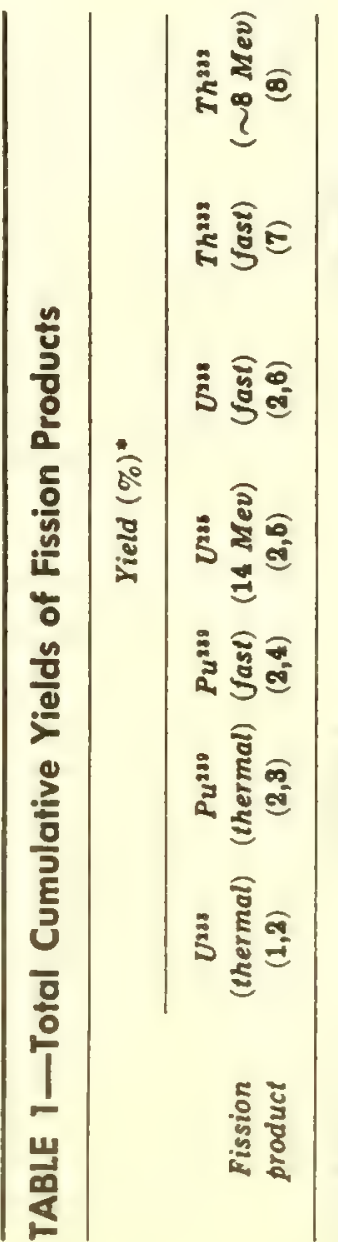

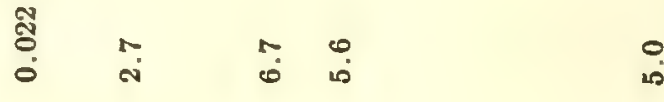

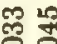

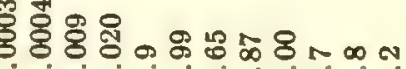

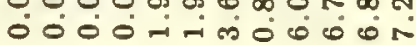

บับ

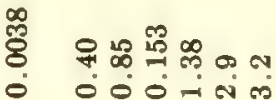

is

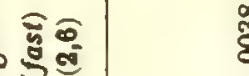

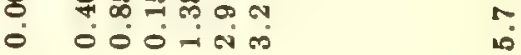

ลิธ

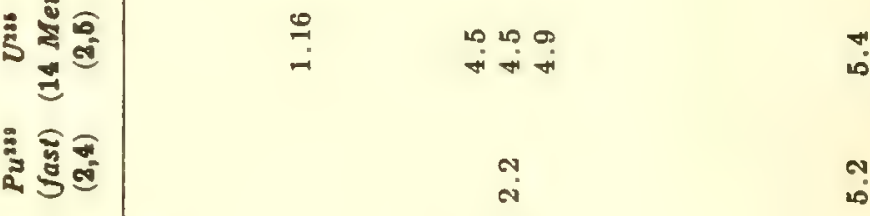

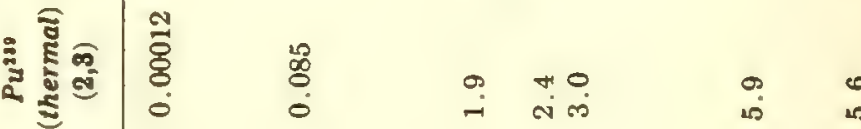

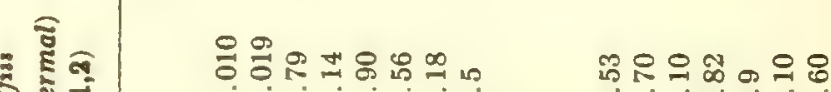

ड्ड

ฝั

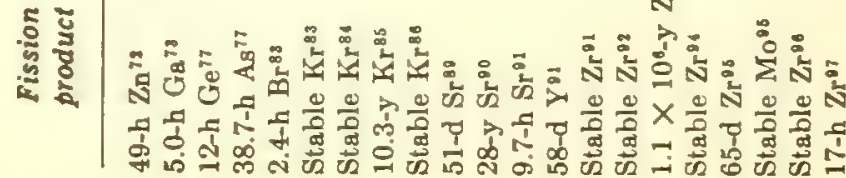




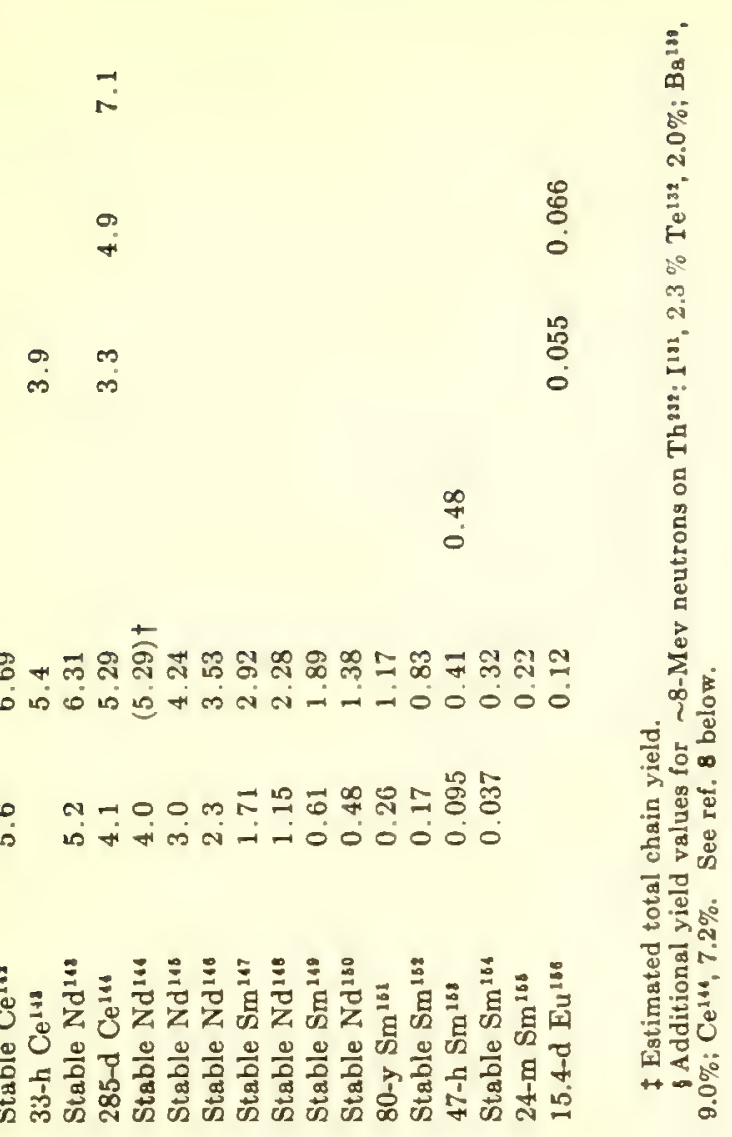

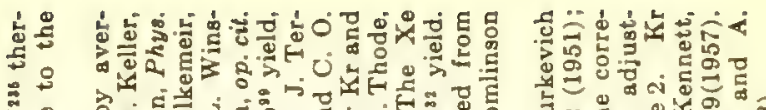

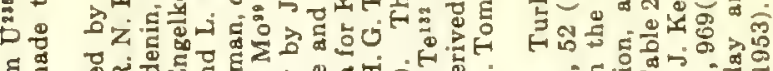
घ 음

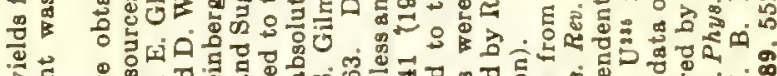

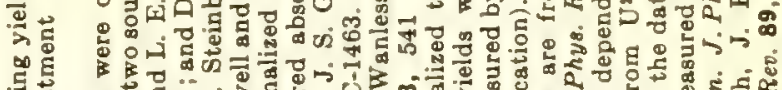

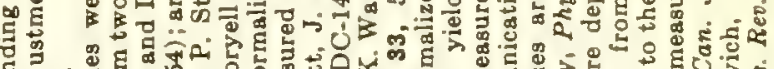

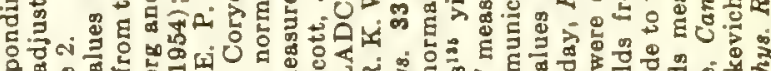

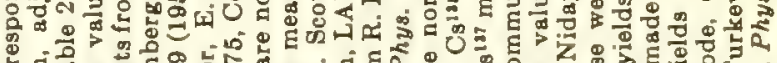

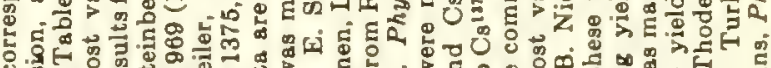

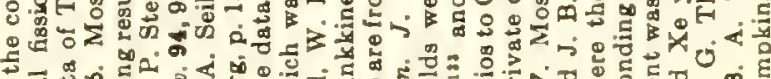

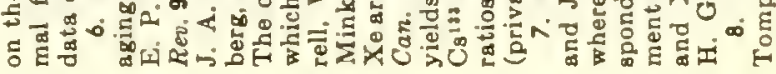

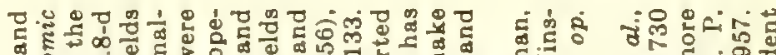

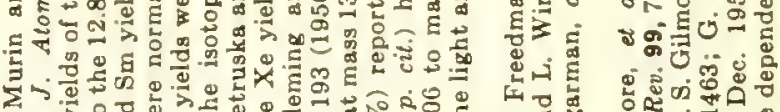
之.

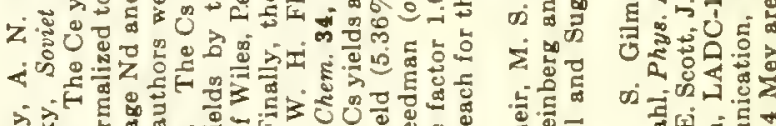

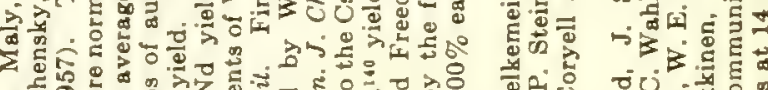

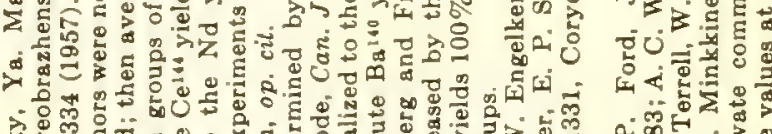

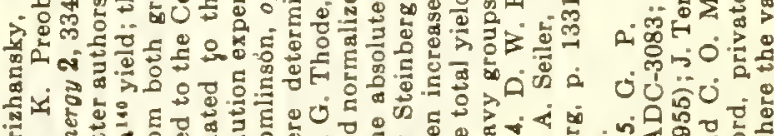

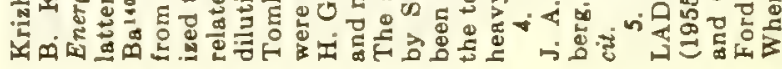

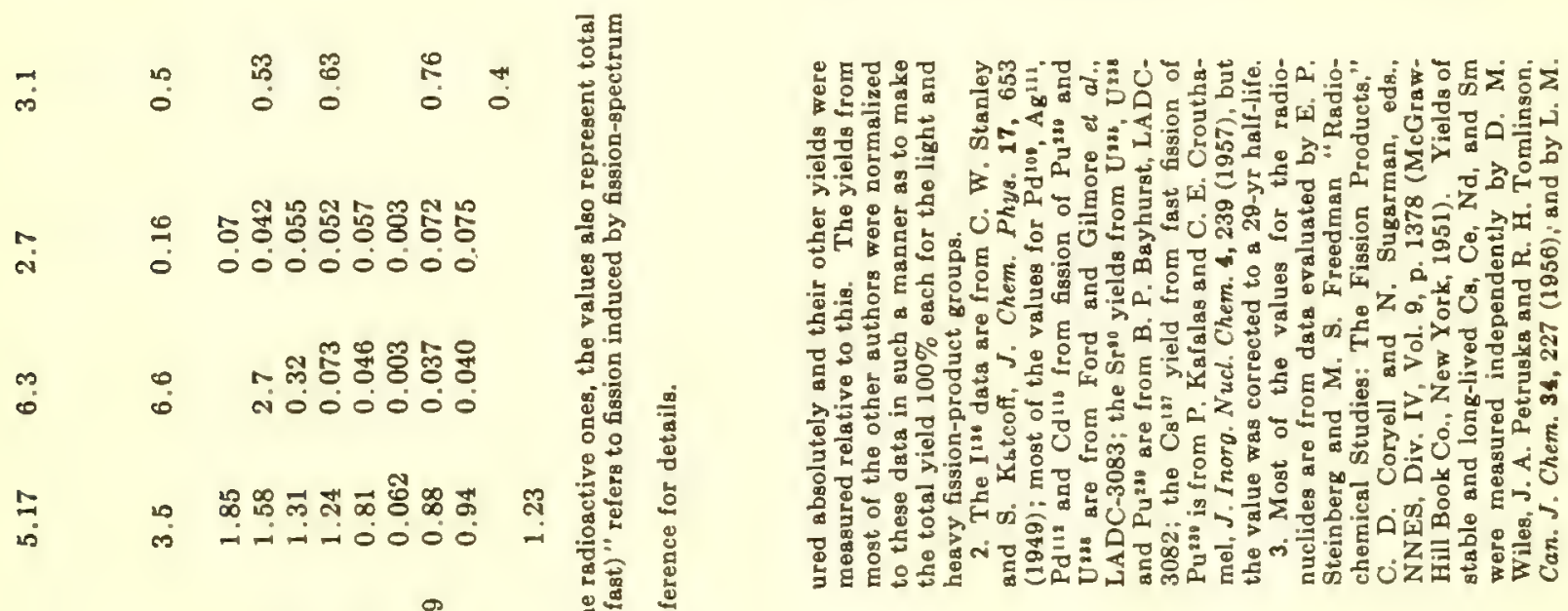

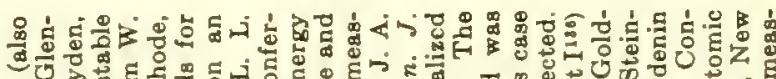

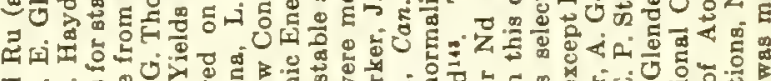

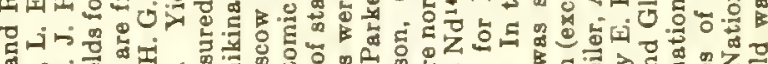

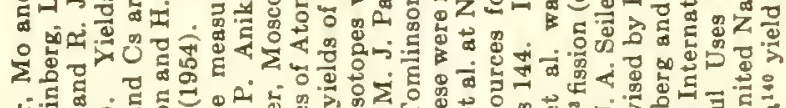

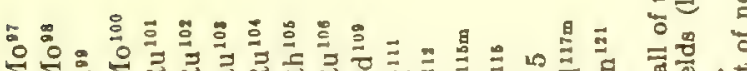

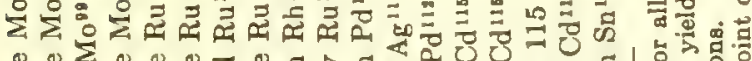

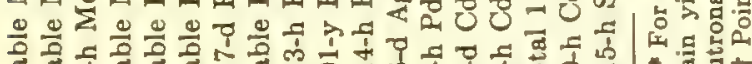

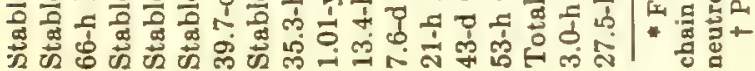

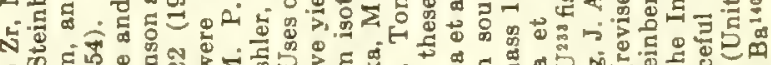

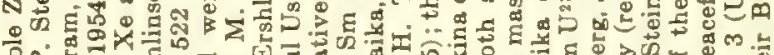

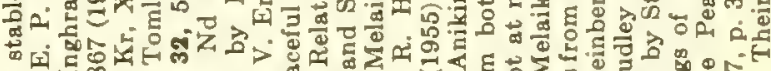
$\triangle$ 玒

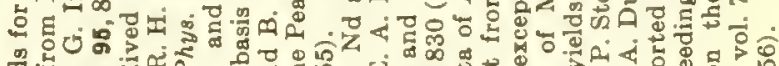

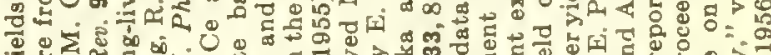

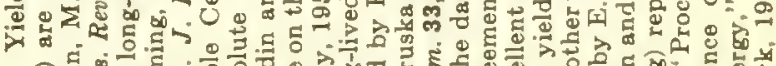
- 


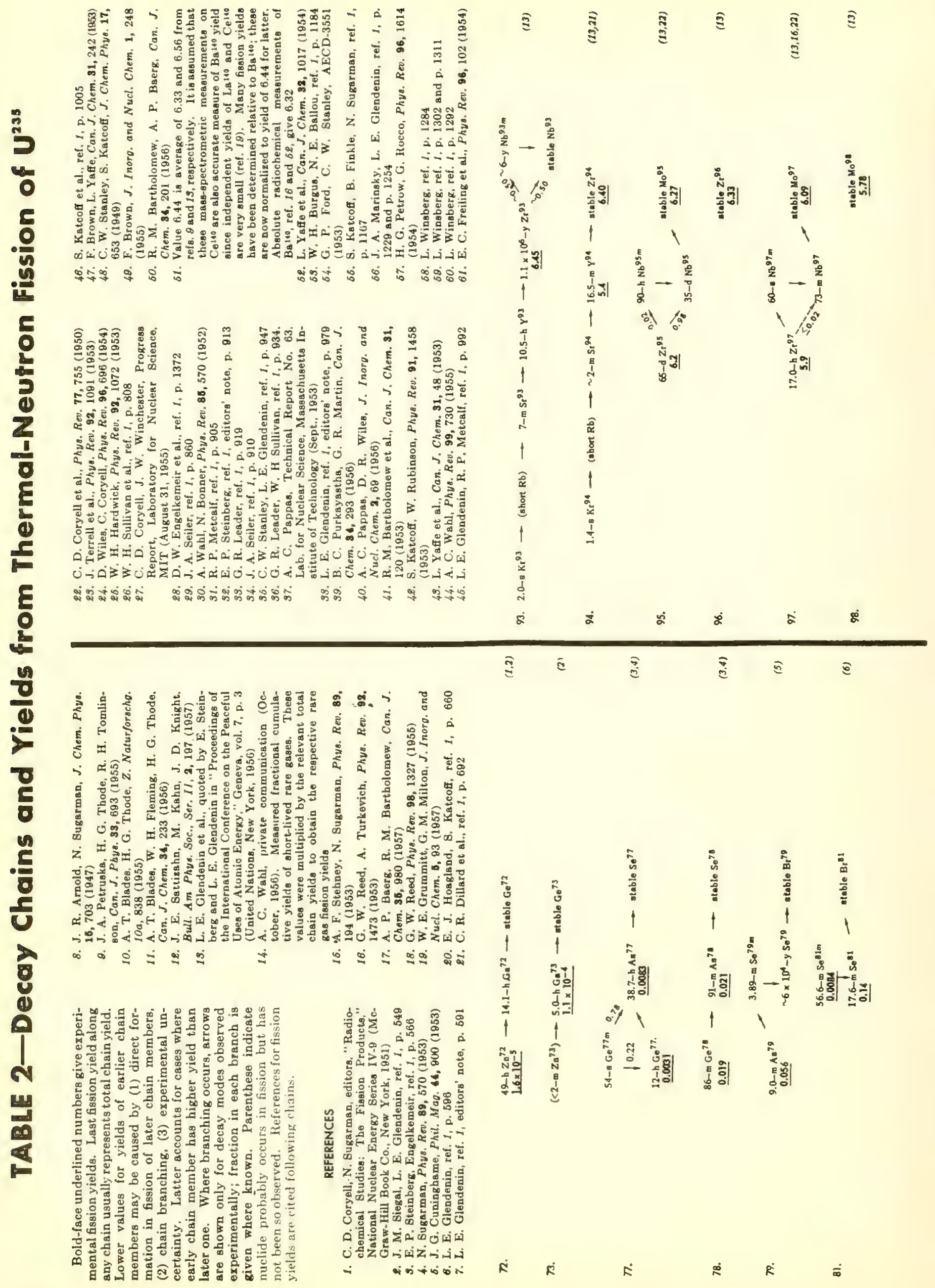




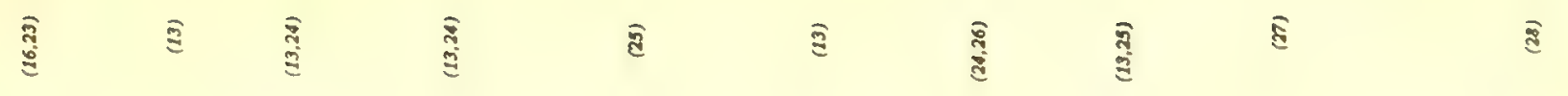
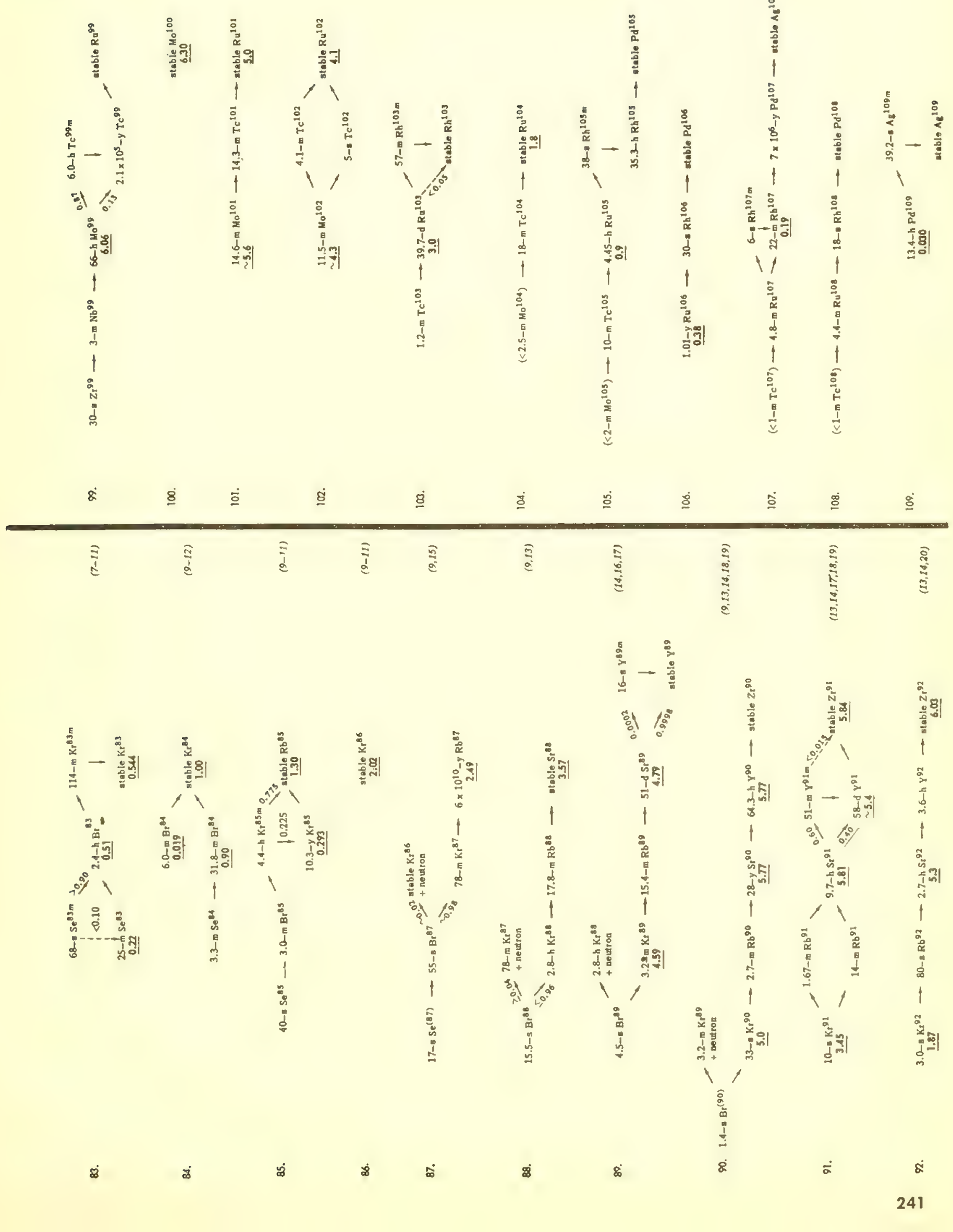


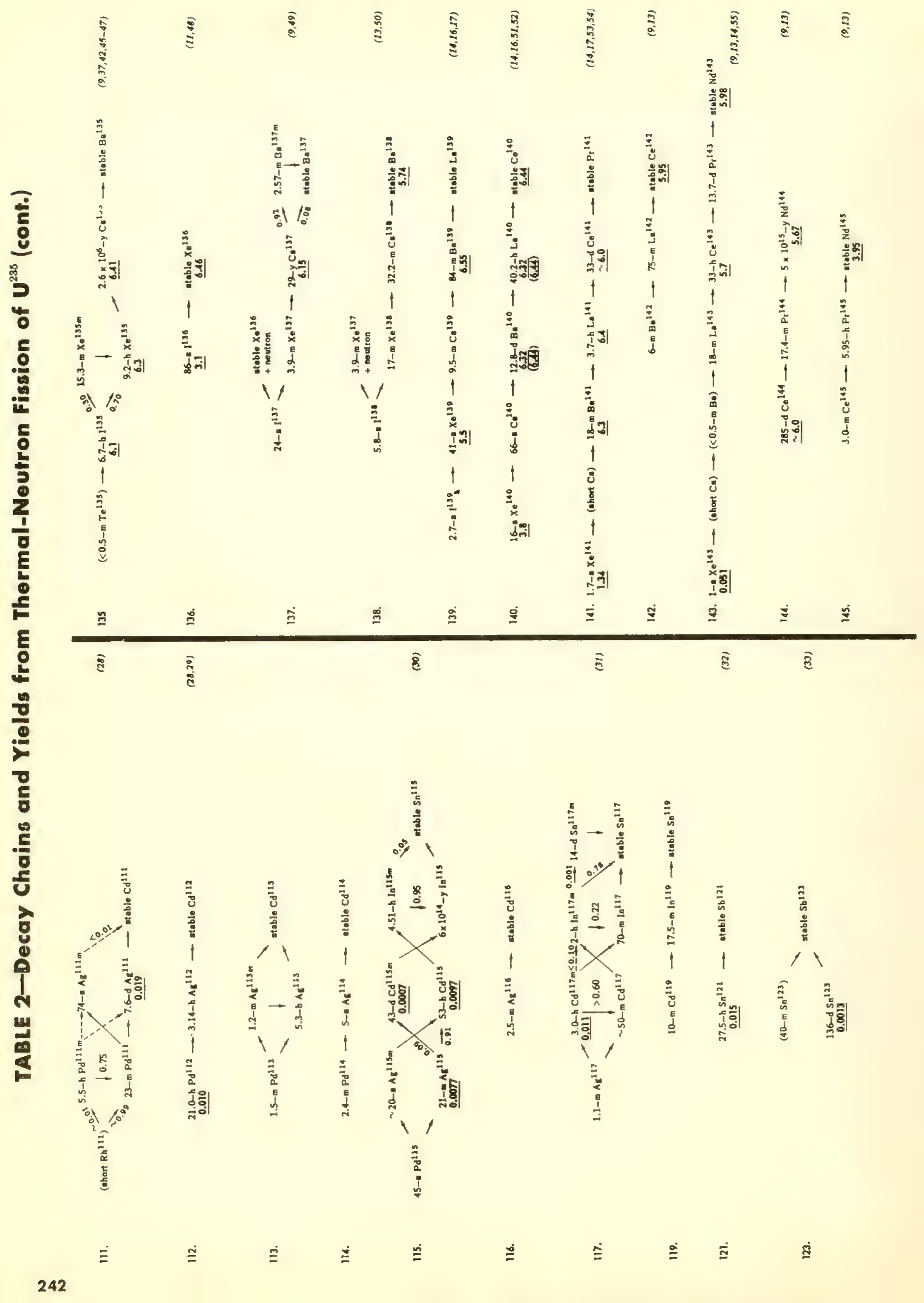




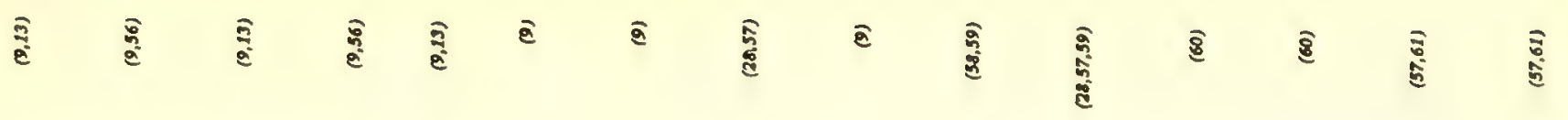

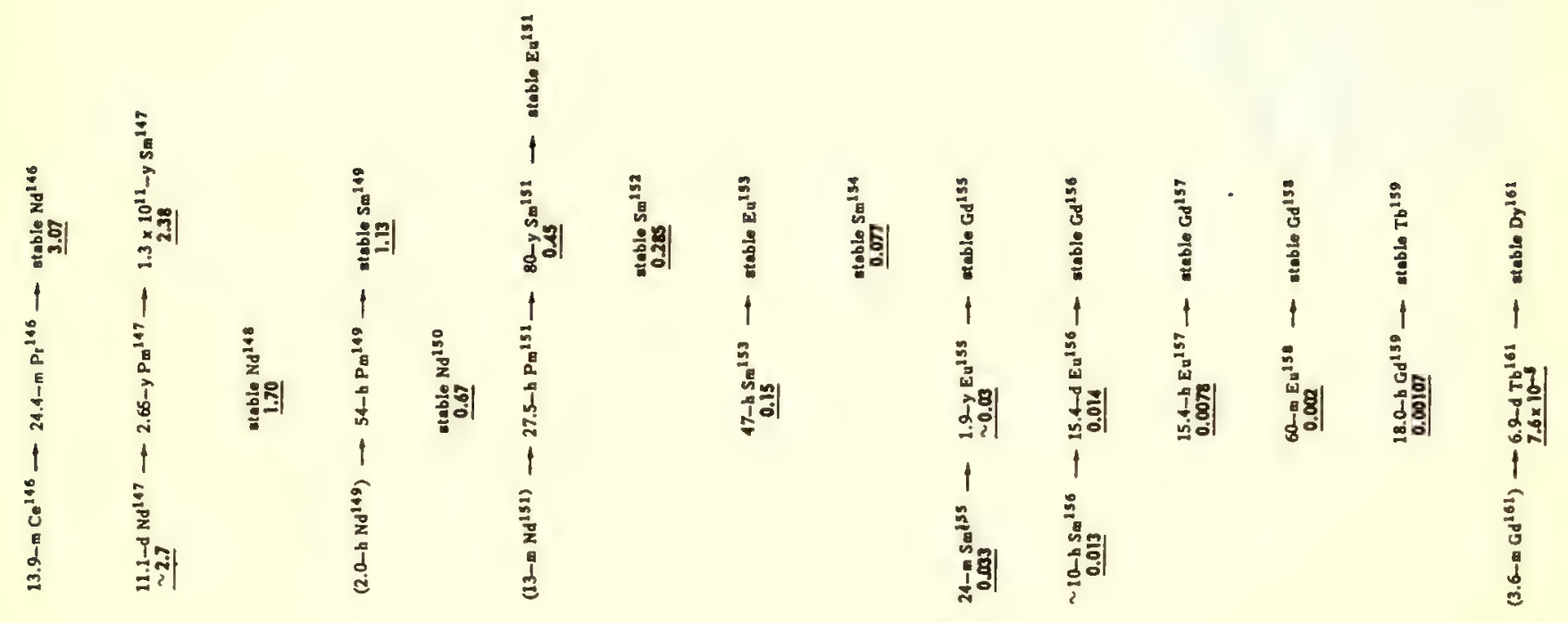

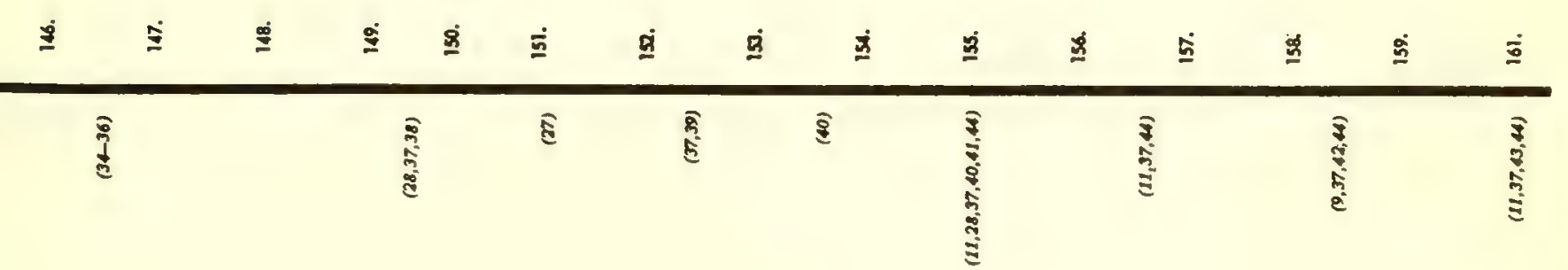

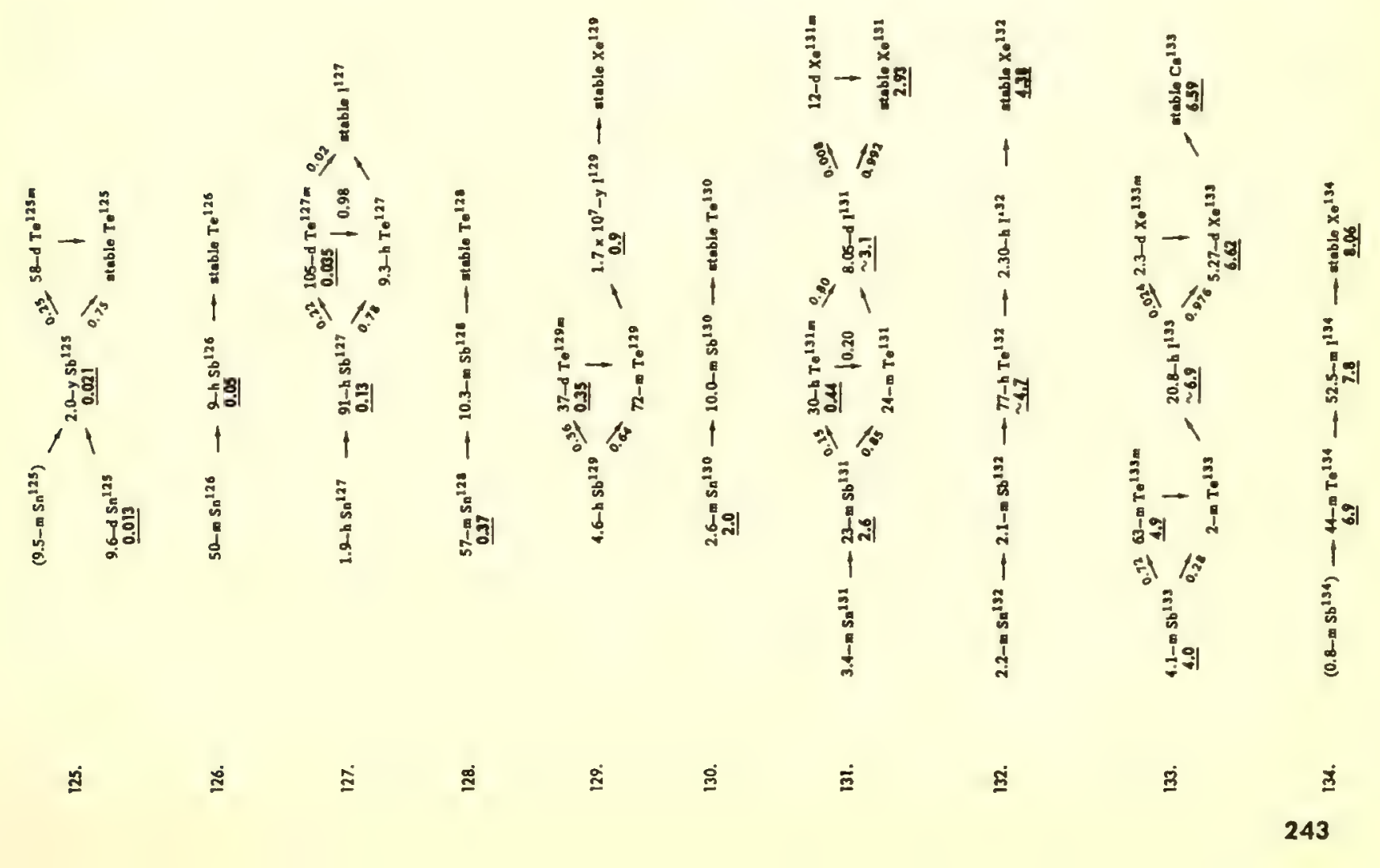




\section{Attenuation of $\gamma$-Rays from an Infinite Plane}

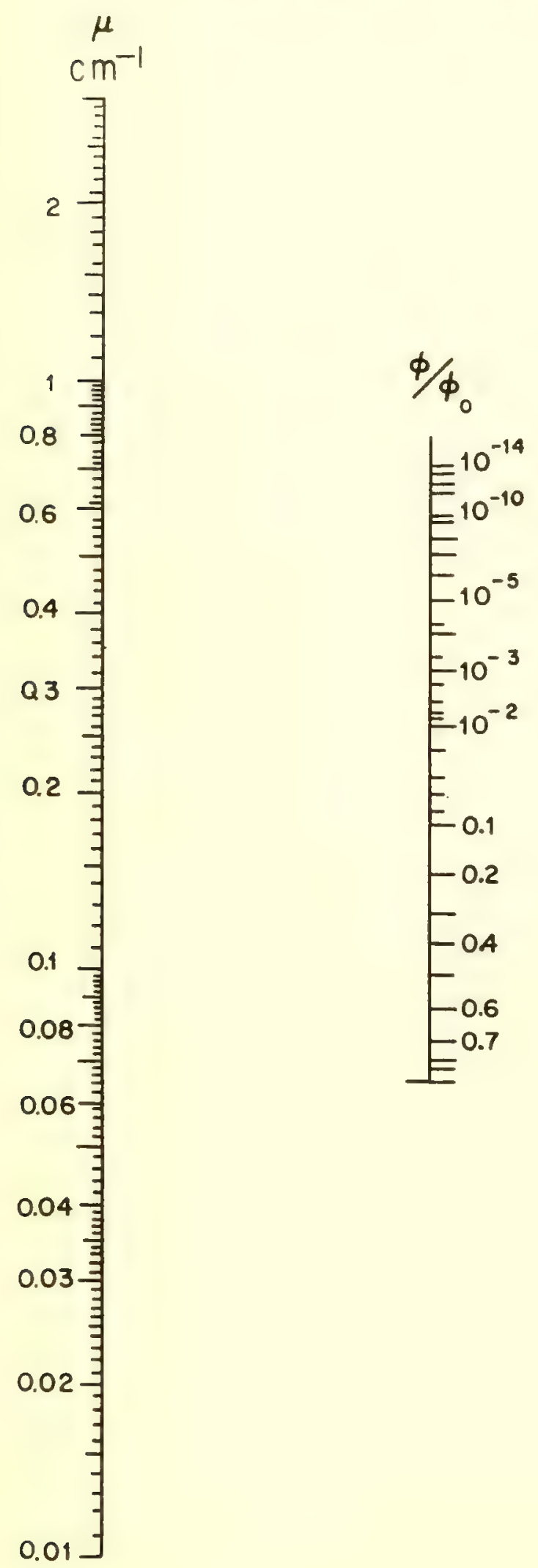

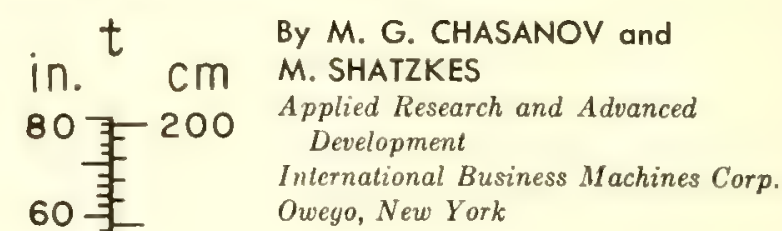

For aN INFINITE PLANE source of gamma rays the attenuation factor $\phi / \phi_{0}$ for materials other than lead is given by

$$
\frac{1}{2}\left[e^{-b}+E_{1}(b)\right]
$$

where $E_{1}(b)$ is a form of the exponential integral (1). In the case of lead, the factor is

$$
\frac{\mathrm{I}}{2}\left[e^{-b}+\frac{E_{1}(b)}{2}\right]
$$

This nomogram solves Eq. 1 for elements other than lead and for gammaray energies to $3 \mathrm{Mev}$. For lead, one can readily determine the attenuation from Eq. 2 .

To calculate attenuation of gamma rays from an infinite plane source, it is necessary to have values for the linear absorption coefficient $\mu$. The linear absorption coefficients can be obtained from curves (2) or tables ( 3 ) of mass absorption coefficients by multiplying by the density of the shield material.

For a shield thickness $t, a$ quantity $b$ is defined as $b=\mu t$. For most elements other than lead, the value $1+b$ for gamma energies up to $3 \mathrm{Mev}$ (2) can be used as an approximation for the buildup factor $B$.

\section{Example}

Problem: How thick must an iron shield be to attenuate the gamma rays from an infinite plane source by a factor of one-tenth?

Procedure: Employing the linear absorption coefficient for iron given by Glasstone (3), $\mu=0.27 \mathrm{~cm}^{-1}$, draw a line from $\mu=0.27$ through $\phi / \phi_{0}=$ -0.1 and read on the $t$ scale the value $6.9 \mathrm{~cm}$.

\section{BIBLIOGRAPHY}

1. T Rockwell III ed "Reactor Shielding Deign Manual," pp. 374-9 (McGra 4-Hill Book Co., Inc., New York, 1956)

2. D. G. Chappell, Nucleonica 14, No. 1, 40 , (1956)

3. S. Glasstone, "Principlea of Nuclear Reactor Engineering," p. 606 (D. Van Nostrand Co. Inc., New York, 1955) 


\section{Gamma-Ray Attenuation with Buildup in Water}

By DAVID G. CHAPPELL, Knolls Alomic Power Laboratory, General Electric Co., Schenectady, New York

This nomogram gives the attenuation factor for a water shielding slab used with a point source of gamma radiation. Two predecessors that do the task for iron and lead have already been presented in Nucleonics Data Sheet No. 18 ("Gamma Attenuation with Buildup in Lead and Iron"- $-\mathrm{NU}$, Jan. '57, p. 52).

Like its predecessors this nomogram includes the effect of buildup-the scattering of degraded radiation into the beam from all points of the slab. Absorption coefficients and buildup factors are taken from the report APEX $176 . *$

Attenuation factor $\pi_{\mathrm{pt}}=B(\mu x) e^{-\mu x}$, where $B$ is the buildup factor for this geometry, $\mu$ is the linear absorption coefficient, and $x$ is the shield thickness. It does not include any allowance for source-to-detector distance. Water density is taken as being equal to $1 \mathrm{gm} / \mathrm{cm}^{3}$.

Examples. (1) By how much will 4-Mev gamma rays from a point source be attenuated in passing through a 16-ft layer of water? The upper of the two sample lines shows that the answer is $7.5 \times 10^{-7}$.

(2) We wish to attenuate $6-\mathrm{Mev}$ photons by a factor of $3.1 \times 10^{-2}$. How thick must a water slab be to do it? The lower of the two sample lines yields the answer: $5.5 \mathrm{ft}$.

$I$ uish to acknowledge the assistance of Miss Janice L. Noble, who drew the nomogram.

* Miscellaneous data for shielding calculations. Compiled by John Moteff. APEX 176 (1954).
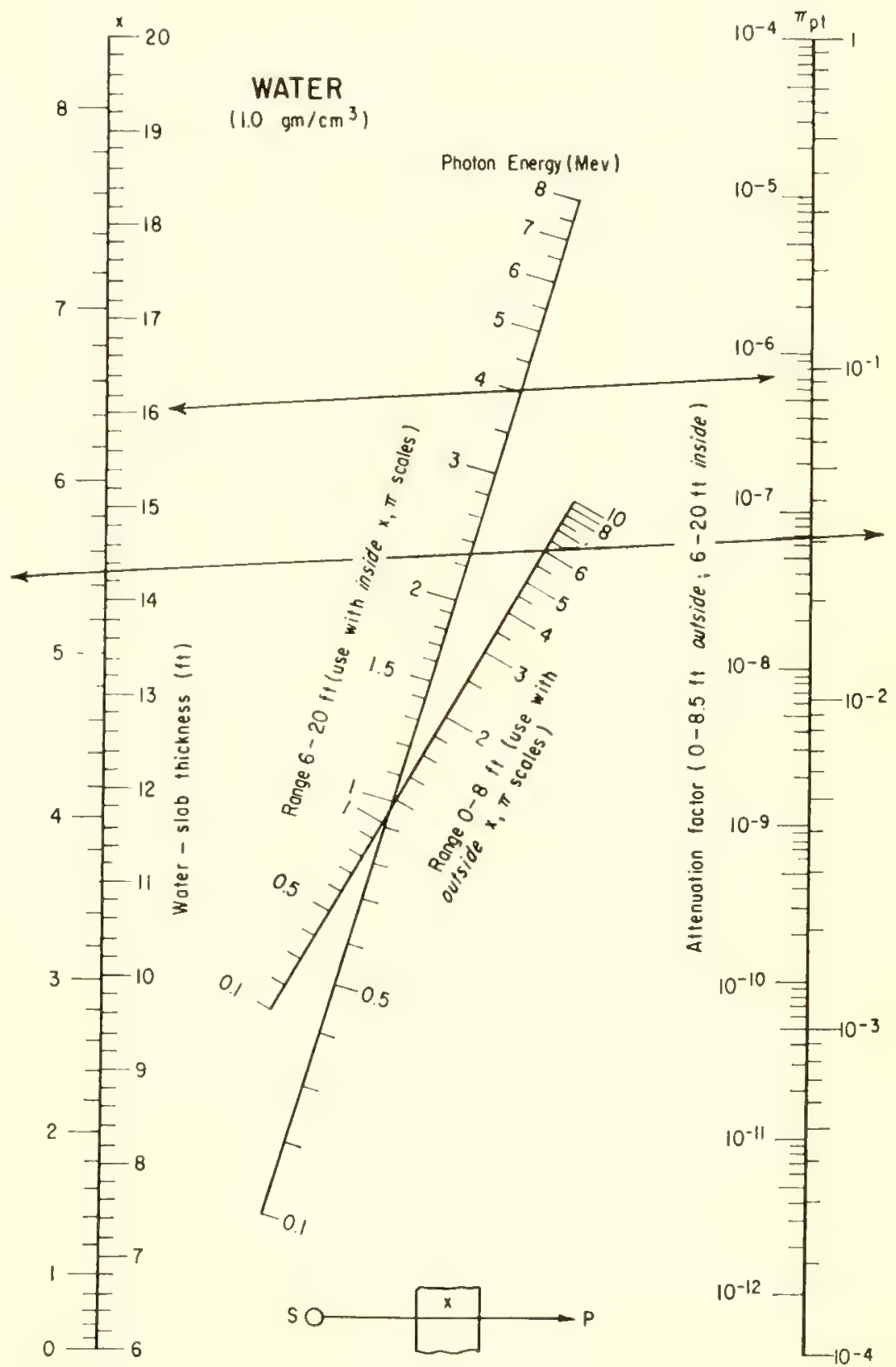


\title{
Fission-Neutron Cross Sections for Threshold Reactions
}

\author{
BY ROBERT S. ROCHLIN \\ General Engineering Laboratory, General Electric Company, Schenectady, New York
}

ThIS DATA SHEET presents published and unpublished data on fission-spectrumneutron activation by the $(n, p),(n, \alpha)$ and $(n, 2 n)$ reactions. We shall refer to these three reactions as threshold reactions, although many of them are exoergic. Although many $(n, \gamma)$ cross sections have been measured (1) for both thermal and fission-spectrum neutrons, data on reactor-neutron activation by the threshold reactions are comparatively scanty.

Cross sections for threshold reactions are needed for calculating activation levels of reactor coolants and components, especially when these reactions produce longer-lived activities then the corresponding $(n, \gamma)$ reactions. For example, in the case of aluminum the $(n, \gamma)$ product decays with a 2.3-min half-life, while the $(n, \alpha)$ product has a half-life of $15 \mathrm{hr}$.

An example of the usefulness of threshold reactions for neutron-activation analysis of materials is the detection of aluminum in silicon, using the $\mathrm{Al}^{27}(n, \alpha) \mathrm{Na}^{24}$ reaction. The $\mathrm{Al}^{27}(n, \gamma)$ $A{ }^{28}$ reaction cannot be used in this case, because $\mathrm{Al}^{28}$ is also produced from silicon by the $\mathrm{Si}^{28}(n, p) \mathrm{Al}^{28} \mathrm{re}$ action. $\mathrm{Na}^{24}$, on the other hand, cannot be produced from silicon by reactor neutrons.

Threshold reactions are also useful for measuring fast-neutron fluxes in nuclear reactors. By using several elements that have different activation thresholds, information can be obtained about the neutron energy spectrum.

Neutrons produced by fission of uranium-235 have a distribution of energies (2) given by

$N(E) d E=(2 / \pi e)^{3 / 2} e^{-E} \sinh (2 E)^{1 / 2} d E$

The cross section for a neutron-induced reaction depends on the energy of the incident neutrons. For fission-spec- trum neutrons, an "effective cross section" is defined as ( $(3)$

$$
\sigma_{e}=P / N F
$$

where $P$ is the number of product nuclei formed per unit time, $N$ is the number of target nuclei, and $F$ is the total flux density of fission neutrons of all energies, whether or not they contribute to the reaction.

Because of the steep slope of the fission spectrum above $2 \mathrm{Mev}$, the effect of moderated neutrons on the spectrum can usually be neglected in the energy range above $2 \mathrm{Mev}$.

Neutron energy thresholds for endoergic reactions are given by $E_{T}=$ $-Q(A+1) / A$, where $A$ is the atomic weight of the target nuclide, and the $Q$ of the reaction can be calculated from isotopic-mass tables (4). Threshold energies are listed in the table. Negative "thresholds" indicate exoergic reactions, for which even thermal neutrons may have a cross section greater than zero. However, even for exoergic reactions, the potential barrier against particle emission (3) usually causes the cross sections to be small for thermal neutrons.

Inthoff ( 5 ) has prepared a nomogram for fission-neutron $(n, p)$ and $(n, \alpha)$ cross sections, based upon the theory described by Hughes ( 3 ). This theory gives rough agreement with many experimental values, but in some cases (e.g., $\mathrm{Ti}, \mathrm{Cs}, \mathrm{Tl}$ ) there are disagreements by factors of 100 or more (6-8).

\section{Experimental Techniques}

In the present work, samples of $\mathrm{Al}$, $\mathrm{Cd}, \mathrm{Co}, \mathrm{Cu}, \mathrm{Fe}, \mathrm{Ge}, \mathrm{Ni}, \mathrm{Sc}$ and $\mathrm{Zn}$ were irradiated in the Brookhaven National Laboratory graphite reactor. The fission-neutron-flux calibration was based upon an assumed effective cross section of $0.60 \mathrm{mb}$ for $\mathrm{Al}^{27}(n, \alpha) \mathrm{Na}^{24}$.
To reduce interference from impurities, Matthey "Specpure" oxide samples were used. To check on interference from activation of impurities by thermal neutrons, samples of each element were irradiated with and without a tight wrapping of cadmium. Since the thermal-neutron flux inside the cadmium wrapping was negligible, the ratio of measured activities of samples with and without cadmium would depart from unity if thermalneutron activation were appreciable. In the present work, calculation of this ratio revealed no activities due to thermal-neutron activation of impurities in any of the samples.

Chemical separations were made to facilitate measurement of weak activities, which would otherwise have been masked by stronger ones.

To obtain unique identification of each reaction product, both gammsray energies and half-lives were measured. Values of photons/disintegration for each observed gamma peak were taken from references 9,10 or 11 . The relative counting efficiency of the $\mathrm{NaI}$ scintillation spectrometer as 8 function of gamma-ray energy was obtained by comparing observed peaks of $\mathrm{Mn}^{66}$ and $\mathrm{Br}^{82}$ gamma rays with their known relative intensities ( 9 ).

$$
* \quad *
$$

$I$ want to thank S I. Friedman for his diligent assistance in operating the spectrometer and calculating data, Miss $B$. A. Thompson and A. Eldridge for making the chemical separations, and W.W. Schultz for continual encouragement and support.

\section{BIBLIOGRAPHY}

1. D. J. Hughes, R. B. Schwartz, "Neutron Cross Sections," BNL-325, 2nd ed. (1958)

2. Handbook 63, Fig. 16 and Table 7 (Nations) Buresu of Standards, U. S. Department of Commerce, Washington, D. C. 1957); also B. E. Watt, Phys. Rev, 87, 1037 (1952)

3. D. J. Hughes, "Pile Neutron Resenrch," Ch. 4 (Addison-Wesley Publishing Co., Cam. 


\begin{tabular}{|c|c|c|c|c|c|c|c|c|c|}
\hline Reaction & Half-life & $E_{r}(M e v)$ & $\sigma_{\cdot}(m b)$ & Rej. & Reaction & Half-life & $E_{T}(M e v)$ & $\sigma_{\theta}(m b)$ & Ref. \\
\hline \multirow[t]{2}{*}{$\mathrm{Al}^{27}(n, p) \mathrm{Mg}^{27}$} & $10.2 \mathrm{~m}$ & 1.90 & 3.43 & 12 & $\mathrm{Ni}^{68}(n, p) \mathrm{Co}^{58}$ & $72 \mathrm{~d}$ & -0.64 & 40. & 16 \\
\hline & & & 2.8 & 13 & & & & 45. & 17 \\
\hline \multirow[t]{3}{*}{$\mathrm{Al}^{27}(n, \alpha) \mathrm{Na}^{24}$} & $15 \mathrm{~h}$ & 3.27 & 0.60 & 12 & & & & 45. & 18 \\
\hline & & & 0.6 & 13 & & & & 140. & 8 \\
\hline & & & 0.44 & 7 & & & & 225. & 14 \\
\hline $\mathrm{Ba}^{132}(n, p) \mathrm{Cs}^{132}$ & $6.2 \mathrm{~d}$ & $\sim 0$ & 5.3 & 14 & $\mathrm{Ni}^{68}(n, p) \mathrm{Co}^{58 \mathrm{~m}}$ & $9.0 \mathrm{~h}$ & -0.64 & 13. & 6 \\
\hline $\mathrm{Ba}^{136}(n, p) \mathrm{Cs}^{136}$ & $13 \mathrm{~d}$ & 2.06 & 0.0015 & 14 & $\mathrm{Ni}^{68}(n, \alpha) \mathrm{Fe}^{6 b}$ & $2.9 y$ & -3.06 & 0.17 & 16 \\
\hline $\mathrm{Be}^{\mathscr{P}}(n, \alpha) \mathrm{He}^{\beta}$ & $0.82 \mathrm{~s}$ & 0.71 & 10. & 13 & $\mathrm{Ni}^{58}(n, 2 n) \mathrm{Ni}^{67}$ & $36 \mathrm{~h}$ & 12.0 & 0.0012 & 15 \\
\hline $\mathrm{B}^{11}(n, \alpha) \mathrm{Li}^{8}$ & $0.84 \mathrm{~s}$ & 7.24 & 0.085 & 13 & $\mathrm{Ni}^{60}(n, p) \mathrm{Co}^{80}$ & $5.4 y$ & 2.07 & $<4.5$ & 14 \\
\hline $\mathrm{Cd}^{110}(n, p) \mathrm{Ag}^{110}$ & $270 \mathrm{~d}$ & 2.12 & $\sim 0.1$ & 8 & & & & 5. & 6 \\
\hline $\mathrm{Cl}^{35}(n, p) \mathrm{S}^{35}$ & $87 d$ & -0.62 & 16. & 19 & & & & $<2$. & 8 \\
\hline \multirow{2}{*}{$\mathrm{Cl}^{33}(n, \alpha) \mathrm{P}^{32}$} & $14.3 d$ & -0.92 & 3.0 & 13 & & & & 0.56 & 15 \\
\hline & & & 4.1 & 7 & $\mathrm{Ni}^{62}(n, \alpha) \mathrm{Fe}^{5 \vartheta}$ & $45 d$ & 0.884 & 0.013 & 15 \\
\hline $\mathrm{Cl}^{37}(n, p) \mathrm{S}^{37}$ & $5.0 \mathrm{~m}$ & 3.6 & 0.24 & 13 & & & & 0.025 & 6 \\
\hline \multirow[t]{3}{*}{$\mathrm{Co}^{68}(n, p) \mathrm{Fe}^{59}$} & $45 d$ & 0.79 & 0.25 & 16 & & & & 0.14 & 8 \\
\hline & & & $\sim 0.3$ & 8 & $\mathrm{O}^{16}(n, p) \mathrm{N}^{16}$ & $7.5 \mathrm{~s}$ & 10.22 & 0.014 & 19 \\
\hline & & & 5.7 & 6 & & & & 0.019 & 19 \\
\hline $\mathrm{Co}^{59}(n, \alpha) \mathrm{Mn}^{56}$ & $2.58 \mathrm{~h}$ & -0.44 & 0.14 & 7 & & & & 0.0185 & 20 \\
\hline \multirow[t]{2}{*}{$\mathrm{Cs}_{8^{133}}(n, \alpha) \mathbf{I}^{130}$} & $12.6 \mathrm{~h}$ & -4.22 & $2.4 \times 10^{-4}$ & $\gamma$ & $\mathrm{O}^{17}(n, p) \mathrm{N}^{17}$ & $4.14 \mathrm{~s}$ & 8.47 & 0.0052 & 19 \\
\hline & & & 5. $\times 10^{-4}$ & 6 & & & & 0.0093 & 20 \\
\hline $\mathrm{Cu}^{63}(n, \alpha) \mathrm{Co}^{60}$ & $5.4 y$ & -1.59 & 0.72 & 8 & $\mathrm{P}^{21}(n, p) \mathrm{Si}^{31}$ & $170 \mathrm{~m}$ & 0.72 & 31.2 & 12 \\
\hline \multirow{2}{*}{$\mathrm{F}^{10}(n, p) \mathrm{O}^{19}$} & $30 \mathrm{~s}$ & 4.21 & 0.5 & 13 & & & & 19. & 19 \\
\hline & & & 0.99 & $\gamma$ & $\mathrm{P}^{31}(n, \alpha) \mathrm{Al}^{28}$ & $2.4 \mathrm{~m}$ & 2.02 & 1.43 & 19 \\
\hline \multirow[t]{2}{*}{$\mathrm{F}^{19}(n, \alpha) \mathrm{N}^{16}$} & $7.5 \mathrm{~s}$ & 1.57 & 4.5 & 19 & & . & & 0.75 & $\gamma$ \\
\hline & & & 4.5 & 7 & $\mathrm{~S}^{32}(n, p) \mathbf{P}^{32}$ & $14.3 \mathrm{~d}$ & 0.95 & 154. & 16 \\
\hline \multirow[t]{4}{*}{$\mathrm{Fe}^{b 4}(n, p) \mathrm{Mn}^{54}$} & $291 d$ & -0.16 & 11. & 16 & & & & 60.3 & 12 \\
\hline & & & 15. & 16 & & & & 30. & 19 \\
\hline & & & 23. & 6 & & & & 21. & $\gamma$ \\
\hline & & & 56. & 8 & $\mathrm{~S}^{34}(n, \alpha) \mathrm{Si}^{31}$ & $170 \mathrm{~m}$ & 1.24 & 3.0 & 19 \\
\hline $\mathrm{Fe}^{64}(n, \alpha) \mathrm{Cr}^{51}$ & $27.8 \mathrm{~d}$ & -0.862 & 0.37 & 6 & & & & 1.2 & $\gamma$ \\
\hline $\mathrm{Fe}^{68}(n, p) \mathrm{Mn}^{58}$ & $2.58 \mathrm{~h}$ & 2.94 & 0.44 & 6 & $\operatorname{Sc}^{4 b}(n, \alpha) \mathrm{K}^{42}$ & $12.5 \mathrm{~h}$ & 0.61 & $<5$ & 8 \\
\hline $\mathrm{Ge}^{70}(n, 2 n) \mathrm{Ge}^{80}$ & $39.6 \mathrm{~h}$ & 11.9 & 1.5 & 8 & $\mathrm{Si}^{28}(n, p) \mathrm{Al}^{28}$ & $2.4 \mathrm{~m}$ & 4.01 & 4. & 19 \\
\hline $\mathrm{Ge}^{72}(n, p) \mathrm{Ga}^{72}$ & $14.1 \mathrm{~h}$ & 3.27 & $<0.01$ & 8 & $\operatorname{Si}^{29}(n, p) \mathrm{Al}^{20}$ & $6.7 \mathrm{~m}$ & 3.1 & 2.7 & 19 \\
\hline $\operatorname{Ge}^{72}(n, \alpha) \mathrm{Zn}^{68}$ & $13.8 \mathrm{~h}$ & -1.12 & $<1$. & 8 & $\mathrm{Ta}^{181}(n, \alpha) \mathrm{Lu}^{178}$ & $22 m$ & $?$ & $8.5 \times 10^{-1}$ & $\gamma$ \\
\hline \multirow[t]{2}{*}{$\mathrm{Mg}^{24}(n, p) \mathrm{Na}^{24}$} & $15 \mathrm{~h}$ & 4.95 & 1.29 & 12 & $\mathrm{Th}^{232}(n, 2 n) \mathrm{Th}^{231}$ & $25.6 \mathrm{~h}$ & 6.3 & 12.4 & 21 \\
\hline & & & 1.0 & 13 & $\mathrm{Ti}^{48}(n, p) \mathrm{Sc}^{48}$ & $85 \mathrm{~d}$ & 1.61 & 4.10 & 6 \\
\hline $\mathrm{Mg}^{25}(n, p) \mathrm{Na}^{25}$ & $62 \mathrm{~s}$ & 3.1 & 2.0 & 19 & $\mathrm{Ti}^{47}(n, p) \mathrm{Se}^{47}$ & $3.4 \mathrm{~d}$ & -0.096 & 0.21 & 6 \\
\hline $\mathbf{M n}^{38}(n, 2 n) \mathbf{M n}^{34}$ & $291 \mathrm{~d}$ & 10.33 & $0.05(?)$ & 15 & $\mathrm{Ti}^{48}(n, p) \mathrm{Sc}^{48}$ & $44 \mathrm{~h}$ & 3.28 & 0.077 & 6 \\
\hline $\mathrm{Mo}^{92}(n, p) \mathrm{Nb}^{92}$ & $10 \mathrm{~d}$ & -0.416 & 1.3 & 6 & $\mathrm{Ti}^{48}(n, \alpha) \mathrm{Ca}^{48}$ & $164 d$ & 2.02 & 0.0055 & 6 \\
\hline $\mathrm{Mo}^{82}(n, \alpha) \mathrm{Zr}^{80}$ & $79 h$ & -3.00 & 0.017 & 6 & $\mathrm{Ti}^{80}(n, \alpha) \mathrm{Ca}^{47}$ & $4.7 d$ & 3.58 & 0.0002 & 6 \\
\hline $\mathrm{Mo}^{95}(n, p) \mathrm{Nb}^{95}$ & $35 \mathrm{~d}$ & 0.150 & $<0.1$ & 6 & $\mathrm{Tl}^{208}(n, p) \mathbf{H g}^{208}$ & $48 \mathrm{~d}$ & -0.297 & 0.002 & 6 \\
\hline \multirow{2}{*}{$\mathrm{Na}^{23}(n, p) \mathrm{Ne}^{23}$} & $40 \mathrm{~s}$ & 3.76 & 0.7 & & $\mathrm{U}^{233}(n, 2 n) \mathrm{U}^{237}$ & $6.6 \mathrm{~d}$ & 6.09 & 4.7 & 15 \\
\hline & & 3.70 & 1.0 & $\begin{array}{c}19 \\
\gamma\end{array}$ & $\mathrm{V}^{51}(n, \alpha) \mathrm{Sc}^{48}$ & $44 h$ & 2.14 & 0.08 & 13 \\
\hline $\mathrm{Na}^{23}(n, 2 n) \mathrm{Na}^{22}$ & $2.6 y$ & 12.98 & 0.006 & 19 & & & & 0.0099 & 7 \\
\hline \multirow{2}{*}{$\mathrm{Na}^{23}(n, \alpha) \mathbf{F}^{20}$} & $11.6 \mathrm{~s}$ & 4.07 & 0.4 & 19 & $\mathrm{Zn}^{84}(n, p) \mathrm{Cu}^{84}$ & $.12 .8 \mathrm{~h}$ & -0.22 & 35. & 8 \\
\hline & & & 0.47 & $\gamma$ & & & & 22. & 6 \\
\hline $\mathrm{Nb}^{93}(n, \alpha) \mathrm{Y}^{90}$ & $64.0 \mathrm{~h}$ & -4.87 & 0.024 & 7 & $\mathrm{Zn}^{67}(n, p) \mathrm{Cu}^{87}$ & $59 \mathrm{~h}$ & -0.214 & 0.27 & 6 \\
\hline & & & o. & $\gamma$ & $\mathrm{Zn}^{88}(n, \alpha) \mathrm{Ni}^{86}$ & $2.56 \mathrm{~h}$ & -0.93 & 0.020 & 7 \\
\hline
\end{tabular}

bridge, Mass. 1953)

4. A. H. Wapstra, Isotopic masses <202, Physica 21, 367 (1955); and J. R. Huizengs, Isotopic masees >201, Physica 21, 410 (1955) 5. W. Inthoff, NoCLEONICs 19, No, 11, 67 (1955)

6. C. E. Melligh, J. A. Payne, R. L. Otlet in "Croc. International Conference on Radioisotopes in Scientific Research," Sept., 1957, Paris (Pergamon Press, London, 1958): based on assumed $\sigma_{a} \Rightarrow 30 \mathrm{mb}$ for $\mathrm{S}^{3 s}(n, p) \mathrm{P}^{3 s}$

7. E. Saeland, K. Samsahl, Joint Establishment for Nuclear Energy Research, Lillestrom. Norway, JENER Report No. 23 and private communication; based on assumed $\sigma_{*}=19$ $\mathrm{mb}$ for $\mathrm{P}^{31}(n, p) \mathrm{Si}^{31}$

8. Values mesured by present suthor and not previously published; based on assumed $\sigma_{\varepsilon}=0.60 \mathrm{mb}$ for $\mathrm{Al}^{27}(n, \alpha) \mathrm{Na}^{24}$

ง. $\mathrm{K}$. Way, et al. "Nuclear Level Schemes,
$A=40$ to $A=92, "$ TID-5300 (1955)

10. J. M. Hollander, et al. "Table of Isotopes," Rev. Mod. Phys. 26, 469 (1953)

11. "Nuclear Data" published monthly (National Research Council, Washington 25 D.C.)

12. R. Richmond, Atomic Energy Research Establishment, Harwell, England, private communication (1957); based on assumed Uass fission cross section of $304 \mathrm{mb}$

19. Ref, s, pp. 100 and 102 ; based on calculating fux near U slab in thermal-neutron beam

14. B. L. Robinson, R. W. Fink, University of Arkansas, private communication; based on assumed $\sigma_{*}=30 \mathrm{mb}$ for $\mathrm{S}^{32}(n, p) \mathrm{P}^{32}$

15. R. P. Schuman, A. C. Mewherter, KAPL-1779 (1957); based on assumption that fissionneutron flux inside uranium receptacle slug equals thermal-neutron fux outside slug
16. G. H. Stafford, L. H. Stein, Nature 172, 1103 (1953); basis for flux messurement not specified

17. C. E. Mellish, J. A. Payne, Nature 178, 275 (1956); based on assumed $\sigma_{0} \Rightarrow 30 \mathrm{mb}$ for $\mathrm{S}^{32}(n, p) \mathrm{P}^{3}$

18. This value, taken from ref. 6 , is sum of $\sigma_{0}=32 \mathrm{mb}$ for direct production of 72-day Coss and 13 rab for production of 9 -hr $\mathrm{Cobs}^{\mathrm{s}}$ which decsys to Cots

19. P. A. Roys, K. Shure, Nuclear $S$ si. and Eno 4536 (1958): based on assumed $\sigma_{0}=0.60$ mb for $\mathrm{Al}^{27}(n, \alpha) \mathrm{Na}^{24}$

20. W. J. Henderson, P. R. Tunnicliffe, Nuclear Sci. and Eno. 3, 145 (1958); Gux calculated from reactor power level

21. J. A. Phillips, AERE-R/R-2366 (1957); based on assumed $\sigma_{0}=60.3 \mathrm{mb}$ for $\mathrm{S}^{32}(n, p) \mathrm{P}^{39}$

28. 8. E. Turner, Anal. Chem. 28, 1457 (1956) 


\title{
X-ray Production with Linear Accelerators
}

\author{
BY MALCOLM H. MacGREGOR \\ Applied Radiation Corp., Walnut Creek, California
}

Linear electron acclerators are particularly useful in their ability to produce intense bremsstrahlung beams. A knowledge of this photon production is important for both irradiation work and for shielding purposes when the electron beam is used directly. The graphs and table presented here are designed for evaluation of photon production and associated shielding problems.

Radiation lengths. If target thicknesses are expressed in radiation lengths, formulas for bremsstrahlung production are essentially the same for all elements. One radiation length is defined as the distance along the beam in which the energy of the typical electron is reduced to $1 / e$ of its original value (1). Figure 1 gives radiation lengths as a function of atomic number.

Forward intensity. The most intense photon radiation occurs directly in line with the electron beam when a target of optimum thickness is placed in the beam. Figure 2 shows these maximum intensities (2). The forward intensity as a function of target thickness is shown in Fig. 3. The curve is for gold at $17 \mathrm{Mev}(3)$, but it applies to all high- $Z$ targets and all electron energies above $5 \mathrm{Mev}$ (4). For low-Z targets and low electron energies, ion-
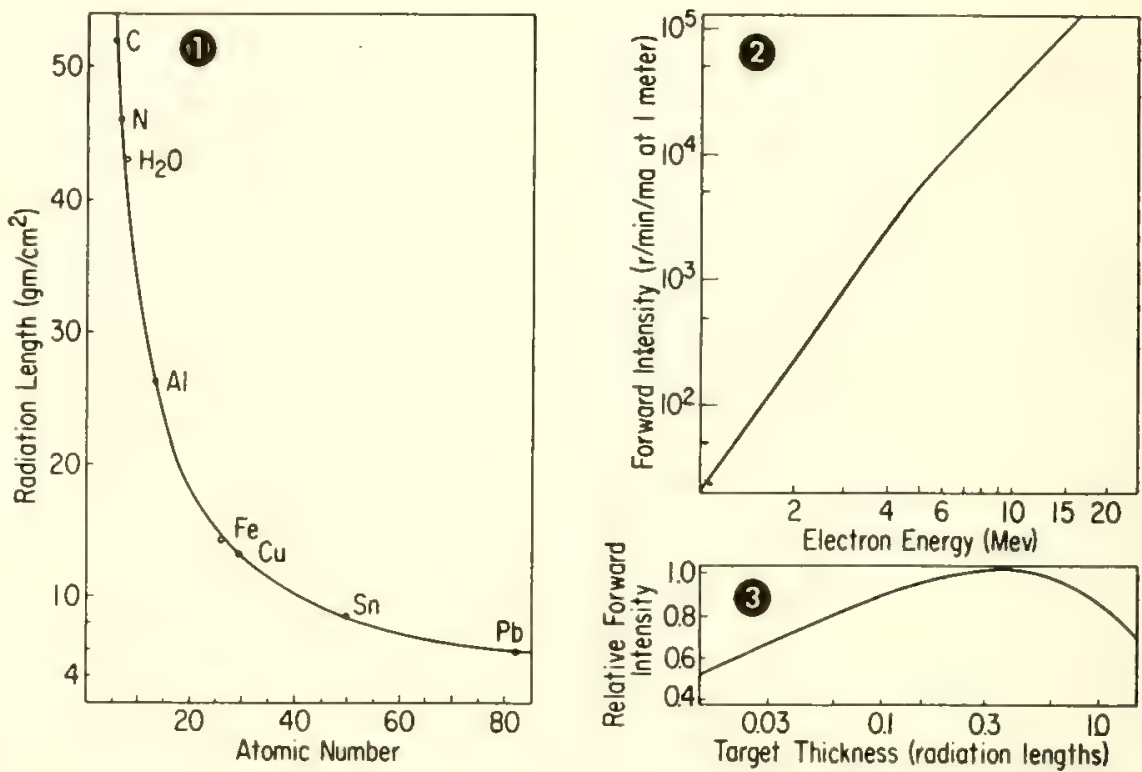

FIG. I shows radiation length as function of atomic number. Expressing target thickness in radiation lengths makes bremsstrahlung-production calculations essentially the same for all targets. FIG. 2 gives maximum photon intensity as a function of electron energy for a high-Z target of optimal thickness. FIG. 3 indicales variation of forward intensity with target thickness

ization losses become increasingly important (see Fig. 6), and the peak in Fig. 3 shifts somewhat toward smaller target thicknesses. Figures 2 and 3

\begin{tabular}{|c|c|c|c|c|c|c|}
\hline \multirow{2}{*}{$\begin{array}{c}\text { Photon Energy } \\
(M \text { ev })\end{array}$} & \multicolumn{6}{|c|}{ Electron Energy (Mev) } \\
\hline & 5.5 & 8.51 & 10.51 & 14.51 & 16.51 & 20.51 \\
\hline 2 & 45.3 & 130 & 208 & 418 & 550 & 868 \\
\hline 4 & 10.6 & 50.1 & 86.2 & 183 & 245 & 396 \\
\hline 6 & $\ldots$ & 22.8 & 46.6 & 108 & 147 & 241 \\
\hline 8 & . & 1.73 & 25.2 & 71.4 & 99.1 & 167 \\
\hline 10 & . & . & 2.10 & 49.1 & 71.4 & 123 \\
\hline 12 & $\ldots$ & $\ldots$ & $\ldots$ & 31.0 & 52.0 & 95.5 \\
\hline 14 & & . & & 2.84 & 34.1 & 75.5 \\
\hline 16 & . & . & . & . & 3.22 & 58.8 \\
\hline 18 & $\ldots$ & $\ldots$ & $\ldots$ & . & $\cdots$ & 40.6 \\
\hline 20 & . & . & $\ldots$ & . & . & 3.97 \\
\hline
\end{tabular}

together enable one to calculate forward photon intensity for a high- $Z$ target and any combination of target thickness and electron energy over the range covered. For a low- $Z$ target, forward intensity is lower. The effect of lower $Z$ can be inserted by using Fig. 5.

Angular distribution. The curves of Fig. 4 enable calculation of intensities in other directions from the forward intensity. In the ranges covered these curves are in good agreement with experiment (3). There is an absence of data for energies above $5 \mathrm{Mev}$, particularly for thick targets. Shielding requirements are difficult to estimate. Probably a safe rule of thumb is that 


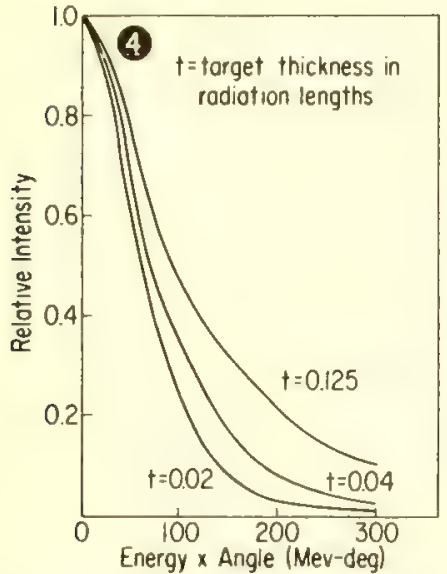

FIG. 4 tells beam width by plotting relative intensity against energy-angle product. FIG. 5 is total conversion efficiency for photon production from electron beam stopped in thick target.
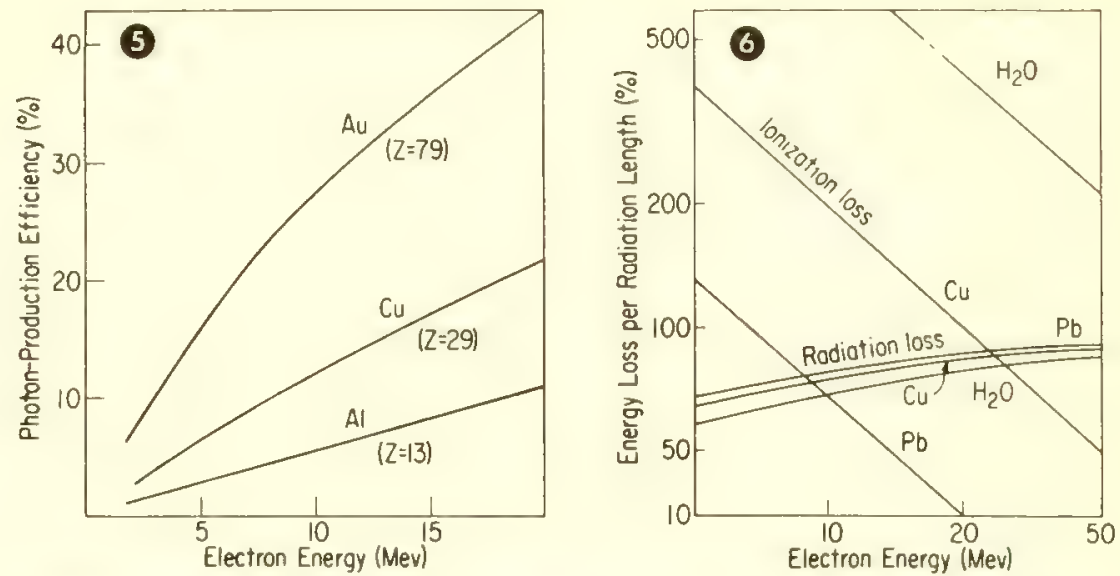

FIG. 6 is thin-target conversion efficiency, given by $\epsilon=t k$, where $t$ is target thickness and $k$ is per cent of electron energy converted into photons per radiation length

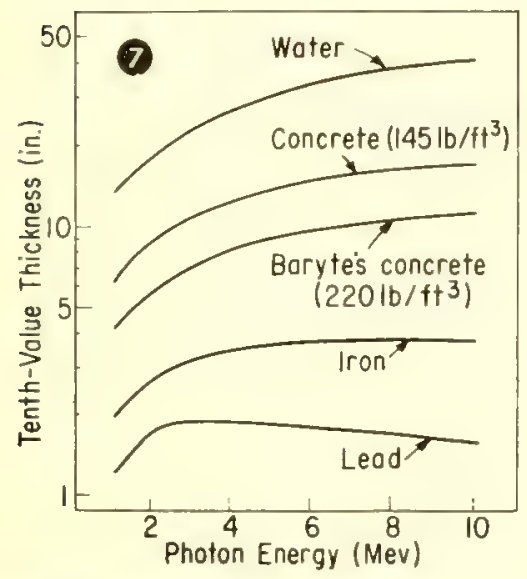

FIG. 7 gives thicknesses of various common shielding materials required to reduce narrow, monoenergetic photon beam to one tenth of its incident intensity. FIG. 8 shows buildup factors for
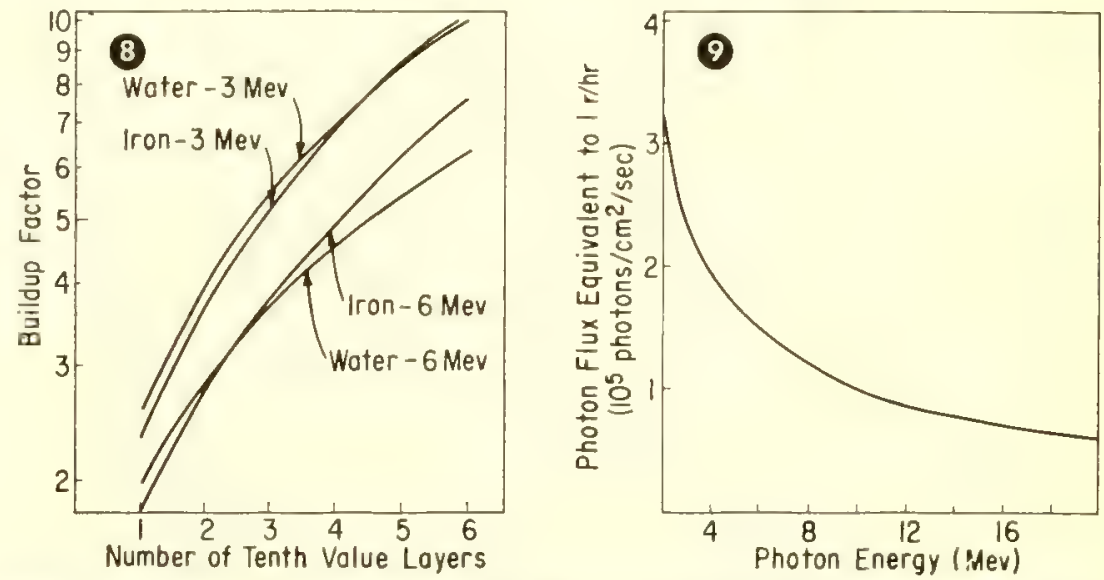

use with FIG. 7 to find actual attenuation in broad-beam geom. efry. FIG. 9 shows equivalence of photon fiux and dose rate to enable treatment of spectrum by energy intervals for electron energies of 10-20 Mev and targets thicker than 0.2 radiation lengths photon intensity at $90 \mathrm{deg}$ can be as high as $5 \%$ of forward intensity.

Total conversion efficiency. Figure 5 gives thick-target photon-production efficiencies (4). For thick targets conversion efficiency varies linearly with atomic number (5). Figure 6 enables computation of conversion efficiency from target thickness $(6)$.

Spectrum shape. The number of photons in each energy interval of a bremsstrahlung spectrum is relatively independent of angle. The spectrum shape is shown by the table ( 7$)$.

Shielding. The narrow-beam absorption coefficients of Fig. $7(8)$ and the buildup factors of Fig. 8 (9) are for shielding calculations. For approximate calculations the spectrum of the table is replaced by an effective photon energy and Figs. 7 and 8 are used at that energy. For 20-Mev electrons the effective photon energy is $\sim 7 \mathrm{Mev}$, and for $6-\mathrm{Mev}$ electrons it is $\sim 3 \mathrm{Mev}$. For more accurate treatment each component of the spectrum is treated separately. Figure 9 shows the equivalence between photon flux and dose rate in air. Figure 9, the table and Fig. 2 can be used together to obtain the forward flux at each energy. Figure 4 gives the flux in any other direction. The $1 / r^{2}$ falloff and the shielding attenuation from Figs. 7 and 8 are then calculated for each energy group. Finally total dose rate is calculated from Fig. 9.

This data sheet is based on Report AM-100 (Applied Radiation Corp., Walnut Creek. Calif., March 13, 1957).

\section{BIBLIOGRAPHY}

1. "American Institute of Physics Handbook," D. E. Gray, ed., p. 8-39 (McGraw-Hill Book Co., New York, 1957)

2. Report AM-102 (Applied Radiation Corp. Walnut Creek, Calif., 1957); NUCLEONiCa 15, No. 11, 178 (1957)

\$. L. H. Lanzl, A. O. Hanson, Phys. Rev. 89, 959 (1953)

4. C. W. Miller, Paper 276 presented at Induatrial Electronics Convention, Oxford, 1954

5. W. W. Buechner, R. J. Van de Graaff, A. E. Burrill A Sperduto, Phus, Rey. T4, 1348 (1948)

6. J. D. Lawson, Nucleonics 10, No. 11, 61 (1952) 7. Nat'l Bu. of Standards Hsandbook 55 (1954) 8. S. Block, private communication

9. J. Moteff, APEX-176 (1954) 

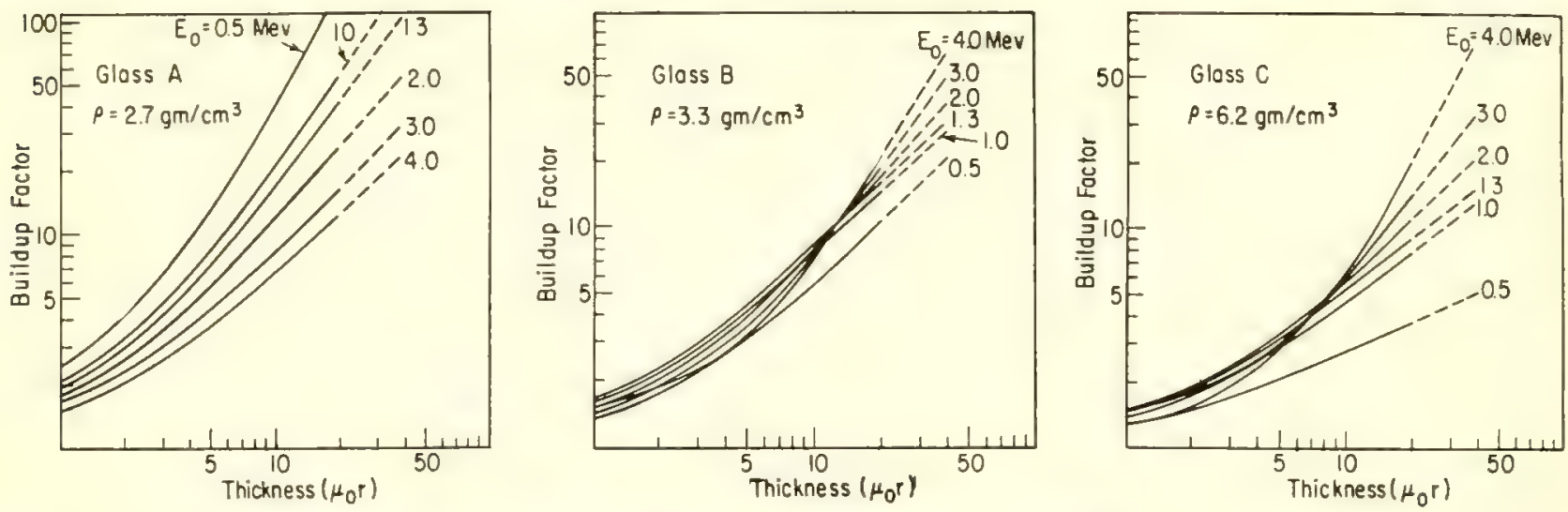

FIG. 1. Computed buildup factors for three glass compositions and six gamma-ray energies

\section{Shielding-Glass Buildup Factors}

\section{By JOHN W. LINDNER, * Pittsburgh Plate Glass Co., Pittsburgh, Pennsylvania}

Gamma-ray buildup factors for three shielding glasses are presented in the accompanying figures. The computations have been based on standard methods (1-4). These data enable the design of a shielding window giving the desired protection at a minimum cost. Previously it has been customary to correct for buildup by adding $\sim 15 \%$ to the computed thickness. The data of Fig. 1-3 show that any rule of thumb like this is valid only for a single photon energy.

Computations. To compute buildup factors for a homogeneous mixture such as glass, it is only necessary to compute an effective atomic number for the glass (1). This can be done by (a) calculating the mean absorption coefficient per electron for the mixture and then (b) finding the element having the same absorption coefficient in the energy range over which Compton scattering is important.

The absorption coefficients of Fig. 2

* Pregent Adpregs: Space Technology Laboratories, Los Angeles, Calif. were calculated from the data of Gladys White Grodstein (5). For energies of 0.1-2.0 Mev, the calculated absorption coefficients of the glasses of densities $2.7,3.3$, and $6.2 \mathrm{gm} / \mathrm{cm}^{3}$ agree to within $\pm 3 \%$ with those of elements having atomic numbers 11,54 , and 73 , respectively. The difference at $4 \mathrm{Mev}$ is still less than $10 \%$.

The dose buildup factors for the three glasses (Fig. 1) are plotted as a function of glass thickness expressed in relaxation lengths.

Glasses. The compositions of the glasses for which the computations were carried out are given in the table. Glasses A and B correspond to Pittsburgh Plate Glasses No. 6740 and No. 4966, respectively. Glasses of corresponding densities produced elsewhere can be considered identical for calculating buildup factors. Differences in composition do not have significant effect on effective atomic number. Glass $\mathrm{C}$ is an experimental glass that corresponds to commercially available $6.2-\mathrm{gm} / \mathrm{cm}^{3}$ glass.
Compositions (wt \%) of Three Shielding Glasses

\begin{tabular}{ccccccccc}
\hline Glass & $\begin{array}{c}\text { Density } \\
\left(\mathrm{gm} / \mathrm{cm}^{3}\right)\end{array}$ & $\mathrm{SiO}_{2}$ & $\mathrm{PbO}$ & $\mathrm{B}_{2} \mathrm{O}_{3}$ & $\mathrm{Na}_{2} \mathrm{O}$ & $\mathrm{BaO}$ & $\mathrm{K}_{2} \mathrm{O}$ & $\mathrm{CeO}_{2}$ \\
\hline $\mathrm{A}$ & 2.7 & 64.8 & - & 3.0 & 6.5 & 15 & 9.0 & 1.7 \\
$\mathrm{~B}$ & 3.3 & 49 & 34.8 & - & 0.5 & - & 14 & 1.7 \\
$\mathrm{C}$ & 6.2 & 5.5 & 82 & 12.5 & - & - & - & - \\
\hline
\end{tabular}

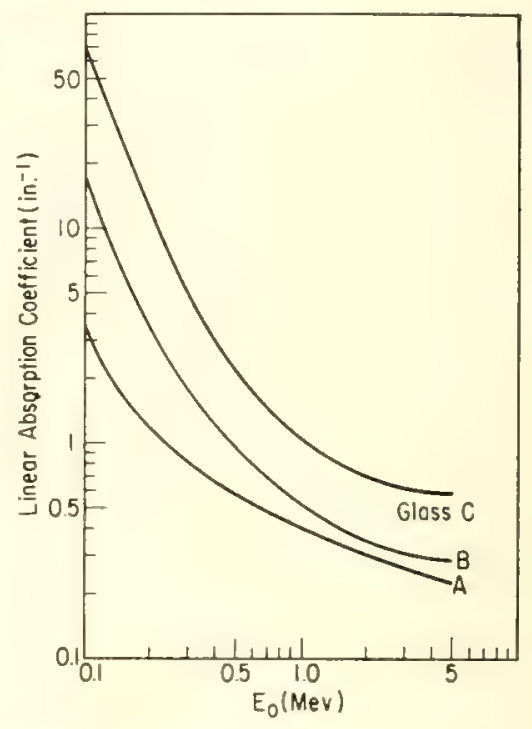

FIG. 2. Calculated coefficients

Accurocy. Goldstein and Wilkins claim an accuracy of $\pm 5 \%$ for their calculations. After making allowance for probable errors in the choice of effective atomic numbers and graphical interpolation, the probable errors in the final results should be $< \pm 15 \%$. However, for shield thickness greater than 20 relaxation lengths, the error may be as great as $\pm 20 \%$.

The author wishes to express his gratitude to Mr. Carlo Del Carlo, who prepared the curves.

\section{BIBLIOGRAPHY}

1. H. Goldstein, J, E. Wilkins, Jr, NYO-3075 (1954)

2. L. V. Spencer, U. Fano, J. Research Nal, Bur. Standards 46, 446 (1951)

3. L. V. Spencer, U. Fano, Phys. Rev. 81, 464 (1951)

4. U. Fano, NUCLEonics 11, No. 8, 8 (1953) and 11, No, 9, 55 (1953)

6. G. W. Grodstein, Nationsl Bureau of Standards Circular 583 (1957) 


\title{
Properties of Zircaloy-2
}

\author{
BY L. S. RUBENSTEIN \\ Bettis Plant, Westinghouse Electric Corporation, Pittsburgh, Pennsylvania
}

The data compilation adjoining summarizes the present knowledge of the corrosion, mechanical and physical properties of Zircaloy-2.

Zircaloy-2 is an alloy of sponge zirconium containing nominally $1.5 \mathrm{wt} \%$ Sn, 0.12 wt $\% \mathrm{Fe}, 0.05$ wt $\% \mathrm{Ni}$ and 0.10 wt $\% \mathrm{Cr}$. Its use in reactors is based on its excellent corrosion resistance, low thermal-neutron-absorption cross section and its structural stability at reactor operating temperatures.

Particularly attractive for reactorfuel cladding is the low thermal-neutron-absorption cross section of 0.22 0.24 barn of Zircaloy-2 (1a) [0.18 for pure zirconium, which has a scattering cross section of $\sim 8$ barns $(1 b)$ ].

Zircaloy-2 is vacuum-melted commercially by a double-consumableelectrode arc-melting technique. It undergoes allotropic transformation from the low-temperature alpha phase (close-packed-hexagonal) to the hightemperature beta phase (body-centered-cubic). This transformation plays an important role in the variation of corrosion and mechanical properties.

It is very easy to weld, but must be protected by welding in controlled inert-atmosphere boxes or by use of efficient inert-gas shields because of the rapid rate of contamination with oxygen and nitrogen when welded in air. Such addition or variation of impurities can cause significant changes in mechanical properties and corrosion resistance.

Zircaloy-2 has been fabricated by almost all of the known metalworking operations. Wire of all gages has been produced by drawing a rod formed by hot rolling or extrusion. Cups have been spun, deep-drawn, flow-turned, back-extruded and impact-extruded. Closed-die forgings have been made and tubing has been formed by both extrusion and welding. Shapes have also been cast using a special vacuummelting and casting technique (2).

\section{Strip Specifications}

The important properties of Zircaloy-2 can be best indicated by the characteristics of the nominal alloy as called for in the military specifications [Mil-Z-19859A (1959)] for acceptable strip as paraphrased below.

Chemical composition. The chemical composition shall be $1.20-1.70 \%$ tin, $0.07-0.20 \%$ iron, $0.05-0.15$ chromium and $0.03-0.08 \%$ nickel. The

TABLE 1-Specifications for Maximum Impurities

\begin{tabular}{lrlr}
\hline Element & $\begin{array}{c}\text { Impurity } \\
\text { level } \\
(p p m)\end{array}$ & Element & $\begin{array}{c}\text { Impurity } \\
\text { level } \\
(p p m)\end{array}$ \\
\hline Aluminum & $\mathbf{7 5}$ & Manganese & 50 \\
Boron & $\mathbf{0 . 5}$ & Nitrogen & 80 \\
Cadmium & $\mathbf{0 . 5}$ & Silicon & 120 \\
Carbon & 270 & Sodium & 20 \\
Cobalt & 20 & Titanium & 50 \\
Copper & 50 & Tungsten & 100 \\
Hafnium & 200 & Uranium- & \\
Hydrogen & 25 & total & 3.5 \\
Lead & 130 & Uranium- \\
Magnesium & 20 & 235 & 0.025 \\
& \multicolumn{4}{c}{0} \\
\end{tabular}

sum of the iron, chromium and nickel contents determined from the average of all analyses made for a single ingot shall fall within the range $0.18-0.38 \%$.

Impurities. Unless otherwise specified, impurity levels of the finished material shall not exceed the limits specified in Table 1.

Mechanical properties. The average hardness of material after annealing shall not exceed the values specified in Table 9 (see foldout adjoining) for the method used.

The material shall conform to the elevated-temperature mechanical pro- perties specified in Table 11 (based on use of vacuum-melted material).

Corrosion properties. Corrosion coupons, after completion of a 14-day test in $750^{\circ} \mathrm{F}, 1,500$-psi steam, shall show the following: (a) a continuous black lustrous temper film; (b) freedom from white or brown corrosion products; (c) freedom from superficial surface stains, cracks, fissures, streaks and blisters; (d) a weight gain of $28 \pm$ $10 \mathrm{mg} / \mathrm{dm}^{2}$.

This work was performed for the U.S. Atomic Energy Commission under contract A T-11-1-GEN-14.

\section{BIBLIOGRAPHY}

1a. G. L. Hartfield, Bettis, pereonal communication (1959). 1b. B. Lustman, F. Kerze, "The Metallurgy of Zirconium," National Nuclear Energy Series Vol. VII-4 (McGraw Hill Book Co., New York, 1955)

2. E. L. Richards, J. H. Hart, W. H. Friske, W. J. Hurford, "The Melting and Fabrication of Zircaloy," 1958 Geneva Conference Paper No. 990

S. R. E. Johnson, W. D. McMullen, in the Bettis Plant Materials Manual (1957)

4. J. H. Keeler, 1956 educational course, ASM, Columbia River Basin

5. J. Weinberg, in "Zirconium Highlights," WAPD-ZH-12 (1958)

6. J, G. Goodwin, The effect of heat treatment on the tensile and corrosion properties of Zircsloy-2, in "Zirconium Highlights," WAPD-ZH-5 (1958)

7. S. Kass, WAPD-TM-97 (1857)

8. W. L. Mudge, Jr., F. Foracher. Mechanical properties of Zircaloy-2, WAPD-101 (1954)

9. R. G. Wheeler, W. S. Kelly. Irradiation of Zircsloy-2 impact specimens containing hydrogen, HW-39805 (1955)

10. W. D. McMullen. Interim report on creep behavior of Zircaloy-2 and Zircaloy-3, WAPD-TM-132 (1958)

11. F R Shober, J. A. Van Echo, L. L. Marsh, Jr., J. R. Keeler. The mechanical properties of zirconium and Zirealoy-2, BMI-1168 (1957)

12. KAPL Progrese Report, Sept.-Oct., 1957 (classified)

13. R. E. Johnson. Torsional properties of Zircaloy-2 and Zircaloy-3, in "Zirconium Highlights," WAPD-ZH-7 (1958)

14. F. Foracher. Effect of cold work on the mechanical properties of Zircaloy-2, WAPD111 (1954)

15. R. S. Kemper, D, L. Zimmerman. Neutron irradiation effects on the tengile properties of Zircaloy-2, HW-52323 (1957)

16. F. Forscher. Summary report on mechanical properties of Zircaloy-3, WAPD-143 (1956)

17. W. D. McMullen, WAPD-Bettis, private communication (1958)

18. J. Intrater Dilatometric investigation of vacuum melted Zircaloy-2, in "Zirconium Highlights," WAPD-2H-7 (1958) 


\section{MECHANICAL PROPERTIES}

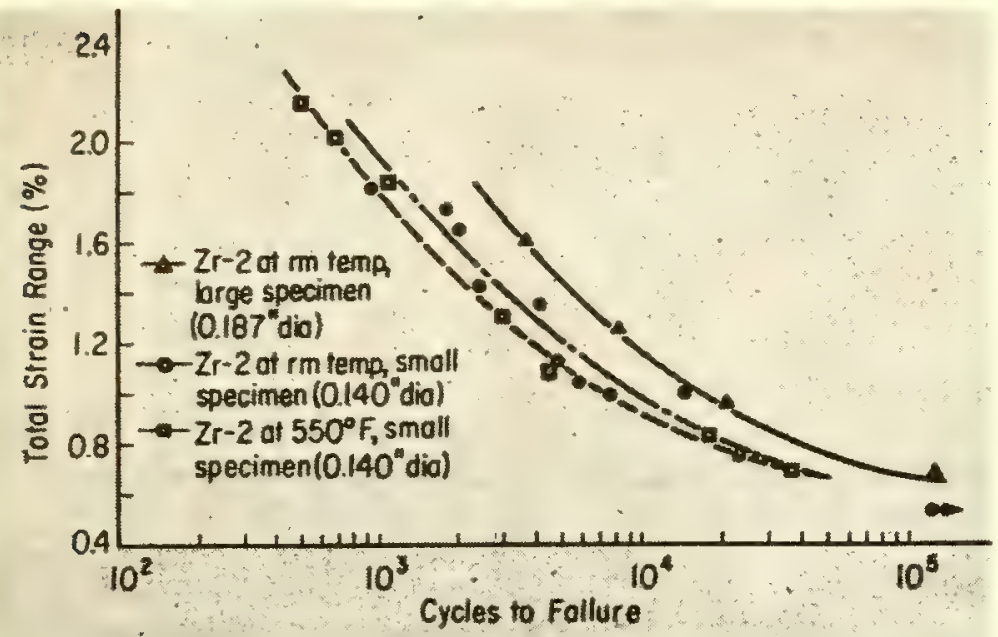

FATIGUE In Table 6 , the fatigue tests were performed at $316^{\circ} \mathrm{F}$ on notched and unnotched specimens with their oxes parallel to the rolling directions. Base-annealed specimens and beta-quenched-plus-annealed $1700^{\circ} \mathrm{C}$ for $1 \mathrm{hr}$ in vacuum, fur nace cooled) specimens were tested. Results indicate a rela. tively high notch sensitivity (8). Cycles to failure for constantstrain fatigue tests (12) are shown at left.

TABLE 8-Effect of Cold Work on

Room-Temperature Properties (Longitudinal Specimens) ( 8 )

\begin{tabular}{ccrc}
$\begin{array}{c}\text { Cold } \\
\text { reduction } \\
(\%)\end{array}$ & $\begin{array}{c}0.2 \% \\
\text { yield } \\
\text { strength } \\
\left(10^{3} \text { psi }\right)\end{array}$ & $\begin{array}{c}\text { Tensile } \\
\text { strength } \\
\left(10^{3} p s i\right)\end{array}$ & $D P H^{*}$ \\
\hline 0 & 66.2 & 82.6 & 210 \\
10 & 81.1 & 92.2 & 228 \\
25 & 90.6 & 103.8 & 231 \\
40 & 96.5 & 107.0 & 243 \\
60 & 97.1 & 111.2 & 256
\end{tabular}

* Diamond-pyramid hardness.

COLD WORKING, IRRADIATION. The effects of cold working on room-temperature longitudinal tensile properties are given in Table $8(8)$. Stress-strain curves along the longitudinal direction at $250^{\circ} \mathrm{C}$ for different amounts of cold work (14) are shown of right. Typical curves for control and irradiated $\left(1.4 \times 10^{21} \mathrm{nvt}\right.$ at $\left.40^{\circ}-60^{\circ} \mathrm{C}\right)$ specimens (15) are shown below.
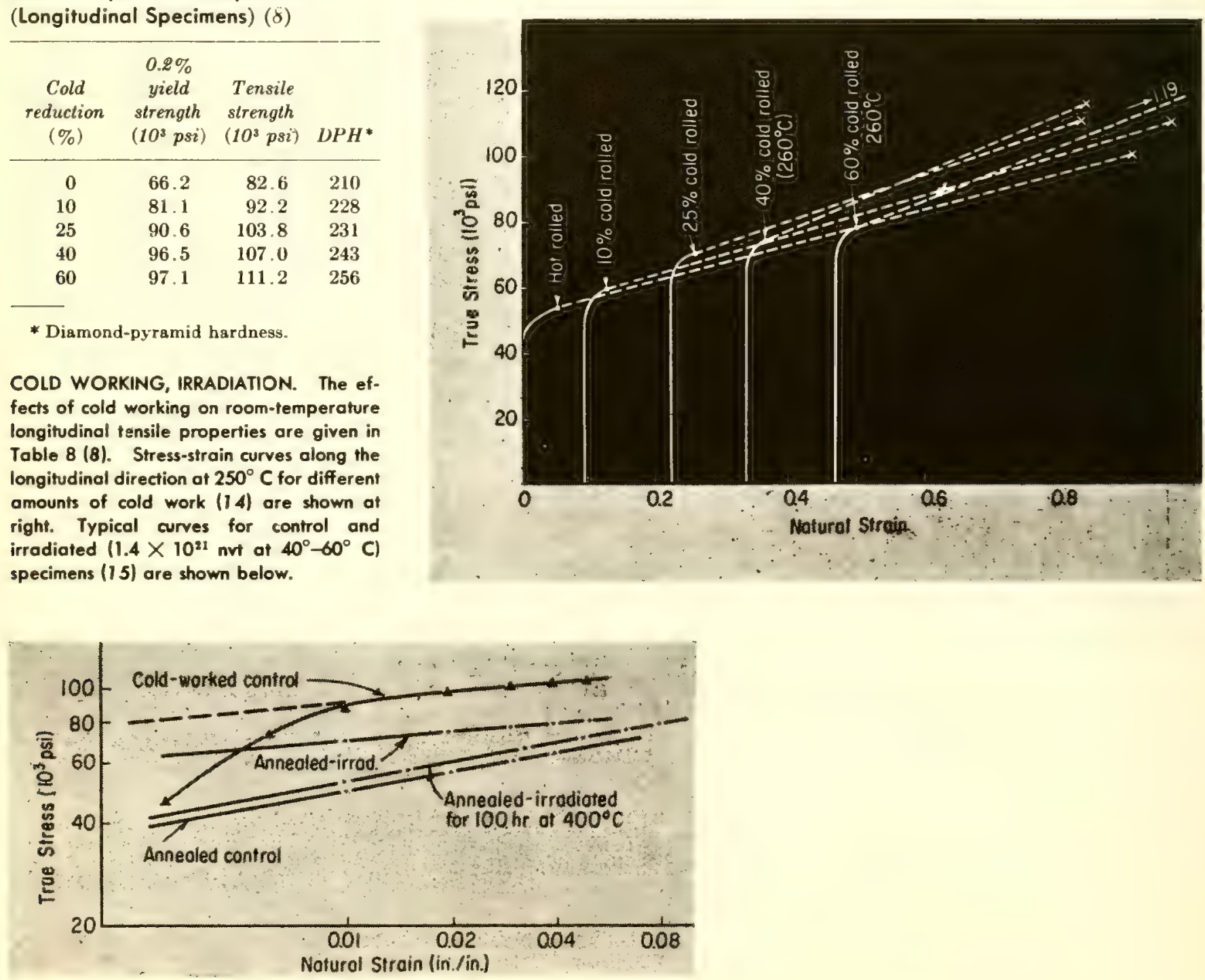
TABLE 6-Fatigue Results at $316^{\circ} \mathrm{C}$

\section{Endurance limi}

(10 $\mathrm{psi}$ )

Treatment
Notch sensitivity Fatigue index at ratio* $10^{8}$ cycles $\dagger$
Beta-quenched and

alpha-annealed

Base-annealed
27.5

11.0

0.80

0.60

28.0

0.85
0.87

* Fatigue ratio = (unnotched fatigue strength) /(tensile strength) † Notch sensitivity index $q=(K,-1) /(K,-1)$, where $K_{f}=$ (unnotched fatigue strength)/(notched fatigue strength) and $\boldsymbol{K}_{t}=$ (theoretical maximum stress at notch) /(nominal stress).

TABLE 9-Hardness Specifications

\begin{tabular}{lc}
\hline \multicolumn{1}{c}{ Test } & Hardness \\
\hline Rockwell B & 98 \\
Rockwell 15T & 92 \\
Rockwell 30T & 80 \\
Rockwell 15N & 68 \\
Brinell (500 kg load) & 158 \\
Brinell (3,000 kg load) & 200 \\
\hline
\end{tabular}

TABLE 10-Compressive Strength $\left(10^{3} \mathrm{psi}\right)(16)^{*}$

\begin{tabular}{lc}
\hline \multicolumn{1}{c}{ Condition } & Compressive strength \\
\hline Room temperature & \\
As-rolled & 184.0 \\
Base-annealed & 183.0 \\
Beta-quenched & 174.4 \\
$260^{\circ} \mathrm{C}$ & \\
As-rolled & 115.2 \\
Base-annealed & 110.7 \\
\hline * Values are sverages of vacuum- and \\
inert-atmosphere-arc-melted Zircaloy-2 at \\
25\% atrain.
\end{tabular}

TABLE 11 -Tensile Specifications for Rolled Sheet, $600^{\circ} \mathrm{F*}$

\begin{tabular}{lccc}
\hline Tensile & $\begin{array}{c}\text { Yield } \\
\text { strength, } \\
0.2 \% \\
\text { of test }\end{array}$ & $\begin{array}{c}\text { offet } \\
\left(10^{3} \mathrm{psi}\right)\end{array}$ & $\begin{array}{c}\text { Elongation } \\
\text { in } \text { \& in. } \\
(\%)\end{array}$ \\
\hline Longitudinal & 31 & 15 & 24 \\
Transverse & 29 & 18.4 & 35
\end{tabular}

* Annealed for $15 \mathrm{~min}$ at $843^{\circ} \mathrm{C}$, air-cooled. All values are minimum acceptable.
TABLE 7-Torsional Properties (13)

\begin{tabular}{cccc}
\hline & $\begin{array}{c}\text { Torsional elastic } \\
\text { modulus } \\
\left(10^{6} \boldsymbol{p s i}\right)\end{array}$ & $\begin{array}{c}\text { Torsional } \\
\text { proportional } \\
\text { limit }(p s i)\end{array}$ & $\begin{array}{c}\text { Torsional } \\
0.2 \% \text { yield } \\
\text { stress }(p s i)\end{array}$ \\
\hline BA & 5.24 & 17,850 & 29,800 \\
BA & 5.29 & 19,450 & 32,100 \\
HR & 5.22 & 13,550 & 29,500 \\
HR & 5.22 & 13,700 & 28,300
\end{tabular}

* BA = base-annealed; HR = hot-rolled.

TORSION. In Table 7, duplicate specimens were evaluated in each case. Bear in mind that base-annealing removes hydrogen from the material (nominally from $50 \mathrm{ppm}$ as hot-rolled to $10 \mathrm{ppm}$ as base-onnealedi.

TABLE 12-Elastic Properties in Tension (11)

\begin{tabular}{|c|c|c|c|}
\hline Condition & $\begin{array}{c}\text { Test } \\
\text { temperature } \\
\left({ }^{\circ} \mathrm{C}\right)\end{array}$ & $\begin{array}{c}\text { Elastic } \\
\text { modulus } \\
\left(10^{6} \text { p8i) }\right.\end{array}$ & $\begin{array}{c}\text { Poisson's } \\
\text { ratio }\end{array}$ \\
\hline \multirow[t]{2}{*}{ Annealed } & 27 & $13.9-14.3$ & $0.368-0.380$ \\
\hline & 150 & $13.3-13.8$ & $0.425-0.460$ \\
\hline \multirow[t]{2}{*}{ Cold-worked } & 27 & 14.5 & $0.382-0.406$ \\
\hline & 150 & $13.9-14.2$ & $0.392-0.432$ \\
\hline
\end{tabular}

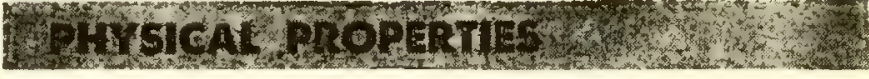

\section{TABLE 13-Physical Properties of Zircaloy-2}

\section{Density (17)}

At $20^{\circ} \mathrm{C}, 6,570 \mathrm{gm} / \mathrm{cm}^{3} \pm 0.0006$ (hot-rolled, vacuum-annealed sampled from eight vacuum-melted ingots)

Electrical resistivity $(1)$

At $21^{\circ} \mathrm{C}, 74 \times 10^{-1} \mathrm{ohm}-\mathrm{cm}\left(29 \times 10^{-1} \mathrm{ohm}\right.$-in. $)$

Thermal conductivity ( 3 )

$\begin{array}{cc}{ }^{\circ} \mathrm{C} & \mathrm{cal} / \mathrm{sec} / \mathrm{cm}^{2} / \\ { }^{\circ} \mathrm{C} / \mathrm{cm} \\ 20 & 0.0279 \\ 100 & 0.0287 \\ 200 & 0.0294 \\ 300 & 0.0306 \\ 400 & 0.0310 \\ 500 & 0.0322\end{array}$

watts $/ \mathrm{cm}^{2} /$
${ }^{\circ} \mathrm{C} / \mathrm{cm}$
0.117
0.120
0.123
0.128
0.130
0.135

$B T U / h r / f^{3} /$

${ }^{\circ} \mathrm{F} / \mathrm{ft}$

6.8

6.9

7.1

7.4

7.5

Coefficient of thermal expansion (1)

Alpha $=6.5 \times 10^{-6} \mathrm{in} . / \mathrm{in} . /{ }^{\circ} \mathrm{C}$, room temperature to $700^{\circ} \mathrm{C}$ Alpha-beta phase transformation temperature limits (vacuum-melted material) (18)

On heating: $815^{\circ}-830^{\circ} \mathrm{C}(\alpha / \alpha+\beta) ; 975^{\circ}-995^{\circ} \mathrm{C}(\beta / \alpha+\beta)$ On cooling: $770^{\circ}-785^{\circ} \mathrm{C}(\alpha / \alpha+\beta) ; 930^{\circ}-960^{\circ} \mathrm{C}(\beta / \alpha+\beta)$ 

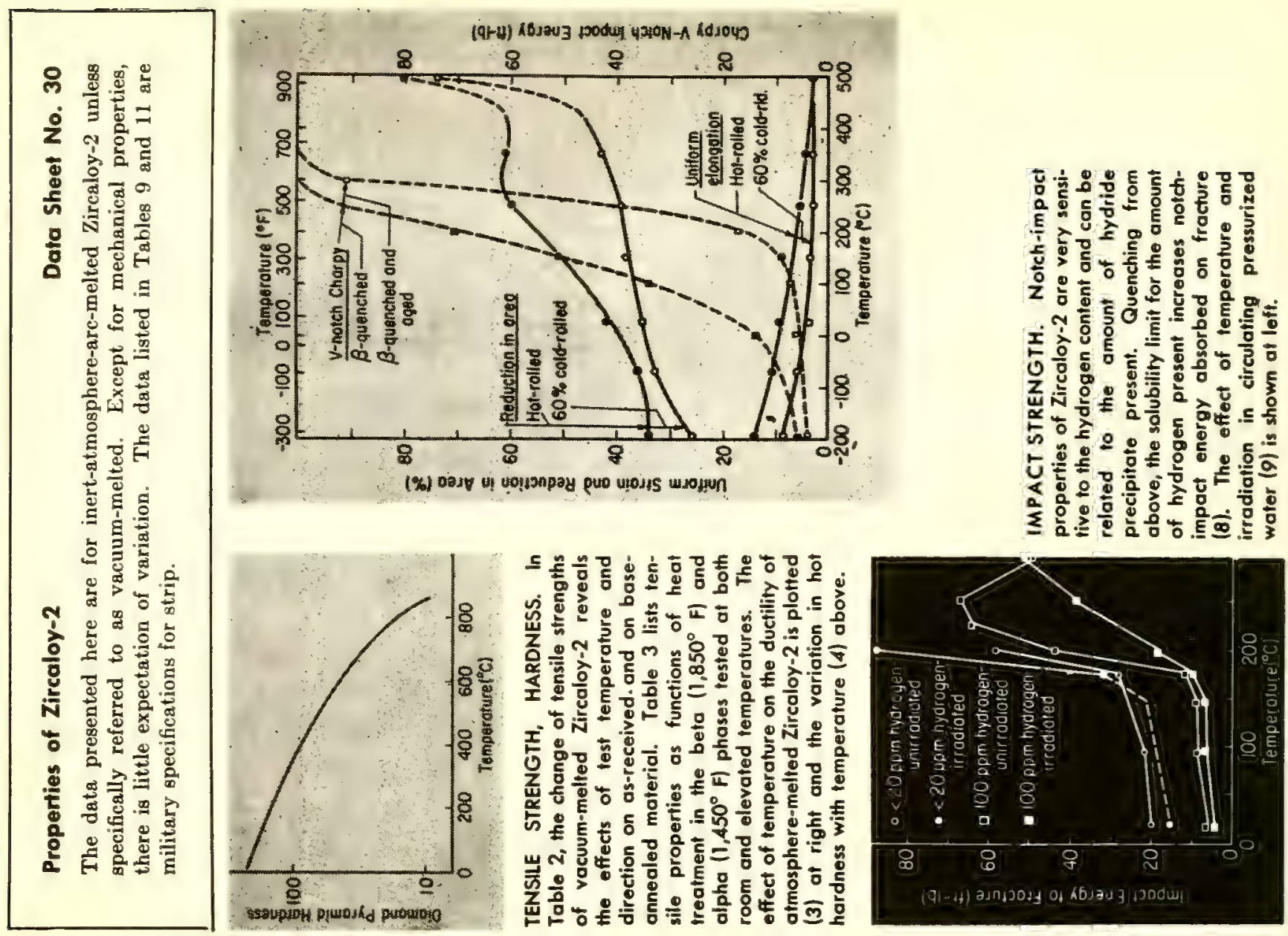

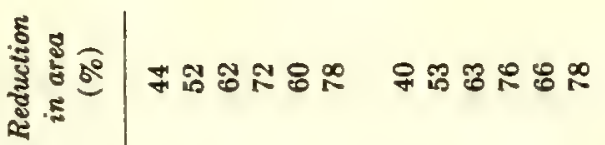

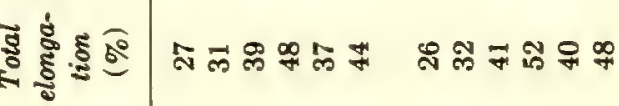

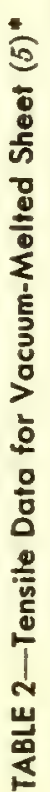

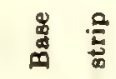

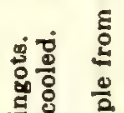

要

这芯

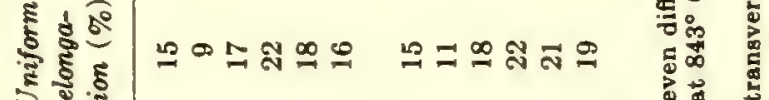

踇

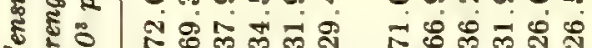

$\rightarrow$ क

ชิำ

sำ

昰

क्षे हैं

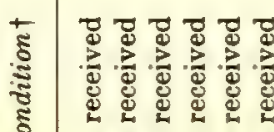

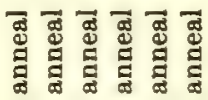

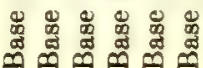

\%ั के

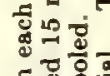

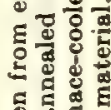

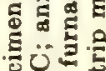

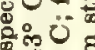

象是

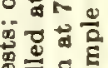

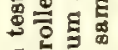

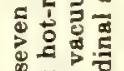

$\div$ \%

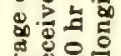

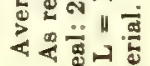

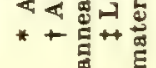

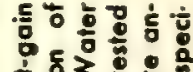

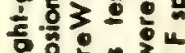

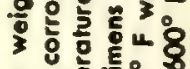

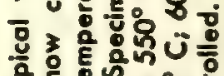

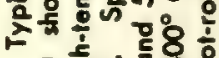

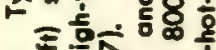

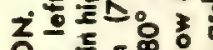
음흐통영 每

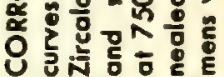

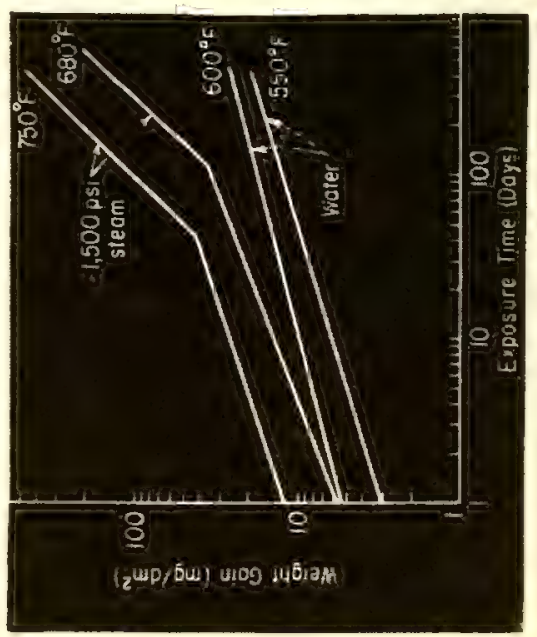



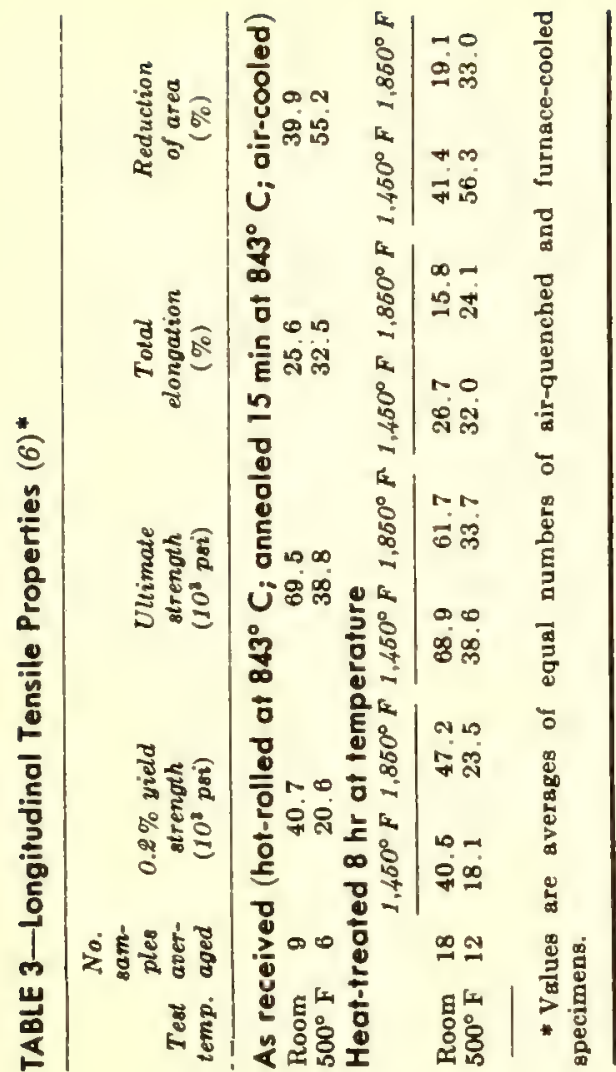

TABLE 4-Creep Properties (10)

\begin{tabular}{|c|c|c|c|c|c|c|c|}
\hline \multirow[b]{2}{*}{$\begin{array}{c}\text { Test } \\
\text { temp. } \\
\left({ }^{\circ} \mathrm{C}\right)\end{array}$} & \multirow[b]{2}{*}{$\begin{array}{c}\text { Stress } \\
\left(10^{3} \mathrm{psi}^{2}\right)\end{array}$} & \multirow{2}{*}{$\begin{array}{c}\text { Plastic } \\
\text { strain on } \\
\text { loading } \\
(\%)\end{array}$} & \multirow[b]{2}{*}{$\begin{array}{l}\epsilon_{0}^{*} \\
(\%)\end{array}$} & \multirow[b]{2}{*}{ (in./in./hr) } & \multicolumn{2}{|c|}{ Rupture } & \multirow{2}{*}{$\begin{array}{c}\text { Reduc- } \\
\text { tion } \\
\text { in area } \\
(\%)\end{array}$} \\
\hline & & & & & $\begin{array}{c}\epsilon^{*} \\
(\%)\end{array}$ & $\begin{array}{c}\text { Time } \\
(h r)\end{array}$ & \\
\hline \multicolumn{8}{|c|}{ As received } \\
\hline 250 & 59.0 & 0.27 & 1.02 & $8.3 \times 10^{-4}$ & 7.64 & 38.2 & 45.7 \\
\hline 250 & 56.0 & 0.23 & 1.23 & $7.1 \times 10^{-5}$ & 7.56 & 468 & 43.9 \\
\hline 250 & 53.0 & 0.25 & 0.80 & $1.2 \times 10^{-6}$ & & $\S$ & \\
\hline 250 & 50,0 & 0.08 & 0.375 & $5.0 \times 10^{-7}$ & & $\S$ & \\
\hline 350 & 51.3 & 0.50 & 0.95 & $2.3 \times 10^{-3}$ & 14.1 & 2 & 53.6 \\
\hline 350 & 45.0 & 0.26 & 0.76 & $3.5 \times 10^{-8}$ & 14.0 & 14.5 & 58.9 \\
\hline 350 & 41.0 & 0.05 & 0.52 & $5.0 \times 10^{-5}$ & 19.2 & 1,087 & 57.0 \\
\hline 350 & 37.5 & 0.02 & 0.40 & $1.6 \times 10^{-6}$ & $15.4+$ & $3,525+8$ & \\
\hline 350 & 35.0 & 0.02 & 0.79 & $6.2 \times 10^{-6}$ & & 8 & \\
\hline 350 & 32.0 & 0.01 & 0.268 & $2.2 \times 10^{-6}$ & & 8 & \\
\hline \multicolumn{8}{|c|}{ Base anneal $20 \mathrm{hr}$ at $750^{\circ} \mathrm{C}$ in vacuum } \\
\hline 250 & 32.0 & 4.67 & & $5 \times 10^{-2}$ & 26.6 & 2.5 & 71.6 \\
\hline 250 & 30.0 & 2.35 & 11 & $2 \times 10^{-6} \dagger$ & & 8 & \\
\hline 250 & 28.0 & 1.53 & 4.0 & $2 \times 10^{-8}+$ & & $\S$ & \\
\hline 250 & 26.0 & 1.859 & 2.55 & $1 \times 10^{-7}$ & & I & \\
\hline \multirow[t]{2}{*}{350} & 27.0 & 5.0 & 13.0 & $4.9 \times 10^{-4}$ & & & \\
\hline & & & 12.4 & $6.2 \times 10^{-1}$ & 21.55 & 88 & 66 \\
\hline 350 & 25.0 & 3.39 & 6.7 & $2.3 \times 10^{-6}$ & & 8 & \\
\hline 350 & 23.0 & 2.09 & & \pm & & q & \\
\hline \multicolumn{8}{|c|}{ Beta quenched $+10 \%$ cold work } \\
\hline 250 & 57.5 & 0.25 & 0.65 & $1.6 \times 10^{-4}$ & 4.9 & 69 & 54 \\
\hline 250 & 56.0 & 0.10 & 0.25 & $3.5 \times 10^{-6}$ & & $\dagger$ & \\
\hline 350 & 51.0 & 0.06 & 0.71 & $1.9 \times 10^{-4}$ & 7.86 & 287 & 52 \\
\hline \multicolumn{8}{|c|}{ Base anneal $+10 \%$ cold work } \\
\hline 250 & 46.0 & 0.08 & 0.48 & $1.15 \times 10^{-2}$ & 6.18 & 14 & 60 \\
\hline
\end{tabular}

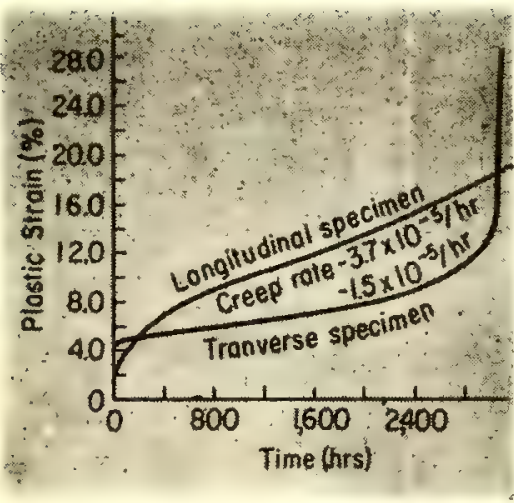

CREEP. Creep properties are given in Table 4 (10). Typical behavior in the rolled product as a function of specimen orientation $(8)$ is indicated at left (conditions: 27,000 psi, $343^{\circ} \mathrm{C}$ ). Creep strength of annealed Zircaloy-2 decreased rapidly with increasing temperature in the range $150^{\circ}-500^{\circ} \mathrm{C}$, the maximum temperature investigated. In Table 5, the stress-rupture-strength properties ore summarized (11).
TABLE 5-Summary of Siress-Ruplure-Strength Properties (11)

\begin{tabular}{lccc} 
& \multicolumn{3}{c}{ Stress to produce rupture in indicated time $\left(10^{2} \mathrm{psi}\right)$} \\
\cline { 2 - 4 }$\left({ }^{\circ} \mathrm{C}\right)$ & $1 \mathrm{hr}$ & $10 \mathrm{hr}$ & $100 \mathrm{hr}$ \\
\hline Annealed sheet, transverse direction & & \\
150 & 35.2 & 32.5 & 30.0 \\
230 & 28.0 & 26.0 & 24.0 \\
345 & 22.2 & 22.0 & 21.8 est. \\
425 & 21.3 & 19.0 & 16.8 \\
500 & 17.7 & 15.1 & 11.4 \\
Cold-worked sheet, transverse direction & & \\
150 & 73.0 & 70.5 & 68.0 \\
230 & 63.0 & 60.5 & 58.0 \\
345 & 50.0 & 46.0 & 43.0 \\
425 & 38.0 & 34.0 & 29.0 \\
500 & 27.5 & 21.5 & 14.5 \\
\hline
\end{tabular}


NOTES 
NOTES 
NOTES 



